\title{
Abstracts of the 16th International E-Congress of the European Geriatric Medicine Society
}

7-9 October 2020

\section{Contents}

Oral Communications on COVID-19

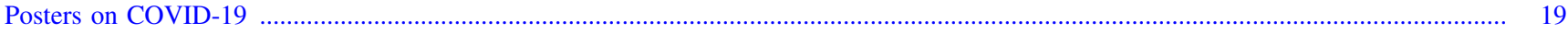

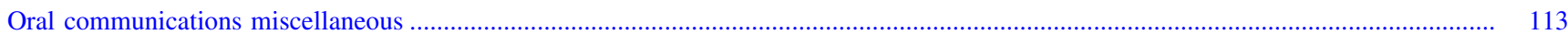

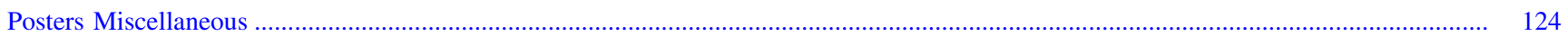




\section{Oral Communications on COVID-19}

\section{Abstract \# 1 \\ Clinical characteristics and outcomes of 821 older patients with SARS-Cov-2 infection admitted to acute care geriatric wards : a multicenter retrospective cohort study}

Lorè Zerah ${ }^{1}$, Édouard Baudouin ${ }^{2}$, Marion Pépin ${ }^{3}$, Morgane Mary ${ }^{4}$, Sébastien Krypciak ${ }^{5}$, Céline Bianco ${ }^{6}$, Swasti Roux ${ }^{7}$, Ariane Gross ${ }^{8}$, Charlotte Toméo ${ }^{2}$, Nadège Lemarié ${ }^{9}$, Antoine Dureau ${ }^{10}$, Sophie Bastiani $^{11}$, Flora Ketz ${ }^{12}$, Clémence Boully ${ }^{13}$, Cédric de Villelongue ${ }^{14}$, Mouna Romdhani ${ }^{15}$, Marie-Astrid de Soutter ${ }^{3}$, Emmanuelle Duron ${ }^{4}$, Jean-Philippe David ${ }^{16}$, Caroline Thomas ${ }^{17}$, Elena Paillaud ${ }^{18}$, Pauline de Malglaive ${ }^{8}$, Eric Bouvard ${ }^{9}$, Mathilde Lacrampe ${ }^{10}$, Elise Mercadier $^{11}$, Alexandra Monti ${ }^{12}$, Olivier Hanon ${ }^{13}$, Virginie FosseyDiaz $^{14}$, Laurianne Bourdonnec ${ }^{15}$, Bruno Riou ${ }^{19}$, Hélène Vallet ${ }^{6}$, Jacques Boddaert ${ }^{20}$

${ }^{1}$ 1. Assistance Publique-Hôpitaux de Paris (APHP), Hôpital La PitiéSalpêtrière, Department of Geriatric Medicine, 75013, Paris, France 2. Sorbonne Université, INSERM, Institut Pierre Louis

d'Épidémiologie et de Santé Publique, UMRS INSERM 1136, 75646, Paris, France, ${ }^{2} 1$. Assistance Publique-Hôpitaux de Paris (APHP), Hôpital La Pitié-Salpêtrière, Department of Geriatric Medicine, 75013, Paris, France, ${ }^{3} 3$. APHP, Hôpital Ambroise Paré, Department of Geriatric Medicine, 92100, Boulogne Billancourt, France 4. Université de Versailles Saint-Quentin en Yvelynes (UVSQ), Université Paris-Saclay, INSERM, CESP, 94800, Villejuif, France, ${ }^{4} 4$. Université de Versailles Saint-Quentin en Yvelynes (UVSQ), Université Paris-Saclay, INSERM, CESP, 94800, Villejuif, France 5. APHP, Hôpital Paul Brousse, Department of Geriatric Medicine, 94800, Villejuif, France, ${ }^{5} 6$. APHP, Hôpitaux Henri Mondor, Department of Geriatric Medicine, 94000, Créteil, France 7. Université Paris Est Creteil, INSERM, IMRB, 94000, Creteil, France, ${ }^{6} 8$. APHP, Hôpital Saint-Antoine, Department of Geriatric Medicine, 75012, Paris, France 9. Sorbonne Université, INSERM, UMRS 1135, Centre d'immunologie et de Maladies Infectieuses (CIMI), 75013, Paris, France, ${ }^{7} 10$. APHP, Hôpital Corentin Celton, Department of Geriatric Medicine, 97132, Issy les Moulineaux, France, ${ }^{8} 11$. APHP, Université de Paris, Hôpitaux Bichat-Beaujon, Department of Geriatric Medicine, 92110, Clichy, France, ${ }^{9} 12$. APHP, Sorbonne Université, Hôpital Tenon, Department of Geriatric Medicine, 75020, Paris, France, ${ }^{10} 13$. APHP, Sorbonne Université, Hôpital Rothschild, Department of Geriatric Medicine, 75012, Paris, France, ${ }^{11} 14$. APHP, Université Paris Sud, Hôpital Béclère, Department of Geriatric Medicine, 92140, Clamart, France, ${ }^{12} 15$. APHP, Sorbonne Université, Hôpital Charles Foix, Department of Geriatric Medicine, 94205, Ivry sur Seine, France, ${ }^{13} 16$. APHP, Hôpital Broca, Department of Geriatric Medicine, 75013, Paris, France 17. Université ParisDescartes, Sorbonne Paris-Cité, 75006, Paris, France, ${ }^{14} 18$. APHP, Université de Paris, Hôpital Bretonneau, Department of Geriatric Medicine and Palliative Care, 75018, Paris, France, ${ }^{15} 19$. APHP, Université de Paris, Hôpital Bretonneau, Department of Psychogeriatric Medicine, 75018, Paris, France, ${ }^{16} 6$. APHP, Hôpital Henri Mondor, Department of Geriatric Medicine, 94000, Créteil, France, ${ }^{17} 8$. APHP, Hôpital Saint-Antoine, Department of Geriatric Medicine, 75012, Paris, France, ${ }^{18} 7$. Université Paris Est Creteil, INSERM, IMRB, 94000, Creteil, France 20. APHP, Paris Cancer Institute CARPEM, Department of Geriatric Medicine, Hôpital Européen Georges Pompidou, 75015, Paris, France, ${ }^{19} 21$. Sorbonne Université, UMRS INSERM 1166, 75013, Paris, France 22. APHP, Hôpital La Pitié-Salpêtrière, Department of Emergency Medicine, 75013, Paris, France, ${ }^{20} 1$. Assistance Publique-Hôpitaux de Paris (APHP), Hôpital La Pitié-Salpêtrière, Department of Geriatric Medicine, 75013, Paris, France 9. Sorbonne Université, INSERM,
UMRS 1135, Centre d'immunologie et de Maladies Infectieuses (CIMI), 75013, Paris, France

Background: There is limited information describing the characteristics and outcomes of hospitalized older patients with confirmed coronavirus disease 2019 (COVID-19).

Methods: We conducted a multicentric retrospective cohort study in 13 acute COVID-19 geriatric wards, from March 13 to April 15, 2020 , in Paris area. All consecutive patients aged $\geq 70$ years, with confirmed COVID-19, were enrolled.

Results: Of the 821 patients included in the study, the mean (SD) age was 86 (7) years; $58 \%$ were female; $85 \%$ had $\geq 2$ comorbidities; $29 \%$ lived in an institution; and the median (interquartile range) Activities of Daily Living Scale (ADL) score was 4 [2-6]. The most common symptoms at COVID-19 onset were asthenia (63\%), dyspnea (45\%), dry cough (45\%), fever (45\%) and delirium (25\%). The in-hospital mortality was $31 \%$ (95\% confidence interval [CI] 27-33). On multivariate analysis, at COVID-19 onset, the probability of in-hospital mortality was increased with male gender (odds ratio [OR], 1.85; 95\% CI 1.30-2.63), ADL score < 4 (OR, 1.84; 95\% CI 1.25-2.70), asthenia (OR 1.59; 95\% CI 1.08-2.32), quick Sequential Organ Failure Assessment score $\geq 2$ (OR, 2.63; 95\% CI 1.64-4.22) and specific COVID-19 anomalies on chest computerized tomography (OR, 2.60; 95\% CI 1.07-6.46).

Conclusions: This study provides new information about older patients with COVID-19 who are hospitalized. A quick bedside evaluation at admission of sex, functional status, systolic arterial pressure, consciousness, respiratory rate and asthenia can identify older patients at risk of unfavorable outcomes.

\section{Abstract \# 2 \\ Prognostic value of sleep disturbances in COVID-19 patients Giulia Cesaroni ${ }^{1}$, Francesca Mazzeo ${ }^{2}$, Flaminia Coccia ${ }^{2}$, Renzo Rozzini $^{1}$ \\ ${ }^{1}$ Fondazione Poliambulanza Istituto Opsedaliero, ${ }^{2}$ Fondazione Poliambulanza Istituto Ospedaliero}

Introduction: The major complications of COVID-19 are respiratory, renal and cardiac. Neurological symptoms are also described, such as agitation, confusion and sleep disturbances [1-2]. Aim of this study is to evaluate sleep disorders in a large group of hospitalized patients with respiratory failure due to COVID-19 and assess their potential association with prognosis [3].

Method: During the period between March 1 and April 30 2020, 1337 patients affected by COVID-19 [4] were admitted to our hospital (Fondazione Poliambulanza Istituto Ospedaliero, Brescia-Italy). Sleep difficulties were reported by nurses on the electronic records of the ward; on the basis of the severity of sleep disturbances physicians prescribed appropriate therapy (i.e. benzodiazepines), using a standard protocol. For the purposes of this study, we reported the presence of sleep disturbances when they required drug treatment.

Results: Among 1337 patients, 20.4\% had sleep disturbances. Sleep disturbances were higher in older patients $(9.5 \%, 20.1 \%, 27.1 \%$ respectively in $<60,61-70,71-80,>81$ years old) and in severe infection. Sleep disturbances were associated with in-hospital mortality also after controlling for potential confounders (SARS-CoV-2 infection severity, sex, age and age-related diseases, i.e. coronary heart diseases and CKD) (HR: 2.1, CI 95\% 1.6-2.7).

Conclusion: Our data show that sleep disturbances in hospitalized COVID-19 patients is associated in hospital mortality. The reasons of the association is not completely known: nevertheless insomnia should be considered a poor prognostic marker in COVID-19 patients. 


\section{Abstract \# 3}

Effectiveness and security of baricitinib in COVID-19 hospitalized older adults with pneumonia

Borja Gil García ${ }^{1}$, Ana Pérez Fernández-Rius ${ }^{1}$, Laura Plaza Carmona $^{1}$, Belén Roldán García ${ }^{1}$, Rubén Alcantud Córcoles ${ }^{1}$, Rita López Bru ${ }^{1}$, Victoria Sánchez-Flor Alfaro ${ }^{1}$, David Caldevilla Bernardo $^{2}$, Almudena Avendaño Céspedes ${ }^{1}$, Marta Mas Romero ${ }^{1}$, Rafaela Sánchez Simón-Talero ${ }^{3}$, Lourdes Sáez Méndez ${ }^{4}$, Luis Romero Rizos ${ }^{1}$, Ginés Sánchez Nievas ${ }^{5}$, Pedro Abizanda Soler ${ }^{1}$

${ }^{1}$ Department of Geriatrics, Complejo Hospitalario Universitario of Albacete, Albacete, Spain, ${ }^{2}$ Department of Radiology, Complejo Hospitalario Universitario of Albacete, Albacete, Spain, ${ }^{3}$ Department of Neumology, Complejo Hospitalario Universitario de Albacete, Albacete, Spain, ${ }^{4}$ Department of Internal Medicine, Complejo Hospitalario Universitario of Albacete, Albacete, Spain, ${ }^{5}$ Department of Rheumatology, Complejo Hospitalario Universitario of Albacete, Albacete, Spain

Introduction: Older adults are the population at the highest risk for Covid-19 adverse outcomes. This work describes the outcomes after the use of Baricitinib as a COVID-19 treatment in older adults with pneumonia.

Methods: Post-authorization retrospective study. The COVID-AGE study included all patients with an age 69 or older hospitalized from March 9 th, until April 20 th $2020(\mathrm{n}=576)$ with Covid in an older adults Covid Unit. In this work we included 59 patients treated with Baricitinib, among the sample of 576, describing the results in terms of mortality, health outcomes and adverse effects.

Results: Mean age was 80.2 years (SD 5.8), and 37.3\% were female. Mean time from hospitalization to baricitinib initiation was 6.9 days, and mean treatment days were 4.4 days. 46 patients received 3 or more doses, with a mean total dose of $10.6 \mathrm{mg}$ (SD 6.5). Baricitinib use at any dose was associated with lower mortality during hospitalization, $23.7 \%$ vs $36.3 \%$ ( $p=0.056$ ). Furthermore, the patients that received at least three baricitinib doses had a significant decrease in crude mortality $(15.2 \%$ vs $36.7 \%$; $p=0.003)$, and a lower mortality risk adjusted by age, OR $0.33(95 \%$ CI $0.15-0.76 ; \mathrm{p}=0.009)$. In this group, 28 patients $(60.9 \%)$ presented improvement in chest $\mathrm{X}$-ray. Reduction in inflammatory biomarkers after the treatment was detected in this last group. 13 of the 59 patients $(22 \%)$ presented adverse outcomes, being the most frequent cardiovascular, infections and gastrointestinal.

Key conclusions: Baricitinib is effective and safe for older adults hospitalized for Covid-19 pneumonia.

\section{Abstract \# 4 \\ Clinical and baseline characteristics of octogenarian and nonagenarian patients with COVID-19: a descriptive study}

Javier Montero Muñoz ${ }^{1}$, Pablo Enrique Solla Suárez ${ }^{1}$, Cristina Guirola García-Prendes ${ }^{1}$, Francisco Jiménez Muela ${ }^{1}$, Laura Gómez Armas $^{1}$, Marta Martínez Rivera ${ }^{1}$, Alberto Domínguez Bravo ${ }^{1}$, José Gutiérrez Rodríguez ${ }^{1}$

${ }^{1}$ Área de Gestión Clínica de Geriatría, Hospital Monte Naranco, Oviedo, Asturias, Spain

Introduction: The elderly population is the most vulnerable to severe acute respiratory syndrome coronavirus 2 (SARS-CoV-2) infection. However, there are currently few observational studies focused on octogenarian and nonagenarian patients. The objective of this study is to describe clinical and baseline characteristics of very elderly patients hospitalized by coronavirus disease 2019 (COVID-19) in an Acute Geriatric Unit (AGU).
Methods: A cross-sectional study was carried out with data from patients older than 80 years admitted to an AGU with a laboratoryconfirmed diagnosis of COVID-19. The variables collected were: sociodemographic characteristics, baseline situation, symptoms, comorbidity and mortality rate.

Results: 58 patients were recruited. The mean age was $88.3 \pm 5.4$ years, $69 \%$ were women, $65.5 \%$ had moderate-severe cognitive impairment and mean Barthel Index was $40.6 \pm 36.70 .7 \%$ were institutionalized. The main symptoms were fever $(60.3 \%)$, dyspnea $(53.4 \%)$, deterioration of functional condition $(50 \%)$, cough $(41.4 \%)$ and delirium (19\%). The most frequent comorbidities were cardiovascular disease $(75.9 \%)$, hypertension $(74.1 \%)$, chronic kidney disease $(50 \%)$, dyslipidemia $(46.6 \%)$ and diabetes mellitus $(29.3 \%)$. The mortality rate was $41.4 \%$.

Key conclusions: Our almost nonagenarian cohort presents a majority of women, with functional dependence, cognitive impairment and predominance of cardiovascular comorbidities. The relatively high presence of atypical symptoms such as deterioration of functional condition or delirium highlights the value of not focusing exclusively on the typical COVID-19 symptoms (fever, dyspnea and cough) in the elderly patient, in whom the diagnosis may be delayed and challenging.

\section{Abstract \# 5}

Age and intensity of psychopathological symptoms in COVID-19 survivals

Natalia Kotovskaya ${ }^{1}$, Tatiana Manevich ${ }^{2}$, Alexander Rozanov ${ }^{2}$, Valentina Ostapenko ${ }^{2}$, Anna Schedrina ${ }^{2}$, Andrey Izyumov $^{2}$, Ksenia Eruslanova $^{2}$, Natalia Deryugina ${ }^{3}$, Yulia Kotovskaya ${ }^{2}$, Olga Tkacheva $^{2}$, Igor Belokrylov ${ }^{1}$

${ }^{1}$ RUDN University, Moscow, Russia, ${ }^{2}$ Pirogov RNMU, RCRCG, Moscow, Russia, ${ }^{3} \mathrm{MSU}$, Moscow, Russia

Introduction: The aim of the study was to investigate the severity of psychopathological symptoms in previously healthy patients of different age groups survived moderate-to-severe COVID-19 pneumonia.

Methods: Before the discharge from the hospital patients who gave informed consent filled in the Symptom Checklist-90-R designed to assess the 11 symptoms dimensions: somatization (SOM), obsessivecompulsive (O-S), interpersonal sensitivity (INT), depression (DEP), anxiety (ANX), hostility (HOS), phobic anxiety (PHOB), paranoid ideation (PAR), psychoticism (PSY). Cognitively impaired patients were not included into the study.

Results: The study involved 106 patients aged 26-84 years: 44 (24 men, 20 women) $<65$ years (median age 55) and 62 (34 men, 28 women) patients $>65$ years (median age 78.5). In both groups, symptoms were detected on the scales of SOM, DEP, ANX, HOS, PAR, and no symptoms on PSY and PHOB scales were reported. Despite a similar pattern of symptoms, most of them tended to be more pronounced in $65+$ patients vs $<65$ years. Older vs younger patients had insignificantly lower score on SOM (0.96 (IQR 0.44) vs 0.89 (IQR 0.25) and PAR 0.22 (IQR 0.11) vs 0.24 (IQR 0.17), and higher score on DEP 0, 53 (IQR 0.26) vs 0.45 (IQR 0.35) and ANX 0.52 (IQR 0.25 ) vs 0.39 (IQR 0.26 ), and significantly higher scores on HOS 0.58 (IQR 0.55) vs 0.31 (IQR $=0.25), \mathrm{p}=0.02$, and $\mathrm{O}-\mathrm{S} 0.60$ (IQR 0.35 ) vs 0.33 (IQR 0.21), $\mathrm{p}=0.01$.

Conclusions: The results suggest that survivors after moderate-tosevere COVID-19 pneumonia potentially need further psychological rehabilitation and psychiatric assessment. 


\section{Abstract \# 6 \\ Typical and atypical presenting symptoms of COVID-19 in elderly patients}

Quoc Duy Nghiem ${ }^{1}$, Héloïse Keravec ${ }^{2}$, Antonio Rainone ${ }^{1}$, Sihem Oukbir-Ferrag $^{1}$, Cristiano Donadio ${ }^{1}$, Bruno Oquendo $^{1}$, Carmelo Lafuente-Lafuente $^{1}$, Joël Belmin ${ }^{1}$

${ }^{1}$ APHP, Sorbonne Université, Hôpital Charles Foix, Ivry-sur-Seine, France, ${ }^{2}$ APHP, Sorbonne Université, Hôpital Rothschild, Ivry-surSeine, France

Background: Few data is available regarding the clinical presentation of COVID-19 in elderly patients.

Methods: In March 2020, COVID-19 clusters emerged in three geriatric rehabilitation units located in two hospitals, in Paris, France. We followed all patients from these units who developed the disease and recorded any new symptom existing at diagnosis, or occurring in the previous 14 and the following 21 days. A group of SARS-Cov-2negative patients served as controls.

Results: SARS-Cov-2 was detected by PCR in the nasopharyngeal swab of 69 patients (mean age: 86 years), of whom $6(8.7 \%)$ were completely asymptomatic. Among symptomatic patients, at the moment of diagnosis a majority had fever $(71.0 \%)$, malaise-asthenia $(24.6 \%)$ or respiratory symptoms $(66.7 \%)$. In addition, many patients had presented other symptoms in previous days, notably: (a) gastrointestinal $(39.1 \%$, median onset 8 days before diagnosis, IQR -9 to +3 days), including diarrhea $(34.8 \%)$ and nausea-vomiting $(11.6 \%)$; (b) neurological symptoms $(30.4 \%$, median onset 5 days before, IQR -11 to -3 ), including delirium (24.6\%) or new behavioural disturbances $(7.2 \%)$; and (c) others symptoms $(29.0 \%$, median onset 4 days before, IQR -10 to 0 ), like falls (15.9\%) and unexplained decompensation of heart failure (13.0\%). None of those symptoms were observed in similar proportion in 25 control patients, hospitalized during the same period, who tested negative for SARS-

CoV-2.

Conclusions: Less typical symptoms, particularly diarrhea, nauseavomiting, delirium, falls and unexplained decompensation of chronic conditions, are frequent in elderly patients with COVID-19 and tend to appear early, several days before more typical symptoms.

\section{Abstract \# 7 \\ Frailty in COVID-19 geriatric patients: a descriptive study \\ Gonçalo Pereira ${ }^{1}$, Tania Fontainha ${ }^{2}$, Domingas Atouguia ${ }^{3}$, Marilia Fernandes $^{3}$, INes Rego Figueiredo ${ }^{3}$, Heidi Gruner ${ }^{3}$, Antonio Panarra ${ }^{3}$ \\ ${ }^{1}$ Nova Medical School, Universidade Nova de Lisboa, ${ }^{2}$ Nova Medical School, Universidade Nova de Lisboa, ${ }^{3}$ Serviço de Medicina 7.2, Hospital Curry Cabral, Centro Hospitalar Universitário de Lisboa Central}

Introduction: Current Covid-19 guidelines for geriatric patients include frailty assessment, using the Clinical Frailty Scale (CFS), in the decisional algorithms for the allocation of resourcesand treatments. However, more studies are needed evaluating different frailty assessmentscales, such as PRISMA-7, and selected outcomes in geriatric Covid-19 patients.

Methods: A descriptive prospective study of the geriatric patients admitted to a tertiary hospital during the COVID19 pandemia from 11 march to 31 june was performed. Information regarding age, sex, comorbidities, number of drugs taken daily and clinical evolution during hospital stay was collected from the clinical file The CFS and PRISMA-7 score was determined for each patient. A patient was considered frail when $\mathrm{CFS} \geq 5$ or PRISMA $\geq 3$.
Results: Of a total of 151 patients, 72 patients were included in this study. The average age was 84 years, $58 \%$ were females, they had an average of 2.8 days of symptoms until being admitted to Hospital. The average number of comorbidities was 5.5 and average number of drugstaken by the aptients was 7.2, the average length of stay 11.32 days and mortality was $8 \%$ (12 patients), 9 were admitted in the ICU. Concerning frailty $71 \%$ had a PRISMA $\geq 3$ and $54 \%$ CFS $\geq 5$. The number of frail patients increased with age. Concerning frail vs. nonfrail patients, no differences were found in the average number of drugs taken, the average number of comorbidities presen $t$ and the average number of symptomatic days until hospital admission.

Discussion: Use of frailty scores in the elderly at admition in the emergency department is essential. New and easy to aplly scores are needed.

\section{Abstract \# 8}

COVID-19: risk factors for severe disease and death in patients aged 70 and over: a retrospective study

Katleen Fagard ${ }^{1}$, Els Devriendt ${ }^{1}$, Evelien Gielen ${ }^{1}$, Johan Flamaing ${ }^{1}$

${ }^{1}$ Dpt of Geriatric Medicine, University Hospitals Leuven, Leuven, Belgium

Introduction: The COVID-19 pandemic called for a rapid reorganization of hospital care. In our hospital, the clinical frailty scale (CFS) was introduced as a result of these reorganisations. A retrospective analysis was performed to investigate whether typical geriatric risk factors, such as frailty, have an added value in addition to conventional risk factors in predicting severe COVID-disease.

Methods: In patients aged 70 years and over, the family was contacted by telephone and asked to complete an online geriatric assessment questionnaire, from which the CFS was scored by the geriatric team. Additional data were collected from the electronic medical records. Baseline characteristics were described with descriptive statistics. Associations were analysed with univariable analyses.

Results: One hundred and five patients were included, mean age 81.9 ( \pm 7.19$)$ years. CFS scores were $1-3$ in 28 patients, $4-6$ in 60 patients, and 7-9 in 17 patients. Univariable analysis showed age, CFS, Charlson Comorbidity Index (CCI), age adjusted CCI (ACCI) and cognitive decline associated with in-hospital mortality. Male gender, obesity, cardiovascular disease, chronic pulmonary disease, diabetes, cancer and hypertension were not significantly associated. Male gender, presence of respiratory symptoms at admission, and COPD were associated with high early warning scores (EWS $\geq 7$ ). The first two were associated with high oxygen need ( $\geq 6$ litres).

Conclusion: Through action of the geriatric team at the time of rapid changes in the hospital, the frailty concept was introduced in the COVID-hospitalization units. A retrospective analysis shows that geriatric risk factors exceed conventional risk factors for severe COVID-disease and death.

\section{Abstract \# 9}

Prevalence of delirium among old and frail patients admitted to hospital with Covid-19

Håkon Ihle-Hansen ${ }^{1}$, Marius Myrstad ${ }^{1}$

${ }^{1}$ Medical department and department of medical research, Bærum Hospital, Vestre Viken Hospital Trust

Introduction: Delirium is a serious condition with high prevalence among acutely ill older patients admitted to hospital. In patients with Covid-19, delirium might complicate both treatment and adherence to 
isolation measures. Infections are among the most common precipitating factors for delirium, but the prevalence of delirium in patients admitted to hospital due to Covid-19 is unknown.

Methods: This was a prospective cohort study in a non-university hospital in Norway. All patients admitted during the 60 first days of the local Covid-19 outbreak, from March 9th to May 7th 2020 were consecutively included. We diagnosed delirium retrospectively based on information found in the patient records. We classified cases as delirium when all DSM-5 diagnostic criteria for delirium were fulfilled. Cases with delirium symptoms and evidence of acute onset of symptoms, but who did not fulfill all diagnostic criteria, were classified as subsyndromal delirium.

Results: In all, 52 patients aged $\geq 60$ years (mean age 76.1 years, 31 $(60 \%)$ men) were included. Mean Clinical Frailty Scale (CFS) score was $3.4,17$ patients $(33 \%)$ were frail (CFS score $\geq 5$ ). Out of these, $15(29 \%)$ had delirium and six $(12 \%)$ had subsyndromal delirium. The prevalence of delirium was $17 \%$ in patients aged 60-69 years, $25 \%$ in those aged $70-79$, and $44 \%$ in patients aged $\geq 80$ years. In frail patients, the prevalence of delirium was $53 \%$.

Key conclusions: Delirium was highly prevalent among patients aged $\geq 60$ years admitted to hospital with Covid-19. The prevalence increased with increasing age and frailty.

\section{Abstract \# 10 \\ (Un)preparedness of the health team in caring for the elder person during the COVID-19 pandemic}

Gildasio Souza Pereira ${ }^{1}$, Nildete Pereira Gomes ${ }^{2}$, Rute dos Santos Sampaio $^{3}$, Lélia Mendes Sobrinho de Oliveira ${ }^{1}$, Larissa Chaves Pedreira $^{1}$, Márcio Ribeiro Melhor ${ }^{4}$, Arianna Oliveira Santana Lopes ${ }^{1}$

${ }^{1}$ Escola de Enfermagem da Universidade Federal da Bahia, Brazil, ${ }^{2}$ Escola de Enfermagem da Universidade Federal da Bahia, Brazil, ${ }^{3}$ Departamento de Fisioterapia da Universidade Federal da Bahia, Brazil, ${ }^{4}$ Escola de Medicina da Universidade Federal da Bahia, Brazil

Introduction: The lack of qualification and adequate preparation of health professionals can lead to incorrect conduct, as well as generate health damages, especially for the elderly.

Objective: to report a case of professional unpreparedness in the assistance of an elder person in times of pandemic in Bahia, Brazil. Case report: Elder, female, 74 years old, hypertensive, diabetic and cardiac patient was taken by her children to the Emergency Care Unit for presenting respiratory distress. Upon arrival, without any further evaluation, the physician suspected COVID-19 and referred her to a Coronavirus Unit, where the nursing team promptly administered fluids. This generated a ventricular overload that precipitated a cardiac arrest for $10 \mathrm{~min}$, requiring orotracheal intubation and use of vasoactive drugs for 15 days. Initial tests were also performed, which resulted negative for COVID-19. She was tested again after 3 days, this time with results positive for COVID-19. The elder woman has remained hospitalized for 30 days, afebrile, sleepy, uncooperative and will have to undergo a tracheostomy. As she was not entitled to a companion during this period, the children were informed about the situation and the pacient did not have any psychological support provided by the hospital.

Conclusion: Regarding the coronavirus pandemic, many professionals are currently underprepared to act effectively, and, especially when concerning an elderly population, the need for a differential diagnosis increases, as COVID-19 is not the only condition the elderly may present.

Keywords: Aged; Coronavirus Infections; Pandemics; Patient Care Team.

\section{Abstract \# 11}

\section{The presenting features of COVID-19 in elderly patients}

Mark Vettasseri ${ }^{1}$, Jennifer Smith ${ }^{1}$, Sylvia Pytraczyk ${ }^{1}$, Jonathan Sheldrake $^{1}$, Bushra Muzammil ${ }^{1}$, Vivek Ramburuth ${ }^{1}$

${ }^{1}$ Nottingham University Hospitals

Introduction: Coronavirus disease (COVID-19) typically presents with respiratory symptoms and fever. However, as elderly patients can develop atypical symptoms with any disease, this project aimed to characterise the presenting features of COVID-19 in the elderly.

Methods: The clinical records of 95 patients with COVID-19 patients, admitted to a Care of the Elderly department of a UK Teaching Hospital were retrospectively reviewed. The primary presenting complaints, admission symptoms, biochemical and radiographic abnormalities were identified in each case.

Results: The median patient age was 82 years and $86 \%$ had a clinical frailty score of 6 or greater. $76 \%$ had positive COVID-19 throat swabs with the remainder diagnosed clinically by their attending physician. The common primary presenting complaints were dyspnoea (32\%), confusion (28\%), falls (23\%), fever (15\%), cough (12\%) and gastrointestinal upset $(11 \%)$. The commonly recorded symptoms during admission clerking were dyspnoea (47\%), hypoxia (43\%), cough $(37 \%)$ and fever $(35 \%)$. $21 \%$ had no respiratory symptoms at admission. Delirium was diagnosed in $46 \%$ of cases. $52 \%$ of patients had a lymphopaenia, $40 \%$ a CRP greater than $100 \mathrm{mg} / \mathrm{L}, 26 \%$ had acute kidney injury and $18 \%$ thrombocytopaenia.Bilateral interstitial changes (29\%) and focal consolidation (21\%) were the most frequent chest X-ray changes. $12 \%$ revealed bilateral ground glass changes. $21 \%$ of patients had no radiographic abnormalities on admission.

Key conclusions: While the majority of elderly patients presented with typical COVID-19 respiratory symptoms, other less characteristic symptoms and signs were also frequently identified. Clinicians must be mindful of atypical presentations to recognise COVID-19 in elderly patients.

\section{Abstract \# 12}

\section{Clinical presentation of COVID-19 in older people}

Jennifer S. Pigott ${ }^{1}$, Catherine Jackman ${ }^{1}$, Nitzan Lindenberg ${ }^{1}$, Lara Beaumont ${ }^{1}$, Samit Patel ${ }^{1}$, Kristi Sun ${ }^{1}$, Nicholas Bentley ${ }^{1}$, Bethan England $^{1}$, Ruth Law ${ }^{1}$, Kunal Shah ${ }^{1}$

${ }^{1}$ Whittington Health NHS Trust, London UK

Introduction: The COVID-19 global pandemic has disproportionately affected older and comorbid populations [1]. There have been early indicators that the clinical presentation in this population may not be typical of the disease more generally [2]. The discrepancy has implications for public health measures such as the UK 'test and trace' initiative.

Methods: Electronic health records were reviewed to collect data on all inpatients over 70 years of age at the Whittington Hospital, London, suspected of having COVID-19, between 3rd March and 22nd April $2020(\mathrm{n}=324)$. Demographic details, comorbidity and symptoms at presentation were recorded. Investigations were reviewed: blood test results (renal function and vitamin D), thoracic imaging and swab results for Sars-Cov-2 Polymerase Chain Reaction (PCR). Outcomes were recorded (death or discharge).

Results: 374 swabs were undertaken in the studied population, 183 of which were positive for Sars-Cov-2. $72 \%$ had typical symptoms of COVID-19, though many presented with frailty syndromes such as falls $(16 \%)$ or reduced levels of functioning $(21 \%)$, including reduced consciousness, mobility or oral intake. $34 \%$ had delirium at presentation or recorded on their discharge summary. A further 52 patients 
had imaging findings suggestive of COVID-19 despite negative PCR; of these 10 (19\%) had delirium.

Key conclusions: This study supports previous suggestions that many elderly patients with COVID-19 present with frailty syndromes such as falls and delirium, though the majority did report classic symptoms. The rates of delirium were higher in those with positive PCR than those with radiological diagnosis but negative PCR. Further data analysis is planned.

References:

1. Lithander FE, Neumann S, Tenison E, Lloyd K, Welsh T J, Rodrigues J C L, Higgins J P T, Scourfield L, Christensen H, Haunton V J, Henderson E J, COVID-19 in older people: a rapid clinical review, Age and Ageing, 2020.

2. D'Adamo H, Yoshikawa T, Ouslander JG. Coronavirus disease 2019 in geriatrics and Long-term care: the ABCDs of COVID-19. Journal of the American Geriatric Society, 2020.

\section{Abstract \# 13 \\ The presenting features of COVID-19 in elderly patients \\ Mark Vettasseri ${ }^{1}$, Jennifer Smith ${ }^{1}$, Sylvia Pytraczyk ${ }^{1}$, Jonathan Sheldrake $^{1}$, Mohammed Ahmed ${ }^{1}$, Bushra Muzammil ${ }^{1}$, Vivek Ramburuth ${ }^{1}$ \\ ${ }^{1}$ Nottingham University Hospitals}

Introduction: Coronavirus disease (COVID-19) typically presents with respiratory symptoms and fever. However, as elderly patients often develop atypical symptoms with any disease, this project aimed to characterise the presenting features of COVID-19 in the elderly.

Methods: The clinical records of 95 patients with COVID-19 patients, admitted to a Care of the Elderly department of a UK Teaching Hospital were retrospectively reviewed. The primary presenting complaints, admission symptoms, biochemical and radiographic abnormalities were identified in each case.

Results: The median patient age was 82 years and $86 \%$ had a clinical frailty score of 6 or greater.76\% had positive COVID-19 throat swabs. The remainder were diagnosed clinically by their attending physician. The common primary presenting complaints were dyspnoea (32\%), confusion $(28 \%)$, falls $(23 \%)$, fever $(15 \%)$, cough (12\%) and gastrointestinal upset (11\%). The commonly recorded symptoms during admission clerking were dyspnoea (47\%), hypoxia (43\%), cough $(37 \%)$ and fever $(35 \%)$. $21 \%$ had no respiratory symptoms at admission. Delirium was diagnosed in $46 \%$ of cases. $52 \%$ of patients had a lymphopaenia, $40 \%$ a CRP greater than $100 \mathrm{mg} / \mathrm{L}, 26 \%$ had acute kidney injury and $18 \%$ thrombocytopaenia.Bilateral interstitial changes $(29 \%)$ and focal consolidation $(21 \%)$ were the most frequent chest x-ray changes. $12 \%$ revealed bilateral ground glass changes. $21 \%$ of patients had no radiographic abnormalities on admission.

Key conclusions: While the majority of elderly patients presented with typical COVID-19 respiratory symptoms, other less characteristic symptoms and signs were also frequently identified. Clinicians must be mindful of atypical presentations to recognise COVID-19 in elderly patients.

\section{Abstract \# 14 \\ Delirium may be the only symptom in older adults with COVID- 19 disease}

Aine McGovern ${ }^{1}$, Fraser Brooks ${ }^{1}$, Thomas Downs ${ }^{1}$, Megan Drennan ${ }^{1}$, Erin Frizzell ${ }^{1}$, Laura Knox ${ }^{1}$, Hazel Miller ${ }^{1}$

${ }^{1}$ Department of Geriatric Medicine, Glasgow Royal Infirmary
Introduction: The importance of delirium in older adults with COVID-19 disease is now recognised. Clinical experience reveals it is sometimes the only symptom on diagnosis. We examine the associates and natural history of the disease in this group.

Methods: We used a prospectively gathered database of consecutive patients in a Scottish geriatric unit with COVID-19 disease, with retrospective data augmentation using the electronic medical record. We identified those who had delirium on diagnosis without "classical symptoms" of fever, shortness of breath or cough and examined their correlates and outcomes.

Results: 158 individuals were studied, 17 (11\%) had only delirium on diagnosis. They were a similar age to those with classical symptoms $(85.4 \pm 6.3$ vs $82.2 \pm 7.7$ years, $p=0.10)$. Clinical Frailty Scale (median, IQR) was the same between groups at 6 (6-7). 30 day mortality was similar in both groups $(2 / 17$ (12\%) versus $24 / 141$ $(17 \%), \mathrm{p}=0.58)$. Data was missing for one individual. $3 / 16(19 \%)$ went on to experience classical symptoms in the next fortnight, $5 / 16$ (31\%) developed an oxygen requirement, 3/16 ( 19\%) diarrhoea, 2/16 $(13 \%)$ seizure. Of the two who died, 1 person developed classic COVID-19 disease and the other deteriorated after a seizure.

Conclusions: Delirium is the only symptom at presentation in a significant number of older adults with COVID-19 disease. This group do not seem to be different in characteristics or outcome to those with classical symptoms. $80 \%$ do not go on to develop classical symptoms, highlighting the importance of clinician awareness of delirium as a symptom of COVID-19.

\section{Abstract \# 15 \\ Chest ultrasound for detection of COVID-19 pneumonia in nursing homes: feasibility study}

Andrea Ticinesi ${ }^{1}$, Antonio Nouvenne ${ }^{1}$, Alberto Parise ${ }^{1}$, Beatrice Prati $^{1}$, Marcello Esposito ${ }^{2}$, Valentina Cocchi ${ }^{2}$, Emanuele Crisafulli ${ }^{2}$, Annalisa Volpi ${ }^{3}$, Sandra Rossi ${ }^{3}$, Marco Baciarello ${ }^{4}$, Elena Giovanna Bignami $^{4}$, Tiziana Meschi ${ }^{4}$

${ }^{1}$ Geriatric-Rehabilitation Department, Azienda OspedalieroUniversitaria di Parma, Parma, Italy, ${ }^{2}$ Post-Graduate Specialization School in Emergency-Urgency Medicine, Department of Medicine and Surgery, University of Parma, Parma, Italy, ${ }^{3}$ Emergency-Urgency Department, Azienda Ospedaliero-Universitaria di Parma and Azienda Unità Sanitaria Locale di Parma, Parma, Italy, ${ }^{4}$ Department of Medicine and Surgery, University of Parma, Parma, Italy

Background: Bedside chest ultrasound is an accurate tool improving the diagnostic process of respiratory diseases. This study aims to evaluate the feasibility of chest ultrasound in nursing homes for detecting coronavirus disease-19 (COVID-19)-related pneumonia. Methods: Eighty-three older patients (age $85 \pm 8$ ) presenting mild to moderate respiratory symptoms or fever, residing in five nursing homes in Northern Italy with previous cases of documented COVID19 , were enrolled in this pragmatic, descriptive, feasibility study. Chest ultrasound was performed at the bedside by a mobile team of hospital specialists with certified expertise in thoracic ultrasonography, following a systematic approach. Presence of ultrasonographic signs of interstitial pneumonia, including focal comet-tail artifacts (Blines), diffuse B-lines, subpleural consolidations and pleural line indentation was detected. The specialist team integrated ultrasound data with clinical and anamnestic information, and gave personalized therapeutic advice for each patient, according to the possible presence of COVID-19 pneumonia.

Results: The most frequent reasons for ultrasound evaluation were fever (63\% of participants) and mild dyspnea (40\%). Fifty-six patients $(67 \%)$ had abnormal ultrasound findings. The most common patterns were presence of multiple subpleural consolidations (32 patients) and 
diffuse B-lines (24 patients), with bilateral involvement. A diagnosis of suspect COVID-19 pneumonia was made in 44 patients, and six of them required hospital admission. Twelve patients had ultrasound patterns suggesting other respiratory diseases, including aspiration pneumonia, heart failure and COPD exacerbation.

Conclusions: In nursing home residents, screening of COVID-19 pneumonia with bedside chest ultrasonography is feasible and may represent a valid diagnostic aid for an early detection of COVID-19.

\section{Abstract \# 16}

COVID-19 deaths in Irish nursing homes: exploring variation and association with the adherence to National Regulatory Quality Standards

Roman Romero-Ortuno ${ }^{1}$, Sean Kennelly ${ }^{2}$

${ }^{1}$ Trinity College Dublin and St James's Hospital Dublin, Ireland, ${ }^{2}$ Tallaght University Hospital, Dublin, and Trinity College Dublin, Ireland

Introduction: The COVID-19 pandemic has disproportionately affected nursing home residents worldwide, with Ireland having one of the highest reported proportions of COVID-19 deaths in this setting. In Ireland, the publication of a 'league table' of crude number of deaths in affected facilities sparked controversy on grounds of being potentially inaccurate and unhelpful.

Methods: We reviewed these published unofficial mortality data together with official data on quality standards published by the Irish regulator.

Results: There was substantial disagreement between the crude number of deaths and the mortality proportion per 100 beds. The association between crude number of deaths and maximum occupancy was significant with a moderate effect size (Spearman's rho $=$ $0.38, \mathrm{p}<0.001, \mathrm{n}=146)$. We found no significant association between occupancy-adjusted mortality and percentage of non-compliance with inspection standards (Spearman's rho $=-0.09, \mathrm{p}=$ $0.315, \mathrm{n}=140$ ). Specifically, we found no association between compliance with staffing, governance/management, premises and infection control. Counterintuitively, there was a mildly significant association between higher compliance with staff training and higher occupancy-adjusted mortality. A multivariate regression analysis on $\mathrm{n}$ $=140$ suggested a mild effect of higher overall non-compliance with lower adjusted mortality.

Conclusions: League tables with crude COVID-19-related nursing home deaths are likely to be unhelpful and data should at least be adjusted for the size of the facilities. Even in the latter case, extrapolation to quality of care is likely to be inappropriate. Much more research is needed to shed light into this complex topic and for this we urgently need a minimum dataset for care homes in Ireland.

\section{Abstract \# 17 \\ The role of frailty in predicting in-hospital mortality in older adults with SARS-COV-2 infection}

Pamela Carrillo Garcia ${ }^{1}$, Javier Jaramillo Hidalgo ${ }^{2}$, Blanca Garmendia Prieto $^{2}$, Isabel Lozano Montoya ${ }^{2}$, Giovanna Cristofori ${ }^{2}$, Maribel Quezada Feijoó ${ }^{2}$, Jorge Artero Ortiz ${ }^{2}$, Sagrario Pérez Delgado $^{2}$, Mónica Ramos Sánchez ${ }^{2}$, Javier Gómez Pavón ${ }^{2}$

${ }^{1}$ Hospital Central de la Cruz Roja. San josé y Santa Adela, ${ }^{2}$ Hospital Central de la Cruz Roja. San José y Santa Adela

Introduction: Mortality in SARS-CoV-2 infection is elevated, especially in elderly. There are many factors associated with this mortality. The aim of this study is to determine the role of frailty as a predictor of in-hospital mortality in older adults with SARS-COV-2 infection.

Methods: We designed an observational cohort study.We included patients aged 75 and older admitted to an Acute Geriatric Unit with diagnosis of SARs Cov-2 infection between March and April 2020The following variables were examined: demographic data, clinical history, comorbidity (Charlson index). Functionality (Barthel Index), cognitive abilities (GDS) geriatrics syndromes, frailty and polypharmacy . Pneumonia Severity: CURB 65 score was collected. Results: 200 patients were recruited (mean age $86 \pm 6.5$ ). 64.5\% came from nursing homes. Comorbidities: Hypertension arterial $68.6 \%$, stroke $41.3 \%$, dementia $38.8 \%$, diabetes mellitus $24.8 \%$, atrial fibrillation $24 \%$ and hearth failure $23.1 \%$. The most previous prescribed drugs: beta blockers $28 \%$ and angiotensin converting enzyme (ACE) inhibitors 26\%. Geriatrics syndromes: $72 \%$ had some degree of fragility; $80.6 \%$ functional dependency. The mortality was $39,5 \% /$ male $48.7 \%$. Using multivariate analysis, the following variables were predictive of in-hospital mortality: severe frailty $(\mathrm{p}<0.006)$ and high score in CURB $65(\mathrm{p}<0.001)$. ACE inhibitors had a protective effect OR: 1.1. $(\mathrm{p} \leq 0.013)$

Conclusions: Frailty and CURB-65 score are valuable as a predictor of in-hospital mortality in older adults with SARS-COV-2 infection. The use of ACE inhibitors didn't increase the risk of mortality.

\section{Abstract \# 18}

Delirium is a presenting symptom of COVID-19 infection in frail adults over 65 years of age: a Cohort Study of 322 hospitalized and 535 community-based older adults

Maria Beatrice Zazzara ${ }^{1}$, Rose Penfold ${ }^{2}$, Amy L. Roberts ${ }^{3}$, Karla A. Lee $^{4}$, Hannah Dooley ${ }^{3}$, Carole H. Sudre ${ }^{5}$, Carly Welch ${ }^{6}$, Ruth C. E. Bowyer ${ }^{3}$, Alessia Visconti ${ }^{3}$, Massimo Mangino ${ }^{7}$, Maxim B. Freydin $^{3}$, Julia S. El-Sayed Moustafa ${ }^{3}$, Kerrin Small ${ }^{3}$, Benjamin Murray $^{8}$, Marc Modat ${ }^{5}$, Jonathan Wolf ${ }^{9}$, Sebastien Ourselin ${ }^{5}$, Finbarr C. Martin ${ }^{10}$, Claire J. Steves ${ }^{3}$, Mary Ni Lochlainn ${ }^{11}$

${ }^{1} \mathrm{MD}$, Department of Twin Research and Genetic Epidemiology, King's College London, St Thomas' Hospital, London, SE1 7EH, Department of Gerontology, Neuroscience and Orthopedics, Sacred Heart Catholic University, Rome, Italy, ${ }^{2} \mathrm{BMBCh}$, Department of Twin Research and Genetic Epidemiology, King's College London, St Thomas' Hospital, London, SE1 7EH, ${ }^{3} \mathrm{PhD}$, Department of Twin Research and Genetic Epidemiology, King's College London, St Thomas' Hospital, London, SE1 7EH, ${ }^{4} \mathrm{MSc}$, Department of Twin Research and Genetic Epidemiology, King's College London, St Thomas' Hospital, London, SE1 7EH, ${ }^{5} \mathrm{PhD}$, School of Biomedical Engineering and Imaging Sciences, King's College London, Westminster Bridge Road, SE17EH, London, UK, ${ }^{6} \mathrm{MRes}$, Institute of Inflammation and Ageing, University of Birmingham, B15 2TT, ${ }^{7} \mathrm{PhD}$, Department of Twin Research and Genetic Epidemiology, King's College London, St Thomas' Hospital, London, SE1 7EH; NIHR Biomedical Research Centre at Guy's and St Thomas' Foundation Trust, London SE1 9RT, UK. ${ }^{8} \mathrm{MSc}$, School of Biomedical Engineering and Imaging Sciences, King's College London, Westminster Bridge Road, SE17EH, London, UK, ${ }^{9}$ MA, Zoe Global Limited, 164 Westminster Bridge Road, London SE1 7RW, $\mathrm{UK},{ }^{10} \mathrm{MD}$, Population Health Sciences, King's College London, Westminster Bridge Road, SE17EH, London, UK, ${ }^{11} \mathrm{MBBCh}$ BAO, Department of Twin Research and Genetic Epidemiology, King's College London, St Thomas' Hospital, London, SE1 7EH

Introduction: Frailty, increased vulnerability to physiological stressors, is associated with adverse outcomes. COVID-19 exhibits a more severe disease course in older, comorbid adults. Understanding of 
atypical presentations in frail, older adults is critical to early identification and containment of COVID-19 infection.

Methods: Observational study using two cohorts: (i) all patients aged 65 years and over with unscheduled admission to a London teaching hospital between March 1st, 2020 and May 5th, 2020; COVID-19 infection confirmed by RT-PCR of nasopharyngeal swab $(n=322)$. (ii) self-report data for community-based adults aged 65 and over enrolled in the COVID Symptom Study mobile application between March 24th (application launch) and May 8th, 2020; reported testpositive for COVID-19 $(\mathrm{n}=535)$. Multivariable logistic regression analysis performed on age-matched samples to ascertain association of frailty with symptoms of confirmed COVID-19.

Results: (i) Hospital cohort: after age-matching, there was a significantly higher prevalence of delirium in the frail sample (P-value: 0.013; Odds Ratio (OR) (95\% Confidence Interval (CI)) = 3.22 (1.44, $7.21)$ ), with no difference in fever or cough. (ii) Community-based cohort: there was a significantly higher prevalence of probable delirium in frailer, older adults $(\mathrm{P}$-value 0.038; OR $(95 \% \mathrm{CI})=2.29$ $(1.33,4.00))$, and also fatigue and shortness of breath.

Key conclusions: This study demonstrates a higher prevalence of delirium as a COVID-19 symptom in older adults with frailty. This emphasizes need for both systematic frailty assessment and delirium screening in acutely ill older patients in hospital and community settings. Clinicians should suspect COVID-19 in frail adults presenting with delirium.

\section{Abstract \# 19 \\ Hospital doctors experience of training during the Covid19 pandemic; what solutions are there? \\ Aria Khani ${ }^{1}$, Nikoo Aziminia ${ }^{1}$, Colette Smith ${ }^{2}$, Ameet Bakhai ${ }^{1}$, Clifford Lisk ${ }^{1}$ \\ ${ }^{1}$ Barnet Hospital BH, ${ }^{2}$ University College Hospital UCH}

The Coronavirus pandemic has changed all facets of our lives ranging from how we work, relax and travel. This includes an impact on the delivery medical training given the need for strict social distancing measures as lecture theatres can potentially become a medium for coronavirus spread. We aimed to explore hospital doctors experience of the delivery of training and new ways of working during this period. Hospital doctors at a UK NHS foundation trust were asked to complete a questionnaire on how they learnt about COVID19, their experience of the delivery of teaching and ways of working. 204 trainees took part; $39 \%$ were male with respondents being White $(50 \%)$, Asian $(25 \%)$ and black (3\%). Trainees got updates and guidance about COVID19 from their employer (61\%), TV news programmes $(54 \%)$ and social media $(45 \%) .73 \%$ of trainees indicated that the delivery of teaching had changed during COVID19. The commonest methods of delivery of teaching were through Microsoft teams $(66 \%)$, webinars $(27 \%)$ and zoom $(25 \%) .17 \%$ reported face to face teaching with social distancing measures. $63 \%$ reported new ways of working including increased use of telephone and video consultations, zoom and Microsoft teams' multidisciplinary meetings and new rotas. The COVID19 pandemic has allowed the rapid transition from face to face teaching to using video collaborative tools for the delivery of teaching and healthcare. This allows trainees and trainers to access teaching sessions remotely thereby accessing training at a time of convenience and reducing travel time for the delivery of regional training days.

\section{Abstract \# 20}

Older patients with urgent need for intensive care due to Covid19. Implementing geriatric aspects in triage decisions

Heinrich Burkhardt ${ }^{1}$, Uwe Sperling ${ }^{1}$

${ }^{1}$ Universitätsmedizin Mannheim - Geriatrisches Zentrum

In expecting large scale need for intensive care unit at the beginning of the covid19 pandemic, a local conference in an urban metropolitan area in south-west Germany was held to find and consent possible triage criteria for allocation of intensive care unit capacities in case of severe shortage of these capacities. Among heads of intensive care units, emergency departments and local ethic experts the department of geriatrics was asked for possible support in refining decision criteria with special regard to identify geriatric vulnerability and translate this in expected prognosis for severe course of lung crisis in the covid19-disease. Beside the qSOFA-score, patient preferences, comorbidity with terminal diseases, aspects of frailty and cognitive impairment were implemented. Those clinical aspects were translated in short and easy to assess control questions. Hereby the identification of both older patients with preserved resources (fit) and older patients already-independent from covid19-in an end of live situation (palliative aspects first) should be possible. A short check-list and a comment explaining the use of the checklist were developed. The algorithm and the check-list were accepted by the local committee and will be evaluated by retrospective interviews and case analysis for usefulness in clinical practice.

\section{Abstract \# 21 \\ Cardiological complications in geriatric population hospitalized during the COVID-19 pandemic.}

Mónica Ramos Sánchez ${ }^{1}$, Isabel Lozano Montoya ${ }^{2}$, Javier Jaramillo Hidalgo $^{2}$, Rocio Toro Cebada ${ }^{3}$, Eva Fernández de la Puente ${ }^{2}$, Giovanna Cristofori ${ }^{4}$, Pamela Carrillo García ${ }^{4}$, Maribel Quezada Feijoó $^{5}$, Rocío Ayala Muññoz ${ }^{5}$, Francisco Javier Gómez Pavón ${ }^{2}$

${ }^{1}$ Department of Cardiology, Hospital Central de la Cruz Roja. Universidad Alfonso X el sabio. Madrid, Spain, ${ }^{2}$ Department of Geriatrics, Hospital Central de la Cruz Roja. Universidad Alfonso X el sabio. Madrid, Spain, ${ }^{3}$ Universidad de Cádiz, ${ }^{4}$ Department of Geriatrics. Hospital Central de la Cruz Roja. Madrid, Spain, ${ }^{5}$ Department of Cardiology. Hospital Central de la Cruz Roja. Universidad Alfonso X el sabio. Madrid, Spain

Introduction: COVID-19 infection may associate cardiac complications such as an acute cardiovascular syndrome (ACS), a decompensation of previous cardiopathies, new onset atrial fibrillation (AF) or venous and ischemic embolic events (EE). We sought to analyze the incidente of cardiac events during the pandemic in a cohort of geriatric patients.

Methods: Observational longitudinal-sectional observational study. 200 patients $\geq 75$ years of age with confirmed diagnosis by PCR or with a high clinical suspicion of COVID-19 admitted to the Geriatrics Service from March to April 2020 were included. Congestive heart failure (CHF) was defined as compatible symptoms and findings on chest radiography and exploration. ACS was considered when there was chest pain, troponin elevation and/or changes on the ECG. Venous and arterial embolic events were confirmed by $\mathrm{CT}$ angiography or ultrasound.

Results: Mean age was $86 \pm 6.5 .79(39.5 \%)$ patients died, with highest mortality in males $(48.7 \%) .64 .5 \%$ were institutionalized in nursing homes. The mean hospital stay was $12.3 \pm 8$ days, and time from symptom onset to hospitalization was $5.7 \pm 3.7$ days. All patients received EE prophylaxis. Cardiac events occurred in $37 \%$. 
CHF was the most prevalent in $13.2 \%$, followed by AF $7.6 \%$. EE were: Pulmonary embolism with $7.6 \%$ followed by deep vein thrombosis $1.5 \%$. $4.1 \%$ presented a stroke followed by peripheral arterial ischemia in $0.5 \%$.

Conclusions: Exacerbation of CHF was the most frequent cardiac complication in geriatric patients affected by COVID-19, followed by pulmonary embolism and AF.

\section{Abstract \# 22 \\ 'Online' Supportive Conversations and Reflective Sessions (OSCaRS) in care homes during the COVID-19 pandemic.}

Jo Hockleyu ${ }^{1}$, Lucy Johnston ${ }^{2}$, Julie Watson ${ }^{1}$, Susan Shenkin ${ }^{3}$

${ }^{1}$ University of Edinburgh, ${ }^{2}$ Edinburgh Napier University, ${ }^{3}$ University of Edinburgh/Royal Infirmary Edinburgh

Introduction: COVID-19 Trauma Guidance suggests opportunities for structured, time-limited discussions about challenging experiences should be offered. It is unknown if such discussions can be effectively delivered online by palliative care specialists to support care home (CH) staff in relation to death/dying. Funded by Scotland's Chief Scientist Office COVID-19 'rapid research' fund, online OSCaRS is being piloted.

Methods: Fortnightly OSCaRS delivered to small groups of $\mathrm{CH}$ staff via a secure online platform in three local $\mathrm{CHs}$ over 10 weeks. Sessions are digitally recorded. The shortened version of the Chesney coping self-efficacy questionnaire is completed by all staff pre/post. Additional post-study questions asked of OSCaRS participants and indepth staff interviews will be undertaken $(\mathrm{n}=10)$. Thematic analysis of the recorded sessions and interviews will be undertaken and related to the staff questionnaire and context of each $\mathrm{CH}$.

Results: New learning on the feasibility and acceptability of providing OSCaRS to frontline staff. The benefit of OSCaRS to $\mathrm{CH}$ staff coping mechanisms, team cohesion and communicaton with relatives during the COVID-19 pandemic will be presented.

Key conclusions:The analysis will inform future practice, and an Implementation Guide for OSCaRS in $\mathrm{CHs}$ will be produced. Key learning on the potential for online support in relation to death/dying during the pandemic and beyond will contribute to future education, training and staff wellbeing resources. It will also inform the role of such sessions in developing individual coping mechanisms and team working alongside communication with relatives during lockdown.

\section{Abstract \# 23 \\ Epidemiological features of residents in long-term care facilities (LTCF) during the outbreak of Covid-19 In Spain}

Ana Pérez Fernández-Rius ${ }^{1}$, Borja Gil García ${ }^{1}$, Rubén Alcantud Córcoles $^{1}$, Laura Plaza Carmona ${ }^{1}$, Belén Roldán García ${ }^{1}$, Cristina Gómez Ballesteros ${ }^{1}$, Marta Mas Romero ${ }^{1}$, Almudena Avendaño Céspedes $^{1}$, Melisa López Utiel ${ }^{1}$, Sara Celaya Cifuentes ${ }^{1}$, Luz María Peña Longobardo ${ }^{2}$, Alicia Noguerón García ${ }^{1}$, Rafael García Molina ${ }^{1}$, Juan Oliva Moreno ${ }^{2}$, Pedro Abizanda Soler ${ }^{1}$

${ }^{1}$ Department of Geriatrics, Complejo Hospitalario Universitario of Albacete, Albacete, ${ }^{2}$ Department of Economic Analysis and Finance, University of Castilla-La Mancha, Toledo, Spain

Introduction: Long-Term Care Facilities (LTCFs) are high-risk settings for respiratory disease outbreaks, and older adults are the population group accounting for the large majority of severe Covid19 cases.

Methods: This epidemiological study includes 198 residents and 264 workers of a LTCF in Albacete, Spain, between 2020 March 6 and
April 5. We also include global data of 1062 residents from other six LTCFs of Albacete for the same period of time. This work describes epidemiological features of residents in LTCF, including demographic, clinical, cognitive, functional and nutritional data, analyzing mortality, residents and personnel characteristics, and associated costs.

results: Pooled mortality rate for 1 -month pandemic was $15.6 \%$, compared with $18.1 \%$ annual mortality for $2019.34 .3 \%$ was the percentage of probable Covid-19 infected residents. Patients probably infected were older, frail, and with a worse functional situation than those without Covid-19 suspect. The most common symptoms were fever, cough and dyspnea. Male older residents, with worse functionality, and higher comorbidity had higher mortality. During the 1-month period analyzed, $65(24.6 \%)$ workers leaved, mainly with Covid-19 symptoms, and 69 new workers were contracted. Costs associated with the Covid-19 in the Facility were estimated at $€$ $276,281 /$ month, mostly caused by resident hospitalizations, worker leaves, replacement of the staff, and intervention of healthcare professionals.

Key conclusions: Residents in LTCFs, mainly those older, frail and with worse functional status, were at higher mortality risk during the Covid-19 pandemic. Personal and economic costs were high, and may have been reduced with a structured outbreak plan.

\section{Abstract \# 24}

Abstract 4. COVID-19 impact on elderly demographics, hospital admissions and health care resources in SPAIN.

Ainhoa Esteve Arrien ${ }^{1}$, Pedro Ortiz Nuñez ${ }^{2}$, Concepción Jiménez Rojas $^{3}$, Barbara Perez Pena ${ }^{4}$, Jesús Lopez Arrieta ${ }^{5}$, Cristina Alonso Bouzon $^{6}$

${ }^{1}$ Hospital Universitario Infanta Leonor, Madrid, Spain, ${ }^{2}$ ndependent, ${ }^{3}$ Hospital Central Cruz Roja, Madrid, Spain, ${ }^{4}$ Clinica Psicogeriátrica Josefina Arregui, Navarra, Spain, ${ }^{5}$ Hospital Universitario La PazCantoblanco, ${ }^{6}$ Hospital Universitario de Getafe, Madrid, Spain

Introduction: Spain has suffered a great burden by Coronavirus global pandemic during 2020 with over 240,000 confirmed cases.

Methods: National census data published in 2020 regarding 2019 were reviewed. Mortality Excess Rates were reviewed with data of 4th June 2020. Mortality and Hospital-Admission-Rates (HAR) were reviewed and percentages calculated regarding general and elderly populations, and health care resources used the previous years published by the National National Summary of Hospital Morbidity-2018 (Encuesta Nacional Morbilidad Hospitalaria-ENMH) in Spain.

Results: Over a population of $47,026,208,0.26 \%$ of the general population have died during COVID19-pandemic from January to June 2020 detecting a $55.8 \%$ excess of mortality regarding an estimation based on previous years. The percentages of people who have died by age groups has been of $0.03 \%$ of $<65 \mathrm{yr}, 0.35 \%$ of people between $65-74 \mathrm{yr}$ and $2.05 \%$ of the population over 75 years. Regarding Autonomous Communities, Madrid accounts for the greatest burden of deaths and has lost $3.42 \%$ of their general population and $3.06 \%$ of their $>74 \mathrm{yr}$ population, followed by Castilla $\mathrm{La}$ Mancha that has lost $2.95 \%$ of their $>74 \mathrm{yr}$ population, Catalonia lost $2.73 \%>74 \mathrm{yr}$ population, Castilla y Leon lost $2.03 \%>74 \mathrm{yr}$ population. Regarding hospital admissions, during 8 weeks admissions have supposed $2.54 \%$ of yearly general admissions in Spain with the greatest burden in Madrid with 5.80\%, Castilla La Mancha 5.38\%, La Rioja $4.22 \%$, Castilla y León $3.23 \%$ and Catalonia 3.18\% due to COVID19.

Conclusions: COVID 19 has supposed an overwhelming lost of $>$ $74 \mathrm{yr}$ population in Spain and a significant percentage of yearly hospital admissions. 


\section{Abstract \# 25 \\ Frailty, clinical presentation and mortality in elderly patients admitted in intermediate-care hospital}

Núria Molist-Brunet ${ }^{1}$, Emma Puigoriol-Juvanteny ${ }^{2}$, Matilde BarnetoSoto $^{1}$, Rosa Torres-Allepuz ${ }^{1}$, Ferran Roca-Carbonell ${ }^{1}$, Nadina Latorre-Vallbona ${ }^{1}$, Joan Espaulella-Panico ${ }^{1}$

${ }^{1}$ Hospital Universitari de la Santa Creu de Vic, ${ }^{2}$ Hospital Universitari de Vic

Introduction: Older adults with coronavirus disease 2019 (COVID19) face an increased risk of adversehealth outcomes including mortality. Additionally, this patient profile can have an atypical clinical presentation. However, the association of age and frailty with clinical outcomes in older COVID-19 patients remains unclear.

Objective: to analyse clinical presentation and to determine the association between frailty and short-term mortality in older adults hospitalized for COVID-19.

Methods: This was a restrospetive, descriptive and observational study. Patients older than 75 admitted in a intermediate-care hospital were included. Data were collected from 15th March to 20th May. Collected data: demographic and biochemical variables, co-morbidities, symptoms and treatment were extracted from electronic medical records. Frailty, graded according to the Rockwood Clinical Frailty Scale (CFS).

Results: 122 patients with COVID-19 diagnosis were included (mean age 86 , SD 5.81; 50.8\% female). Median CFS score of 5 (range 2-9). Regarding clinical presentation, 28 patients $(22.95 \%)$ did not present any typical symptom (fever, cough or dyspnoea) and $21(17.21 \%)$ of them presented hipoactivity without any typical symptom. The most prevalent clinical complications were pneumonia $(n=69(56.55 \%))$ and delirium $(\mathrm{n}=51(41.80 \%)) .54$ patients died $(44.26 \%)$. As age and frailty of patients increases, so does the mortality rate $(\mathrm{p}<0.05)$. Till $67.35 \%$ of patients who were discharge suffered a functional impairment. Among atypical presentation, delirium was associated with a higher mortality $(\mathrm{p}<0.05)$.

Key conclusions: Elderly patients with COVID suffered high mortality and discapacity. Frailty is a prognostic marker in these patients. A relevant part of elderly patients have an atypical clinical presentation

\section{Abstract \# 26 \\ A new barrier to COVID-19 transmission in nursing home residents: the successful experience of 17 facilities in France \\ Joël Belmin ${ }^{1}$, Nathavy Um Din ${ }^{2}$, Cristiano Donadio ${ }^{2}$, Maurizio Magri $^{2}$, Quoc Duy Nghiem², Bruno Oquendo ${ }^{3}$, Carmelo Lafuente- Lafuente $^{4}$ \\ ${ }^{1}$ Sorbonne Université, ${ }^{2}$ Hôpital Charles Foix, ${ }^{3}$ Sorbonne Universisté, ${ }^{4}$ Sorbonne Université, Paris, France}

Context: During the COVID-19 pandemic wave that hit France in March-April 2020, staff members of some French nursing homes decided to confine themselves with their residents on a voluntary basis to reduce the risk of entry of SARS-CoV-2 in the facility.

Objective: To investigate COVID-19 related mortality in French nursing homes that had an experience of staff confinement (NH-SC) with residents.

Methods: Case-control study conducted from March 1 and May 11, 2020 in French nursing homes. NH-SC were identified from the media. COVID-19 cases among residents and the related mortality. COVID-19 cases were diagnosed by primary care or hospital physicians, and were recorded by a telephone interview with the directors for NH-SC, and by a nationwide declaration survey to health authorities for all the facilities.

Results: We identified 17 nursing homes in which 794 staff members confined themselves to the facility with their 1250 residents. Only one NH-SC (5.8\%) had cases of COVID-19 among the residents compared with 4599 facilities $(48.3 \%)$ in the national survey (OR: 0.07 , $95 \%$ CI $0.01-0.50, \mathrm{p}<0.001)$. Five residents $(0.4 \%)$ in the NH-SCs had COVID-19 in comparison with $62,368(9.0 \%)$ in the national survey (OR: $0.03,95 \%$ CI $0.02-0.10, \mathrm{p}<0.001$ ). Five residents $(0.4 \%)$ in the NH-SCs died because of COVID-19 in comparison with $12,516(1.8 \%)$ in the national survey (OR: $0.22,95 \%$ CI $0.09-0.53$, p $<0.001)$.

Conclusion: Self-confinement of staff members with residents seems to be effective in protecting nursing home residents from mortality related COVID-19.

\section{Abstract \# 27}

Nosocomial spread of SARS-CoV-2 in a rehabilitation facility: the silent threat of asymptomatic patients with high viral load?

Pauline Putallaz ${ }^{1}$, Wanda Bosshard ${ }^{1}$, Matthaios Papadimitriou ${ }^{2}$, Laurence $\mathrm{Senn}^{3}$, Chistophe Büla ${ }^{1}$

${ }^{1}$ Service de Gériatrie et Réadaptation Gériatrique, CHUV (Centre Hospitalier Universitaire Vaudois), Lausanne, Switzerland, ${ }^{2}$ Service des Maladies Infectieuses, CHUV (Centre Hospitalier Universitaire Vaudois), Lausanne, Switzerland, ${ }^{3}$ Responsable de l'unité Hygiène, Prévention et Contrôle de l'Infection, Service des Maladies Infectieuses, CHUV (Centre Hospitalier Universitaire Vaudois), Lausanne, Switzerland

Introduction: Severe acute respiratory syndrome coronavirus 2 (SARS-CoV-2) infection spreads rapidly within skilled nursing facilities but little is known about its spread in rehabilitation facilities. We assessed nosocomial transmission, adequacy of symptom-based screening and specificities of COVID-19 older patients in a 95-bed geriatric rehabilitation facility.

Methods: Retrospective case series analysis of patients who tested positive for SARS-CoV-2 (rRT-PCR) in our rehabilitation facility. Typical (fever, cough, shortness of breath) and atypical (malaise, increased confusion, rhinorrhea, anosmia, dizziness, headache, nausea, or diarrhea) symptoms present over the preceding and following days were recorded. The viral load at the time of diagnosis was reported.

Results: A total of 34 patients (median age 86 years, range 66-98, $67 \%$ female) tested positive for SARS-CoV-2 between March 15th and April 23rd, 2020. Nosocomial transmission occurred in 32 (94\%), and $22(65 \%)$ had a viral load $>106$ copies $/ \mathrm{ml}$. At the time of testing, only $21 / 34(62 \%)$ patients were symptomatic (18 typical and 3 atypical). Among the remaining 13/34 (38\%) asymptomatic patients, $9(69 \%)$ subsequently developed symptoms (5 typical and 4 atypical) after a median of 2 days. A high viral load $(>106 \mathrm{cp} / \mathrm{ml})$ was observed in $9 / 13(69 \%)$ initially asymptomatic patients. During the same period, 19 staff members (incl. residents, nurses, physical and occupational therapists, dieticians, and housemaids) were diagnosed with COVID-19, all symptomatic.

Conclusion: The rapid and widespread transmission of SARS-CoV-2 and the high prevalence of asymptomatic patients with high viral load at the time of testing, all support an extended screening approach in similar rehabilitation facility. 


\section{Abstract \# 28}

The clinical frailty scale and in-hospital mortality in older hospitalized COVID-19 patients in the Netherlands; the COVIDOLD study.

\section{The COVID-OLD Project Group The COVID-OLD Project Group}

${ }^{1}$ LUMC, Erasmus MC, Catharina Ziekenhuis, Amsterdam UMC, Reinier de Graaf, Alrijne Ziekenhuis, Deventer Ziekenhuis, ETZ, Franciscus Gasthuis, Gelre Ziekenhuizen, MC Leeuwarden, OLVG, Spaarne Gasthuis, UMCG, UMC Utrecht, VieCuri MC

Introduction: In the Netherlands, $89 \%$ of all patients who died from COVID-19 infection were 70 years or older. Clinical decision guidelines on hospitalization of patients have been based on the Clinical Frailty Scale (CFS), but no data are available yet on the predictive value of the CFS in this setting. We aim to develop and validate a prediction instrument based on the CFS for in-hospital mortality in older patients with COVID-19 infection.

Methods: The COVID-OLD study (www.covidold.nl) is an ongoing Dutch multi-center cohort study of 22 hospitals. Hospitalized patients of 70 years and older with COVID-19 infection were included. We collected data on demographics, comorbidities, disease severity, CFS, and in-hospital mortality. Here we present preliminary results of 3 hospitals, during the conference we expect to present analyses in over 2000 patients.

Results: A total of 243 patients were included. The median age was 76 years (IR 73-83), median CFS was 3 (IR 2-5). Most patients had few comorbidities and ADL-independency. In total, $40 \%(\mathrm{~N}=98)$ of patients died during hospital admission, ranging from $27 \%$ (CFS 1-3) to $55 \%$ (CFS 6-8). After correction for age and sex, the CFS was independently associated with in-hospital mortality (Odds Ratio of 2.9 (95\% CI 1.2-6.5) for CFS 6-8 versus CFS 1-3).

Conclusions: In-hospital mortality is high among older patients hospitalized with COVID-19 infection in the Netherlands and CFS is an independent predictor for this mortality. These findings may help in shared decision-making regarding hospitalization and advance care planning.

\section{Abstract \# 29}

\section{Characteristisc of elderly patients with COVID-19}

Isabel Arnau-Barrés ${ }^{1}$, Marta Herrero Torrus ${ }^{1}$, Ana Pascual-Dapena ${ }^{2}$, Javier Meseguer Donlo ${ }^{2}$, Olga Vázquez Ibar ${ }^{1}$, Robert GüerriFernandez ${ }^{3}$, Lizzeth Canchucaja Gutarra ${ }^{1}$

${ }^{1}$ Geriatrics Department, ${ }^{2}$ Universitat Pompeu Fabra School of Medicine, ${ }^{3}$ Infectious Diseases Department

Objectives: To describe the characteristics of elderly patients affected by COVID-19 hospitalized at a Geriatric facility (GF).

Materials and methods: We conducted a retrospective study of patients hospitalized in a GF affected by COVID-19. We reviewed the records of all patients hospitalized from March to May 2020. A multivariable logistic regression model was fitted for the prognostic factors for mortality including in the model those variables with a $\mathrm{p}<$ 0.10 in the univariate model.

Results: We identified 50 patients with a mean age of 88 years (SD 8.04). 17 men (34\%).Of all patients included 18 (37.5\%) lived at home and $28(58.33 \%)$ at nursing homes and $2(4.17 \%)$ in a long term care facility. $33(66 \%)$ were dependent for the activities of daily life. $25(50 \%)$ of the patients had cognitive impairment. The average comorbidity Charlson index was 4 (IQR 3-7). The main symptoms were: fever $36(73.47 \%)$, cough $21(42.9 \%)$, dyspnoea $27(55 \%)$ and $10(20 \%)$ gastrointestinal symptoms.Treatment was given in 43 (86\%)cases: hidroxicloroquine $34(72 \%)$, azithromycin $41(87 \%)$, corticosteroids $21(42 \%)$ and heparine in 39 (82\%). All of them needed oxygen al least in the first days of hospitalization. A total of $27(54 \%)$ died. When analysing risk factors for death in a multivariable logistic regression, troponin presented an OR $1.14(95 \% \mathrm{CI}$ 1.01-1.29; $\mathrm{p}=0.034)$ and the highest value of creatinine OR $3.2(95 \%$ CI 1.18-9.01; $\mathrm{p}=0.021$ ) and C-Reactive Protein 1.09 (95 CI $1.01-1.17 ; \mathrm{p}=0.017)$ when adjusting by age, sex and severity at admission.However we did not find any association with ferritin, D-dimer, or serum albumin.

Conclusions: Overall mortality was associated with being male, with higher levels of troponin at admission and higher levels of creatinine during hospitalization. No association with inflammatory or procoagulant parameters were found.

\section{Abstract \# 30 \\ Nutritional assessement and intervention of hospitalized COVID- 19 older patients \\ Sofia Duque ${ }^{1}$, Mariana Saraiva ${ }^{2}$, Cândida Fonseca ${ }^{1}$, Luís Campos ${ }^{1}$ \\ ${ }^{1}$ COVID-19 Ward, Internal Medicine Department, São Francisco Xavier Hospital, Occidental Lisbon Hospital Centre, ${ }^{2}$ COVID-19 Ward, Nutrition Department, São Francisco Xavier Hospital, Occidental Lisbon Hospital Centre}

Introduction: Older patients have a higher risk of malnutrition and COVID-19. COVID-19 can lead to malnutrition and this condition is possibly related to higher mortality and functional decline. Therefore, nutritional assessment and intervention (NAI) cannot be underestimated in COVID-19 older patients.

Methods: Taking the example of a COVID-19 ward, the authors identify the barriers to NAI and the strategies used to overcome those barriers.

Results: Many barriers were identified: ward isolation conditions; personal protective equipment; limited availability of assessment devices; limited time and restricted access to patients, preventing direct contact of some healthcare professionals (HCP) like nutritionists; COVID-19-related symptoms like anorexia, olfactory \& gustatory dysfunctions, fatigue, dyspnea, swallowing difficulties and reduced food intake; immobility-related constipation; meals disposable packaging and limited choices; HCP nutrition illiteracy. Some strategies to overcome these barriers are: increasing awareness of HCP about nutrition; inclusion of nutritional parameters in clinical history checklists; engagement of a multidisciplinary team and improvement of communication between HCP who have direct access to the patient and nutritionists; access to new technologies like smartphones and tablets that enable communication between the patient, the family, the nutritionist and the other HCP; control of gastrointestinal symptoms; adaptation of food and water consistence and texture; personalization of the diet; prescription of nutritional supplements; motivational messages to improve intake.

Key conclusions: COVID-19 pandemic can be seen as an opportunity to increase awareness about nutrition and to develop innovative and creative strategies that improve NAI, potentially reducing mortality and improving functional rehabilitation of COVID-19 patients.

\section{Abstract \# 31}

Exploring how nursing home staff in Ireland manage responsive behaviours in residents with dementia, during the Covid-19 pandemic

Elizabeth O'Donnell ${ }^{1}$

${ }^{1}$ Lancaster University 
Introduction: Agitation, aggression and wandering are responsive behaviours associated with dementia that are frequently treated with antipsychotic drugs, despite evidence of limited effectiveness and an increased risk of death. This research aims to understand how responsive behaviours are managed, using both pharmacological and non-pharmacological approaches, by nursing home staff in the Republic of Ireland during the Covid-19 pandemic. A further objective is to examine the impact of social isolation, due to Covid-19 restrictions, on responsive behaviour in residents with dementia.

Methods: After receiving ethics approval from Lancaster University Faculty of Health and Medicine Research Ethics Committee (FHMREC), an online questionnaire was distributed to nursing homes in Ireland $(n=443)$, to be completed during the period of Covid-19 restrictions, by care home managers, nurses and healthcare assistants. A sample of participants were invited to take part in an interview to explore their responses in greater depth. Concurrent analysis of qualitative data was informed by thematic analysis.

Results: Preliminary findings indicate that during lockdown, the absence of family visits and social interaction, resulted in cognitive decline, low mood and an increase in responsive behaviours in some residents with dementia. Nursing home staff expressed concerns regarding inadequate external support during the Covid-19 pandemic and highlighted the need for well-trained staff, familiar with residents needs, to manage responsive behaviours effectively.

Conclusions: The findings will inform policy and practice changes to support nursing homes and facilitate adoption of non-pharmacological approaches to manage responsive behaviours in residents with dementia.

\section{Abstract \# 32 \\ Psychosocial and emotional impact of COVID-19 visitor restrictions to nursing homes on families of residents with cognitive impairment: A cross-sectional study as part of the Engaging Remotely in Care (ERiC) project}

Rónán O'Caoimh ${ }^{1}$, Mark R O’Donovan ${ }^{1}$, Margaret P Monahan ${ }^{2}$, Caroline Dalton O'Connor ${ }^{2}$, Catherine Buckley ${ }^{3}$, Caroline Kilty ${ }^{2}$, Serena Fitzgerald ${ }^{2}$, Irene Hartigan ${ }^{2}$, Nicola Cornally ${ }^{2}$

${ }^{1}$ Department of Geriatric Medicine, Mercy University Hospital, Cork, Ireland, ${ }^{2}$ Catherine McAuley School of Nursing and Midwifery, University College Cork, Cork City, Ireland, ${ }^{3}$ Northridge House Education and Research Centre, St. Lukes Home, Cork City, Ireland

Introduction: COVID-19 has disproportionately affected older adults, including residents in nursing homes, where visiting restrictions are in effect. These may negatively affect visitors. We examined the effects of COVID-19 visiting restrictions on measures of perceived loneliness, well-being and carer quality of life (QoL) amongst visitors of residents with and without cognitive impairment $(\mathrm{CI})$ in Ireland.

Methods: We created a cross-sectional online survey. Loneliness was measured with the UCLA brief loneliness scale, psychological wellbeing with the WHO Well-being Index and carer QoL with the Adult Carer QoL Questionnaire (support for caring sub-scale). The link was circulated through university mail lists and targeted social media accounts in June 2020

Results: In all, 221 responses were received. Most (91.8\%) identified themselves as immediate family and most were female $(81.4 \%)$. Only $12 \%$ were aged $\geq 65$ years; the majority $(69 \%)$ were aged between 45 and 64 . Most (79.6\%) identified that their resident had CI. One-third $(35 \%)$ indicated that restrictions had impaired communication with nursing home staff. Median loneliness scores were 4/9, well-being scores 56/100 and carer QoL scores 9/15. Visitors of those with CI reported significantly lower well-being $(\mathrm{p}=0.009)$ but no difference in loneliness $(\mathrm{p}=0.2)$ or Qol $(\mathrm{p}=0.4)$. Linear regression modelling adjusting for age, sex and location confirmed that CI independently predicted lower well-being $(\mathrm{p}=0.03)$.

Key conclusions: This survey suggests that many nursing home visitors experience low psychosocial and emotional well-being during the COVID-19. Visitors of residents with CI report significantly poorer well-being. Research is required to understand if this is due to disrupted caregiving roles resulting from the restrictions imposed.

\section{Abstract \# 33}

Co-morbidity and COVID-19 in a cohort of 274,356 older adults: is age really the key driver?

Jane Masoli $^{1}$, Joao Delgado ${ }^{2}$, Luke Piling ${ }^{2}$, Janice Atkins ${ }^{2}$, Chai-Ling $\mathrm{Kuo}^{3}$, George Kuchel ${ }^{3}$, David Melzer ${ }^{4}$

${ }^{1}$ University of Exeter Medical School, Royal Devon and Exeter NHS Foundation Trust, ${ }^{2}$ University of Exeter Medical School, ${ }^{3}$ University of Connecticut, ${ }^{4}$ University of Exeter Medical School, University of Connecticut

Introduction: It is unclear whether the preponderance of common diagnoses such as diabetes and hypertension in COVID-19 confer risk or reflect high population prevalence. Associations between dementia and COVID-19 could be a care home effect. The ApoE e4e4 (homozygous) genotype is highly associated with dementia. We aimed to estimate associations between pre-existing diagnoses and COVID-19 and clarify potential genetic pathways via ApoE e4e4 for dementia. Methods: UK Biobank (England) participants baseline (2006-2010), plus secondary care data to 2017. Demographic, pre-existing diagnoses and ApoE genotype association tested with COVID-19 status (16th March-14th April 2020) in logistic models, adjusted for demographics, study site and other diagnoses.

Results: $\mathrm{N}=274,356$ participants aged $65+$, including $448(0.16 \%)$ hospitalized COVID-19 patients. Common co-morbidities were hypertension $(58.5 \%)$, CHD $(21.1 \%)$, falls or fragility fractures (30.6\%), and type 2 diabetes (19.6\%). In adjusted models, COVID-19 patients were more likely than other participants to have pre-existing dementia $(\mathrm{OR}=3.07$ 95\%; CI 1.71-5.50), $\mathrm{COPD}(\mathrm{OR}=1.82 ; \mathrm{CI}$ 1.33-2.49), depression $(\mathrm{OR}=1.81$; CI 1.36-2.40), type 2 diabetes $(\mathrm{OR}=1.70$; CI 1.30-2.21), chronic kidney disease and atrial fibrillation, hypertension $(\mathrm{OR}=1.29$; $\mathrm{CI} 1.04-1.59)$. $\mathrm{CHD}(\mathrm{OR}=0.92 ; \mathrm{CI}$ 0.71-1.20) prevalence was similar in COVID-19 patients and other participants. ApoE e4e4 homozygotes were more likely to be COVID-19 test positives ( $\mathrm{OR}=2.31$; CI 1.65-3.24) compared to e3e3 homozygotes.

Conclusion: Specific co-morbidities are disproportionally common in older adults who develop severe COVID-19. These results do not support simple age-based targeting to prevent severe COVID-19 infection. Dementia has a plausible genetic pathway of increased COVID-19 risk and is not just a care home association.

\section{Abstract \# 34 \\ Trends of admissions to a large academic urban hospital during the COVID-19 outbreak in Italy: focus on older patients \\ Andrea Ticinesi ${ }^{1}$, Antonio Nouvenne ${ }^{1}$, Nicoletta Cerundolo ${ }^{1}$, Beatrice Prati ${ }^{1}$, Loredana Guida ${ }^{1}$, Giulia Chiussi ${ }^{1}$, Ilaria Morelli ${ }^{1}$, Giampiero Castaldo ${ }^{1}$, Alberto Parise ${ }^{1}$, Bruno Agozzino ${ }^{1}$, Dario Magnani ${ }^{1}$, Erminia Ridolo ${ }^{2}$, Angela Guerra ${ }^{2}$, Tiziana Meschi ${ }^{2}$ \\ ${ }^{1}$ Azienda Ospedaliero-Universitaria di Parma, Parma, Italy, ${ }^{2}$ Department of Medicine and Surgery, University of Parma, Parma, Italy}


Background: Northern Italy faced a severe outbreak of coronavirus19 associated disease (COVID-19), that put the public healthcare system into stress for unprecedented overflow of patients requiring hospital care. Our aim was to track the trends of hospitalizations for suspect COVID-19 at our academic institution, with particular focus on older age $(\geq 75)$.

Methods: Admissions for suspect COVID-19, defined as presence of fever or respiratory symptoms and CT imaging suggestive for alveolar-interstitial pneumonia, and discharges were tracked on a daily basis from February 28 to May 13th, 2020, at the Geriatric-Rehabilitation Department of Parma University-Hospital, Emilia-Romagna region. Age, gender, coronavirus-19 testing and outcomes were recorded. To better analyze demographic trends, the outbreak period was split into two phases (exponential growth, from Feb 28 to Mar 22, and plateau/degrowth, from Mar 23 to May 13).

Results: The number of patients admitted with suspect COVID-19 was 1634 (816 in the growth phase and 818 in the degrowth phase). Patients admitted during the second phase were older (age $77 \pm 14$ vs $70 \pm 14, \mathrm{p}<0.001)$, most frequently female $(52 \%$ vs $40 \%, \mathrm{p}<0.001)$ and had a lower prevalence of positive swabs for coronavirus-19 $(59 \%$ vs $75 \%, \mathrm{p}<0.001)$, but similar mortality ( $28 \%$ vs $27 \%)$. Older patients (age $\geq 75$ ) had a significant trend towards higher mortality ( $\mathrm{p}$ $<0.001)$ and prevalence of positive swabs $(\mathrm{p}<0.001)$

Conclusions: In our experience, older patients, especially of female gender, were more frequently infected by coronavirus-19 during the second phase of the outbreak. This circumstance may be useful for establishing preventive measures targeted at the older population in future outbreaks.

\section{Abstract \# 35 \\ A systematic review assessing the under-representation of elderly adults in COVID-19 trials \\ Yaara Leibovici Weissma ${ }^{1}$ \\ ${ }^{1}$ Rabin Medical Center}

Objectives: To assess the relevance of clinical trials on COVID-19 for elderly adults

Design: Systematic review.

Setting: Randomized controlled trials (RCTs; $n=12$ ) comparing therapeutic or prophylactic interventions registered and/or published since December 2019.

Participants: Adults with COVID-19.

Measurements: Exclusion criteria that could limit participation of elderly adults were assessed, as well as adjusted analyses on age. Mean participant ages were reported and compared with observational studies.

Results: Twelve RCTs assessing drug therapy for COVID-19 were included. Mean age of patients included in RCTs was 56.3 years. One trial reported a subgroup analysis in patients $\geq 65$. An upper age limit was applied in three published trials $(25 \%)$ and in 200/650 (31\%) trials registered at clinicaltrials.gov. Patients were excluded for liverfunction abnormalities in nine trials, renal disease in seven, cardiac disease or risk of torsade de pointes in six, and four for cognitive or mental criteria. Only three trials allowed a family member to provide consent. Patients enrolled in RCTs were on average 20 years younger than those included in large $(n \geq 1000)$ observational studies. Seven studies had as their primary outcome a clinical endpoint, but none reported cognitive, functional or quality of life outcomes or need for rehabilitation or long-term care facility placement.

Conclusion: Elderly patients are clearly underrepresented in RCTs, although they comprise the population hardest hit by the COVID-19 pandemic. No data were found on long-term outcomes. Future investigations should target this vulnerable population.

\section{Abstract \# 36}

A snapshot of comprehensively assessed COVID-19 patients in a North-Italian Emergency Department: multidimensional frailty or organ-related factors and chronological age?

Erik Lagolio $^{1}$, I. Becker ${ }^{2}$, Jacopo Demurtas ${ }^{3}$, Stefania Bottone ${ }^{4}$, Laura Spadafora ${ }^{4}$, Cristina Cocino ${ }^{4}$, AM. Meyer ${ }^{5}$, L. Pickert ${ }^{6}$, V. Burst $^{6}$, T. Benzing ${ }^{7}$, Maria Cristina Polidori ${ }^{7}$

${ }^{1}$ Emergency Medicine (A\&E) -As12 - H Santa Corona, Pietra Ligure and First Aid, H Santa Maria Misericordia, Albenga, Italy, ${ }^{2}$ Insitute of Medical Statistics, University of Cologne, Faculty of Medicine and University Hospital, Cologne, Germany, ${ }^{3}$ Primary Care Department USL Toscana Sud Est, AFT Orbetello, ${ }^{4}$ Emergency Medicine (A\&E) -Asl2 - H Santa Corona, Pietra Ligure, Italy, ${ }^{5}$ Ageing Clinical Research, Department of Internal Medicine and Center for Molecular Medicine, Cologne, University of Cologne, Faculty of Medicine and University Hospital Cologne, Cologne, Germany, ${ }^{6}$ Ageing Clinical Research, Department of Internal Medicine and Center for Molecular Medicine, Cologne, University of Cologne, Faculty of Medicine and University Hospital Cologne, Cologne, Germany, ${ }^{7}$ Cologne

Excellence Cluster on Cellular Stress-Responses in Aging-Associated Diseases (CECAD), University of Cologne, Faculty of Medicine and University Hospital Cologne, Cologne, Germany

Background and aim: The current COVID-19 pandemic is mowing down older generations and exhausting world economies. To date, there is no efficacious strategy to predict outcomes, stratify patients at risk and allocate healthcare resources appropriately. Our aim is to demonstrate that chronological age and organ-related criteria might not be as determinant as multidimensional frailty to characterize patients' risk for poor outcomes.

Patients and methods: To reach the above purpose, we retrospectively assessed the clinical, psychosocial and functional characteristics of older patients with COVID-19 disease experiencing different outcomes from hospitalization to admission to intensive care unit (ICU), to treatment with non invasive versus invasive ventilation, to death. The patient collective under investigation was admitted to the Emergency Department of Pietra Ligure city, Italy between March 1, 2020 and April 15, 2020. A total of 118 consecutive older patients (47F, $73 \pm 14$ years) with swab-positive COVID-19 disease underwent at admission clinical examination, social assessment as well as Braden and Conley scales. Duration, frequency, cause and type of ventilation therapy among other outcomes were recorded.

Results: The patients assumed 4 drugs on average (median: 1-7) and had 1-3 concomitant diseases. The Braden score ranged between 11 and 20 and the Conley scale between 0 and 4. Eighteen\% lived in long-term facilities. Ventilation therapy (V) was received by 99 patients, $72 \%$ of total sample not invasive and $12 \%$ invasive. Although statistics is still ongoing at the time of admission, a preliminary analysis shows that self-sufficient patients were significantly more likely to be admitted to ICU and invasively ventilated than functionally impaired patients $(\mathrm{p}>0.04)$.

Conclusion: Despite the retrospective and preliminary nature of the data, there are some hints that multidimensional frailty, as a surrogate marker of biological age or at least an important factor of it, functions, plays a role in COVID-19 patients' trajectories.Further analysis are needed to establish meaningful correlations between ventilation therapies and multidimensional frailty. Also, the ongoing analyses are aimed at verifying if severely disabled patients were allocated to resource in the name of "clinical reasonableness" or "soft utilitarian" approaches. A comprehensive geriatric assessment, exploring domains beyond chronological age, hypoxemia and lung CT should be routinely performed and guide triaging algorithms in COVID-19 as well as several other life-threatening diseases. 


\section{Abstract \# 37}

How does research for older people need to change in a world with COVID-19 - findings from an international survey

Sarah Richardson ${ }^{1}$, Carmille Carroll ${ }^{2}$, Jacqueline Close $^{3}$, Adam Gordon $^{4}$, John O'Brien ${ }^{5}$, Terence Quinn ${ }^{6}$, Lynn Rocheste ${ }^{7}$, Avan Sayer $^{1}$, Susan Shenkin ${ }^{8}$, Nathalie van der Velde ${ }^{9}$, Jean Woo ${ }^{10}$, Miles Witham ${ }^{1}$

${ }^{1}$ AGE Research Group, Translational and Clinical Research Institute, Biomedical Research Building, Campus for Ageing \& Vitality, Newcastle University, ${ }^{2}$ University of Plymouth, Faculty of Health, ${ }^{3}$ Neuroscience Research Australia and Prince of Wales Clinical School, University of New South Wales, Sydney, Australia, ${ }^{4}$ Division of Medical Sciences and Graduate Entry Medicine, University of Nottingham; NIHR Applied Research Collaboration - East Midlands (ARC-EM), Nottingham. UK; University Hospitals of Derby and Burton NHS Foundation Trust, Derby, UK, ${ }^{5}$ Department of Psychiatry, University of Cambridge and Cambridgeshire and Peterborough NHS Foundation Trust, Cambridge, ${ }^{6}$ Institute of Cardiovascular and Medical Sciences, University of Glasgow, ${ }^{7}$ Brain and Movement Research Group, Translational and Clinical Research Institute, Biomedical Research Building, Campus for Ageing \& Vitality, Newcastle University, ${ }^{8}$ Geriatric Medicine, Usher Institute, University of Edinburgh, ${ }^{9}$ Department of Internal Medicine, Section of Geriatric Medicine, Amsterdam Public Health Research Institute, Amsterdam UMC, University of Amsterdam, ${ }^{10}$ Department of Medicine \& Therapeutics, Institute of Ageing, The Chinese University of Hong Kong

Introduction: Older people are disproportionately affected by the COVID-19 pandemic, which has had a profound impact on research as well as clinical service delivery. This survey aimed to identify priorities for COVID-19 research and key challenges in continuing research with older people, both during and after the current pandemic

Methods: The online survey contained five multiple-choice demographic questions and five free-text responses seeking views on research priorities, barriers to research delivery, and lessons learned. The survey was open from 8th to 16th May 2020, and was distributed via professional networks of clinicians and academics specialising in the care of older people and was shared on Twitter. Data were analysed using SPSS v24, and free-text responses were grouped into themes emerging from the data.

Results: 267 researchers and clinicians completed the survey: $83 \%$ from the UK. The top three research priorities identified were: management of COVID-19 and its complications, epidemiology of COVID-19 including its specific presentation and longer-term consequences in older people, and the wider societal impact of pandemic restrictions. Respondents identified that research needs to embrace remote working, with technology and methods to support this, whilst including older people living with frailty, cognitive impairment, multimorbidity, and those living in care homes. Respondents highlighted new opportunities including the ability to collaborate more widely and to design and deliver research efficiently at scale.

Conclusions: Profound changes are required in the way that we design and deliver research. This provides an opportunity to enhance all research with older people in the future.

\section{Abstract \# 38 \\ Effect of the COVID-19 epidemic on physical activity in community-dwelling older adults in Japan: A cross-sectional online survey}

Minoru Yamada ${ }^{1}$, Hidenori Arai ${ }^{2}$
${ }^{1}$ University of Tsukuba, ${ }^{2}$ National Center for Geriatrics and Gerontology

Objective: The objective of this study was to investigate changes in physical activity (PA) between January (before the COVID-19 epidemic) and April (during the COVID-19 epidemic) 2020 in community-dwelling older adults in Japan.

Design: Cross-sectional online survey

Setting and subjects: From April 23 to 27, 2020, an online survey was completed by 1600 community-dwelling older adults in Japan. Methods: We assessed the frailty status using the Kihon checklist, and other demographics and asked questions regarding PA at two time points: January and April 2020. We defined the total PA time (minutes) per week based on activity frequency and time.

Results: The study participants' mean age, proportion of women, and prevalence of frailty were $74.0 \pm 5.6$ years, $50 \%(\mathrm{n}=800)$, and $24.3 \%$ ( $\mathrm{n}=388$ ), respectively. We found a significant decrease in total PA time in April 2020 (median [interquartile range (IQR)], 180 [0-420]) when compared to January 2020 (median [IQR], 245 [90-480]) ( $<<0.001)$. We also performed a subgroup analysis according to the frailty category; total PA time significantly decreased in April 2020 when compared to January 2020 for all frailty categories $(\mathrm{P}<0.001)$.

Conclusion: In conclusion, due to the COVID-19 epidemic, the total PA time in April 2020 significantly decreased compared to that in January 2020 in older adults. This finding may lead to a higher incidence of disability in the near future in older people.

\section{Abstract \# 39}

Converting our face-to-face research to virtual assessmentsthe experience of the life after falls team

Chun Fatt Lau ${ }^{1}$, Nur Husna Shahimi ${ }^{2}$, Nurul Farhana M Johdi ${ }^{3}$, Ainoriza M Aini ${ }^{3}$, Einly $\mathrm{Lim}^{2}$, Maw Pin Tan ${ }^{1}$

${ }^{1}$ Faculty of Medicine, University of Malaya, ${ }^{2}$ Faculty of Engineering, University of Malaya, ${ }^{3}$ Faculty of Built Environment, University of Malaya

Introduction: The first case of COVID-19 in Malaysia was detected on 24 January 2020. This severely impacted the recruitment and follow-up rates of the Life After Falls (LiAF) study, with recruitment coming to a total standstill since the lockdown started on 18 March 2020. We describe the process by which we switched our research data collection from face-to-face to virtual assessments.

Methods: Communication between the team was carried out through two video conference meetings, social media messaging (WhatsApp Inc., USA) and electronic mail. Investigators first looked through the data collection process to identify variables that could immediately be converted to telephone or video calls. Variables that were not possible to be acquired virtually were then listed. Investigators were presented with the second list and a list of questionnaires or alternative methods for measuring these variables were proposed. The final survey questionnaire was finalized through group consensus.

Results: Sociodemographics, falls history, activities of daily living, social participation, medical history, psychological status, falls efficacy, quality of life and physical activity questions remain unchanged. Cognitive assessments, physical performance and $\mathrm{OH}$ assessments were switched to the Picture Memory Impairment Scale, Telephone Mobility Assessment Questionnaire and Orthostatic Hypotension Questionnaire respectively. Advice on lifestyle, medication changes, home-based exercises and cognitive training were provided based on individual findings.

Conclusion: Persevering with research progress during the pandemic ensures older people with falls continued to be assessed. A research outpost is established to complete the visual, postural blood pressure, 
memory and physical assessments a safe distance away from the hospital.

\section{Abstract \# 40 \\ Developing a pandemic-friendly questionnaire for the transforming cognitive frailty into self-sufficiency (AGELESS) Longitudinal Cohort Study}

Kiirtaara Aravindhan ${ }^{1}$, Moses Raj Muraly ${ }^{1}$, Sumaiyah Mat ${ }^{1}$, Maw Pin $\operatorname{Tan}^{1}$

${ }^{1}$ Faculty of Medicine, University of Malaya

Introduction: After a 3-year funding drought, the Ministry of Education awarded a Long-term Research Grant Scheme funding to merge the Towards Unusual Ageing (TUA) and Malaysian Elders Longitudinal Research (MELoR) studies into a single longitudinal cohort, now called Transforming Cognitive Frailty into Self-Sufficiency (AGELESS). Initiating longitudinal study of ageing during the COVID-19 pandemic ruled out traditional methods of public engagement and face-to-face assessments. A virtual survey questionnaire was, therefore, developed using a modified Delphi process. Methods: Draft documents were circulated via e-mail, with WhatsAppTM reminders. A face-to-face version of the survey developed during two workshops prior to the lockdown was disseminated to all 50 AGELESS investigators, who were asked to rank each section by importance, appropriateness and to provide free text comments. Changes were made according to suggestions after each round.

Results: A total of 213 comments and suggestions were received after three modified Delphi rounds over a 4-week period. The initial draft survey questionnaire contained 18 sections. The final survey was divided into four different questionnaires: new recruits, main questionnaire, healthcare utilization and mobility to be completed over three virtual interview sessions. Sections were reordered according to importance. Anthropometric, physical performance and frailty assessments were replaced with self-reported measures. Cognitive assessments were replaced with online friendly versions.

Conclusion: Moving to virtual surveys will enable us to capture the effects of a major global event on the lives of older Malaysians. Training of enumerators on virtual interview techniques and piloting are now underway prior to full scale implementation on all 4500 participants.

\section{Abstract \# 41 \\ Physical activity of people over 60 during the Covid-19 pandemic-impact on health-related quality of life \\ Tania Zieschang ${ }^{1}$, Sandra Lau ${ }^{1}$, Michel Hackbarth ${ }^{1}$, Jessica Koschate $^{1}$ \\ ${ }^{1}$ Carl von Ossietzky University Oldenburg, Geriatric Medicine}

Introduction: In order to flatten the infection rate during the Covid19 pandemic enormous restrictions were imposed on social life and organized sports activities. Particularly people above the age of 60 years were designated as a high-risk group for a severe course of the disease. This group might have restricted their range of action more than others, which af-fects their daily physical activity (PA). Reduced PA affects not only physical capacities, but also quality of life. Therefore, the study aims at describing changes in physical activity and the potential impact on health-related quality of life (HQOL).

Methods: Persons (60+) were asked about their PA, participation and HQOL (SF-12) before, during and after the pandemic-related restrictions. Subjective appraisal of risk and coping strategies with the restrictions are recorded.
Results: ANOVA will be used to assess differences in PA, participation and HQOL before, during and after the restrictions. Differences of these parameters will be correlated among each other. Level of significance will be set to $\alpha=5 \%$.

Discussion: Based on the results of the data collection, the effects of the pandemic-related restrictions on PA and the impact on HQOL will be assessed. Subjective appraisal of older people for the situation will be evaluated. Potentially, activities can be identified to mitigate changes in HQOL and recommendations for PA during a potential second wave of the pandemic can be made.

\section{Abstract \# 42 \\ Virtual geriatric clinics and the COVID-19 catalyst: a systematic review \\ Robert P. Murphy ${ }^{1}$, Karen A. Dennehy ${ }^{1}$, Maria M. Costello ${ }^{1}$, Evelyn P. Murphy ${ }^{1}$, Conor S. Judge ${ }^{1}$, Martin J. O’Donnell ${ }^{1}$, Michelle D. Canavan $^{1}$ \\ ${ }^{1}$ Galway University Hospital}

The current COVID-19 health crisis has led to many innovative healthcare solutions around interacting with patients, particularly older people who have been advised to avoid the hospital setting and self-isolate. Virtual geriatric clinics have become increasingly utilised to complete outpatient consultation for older adults who are unable to attend in-person. The scope of virtual clinics has been rapidly expanding, but concerns exist about feasibility of such virtual consultations for older people. The aim of this systematic review is to describe the satisfaction, clinic productivity, clinical benefit, satisfaction and costs associated with the virtual geriatric clinic model of care.

Methods: A systematic review of PubMed, MEDLINE and CINAHL databases was conducted up to April 2020. Two independent reviewers extracted the information. Four subdomains were focused on: satisfaction with the virtual geriatric clinic, clinic productivity, clinical benefit to patients, costs and any challenges associated with the virtual clinic process.

Results: Nine studies with 975 patients met our inclusion criteria. All were observational studies. Seven studies reported patients were satisfied with the virtual geriatric clinic model of care. Productivity outcomes included reports of cost-effectiveness, savings on transport, and improved waiting list metrics. Clinical benefits included successful polypharmacy reviews, and reductions in acute hospitalisation rates. Varying challenges were reported for both clinicians and patients in eight of the nine studies. Hearing impairments and difficulty with technology added to anxieties experienced by patients. Physicians missed the added value of a thorough physical examination and had concerns about confidentiality.

Conclusion: Virtual geriatric clinics demonstrate evidence of productivity, benefit to patients, cost effectiveness and patient satisfaction with the treatment provided despite the challenges incurred. In the current suboptimal pandemic climate, virtual geriatric clinics may provide a means to provide continuity of care, if adopted with the above considerations in mind.

\section{Abstract \# 43}

Quality-of-life during COVID-19 quarantine: the impact of lifespace reduction in older adults

Márlon Aliberti ${ }^{1}$

${ }^{1}$ University of Sao Paulo Medical School 
Background: Although physical distancing might be a powerful weapon to flatten the curve from the COVID-19 epidemic, older adults may adapt poorly to the changing social and health structures that accompany it. Objectives: We investigated the factors associated with the impact of COVID-19 pandemic in the quality-of-life of community-dwelling older adults.

Methods: Prospective study comprising 557 older adults aged $\geq 65$ years, without dementia, from four different health centers in Sao Paulo, Brazil. Participants were followed by structured telephone interviews every 2 weeks since the quarantine has been ordered. Older adults reported the level of impact (not at all, to some extent, or a great extent) the coronavirus pandemic in their quality-of-life. The primary variables of interest were the Life-Space Assessment (LSA) significant reduction (before vs. during the quarantine) and frailty, assessed by the FRAIL scale. Comprehensive geriatric assessment also included sociodemographic factors, comorbidities, cognitive performance, activities of daily living, gait difficulty, physical activity, loneliness, depression, and anxiety.

Results: Participants had a mean age of $80 \pm 7.5$ years, $65 \%$ were women, $15 \%$ were living alone, $33 \%$ were frail, and $79 \%$ fulfilled criteria for a clinically significant LSA reduction ( $\geq 5$ points). The coronavirus pandemic affected the quality-of-life of $77 \%$ of older adults. In adjusted logistic regression, a significant LSA reduction was associated with higher levels of impact in quality-of-life $(\mathrm{OR}=2.2$; 95\% CI 1.3-3.6). Frailty modified the effect of LSA reduction in quality-of-life ( $\mathrm{P}$ for interaction $=0.03$ ). Among frail individuals, the impact of LSA reduction in quality-of-life was almost doubled $(\mathrm{OR}=$ $4.20 ; 95 \% \mathrm{CI} 2.36-7.50)$ than among those non-frail $(\mathrm{OR}=2.18 ; 95 \%$ CI 1.33-3.58).

Conclusions: The COVID-19 pandemic is affecting the quality-oflife of the majority of older adults. The LSA reduction is strongly associated with quality-of-life during COVID-19 quarantine, particularly among those older people who are frail.

\section{Abstract \# 44 \\ Burden and anxiety in caregivers of Alzheimer's disease patients during the COVID-19 pandemic: a cross-sectional study}

Gelanys Castro-Tejada ${ }^{1}$, Franck Diaz-Garelli ${ }^{2}$, Alicia H. Mauricio $\mathrm{C}^{3}$, Angel L. Rodriguez-Lockward ${ }^{4}$, Issaell Hernandez ${ }^{4}$, Jesabelle Dominguez ${ }^{4}$, Karina Gomez $\mathrm{R}^{4}$, Karla Diaz Mesa ${ }^{5}$, Lisemaine Dieuveuil ${ }^{4}$, M. Rodriguez Tavarez ${ }^{4}$, Melani Peña ${ }^{4}$, Martin Medrano ${ }^{4}$

${ }^{1}$ Pontificia Universidad Católica Madre y Maestra, ${ }^{2}$ University of North Carolina at Charlotte, 9201 University City Blvd, Charlotte, NC 28223. USA. (704) 687-8622, ${ }^{3}$ Pontificia universidad católica madre y maestra, Autopista Duarte Km 1 1/2, Santiago De Los Caballeros 51000, ${ }^{4}$ Pontificia Universidad Catolica Madre y Maestra. Autopista Duarte Km 1 1/2, Santiago De Los Caballeros 51000 (809) 580-1962, ${ }^{5}$ INTEC

Introduction: Alzheimer's disease (AD) advancement imposes tremendous burdens on caregivers, which are increased by the patient's dependency demand [1]. Confinement and social interaction disruption increase the risk of mental health problems such as anxiety, insomnia, paranoia, panic, aggression, and depression [2]. Limitations on occupational, social, familial, and personal activities are known to increase the physical and emotional burden of the caregiver [3]. The COVID-19 pandemic has exacerbated these limitations for all. We aimed to investigate the impact of confinement circumstances on the caregivers of AD patients hypothesizing that the superimposed burden can affect their mental health.

Methods: A web-based survey on a cohort accessed from the National Institute on Aging Late-Onset Alzheimer's Disease Family Study [4] dataset. We applied the short form Zarit Burden Interview
(ZBI) and Beck Anxiety Inventory (BAI) with primary outcome measures: burden and anxiety scores.

Results: A total of 1709 individuals were eligible, and 198 (11.6\%) responded. Participants' ages ranged from 18 to 76 years, $82.3 \%$ female, $52.5 \%$ married or partnered, $8.1 \%$ were professional caregivers, and $64.6 \%$ mother/father. The mean time as a caregiver was $3.27 \pm 1.15$, with $11.3 \pm 4.1$ years of education. The caregiver's mean burden score was $18.3 \pm 6$ which correspond to an intense burden. Anxiety score was $14.8 \pm 11.2$ consistent with mild anxiety level. A X2 test found statistical significance between age and burden with a $\mathrm{p}=0.008$.

Conclusions: Caregivers had an intense burden. Anxiety levels during the pandemic associated with this burden; therefore, health professionals should include a thorough caregiver assessment after the confinement is over.

\section{References:}

1. Hoffman D, Zucker H. A Call to Preventive Action by Health Care Providers and Policy Makers to Support Caregivers. Prev Chronic Dis 2016; 13:160233

2. Haney, Craig. "Mental Health Issues in Long-Term Solitary and 'Supermax' Confinement." Crime \& Delinquency, vol. 49, no. 1, Jan. 2003, pp. 124-156, https://doi.org/10.1177/0011128702239239.

3. Caregiving. Centers for Disease Control and Prevention. https:// www.cdc.gov/aging/caregiving/index.htm accessed 06/15/2020

4. Lee, Joseph $\mathrm{H}$ et al. "Identification of novel loci for Alzheimer disease and replication of CLU, PICALM, and BIN1 in Caribbean Hispanic individuals." Archives of neurology vol. 68, 3 (2011): 320-8. https://doi.org/10.1001/archneurol.2010.292

\section{Abstract \# 45 \\ Non-COVID 19 related mortality rates in a nursing home during the COVID-19 pandemic}

Julia Tua ${ }^{1}$, Francesca Farrugia ${ }^{1}$, Lara Camilleri ${ }^{1}$, Doriella Galea ${ }^{1}$, Jessica Camilleri ${ }^{1}$, Stephanie Dalli ${ }^{1}$, Ronald Fiorentino ${ }^{1}$, Antoine Vella $^{1}$

${ }^{1}$ St. Vincent de Paule Residence

Background: Non-COVID 19 related rise in mortality rate was a phenomenon noted internationally during the COVID-19 pandemic, especially in the geriatric population. St. Vincent de Paul Residence (SVPR) is a large nursing home in Malta for highly dependent, frail persons requiring 24-h medical supervision. COVID-19 was first locally transmitted in Malta in mid-March, when several restrictive measures were put into place to contain the spread of COVID-19 within the facility. Up until May 2020, there was one case of COVID19 amongst 988 residents, and no COVID-19 related deaths at SVPR. Despite this, we hypothesized that the mortality rate during the COVID-19 pandemic will be significantly higher at SVPR than previous years in view of non-COVID-19 related deaths.

Aim: We aimed to compare mortality rates amongst residents of three consecutive years at SVPR, to assess for a rise in mortality rate during the COVID-19 pandemic.

Research Method: This retrospective study was carried out by collecting data from local software, medical records at SVPR.

Results: so farResults from June 2020 are still being collected. There was no significant spike in mortality rate during the COVID-19 pandemic compared to the previous 2 years.

Conclusion: The restrictive measures put in place at SVPR to contain COVID-19 did not increase the Non-COVID related mortality rate, with no spike in deaths compared to previous years. 


\section{Abstract \# 46}

Palliative care of elderly cancer patients during the COVID-19 pandemic: opportunities in the wake of crisis

Yakir Rottenberg $^{1}$, Adir Shaulov ${ }^{2}$

${ }^{1}$ Faculty of Medicine, Hebrew University of Jerusalem, Israel; Department of Oncology, Hadassah Medical Center, Jerusalem, Israel., ${ }^{2}$ Faculty of Medicine, Hebrew University of Jerusalem, Israel; Department of Hematolgy, Hadassah Medical Center, Jerusalem, Israel.

Background: During the 1st wave of the COVID-19 pandemic, death rates in Israel were low. However, the mortality rates of elderly COVID-19 patients were demonstrably higher, resulting in a changing atmosphere and different palliative care needs for elderly COVID19 and non-COVID-19 patients. This crisis may highlight the need for palliative care and clarification of treatment goals among elderly patients, especially those with cancer.

Methods: Description of the palliative care team's experience at the Hadassah Medical Center, a tertiary cancer center (900 beds) for the Jerusalem district, Israel.

Results: Social isolation, virtual meeting and loneliness placed constraints on efficient communication among the triad of patients, families and health care professionals. Fear of infection with a potentially lethal disease and infected from caregivers added to patient stress. In addition, information for evaluation of disease status critical for defining future goals was limited due to deferred treatments, clinic appointments and imaging. Deferred clinic appointments and virtual meeting also constrained in depth discussions with patients leaving less opportunities for defining goals of care.

Conclusions: During the 1st wave of the COVID-19, various barriers to effective palliative care have been reported among elderly cancer patients despite potential opportunities during this crisis. In the light of potential further waves of the COVID-19, the clinical ramifications of our findings suggest that early defining treatment's goals and optimizing planning for palliative care delivery are needed.

\section{Abstract \# 47 \\ SARS-COV-2 pandemic prosess effect in invidiuals aged 60 years and older and coping skills: Turkey example \\ Sevnaz Şahin ${ }^{1}$, Nurgül Kocakoç ${ }^{1}$, Buğçe Baybaş ${ }^{1}$, Cemil Yavuz ${ }^{2}$, Fehmi Akçiçek $^{1}$, Fisun Şenuzun Aykar ${ }^{3}$ \\ ${ }^{1}$ Ege University, ${ }^{2}$ Muğla Sıtkı Koçman University, ${ }^{3}$ İzmir Tınaztepe University}

Introduction: In March 2020, quarantine was launched in most countries for the SARS-COV-2, which was declared as pandemic by WHO. It was planned to determine the effect and coping methods of pandemic and quarantine on elderly individuals.

Materials and methods: It is planned as a descriptive and crosssectional research. The data were collected via e-survey method on Ege University 3AU social media accounts. Research data, SPSS 22.0 statistics program was evaluated.

Findings: Of the 1056 individuals aged 60 and more with an average age of $67,71 \%$ are women, $56.9 \%$ are university graduates, $22.5 \%$ live alone. The most important problems in quarantine are being unable to shop $19.4 \%$, loneliness $12.97 \%$. The most important support is phone calls with family members $(18.7 \%)$. In quarantine, $21.1 \%$ of them had health problems at least once, $13.7 \%$ never exercised at home, and 55\% watched news continuously. Affected by quarantine is X 6.23, quality of life X 5.9, anxiety levels X 5.94, coping levels X 7.76. A positive correlation was found between anxiety (r: 0.200 , p:
0.00 ) and quarantine exposure (r: 0.130, p: .00) and following the news.Coping (t: -0.369 , p: 0.712$)$ and quarantine-affected situations $(\mathrm{t}:-0.196, \mathrm{p}: 0.571)$ were significant in favor of women. As the number of health problems experienced increased, the quality of life score (p: 0.019) and the level of exposure to quarantine (p: 0.001) decreased.

Conclusion: Pandemic and quarantine have affected the age of 60 and over. Social connections and technology have gained importance in dealing with the process.

\section{Abstract \# 48}

\section{Impact of COVID-19 pandemic in an orthogeriatric unit}

Ś́lvia Balhana $^{1}$, Afonso Cardoso ${ }^{2}$, Lia Marques ${ }^{1}$, Armando Pereira ${ }^{2}$, José Lomelino Araújo ${ }^{1}$, Paulo Rego ${ }^{2}$

${ }^{1}$ Internal Medicine, Hospital Beatriz Ângelo, ${ }^{2}$ Orthopedic surgery, Hospital Beatriz Ângelo

Introduction: COVID-19 pandemic forced hospitals to reassign resources. We seek to understand the effects of our hospital contingency plan on the assessment of patients admitted to orthogeriatric unit (OGU), which treats hip fractures in patients over 65 years. Objective: Determine the impact of COVID-19 pandemic on our OGU.

Methods: Retrospective case control study of patients admitted to OGU during March-April 2020 (COVID-19 group) compared to the same period of 2019 (control group).

Results: 59 patients were admitted in 2019 and 36 patients in 2020 Average patient age was 82.9 years and mostly women $(75 \%)$. Between the two groups there were no statistical differences in age $(\mathrm{p}$ $=0.823)$, gender $(\mathrm{p}=0.526)$, nutritional assessment $(\mathrm{p}=0.719)$, physiotherapy intervention $(\mathrm{p}=0.811)$ or length of stay $(\mathrm{p}=0.531)$. Time to surgery was $<48 \mathrm{~h}$ in $20 \%$ patients in 2019 and $31 \%$ in 2020 $(\mathrm{p}=0.230)$. Medical complications were higher in $2020(\mathrm{p}=0.028)$, mostly urinary and respiratory infections $(21 \%$ in 2019 Vs $39 \%$ of hospitalizations in 2020). Three patients were diagnosed with COVID-19. The in-hospital mortality rate was of 5\% in 2019 and $12 \%$ in $2020(\mathrm{p}=0.078)$. There were less social assessments $(\mathrm{p}=$ $0.008)$ and less in-home rehabilitation $(\mathrm{p}=0.026)$ in the COVID-19 group.

Key conclusions: During COVID-19 pandemic, the number of patients admitted to our OGU was substantially lower, there was an increase in medical complications and a decrease in social assessments and in in-home rehabilitation. There was a higher mortality rate, although not statistically significant. These findings will help guide health care in this challenging environment.

\section{Abstract \# 49}

The impact of COVID-19 on activity and involvement in social life and quality of life among people 60+ living in Poland, depending on the symptoms of depression

Marta Lewandowicz ${ }^{1}$, Sylwia Kropińska ${ }^{2}$, Sławomir Tobis ${ }^{2}$, Aleksandra Kaluźniak-Szymanowska ${ }^{2}$, Roma KrzymińskaSiemaszko $^{2}$, Katarzyna Wieczorowska-Tobis ${ }^{2}$

${ }^{1}$ Poznan Uniwersity of Medical Sciences, ${ }^{2}$ Poznan University of Medical Sciences

The world is plunging into the COVID-19 pandemic, which is why unprecedented restrictions have been imposed, also on social relations. Social distance measures have been introduced to limit social interaction, contributing to the slow transmission of coronavirus (COVID-19). In older people, isolation and the implications of social 
distancing are a particular challenge for their physical activity, mental health and social relations [3], and can have a number of adverse effects through the progress of social isolation, loneliness and a sedentary lifestyle [9]. Social isolation leads to a wide range of psychological symptoms, including depression and anxiety, and negatively affects the quality of life [3]. The study evaluated the prevalence of depressive symptoms, activity and involvement in social life, as well as the quality of life before and after the outbreak. Methods: The study included 168 people over 60 years of age (125 females) who voluntarily completed an online survey. The presence of symptoms of depression was assessed using the Yesavage 15-point Geriatric Depression Rating Scale. On its basis, the subjects were divided into two groups (people without depressive symptoms-0-5 points and people with symptoms of depression $>5$ points). The quality of life was rated on a 10-point scale, where 0 points meant the worst possible, and 10-the best imaginable. Activity and involvement in social life were also assessed on a 10-point scale, where 0 said that the examined person was not able to get out of bed alone, and 10 points indicated the lack of any restrictions on activity. Both factors were assessed before and during the pandemic.

Results: $31.5 \%$ of the respondents had depressive symptoms (among them, $94.3 \%$ were women). In people living alone, the symptoms of depression were significantly more frequent $(p<0.001)$ than among married ones. Activity and involvement in social life deteriorated significantly as a result of COVID-19 in all subjects $(\mathrm{p}<0.001)$, among people with depressive symptoms $(\mathrm{p}<0.0001)$ and persons without depressive symptoms $(\mathrm{p}<0.0001)$. The mean scores before the outbreak of the pandemic in both groups were similar and amounted to $8.4 \pm 2.1$ points for subjects without depressive symptoms and $8.0 \pm 2.1$ points for those with symptoms of depression. As a consequence of the epidemic, people with symptoms of depression showed significantly worse activity and involvement in social life than people without depressive symptoms $(6.2 \pm 2.4 ; 7.4 \pm 2.4 ; \mathrm{p}<$ 0.001 , respectively).Similar results were obtained for the quality of life. The COVID-19 outbreak significantly affected the quality of life in all subjects $(\mathrm{p}<0.001)$, those with depressive symptoms $(\mathrm{p}<$ $0.001)$ and no symptoms $(\mathrm{p}<0.001)$. People with depression indicated on average a significantly lower quality of life than people without depression, both before and during the pandemic (before the pandemic: $7.4 \pm 1.7 ; 8.2 \pm 1.6 ; \mathrm{p}<0.001$; during a pandemic: $5.4 \pm$ $1.8 ; 6.6 \pm 1.8 ; \mathrm{p}<0.001$, respectively).

Conclusion: Social isolation associated with the COVID-19 pandemic has worsened the quality of life and has reduced the activity and involvement of older people in social life. Symptoms of depression among people over 60 years of age resulted in a significantly stronger deterioration in the quality of life and involvement in social life during the pandemic compared to subjects who did not have these symptoms.

\section{Abstract \# 50 \\ The impact psychological resilience on development of coronavirus anxiety and sarcopenia in older adults during COVID-19 Pandemic}

Sumru Savas ${ }^{1}$, Asli Kilavuz ${ }^{1}$, Özlem Kuman Tunçel ${ }^{2}$, Zehra Kosuva Öztürk $^{1}$, Fehmi Akçiçek ${ }^{1}$

${ }^{1}$ Geriatrics Section, Internal Medicine Department, School of Medicine, Ege University, Izmir, Turkey, ${ }^{2}$ Department of Psychiatry, School of Medicine, Ege University, Izmir, Turkey

Aim: Older adults faced substantial problems such as loneliness, anxiety, and depression in the pandemic by severe acute respiratory syndrome Coronavirus 2. After a challenge or stress, resilience might promote adaptation, resistance, or recovery of mental-physical health, and resilience might be a substantial preventive factor for older adults in the pandemic era. We hypothesized that older people with better psychological resilience might have adapted the pandemic process better. So, we aimed to evaluate Coronavirus anxiety and psychological resilience and to screen sarcopenia in older patients.

Methods: We included 40 older patients who were evaluated between January 2019 and September 2019 with the Resilience Scale for Adults (RSA), handgrip strength (HGS), and social support scale (SSS) in our prior study. We applied SARC-F, Coronavirus anxiety scale (CAS), and a questionnaire prepared by researchers, via a phone call for this study.

Results: A total of 40 patients were included. Mean age, education years, the number of chronic diseases-medications used, HGS, PRS score, BMI, and SSS score of the patients evaluated before the pandemic were: $73.3 \pm 5.6$ (years), $9.7 \pm 4.1$ years, $1.7 \pm 1.2,3.6 \pm 3.3$, $22.5 \pm 7.3(\mathrm{~kg}), 128.8 \pm 14.1,29 \pm 5.9\left(\mathrm{~kg} / \mathrm{m}^{2}\right), 9 \pm 5.1$. Mean SARC-F, and CAS scores were: $1.8 \pm 1.7$, and $0.9 \pm 2.02$. Mean resilience score negatively correlated with CAS $(r=-0.423, p=$ $0.020)$. CAS score was also negatively correlated with the number of comorbidities-medications, and positively correlated with HGS $(\mathrm{r}=-$ $0.530, p=0.002 ; \mathrm{r}=-0.533, \mathrm{p}=0.002 ; \mathrm{r}=0.485, \mathrm{p}=0.020)$. SARCF negatively correlated with HGS $(r=-0.574, \mathrm{p}=0.003)$.

Conclusions: In our sample consisting of highly resilient older people, higher resilience scores were associated with lower anxiety.

\section{Abstract \# 51}

Monitoring the senior population with comorbidities during the COVID-19 pandemic in Brazil

Cláudia Fernanda Trindade Silva ${ }^{1}$, Monaliza Lemos Souza ${ }^{1}$, Larissa Chaves Pedreira ${ }^{1}$, Clara Magalhães Vasconcelos ${ }^{1}$

${ }^{1}$ Universidade Federal da Bahia - UFBA

Introduction: The COVID-19 pandemic demanded social distance measures due to the high transmissibility of Sars-CoV-2. The fear of contracting the virus has removed seniors from health services, affecting the medical monitoring of comorbidities such as diabetes, heart disease and cancer. The senior population is part of the risk group for the development of the severe form of COVID-19. In this context, it is questioned how care has been conducted for seniors with comorbidities, which need follow-up.

Methodology: This is free communication with the objective of reflecting the health of the elderly population during the pandemic by Sars-CoV-2.

Results: The fear of contracting COVID-19 in health services decreased the demand for assistance to this population, who have chronic diseases that need strict monitoring. Consequently, there is a significant increase in mortality at home, related to cardiovascular diseases and neoplasms, as well as mental disorders caused by confinement. In Brazil, telemedicine has been used as an alternative to maintain the monitoring of these seniors, and our Unified Health System (SUS) provides long-term drugs for home delivery of those registered. However, the alternative is limited to those who have a telephone and a home close to the health units. Home visits, scheduling appointments for follow-up appointments, as well as telemonitoring are viable alternatives in times of pandemic, however access strategies must be thought of for people living in rural, riverside or island communities.

Conclusion: Primary care and family doctors must be more vigilant monitoring the senior person who is not contaminated COVID-19. It is important to implement and strengthen strategies for teams to access communities, given the size of the Brazilian territory and the peculiarities of each region. 


\section{Abstract \# 52 \\ Covid-19: adapting the geriatric organisations to respond to the pandemic}

Luc Goethals ${ }^{1}$, Thomas Célarier ${ }^{2}$, Ludovic Lafaie ${ }^{2}$, Nathalie Barth ${ }^{3}$, Baptiste Gramont $^{2}$, Etienne Ojardias ${ }^{2}$, Bienvenu Bongue ${ }^{1}$

${ }^{1}$ Chaire santé des Ainés, Université Jean Monnet, Saint-Etienne, France, ${ }^{2}$ Department of Clinical Gerontology, University Hospital of Saint-Etienne, France, ${ }^{3}$ Gérontopôle Auvergne Rhône-Alpes, SaintEtienne, France

Background: In France, $89 \%$ of hospital deaths are among people aged 65 and over. Deaths in nursing homes account for almost $40 \%$ of all deaths. French hospital geriatric departments are mobilized to make the patient flow easier, ensuring continuity of care, and coordinating all healthcare professionals. This pandemic required a major and rapid reorganisation of all the healthcare offer in general and in geriatrics in particular, to optimize the patient flow

Method: We report the impact of the pandemic of Covid-19 on geriatric wards and describes the whole reorganisation implemented to be able to receive Covid patients, as to maintain the usual care of non-Covid patients.

Results: Our teams were able to design complete flow for older Covid+ patients in just a few weeks with the creation of dedicated services, geriatric Covid-19 telephone hotlines, dedicated beds for patients from nursing homes, and mobile geriatric teams visiting nursing homes.The creation of a specific Covid-19 telephone hotline allowed a direct link between structures such as nursing homes and the hospital centre. Associated with this new organization, mobile teams propose medical care to nursing home patients at their places of residence. The close city/hospital link enables to reduce the risk of epidemics in nursing homes through training interventions, particularly on the barrier gestures to adopt during this epidemic.

Key conclusion: Given the situation and particularly in nursing homes, an adaptation of dedicated hotlines and mobile hospital teams improved the management of the patient's flow. The sustainability of these services seems necessary.

\section{Abstract \# 53 \\ A telemedicine management program for older people at risk of osteoporotic fractures during COVID-19 pandemic}

Ekaterini Zigoura ${ }^{1}$, Annarosa Floris ${ }^{1}$, Alberto Cella ${ }^{1}$, Barbara Senesi ${ }^{1}$, Camilla Prete, Alberto Pilotto ${ }^{2}$

${ }^{1}$ (1) Department of Geriatric Care, Orthogeriatrics and Rehabilitation, Galliera Hospital, Genova, Italy; ${ }^{2}(1)$ Department of Geriatric Care, Orthogeriatrics and Rehabilitation, Galliera Hospital, Genova Italy; ${ }^{2}$ Department of Interdisciplinary Medicine, University of Bari, Italy

Introduction: Osteoporotic fractures are a leading cause of morbidity and loss of functional independence in older people. During COVID19 pandemic, ordinary visits were not possible so, we developed a telemedicine management program, in order to provide the best care to our older patients at risk of osteoporotic fractures.

Methods: From March 16 to June 22, 285 patients were contacted 10 days prior to their scheduled visit. They were informed that an e-visit was possible, and were asked to email their queries and the results of their exams. The possibility of a 2 way video interaction, using a dedicated connection, was also offered thorough a dedicated electronic system (jitsi meet/Galliera). The densitometric scan (DXA) was replaced by clinical history and fracture prediction tools. A specific on-line questionnaire was elaborated to this purpose.

Results: All 285 patients have replied (mean age 76.9 years; $10 \%$ males). No one referred COVID-19 infection. Of the 115 vitamin D serum dosages received nobody presented vitamin D deficiency. Due to the lock-down, the infusions of zolendronic acid were not possible and the shift to other drugs was advised in 18 cases. 23 patients had temporally interrupted their pharmacologic treatment to prevent osteoporosis and were encouraged to continue. Patients in denosumab or teriparatide treatment were monitored. Medical report, receipts for drugs and a new follow-up date were sent by email.

Key conclusions: This telemedicine management program was well accepted and represented a bridge, between health care providers and older osteoporotic patients, in order to guarantee an effective care.

\section{Posters on COVID-19}

\section{Abstract \# 1}

Hypothesis of the use of neuroaminidase IN COVID 19 disease Mario Limodio ${ }^{1}$, Roberto Menicagli ${ }^{2}$, Marta Limodio ${ }^{3}$

${ }^{1}$ Spaziani Hospital Infective diseases UOC, ${ }^{2}$ Romabiomed Research Lab, ${ }^{3}$ Asst Frosinone Italy

MERS SARS COVID-19, (CoV).causes severe acute, often fatal respiratory syndromes and death, The $-\mathrm{CoV} S$ proteins contain an $\mathrm{N}$-terminal RBD. Our study allowed us to verify how this type of protein has a configuration that is structurally similar to glycoproteins of the "MUCINS" type with the final part consisting of saccharide groups, exactly sialic acid, that binds to the receptors of the host cellsTheir affinity to ACE 2 receptors is similar to other viruses; in this case the sialic acid present in, $(\mathrm{CoV})$, as the terminal part of the external neuroaminidase glycoprotein type, binds the ACE receptors. We used the neuroaminidase enzyme to break the bound that links the sialic acid with the rest of the glycoprotein making it impossible to link the virus to the host cell It 's very important to note, to understand the various pathological expressions of the virus in patients, as the endogenous production of neuroaminidase decreases in various pathological or physiological conditions as the age; this fact could explain why children very rarely get infectedWe conducted our preliminary tests on mucus secreted by the nose that contains many corona viruses We added the neuroaminidase and after we determinated the liberated sialic acid. The results showed that the amounts of free sialic acid increased by $90 \%$ and confirmed that the enzyme breaks the bonds between sugars and proteins, thus inactivating the virus' diffusive capacity. The other important result is that introduced neuroaminidase can compete with ACE2 receptors and further inhibit the binding of the virus to host cells.

\section{Abstract \# 2}

\section{Pharmacologic mediated unilateral fixed mydriasis}

Julie McCarthy ${ }^{1}$, Atef Michael $^{2}$

${ }^{1}$ Mary Stevens Hospice, Stourbridge, UK, ${ }^{2}$ Russells Hall Hospital, Dudley, UK

Case report: A 79-year-old lady was admitted with shortness of breath. The patient had a diagnosis of adenocarcinoma of the lung on a background of COPD. She was started on nebulised salbutamol 2.5 $\mathrm{mg}$ and ipratropium bromide $500 \mathrm{mcg}$. On Day 5 , it was observed that the patient had a fixed $6 \mathrm{~mm}$ dilated left pupils and the right pupil was $3 \mathrm{~mm}$. In the previous day the pupils were non-dilated, equal and reactive bilaterally. The patient was not on mydriatic eye drops . On examination there were no other lateralising neurological signs; cranial nerves as well as motor exam of the upper and lower limbs were normal, apart from the dilated fixed left pupil. After clinically 
excluding an acute neurological event, the mydriatic effect of ipratropium anti-muscarinic action was considered to be a potential cause for the fixed dilated pupil, however this did not explain the unilateral involvement. The patient was observed while using the nebuliser and it was noticed that she could not tolerate the mask affixed to her face to form an adequate seal, rather, she was holding it aloft and favouring her right side, such that the mist dispersed more readily to the left; this explained the unilateral manifestations. The assumption was subsequently proven upon discontinuing the ipratropium, when the pupils size, symmetry and reactivity returned to normal after around $24 \mathrm{~h}$, in keeping with timescales documented in previous case reports.

Discussion: Ipratropium bromide, as an antagonist of muscarinic acetylcholine receptors, causes mydriasis due to unopposed action of the dilator pupillae muscle, and cycloplegia due to ciliary muscle relaxation. The ocular complications of ipratropium bromide are due to the local effect of the medication; i.e. when the aerosolised ipratropium comes into contact with the eyes. The incidence of ipratropium induced ocular complications is between $1 \%$ to $<1$ per 1000 patients. Whilst there have been some case reports of ipratropium causing mydriasis, it remains in practice, a poorly recognised adverse drug reaction (ADR). Patients must be instructed in the correct administration of ipratropium nebuliser to avoid its release into the eye. Clinicians need to be aware of the uncommon mydriatic side effect of ipratropium to avoid unnecessary imaging and other investigations.

\section{Abstract \# 3}

\section{Hip fracture and the urinary bladder}

Atef Michael ${ }^{1}$, Arlinda Lago ${ }^{1}$

${ }^{1}$ Russells Hall Hospital, Dudley, UK

Introduction: Some older people have falls, resulting in hip fracture, while going to the bathroom to urinate, or coming back. The aim of this study is to analyse the "phenomenon" of hip fracture related to the urinary bladder.

Methods: Prospective observational study of consecutive patients admitted to a UK teaching hospital with hip fracture in an 8 months period. Patients, notes and electronic records were reviewed; data were downloaded on excel sheet and analysed using descriptive statistics.

Results: 377 hip fracture patients were admitted in the study period, of whom 51 patients $(14 \%)$ had falls related to the bathroom. Of these $20 \%(10 / 51)$ male and $80 \%$ (41/51) female with average age of 81.6 and 82.8 years respectively. $51 \%(26 / 51)$ had urinary incontinence. $51 \%(26 / 51)$ had hypertension and 31\% (16/51) had osteoarthritis. $67 \%(34 / 51)$ were on $>4$ drugs, $55 \%(28 / 51)$ were on antihypertensives, $22 \%(11 / 51)$ were on diuretics and $31 \%(16 / 51)$ were on an antidepressant. Falls were multifactorial; frailty in $41 \%$ (21/51) and postural hypotension in $24 \%(12 / 51)$ were considered the most contributing factors.

Discussion: The number of patients who had fractures related to the urinary bladder could be underestimated as some older people with dementia were found on the floor with no reliable history; many of them could have been going to the bathroom. There are many reasons why elderly people might fall while going to the bathroom including postural hypotension, rushing to avoid urge incontinence, hypnotic or extrapyramidal side effects of medications, not using the mobility aids and poor lighting conditions.Conclusion: In this study $14 \%$ of falls resulting in hip fracture was attributed to the urinary bladder. Urinary bladder symptoms are a frequently forgotten risk factor for falls and fractures. To reduce the risk in this group of patients, urinary tract symptoms need to be explored, diagnosed and treated. Medications; specially antihypertensives and CNS active drugs should be rationalized and diuretics are to be avoided if possible.

\section{Abstract \# 4 \\ Pharmacological Management of COVID-19 infection in older patients \\ Javier Jaramillo-Hidalgo ${ }^{1}$, Isabel Lozano-Montoya ${ }^{1}$, Pamela Carrillo- García $^{1}$, Blanca Garmendia-Prieto ${ }^{1}$, Maribel Quezada-Feijoó ${ }^{1}$, Monica Ramos-Sanchez ${ }^{1}$, Giovanna Cristofori ${ }^{1}$, Eva Fernandez De La Puente ${ }^{1}$, Gemma Cuesta-Castellón ${ }^{1}$, Javier Gómez-Pavón ${ }^{1}$ \\ ${ }^{1}$ Hospital Central De La Cruz Roja}

Objectives: No evidence about pharmacological management of COVID-19 is available today. We aim to describe the different treatments prescribed, mean duration and incidence of drug side effects in older patients affected by COVID-19.

Methods: Descriptive ambispective study. Older adults ( $\geq 75$ years) consecutively admitted to an Acute Geriatric Unit diagnosed with COVID-19 respiratory tract infection/pneumoniae from March 15 to 15, 2020 in Madrid, Spain. Treatment and side effects reported in electronic clinical records were collected.

results: 200 patients were included ( $61 \%$ women, $86 \pm 6.53$ years). 87.9\% with COVID-19 pneumonia (CURB 65: $2.21 \geq 1.04$ ) and $69 \%$ with polypharmacy. The most common drugs prescribed were: $82.7 \%$ hydroxychloroquine (mean duration $7.4 \pm 3.0$ days), 56.9\% azithromycin (mean duration $3.7 \pm 1.3$ days), $11 \%$ lopinavir/ritonavir (mean duration $7.0 \pm 4.3$ days), $2 \%$ duronavir/cobicistat; $15 \%$ recieved antiretroviral treatment + hydroxychloroquine. Drug side effects was reported in $3.9 \%$ patients treated with lopinavir/ritonavir and $11.5 \%$ treated with hydroxychloroquine (diarrhea $2.9 \%$, vomiting $2.4 \%$, hypoglycemia $1.9 \%$ ).

Conclusion: Hydroxychloroquine is the most frequent drug used to treat COVID-19 in this population and it is associated with common pharmacologic adverse effects, particularly affecting gastrointestinal system.

\section{Abstract \# 5}

\section{Delirium in older patients with COVID-19}

Alessandra Marengoni ${ }^{1}$, Alberto Zucchelli ${ }^{1}$, Giulia Grande ${ }^{2}$, Laura Fratiglioni $^{2}$, Debora Rizzuto ${ }^{3}$

${ }^{1}$ University of Brescia, ${ }^{2}$ Karolinska Institutet, ${ }^{3}$ Karolinska Instituet

Background: It is well-known that delirium is a frequent condition in medical wards and has a strong negative prognostic impact among hospitalized older patients. The aim of this study was to evaluate prevalence of delirium in older patients admitted to hospital with a suspected diagnosis of COVID-19 and its effect on mortality.

Methods: 91 patients, aged 70-years and older, consecutively admitted to an acute geriatric ward in a designated hospital in Montichiari (Brescia, Northern Italy) from March 8th to April 17th, 2020 were included. Nasal and pharyngeal swab samples were collected at hospital admission. COVID-19 cases were confirmed by reverse transcriptase-polymerase chain reaction assay for SARS-Cov-2 RNA. Delirium was diagnosed according to the DSM-V criteria. The number of chronic diseases was calculated among a pre-defined list of 60. The pre-disease Clinical Frailty Scale (CFS) was assessed at hospital admission; patients were scored from 1 "very fit" to 9 "terminally ill". In the CFS the degree of frailty corresponds to the degree of dementia if the latter is present (4). The study was notified to the Ethical Committee of the Brescia County. 
Results: Of the total sample, 39 patients died, 52 were discharged and 3 were transferred to ICU. Twenty-five patients (27.5\%) had delirium. Seventy percent of patients with delirium died during hospitalization compared to $31.8 \%$ of those without delirium. Patients with delirium were older $(81.7$ vs. 78.6 years, $\mathrm{p}<0.02)$ and more likely to be frail $(\mathrm{CSF}$, median $=5 ;$ IQR $=3-6$ vs. $3 ; 2-4, \mathrm{p}<0.001)$. Patients with or without delirium did not differ for type and frequency of COVID-19 symptoms and peripheral oxygen saturation at admission and need of non-invasive ventilation. In a multivariate logistic regression model adjusted for age, sex, number of chronic diseases, frailty, and arterial blood oxygen saturation at admission, patients with delirium were four times more likely to die during hospital stay compared to those without delirium $(\mathrm{OR}=3.98 ; 95 \%$ CI $1.05-17.28)$.

Conclusions: These findings suggest that delirium should be interpreted as an alarming sign indicating a severe infection, and, as such, used as prognostic indicator in older persons affected by COVID-19, and that delirium precipitating factors should be systematically explored in the clinical setting. Guidelines on COVID-19 should specifically address older persons' needs including prevention and management of delirium.

\section{Abstract \# 6 \\ Atypical symptoms of SARS-COV-2 infection and its association with mortality in older adults}

Isabel Lozano-Montoya ${ }^{1}$, Maribel Quezada- Feijoó ${ }^{2}$, Javier JaramilloHidalgo ${ }^{1}$, Blanca Garmendia-Prieto ${ }^{1}$, Pamela Carrillo-García ${ }^{3}$, Beatriz Pallardo-Rodil ${ }^{1}$, Gloria Izquierdo-Zamarriego ${ }^{1}$, Cristina Gianella-Blanco ${ }^{1}$, Saleta Goñi-Roson ${ }^{1}$, Mónica Ramos-Sánchez ${ }^{2}$, Javier Gómez-Pavón ${ }^{1}$

${ }^{1}$ Servicio de Geriatría, Hospital Universitario Central de la Cruz Roja, ${ }^{2}$ Servicio de Cardiología, Hospital Universitario Central de la Cruz Roja, ${ }^{3}$ Servicio de Geriatría, Hospital Universitario Central de la Cruz Roja)

Objective: To describe clinical characteristics of SARS- CoV-2 infection and its association with mortality in older adults admitted to an Acute Geriatic Unit in a Secondary teaching Hospital in Madrid, Spain.

Methods: Unicenter, descriptive ambispective longitudinal study. Older adults ( $\geq 75$ years) consecutively admitted to an Acute Geriaric Unit diagnosed with SARS-COV-2 infection (confirmed by RT-PCR or suspected by clinical and imaging) from March 15 to May 15, 2020 were included. Sociodemographic data, geriatric assessment (Barthel Index-BI, Clinical Frailty Scale-CFS, Global Deterioration ScaleGDS, length of hospital stay and symptoms were collected from the electronic medical records.

Results: 200 patients were included (61\% women, $86 \pm 6.53$ years). Length of hospital stay (12.3 \pm 8 days). The median time from onset of symptoms to first hospital admission was $5.7 \pm 3.7 .39 .7 \% \mathrm{BI} \leq$ $60,30.1 \%$ GDS $\geq 5,73 \%$ CFS $\geq 5$. The most common symptoms at onset of illness were: dyspnoea $63.1 \%$, fever $57.5 \%$, anorexy $55.1 \%$, cough $47.8 \%$, delirium $39.1 \%$, falls $9.2 \%$ and diarrhea $5.3 \%$. Global in-hospital mortality was $39.9 \%$. Delirium was independently associated with mortality $(22.2 \%$ vs $16.3 \%, \mathrm{p}=0.001)$.

Conclusion: Atypical symptoms (anorexy, delirium and falls) are common as clinical presentation of SARS-CoV-2 infection in older patients admitted to hospital. Delirium was highly prevalent and it was found to be a predictor of in-hospital mortality.

\section{Abstract \# 7}

The radiographic pulmonary ground-glass findings need careful evaluation

Sara Gabriele ${ }^{1}$, Sergio Minervini ${ }^{1}$, Roberto Scheisi ${ }^{1}$, Renzo Girardello $^{1}$

${ }^{1}$ Department of Medicine, Section of Geriatrics, Rovereto Hospital, Azienda Provinciale per i Servizi Sanitari (APSS) di Trento, Italy.

Common radiology findings of COVID-19 include interstitial thickening and multilobe ground-glass opacities (GGO) with peripheral and lower lung zone distribution. We describe an 81-year-old woman with diarrhea and features of COVID-19 on chest high-resolution computed tomography (HRCT). No symptoms of upper and lower respiratory tract infection or influenza-like symptoms were described. Either COVID-19 IgM + IgG Rapid Test Kit and the nasopharynx swab test resulted in negative; however, she was admitted to COVIDunit and considered infected for gastroenteric syndrome and pattern of interstitial pneumonia. Focusing on her history, we discovered that the pulmonary fibrosis was already known in her clinical data, probably caused by Rheumatoid Arthritis and Amiodarone. Furthermore, she has been suffering from chronic colitis with periodic reactivation. In conclusion, even during a worldwide outbreak of COVID-19, the radiographic findings need to be correlated with symptoms, history, physical exam, laboratory, and microbiological tests. Specifically, this case highlights that in a patient population with a low pretest probability of COVID-19 infection, the typical GGO imaging features should be interpreted with caution.

\section{Abstract \# 8 \\ Age-related differences in clinical presentation of COVID-19 and factors associated with mortality: a retrospective analysis of patients hospitalized during the pandemic peak}

Andrea Ticinesi ${ }^{1}$, Antonio Nouvenne ${ }^{1}$, Nicoletta Cerundolo ${ }^{1}$, Loredana Guida ${ }^{1}$, Giulia Chiussi ${ }^{1}$, Ilaria Morelli ${ }^{1}$, Giampiero Castaldo $^{1}$, Alberto Parise ${ }^{1}$, Bruno Agozzino ${ }^{1}$, Dario Magnani ${ }^{1}$, Erminia Ridolo $^{2}$, Beatrice Prati ${ }^{1}$, Angela Guerra ${ }^{2}$, Anna Nardelli ${ }^{1}$, Tiziana Meschi ${ }^{3}$

${ }^{1}$ Azienda Ospedaliero-Universitaria di Parma, Parma, Italy, ${ }^{2}$ Department of Medicine and Surgery, University of Parma, Parma, Italy, ${ }^{3}$ Department of Medicine and Surgery, University of Parma

Background: The clinical presentation of several infective diseases is different in older than adult patients. Our aim was to compare the clinical presentation of coronavirus-19 disease (COVID-19) across age groups $(\leq 55,56-65,66-75,76-85,>85)$ of patients hospitalized at Parma University-Hospital, Italy, and identify predictors of adverse outcomes.

Methods: We retrospectively reviewed the clinical records of 594 patients admitted for suspect COVID-19 (respiratory symptoms or fever plus chest CT positive for alveolar-interstitial pneumonia) during the pandemic peak. Data on symptoms and their duration, extension of lung parenchyma involvement at CT, baseline lab tests at ward admission, comorbidities, medications, CHA2DS2-Vasc score, and outcomes were collected.

Results: At admission, older patients had lower prevalence of fever and cough, and higher prevalence of dyspnea ( $\mathrm{p}$ for trend $<0.001$ for all symptoms). Older patients sought hospital care earlier than adults (duration of symptoms before hospitalization $6 \pm 4$ days for age $>85$ vs $9 \pm 6$ days for age $<55, \mathrm{p}<0.001)$, higher chest $\mathrm{CT}$ visual scoring extension, neutrophil counts, blood creatinine, International Normalized Ratio, C-reactive protein and D-dimer, and lower $\mathrm{PaO}_{2}$ at arterial blood gas analysis and hemoglobin levels ( $\mathrm{p}$ for trend $<0.001$ 
for all tests). They also had higher number of comorbidities, medications and CHA2DS2Vasc score. In a stepwise linear regression model, the factors significantly $(\mathrm{p}<0.05)$ associated with mortality were $\mathrm{PaO}_{2}(\beta=-0.239)$, CHA2DS2Vasc score $(\beta=0.205)$, D-dimer $(\beta=0.144)$, creatinine $(\beta=0.106)$ and $C$ T visual scoring extension $(\beta$ $=0.116$ ).

Conclusions: COVID-19 has a different clinical presentation in older patients. Laboratory tests, CT parameters and CHA2DS2Vasc score may provide useful prognostic information.

\section{Abstract \# 9 \\ Delirium in COVID-19: an analysis of a large group of patients admitted to an academic hospital in Northern Italy}

Andrea Ticinesi ${ }^{1}$, Alberto Parise ${ }^{1}$, Nicoletta Cerundolo ${ }^{1}$, Angela Guerra $^{2}$, Loredana Guida ${ }^{1}$, Giulia Chiussi ${ }^{1}$, Ilaria Morelli ${ }^{1}$, Giampiero Castaldo $^{1}$, Alberto Parise ${ }^{1}$, Bruno Agozzino ${ }^{1}$, Dario Magnani ${ }^{1}$, Erminia Ridolo ${ }^{2}$, Beatrice Prati ${ }^{1}$, Anna Nardelli ${ }^{1}$, Antonio Nouvenne ${ }^{1}$, Tiziana Meschi ${ }^{2}$

${ }^{1}$ Azienda Ospedaliero-Universitaria di Parma, Parma, Italy, ${ }^{2}$ Department of Medicine and Surgery, University of Parma, Parma, Italy

Background: Delirium is a well-known complication of acute infections in geriatric patients. The incidence and clinical correlates of delirium in patients with coronavirus disease-19 (COVID-19) are still poorly investigated.

Methods: We retrospectively reviewed the clinical records of 673 patients hospitalized for suspect COVID-19 (respiratory symptoms or fever plus chest CT positive for alveolar-interstitial pneumonia) during the pandemic peak in Parma, Italy. Delirium during stay was defined in accordance with the Confusion Assessment Method criteria. Data on age, gender, clinical presentation of COVID-19, comorbidities, drugs, functional performance, extension of lung parenchyma involvement at chest CT and baseline lab tests were collected. The outcome (survival vs death) was also recorded.

Results: Delirium was detected in 65 patients $(9.7 \%$ of the population). Patients who developed delirium were in average older (age 83 \pm 10 vs $72 \pm 15, \mathrm{p}<0.001)$, had a higher number of chronic comorbidities $(3.7 \pm 1.9$ vs $2.9 \pm 2.1, \mathrm{p}<0.01)$, and chronic medications $(5.6 \pm 3.4$ vs $4.0 \pm 3.3, \mathrm{p}<0.001)$, and were less performant (frequency of autonomy before hospitalization $25 \%$ vs $66 \%$, p < 0.001). The baseline clinical presentation of COVID-19 was also different in those who developed delirium, with lower frequency of cough, higher burden of acidosis at blood gas analysis, higher serum sodium, lactate dehydrogenase, creatine-phosphokinase, D-dimer and partial thromboplastin time (age- and sex-adjusted p-values $<0.01$ ). Delirium was also associated with a higher mortality (46\% vs $22 \%$, p $<0.001)$.

Conclusions: Delirium is a common complication of COVID-19, especially in older patients with disability, multimorbidity and polypharmacy. Several laboratory alterations typical of COVID-19 may be associated with a higher risk of delirium.

\section{Abstract \# 10 \\ Guillai-Barré Syndrome as first symptom of Covid-19. Case reports}

Axelle Stockmans ${ }^{1}$, Hilde Baeyens ${ }^{1}$, Leen Derez ${ }^{1}$, Kyri Van Hecke ${ }^{1}$, Jean-Pierre Baeyens ${ }^{2}$

${ }^{1} \mathrm{AZ}$ Alma Eeklo, ${ }^{2} \mathrm{AZ}$ Alma Eeklo, Be; Univ.Lux.
A lady ( 84 y) was admitted because of frequent falls the last days. The second day of admission she was not longer able to stand on her legs. The next day she was not able to take her food of her GSM. This progressive ascending paralysis was symptomatic for a Guillain-Barré Syndrome. A lumbar puncture confirmed the albumin-cytologic dissociation. There was no fever and no respiratory symptoms. Because of a paper about this subject in the NEJM, a swap test was performed and was Positive for Covid-19. With the classical therapy with gammaglobulines she recovered in a few weeks. She did not develop any respiratory symptoms, nor GI symptoms. A second lady (92y), was admitted already 2 weeks with COVID respiratory disease. While recovering from her COVID, with declining need for oxygen, she had again fever and falls. Few days later, she developed dysestesias in fingers and toes. She could not take her drink by herself any more. A progressive paralysis of legs and arms was apparent. A LP indicates an albumin-cytologic dissociation. She recovered fully after therapy with gammaglobulines. Guillain-Barré Syndrome is generally associated with viral infections and vaccinations. It is not surprising to see this syndrome associated with Covid-19, but in the first case it was the only symptom!

\section{Abstract \# 11 \\ Characteristics and determinasnts of outcomes among older adults with COVID-19 infection treated in University Malaya Medical Centre (UMMC), Malaysia}

G. H. M. Pang ${ }^{1}$, C. N. Thiam ${ }^{1}$, K. Hasmukharay ${ }^{2}$, W. C. Lim ${ }^{1}$, A. Abdullah $^{3}$, C. C. $\mathrm{Ng}^{1}$, N. I. Saedon ${ }^{4}$, M. P. Tan ${ }^{4}$, H. M Khor ${ }^{4}$, A. V. Chin ${ }^{4}$, K. M. Tan ${ }^{4}$, S. B. Kamaruzzaman ${ }^{4}$, T. Ong ${ }^{4}$

${ }^{1}$ Ministry of Health Malaysia, ${ }^{2}$ University Malaya Medical Center, ${ }^{3}$ Pusat Perubatan Pakar UITM, ${ }^{4}$ University Malaya Medical Centre

Background: The Covid-19 pandemic poses high risk to older adults, leading to atypical presentation, higher morbidity and mortality world-wide.

Methods: Retrospective analysis of 26 Covid-19 patients aged 65 or more treated in UMMC from 01.02.2020 to 15.4.2020. All data were traced from electronic medical record. Basic demographics and clinical characteristics were analysed for association with in-patient mortality, critical illness (defined as Covid-19 category $4 \& 5$, cytokine release syndrome), and presence of complication (defined as delirium, deconditioning, pressure ulcer, pneumonia, and thromboembolism).

Results: Patients with higher C-reactive protein CRP (median $203.5 \mathrm{mg} / \mathrm{L}$, IQR $138.4 \mathrm{mg} / \mathrm{L}, \mathrm{p}=0.019$ ), higher ferritin (median $1868.1 \mathrm{ug} / \mathrm{L}, \mathrm{IQR} 1381.8 \mathrm{ug} / \mathrm{L}, \mathrm{p}=0.02$ ) were significantly associated with in-patient mortality. Clinical frailty score (CFS) 6-9, male gender, nursing home resident status, basic ADL, lower absolute lymphocyte count (ALC) were not associated with in-patient mortality. Only higher ferritin (mean $1590 \mathrm{ug} / \mathrm{L}$, SD $906.8 \mathrm{ug} / \mathrm{L}, \mathrm{p}=$ 0.004) was associated with critical illness whereas age, gender, CFS score, lower ALC, higher CRP were not significantly associated with critical illness. Patients more frail CFS 6-9, $\mathrm{n}=5$ (19.2\%) vs CFS $1-5, \mathrm{n}=21(80.8 \%), \mathrm{p}=0.045$ and higher CRP (mean $121.8 \mathrm{mg} / \mathrm{L}$, SD $75.0 \mathrm{mg} / \mathrm{L}, \mathrm{p}=0.01$ ) were associated with development of complication. Age, gender, lower ALC, higher ferritin were not associated with presence of complication. The commonest complications were delirium and deconditioning.

Conclusion: Biomarkers such as higher CRP and ferritin were associated with adverse clinical outcomes in post Covid-19 infection. 


\section{Abstract \# 12 \\ Association between hypernatraemia and mortality in older people hospitalized for COVID-19: a prospective study}

Francesco Bolzetta ${ }^{1}$, Monica Maselli ${ }^{1}$, Marino Formilan ${ }^{1}$, Flavio Busonera ${ }^{1}$, Paolo Albanese ${ }^{1}$, Federica Chiaromanni ${ }^{1}$, Antonietta Romano $^{1}$, Nicola Veronese ${ }^{2}$

${ }^{1}$ Medical Department, Geriatric Unit, Azienda ULSS (Unità Locale Socio Sanitaria) 3 "Serenissima", Dolo-Mirano District, Italy, ${ }^{2}$ Azienda ULSS 3 (Unità Locale Socio Sanitaria) "Serenissima", Primary Care Department, Dolo-Mirano District, Venice, Italy

Background: Coronavirus disease 19 (COVID-19) is a global outbreak. This infectious disease seems to be associated with a higher rate of dehydration and hypernatraemia. However, the clinical meaning of hypernatraemia in COVID-19 is still unknown. Given this background, the aim of this work is to investigate if serum sodium at admission can predict mortality in older people hospitalized for COVID-19.

Methods: Data were collected in the Geriatric Section of the Dolo Hospital, ULSS 3 "Serenissima", Venice from 31st March to 01st May 2020. Serum sodium levels were categorized as $<135$ (hyponatraemia), 135-145 (normal) and $>145$ (hypernatraemia) $\mathrm{mEq} / \mathrm{L}$. Vital status was assessed using administrative data. Cox's regression analysis, adjusted for potential confounders, was used for assessing the strength of the association between serum sodium levels and mortality. The data were reported as hazard ratio (HR) with $95 \%$ confidence intervals (CIs).

Results: 84 older people (mean age: 84.1 years; females $=61.9 \%$ ) were included. Hypernatraemia was present in the $42.8 \%$ of the people included. People with hypernatraemia were significantly older than other people. Taking people with normal sodium levels as reference and adjusting for 15 potential confounders, people with hypernatraemia reported a significant higher risk of mortality $(\mathrm{HR}=$ 3.06; 95\% CI 1.04-9.96)

Conclusions: Our paper shows that hypernatraemia is a common condition in COVID-19 and significantly associated with poor prognosis in older people hospitalized for COVID-19, suggesting that a good hydration is important for a good prognosis during the clinical course of this condition.

\section{Abstract \# 13 \\ Prognostic value of c-reactive protein in older people hospitalized for COVID-19: a prospective study}

Marino Formilan ${ }^{1}$, Francesco Bolzetta ${ }^{1}$, Monica Maselli ${ }^{1}$, Flavio Busonera $^{1}$, Paolo Albanese ${ }^{1}$, Federica Chiaromanni ${ }^{1}$, Antonietta Romano $^{1}$, Nicola Veronese ${ }^{2}$

${ }^{1}$ Medical Department, Geriatric Unit, Azienda ULSS (Unità Locale Socio Sanitaria) 3 "Serenissima", 30031, Dolo, Mirano District, Italy, ${ }^{2}$ Azienda ULSS 3 (Unità Locale Socio Sanitaria) "Serenissima", Primary Care Department, Dolo-Mirano District, Venice, Italy

Background: Coronavirus disease 19 (COVID-19) is a global outbreak. Inflammation seems to have an important role in the course of this disease, but if elevated plasma C-reactive protein (CRP) levels can predict mortality in older people is still unknown. Given this background, the aim of this work is to investigate if plasma CRP levels at admission can predict mortality in older people hospitalized for COVID-19.

Methods: Data were collected in the Geriatric Section of the Dolo Hospital, ULSS 3 "Serenissima", Venice from 31st March to 01st May 2020. Plasma CRP levels were categorized in tertiles $(<38$, $38-98,>98 \mathrm{mg} / \mathrm{l})$. Vital status was assessed using administrative data. Cox's regression analysis, adjusted for potential confounders, was used for assessing the strength of the association between plasma CRP levels and mortality. The data were reported as hazard ratio (HR) with $95 \%$ confidence intervals (CIs).

Results: 84 older people (mean age: 84.1 years; females $=61.9 \%$ ) were included. No significant differences in terms of demographic and clinical characteristics emerged across CRP tertiles. Taking people in the lowest tertile as reference and adjusting for 15 potential confounders, people in the highest CRP levels tertile reported a significant higher risk of mortality ( $\mathrm{HR}=5.49$; 95\% CI 2.01-14.97). Each increase in $10 \mathrm{mg} / \mathrm{l}$ of CRP was associated with an increased risk of mortality of $9 \%(\mathrm{HR}=1.09$; $95 \%$ CI 1.04-1.14).

Conclusions: Our paper shows that CRP is strongly associated with poor prognosis in older people hospitalized for COVID-19 suggesting that inflammation may play a pivotal role in the pathogenesis of this infectious disease.

\section{Abstract \# 14}

\section{UNderstanding COVid in the VulnERable (UNCOVER)- experience from a tertiary centre in Malaysia}

Kejal Hasmuk ${ }^{1}$, Nor Izzati Saedon ${ }^{1}$, Terence Ong ${ }^{1}$, Shahrul Bahyah Kamaruzzaman ${ }^{1}$, Hui Min Khor ${ }^{1}$, Kit Mun Tan ${ }^{1}$, Maw Pin Tan ${ }^{1}, \mathrm{Ai}$ Vyrn Chin ${ }^{1}$, Wan Chieh Lim ${ }^{1}$, Siew Ping Loh ${ }^{1}$, Chiann Ni Thiam ${ }^{1}$, Aimy Abdullah ${ }^{1}$, Chai Chen $\mathrm{Ng}^{1}$, Gordon Pang Hwa Mang ${ }^{1}$, Anjanna Kukreja $^{2}$, Juita Sirimanne Dewi ${ }^{3}$, Bushra Megat Johari ${ }^{2}$, Han Cheng $\mathrm{Ong}^{4}$, Sazali Basri ${ }^{4}$, Pui Li Wong ${ }^{4}$, Sharifah Faridah Syed Omar ${ }^{4}$, Asma Sohail $^{2}$, Sasheela Ponnampalavanar ${ }^{2}$, Adeeba Kamarulzaman ${ }^{2}$

${ }^{1}$ Geriatric Unit, University Malaya Medical Centre (UMMC), ${ }^{2}$ Infectious Disease Unit, University Malaya Medical Centre (UMMC), ${ }^{3}$ Medical Department, Hospital Sarikei, Sarawak, Malaysia, ${ }^{4}$ Infectious Disease Unit, University Malaya Medical Centre (UMMC)

Introduction: The COVID-19 pandemic has unveiled the vulnerability and resilience of older persons globally. We aim to describe their atypical presentations and clinical characteristics.

Methods: In this retrospective, single-centre study, we included all confirmed cases of SARS-CoV2 aged $\geq 65$ in University Malaya Medical Centre. Demographics, clinical presentations and treatment data were extracted from electronic medical records.

Results: As of April 30th 2020, there were 138 COVID-19 cases confirmed by rt-PCR of which $26(18.8 \%)$ were aged $\geq 65$.The average age was 76.2 years (SD8.2). The mean Charlson Comorbidity Index (CCI) was 5 (SD 1.8) with hypertension being the most common $(19[73.1 \%])$ followed by diabetes $(16[61.5 \%])$ and ischemic heart disease (5[19.2\%]). The average length of stay was 27.9 days (SD:16.9). Frailty assessments revealed $12(50 \%)$ having Clinical Frail Scale (CFS) of $\geq 5$. The median day of illness at presentation was 4.5 (IQR:6).Twenty-three $(88.5 \%)$ were symptomatic at presentation; 7 (26.9\%) presented with new onset delirium, 10 (38.5\%) with lethargy and loss of appetite, $5(19.2 \%)$ with diarrhoea, abdominal discomfort, nausea and only $8(30.8 \%)$ and $15(57.7 \%)$ presented with shortness of breath and cough respectively. Three (11.5\%) remained asymptomatic throughout hospitalization. The median laboratory parameters on presentation were: white cell count 6.9 (IQR: 5.9-9.9), absolute lymphocyte count (ALC) 1.28 (IQR 1.03-2.11) and C-Reactive Protein 35.7 (IQR 9.3-90.4). Median duration of viral shedding was $20 \cdot 5$ days (IQR16.5-26.25). The longest observed duration of viral shedding was 57 days, in our sole nonagenarian. We had twenty $(76.9 \%)$ survivors.

Key conclusions: Cases were first detected primarily through contact tracing. Delirium, lethargy and gastrointestinal symptoms were common, with respiratory symptoms often absent. Contact tracing and 
a high index of suspicion appears vital in addressing COVID-19 in older adults.

\section{Abstract \# 15 \\ Age as the strongest risk factor for mortality in COVID-19 PAtients \\ Francesca Mazzeo ${ }^{1}$, Giulia Cesaroni ${ }^{1}$, Alberto Zaniboni ${ }^{1}$, Marco Trabucchi ${ }^{1}$, Angelo Bianchetti ${ }^{2}$, Anette Ranhoff ${ }^{3}$, Renzo Rozzini ${ }^{1}$ \\ ${ }^{1}$ Fondazione Poliambulanza Istituto Ospedaliero, ${ }^{2}$ Istituto Clinico Sant'Anna, ${ }^{3}$ University of Bergen - Diakonhjemmet Hospital}

Introduction: COVID-19 shows a considerably higher mortality rate in elderly persons, with chronic diseases and frailty. This study assesses the independent effect on mortality of age-related diseases and age itself.

Methods: Data were collected in our hospital Fondazione Poliambulanza Istituto Ospedaliero (Brescia), between March 1 and April 30, 2020. 1416 patients (mean age: $68.3+13.9$, males $=$ $934.66 \%$ ) with interstitial pneumonia were assessed.

Results: In-hospital mortality was $20.8 \%(\mathrm{n}=257$, age $79.8+8.1$ ). Associated with in-hospital mortality were: old age, i.e. age $(>85$ years) (HR 10.7; 95\% CI 7.0-16.5), gender (HR 1.6; 95\% Cl 1.2-2.0), platelet count $(<150.000 /$ micrlL) (HR $1.8 ; 95 \% \mathrm{Cl} 1.4-2.4)$, C-Reactive Proteine $(>100 \mathrm{mg} / \mathrm{l})(\mathrm{HR} 3.9 ; 95 \% \mathrm{Cl} 1.4-2.4)$, Lactate Dehydrogenase ( $>150$ U/l) (HR 4.9; 95\% Cl 3.7-6.5), Creatine Kinase $(>100 \mathrm{U} / \mathrm{dl})(\mathrm{HR} 3.6 ; 95 \% \mathrm{Cl} 2.8-4.7)$, serum albumin $(<3.0$ $\mathrm{gr} / \mathrm{dlm})(\mathrm{HR} 1.8 ; 95 \% \mathrm{Cl} 1.3-2.3), \mathrm{PaO} 2 / \mathrm{FiO} 2$ (per point increase) (HR 0.992; 95\% Cl 0.991-0.993), diabetes mellitus (HR 1.3; $95 \% \mathrm{Cl}$ 1.0-1.7), coronary disease (HR 2.3; $95 \% \mathrm{Cl} 1.8-3.0$ ), acute renal disease (HR 2.4; $\mathrm{Cl} 95 \%$ 1.8-3.2) and chronic kidney disease (HR 4.4; $95 \% \mathrm{Cl} 3.3-5.7)$. When patients with $<65$ years old are the reference different age classes maintain their independent negative risk of mortality (65-69 HR: $1.695 \%$ CI 1.0-2.7; 70-74, HR: $3.395 \%$ CI 2.0-5.6; 75-79: HR: 3.9 95\% CI 2.3-6.5; 80-84: HR 5.4 95\% CI 3.2-9.2; 85+: HR 5.7, 95\% CI 3.3-9.9).

Key conclusions: Age has an impressive independent association with mortality (more than frailty or comorbidity). This fact should be taken in prevention, prognosis and medical decisions.

\section{Abstract \# 16 \\ Delirium: clinical presentation and outcomes in a series of elderly Covid-19 patients \\ Renzo Rozzini ${ }^{1}$, Angelo Bianchetti ${ }^{2}$, Giulia Cesaroni ${ }^{3}$, Flaminia Coccia $^{1}$, Marco Trabucchi ${ }^{1}$ \\ ${ }^{1}$ Fondazione Poliambulanza Istituto ospedaliero, ${ }^{2}$ Istituto clinico Sant'Anna, ${ }^{3}$ Fondazione Poliambulanza Istituo ospedaliero}

Introduction: Aim of this cases report is to describe the clinical characteristics and outcomes of a series of 14 older patients (males 11) consecutively admitted in non-ICU ward because of SARS-CoV2 infection who developed hypo- or hyperkinetic delirium.

Methods: Clinical characteristics (somatic and psychic diseases, and function) are assessed. Delirium was detected through 4At (assessment test for delirium and cognitive impairment).

Results: Hyperactive delirium with aggression and agitation was observed in 6/14 patients, The overall mortality rate was $71 \%(10 / 14$ patients). The 6 patients with hypokinetic delirium died. Among the 8 patients with hyperkinetic delirium 4 died. Among the 4 survivors we observed two different clinical patterns: 2 patients had dementia but not ARDS, and 2 patients had no dementia, but ARDS.

Conclusions: All subjects developing hypokinetic delirium died. This form of delirium is associated with worse outcomes, particularly among patients affected by dementia [1]. Hypokinetic delirium needs to be considered a marker of poor prognosis even in previously fit patients. Hyperkinetic delirium in cognitively unimpaired patients with mild ARDS has a better prognostic value than hypokinetic delirium in those with the same lung impairment. Based on our observations we hypothesize that delirium subtypes may markers of biological severity of precipitating disease in COVID-19 patients too. References:

[1] Rosgen BK, Krewulak KD, Stelfox HT, et al. The association of delirium severity with patient and health system outcomes in hospitalized patients: a systematic review. Age Ageing. 2020 Apr 28. pii: afaa053. https://doi.org/10.1093/ageing/afaa053. [Epub ahead of print]

\section{Abstract \# 17}

Is viral load a marker of disease severity and adverse clinical outcome in Older adults with COVID-19 infection?

Chiann Ni Thiam ${ }^{1}$, Kejal Hasmukharay ${ }^{2}$, Gordon Hwa Mang Pang ${ }^{1}$, Wan Chieh Lim ${ }^{1}$, Chai Chen $\mathrm{Ng}^{1}$, Aimy Abdullah ${ }^{3}$, Nor Izzati Saedon ${ }^{4}$, Kit Mun Tan ${ }^{4}$, Hui Min Khor ${ }^{4}$, Ai-Vyrn Chin ${ }^{4}$, Maw Pin $\mathrm{Tan}^{4}$, Shahrul Bahyah Kamaruzzaman ${ }^{4}$, Terence Ing Wei Ong ${ }^{4}$

${ }^{1}$ Ministry of Health Malaysia, ${ }^{2}$ University of Malaya Medical Centre, ${ }^{3}$ Pusat Perubatan Pakar, UITM, ${ }^{4}$ University Malaya Medical Centre

Background: Older adults with COVID-19 infection have worse clinical outcomes. Real-time PCR (RT-PCR) assay indicated by the cycle threshold $(\mathrm{Ct})$ not only can confirm COVID-19 infection but also determine the viral load (inversely proportional to $\mathrm{Ct}$ Value). This study aims to look for an association of viral load with disease severity and adverse clinical outcome.

Methods: Data were extracted from an observational study of older adults diagnosed with COVID-19 infection treated in the University Malaya Medical Centre (UMMC) from 1 March 2020 to 31 May 2020. Disease severity is based on clinical symptoms either asymptomatic, mild respiratory symptoms, mild to moderate symptoms with pneumonia, or severe symptoms. Adverse clinical outcomes include mortality, prolonged hospitalization, inpatient complications, and functional disabilities.

Results: Out of 26 patients, 9 (34.6\%) were asymptomatic or had mild symptoms, $17(65.4 \%)$ had pneumonia or severe symptoms. 6 (23.1\%) died, $23(88.5 \%)$ had prolonged hospitalization, 11 (42.35) had inpatient complications, and $6(23.1 \%)$ developed new functional disabilities. Patients who had pneumonia or severe symptoms $(\mathrm{M}=$ $21.73, \mathrm{SD}=5.53$ ) compared to asymptomatic patients or with mild symptoms $(\mathrm{M}=23.06, \mathrm{SD}=4.81)$ did not have significantly lower $\mathrm{Ct}$ value, $\mathrm{t}^{21}=0.571, \mathrm{p}$-value $=0.574$. Ct value was significantly lower in patients who died $(\mathrm{M}=19.58, \mathrm{SD}=1.56)$ than those survived $(\mathrm{M}=$ 23.12, $\mathrm{SD}=5.77), \mathrm{t}(20.5)=2.3, \mathrm{p}$-value $=0.032$, and patients developed inpatient complications $(M=19.78$, SD 3.18) than those $\operatorname{did} \operatorname{not}(\mathrm{M}=23.74, \mathrm{SD}=5.77), \mathrm{t}(20.7)=2.12$, $\mathrm{p}$-value $=0.047$.

Conclusion: Despite the lack of association with disease severity, viral load was weakly associated with mortality and inpatient complications. Therefore, further studies on other predictors are recommended to guide the management of COVID-19 infection in older adults. 


\section{Abstract \# 18}

\section{Arrhytmias and COVID 19: another clinical sign.}

Carmen Elías de Molins Peña ${ }^{1}$, Jorge Eduardo Corrales Cardenal ${ }^{1}$, Julia Castillo Garcia ${ }^{1}$, Beatriz Gamboa Huarte ${ }^{2}$

${ }^{1}$ Hospital Nuestra Señora de Gracia, Zaragoza, Spain, ${ }^{2}$ Hospital Nuestra Señora de Gracia

Introduction: 87 years old patient, personal history of: high blood pressure, type 2 diabetes, transient ischemic accident, chronic anaemia. In treatment with: omeprazole, metformin, acetylsalicylic acid (ASA), trazodone and finasteride. Geriatric assesment: widower, lives alone, no family support. Barthel's index 95/100, VIG-Frail: 0, 26 (Low frailty). No cognitive impairment.

Case study: Admitted in an acute care geriatric ward in April with respiratory symptoms: cough, dispnea in absence of fever. Chest x-ray shows a condensation in the right middle lobe, without changes in blood tests, and two negative swab test for COVID 19, despite his close contact with a COVID 19 (+) patient; was treated as a community-acquired pneumonia, and discharged without a clear radiological improvement. A month later re-admitted, first episode of atrial fibrillation, high levels of troponin, without an acute coronary syndrome and lymphopenia, high levels of D-Dimer and lactate deshydrogenase. Chest $\mathrm{x}$-ray showed bilateral peripheral opacities, described as interstitial pattern. Swab test persisted negative.Thoracic CT showed pulmonary fibrosis pattern, bilateral areas of ground glass pattern predominantly in right hemitorax. Serology for COVID 19 was positive. Due high suspicion of infection started empirical treatment for COVID 19: hydroxychloroquine, azithromycin, ceftriaxone, low molecular weight heparin. During follow up, swab test persisted negative, and light improvement in chest x-rays. DiscussionArrhytmias has been described as a cardiac clinical sign in COVID 19 infection, such as atrial fibrillation. Considered this case as a false negative, given the clinical manifestations, blood tests alteration and suggestive imaging and persistently negative swab test.

\section{Abstract \# 19 \\ Arterial ischemia and COVID 19-Atypical case \\ Jorge Eduardo Corrales Cardenal ${ }^{1}$, Julia Castillo García ${ }^{1}$, Carmen Elías de Molins Peña ${ }^{1}$, Beatriz Gamboa Huarte ${ }^{1}$ \\ ${ }^{1}$ Hospital Nuestra Señora de Gracia, Zaragoza, Spain}

95 years old patient, personal history: high blood pressure, chronic renal failure. In treatment with: paracetamol and trazodone. Geriatric assesment: Lived at home with his wife, severe dependency for daily life activities, Barthel's index: 15/100. Mild cognitive impairment. VIG-Frail: 0.44 , moderate risk of mortality in a year. Starts with gangrene in his right foot toes, without knowing when it started. No fever, cough, chest pain or risk contact with a COVID 19 patient. Swab test in emergency room being negative. Assessed by vascular surgery which ruled out revascularization considering the case as a irreversible lower limb ischemia, if worsening consider amputation.Treated at first with anticoagulants + broad spectrum antibiotics, ischemia progressed to the heel in a few days, presented fever once, blood cultures were drawn being negative. Family at first preferred a conservative approach over amputation, with pain management but due to clinical stability, decided to amputate. The patient persisted asymptomatic, and a new swab test to discard COVID19 infection was made before surgery being Positive. Pre surgical Chest X-ray showed left lung infiltrates, and increased acute phase reactants and hepatic enzymes. Surgery was ruled out, and started treatment with Ceftriaxone + Azythromicin. Progressive worsening: - Fever, low oxygen saturation, low level of consciousness.- Chest X-Ray: bilateral multilobar condensations. Adequacy of the therapeutic effort is decided due to refractory symptoms. Arterial thrombotic complications in COVID 19 patients is described. In geriatrics patients the presentation might be atypical and there could be a higher number of false negative testing.

\section{Abstract \# 20 \\ A COVID-19 SURVIVOR: a case report of a 91-year-old male with chronic respiratory insufficiency with co-infection by SARS- Cov-2 and rhinovirus}

NGOULIE Reine ${ }^{1}$

${ }^{1}$ Department of Internal Medicine, CHU Brugmann, Université Libre de Bruxelles, Brussels, Belgium

Abstract: In December 2019, Wuhan authorities in china declared the first cases of pneumonia of an unknown origin. It is now well established that the cause is a new coronavirus, SARS-CoV-2. The disease, COVID-19, progressed to a pandemic. In this case report, we describe the first patient hospitalised with COVID-19 in CHU Brugmann, a Belgium university hospital. A 91-year-old male presented initially with anorexia and vomiting, then with cough and acute respiratory insufficiency. The patient already suffered from chronic restrictive respiratory insufficiency with sequelae of a previous tuberculosis infection. Other relevant past medical history consisted in arterial hypertension, protein malnutrition and functional disabilities. A bacterial co-infection had been reasonably excluded by extended microbiological and serological testing. In addition, no significant reduction of blood inflammatory level was observed after a cure with an empirical broad-spectrum antibiotic. With additional investigations, we concluded in a viral pneumonia due to a co-infection by SARS-COV-2 and rhinovirus. The patient was discharged after 25 days.

Message: The risk of death in hospitalized patients with COVID- 19 increases with age. According to currently available data in Belgium, $90 \%$ of deceased hospitalized patients are over 64 years old. This patient, aged over 90 years old with COVID-19, survived in spite of other potential severity criteria such as co-infection with rhinovirus, chronic restrictive respiratory insufficiency, arterial hypertension and geriatric syndromes. It would be interesting to further investigate the general profile of the elderly population who underwent a worse disease evolution to gain insight of the risk factors.

\section{Abstract \# 21 \\ Stereotype of Covid-19 pathology at the senior patient with associated comorbidities}

Sabinne Taranu ${ }^{1}$, Anca Iuliana Pislaru ${ }^{2}$, Adina Carmen Ilie ${ }^{3}$, Ioana Alexandra Sandu ${ }^{2}$, Ramona Stefaniu ${ }^{2}$, Anna Marie Herghelegiu ${ }^{4}$, Catalina Raluca Nuta ${ }^{4}$, Ovidiu Lucian Bajenaru ${ }^{4}$, Gabriel Ioan Prada ${ }^{4}$, Ioana Dana Alexa ${ }^{2}$

${ }^{1}$ CI Parhon Hospital Iasi, ${ }^{2}$ University of Medicine and Pharmacy Iasi, ${ }^{3}$ University of Medicine and Pharmacy, ${ }^{4}$ University of Medicine and Pharmacy Bucharest

COVID-19 is an infectious pathology that predisposes the senior patients to typical and majoritar symptomatic forms. In a pandemic context, all specialties, but especially surgical ones, suffer the impasse of therapeutic delays. We present the case of a 67 -year-old patient, with neurological, cardiovascular and pulmonary comorbidities, no urological history, difficult to manage anamnestic. He arrived at the emergency room, accusing macroscopic hematuria, additionally associating cough without expectoration, in conditions of afebrility 
and the patient was directed to the Urology Service. He was collected RT-PCR SARS CoV-2, in accordance with the rules of the pandemic context, certifying a negative result, with the opportunity of urological visa surgery. Postoperative, the patient was hospitalized in the ICU ward and the respiratory function deteriorated, with the tendency to desaturate, with the intensification of cough, requiring invasive mechanical ventilation. The culture of the tracheobronchial aspirate objectified a bacterial infection, corrected treated with antibiotic, but the patient develops intestinal symptoms, resulting in bacteriosis (Clostridium difficile). The aggravating spectrum of postoperative respiratory symptoms and the uncertain spectrum of intestinal symptoms led to the need for retesting for SARS CoV-2 pathology, with a positive result. We draw attention to the stereotypy in the manifestation and evolution of COVID-19 pathology in the senior and fragile patient. In the conditions of a previous negative result, only the symptomatic storm and the rapid degradation of the general condition claim the need for retesting. The insidious onset of respiratory phenomena and the false-negative or harvested test during the incubation period allowed surgery.

\section{Abstract \# 22}

Covid-19 case series: Geriatric Giants presentations prevail.

Catriona Reddin ${ }^{1}$, Tomas O. Flatharta ${ }^{1}$, Karen Dennehy ${ }^{1}$, Caoimhe Hanrahan ${ }^{1}$, Gearoid Coughlan ${ }^{1}$, Clodagh Mcdermott ${ }^{1}$, Michelle Canavan $^{1}$, Ruairi Waters ${ }^{1}$

${ }^{1}$ Department of Geriatric Medicine, University College Hospital Galway

Introduction: As of the 4th of June, there have been 2, 211, 148 of those cases reported in Europe (1). In Ireland, 18.39\% of reported cases were 75 years of age or older, as of 2 nd June (2). The ECDC have described the case definition to include the following clinical criteria; cough, shortness of breath, fever, anosmia/ageusia/dysguesia (3). However, we must be cognizant that the older population may present atypically. Studies have shown that confusion is a common feature (4).

Methods: We present two cases of covid-19 presenting to a tertiary hospital under the care of a geriatric team.

Results: Case 1 is an 84 year old gentleman who presented with acute onset confusion and pyrexia. He had no respiratory symptoms. Collateral history confirmed that he was premorbidly fully independent with ADLs and had normal cognitive function. His past medical history includes ischaemic heart disease and prostate cancer. His chest $\mathrm{X}$-Ray demonstrated bibasal infiltrates and he was lymphopenic (0.6). Case 2 is a 78 year old gentleman who presented following a witnessed fall. This occurred following a three day history of rigors and diaphoresis with fluctuating confusion in the preceding $24 \mathrm{~h}$. There were no respiratory symptoms. Past medical history includes hypertension and glaucoma. Chest X-Ray demonstrated bilateral infiltrates and lymphocyte was normal (1.7).

Conclusions: Atypical disease presentations in this age population is a well-recognized phenomenon that has preceded this pandemic. Age over 60 and underlying cardiac co-morbidity are associated with higher mortality ${ }^{5}$. It is imperative that we remain vigilant of atypical presentations.

\section{References:}

1. WHO. Coronavirus disease (Covid-19) Situation report 136 [Internet]. World health organisation; 2020 Jun. Available from: https://www.who.int/docs/default-source/coronaviruse/situationreports/20200604-covid-19-sitrep-136.pdf?sfvrsn=fd36550b_2 2. HPSC. Epidemiology of COVID-19 in Ireland [Internet]. 2020 Jun. Available from: https://www.hpsc.ie/a-z/respiratory/coronavirus/ novelcoronavirus/casesinireland/epidemiologyofcovid-19inireland/
COVID-19_Daily_epidemiology_report_(NPHET)_04062020_ v1_website.pdf

3. European Centre for Disease Prevention and Control. Case definition coronavirus disease 2019 (COVID-19) [Internet]. 2020. Available from: https://www.ecdc.europa.eu/en/covid-19/ surveillance/case-definition

4. Mao L, Jin H, Wang M, Hu Y, Chen S, He Q, et al. Neurologic Manifestations of Hospitalized Patients With Coronavirus Disease 2019 in Wuhan, China. JAMA Neurol [Internet]. 2020 Apr 10 [cited 2020 Jun 5]; Available from: https://jamanetwork.com/journals/ jamaneurology/fullarticle/2764549

5. Du R-H, Liang L-R, Yang C-Q, Wang W, Cao T-Z, Li M, et al. Predictors of mortality for patients with COVID-19 pneumonia caused by SARS-CoV-2: a prospective cohort study. Eur Respir J. 2020 May; 55(5):2000524.

\section{Abstract \# 23}

\section{Symptomatology at the end of life with COVID-19}

Lisa Kelly ${ }^{1}$, Noreen O'Shea ${ }^{1}$, Mahrukh Azhar ${ }^{1}$, Sharon Beatty ${ }^{1}$, Jennifer Brennock ${ }^{1}$, Eileen Mannion ${ }^{1}$, Dympna Waldron ${ }^{1}$

${ }^{1}$ Galway University Hospital, Ireland

Introduction: Patients with COVID-19 infection can deteriorate rapidly, over $\mathrm{h}$, with regards oxygen and care needs [1]. For those patients without medical reversibility from COVID-19, the terminal phase of their illness can be variable [2]. We describe the clinical course of the dying phase of three patients referred to palliative care services within a tertiary hospital to outline the heterogeneity within this cohort and the need to be vigilant for escalating symptoms at the end of life (EoL).

Methods: Retrospective examination of clinical notes of three patients with COVID-19 referred to our specialist palliative care service to determine symptomatology and medication requirements at the EoL.

Results: Our patients suffered predominantly from dyspnoea and agitation in their EoL phase. Medication requirements to achieve symptom control varied considerably despite the fact that all patients were opioid naive. Within the last $48 \mathrm{~h}$ of life our patients required a mean of $28 \mathrm{mg}$ Morphine Sulphate sub-cutaneous (s/c), as-required (prn) (range 12.5-42.5 mg) for management of dyspnoea and a mean of $28 \mathrm{mg}$ Midazolam (range 12.5-55 mg) s/c, prn for agitation. In contrast, secretions were less prominent in this cohort.

Key conclusions: Dyspnoea and terminal agitation were key symptoms experienced by patients with COVD-19 at the EoL. Their symptoms tended to escalate quickly and required careful monitoring to ensure adequate administration of prn medication to maintain symptom control. Obstacles included the need for strict isolation with full Personal Protective Equipment (PPE) and the need for staff to minimise contact time as much as possible.

\section{References:}

1. Zhou F, Yu T, Du R, Fan G, Liu Y, Liu Z et al. Clinical course and risk factors for mortality of adult inpatients with COVID-19 in Wuhan, China: a retrospective cohort study. The Lancet. 2020; 395 (10229):1054-1062.

2. Lovell N, Maddocks M, Etkind S, Taylor K, Carey I, Vora V et al. Characteristics, Symptom Management, and Outcomes of 101 Patients With COVID-19 Referred for Hospital Palliative Care. Journal of Pain and Symptom Management. 2020; . 


\section{Abstract \# 24}

Shcherbinskaya L. S., Zelenko A. V., Siniakova O. K., Semushina E. A., Zhilevich L. A. 1 Assessment of the functional state of the organism of the centenarians of the Republic of Belarus Scientific and Practical Center of Hygiene, Minsk, Belarus, Republican Ho

Andrey Zelenko ${ }^{1}$, Olga Siniakova ${ }^{1}$, Elena Semushina ${ }^{1}$, Ludmila Zhilevich $^{2}$

\section{${ }^{1} \mathrm{SPCH},{ }^{2}$ RHDWW named P.M. Masherov}

The World Health Organization predicts an increase in the proportion of people over 60 by 2050 . by $10 \%$. Health problems are noncommunicable diseases. Also, depression caused by poverty and loneliness can aggravate the course of pathological processes. We examined 36 centenarians (people over 90 years old) using methods of prenosological diagnosis. The average age is $91.81 \pm 0.26$ years, of which 10 are women and 26 are men. All patients underwent the following studies:volumetric sphygmography to determine the stiffness of the vascular wall, peripheral vascular patency by volumetric sphygmography; determination of the adaptive capabilities of the body, vegetative and central regulation, assessment of the energy resources of the body, functional state state by assessing heart rate variability; determination of body weight, body composition in percentage terms, metabolic age using the bioimpedance method.The changes in the functional state and its reserves was identified before the COVID-19 pandemic in our study. Identified changes can be considered as processes, as a result of which regulatory systems are stressed in the absence of complaints and which can lead to a breakdown of adaptation, i.e. pathological process or exacerbation of existing diseases. We plan to conduct these studies again after the pandemic and the lifting of restrictive measures. An assessment will be given of the functional state of the organism, its adaptive capabilities. The data obtained can be useful in predicting measures to reduce epidemiological risks, taking into account the development of probabilistic psychoemotional conditions in the elderly population in territories exposed to adverse biological factors.

\section{Abstract \# 25 \\ Case report: bedside lung ultrasound monitoring in a patient diagnosed with COVID 19 \\ Maurizio Magri ${ }^{1}$, Cristiano Donadio ${ }^{1}$, Carmelo La Fuente ${ }^{1}$, Joël Belmin $^{1}$ \\ ${ }^{1}$ Hopital Charles-Foix}

Background: SRAS-nCoV-19 virus has become a worldwide pandemic since first being described at the end of 2019. Virus infection results in a distinctive inflammatory interstitial lung disease that may lead to severe acute respiratory syndrome, especially in older patients. Method: We performed lung ultrasound in a patient with diagnosis of COVID-19 at day 2, day 8 and day 10 to monitor the evolution of the lung lesions and to asses the feasibility of bedside ultrasound in the disease management. Lung scans were performed using a portable ultrasound (PHILIPS-LUMIFY) by two investigators trained in rapid lung ultrasound.

Result: Ultrasound images demonstrated the hyperechoic vertical artifacts (B-lines) commonly present in lung interstitial involvement. In case of underlying pneumonia, when there are several B-lines, we can detect the presence of "curtain sign". The ultrasound features were often bilateral and the number of $\mathrm{B}$ waves has changed according to the progression of the disease.

Conclusion: Pulmonary ultrasound is a safe and easy diagnostic instrument to detect unilateral, bilateral, diffuse (curtain sign) B-lines or focal consolidations. In this context, lung ultrasound at the bedside plays an important role.Recognize the typical lung features of SRASnCoV-19 and quickly define the prognosis allows us to deploy the right resources for each patient; it becomes crucial when the available resources are not enough, as happened during this global pandemic.

\section{Abstract \# 26 \\ Pancytopenia due to SARS-CoV-2 \\ Patricia López Pardo ${ }^{1}$, Raquel Martín Pozuelo Ruiz de Pascual ${ }^{1}$, Pedro López-Dóriga Bonnardeaux ${ }^{1}$ \\ ${ }^{1}$ Geriatric Department. Hospital Universitario de Getafe}

87-year-old woman referred from the emergency room for a four-day evening fever without another associated clinic. It was a patient with no history of interest, independent for the basic activities of daily life and without cognitive impairment. Physical examination revealed crackles in both lung bases, and chest X-rays revealed bilateral infiltrates compatible with COVID. Analytically, unknown pancytopenia stood out: 1730 leukocytes (neutropenia and lymphopenia of 760 in both series), 94, 000 platelets and hemoglobin of $11.5 \mathrm{~g} / \mathrm{dl}$. Hematology evaluation was requested, which carried out smears compatible with the infectious process: $45 \%$ of the segmented population with reinforced granulation and some hyposegmented forms with some crooks, $44 \%$ small lymphocytes and some activated lymphocyte, $11 \%$ monocytes with abundant vacuoles. Given these findings, a PCR was performed for SARS-CoV-2, which was positive and entered for respiratory surveillance and analytical control. The patient did not wish to receive compassionate use treatment for COVID-19. After $48 \mathrm{~h}$, she presented a spontaneous analytical improvement with practical recovery of the parameters.

\section{Abstract \# 27}

\section{First case of COVID-19 in a local hospital}

Raquel Ortés Gómez ${ }^{1}$, Guadalupe Lozano Pino ${ }^{1}$, Estela Villalba Lancho $^{1}$, Jean Carlo Heredia Pons ${ }^{1}$

${ }^{1}$ Virgen del Puerto Hospital. Plasencia (Cáceres, Spain)

Introduction: We report the first case of COVID-19 at Virgen del Puerto Hospital, Plasencia (Cáceres, Spain).

Methods: Mr. D., an 86-year-old man, had a history of hypertension, dyslipidemia, peripheral vertigo and osteoarthritis. He was independent for ADL, with a score of 5 on FAC scale and a score of 2 on Pfeiffer test. He lived alone at home. The patient was admitted to hospital on February 28, 2020, with a subcapital fragility hip fracture. He started with voiding discomfort on March 2 and with fever on March 3. He remains afebrile with antipyretics and he underwent surgery on March 4.

Results: Mr. D. continued days after surgery with fever and he began with abdominal discomfort in relation to intestinal pseudoobstruction. On March 6 we started empirical antibiotic treatment. On March 9 we observed pancytopenia and it was described on abdominal computed tomography (CT) scan dolicosigma and incipient consolidations in lower lobes lung. We indicated airborne precautions and, after ruling out other viral infections, we request PCR SARS-CoV-2 on March 10 with positive result. In the following days Mr. D. worsened with respiratory symptoms despite he received treatment with antibiotics, Hydroxychloroquine, Lopinavir/Ritonavir, Interferon- $\beta$ and oxygen therapy. Intensive support was dismissed and Mr. D. died on March 18.

Key conclusions: COVID-19 is a life-threatening disease with different forms of presentation and that most frequently affects the 
elderly population. In the case we report fever was present from de beginning but respiratory symptoms were not present until the end.

\section{Abstract \# 28 \\ Thoracic CT and COVID 19. Imaging and False negatives in COVID 19.}

Julia Castillo García ${ }^{1}$, Jorge Eduardo Corrales Cardenal ${ }^{2}$, Carmen Elias de Molins Peña ${ }^{3}$, Beatriz Gamboa Huarte ${ }^{3}$

${ }^{1}$ Hospital Nuestra Señora de Gracia de Zaragoza, ${ }^{2}$ Hospital Nuestra señora de Gracia de Zaragoza, ${ }^{3}$ Hospital Nuestra Señora de Gracia

Introduction: 81 years old. Personal history: High blood pressure, moderate high pulmonary pressure, ischemic cardiopathy, diastolic heart failure, chronic renal failure. Treatment with: Adiro, furosemideGeriatric assessment: Institutionalized, widower. Moderate dependency for daily life activities, Barthel's index: 45/100. Mild cognitive impairment.

Case study: In march is admitted to the acute geriatric ward with a left inferior lobe pneumonia without changes in blood test and a negative swab test for COVID 19. Chest X-Ray with an extensive left hemithorax condensation with no improvement despite broad spectrum antibiotic (ertapenem). During admission, no changes on imaging and two more swab test came back negative with negative serology for COVID 19 infection. Presented respiratory distress with bronchospasm that subsided with corticosteroid therapy. Given the poor evolution of the patient, a thoracic CT scan was performed; presenting bilateral patched and extensive alveolar occupation with a ground glass pattern compatible with COVID-19.Clinical worsening with low oxygen saturation, with a poor response to rescue treatment, presenting adult respiratory distress syndrome. Chest X-ray: bilateral multilobar condensations. Due to refractory syndrome and progressive worsening palliative care approach was used with end of life stage. Given the high suspicion of COVID 19 infection a post-mortem swab test was made with a positive result.

Discussion: The sensitivity of Thoracic CT to diagnose COVID-19 has been reported as high and can predate a positive viral laboratory test, such as the case described. Considering this case at first as a false negative due to clinical and imaging findings with negative laboratory test.

\section{Abstract \# 29}

Atypical clinical manifestations of COVID-19 infection in a family cluster: Facial nerve palsy and diarrhea

Yaohua Chen ${ }^{1}$, Céline Derollez ${ }^{2}$, Tifanie Alberto ${ }^{2}$, Marie-Anne Mackowiak $^{2}$

${ }^{1}$ Department of Geriatric, ${ }^{2}$ Memory clinic

Introduction: COVID-19 emerged as a viral infectious agent of severe respiratory syndrome with high contagiousness and ongoing global pandemic. Atypical manifestations, e.g. other than respiratory symptoms started to be reported.

Methods: We reported of a family cluster of atypical clinical manifestations of COVID-19.

Results: 84-year-old frail woman was admitted to our geriatric dedicated COVID-19 inpatient ward, due to intractable diarrhea and deconditioning. She was frail and had a long list of comorbidities, such as mild cognitive impairment, chronic skin sores, obesity, falls, arthritis and depression. Prior to the hospitalization, she remained in a rehabilitation center for 2 months, until discharge following a negative swab for SARS-Cov2. The diagnosis of COVID-19 was finally confirmed in the geriatric unit with her mother by naso-pharyngeal and feces RT-PCR assays. Her 57-years-old woman took care of her during the week interval between discharge from rehabilitation and admission in our unit. Her daughter was admitted in neurological inpatient ward 1 week later because of acute isolated left facial nerve palsy. Diagnosis of COVID-19 was confirmed in both cases while no major respiratory syndrome was reported.

Conclusions: Clinicians should be aware of atypical manifestations due to COVID-19. Data remained poor regarding atypical presentation among older and frail patients. It seems that in a relatively large proportion of these patients, they only experienced minor symptoms. Preventive measures and social isolation of contact-cases are key strategies to arrest the pandemic. These cases will also be helpful for the understanding of pathophysiology of the virus.

\section{Abstract \# 30}

Glucocorticoid treatment in COVID-19 bilateral pneumonia-a clinical case report

Itxaso Marín-Epelde ${ }^{1}$, Agurne García-Baztán ${ }^{1}$, Isabel RodríguezSánchez ${ }^{2}$, Belén González-Glaría ${ }^{1}$, María Gonzalo-Lázar ${ }^{1}$, César Cuevas-Lara $^{3}$, Marina Sánchez-Latorre ${ }^{1}$, Nancy Jeanette GonzalesMontalejo $^{1}$, Álvaro Casas-Herrero ${ }^{1}$, Fabricio Zambom-Ferraresi ${ }^{3}$

${ }^{1}$ Complejo Hospitalario de Navarra, Pamplona, Spain, ${ }^{2}$ Hospital Clínico San Carlos, Madrid, Spain, ${ }^{3}$ "Complejo Hospitalario de Navarra (CHN)", "Universidad Pública de Navarra (UPNA)", "IdiSNA", Pamplona, Navarre, Spain.

Glucocorticoids seem to be an effective treatment for cytokine storm activation in COVID-19 patients [1]. We present an 84 year-old male (Barthel Index 100/100) with a personal history of hypertension, type 2 diabetes mellitus, hypertensive cardiopathy with preserved ejection fraction, anticoagulated atrial fibrillation and obstructive sleep apnea syndrome who presented with cough, respiratory secretions, dyspnea and fever. He was admitted with bilateral COVID-19 pneumonia. Analytically, he presented with lymphopenia (600/L), thrombocytopenia $(105,000 / \mathrm{L})$, elevated D-Dimer levels (2015 FEU/mL), elevated ferritin levels (721 microg/L), low sodium levels $(132 \mathrm{mEq} /$ $\mathrm{L}$ ), worsening of renal function (creatinine $2.14 \mathrm{mg} / \mathrm{dL}$ ) and elevated CRP levels $(134.7 \mathrm{mg} / \mathrm{L})$ with no procalcitonin elevation. He was started on Hydroxicloroquine and antibiotics on admission. On day 11 , he suffered a worsening of the respiratory situation, with increasing oxygen necessities. The chest X-Ray performed also showed a worsening of the bilateral reticular pneumonia, with the association of consolidations. Thus, glucocorticoids at high doses were initiated ( $125 \mathrm{mg} /$ day for 3 days) and Tocilizumab $(600 \mathrm{mg})$ was administered. After said treatment, he gradually improved, both subjectively (dyspnea) and objectively (as shown by $\mathrm{Pa} / \mathrm{FiO} 2$ evolution and clearing of the bibasilar rales on auscultation). X-Ray improvement was seen on day 17 , with resolution of the previously seen consolidations on the right side and a very slight persistence of reticular affectation on the left. Analytically, lymphopenia (900/L), elevated levels of D-Dimer (1563 FEU/mL), elevated ferritin levels (622 microg/L) and slightly elevated CRP levels $(19 \mathrm{mg} / \mathrm{dL})$ persisted, but platelet count, renal function and LDH levels had normalized.

\section{Abstract \# 31 \\ Comparative characteristics of Russian young and old patients with SARS-CoV-2}

Maria Karnaushkina $^{1}$, Elena Antonova ${ }^{2}$, Marina Smorzhevskaya ${ }^{2}$, Svetlana Topolyanskaya ${ }^{1}$ 
${ }^{1}$ First Moscow State Medical University, ${ }^{2}$ War Veterans Hospital N3

Introduction: According to some reports, older people are at highest risk from SARS-CoV-2, but Russian data on SARS-CoV-2 severity are not available.

Methods: 155 patients were enrolled in this study. Patient's age varied from 26 to 94 years: $41.3 \%$ of patients were $<60$ years, $34.2 \%-60-74$ years, $24.5 \%-\geq 75$ years. $52.9 \%$ of patients were men.

Results: Upon enrollment $47.4 \%$ of very elderly ( $\geq 75)$ and $21.9 \%$ of young $(<60)$ patients had grade 3-4 clinical severity $(\mathrm{p}=0.007)$. Most laboratory parameters in very elderly patients were worse than in young: hemoglobin levels were 121 и $135 \mathrm{~g} / \mathrm{l}$, respectively ( $\mathrm{p}=$ $0.0001)$, leukocytes -10.4 and $6.5(\mathrm{p}=0.00007)$, neutrophils -8.5 and $4.7(\mathrm{p}=0.00005)$, total serum protein-60.3 and $66.0(\mathrm{p}=$ $0.00001)$, albumin -30.2 and $35.9(\mathrm{p}=0.000001)$, urea-11.0 and 6.6 $(\mathrm{p}=0.004)$, creatinine-132 and $98.0(\mathrm{p}=0.0001)$, total bilirubin16.8 and $10.2(\mathrm{p}=0.0003)$, calcium-0.72 and $1.1(\mathrm{p}=0.0001)$, D-dimer - 1716.6 and $1253.4(\mathrm{p}=0.07)$. There were significant differences in disease worsening during follow-up: $89.5 \%$ of patients 75-94 years and $51.6 \%$ of young patients have worsened ( $\mathrm{p}=$ $0.0001)$. Follow-up computed tomography showed that $94.7 \%$ of patients 75-94 years old had severe lung damage (grade 3-4), while among young patients this indicator was $62.5 \%(\mathrm{p}=0.0002) .86 .8 \%$ of patients $75-94$ years old and $29.7 \%$ of young died during followup; discharged from hospital- $-70 \%$ of young and $13 \%$ of very elderly patients $(\mathrm{p}<0.00001)$.

Key conclusions: In very elderly patients SARS-CoV-2 is more severe and is associated with clinical severity, CT progression and worsening of laboratory parameters and ultimately results in death of patients.

\section{Abstract \# 32}

The role of Chest radiography on the management of COVID-19 Andreia Teles Ribeiro ${ }^{1}$, Gonçalo Freire ${ }^{2}$

${ }^{1}$ USF Descobertas, ACES Lisboa Ocidental e Oeiras, ARS Lisboa e Vale do Tejo, ${ }^{2}$ Serviço Radiologia, Hospital Beatriz Ângelo, Loures, Lisboa

Introduction: Amidst the COVID-19 pandemic, organization is essential to boost healthcare quality and reduce mortality rates, especially in the elderly. Typically, COVID-19 diagnosis is made through real-time reverse transcription polymerase chain reaction (RT-PCR), but results take about $6 \mathrm{~h}$ to be available.

Methods: For this paperwork, a literature review was undertaken in PubMed and CENTRAL with the terms COVID-19 [MESH] and Radiography [MESH]. Furthermore, radiographic COVID-19 categorization by the British Society Thoracic Imaging is explained and illustrated.

Results: Chest tomography is the gold-standard to study lung parenchyma abnormalities. However, it is expensive and timeconsuming. Although CXR is less sensitive, it can demonstrate findings suggestive of COVID-19 such as peripheral and bilateral ground glass opacities. It can also have an important role on risk stratification, distinguishing patients with increased risk for hospital admission and intubation. When the CXR is normal, patients can more confidently have medical release with isolation measures while waiting for the RT-PCR test results. Furthermore, in the geriatric population the differential diagnosis of COVID-19 is extensive. While it is essential to make a good clinical history and physical examination, CXR is able to diagnose other diseases such as cardiac insufficiency, pleural effusion or pneumothorax.

Conclusion: Chest radiography is useful for both clinicians and patients, has it can detect findings suggestive of COVID-19 as well as other differential diagnosis. In addition, a normal CXR decreases the length of stay in the Emergency Department for those who have mild clinical disease, decreasing the risk of nosocomial infections.

\section{Abstract \# 33 \\ Mortality factors in patients older than 80 years admitted for COVID-19 to an acute geriatric unit}

Javier Montero Muñoz ${ }^{1}$, Pablo Enrique Solla Suárez ${ }^{1}$, Cristina Guirola García-Prendes ${ }^{1}$, Francisco Jiménez Muela ${ }^{1}$, Laura Gómez Armas $^{1}$, Marta Martínez Rivera ${ }^{1}$, Alberto Domínguez Bravo ${ }^{1}$, José Gutiérrez Rodríguez ${ }^{1}$

${ }^{1}$ Área de Gestión Clínica de Geriatría, Hospital Monte Naranco, Oviedo, Asturias, Spain

Introduction: Several prognostic factors have been described in severe acute respiratory syndrome coronavirus 2 (SARS-CoV-2) infection. However, no studies analyze the impact of known factors associated with poor outcomes in older patients like functional dependence or cognitive impairment. The objective of this study is to identify factors associated with in-hospital mortality in patients older than 80 years admitted with diagnosis of coronavirus disease 2019 (COVID-19).

Methods: a retrospective observational study was carried out with data from patients older than 80 years admitted to an Acute Geriatric Unit with a laboratory-confirmed diagnosis of COVID-19. Sociodemographic, functional, cognitive, comorbidity and laboratory variables were collected. An univariate analysis to identify mortality associated factors was performed.

Results: 58 patients were recruited. The median age was 89 (85-92), $69 \%$ were women, $65.5 \%$ had moderate-severe cognitive impairment and median previous Barthel Index was 35 (0-73.8). 70.7\% were institutionalized. The mortality rate was $41.4 \%$. Factors associated with mortality were Barthel Index $<40(\mathrm{OR}=3.8$ [1.2-12.2]), cognitive impairment $(\mathrm{OR}=5[1-25.3])$, chronic kidney disease $(\mathrm{OR}=$ 3.2 [1.1-9.7]), white blood cells count $(\mathrm{p}=0.037), \mathrm{LDH}(\mathrm{p}=0.019)$, procalcitonin $>0.5 \mathrm{ng} / \mathrm{mL}(\mathrm{OR}=3.9[1.03-15])$ and viral load $>100.000$ copies $/ 1000$ cells $(\mathrm{OR}=5.5[1.4-22.1])$.

Key conclusions: Our results suggest that cognitive and functional impairment could be prognostic factors in COVID-19.This strengthens the value of comprehensive geriatric assessment in decision making given a new outbreak. Also, the association observed with bacterial superinfection markers or viral load and mortality could help identify those elderly patients with more potential to benefit from certain treatments like antibiotics or antiviral therapy.

\section{Abstract \# 34}

Geriatric inpatients with COVID-19: mortality rate and risk factors of death

Cedric Mahiat ${ }^{1}$, Séverine Henrard ${ }^{2}$, Isabelle Gilard ${ }^{3}$, Nicolas Lanthier $^{3}$, Peter Starkel ${ }^{3}$, Isabelle De Brauwer ${ }^{3}$, Pascale Cornette ${ }^{3}$

${ }^{1}$ St-Luc University Hospital, Brussels, ${ }^{2}$ UCLouvain, ${ }^{3}$ CUSLuc

Introduction: No cohort study so far has reported the in-hospital mortality (IHM) rate of geriatric patients with COVID-19.

Methods: Retrospective cohort study of the first 50 patients aged $\geq$ 75 years admitted to the geriatric wards of a Belgian academic hospital with PCR-confirmed COVID-19 between March 11 and April 17, 2020. The main endpoint (death in hospital or discharge alive) was documented in all patients. Factors associated with in-hospital death were assessed in multivariable Cox's regression. 
Results: Before hospital admission, the 50 inpatients (median age: 88 years; $54 \%$ men; $46 \%$ living in nursing home) had cognitive impairment $(54 \%)$, malnutrition $(40 \%)$, major polymedication $(\geq 10$ daily drugs: $36 \%)$ and were severely/very severely frail $(52 \%)$ according to the clinical frailty score [1] (median 7; range 4-8). In $36 \%$ of the patients, the first COVID-19 manifestation was atypical (severe fatigue, fall, diarrhoea, delirium-related symptoms) without fever or respiratory symptom. The IHM rate was $52 \%$ as 26 patients died in hospital and 24 patients were discharged alive. The risk of inhospital death was associated $(\mathrm{p}<0.05)$ with age (Hazard ratio (HR) $=1.10$ per year $)$, chronic respiratory disease $(\mathrm{HR}=4.72)$, marked lung infiltrates $(\mathrm{HR}=3.11)$ and cerebral apathy $(\mathrm{HR}=2.47)$ upon COVID-19 diagnosis, but not associated ( $\mathrm{p}>0.15)$ with male gender, nursing home residency, cognitive impairment, malnutrition and severe/very severe frailty.

Conclusions: This is the first cohort study with complete follow-up until hospital discharge in geriatric inpatients with COVID-19. Further research is needed to determine the IHM and its predictors in these high risk inpatients.

1. Rockwood K, Song X, MacKnight C, Bergman H, Hogan DB, McDowell I et al. A global clinical measure of fitness and frailty in elderly people. CMAJ. 2005; 173(5):489-95.

\section{Abstract \# 35 \\ In-hospital mortality rates in older COVID-19 inpatients: a literature review}

Cédric Mahiat ${ }^{1}$, Christophe De Terwangne ${ }^{1}$, Isabelle De Brauwer ${ }^{2}$, Séverine Henrard ${ }^{1}$, Benoit Boland ${ }^{2}$

${ }^{1}$ UCLouvain, ${ }^{2}$ CUSLuc

Background: Little is known at the end of spring 2020 about inhospital mortality (IHM) rates of older inpatients with COVID-19. Valid IHM rate calculations require a cohort study design with complete follow-up of all patients until hospital discharge. The aim of this study was to describe IHM rates in older patients ( $\geq 65$ years) with COVID-19.

Methods: Medical literature review from December 1, 2019 to June 17, 2020 of cohort studies including older patients (OP) with COVID19. The outcome was the patient's vital status (death or alive) upon hospital discharge.

Results: Twenty cohort studies selected and compared OP with the outcome at a certain hospital date (censoring bias). Four cohort studies completed the OP hospital follow-up, allowing calculation of the IHM rate. The IMH rate was $26.2 \%$ in 65 OP of the Pulmonary hospital in Wuhan [1], 34.5\% in 55 OP of the Zhongnan hospital in Wuhan [2], 35.3\% in 17 OP of the Dabieshan Medical Center in Huanggang [3] and $41.0 \% \%$ in $117 \mathrm{OP}$ of the Chelsea \& Westminster hospital in London [4]. Pooling these four cohorts (710 COVID-19 inpatients), we calculated an average IHM rate of $35.4 \%$ [95\% CI $29.6-41.3 \%$ ] in the 254 OP, which was six times higher than the IHM rate of 5.9\% [95\% CI 3.6-8.2\%] in the 456 younger patients.

Conclusion: Few COVID-19 cohort studies which included older patients have reported the vital status of all patients at hospital discharge. Completed cohorts are deeply needed to determine the IHM rates in this high risk population.

1. Du RH, Liang LR, Yang CQ, Wang W, Cao TZ, Li M et al. Predictors of mortality for patients with COVID-19 pneumonia caused by SARS-CoV-2: a prospective cohort study. Eur Respir J. 2020; 55(5):2000524.

2. Chen T, Dai Z, Mo P, Li X, Ma Z, Song S et al. Clinical characteristics and outcomes of older patients with coronavirus disease 2019 (COVID-19) in Wuhan, China (2019): a single-centered, retrospective study. J Gerontol A Biol Sci Med Sci. 2020.
3. Yao Q, Wang P, Wang X, Qie G, Meng M, Tong X et al. A retrospective study of risk factors for severe acute respiratory syndrome coronavirus 2 infections in hospitalized adult patients. Pol Arch Intern Med. 2020; 130(5):390-9.

4. Khalil K, Agbontaen K, McNally D, Love A, Mandalia S, Banya W et al. Clinical Characteristics and 28-Day Mortality of Medical Patients Admitted with COVID-19 to a Central London Teaching Hospital. J Infect. 2020.

\section{Abstract \# 36}

\section{COVID 19-Refocus your lens}

Arne Heyns ${ }^{1}$, Koen Peers ${ }^{2}$, Johan Flamaing ${ }^{3}$, Evelien Gielen ${ }^{3}$, Jolan Dupont $^{3}$, Hilde Beyens ${ }^{4}$, Jos Tournoy ${ }^{3}$

${ }^{1}$ Department of Physical Medicine and Rehabilitation, University Hospitals Leuven, Leuven, Belgium., ${ }^{2}$ University Hospitals Leuven, Department of Physical and Rehabilitation Medicine, Herestraat 49, B-3000 Leuven, Belgium; KU Leuven - University of Leuven, Department of Development and Regeneration, Herestraat 49, Box 805 , B-3000 Leuven, Belgium, ${ }^{3}$ Gerontology \& Geriatrics, Department of Public Health and Primary care, KU Leuven, Herestraat 49, 3000 Leuven, Belgium; Department of Geriatric Medicine, UZ Leuven, Herestraat 49, 3000 Leuven, Belgium, ${ }^{4}$ University Hospitals Leuven, Department of Physical and Rehabilitation Medicine, Herestraat 49, B-3000 Leuven, Belgium

Introduction: COVID-19 created an unforeseen pandemic, mainly afflicting an older population. As an unknown disease, it lead to a high number of hospitalizations with scarce information on consequences on physical and mental health. The aim of this study was to compose and implement a short and feasible multi domain screening assessment to evaluate hospitalized COVID-19 patients to determine their further needs for adequate care and rehabilitation.

Methods: A multidisciplinary expert panel agreed on a fixed combination of existing screening tools, taking into account the necessary hygienic aspects. It was gradually implemented with medical stable inpatients in the University Hospitals Leuven (Belgium) and data were analysed retrospectively.

Results: Hand grip strength, timed chair stand test (TCS), Barthel index, swallowing screening, Nutritional Risk Screening 2002, HADS (Hospital Anxiety and Depression Scale) and MoCA (Montreal Cognitive Assessment) were agreed upon. Preliminary analysis showed that only $6 \%$ of the older ( $>70$ year) patients were able to perform the TCS within $14 \mathrm{~s}$. Around one third of patients showed significant anxiety or depressive symptoms. $75 \%$ had a score of 26 or less on the MoCA. $62 \%$ of older patients demonstrated severe functional impairment (Barthel $<9$ ).

Conclusion: A large majority of hospitalized COVID-19 patients show problems or deficits in either physical, functional or mental domains, demanding adequate therapy and follow up after discharge. Especially the impact on older persons and their strength was striking, implying the need for a good discharge planning with adequate follow-up and evaluating the need for in- or outpatient rehabilitation programs.

\section{Abstract \# 37 \\ Mortality rate and the factors associated to it due to COVID-19 infection in people aged 90 or more in a Spanish hospital}

Concepción Lobato Cassinello ${ }^{1}$, María De La Puente Martín ${ }^{1}$, Sasha Raquel Botbol Moreno ${ }^{2}$, Soledad Domínguez Mendoza ${ }^{2}$, Carmen Navarro Ceballos ${ }^{2}$, Lourdes Del Rosario Evangelista Cabrera ${ }^{2}$, Maria 
del Rosario García Martín², Maria Jesús Molina Hernández², Jaime Rodríguez Salazar ${ }^{2}$, Karina Liz Quiñones ${ }^{2}$

${ }^{1}$ Department of Geriatrics, Hospital Universitario Severo Ochoa (Leganés), ${ }^{2}$ (Department of Geriatrics, Hospital Universitario Severo Ochoa (Leganés))

Introduction: Evaluate the mortality rate due to COVID-19 infection and the factors associated to it in people aged 90 or more.

Methods: Cross-sectional descriptive analysis from patients admitted in a Madrid hospital, from 1st of March to May 15th, 2020. Statistical analysis: chi-square for categorical variables and t-test and U MannWhitney for quantitative variables. Multivariate analysis: Binary logistic regression ( $\mathrm{p}<0.05$, CI 95\%). SPSS V. 26.0.

Results: 58 patients were included, mean age $92.7 \pm 2.8$ years, woman (65.5\%). 27.8\% had dementia. $41.1 \%$ came from nursing homes. Comorbidity estimated by Charlson Index $X=2.6 \pm 1.6 .56$ patients had polypharmacy ( $\mathrm{X}=8.9 \pm 3.3$ drugs); $34.5 \%$ were taking ACEI and $27.6 \%$ anticoagulant theraphy before admission. The main symptom was dyspnea (other evaluated were fever, delirium or gastrointestinal symptons). $45.6 \%$ showed bilateral pneumonia. Inpatient mortality was $32.8 \%$ during the admission. In the bivariate analysis, a higher in-hospital mortality was associated with being male $(\mathrm{p}=$ $0.042)$, admission in March ( $\mathrm{p}=0.009)$, having dyspnea $(\mathrm{p}=0.006)$, or dementia $(\mathrm{p}=0.029)$. In the multivariate analysis it remained having dyspnea (OR:4.6; 95\% CI 1.1-19.5; $\mathrm{p}=0.04$ ).

Key conclusions: At that time, Madrid had 64408 positive COVID tests [1]. The series have shown that as you get older, your risk for severe illness from COVID-19 increases and the mortality rates are higher in elderly people [2]. The current analysis in our sample shows a high mortality rate, but none of the previous conditions evaluated are related to increased it, so attending to the symptons, like dyspnea, is important to graduate severity.

References:

[1] https://www.isciii.es/QueHacemos/Servicios/VigilanciaSalud PublicaRENAVE/EnfermedadesTransmisibles/Documents/INFORMES/ Informes $\% 20$ COVID-19/Informe $\% 20 n^{\circ} \% 2031 . \% 20$ Situación $\% 20 \mathrm{de}$ $\% 20$ COVID-19\%20en\%20España $\% 20 \mathrm{a} \% 2014 \% 20 \mathrm{de} \% 20$ mayo $\%$ 20de\%202020.pdf

[2] https://www.cdc.gov/coronavirus/2019-ncov/specific-groups/ high-risk-complications.html

\section{Abstract \# 38}

\section{Atypical presentations of COVID-19 in a frail population}

Victoria Dunnett-Kane ${ }^{1}$, Neethu Billy Graham Mariam ${ }^{1}$, Robert Gillot $^{1}$, Jessica Newton ${ }^{1}$, Muhammad Haris ${ }^{1}$, Ankita Prasad ${ }^{1}$, Lauren Wentworth $^{1}$

${ }^{1}$ Manchester Foundation Trust

Background: The most commonly reported symptoms of COVID-19 are cough, fever and shortness of breath [1]. It is well known that older patients present atypically with infections [2]. We aimed to study the clinical features of COVID-19 in such a population.

Methods: We reviewed 70 randomly selected patients treated on a COVID ward at Trafford Hospital, accepting patients with a 'Not for Resuscitation' decision.

Results: $39 \%$ patients were male. The median age was 85 and CFS 6 . The most common presentations in patients who tested positive within 14 days of admission were: $48 \%$ confusion, $27 \%$ with a fall, $27 \%$ shortness of breath, $22 \%$ fever, $18 \%$ reduced oral intake and $9 \%$ generally unwell. In the cohort of patients who tested positive after 14 days of admission $50 \%$ had a cough, $31 \%$ social swabs, $23 \%$ fever, $20 \%$ shortness of breath, $12 \%$ generally unwell.
Key conclusions: Our data suggests that frail and older populations present with atypical symptoms, which has implications on the testing strategy in this cohort. The difference in presentations with COVID19 between patients who tested positive 14 days following admission may represent the current testing criteria in swabbing inpatients for COVID-19. It is of note that hyperactive delirium is more commonly reported in hospital inpatients, whereas features of hypoactive delirium such as reduced oral intake were more commonly noted in our cohort[3].

References:

1. Docherty Annemarie B, Harrison Ewen M, Green Christopher A, Hardwick Hayley E, Pius Riinu, Norman Lisa et al. Features of 20133 UK patients in hospital with covid-19 using the ISARIC WHO Clinical Characterisation Protocol: prospective observational cohort study BMJ 2020; 369 :m198

2. Corretge, M. \& Doubal, F. \& Mead, G. Atypical presentation of disease in older age: A review. CME Journal Geriatric Medicine. 2010; 12. 128-140.

3. van Velthuijsen EL, Zwakhalen SMG, Mulder WJ, Verhey FRJ, Kempen GIJM. Detection and management of hyperactive and hypoactive delirium in older patients during hospitalization: a retrospective cohort study evaluating daily practice. Int $\mathrm{J}$ Geriatr Psychiatry. 2018; 33 (11):1521-1529. https://doi.org/10.1002/gps. 4690

\section{Abstract \# 39 \\ Lymphopaenia in an elderly cohort of patients with COVID-19 background}

Victoria Dunnett-Kane ${ }^{1}$, Neethu Billy Graham Mariam ${ }^{1}$, Elizabeth Drysdale $^{1}$, Jolijn Arits ${ }^{1}$, Amal Samsudeen ${ }^{1}$, Lauren Wentworth ${ }^{1}$

${ }^{1}$ Manchester Foundation Trust

Background: Lymphopaenia has been described as a prognostic factor of mortality and length of stay in older hospitalised patients [1]. It is also a frequently noted biochemical feature of COVID-19 [2]. We aimed to study the rates of lymphopaenia in an elderly cohort of hospitalised patients with COVID-19.

Methods: We reviewed 70 randomly selected patients admitted to a specialist COVID-19 unit between 3/4/20 and 30/4/20, all of whom had 'Not For Resuscitation' decisions. We compared baseline lymphocyte count and lymphocyte count after testing positive for COVID-19.

Results: The median age of our patients was 85 , with an in-hospital mortality of $26 \%$. We excluded 6 patients who did not have baseline lymphopaenia counts recorded. $9 \%$ of patients were lymphopaenic at baseline. $68 \%$ of patients with a normal lymphocyte became lymphopaenic during admission for COVID-19. The median length of stay for patients who became lymphopaenic was 41.5 days, compared to 35.4 days for those who did not become lymphopaenic.

Key conclusions: Although lymphopaenia is common in an elderly cohort, from our patient population it appears that lymphopaenia is an indicator of COVID-19 infection. From our data, lymphopaenia appears to be associated with longer length of stay. Further research in larger cohorts is required to establish the prognostic value of lymphopenia in older patients with COVID-19.

\section{References:}

1. Rubio-Rivas, M., Formiga, F., Grillo, S. et al. Lymphopenia as prognostic factor for mortality and hospital length of stay for elderly hospitalized patients. Aging Clin Exp Res 28, 721-727 (2016).

2. Tan, L., Wang, Q., Zhang, D. et al. Lymphopenia predicts disease severity of COVID-19: a descriptive and predictive study. Sig Transduct Target Ther 5, 33 (2020). 


\section{Abstract \# 40 \\ A retrospective review of Covid-19 symptoms in an in-patient cohort from $48 \mathrm{~h}$ prior to PCR swab.}

Anna McKeogh ${ }^{1}$, Siofra Bennett ${ }^{1}$, Mary Randles ${ }^{1}$, Daniel Schmidt ${ }^{1}$

${ }^{1}$ Mallow General Hospital

Background: COVID-19 is a novel corona virus first detected in China in 2019. Common symptoms include fever, cough, and dyspnoea. [1, 2, 3] Based on this the Health Protection and Surveillance Centre (HPSC) Ireland published an algorithm for screening of Covid-19. [4] The older adult population may have atypical presentations. [5, 6] We describe a cohort of older adults who developed nosocomial Covid-19 infection in Ireland. The aim was to determine if they would have met the hPC criteria at the time of diagnosis and if expansion might facilitate earlier diagnosis.

Method: This retrospective case review (cohort study) included patients with Covid-19 confirmed by PCR in a level two Irish Hospital. Medical and nursing notes, for $48 \mathrm{~h}$ prior to the decision to test for Covid-19 were reviewed and symptoms recorded.

Results: Among the 12 confirmed cases, the median age was 81.5 years. Testing was most commonly due to contact tracing (58\%) and pyrexia $>38.1(58 \%)$. In the $48 \mathrm{~h}$ prior to testing the most common symptoms were; fever $(58 \%)$, nausea and vomiting (50\%), cough $(42 \%)$, delirium (42\%), diarrhoea (42\%) and, dyspnoea (33\%). Seven patients $(58 \%)$ would have met hPC criteria.

Conclusion: In our cohort of older adults, only 58 percent would have met national screening criteria. It is notable that expansion to include delirium, or GI symptoms would have resulted in a diagnostic yield of 75 percent (9/12). Although we acknowledge the number of patients is small, this analysis highlights the importance of vigilance for atypical symptoms of Covid-19 in older adults.

References:

1. Clinical characteristics of coronavirus disease 2019 in China. Guan W-J, Ni Z-Y, Hu Y, et al. N Engl J Med. 2020:0. [Google Scholar]

2. Clinical characteristics of 138 hospitalized patients with 2019 novel coronavirus-infected pneumonia in Wuhan, China. Wang D, Hu B, Hu C, et al. JAMA. 2020; 323:1061-1069. [Google Scholar]

3. Guan W, Ni Z, Hu Y, et al. Clinical characteristics of coronavirus disease 2019 in China. N Engl J Med. https://doi.org/ 10.1056/NEJMoa2002032.

4. hPC Assessment and testing pathways for Covid-19 - Assessment and testing pathway for use in a Hospital Setting. Published by hPC Ireland. Publication date 2.6.2020. Accessed 22.6.2020 https://www.hpsc.ie/a-z/respiratory/coronavirus/novelcoronavirus/ algorithms/COVID-19\%20Assessment $\% 20$ and $\% 20$ testing $\% 20$ pathway\%20for\%20Hospital\%20Setting.pdf

5. Jung, YJ, Yoon JL, Kim h, Lee AY, Kim MY, Cho JJ. Atypical clinical presentation of geriatric syndrome in elderly patients with pneumonia or coronary artery disease. Ann of Geri Med and Res. 2017; 21(4):158-63

6. Malone ML, Hogan TM, Perry A, Biese K, Bonner A, Pagel P, Unroe KT. COVID-19 in older adults - Key points for emergency department providers. J of Geri Emerg Med. 2020; 1(4):1-11

\section{Abstract \# 41 \\ Outcomes in a frail population with COVID-19 in a secondary care setting}

Neethu Billy Graham Mariam ${ }^{1}$, Victoria Dunnett-Kane ${ }^{1}$, Tim Seers ${ }^{1}$, Corina Fankhauser ${ }^{1}$, Alaa Al-Salihi ${ }^{1}$, Lauren Wentworth ${ }^{1}$

${ }^{1}$ Manchester Foundation Trust
Introduction: Current evidence suggests older patients with COVID19 have worse outcomes [1] than younger patients. We reviewed the characteristics and prognostic factors in a frail population with COVID-19 in a secondary care setting.

Methods: 70 randomly selected patients treated on a COVID-19 ward at Trafford Hospital, only accepting patients with a 'Not for Resuscitation' decision were reviewed. Patient characteristics including the Rockwood Clinical Frailty Score (CFS) [2] were recorded. Primary outcomes were death and discharge destination.

Results: $39 \%$ of patients were male. The median age and CFS were 85 and $6(4-8)$ respectively. $50 \%$ of patients had hypertension, $31 \%$ had type 2 diabetes, $30 \%$ had a CVA and $29 \%$ had dementia. $23 \%$ of patients died on the COVID unit whilst $33 \%$ of patients were transferred to another ward and $44 \%$ were discharged home or to a place of care. Of those who died, $61 \%$ were male. A further 2 patients died in hospital following transfer to other wards. The median CFS was 6 for those who died, 6 for patients who were transferred to another ward, 5 for patients discharged home and 7 for those who were discharged to a care facility.

Key conclusions: We reviewed a moderately frail population and found that a quarter died as inpatients. Although under $40 \%$ of the cohort were male, over $60 \%$ of mortalities were in male patients.

References:

1. Liu K, Chen Y, Lin R, Han K. Clinical features of COVID-19 in elderly patients: A comparison with young and middle-aged patients. J Infect. 2020; 80 ${ }^{6}$ :e14-e18. https://doi.org/10.1016/j.jinf.2020.03.005 2. Rockwood K, Song X, MacKnight C, et al. A global clinical measure of fitness and frailty in elderly people. CMAJ. 2005; $173^{5}: 489-495$. https://doi.org/10.1503/cmaj.050051

\section{Abstract \# 42}

\section{Acute kidney injury in older patients with COVID-19}

Neethu Billy Graham Mariam ${ }^{1}$, Victoria Dunnett-Kane ${ }^{1}$, Robert Gillott ${ }^{1}$, Rosalind Kelly ${ }^{1}$, Matthew Dawson ${ }^{1}$, Natalie Woodhouse ${ }^{1}$, Lauren Wentworth ${ }^{1}$

\section{${ }^{1}$ Manchester Foundation Trust}

Introduction: Acute kidney Injury (AKI) is a common complication in hospitalised adults [1, 2]. COVID-19 infection has a well-documented association with AKI. One study estimated the prevalence in hospitalised patients around $36.6 \%$, with higher rates in older people, and found it to be associated with poorer prognosis [3]. This appears to be secondary to a complex mechanism involving virus-mediated injury, angiotensin activation, cytokine storm, hypercoagulation and microangiopathy[4]. We aimed to establish the rates of AKI in an elderly cohort of COVID-19 patients.

Methods: We reviewed 70 randomly selected patients treated on a COVID unit in Trafford General Hospital, which accepted patients with a 'Not for Resuscitation' decision.

Results: The median age of our patients was 85 , with an in-hospital mortality of $26 \%$. 15 (21\%) had a documented AKI. Of these, $80 \%$ were stage $1,13 \%$ stage 2 , and 1 patient had a stage 3 AKI. An additional 17 patients (24\%) had renal function impairment compared to their baseline which did not classify as an AKI. Eight (53\%) of the patients who had an AKI died in hospital.

Key conclusions: Although our cohort of elderly patients did not demonstrate high rates of AKI compared to the literature, almost half of patients demonstrated some level of derangement compared to baseline. Furthermore, the all-cause in-hospital mortality in the small number of patients with AKI is much higher than the overall mortality. More research needs to be conducted into the pathophysiology and prognostic value of AKI in COVID-19. 


\section{References:}

1. Chia-Ter Chao, Hung-Bin Tsai, Yu-Feng Lin, Wen-Je Ko, Acute kidney injury in the elderly: Only the tip of the iceberg, Journal of Clinical Gerontology and Geriatrics, Volume 5, Issue 1, 2014, Pages $7-12$

2. Jie-Qiong Liu1, 2, Guang-Yan Cai1, Shuang Liang1, Wen-Ling Wang1, Si-Yang Wang1, Fang-Lei Zhu1, Sa-Sa Nie1, Zhe Feng1, Xiang-Mei Chen 1 . Characteristics of and risk factors for death in elderly patients with acute kidney injury: a multicentre retrospective study in China.

3. Hirsch J, Ng J, Ross D, Sharma P, Shah H, Barnett R, et al. Acute kidney injury in patients hospitalized with COVID-19. Kidney International. 2020 July; $98^{1}$ : 209-218.

4. Batlle D, Soler M, Sparks M, Hiremath S, South A, Welling P, et al. Acute Kidney Injury in COVID-19: Emerging Evidence of a Distinct Pathophysiology. JASN. 2020 May;: Online ahead of print.

\section{Abstract \# 43 \\ Clinical complications and mortality from Covid-19 in the elderly during admission to a geriatric unit in Teruel, Spain.}

Miguel Sánchez ${ }^{1}$, Carmen Oquendo ${ }^{1}$, Magdalena Linge ${ }^{1}$, Maria Elena Castro ${ }^{1}$, Marta Mercedes Gonzalez ${ }^{1}$, Carlos Gala ${ }^{1}$, Mercedes Forcano ${ }^{1}$

${ }^{1}$ Unidad Geriatría. Hospital San José Teruel

Introduction: Epidemiological and clinical features of COVID-19 patients have been reported, however a specific clinical evolution and the mortality risk factors associated have not been well described in elderly patients. In this paper, we analyze the clinical presentation, complications, and its correlation with mortality during admission in an acute geriatric ward.

Material and methods: In this retrospective, observational study, we included all the COVID-19 cases diagnosed by RT- PCR. We described initial symptoms, complications, and its correlation with mortality in the sample.

Outcome: 43 patients were included. The most common symptoms were fever $(72.7 \%)$, shortness of breath $(67.4 \%)$ and muscle pain (66.7\%). According to x-ray findings, $11.6 \%$ of patients showed bilateral pneumonia, $27.9 \%$ of patients showed multiple mottling and ground-glass opacity. The most important complications developed were physical deconditioning $(60.5 \%)$, delirium $(41.9 \%)$ and deterioration of the renal function $(39.5 \%)$. $37.2 \%$ of patients died during the hospitalization. Mortality by groups showed: $40 \%$ among people over 85 years old, $40 \%$ were women, $40,9 \%$ had dementia, $37.5 \%$ were care home residents and $41.4 \%$ had pathological $\mathrm{x}$-rays. The association between those variables and mortality were not statistically significant $(\mathrm{p}>0.05)$.

Conclusion: The most common symptoms are fever and dyspnea. Patients during admission usually have complications such as physical deconditioning and delirium. Even though there was not a statistically significant relation between mortality and the variables studied, there was a slightly higher mortality in patients older than 85 , care home residents, women, those with dementia, and pathological $\mathrm{x}$-ray findings.

\section{ABSTRACT \# 44}

\section{COVID-19 and aging: underlying factors of severity}

Maria Neves ${ }^{1}$, Leonor Vasconcelos de Matos ${ }^{1}$, Ana Vasques ${ }^{1}$, Joana Granado $^{2}$, Ana Esteves ${ }^{3}$, Ana Barradas ${ }^{3}$, Ana Pedroso ${ }^{4}$, Luciana Frade $^{4}$, Susana Peres ${ }^{2}$, Susana Jesus ${ }^{4}$, Cândida Fonseca ${ }^{4}$, Kamal Mansinho $^{2}$
${ }^{1}$ Department of Medical Oncology Hospital São Francisco Xavier, Centro Hospitalar Lisboa Ocidental, Lisbon, Portugal, ${ }^{2}$ Department of Infectious Diseases, Hospital Egas Moniz, Centro Hospitalar Lisboa Ocidental, Lisbon, Portugal, ${ }^{3}$ Department of Internal Medicine, Hospital Egas Moniz, Centro Hospitalar Lisboa Ocidental, Lisbon, Portugal, ${ }^{4}$ Department of Internal Medicine, Hospital São Francisco Xavier, Centro Hospitalar Lisboa Ocidental, Lisbon, Portugal

Introduction: Mortality related to COVID-19 is higher in the elderly population. The purpose of this study was to compare the clinical features of elderly (EG) and young and middle-aged (YM) patients with COVID-19 and analyze mortality predictors.Methods: Retrospective, single center study including patients hospitalized due to COVID-19 infection, from 13 March to 15 June 2020. Results: Of the 195 patients analyzed, $50.8 \%$ were female, $111(56.9 \%)$ patients had $\geq 65$ years $(\mathrm{EG})$ (median age $79[65 ; 94])$, of which $45 \%$ had $\geq 80$ years. 84 patients were YM (median age 50 [22; 54]). EG had multimorbidity: hypertension, diabetes, heart failure and coronary disease $(\mathrm{p}<0.001)$. At admission Early Warning Score-2 $(\mathrm{p}<0.001)$, c-reactive protein (CRP), D-dimer, creatinine, anemia and lymphopenia were higher in EG. Median time of hospitalization was higher in EG (14 vs 10 days, $p=0.004$ ). Complications were more common in $\mathrm{EG}$, bacterial infection (56\% vs $32 \%$ ) and acute kidney injury (31 vs $19 \%)$. There were no significant differences in admission to intensive care. There were 18 deaths (mortality 9, 23\%), 16 in EG, $13(81 \%)$ among patients aged $\geq 80$ years, 2 deaths in YM ( $\mathrm{p}=0.004)$. Modified Early Warning Score (MEWS) at admission (OR 1.60, 95\% CI 1.07-1.37, $\mathrm{p}=0.021$ ) and CRP above $5 \mathrm{mg} / \mathrm{dL}$ (OR 2.12, 95\% CI $1.13-26.26, \mathrm{p}=0.034)$ were independent predictors of mortality in EG. Conclusion: EG were at higher risk for complications and had higher mortality. Identification of specific scores of severity for this population, like MEWS at admission and CRP, is essential to ensure that best care is provided to these patients.

\section{ABSTRACT \# 45}

\section{COVID 19-Clinical Picture in the Elderly Population: A Qualitative Systematic Review}

Agnieszka Neumann-Podczaska ${ }^{1}$, Salwan Al-Saad ${ }^{1}$, Lukasz Karbowski ${ }^{1}$, Michal Chojnicki ${ }^{2}$, Slawomir Tobis ${ }^{3}$, Katarzyna Wieczorowska-Tobis ${ }^{1}$

${ }^{1}$ Geriatrics Unit, Chair and Department of Palliative Medicine, Poznan University of Medical Sciences, Poznan, Poland, ${ }^{2}$ Department of Biology and Environmental Protection, Poznan University of Medical Sciences, Poznan Poland, ${ }^{3}$ Occupational Therapy Unit, Chair of Geriatrics and Gerontology, Poznan University of Medical Sciences, Poznan Poland

Introduction: Large volumes of recently published COVID-19 data makes it strenuous to effectively assess patterns in disease presentation and key clinical features in the older population. A concise qualitative summary of clinical characteristics, in light of most recently published data, allows for improved understanding, better clinical judgement, and guides efforts towards insufficiently researched aspects of disease.

Methods: Qualitative systematic review was performed concerning all articles published from 1st of January to 1st of June 2020, using the keywords COVID-19 and SARS-CoV-2 followed by the generic terms elderly, older adults or older individuals. Following the extraction of data, summarized findings were then given confidence scores by an adaptation of the STROBE statement and CERQual approach.

Results: In total, 1598 articles were screened, of which 20 studies were included in the final analysis, pertaining to 4965 older COVID19 patients ( $\geq 60$ years old). Fever, cough, dyspnea, fatigue, and 
sputum production were the most common symptoms observed. Changes in laboratory findings indicate lymphopenia, inflammation, and occasional organ damage. Renal injury was the most common complication. Roughly $70 \%$ of the older adults required supplementary oxygen, while invasive mechanical ventilation was required in almost a third of the patients. Patients with recorded outcome had a mortality rate of $20.0 \%$ (907/4531). Confidence in findings varied.

Conclusion: Variety in symptom presentation is to be expected, and abnormalities in laboratory findings are present. Risk for mortality is evident, and attention to the need for supplementary oxygen and possible mechanical ventilation is advised. Presented literature may allow for the construction of better predictive models of COVID-19 in older populations.

\section{Abstract \# 46}

\section{Hospital care in geriatric patiens sars Covid 19 infected}

Pilar Ochoa Calvo ${ }^{1}$, J. Eduardo Corrales Cardenal ${ }^{2}$, Claudia P. Murillo Erazo ${ }^{2}$, Sofia Solsona Fernandez ${ }^{1}$

\section{${ }^{1}$ GERIATRA, ${ }^{2}$ MIR GERIATRIA}

Objectives: Description of the COVID 19 infected geriatric patient hospitalized. Assessment of the specific hospital care given during this period.

Method: Descriptive, retrospective study. Sample: all patients admitted in Geriatric Unit from March to May 2020 with firm diagnosis of COVID-19 (PCR and serology test).

Variables: Demographic, clinical and radiological presentation; treatment received; psychological care; geriatric assessment (frailty, functional, cognitive) and severity criteria. Stadistic program SPSS.

Results: N: 41 patients. 68, 32\% women. Mean age: 87.54 years. Nursing home: $73.3 \%$. Average hospital stay: 15.98 days. At discharge: Death: $24.4 \%$. Nursing home: $56.1 \%$.Barthel index (median): 30/100, Pfeiffer test (median) 8 errors, Fragility: Mild (29.3\%), Moderate (34.1\%), Severe (36.6\%)NECPAL: $43.9 \%$ positive. Severity of illness (CURB-65): 1 pt: 36.6\%; 2 pt: $46.3 \%$; 3 pt: $17.1 \%$. Q-SOFA: 0 (65.9\%). Initial Symptoms: $53.7 \%$ Fever; $29.3 \%$ Dyspnoea; $24.4 \%$ Cough; $24.4 \%$ Falls; $12.2 \%$ Delirium. XRay: $51.2 \%$ not suggestive.Treatment (46.3\%): Azithromycin (89.5\%); Cephalosporins (63.2\%); Hydroxychloroquine (57.9\%).Complications: $58.5 \%$ without any complications, during hospitalized.Psychological assistance: $80.5 \%$. Methods: video calls (average 3 per patient), grieving calls (1 per family who needed)Follow up (1 month): Re-Hospitalized (9.8\%); Death (17.1\%).

Conclusions: The pattern patient (Covid 19 infected): Women elder than 80 , with moderate dementia and severe dependence, living in a nursing home, and palliative care subsidiaries. The most have non typical Covid 19 symptoms neither radiological criteria. Psychological assistance, using technological aids, had benefits, for both, families and patients.

\section{Abstract \# 47 \\ Delirium recognition and mortality in older inpatients with COVID-19 disease}

Aine McGovern ${ }^{1}$, Fraser Brooks ${ }^{1}$, Drennan Megan ${ }^{1}$, Erin Frizzell ${ }^{1}$, Laura Knox $^{1}$, Quinn Terry ${ }^{1}$, Hazel Miller ${ }^{1}$

${ }^{1}$ Department Of Geriatric Mediicne, Glasgow Royal Infirmary

Introduction: Delirium is associated with poor outcomes. Early reports of covid-19 in older adults suggest delirium is common. Our primary aim was to assess the prevalence and outcomes of delirium in COVID-19. A recent national delirium audit suggested delirium may be under-diagnosed in frailer individuals. We also assessed the relationship between frailty and delirium recognition.

Methods: This was a retrospective observational study of consecutive patients with community or hospital acquired COVID-19 in a Scottish geriatric unit. "Recognised Delirium" was identified if delirium or cognitive change was described in medical notes, 'Unrecognised Delirium' if delirium screening suggested delirium but there was no description of cognitive change. Frailty was assessed using the Clinical Frailty Score (CFS) and compared across the three groups: no delirium, recognised delirium, unrecognised delirium.

Results: 158 individuals were studied. Average age was $82.5 \pm 7.6$ years, $61 \%$ were female. 67 (42\%) had recognised delirium, $23(15 \%)$ unrecognised delirium and $68(24 \%)$ no delirium. Mortality was lower in those with delirium than those without, but the difference was not statistically significant (12 versus $22 \%, \mathrm{p}=0.099)$. People with unrecognised delirium had higher mortality than those with recognised delirium (30 versus $6 \%, \mathrm{p}=0.002$ ). CFS (median, IQR) was 6 (6-7) without delirium, 6 (6-7) with recognised delirium and 7 (6-7) with unrecognised delirium.

Conclusions: Delirium is common in COVID-19. The highest mortality was in people with unrecognised delirium. They had a slightly higher frailty score which may have impacted on delirium recognition. Screening for delirium at COVID-19 diagnosis may help improve care for this vulnerable group.

\section{Abstract \# 48 \\ Multidimensional frailty, not chronological age, is associated to outcomes in respiratory diseases: Implications for the COVID- 19 pandemic}

Ulla Matthissen ${ }^{1}$, Verena Rehbaum ${ }^{1}$, Lena Pickert ${ }^{1}$, Anna Maria Meyer $^{1}$, Annelie Goldbeck ${ }^{1}$, Volker Burst ${ }^{1}$, Thomas Benzing ${ }^{1}$, Maria Cristina Polidori ${ }^{1}$

${ }^{1}$ Ageing Clinical Research, Department II of Internal Medicine and Center for Molecular Medicine Cologne, University of Cologne, Faculty of Medicine and University Hospital Cologne, Cologne, Germany

Background: Due to the current COVID-19 pandemic, medical professionals around the world are facing a variety of challenges. In some countries it has not been possible to provide all required ventilation therapy due to lack of resources. Difficult decisions had to be made with respect to triaging and chronological age was often used as a criterion. The aim of the present study was to retrospectively assess the clinical characteristics of older patients with respiratory diseases experiencing different outcomes from hospitalization to admission to intensive care unit (ICU), to treatment with non-invasive versus invasive ventilation, to death.

Patients and Methods: The patient collective under investigation is based on four prospective studies conducted at the University Hospital of Cologne between 2016 and 2020. A total of 1.276 older multimorbid patients previously recruited in prospective studies in the emergency department (ED), the ICU and the internal acute care unit were screened for the presence of respiratory diseases as main diagnosis (acute or chronic pulmonary disease) as well the availability of data from a Comprehensive Geriatric Assessment (CGA) with prognosis calculation using the Multidimensional Prognostic Index (MPI) at admission. The MPI considers the functional, social, physical and psychological aspects of the patients and provides continuous values between 0 and 1 , which can be subdivided into three risk groups for mortality and rehospitalization, MPI-1 (0.00-0.33), MPI-2 (0.34-0.66) and MPI-3 (0.67-1.00). Furthermore, acute respiratory stress syndromes (ARDS), tracheotomies, duration, frequency and 
cause of ventilation therapy, infections of the respiratory tract and antibiotics/ virustatics were recorded.

Results: Of the patients' records screened, 237 (M 146, F 9178.1-5.9 years)met inclusion criteria. Ventilation therapy (V) was received by $46(19.4 \%)$ of the patients. The patients who received $\mathrm{V}$ tended to be chronological younger than non-ventilated patients (NV) (median 76.5 (70-82) vs. 78.0 (75-82) years; p-value 0.073). Patients in the V group were significantly more male than those in the $\mathrm{NV}$ group ( $73.9 \%$ vs. $26.1 \%$; p-value 0.039 ). The mean MPI value at admission was $0.61( \pm 0.20)$ in $\mathrm{V}$ patients and $0.50( \pm 0.17)$ for NV patients $(\mathrm{p}$ $<0.001)$. V patients belonged more often to the MPI-3 than NV patients $(41.3 \%$ vs. $22.0 \%)$ and less to the MPI- 1 group than NV patients $(10.9 \%$ vs. $20.4 \%$; $\mathrm{p}=0.020)$. V patients' length of hospital stay was significantly longer than in NV patients' (14.0 vs. 9.0 days; $p$ $=0.006)$ and $\mathrm{V}$ patients had a significant higher heartbeat at admission - prior to being ventilated - than NV patients $(92.0 / \mathrm{min}$ vs. $77.0 /$ min; $p=0.039)$. Thirty-seven $\%$ of $\mathrm{V}$ patients suffered from a hospital acquired pneumonia before they had to be ventilated $(16.8 \%$ of $\mathrm{NV}$ patients; $\mathrm{p}=0.003$ ).

Conclusion: Despite the retrospective nature of the data, a correlation between MPI and ventilation therapies could be observed in older patients affected by respiratory disease, suggesting that multidimensional frailty as a surrogate marker of biological age is a major determinant of patients trajectories for respiratory outcomes. As ventilated patients were younger than nonventilated patients, biological age appears more predictive than chronological age as far as poor outcomes are concerned in this patient population. Further research is needed to establish meaningful correlations between ventilation therapies and MPI, but multidimensional frailty should be systematically addressed in life-threatening conditions such as the COVID-19 disease in order to meet correct clinical and ethical decisions.

\section{Abstract \# 49 \\ The show must go on: teaching geriatric medicine during Covid- 19 lock-down}

Marit Stordal Bakken ${ }^{1}$, Anette Hylen Ranhoff ${ }^{1}$, Susanne Sørense Hernes $^{1}$, Elisabeth Skaar $^{2}$, Katinka Alme ${ }^{3}$

${ }^{1}$ University of Bergen, Norway, ${ }^{2}$ Haukeland University Hospital, Bergen, Norway, ${ }^{3}$ Haraldsplass Deaconess Hospital, Bergen, Norway

Background: The Covid-19 lock-down in Norway coincided with the most intensive period of geriatric teaching for medical students at the University of Bergen. The geriatric curriculum for the 6th (out of 12) semester medical students (gerMED6) is a well-structured package consisting of interrelated, highly interactive group sessions and lectures. Four case-based learning (CBL) sessions constitute the cornerstone of gerMED6, in each of which a selected core topic (acute functional decline, delirium, falls, and frailty) is thoroughly discussed. Additionally, a team-based learning (TBL) session (interface between depression and dementia), and traditional lectures (infections and dyspnoea as well as topics already mentioned) are provided. The curriculum, with explicit theoretical and practical learning objectives, is readily available for the students at semester start.

Method: The pre-existing 2-month gerMED6 module was converted to digital platforms. From March-May 2020, the (166) students were offered real time CBL and TBL interactive sessions; lectures where provided by video. An online anonymous feedback form was used to evaluate the course (learning goals, content, and organization).

Results: Eighty-three students (50\%) responded. Ninety-nine percent reported that the curriculum was at a suitable level; $82 \%$ reported that the educational gain was good or very good (35 and $47 \%$, respectively). This was supported by brief questions showing the students had reached the core learning objectives.

Conclusion: Converting the gerMED6 to digital platforms was successful. The students emphasised the clinical relevance, structure, coherence and information as important. Digital elements will undoubtedly be a part of our future teaching of geriatric medicine.

\section{Abstract \# 50}

Follow-up of patients after acute COVID-19: results on persistent symptoms and control imaging findings

Alpay Medetalibeyoglu ${ }^{1}$, Gulistan Bahat ${ }^{2}$, Yunus Catma ${ }^{1}$, Meryem Merve Oren ${ }^{3}$, Emine Bilge Caparali ${ }^{4}$, Sena Bayrakdar ${ }^{4}$, Seniha Basaran ${ }^{5}$, Naci Senkal ${ }^{1}$, Murat Kose ${ }^{1}$, Mustafa Erelel ${ }^{6}$, Mehmet Akif Karan $^{2}$, Tufan Tukek ${ }^{1}$

${ }^{1}$ The Medical Faculty of Istanbul University, Department of Internal Medicine, Division of General Internal Medicine, ${ }^{2}$ The Medical Faculty of Istanbul University, Department of Internal Medicine, Division of Geriatrics, ${ }^{3}$ The Medical Faculty of Istanbul University, Department of Public Health, ${ }^{4}$ The Medical Faculty of Istanbul University, Department of Internal Medicine, ${ }^{5}$ The Medical Faculty of Istanbul University, Department of Infectious Diseases, ${ }^{6}$ The Medical Faculty of Istanbul University, Department of Chest Diseases

Background/Objective: Covid-19 may have consequences that may continue to affect the suffered patients in the early post-infectious period and later in life. We aimed to report the much-needed data on the follow-up of affected patients.

Methods: 665 recovered Covid-19 patients who were admitted to our follow-up outpatient clinic were included in the study. They were evaluated by physicians from different disciplines. Their clinical characteristics and detailed laboratory examination were recorded. Computed tomography (CT) or X-ray was performed as control chest imaging by their risk of pulmonary involvement.

Results: The median age was 46 years, and 346 (53\%) were males. We assessed patients at a median of 47 days after the recovery. About half $(42 \%)$ of the patients were suffering from one or more ongoing symptomatology other than the fever. The most common symptoms were fatigue (14\%), dyspnea (12\%\%), and dry cough $(11.4 \%)$. The detailed laboratory investigation revealed that there were few patients with laboratory abnormalities that may be associated with Covid-19 disease.More than $1 / 5$ of the patients who underwent control imaging showed some imaging abnormalities in the early post-acute period. Pulmonary abnormalities were detected in almost $15 \%$ of patients that had normal imaging on admission. In those that had ground-glass or fibrotic pathology on control imaging, persistent fatigue, and dry cough was more common ( $\mathrm{p}=0.03, \mathrm{p}=0.04$, respectively).

Conclusion: There was a significant prevalence of persistent symptoms in the early post-recovery period after Covid-19. These results suggest that a close follow-up for consequences of Covid-19 is needed.

\section{Abstract \# 51}

\section{Dysgeusia after covid as a cause of refusal to ingest}

Yanira Aranda Rubio ${ }^{1}$, Gemma Cuesta $^{1}$, Saleta Goñi ${ }^{1}$, Ivón Rivera ${ }^{1}$, Rocio Gomez del Río ${ }^{1}$, Carmen Alcaraz-L ${ }^{2}$

${ }^{1}$ Hospital Central de la Cruz Roja, ${ }^{2}$ Hospital General Universitario Santa María del Rosell

87-year-old man with a history of type 2 diabetes mellitus and high blood pressure. He was not cognitively impaired and walked independently. He was ill with COVID-19 last March with diffuse 
bilateral pulmonary opacities predominantly peripheral and pneumonic in appearance associated with a diffuse prominence of the bronchovascular network. CURB65 scale: 3 points. Hydroxychloroquine treated. After this episode, in subsequent medical consultation, the patient described loss of appetite after having suffered Covid-19 with dysgeusia associated with anosmia. Weight loss of 15 kilos in 3 months. Endoscopy study was completed without significant alterations; Abdominal CT with a result of cholelithiasis; Brain CT without pathology; Chest CT scan shows extensive bilateral lung involvement with fibrosis in the lower lobes; Analytics without significant findings. Given the result of complementary tests, the patient is diagnosed with dysgeusia as a secondary complication of the covid19 virus. The COVID-19 pandemic represents a new challenge for global health. In this clinical case, the importance of its secondary complications stands out. Coronoviruses not only affect the respiratory system, but also have deleterious effects on the central nervous system. $\mathrm{CoV}$ is reported to be found in the brain or cerebrospinal fluid. The pathobiology of these neuroinvasive viruses is fully known.The well-recognized symptoms of COVID-19 include fever, cough, dyspnea, sputum production, myalgia, arthralgia, headache, diarrhea, nausea/vomiting, and sore throat.There is growing evidence that olfactory dysfunction can occur in patients with COVID-19. Taste depends on the activity of specialized epithelial cells, the taste cells, which are found mainly in the mucosa of the tongue.The frequency of smell and taste disorders is high $(49.8 \%$ (95\% confidence interval, $8.2-91.5 \%, \mathrm{I}^{2}=99.6 \%$ ) as other symptoms, so recent Studies indicate that at least anosmia could be included in the symptom lists of COVID-19. Although there is promising evidence, it is premature to conclude that smell and taste disorders are strongly associated with the diagnosis of COVID-19. Multiple cross-sectional studies have shown that the incidence rate of olfactory dysfunction in COVID-19 patients ranges from 33.9 to $68 \%$ with a female predominance. The early olfactory recovery rate in recent studies is $44 \%$.Recent findings could show that SARS-CoV-2 could enter taste cells through Angiotensin Converting Enzyme 2 and as a consequence, the normal functioning of these sensory cells would be disrupted. For its treatment, due to neuronal involvement, treatments such as pregabalin are recommended.

\section{Abstract \# 52 \\ A profile of Frailty, Disease Severity and Outcome among hospitalised Irish older adults with Covid-19}

Elizabeth Moloney ${ }^{1}$, Rónán O'Caoimh ${ }^{1}$, Paul Gallagher ${ }^{2}$, Emer Ahern $^{2}$, Joe Eustace ${ }^{3}$, Kieran O'Connor ${ }^{1}$, Catherine O'Sullivan ${ }^{1}$, Keith McGrath $^{1}$, Eoin Duggan ${ }^{1}$, Susan Lapthorne ${ }^{2}$, Rachel Barry ${ }^{2}$, Corinna Sadlier ${ }^{2}$, Mary Horgan ${ }^{2}$, Eamonn Faller ${ }^{2}$, Denis O'Mahony ${ }^{2}$, Norma Harnedy $^{2}$, Pádraigín O'Sullivan ${ }^{2}$, Eimear Tracey ${ }^{4}$, Arthur Jackson $^{5}$, Michael O'Connor ${ }^{6}$, Marianne Nolan ${ }^{2}$, Eve Stanley ${ }^{2}$

${ }^{1}$ Mercy University Hospital, ${ }^{2}$ Cork University Hospital, ${ }^{3} \mathrm{HRB}$ Clinical Research Facility UCC, ${ }^{4}$ Saint Finbarrs Hospital, Cork, ${ }^{5}$ Mercy University Hospital, Cork, ${ }^{6}$ Cork University Hospital, Cork

Background: Older adults and those with multiple co-morbidities are reported to have more severe clinical manifestations and higher mortality with COVID-19 infection. Detailed examination of patient profiles is required to better understand the clinical course of COVID19 in older patients. Aim: To examine the characteristics and outcomes of hospitalised older adults with COVID-19.Design: Retrospective, multi-centre, cohort observational study

Methods: Data from sixty-nine hospitalised patients aged over 70 years with reverse transcriptase polymerase chain reaction-confirmed COVID-19 at three Irish hospitals were collected from health records. Demographics, weight, symptom profile, COVID-19 severity level based on Irish Thoracic Society guidelines, Clinical Frailty Scale (CFS), Cumulative Illness Rating Scale-Geriatric (CIRS-G) scores, laboratory, poly-pharmacy and radiological data were reviewed. Results: The mortality rate among patients was $23.2 \%(\mathrm{n}=16)$. Median age of survivors was 81.5 years (IQR 76.5-86.5). Mean CFS and CIRS-G scores were $5 \pm 1.6$ and $8.19 \pm 4.4$. Most patients $(\mathrm{n}=$ $56,81.1 \%$ ) were categorised as mild COVID-19 cases. Only five patients $(\mathrm{n}=5,7.2 \%)$ were asymptomatic. Atypical symptoms were common, including diarrhoea $(10.1 \%)$ and anorexia (10\%). Delirium was noted in almost one-third of patients $(n=21,30.4 \%)$. Seven patients $(\mathrm{n}=7,10.1 \%)$ required intubation and intensive care unit admission. Over $1 / 3$ of delirious patients died $(n=8,38 \%)$. Frail patients were older $(\mathrm{P}=0.005)$, more likely to have dementia $(\mathrm{P}=$ $0.04)$ and required more ventilatory support than non-frail patients $(\mathrm{P}$ $=0.001$ ).

Conclusion: Despite mild COVID-19 symptoms, mortality and delirium rates remained high in our study. Multifaceted patient assessment, including frailty and comorbidity measurement, helped build a detailed profile of these hospitalised older COVID-19 patients.

\section{Abstract \# 53}

Effectiveness of tocilizumab in patients with SARS-Cov-2 infection in an intensive care unit

Elena Prado-Mel ${ }^{1}$, Eva Alfaro-Lara ${ }^{1}$, Ana Belén Guisado-Gil ${ }^{1}$, Laura Herrera-Hidalgo ${ }^{1}$, Maria Victoria Gil Navarro ${ }^{2}$

${ }^{1}$ Pharmacy Service. Virgen del Rocio Universitary Hospital, ${ }^{2}$ Pharmacy Service.Virgen del Rocio Universitary Hospital

Objective: to analyze the characteristics of the patients treated with tocilizumab, as well as its effectiveness in the treatment of SARSCoV-2 infection.

Material and methods: Patients admitted to the intensive care unit (ICU) with severe SARS-CoV-2 infection, who received tocilizumab, according to the indications established by the Spanish Agency for Medicines and Health Products (AEMPS) during the months of March-May 2020 were included. The variables were collected: sex, age, weight, height, body mass index (BMI). As risk factors for cardiovascular disease, the presence of hypertension, diabetes and dyslipidemia was recorded. If the patient suffered from another underlying chronic disease (heart failure, chronic kidney failure, cirrhosis, liver transplantation, oncological disease, chronic obstructive pulmonary disease, asthma, bronchiectasis, neurological disease), it was classified as high-risk comorbidity. The date of onset of symptoms of SARS-CoV-2 infection and the date of administration of tocilizumab, the dose and the number of administrations were collected. As a primary objective, the variation of the acute phase reactants (CRP, fibrinogen, lymphocytes and D-dimers, before and 5 days after the first dose) were collected. The t-student test was applied for paired samples $(\mathrm{p}<0.05)$. The secondary objectives were the stay in the ICU (days) and the number of deaths. The data were analyzed with the SPSS statistical package.

Results: 18 patients were included, 15 men. The mean age was 66.77 years $(47-80)$. The mean BMI was $29.55 \mathrm{Kg} / \mathrm{m}^{2}, 27.77 \%$ of the patients were obese and $50 \%$ suffered from pre-obesity (BMI: 27-29.9). Regarding cardiovascular risk factors, 11 were hypertensive, 5 were diabetic and 10 were dyslipidemic. Six patients had highrisk comorbidity and 4 had no risk factors or comorbidity. The administration of tocilizumab with respect to the onset of symptoms presented a median of 9 days (1-16). The mean dose received was $600 \mathrm{mg}$ ( 4 received 3 doses, 5 patients 2 doses and 9 patients 1 dose.) At 5 days after the first dose, on average, CRP decreased $207.8495 \%$ CI [147.77-267.90], fibrinogen decreased 2.73 95\% CI [1.52-3.93], and lymphocytes increased $0.31195 \%$ CI [0.12-0.49], all reaching 
statistical significance. The D-dimers increased on average 5254.66 95\% CI [ $(-4158.06$ to 14667.40$)](\mathrm{p}<0.251)$. Finally, the mean stay in the ICU was 17.5 days (2-48). There were 6 deaths, of which 4 had high-risk comorbidity, 5 were hypertensive and dyslipidemic, and only 1 was diabetic.

Conclusions: A majority of the patients admitted to the ICU who received tocilizumab are obese or pre-obese older than 65 years, hypertensive and dyslipidemic. The use of tocilizumab is statistically significantly associated with an increase in lymphocytes and a decrease in fibrinogen and CRP at 5 days after the first dose, but not with D-dimers. The number of deaths was low. Hypertension and dyslipidemia stand out as prevalent risk factors among the deceased. More studies are needed to evaluate the efficacy of tocilizumab.

\section{Abstract \# 54}

How does SARS-CoV-2 targets the elderly patients? Potential mechanisms increasing disease severity

Annemieke Smorenberg ${ }^{1}$, Edgar J. G. Peters ${ }^{2}$, Paul L. A. van Daele ${ }^{3}$, Esther J. Nossent ${ }^{4}$, Majon Muller ${ }^{1}$

${ }^{1}$ Department of internal geriatric medicine, Amsterdam UMC location VU medical center, De Boelelaan 1117, $1081 \mathrm{HV}$, Amsterdam, the Netherlands, ${ }^{2}$ Amsterdam UMC, Vrije Universiteit Amsterdam, Department of Internal Medicine, Section of Infectious Diseases, Amsterdam Institute for Infection and Immunity, De Boelelaan 1117, $1081 \mathrm{HV}$, Amsterdam, The Netherlands, ${ }^{3}$ Department of allergology and clinical immunology, Erasmus Medical Center, Doctor Molewaterstraat 40, 3015 GD, Rotterdam, the Netherlands, ${ }^{4}$ Department of pulmonary medicine, Amsterdam UMC - location VU medical center, De Boelelaan 1117, 1081 HV, Amsterdam, the Netherlands

Introduction: Among COVID-19 cases, especially the (frail) elderly show a high number of severe infections, hospital admissions, complications, and death. The highest mortality is found between 80 and 89 years old. Why do these patients have a higher risk of severe COVID-19? In this review we address potential mechanisms regarding viral transmission, physical reserve and the immune system, increasing the severity of this infection in elderly patients. Observations: First, the spread of COVID-19 may be enhanced in elderly patients. Viral shedding may be increased, and early identification may be complicated due to a typical disease presentation and limited testing capacity. Applying hygiene and quarantine measures, especially in patients with cognitive disorders including dementia, can be challenging. Additionally, elderly patients have a decreased cardiorespiratory reserve and are more likely to have co-morbidity including atherosclerosis, rendering them more susceptible to complications. The aging innate and adaptive immune system is weakened, while there is a pro-inflammatory tendency. The effects of SARS-CoV-2 on the immune system on cytokine production and T-cells, further seem to aggravate this pro-inflammatory tendency, especially in patients with cardiovascular comorbidity, increasing disease severity.Conclusions: The combination of all factors mentioned above contribute to the disease severity of COVID-19 in the older patient. While larger studies of COVID-19 in elderly patients are needed, understanding the factors increasing disease severity may improve care and preventative measures to protect the elderly patient at risk for (severe) COVID-19 in the future.

\section{Abstract \# 55}

First wave of COVID-19 in the oldest-old in the acute hospital in Belgium: a retrospective multicenter study describing clinical characteristics, treatment limitation decisions and in-hospital mortality related to frailty and respiratory failure

Ruth Piers ${ }^{1}$, Wim Janssens ${ }^{1}$, Katrien Cobbaert ${ }^{2}$, Inge Pattyn ${ }^{3}$, Ine Westhovens $^{4}$, Han Martens ${ }^{5}$, Katrien Van Puyvelde ${ }^{6}$, Sandra Maertens ${ }^{7}$, Vinciane Guyssens ${ }^{8}$, Hilde Baeyens ${ }^{9}$, Griet Buyck ${ }^{10}$, Eva De Raes ${ }^{2}$, Marie Louise Van Leeuwen ${ }^{4}$, Veerle Mouton ${ }^{3}$, David Dedecker ${ }^{5}$, Eva Van Braeckel ${ }^{1}$, Dominique Benoit ${ }^{1}$, Nele Van Den Noortgate $^{1}$

${ }^{1}$ Ghent University Hospital, ${ }^{2} \mathrm{AZ}$ Delta, ${ }^{3} \mathrm{AZ}$ Groeninge Kortrijk, ${ }^{4}$ Jessa Ziekenhuis Hasselt, ${ }^{5}$ AZ Sint Lucas Gent, ${ }^{6}$ Stedelijk Ziekenhuis Aalst, ${ }^{7} \mathrm{AZ}$ Damiaan Oostende, ${ }^{8} \mathrm{AZ}$ Blasius Dendermonde, ${ }^{9} \mathrm{AZ}$ Alma Eeklo, ${ }^{10} \mathrm{AZ}$ Sint Jan Brugge

Introduction: There is lack of knowledge on treatment limitation decisions (TLD), and outcome linked to frailty in older people hospitalized with corona virus disease 2019 (COVID-19).

Method: A retrospective cohort study in patients 80 plus admitted in 10 acute hospitals in Belgium in March and April 2020.

Results: From 711 patients, $62.9 \%$ were home-dwelling, $37.1 \%$ from a nursing home/service flat. Eighty\% had cardiovascular comorbidity, $40 \%$ dementia, metabolic disease or kidney disease. Fifty-four\% were moderately or severely frail (clinical frailty scale (CFS) 6 and 7), 3\% had CFS 8-9 2 weeks before admission. Early (within $72 \mathrm{~h}$ after admission) TLD to not refer to intensive care unit (ICU) in case of deterioration were taken in $473(66.5 \%)$ patients; significantly associated with CFS $(\mathrm{p}<0.001)$ : TLD ranging from $12 \%$ in CFS 1 to $88 \%$ in CFS 7 and $100 \%$ in CFS 8-9. Overall in-hospital mortality was $34.6 \%$ with main cause of death respiratory failure (81.2\%). In 373 patients without respiratory failure, in-hospital mortality ranged from $0 \%$ (CFS 1) to $14 \%$ (CFS 7) and $72.7 \%$ in CFS $8-9$ (p > 0.001). In 337 patients with respiratory failure, in-hospital mortality was $60 \%$, regardless of CFS score $(\mathrm{p}=0.222)$. All-cause in-hospital mortality did not differ between ICU treated patients versus non-ICU treated patients in univariate analysis. Multivariate analyses are currently being performed.

Conclusions: Early TLD were taken frequently in older hospitalized COVID-19 patients, significantly associated with the level of frailty. First analyses show an association between frailty and in-hospital mortality in mild but not in severe SARS-CoV-2 infection.

\section{Abstract \# 56 \\ Failing the frail-the need to broaden the COVID-19 case definition for geriatric patients}

Clare Hunt ${ }^{1}$, Flora Olcott ${ }^{1}$, Terrence Chan ${ }^{1}$, George William ${ }^{1}$

${ }^{1}$ Dartford and Gravesham NHS Trust

Introduction: The criteria for possible SARS-CoV-2 infection focuses on evidence of pneumonia, acute respiratory distress syndrome, anosmia/change in sense of taste, fever $\geq 37.8{ }^{\circ} \mathrm{C}$, new persistent cough and breathlessness, as outlined by Public Health England. Triage of patients in the hospital setting typically relies on these symptoms. As elderly people with infections often present atypically there is a need to increase understanding of how COVID19 affects this population.

Method: This retrospective observational study looks at patients aged 70 and over who were admitted to a district general hospital in SouthEast England between March 15th and May 31st 2020 and tested positive for SARS-CoV-2 on admission. Clinical characteristics and 
observations were collected from their initial hospital electronic record and analysed.

Results: A total of 225 patients were included, of whom only $11.5 \%$ $(n=26)$ presented with the trio of cough, fever and breathlessness. However, $68(30.2 \%)$ did not present with any of these symptoms (p $<0.001)$. The most frequent atypical complaints were 'generally unwell' ( $\mathrm{n}=46,20 \%)$, falls $(\mathrm{n}=43,19 \%)$ and confusion $(\mathrm{n}=37$, $16 \%$ ). In comparison to patients presenting with a temperature $\geq 37.6$ ${ }^{\circ} \mathrm{C}(\mathrm{n}=73,32.4 \%), 81(36.0 \%)$ recorded a normal temperature of $36.5-37.5^{\circ} \mathrm{C}(\mathrm{p}>0.05)$ and $20.4 \%(\mathrm{n}=46)$ had a temperature $<36.4$ ${ }^{\circ} \mathrm{C}(\mathrm{p}=0.0003)$.

Conclusion: A significant proportion of geriatric patients with COVID-19 presented with atypical symptoms and observations. The relatively high number of falls in this cohort highlights the need for thorough history-taking, as well as accurate rapid diagnostic testing for SARS-CoV-2 to allow effective infection control in the hospital setting.

\section{Abstract \# 57 \\ Diagnostic utility of baseline chest radiography among older patients with Coronavirus Disease 2019 (COVID-19)}

Patrick Crowley ${ }^{1}$, Elizabeth Moloney ${ }^{1}$, Ronan O. Caoimh ${ }^{1}$

${ }^{1}$ Mercy University Hospital (MUH)

Introduction: While reverse-transcriptase polymerase chain reaction (RT-PCR) is the diagnostic reference standard for COVID-19, the test is limited by high false negative rates and delay in reporting results [1]. Radiology is an essential aid to diagnosis and prognostication [1]. Although computed tomography (CT) is the most sensitive and specific modality [1], it is limited by increased radiation exposure and issues relating to availability and infection control [2]. While existing studies report relatively poor sensitivity for chest radiography $[2,3$, 4], older patients often present with more advanced lung involvement $[1,3,4]$. The purpose of this study is to evaluate the diagnostic utility of baseline chest radiography among older patients with COVID-19. Methods: This retrospective study involved patients over 70 years of age who were diagnosed with COVID-19 by RT-PCR between 27 th February and 24th April 2020 at three hospitals in Cork, Ireland. Chest radiography findings were based on the initial radiology report. Results: 69 patients were diagnosed with COVID-19. 40 (58\%) were male and $29(42 \%)$ were female. The mean age was 80.65 . Of the 62 patients who had baseline chest radiography, 9 (14.5\%) were radiologically negative while $53(85.5 \%)$ had reported abnormalities consistent with COVID-19. Males $(89.2 \%)$ were more likely to have abnormal findings than females (80\%). 51\% had bilateral lung involvement. $24.5 \%$ had only right-sided involvement. $24.5 \%$ had only left-sided involvement. $75.5 \%$ had predominantly mid-lower zone involvement. $3.8 \%$ had only upper zone involvement. $20.7 \%$ involved both upper and lower zones. Key

Conclusions: Baseline chest radiography is a useful tool for diagnosing COVID-19 among older patients.

References:

1. Cozzi D, Albanesi M, Cavigli E, Moroni C, Bindi A, Luvara S, Lucarini S, Busoni S, Mazzoni LN and Miele V. Chest X-ray in New Coronavirus Disease 2019 (COVID-19) Infection: Findings \& Correlation With Clinical Outcome. Radiol Med (2020) Jun 9: 1-8 https://doi.org/10.1007/s11547-020-01232-9

2. Wong HYF, Lam HYS, Fong AHT, Leung ST, Chin TWY, Lo CSY, Lui MMS, Lee JCY, Chiu KWH, Chung TWH, Lee EYP, Wan EYF, Hung IFN, Lam TPW, Kuo MD and Ng MY. Frequency and Distribution of Chest Radiographic Findings in Patients Positive for COVID-19. Radiology (2020) Aug; 2962 :E72-E78. https://doi.org/ 10.1148/radiol.2020201160
3. Gatti M, Calandri M, Barba M, Biondo A, Geninatti C, Gentile S, Greco M, Morrone V, Piatti C, Santonocito A, Varello S, Bergamasco L, Cavallo R, Di Stefano R, Riccardini F, Boccuzzi A, Limerutti G, Veltri A, Fonio P and Faletti R. Baseline Chest X-ray in Coronavirus Disease 19 (COVID-19): Association with Clinical and Laboratory Data. Radiol Med (2020) Sept 7: 1-9 https://doi.org/10.1007/ s11547-020-01272-1

4. Ippolito D, Pecorelli A, Maino C, Capodaglio C, Mariani I, Giandola T, Gandola D, Bianco I, Ragusi M, Franzesi CT, Corso R and Sironi S. Diagnostic Impact of Bedside Chest X-ray Features of 2019 Novel Coronavirus in the Routine Admission at the Emergency Department: Case Series from Lombary Region. Eur J Radiol. (2020) Aug 129: 109092.

\section{Abstract \# 58 \\ Recognition and characterization of the frail older adults in Belo Horizonte, Brazil during COVID19 pandemic}

Maria Carolina Vieira ${ }^{1}$, Cassio Mota ${ }^{2}$, Camila Barbosa Mota ${ }^{3}$, Ana Julia Santos ${ }^{3}$, Patricia Mattoso ${ }^{3}$

${ }^{1}$ FELUMA, ${ }^{2}$ PUCMG, ${ }^{3}$ Supere Senior Center

The recognition and characterization of the frail older adults is essential in Covid-19's fight strategies. They are critical to develop a special plan for geriatric care due to the high vulnerability and preexisting health conditions of this group. The "shared decision making" with the patient and family is particularly important in this context. Within a Project of integrated assistance to older people at risk of functional decline in the COVID-19 pandemic in Belo Horizonte, a cross-sectional this study was carried out with the aim of identifying fragile older people and broadening the understanding of preferences, care models in this group. In the quantitative step, the self-administered questionnaire with easy access and understanding made available on electronic means applied to 410 older adults was used. Three hundred eighty one was recruited to this study. In the qualitative step, 50 older adults participated in semi-structured interviews by videoconference, telephone call or presential encounters. Specialized care for the older adults involves appropriate communication strategies, shared decisions and prognosis definitions. Understanding the specificities of this group and anticipating advance directives can mitigate the overload in emergency care and intensive care units. Key words: frailty, screening, covid19, healthy policy, care planning.

\section{Abstract \# 59}

Virtual (Telephone/video) clinics as an outpatient clinic alternative for older people in the COVID 19 pandemic

Atef Michael ${ }^{1}$

${ }^{1}$ Russells Hall Hospital, Dudley, UK

Introduction: During the COVID 19 pandemic, hospitals have had a heavy workload and obviously represent an environment with potential high COVID 19 antigen load. To reduce the risk of spread of infection many outpatient clinics in the NHS hospitals across the UK, have been cancelled/postponed, and a follow up alternative has to be found. An attractive alternative is telemedicine; a virtual clinic as a telephone or video consultations. The advantages and disadvantages of the virtual clinic and when it can and cannot be used in older people will be briefly discussed. The lists are not exhaustive, but represent general and broad outlines and consideration to guide practice and decision-making. Virtual clinics can be used to: 
- Review the results of previously requested investigations and decide further action.

- Know the impact of newly started or reduced medications• Assess recovery following operative procedures.

- Triage and prioritize patients to identify those who need a face-toface consultation.

- Notice incompleteness, incorrectness and ambiguities of previous plans in the discharge summaries or clinic letters $\bullet$ Follow up of some previously diagnosed chronic diseases.

- Treat some acute minor conditions e.g. common cold Virtual clinics cannot be used in:

- First outpatient clinic consultation in many disease conditions .

- Medical emergencies or markedly unwell patients.

- Patients with red flags.

- Patients with suspicion of malignancy.

- Assessment of patients with neurologic deficit Advantages of virtual clinics:

- To save older people, relatives and carers the time, transport expenditure and effort and may be travelling long distances.

- To provide more convenient care $\bullet$ To reduce the risk of exposure of older people to infection.

- To save physicians and health care professionals time and movement between hospital sites or clinics.

- To reduce unnecessary attendance at the hospital and increase the outpatient clinic capacity.

- To reduce carbon emissions Disadvantage of virtual clinics:

- There could be technological, societal or professional barriers.

- Not all older patients have access to video consultation facilities.

- It might not be a practical option for older people who are hard of hearing or with poor vision or who have some memory impairment or lack of dexterity.

- Inability to examine the patient and missing physical findings.

- Possibility of missing serious pathology; this can represent medico legal liability.

- Virtual clinics could be time consuming. And in case of telephone calls:

- Physicians are unable to use "Looks well "or "looks unwell" valuable summative clinical impression. .

- Inability to assess body language.

- Inability to observe crucial physical.

- Inability to objectively have evidence of improvement or otherwiseConclusions:

- The face-to-face medical consultation remains the gold standard however there could be situations when an alternative may be considered more appropriate.

- Virtual clinic is a viable option to be considered for follows up of older patients during the COVID 19 pandemic.

- Video consultations, compared to the telephone ones, allow more interaction with patients and have the advantage of more visual clues and would provide much more information. However many elderly may not be geared to use such technology.

- The decision to have a virtual clinic should be individualised.

- The physicians need to be aware of the limitations and the medico legal consequences.

- It is important to share a summary of the virtual consultation outcome with the patient, e.g. via a letter or an email.

- The virtual clinics will be refined, modified and improved with guidelines to identify indications and "contraindications" to ensure safe effective clinical care.

\section{Abstract \# 60}

Lance-Adams syndrome in an octogenarian woman with SARS COV-2 pneumonia: case report

Pamela Lisette Carrillo Garcia ${ }^{1}$, Blanca Garmendia Prieto ${ }^{2}$, Sagrario Pérez Delgado ${ }^{3}$, Jorge Artero Ortiz ${ }^{4}$, Javier Gómez Pavón ${ }^{3}$

${ }^{1}$ Hospital Central de La Cruz Roja. San José y Santa Adela, ${ }^{2}$ Hospital Central dela Cruz Roja. San José y Santa Adela, ${ }^{3}$ Hospital Central de la Cruz Roja. San José y Santa Adela, ${ }^{4}$ Hospital Central de la Cruz Roja. San José y Santa Adela.

Introduction: Lance-Adams syndrome (LAS) is a condition characterized by development of chronic post-hypoxic action myoclonus due to a temporary lack of or inadequate brain oxygen supply 4 . LAS is a rare complication 3.Clinical presentation is action myoclonus and problems in cognitive function $4,5,6$.

Case report: An 82-year-old woman was admitted having presented SARS-CoV-2 pneumonia. After clinical, radiological and analytical worsening with signs of acute respiratory distress syndrome required admission to ICU, Orotracheal intubation was needed for 6 days. During her staying in the ICU, she had many complications: Pneumomediastinum and probable interstitial emphysema in possible relationship with barotrauma, acute renal failure, and bacterial superinfection .She received the following treatments: Lopinavir/Ritonavir, Hydroxychloroquine, Ceftriaxone, Azithromycin, Corticosteroids and Enoxaparin. After staying in the ICU, she presented tremor in right upper limb that radiated to the lower limb on the same side, which difficulted the ambulation. Brain MRI: oval areas of ischemia in protuberance, midbrain and semioval center. Metabolic disorders were ruled out.She was evaluated by t neurologist and was diagnosed Lance-Adams Syndrome. Treatment with clonazepam. The patient received physical rehabilitation treatment.

Conclusions: Many cases of LAS are related to cardiac arrest and subsequent resuscitation; in this case myoclonus is described in a patient who presented hypoxemic state without cardiac arrest. Pharmacological treatment has not been established. In our patient clonazepam was sufficient to control the myoclonus.We emphasize that early diagnosis is necessary in order to start appropriate treatment: pharmacological and rehabilitation .

\section{Abstract \# 61}

Early digital rehabilitation program for hospitalized COVID-19 patients

Yael Adoni Tamir ${ }^{1}$, Ron Cialic ${ }^{1}$, Orly Barak ${ }^{1}$

${ }^{1}$ Tel Aviv Sourasky Medical Center, Tel Aviv, Israel; Sackler Faculty of Medicine, Tel Aviv University, Tel Aviv

Introduction: The COVID-19 pandemic presented us with unique challenges. COVID-19 patients are prone to develop severe respiratory failure and debilitating deconditioning. In addition, COVID-19 patients are physically and socially isolated. The care of these patients necessitate innovative approach.. We present a model, based on combined digital and hands-on tool, designed to provide early comprehensive rehabilitation for isolated patients.

Methods: Bites $\odot$ is a digital platform for sharing video-based interactive presentations. We used Bites to create a collection of education and training videos encompassing various biopsychosocial domains. These videos are sent to the patient via smart phone, enabling to deliver content tailored to the patient needs. A multidisciplinary based protocol directs the usage of this platform in an earlyrehabilitation program, comprised of digital and hands-on interventions. 
Results: We created a collection of professional education and training videos in the following fields: Medicine, Physiotherapy, Occupational therapy, Nutrition, Social Services, Speech therapy. As the epidemic curve in the country was successfully flattened, the large scale implementation of the program was not executed. Accounting for the high likelihood of a second COVID-19 wave, the potential for this early rehabilitation tool is high.

Key conclusions: The special situation of isolated and deconditioned patients presents a unique challenge. Implementation of an early combined digital/hand-on rehabilitation program can improve the well-being and outcomes of those who can participate in such a program. The program is currently under adjustment to be used to provide early rehabilitation in the non-COVID-19 hospitalized patients and in the post-hospital period as well.

\section{Abstract \# 62}

Recognition and characterization of the frail older adults in Belo Horizonte, Brazil during COVID19 pandemic

Maria Vieira ${ }^{1}$, Cassio Mota ${ }^{2}$, Camila Barbosa Mota ${ }^{3}$, Ana Julia Santos $^{3}$, Cadjija Soares ${ }^{3}$, Patricia Mattoso ${ }^{3}$

\section{${ }^{1}$ FELUMA, ${ }^{2}$ PUCMINAS, ${ }^{3}$ SUPERE SENIOR CENTER}

The recognition and characterization of the frail older adults is essential in Covid-19's fight strategies. They are critical to develop a special plan for geriatric care due to the high vulnerability and preexisting health conditions of this group. The "shared decision making" with the patient and family is particularly important in this context. Within a Project of integrated assistance to older people at risk of functional decline in the COVID-19 pandemic in Belo Horizonte, a cross-sectional this study was carried out with the aim of identifying fragile older people and broadening the understanding of preferences, care models in this group. In the quantitative step, the self-administered questionnaire with easy access and understanding made available on electronic means applied to 410 older adults was used. Three hundred eighty one was recruited to this study. In the qualitative step, 50 older adults participated in semi-structured interviews by videoconference, telephone call or presential encounters. Specialized care for the older adults involves appropriate communication strategies, shared decisions and prognosis definitions. Understanding the specificities of this group and anticipating advance directives can mitigate the overload in emergency care and intensive care units.

Keywords: frailty, screening, covid19, healthy policy, care planning.

\section{Abstract \# 63 \\ Management of COVID-19 pandemic in a Maltese Non-Acute Hospital; lessons learnt from Karin Grech Rehabilitation Hospital}

Maria Bonnici ${ }^{1}$, Daniel Debattista ${ }^{1}$, Clarissa Zehlicke ${ }^{2}$, Claire Callus ${ }^{1}$, Michelle Grech ${ }^{1}$, Rebecca Ceci Bonello ${ }^{1}$, Indika Thilan Perera $^{1}$, John Cordina ${ }^{3}$

${ }^{1}$ Karin Grech Hospital, Malta, ${ }^{2}$ Karin Grech Hospital-Malta, ${ }^{3}$ Karin Grech hospital - Malta

Introduction: Karin Grech Hospital (KGH) is Malta's main rehabilitation hospital with over 300 in-patient beds, outpatients and multidisciplinary clinics. During the COVID-19 pandemic, KGH set up its own pandemic risk modification plan which met the hospital specific needs without deviating from the national practice guidelines stream.
Method: The management plan was designed in a triple axis fashion; Professional \& Clinical: Staff received training and simulation for COVID-19 care and prevention. Admissions were suspended until a safe admission pathway was drafted. Outpatient clinics were stopped. Protocols for isolation, contact tracing and quarantining of inpatients were developed.Managerial: A task force was created to develop protocols. Rosters were adjusted and meetings conducted virtually to minimize staff contact.Infrastructural: A ward equipped for isolation of COVID-19 cases was added, and necessary personal protective equipment supplied. Staff was split into COVID-19 and Non COVID19 teams to prevent cross infection.

Results: Staff felt prepared to confront the pandemic before the onset of the COVID-19 outbreak in Malta. Suspension of admissions until a safe pathway was created decreased the number of positive patients. The few cases of COVID-19 positive patients were quickly identified and isolated, without spread within wards. Staff shortages due to mandatory quarantine presented significant challenges which were overcome with good team-work and planning. Despite the staff shortages and restrictions patient rehabilitation was achieved.

Conclusion: Thorough planning, safe practice, and a willingness to adapt to challenges lead to the successful management and containment on COVID-19 cases, while maintain a rehabilitation in-patient service.

\section{Abstract \# 64}

Hospital trainees" worries, percieved sufficiency of information and reported psychological health during the Covid19 pandemic

Aria Khani ${ }^{1}$, Nikoo Aziminia ${ }^{1}$, Colette Smith ${ }^{2}$, Ameet Bakhai ${ }^{1}$, Clifford Lisk ${ }^{1}$

${ }^{1}$ Barnet Hospital BH, ${ }^{2}$ University College Hospital UCH

Introduction: During the A/H1N1 influenza pandemic, healthcare workers presented frequent concerns regarding their health, their families health as well as high levels of psychological distress. We assessed hospital trainees' concerns, perceived sufficiency of information, behaviour and reported psychological health during the COVID 19 pandemic.

Method: Between May 1st and 23rd 2020, 204 trainees of an NHS Foundation trust completed a questionnaire regarding worries and concerns about the new COVID19 pandemic.

Results: 92\% looked after COVID19 patients. 92\% were worried about COVID19; the most frequent concern was that of family and friends dying from COVID19 (75\%). 22\% reported infection with COVID19. $7 \%$ of trainees were so concerned about COVID19 infection that they would avoid going to work. Perceived sufficiency of information about COVID19 was moderately high. 26\% reported that they were able to socially distance at work compared to $94 \%$ able to socially distance outside work. $42 \%$ reported that their psychological health had been affected by their work with the commonest being anxiety $(57 \%)$, emotional distress $(51 \%)$ and burnout $(38 \%)$. $96 \%$ think it is important to have a service that provides psychological support during this pandemic; only $62 \%$ would consider its use at work.

Conclusions: A significant proportion of hospital trainees are worried about the COVID19 pandemic with high levels of reported psychological distress. Given that almost a third would not use psychological support services at work, hospital leaders and liaison psychiatry need to explore the reasons for not wanting to use services at work and consider providing psychological services outside work. 


\section{Abstract \# 65 \\ Does clean site elective endoscopy increase risk Of Covid-19 transmission?}

Sachan Maruthan ${ }^{1}$, Suji Kim ${ }^{1}$, Aria Khani ${ }^{2}$, Kalpesh Bershedas ${ }^{3}$

${ }^{1}$ General Medicine, Barnet Hospital, Royal Free London NHS Trust,

${ }^{2}$ Acute Internal Medicine, Barnet Hospital, Royal Free London NHS Trust, ${ }^{3}$ Gastroenterology, Barnet Hospital, Royal Free London NHS Trust

Introduction: The COVID-19 pandemic has led to significant service reorganisation within the National Health Service (NHS). Hadley Wood, a non-acute private hospital, is now offering NHS endoscopy for the Royal Free London Trust. New green-site recommendations now include a 7 day isolation period, swab testing three days prior to endoscopy, temperature check and questionnaire upon arrival. We hypothesize that patients are not subjected to an increased risk of contracting COVID-19 from endoscopy and would not require routine swabbing.

Methods: A single-centre retrospective analysis was performed on patients requiring high priority endoscopy from 13/04/2020 to 14/5/ 2020. Telephone surveys were conducted at least 7 days post endoscopy by 2 researchers screening for COVID-19 symptoms after endoscopy, date of symptom onset and transmission risk behaviours including GP visits, social distancing measures, and the frequency of leaving their home.

Results: 62 patients were included in the analysis; four were excluded due to non-response (2), non COVID-19 related death (1) and previous RT-PCR positive COVID-19 (1). One patient reported typical COVID-19 symptoms within 14 days post endoscopy (1.61\%). However, this patient had been in public on four occasions post endoscopy. 61 of 62 patients were asymptomatic. None had routine swabbing prior to endoscopy.

Conclusions: This study illustrates no significant increased risk of contracting COVID-19 from green-site endoscopy despite the lack of routine RT-PCR testing pre-endoscopy. This study proposes that swab testing prior to green-site endoscopy is not routinely required which is of benefit as general consensus is that increased visits to hospital may increase the risk of COVID-19.

\section{Abstract \# 66 \\ A rapid response network for COVID-19 management in nursing homes}

Flamaing Johan ${ }^{1}$, Tournoy Jos ${ }^{1}$, Van Pottelbergh Gijs ${ }^{2}$, Helsen Tuur ${ }^{3}$, Nelde Annke ${ }^{3}$, Claes Helena ${ }^{4}$, Van Nuffel Willem ${ }^{5}$, Charlier Filip ${ }^{6}$, Tambeur Wim ${ }^{7}$, Balcaen Koen ${ }^{8}$, Van Cleynenbreugel Evelyn ${ }^{9}$, Van Parys Veerle ${ }^{9}$, Michiels Dominik ${ }^{10}$, Mertens Reini ${ }^{10}$, Ampe Jan ${ }^{11}$

${ }^{1}$ Dept. Geriatric Medicine, UZ Leuven; Dept. Public Health and Primary Care, KU Leuven, ${ }^{2}$ Dept. Public Health and Primary Care, KU Leuven, ${ }^{3}$ Dept. Geriatric Medicine, HH Ziekenhuis, Leuven, ${ }^{4}$ General Practitioner, Oud-Heverlee, Belgium, ${ }^{5}$ General Practitioner, Rotselaar, Belgium, ${ }^{6}$ General Practitioner, Binkom, Belgium,

${ }^{7}$ Hospital Director, UZ Leuven, ${ }^{8}$ Nursing Director, UZ Leuven,

${ }^{9}$ Dept. Geriatric Medicine, UZ Leuven, ${ }^{10}$ Dept. quality of care, UZ Leuven, ${ }^{11}$ Nursing Director, HH Ziekenhuis, Leuven

Rationale: COVID-19 entered Belgium in the beginning of March 2020 and resulted in organization of hospital surge capacity to accommodate COVID-patients. Nursing homes (NH) in Belgium closed their doors for visitors on March 17th, 2020. With the focus on hospital COVID-management, the situation in nursing homes remained under the radar until COVID-outbreaks and deaths were increasingly reported from the end of March on. By the end of May more COVID-related deaths have occurred in nursing homes $(\mathrm{n}=$ 4742) than in hospitals $(n=4488)$.This Abstract reports the goals and results of the rapid response network for COVID-19 management in nursing homes (RRNWNH), initiated in the beginning of April 2020 in the Leuven region, Belgium.

Methods: The RRNWNH consisted of 3 main partners: hospitals ( $\mathrm{n}=$ $2)$, NH management $(\mathrm{n}=58)$ and coordinating general practitioners (CGP's) of NH $(\mathrm{n}=58)$. A steering committee of hospital nursing directors $(n=2)$, geriatricians $(n=3)$, CGP's $(n=4)$ and clinical process managers $(n=4)$ coordinated actions in the RRNWNH. These actions consisted of creating a website, monitoring tool and an intervention and follow-up log tool for the RRNWNH partners, a hotline for CGP's, an on demand outreach Nose Throat Ear physician swabteam, educational webinars and intervisions with $\mathrm{NH}$, onsite medical and infection control advice, secondment of hospital nurses and PPE purchase/exchange.

Results: Weekly online meetings were set up with the partners of the RRNWNH and the steering committee to discuss COVID-outbreaks and actions. The website offers $\mathrm{NH}$ all necessary contact information and links to educational material and network reports. The monitoring tool was used by $31 \mathrm{NH}(1-33$ registrations per $\mathrm{NH})$ depicting COVID-cases and mortality in residents and health care workers (HCW), HCW absence and personal protective equipment (PPE) needs. The intervention and follow-up log tool allowed to communicate and coordinate interventions in the different NH's. This allowed medical (geriatricians and CGPs) and infection control advice by advanced clinical practice nurses on site. CGP's contacted geriatricians, gerontopsychiatrists, palliative care physicians and hospital epidemiologists also via the hotline for specific urgent matters. The swabteam screened 1337 residents and $1534 \mathrm{HCW}$ in $16 \mathrm{NH}$ with COVID-outbreaks. Weekly infection control webinars were followed by $\pm 20 \mathrm{NH} .27$ hospital nurses worked 118 shifts in $7 \mathrm{NH}$ with HCW shortage due to COVID-outbreaks. FFP2 sterilization and apron purchase was centralized for several $\mathrm{NH}$.

Conclusion: Our RRNWNH was perceived as an urgent need and added value for a vulnerable sector in this COVID crisis. The network enabled the detection of COVID-related problems in $\mathrm{NH}$, prioritization of and response to specific medical, infection control, educational, staffing and material needs. The RRNWNH forms the basis for a more preventive approach in the future and for the development of a long-lasting integrated care network between the partners involved.

\section{Abstract \# 67}

Lessons learnt from COVID-19 related end of life care - a review of deaths from a london district general hospital

Antony Zacharias ${ }^{1}$, Resham Arain ${ }^{1}, \mathrm{Ku}$ Shah $^{1}$, Sheena Mitchell ${ }^{1}$

${ }^{1}$ The Whittington Hospital, London

Introduction: In the United Kingdom in 2020, SARS-CoV-2 is the leading cause of death, and age is the most predictive factor $[1,2]$. We present a review of such deaths from geriatric wards in a district general hospital between 15th March and 30th April 2020.

Method: Structured judgement reviews were retrospectively completed for 18 patients using physical and electronic records. Avoidability of death and care quality were graded using the 'Avoidability of Death Judgement Score' and 'NCEPOD Classification of Care' [3, 4].

Results: The mortality rate was greater for patients with, compared to those without, COVID-19 (26.21\% versus $15.29 \%)$. The mode avoidability score was 6 (range 5-6). All patients had an appropriate treatment escalation plan. Sixteen were recognised as 'end of life' (EoL) and received anticipatory medications. $37.5 \%$ were not referred 
to palliative care due to rapid deterioration. Those that were, received anticipatory medications more proactively ( $80 \%$ versus $62.5 \%$ ), and fewer continued 'active management' (40\% versus $75 \%)$. Next of kin were regularly updated in 15 cases $(83.3 \%)$ with facilitated video calls. In six cases, ward moves led to reduced quality of care, namely miscommunication of target oxygen saturations and care shifting from comfort prioritisation. Deprivation of Liberty Safeguards were submitted for only $27.3 \%$ of patients that lacked capacity.

Conclusions: Our review demonstrates a high standard of EoL care provided in uncertain times. Early involvement of the multidisciplinary team improved care, whereas inter-ward transfers tended to detract from this. Greater detail will be available in our final presentation.

\section{References:}

[1] Campbell A, Caul S. Deaths involving COVID-19, England and Wales. Office for National Statistics 2020.

[2] The Economist. Tracking covid-19 excess deaths across countries. The Economist 2020.

[3] Confidential Enquiry into Patient Outcome N, Death. NCEPOD grading system for use by case reviewers. NCEPOD 2020. [4] Hutchinson A. Using the structured judgement review method 2016.

\section{Abstract \# 68 \\ Prognostic value of lung ultrasound in older nursing home residents affected by COVID-19}

Nicola Veronese ${ }^{1}$, Luca Gino Sbrogiò ${ }^{1}$, Roberto Valle ${ }^{1}$, Laura Marin $^{1}$, Elena Boscolo Fiore ${ }^{2}$, Andrea Tiozzo ${ }^{1}$

${ }^{1}$ ULSS 3 Serenissima, ${ }^{2}$ Chioggia Nursing Home

Background: Lung ultrasound (LUS) imaging may play an important role in the management of patients with COVID-19-associated lung injury, particularly in some special populations, such as pregnant women. However data regarding the prognostic role of the LUS in nursing home residents, one of the most affected populations by COVID-19, are not still available.

Methods: Nursing home residents were followed-up with a LUS from 08th April to 14th May 2020 in Chioggia, Venice. COVID-19 was diagnosed through a nasopharyngeal swab. LUS results were scored according to previous works on COVID-19 pneumonia using a 12-zone method, with a score from 0 to 12 , higher scores reflecting more pneumonia severity. Mortality was assessed using administrative data. Data regarding accuracy (and related parameters) were then reported.

Results: Over 175 nursing home residents, 48 (mean age: 84.1 years; 39 females) were affected by COVID-19, with 12 died during the follow-up period. The mean LUS score was 3 . The area under the curve of LUS in predicting mortality was 0.603 (95\% confidence intervals: $0.419-0.787)$. Taking score $>4$, the sensitivity in predicting mortality was $58.33 \%$, specificity $63.89 \%$, a positive likelihood ratio of 1.62 and a negative of 0.65 .

Conclusions: LUS is able to significantly predict mortality in nursing home residents affected by COVID-19, indicating that this simple tool can be routinely used in this setting instead of more invasive techniques available only in hospital.

\section{Abstract \# 69 \\ Hospital doctors perspectives of social distancing and solutions during The Covid19 pandemic}

Aria Khani ${ }^{1}$, Nikoo Aziminia ${ }^{1}$, Colette Smith ${ }^{2}$, Ameet Bakhai ${ }^{1}$, Clifford Lisk ${ }^{1}$
${ }^{1}$ Barnet Hospital BH, ${ }^{2}$ University College Hospital UCH

In response to the COVID19 pandemic, the UK government announced social distancing as part of measures designed to reduce the spread of coronavirus. We aimed to assess perspectives of hospital doctors in training on social distancing and solutions for their workplace in hospital.Between 1st and 23rd of May 2020, trainees at a UK NHS foundation trust were asked to complete a questionnaire regarding social distancing and perceived solutions for the workplace.204 trainees responded. 60\% were female with $37 \%$ of respondents being Specialist Registrars. 26\% reported that they socially distanced themselves at work compared to $94 \%$ socially distancing themselves outside work. The commonest reasons for not socially distancing at work were stated as "unable to socially distance during ward rounds" (72\%) and "unable to socially distance during board rounds due to the size of the room" (68\%). Options suggested to enable social distancing at work include larger offices (85\%), more computers $(76 \%)$, larger recreational spaces (62\%), EPR access at home $(49 \%)$ and remote ward rounds $(38 \%)$. With the ongoing pandemic, social distancing measures are likely to be required for some time. It is therefore important that healthcare leaders consider potential solutions offered by their staff as part of measures at work to reduce the spread of coronavirus in healthcare settings.

\section{Abstract \# 70 \\ Healthcare workers experience of the Covid19 pandemic: perspectives from the frontline}

Aria Khani ${ }^{1}$, Nikoo Aziminia ${ }^{1}$, Colette Smith ${ }^{2}$, Ameet Bakhai ${ }^{1}$, Clifford Lisk ${ }^{1}$

\section{${ }^{1}$ Barnet Hospital BH, ${ }^{2}$ University College Hospital UCH}

The COVID19 pandemic has seen a death toll of 360, 089 deaths globally. This raises concerns about the mental health and wellbeing of Healthcare workers (HCWs) caring for patients with COVID19. We aimed to assess HCWs concerns, perceived sufficiency of information and reported psychological health during COVID19. Between 1st and 31st May 2020; $410 \mathrm{HCWs}$ of an NHS Trust completed a questionnaire regarding concerns, perceived sufficiency of information and reported psychological health during the COVID19 pandemic.There was a preponderance of females (307) with hospital doctors in training (HD) (204), nurses and health care assistants (NHCAs) (125) and Allied health professionals (AHPs) (81).The majority of HCWs had looked after COVID19 patients; HD 92\%, NHCAs 79\%, AHP 77\%. The most frequent concerns across all HCW staff groups was that of family and friends dying from COVID19, family and friends being infected with COVID19 and being infected with COVID19 and transmitting it to family and friends. HCWs were less worried about themselves dying from COVID19; HD 22\%, NHCAs $35 \%$, AHP 25\%.The most frequent reported psychological health concerns amongst the 3 staff groups were anxiety, emotional distress, burnout and depression.HCWs experienced high levels of anxiety about the pandemic with their degree of worry mainly pertaining to the health of their family and friends. It is therefore important for hospital leaders to focus on wellbeing programmes for HCWs, their families and friends to ensure that they can function at a high level in the workplace in times of extreme distress during pandemics. 


\section{Abstract \# 71}

The realization of a special cohort unit in the prevention of a general outbreak of SARS CoV2 in Flemish nursing homes

Gillis Katrin ${ }^{1}$, Servotte Hilde ${ }^{2}$, Lievens Serge ${ }^{2}$, Cuvelier Henk ${ }^{3}$, Nieberding Philip ${ }^{3}$, Saegeman Veroniek ${ }^{3}$

${ }^{1}$ Odisee University College, ${ }^{2}$ vzw Samen-Ouder, ${ }^{3} \mathrm{AZ}$ Nikolaas

During the COVID-19 pandemic, the Flemish Agency for Care recognized two special cohort units for COVID-19 positive elderly people residing in nursing homes. The aim of the operationalization of special cohort units was fourfold: the protection of residents and caregivers in nursing homes, prevention of an overcapacity in regional hospitals, providing optimal and more specialized care for COVID-19 positive residents of whom the health status deteriorates and who declared having no willingness for hospital admission and reducing the workload and anxiety among caregivers in nursing homes.From the early stage on of the pandemic, residents from a consortium of six nursing homes (580 residents) who were diagnosed as COVID-19 positive were allocated to one special cohort ward at one location. On the other hand older COVID-19 positive hospitalized patients who could not return to the home setting, could be discharged towards this special cohort unit as long as isolation was required. To realize this approach, four essential conditions needed to be fulfilled: direct availability of the right infrastructure according to the principles of barrier nursing, featuring a source for protection materials, the presence of a competent team of experts and caregivers, and finally no-impact policy on other care departments in the nursing homes or the hospital. A strong collaboration between the consortium, the regional hospital and primary care practitioners made the implementation successful and prevented a further outbreak of COVID-19 in the nursing homes. Important insights and challenges for shared clinical primary and secondary care for older adults were gained.

\section{Abstract \# 72 \\ Older patients' perspectives and experience of hospitalization during the COVID-19 pandemic - a qualitative explorative study \\ Dorthe S. Nielsen ${ }^{1}$, Rikke Frøslev Hansen ${ }^{2}$, Sanne Have Beck ${ }^{2}$, Jette Wensien $^{2}$, Tahir Masud ${ }^{3}$, Jesper Ryg ${ }^{2}$ \\ ${ }^{1}$ Department of Geriatric, Migrant Health Clinic, Odense University Hospital, University of Southern Denmark, ${ }^{2}$ Department of Geriatric Medicine, Odense University Hospital, Odense, Denmark, \\ ${ }^{3}$ Department of Geriatric Medicine, Nottingham University}

Aim: The aim of this study was to gain in-depth knowledge and an increased understanding of how isolation from close relatives and carers, during the COVID-19 pandemic, impacted older patients during hospitalization in a Geriatric department.

Method: The study was conducted as a qualitative study using qualitative explorative interviews and a phenomenologicalhermeneutic approach. The phenomenological-hermeneutic approach was chosen to get an understanding of the older participants' perspectives and lived experiences. Complete interviews were available for 11 patients -six men and five women, between 69 and 91 years of age.

Results: The analysis revealed five main themes: (1) COVID-19 creates fear and anxiety, (2) Relationships become strained, (3) The transformation from being "a person" to being "a patient", (4) Virtual contact can't replace the real world, and (5) The environment of care. All identified themes were connected to participants' narratives about being isolated with no contact to close relatives or carers. The co-creation during data collection, consisting of ongoing conversations and questioning of the older patients, was challenged several times because the interviews had to be interrupted or shortened due to ethical considerations regarding the individual person's needs and conditions.

Conclusions: Our study provided in-depth knowledge about how older patients experienced hospitalization during the COVID-19 pandemic in a Danish hospital. The results emphasize that compassionate care includes a willingness to listen to patients' narratives and to imagine life as the older patient depicts it, which can lead to a better understanding of the individual person's needs, and increase compassionate caring.

\section{Abstract \# 73}

\section{Rekindling the spark}

Judith Ellis ${ }^{1}$

\section{${ }^{1}$ UK Nurse in Covid Nightingale Hospital}

Caring for elderly people recovering from Covid requires far more than addressing physical rehabilitation and waiting for that negative covid swab that is that vital ticket for discharge home or return to a care setting. As patients arrive on the Nightingale Manchester wards they are all exhausted, wary or indeed scared and for some it is even more upsetting. Staring into their eyes you observe a blankness that I have only witnessed before in those who have undergone prolonged cancer treatment or indeed in the eyes of those escaping war zones in low income countries. Still alive the spark of life has disappeared. The look has been described as 'soul death'. Soul death is allowing the sufferers to block out painful memories as a means of protection, allowing a perfusing numbness, an all consuming desire to sleep and be unaware of the world around.The scared, afraid and confused we can reassure, comfort and calm and indeed distract at times of added stress but for those with that scary and somehow inhuman blank stare or who just continuously appear asleep, the challenge for the caring team is how to rekindle that spark. The Family Liaison Team at the Manchester Nightingale covid hospital was established to facilitate communication between families and their medically isolated loved ones, but their role has become fundamental to the rehabilitation of elderly covid patients. Rehabilitation is dependent upon a desire to recover, a desire to re-engage with the world, a desire to live.

\section{Abstract \# 74 \\ Mortality from COVID-19 in the elderly: a perspective from a care of the older person unit in a london hospital during the COVID-19 pandemic}

Rosanna Sasson ${ }^{1}$, Devesh Shah ${ }^{1}$, Naomi Head ${ }^{1}$, Motoko Otomo ${ }^{1}$, Oscar Lancaster ${ }^{1}$, Jamie Addlestone ${ }^{1}$, Antony P Zacharias ${ }^{1}$, Phoebe Sharratt $^{1}$, Kristi Sun ${ }^{1}$, Johnny Swart ${ }^{1}$

${ }^{1}$ The Whittington Hospital, London

Introduction: COVID-19 is the leading cause of death in the United Kingdom in 2020, especially in elderly populations [1]. We present data from a Care of the Older Person (COOP) unit to compare patient demographics and mortality to the previous year.

Methods: Data was collected from electronic records of all patients admitted onto the COOP unit between 15/03 and 30/04 in 2019 and 2020. Admissions were classified as COVID-19 positive based on high clinical suspicion or swab result and compared for age, sex and mortality.

Results: Between 15/03-30/04/2020, there were 269 admissions. $164 / 269(61.0 \%)$ were diagnosed with COVID-19. Mean age was 75.2 years: 75.7 if COVID-19 positive vs. 74.4 if negative. Overall, 130/269 (48.3\%) were male: $87 / 164(53.0 \%)$ if COVID-19 positive 
vs. $43 / 105(41.0 \%)$ if negative. In total, 51/269 (19.0\%) died in hospital. Mortality rates were 38/164 (23.2\%) if COVID-19 positive compared to 13/105 (12.4\%) if COVID-19 negative. In the 2019 comparator period, there were 219 admissions, with mean age 75.7 years. $101 / 219(46.1 \%)$ were male, with inpatient mortality rates of $22 / 219(10.5 \%)$.

Conclusion: During the COVID-19 pandemic, there were increased admissions to the COOP wards. The admissions were of a similar average age and gender proportion to 2019. As expected, COVID-19 almost doubled the observed mortality rate. COVID-19 negative admissions had a similar mortality rate to the 2019 cohort.

[1] Campbell A, Caul S. Deaths involving COVID-19, England and Wales. Office for National Statistics 2020.

\section{Abstract \# 75 \\ A moving story: the impact of ward moves and length of stay in a care of the older person unit in a london hospital during the COVID-19 pandemic}

Antony P. Zacharias ${ }^{1}$, Rosanna Sasson ${ }^{1}$, Oscar Lancaster ${ }^{1}$, Naomi Head $^{1}$, Motoko Otomo ${ }^{1}$, Jamie Addlestone ${ }^{1}$, Kristi Sun ${ }^{1}$, Devesh Shah $^{1}$, Phoebe Sharratt ${ }^{1}$, Johnny Swart ${ }^{1}$

${ }^{1}$ The Whittington Hospital, London

Introduction: Multiple ward moves increase the risk of hospitalacquired infection, length of stay (LOS) and mortality [1, 2]. Given the high transmissibility of SARS-CoV-2, we investigated the effects of ward moves on elderly patient outcomes during this pandemic.

Methods: Data was collected from electronic records of all patients admitted onto the Care Of The Older Person (COOP) unit between 15/03 and 30/04 in 2019 and 2020. All ward moves from the emergency department onwards were counted. We classified 1 to 2 moves as 'low', and 3 or more moves as 'high'. Admissions were classified as COVID-19 positive based on high clinical suspicion or swab result. Admissions resulting in death were excluded from LOS analysis.

Results: Between 15/03 and 30/04/2020, there were 269 admissions of which 164/269 (61.0\%) were COVID-19 positive. Mean LOS in COVID-19 positive admissions was longer than in negative admissions (12.5 vs. 8.0 days). In 2019 there were 219 admissions with a mean LOS of 9.0 days. 'High' ward moves correlated with increased mortality compared to 'low' ward moves in COVID-19 positive $(28.6 \%$ vs. $21.3 \%$ ), and negative admissions (18.8\% vs. $11.2 \%)$. Comparatively, in 2019, 'high' ward moves correlated with increased mortality from $9.9 \%$ to $11.8 \%$.Longer LOS also correlated with 'high' ward moves in both COVID-19 positive (10.7 vs. 14.5 days) and negative admissions (7.4 vs. 13.1 days).

Conclusion: COVID-19 admissions had a longer average LOS and increased in-patient mortality than the same time period in 2019. Three or more ward moves correlated with increased LOS and mortality, regardless of COVID status.

[1] Stowell A. et al. (2013). Hospital out-lying through lack of beds and its impact on care and patient outcome. Scandinavian Journal of Trauma, Resuscitation and Emergency Medicine, 21, 17

[2] Perimal-Lewis L, Li J.Y, Hakendorf P.H, Ben-Tovim D.I, Qin S. and Thompson C.H. (2013). Relationship between in-hospital location and outcomes of care in patients of a large general medical service. Internal Medicine Journal, 43, 712-716

\section{Abstract \# 76}

Observations from care home admissions to a care of the older person unit in a london hospital during the COVID-19 pandemic

Devesh Shah ${ }^{1}$, Rosanna Sasson ${ }^{1}$, Kristi Sun ${ }^{1}$, Phoebe Sharratt ${ }^{1}$, Motoko Otomo ${ }^{1}$, Naomi Head ${ }^{1}$, Oscar Lancaster ${ }^{1}$, Jamie Addlestone ${ }^{1}$, Antony Zacharias ${ }^{1}$, Johnny Swart ${ }^{1}$

${ }^{1}$ The Whittington Hospital, London

Introduction: In 2020, care homes reported a high burden of infection and mortality rates from COVID-19 [1]. We compared admissions from care homes to our Care of the Older Person (COOP) unit during the pandemic against an equivalent period in 2019 .

Methods: Data was collected from electronic records of all patients admitted to our unit between 15/03 and 30/04 2019 and 2020 . Admissions were classified as COVID-19 positive based on high clinical suspicion or swab result.

Results: Between 15/03 and 30/04/2020, there were 269 admissions of which $30(11.1 \%)$ were from care homes (mean age 80.6 vs. 75.2 from home). Overall 20/30 (66.7\%) of care home admissions were COVID-19 positive compared to 124/207 (59.9\%) admitted from home. In 2020, COVID-19 positive admissions from care homes had a mortality rate of $8 / 20(40.0 \%)$ vs. $2 / 10(20 \%)$ in negative admissions. In 2019, 1/20 (5.0\%) patients admitted from a care home died. In admissions from home, the mortality rate was $25 / 124(20.0 \%)$ if COVID-19 positive vs. 9/83 (10.8\%) if COVID-19 negative. In care home admissions, average length of stay (LOS) was similar between 2019 and 2020 (10.5 vs. 10.4 days). LOS was 13.5 days if COVID-19 positive vs 4.5 days if negative.

Conclusion: Mortality rates in COVID-19 positive admissions from care homes were double that of patients admitted from home. LOS was disproportionately higher in COVID-19 positive vs. COVID-19 negative care home admissions.

[1] - "Number of deaths in care homes notified to the Care Quality Commission, England", Office For National Statistics, 02/06/2020 (Accessed 08/06/2020).

\section{Abstract \# 77}

Developing a data $\&$ innovation platform for care homes in Scotland

Lucy Johnston ${ }^{1}$, Jo Hockley ${ }^{2}$, David Henderson ${ }^{1}$

${ }^{1}$ Edinburgh Napier University, ${ }^{2}$ Department of Primary Palliative Care, Usher Institute, University of Edinburgh

Introduction: The COVID-19 pandemic has increased the need for reliable, real time data on the care of older people. We aimed to identify current data availability, challenges, and priorities for future research and innovations in Care Homes.

Methods: Scoping fieldwork in six Lothian (Scotland) Care Homes included (a) 'audit' of all data items currently collected through paper or electronic care planning and (b) interviews with care home managers to discuss (i) availability /use of data; (ii) gaps; (iii) digital capabilities; and (iv) priorities for future research.

Results: All homes collected data routinely on dependency, nutrition, weight, falls, pressure sores and infections, and as required on wounds, frailty, bowels or fluid intake. Other data items are also recorded (e.g. pain, sleep, observations) that can identify changes in residents' condition in real time. There was significant variation between homes in recording processes and assessment tools. Data are often used as 'standalone' pieces of information rather than creating a holistic/longitudinal view of each resident. Priorities for future research included standardising documentation, recording non-care tasks, identifying change in residents' condition, and supporting staff. 
Key conclusions: Care homes can be data heavy, but there is significant heterogeneity in the data, and it is mostly used to direct immediate resident care. There is an opportunity for a National Care Home Data Platform, with strong foundations. This is essential to: provide a robust data source for policy and planning; enable real time digital connectivity between care homes and health \& social care providers; and inform future innovative technologies.

\section{Abstract \# 78 \\ SARS-CoV-2 inpatient setting isolation: collateral damage among geriatric patients}

Victoria Garay-Airagui ${ }^{1}$, Maria Gebauer-Campos ${ }^{2}$, Carla GàmezAsunción $^{2}$, Carlos Alfredo Colato-López ${ }^{3}$, Diego Sepúlveda-Moya ${ }^{3}$

${ }^{1}$ Medical Specialize in Geriatrics. University Hospital of Guadalajara (Guadalajara, Spain), ${ }^{2}$ Internal Medicine Resident of Geriatrics. University Hospital of Guadalajara (Guadalajara, Spain), ${ }^{3}$ Medical Specialize in Geriatrics. University Hospital of Guadalajara (Guadalajara, Spain)

Introduction: We present a male 85-years-old patient (hypertension, chronic plaquetopenia, prostatic adenocarcinoma, depressive mayor illness) who is admitted in acute-geriatrician unit with the diagnosis of no-consolidative SARS-Cov-2 respiratory infection. Geriatrician assessment: Barthel Index 50/100, moderate cognitive impairment, Global Deterioration Scale 6, nursery-home for 3 years. Polypharmacy was presented.

Methods: The patient was treated following the in-hospital protocol for SARS-Cov-2 available in our hospital in April 2020 (hydroxychloroquine and azithromycin) and bacterial secondary infection suspicion was treated by cefalosporines. Fluidtherapy was needed for accute renal kidney. No other focus of infection.Hospitalization setting, acute stressful episode and disciplined isolation measures, trigger an intensive acute confusional syndrome. In this context, the patient suffers a craneo encefalic traumatism that requires a CT-Scan and neuroleptic management (Initially risperidone with extrapyramidal side effects). Benzondiazepines and antidepressants were retired. A diarly difficult assessment was performed despite the fluctuating nature of the vascular cognitive impairment established. Anticoagulation treatment according to SARS-Cov-2 prothrombic status was contraindicated because plaquetopenia and craneo encefalic traumatism.

Results: After 20-days of hospitalization, polypharmacy was reduced and optimized, reaching a controlled sleep-wake cycle with a combination of quetiapine and mirtazapine. Not aripripazole via intramuscular were needed at the time of discharge. Respiratory complications were not performed.Key conclusions:-Isolation protocol is not fixed to geriatric patients: They would suffer more inhospital complications, prolonged hospitalizations, loss of functionality, acute confusional syndrome, and lack of quality care.

\section{Abstract \# 79 \\ Avoiding burnout of the care home workforce during the COVID- 19 pandemic and beyond: sharing national learning and local initiatives \\ Cari Malcolm ${ }^{1}$, Lucy Johnston ${ }^{1}$, Jo Hockley ${ }^{2}$, Susan D. Shenkin ${ }^{3}$ \\ ${ }^{1}$ ENU, ${ }^{2}$ Usher Institute, University of Edinburgh, ${ }^{3}$ University of Edinburgh}

Introduction: COVID-19 in care homes has heightened the risk of staff burnout, undermining already problematic staff retention and low morale. There has been an associated proliferation of resources and online initiatives to support frontline workers, however, few of these are directly targeted at the care home workforce. Care home workers are highly skilled in caring for people with complex needs, but have very variable levels of formal training, and just over half of care homes in Scotland include registered nurses. This project will rapidly collate existing resources and identify, direct from care home workers, their best practice, initiatives, and resources used to support resilience and retention during this pandemic and moving forward.

Methods: (1) Rapid review of care home specific evidence and resources (including published research and social media); (2) Online survey of Enabling Research in Care Homes (ENRICH) members across Scotland $(n=55)$; (3) Case studies within six care homes to identify what is working well and what is not in terms of promoting resilience and emotional support.

Results: The rapid review has identified a wide range of resources directed at supporting staff working in care homes; the survey and case studies will provide data on the key learning and resources that have supported staff, and outline the challenges identified.

Key conclusions: This comprehensive review of resources and initiatives will make a valuable contribution to policy and practice designed to reduce burnout and foster retention not just in care homes but more widely across health and social care.

\section{Abstract \# 80 \\ Inpatient management of the older adults with COVID-19 infections in a University Hospital in Kuala Lumpur-an observational study}

Wan Chieh Lim ${ }^{1}$, Ai-vyrn Chin ${ }^{2}$, Shahrul Bahyah Kamaruzzaman ${ }^{2}$, Maw Pin $\operatorname{Tan}^{2}$, Kit Mun Tan², Hui Min Khor ${ }^{2}$, Nor 'Izzati Saedon², Kejal Hasmukharay ${ }^{2}$, Chiann Ni Thiam ${ }^{1}$, Gordon Hwa Mang Pang ${ }^{1}$, Aimy Abdullah ${ }^{1}$, Chai Chen $\mathrm{Ng}^{1}$, Terence Ing Wei Ong ${ }^{2}$

${ }^{1}$ Ministry of Health Malaysia, ${ }^{2}$ University of Malaya Medical Centre

Introduction: Our knowledge on the treatment of older adults diagnosed with COVID-19 is evolving. In this study, we share our experience managing a cohort of older adults with COVID-19.

Methods: An observational study involved 25 patients aged $\geq 60$ years old diagnosed with COVID-19 who received treatment in a hospital in Kuala Lumpur. Data was traced from electronic medical records, and looked at inpatient management instituted.

Results: 6 patients (24\%) died. 11 patients (44\%) received oxygen support, out of which $5(20 \%)$ received positive pressure ventilation. 14 patients $(56 \%)$ did not receive antiviral treatment: 9 of them had mild or no symptoms (all survived), and 5 received palliative care (all died). 11 patients (44\%) received antiviral treatment: 9 patients received 1-9 days' course of Hydroxychloroquine (mode of 5 days). 5 patients received Lopinavir/Ritonavir for 2-7 days (mode of 2 days). 4 patients received a single dose of Toclizumab. All patients who were actively treated and required oxygen support were started on Lopinavir/Ritonavir and/or Toclizumab. 5 patients who received Hydroxychloroquine (55.5\%) had prolonged QT intervals. 1 patient who received antiviral treatment died. 17 patients received Geriatric co-management, and 8 were transferred to the Geriatrics ward for further management.

Key conclusions: There was no consistent management plan delivered to our patients. Prognostication is important in determining treatment goals and outcomes. Caution is advised in treating older adults with Hydroxychloroquine due to risk of prolonged QT intervals. There is a role for Geriatric co-management in dealing with complex care issues that may arise. 


\section{Abstract \# 81 \\ Preventing loneliness among nursing home residents during the COVID-19 pandemic. A case study from Poland \\ Urszula Kłosińska ${ }^{1}$, Piotr Seiffert ${ }^{2}$, Jarosław Derejczyk ${ }^{3}$, Grzegorz Derejczyk $^{3}$ \\ ${ }^{1}$ Faculty of Psychology, SWPS University, Wrocław, Poland, ${ }^{2}$ Geriatric Ward, Municipal Hospital in Chorzów, Poland, ${ }^{3}$ Senior Residence 'Green Corner', Mysłowice, Poland}

Introduction: Due to the COVID-19 outbreak in Poland nursing homes prohibited all visits since the 11th of March 2020. Significant lack of contact with families can increase feelings of loneliness and anxiety among nursing home residents. The aim of this study is to present methods of providing contact between residents and their relatives during this isolation.

Methods: Our study took place in the polish nursing home for the elderly. 88 residents were constantly assessed by a geriatrician and a psychologist from the beginning of quarantine up to 30th of May. Based on its assessment we planned a therapeutic strategy for the time of expected lockdown.

Results: Severely restricting family visits was the most painful effect of the quarantine reported by the residents. $45 \%$ of them reported an intense longing for relatives. $34 \%$ of all residents needed additional pharmacological intervention due to increased agitation, insomnia, or depressiveness. We implemented the following strategies to maintain the contact between residents and their relatives: smartphone/tablet videoconferences, meetings through the window, memory books prepared by the families, support lists from families, video wishes for relatives, 'hug-time' screen, and safe outdoor meeting space. We are still in the process of assessing the effectiveness of these methods.

Key conclusions: There are multiple effective methods that can be used to provide contact between residents and their families even if visits in the nursing home are not allowed. Smartphone videoconferences and meeting through the window were not effective because of vision and hearing limitations.

\section{Abstract \# 82}

Psychological evaluation of the healthcare workers in a Geriatric Unit during the Covid 19

Tatiana Zuinen ${ }^{1}$, Vanessa Willems ${ }^{1}$, Marie-Noëlle Geurt ${ }^{1}$, Sandra Higuet $^{1}$

${ }^{1} \mathrm{CHU}$ Charleroi

Introduction: Since the outbreak of SARS-CoV-2 in 2020 in Belgium, healthcare workers have been in the frontline of the disease. They are the primary ressource that we need to fight this virus, but the psychological environnement in which they had evolved is full of stress factors. In order to anticipate a potential nervous breakdown, we have chosen to record their perception of the situation/state of mind.

Methods: On a voluntary based participation, two questionnaires were administrated to healthcare workers in geriatric non-covid unit of ISPPC of Charleroi. We have used the Perceived Stress Scale 14 (PSS) Questionnaire to evaluate the perceived stress within the month, and the Brief Resilent Coping Scale (BRCS-2004) to determine the presence of helpfull strategies in the health workers'psychological functionning.

Results: 31 healthcare workers have participated to our survey with $77.41 \%$ of women and the average age was 37.26 years old, $58 \%$ of nurses, $9.6 \%$ of physiotherapists, $6 ., 45 \%$ of physicians. Regarding PSS 14 score, with an average score of $27.65 / 56$ we observed that the healthcare workers were not overstressed by the situation. We can observe that younger healthcare workers $(<35$ years old $)$ perceived more stress $(30.5 / 56)$ than the older one ( $>45$ years old) with an average score of $21.4 / 56$. They represent $41.9 \%$ of our sample. With a $13.84 / 20$ of average score at the BRCS, the sample could be seen as medium resilient copers and neither coping strategies is more used one from another.

Conclusion: This observational analyse tend to show that younger healthcare workers have perceived this situation as more stressfull than their older collegues and it therefore suggests that we should focus our future psychological interventions more on the youngest healthworker, especially in case of a second wave.

\section{Abstract \# 83}

Supporting staff through the Covid-19 pandemic-a quality improvement initiative

Sherena Nair ${ }^{1}$, Shan Liung Liew ${ }^{2}$, Atif Nasrullah ${ }^{1}$, Muhammad Iqbal $^{1}$

${ }^{1}$ Leeds Teaching Hospitals NHS Trust, ${ }^{2}$ Calderdale and Huddersfield NHS Trust

This quality improvement project was undertaken during the outset of Covid-19 to promptly identify the support needed for staff managing elderly patients with Covid-19 infections. Due to the nature of rotating medical teams and rapidly evolving guidance, this project enabled the team to work cohesively and optimally during such unprecedented times. A semi-structured survey was undertaken with feedback gathered from 20 members of staff on a designated covid ward including doctors, nurses, clinical support workers, and domestic staff. A 'Lessons Learned Log' was also created over a week to understand the pressures and issues faced by the team in order to put into place relevant support strategies swiftly. Responses from the survey were subsequently analysed into thematic headings. This initiative highlighted that communication about changes in Covid-19 guidance was crucial in allaying anxieties of the team. Notice about wards becoming designated covid areas despite rapid turnover of patients enabled staff to prepare themselves psychologically. Debrief sessions after deaths of patients also helped with the distress experienced by staff. Key strategies were discussed in the daily safety huddle with input from relevant specialties such as palliative care and liaison psychiatry to further enhance patient care. This project has led to a robust system in place to support staff on covid wards to care confidently and compassionately for elderly patients with covid-19.

\section{Abstract \# 84}

A new barrier to COVID-19 transmission in nursing home residents: the successful experience of 17 facilities In France

Nathavy Um Din ${ }^{1}$, Cristiano Donadio ${ }^{1}$, Maurizio Magri ${ }^{1}$, Quoc Duy Nghiem $^{1}$, Bruno Oquendo ${ }^{2}$, Carmelo Lafuente-Lafuente ${ }^{2}$, Sylvie Pariel $^{3}$, Joël Belmin ${ }^{2}$

${ }^{1}$ Service universitaire de gériatrie, Hôpital Charles Foix, Groupe hospitalier APHP Sorbonne Université, Ivry-sur-Seine, France, ${ }^{2}$ Service Universitaire de Gériatrie, Hôpital Charles Foix, Groupe Hospitalier APHP Sorbonne Université, Ivry-sur-Seine, France; Faculté de Médecine Sorbonne Médecine, Paris, France, ${ }^{3}$ Service Universitaire de Gériatrie, Hôpital Charles Foix, Groupe Hospitalier APHP Sorbonne Université, Ivry-sur-Seine, France

Introduction: COVID-19 is a major threat to nursing homes. During the COVID-19 pandemic that hit France in March and April 2020, staff members of some French nursing homes decided to confine 
themselves with their residents to reduce the risk of entry of SARSCoV-2 in the facility.

Methods: This was a case-control observational study. Nursing homes that had an experience of staff confinement (NH-SCs) with residents were identified from the media. Their directors were surveyed via a telephonic interview to investigate the number of proven and possible COVID-19 cases among their residents and staff from March to May 2020. The results were compared with those obtained from a national survey in all French nursing homes conducted during the same period.

Results: We identified 17 NH-SCs in which 794 staff members confined themselves with their 1, 250 residents. Only one NH-SC (5.8\%) had cases of COVID-19 among the residents compared with 4 , 600 nursing homes $(65.8 \%)$ in the national survey $(p<0.001)$. Five residents $(0.4 \%)$ in the NH-SCs had proven or possible COVID-19 in comparison with $62,373(11.1 \%)$ in the national survey $(\mathrm{p}<0.001)$. Five residents $(0.4 \%)$ in the NH-SCs died because of COVID-19 in comparison with $12,521(2.2 \%)$ in the national survey $(\mathrm{p}<0.001)$. Twelve staff members (1.6\%) in the NH-SCs had proven or possible COVID-19 in comparison with 29, 463 (7.9\%) in the national survey $(\mathrm{p}<0.001)$.

Key conclusions: Self-confinement of staff members with nursing home residents seems to be effective in protecting them from COVID-19 and related mortality.

\section{Abstract \# 85 \\ Geriatric syndromes and SARS-COV-2 side effects: a difficult relationship}

Carla Gamez Asuncion ${ }^{1}$, María Gebauer Campos $^{1}$, Victoria Garay Airaghi $^{2}$, Carlos Alfredo Colato López ${ }^{2}$, Diego Luis Sepulveda Moya $^{2}$

${ }^{1}$ Internal Medicine Resident of Geriatrics. University Hospital of Guadalajara (Guadalajara, Spain), ${ }^{2}$ Medical Specialize in Geriatrics. University Hospital of Guadalajara (Guadalajara, Spain)

Introduction: We present a male 83 years-old patient (Hypertension, dislypemia, long-term epilepsy treated with sodium valproate) who was admitted in acute-geriatrician unit with the diagnosis of bilateral pneumonia SARS-Cov-2 positive. Subacute deterioration referred by hisfamily consists on: Mutism over and authoritarian personality and remarkable cognitive rigidity, recent widowhood and institutionalization. Secondary loss of functionality and fragility.

Methods: Our patient was treated following the in-hospital protocol for SARS-Cov-2 available in our hospital in March 2020 (hydroxychloroquine and azithromycin) and bacterial secondary infection suspicion was treated by cefalosporines. Prolonged respiratory insufficiency and d-dimer testing forced to study a possible pulmonary thromboembolism (Discarded by Angio-TC Scan). Hypoactive delirium were performed. Multidisciplinary approach included: Infectious process solution, hydroelectrolite-disbalance correction (Hypernatremia), sodium valproate test-levels (Correct), electroencephalogram study (Normal) and CT-Scan (Cortical-Subcorticalatrophy and chronic vascular disease). Hypertransaminasemia (During SARS-CoV-2 infection) were performed and antiepileptic treatment was managed with levetiracetam.

Results: The patient presented a good respiratory clinical evolution. It was impossible to improve his poor oral intake and a proper rehabilitation program. He was discharged withoptimized antiepileptic treatment, added to vortioxetine initial doses and an intensive geriatrician follow-up.

Key conclussions: In this SARS-CoV-2 pandemia we not only have seen respiratory/organ failure events, we alsohave had to manage secondary effects of isolation and torpid presentations of geriatricsyndromes. Unfortunately these problems are invisible for most SARS-CoV-2 health policies.-The future functional recovery is limited in the pandemic scenario, a frustrated plan of carefrom a geriatric point of view.

\section{Abstract \# 86}

Impact of Social Isolation due to COVID-19 in older people: mental and physical effects and recommendations.

Patricia Perez-Rodriguez ${ }^{1}$, Isabel Rodriguez-Sanchez ${ }^{2}$, Walter Sepulveda-Loyola ${ }^{3}$, Felipe Ganz ${ }^{4}$, Rocio Torralba ${ }^{5}$, Daniel V. Oliveira $^{6}$, Leocadio Rodriguez-Mañas ${ }^{7}$

${ }^{1}$ Geriatrics Department. Puerta de Hierro University Hospital, Madrid, Spain., ${ }^{2}$ Geriatrics Department. Complejo Hospitalario de Navarra, Spain., ${ }^{3}$ Program of Masters and Doctoral degree in Rehabilitation Sciences, Londrina State University, ${ }^{4}$ Faculty of Rehabilitation Medicine, University of Alberta, Edmonton, Canada, ${ }^{5}$ Psychiatry Department. Hospital Universitario Ciudad Real, Ciudad Real, Spain, ${ }^{6}$ Maringá University Center. Health Promotion Department. Maringá, Paraná, Brazil, ${ }^{7}$ Geriatrics Department. Hospital Universitario de Getafe, Madrid, Spain

Introduction: The aim of this study was to review the impact of social isolation during COVID-19 pandemic on mental and physical health of older people and the recommendations for patients, caregivers and health professionals.

Methods: A narrative review was performed including 20069 noninstitutionalized community-living people from ten descriptive crosssectional papers. Articles since 2019 to 2020 published on Pubmed, Scielo and Google Scholar databases with the following MeSh terms ('COVID-19', 'coronavirus', 'aging', 'older people', 'elderly', 'social isolation' and 'quarantine') were included. The studies not including people over 60 were excluded. Guidelines, recommendations, and update documents from different international organizations related to mental and physical activity were also analyzed.

Results: Forty-one documents have been included in this narrative review, involving a total of 20.069 individuals, 58\% women, from Asia, Europe and America. Thirty-one articles includedrecommendations and ten addressed the impact of social distancing on mental or physical health. Anxiety, depression, poor sleep quality and physical inactivity were the main outcomes reported during the isolation period. The main recommendations were centered in cognitive strategies and increasing physical activity levels using technology and apps, online videos and telehealth.

Conclusion: Both mental and physical health in older people are negatively affected duringthe social distancing for COVID-19. A multicomponent program with exercise and psychological strategies is highly recommended for this population during the confinement. Future investigation in this area is needed.

\section{Abstract \# 87}

The maiden voyage of the corrib ward: shining a spotlight on older adult care during a pandemic - an Irish perspective

Dr. Karen Dennehy ${ }^{1}$, Caoimhe Hanrahan ${ }^{1}$, Catriona Reddin ${ }^{1}$, Clodagh McDermott ${ }^{1}$, Dr. Ruairi Waters ${ }^{1}$, Dr. Michelle Canavan ${ }^{1}$

${ }^{1}$ Galway University Hospital, Ireland

Introduction: During the COVID 19 pandemic a higher case-fatality ratio, has been observed in the older patient ( $>80$ yrs)in both Chinese $(14.8 \%)$ and Italian populations (21\%). (1) (2) As geriatricians we knew we had the ability to dramatically impact clinical decision making during this pandemic. One of the cornerstones of caring for 
the elderly is identifying patients maximally benefiting from goal orientated multidisciplinary care (3).

Methods: We introduced a geriatric COVID 19 service.This team consisted of geriatric consultants, trainees, multi-disciplinary team and advanced nurse practitioners. We admitted, treated and rehabilitated all query and confirmed COVID 19 geriatric patients over a 12 weeks period in our tertiary referral centre. All patients were admitted and clinical frailty scale score assigned. An early and honest discussion around goals of care were conducted in collaboration with patients and their families.

Results: Initially, we analysed the first 100 patients through our service for this Abstract. Comprehensive geriatric assessments were completed on everyone. Seventy-five percent of patients were admitted from home, $21 \%$ from nursing homes and $4 \%$ from respite care.The average age of our patient cohort was 82 years. Average CFS was 5. Seven patients were palliated with end of life care implemented by our team, their average CFS was higher (8).

Conclusions: We feel our care positively impacted on the care of older patients during this pandemic.Fatality number were reduced in this cohort.Older adults care is optimized when treated by the physicians that have experience with their unique needs.

\section{Abstract \# 88 \\ Would you trust a diagnostic procedure with $100 \%$ specificity and $96 \%$ sensitivity?}

Van den Eeckhaut Lisa ${ }^{1}$, Lemper Jean-Claude ${ }^{1}$, Rebel Sonja ${ }^{2}$, Barbé Kurt $^{3}$, Beyer Ingo ${ }^{4}$

${ }^{1}$ Geriatric Rehabilitation Centre Scheutbos, Silva Medical, Brussels; Geriatric Department, University Hospital (UZ) Brussels, Vrije Universiteit Brussel (VUB), Belgium, ${ }^{2}$ Geriatric Rehabilitation Centre Scheutbos, Silva Medical, Brussels, Belgium, ${ }^{3}$ Biostatistics \& Medical informatics, Faculty of Medicine and Pharmacy, (VUB), Belgium, ${ }^{4}$ Geriatric Rehabilitation Centre Scheutbos, Silva Medical, Brussels; Geriatric Department, University Hospital (UZ) Brussels, Vrije Universiteit Brussel (VUB); Frailty in Ageing Research Department, Gerontology, Faculty of Medicine and Pharmacy, (VUB), Belgium

Introduction: Nasopharyngeal swab (NPS) RT-PCR SARS-CoV-2 detection has $100 \%$ specificity and $70 \%$ sensitivity $[1,2]$. We report a patient with four negative swabs.

Methods: 94-year-old woman transferred for wrist-fracture rehabilitation on March 13th. Prior history: knee-prosthesis, osteoporosis, falls, cognitive deterioration. She took no medication. Examination revealed cognitive deficits and wrist plaster. WBC (neutrophils) were slightly increased, CRP normal. Six days later she developed dyspnoea and cough, but no fever, crackles or desaturation. NPS was negative. She remained clinically stable. Day 7 after symptom onset showed crackles over the left lung, $\mathrm{T}^{\circ} 37.6^{\circ} \mathrm{C}, 89 \% \mathrm{SaO}_{2}$, normal WBC (lymphopenia). X-ray revealed lower left pneumonia. NPS was negative. Influenza, RSV, Mycoplasma, Chlamydia-pneumoniae were ruled out, blood cultures negative, sputum not obtained. Amoxicillin/clavulanate was initiated, shifted to piperacillin/tazobactam and clarithromycin added for persistent fever. NPS was negative (day 12). There was no clinical improvement, pneumonia worsened, and the patient was transferred for CT (day 19), showing non-specific fivelobe-involvement. NPS was negative. She slowly recovered clinically and radiologically. SARS-CoV-2 antibody testing became available in Belgium revealing specific $\mathrm{IgG}$.

Results: Serologically-confirmed COVID-19 in a patient with 4 negative RT-PCR results. Recently published timing-dependent sensitivity [3] indicates probabilities of negative RT-PCR in our patient of $0.4,0.3,0.5$ and 0.7 respectively, resulting in a cumulative probability of 0.04 , or cumulative sensitivity of $96 \%$.

Conclusions: Serial testing was unable to detect SARS-CoV-2 in our patient despite a cumulative sensitivity of $96 \%$. While positive tests identify COVID-19 patients, negative testing is less conclusive. When suspicion is high, a negative test cannot rule out the disease.

\section{References:}

1. Sethuraman N, Jeremiah SS, Ryo A. Interpreting Diagnostic Tests for SARS-CoV-2. JAMA. 2020; 323(22):2249-2251. https://doi.org/10.1001/jama.2020.8259

2. Watson J, Whiting PF, Brush JE. Interpreting a covid-19 test result. BMJ 2020; 369:m1808. https://doi.org/10.1136/bmj.m1808

3. Kucirka LM, Lauer SA, Laeyendecker O, Boon D, Lessler J. Variation in False-Negative Rate of Reverse Transcriptase Polymerase Chain Reaction-Based SARS-CoV-2 Tests by Time Since Exposure. Ann Intern Med 2020; May 13; M20-1495. https://doi.org/10.7326/M20-1495

\section{Abstract \# 89 \\ Covid-19 in nursing homes: problems and measures taken by crisis teams}

Lisa van Tol ${ }^{1}$, Margot de Waal ${ }^{2}$, Sarah Janus ${ }^{3}$, Sytse Zuidema ${ }^{4}$, Monique Caljouw ${ }^{5}$, Wilco Achterberg ${ }^{6}$

${ }^{1} \mathrm{PhD}$ Candidate Leiden University Medical Center, ${ }^{2}$ Senior Researcher Leiden University Medical Center, ${ }^{3}$ Post Doc Researcher Univeristy Medical Center Groningen, ${ }^{4}$ Professor Univeristy Medical Center Groningen, ${ }^{5}$ Assistant professor Leiden University Medical Center, ${ }^{6}$ Professor Leiden University Medical Center

Background: The Covid-19 pandemic sets nursing homes with great challenges. They have to make quick decisions based on limited resources and installed crisis teams, to inventorise problems, and set up measures. This study aims to qualitatively map problems and measures taken in Dutch nursing homes, and to explore what policy experts would favor when a Covid-19 outbreak occurs.

Methods: Nursing home organizations' $(\mathrm{N}>40)$ crisis team minutes are collected weekly from March 2020 onwards. From these minutes, text passages about encountered problems, measures taken, and their impact are selected and systematically coded. Weekly, thematic analysis of selected passages is performed and results are summarized. Next, multidisciplinary expert panels (care professionals, managers and client representatives), prioritize measures they think are most important or urgent to take at a new Covid-19 outbreak.

Results: Nursing homes mostly face problems and take measures regarding: personnel scheduling and personnel's wellbeing (27\% of selected text passages), personal protective equipment (16\%), visitors- and door policy (15\%), hygiene and care (12\%), nursing home beds and seclusion and isolation of clients $(10 \%)$ and clients' and their loved ones well-being (9\%). However, the percentages of text passages per theme differ weekly. Panel experts indicate diverse measures to be important or urgent for (future) outbreaks.

Conclusion: Crisis team minutes are a valuable and very up to date data source that is available without requesting much time or effort. They show a large variety in problems and measures taken within nursing homes.

\section{Abstract \# 90}

Frailty inpatient management and outcomes in Covid-19: experience from A University Hospital

Chai Chen $\mathrm{Ng}^{1}$, Wan Chieh Lim ${ }^{1}$, Kejal Hasmukharay ${ }^{2}$, Chiann Ni Thiam ${ }^{1}$, Gordon Pang ${ }^{1}$, Aimy Abdullah ${ }^{2}$, Siew Ping Loh ${ }^{1}$, Nor Izzati 
Saedon $^{2}$, Kit Mun Tan ${ }^{2}$, Shahrul Bahyah Kamaruzzaman ${ }^{2}$, Ai-Vyrn $\mathrm{Chin}^{2}$, Maw Pin Tan ${ }^{2}$, Hui Min Khor ${ }^{2}$, Terence Ong ${ }^{2}$

${ }^{1} \mathrm{MOH},{ }^{2} \mathrm{MOE}$

Introduction: Frailty is a predictor of multiple adverse inpatient outcomes. The aim of our study is to examine frailty, using Clinical Frailty Scale (CFS), its association with inpatient management and outcomes in COVID-19.

Methods: This is a retrospective cohort study of older adults aged above 65, diagnosed with COVID-19 infection, treated in the UMMC, where we compared patients with CFS 1-5 (non-frail to mildly frail) $(\mathrm{n}=14)$ against those with CFS 6-8 (moderate to severely frail) $(\mathrm{n}=$ 12).

Results: As of April 2020, we have total 26 hospitalised older adults. The mean age was $82^{6}$ in patients with CFS $6-8$, which was significantly higher than those with CFS $1-5$ [71(5); $\mathrm{p}=0.000]$. Overall $58 \%$ of those with CFS 6-8 $(n=7)$ presented from institutional care setting. Almost half of those with increasing frailty presented with pneumonia requiring oxygen. Our data revealed that all of those with CFS 6-8 were co-managed by geriatric care $(\mathrm{p}=0.001)$ and potentially managed to get their care planned in advanced $(p=007)$ with non-active resuscitation order issued in time $(\mathrm{p}=0.00)$. Those with CFS 1-5 were more likely to receive critical care $(p=0.017)$ and antiviral therapy $(p=0.000)$. Inpatient mortality was higher with increasing frailty, with total 5 out of 6 death were reported in those with CFS 6-8. The median length of hospital stay was 27 (IQR 15-40), which was the same between the CFS categories, $p=0.695$. Our data revealed worsening physical health status in both categories.

Conclusion: Frailty is an important component of COVID-19 management. There are guidelines that guide management but adherence to it requires individualised decision making.

\section{Abstract \# 91 \\ Frequency and intensity of medical interventions in a nursing home during COVID-19 PAndemic \\ Patrick Doyle $^{1}$, Sean Kennelly ${ }^{1}$, Dan Ryan ${ }^{1}$, Desmond O. Neill ${ }^{1}$ \\ ${ }^{1}$ Peamount Healthcare and TCD, Dublin, Ireland}

Introduction: The COVID-19 pandemic has disproportionately affected nursing home residents internationall y[1], with $62 \%$ of COVID-10 related deaths in Ireland occurring in residential care facilities. The increased care needs of nursing home residents with COVD-19 has stimulated discussions related to transfer to hospitals for higher intensities of care. However little focus has been given to calculating physician care needs of residents who remain in the nursing home, an ethical imperative for advance care planning which ensures adequate medical care for those not transferring. This study profiled the frequency and intensity of medical intervention.

Methods: Retrospective review of 51 residents' healthcare records from January-May 2020. We assessed the frequency and intensity of medical intervention, compared pre-pandemic and pandemic periods. Results: Of 51 residents (31 women, mean age 84.1+8.2), 19 (37\%) acquired COVID-19. Most residents were maximum dependency $(\mathrm{n}=$ $32,(55 \%))$ with over half diagnosed with dementia $(\mathrm{n}=31,51 \%)$ and $29(47 \%)$ with stroke. The average number of medical interventions per month for all residents more than doubled, from 72 during the prepandemic period to 155 during the 3 -month pandemic period: chisquare $(1, \mathrm{~N}=227)=7.27, \mathrm{p}=0.007$. Night-time and week-end interventions increased by $268 \%$ (19 vs 70 ).

Key conclusions: The total frequency of physician interventions for nursing home residents increased significantly during the COVID-19 pandemic. This highlights the importance of ensuring increased access to physicians, including night-time and week-ends, in pandemic planning for nursing home residents.

\section{References:}

1. Fallon A, Dukelow T, Kennelly SP, O’Neill D. COVID-19

in Nursing Homes QJM 2020; hcal36.https://doi.org/ 10.1093/qjmed/hcaa136

\section{Abstract \# 92}

The implementation of polish national recommendations against COVID-19 outbreak in long-term care facilities - a case study.

Piotr Seiffert ${ }^{1}$, Urszula Kłosińska ${ }^{2}$, Rafał Słowiński ${ }^{3}$, Barbara Maślanka-Seiffert ${ }^{4}$, Grzegorz Derejczyk ${ }^{5}$, Jarosław Derejczyk ${ }^{5}$

${ }^{1}$ (1)Senior Residence 'Green Corner', Mysłowice, Poland (2) Geriatric Ward, Municipal Hospital in Chorzów, Poland, ${ }^{2}(1)$ Senior Residence 'Green Corner', Mysłowice, Poland (2) Faculty of Psychology, SWPS University, Wrocław, Poland, ${ }^{3}$ Palliative and Long-term Care Centre "Caritas", Stare Siołkowice, Poland, ${ }^{4}(1)$ Department of Gastroenterology and Hepatology, Medical University of Silesia, Katowice, Poland (2) Geriatric Ward, Municipal Hospital in Chorzów, Poland, ${ }^{5}$ Senior Residence 'Green Corner', Mysłowice, Poland

Introduction: National recommendations against the COVID-19 outbreak in long-term care facilities (LTCF) differ worldwide. The aim of the study is to present protective methods of two LTCF in relation to the polish recommendations.

Methods: We made an interview with the employees of two LTCF in Poland - the public (P-LTCF) and the commercial one (C-LTCF). They were asked about the dates of applying recommendations and other protective methods.

Results: Some of the polish recommendations were specific obligations, while others were suggestions with a wide range of arbitrariness. Following activities: family visits prohibition, personal protective equipment obligation and staff health monitoring had been implemented in both LTCF earlier than the strict official recommendations were made. Educational training was applied after the recommendation had been announced in both facilities. Both facilities took additional effort: only full-time employees limitation, isolation area for new/coming back residents, residents cohorting, meals in rooms only, separate entrance for staff, obligatory staff temperature measurement, ensuring reserve of supplies. P-LTCF conducted widespread PCR screening, while C-LTCF examined selected staff and residents. The C-LTCF implemented also: admissions canceling, the disinfectant gate on the entrance, floor disinfection mats, UVC exposure of packages, Tele-Holter ECG.Key conclusion: All the strategies were implemented at the early stage of the pandemic. Part of the restrictions differed in both places, mostly because of the lack of detailed official instructions. Some methods were impossible to implement in the public LTCF, while others in the commercial one. No COVID-19 patient was diagnosed in any of the LTCFs.

\section{Abstract \# 93}

Does a geriatrician-led telephone advice line optimise care for frail older people during the COVID-19 pandemic?

Celina Handalage ${ }^{1}$, Alice Jundi ${ }^{2}$, Rebecca Anthony ${ }^{2}$, Gurjit Chhokar $^{2}$, Eileen Burns ${ }^{2}$

${ }^{1}$ Leeds Teaching Hospitals Trust (LTHT), ${ }^{2}$ LTHT

Introduction: The COVID-19 pandemic required a change in practice across primary and secondary care. We recognised the need for community-based healthcare professionals working with frail older 
people to have rapid access to specialist advice. A telephone line was set up operating everyday 08:00-21:00. It was staffed by geriatricians and the focus was on advance care planning, admissions avoidance and delivering community-based treatment.

Methods: Electronic patient records were reviewed from 21st April to 29th May 2020. Data was collected retrospectively from 28 weekdays including the timing and source of the call, details of advance care plans and outcomes for the patients.

Results: Data was collected from 160 patients. The average age of patients was 85 years. $15 \%$ of those who were tested were positive for COVID-19. In terms of advanced care planning, $43 \%$ of all calls had preferred place of care (PPOC) and $27 \%$ had resuscitation status discussed. Whilst the majority of calls came from GPs, $11 \%$ of calls came from community matrons, $5 \%$ from paramedics and there were a variety of other users.

Key conclusions: The data showed there was a need for supporting colleagues to help fulfil a person's wish to remain at home and to access the most appropriate hospital service when a decision was made to admit. This extended beyond patients with COVID-19. The data are being used to inform how this advice line can be implemented as a more permanent feature of our clinical service.

\section{Abstract \# 94 \\ Investigating the impact of Covid-19 on hip fracture management in a Tertiary Referral Hospital}

Michael Curran ${ }^{1}$, Nur Atikah Mohd Asri ${ }^{1}$, Pamela Hickey ${ }^{1}$, Brian Lenehan $^{1}$, Fibarr Condon ${ }^{1}$, Jude Ryan ${ }^{1}$

${ }^{1}$ University Hospital Limerick

Introduction: Covid-19 has caused unprecedented challenges the world over. On average 360 patients are treated for hip fractures in University Hospital Limerick (UHL) annually. Numerous improvements have been implemented in our service in recent years but Covid-19 has shifted this dynamic.

Methods: Data was collected from the Irish Hip Fracture Database (IHFD) and UHL Patient Administration System from March 1st until May 1st 2020. It was compared with data from the same timeframe in 2019. Statistical analysis was performed using SPSS.

Results: Demographic characteristics were comparable between both cohorts. The average length of stay (LOS) was 8 days compared to 15 days in 2019. This was primarily due to changes in hospital policy and an increased onus to discharge patients during the Covid-19 crisis. Strikingly the inpatient mortality rate at 30 days during the Covid-19 period was $13.4 \%(n=7)$ compared to just $1.7 \%(n=1)$ in 2019. Subsequent analysis from the 2019 data however unearthed a 60 day mortality rate of $25 \%$. This would suggest that subsequent analysis of mortality rates from 2020 will increase further. Readmission rates are also higher during the current pandemic with $10.5 \%$ of patients requiring readmission within 30 days compared to $5 \%$ at 30 days in 2019. Interestingly 10 patients were admitted from nursing homes compared to just two patients in 2019.

Conclusion: Increased demand for beds during Covid-19 crisis led to earlier discharge from our institution. Provisional analysis suggests this subsequently increased both readmission and mortality rates.

\section{Abstract \# 95 \\ Eight nursing-home-specific, COVID lectures attended by mean 330 staff per session}

Dr. Dan James Ryan ${ }^{1}$, Aoife McFeely ${ }^{2}$, Cathy Payne ${ }^{3}$, Karen Charnley $^{4}$, Sean Kennelly ${ }^{1}$, Mary Flanagan ${ }^{5}$
${ }^{1}$ Tallaght University Hospital and Trinity College Dublin, ${ }^{2}$ Tallaght University Hospital, ${ }^{3}$ All-Ireland Institute Hospice and Palliative Care, ${ }^{4}$ All-Ireland Institute of Hospice and Palliative Care, ${ }^{5} \mathrm{Our}$ Lady's Hospice and Palliative Care

Nursing homes, isolated from academic centres, have contended with rapidly-changing guidelines and modified clinical practice. An interactive, videoconference-facilitated education series was commenced to provide up-to-date guidance to nursing homes through a collaboration between Geriatrics Department, Tallaght University Hospital, Dublin and the All-Ireland Institute of Hospice and Palliative Care. The format was a brief didactic lecture but primarily focused on $\mathrm{Q}$ and A designed to address the COVID issues of the week. The first session, "Anticipatory Prescribing" was viewed by 300 nursing home healthcare workers and GPs. Attendances rose with COVID case rates to 550 mid-pandemic. At submission, eight COVID lectures were attended by a mean of 330 nursing homesrelated staff. Topics covered include a microbiologist's and then a respiratory consultant perspective on COVID, advance care planning, outbreak management and as the pandemic weaned; nutritional and swallow challenges in COVID, IT solutions to isolation/rehabilitation and then reopening nursing homes. Each week registrants were requested to input their "burning issue", which was addressed through Q and A. Using word-cloud software the primary issues in March, pre-pandemic, were end of life care, anticipatory medication stocks and syringe drivers. In April, mid-pandemic, the issues were PPE, managing COVID and Family. In late May, post-pandemic, focus changed to rehabilitation, isolation, re-accessing services. This lecture series was very well attended, provides a curriculum and highlights the success of videoconference lectures. Through question submission we provide a summary of the primary nursing home issues at differing time-points in a pandemic, albeit influenced by the lecture topic.

\section{Abstract \# 96}

\section{Vasculitis associated to SARS-COV2 infection}

Maria Jose Martin Legorburo ${ }^{1}$, Esther Espinosa Gimeno ${ }^{1}$, Carla Gamez Asunción ${ }^{1}$, Maria Gebauer Campos ${ }^{1}$, Viviana Oscullo Yepez ${ }^{1}$, Juan Rodriguez Solis ${ }^{1}$

${ }^{1}$ Hospital General Universitario de Guadalajara (Guadalajara).

Introduction: Different dermatological manifestations associated with COVID-19 infection have been published. Its frequency is variable (between 0.2 and $20.4 \%$ of infected patients), as well as the time to appear. The most frequent are: purpuric exanthema, vesicles, urticarial eruptions. 1

Clinical case: Here we present the case of a 90-year-old man with predominantly acral purpuric lesions in upper and lower limbs, 5 weeks after being hospitalized for Covid-19 pneumonia. Institutionalized patient with severe dependence for basic activities of daily living (ADLs) and mild cognitive impairment. Personal history: atrial fibrillation (AF), chronic renal disease. Admission to hospital for decompensated heart failure. After clinical improvement during admission, acral purpura and necrotic vascular lesions appear in 4 extremitiesEcoDoppler of both legs and echocardio, rules out other thromboembolism complications. We performed skin biopsy, small and medium vessel leukocytoclastic vasculitis, with isolated secondary intravascular fibrin thrombo. Coagulation tests to be highlighted are: D-Dimer elevation (7MG/L), antithrombin III deficiency $(78 \%), \mathrm{C}$ protein deficiency $(71 \%)$, Factor VIII elevation (318\%), Lupus anticoagulant: weak positive. The vasculitis study highlights: positive ANA, C3 complement low.Empirical treatment with high-dose corticotherapy was initiated and heparin was maintained at therapeutic doses due to suspected post SARS-COV2 
vasculitis.Conclusions:-Patients with SARS-COV 2 infection who develop vasculitis-like skin manifestations is constantly growing.Although it is more common in children, it can also be developed in adults and the elderly.2-Multifactorial physiopathology still unknown, related to states of hypercoagulability, innate immunity and activation of cytokines by monocytes/macrophages and to the ECA2 receptor. Bibliography:

1. Castelnovo L, Capelli F, Tamburello A, Faggioli PM, Mazzone A. Symmetric cutaneous vasculitis in COVID-19 pneumonia. J Eur Acad Dermatol Venereol. 2020; 10.1111/jdv.16589.

2. Criado PR, Abdalla BMZ, de Assis IC, van Blarcum de Graaff Mello C, Caputo GC, Vieira IC. Are the cutaneous manifestations during or due to SARS-CoV-2 infection/COVID-19 frequent or not? Inflamm Res. 2020; 1-12.

\section{Abstract \# 97 \\ Profiling COVID-19 infection in Irish nursing homes}

Aoife Fallon ${ }^{1}$, Adam Dyer ${ }^{2}$, Ruth Martin ${ }^{3}$, Siobhan Kennelly ${ }^{3}$, Alan Martin $^{4}$, Desmond O'Neill ${ }^{1}$, Sean Kennelly ${ }^{1}$

${ }^{1}$ Tallaght University Hospital, ${ }^{2}$ St James' Hospital, ${ }^{3}$ Connolly Hospital, Blanchardstown, ${ }^{4}$ Beaumont Hospita

Background: SARS-CoV-2 disproportionately affects nursing home (NH) residents, who account for $24-82 \%$ of COVID-19-related deaths. In Ireland, the first NH case of COVID-19 occurred on 16/03/ 2020. A COVID-19 testing program of residents and staff took place from $18 / 04 / 2020$ to $05 / 05 / 2020$.

Aims: To examine characteristics of NHs across three Community Health Organisations (CHOs) in Ireland, proportion with COVID-19 outbreaks, staff and resident symptom profile and resident mortality rates.

Methods: Forty-five NHs across three CHOs were surveyed. Details were collected on number of beds, occupancy, COVID-19 outbreak, date of first laboratory-confirmed COVID-19 case (resident/staff), total symptomatic/asymptomatic cases, and outcomes for residents from $29 / 02 / 2020$ to $22 / 05 / 2020$.

Results: Surveys were returned from $62.2 \%$ (28/45) of NHs (2043 residents, 2303 beds). Three-quarters (21/28) had COVID-19 outbreaks (1741 residents, 1972 beds). Median time from first case of COVID-19 in Ireland to first case in included NHs was 27.0 days. COVID-19 incidence in residents was 43.9\% (764/1741): laboratoryconfirmed in $40.1 \%$ (710/1741), with $27.2 \%$ (193/710) asymptomatic, and clinically-suspected in $3.1 \%$ (54/1741). Resident case-fatality was $27.6 \%(211 / 764)$ for laboratory-confirmed/clinically-suspected COVID-19. Similar proportions of residents in NHs with "early" outbreaks (<28days) vs "late" outbreaks developed confirmed/suspected COVID-19. A lower proportion of residents in NHs with "early" outbreaks recovered compared to those with "late" outbreaks (37.4\% vs $\left.61.7 \% ; \chi^{2}=56.9, \mathrm{p}<0.001\right) .675 \mathrm{NH}$ staff across twentyfour sites developed confirmed/suspected COVID-19; 23.6\% (159/ $675)$ were asymptomatic. There was a significant correlation between the proportion of staff with symptomatic COVID-19 and resident numbers with confirmed/suspected COVID-19 (Spearman's rho = $0.81, \mathrm{p}<0.001)$.

Conclusion: This study demonstrates the impact of COVID-19 on $\mathrm{NH}$ residents and staff. High infection rates lead to challenges in care provision.

\section{Abstract \# 98}

Consequences of impaired coagulation and impact of antithrombotic prophylaxys in elderly patients with coronavirus disease (COVID-19).

Carla Gamez Asuncion ${ }^{1}$, Maria José Martín Legorburo ${ }^{1}$, María Gebauer Campos ${ }^{1}$, Irene Bartolomé Martín ${ }^{2}$, Juan Rodriguez Solís ${ }^{2}$

${ }^{1}$ Internal Medicine Resident of Geriatrics. University Hospital of Guadalajara (Guadalajara, Spain), ${ }^{2}$ Medical Specialize in Geriatrics. University Hospital of Guadalajara (Guadalajara, Spain)

Background: The SARS-COV-2 pandemic has particularly affected the elderly. These have been treated with anticoagulants in a preventive way. We reviewed those patients on anticoagulant treatment and those who developed COVID-19 with adverse events.

Methods: Retrospective study of all patients older than 80 years with a diagnosis of SARS-COV-2 discharged from a University Hospital in Spain from March 1 to May 31, 2020.

Results: 91 patients (100\% analyzed). Median age 84.5 (81.2-89.8). Women $37.8 \%$. Institutionalized $32.2 \%$. Charlson median 2 (1-2). Independent $41.8 \%$, severe dependency $22 \%$; without cognitive impairment $70.3 \%$, moderate dementia $19.7 \%$. Anticoagulated $32.9 \%$; heparin (LMWH) during hospitalization $88.9 \%$ (64.1\% prophylactic, $28.3 \%$ anticoagulant dose); thrombosis $4.4 \% ; 11 \%$ bleeding; exitus $32.2 \%$.We found no relationship between anticoagulant treatment prior to the disease and mortality or thrombotic events. However, patients on anticoagulants before COVID-19 suffered more bleeding episodes (OR 3.1: 95\% CI 13-7.3; p = 0.008). Heparin administration was only associated with the probability of thrombotic disease $(p=0.026)$. We did not find a significant association between heparin dose, bleeding ormortality.

Key conclusions: About a third of the sample died and the only association found was COVID-19.-In this sample, prior anticoagulation had no protective effect on patients and increased the risk of bleeding.-According to the literature, it is safer to use heparin than other anticoagulants, and it has also been shown to prevent thrombosis. It has no influence on mortality

\section{Abstract \# 99}

How to improve the quality of care for older people with COVID19? From the acute care unit to long-term care facilities

Yaohua Chen ${ }^{1}$, Cédric Gaxatte ${ }^{1}$, Anne Charpentier ${ }^{1}$, Victoire Leroy ${ }^{1}$, Pascaline Cassagnaud ${ }^{2}$, Isabelle Delabrier ${ }^{1}$, Jean Roche ${ }^{1}$, Domnique Huvent-Grelle ${ }^{1}$, Bénédicte Simovic ${ }^{1}$, Catherine Gires ${ }^{1}$, Eric Boulanger ${ }^{1}$, Jean-Baptiste Beuscart ${ }^{1}$, François Puisieux ${ }^{1}$

${ }^{1}$ Department of Geriatrics, ${ }^{2}$ Memory clinic

Introduction: Although the mortality rate due to COVID-19 is lower than $3 \%$ in some countries, it raises to $15 \%$ for people aged over 80 . The department of Geriatrics and the care in older persons are facing a great challenge in this difficult time.

Methods: In France and particularly in the North, the highest infection wave stroke around end March and early April. We described how the department of Geriatrics in Lille (Northern France) reimplemented human resources respond to the needs in the acute care and in the long-term care facilities (LTCF) from March to April 2020.

Results: In the acute care setting, geriatricians created and run two inpatient wards specifically dedicated to older and frail patients and suspected of COVID-19. The mean age was 84 years old, $40 \%$ of them lived in LTCFs. At discharge, one third returned home, one quarter was transferred to a dedicated geriatrics COVID-19 rehabilitation ward. Specific geriatric care was delivered including nutrition, mobility, prevention of delirium, ethic concertation and participation 
to clinical trials if relevant. Some of the process were adopted by other younger inpatient wards, especially ethical concertation. As a tertiary center, we opened a hotline for LTCFs seven days a week and implemented "screening on wheels".

Conclusion: Our team achieved an efficient way of organization of the whole geriatric sector during the pandemic. The department of Geriatrics has a crucial role to play in the referral and the organization of care in the entire region. The post-Covid world will need transdisciplinarities and collaboration.

\section{Abstract \# 100 \\ Patterns of geographical distribution of COVID-19 infections and deaths-possible explanations}

Gabriel-Ioan Prada ${ }^{1}$, Catalina Raluca Nuta ${ }^{2}$, Ioana Dana Alexa ${ }^{3}$, Ovidiu-Lucian Bajenaru ${ }^{1}$, Rozeta Draghici ${ }^{4}$, Alexandra Rusu ${ }^{4}$, Adina Ilie $^{3}$, Anca Pislaru ${ }^{3}$, Ioana Sandu ${ }^{3}$, Ramona Stefaniu ${ }^{3}$, Anna Marie Herghelegiu $^{1}$

${ }^{1}$ University of Medicine and Pharmacy „Carol Davila”, Bucharest, Romania National Institute of Gerontology and Geriatrics „Ana Aslan”, Bucharest, Romania, ${ }^{2}$ University of Medicine and Pharmacy „Carol Davila”, Bucharest, Romania, ${ }^{3}$ University of Medicine and Pharmacy „Gr.T. Popa”, Iassi, Romania, ${ }^{4}$ National Institute of Gerontology and Geriatrics „Ana Aslan”, Bucharest,

RomaniaEpidemiologic characteristics of the new coronavirus infection named COVID-19 took by surprise the whole medical community as well as other authorities and general population worldwide. It has a specific behavior that transformed the infection initially localized in a single country in Asia into a real pandemic, situation never faced by mankind since 1918 . There have been many data regarding this COVID-19 but for the time being it seems to be too early to draw some final conclusions regarding the complete epidemiologic characterization of this virus as well as effectiveness of various therapeutic approaches available, including development of some new vaccines. One striking aspect that maybe did not receive enough attention is a huge difference regarding prevalence of infections and deaths rates due to or related to COVID-19 between developed countries and developing Eastern European countries: 20-40 times lower in the latter. It is interesting to note that this pattern can be observed even in the immigrant communities that originated in Eastern European countries but lived in developed countries. Several populational characteristics are reviewed, including the compulsory anti-tuberculous (BCG) vaccination that was performed in all Eastern European countries, unlike the situation of developed countries (in Europe and other continents).

\section{Abstract \# 101 \\ Acute presentations of nursing home residents to hospital during COVID-19 pandemic \\ Laura Bailey $^{1}$, Alice O'Donoghue ${ }^{1}$, Alan Martin ${ }^{2}$ \\ ${ }^{1}$ Beaumont Hospital, Dublin, ${ }^{2}$ Medicine for the Elderly, Beaumont Hospital, Dublin}

Introduction: The impact of COVID-19 has disproportionately affected nursing home residents across the world. In the catchment area of our tertiary hospital, we have over 2500 nursing home beds. Our aim was to look at the demographics of these patients, their presenting complaints, outcome, clinical characteristics and rate of infection.
Methods: We used admission data from ED and the COVID assessment unit to gather patient data over a 10 week period. We then used PIPE laboratory software and McKesson Radiology for results of investigations and clinical course.

Results: 78 residents were admitted via the COVID assessment unit during this time. 12 patients passed away, and 9 of these tested positive for COVID-19. 64\% of patients tested positive or where treated as such due to high clinical suspicion.The age groups were quite evenly spread with 22 under 70, 24 aged 70-79, 24 aged 80-89 and 12 at age $90+$. All cause mortality rates for the groups were $13.6 \%, 20 \%, 16.6 \%$ and $8.33 \%$ respectively. The mortality rate in positive patients was over three times higher than in negative patients; $21 \%$ vs $7 \%$. Average lymphocyte count for positive patients on day of admission was 1.17 , with an average lowest value during their stay of 0.91 . For negative patients, the results were 1.48 and a lowest value of 1.16. Interestingly, the average CRP of a non-COVID patient on admission was 137.8 compared to 99.9 for COVID positive admissions.The most common presenting symptoms included: dyspnoea, anorexia, malaise, fever and cough.Of the patients presenting with non-COVID-19 symptoms, falls, abdominal pain, urinary sepsis and fractures were most common.

Conclusions: Many nursing home residents were admitted during the COVID pandemic for other medical reasons and this is important given that many issues may be reversible. The long length of stay of many of our patients warrants further investigation and care planning. Our findings in laboratory data and symptoms reflect the findings of other institutions in this patient group.

\section{Abstract \# 102}

\section{COVID-19 in care homes: a unique Malaysian experience}

\section{Hakimah Sallehuddin ${ }^{1}$, Kejal Hasmu ${ }^{2}$, Maw Pin Tan ${ }^{3}$}

${ }^{1}$ Department of Medicine, Faculty of Medicine and Health Sciences, University Putra Malaysia, Malaysia, ${ }^{2}$ Geriatric Unit, Department of Medicine, University Malaya Medical Center, Malaysia, ${ }^{3}$ Geriatric Unit, Department of Medicine, Faculty of Medicine, University of Malaya, Malaysia

Malaysia is only starting to age in 2020 where it becomes a home for 2.4 million older adults aged over 65 years. As the population ages, the number of older persons with frailty and multimorbidity will increase. Around 1\% of Malaysian older persons were cared for in the care homes. COVID-19 impacted care homes in Malaysia differently in a way that it has more than 1400 care homes but only a quarter of that number was registered under the Malaysian law. The long-term care facilities in Malaysia were fragmented and multiple stakeholders were involved in providing the care, primarily non-government organizations (NGO), religious organizations and private operators. Therefore, urgent action was required to prevent the infection from entering and spreading in these care homes. This included guideline development and a mass screening by the Malaysian Ministry of Health. Published data by the government showed that infection occurred in $8 \%$ (15 out of 267 ) of care home screened. The residents and staffs of care homes for COVID-19 were screened with overall infection rate of $0.2 \%$ ( 26 out of 11,049$)$. Eighteen out of $26(85.7 \%$ ) of the infected persons were asymptomatic. Only five deaths have occurred from COVID-19 at care homes as of 7th May 2020. Malaysia is entering recovery phase from COVID-19 from 10th June 2020 with on-going precautionary necessary in care homes. Homes remain in lockdown despite relaxation in other sectors with no apparent solution to reopening homes to new admissions and visitors. 


\section{Abstract \# 103}

How are telephone triage services used by older persons over the age of 65 during the Covid-19 pandemic? A longitudinal analysis.

Farah Islam ${ }^{1}$, Koen Milisen ${ }^{2}$, Ewa Kocot ${ }^{3}$, Christoph Sowada ${ }^{3}$, Marc Sabbe $^{4}$

${ }^{1}$ Department of Public Health and Primary Care, Faculty of Medecine, KU Leuven, Leuven, Belgium; Institute of Public Health, Faculty of Health Sciences, Jagiellonian University, Kraków, Poland, ${ }^{2}$ Department of Public Health and Primary Care, Faculty of Medecine, KU Leuven, Leuven, Belgium; Department of Geriatric Medicine, University Hospitals Leuven, Leuven, Belgium, ${ }^{3}$ Institute of Public Health, Faculty of Health Sciences, Jagiellonian University, Kraków, Poland, ${ }^{4}$ Department of Public Health and Primary Care, Faculty of Medecine, KU Leuven, Leuven, Belgium; Department of Emergency Medicine, University Hospitals Leuven, Leuven, Belgium

Background: The use of telephone triage services for managing patients remotely has rapidly escalated during the Covid-19 pandemic. Older segments of the population are amongst the most complex population groups to manage using these services, and they are also amongst those who are at the highest risks for experiencing negative health-related consequences due to Covid-19. As part of the TRANS-SENIOR international training and research network, we seek to compare the use of telephone triage services by older persons (over 65 years of age) before-, during, and after the Covid-19 pandemic.

Methods: Data will include an estimated 30,000 call records from the 1733-out-of-hs telephone triage call center in Belgium (more information available at: https://www.health.belgium.be/en/health/need -call-doctor-call-1733) obtained 3 months prior to-, during-, and after the Covid-19 pandemic peak. All calls concerning medical assistance for older patients (over 65 years of age) between December 1, 2019 and August 30, 2020 will be included for analysis. Variables related to patient-related characteristics and user-trends (i.e. age, sex, time of day, location, estimated level of urgency, dispatch outcome) will be explored using descriptive, comparative, and longitudinal analysis.

Results: Data collection is currently in process. Findings will be analyzed and made available for presentation at the EuGMS 2020 digital congress. Conclusion: The current research will allow us to better understand how telephone triage services are used by older persons during the ongoing Covid-19 pandemic.

\section{Abstract \# 104 \\ Case series: clinical presentations of COVID-19 in frail older adults}

Morgan Bailey ${ }^{1}$, Gareth Knott ${ }^{1}$, Charlotte Kirk ${ }^{1}$

${ }^{1}$ WSHFT

Introduction: Non-specific or atypical presentation is extremely common in frail older adults when acutely unwell. Case reports have described frail older people with COVID-19 presenting with falls and delirium, and lacking the typical features of fever, cough and shortness of breath (SOB) $[1,2]$. We present a 50 patient case series describing presenting features of COVID-19 illness in an older frail population.

Methods: A retrospective case note review was undertaken of 50 patients aged 65 and over with an admission Clinical Frailty Scale [3] score of 5-8 admitted to Worthing Hospital, England, between 12th March and 1st May 2020 with a positive reverse transcription- polymerase chain reaction nasopharyngeal swab for severe acute respiratory syndrome coronavirus 2 .

Results: In our cohort, presenting with falls, delirium and functional decline was as likely as with fever, cough and SOB. 30\% of our cohort did not have any of the typical symptoms of fever, cough or SOB at presentation. Half of patients were delirious at presentation. Conclusions: We highlight the need for continued vigilance when assessing frail older adults currently presenting with frailty syndromes, who may be less like to manifest typical COVID-19 symptoms. Considering "Could this be COVID-19?" will impact upon patients' diagnosis, treatment and clinical pathway. Prior to universal admission testing in the NHS in England, we locally adapted our case screening criteria for potential COVID-19 to include presentations with otherwise unexplained delirium.

1. Tay h, Harwood R. Atypical presentation of COVID-19 in a frail older person. Age Ageing. 2020;

2. Neerland BE, Dobloug A, Nore KG, Mikaelsen EE, Halsen A, Ahmed MV. Covid-19 pavist hos eldre kvinne med akutt funksjonssvikt. Tidsskr den Nor Laegeforening. 2020;

3. Rockwood K, Song X, MacKnight C, Bergman H, Hogan DB, McDowell I, et al. A global clinical measure of fitness and frailty in elderly people. CMAJ. 2005;

\section{Abstract \# 105}

Biochemical markers of severity and outcome in the older patient with COVID-19

Abisoye O. Akintimehin ${ }^{1}$, David Smithard ${ }^{1}$, Penelope J. Teoh ${ }^{1}$

${ }^{1}$ Lewisham and Greenwich NHS Foundation Trust

Introduction: Markers commonly used to prognosticate in COVID19 include d-dimer, ferritin and troponin. The aim of this project was to compare biochemical markers and outcome in older and younger COVID-19 patients.

Methods: We reviewed patients admitted to our District General Hospital between 2nd April 2020 and 23rd April 2020. Those $\geq 75$ years who were diagnosed with COVID-19 (by either swab or clinical picture) were included. Their admission dependency, frailty score, initial serum d-dimer, ferritin and troponin were collected. Clinical frailty score, mortality, discharge destination, and dependency on discharge were recorded. These patients were gender-matched 1:1 to controls (adults $<75$ years admitted with COVID-19).

Results: Those $\geq 75$ years are equally likely to be diagnosed with COVID-19 from a respiratory viral swab than those $<75 \mathrm{yrs}(79.0 \%$ vs $81.0 \% \mathrm{p}=0.724)$. Those $\geq 75$ years had slightly lower initial mean serum ferritins $(1352 \mathrm{ug} / \mathrm{L}$ vs $1476 \mathrm{ug} / \mathrm{L}, \mathrm{p}=0.896)$ and d-dimers (3188 ng/L vs $3398 \mathrm{ng} / \mathrm{L}, \mathrm{p}=0.370$ ). They have significantly higher serum troponins $(88.1 \mathrm{ng} / \mathrm{L}$ vs $35.9, \mathrm{p}=0.007)$. Patients $\geq 75$ years are significantly more likely to die during admission $(55.0 \%$ vs $25.0 \%, \mathrm{p}<0.000015$ ). Only $12.9 \%$ of patients $\geq 75$ years had a change to their discharge destination. Using logistic regression, laboratory markers and frailty score did not predict an increase in support on discharge, however $\mathrm{d}$-dimer $(\mathrm{p}=0.039)$ and age $\geq 75$ years $(\mathrm{p}=$ $0.005)$ predicted mortality.

Conclusions: Older patients with COVID-19 have slightly lower ferritins and d-dimers than younger patients, but significantly higher troponins. D-dimer predicts mortality and however, despite a high mortality, a small proportion required a change to their discharge destination. 


\section{Abstract \# 106}

Functional impairment after COVID-19, is there recovery?

Ester Hoyos Alcañiz ${ }^{1}$

${ }^{1}$ Geriatrics

Introduction: COVID-19 disease has led to prolonged admissions in elderly people, which entails a loss of the ability to independently in activity of their daily life.

Methods: The case of a 78-year-old man admitted by COVID-19 in the period from March to June 2020 is reported.

Results: A 78-year-old male patient without comorbidity, independent, went to the Emergency Department on 03/11/2020. He presents dyspnea associated with non-productive cough and fever of 48-h. In Chest radiography, a mixed pattern of diffuse and bilateral distribution, compatible COVID-19 infection. SARS CoV-2 PCR was performed, being positive. He entered the Geriatrics plant starting treatment on 03/13 with Dolquine and Kaletra and, given the severity data, corticosteroid treatment (03/20) and Tocilizumab (3 doses, 03/17). The patient presented bad clinical evolution. ICU admission is decided. During his stay in the ICU (03/20-07/07), the patient presented a numerous medical and surgical complications. The patient required orotracheal intubation on admission and subsequent percutaneous tracheostomy. On 02/04, he presented refractory shock secondary to sepsis of abdominal origin, suffering a cardiorespiratory stop, requiring maneuvers of reanimation for $25 \mathrm{~min}$. Subsequently, hypoxic-ischemic encephalopathy. In thoracoabdominal scanner 03/04, colonic perforation at the junction of the descending colon with sigma. Surgical intervention was decided on 04/23. In this context, numerous episodes of atrial fibrillation, initiating treatment with Amiodarone and anticoagulant. As complications of prolonged admission, the patient presents episodes of repeated urine retention, pressure ulcer in the sacral region grade II, pressure ulcer in both knees due to prone position and tetraparesis of the critically ill patient.The patient was admitted to our mid-stay unit for functional on $06 / 06$, presenting $1 / 5$ strength in all limbs.

Conclusions: Geriatricians must pay special attention to the functional recovery of these patients since it is essential to improve their quality of life.

\section{Abstract \# 107 \\ Monitoring of assisted elderly people during COVID-19 times at a State Reference Center in Bahia}

Lélia Mendes Sobrinho de Oliveira ${ }^{3}$, Rosângela Souza Oliveira ${ }^{3}$, Nildete Pereira Gomes ${ }^{1}$, Larissa Chaves Pedreira ${ }^{1}$, Márcio Ribeiro $\mathrm{Melho}^{2}$, Arianna Oliveira Santana Lopes ${ }^{1}$

${ }^{1}$ Escola de Enfermagem da Universidade Federal da Bahia, Brazil, ${ }^{2}$ Escola de Medicina da Universidade Federal da Bahia, Brazil, Centro de Referência Estadual de Atenção à Saúde do Idoso. Bahia, Brasil $^{3}$

Introduction: The COVID-19 pandemic has implications for health institutions' practices. Objective: To report the work process at a Reference Center in Bahia-Brazil at the time of the COVID-19 pandemic.

Methodology: This is an account of the experience of monitoring elderly people assisted at a Reference Center in Bahia-Brazil, during a COVID-19 pandemic.

Experience report: With suspension of presential consultations, the center developed a service for clinical follow-up and monitoring of assisted elderly people via telephone. Doctors and nurses access the schedule of patients for the day, observe their medical records and manage their clinical condition through a telephone call. In contact with family members of the elder, professionals carry out research on the presence of respiratory symptoms, general clinical status and use of med ications. Health guidance on preventive practices for COVID19 is carried out, as well as emphasizing the importance of remaining linked to a primary care unit. The center offers face-to-face assistance to family members who are protected, a bus during the morning shift, provision of medical prescriptions, various reports and guidance on questions about care during this period.

Conclusion: It can be concluded that during pandemic times it is fundamental to adequate the work process developed at the Reference Center for the Elderly, seeking to guarantee the preservation of their monitoring and guidance facing new health challenges. Keywords: Elderly; Coronavirus; Pandemics.

\section{Abstract \# 108 \\ Multidisciplinary management after COVID pandemic in nursing} home

Cedeño Veloz Bernardo Abel ${ }^{1}$, Marina Sanchez Latorre ${ }^{1}$, ChenHui Chen $^{1}$, Ana Sofía Pozo Vico ${ }^{1}$, Nancy Jeanette Gonzales Montejo ${ }^{1}$, Maria Fernanda Ramon Espinoza ${ }^{1}$, Itxaso Marín Epelde ${ }^{1}$

${ }^{1}$ Complejo Hospitalario de Navarra

Introduction: There is growing international evidence that people living in socio-health centers are especially vulnerable to severe SARS-CoV-2 infections and are experiencing high mortality rates. Due to its characteristics (advanced age, functional and mental difficulty, comorbidities ...), the consequences of both the disease and the problems derived from confinement itself secondary to social isolation, immobility and the anxiety generated are especially important. Multidisciplinary management is necessary to prevent these complitations.

Methods: Narrative review of Management was performance by the authors in order to make recomendatios to the nursing home in our clinical area.

Results: 16 recommendations were done by the groups. These are relative to medical follow-up (with analytics and radiography), anticoagulant and corticoids management, screening of functional and cognitive decline, malnutrition, delirium, anxiety, depression and polipharmafacy. For of all these problem, practical advice (based on exercise with Vivifrail, cognitivie stimulation, nutrition with suplement prescripcion, management polypharmacy with STOP-START criteria, management frecuent comorbilitis such hypertension, diabetes; reduccing risk of falling and coordination between primary care and geriatrics) were recommended in order to improve residents health status

Conclusion: After the first wave of COVID pandemic, management of residents in nursing homes is still a big challenge. Practical recommendations are the base to improve residents management.

\section{Abstract \# 109}

\section{EuGMS SIG on GR Symposium}

Achterberg Wilco ${ }^{1}$, Grund Stefan ${ }^{2}$

${ }^{1}$ Department of Public Health and Primary Care, Leiden University Medical Center, Hippocratespad 21 Postzone V0-P, Room V6-76, Postbus 9600, 2300 RC, Leiden, The Netherlands., ${ }^{2}$ Center for Geriatric Medicine, Agaplesion Bethanien Hospital Heidelberg,

Presentation 1: Wilco Achterberg (NL): „Pan-European Study on Outcome and Service in Geriatric Rehabilitation in Times of COVID19 Pandemic".The COVID-19 pandemic is hitting the world hard, and older and more vulnerable persons are more likely to get very ill 
when infected. Older and multimorbid patients have the highest morbidity and mortality. The majority of patients that are admitted to the hospital are old ( $>70$ years). Some of them have been, or are being, admitted to Intensive Care Units/ventilators. Many patients stay on the ICU/hospital for many weeks, and may have physical problems such as pulmonary problems, deconditioning/sarcopenia, pressure ulcers, cognitive problems and psychological problems because of many weeks of social isolation. Many of these patients had multi-organ failure. (1-4) To get insight into the course of functional and medical recovery in (geriatric) rehabilitation patients affected by COVID-19 in Europe, we initiated this international multi-center observational cohort study. In this session we present the protocol and first results of the,, Pan-European Study on Outcome and Service in Geriatric Rehabilitation in Times of COVID-19 Pandemic".

1. Sun P, Qie S, Liu Z, et al. Clinical characteristics of 50, 466 hospitalized patients with 2019-nCoV infection. J Med Virol 2020; Feb 28. https://doi.org/10.1002/jmv.25735.

2. Wang YXJ, Liu WH, Yang M, Chen W. The role of CT for Covid19 patient's management remains poorly defined. Ann Transl Med 2020; 8:145.

3. Liu Y, Yang Y, Zhang C, et al. Clinical and biochemical indexes from $2019 \mathrm{nCoV}$ infected patients linked to viral loads and lung injury. Sci China Life Sci 2020; 63:364-374.

4. Wilson N, Kvalsvig A, Barnard LT, Baker MG. Case-fatality risk estimates for COVID-19 calculated by using a lag time for fatality. Emerg Infect Ids 2020; 26(6).

Presentation 2: Stefan Grund (DE): "Developing geriatric rehabilitation in Europe - Aims and current projects of the EuGMS SIG-on GR "Due to demographic changes and the special needs of older people, especially older multimorbid patients after acute hospital stay, the interest in geriatric rehabilitation services is rising in all European countries. To react on this development the EuGMS Special Interest Group on Geriatric Rehabilitation was founded at the EuGMS-Congress in Berlin in 2018. Once the "European consensus on core principles and future priorities for geriatric rehabilitation ..." was reached (1), the EuGMS Special Interest Group on Geriatric Rehabilitation defined areas of further research and action to develop geriatric rehabilitation in Europe. Because of our knowlegde about the heterogenity of geriatric rehabilitation services across Europe (2) we are working on a "Guideline on Best Practice in geriatric rehabilitation". In this presentation the audience will first get an overview about the content and status of all current SIG on GR projects, adaptions due to COVID-19 pandemic included. Additionally the first results of the working process on the „Guideline on Best Practice in Geriatric Rehabilitation" will be presented.

1. Grund, S., Gordon, A.L., van Balen, R. et al. European consensus on core principles and future priorities for geriatric rehabilitation: consensus statement. Eur Geriatr Med 11, 233-238 (2020). https://doi.org/10.1007/s41999-019-00274-12.

2. Grund, S., van Wijngaarden, J.P., Gordon, A.L. et al. EuGMS survey on structures of geriatric rehabilitation across Europe. Eur Geriatr Med 11, 217-232 (2020). https://doi.org/10.1007/ s41999-019-00273-2

\footnotetext{
Abstract \# 110

Increasing access to and safety of nursing home by "infrared remote monitoring system" during the COVID-19 infection pandemic: a case from Japan

Kazuko Obayashi ${ }^{1}$, Naonori Kodate ${ }^{2}$, Shigeru Masuyama ${ }^{3}$

${ }^{1}$ Nihon Fukushi University, ${ }^{2}$ University College Dublin, ${ }^{3}$ Tokyo Medical University
}

1. Introduction: The risk associated with COVID-19 infection is considered to be moderate to high among those interacting indoors, particularly older people. As a result, they refrain from accessing various services including nursing homes. In order to overcome this barrier to access and create a safer environment for care professionals, a nursing home in Tokyo adopted isolation measures to reduce the frequency of contact. In a nursing facility in Tokyo, a remotely controlled infrared monitoring system was introduced, and its impact and effectiveness were tested.

2. Methods: A monitoring system was set up for 38 older adults, and changes in the frequency of visiting the room and the workload of care professionals were compared before and after the introduction of the devices.

3. Results: The monitoring system decreased the frequency of room visits by care staff at night from 22.9 to 10.9 times, as it provided the visual information about the residents' status quo remotely. The system allowed the facility to welcome five potentially Covid 19 infected residents as a result. The fatigue level of night-shift care staff also showed a decrease. A remarkable effect was found particularly towards the end of their shifts when fatigue levels reach the highest. 4. Key conclusions: The remote monitoring system decreased the physical and mental burden on care staff by reducing the frequency of their visit to residents' rooms at night. The system can be highly effective in tackling the challenge of Covid19 by mitigating the barrier to people's access to care home facilities. This study was in part supported by the Japan Keirin Autorace Foundation. We would like to acknowledge the support and dedication of care professionals.

\section{Abstract \# 111}

The presentation of a COVID-19 positive patient in a 1000- bed long term care facility in Malta

F. Farrugia ${ }^{1}$, D. Galea ${ }^{1}$, M. Debono ${ }^{1}$, L. Camilleri ${ }^{1}$, J. Tua ${ }^{1}$, S. Dalli ${ }^{1}$, A. Vella ${ }^{1}$, R. Fiorentino ${ }^{1}$

${ }^{1}$ St. Vincent de Paul long term care facility, Luqa, Malta

Introduction: This case report details the presentation and management of the only patient diagnosed with COVID-19 in a 1000-bed long term care facility which houses frail patients who require $24 \mathrm{~h}$ nursing and medical care and the measures undertaken in order to limit the spread of COVID-19.

Case Presentation: The patient was an 88-year old lady who was a known case of diabetes mellitus, obesity, asthma, hypertension, hip fracture and congestive heart failure who experienced sore throat. All her parameters were normal and she was never febrile. A COVID-19 nasopharyngeal swab was positive. Management: The management of this patient required a holistic approach from all the teams within the facility in order to treat the patient as well as contain the spread of COVID-19 within a vulnerable population. The measures which were enacted in a timely fashion before the case of COVID-19 was registered in the facility were effective in containing the spread. These included the thermal screening of all staff entering the facility, all elective appointments outside the facility were postponed, contact tracing of staff and low threshold for swabbing for staff and patients. To date only one resident contracted COVID-19 from the entire facility. Immediate action was undertaken for medical isolation of the patient and was nursed in another ward catered for residents with COVID-19 within the facility and made an uneventful recovery.

Conclusions: Effective and timely measures in infection control were crucial in preventing COVID-19 spread to residents and in between residents. 


\section{Abstract \# 112 \\ Atypical presentations of COVID-19 in care home residents presenting to secondary care: a UK Single Centre Study}

Mark James Rawle ${ }^{1}$, Deborah Lee Bertfield ${ }^{2}$, Simon Edward Brill ${ }^{3}$

${ }^{1}$ Department of Geriatric Medicine, Barnet Hospital, Royal Free London NHS Foundation Trust \& MRC Unit for Lifelong Health and Ageing at UCL, ${ }^{2}$ Department of Geriatric Medicine, Barnet Hospital, Royal Free London NHS Foundation Trust, ${ }^{3}$ Department of Respiratory Medicine, Barnet Hospital, Royal Free London NHS Foundation Trust

Purpose: The United Kingdom (UK) care home population has experienced high mortality during the COVID-19 pandemic [1]. Atypical presentations of COVID-19 are being reported in older adults and may pose difficulties for early isolation and treatment, particularly in institutional care settings $[2,3]$. We aimed to characterise the presenting symptoms and associated mortality of COVID19 in older adults, with a focus on care home residents and older adults living in the community.

Methods: This was a retrospective cohort study of consecutive inpatients over 80 years old hospitalised with PCR confirmed COVID-19 between 10th March 2020 and 8th April 2020. Symptoms at presentation, including those associated with frailty, were analysed. Differences between community dwelling and care home residents, and associations with mortality, were assessed using between-group comparisons and logistic regression.

Results: Care home residents were less likely to experience cough $(46.9 \%$ vs $72.9 \%, p=0.002)$ but more likely to present with delirium $(51.6 \%$ vs $31.4 \%, \mathrm{p}=0.018)$, particularly hypoactive delirium $(40.6 \%$ vs $24.3 \%, p=0.043)$. Mortality was more likely in the very frail (OR $1.25,95 \%$ CI $1.00,1.58, \mathrm{p}=0.049)$ and those presenting with anorexia (OR 3.20, 95\% CI 1.21, 10.09, $\mathrm{p}=0.028$ ). There were no differences in either mortality or length of stay between those admitted from care homes and community dwelling older adults. Conclusion: COVID-19 in those over 80 does not always present with typical symptoms, particularly in those admitted from institutional care. These individuals have a reduced incidence of cough and increased hypoactive delirium. Individuals presenting atypically, especially with anorexia, have higher mortality.

References:

1. Office for National Statistics. Deaths involving COVID-19 in the care sector, England and Wales: deaths occurring up to 1 May 2020 and registered up to 9 May 2020 (provisional). 2020 [3rd June 2020]; Available from: https://www.ons.gov.uk/peoplepopulationand community/birthsdeathsandmarriages/deaths/articles/deathsinvolving covid19inthecaresectorenglandandwales/deathsoccurringupto1may 2020andregisteredupto9may2020provisional.

2. Docherty AB, Harrison EM, Green CA, Hardwick HE, Pius R, Norman L, et al. Features of 20133 UK patients in hospital with covid-19 using the ISARIC WHO Clinical Characterisation Protocol: prospective observational cohort study. BMJ. 2020 May 22; 369:m1985.

3. Bianchetti AR, R; Guerini, F; Boffelli, S; Ranieri, P; Minelli, G; Bianchetti, L; Trabucchi, M. Clinical presentation of COVID19 in dementia patients. J Nutr Health Aging. 2020.

\section{Abstract \# 113}

Experience of COVID-19 in a UK older adult rehabilitation hospital, an observational case series

Morven McElroy ${ }^{1}$, Terry Quinn ${ }^{1}$, Carol McCarthy ${ }^{1}$, Greg Waddell ${ }^{1}$, Ewen Cameron ${ }^{1}$, Kathyrn McIlroy ${ }^{1}$, Aine McGovern ${ }^{1}$

${ }^{1}$ Department of Medicine for the Elderly, Glasgow Royal Infirmary, NHS Greater Glasgow and Clyde

Introduction: The effects of the COVID-19 viral pandemic have been felt across all healthcare settings. We describe epidemiology, outcomes and learning from an outbreak of COVID-19 in an older adult rehabilitation unit.

Methods: Lightburn hospital is a 56 bedded rehabilitation hospital that serves, but is geographically remote from, a large urban teaching hospital in Glasgow, UK. Cohort inception was first diagnosis of COVID-19. The treating clinical team collected demographic, clinical and laboratory features prospectively, augmented by reference to electronic medical records. We performed follow-up for outcomes of death, discharge and readmission.

Results: Index case was diagnosed clinically on 18th March and follow-up data are available until 25th May. Over a 5-week period, of 51 inpatients, 29 (57\%) developed COVID-19. Median age:84 (range 74-100); 18 (62\%) female; median Clinical Frailty Scale:6 (range 5-8). Fourteen (48\%) had classical COVID-19 symptoms of cough and dyspnoea, 7 (24\%) had delirium, $3(10 \%)$ were asymptomatic. Viral PCR testing was positive in $29(100 \%)$, median time to negative viral status: 20 days (range 7-25). Median length of inpatient stay following diagnosis was 16.5 days (range $0-53$ ). Over the period of follow up, 8 (28\%) patients died, $10(35 \%)$ returned home and none were readmitted.

Key Conclusion: Our single centre experience of COVID-19 highlights several issues relevant to older adult rehabilitation settings that are discussed in more depth:potential for nosocomial infection, nonspecific presentations of COVID-19 in older adults, patterns of viral positivity, complexity of discharge planning and heterogeneity in outcomes.

Keywords: COVID-19, Rehabilitation, Older people, Organisation of care

\section{Abstract \# 114 \\ Covid -19 in Dutch long-term care facilities: a cohort study on symptomatology, mortality and risk factors}

Jeanine Rutten ${ }^{1}$, Anouk van Loon ${ }^{1}$, Martin Smalbrugge ${ }^{1}$, Janine van Kooten $^{1}$, Cees Hertogh ${ }^{1}$, Laura van Buul ${ }^{1}$

${ }^{1}$ Amsterdam UMC

Background: Many reports on coronavirus disease 2019 (Covid-19) have highlighted that older people are more susceptible to severe illness and have a higher mortality risk. Yet, research on Covid-19 symptomatology, mortality and risk factors in a large group of longterm care facility (LTCF) residents is lacking.

Methods: In this prospective cohort study, we collected data of Dutch LTCF residents with clinically suspected Covid-19 via electronic health records, between March 18th and May 13th, 2020, . Registration was performed on diagnostic status (Covid-19: confirmed/ ruled out) and symptomatology ((a)typical and other symptoms). Data on mortality and mortality risk factors (age, gender and comorbidities) were extracted from usual care data.

Results: In our sample with clinically suspected COVID-19 residents $(\mathrm{N}=4007)$, Covid-19 was confirmed in 1538 residents $(38 \%)$. Symptomatology did not differ between residents with confirmed Covid-19 and ruled out Covid-19. Yet, residents with confirmed 
Covid-19 were three times more likely to die within 30 days (42\% versus $15 \%$ ). Within this group mortality was higher for men than for women (HR: 1.8) and we observed a higher mortality for Dementia (HR 1.3), reduced kidney function (HR 1.4) and Parkinson's Disease (HR 1.5), even when corrected for age, gender and comorbidities.

Conclusion: In clinically suspected COVID-19 LTCF residents there is overlap in symptoms between confirmed Covid-19 and ruled out COVID-19. The mortality risk in confirmed Covid-19 residents was 3 times higher than in residents where COVID-19 was ruled out, especially for men. This increased risk could not be explained by differences in age, gender and comorbidities.

\section{Abstract \# 115 \\ Case fatality rate in Belgian Care Homes: search for underlying reasons}

Nele Van Den Noortgate ${ }^{1}$, Filip Vandaele ${ }^{2}$, Dominique Roodhooft ${ }^{3}$

${ }^{1}$ Ghent University Hospital, ${ }^{2}$ VZW Zorgsaam, ${ }^{3}$ VZw zorgsaam

Background: Care homes in Belgium has been confronted with high case fatality rate from COVID-19 during the pandemic in Belgium. This analysis of data aims to give insight in underlying reasons.

Methods: Observational study from 19/03 till 13/05 in a care home organisation, representing 2205 residents and 1920 staff members in Flanders, Belgium. Following data were collected: residents with possible/proven COVID, hospital admissions, mortality, tested residents and staff, personal protective equipment (PPE). Descriptive analysis was performed using SPSS 26.

Results: A total of 386 residents (17\%) were isolated due to a possible/proven infection. A trend to less hospitalised residents in 2020 ( $\mathrm{n}=196 ; 24 \%$ COVID related) compared to 2019 same period $(\mathrm{n}=$ $226)(p=0.06)$ was noted. Significant more residents died $(n=173$; 60 posssible COVIDrelated) compared to $2019(\mathrm{n}=118 ; \mathrm{p}=0.004$; excess mortality 46, 6\%). Between 3 and 8 April, 130 of 550 (23.6\%) low symptomatic residents and their contacts tested positive. Blanked testing by government afterwards registered 37 of 1987 positive cases $(1.86 \%)$. Among staff members, $105 / 2166(4.8 \%)$ tested positive. In care homes where contact testing and FFP2 masks were introduced, infection rate was only $10 \%$ compared to $30 \%$ in early infected homes.

Conclusion: Testing residents and staff with low symptomatic disease and their contacts together with provision of PPE declined infection rate in the nursing homes. The availability of blanket testing through official organisations started after the steep rise in infections. Despite the high number of infected residents, the hospitalisation of patients stayed below the annual rate.

\section{Abstract \# 116 \\ Referrals of patients at Saint Vincent de Paul Long term care facility (SVP) to the Accident and Emergency Department at Mater Dei Hospital in COVID-19 and non-COVID-19 eras.}

Doriella Galea ${ }^{1}$, Francesca Farrugia ${ }^{1}$, Julia Tua ${ }^{1}$, Lara Camilleri ${ }^{2}$, Melanie Debono ${ }^{2}$, Jessica Camilleri ${ }^{2}$, Ronald Fiorentino ${ }^{1}$

${ }^{1}$ Saint Vincent de Paul Long Term Care Facility, ${ }^{2}$ Saint Vincent de Paul Long Term Facility

Background: The pandemic of COVID-19 has affected a number of practices at SVP in order to minimise exposure of vulnerable patients. A transfer to the acute hospital involved quarantining the patient for 14 days away from his/her usual ward (environment). This might have affected the clinical decision of who to refer to A\&E. April-May
2019 and April-May 2020 refer to non-COVID-19 and COVID-19 eras respectively.

Aim: To establish whether there was a change in number and type of referrals to $\mathrm{A} \& \mathrm{E}$ when comparing referrals done in the two eras.

Method: All patients who were transferred to A\&E from SVP in April-May 2019 and April-May 2020 were included in the study. The search was carried out via iSOFT. Patients' demographics, investigations and diagnosis were looked at.

Results: In April-May 2019 a total of 61 patients were referred (2 male patients referred twice giving a total of 63 referrals) as opposed to 39 patients (one female patient transferred twice giving a total of 40 referrals) in April-May 2020. In 2019 there were 34 admissions (54\%) as opposed to $25(64.1 \%)$ in 2020.26 males (42.6\% of referred patients) were referred in 2019 as opposed to 18 males $(43.1 \%)$ in 2020.7 of the patients referred in 2019 died as opposed to 3 patient in 2020.

Conclusion: It seems that less patients were referred in 2020 possibly to avoid transfer of vulnerable to the acute hospital where there were COVID-19 positive patients.

Limitations: A short time-frame was included for the study.

\section{Abstract \# 117 \\ Analysis of mortality data in a long-term-care unit during the COVID-19 epidemic in France}

Isabelle Gantois ${ }^{1}$, Caroline Masurel ${ }^{1}$, Menel Msolly ${ }^{1}$, Carlos Labat ${ }^{2}$, Hubert Tronel ${ }^{1}$, Marie-Christine Godart ${ }^{1}$, Cecile Billon ${ }^{1}$, Aurelie Bannay $^{1}$, Nicolas Jay ${ }^{1}$, Athanase Benetos ${ }^{3}$

${ }^{1}$ University Hospital of Nancy, Université de Lorraine, ${ }^{2}$ DCAC Inserm U1116, Université de Lorraine, ${ }^{3}$ University Hospital of Nancy, Université de Lorraine; France DCAC Inserm U1116, Université de Lorraine

The COVID-19 epidemic has had a major impact among residents of nursing homes (NH) and long-term care units (LTC). In France, it is estimated that more than $10,000 \mathrm{NH}$ and LTC residents died from COVID-19. However, for several reasons (lack of systematic biological tests, population with several comorbidities and high mortality rates), the real impact of this pandemic on mortality is not really known.Objective: To analyse the mortality data in an LTC department during the COVID-19 epidemic and to compare these data with the mortality data in this department during the same time of year, in 2018 and 2019.

Methods: This study was carried out prospectively in the 105 residents (median age 84 years, $56 \%$ women) of the LTC department of Nancy University Hospital present in this structure on March 10, 2020, at least 15 days before the presence of the very first COVID-19 symptoms in the LTC unit. The monitoring ended on May 31, 2020 (total duration of FU 12 weeks). Mortality data were compared with those of residents who were present in the same department on March $10,2018(\mathrm{n}=106$, median age 86.7 years, $67 \%$ women $)$ and $2019(\mathrm{n}=$ 103, median age 87.0 years, $60 \%$ women) (same FU period).

Results: Among the 105 residents of the 2020 cohort, 58 (55\%) became positive for COVID-19 during the FU period. During this period, 27 residents of this cohort died. The table below presents the characteristics in the 4 groups according to the vital state and the COVID-19 state (rt-PCR).SEE TABLE: https://aristeaint-my.share point.com/:w:/g/personal/testa_aristea_com/EUDutoy8M9RLIWOVI eVPd2kB0WkVoHdzJOD81FRDfYaGbQ?e = S4Xva0. Comparatively, in similar FU periods, we observed 16/106 (15\%) deaths in 2018 (median age: 88 years, women $62.5 \%$ ) and $12 / 103(12 \%)$ deaths in 2019 (median age 82 years women $58 \%)(\mathrm{p}<0.02$ compared to the 2020 mortality). 12 of the 19 deaths associated with COVID-19 occurred during the pandemic peak period (April 8-25). 
Conclusions: Between March 10 and May 31, the death rates of COVID-19 positive subjects were twice as high as that of COVID-19 negative subjects of the same cohort and at mortality rates during the same period during the previous 2 years. In this relatively small cohort of institutionalized people, age, sex and number of co-morbidities were not related to the risk of contracting or dying from coronavirus-19.

\section{Abstract \# 118}

\section{COVID 19: dementia. the great forgotten}

Ana Sofía Pozo Vico ${ }^{1}$, Belén González Glaría ${ }^{1}$, Nancy Gonzales Montejo ${ }^{1}$, María Fernanda Ramón Espinoza ${ }^{1}$, Itxaso Marín Epelde ${ }^{1}$, Chenhui Chen ${ }^{1}$, Marina Sánchez Latorre

${ }^{1}$ Servicio de Geriatría. Complejo Hospitalario de Navarra

Introduction: Coronavirus disease 2019 pandemic has produced devastating consequences among elderly. A silent surge in fatalities in nursing homes has been echoed by government agencies, but we have ignored the great impact of household confinement on dementia patients and their caregivers. This emergency situation has exposed unmet primary health needs.

Methods: Dementia group of SNGG (Sociedad Navarra de Geriatría y Gerontología) design a Five-Minute-Survey to identify the main problems that caregivers had faced in the home care of dementia patients. It was distributed by mailing among AFAN (Asociación de Familiares de Enfermos de Alzheimer) members and users of some daily centers.

Results: 106 people answers the survey with $74.5 \%$ women. The most frequent age range of caregivers was 46-65 years old and they have lived with the patient at least 1 year. Mid-afternoon was the most complicate moment. The most stressful situations were being housebound (56\%) and shower time (30\%) and caregivers felt lonelier when they had to perform new activities. In respect of the impact: $48 \%$ patients experienced functional and cognitive impairment, and caregivers felt anxiety (57\%), mood changes (47\%) and sleep disorders (34\%). Finally, caregivers suggest having MORE SUPPORT AND UNDERSTANDING would have felt them more relieved. Conclusions: After analyzing the situation, we have detected a serious communication failure and a clear lack of support to caregivers. To sum up, in spite of the catastrophic consequences of this setback, we hope we grasp the opportunity to respond with the right care, at the right time, in the right ways.

\section{Abstract \# 119 \\ The show must go on: teaching communication skills during Covid-19 lock-down}

Katinka Nordheim Alme ${ }^{1}$, Gunnar Tschudi Bondevik ${ }^{2}$, Sharline Riiser $^{3}$, Inga-Cecilie Sørheim ${ }^{3}$

${ }^{1}$ University of Bergen, ${ }^{2}$ Department of Global Public Health and Primary Care, University of Bergen 2. National Centre for Emergency Primary Health Care, NORCE Norwegian Research Centre, Bergen., ${ }^{3}$ Department of Global Public Health and Primary Care, University of Bergen, Bergen, Norway.

Background: Because of the Covid-19 lock down all teaching activities on campus were cancelled this spring. As a replacement, much of the planned courses were held using digital platforms. We launched a 1 week digital course in communication skills for 6th semester medical students.
Methods: The evaluation is based on the teacher comments and a structured student feedback form consisting of two qualitative questions (Q1: "What was good with the communication course?" and Q2: "What could be better") and three with a likert scale from 1-5 where 1 was "bad" and 5 was "best" to rate the $(\mathrm{Q} 3)$ course, $(\mathrm{Q} 4)$ the lectures and (Q5) the group sessions respectively. The data from the qualitative questions are picked out non-systematically.

Results: 54 of 80 students answered the questionnaire. On question 3 $58 \%$ and $40 \%$ gave a score of 4 and 5 respectively. On question $456 \%$ and $36 \%$ rated the lectures as 4 and 5 . And $70 \%$ rated the group session as "best" (Q5). The mix of class and group-sessions and video and live was evaluated as positive.

Conclusion: Launching the communication course on a digital platform was evaluated as a success. One of the most important factors seem to be a wells structured framework in the curriculum and information to the students. The biggest concerns before and during this course was information security and student care.

\section{Abstract \# 120}

Survey of smart device use in hospitalised older adults to inform design of virtual outpatient clinics.

Greg Waddell ${ }^{1}$, Lesley Arends ${ }^{1}$, Brooke Marron ${ }^{1}$, Michael Rodger ${ }^{1}$

${ }^{1}$ NHS Greater Glasgow and Clyde

Introduction: Urgent reorganisation of services in response to COVID-19 has taken advantage of virtual consultations-allowing many face-to-face clinics to be conducted remotely. Implementation relies on our population's ability to access and operate smart technology. Exploring practicalities with hospitalised older adults informs our virtual clinic design and protects against disadvantaging this group.

Methods: Survey of older adults $(\mathrm{n}=30)$ within Department of Medicine for the Elderly in an acute teaching hospital. Convenience sample.

Exclusions: Those treated under Adults with Incapacity, unable to engage due to acuity of illness, or approaching end of life.

Results: Mean age: $78 \pm 11.7$ years. Whilst $87 \%(26 / 30)$ had access to a mobile device - only $40 \%$ had access whilst in hospital. The commonest use was audio calls (at least daily in 54\%). In comparison, $88 \%$ had never used video-calling. Those with the highest self-reported competence in using their devices typically limited their use to audio calls. $35 \%$ felt limited by impaired vision; $23 \%$ by impaired hearing. When prompted, only $62 \%$ could switch on and unlock their device - and only 2 could access the virtual clinic test page (launch browser and manually enter URL).

Conclusions: Hospitalised older adults would be less able to access virtual clinic follow-up. Whilst some technical, sensory and competence issues could be overcome with facilitated access (eg Near Me hubs)—it cannot adequately replace face-to-face appointments for this group at present.

\section{Abstract \# 121}

Behind all the headlines, making sense of clinical practice for older people at the Ashford and St Peters NHS Foundation trust, United Kingdom during COVID-19 crisis

Rashid Mahmood ${ }^{1}$, Agustin Aranda-Marteniz ${ }^{1}$, Amir Manzoor ${ }^{1}$, Radcliffe Lisk ${ }^{1}$

${ }^{1}$ Ashford and St Peters NHS Foundation Trust, Chertsey, United Kingdom 
Introduction: Health services across the globe have been stretched beyond limit due to COVID-19 pandemic. Review of the performance during this period should give us valuable insight how they have performed and this should help plan future care models.

Method: Data of all over 75 years olds patients presenting to our hospital from March-May 2020 was collected and compared with the same time period from 2019.Results:Variables: March-May 2019 March-May $2020 \%$ increase or decreaseED attendance (n) 4876 3568 26.9\% decreaseCOVID19 Positive (n) $0 \quad 317 \quad 317 \%$ increaseConversion to admission $50.9 \% 59.1 \% \quad 16.1 \%$ increaseInpatient deaths (n) $20529744.9 \%$ increase (avg - 68.33/month) (avg—99/month) Crude mortality rate $8.2 \%$ (avg) $13.1 \%$ (avg) $59.6 \%$ increaseNon elective length of stay (days) 7.09 (avg) 7.18 (avg) No real change 30 day readmission $22.46 \%$ (avg) 22.73 (avg) No real changeCOVID-19 peak period-April 2020:Variables: April 2019 April 2020 (COVID-19 peak) \% increase or decreaseED attendance (n) $1587 \quad 101035 \%$ decreaseCOVID-19 Positive (n) $0212212 \%$ increase Conversion to admission $49.3 \% \quad 65.3 \% \quad 32.5 \%$ increase Inpatient deaths (n) $58131 \quad 125.8 \%$ increase Crude mortality rate $7.0 \% 19.1 \% 172.8 \%$ increase Non elective length of stay (days) 6.89 5.950 .94 decrease 30 day readmission $23.9 \% \quad 21.1 \% \quad 11.71 \%$ decreaseAmong the over 75s COVID-19 +ve patients (317), there were 139 deaths (mortality rate $43.8 \%$ ).

Conclusion: Our review has confirmed reduction in ED attendance but patients were more likely to be admitted to hospital with significant rise in the mortality. As expected, older patients especially above the age of 75 years were worse affected during COVID-19 pandemic.

\section{Abstract \# 122 \\ Hip fractures outcomes during COVID-19 pandemic in a district general hospital in United Kingdom \\ Radcliffe Lisk ${ }^{1}$, Carley Allen ${ }^{1}$, Hazel Watters ${ }^{1}$, Keefai Yeong ${ }^{1}$, Rashid Mahmood ${ }^{1}$ \\ ${ }^{1}$ Ashford and St Peters NHS Foundation Trust, Chertsey, United Kingdom}

Introduction: Pandemics, like COVID-19, test the resilience of tested healthcare models. It is important to establish if these models were still capable of delivering high quality care during COVID-19 especially to older people presenting fracture neck of femur (NOF). At Ashford and St Peters NHS Trust, we operate shared care model, where patients are cared for by named orthopaedic surgeon and orthogeriatrician. During pandemic, orthogeriatricians were deployed on COVID-19 wards to support medical division. In newly configured services, NOF patients were moved to non-COVID hospital (Ashford), part of our trust, on day 5-7 postoperatively, and were solely looked after by orthopaedic team without the support of their medical counterpart.

Method: Data of patients with fracture NOF was collected during the COVID-19 period (March-May 2020) and variables were compared with the data available from the same period from 2018 and 2019.

Results: Variables 20182019 COVID-19Numbers $106 \quad 109$ 97COVID-19 Positive $004.1 \%(n=4 / 97)$ Time to Surgery $(<36 \mathrm{~h})$ $84 \% \% 84.3 \% 73.2 \%$ Orthogeriatrician input $(<72 \mathrm{~h}) 100 \% 97.6 \%$ $58.8 \%$ Mobilised out of bed by the day after surgery $88.3 \% 76.7 \%$ $80.2 \%$ Overall hospital length of stay (LOS) (days) 11.0310 .910 .8 Mortality $8.06 \% 2.0 \% 7.20 \%$ Discharge to usual residence $65.6 \%$ $73.3 \% 69.1 \%$

Conclusion: The established care model and protocols developed by Orthogeriatric team were able to provide high quality care in these unprecedented times without the support of orthogeriatricians. Scaled up 7 day therapy, community and social services also helped to maintain same standard of care.

\section{Abstract \# 123 \\ Evaluating perspectives of friends and family of nursing home residents on the nursing home visiting restrictions during COVID-19: a cross-sectional observational study}

Joost David Wammes ${ }^{1}$, Bianca Buurman-van Es ${ }^{1}$, Janet MacNeilVroomen ${ }^{1}$

${ }^{1}$ Department of Geriatrics, Amsterdam University Medical Centres, Amsterdam, The Netherlands

Objectives: The COVID-19 pandemic has caused many nursing homes to prohibit resident visits to prevent viral spread. The perceptions of family members and friends of the nursing home residents is not clear if the potential detrimental effects of isolating residents outweigh the protective effects, and what does the social network view as important during and after the COVID-19 nursing home restrictions. The aim of this study was to capture these perspectives from the social network of nursing home residents.

Methods: In this cross-sectional observational study, family members and friends of nursing home residents were asked to fill in an online survey about COVID-19 nursing home restrictions. A total of 1997 were included in data analyses.

Results: Satisfaction of communication access to nursing home residents was highest when respondents had the opportunity to stay in touch with nursing home residents by nurses informing them via telephone, visits behind glass, and visits outside maintain physical distance. Respondents were concerned that nursing home residents had increased loneliness (76\%), sadness (66\%), and decreased QOL $(62 \%)$ while study respondents reported personal sadness $(73 \%)$ and fear $(26 \%)$. Overall, $40 \%$ of the respondents found the adverse effects of the visiting restrictions outweighed the protective effect for nursing home residents. Respondents expressed the need for increased information, communication options, and better safety protocols.

Conclusion: Both nursing home residents and relatives experienced adverse psychological effects because of COVID-19 visiting restrictions. The psychological symptoms and unmet needs of nursing home residents and informal caregivers should be addressed in health policy.

\section{Abstract \# 124}

Covid-19: a communication crisis?

Jessica Webster ${ }^{1}$, Robert Smith-Baker ${ }^{1}$, Helen Bounds ${ }^{1}$, Caio Redknapp ${ }^{1}$, Sadia Saber ${ }^{1}$, Cianan O'Sullivan ${ }^{1}$

${ }^{1}$ Homerton University Hospital

Introduction: In geriatrics, where conditions such as delirium and dementia are common, collateral information can be vital. Covid-19 restrictions left families unable to visit, meaning telephone and video calling was essential, especially in those vulnerable to disorientation. We investigated whether contact between medical teams and families (updates) were established in Homerton University Hospital during the crisis, and whether this resulted in clinical or positive non-clinical outcomes.

Methods: Electronic patient records (EPR) were searched for all patients $>65$ treated for covid-19 during the pandemic peak $(16 / 03 /$ 2020-30/04/2020). EPR was searched for delirium at admission and/ or during stay, dementia diagnosis, number of updates, and any documented outcomes. 
Results: Mean updates per day was greater in the dementia and delirium on admission populations, 0.61 and 0.58 respectively, than for the general population ( 0.53$)$ but lower for those that developed delirium in hospital $(0.41) .70 \%$ of patients received at least one update, of which $44 \%$ had a documented clinical outcome and $28 \%$ had a positive outcome.

Key conclusions: Communication with families remained a priority during the crisis, and occurred more when patients were less able to do so themselves. Telephone conversations still yielded many clinical and positive outcomes. The familiarity and reorientation that families can provide to patients via video call may benefit both dementia and delirium patients, and a pilot of video calls with a dedicated communications team is ongoing.

\section{Abstract \# 125 \\ Geriatric assessment in elderly patients with COVID-19 during admission to a Geriatric Unit in Teruel, Spain.}

Magdalena Linge Martín ${ }^{1}$, Carmen Oquendo Marmaneu ${ }^{1}$, Miguel Sánchez Ortiz ${ }^{1}$, Esther Álvarez Gómez ${ }^{1}$, Milagritos Margot Vasquez $\mathrm{Camacho}^{1}$, Ana Mateo $\mathrm{Abad}^{1}$, Dionis Carolina Fernández Minaya ${ }^{1}$

${ }^{1}$ Hospital San José, Teruel, Spain

Introduction: Knowing the profile of the patients is useful to provide an individualized attention that adapts to their needs, especially in geriatric patients with COVID-19 where published studies have shown worse clinical results and greater mortality. In this paper, we describe the characteristics of our patients with the aim to understand the repercussions of this infection in elderly patients.

Methods: We conducted a retrospective, observational study. 43 patients with COVID-19 were admitted to our Geriatric Unit at Hospital San Josë between February and May 2020. We collected multiple types of data, including sociodemographic, functional, cognitive variables, comorbidity, risk factors, length of stay and destination upon discharge.Results: 43 patients were included. Average age: 85.3 years old. Gender: men $53.5 \%$, women $46.5 \%$. Place of residence: $25.6 \%$ lived at home and $74.4 \%$ in an elderly residence. Marital status: single $18.6 \%$, married $27.9 \%$, widower 48.8\% . Barthel index: independent $9.3 \%$, slight dependency $27.9 \%$, moderate dependency $16.3 \%$, severe dependency $39.6 \%$, total dependency $6.9 \%$. Comorbidities: High blood pressure $76.7 \%$, nutritional deficit $62.8 \%$, dementia $47.7 \%$. Hospital admission in the last 3 months for pneumonia or flu $25.6 \%$. Average length of stay: 19.67 days. Destination upon discharge: elderly residence $49 \%$, home $12 \% .37 \%$ of the patients died during hospitalization.

Conclusions: In this study, the average patient admitted with COVID-19 was an 85-year-old man, institutionalized, with severe dependence for basic activities of daily living, with high blood pressure and nutritional deficit, with a prolonged length of hospitalization and destination upon discharge to an elderly residence.

\section{Abstract \# 126 \\ First medical students' experience from their participation in a Family Communication Service for hospitalized older patients and their families during the COVID-19 pandemia}

Anaïck Besozzi ${ }^{1}$, Marina Kotsani ${ }^{2}$, Nicolas Gambier ${ }^{3}$, Anna KearneySchwartz $^{2}$, Marie-Jeanne Fourriere ${ }^{2}$, Louise Tyvaert ${ }^{4}$, Marc Braun ${ }^{5}$, Laure Joly ${ }^{6}$

${ }^{1}$ Centre Mémoire de Ressources et de Recherche, CHRU Nancy, France, ${ }^{2}$ Université de Lorraine, CHRU-Nancy, Pôle « Maladies du
Vieillissement, Gérontologie et Soins Palliatifs », F-54000, Nancy, France, ${ }^{3}$ Université de Lorraine, CHRU-Nancy, Department

Introduction: COVID-19 pandemia imposed restrictions in the visit routines of hospitalized older patients, which were though to negatively impact the psychological health of patients and their families. A Family Communication Service (FCS), facilitated by volunteer medical students, was implemented in the Geriatric Department of University Hospital in Nancy, France, to attenuate those consequences. We aimed to evaluate student's experience from their participation.

Methods: Seventeen undergraduate medical students received dedicated training on their mission's outline and on Hygiene rules by both Faculty and Hospital and were equipped with the necessary infrastructure to organize calls and regulated personal objects exchanges between older patients and their families for 1 month (20/4-20/5/ 2020). Finally, an anonymized questionnaire was answered $(\mathrm{N}=15)$. Results: In total, 258 actions took place: 119 students-family phonecalls (actions' organization), 94 patient-family video-calls, 24 patientfamily phone-calls and 22 objects' exchanges. Students reported an 8.8/10 satisfaction degree on the global functioning of the FCS and a 9/10 level of recommendation of the FCS to their peers. Predominant were positive reports on the amelioration of their communication skills with older vulnerable patients (with complex conditions), their families, the geriatric team and their peer students. Working in autonomy and experiencing the human aspect of the doctor-patient relationship, beyond medical conditions, discovering the patient's point of view and their family context were estimated as valuable experiences for their vision of the medical profession.

Key conclusions: Active involvement of undergraduate medical students in activities beyond strict medical duties can be an enriching experience for themselves and their curricula.

\section{Abstract \# 127 \\ Perceived benefits of a novel Family Communication Service for hospitalized older patients and their families during the COVID-19 pandemia.}

Anaïck Besozzi ${ }^{1}$, Marina Kotsani ${ }^{2}$, Nicolas Gambier ${ }^{3}$, Anna KearneySchwartz $^{2}$, Marie-Jeanne Fourrier ${ }^{2}$, Louise Tyvaert ${ }^{4}$, Marc Braun ${ }^{5}$, Laure Joly ${ }^{6}$

${ }^{1}$ Centre Mémoire de Ressources et de Recherche, CHRU Nancy, France, ${ }^{2}$ Université de Lorraine, CHRU-Nancy, Pôle « Maladies du Vieillissement, Gérontologie et Soins Palliatifs », F-54000, Nancy, France, ${ }^{3}$ Université de Lorraine, CHRU-Nancy, Department of Clinical Pharmacology and Toxicology, F-54000, and Université de Lorraine, Faculté de Medecine, F-54000 Nancy, France, ${ }^{4}$ Université de Lorraine, Faculté de Medecine, F-54000, CRAN UMR 7039,

Nancy, France, ${ }^{5}$ Faculté de Médecine, Université de Lorraine, Nancy, France, ${ }^{6}$ Faculté de Médecine, Université de Lorraine and Université de Lorraine and CHRU-Nancy, Pôle «Maladies du Vieillissement, Gérontologie et Soins Palliatifs », F-54000, Nancy, France

Introduction: Hospital visits' limitations imposed by the COVID-19 crisis were considered having negative consequences in hospitalized patient's well-being. We present the perceived benefits of a novel Family Communication Service (FCS) implemented in the Geriatric Department of University Hospital of Nancy, France.

Methods: Volunteer undergraduate medical students, who were trained, suitably equipped and attributed the mission to organize communication actions between older hospitalized patients and their families (20/4-20/5/2020), answered an anonymized questionnaire. Results: In total, 258 actions (205 in non-COVID; 53 in COVID sector) took place for 84 patients (74 non-COVID; 15 COVID) by 17 students: 119 students-family phone-calls (88 non-COVID; 31 
COVID), 94 patient-family video-calls (77 non-COVID; 17 COVID), 24 patient-family phone-calls (22 non-COVID; 2 COVID), 21 regulated personal objects exchanges (18 non-COVID; 3 COVID). Fifteen students answered the questionnaire $(88.2 \%)$. There were strongly perceived important psychological benefits for the patients (psychological comfort, reassurance, joy, elimination of the feeling of abandonment, mood boosting, beneficial contact with young students) and their families (reassurance, serenity, joy, de-stress), who managed to maintain the affective connection and warmly expressed their gratitude. Geriatric teams welcomed being offloaded and reported facilitation to their care duties also by patients' mood amelioration. Visual contact by video-calls was considered an added value and it was suggested to be continued out of the crisis context, along with the recreational occupation for older patients.

Key conclusions: The FCS resulted to be beneficial for older hospitalized patients, their families and the medical/paramedical stuff. Scaling up of some of its aspects merit consideration.

\section{Abstract \# 128 \\ Safety patient circuit in a cardiology intensive care unit during the Covid-19 pandemic period}

Joana Pereira Sousa ${ }^{1}$, Paula Catarino ${ }^{1}$, Maritza Ribeiro ${ }^{1}$, Licínia Carvalheiro $^{1}$, Sílvia Paiva ${ }^{2}$, Maurício Alves ${ }^{2}$, Ana Lúcia Rosa ${ }^{2}$, Óscar Ângela $^{2}$, Filomena Mendes ${ }^{2}$, Jorge Canais ${ }^{3}$

${ }^{1}$ Heart Failure Intensive Care Unit - Centro Hospitalar e Universitário de Coimbra, Portugal, ${ }^{2}$ Coronary Intensive Care Unit - Centro Hospitalar e Universitário de Coimbra, Portugal, Heart Failure Intensive Care Unit/Coronary Intensive Care Unit - Centro Hospitalar e Universitário de Coimbra, Portugal

Introduction: In a time of SARS-CoV-2 pandemic worldwide spread, patients who suffer from acute heart disease, must have a safe environment when admitted into a cardiology intensive care unit. Covid19 pandemic brought mandatory changes into the healthcare institutions, where patients' safety circuits were organized.

Methods: This is a descriptive-observational study of patients with heart conditions (heart failure and acute myocardial infarction), admitted in a cardiac ICU in a Portuguese University Hospital. All the patients were admitted to the emergency department (ED) due to a cardiac event. As SARS-CoV-2 tests were not always available at the $\mathrm{ED}$, patients were transferred to a coronary ICU with six single bedrooms where patients were monitored and stabilized. In contrast, the SARS-CoV-2 test was meanwhile performed. If tests were negative, patients were transferred to a five-bed ICU or a cardiology ward. If tests were positive, patients were transferred to a cardiology ward or ICU in a dedicated Covid-19 building in another area.

Results: Between April and June of 2020, among a total of 406 patients admitted in the ICU, only one asymptomatic patient tested positive to SARS-CoV-2, who was then transferred to Covid-19 coronary facility. Seventy-six (19\%) patients were transferred to the five-bed ICU, 326 (80\%) were transferred to the cardiology ward, and four patients died due to cardiac problems. During this period, none of the healthcare providers got infected by SARS-CoV-2.

Key conclusion: The circuit organization implemented in our University Hospital allowed a safe care environment by healthcare professionals to our patients.

\section{Abstract \# 129}

Rapid redesign of acute services for frail older people during the coronavirus pandemic

Janice Bernardo ${ }^{1}$

${ }^{1}$ The Princess Alexandra Hospital NHS Trust

Introduction: The coronavirus (Covid-19) pandemic presents particular risks for frail older people presenting for acute assessment in Emergency Departments or admitted to hospital. We responded by rapid service redesign to enhance capacity to assess and treat individuals whilst avoiding or minimising hospital exposure. This needed hospital, community and social care to share information, decisions and ongoing care and supervision without the patient moving about. Methods: Plan- Do-Study-Act method was used to rapidly test, refine and implement service redesign. Changes included procedures for accessing services, reducing duplication of assessments, changing the scope and routines for health professionals, and incorporating volunteer support.

Results: Use of the new Clinical Frailty Scale App plus digital application were upskilled staff enabling each provider to preform and share expert assessments of frail people, improving quality and saving time. Primary care data interoperability and access to health and care system capacity report were authorised. A multiagency rapid access virtual clinic enabled quicker responses, sharing of these assessments and agreeing care plans without patients attending hospital. A Frailty Clinical Navigator was introduced in a COVID dedicated and non-COVID Emergency Department (ED). Discharge medications and equipment delivery were supported by voluntary sector.

Key conclusion: Integrated working between health, social and voluntary services is imperative to deliver the right care at the right time. Frailty specific innovative roles need to be further explored creating more workforce flexibility and sustainability. Data interoperability must be prioritised.

\section{Abstract \# 130 \\ Reconfiguration of Community Health Services Medicines Training due to COVID-19}

John Bartoli-Abdou ${ }^{1}$, Temitope Odetunde ${ }^{2}$, Peter Brown ${ }^{2}$, Lelly $\mathrm{Oboh}^{3}$, Nicola Torrens ${ }^{3}$, Jennifer M. Stevenson ${ }^{4}$

${ }^{1}$ Guy's \& St Thomas' NHS Foundation Trust, and Institute of Pharmaceutical Science, King's College London, ${ }^{2} 1$ Guy's \& St Thomas' NHS Foundation Trust, ${ }^{3}$ Guy's \& St Thomas' NHS Foundation Trust, ${ }^{4}$ Guy's \& St Thomas' NHS Foundation Trust, Institute

Introduction: Guy's and St. Thomas' NHS Foundation Trust Integrated Local Services (ILS) delivers home care to vulnerable patients; a large part of which involves support with medicines. At the onset of the COVID-19 Pandemic ILS rapidly reconfigured to relieve pressure on the acute hospitals. For ILS staff to safely support medicines use, training is usually delivered in small groups and competence assessed in patients' homes. To minimise patient and staff risk of potential exposure to COVID-19 new training and assessment were developed to up skill redeployed staff (nursing assistants and rehabilitation support workers), the outcomes of which are described.

Methods: Two face to face training sessions were consolidated into one, and three simulated Objective Structured Clinical Examinations (OSCE) assessments of medicines administration were developed to replace two supervised medication administration competency assessments. Labelled medicines were obtained from King's College 
London's (KCL), and their facilities utilised to allow physical distancing.

Results: Over two days, training was delivered to 33 staff, and OSCEs completed by 18 candidates; no candidate passed. $78 \%$ made a clinical decision outside of their scope of practice, $56 \%$ administered an incorrect dose, and $66 \%$ administered an expired medication. The majority of candidates followed the correct procedure when medication was refused $(78 \%)$.

Key conclusions: OSCEs were a useful educational tool where candidates can be objectively observed without intervention, and direct feedback on errors provided. Additional training on the handling and administration of medicines was required for all candidates. Training and OSCEs will be redesigned to meet the needs identified. Collaboration with academic partners is beneficial.

\section{Abstract \# 131 \\ COVID-19 impact on functional outcomes in the elderly}

Ana Filipa Teixeira Ferreira ${ }^{1}$, Sílvia Pacheco de Sousa ${ }^{1}$, Maria Margarida Luís ${ }^{1}$, Vítor Neves Lopes ${ }^{2}$, Mari Mesquita ${ }^{1}$

${ }^{1}$ Centro Hospitalar Tâmega e Sousa, ${ }^{2}$ Centro Hospitalar e Universitário de São João

Introduction: The management of COVID-19 and non-COVID-19 patients during the pandemic in hospitalized elderly patients is an evolving challenge. Hospitalization often marks the beginning of a downward trajectory characterized by declining function, institutionalization and death. Functional status may be impacted by COVID-19.

Methods: The sample includes all the geriatric patients admitted to the general wards of the Internal Medicine Department of Hospital Padre Américo, in Penafiel, Portugal, in April 2020. The sample is sequential and non-randomized. The functional status was evaluated using the Katz Index of Independence in Activities of Daily Living on admission and on discharge. All patients admitted were tested for COVID-19 using RT-PCR detection of SARS-CoV-2. The data was analyzed using SPSS analysed by SPSS version 26 for MacOS (SPSS Inc., Chicago, IL, USA). Means of 2 continuous normally distributed variables were com $\neg$ pared by independent samples Student's t test. A value of $\mathrm{P} \leq 0.05$ was considered significant.

Results and key conclusions: 232 patients (54.5\% female), with 80.2 $( \pm 7.87)$ years old were admitted. 70 patients $(30.2 \%)$ tested positive for SARS-CoV-2. 66 patients $(28.4 \%)$ died, with 20 (8.62\%) infected with COVID-19. The groups of patients with and without COVID-19 were comparable in terms of age, Charlson Index Score and main diagnosis $(\mathrm{P}>0.05)$. The mean Katz difference between the moments of discharge and admission was not significant $(\mathrm{p}=0.447)$. COVID19 does not seem to affect functionality in geriatric patients with mild forms of infection. Quality of care is assumed to have been similar in both groups.

\section{Abstract \# 132 \\ Investigation of factors affecting death and survival in geriatric patients infected With COVID-19: a pandemic hospital experience}

Rabia Bag Soytas ${ }^{1}$, Damla Unal ${ }^{1}$, Pinar Arman ${ }^{1}$, Veysel Suzan ${ }^{1}$, Tugce Emiroglu Gedik ${ }^{2}$, Gunay $\mathrm{Can}^{3}$, Bora Korkmazer ${ }^{4}$, Ridvan Karaali $^{5}$, Sermin Borekci ${ }^{6}$, Mert Ahmet Kuskucu ${ }^{7}$, Hakan Yavuzer ${ }^{1}$, Deniz Suna Erdincler ${ }^{1}$, Alper Doventas ${ }^{1}$

${ }^{1}$ Istanbul University-Cerrahpasa, Cerrahpasa Faculty of Medicine, Department of Internal Medicine, Division of Geriatrics, Istanbul,
Turkey, ${ }^{2}$ Istanbul University-Cerrahpasa, Cerrahpasa Faculty of Medicine, Department of Internal Medicine, Division of Geriatrics, Istanbul, Turkey), ${ }^{3}$ Istanbul University-Cerrahpasa, Cerrahpasa Faculty of Medicine, Department of Public Health, Istanbul, Turkey), ${ }^{4}$ Istanbul University-Cerrahpasa, Cerrahpasa Faculty of Medicine, Department of Radiology, Istanbul, Turkey, ${ }^{5}$ Istanbul UniversityCerrahpasa, Cerrahpasa Faculty of Medicine, Department of Infectious Diseases, Istanbul, Turkey), ${ }^{6}$ Istanbul UniversityCerrahpasa, Cerrahpasa Faculty of Medicine, Department of Pulmonary Diseases, Istanbul, Turkey, Istanbul UniversityCerrahpasa, Cerrahpasa Faculty of Medicine, Department of Medical Microbiology, Istanbul, Turkey

Objective: We aimed to investigate the factors affecting the mortality of patients aged $\geq 65$ who were hospitalized with COVID- 19 .

Method: From 11 March to 28 May 2020, patients who were hospitalized with COVID-19 are included. Demographic characteristics, comorbidities, number of drugs, laboratory datas and oxygen saturations were recorded at the time of application, 5th day of treatment and at discharge. CURB65 (Confusion, Urea, Respiratory rate, Blood pressure, Age $\geq 65$ ) scores were calculated. The hospitalized days numbers, ECG changes, presence of delirium and pressure ulcer, oxygen treatment types and drugs used for COVID-19 recorded.

Results: 218 patients (112 men, 106 women) were included in the study. The patients were divided into 3 groups as 65-74 (n:107), $75-84$ (n:86) and $\geq 85$ (n:25) years. 52 of patient (\%24) were died (34 men, 18 women). While the mortality rate of men was higher than women (p:0.021), no significant relationship was found between age groups and death. Dispnea (p:0.002), polipharmacy (p:0.022), malignancy $(p<0.001)$ and dementia (p:0.024) were associated with death. There was a relationship between hospitalized days numbers at intensive care $(\mathrm{p}<0.001)$, and clinics $(\mathrm{p}<0.001)$, intubated days (p:0.011), application oxygen saturation $(\mathrm{p}<0.001)$ and mortality. Neutrophil/lymphocyte ratio ( $\mathrm{p}<0.001$ ), lymphocyte (p:0.011) on admission and the highest values of procalcitonin (p:0.026), creatinin ( $\mathrm{p}: 0.006)$ and LDH $(\mathrm{p}<0.001)$ were associated with death. Highflow oxygen therapy was found to have a protective effect on mortality (p:0.012).

Conclusion: Covid-19 is more mortal in older adults. It is important to be aware of the factors affecting mortality in order to prepare for a possible second wave.

\section{Abstract \# 133}

Use of telephone consultations to avoid Hospital Admission-the COVID Frailty Hotline

\section{Mary Buckley ${ }^{1}$, Riaz Moola ${ }^{1}$, David Robinson ${ }^{1}$, Rory Nee ${ }^{1}$}

${ }^{1}$ St. James's Hospital, Dublin, Ireland

Introduction: St James Hospital is the largest hospital in Ireland with 1,000 inpatient beds. In 2017 there were 48,000 ED attendances and 23,550 inpatient discharges. COVID-19 was predicted to overwhelm our services with numerous contingency plans put in place across the hospital.

Methods: With the aim of reducing crisis ED attendance, hospital admissions and facilitating ED avoidant medical admission of frail older adults in need of hospital care, a dedicated phone line to a consultant geriatrician was circulated to all GPs in the catchment area. The phoneline operated during working hour. Telephone advice was provided with urgent Day Hospital reviews as needed.

Results: 19 GPs requested 31 consults during the months of April and May. The average age was 84 years. The average Clinical Frailty Scale was 6, indicating moderate frailty. Outcomes include ED avoidant medical admission (6), next day review via Day Hospital (7), domiciliary visit (3), or telephone advice ${ }^{15}$. The admission avoidance 
rate was $80 \%$ and ED avoidance rate was $100 \%$. 8 patients were nursing home residents and 5 were known COVID positive. Consultations included dementia with BPSD (14), delirium (3), severe anxiety (2), one resulting in a fixed delusion that he had COVID. Other consultations included - back pain, movement disorders, falls, labile blood pressure and polypharmacy.

Conclusion: The COVID Frailty Hotline is effective at reducing crisis ED attendance and hospital admissions. $80 \%$ of hospital admissions were avoided in these cases with ED avoidant direct admissions facilitated for the $20 \%$ in need of hospital care.

\section{Abstract \# 134 \\ Departmental review of clinical characteristics of Covid19 presentation in an older hospitalized cohort.}

Alanna Keena ${ }^{1}$, Shelina Seebah ${ }^{1}$, StJohn Kelliher ${ }^{2}$, Ruth Daunt ${ }^{1}$, Alice O’Donoghue ${ }^{1}$, Colm Byrne ${ }^{1}$, Linda Brewer ${ }^{1}$

${ }^{1}$ Beaumont Hospital, ${ }^{2}$ UCC

Introduction: The Covid19 pandemic reached Ireland on 29th February 2020 and within weeks spread throughout hospitals. We examined clinical characteristics of select frail, older adults with Covid19 on three geriatrician-governed wards mid March to mid April 2020.

Methods: Patients with Covid19 were randomly selected. Their charts, computerised blood tests and chest x-rays (CXRs) were reviewed. Anonymized data were imported into excel for analysis.

Results: 20 patients were included, all aged $>70$ y (median $82.5 \mathrm{y}$ ). $75 \%$ were male, $95 \%$ had Clinical Frailty Score (CFS) $\geq 4$ at baseline. None were diagnosed with Covid19 on admission. $60 \%$ were clinically asymptomatic at diagnosis. $70 \%$ had oxygen saturations $>$ $94 \%$, a further $15 \%$ were noted hypoxic and $5 \%$ were already on oxygen. $75 \%$ had temperature $\leq 38{ }^{\circ} \mathrm{C} .80 \%$ and $60 \%$ had abnormal $\mathrm{CRP}$ and lymphocyte counts respectively. Highest CRP and lymphocyte counts were recorded at a median of 5.2 and 3.2 days post diagnosis, respectively. CXR findings and oxygen saturation drops were most severe at a median of 1.1 and 2.6 days, respectively. $100 \%$ appropriately had 'do not attempt resuscitation' status clearly documented in notes. Palliative medication was commenced at a median of 6.3 days. $40 \%(\mathrm{n}=8)$ died, $25 \%$ were discharged home, $10 \%$ to rehabilitation and $10 \%$ to a nursing home.

Conclusion: Over half of patients were asymptomatic and were diagnosed with Covid19 by ward swab 'sweep', proving a useful method in identifying and controlling covert hospital outbreaks amongst older people. Over one-third died from Covid19 related illness, with high frailty burden impacting on the high mortality rate.

\section{Abstract \# 135 \\ Seroprevalence of anti-SARS-CoV-2 antibodies in the Health Workers in Hospitals and in Nursing Homes in East France.}

Sophie Siegrist ${ }^{1}$, Hajare Sarah Mjahed ${ }^{2}$, Carlos Labat ${ }^{3}$, Athanase Benetos $^{4}$

${ }^{1}$ University Hospital of Nancy, Université de Lorraine, Agence Régionale de Santé Grand Est, France, ${ }^{2}$ University Hospital of Nancy, Université de Lorraine, France, ${ }^{3}$ Inserm U1116 DCAC, Université de Lorraine, France, ${ }^{4}$ University Hospital of Nancy, Université de Lorraine, Inserm U1116 DCAC, Université de Lorraine, France

Introduction: Health workers (HWs) are at the front line of the Covid-19 outbreak response. Despite safe work practices, it's believed that they are at higher exposure risk to Covid-19 than the general population. In France, testing of HWs has so far been restricted to symptomatic individuals leading to underestimation of the real impact of the disease to the HWs. Here, we estimate the seroprevalence of anti-SARS-CoV-2 antibodies in the of HCWs working in hospitals and in nursing homes (NHs) of the Metz county in Lorraine region, one of the most affected regions in France.

Methods: During the epidemic, HWs, volunteers, symptomatic and asymptomatic, were screened for anti-SARS-Cov-2 antibodies using a rapid immunodiagnostic test. The results of this screening test as well as the information from self-administered, standardized questionnaires covering different topics were collected to perform a retrospective study on seroprevalence of anti-SARS-CoV-2 antibodies within this Covid-19 exposed population.

Results: 105 participants were enrolled in this study. The mean time from symptom onset to blood sample testing was $34 \pm 16$ days. Rapid immunodiagnostic test detected antibodies in 13/105 (12.4\%) of the HCWs and among them only $59 \%$ were symptomatic. Our results showed that working in NHs displays a significantly higher seropositivity rate.SEE TABLE: https://aristeaint-my.sharepoint. com/:w:/g/personal/testa_aristea_com/ER11L_MG48ZIm4GPjy2zY TYBKmcwQD2ub5qnarpFhVTE9w?e $=$ s4Cp8c.

Conclusion: Our results highlight a higher rate of Covid-19 exposure within HWs NHs. Large SARS-Cov-2 seroepidemiological data are needed to understand transmission dynamics. This may allow to explain the difference in seropositivity rate seen between health establishments and therefore to adapt guidance to better control measures in the different workplaces.

\section{Abstract \# 136 \\ Reflections of a care home provider on the COVID-19 pandemic: A catalyst for integrated care}

Tanya Coles ${ }^{1}$, Samantha Crawley ${ }^{1}$, Fionna Martin ${ }^{2}$, Peter Diem ${ }^{2}$, Jay Patel $^{3}$, Jackie Masterton ${ }^{4}$, Jan Noble ${ }^{5}$, Dan Wilson ${ }^{6}$, Clarissa Perks ${ }^{7}$, Tania Kalsi ${ }^{2}$

${ }^{1}$ Excelcare, ${ }^{2}$ Guys \& St Thomas' NHS Foundation Trust, ${ }^{3}$ Hetherington Group Practice, ${ }^{4}$ Prentis Medical Centre, ${ }^{5} \mathrm{St}$ Christopher's Hospice, ${ }^{6}$ Kings College Hospital NHS Foundation Trust, ${ }^{7}$ Quay Health Solutions GP Care Home Service

Introduction: The COVID-19 pandemic has disproportionately affected frail older people living in care homes. In the United Kingdom, care homes are largely run by private providers with healthcare accessed through general practitioners and community/ hospital services. As a care home provider, there is a need not only to reflect on the challenges, but also the gains during the pandemic.

Methods: We reflect our perspective on how the change in working relationships between our care homes in South London and local healthcare services produced different processes of care.

Results: There was increased collaboration between professionals from different organisations, greater good-will, understanding and respect. There was innovation in healthcare access for residents, moving from sometimes slow or restrictive access to more rapid and flexible assessments. Local healthcare professionals enhanced their support to care homes. In turn, care homes supported the health system in delivering high quality healthcare, supporting transitions of care and increasing provision of end of life care. Care home multidisciplinary meetings were rapidly established with membership from care homes, consultant geriatricians, primary care, pharmacists and palliative care. Through this collaborative approach, we saw improved access to geriatric medicine and palliative care expertise, improvements in shared electronic records, personalised advance care 
planning, greater numbers receiving end of life in their preferred place of death and reduced hospital admissions.

Conclusions: Whilst the COVID-19 pandemic has had a devastating impact on care homes and their residents, it has offered opportunities to build better integrated and collaborative working relationships between care homes and healthcare.

\section{Abstract \# 137 \\ Psychological effects Of COVID-19 epidemic among hemodialysis patients}

Irem Pembegül ${ }^{1}$, Özgül Balseven ${ }^{1}$

${ }^{1}$ malatya Education and Research Hospital

Abstract: The corona virus epidemic (COVID-19), which affects the whole world, is an internationally concerned public health emergency and poses a challenge to psychological resilience. With the COVID19 outbreak, an atmosphere of global anxiety and depression arose. Individuals at the stage of end-stage renal failure (ESRD); faced with the obligation to depend on a machine and/or process in order to survive. In addition, it is possible that the pandemic process will cause more severe damage in patients undergoing hemodialysis (HD) due to ESRD. The aim of this study was to analyze the symptoms of depression, anxiety, and stress in hemodyalysis patients during the COVID-19 pandemic.

Materials and methods: 149 in hemodyalysis patients aged 26-92 years $(M=60.81 \pm 15.24), 54.4 \%$ of them were men. They filled out the Depression, Anxiety and Stress Scales (DASS-21). In the adaptation process of the Fear of COVID-19 Scale were performed.

Results: This study included 149 respondents from Malatya. In total, $72.5 \%$ of respondents rated the psychological impact of the outbreak as moderate or severe; $14.8 \%$ reported moderate to severe depressive symptoms; $57.7 \%$ reported moderate to severe depression symptoms; $57.7 \%$ reported moderate to severe anxiety levels and $27.5 \%$ reported moderate to severe stress levels. the Fear of COVID-19 Scale reported $19.66 \pm 9.49$ and covid fear scores positively correlated with age, depression and anxiety scores $(\mathrm{p}<0.001, \mathrm{p}<0.001$, and $\mathrm{p}<0.001$, respectively).

Discussion: In addition to the frequently encountered psychological problems in hemodialysis patients, increased psychosocial effects should be taken into consideration during the COVID 19 pandemic period.

\section{Abstract \# 138 \\ Rapid establishment of geriatrician-led end of life ward for Covid-19}

Rosemary Arnott ${ }^{1}$, Anna Lewis ${ }^{1}$, Daniela Berbecila ${ }^{1}$, James Deery ${ }^{1}$, Lisa Wells ${ }^{1}$, Mun Hoe Poon ${ }^{1}$

${ }^{1}$ Nottingham University Hospitals NHS Trust, UK

Introduction: Palliation and end of life care were needed for many frail patients with Covid-19.

Methods: A dedicated ward was opened at short notice, led by geriatricians with palliative care input. Retrospective data was collected for consecutive patients admitted to the ward between 3rd April and 26th May 2020 and qualitative data regarding staff experience using a questionnaire.

Results: Of the 168 patients (55\% male) admitted, $48.8 \%$ came directly from Emergency Department and admission areas and the remainder from inpatient wards. Time spent on the ward ranged from $10 \mathrm{~min}$ to 17 days (median length of stay $43 \mathrm{~h}$ ). $75 \%$ had Clinical Frailty Scale score of 6 or more. 150 died, with $75 \%$ naming Covid19 as primary cause.Ward staff from non-palliative care background reported increased confidence in many aspects of palliation including assessing comfort, relieving symptoms and in supporting relatives. Staff apprehension about working on a Covid-19 ward eased once the ward was established.Qualitative themes emerging from staff feedback included: professional competency, team work, emotional response, care and consistency in a time of change.

Conclusion: Careful gatekeeping ensured that our ward cared for appropriate older patients living with frailty. The formation of a specialist unit over a short period of time created a series of logistical and management challenges. The emotional burden felt by staff was prominent in feedback. These challenges were outweighed by the personal and professional development of staff coupled with the strong sense of teamwork, pride and enthusiasm felt in providing high quality care.

\section{Abstract \# 139}

Administration of medication for Parkinson's disease in older adults admitted with COVID-19 to barnet hospital: a quality improvement project

Ladan Akbarian-Tefaghi ${ }^{1}$, Darshan Nagaria ${ }^{1}$, Anuriti Aojula ${ }^{1}$, Mark Rawle $^{1}$

${ }^{1}$ Royal Free London NHS Foundation Trust, UK

Introduction: It is crucial for patients with Parkinson's disease (PD) to receive their medications in a timely manner as delays can cause worsening symptoms. 1 Parkinson's UK launched the 'Get it on Time' campaign to raise awareness and have advised that patients continue taking their medications as prescribed during the COVID-19 pandemic. 2 During the pandemic we conducted the second round of a Quality Improvement Project (QIP) in Barnet Hospital (United Kingdom) that had initially identified significant delays in PD medication administration among acute admissions, adapted to identify its impact on COVID-19 patients.

Methods: Audit of patients with PD and a community prescription of medications to treat PD, admitted via Barnet Hospital emergency department over 3 months. We collected data on times to PD medication prescription and administration (including rationale for delays) and COVID-19 PCR status. Any deviation $\geq 1 \mathrm{~h}$ from community prescription time was categorised as a delay.

Results: Data were collected for 28 patients, 12 of whom (43\%) had PCR positive COVID-19. Patients with COVID-19 had increased delays in administration of PD medication compared to controls (median $3.75 \mathrm{~h}$ versus $1.2 \mathrm{~h}, \mathrm{p}<0.05$ ). This was primarily due to delay in obtaining correctly dosed rotigotine patches in critical illness. Overall delay frequency was similar to data collected prior to cycle one of our QIP $(59 \%$ versus $69 \%, \mathrm{p}=0.53)$.

Conclusions: PD patients with COVID-19 experienced longer medication delays. Our data demonstrate the need to improve local availability of rotigotine patches, and implement further education on this aspect of PD care among staff. References:1. Grissinger M. Delayed Administration and Contraindicated Drugs Place Hospitalised Parkinson's Disease Patients at Risk. P T. 2018; 43(1):10-39.2. Parkinson's UK (26/6/20). Understanding coronavirus and Parkinson's. https://www.parkinsons.org.uk/news/understandingcoronavirus-and-parkinsons. 


\section{Abstract \# 140 \\ Reduced use of empiric drug treatments for severe COVID-19 in older patients}

Maria Muñoz Garcia ${ }^{1}$, Elisabet Sánchez García ${ }^{2}$, Eva Delgado Silveira ${ }^{1}$, Hilario Martínez Barros ${ }^{1}$, Jesús Mateos del Nozal ${ }^{2}$, Ana María Álvarez Díaz ${ }^{1}$, Alfonso Cruz Jentoft ${ }^{2}$

${ }^{1}$ Pharmacy Department. Hospital Ramón y Cajal, ${ }^{2}$ Geriatric Service. Hospital Ramón y Cajal

Aim: To compare empsiric drug treatments used for severe SARs.Cov2 infection (COVID-19) in patients below and over 70 years of age.

Methods: We performed an observational retrospective study of. all patients admited to a large hospital in Madrid with confirmed severe COVID-19 between 2nd March and 25th May. All patients with confirmed COVID-19 were identified from medical records. We assessed age, gender, in-hospital mortality and empiric drugtreatments used according to evolving hospital protocols. All drugs were used off-label, as there was no approved treatment for this condition. Results: 2497 hospitalized patients with confirmed COVID-19 were identified (mean age 67.2 years, range $0-104,60.3 \%$ males), being $1238(49.6 \%) \geq 70$ years old and $694(27.8 \%) \geq 80$ years. Mean age of the whole sample was 67.2 years ( $0-104$ years), $60.3 \%$ were men. Differences between those over and below 70 years of age were observed in the use of drugs: hidroxocloroquine $(84.5 \% \geq 70$ years vs $88.7 \%$ in younger patients, $\mathrm{p}<0.001)$; lopinavir/ritonavir $(60 \%$ vs $75.2 \%, \mathrm{p}=0.0001)$; azitromicine $(44.7 \%$ vs $55.2 \% \mathrm{p}=0.000)$; and remdesivir $(12.3 \%$ vs $25.3 \%, \mathrm{p}=0.000)$. We didn't find differences in the use of tocilizumab (1.6\% vs $2.3 \%)$ or pulses of corticoids $(9.8 \%$ vs $7.8 \%$ ). Overall mortality rate was $20.1 \%$, being higher in older $(32.9 \%)$ than younger patients $(7.5 \%, \mathrm{p}=0.0001)$, with no differences according to gender.

Conclusions: Almost half of the patients admitted to our hospital for severe COVID-19 were 70 years or older. Differences were observed in the rates of use of empiric drug treatments for COVID-19, with a reduced use of most drugs in older patients.

\section{Abstract \# 141 \\ Lung ultrasound comparison between symptomatic and asymptomatic older patients with COVID-19 infection. A cross-sectional multicentre preliminary report.}

Chukwuma Okoye ${ }^{1}$, Alessandra Fabbri ${ }^{1}$, Daniela Guarino ${ }^{1}$, Antonio Polini $^{1}$, Tommaso Riccioni ${ }^{1}$, Rachele Antognoli ${ }^{1}$, Valeria Calzolaro ${ }^{1}$, Ludovica Zisca ${ }^{2}$, Lucia Visaggi ${ }^{3}$, Camilla Bianchi ${ }^{4}$, Pasquale Morano $^{4}$, Fabio Monzani ${ }^{5}$

${ }^{1}$ Geriatrics Unit, Department of Clinical and Experimental Medicine University of Pisa, ${ }^{2}$ Presidio Ospedaliero "I Fraticini", Azienda USL Toscana Centro, Firenze (Italy), ${ }^{3}$ Geriatric Unit, Department of Internal Medicine, Geriatrics and cardio-vascular pathology, Azienda Ospedaliera Universitaria Federico II, Napoli, ${ }^{4}$ Presidio Ospedaliero Anna Torrigiani, Azienda USL Toscana Centro, Firenze, ${ }^{5}$ Geriatrics Unit, Department of Clinical and Experimental Medicine, Azienda Ospedaliera Universitaria Cisanello, Pisa

Introduction: Recent studies have confirmed the potential role of lung ultrasound (LUS) in the diagnostic approach of COVID19 in patients severely ill; however, scanty data regarding asymptomatic patients are reported in the literature. The aim of the present study is to compare LUS features between older than 65 y.o inpatients admitted in an acute tertiary care hospital setting and asymptomatic residents of two intermediate care hospitals.
Methods: In the first part of the study we enrolled older than 65 years old patients admitted in COVID-19 acute unit of Cisanello Hospital (Pisa, Italy). In the second part of the study we evaluated asymptomatic older residents within 10th and 14th day from the PCR nasal swab test diagnosis of COVID-19 infection in two intermediate care hospitals in Florence.

Results: We included 51 COVID-19 inpatients (mean age $82 \pm 13$ ). $26(51 \%)$ were asymptomatic, 25 (49\%) presented typical COVID-19 symptoms. Asymptomatic COVID19 patients were more frequently females $21(80.8 \%)$ vs $10(32.3 \%), \mathrm{p}<0.001$, older ( $85 \pm 9.5$ vs 78.9 \pm 15.3 yo; $\mathrm{p}=0.04$ ) and more functionally impaired (ADL $2.26 \pm 2.5$ vs $4.20 \pm 2.3, \mathrm{p}=0.009$ ) than those with symptoms, but with a lower burden of comorbidities (CIRS-c $1.1 \pm 0.8$ vs $2.5 \pm 1.8, \mathrm{p}=0.003$ ). Pulmonary consolidations were present in $3 / 26(11.5 \%)$ asymptomatic patients vs $5 / 25(20 \%)$ symptomatic $(\mathrm{p}=0.46)$. Bilateral B-lines were frequently found in symptomatic patients (respectively 24 (96\%) vs $17(65.4 \%) \mathrm{p}=0.006$.

Key Conclusions: In our study no differences between asymptomatic and symptomatic older patients with COVID-19 infection were found in terms of the number of pneumonic consolidations or subpleural nodules. Symptomatic older patients with COVID19 were more likely male, younger, and less functionally impaired but with a higher burden of comorbidities compared to asymptomatic.

\section{Abstract \# 142 \\ COVID-19 outbreak and course-experiences from the City of Zurich Nursing Homes \\ Heike Geschwindner ${ }^{1}$, Céline Mbilo ${ }^{1}$, Gabriela Bieri-Brüning ${ }^{2}$ \\ ${ }^{1}$ city Of Zurich Nursing Homes, ${ }^{2}$ Geriatrics Department, City Of Zurich Nursing Homes}

Introduction: Even though prevention measures have been implemented early during COVID-19 pandemic there have been six Coronavirus outbreaks in the City of Zurich Nursing Homes in total. Residents and employees who tested positive or had contact with infected persons were identified as presumptive infection sources. However, two sources kept unknown. At the beginning of April, there were a series of outbreaks in two nursing homes, which were difficult to contain. A stringent cohort testing of all residents and employees in affected wards was carried out. Thus resulted in the detection of a high percentage of asymptomatic residents tested positive.

Methods: To identify the spreading of infection retrospectively as well as to describe the outbreaks in chronological order we analyse data available from both residents and employees. Case series of 121 old aged patients with asymptomatic, mild, severe and critical courses will be analysed to gain further knowledge with respect to this specific population.

Results: The preliminary results show that beside the high proportion of asymptomatic patients there are many oligosymptomatic patients, as well as a high proportion of critical cases and deaths.

Conclusions: Our experiences show that $40 \%$ of virus carriers are asymptomatic upon diagnosis. For this reason, broad testing is needed in nursing home wards with COVID-19 infections. This approach is worth the effort, due to the high lethality of the COVID-19 virus in nursing homes. 


\section{Abstract \# 143}

Legal aspect of ethical dilemmas during the COVID-19 crisis Maro Mpalta ${ }^{1}$, Marina Kotsani ${ }^{2}$

${ }^{1}$ Supreme Court Lawyer, Msc on Bioethics, Aristotle University of Thessaloniki, Medical School, Thessaloniki, Greece, ${ }^{2}$ Université de Lorraine, CHRU-Nancy, Pôle « Maladies du Vieillissement, Gérontologie et Soins Palliatifs », F-54000, Nancy, France

Introduction: Human life is the ultimate legitimate right of all. Still, this principal has been put into challenge during the COVID-19 pandemic due to moral and ethical dilemmas raised from scarce health care resources, especially regarding older patients' care.

Methods: Review of medical literature, International legal texts (Universal Declaration Of Human Rights (article 3), International Covenant on Civil and Political Rights (article 6), Charter Of Fundamental Rights of the EU (article 2), American Convention on Human Rights (article 4), African Charter on Humans' and Peoples' Rights (article 4), Oviedo Convention) and the Universal Declaration on Bioethics and Human Rights (UDBHR) (articles 3, 4, 5, 10) with regards to dilemmas of health professionals during the COVID-19 pandemic

Results: The right of life is absolute irrespectively any conditions. Forced choices of (non)-allocation of scarce infrastructure still constitute homicide in legal terms. Basic principles of the UDBHR have been challenged during the COVID-19 pandemic, such as the principles of beneficence, nonmaleficence, justice and the respect of autonomy. Frail older patients may be more vulnerable to negative triage practices, especially in cases where specialized care for older people is undervalued by national health care systems.

Key conclusions: The COVID-19 pandemic revealed a possibly underlying predisposition to unfavorable for the older patient distribution of limited health care resources. Geriatric medicine principles should be applied in and out of the context of sanitary crisis, may contribute to the formation of positive attitudes towards older age and may aid distributive justice and medically and ethically difficult decision making.

\section{Abstract \# 144 \\ COVID-19 pandemic response in Greece: awareness and concerns of primary health care professionals.}

Magda Gavana ${ }^{1}$, Dimitra Iosifina Papageorgiou ${ }^{1}$, Anna-Bettina Haidich $^{2}$, Stamatia Kokkali ${ }^{3}$, Persefoni Talimtzi ${ }^{4}$, Aristofanis Paganas $^{5}$, Martha Andreou ${ }^{6}$, Despoina Symintiridou ${ }^{7}$, Vesela Yakimova-Polyzou ${ }^{8}$, Emmanouil Smyrnakis ${ }^{9}$

${ }^{1}$ Laboratory of Primary Health Care, General Practice and Health Services Research, Aristotle University of Thessaloniki, Greece, ${ }^{2}$ Laboratory of Hygiene, Social-Preventive Medicine and Medical Statistics, Medical School, Aristotle University of Thessaloniki, Thessaloniki, Greece, ${ }^{3} \mathrm{Nea}$ Kallikrateia Heatlh Center, Prefecture of Chalkidiki, Greece, ${ }^{4}$ Laboratory of Hygiene, Social-Preventive Medicine and Medical Statistics School of Medicine, Medical School, Aristotle University of Thessaloniki, Prefecture of Thessaloniki, Greece, ${ }^{5}$ Litochoro Health Center, Prefecture of Pieria, Greece, ${ }^{6}$ Health Center of Avdira, Prefecture of Xanthi, Greece, ${ }^{7} 1$ st TOMY Serron, Prefecture of Serres, Greece, ${ }^{8}$ Pyli Health Centre, Prefecture of Trikala, Greece, ${ }^{9}$ Laboratory of Primary Health Care, General Practice and Health Services Research, Aristotle University of Thessaloniki, Greece. On behalf of the Aristotle University of Thessaloniki Primary Health Care Research Network

Introduction: During COVID-19 pandemic long term patients and elderly patients, the main users of primary health care (PHC) services, were the groups at major risk. In Greece PHC was not engaged in the management of the pandemic to the same degree as hospitals. This study investigates the awareness and concerns of primary healthcare workers (PHCW) regarding the COVID-19 pandemic.

Methods: A cross-sectional survey was conducted in public PHC units in Greece. A web-based 18-items questionnaire was administered, exploring HCWs' demographics, awareness level and knowledge of the pandemic preparedness plan and their work or nonwork related concerns.Results: 444 PHCWs participated in the study, most were female $(67.1 \%)$ with a mean age of 44.4 (SD:9.2) years old and a mean working experience of 11.9 (SD:9.8) years. Participants being or living with high-risk individuals (e.g. elderly) showed less awareness $(p=0.006,95 \%$ CI $-0.95,-0.16)$. PHCWs in rural settings show more situation awareness, compared to PHCWs in urban areas $(\mathrm{p}=0.008,95 \% \mathrm{CI} 0.28,1.92)$. Older PHCWs have less concerns than younger PHCWs $(\mathrm{p}=0.025,95 \% \mathrm{CI}-0.08,-0.005)$. Second line PHCWs are also less concerned than first line physicians $(\mathrm{p}=0.028,95 \%$ CI $-1.49,-0.08)$. First line non-physicians PHCWs know less about the preparedness plan than their physician counterparts $(\mathrm{p}<0.001,95 \% \mathrm{CI}-0.97,-0.38)$. Same applies for participants being or living with high-risk individuals $(\mathrm{p}=0.025,95 \%$ $\mathrm{CI}-0.37,-0.03)$. Conclusions: In view of the second wave of the pandemic, PHC structures and PHCWs should be empowered to actively participate in treating and monitoring high risk and geriatric outpatients in PHC settings.

\section{Abstract \# 145}

Admissions to Hospital from Local Care Homes During COVID19 Pandemic

Jennifer S. Pigott ${ }^{1}$, Celia Bielawski ${ }^{1}$

${ }^{1}$ Whittington Health NHS Trust, London UK

Introduction: The COVID-19 global pandemic has disproportionately affected care home residents. Internationally reports range from 19 to $72 \%$ of COVID-19 deaths occurring in care homes [1]. Whittington Health NHS Trust, Care of Older People department provide an integrated ageing service which supports Islington Care Homes. Enhanced advanced care planning specifically to cover COVID- 19 was undertaken in February 2020.

Methods: Data was collected for patients admitted from Islington Care Homes to the Whittington Hospital during the peak of the UK pandemic (1/3/20-31/5/20), and an equivalent time period from 2019. Demographics, clinical diagnoses, treatments, and outcomes were collected.

Results: 19 Islington Care Homes admissions occurred, with two admitted twice. A further 4 were able to return home following treatment in the Emergency Department. All patients received treatment in hospital that could not have been provided in the community. 6 of the admissions tested positive for Sars-Cov-2 on PCR with another being diagnosed with COVID-19 based on imaging and clinical features but with a negative swab result. 2 of those with COVID-19 and 1 without COVID-19 died in hospital. During the same time window in 2019 there were 45 admissions from Islington Care Homes to the Whittington Hospital; 5 died in hospital. The age profile was younger in 2020 (median 78 vs 84 years).

Key conclusions: Integrated services support advanced care planning in care homes to avoid unnecessary hospital admissions and facilitate end of life care in preferred place of death. We demonstrated this during the COVID-19 pandemic, particularly for the elderly.

\section{References:}

[1] Comas-Herrera A, Zalakaín J, Litwin C et al. Mortality Associated with COVID-19 Outbreaks in Care Homes: Early International Evidence. 
https://ltccovid.org/wp-content/uploads/2020/04/Mortality-associatedwith-COVID-26-April-1.pdf.

\section{Abstract \# 146 \\ Management of suspicious for COVID 19 patients at HEALTH CENTER of VARI}

Rev. Dr Michael Dandoulakis ${ }^{1}$, Paolo Sklavounos ${ }^{2}$, George Varsamis $^{2}$, Panagiotis Bouras ${ }^{2}$

${ }^{1}$ Health Center of VARI, ${ }^{2}$ Health Center of Vari

Purpose: The purpose of this study is to study of the management of suspected cases for COVID 19 in the Open Clinic (OC) of H.C. of Varis, during the period of LOCDDOWN.

Material-Methods: In the period from 23/3/2020 there was recording of all teleconferences relating to incidents suspected of COVID 19, in a special EXCELL file, for the purpose of telephone communication in the coming days.

Results: The period from $23 / 3 / 2020$ to $13 / 4 / 2020$ telephone contact was made with 18 patients, $10(55,5 \%)$ women and $8(44,5 \%)$ men. ICPC-2 diagnoses were as follows: R74:13 (72, 2\%), R05:1 (5\%), R21:1, R78:3 (17, 8\%), D10:1 (5\%), .In 10 of them there was telephone contact "1time and 6 of them were made telephone contact on the 6th day. The outcome in all 18 incidents was healing.

Conclusions: The management of cases with symptoms of viral upper respiratory infection in K Y Varis can be considered successful. All incidents were treated and in most of them there was successful telephone communication and monitoring. This experience prepares the health professionals of Health Center of Varis for the successful treatment of the second wave of the Covid19 pandemic next fall.

\section{Abstract \# 147}

Following flu and COVID through a big nursing homes network

Hay Paul-Emile ${ }^{1}$, Armaingaud Didier ${ }^{1}$, Josseran Loïc ${ }^{2}$, Delespierre Tiba $^{3}$

${ }^{1}$ Korian, ${ }^{2}$ APHP - CESP-EQ01 UMRS 1018 INSERM, ${ }^{3}$ Korian CESP-EQ01 UMRS 1018 INSERM

Introduction: Korian ${ }^{\circledR}$ group is specialized in care and support for fragile people. Its professional data warehouse is operational today in 298 French nursing homes (NH) and hosts all residents' data including the residents' care narratives fed on a daily basis. In the past, we designed syndromes by using the standard query language. As COVID cases were first identified, we enriched at the end of February our flu syndrome with coronavirus-like terms, the objective being to follow the COVID propagation from the beginning and delineate whenever possible the afflicted population characteristics.

Methods: We designed a NH residents' cohort by collecting all active older than 60, in the database 2 weeks before 09/30/2019, and followed them until $06 / 01 / 2020$, cutting the 32 weeks period in two: until 03/01/2020 and after.We built sociodemographic and syndromic characteristics as well as administrative and autonomy statuses. We studied the residents' profile on 09/30/2019, then 22 weeks later, on 03/02/2020, and finally, 13 weeks later, on 06/01/2020.

Results: Among our 21111 nursing homes residents cohort, 7516 (35.6\%) got an ARI-ILI syndrome, 1885 (25.1\%) before March, 5631 (74.9\%) after. 4399 (20.8\%) died from all causes, 2638 (60\%) before March, $1791(40 \%)$ after. In addition, $5800(27.5 \%)$ were hospitalized, $3984(68.7 \%)$ before March and 1816 (31.3\%) after.ConclusionCOVID period showed a significant excess mortality ( $\mathrm{p}$-value Chi2-test $=3.5 \mathrm{e}_{-} 15$ ). It also showed a significant decrease in hospitalizations (p-value Chi2-test $<2.2 \mathrm{e}_{-} 16$ ) in a context of limited access to hospitals.

\section{Abstract \# 148 \\ Burnout syndrome among healthcare professionals in DR C.I. Parhon Hospital Geriatric Department During The COVID 19 Pandemic.}

Ioana Alexandra Sandu ${ }^{1}$, Ramona Stefaniu ${ }^{1}$, Adina Carmen Ilie ${ }^{1}$, Anca Iuliana Pîslaru ${ }^{1}$, Călina Anda Sandu ${ }^{2}$, Anne Marie Herghelegiu $^{3}$, Catalina Raluca Nuta ${ }^{3}$, Ovidiu Lucian Bajenaru ${ }^{3}$, Gabriel Ioan Prada ${ }^{3}$, Ioana Dana Alexa ${ }^{1}$

1 "Grigore T. Popa" University of Medicine and Pharmacy Iasi, ${ }^{2}$ "Sf. Spiridon" Emergency Clinical Hospital, Iaşi, "3 "Carol Davila" University of Medicine and Pharmacy, Bucharest

Introduction: The medical world in 2020 is facing a new public health crisis that threatens the world globally with the emergence and spread of the new coronavirus- SARS-COV-2. Despite rigorous efforts for home isolation and quarantine, the incidence of COVID-19 is constantly increasing globally. Consequently, the medical staff is subjected to an overwhelming workload to which is added the exhaustion/lack of personal protective equipment, lack of specific drugs and feelings of inadequacy, and aggressive media coverage. All mentioned can contribute to the mental burden of these healthcare workers.

Methods: We conducted a prospective, hospital-based study, to determine the presence of burnout among medical staff. We applied a burnout questionnaire to all medical staff involved in the monitoring and treatment of patients suspected of COVID 19 (doctors, resident doctors, and nurses), in the Geriatrics and Gerontology department of Dr C.I Parhon hospital.

Results: As the COVID 19 pandemic continues, very important clinical strategies are needed to be able to support healthcare professionals. It is crucial to understand the psycho-emotional impact of the COVID-19 outbreak among them in order to maintain their psychological well-being in particular, given that a considerable proportion of health workers have reported symptoms of depression, anxiety, insomnia and suffering.

\section{Abstract \# 149 \\ Comparative study of the patient examined at the Open Clinic of Health Center of Vari during the period March-April 2019 and 2020}

Michael Dandoulakis ${ }^{1}$, Panagiotis Bouras ${ }^{1}$, Eirini Siafi ${ }^{1}$, Paolo Sklavounos ${ }^{1}$, George Varsamis ${ }^{1}$

${ }^{1}$ Health Center of Vari

Purpose: The purpose of the study is to carry out a comparative study of the cases examined in the period March-April 2019 and 2020 in order to study the effect of the Covid19 pandemic on the incidence of diseases at the Open Clinic (O.C.) of Health Center (H.C.) of Vari. Material-Methodology: The book of the O.C. of H.C. of Vari was used, in order to measure all patients examined in the period MarchApril 2019 and 2020. Items were processed by using an Excel 2007 spreadsheet.

Results: PATIENTS EXAMINED AT H.C.VARI 20192020 4/3-22/ 3519 238-281-54\%23/3-4/5 917,225-692-75\%. During the period $4 / 3-4 / 5 / 2019$ were examined 1436 patients. From $4 / 3$ to $22 / 3$ were examined 519 patients and from $23 / 3$ to $4 / 5$ were examined 917 patients. In the corresponding period of 2020 were examined 238 and 225 patients, that is $281(-54 \%)$ and $692(-75 \%)$. 
Conclusions: There is a significant reduction in patients who have been examined in the O.C. of H.C. of Vari, compared to the same period last year. The same finding has been found in other health care units. This phenomenon is under study in order to discover the causes that cause it.

\section{Abstract \# 150 \\ Old lives matter-inpatients from nursing homes in a COVID-19 ward. \\ Pedro Oliveira ${ }^{1}$, Luciana Silva ${ }^{1}$, Raquel Moura ${ }^{1}$, Mariana Santos ${ }^{1}$, Leonor Silva ${ }^{1}$, Tiago Costa ${ }^{1}$, Mariana Fidalgo ${ }^{1}$, Pedro Salvador ${ }^{1}$, Agripino Oliveira ${ }^{1}$, Rafaela Veríssimo ${ }^{1}$ \\ ${ }^{1}$ Centro Hospitalar Vila Nova de Gaia/Espinho}

Introduction: In nursing homes [NH], during COVID-19 pandemic, residents with advanced age and frequent chronic underlying health conditions are at high-risk for severe outcomes.

Aim: Characterization of NH inpatients profile admitted to a COVID19 ward.

Methods: This is a prospective observational study in a tertiary Portuguese hospital. There were analysed elderly [ $\geq 80$ years] inpatients between March 17th and May 31st 2020. Data was acquired from the hospital information systems. Patients were classified within a clinical frailty scale as no frail [CFS 1-4] and frail [CFS 5-8]. The number of comorbidities of inpatients from nursing homes was measured to evaluate the impact in COVID-19 mortality. Chi-square and Relative Risk [RR] were used and a p-value $<0.05$ was considered to be statistically significant.

Results: There were 283 hospital admissions which $47.3 \%$ were elderly and $51.9 \%$ females. Of those, $32.9 \%$ of the patients came from $\mathrm{NH}$ and frailty was identified in $87.1 \%$ of the episodes. The overall mortality was $33.6 \%$ and considering patients from $\mathrm{NH}$ the $\mathrm{RR}=$ 1.83. These inpatients were also associated with frailty $[R R=3.76]$, multimorbidity $[R R=1.27]$ and were elderly $[R R=3.12]$.

Conclusion: The high mortality rate found in patients from $\mathrm{NH}$ could be associated with the coexistence of frailty, multimorbidity and elderly. Proactive steps by $\mathrm{NH}$ facilities to identify and exclude potentially infected staff and visitors, actively monitoring for potentially infected patients, and implement appropriate infection prevention and control measures are needed to prevent the incoming of Covid-19.

\section{Abstract \# 151}

\section{Elderly and COVID-19 in a tertiary hospital}

Pedro Salvador ${ }^{1}$, Raquel Moura ${ }^{1}$, Mariana Santos ${ }^{1}$, Leonor Silva ${ }^{1}$, Luciana Silva ${ }^{1}$, Tiago Costa $^{1}$, Mariana Fidalgo ${ }^{1}$, Pedro Oliveira ${ }^{1}$, Agripino Oliveira $^{1}$, Rafaela Veríssimo

${ }^{1}$ Centro Hospitalar de Vila Nova de Gaia e Espinho

Background: The COVID-19 pandemic sets many challenges on patient care. Advanced age has previously shown to predict the increased inpatient mortality and length of stay. However, it is still unknown if this finding is the same regarding COVID-19.

Objective: Evaluate the elderly inpatients admitted with COVID-19 in a tertiary hospital and how they relate with efficiency indicators. Methods: Prospective observational study involved all adult inpatients with COVID-19, admitted between March 17th and May 31st 2020. Patients $\geq 80$ years were included in the elderly group. Data regarding patient's comorbidity, frailty, length of stay and in-hospital mortality was obtained. Frailty was determined using the clinical frailty scale (CSF) classifying patients as frail or no frail (CFS 1-4 or
5-8, respectively). We used multivariate analysis to evaluate elderly interaction with other variables. All tests were 2-sided, considered a p-value $<0.05$ to be statistically significant and a $95 \%$ confidence interval.

Results: There were included 283 episodes, $47.3 \%$ on elderly group and $51.9 \%$ females. Frailty was identified in $44.2 \%$ of all episodes. The overall mortality was $33.6 \%$. The elderly group was associated with higher mortality [OR 1.91] as well as frailty [OR 7.14] and multimorbidity [OR 3.81]. No association was found with the length of stay.

Conclusion: The high mortality rate we found in subjects $\geq 80$ years could be partly related to the coexistence of frailty and multimorbidity. Continued evaluation of COVID-19 in large diverse populations is important to identify individuals at risk and improve clinical outcomes.

\section{Abstract \# 152}

\section{Frailty and Covid-19 in a tertiary hospital}

Luciana Silva ${ }^{1}$, Mariana Santos ${ }^{1}$, Raquel Moura ${ }^{1}$, Leonor Silva ${ }^{1}$, Tiago Costa $^{1}$, Mariana Fidalgo ${ }^{1}$, Pedro Oliveira ${ }^{1}$, Pedro Salvador ${ }^{1}$, Agripino Oliveira $^{1}$, Rafaela Veríssimo ${ }^{1}$

\section{${ }^{1}$ Centro Hospitalar Vila Nova de Gaia/Espinho}

Background: Frailty predicts inpatient mortality and length of stay, but its link to COVID-19 is under researched.

Objective: Evaluate and compare the frailty in the COVID-19 ward and Internal Medicine ward.

Methods: Prospective Observational Study in a tertiary Portuguese hospital. Were analyzed every inpatients between March 17th to May 31st 2020. Data was acquired from the hospital information systems. Patients were classified within a clinical frailty scale as no frail (CFS 1-4) and frail (CFS 5-8). Chi-square and t-test were used and a p-value $<0.05$ was considered to be statistically significant. The Relative Risk was also performed.

Results: There were 814 hospitalization episodes, which $65.2 \%$ belonged to medicine ward. There were $52.5 \%$ females and the mean age was 81 years old. The age group $\geq 65$ years old represented $81.8 \%$ of the admissions and the age group $\geq 80$ was $55 \%$. Of all episodes, $57.7 \%$ of them were frail patients, of which $26.4 \%$ belongs to COVID-19 ward. The length of stay was lower for internal medicine ward [10.46 $\mathrm{p}=0.0001]$ but in frailty patients there was no difference. The overall mortality was $26.7 \%$ and there was no difference between wards. If we consider the frail patients, in COVID-19 ward the Relative Risk was 1.43 .

Conclusion: In this study frailty is linked to mortality in COVID-19 patients, but not to length of stay. More prospective work is needed to confirm this outcome and understand how to improve it.

\section{Abstract \# 153}

Telehealth prevention action by medical students for community dwelling older adults during covid19 confinement

Abraham Eliane $^{1}$, Gambier Nicolas ${ }^{2}$, Orsini Françoise ${ }^{1}$, Abraham Aurélie $^{2}$, Besozzi Anaick ${ }^{3}$, Tyvaert Louise ${ }^{2}$, Braun Marc ${ }^{2}$, Joly Laure $^{2}$

${ }^{1}$ Réseau G. Cuny, ${ }^{2}$ Université de Lorraine, ${ }^{3} \mathrm{CHRU}$ Nancy

Introduction: During COVID19 pandemic, population of community-dwelling older adults was particularly isolated from the classic healthcare system. Undergraduate medical students served as a liaison with ambulatory network healthcare system. The aim of this action was to prevent and detect medical, social, or mental problems in this 
population of Nancy Metropole in Lorraine, allowing to order rapidly and relieve general practitioners'workload.

Methods: The students had to phone frail people in order to provide them social contact according to a well-defined protocol written by the Territorial Platform of Support (PTA) of Grand Nancy, after receiving dedicated training to strategic, safe, smart, and resourceconscious way. They had to send a weekly report to the PTA physicians, and can contact them immediately (by phone or mail) as often as they needed. The PTA physicians can then take action accordingly, in conjunction with the general practitioner.

Results: 145 students volunteered for 10 weeks, performed a telephone-based health survey. Each student called from 3 to 5 patients, from one to five times a week for each patient, so that the call duration was about $6 \mathrm{~h} /$ week per student. More than 700 patients were included in the action.

Key Conclusions: This project certainly prevented many health issues from occurring among aged people and enabled undergraduate medical students to consolidate their plan of career development. There is necessary an impact of COVID on medical education, and those students, who weren't in direct contact with patients, showed their collaborative spirit and enhance their medical skills in a completely new and innovative manner.

\section{Abstract \# 154 \\ Use of monoclonal antibodies in octogenarian patients with COVID-19. About a case.}

Magdalena Linge Martín ${ }^{1}$, Julien Paola Caballero Castro ${ }^{2}$, Dionis Carolina Fernández Minaya ${ }^{3}$, Jenifer Peinado García ${ }^{2}$, Juana María Vicario Bermúdez ${ }^{2}$

${ }^{1}$ Hospital San José, Teruel, Spain, ${ }^{2}$ Hospital Obispo Polanco, Teruel, Spain, ${ }^{3}$ Hospital San José, Teruel. Spain

Introduction: Tocilizumab is a monoclonal antibody indicated in patients with severe involvement of the T-CAR cell-induced cytokine release syndrome. Linked to this indication, it has been used against COVID-19 despite the lack of clinical trials.

Methods: Analysis of the case of an 83-years-old man admitted in March 2020 due to COVID-19 infection. He presented a torpid evolution despite specific treatment, receiving treatment with tocilizumab, presenting clinical improvement with reduction of oxygen therapy and allowing the hospital discharge.

Results: 83-years-old man with a history of dyslipidemia, without toxic habits, independent for basic activities of daily living (Barthel index 100/100) who was admitted to hospital for fever, dry cough, myalgia and arthralgia for five days of evolution. COVID-19 infection was diagnosed by PCR and specific treatment was established with lopinavir/ritonavir, hydroxychloroquine and ceftriaxone. The patient presented torpid evolution, needing intravenous corticosteroid and oxygen therapy at high flows to maintain adequate saturations. Given the lack of improvement, tocilizumab was administered in a single dose, after $48 \mathrm{~h}$ of the same became a favorable evolution, being able to decrease the flows of oxygen therapy and improving auscultation. The patient discharged at home with oxygen therapy in 2-liter nasal glasses per minute.

Conclusions: Tocilizumab may be a potential treatment in severe and critical cases of patients with COVID-19 due to its mechanism of action by interrupting the release of cytokines, however, it is necessary to carry out controlled clinical trials that evaluate the safety and efficacy of this drug for this indication.

\section{Abstract \# 155}

A french geriatric team created to support the frail elderly during the COVID-19 epidemic

Chen Yaohua ${ }^{1}$, Puisieux Francois ${ }^{1}$

${ }^{1}$ CHRU LILLE

Introduction: COVID-19 has particularly affected nursing homes $(\mathrm{NH})$. One of the challenges was the difficulties on achieving realtime reverse transcription-PCR (rRT-PCR) test. In the University hospital of LILLE (FRANCE), we first created a 24/7 hotline to support NHs' medical team on March 17, 2020. Later, a mobile testing team was created on April 8, 2020 to provide rapid diagnostic assistance and to avoid admissions to the emergency rooms, for 121 NHs.

Materials and methods: The team consists of one leading nurse, 9 nurses, one advanced-skilled nurse for the coordination, and one geriatrician with advanced skills in infectious diseases. The objectives are providing support for early diagnosis and care; organization of cohorting and electronic recording system; offering training for nurses about sampling and tracing contact for the future massive screening of residents and health workers. Sampling techniques include upper respiratory tract specimens, direct stool or swab sampling for rRTPCR and blood sampling for serology.

Results: For residents of 43 NHs: Different testing tools: 1636 samples for rRT-PCR: 215 nasopharyngeal aspirates (128 negative, 87 positive); 1401nasopharyngeal swab (1304 negative, 90 positive); 20 anal specimens (all negative); and 134 serologies (34 positive out of 80 available). 46 residents underwent different testings. Reasons for testing: 1380 for contact tracing, 107 for diagnoses and 102 for follow-up.Training was provided for 60 health workers.

Conclusion: The establishment of this team allows early diagnosis to limit the number of new cases.The feedback of the $\mathrm{NH}$ is positive and the links between $\mathrm{NH}$ and the hospital are strengthened.

\section{Abstract \# 156}

Cognitive, functional and nutritional situation of institutionalized elderly after the COVID pandemic 19.

M. C. Arenas ${ }^{1}$, P. Pérez-Rodríguez ${ }^{1}$, S. Aparicio-Mollá ${ }^{2}$, S. JimenezArmero $^{1}$, C. Bermejo ${ }^{1}$

${ }^{1}$ Geriatrics Service, Puerta de Hierro Hospital, ${ }^{2}$ Geriatrics Nutrition, Puerta de Hierro Hospital

Introduction: The aim of this study was to compare functional, mental and nutritional status before and after COVID19 pandemic, among nursing homes residents in Madrid.

Methods: A cross-sectional study was carried out in four nursing homes that included 435 residents. Functional Ambulation Capacity (FAC), Barthel Index (BI), handgrip strength, Sarc-F score, Global Deterioration Scale (GDS), Mini-Mental State Exam (MMSE), Mini Nutritional Assessment short-form (MNA) were used to measure outcomes.

Results: The median age was 88 years and $78,4 \%$ were women. One hundred ninety $(43,9 \%)$ patients had tested positive for coronavirus. Functional impairment was detected in $21,9 \%$ of patients who walked independently (FAC scale 4 or 5), in $40 \%$ of those needing supervision (FAC 3 ) and in 33, 6\% of those needing assistance. Comparing BI showed functional impairment in the $26,7 \%, 36,7 \%$ and $48,5 \%$ of residents with mild, moderate and severe dependence. Handgrip strength was low in $90,5 \%$ of the residents and $75,63 \%$ had sarcopenia according to Sarc-F score. We observed cognitive decline in $44,4 \%, 75 \%$ and $40 \%$ of residents with GDS 3,4 , and 5 respectively and also $45 \%$ of participants with mild cognitive impairment in the 
MMSE. Onset of depressive symptoms was found in 53, 5\% of participants. Malnutrition status increased 36, 78\% after COVID pandemia and 38, 39\% had lost weight.

Key Conclusions: We observed a significative impact in functional, mental and nutritional status in elderly, living in nursing homes after COVID-19 pandemic.

\section{Abstract \# 157 \\ Case study: covid infection in elderly \\ Soledad Dominguez Mendoza ${ }^{1}$, Maria De La Puente Martin ${ }^{1}$, Concepcion Lobato Cassinello ${ }^{1}$, Sasha Botbol ${ }^{1}$, Carmen Navarro Ceballos ${ }^{1}$ \\ ${ }^{1}$ hospital Universitario Severo Ochoa}

Background: Elderly patients show an increased risk of complications due to COVID infection.

Clinic Case: An 88 years old male patient with personal history of high blood pressure and anticoagulated atrial fibrillation. Last hospital admission was due to respiratory failure secondary to bilateral pneumonia by SARS-COV2 that required continuous oxygen home therapy and switched to enoxaparine (previously acenocumarol). Ten days later, he returned due to increased dyspnea, hemoptoic expectoration and fever. His blood test revealed creatinine $5,56 \mathrm{mg} / \mathrm{dl}$, PCR elevation and leukocytosis. Chest $\mathrm{x}$-ray showed improvement of previous bilateral infiltrates. Abdominal CAT performed because of acute kidney failure and hematuria displayed retroperitoneal hematoma which required red blood cell transfusion. Hence, anticoagulation was withdrawn during admission. Chest CAT performed because of progressive increased dyspnea and high d-dimero values revealed bilateral pulmonary embolism with signs of pulmonary hypertension and right ventricular overload. This event forced reintroduction of enoxaparine and exhaustive anticoagulation control. Additionally, due to erratic INR control, acenocumarol was switched to dabigatran at discharge.

Conclusion: COVID infection has several manifestations along its evolution. Multiple complications could arise because of virus itself and additionally to its treatment.

\section{Abstract \# 158 \\ SVAMA-MPI and mortality/hospitalization in nursing home residents affected by COVID-19}

Nicola Veronese ${ }^{1}$, Vanni Stangherlin ${ }^{1}$, Paola Mantoan ${ }^{1}$, Marco Chiavalin $^{1}$, Florina Tudor ${ }^{1}$, Alberto Pilotto ${ }^{2}$, Gianfranco Pozzobon ${ }^{1}$

${ }^{1}$ ULSS 3 Serenissima, Venice, Italy, ${ }^{2}$ E.O. Galliera, Genova, Italy

Background: Coronavirus-19 disease (COVID-19) is a widespread condition in nursing homes (NHs). The MPI (Multidimensional Prognostic Index)-SVaMA is a well-calibrated prognostic tool in older adults, but no research was made regarding its importance in $\mathrm{NH}$ residents during COVID-19 pandemic. Therefore, the primary aim of this study was to assess whether MPI-SVaMA can predict mortality and hospitalization, during a period of 90 days in COVID$19 \mathrm{NH}$ residents.

Methods: Nursing home residents affected by COVID-19, in the territory of the Azienda Unità Locale Socio Sanitaria 3, Venice, Italy. The MPI-SVaMA was developed considering nine different domains and 55 items, with a score from 0 to 1 , higher values indicating higher risk of negative outcomes. Hospitalization and mortality were assessed using administrative data. A Cox's regression analysis was used reporting the results as hazard ratios (HRs) with 95\% confidence intervals (CIs).
Results: Among 3, 850 NHs residents, 331 affected by COVID-19 were included (mean age: 86 years; $72.8 \%$ females). Over the 90 days of follow-up, we observed 109 deaths and 94 hospitalizations. Taking those with MPI-SVaMA $<0.50$ as reference, an MPI-SVaMA $>0.63$ was associated with a significant higher risk of death/hospitalization $(\mathrm{HR}=1.69 ; 95 \% \mathrm{CI} 1.14-2.51)$, death $(\mathrm{HR}=1.57 ; 95 \% \mathrm{CI}$ 1.01-2.44) and hospitalization (HR $=2.44 ; 95 \%$ CI $1.46-4.06)$. Similar results were evident using MPI-SVaMA as increase in 0.10 points. A MPI-SVaMA value $>0.63$ had a good specificity for detecting these outcomes. CONCLUSION: Higher MPI-SVaMA values were associated with a significant higher risk of mortality and hospitalization, over 3 months of follow-up, among nursing home residents affected by COVID-19 infection.

\section{Abstract \# 159 \\ Rapid deployment of telemedicine use by hospitalists during COVID-19: a pilot model and qualitative study}

Sue Dhamija ${ }^{1}$, Bradley Geyer ${ }^{1}$, Monica Sethi ${ }^{1}$, Tomas Ricalde ${ }^{1}$, Gunjan Dalal ${ }^{1}$, Asra Khan, Rajendra Suvarna

${ }^{1}$ Multicare Auburn Medical Center, Multicare Inpatient Specialists, Washington State, USA

Introduction: A surge in COVID-19 related hospital admissions is expected in the coming months. Hospitals are experiencing staffing shortages, as is our hospitalist group. To address this problem, we aimed to explore the suitability and feasibility of telemedicine service for our community hospitalist group and understand its limitations in a pilot study.

Methods: Four remote hospitalists performed ten telemedicine encounters each onadmitted patients ( $n=40$ total encounters). The patient population consisted of adult acute hospital admissions along with geriatric and adult psychiatry inpatients. Hospitalists situated themselves in our hospitalist office for the purposes of this pilot. Remote hospitalists partnered with a "telepresenter" who is also a hospitalist in our group. The telepresenter hospitalist mobilized the 2-way audio-visual telemedicine cart into the patient room and assisted with performing a physical exam. At completion, all 4 hospitalists were qualitatively interviewed individually and their interviews analyzed to evaluate their experience.

Results: Remote hospitalists were satisfied with their ability to evaluate and communicate with patients using telemedicine. They felt confident knowing that the physical exam was performed by a hospitalist colleague, a "telepresenter." The 2-way audio-visual experience was seamless. As noted by our remote hospitalists, patients found the encounter to be positive and enjoyed continuity of care. Limitations include the rapid response and late call workflow for which back-up systems will need to be implemented. A 5-h moonlighting telepresenter shift will need to be staffed.

Conclusion: Hospitalists found Telemedicine use to be feasible, rapidly deployable, and effective for inpatient rounding.

\section{Abstract \# 160}

Atypical presentations of COVID19 in older adults living with frailty to hospital

Dr Rachael Morris-Smith ${ }^{1}$, Dr Hannah $\mathrm{Li}^{1}$

${ }^{1}$ Weston General Hospital, Weston-Super-Mare, UK

Providing care for older patients living with frailty in the Emergency Department during the first peak of COVID19 demonstrated atypical presentations such as falls and delirium or was an incidental finding on routine hospital swab rather than the documented typical respiratory symptoms. This audit looks at the presentations of patients aged 
over 79 years to hospital who had a positive COVID19 swab result on admission (within four $\mathrm{h}$ of arrival to hospital). The findings suggest $79 \%$ of patients presented with symptoms other than respiratory ones. For $19 \%$ of patients the positive COVID19 swab was found as an incidental finding. Knowledge of these atypical presentations may help identify COVID19 earlier in this group.

\section{Abstract \# 161 \\ Atypical COVID-19 presentations in the Frail Older Population. Dr Rachael Morris-Smith ${ }^{1}$, Dr Hannah $\mathrm{Li}^{2}$ \\ ${ }^{1}$ Weston General Hospital, Weston-Super-Mare, ${ }^{2}$ Weston General Hospital, Weston-Super-Mare, UK}

AimIt is apparent that the SARS-COV-2 virus disproportionately affects the older population and it has been anecdotally reported that COVID-19 presents atypically in this cohort. This can cause difficulty in identifying, treating, and appropriately isolating these patients. This project was therefore undertaken to describe and quantify clinical presentations of confirmed cases of COVID-19 in the older population. MethodThis was a retrospective cohort study of 50 patients who had PCR confirmed COVID-19 between April 1st 2020 and April 27 th 2020 and were over the age of 80.9 patients were excluded who had been tested routinely and did not have symptoms that triggered testing or who initially tested positive elsewhere. Symptoms at presentation were analysed and delirium during admission and clinical frailty scores were also assessed. ResultsOf the 41 patients, the majority did not present with symptoms consistent with WHO case definition of COVID-19. Although respiratory symptoms were the most common presenting complaint (29\%), most patients presented atypically with a fall $(20 \%)$, delirium (15\%), GI symptoms $(12 \%)$ or asymptomatically (12\%). $41 \%$ of patients were febrile, $44 \%$ had a cough, $29 \%$ were breathless and $46 \%$ had abnormal respiratory observations at presentation.ConclusionOverall, older frail patients are more likely to present atypically and may not have the more widely accepted COVID-19 symptoms of cough, shortness of breath, new temperature. A high degree of suspicion should be maintained for COVID-19 in these patients who may only present with frailty syndromes.

\section{Abstract \# 162 \\ COVID-19 and integrated health monitoring and support for institutionalized elderly: ILPI-BH Project}

Jáder Freitas Maciel Garcia de Carvalho ${ }^{1}$, Edgar Nunes de Moraes ${ }^{1}$, Cristiana Ceotto Deslandes ${ }^{2}$, Flavia Lanna de Moraes ${ }^{2}$, Ann Kristine Jansen $^{3}$, Rodrigo Ribeiro dos Santos ${ }^{4}$

${ }^{1}$ Hospital das Clínicas da Universidade Federal de Minas Gerais, ${ }^{2}$ Prefeitura Municipal de Belo Horizonte, ${ }^{3}$ Departamento de Nutrição da UFMG, ${ }^{4}$ Departamento de Clínica Médica da UFMG

Introduction: Mortality due to COVID-19 is high in Long-Term Care Facilities (LTCFs), related the frailty and the difficulty in implementing the actions proposed by health authorities. To reduce this damage, the ILPI-BH Project was created in the city of Belo Horizonte (Brazil), a partnership between the City Hall and the Hospital das Clínicas of the Federal University of Minas Gerais (HC-UFMG). Methods: Actions were implemented to monitor, train, prevent and block outbreaks of COVID-19 like isolation of symptomatic elderly people in a reception unit, universal testing with RT-PCR and assistance in geriatrics and gerontology also for unrelated demands to COVID-19. The Geriatrics and Gerontology Team at HC-UFMG was divided into five groups that took responsibility for 1656 elderly people ( $43.3 \%$ of the institutionalized elderly in the city) from most vulnerable 89 LTCFs. Each group received a smartphone to facilitate contact with the LTCFs. An artificial intelligence tool (Chatbot) capable of identifying and reporting suspected COVID-19 cases daily, was also made available. Classes were organized by web conference with multiprofessional gerontological topics.

Results: From the start of the pandemic in the city to August 2020, 34 LTCF (38.2\%) had outbreaks of COVID-19. 94 elderly people were admitted to the reception unit and $51 \%$ of these had positive RT-PCR. There were 12 registered deaths and an average outbreak lethality rate below $10 \%$.

Key conclusions: With integrated actions it seems possible to minimize the damage caused by COVID-19 in LTCFs and to reduce mortality among institutionalized elderly.

\section{Abstract \# 163}

¿Are falls in older adults related to early diagnosis of Covid-19, can they be used as a predictive risk factor ?

Licelore Ruiz ${ }^{1}$, Victor Fernandez ${ }^{2}$, Carlos Batres ${ }^{2}$, Diego Jaramillo ${ }^{3}$, Rosa Nicosia ${ }^{2}$, Manuel Guzman ${ }^{2}$, Ines De La Rosa ${ }^{2}$, Sonia Franco ${ }^{2}$, Luis Albornoz ${ }^{2}$, Olga Krysiuk ${ }^{2}$, Yoana Fernandez ${ }^{2}$, Maria Hoyas ${ }^{2}$, Maria A. Moreno ${ }^{2}$, Silvia Gonzalez ${ }^{2}$, Carla Luna ${ }^{2}$, Mar Navarrete ${ }^{2}$, Mirea Flores ${ }^{2}$, Beatriz Muño ${ }^{2}$

${ }^{1}$ ORPEA IBERICA.MEDICA, ${ }^{2}$ ORPEA IBERICA., ${ }^{3}$ ECONOMISTA-ASESOR

Objectives: Measure the association of falls in adults, who had symptoms of Covid-19, identifying their association with pathologies, scale of risk of fall, Barthel, poly medication and nutritional status. A descriptive study is performed. Ho: Covid-19 has no influence on the likelihood of falls.

Method: The data of residents with clinical symptoms of Covid-19 is analyzed. And presence of falls in residents with Covid-19 and without. Period March to June 2020. Statistical packages SPSS v.19. Results: The population is 131 residents. $73.28 \%$ High risk of falls. $16 \%$ not at risk of falling. Barthel $34.48 \%$ have moderate/independence. $56 \%$ residents had falls .Falls occur in $70 \%$ of residents at high risk of falling and $30 \%$ of those at medium risk or not. The fall vs Barthel: $45.58 \%$ of residents had a moderate, or independence dependency. The median Death is 12.5 days. $53 \%$ are poly medicated. The fall occurs within $4 \mathrm{~h}$ of administration of medicines in $40.5 \%$ of residents. The pathologies are cardiovascular $82 \%$ Neurological $40 \%$ and Respiratory $22 \%$. $64.8 \%$ of the study's residents have normal nutritional status. $82 \%$ of residents with malnutrition have falls.

Conclusions: The null hypothesis: Covid-19 has no influence on the probability of falls is rejected at all levels of significance. P-value $0.000113 .45 .58 \%$ had a moderate, mild or independence. The profiles with the highest risk of mortality after the fall are residents with liver, kidney pathologies and malnutrition. The study provides support for early identification of suspected Covid-19 in residents with falls.

\section{Abstract \# 164}

A COVID-19 Nursing Home Transmission Study: sequence and metadata from weekly testing in an extensive nursing home outbreak

Judith van den Besselaar ${ }^{1}$, Reina Sikkema ${ }^{2}$, Fleur Koene ${ }^{3}$, Laura van Buul $^{1}$, Bas Oude Munnik ${ }^{2}$, Rene te Witt ${ }^{4}$, Marion Koopmans ${ }^{2}$, Cees Hertogh $^{1}$, Bianca Buurman ${ }^{1}$

${ }^{1}$ Amsterdam UMC, ${ }^{2}$ Erasmus Medical Centre Rotterdam,

${ }^{3}$ Amsterdam Public Health Research Institute, ${ }^{4}$ Eurofins I NMDLLCPL, Rijswijk, The Netherlands 
Background: This study aimed to assess the contribution of asymptomatic and presymptomatic residents and staff in SARS-CoV-2 transmission during a large outbreak in a Dutch nursing home.

Methods: Observational study in a 185-bed nursing home with two consecutive testing strategies: testing of symptomatic cases only, and weekly facility wide testing of staff and residents regardless of symptoms. Nasopharyngeal and oropharyngeal testing with RT PCR for SARs-CoV-2 was conducted with a standardized symptom assessment. Positive samples with a cycle threshold (CT) value below 32 were selected for sequencing.

Results: 185 residents and 244 staff participated. Sequencing identified one cluster. In the symptom-based test strategy period $3 / 39$ residents were presymptomatic compared to $38 / 74$ residents in the period of weekly facility-wide testing (p-value $<0.001$ ). In total, $51 / 59$ (91.1\%) of SARS-CoV-2 positive staff was symptomatic, with no difference between the two testing strategies (p-value 0.763). Loss of smell and taste, a sore throat, headache or myalga was hardly reported in residents compared to staff ( $\mathrm{p}$-value $<0.001$ ). Median Ctvalue of presymptomatic residents was 21.3 , which did not differ from symptomatic (20.8) or asymptomatic (20.5) residents (p-value $0.624)$.

Conclusions: The underreporting of symptoms in residents compared to staff suggests that a/presymptomatic residents could be unrecognized symptomatic cases. However, symptomatic and presymptomatic/unrecognized symptomatic residents both have the same potential for viral shedding. The high prevalence symptomatic staff found in facility wide testing suggests that staff has difficulty to attribute their symptoms to possible SARS-CoV-2 infection. Weekly testing was an effective strategy for early identification of SARS-Cov2 cases, which resulted in fast isolation and mitigation of this outbreak.

\section{Abstract \# 165 \\ Asymptomatic cases and limited transmission of SARS-CoV-2 in residents and healthcare workers in three Dutch nursing homes \\ Laura van Buul ${ }^{1}$, Judith van den Besselaar ${ }^{1}$, Fleur Koene ${ }^{1}$, Bianca Buurman $^{1}$, Cees Hertogh ${ }^{1}$ \\ ${ }^{1}$ Amsterdam University Medical Centre}

Purpose: Many nursing homes worldwide have been hit by outbreaks of the new severe acute respiratory syndrome coronavirus 2 (SARSCoV-2). We aimed to assess the contribution of a- and presymptomatic residents and healthcare workers in transmission of SARSCoV-2 in three nursing homes.

Methods: Two serial point-prevalence surveys, 1 week apart, among residents and healthcare workers of three Dutch nursing homes with recent SARS-CoV-2 introduction. Nasopharyngeal and oropharyngeal testing for SARS-CoV-2, including reverse-transcriptase polymerase chain reaction (rRT-PCR) was conducted with reporting of cycle threshold $(\mathrm{Ct})$. Participants were categorized as symptomatic, presymptomatic or asymptomatic with standardized symptom assessment.

Results: In total, 297 residents and 542 healthcare workers participated in the study. At the first point-prevalence survey, 15 residents tested positive of which one was presymptomatic $(\mathrm{Ct}$ value $>35)$ and three remained asymptomatic ( $\mathrm{Ct}$ value of 23,30 and 32). At the second point-prevalence survey one resident and one healthcare worker tested SARS-CoV-2 positive (Ct value $>35$ and 24 , respectively) and both remained asymptomatic.

Conclusion: This study confirms a- and presymptomatic occurrence of Covid-19 among residents and health care workers. Ct values below 25 suggested that these cases have the potential to contribute to viral spread. However, very limited transmission impeded the ability to answer the research question. We describe factors that may contribute to the prevention of transmission and argue that the necessity of large-scale preemptive testing in nursing homes may be dependent of the local situation regarding prevalence of cases in the surrounding community and infection control opportunities.

\section{Abstract \# 166 \\ Symptom clusters in COVID-19: a potential clinical prediction tool from the COVID Symptom Study App}

Carole H. Sudre $*^{1}$, Karla A. Lee $*^{2}$, Mary Ni Lochlainn ${ }^{2}$, Thomas Varsavsky $^{1}$, Benjamin Murray ${ }^{1}$, Mark S. Graham ${ }^{1}$, Cristina Menni ${ }^{2}$, Marc Modat $^{1}$, Ruth C. E. Bowyer ${ }^{2}$, Long H. Nguyen ${ }^{3}$, David A. Drew $^{3}$, Amit D. Joshi ${ }^{3}$, Wenjie Ma ${ }^{3}$, Chuan-Guo Guo ${ }^{3}$, Chun-Han $\mathrm{Lo}^{3}$, Sajaysurya Ganesh ${ }^{4}$, Abubaka Buwe ${ }^{4}$, Joan Capdevila Pujol ${ }^{4}$, Julien Lavigne du Cadet ${ }^{4}$, Alessia Visconti ${ }^{2}$, Maxim B. Freydin ${ }^{2}$, Julia S. El-Sayed Moustafa ${ }^{2}$, Mario Falchi ${ }^{2}$, Richard Davies ${ }^{4}$, Maria F. Gomez ${ }^{5}$, Tove Fall ${ }^{5}$, M. Jorge Cardoso ${ }^{1}$, Jonathan Wolf ${ }^{4}$, Paul W. Franks $^{6}$, Andrew T. Chan ${ }^{3}$, Tim D. Spector ${ }^{2}$, Claire J Steves**2 ${ }^{2}$, Sébastien Ourselin** 1

${ }^{1}$ School of Biomedical Engineering \& Imaging Sciences, King's College London, Westminster Bridge Road, SE17EH London, UK, ${ }^{2}$ Department of Twin Research and Genetic Epidemiology, King's College London, Westminster Bridge Road, SE17EH London, UK, ${ }^{3}$ Clinical and Translational Epidemiology Unit, Massachusetts General Hospital, MA, USA, ${ }^{4}$ Zoe Global Limited, 164 Westminster Bridge Road, London SE1 7RW, UK, ${ }^{5}$ Lund University Diabetes Centre, Department of Clinical Sciences, Malmö, Sweden, ${ }^{6}$ Department of Twin Research and Genetic Epidemiology, King's College London, Westminster Bridge Road, SE17EH London, UK; Lund University Diabetes Centre, Department of Clinical Sciences, Malmö, Sweden

As no one symptom can predict disease severity or the need for dedicated medical support in COVID-19, we asked if documenting symptom time series over the first few days informs outcome. Unsupervised time series clustering of symptom development was performed on data collected from a training dataset of completed cases enlisted early from the COVID Symptom Study Smartphone application, yielding six distinct symptom presentations $(n=1653)$. Clustering was validated on an independent replication dataset $(\mathrm{n}=$ 1047). Individuals assigned to differing symptom clusters showed differences in the subsequent need for respiratory support in hospital, with risk escalating from $1.5 \%$ in cluster 1 ('flu like with no fever) to $19.8 \%$ in cluster 6 (Severe level 3: Abdominal and Respiratory). Moreover, older, frailer (PRISMA7) individuals and those with multimorbidities were more likely to exhibit the two most severe symptom clusters (Severe level 2: Confusion, and Severe level 3 Abdominal and Respiratory), distinguishable as early as day 3. Investigating prediction of subsequent need for respiratory support, we found that personal characteristics alone, including PRISMA7 frailty, were able to achieve a ROC-AUC of $69.5 \%$. Adding data from the first 5 days of symptom logging after onset, the ROC-AUC of need for respiratory support was substantially improved at $78.8 \%$. Such an approach could be used to monitor at-risk patients and predict medical resource requirements days before they are required, opening the doors for proactive interventions. 


\section{ABSTRACT \# 167}

Nasal irrigation and gargling to prevent transmission of SarsCoV-2 to care home residents-A feasibility-pilot cluster randomised controlled trial

Ryan Buchanan ${ }^{1}$, Kinda Ibrahim ${ }^{1}$, Beth Stuart ${ }^{1}$, Julie Parkes ${ }^{1}$, Helen Roberts ${ }^{1}$, Jeremy Wyatt ${ }^{1}$, Thomas Daniels ${ }^{1}$

${ }^{1}$ University of Southampon

Background: Sars-CoV-2 is transmitted via droplet spread and can be carried in the nasal passages in asymptomatic or mildly symptomatic individuals. There is evidence that hypertonic saline nasal irrigation and gargling (HSNIG) can reduce the viral load of coronaviruses. We present a feasibility/pilot 1:1 cluster-randomised trial of hNIG in care home $(\mathrm{CH})$ staff to protect $\mathrm{CH}$ residents from COVID-19.

Method: Staff in intervention $\mathrm{CHs}$ were asked to administer hNIG each shift. Nominated champions led the implementation. Baseline data, uptake of hNIG and outcomes were collected via self-reported online questionnaires. Barriers and facilitators to the intervention were explored via interview.

Results: We contacted $30 \mathrm{CHs}$ and randomised seven-four to intervention and three to usual practice. One dropped out on being allocated to usual practice and two did not complete data collection. Of these one $\mathrm{CH}$ felt overwhelmed during the pandemic and the other felt that the intervention was no longer needed. Four CHs (2 intervention, 2 control) completed the study. In the two intervention $\mathrm{CHs}$ adherence to the intervention was varied (100\% and $40 \%)$ Facilitators to uptake of the hNIG included: highly motivated champions, strong leadership and integration into daily routines. Reported barriers were a lack of ownership by managers and champions and staff already feeling safe from COVID-19.

Conclusion: We demonstrate that self-administration of hNIG by $\mathrm{CH}$ staff is feasible. A future trial to test effectiveness should not rely solely on self-reported outcome data. Consideration should be given to a stepped-wedge design so all $\mathrm{CHs}$ participate in the intervention.

\section{Abstract \# 168 \\ A real-world analysis of the value of repeated swabbing in COVID-19}

Ealish Swift ${ }^{1}$, Fidaa Natour ${ }^{1}$, Rose Jones ${ }^{1}$, Lauren Morgan ${ }^{1}$, Joshua Hrycaiczuk $^{1}$, Matthew Stuttard ${ }^{1}$, Mohammed Mohammednoor ${ }^{1}$, Nina Holling $^{1}$, Bahar Al-Ramadhani ${ }^{1}$, Orry Swift ${ }^{2}$, Stephanie Carroll ${ }^{1}$, Martin Williams ${ }^{1}$, Peter Muir ${ }^{3}$, Abigail Cannon ${ }^{1}$

${ }^{1}$ University Hospitals Bristol and Weston NHS Foundation Trust, ${ }^{2}$ University of Southampton, ${ }^{3}$ North Bristol NHS Trust

Introduction: Having a nasopharyngeal swab taken for COVID-19 can be distressing and uncomfortable for patients with dementia, and lead to poor sample quality due to lack of compliance. However, repeated swabs are often central to de-escalation, transfer to community hospitals, and repatriation to nursing homes. To act in patients' best interests, healthcare professionals need to know how sensitive the swabs are, and if repeat swabbing is of value. Swab results were compared to clinical suspicion in a hospital environment to analyse the efficacy of repeated swabbing.

Methods: We correlated swab results with the clinical index of suspicion (obtained from patient notes) for 539 COVID-19 samples. Results: Of patients treated empirically as COVID-19 positive, $50 \%$ had a positive result on the first swab, and a further 7\% later tested positive on repeated swabs. $18 \%$ were not reswabbed after an initial negative result but instead were treated clinically, and $25 \%$ never swabbed positive despite repeated attempts.
Key conclusions: Given that only $7 \%$ of suspected COVID patients become positive after an initial negative swab, and that a full $25 \%$ of suspected patients never swab positive, it may be pragmatic to use swabbing as more of a rule-in rather than rule-out measure, avoiding repeat sampling in those in whom this may cause distress. In addition, de-escalation and repatriation protocols requiring re-swabbing prior to discharge may need to be updated to consider the necessity of repeat swabbing in light of these findings.

\section{Abstract \# 169}

Using WhatsApp messaging platform to facilitate rapid multiprofessional, multi-organisational support for care homes during the COVID-19 pandemic

Tania Kalsi ${ }^{1}$, Dan Wilson ${ }^{2}$, Chris Kalafatis ${ }^{3}$, Nick Gough ${ }^{1}$, Christen San Pedro ${ }^{4}$, Samatha Crawley ${ }^{5}$, Emma Hall ${ }^{6}$, Jay Patel ${ }^{7}$, Ade Lawal ${ }^{8}$, Rebecca Dallmeyer ${ }^{8}$

${ }^{1}$ Guys \& St Thomas' NHS Foundation Trust, ${ }^{2}$ Kings College NHS Foundation Trust, ${ }^{3}$ South London and the Maudsley NHS Trust, ${ }^{4} \mathrm{HC}$ One, ${ }^{5}$ Excelcare, ${ }^{6} \mathrm{St}$ Christopher's Hospice, ${ }^{7}$ Hetherington Group Practice, ${ }^{8}$ Quay Health Solutions GP Care Home Service

Introduction: The COVID-19 pandemic has disproportionately affected care homes. Southwark and Lambeth are two inner city London boroughs with high levels of social deprivation that were affected early in the UK's first wave of COVID-19 infections. During the crisis, it became immediately apparent the care homes in these boroughs needed a rapid escalation of support. In response, we developed a multi-specialist care home support network from a wide array of local health and care providers. This consisted of managers and senior staff from local care homes as well as primary and secondary care doctors, nurses, and allied health professionals with expertise in older people's mental and physical health and palliative care. Using WhatsApp messaging (with appropriate Information Governance) the network rapidly provided peer support, disseminate guidelines and provide education. Discussion of individual patients was prohibited and taken off-line to respect confidentiality. We evaluate the utility of WhatsApp in enabling support for care homes in the COVID-19 pandemic.

Methods: Utility was evaluated by (a) messaging activity and (b) user feedback via survey. The survey was distributed to members of the individual WhatsApp groups (Southwark and Lambeth operated separately) using a web-based survey link (Survey Monkey). Questions were asked using five-point Likert scales and free text responses. Results: $48.4 \%$ (31/64) response rates. Response rates were higher in Southwark which differed by having a one GP provider care model (65.8\% vs $42.1 \%, \mathrm{p}=0.04)$. A total of 1696 messages were sent in Southwark compared to 1148 messages in Lambeth. $90.3-96.8 \%$ of respondents reported that the WhatsApp group improved their feeling of being part of team, having shared goals, improved trust, helped to keep up with national guidance and helped navigate and access expertise. $83.9 \%$ felt it helped enable care to be provided in the resident's setting of choice. Free text responses reported benefits including " "coordinated and prompt support", "access to other key professionals quickly to aid clinical decision making", "feeling of support and we were in it together".

Conclusions: Simple messaging platforms to connect professionals with mutual goals was useful during the pandemic, more so when combined with a single GP provider model. 


\section{Abstract \# 170 \\ 90-day mortality by age and frailty status in patients hospitalized due to Covid-19 infection}

Marte Meyer Walle-Hansen ${ }^{1}$, Håkon Ihle-Hansen ${ }^{2}$, Trygve Berge ${ }^{3}$, Marius Myrstad ${ }^{3}$

${ }^{1}$ Department of Medical Research, BS VVHF, 1346 Gjettum, Norway, ${ }^{2}$ Department of Internal Medicine, BS VVHF, 1346 Gjettum, Norway, ${ }^{3}$ Department of Internal Medicine, Department of Medical Research, BS VVHF, 1346 Gjettum, Norway

Introduction: Old and frail people are vulnerable to acute illness. Studies have revealed that in-hospital mortality due to Covid-19 is high and increases with increasing age. We are not aware of any studies that have reported long-term outcome in older people hospitalized with Covid-19 infection. This research aims to study 90-day mortality by age group and premorbid frailty status among patients hospitalized with Covid-19 infection.

Methods: This is a prospective cohort study. Patients included were admitted between March 9th and May 7th 2020, to a non-university public hospital in the Oslo area (Norway). Premorbid frailty status was assessed using the Clinical Frailty Scale (CFS). Patients were categorized into younger fit (age $<70$ and CFS score $<5$ ), older fit (age $\geq 70$ years and CFS score $<5$ ) and frail (CFS score $\geq 5$, all ages) patients.

Results: 74 patients $(58 \%$ men) were hospitalized with Covid-19 infection (median age 71 (range 30-95) years). 40 patients $(54 \%)$ were aged $\geq 70$ years. The overall 90 -day mortality was $21 \%$ (median number of days from hospital admission to death was 11 (mean 12.3, range 0-27 days), 20 patients were frail (CFS score $\geq 5$ ), median CFS score was 2 . Mortality by age and frailty status was $3 \%$ in younger fit, $24 \%$ in older fit and $45 \%$ in frail patients.

Key conclusions: Compared to younger fit hospitalized Covid-19 patients, 90-day mortality was 8 times higher in older fit patients, and 15 times higher in frail patients.

\section{Abstract \# 171 \\ Seroprevalence of SARS-CoV-2 specific antibodies among residents and healthcare workers in 42 nursing homes in Spain}

Victoria Perez ${ }^{1}$, Peggy Ríos-Germán ${ }^{1}$, Irene Serrano ${ }^{2}$, Rosa Nicosia ${ }^{1}$, Silvia Lores ${ }^{1}$, Blanca Díaz ${ }^{1}$

${ }^{1}$ Health Department, Orpea Iberica Group, ${ }^{2}$ Quality Department, Orpea Iberica Group

Introduction: The aim of this study was to determine residents and healthcare workers (HCW) immunity against SARS-CoV-2 in Orpea Iberica Group, a group of nursing homes $(\mathrm{NH})$ in Spain.

Methods: Serum samples for SARS-CoV-2-IgG antibodies were tested in two phases. The first phase took place from April 21th to May 25th 2020 in all residents and in all HCW of the NH. The second phase was carried out for new residents and new HCW from the last day of the first phase until the first of September, 2020. Data have been updated weekly and been reported to the Quality Department of the Group. Seroprevalence of residents living and HCW working, at September 1st, 2020 is presented in this study.

Results: SARS-CoV-2-IgG antibodies were detected in $2230(52.8 \%)$ residents and $895(32 \%) \mathrm{HCW}$ in $38 \mathrm{NH}$. Serorevalence between regions was: Comunidad de Madrid (23 NH): $67.5 \%$ of residents and $44.1 \%$ of $\mathrm{HCW}$ were positive for $\mathrm{IgG}$; and following: Castilla y León $(2 \mathrm{NH}): 35.7 \%$ and $16.7 \%$; Cataluña $(3 \mathrm{NH}): 46.3 \%$ and $28.2 \%$; Andalucía (6 NH) $25.2 \%$ and $11.1 \%$; La Rioja (1 NH): $11.3 \%$ and
11.7\%; Galicia (2 NH) $3.5 \%$ and $0 \%$; Comunidad Valenciana (1 $\mathrm{NH}$ ): $0 \%$ and $1.4 \%$.

Key Conclusions: In our Group of NH, SARS-CoV-2 seroprevalence is high and had a substantial geographical variability. It was higher in Comunidad de Madrid, Castilla y León and Cataluña and lower in the other regions.

\section{Abstract \# 172}

Treatment escalation decisions in patients admitted with COVID19: results of an international multi-centre study

Geriatric Medicine Research Collaborative ${ }^{1}$

${ }^{1}$ University of Birmingham

Introduction: Treatment escalation and limitation (TEAL) decisions are made to prevent treatment that is futile, or against patient wishes. This study set out to assess decision making during the COVID-19 pandemic

Methods: Multi-centre cohort study involving patients aged 18 years and older hospitalised with COVID-19. We used logistic regression to assess impact of age, Clinical Frailty Scale (CFS), sex, multi-morbidity, and country (UK vs. elsewhere) on likelihood of Do Not Attempt Cardiopulmonary Resuscitation (DNACPR) and TEAL decisions, admission to critical care, and end of life care provision. We used Cox regression analysis to assess impact on overall risk of death.

Results: This study included 5766 patients from 55 hospitals in 12 countries; 5133 (89\%) from UK hospitals. Patients were more likely to have DNACPR or TEAL decisions if they were older, more frail, or had greater multi-morbidity. Patients were also less likely to be admitted to critical care and more likely to have had end of life care if they were older and more frail. Patients in the UK were more likely to have DNACPR or TEAL decisions made, less likely to be admitted to critical care, and more likely to have end of life care commenced compared to other countries, independent of age, frailty, and multimorbidity. However, overall risk of death did not differ between the UK and other countries.

Conclusions: There are international differences in decision-making for DNACPR decisions and escalation of care to critical care. Holistic decisions should consider the patient's past wishes and treatment futility.

\section{Abstract \# 173}

Delirium and relative bradycardia is common in patients who die of COVID-19 in hospital

Peter Diem ${ }^{1}$, Tania Kalsi ${ }^{1}$, James Fleet ${ }^{1}$, Nigel Beckett ${ }^{1}$

${ }^{1}$ Guys \& St Thomas' NHS Foundation Trust

Introduction: COVID-19 is dangerous for frail older people. It is important to understand the clinical features particularly in severe cases/non-survivors to identify areas for future clinical support, identify high risk groups as well as areas for future research.

Methods: Retrospective case notes review of COVID-19 deaths in a single institution in London as part of the hospital mortality review/ RCP Structured Judgement Review Process. Presenting symptoms on admission, observations, clinical course systematically reviewed and themes identified.

Results: 41 deaths occurred between 14.3 .20 and 1.6.20. Mean age 82 years, range 57-99. 80\% (33/41) acquired COVID-19 in the community. 8 acquired in hospital. Of community acquired cases, $27 \%$, (9/33) were from care homes. In those presenting with COVID-19 from the community, the most common presentation was delirium 
(75.8\%, 25/33). Less common were respiratory symptoms $(57.6 \%$, $19 / 33)$, fever $(30.3 \%, 10 / 33)$, falls $(27.3 \%, 9 / 33)$ and poor oral intake $(18.2 \%, 6 / 33)$. Only $27.3 \%$ (9/33) presented with classic symptoms of fever, breathlessness and/or cough. Of all COVID-19 deaths, $89.7 \%$ (37/41) had 1+ key comorbidities (PVD, IHD, CVD, heart failure, hypertension, diabetes). $69.2 \%$ (28/41) had/developed AKI. CRP often but not always elevated, rising in most but not all patients. Most had escalating oxygen requirements and worsening hypoactive delirium. Despite severe respiratory compromise, fever and dehydration, $60 \%$ (25/41) only mounted a muted tachycardic response/ were relatively bradycardic (Faget Sign) and remained normotensive/ hypertensive.

Conclusions: 1 . Most older COVID-19 non-survivors don't present classically, delirium is the most common presentation 2. Absence of tachycardia/hypotension despite fever/respiratory compromise might be a good diagnostic indicator of COVID-19.

\section{Abstract \# 174}

\section{Delirium is common in patients hospitalized with COVID-19}

Leiv Otto Watne ${ }^{1}$, Kristian Tonby ${ }^{1}$, Aleksander Holten ${ }^{1}$, Theresa Olavsveengen ${ }^{1}$, Luis Romundstad ${ }^{1}$, Hilde Woien ${ }^{1}$, Bjorn Erik Neerland ${ }^{1}$

${ }^{1}$ Oslo University Hospital

Introduction: Several aspects of COVID-19 are known risk factors of delirium, such as hypoxia, inflammation, heavy sedation and mechanical ventilation. In this cohort study we report the occurrence of delirium among COVID-19 patients admitted to Oslo University Hospital (OUH), Norway.

Methods: All patients above 18 years hospitalized at OUH with confirmed COVID-19 until May 12020 were consecutively included. Delirium was diagnosed according to the DSM-5 criteria.

Result: 168 patients were included. 48 patients $(29 \%)$ had delirium during the hospital stay, and $15(10 \%)$ were delirious at admission. 41 patients were treated in the Intensive Care Unit (ICU) and of these, 30 (73\%) developed delirium. All 25 patients requiring mechanical ventilation developed delirium. In $14(56 \%)$ of these, extubation was postponed because of delirium and 4 patients $(16 \%)$ had to be reintubated. Ten $(77 \%)$ of 13 patients never on mechanical ventilation that died, experienced delirium in the terminal phase of palliative care.

Discussion: Our results show that delirium is common in COVID-19 patients, in particular in the ICU. Delirium is a highly relevant condition in COVID-19 for several reasons. (1) Delirium can be an early symptom of COVID-19. (2) Treatment, including respiratory support, is complicated by non-cooperating patients. (3) Mandatory infection control measures may be difficult to combine with a delirium friendly environment and restrict the ability to involve family members on the wards. This is particularly worrisome since delirium is common in the terminal phase.

\section{Abstract \# 175}

Beyond chronological age: Frailty and multimorbidity predict mortality in patients with coronavirus disease 2019

Alberto Zucchelli ${ }^{1}$, Alessandra Marengoni ${ }^{1}$, Davide Liborio Vetrano $^{2}$, Emanuele Botteri ${ }^{3}$, Andrea Armellini ${ }^{3}$, Franco Nicosia ${ }^{3}$, Giuseppe Romanelli ${ }^{1}$, Andreas Beindorf ${ }^{3}$, Paola Giansiracusa ${ }^{3}$, Emirena Garrafa ${ }^{1}$, Luigi Ferrucci ${ }^{4}$, Laura Fratiglioni ${ }^{2}$, Roberto Bernabei $^{5}$, Graziano Onder ${ }^{6}$
${ }^{1}$ Università degli Studi di Brescia, ${ }^{2}$ Karolinska Institutet, ${ }^{3}$ ASST Spedali Civili Brescia, ${ }^{4}$ National Institute on Aging - USA, ${ }^{5}$ Università Cattolica del Sacro Cuore Roma, ${ }^{6}$ Istituto Superiore di Sanità

Background: The prompt identification of patients affected by coronavirus disease 2019 (COVID-19) at higher risk of death represents a turning point in their clinical management and in the decisionmaking process. We aimed to evaluate whether frailty and multimorbidity predict in-hospital mortality in patients with COVID-19 beyond chronological age.

Methods: We analysed data from 165 patients consecutively admitted to an acute geriatric medicine ward in Brescia, Northern Italy from March 8th to April 17th, 2020, with a suspected diagnosis of COVID19. The infection was confirmed by reverse transcriptase-polymerase chain reaction assay for severe acute respiratory syndrome coronavirus 2 RNA. Pre-disease frailty status was assessed with the 9-point Clinical Frailty Scale (CFS) and categorized as 'no to mild frailty' (< 6 points), and 'moderate to severe frailty' ( $6+$ points). Based on a predefined list of 60 chronic diseases, multimorbidity was defined as the co-occurrence of $\geq 2$ of these chronic diseases in the same patient. Cox regression models were used to evaluate the hazard (HR) of inhospital mortality as a function of frailty and multimorbidity in the whole population and in those aged $70+$ years. The C-statistics of different Cox regression models were used to assess the increment in accuracy in the prediction of in-hospital mortality provided by adding information on the CFS and multimorbidity to chronological age.

Results: The median length of stay of the whole sample population was 10 days (IQR 7-17). Among the 165 patients, 112 were discharged, 11 were transferred to intensive care units and 42 died. Patients who died were older $(81.0$ years vs. 65.2 years, $\mathrm{p}<0.001)$, more frequently multimorbid $(97.6 \%$ vs. $52.8 \% ; \mathrm{p}<0.001)$ and more likely to be frail $(37.5 \%$ vs. $4.1 \%$; p $<0.001)$. Less than $2.0 \%$ of patients without multimorbidity and frailty, $28 \%$ of those with multimorbidity only and $75 \%$ of those with both multimorbidity and frailty died during hospitalization. In survival analyses, adjusted for age, sex, and education, each unitary increment in the CFS score was associated with a significantly higher risk of in-hospital death in the whole sample (HR $=1.31 ; 95 \%$ CI $1.05-1.62)$ and in patients aged $70+$ years $(\mathrm{HR}=1.29 ; 95 \%$ CI $1.04-1.62)$, whereas each increment in the number of chronic diseases was not significantly associated with higher risk of death. The addition of the CFS to chronological age and sex increased in-hospital mortality prediction by $9.4 \%$ in those aged $70+$ years. The highest positive predictive value was due to moderatesevere frailty ( 0.75 with a positive likelihood ratio of 9.15$)$, whereas the highest negative predictive value was linked to multimorbidity (0.98 with a negative likelihood ratio of 0.05 ).

Conclusions: Frailty identifies patients with COVID-19 at risk of inhospital death independently of age. The significant prognostic value of frailty is maintained in persons aged 70 years and older. Multimorbidity also contributes to mortality risk stratification, especially because of the very low probability of death in its absence. Systematic screening for frailty and multimorbidity in patients with COVID-19 may help to identify high-risk individuals in need of early intervention and to develop and validate clinical decision support tools.

\section{Abstract \# 176}

Luc Dauchet, Eric Boulanger, Emmanuel Hirsch, Aline Meirhaeghe, Philippe Amouyel

Luc Dauchet ${ }^{1}$, Eric Boulanger ${ }^{1}$, Emmanuel Hirsch ${ }^{2}$, Aline Meirhaeghe $^{1}$, Philipper Amouyel ${ }^{1}$

${ }^{1}$ Univ Lille, Inserm, CHU Lille, Institut Pasteur de Lille, U1167 RIDAGE Facteurs de risque et déterminants des maladies liées à l'âge, 
59000 Lille., ${ }^{2}$ Département de recherche en éthique, Université ParisSaclay.

Introduction: The end of the COVID-19 pandemic lockdown raised anxiety especially for aged people. To alleviate it, we created a score estimating the relative risk to be infected by SARS-COV2 and to die from it according to age, sex and body mass index (BMI).

Methods: We used the cumulative number of deaths of the largest most severely affected French region, the Île de France (IDF) excluding people living in institutions, on April 22 2020, grouped by 10 -year age classes. To approximate the impact of sex we calculated a standardized mortality ratio for men (1.49) and women (0.65). The influence of BMI was taken into account using data from an English cohort [1].

Results: The COVID-SCORE applied to individuals without known comorbidities, and non-pregnant women. A publication [2] identified an agexBMI interaction that prompted us to exclude individuals with BMI $>40 \mathrm{~kg} / \mathrm{m}^{2}$. The COVID-SCORE is available on https://www.covid-score.fr. The COVID-SCORE ranged from $<0.01$ to 61.08. For example, a woman, aged $60-70$ with a $25 \leq \mathrm{BMI}<30$ had a COVID-SCORE of 1.0 corresponding to the mean risk of the population and a man aged 70 to 80 with a $30 \leq \mathrm{BMI}<35$ had a COVID-SCORE of 7.0. We defined a simple five-coloured scale and delivered specific recommendations according to each risk colour.

Conclusion: The COVID-SCORE is a public heath tool empowering individuals to make weighted decisions for daily situations as to meet family or to have collective and working activities, to bring back a bit of serenity in a highly anxiety inducing context.

[1] Bacon S et al https://doi.org/10.1101/2020.05.06.20092999

[2] Kass DA et al https://doi.org/10.1016/S0140-67362031024-2

\section{Abstract \# 177 \\ Telecardiology in geriatric patients: a control measure in times of COVID-19}

Mónica Ramos Sánchez ${ }^{1}$, Javier Jaramillo Hidalgo ${ }^{2}$, Isabel Lozano Montoy ${ }^{3}$, Giovanna Cristofori ${ }^{4}$, Eva Fernández de la Puente ${ }^{5}$, Blanca Garmendia Prieto $^{6}$, Pilar Belén Chaves Pérez ${ }^{7}$, Maribel Quezada Feijoó $^{8}$, Rocio Ayala Muñoz ${ }^{8}$, Francisco-Javier Gómez Pavón ${ }^{5}$

${ }^{1}$ Department of Cardiology; Hospital Central de la Cruz Roja. Universidad Alfonso X el sabio. adrid, Spain, ${ }^{2}$ Department of Geriatrics; Hospital Central de la Cruz Roja. Universidad Alfonso X el sabio. Madrid, Spainn), ${ }^{3}$ Department of Geriatrics; Hospital Central de la Cruz Roja. Universidad Alfonso X el sabio. Madrid, Spain),

${ }^{4}$ Department of Geriatrics; Hospital Central de la Cruz Roja. Madrid, Spain, ${ }^{5}$ Department of Geriatrics; Hospital Central de la Cruz Roja. Universidad Alfonso X el sabio. Madrid, Spain, ${ }^{6}$ Department of Geriatrics; Hospital Central de la Cruz Roja. Madrid, Spain, ${ }^{7}$ Universidad Complutense de Madrid, Spain, ${ }^{8}$ Department of cardiology; Hospital Central de la Cruz Roja. Universidad Alfonso X el sabio. Madrid. Spain

Introduction: The SARS-COVID-19 pandemic has led to a reorganization of traditional medical care. Telemedicine could be an intermediate solution. We sought to analyze the characteristics of patients who received telecardiology consultations as follow-up of their heart disease, the prevalence of mortality and hospital admissions during the pandemic period.

Methods: Retrospective longitudinal cohort study. 300 patients with appoinment in cardiology consultations during the pandemic period (from March 13 to May 13, 2020) aged $\geq 70$ years were included. During telephone consultation the following variables were collected: age, type of heart disease, changes in management generated by the consultation, hospitalizations due to heart disease, mortality and causes, and COVID infections.
Results: $\mathrm{N}=300,55.3 \%$ women, mean age $83.07 \pm 6.7$. The most frequent reason for cardiology consultation was due to arrhythmias/conduction disorder $(36 \%)$. $88 \%$ of the patients remained without medical changes, $6 \%$ required treatment adjustment and $2.3 \%$ were discharged by telephone. $90,6 \%$ of the patients did not need to go to the emergency department, $4 \%$ came due to symptoms compatible with COVID, and only $1.7 \%$ came because of cardiological worsening. $5.6 \%$ of our patients suffered from COVID infection. $2.3 \%$ of patients died, out of which $0.3 \%$ (1) were due to cardiopathies, $0.7 \%$ (2) from COVID-19 and $1.3 \%$ (4) from other causes. Conclusion: Telemedicine is a useful tool to support the monitoring of patients with stable heart disease. Our patients suffered a low percentage of cardiac decompensations and mortality, as well as a low COVID-19 infection and mortality rate.

\section{Abstract \# 178}

Ageism never gets old: preliminary results of an ongoing survey to understand prejudice and attitudes towards the elderly in the era of COVID-19

Caglar Cosarderelioglu, Aysenur Ceylan ${ }^{2}$, Emine Gemci ${ }^{1}$, Busra Yurumez Korkmaz ${ }^{1}$, Seher Yigit ${ }^{1}$, Volkan Atmis ${ }^{1}$, Ahmet Yalcin ${ }^{1}$, Sevgi Aras ${ }^{1}$, Murat Varli ${ }^{1}$

${ }^{1}$ Ankara University School of Medicine, Department of Internal Medicine, Division of Geriatrics, ${ }^{2}$ Ankara University School of Medicine, Department of Internal Medicine

Introduction: Ageism against the elderly differs from other forms of discrimination in that every human being is inevitably a subject of it as they live long enough. In the era of COVID-19, as the older population is under the spotlight, ageism can be encountered in various aspects of life. To assess society's prejudice and attitudes toward the elderly during the pandemic and to understand whether ageism is related to nonfactual information spread, we designed an online survey. To the best of our knowledge, this is the first study addressing these questions based on responses to a survey.

Methods: We designed a cross-sectional online survey consisting of 26 questions to assess/understand the perception and attitudes of Turkish population toward elderly during the pandemic. Among the questions, 5 of them were demographic, rest of them questioned false beliefs and community's source of information about COVID-19, attitudes to aged, and awareness of ageism.

Results: 550 participants enrolled in the study. $65.3 \%$ of them are women. $94.3 \%$ of participants are under 65 years old. $81.1 \%$ of participants never heard about ageism term. Surprisingly, $23 \%$ falsely believe that older people spread the virus more than young ones. There is a significant relation between not knowing ageism and false beliefs $(p=0.04)$. As can be expected, false information is more common at low education levels. On the other hand, $51 \%$ of respondents think that health services during the pandemic should be prioritized according to age with $35 \%$ thinking that $<55$ years should be favored. Finally, although almost everyone (\%92.4) agrees that elderly experience psychological distress during the pandemic, only $50 \%$ of respondents take action to reduce anxiety of their elderly relatives.

Key conclusions: A society is measured by how it cares for its "older" citizens' says WHO. In this regard, we tried to understand how our society approaches the elderly and cares for them throughout COVID-19 pandemic. Our results suggest that educating the society about facts and prevention of misinformation may reduce ageism. Finally, we believe that increasing awareness is our duty as healthcare providers until the next pandemic. 


\section{Abstract \# 179 \\ Geriatric giants present in the suspected COVID-19 population in Galway University Hospital}

Clodagh Marie McDermott ${ }^{1}$, Caoimhe Hanrahan ${ }^{1}$, Karen Dennehy ${ }^{1}$, Edel Mannion ${ }^{1}$, Ruairi Waters ${ }^{1}$, Michelle Canavan ${ }^{1}$

${ }^{1}$ Department of Geriatric Medicine, Galway University Hospital

The morbidity and mortality associated with the new coronavirus (COVID-19) is higher among the elderly. (1) Research has shown that employment of a comprehensive geriatric assessment (CGA) in the care of an acutely unwell older person, improves survival as it identifies co-existing conditions and guides management. (2) The aim of this review is to identify the geriatric syndromes or "giants" 3 affecting older individuals presenting to Galway University Hospital (GUH) with suspected or confirmed COVID-19 infection. This includes incontinence, constipation, malnutrition, delirium, falls and polypharmacy. A dedicated COVID-19 Geriatrics team was created. Criteria for admission under the care of this team included being aged $>75$ years and one or more of the following; confirmed COVID-19 infection, fever $>38 \mathrm{C}$, new or changed cough, new or increased dyspnoea, new or increased oxygen requirement, new opacifications on a chest $\mathrm{x}$-ray, nursing home resident. Data was collected on admission from this cohort between April and May 2020 via a developed CGA.133 patients were included in this evaluation. 50\% had urinary incontinence on admission with $37 \%$ reporting constipation. 41 patients had evidence of delirium on admission with 11 patients requiring chemical sedation. $27 \%$ had a poor nutritional status with only $3 \%$ having pressure ulcers. 16 patients suffered a fall on admission to GUH. Polypharmacy was the most prevalent geriatric syndrome affecting $96 \%$ of patients. A significant number of older patients admitted with confirmed or suspected COVID-19 had coexisting geriatric syndromes. Utilising the CGA allowed accurate assessment of, and care planning for, these older persons. (4) .

Bibliography:

1. Liu K, Chen Y, Lin R, Han K. Clinical features of COVID-19 in elderly patients: A comparison with young and middle-aged patients. Vol. 80, Journal of Infection. W.B. Saunders Ltd; 2020. p. e14-8.

2. Ellis G, Whitehead MA, Robinson D, O'Neill D, Langhorne P. Comprehensive geriatric assessment for older adults admitted to hospital: Meta-analysis of randomised controlled trials. BMJ. 2011 Nov 19; 343 (7832): 1034.

3. British Geriatrics Society. A giant of geriatric medicine - Professor Bernard Isaacs (1924-1995) Post 1 [Internet]. [cited 2020 Jun 8]. Available from: https://www.bgs.org.uk/a-giant-of-geriatricmedicine-professor-bernard-isaacs-1924-1995-post-14. National Clinical Programme for Older People. Comprehensive Geriatric Assessment A Summary Adapted from Specialist Geriatric Team Guidance on Comprehensive Geriatric Assessment National Clinical Programme for Older People (2016).

\section{Abstract \# 180 \\ Predictors of in-hospital mortality in very old patients with COVID-19: a monocentric retrospective study}

Aline Mendes ${ }^{1}$, Christine Serratrice ${ }^{2}$, François Herrmann ${ }^{3}$, Laurence Genton $^{4}$, Samuel Périvier ${ }^{3}$, Max Scheffler ${ }^{5}$, Thomas Fassier ${ }^{2}$, Philippe Huber $^{3}$, Marie-Claire Jacques ${ }^{3}$, Virginie Prendki ${ }^{2}$, Xavier Roux $^{2}$, Katharine Di Silvestro ${ }^{2}$, Véronique Trombert ${ }^{2}$, Stephan Harbarth $^{6}$, Gabriel Gold ${ }^{3}$, Christophe E. Graf ${ }^{7}$, Dina Zekry ${ }^{2}$

${ }^{1}$ Division of Geriatrics, University Hospital of Geneva, ${ }^{2}$ Divison of Internal Medicine for the aged, University Hospitals of Geneva, ${ }^{3}$ Division of Geriatrics, University Hospitals of Geneva, ${ }^{4}$ Clinical
Nutrition, University Hospitals of Geneva, ${ }^{5}$ Division of Radiology, University Hospitals of Geneva, ${ }^{6}$ Division of Infectious Diseases and Infection Control Program, University Hospitals of Geneva and Faculty of Medicine, ${ }^{7}$ Division of Internal Medicine and Rehabilitation, University Hospitals of Geneva

Introduction: Predictors of in-hospital mortality related to COVID19 in the oldest old are unknown but probably different from the general population.

Methods: Monocentric retrospective study of patients aged 65 years and older hospitalized in acute geriatric wards for COVID-19. Data from hospital admission was collected from the electronic medical records. Multiple logistic regression and Cox proportional-hazard models were used to predict mortality.

Results: Of a total of 235 Caucasian patients, $43 \%$ were male, with a mean age of $86 \pm 6.5$ years. Seventy-six patients (32\%) died during their hospital stay. Hypertension, cognitive impairment, heart failure, chronic kidney disease, and diabetes were the most prevalent comorbidities. Male sex was associated with mortality in this group but not differences in age. Non-survivors had a higher rate of heart failure $(p=0.044)$ and bilateral and peripheral infiltrates on chest radiographs $(\mathrm{p}=0.001)$. A multivariable Cox regression model showed that male sex (HR 3.84, 95\% CI 1.93-7.62, p = 0.0001), increased fraction of inspired oxygen (HR 1.06, 95\% CI 1.03-1.09, $\mathrm{p}$ $<0.0001)$ and the presence of crackles at clinical status performed at admission (HR 2.84, 95\% CI 1.23-6.57, $\mathrm{p}=0.0145$ ) were the best predictors of mortality, while the absence of functional decline was protective (HR 0.98, 95\% CI 0.97-0.99, $\mathrm{p}=0.0003$ ).

Conclusions: In the oldest old hospitalized for COVID-19 male sex, crackles, a need for a higher fraction of inspired oxygen and functional decline at admission were independent risk factors of mortality. These routinely collected parameters, and not differences in age, should be taken into account to evaluate prognosis in the oldest old hospitalized for COVID-19.

\section{Abstract \# 181}

Epidemiological aspects of COVID-19 in N. Macedonia-highest mortlity rate in elderly

Biljana Petreska-Zovic ${ }^{1}$

${ }^{1}$ PHI Specialized Hospital for Geriatric and Palliative Medicine, Skopje

Introduction: First case of COVID-19 in N.Macedonia was registred on February 26th 2020 - a female patient returning from Italy. Until June 7 th $0,14 \%$ of the population was infected or, in absolute number, 3025 people. Methods: statistical analysis of the official data from Ministry of health.

Results: On population 2083379 people, total cases 3025, active $1226(0,05 \%$ of the population), deaths 153 ( 73 per $1 \mathrm{M})$-case fatality rate $5,18 \%$ (global world case fatality rate $5,76 \%$ ). Recovered 1646 . Total tests 36325 (17 423 per $1 \mathrm{M}$ ). Demographic data: total cases 3025: by gender-male 1453 (48\%), female 1572 (52\%); by age-0-9 148 (5\%), 10-19 yrs 148 (5\%), 20-29 yrs 393 (13\%), 30-39 yrs 533 (17\%), 40-49 yrs 540 (18\%), 50-59 yrs 598 (20\%), 60+ yrs 665 (22\%). Total deaths 153: by gender: 100 male $(65.4 \%), 53$ female (34.6\%). By age: 20-29 yrs 2 (1 male, 1 female), 30-39 yrs 2 (2male), 40-49ysr 12 (10 male, 2 female), 50-59 yrs 33 (23 male, 10 female), 60-69 yrs 48 (33 male, 15 female), 70-79 yrs 39 (26 male, 13 female), 80-89 yrs 15 (11 male, 4 female), 90+ yrs 2 ( 2 male). Total deaths of patients $60+\mathrm{yrs}-104$ or $68 \%$ of all deaths.

Key conclusion: Although only $22 \%$ of total cases of COVID-19 in N.Macedonia are at the age of 60 and above, which is similar to the younger age groups, they have the highest mortality $-68 \%$ of total 
deaths. This imposes the necessity of strong preventive measures for this age group.

\section{Abstract \# 182 \\ Higer mortality excess was found in younger departments in France during the COVID-19 pandemic \\ Emmanuel Gonzalez-Bautista ${ }^{1}$, Claudia Lorena Vargas-Aleman ${ }^{2}$ \\ ${ }^{1}$ Gérontopôle, Toulouse University Hospital, France., ${ }^{2}$ Independent Researcher}

Background: Surveillance for COVID-19 in France included the mortality excess (ME) at sub-national levels. The most significant proportion of fatalities occurred in older adults. However, there has been little evidence using an ecological (aggregate) approach. We aimed to test if the departments with a higher proportion of older adults reached a higher mortality excess in France and if they maintained a mortality excess for longer during the pandemic period. Methods: We used the data published by Geodes Public Health aggregated at the department level ( $n=100$ for Metropolitan France). Five levels of mortality excess in the French population were defined comparing expected versus observed mortality (EuroMomo model, 16-22 March to 11-17 May of 2020). The departments were ordered based on their proportion of the population aged $60+$. We obtained simple and complex absolute and relative measures of inequality of the maximum level of ME and the number of weeks with ME.

Results: The maximum level of mortality excess of the youngest French departments was, on average, thrice that of the oldest departments. The youngest French departments reported mortality excess an average of 5 weeks longer than the oldest departments. Significant inequality was consistent using simple and complex, absolute and relative measures of inequality.

Conclusion: Highest levels of mortality excess, and during a more extended period, were found in departments with a relatively younger population. A higher proportion of older adults in the population might be associated with a third socio-economical "protective" variable. Further multidisciplinary analyses are necessary to explain these health inequalities.

\section{Abstract \# 183 \\ Clinical features in elderly patients with coronavirus disease (COVID-19) hospitalized in Guadalajara, Spain}

Carla Gamez Asuncion ${ }^{1}$, Maria José Martin Legorburo ${ }^{1}$, María Gebauer Campos ${ }^{1}$, Irene Bartolomé Martín ${ }^{2}$, Juan Rodríguez Solís ${ }^{2}$

${ }^{1}$ Internal Medicine Resident of Geriatrics. University Hospital of Guadalajara (Guadalajara, Spain), ${ }^{2}$ Medical Specialize in Geriatrics. University Hospital of Guadalajara (Guadalajara, Spain)

Background: The coronavirus disease (COVID-19), caused by novel coronavirus (SARS-CoV-2) occurs in persons of all ages but has specially impacted the elderly people.

Methods: Retrospective study of all patients older than 80 years with a diagnosis of SARS-COV-2 discharged from a University Hospital in Spain from March 1 to May 31, 2020.Results: 91 patients (100\% analized). Median age 84, $5(81,2-89,8)$. Women 37, 8\%. Institutionalized $32,2 \%$. Charlson median 2 (1-2). Independent $41,8 \%$, severe dependency $22 \%$; no cognitive decline $70,3 \%$, moderate dementia 19,7\%. Pneumonia 75, 8\%. Hidroxicloroquine + Azithromycin 66, 7\%; Lopinavir/Ritonavir + Hidroxicloroquine + Azithromycin 12, 1\%; steroids 42, 9\%; support treatment $19,8 \%$, heparin (LWMH) 88, 9\%; delirium 33, 9\%; exitus 32, $2 \%$.None association was found between mortality and age, gender, comorbidity, functional or mental condition. We only found relation with delirium and mortality (OR 1, 1: IC95\% 1, 5; p = 0, 003) but the presence of pneumonia or coagulation complications didn't increase the mortality. Heparin dose, steroids or the treatment elected also were not related with mortality.The treatment was associated with Barthel index $(\mathrm{p}=0,001)$.

Key conclussions: A third of the sample died during hospitalization. It is remarkable that we couldn't associate it with functional condition, only with presence of delirium. It could be consequence of the sample size. The treatment elected by the physician might be based on the previous condition and independence of the patient, with no differences in the prognosis.

\section{Abstract \# 184 \\ Old frequent users in a tertiary hospital Portuguese reality at a glance}

Guilherme Magalhaes ${ }^{1}$, Sara Dias ${ }^{2}$, Rita Alves ${ }^{2}$, Marilia Fernandes ${ }^{2}$, Diogo Drummond Borges ${ }^{2}$, Ines Rego Figueiredo ${ }^{2}$, Jorge Fernandes ${ }^{2}$, Pedro Fiuza ${ }^{2}$, Heidi Gruner ${ }^{2}$, Antonio Panarra ${ }^{2}$

${ }^{1}$ UNidade de Cuidados Paleativos, Centro Hospitalar Universitário Lisboa Central, ${ }^{2}$ Serviço de Medicina 7.2, Hospital Curry Cabral, Centro Hospitalar Universitário de Lisboa Central

Introduction: Expected death has no medical definition or consensus, but pertains to an acute or gradual clinical situation or condition that will not or cannot return or resume. The expected death and deterioration in the patient's health is often due to advanced disease, limitation of medical treatment and terminal illness, but the controversial emerges when some patients may prefer not to undergo lifesustaining treatments. The probability is associated with general indicators of decline and increasing needs, as the Gold Standard Framework Prognostic Indicator Guidance points.

Methods: Prospective descriptive study of the patients admitted to a tertiary Hospital with COVID during the Pandemia from 11 March to 31 May. Patients who died were included and grouped according to admittance to Intensive Care Units (ICU) and compared according to demographic data, frailty scales, palliative Performance Scale version 2 comorbidities, used medication, admittance to hospital in the previous 6 month, and average length of stay.

Results: There were 28 patients included, of those 16 were admitted to the ICU. The majority were between 75 and $85 \mathrm{y}$, none above $85 \mathrm{y}$. Average CFS was 2.6 vs 5.9, PPSV2 74.6 vs 44.7, PRISMA7 1.4 vs 4.2, Charlson index 1.6 vs 5.9 , number of drugs 4.8 vs 8.7 , recent admissions similar (2), lenght of stay 6.5 vs 7.2 . Of these only 1 patient, was the cause of death not related to COVID infection.

Discussion: In face of an unprecedented humanitarian crisis, research is important to evaluate and characterize the risk factors associated with the patients with expected death, and may contribute to better clinical decision and resource management. Our study showed that patients that died with higher disease burden were less likely to be admitted to the ICU.

\section{Abstract \# 185}

Association between delirium, impaired consciousness, and adverse outcomes in hospitalized patients with COVID-19.

Flavia Garcez ${ }^{1}$, Márlon Aliberti ${ }^{1}$, Thiago Avelino-Silva ${ }^{1}$

${ }^{1}$ University of Sao Paulo Medical School

Objectives: To investigate the occurrence of delirium and impaired consciousness in hospitalized patients with COVID-19 and explore their association with adverse outcomes. 
Methods: Longitudinal observational study in a tertiary university hospital dedicated to the care of severe cases of COVID-19 in Sao Paulo, Brazil. We included 707 patients aged $\geq 50$ years consecutively admitted to the hospital between March and May 2020. We completed detailed reviews of electronic medical records to collect our data. We identified delirium using the Chart-based Delirium Identification Instrument (CHART-DEL) and defined delirium as prevalent when present on admission. We also classified patients according to level of consciousness on admission using the Richmond Agitation-Sedation Scale. Our outcomes of interest were in-hospital death, length of stay, admission to intensive care, and ventilator utilization. We estimated the associations of delirium and impaired consciousness with outcomes using regression models adjusted for sociodemographic factors, Charlson comorbidity index, antihypertensive drugs, signs, and symptoms related to COVID-19.

Results: Overall, we identified delirium in 232 participants (33\%). On admission, $86(16 \%)$ were delirious, and 203 (29\%) had impaired consciousness. We observed 263 deaths (37\%) in our sample, and inhospital mortality reached $51 \%$ in patients who experienced delirium. Both prevalent delirium and overall delirium were associated with inhospital death, with an adjusted hazard ratio (aHR) of $1.81(95 \%$ confidence interval [95\% CI ] 1.25-2.62) and adjusted odds ratio (aOR) of 1.57 (95\% CI 1.02-2.42), respectively. In-hospital mortality in hyperalert, lethargic/stuporous and comatose patients was respectively $74 \%(\mathrm{aHR}=2.63 ; 95 \%$ CI $1.34-5.17), 56 \%(\mathrm{aHR}=2.53 ; 95 \%$ CI $1.55-4.14)$, and $74 \%$ (aHR $=3.35$; 95\% CI 1.61-7.0). Delirium was also associated with increased length of stay, admission to intensive care, and ventilator utilization.

Conclusion: Delirium and impaired consciousness were strongly associated with in-hospital death in adults aged $\geq 50$ years with COVID-19. Clinicians should include delirium and consciousness evaluations in their routine assessments of severity and prognosis in this population, despite the pandemic-related difficulties for patient care.

\section{Abstract \# 186 \\ Mental health, aggression, and COVID-19: impact of lockdown measures on mental health and domestic violence victimization in older adults}

Anne Nobels ${ }^{1}$, Egon Robert ${ }^{1}$, Lotte De Schrijver ${ }^{1}$, Sabine De Moor ${ }^{2}$, Joke Depraetere $^{2}$, Evelyn Schapansky ${ }^{2}$, Christophe Vandeviver ${ }^{2}$, Ines Keygnaert ${ }^{1}$

${ }^{1}$ International Centre for Reproductive Health, Department of Public Health and Primary Care, Ghent University, Ghent, Belgium,

${ }^{2}$ Department of Criminology, Criminal Law and Social Law, Ghent University, Ghent, Belgium

Introduction: To contain the COVID-19 pandemic, governments around the world restricted social and physical contact by issuing lockdown and social-distancing measures. Older adults, being considered a risk group for COVID-19, often follow more strict rules. Yet, lockdown measures may induce mental health problems and increase the occurrence of domestic violence (DV). We examine the effects of lockdown measures on mental health and DV in older adults during the COVID-19 pandemic.

Methods: In the first wave of this longitudinal cohort study, an online self-report questionnaire on mental health and aggression was administered to a non-probabilistic sample of participants living in Belgium, Germany and Portugal. Participants were sampled through national media, social media, and snowballing procedures. This Abstract reports on Belgian events that occurred between 13 March and 13 April 2020. The second wave of data-collection is currently ongoing.
Results: 358 participants aged 65 years and older were included in the analysis. $15 \%$ of respondents experienced DV with $14 \%$ experiencing psychological violence, $1 \%$ physical violence and $1 \%$ sexual violence. One respondent disclosed an (attempted) rape. Compared with non-victimized respondents, DV victims reported more stress $(54 \% \mathrm{v}$ $24 \%)$, more sedative use (35\% v 13\%) and showed more often symptoms of Acute Stress Disorder (33\% v 8\%).

Key conclusions: DV in older adults appears to be common, linked with lockdown measures due to COVID-19, and associated with adverse mental health outcomes. These findings highlight the possible need for public health measures and sociocultural changes preventing DV and improving mental health during lockdown in older adults.

\section{Abstract \# 187 \\ Compliance elderly to the directions to stay at home during the covid 19 lockdown in Greek province}

Martha Andreou ${ }^{1}$, Naili Tersenidou ${ }^{1}$, Ioanna Aggelidou ${ }^{1}$, Riola Tersenidou ${ }^{1}$, Eleni Paraskevopoulou ${ }^{1}$, Despoina Xatzipanagiotou ${ }^{2}$

${ }^{1}$ Health Center of Avdira Xanthis, ${ }^{2}$ Health Directorate of Xanthi Region

Introduction: The Prefecture of Xanthi is the second one in Greece in positive Covid 19 samples (over 200) and deaths (17). The present study recorded the compliance, of elderly patients in two Xanthi's Municipalities, to the instructions of EODY (National Public Health Organization) for quarantine from March 15 to May 31.Methods: Using a structured questionnaire, we recorded age, gender, presence of partner and children, education, income, disease information, how to procure medicines and food, if they spent Easter/Bayram holiday alone and consent to the strict measures taken.Results: 265 elderly agreed to participate, of which $63.4 \%$ were women aged $73.4 \pm 5.9$ years, the majority were married $(71.7 \%)$, compulsory educated $(45.7 \%)$ with more than two children $(78.4 \%)$ and income $<15000$ euros. Television was the main source of information (89.8\%), while $85.7 \%$ spent Easter/Bayram alone. The supply of medicines $(77,8 \%)$ and food $(40 \%)$ was made to order. There was a strong correlation between children's presence and elderly compliance $(\mathrm{p}=0.019)$. Almost all of them agreed with the strict measures taken (99.2\%). Key conclusions: We found a high rate of compliance of the elderly in the lockdown period which agrees with almost zero positive people in the region (3) in contrast to other Xanthi' s Municipalities. The elderly should be informed to comply with personal protective measures by their family physicians as there is a large viral load in the area.

\section{Abstract \# 188}

Was COVID already spreading in Belgium on 25th of February?

Hilde Baeyens ${ }^{1}$, J. P. Baeyens ${ }^{1}$, Christine Balcaen ${ }^{2}$, Leen Derez ${ }^{1}$, Anne Piette ${ }^{3}$, Kyri Van Hecke ${ }^{1}$

${ }^{1}$ AZ Alma, Eeklo, ${ }^{2}$ Bassevelde, ${ }^{3}$ Az Alma, Eeklo

On 3rd of February, the first Belgium COVID-patient arrived from Wuhan. On the 29 of February, a citizen returning from France was COVID-positive. On 13th of March, Belgium went in lock down. On 25th of February, a 90-year old lady was admitted to our geriatric ward. She had anorexia for 1 week and gait problems. She was known with bronchiëctasia and coughing. On admission (day 8 from beginning of symptoms) crp was $463 \mathrm{mg} / \mathrm{dl}$, mild lymphopenia $\left(1,16.10^{3}\right.$ / $\mu \mathrm{L}$ ) was present, oxygen saturation $95 \%$ (2-liter oxygen per minute). She was treated with beta-lactam antibiotics, moxifloxacin, and antithrombotic prophylaxis. On day 11, she developed bilateral 
pneumonic infiltrates with high oxygen demand. Droplet isolation was installed. Because of persistent fever on day 16, a naso-pharyngeal swab for PCR COVID and other respiratory pathogens (virus, bacteria, fungi) was negative. On day 26, her lymphopenia disappeared, and oxygen demand reduced. She went home on day 66, still needing oxygen and not able to walk due to severe sarcopenia. On day 86 , she was referred to our geriatric day hospital. COVID-IgG antibodies were slightly positive: 7, $6 \mathrm{AU} / \mathrm{ml}$. Refrigerated serum of day $32(19 / 3)$ was strongly positive: $76,6 \mathrm{AU} / \mathrm{ml}$. Her grandson went skiing in Livigno (Lombardy) from 7th till 13th February and joined the football match in Rome on 18th of February (AA Gent - AS Roma). At home he got respiratory symptoms and on 17/3 he stayed at home in quarantine. Conclusion: On 25th of February, COVIDdisease was already in our hospital in Eeklo, Belgium.

\section{ABSTRACT \# 189}

The Multidimensional Prognostic Index (MPI) For The Prognostic Stratification Of Older Inpatients With COVID-19: a multicenter study of the italian Geriatric Society Hospital and Community (SIGOT)

Alberto Pilotto ${ }^{1}$, Nicola Veronese ${ }^{2}$, Margherita Azzini ${ }^{3}$, Giovanni Cenderello ${ }^{4}$, Alberto Castagna ${ }^{5}$, Andrea Pilotto ${ }^{6}$, Romina Custureri ${ }^{7}$, Simone Dini ${ }^{7}$, Sara Tita Farinella ${ }^{4}$, Giovanni Ruotolo ${ }^{5}$, Andrea Padovani ${ }^{6}$, Alberto Cella ${ }^{7}$

${ }^{1}$ 1) Department Geriatric Care, Orthogeriatrics and rehabilitation, Galliera Hospital, Genova Italy; 2) Department of Interdisciplinary Medicine, University of Bari, Italy, ${ }^{2} 3$ ) Department of Primary Care, District 3, ULSS 3, Venice, Italy, ${ }^{3} 4$ ) Geriatrics Unit, “” Mater Salutis “" Hospital, Legnago ULSS 9 Scaligera, Verona, Italy, ${ }^{4} 5$ ) Infectious Disease Unit, Sanremo Hospital, ASL 1 Imperiese, Sanremo, Italy, ${ }^{5}$ 6) Geriatrics Unit, "Pugliese Ciaccio" Hospital, Catanzaro, Italy, ${ }^{6} 7$ ) Neurology Unit, Department of Clinical and Experimental Sciences, University of Brescia, Italy, ${ }^{7} 1$ ) Department Geriatric Care, Orthogeriatrics and rehabilitation, Galliera Hospital, Genova Italy “

Background: The recent diffusion of the coronavirus-19 disease (COVID-19) has reached proportions to be declared a pandemic disease, particularly in older people. This pandemic created important issues in terms of prognostic evaluation. Multidimensional prognostic index (MPI) is widely used as prognostic tool in geriatric medicine. However, there are no studies yet regarding the use of MPI to predict prognosis in older COVID-19 patients.

Methods: Older hospitalized patients (age $>65$ years) affected by COVID-19 across five Italian hospitals were included from 26th April to 19th June 2020. At baseline, a comprehensive geriatric assessment was made and the MPI calculated accordingly. Clinical, bio-humoral and instrumental information related to COVID-19 were also collected. A follow-up period was proposed.

Results: 104 Italian participants hospitalized for COVID-19 (mean age: $81.1 \pm 8.0$; range: $65-95$ years; females $=66.3 \%$ ) were included Their mean MPI value was $0.56 \pm 0.25$, with $21.4 \%$ in the MPI low risk, $28.2 \%$ in the moderate and the $45.6 \%$ in the high risk MPI category. Among clinical signs investigated, cough was significantly more common in the MPI 1 compared to the MPI 3 category (63.6 vs. $27.7 \%, p=0.003$ ). Regarding vaccination history, people in MPI presented a higher prevalence of anti-pneumococcal vaccination $(22.7 \%$ vs. $4.3 \%, p=0.02)$ than those in MPI 3. Finally, regarding therapeutically approach, people in MPI 1 were more frequently treated with anti-retroviral medications than their counterparts $(\mathrm{p}=$ 0.03 ) and were more frequently exposed to non-invasive ventilation (54.5 in MPI 1 vs. $10.6 \%$ in MPI 3, p = 0.003).

Conclusions: Older people hospitalized for COVID-19 have a high prevalence of frailty making this condition of importance for clinical- decision making. Physicians had the propensity of using more frequently non-invasive ventilation and anti-retroviral medications in less frail patients. The prognostic role of anti-pneumococcal vaccination should be explored during the follow-up period.

\section{Abstract \# 190}

Awareness of caregivers of elderly patients for COVID-19 pandemia

\section{Guzin Cakmak $^{1}$, Zeynel Abidin Ozturk ${ }^{1}$}

${ }^{1}$ Gaziantep University, Faculty of Medicine, Department of Internal Medicine, Division of Geriatrics

Introduction: During the COVID-19 pandemic, the disease caused by the new coronavirus, older people are especially vulnerable to severe illness. Caregiving to elderly patients become harder than before, and being well-informed is more critical in this duration.

Methods: This cross-sectional study was conducted for a period of 2 months, from March to May 2020. A questionnaire interrogated awareness and knowledge levels of caregivers. The questionnaire has consisted of general information about pandemic and demographic data. Participants answered the questions from the internet. SPSS-22 was used for data analysis.

Results: The study population was composed of 79 women and 21 men. Sixty-nine percent of the patients were suffered from dementia. $30 \%$ of the participants were responsible for caregiving for more than 10 years. $76 \%$ of the caregivers were willing to take more information. $33 \%$ of the caregivers were taking the information from a single source. General knowledge status about pandemics were found to be correlated to education level ( $\mathrm{p}<0.01, \mathrm{r}=0.397)$.

Key conclusions: Caregiving to elderly patients is an essential responsibility in the pandemic. Education levels of caregivers could affect the health status of geriatric patients in this duration. Prospective studies will be better to explain the relationship between health outcomes for geriatric patients and the education levels of their caregivers.

\section{Abstract \# 191 \\ Diagnosis, surveillance and control in the transition phase of the pandemic covid19 in Castilla y León (Spain)}

Leticia Sierra_martínez ${ }^{1}$, Iker Sierra-Martínez ${ }^{2}$, Rosario MartínezFuerte $^{3}$, Gonzalo Calderon-Calvo ${ }^{3}$, Natalia Sanz-González ${ }^{4}$

${ }^{1}$ Valladolid Este Primary Assistance Gerency, Valladolid, Spain,

${ }^{2}$ Traumatology Department, Hospital of Medina de Campo

(Valladolid), Spain, ${ }^{3}$ Pilarica Health Center, Valladolid Este Primary Assistance, Valladolid, Spain, ${ }^{4}$ Parquesol Senior Center

In the community of Castilla y León a diagnosis, surveillance and control strategy is being established in the transition phase of the COVID-19 pandemic, of the patients and their close contacts. In all Rural and urban health centers, they will have a Public Health manager who will guarantee that the epidemiological follow-up of cases of COVID19 and contacts of the Health center is adequate.Result of this monitoring on June 20, 2020: Population: 2,320,242 Possible cases last 7 days: 673Possible cases last 14 days: 1354 Incidence c/10000 inhabitants. Possible cases last 7 days:2.9 Incidence c/10000 inhabitants. Possible cases last 7 days: 5.8 Patients with per performed last 7 days: 8735 Patients with pcr performed last 14 days: 19,664 No. of PCRs/possible cases in the last 7 days: 13 No. of PCRs/possible cases last 14 days: 14 Suspicious cases with 1st PCR in the last 7 days 634 Suspected cases with 1st PCR in the last 14 days: $1255 \%$ Suspected cases with PCR performed in the last 7 days: 
94\% Suspected cases with PCR performed in the last 14 days:93Patients with pcr + last 7 days:134 Patients with per + last 14 days: 292PCR Incidence Rate $+/ 10000$ inhabitants. Last 7 days:0.58PCR Incidence Rate $+/ 10,000$ inhabitants. Last 14 days: 1.26.

Conclusion: The incidence of covi-19 in Castilla y León not only remains at the lowest figures in recent months, but also begins to decrease in the number of infected people showing the light at the end of the tunnel.

\section{Abstract \# 192 \\ Characteristics of patients older than 90 years hospitalized for COVID-19.}

Sasha Botbol ${ }^{1}$, Maria Jesus Molina ${ }^{2}$, Carmen Navarro ${ }^{2}$, Maria Rosario Garcia ${ }^{2}$, Concepcion Lobato ${ }^{2}$, Soledad Dominguez ${ }^{2}$, Maria De la Puente ${ }^{2}$, Lourdes Evangelista ${ }^{2}$, Karina Quiñones ${ }^{2}$, Jaime Rodriguez $^{2}$

${ }^{1}$ Hospital Severo Ochoa Leganes, Madrid., ${ }^{2}$ Hospital Severo Ochoa Leganes, Madrid

Introduction: SARS-COV2 infection presents a wide spectrum of clinical presentation, ranging from mild to severe symptomatic forms. Most fatal cases occurred in the older population or with associated medical comorbilities.

Objectives: To describe the characteristics of patients older than 90 years who were hospitalized for COVID-19.

Materials and Methods: Descriptive, cross-sectional study of patients over 90 years, who were admitted at the Hospital Severo Ochoa, Madrid, from March to May 2020. Variables analyzed: Demographics: (Age, Sex, Social status), Functional: Barthel Index (B.I) and Functional Ambulation Classification (FAC), Mental: dementia, Clinical: Charlson Index (Ch.I), and In-hospital mortality. Statistical Analysis: SPSS V25.

Results: 58 patients.Mean age $92.67 \pm 2.80,65.5 \%$ women. $58.9 \%$ lived at home.Mean Ch.I of $2.55 \pm 1.60$, the mean number of drugs $8.98 \pm 3.33$. FAC 5: $43.3 \%$, with B.I mean 61.80 . $67.2 \%$ had no previous diagnosis of dementia. Dyspnea was the main symptom $58.6 \%$. In the emergency area, $46.6 \%$ required oxygen therapy, and $72.4 \%$ at admission. The nasopharyngeal PCR result positive in 93.1\%. Mean value of DDimer 3.12; Ferritin 585; LDH 236 and PCR 87. Findings in the chest X-ray evidenced a bilateral infiltrate in 45.6\%.The most used treatments were: Dolquine 87.9\%, Azithromycin $65.6 \%$, Kaletra 39 , 6\%, corticosteroids $35 \% .30 .3 \%$ required oxygen therapy at home. In-hospital mortality was $32.8 \% .36 .8 \%$ presented at least one admission in the following 2 months.

Conclusions: In our sample, patients over 90 years, presents a good functional situation and a lower rate of dementia. Mortality rate in this group is high.

\section{Abstract \# 193 \\ COVID 19 experience in a geriatric Hospital in Italy \\ Massimiliano Fedecostante ${ }^{1}$, Valentina Cesari ${ }^{1}$, Moira Lucarelli ${ }^{1}$, Antonia Scrimieri ${ }^{1}$, Mariagiovanna Cozza ${ }^{1}$, Erika Ciarrocchi ${ }^{1}$, Barbara Carrieri ${ }^{1}$, Fabrizia Lattanzio ${ }^{2}$, Antonio Cherubini ${ }^{1}$ \\ ${ }^{1}$ Geriatria, Accettazione Geriatrica e Centro di ricerca per l'invecchiamento, IRCCS INRCA, Ancona, Italy, ${ }^{2}$ Lattanzio Scientific Direction, IRCCS INRCA, Ancona, Italy}

Introduction: SARS-CoV-2 infection might lead to severe disease especially in older adults. During the SARS-CoV-2 epidemic our geriatric hospital was specifically dedicated to the care of older patients with COVID-19. The aim of this study is to present our clinical experience.

Methods: We analyzed data of all COVID-19 patients admitted to our hospital between March and April 2020. All the main clinical, laboratory and instrumental findings were collected.

Results: we evaluated 147 patients. Seventy patients were male (47.6\%), 77 female (52.4\%). Mean age: 86.7 (range 70-100). Mean length-of-stay 18.1 days (range 0-56). Forty four (29.9\%) were completely disabled. Half of the patients were suffering from cognitive decline/dementia $(73,49.7 \%)$. During the hospitalization 79 $(53.7 \%)$ patients died. In a preliminary analysis only cognitive status significantly influence in-hospital mortality (OR for cognitive decline/ dementia: 2.39 CI 1.15-4.97).

Key conclusions: Our preliminary analysis showed that among older adults, age itself seemed not to influence outcomes of COVID-19 patients, while other factors such as cognitive status are involved. Further analysis including more clinical, laboratory and instrumental data will be conducted.

\section{Abstract \# 194}

Physical-social activity, and social support during lock-down period because of Covid-19 pandemic in a city center

Sumru Savas ${ }^{1}$, Asli Kilavuz ${ }^{2}$

${ }^{1}$ Geriatrics Section, Internal Medicine Department, School of Medicine, Ege University, Izmir, Turkey, ${ }^{2}$ Geriatrics Section, Internal Medicine Department, School of Medicine, Ege University, Izmir, Turkey

Aim: In Post- Pandemic Era older people stayed at home because of COVID-19 pandemic, and they faced problems such as loneliness, anxiety, limited access to health care. We investigated older people in terms of social support, social activity, and physical activity (PA) during the lock-down.

Methods: Older people aged $\geq 65$ years were enrolled. Participants completed online questionnaire prepared by researchers (or a relative of elderly). The questionnaire included age, gender, education, social activity, exercise, and leisure time activity, diseases, medications, and COVID-19 related questions, and SARC-F.

Results: A total of 137 people $(63.5 \%$ males) were included. During lock-down, they were with spouse $(57.7 \%)$, alone $(16.1 \%)$, caregiver $(2.2 \%), 47.4 \%$ were visited by relatives (outside with social space and mask (19\%), at home with social space $(6.6 \%)$, at home social space and mask (19.7\%)). Only $14.6 \%$ had difficulty in getting their needs, and $78.8 \%$ got help from others, $7.3 \%$ did not make video calls/or meetings. Regular PA was made by $43.1 \%$. Weight did not change $(48.9 \%)$, increased $(34.3 \%)$, decreased $(13.1 \%)$. They feel better (3.6\%), the same $(56.9 \%)$, weaker $(39.4 \%)$ than in prepandemic period in terms of muscle strength. After lock-down has ended, they feel no difference $(44.5 \%)$, tired $(27.7 \%)$, anxious (16.8\%). $1.5 \%$ were diagnosed Covid-19 positive, and $0.7 \%$ were hospitalized. All of participants had SARC-F scores $\geq 4$.

Conclusion: Though most of older people in our sample indicated sufficient social support, and careful social activity, and regular PA, they all had high SARC-F scores which are predictive of sarcopenia and poor outcomes. 


\section{Abstract \# 195 \\ The geriatric population COVID-19 observational study (GeroCovid): protocol and update of an ongoing multisetting, multipurpose and multicentric initiative}

Susanna Del Signore ${ }^{1}$, Caterina Trevisan ${ }^{2}$, Stefano Fumagalli ${ }^{3}$, Pietro Gareri $^{4}$, Alba Malara ${ }^{5}$, Enrico Mossello ${ }^{3}$, Stefano Volpato ${ }^{6}$, Gianluca $\mathrm{Zia}^{1}$, Raffaele Antonelli Incalzi ${ }^{7}$

${ }^{1}$ Bluecompanion 1td, London, UK, ${ }^{2}$ Geriatrics Division, Department of Medicine (DIMED), University of Padua, Italy, ${ }^{3}$ Geriatric Intensive Care Unit, Department of Experimental and Clinical Medicine, University of Florence, Italy, ${ }^{4}$ Center for Cognitive Disorders and Dementia - Catanzaro Lido, ASP Catanzaro, Italy, ${ }^{5}$ Scientific Committee of National Association of Third Age Residences (ANASTE) Calabria, Lamezia Terme (Catanzaro), Italy, ${ }^{6}$ Department of Medical Science, University of Ferrara, Ferrara, Italy, ${ }^{7}$ Unit of Geriatrics, Department of Medicine, Campus Bio Medico University and Teaching Hospital, Rome, Italy

Introduction: Despite the growing evidence on COVID-19, there are still many gaps in the understanding of this disease, especially concerning older people, who are the most affected by the pandemic.

Methods: The GeroCovid e-Registry is a European de-identified clinical data electronic registry of COVID-19 patients aged $\geq 60$ years, observed retrospectively or prospectively since March 1st, 2020. This study has been promoted by the Italian Society of Gerontology and Geriatrics and involves investigational sites across Italy, Spain and Norway. GeroCovid will explore the impact of COVID-19 in the following care settings: acute wards, nursing home, geriatric outpatient and home care, and dementia outpatient clinic. A common framework, adapted for the data collection in different settings, will evaluate: risk factors, clinical presentation and outcomes in COVID-19 inpatients; best strategies to prevent contagion in nursing home; impact of COVID-19 and social isolation on emotional, neuropsychological, functional and physical health; and, effects of telemedicine on health and management of dementia patients.

Results: As of June 30th, 70 investigational sites have obtained their Ethical Committee approval and 477 cases (age $82.9 \pm 11.0$ years; $51 \%$ men) have been recorded in the e-registry. The current inclusion rate is $9-10$ patients/day. The clinical follow-up of prospective cases and data collection will end on September 30th and preliminary results will be available in October 2020 .

Key conclusions: GeroCovid will explore relevant aspects of COVID-19 with high-quality and comprehensive data on older adults' health. This will help optimizing COVID-19 prevention and management, with practical implications for ongoing and possible future pandemics.

\section{Abstract \# 196 \\ Covid-19 outbreak in a geriatric department. An observational Study}

Solvejg Henneberg Pedersen ${ }^{1}$

${ }^{1}$ Holbaek Sygehus

Covid-19 outbreak in a geriatric ward-an observational study Solvejg Henneberg Pedersen, MD (1), Aras Kamal Mohammad Ali, MD (1), Ida KAppel Buhl, MD, ph.d (1), Louise B. Jessen, MD (1), Susanne Dam Nielsen, MD, DMSc, professor 2)1: Geriatric Section, Medical Department, Holbaek Hospital 2: Department of infectiousDsease, Rigshospitalet

Introduction: Covid19 affects everyone, but outcome severity has been reported to be worse in elderly and comorbid patients. Geriatric patients are known to have atypical disease presentation that might impair timely diagnosis.

Material: During a period spanning from March 23rd to April 21nd 2020 a geriatric ward experienced an outbreak of Covid19. The ward had 9 hospital rooms and a total capacity of 24 beds. On April 21st the ward was shut down, all patients and staff were tested, and patients transferred to other units.

Result: At total of 12 patients was tested SARS-CoV-2 positive during the period or on readmission within 7 days after discharge. The outbreak was located to 3 of the 9 hospital rooms, one single-occupancy room (SOR) and two quadruple-occupancy rooms (QOR). No detectable transmission from the patient in SOR was identified. In one QOR (Room A) a total of 6 patients were tested positive (5 being clinically probable index cases), and in the other QOR (Room B) a total of 5 patients were tested positive (1 being a probable index case). Of the 12 patients 6 had been tested negative at admission, 5 of these were among probable index cases. Of the 12 patients 3 were found at the screening on April 21st. Of the 12 patients 6 had atypical/no symptoms of Covid-19 disease.

Discussion: Prevention of disease spread depends on testing being accurate. The high number of probable false negative tests at admission in this geriatric population increases risk of transmission to other patients.

\section{Abstract \# 197 \\ The Role of Hospital-at-Home Service in surveillance of COVID- 19 elderly patients}

Catarina Cabral ${ }^{1}$, Maria José Campos ${ }^{1}$, Maria Manuela Soares ${ }^{1}$, Nuno Pinheiro $^{1}$, Vanisa Rosário ${ }^{1}$, Margarida Salgueiro ${ }^{1}$, Carla Fernandes ${ }^{1}$, António Carvalho ${ }^{1}$, Kamal Mansinho ${ }^{1}$

${ }^{1}$ Centro Hospitalar de Lisboa Ocidental

Introduction: Health services faced reorganization during COVID19. The Hospital-at-Home Service (HHS) followed COVID-19 patients through a daily phonecall and test making. The elderly population was one of our major concerns.

Material and Methods COVID-19 patients $>65$ years old (YO) were organized by type of follow-up: home, routine procedure, active hospitalization and after discharge, from March 15th to June 29th, 2020. A demographic characterisation was carried out, using Microsoft Office - Excel 2013.

Results: HHS followed 419 patients: 73 (17.4\%) were $>65$ YO, 31 men (42.4\%; M: 72, 3YO) and 42 women (57.5\%; M: 74.9 YO). At the Emergency Room (ER), 30 patients (40.5\%; M: 72.2 YO) tested positive, with no admission criteria: 12 men (40.0\%; M: $70.3 \mathrm{YO})$ and 18 women (60.0\%; M: 75.1 YO). Two of these patients were sent back to the ER through quick articulation with first responders for worsening of symptoms: males, 76 and 71 YO. Only one survived. Six of the 73 elderly (8.33\%; M: 72.3 YO) tested positive on ambulatory. Of the 419 patients, 86 were admitted to the hospital: 37 (43.0\%) were $>65$ YO (16 men (43.3\%; M:73.8YO) and 21 women (56.8\%; M:7 5.3 YO)). None were re-admitted. Microbiologic cure was sought after discharge: 28 patients are cured. One patient died after discharge at home.

Conclusions: Almost $20 \%$ of the patients on surveillance were elderly, a frail age group and at higher risk of intrahospital complications. HHS avoided unnecessary hospitalizations and assured a close surveillance and rapid response to clinical deterioration. It was an asset during the COVID-19 pandemic when outpatient support is scarce. 


\section{Abstract \# 198}

COVID-19 infection in home-cared versus nursing home geriatric patients: Protocol

David Prescott ${ }^{1}$, Ana Isabel Brochado ${ }^{1}$, Vânia Pereira ${ }^{1}$, Maria João Serpa $^{1}$, André Rodrigues ${ }^{1}$, Olga Pereira ${ }^{1}$, Vasco Evangelista ${ }^{1}$, Raquel Almeida ${ }^{1}$, Lia Marques ${ }^{1}$, José Lomelino Araújo ${ }^{1}$

${ }^{1} \mathrm{HBA}$

Coronavirus disease (COVID-19) is an infectious disease caused by the newly discovered 2019 coronavirus. It was named Severe Acute Respiratory Syndrome Coronavirus 2 (SARS-CoV-2) due to its' capability to induce an extreme systemic inflammatory response, particularly in young adults. However, the geriatric population is particularly affected by this novel infection, given its' established fragility and high rate of comorbidities. This is a retrospective observational study protocol. We plan to include all the elderly adults (> 65 years old) admitted in the COVID-19 dedicated ward of a district hospital, diagnosed with SARS-CoV-2 infection, from March to June 2020. We will analyse variables as continuous numerical or categorical values, as appropriate. All analyses will be presented with a 95\% confidence interval. Parametric or non-parametric tests will be used according to population distribution. All quantitative data will be qualitatively interpreted. Statistical significance will be considered if Pearson $<0.05$. Subgroup and sensitivity analysis will be done as needed.We will compare Home-cared with Nursing Home geriatric patients and analyse all data on percentage of total COVID admissions, symptoms at arrival, lab analysis, oxygen needs, therapy, number of days of active and social admission, disease complications, final outcome, functional status, dependence in activities of daily living and frailty scores. We also plan to collect data on adverse events of therapy in both groups.We plan to interpret the impact this pandemic has produced in older populations, while also identifying the needs, differences and disease burden these patients have according to residence at admission.

\section{Abstract \# 199 \\ Which parameters affected caregiver awareness in COVID-19 pandemic? \\ Guzin Cakmak $^{1}$, Zeynel Abidin Ozturk ${ }^{1}$ \\ ${ }^{1}$ Gaziantep University, Faculty of Medicine, Department of Internal Medicine, Division of Geriatrics}

Introduction: COVID-19 (new coronavirus) pandemic inversely affected too many areas world-wide such as health care, economics, industry, tourism, etc. In this study, we aimed to clarify the parameters which influenced the attachment of caregivers to measures.

Methods: This cross-sectional study was conducted for a period of 2 months, from March to May 2020. A questionnaire interrogated awareness levels of caregivers for the COVID-19 pandemic. The questionnaire consisted of general information about pandemic and demographic data. Participants were asked for this questionnaire on the internet and telephone conversation. We also evaluated the life quality of caregivers with EQ-5D. SPSS-22 was used for data analysis.

Results: The study population was composed of 79 women and 21 men. Caregivers aged between 25 and 39 were more adherent to social isolation measurement $(\mathrm{p}=0.031)$ than others. The worst supporter of this measure was aged $18-24(\mathrm{p}=0.001)$. The participants used more than one resource for being informed about the COVID-19 pandemic were more adapted to distance education than others $(p=0.022)$. They also supported the home office style working than others $(\mathrm{p}=0.039)$. Caregivers that were willing to gain more information about pandemics were more adherent to social isolation ( $\mathrm{p}$ $<0.01)$. The life quality of the caregivers who were afraid of infecting their patients was diminished significantly $(\mathrm{p}<0.01, \mathrm{r}=$ 0.310).

Conclusion: For the management of pandemic, public awareness, and their demand to adhere to preventive measures are crucial. Health care workers are in a vital position for informing the public, mostly caregivers. More studies could be useful in this area.

\section{Abstract \# 200 \\ Symptoms and final diagnoses of patients referred to one of the Covid-19 dedicated hospitals in Poland- a cross-sectional study}

Agnieszka Kasiukiewicz ${ }^{1}$, Zyta Beata Wojszel ${ }^{1}$, Miroslaw Kazberuk ${ }^{2}$, Julita Sitkiewicz ${ }^{3}$, Julita Olszewska ${ }^{4}$

${ }^{1}$ Department of Geriatrics, Medical University of Bialystok, Hospital of the Ministry of Interior and Administration in Bialystok, Bialystok, Poland, ${ }^{2}$ Medical University of Bialystok, Hospital of the Ministry of Interior and Administration in Bialystok, Bialystok, Poland, ${ }^{3}$ Hospital of the Ministry of Interior and Administration in Bialystok, Bialystok, Poland, ${ }^{4}$ Hospital of the Ministry of Interior and Administration in Bialystok, Bialystok, Poland

Objective: The health care system in Poland has been adapted to SARS-CoV2 outbreak and 22 hospitals were transformed into facilities dedicated to patients with Covid-19. The aim of the study was to characterize patients referred to one of them as suspected/confirmed to have Covid-19.

Methods: A cross-sectional study of patients referred to the Hospital of the Ministry of Interior and Administration in Bialystok (160 beds) between 14th Apr and 30th June 2020.

Results: A total of 193 patients (median 65y, IQR-46; 79) were referred to the hospital in the study period, 33 of whom required medical assistance while in quarantine. $114(59 \%)$ of them were in need for hospitalization because of general health status (median $73 \mathrm{y}$, IQR-60, 5; 83). $80(41 \%)$ were brought by the ambulance, $48(25 \%)$ transferred from other hospitals, $23(12 \%)$ referred by GPs, and 35 $(18 \%)$ came without a referral. The main primary diagnoses were: fever $(37 \%)$, pneumonia (22\%), stenocardia or dyspnea $(23 \%)$, and trauma (6\%). 40 (21\%) patients had no symptoms typical for Covid19. Only 26 patients $(16 \%)$ were confirmed with RT PCR test as Covid-19 cases, 22 of whom were hospitalized. Other infectious diseases and acute cardiologic disorders proved to be the cause of the symptoms in the majority of cases.

Key conclusions: In most patients referred during an epidemic to a studied hospital dedicated to Covid-19, no Sars-CoV 2 infection was confirmed. Those requiring hospitalization were significantly older, and symptoms that led to admission were caused mainly by other infections or acute cardiac conditions.

\section{Abstract \# 201 \\ Medical burden and origin of infection of geriatric Covid-19 patients-a cross-sectional study}

Agnieszka Kasiukiewicz ${ }^{1}$, Zyta Beata Wojszel ${ }^{1}$, Miroslaw Kazberuk $^{2}$, Julita Sitkiewicz ${ }^{3}$, Julita Olszewska ${ }^{4}$

${ }^{1}$ Department of Geriatrics, Medical University of Bialystok, Hospital of the Ministry of Interior and Administration in Bialystok, Bialystok, Poland, ${ }^{2}$ Medical University of Bialystok, Hospital of the Ministry of Interior and Administration in Bialystok, Bialystok, Poland, ${ }^{3}$ Hospital of the Ministry of Interior and Administration in Bialystok, Bialystok, 
Poland, ${ }^{4}$ Hospital of the Ministry of Interior and Administration in Bialystok

Objective: To study characteristics of hospitalized patients diagnosed with Covid-19, to evaluate their medical outcomes and origin of infection.

Methods: A cross-sectional study of patients diagnosed with Covid19 and admitted to the Covid-19 dedicated Hospital in Bialystok. Data on patients' medical history, functional state, and possible origin of infection were collected in the period 14 Apr-30 Jun 2020.

Results: A total of 26 of 193 patients referred to the hospital during the study period were diagnosed with Covid-19, 22 of them were admitted to the hospital; median age-71 $(64 ; 85)$ years. They were burdened with chronic disorders: hypertension $(63 \%)$, heart failure $(55 \%)$, coronary artery disease $(41 \%)$, and diabetes $(18 \%) .4$ patients had symptoms of delirium during hospitalization. $8(31 \%)$ patients required intensive care, and they were older (median age 83, 5 (63-93) y vs $70(57 ; 80)$ y), with a higher number of co-morbidities; 3 patients died. Only $10(48 \%)$ of hospitalized patients were independent in IADL (all patients $<60$ y.o. and $35 \%$ of geriatric patients) and got infected with Covid-19 from the spouse (2), friends (2) or from an unknown source (6). Disabled older persons, not able to walk outside the home, got infected from family, including those banned together in quarantine (8), caregivers (2), and in a palliative care facility (2). Two older persons consequently avoided social contacts, however, they were infected by caregivers.

Key conclusions: Hospitalized Covid-19 patients are vulnerable and mainly geriatric population. Particular attention should be paid to appropriate strategies for protecting these persons from infection as, as they get infected mainly from family and caregivers.

\section{Abstract \# 202}

Analysis of the impact of the 2019 coronavirus epidemic (CoViD19) on the presentation and crowding of patients for minor traumas at Lombardy ED

Gabrieleq Savioli ${ }^{1}$, Iride Francesca Ceresa ${ }^{1}$, Paolo Maggioni ${ }^{2}$, Elena Novara $^{2}$, Tonia Persiano ${ }^{2}$, Francesca Grulli ${ }^{2}$, Viola Novelli ${ }^{3}$, Alba Muzzi $^{3}$, Giovanni Ricevuti ${ }^{2}$, Enrico Oddone ${ }^{4}$, Maria Antonietta Bressan $^{5}$

${ }^{1}$ Emergency Department, IRCCS Policlinico San Matteo, 27100 Pavia, Italy, ${ }^{2}$ University of Pavia, 27100 Pavia, Italy, ${ }^{3}$ Medical Direction, IRCCS Policlinico San Matteo, 27100 Pavia, Italy, ${ }^{4}$ Assistant Professor, Department of Public Health, Experimental and Forensic Medicine, University of Pavia, 27100 Pavia, Italy., ${ }^{5}$ Past Director Emergency Department, IRCCS Policlinico San Matteo, 27100 Pavia, Italy.

Background: Italy's 2019 coronavirus epidemic (CoViD-19) originated in southwestern Lombardy, on February 21, 2020. The Fondazione IRCCS Policlinico San Matteo di Pavia has been involved in the management of the outbreak since its beginning. All the hospitals involved have experienced a huge increase in crowding, while witnessing a reduction in the presentation of minor emergencies. These had been pointed out by some authors as one of the causes of crowding and included in the crowding input factors.

Purpose: As part of a study that wanted to assess the impact of the CoViD pandemic on geriatric minor emergencies we evaluated the geriatric population that went to the ER for minor trauma.

Materials and Methods: We evaluated all patients aged 75 years and older who accessed our emergency room for minor traumas from February 22 to May 1, 2020 and during the same period of the previous year.

Results and discussion: We enrolled 397 patients. There was a severe reduction in the total number of accesses for minor trauma:
120 in the CoViD period and 277 in the previous year. Gender, age and vital parameters were overlapping. Patients in the CoViD pandemic tended to be more frequently carried by ambulance and 118 compared to the reference period ( $67 \%$ vs $44 \%$, the remaining half by independent means). The priority codes for medical examination were not different $(94 \%$ minor emergencies, $4 \%$ non-urgencies in the pandemic period vs 95 and 3\%). During the pandemic, the severity codes at discharge were higher (yellow and red) with a slightly higher frequency than in the reference period (9\% vs 3\%) and patients more frequently required hospitalization ( $21 \%$ vs $10 \%)$. Rarely this category of patients presents altered crowding indexes. Crowding input factors are however lower in the pandemic period: reduced attendants (120 vs 277) and reduced average waiting times (51 min vs $75 \mathrm{~min}$ ). The percentage of patients who exceeded the waiting time target by priority code of medical examination is also reduced (3\% vs 5\%). Crowding throughput factors worsened only slightly: LOS (394 vs $248 \mathrm{~min}$ ). Crowding output factors are also slightly worsened: the percentage of access block is higher during the pandemic (9\% vs 5\%). The Total Access Block Time is significantly higher in the CoViD period (6059 vs $2535 \mathrm{~min}$ ). Crowding for patients who came for all causes of our ED was higher in the pandemic period compared to the reference period $(\mathrm{OR}=3.95)$.

Conclusion: The epidemic led to a drastic reduction of accesses due to minor geriatric trauma, without any effect on overall ED crowding. Patients have more frequently the need for hospitalization and more severe exit codes. Acknowledgements: we would like to thank all employees of the IRCCS Policlinico San Matteo Foundation for their extraordinary efforts during the pandemic.Keywords: COVID-19, minor trauma, ER, Triage, Emergency Department.

\section{Abstract \# 203}

Analysis of the impact of the 2019 coronavirus epidemic (CoViD 19) on the presentation and crowding of patients for neurological disorders at a Lombardy ED.

Gabriele Savioli ${ }^{1}$, Iride Francesca Ceresa ${ }^{1}$, Paolo Maggioni ${ }^{2}$, Francesca Grulli $^{2}$, Elena Novara ${ }^{2}$, Tonia Persiano ${ }^{2}$, Viola Novelli ${ }^{3}$, Alba Muzzi ${ }^{3}$, Giovanni Ricevuti ${ }^{4}$, Enrico Oddone ${ }^{5}$, Maria Antonietta Bressan $^{6}$

${ }^{1}$ Emergency Department, IRCCS Policlinico San Matteo, 27100 Pavia, Italy, ${ }^{2}$ University of Pavia, 27100 Pavia, Italy, ${ }^{3}$ Medical Direction, IRCCS Policlinico San Matteo, 27100 Pavia, Italy, ${ }^{4}$ University of Pavia, 27100 Pavia, Italy., ${ }^{5}$ Assistant Professor, Department of Public Health, Experimental and Forensic Medicine, University of Pavia, 27100 Pavia, Italy., ${ }^{6}$ Past Director Emergency Department, IRCCS Policlinico San Matteo, 27100 Pavia, Italy.

Background: The coronavirus 2019 (CoViD -19) epidemic in Italy originated in the south-west of Lombardy, on February 21, 2020. The Fondazione IRCCS Policlinico San Matteo di Pavia has been involved in the management of the outbreak since its beginning. Crowding has been defined as a worldwide problem and is a cause of reduced quality of care and patient satisfaction. It is due and identified through three orders of factors: those at the access (input); those related to the patient's process (throughput); and those at the exit from the ED (output). The latter are considered the main responsible for Crowding. Purpose: As part of a study that wanted to assess the impact of the CoViD pandemic on time-dependent diseases in geriatric patients we evaluated the geriatric population that went to the ER for neurological disorders.

Materials and Methods: We evaluated all patients aged 75 years and older who were accessing our emergency room for neurological disorders from February 22 to May 1, 2020 and during the same period of the previous year. 
Results and discussion: We enrolled 530 patients. There was only a slight reduction in the total number of accesses for acute neurological disorders: 243 in the CoViD period and 287 in the previous year. The vital parameters were overlapping without statistically significant differences. Patients in the CoViD pandemic tend to be more frequently carried by ambulance and 118 compared to the reference period (90\% vs 64\%, the remaining half autonomous). Patients in the CoViD pandemic have higher priority to medical examination and severity to discharge codes (yellow and red) more frequently than in the reference period ( $79 \%$ vs $65 \%$ respectively) and more frequently need hospitalization ( $67 \%$ vs $54 \%$ ). The Crowding input factors are slightly lower in the pandemic period: the patients are slightly reduced (243 vs 287) as the average waiting times (52 min vs $86 \mathrm{~min}$ ) accesses. The percentage of patients who exceeded the waiting time target by code of priority to the medical visit is also reduced (19\% vs $30 \%$ ). Crowding throughput factors worsened: LOS (766 vs $563 \mathrm{~min}$ ). Crowding output factors also worsened: the percentage of access block is higher during the pandemic ( $21 \%$ vs $13 \%)$. The Total Access Block Time is significantly higher in the CoViD period both for the Visiting Rooms (53, 117 vs 27, $688 \mathrm{~min}$ ) and for the Holding Area (14, 401 vs $3,350 \mathrm{~min})$.

Conclusion: the epidemic has led only to a slight reduction in access for acute neurological disorders; we must therefore think that this disease does not suffer major deflections in the pandemic period for the geriatric population. Patients are more frequently in need of hospitalization and more severe exit codes. The pandemic period has presented a worsened crowding for these patients due to the Exit Block, resulting in a greater workload for emergency room workers. Acknowledgements: We would like to thank all employees of the IRCCS Policlinico San Matteo Foundation for their extraordinary efforts during the pandemic.

Keywords: COVID-19, Acute Neurological Disorders, Time Dependent Pathologies, First Aid, Triage, Emergency Department, crowding.

\section{Abstract \# 204 \\ Analysis of the impact of the 2019 coronavirus epidemic (CoViD - 19) on the presentation and crowding of Patients for chest pain at a Lombardy ED.}

Gabriele Savioli ${ }^{1}$, Iride Francesca Ceresa ${ }^{2}$, Francesca Grulli ${ }^{3}$, Paolo Maggioni $^{3}$, Tonia Persiano ${ }^{3}$, Elena Novara ${ }^{3}$, Viola Novelli ${ }^{4}$, Alba Muzzi $^{4}$, Giovanni Ricevui ${ }^{5}$, Enrico Oddone ${ }^{6}$, Maria Antonietta Bressan $^{7}$

${ }^{1}$ Emergency Department, IRCCS Policlinico San Matteo, 27100 Pavia, Italy, ${ }^{2}$ Keywords: COVID-19, Chest Pain, Time Dependent Pathologies, First Aid, Triage, Emergency Department, crowding., ${ }^{3}$ University of Pavia, 27100 Pavia, Italy., ${ }^{4}$ Medical Direction, IRCCS Policlinico San Matteo, 27100 Pavia, Italy, ${ }^{5}$ Department of Drug Science, University of Pavia, Italy, - Saint Camillus International University of Health Sciences - Rome - Italy, ${ }^{6}$ Assistant Professor, Department of Public Health, Experimental and Forensic Medicine, University of Pavia, 27100 Pavia, Italy., ${ }^{7}$ Past Director Emergency Department, IRCCS Policlinico San Matteo, 27100 Pavia, Italy

Background: The 2019 coronavirus epidemic (CoViD -19) in Italy originated in the southwest of Lombardy, on February 21, 2020. The Fondazione IRCCS Policlinico San Matteo di Pavia has been involved in the management of the outbreak since its beginning. Crowding has been defined as a worldwide problem and is a cause of reduced quality of care and patient satisfaction. It is due and identified by three orders of factors: those at the access (input); those related to the patient's process (throughput); and those at the exit from the PS (output). The latter are considered the main responsible for Crowding.
Purpose: As part of a study that wanted to assess the impact of the CoViD pandemic on time dependent diseases we evaluated the geriatric population who went to the ER for chest pain.

Materials and Methods: We evaluated all geriatric patients accessing our PS for chest pain from February 22 to May 1, 2020 and during the same period of the previous year. Results and discussion: We enrolled 405 patients. There was a severe reduction in the total number of accesses for chest pain: 144 in the CoViD period and 261 in 2019. The vital parameters were overlapping without statistically significant differences. In both periods there was no gender prevalence (M 50\% in 2019 and 55\% in 2020) or age difference (average 82 in 2019 and 83 in 2020). Patients in the CoViD pandemic tend to be more frequently carried by ambulance and 118 compared to the reference period $(74 \%$ vs $55 \%$, the remaining half by independent means). Priority codes for medical examination were not different. CoViD pandemic patients have higher discharge severity codes (yellow and red), more frequently than in the reference period $(40 \%$ vs $30 \%$ ) and they also more frequently need hospitalization (39\% vs $31 \%$ ). Crowding input factors are lower in the pandemic period: both the patients (144 vs 261) and average waiting times (62 min vs 79 min) are reduced. Also the percentage of patients who exceeded the waiting time target by priority code is lower during the pandemic ( $31 \%$ vs $45 \%)$. The Crowding throughput factors have instead worsened: LOS (720 vs $501 \mathrm{~min}$ ). The Crowding output factors also worsened: the percentage of access block is higher during the pandemic (17\% vs $8 \%$ ). The Total Access Block Time is significantly higher in the CoViD period both for the Visiting Rooms $(25,185$ vs 12,014 min) and for the Holding Area (13,341 vs $1975 \mathrm{~min})$. The interpretation of the data must also take into account the increased incidence of late heart attacks highlighted by the literature in the period of the epidemic and also published by a group of our clinic. Conclusion: the epidemic has led to a reduction of accesses for chest pain, especially self-reported. Patients had more frequently the need for hospitalization and more severe exit codes. The pandemic period presented a worse Crowding for these patients due to the Access Block.

Acknowledgements: We would like to thank all employees of the IRCCS Policlinico San Matteo Foundation for their extraordinary efforts during the pandemic.

Keywords: COVID-19, Chest Pain, Time Dependent Pathologies, First Aid, Triage, Emergency Department, crowding.

\section{Abstract \# 205}

Analysis of the impact of the 2019 coronavirus epidemic (CoViD 19) within the elderly patient population on the presentation mode and crowding at a Lombardy ED.

Gabriele Savioli $^{1}$, Iride Francesca Ceresa ${ }^{1}$, Paolo Maggioni ${ }^{2}$, Elena Novara $^{2}$, Francesca Grulli ${ }^{2}$, Tonia Persiano ${ }^{2}$, Viola Novelli ${ }^{3}$, Alba Muzzi $^{3}$, Giovanni Ricevuti ${ }^{4}$, Enrico Oddone ${ }^{5}$, Maria Antonietta Bressan $^{6}$

${ }^{1}$ Emergency Department, IRCCS Policlinico San Matteo, 27100 Pavia, Italy, ${ }^{2}$ University of Pavia, 27100 Pavia, Italy, ${ }^{3}$ Medical Direction, IRCCS Policlinico San Matteo, 27100 Pavia, Italy, ${ }^{4}$ Department of Drug Science, University of Pavia, Italy, - Saint Camillus International University of Health Sciences - Rome - Italy,

${ }^{5}$ Assistant Professor, Department of Public Health, Experimental and Forensic Medicine, University of Pavia, 27100 Pavia, Italy., ${ }^{6}$ Past Director Emergency Department, IRCCS Policlinico San Matteo, 27100 Pavia, Italy.

Introduction: Crowding in ED is a cause of reduced quality of care and patient satisfaction. It is due and identified by three orders of factors. Access factors (input): the number of accesses, average 
waiting times, percentage of waiting times longer than expected. Factors related to the patient's process usually analyzed through the total length of stay in ED (Length of Stay). The factors at the exit from the ED (output) are the main ones responsible for Crowding. They are usually measured with the exit block. This is defined by the length of stay in PS more than $8 \mathrm{~h}$ before admission. The exit block can be expressed as a percentage of patients who suffer it or the total time of exit block in each time frame.

Purpose: As part of a study to assess the impact of the CoViD pandemic on time-dependent diseases, we evaluated the population who went to the Emergency Room for acute neurological disorders during the period of the CoViD pandemic from 23 February to 1 May 2020 and compared it with the population accessing our Emergency Room during the same period of the previous year.

Materials and Methods: We evaluated all patients over 75 years of age who needed access to our emergency room from February 22 to May 1, 2020. We then compared this population with those over 75 years of age who were accessing our emergency room during the same period of the previous year. Results and discussion: We enrolled 4891 patients. There was a severe reduction in the total number of elderly accesses: 1910 in the CoViD period and 2981 in the previous year. However, this decrease $(-36 \%)$ was lower than that of the general population (about $-50 \%$ ). The vital parameters were overlapping without statistically significant differences. In both periods a slight female prevalence was confirmed (about 55\%). The average age (84 years) was superimposable. Patients in the CoViD pandemic tend to appear more frequently accompanied by ambulance and 118 compared to the reference period ( $78 \%$ vs $38 \%$, the remaining half by independent means). Patients in the CoViD pandemic have high (yellow and red) priority and discharge severity codes with only slightly higher frequency compared to the reference period $(50 \%$ vs $44 \%$ and $43 \%$ vs $25 \%$ respectively) and more frequently need hospitalization (54\% vs $31 \%$ ). The Crowding input factors are lower in the pandemic period: reduced patients (1910 vs 2981) and reduced average waiting times (62 min vs $92 \mathrm{~min}$ ). Reduced also the percentage of patients who exceeded the waiting time target by code of priority to the medical examination (16\% vs 26\%). Crowding throughput factors worsened: LOS (853 vs $485 \mathrm{~min}$ ). Crowding output factors also worsened: the percentage of access block is higher during the pandemic (30\% vs 10\%). The Total Access Block Time is significantly higher in the CoViD period both for the Visiting Rooms (632,738 vs $199,073 \mathrm{~min}$ ) and for the Holding Area (80,253 vs 38,420 min). Conclusion: the epidemic has led to a reduction in the access of elderly patients, although to a lesser extent than the general population, Especially self-reported patients. Patients have more frequent hospitalization needs and more severe exit codes. The period of the pandemic presented a worse crowding for these patients due to the Exit Block resulting in a prolonged stay in the ER.

Acknowledgements: we would like to thank all employees of the IRCCS Policlinico San Matteo Foundation for their extraordinary efforts during the pandemic.

Keywords: COVID-19, crowding, Time Dependent Pathologies, First Aid, Triage, Emergency Department.

\section{Abstract \# 206 \\ Need for urgency and emergency care of elderly patients at a Lombardy ED during the 2019 coronavirus epidemic (CoViD -19) \\ Gabriele Savioli ${ }^{1}$, Iride Francesca Ceresa ${ }^{1}$, Paolo Maggioni ${ }^{2}$, Elena Novara $^{2}$, Francesca Grulli ${ }^{2}$, Tonia Persiano ${ }^{2}$, Viola Novelli ${ }^{3}$, Alba Muzzi $^{3}$, Giovanni Ricevuti ${ }^{4}$, Enrico Oddone ${ }^{5}$, Maria Antonietta Bressan $^{6}$}

${ }^{1}$ Emergency Department, IRCCS Policlinico San Matteo, 27100 Pavia, Italy, ${ }^{2}$ University of Pavia, 27100 Pavia, Italy., ${ }^{3}$ Medical Direction, IRCCS Policlinico San Matteo, 27100 Pavia, Italy, ${ }^{4}$ Department of Drug Science, University of Pavia, Italy, - Saint Camillus International University of Health Sciences - Rome - Italy, ${ }^{5}$ Assistant Professor, Department of Public Health, Experimental and Forensic Medicine, University of Pavia, 27100 Pavia, Italy, ${ }^{6}$ Past Director Emergency Department, IRCCS Policlinico San Matteo, 27100 Pavia, Italy.

Background: The 2019 coronavirus epidemic (CoViD -19) of Italy originated from Codogno, an Italian municipality of 15,978 inhabitants in the province of Lodi, in the south-west of Lombardy, on February 21, 2020. Codogno is about $50 \mathrm{~km}$ from Pavia, home of one of the largest educational hospitals in the region, the IRCCS Policlinic San Matteo Foundation. The hospital has been involved in the management of the outbreak since its beginning. The elderly is a fragile subject and represents the population with the highest mortality within the pandemic. Due to its fragile condition and the coexistence of chronic diseases, it is also one of the populations with the highest access to emergency departments (ED). During the CoViD period, the elderly had to turn to EDs not only for CoViD cases but also for the exacerbation of their underlying diseases.Purpose: to assess the impact of the CoViD pandemic concerning the access motives of the elderly population (> 75 years) who went to the Emergency Room during the CoViD pandemic period from 23 February to 1 May 2020, compared to the population accessing our Emergency Room during the same period of the previous year.

Materials and Methods: We evaluated all patients over 75 years of age who needed access to our emergency room from February 23 to May 1, 2020. We then compared this population with the population over 75 years of age who were accessing our emergency room during the same period of the previous year.

Results and discussion: We enrolled 4891 patients. There has been a severe reduction in the total number of elderly accesses: 1910 in the CoViD period and 2981 in the previous year. However, this decrease $(-36 \%)$ was lower than the one concerning the general population (about $-50 \%$ ). The causes for access are shown in the table below: ACC. CAUSES 20192020 ACCESS CAUSES $20192020 \% \% \%$ \%Dyspnea 13.59 18.0 Fever-sepsis 3, 1914, 19 Minor trauma 9, 196, 28 Neurological 9, 2912, 46 Trauma 9, 268, 64 L syncope 3, 663, 09 Psychic disorders 0, 27 0.47 Headache 0, 700, 21 Thoracic pain 8, 557, 43 Lumbar pain 0, 970, 42 Abdominal pain 9, 336, 02 Injuries/Burns 1, 20 0, 47Minor signs 15, 1310, 58 vital parameters 3, 252, 09 Specialistic 3, 492, 25 Palpitation 3, 42 2, 62 Bleeding 4, 833, 82 Intoxication $0,200,05$ The vital parameters were superimposable without statistically significant difference. The main cause of access during the pandemic was the dyspneic symptomatology followed by fever. The period of comparison coincides with the flu peak of 2019. The reasons for access that have suffered the greatest reduction were those for minor medical symptoms and specialist visits. The other reasons for access showed a modest and less marked reduction than that suffered by other age groups.

Conclusion: the epidemic has led to a reduction in access for elderly patients, although less than the general population. The most frequent reasons for access were compatible with $\mathrm{CoViD}$ symptoms in more than a third of the accesses. However, the elderly still had to access EDs frequently due to the exacerbation of their chronic pathologies. Acknowledgements: we would like to thank all employees of the Fondazione IRCCS Policlinico San Matteo for their extraordinary commitment during the pandemic.

Keywords: COVID-19, Need for urgency and emergency care, crowding, Time Dependent Pathologies, First Aid, Triage, Emergency Department 


\section{Abstract \# 207}

Comparison of clinical characteristics and outcome measures of PCR-positive and PCR-negative patients diagnosed as Covid19: analyses focusing on the older adults

Alpay Medetalibeyoglu ${ }^{1}$, Gulistan Bahat ${ }^{2}$, Naci Senkal ${ }^{1}$, Murat Kose $^{1}$, Yunus Catma, Yusuf Yesil ${ }^{3}$, Emine Bilge Caparali ${ }^{1}$, Sena Bayrakdar $^{1}$, Sevim Mese ${ }^{4}$, Ali Agacfidan ${ }^{4}$, Mustafa Oral Oncul ${ }^{5}$, Mehmet Akif Karan ${ }^{2}$, Tufan Tukek ${ }^{1}$

${ }^{1}$ Istanbul University Istanbul Faculty of Medicine, Department of Internal Medicine, Istanbul, TURKEY, ${ }^{2}$ Istanbul University Istanbul Faculty of Medicine, Department of Internal Medicine, Division of Geriatrics, Istanbul, TURKEY, ${ }^{3}$ Istanbul University Istanbul Faculty of Medicine, Department ofBiochemistry, Istanbul, TURKEY, ${ }^{4}$ Istanbul University Istanbul Faculty of Medicine, Department of Microbiology, Istanbul, TURKEY, ${ }^{5}$ Istanbul University Istanbul Faculty of Medicine, Department of InfectiousDiseases, Istanbul, TURKEY

\section{Abstract:}

Purpose: While the definitive diagnosis of Covid-19 relies on PCR confirmation of the virus, the sensitivity of this technique is limited. The clinicians had to go on with the clinical diagnosis of Covid-19 in selected cases. We aimed to compare PCR-positive and PCR-negative patients diagnosed as Covid-19 with a specific focus on older adults. Methods: We studied 601 hospitalized adults. The demographics, comorbidities, triage clinical, laboratory characteristics, and outcomes were noted. Differences between the PCR $(+)$ and $(-)$ cases were analyzed. An additional specific analysis focusing on older adults $(\geq$ 65 years $)(n=184)$ was performed.

Results: The PCR confirmation was present in 359 (59.7\%). There was not any difference in terms of age, sex, travel/contact history, hospitalization duration, ICU need, the time between first symptom/ hospitalization to ICU need, ICU days, or survival between PCRpositive and negative cases in the total study group and older adults subgroup. The only symptoms that were different in prevalence between PCR confirmed and unconfirmed cases were fever $(73.3 \%$ vs. $64 \%, \mathrm{p}=0.02)$ and fatigue/myalgia $(91.1 \%$ vs. $79.3 \%, \mathrm{p}=0.001)$. Bilateral diffuse pneumonia was also more prevalent in PCR confirmed cases $(20 \%$ vs. $13.3 \%, \mathrm{p}=0.03)$. In older adults, the PCR $(-)$ cases had more prevalent dyspnea $(72.2 \%$ vs. $51.4 \%, \mathrm{p}=0.004)$, less prevalent fatigue/myalgia $(70.9 \%$ vs. $88.6 \%, \mathrm{p}=0.002)$.

Conclusion: The PCR (+) and (-) cases displayed very similar disease phenotypes, courses, and outcomes with few differences between each other. The presence of some worse laboratory findings may indicate worse immune protective response in PCR (-) cases.

\section{Abstract \# 208 \\ Demographic details of patients referred to the Social Prescribing Program in The Mercer's Institute for Successful Aging (MISA) at St James's Hospital, Dublin \\ Bernie McGowan ${ }^{1}$, Mary Buckley ${ }^{1}$, Gerard Boyle $^{2}$, David Robinson ${ }^{1}$ \\ ${ }^{1}$ Mercer's Institute for Successful Ageing (MISA), St James's Hospital, Dublin $8,{ }^{2}$ Dept of Medical Physics \& Bioengineering (MPBE), St James's Hospital, Dublin 8}

Introduction: Social prescribing is a means for health professionals to refer patients to a link worker, who can help connect them to resources in their community often provided by the voluntary sector, with a view to improving their health and wellbeing. With financial support from Sláintecare, the Mercer's Institute for Successful Ageing (MISA) at St James's Hospital, Dublin have introduced the first hospital based social prescribing program in Ireland.
Method: All information pertaining to patients referred to the Social Prescribing Co-ordinator is recorded on a PSPP database. The information collected includes demographic details, sources of referral, reason for referral, home circumstances, health care packages where relevant. The results of an analyses of data on the first 40 patients referred to the social prescribing program between May 1st 2020 and July 31 st 2020 when COVID-19 restrictions were in place are as follows.

Results: The mean age of the group is $79.8 \mathrm{yrs}$ (Std dev \pm 6.3 ), In total 28 patients $(69 \%)$ were female and $12(31 \%)$ were male. A total of 28 patients $(69 \%)$ were living alone, a further 7 patients $(17 \%)$ were living with a partner and the remaining $6(14 \%)$ were living with a son or daughter. In total 24 patients $(59 \%)$ were referred due to social isolation and loneliness, a further $7 \%$ with anxiety depression and low mood as a result of isolating.

Conclusion: Social Prescribing as a non-medical response to health and well being challenges should continue and be extended to operate alongside other established medical referral pathways.

\section{Abstract \# 209}

Prioritising the assessment of referrals to the Social Prescribing program in The Mercer's Institute for Successful Aging (MISA), St James's Hospital, Dublin 8.

Bernie McGowan ${ }^{1}$, Mary Buckley ${ }^{1}$, Gerard Boyle ${ }^{2}$, David Robinson ${ }^{1}$

${ }^{1}$ Mercer's Institute for Successful Ageing (MISA), St James's Hospital, Dublin 8, ${ }^{2}$ Dept of Medical Physics \& Bioengineering (MPBE), St James's Hospital, Dublin 8

Introduction: During the COVID 19 pandemic social prescribing link co-ordinators are moulding their role to find alternative ways of supporting the person referred to their service. With many community based services for older people closed at present it is more challenging to discharge people from the SP service which may result in prioritising consultations and waiting lists.

Method: A review of the first 40 patients referred to the Care of the Older People SP programme at St James's Hospital was carried out. Preliminary results would indicate that referrals may be need to be prioritised in the following order.

Results: (Taking into consideration the presence or not of a home care package (HCP) and the number of hours included in the HCP) 1 . Person with a diagnosis of dementia, living alone with co-morbidities and no family support 2 . Person without a diagnosis of dementia, living alone with co-morbidities and no family support3. Person with a diagnosis of dementia, living alone with co-morbidities and family support (not live in) 4. Person without a diagnosis of dementia, living alone with co-morbidities and family support (not live in) 5. Person with a dementia, living with a carer (partner or son/daughter) with comorbidities 6. Person without a diagnosis of dementia, living with a carer (partner or son/daughter) with co-morbidities.

Conclusion: When prioritising the urgency of addressing referrals to the social prescribing program it is useful to have indicators but also important to recognise that each case needs to be assessed on an individual basis

\section{Abstract \# 210 \\ Health changes following the outbreak of COVID-19 across sex and European regions. Preliminary results from the 2020 SHARE COVID-19 survey}

Karen Andersen-Ranberg ${ }^{1}$, Lasse Lybecker Scheel-Hincke ${ }^{2}$, Linda Juel Ahrenfeldt ${ }^{2}$ 
${ }^{1}$ Unit of Epidemiology, Biostatistics and Biodemography, Dept. of Public Health, University of Southern Denmark; Geriatric Research Unit, Dept. of Geriatric Medicine, Odense University Hospital; Dept. of Clinical Research, University of Southern Denmark, Odense, Denmark, ${ }^{2}$ Unit of Epidemiology, Biostatistics and Biodemography, Dept. of Public Health, University of Southern Denmark, Odense, Denmark

Background: The novel coronavirus (COVID-19) has spread throughout the world. Protective measures such as good hygiene, social distancing and isolation have effectively limited virus spread. How these behaviors affect our health are unknown. We examined changes in physical and mental health following the outbreak of COVID-19 across sex and European regions in middle-aged and older adults. Methods: Cross-sectional, cross-European study including 23,084 men and 31,443 women aged $50+$ years participating in the Survey on Health, Ageing and Retirement in Europe (SHARE) Corona Survey 2020. Sex and regional differences in self-reported changes in health measures before and during the COVID-19 outbreak were investigated using logistic regression models adjusted for potential confounders.

Results: Women had significantly higher odds than men for worsened self-rated health $(\mathrm{OR}=1.19)$, for doing activities less often (ever left home $(\mathrm{OR}=1.28)$, going shopping $(\mathrm{OR}=1.85)$, going out for a walk $(\mathrm{OR}=1.36)$, meeting $5+$ people outside home $(\mathrm{OR}=1.55)$, visiting family members $(\mathrm{OR}=1.35)$ ), and for having more mental health problems (felt more nervous $(\mathrm{OR}=1.20)$, depressed $(\mathrm{OR}=1.22)$, and lonely $(\mathrm{OR}=1.29)$ ). Large regional differences were observed with Northern Europeans having the smallest changes and Southern Europeans having the largest changes across all measures.

Conclusions: Preliminary results from the SHARE Corona survey show considerable sex and regional differences in changes in selfreported health from before to during the COVID-19 outbreak. In line with previous results based on SHARE studies, women and participants living in Southern Europe suffer more from the effects of the COVID-19 pandemic.

\section{Abstract \# 211 \\ A fuller picture of COVID-19 prognosis: the added value of vulnerability measures to predict death in hospitalized older adults}

Márlon Aliberti ${ }^{1}$, Kenneth Covinsky ${ }^{2}$, Flavia Garcez ${ }^{3}$, Pedro Curiati ${ }^{1}$, Murilo Dias ${ }^{3}$, Wilson Jacob-Filho ${ }^{3}$, Thiago Avelino-Silva ${ }^{3}$

${ }^{1}$ University of São Paulo Medical School; Hospital Sirio-Libanês Research Institute, ${ }^{2}$ University of California, San Francisco, ${ }^{3}$ University of São Paulo Medical School

Background: Although older adults constitute most hospitalizations and deaths from COVID-19, conventional triage tools in acute care settings ignore key aspects of geriatric vulnerability. Objective: To estimate the usefulness of adding a rapid geriatric vulnerability screening to an illness acuity tool to predict mortality in hospitalized COVID-19 patients.

Methods: This is a cohort study comprising 1565 patients aged $\geq 50$ years consecutively admitted to a large university hospital dedicated to COVID-19 care in Sao Paulo, Brazil, between March 30 and June 30,2020 . We assessed geriatric vulnerability using the PRO-AGE scoring system $(0-7$; higher $=$ worse $)$, a validated and easy-to-administer screening tool that rates physical impairment, recent hospitalization, acute mental change, weight loss, and fatigue. Baseline covariates included age, sex, Charlson comorbidity score, and the National Early Warning Score (NEWS), a well-known illness acuity tool based on physiological parameters. Our outcome was time-todeath within 60 days from admission.Results: Patients had a median age of 66 years, and $57 \%$ were male. The cumulative incidence of 60-day mortality ranged from 20 to $69 \%$ across quartiles of PROAGE. In Cox proportional-hazards models, compared with PRO-AGE scores 0-1 (lowest quartile), the adjusted hazard ratios $(95 \% \mathrm{CI})$ for 60-day mortality for PRO-AGE scores $2-3$, 4, and 5-7, were 1.7 (1.3-2.2); 2.1 (1.6-2.8); and 3.1 (2.3-4.0), respectively. The PROAGE predicted different mortality risk levels within each stratum of NEWS and markedly improved the discrimination of predictive models including demographics, Charlson comorbidity score, and the NEWS.

Conclusions: In this large sample of patients admitted to the hospital with COVID-19, measuring geriatric vulnerability in addition to illness acuity improved accuracy to predict mortality. Providers in fastpaced acute care settings should be aware that combining practical tools like PRO-AGE and NEWS may help stratify the risk of death from COVID-19 better than using illness acuity alone.

\section{Abstract \# 212}

The frail elderly population as the principal focus of the NHS Nightingale North West COVID-19 Field Hospital

Trent Herdman ${ }^{1}$, Timothy Seers ${ }^{1}$, Cassandra $\mathrm{Ng}^{1}$, Rebecca Davenport $^{1}$, Sarah Sibley ${ }^{1}$, Stephen Mannion ${ }^{1}$, Sooria Balasegaram ${ }^{2}$, Anthony Redmond ${ }^{1}$

${ }^{1}$ NHS Nightingale Hospital North West, Manchester UK, ${ }^{2}$ National Infection Service, Public Health England, UK

Introduction: COVID-19 field hospitals have been established in the UK to support the surge capacity of the National Health Service while protecting the community from onward infection. We investigated the impact of frailty on such a hospital over time.

Methods: We described the patient population of the NHS Nightingale North West (NNW) field hospital in April-June 2020 from medical records including clinical frailty score (CFS). We analysed predictors of mortality using logistic regression and admission duration using Cox's regression, and described trends in frailty prevalence over time using linear regression.

Results: 104 COVID-19 patients were admitted from hospitals in North West England, 74\% with moderate-to-severe frailty (CFS $>5$ ). 84 were discharged, 14 transferred to other hospitals, and six died on site. High C-reactive protein $(\mathrm{CRP})>50 \mathrm{mg} / \mathrm{dl}$ predicted 30 -day mortality (aOR $11.3,95 \%$ CI $3.1-41.4, \mathrm{P}<0.001$ ), with diabetes and admission Early Warning Score retained in the final explanatory model. Patients with CFS $>5$ had a 10-day median admission, vs. 7-day for CFS $\leq 5$ and half the likelihood of discharge on a given day (HR 0.53; 95\% CI 0.32-0.88, P = 0.015). CRP > $50 \mathrm{mg} / \mathrm{dl}$ and nosocomially acquired COVID-19 also predicted admission duration. Consequently, while frailty on admission was steady over time, rate of discharge was lower, so prevalence of CFS $>5$ increased from $64 \%$ initially to $90 \%$ in the final week (non-zero slope $\mathrm{P}<0.001$ ). Conclusions:The NNW population was characterized by high levels of frailty, which increased over time, with operational implications. Adapting to the needs of this population will improve NNW if it is needed again.

\section{Abstract \# 213}

Assessing the impact of covid-19 and frailty in older people

Daniel Aldcroft ${ }^{1}$, Anna Fearon ${ }^{2}$, Ryan George ${ }^{3}$, Eleni Malloupa ${ }^{4}$, Cassandra $\mathrm{Ng}^{5}$

${ }^{1}$ Clinical Fellow, Wythenshawe Hospital, Manchester University NHS Foundation Trust, ${ }^{2}$ Senior Frailty Nurse, Wythenshawe 
Hospital, Manchester University NHS Foundation Trust, ${ }^{3}$ Clinical Scientist, Wythenshawe Hospital, Manchester University NHS Foun

Introduction: COVID-19 has significantly impacted the lives of older adults, with frail populations disproportionately affected. We aimed to develop deeper understanding of this impact on older people, and how frailty influenced outcomes.

Methods: Patients aged 65 years and above who tested COVID-19 positive between 1st March and 27th July 2020 in Wythenshawe Hospital were identified by our Clinical Scientist. We analysed a smaller cohort of patients (obtaining data from Electronic Patient Records), assessing parameters including length of stay, 30-day mortality, level of dependency, imaging, and admission frailty scores such as Charlson Comorbidity Index (CCI) and Rockwood Clinical Frailty Score (CFS).

Results: 556 adults aged 65-99 years old tested positive for COVID19. $49.6 \%$ were male and $60 \%$ were classed as community-onset. Subgroup analysis demonstrated over $40 \%$ were frail. The majority of older patients exhibited typical COVID-19 symptoms, with approximately $60 \%$ displaying characteristic chest radiograph appearances. Higher CCI and CFS scores were correlated with increased mortality. Older males experienced a higher death rate $(53 \%)$. There is a greater degree of dependency following COVID-19 in frail patients, often requiring transfer to non-acute hospital settings linked to our Trust for discharge planning and rehabilitation. Conclusion: COVID-19 leads to higher mortality and morbidity in older adults. Frailty is important in predicting outcomes, with higher CCI/CFS scores, and male gender, all associated with increased mortality. Frail older survivors were more likely to have increased dependency levels, requiring complex discharge planning and social support. Using non-acute hospitals was crucial in ensuring capacity for acute admissions.

\section{Abstract \# 214 \\ Age and frailty are associated with adverse outcomes in patients with COVID-19: results of a multi-centre international cohort study}

Geriatric Medicine Research Collaborative

${ }^{1}$ University of Birmingham

Introduction: Increased mortality has been demonstrated in older adults with COVID-19, but the effect of frailty has been unclear. Methods: Multi-centre cohort study involving patients aged 18 years and older hospitalised with COVID-19, using routinely collected data. We used Cox regression analysis to assess the impact of age, frailty, and delirium on the risk of inpatient mortality, adjusting for sex, illness severity, inflammation, and co-morbidities. We used multinomial logistic regression analysis to assess the impact of age, Clinical Frailty Scale (CFS), and delirium on risk of increased care requirements on discharge, adjusting for the same variables.

Results: Data from 5711 patients from 55 hospitals in 12 countries were included (median age 74, IQR 54-83; 55.2\% male). The risk of death increased independently with increasing age ( $>80$ vs $18-49$ : HR 3.25 , CI 2.37-4.46), frailty (CFS 8 vs $1-3$ : HR 2.86 , CI $2.19-3.73$ ) inflammation, renal disease, cardiovascular disease, and cancer, but not delirium. Age, frailty (CFS 8 vs $1-3$ : OR 7.02, CI 4.59-10.75), delirium, dementia, and mental health diagnoses were all associated with increased risk of higher care needs on discharge. Likelihood of adverse outcomes increased across all grades of CFS from 4 to 9 .

Conclusions: Age and frailty are independently associated with adverse outcomes in COVID-19. Risk of increased care needs was also increased in survivors of COVID-19 with frailty or older age .

\section{Abstract \# 215}

Geriatric population infected by SARS-CoV2: characterisation of patients managed in primary healthcare

Marina Faria ${ }^{1}$, Ana Esteves ${ }^{2}$, Catarina Carvalho ${ }^{3}$, Mariana Santos ${ }^{4}$

${ }^{1}$ Unidade de Saúde Familiar Lusa, ${ }^{2}$ Unidade de Saúde Familiar Conde de Oeiras, ${ }^{3}$ Unidade de Cuidados de Saúde Personalizados de Lindaa-Velha, ${ }^{4}$ Unidade de Saúde Familiar Linha de Algés

Introduction: The elderly are the most vulnerable to COVID-19, mainly those with chronic disorders. In March 2020, Portugal created a platform, Trace COVID-19, allowing the monitoring of SARS$\mathrm{CoV} 2$ positive patients by primary healthcare. The patients are monitored through daily telephone contacts carried out by the physicians. The goal of this study is to characterise the geriatric population tracked through this platform.

Methods: An observational retrospective study was carried out examining the geriatric population monitored through Trace COVID19 by four Healthcare Centers in Oeiras. Patients were older than 65 years, with a positive test for SARS-CoV2 and signed as cured or died due to COVID-19, between March 26th and August 31st 2020. A descriptive analysis of sociodemographic characteristics and comorbidities was performed.

Results: 42 patients were included, with an average of 73 years, the majority women. About $85.7 \%$ had comorbidities, most commonly hypertension and dyslipidemia. $54.8 \%$ were symptomatic, most commonly dry cough and myalgia. $85.7 \%$ required medical observation and $26.2 \%$ required hospitalisation. $54.8 \%$ were unaware of an epidemiological link. $11.9 \%$ lived in institutions. There were 6 deaths, mainly women, whose average age was 86 years.

Conclusions: There were a large number of asymptomatic patients. Respiratory disease was not the most prevalent comorbidity; out of the three symptoms used as test criteria in Portugal, only cough appeared to be a frequent symptom. Few patients needed hospitalization. This study requires a critical review since the most severe cases were followed up at hospital care and not in primary healthcare.

\section{Abstract \# 216}

\section{Late viral RNA shedding in SARS-Cov geriatric patients}

Pedro Fiuza ${ }^{1}$, Marilia Fernandes ${ }^{1}$, Sara Dias ${ }^{1}$, Diogo Drummond Borges ${ }^{1}$, Andreia Amaral ${ }^{2}$, Luisa Azevedo ${ }^{3}$, Heidi Gruner ${ }^{1}$, Antonio Panarra $^{1}$

${ }^{1}$ Serviço Medicina 7.2 Centro Hospitalar Lisboa Central, ${ }^{2}$ Serviço de Medicina 2.4 Centro Hospitlar Universitario Lisboa Central, ${ }^{3}$ Serviço de Medicina 1.2 Centro Hospitalar Lisboa Central

Introduction: Late viral RNA shedding in SARS-Cov patients is a reality with socioeconomic repercussions, the question is if age plays a role. Objective: Evaluation of factors associated with prolonged viral RNA shedding in SARS-Cov elderly patients admitted to a tertiary hospital, during the COVID-19 outbreak in 2020.

Methods: Retrospective descriptive study of 107 patients admitted to the ward that had an average of 23.1 days of viral shedding, comparing those with early ( $<15$ days after illness onset) and late $(>15$ days after illness onset) RNA shedding, according to age. Evaluated parameters comparing the older with $\geq 65$ years $(\mathrm{O})$ and the younger adults (Y) were gender, comorbidities and medication, symptoms onset and emergence department (ER) provenience, average length of stay, intensive care admittance (ICU) and mechanical ventilation.

Results: There were $72(67.2 \%)$ with prolonged viral RNA shedding of which 35 older $(\mathrm{O})$ and 37 younger $(\mathrm{Y})$ patients. Comparing the two groups concerning male gender $51,4 \%(\mathrm{O}) / 83,8 \%(\mathrm{Y})$, average age $79.14(\mathrm{O}) / 48.2(\mathrm{Y})$ years, average number of comorbidities was 
$5.47(\mathrm{O}) / 2.72(\mathrm{Y})$, and the average number of used medication was $7.82(\mathrm{O}) / 3,59(\mathrm{Y})$. The average duration of symptoms onset to hospital admission was $8.32(\mathrm{O}) / 4.89(\mathrm{Y})$ days and provenience from the ER was $60 \%(\mathrm{O}) / 54.1 \%(\mathrm{Y})$ of the patients. The average length of stay was $19.6(\mathrm{O}) / 12.7(\mathrm{Y})$ days of which admitted to ICU were $34.2 \%(\mathrm{O}) / 43.2 \%(\mathrm{Y})$ patients and submitted to mechanical ventilation were $31.4 \%(\mathrm{O}) / 32.4 \%(\mathrm{Y})$.

Discussion: The elder patients had a female prevalence, more comorbidities and average number of medications. Though the average length of stay was longer there were viewer ICU admissions, but almost the same amount of patients underwent mechanical ventilation.

\section{Abstract \# 217 \\ EUGMS 2020:Geropsychiatric care during Coronavirus 2019 (COVID-19) outbreak in Turkey

\begin{abstract}
Mehmet Ilkin Naharci ${ }^{1}$, Bilal Katipoglu ${ }^{1}$, Ilker Tasci ${ }^{2}$
${ }^{1}$ Division of Geriatrics, University of Health Sciences, Gulhane Faculty of Medicine and Gulhane Training and Research Hospital, Ankara, Turkey, ${ }^{2}$ Department of Internal Medicine, University of Health Sciences, Gulhane Faculty of Medicine and Gulhane
\end{abstract}

Introduction: We aimed at examining the impacts of COVID-19 pandemic on older adults' daily living and the burden of geropsychiatric care issues in Turkey. We also proposed several solutions to ease the period of social isolation.

Methods: To examine the available information on geropsychiatric care in Turkey, we performed a literature search in local databases and PubMed starting from March through June 2020 and examined the local media releases.

Results: The national budget explicitly covered the entire costs of screening, hospital admissions, and treatment for COVID-19. To provide maintenance medication, the governor's emergency order provided access to a 30-day refill of prescription medications for chronic conditions from the local pharmacies. Outpatient appointments were mostly suspended. As for nursing home care, the Turkish Ministry of Health released and updated a guideline to reduce the impact of the outbreak on these settings. The outbreak led to an increased demand for home health care, especially in some nonhomebound older adults avoiding hospital admissions. Concerning the engagement with novel preventive measures, many older individuals have initially failed to engage in home stay and attempted to go out for various reasons such as exercising, meeting friends, dog walking, and shopping.

Key conclusions: This latest outbreak has shown the lack of adequate, human-centered management plans in the context of elderly care during a pandemic. Extraordinary measures taken by the authorities should cover the physical, mental, and social needs of older people, as well as the involvement of elderly organizations during decision making.

\section{Abstract \# 218}

\section{Are older people adequately profiled in COVID-19 training?}

Amy Lynch ${ }^{1}$, Desmond O’Neill ${ }^{1}$

${ }^{1} \mathrm{TCD}$, Dublin, Ireland

Introduction: Older people represent the key demographic affected by mortality and morbidity of COVID-19 but there are concerns that this vulnerability has not been accorded due prominence by international health bodies such as the WHO. This may reflect a wider failure by the profession in general: we assessed the relative prominence and timeliness of focused presentations on older people by four postgraduate medical colleges in the UK and Ireland.

Method: We analysed schedules and content clinical update teaching presentations and videocasts to calculate proportion of topics specifically addressing older people, and their scheduling over a 14-week period spanning the beginning and evolution of the pandemic.

Results: Of 80 talks given by the 4 colleges, only seven (9\%) directly related to older people. A specific focus on older people appeared on the seventh week of weekly clinical updates in one college, and on the second week for another, no clear dates were attached to talks given by the third college, and no presentation featured on the COVID-19 presentations of the fourth college.

Key conclusions: Adequate and timely education for physicians in the UK and Ireland is not proportionate in timing or content to the burden of morbidity and mortality of older people from COVID-19 . A learning outcome of this pandemic should be to encourage stronger joint working with geriatric medicine and a closer proportionality between the populations most affected in the content and scheduling of medical postgraduate educational initiatives for emergent crises, disaster and pandemic planning.

\section{Abstract \# 219}

The importance of trace COVID-19: the Portuguese surveillance platform

Andreia Teles Ribeiro ${ }^{1}$, Ricardo Simões de Araújo ${ }^{1}$, Valter Moreira ${ }^{1}$

${ }^{1}$ USF Descobertas, ACES Lisboa Ocidental e Oeiras, ARS Lisboa e Vale do Tejo

Introduction: Coronavirus disease 2019 (COVID-19) spreads from person-to-person through droplet and contact transmission. In Portugal, Public Health has created a platform called Trace COVID-19 to monitor and follow patients with confirmed disease, with suspected symptoms or who have been exposed in closed contact to other patients with the disease.

Methods: We provide a description of the Trace COVID-19 platform and its function. We also report the results of our unit in terms of number of patients followed, referrals to the emergency service, patients with confirmed disease and number of recoveries.

Results: It's considered a suspected case of COVID-19 when a person has symptoms, and exposed when we find contact with a positive case. In both cases, the patient is inserted in the platform TraceCOVID-19 to be followed up. In exposed patients they are considered in active surveillance and monitored daily by Public Health professionals to re-assure of the quarantine measures and do contact tracing. In suspected cases, is required an overactive surveillance made by General Practitioners, and a diagnostic test is prescribed. In this case, each person is contacted daily to monitor their symptoms, if there is a necessity for observation (either in the community or in the hospital) and to answer to the needs of the patients.

Key conclusions: This platform enabled clinicians to follow the patients at home, saving resources in hospitals and primary care centers, prioritizing the patients who need to be evaluated, and giving patients a regular contact to answer their needs.

\section{Abstract \# 220}

Age, ageing and age-itation in the Age of COVID-19: the necessary combination of the geriatric medicine and public health approaches

Prof A. Mark Clarfield

Ben-Gurion University of the Negev, Beersheva, Israel 
COVID-19, the illness caused by the SARS-CoV-2 virus, has reached pandemic proportions and is greatly affecting countries around the world. Although the virus can cause disease in anyone, it is particularly dangerous for those with various "co-morbidities " such as heart disease, hypertension, diabetes, obesity and others. Furthermore, advancing age (from about 60 on) even in those older persons without any accompanying illnesses, is a strong and independent risk factor for pneumonia, need for an ICU bed and death from COVID-19. Although intuitively it makes sense that older persons would be more at risk from such viral threats, this is not always the case. For example, the 1918-19 influenza pandemic spared older persons and the SARS-CoV-2 does not seem to affect children. Obviously we still have much to learn about this new virus and its age-related predilections.

However, given the clear danger of this virus to older persons it is essential to find ways to protect at-risk persons from the virus while at the same time not harming, more than absolutely necessary, their essential freedoms. Furthermore we must at the same time take into account their social/psychological needs. Even in the absence of physical disease societal measures taken to protect society in general can wreak psychological havoc on isolated seniors.

In our approach we must keep in mind that the older population is extremely heterogeneous - ranging chronologically anywhere from about 60 up until the very outer ranges of the maximum life span (122). On average, each age cohort from "young-old" (60-79) to "old-old $(80+)$ is different and within each group there are also huge variations relating to SES, illness exposure and burden. Furthermore, the situation of almost older persons in high income countries (HICs) differs significantly from almost every elder in the poorer regions of the world, ie those which are low income (LICs). One example re COVID-19: a much higher proportion of older persons in the LICs live in multi-generational households making the idea of "social distancing" far less feasible than in HICs.

But working in the opposite direction, disadvantaging older person in HICs, a much higher proportion live in long-term institutions which for obvious reasons constitute a very large proportion of COVID-19 related deaths in these countries. As well, for those elders in the community, lockdown policies of HICs which are effective in "flattening the curve" can also flatten the ability of older persons to cope with the resultant social isolation which for the most part does is not seen in LICs

As such, our approach to the risks to older persons during this pandemic must take many factors into account: biology, clinical medicine, epidemiology and public health. The blanket is indeed not large enough for all but we must place it as judiciously as possible in order to protect, cover and keep warm as much as possible of the body politic. Until the successful advent of a vaccine effective in older persons, there is much to be done. An approach which will combine the expertise of geriatric medicine with time honoured field of public health is essential to knitting this protective cloth.

\section{Abstract \# 221 \\ Frailty status is not associated with unawareness of protective measures against COVID19: the Mexican experience}

Virgilio Hernández-Ruiz ${ }^{1}$, Blanca Estela López-Graciano ${ }^{1}$, José Alberto Avila-Funes ${ }^{2}$

${ }^{1}$ Geriatrics Department. Instituto Nacional de Ciencias Médicas y Nutrición Salvador Zubirán, Mexico City, Mexico, ${ }^{2}$ Geriatrics Department. Instituto Nacional de Ciencias Médicas y Nutrición Salvador Zubirán, Mexico City, Mexico/Univ. Bordeaux, Inse

Introduction: COVID-19 disproportionally affects older adults. Within this already vulnerably population, frail individuals may be at greater risk of inadvertently not knowing public health interventions. Hence, the objective of the study was to determine if frailty status is associated to unawareness of protective measures for the pandemic, and if living alone modified the association.

Methodology: cross-sectional study including 292 patients from a Geriatrics outpatient clinic of a university-affiliated tertiary-care hospital in Mexico City whose consult was cancelled during mandatory lockdown. A structured telephonic survey was applied. Frailty status was determined using the FRAIL scale (Morley J., et al). Direct questions concerning awareness of governmental indicated protective measures were posed (mandatory lockdown, hand hygiene, and social distancing), as for the "living alone" status. Multinomial logistic regression models were performed to determine the researched association.

Results: mean age 83.1 ys ( \pm 7.2$), 72.3 \%$ were women, and $7 \%$ lived alone. Pre-frail status was found in $44.6 \%$ and $22.3 \%$ of the participants were frail. The unadjusted multinomial logistic regression models found no statistically significant association between frailty status and unawareness of protective measures for COVID-19: hand hygiene $(\mathrm{p}=0.263)$, mandatory lockdown $(\mathrm{p}=0.891)$, and social distancing $(\mathrm{p}=0.49)$. Living alone status did not modified the obtained results.

Conclusion: Frailty status was not associated to unawareness of protective measures against COVID-19 and living alone did not modified the observed results. These findings may reflect that public communication strategies in Mexico may permeate even to the more vulnerable populations. Thus, efforts to maintain positive public health messages may be advantageous.

\section{Abstract \# 222}

Geriatric status in the period of social distancing during COVID19outbreak: the role of online physical training

Tatsiana Alenskaya ${ }^{1}$, Andrey Ilnitski $^{2}$, Kirill Prashchayeu ${ }^{2}$

${ }^{1}$ Vitebsk State Medical University, Belarusian Republican Gerontological Public Association, ${ }^{2}$ Belarusian Republican Gerontological Public Association

The COVID-19 outbreak and social distancing as measure of prevention of quick spreading of infection posed challenges for geriatricians to improve measures of medical and social adaptation of elders.

Materials and methods: Vitebsk State Medical University with Belarusian Gerontological Association since March 30, 2020 carried out interdisciplinary online measures which included online clinical counseling and remote physical training of elders (mean age was 71.3 \pm 3.5 years) to maintain their geriatric status. Online counselling was carried out by geriatricians and ergotherapists to create medical, social, physical, psychological programs of rehabilitation and adaptation. They created special complexes of adaptive physical training which were posted on the websites of territorial social service centers. Each territorial center was assigned a specialist in physical rehabilitation which made daily online group classes on Viber, Skype or Phone. Printed materials were also produced and send by post. Respiratory gymnastics was a mandatory element of all programs. At the request of older persons, adapted complexes were also prepared individually.

Results: Online training with active movement was more popular among women. Men preferred the online game of checkers or chess, which were passive but improved the level of cognition. During 14-days training we observed not improving, but maintenance of the levels of cognition, mobility, prevention of falls. The level of depression and moral status statistically improved, $\mathrm{p}<0,05$. 
Conclusion:s Online physical training allowed to prevent the worsening of geriatric status during social distancing, especially concerning falls, depression and moral status.

\section{Abstract \# 223 \\ Depression of elderly people in Patras during the lockdown measures of COVID-19}

Manolis Mentis ${ }^{1}$, Katerina Athanasopoulou ${ }^{2}$, Konstantinos Stolakis ${ }^{3}$, Georgios Kolokithas ${ }^{4}$, Sofia Plota ${ }^{4}$, Christina Sirmi ${ }^{4}$, Eugenia Chroni $^{1}$, Eeleni Giannakou ${ }^{1}$, Elias Panagiotopoulos ${ }^{3}$

${ }^{1}$ University of Patras (U.P), GREECE, ${ }^{2}$ E.u.Dia. PATRA, GREECE, ${ }^{3}$ University of Patras (U.P), GREECE, E.u.Dia. PATRA, GREECE,

${ }^{4} 3 \mathrm{~d}$ Open Care Centre of Patras (3d O.C.C.P), GREECE

Introduction: The COVID-19 pandemic has affected the elderly, as it has changed their daily life at all levels (activities, health stress, emotional state, social relationships).

Aim: The aim of the study was to investigate the existence of depression in elderly people due to the emergency lockdown measures.

Method: The cross-sectional study was conducted on April 13-22, by telephone interviews in a sample of 65 elderly ( $81.5 \%$ response rate), randomly selected from an Open Care Centre. The Depression Geriatric Scale was used to investigate depression (Cronbach's a $=$ 0.788).

Results: The mean age of the sample was 71.88 years $(\mathrm{SD} \pm 5.92)$ and $80 \%$ were women. The mean value of their self-perceived health on a ten-point scale where 10 stood for "best possible" was 7.24 (SD $\pm 1.53)$. Respectively, the mean value of their self-perceived emotional state was $7.18(\mathrm{SD} \pm 1.73)$, the mean value of their perceptions regarding the extent to which coronavirus changed their life was 8.62 $(\mathrm{SD} \pm 1.22)$ and their average compliance with the State's guidelines was 9.59 ( $\mathrm{SD} \pm 0.26)$. Depression was negatively correlated with self-perceived health $(\mathrm{r}=-0.438, \mathrm{p}=0.001)$, emotional state $(\mathrm{r}=$ $-0.588, \mathrm{p}=0.000)$ and positively correlated with change in life $(\mathrm{r}=$ $0.377, p=0.005)$. Finally, the participants reported activities they missed during the lockdown, highlighting the "absence of a sense of freedom and walks", "activities in Open Care Centre" and "contact with family".

Conclusions: The research pointed out the negative emotional impact of the lockdown in elderly people and the need of community care services.

\section{Abstract \# 224 \\ Interest in meta-research in geriatric medicine: a survey of members of the European Geriatric Medicine Society}

Nicola Veronese ${ }^{1}$, Gabriel Torbahn ${ }^{2}$, Jacopo Demurtas ${ }^{3}$, Charlotte Beaudart ${ }^{4}$, Pinar Soysal ${ }^{5}$, Alessandra Marengoni ${ }^{6}$, Susan Shenkin ${ }^{7}$, Mirko Petrovic ${ }^{8}$, Cornel Sieber ${ }^{9}$, Antonio Cherubini ${ }^{10}$, Lee Smith ${ }^{11}$, Stefania Maggi ${ }^{12}$

${ }^{1}$ University of Palermo, ${ }^{2}$ Friedrich-Alexander-Universität ErlangenNürnberg, Nuremberg, ${ }^{3}$ USL Grosseto, ${ }^{4}$ University of Liege,

${ }^{5}$ Bezmialem Vakif University, ${ }^{6}$ University of Brescia, ${ }^{7}$ University of Edinburgh, ${ }^{8}$ Ghent University, ${ }^{9}$ Friedrich-Alexander-Universität Erlangen-Nürnberg, ${ }^{10}$ IRCCS INRCA, Ancona, ${ }^{11}$ Anglia Ruskin University, ${ }^{12}$ Consiglio Nazionale delle Ricerche

Purpose: There has been an exponential increase in meta-research, especially in the branch dealing with systematic reviews [SRs] and meta-analyses [MAs]. However, the knowledge regarding these topics in geriatric medicine is still poorly explored. We therefore undertook a survey of the current knowledge and needs in meta-research in geriatrics.

Methods: A short survey (taking approximately 5 min to complete) was freely available on the European Geriatric Medicine Society (EuGMS) website and disseminated via social networks by the EuGMS and the authors of the survey. The questionnaire was available during the whole year of 2019. The questionnaire specifically addressed demographic information, previous research activities and the knowledge of the participants on meta-research in geriatric medicine.

Results: The survey was completed by 291 participants from 36 different countries of about 3,000 EuGMS members (age 55-64 years; $51.5 \%$ female; mainly from Italy and Germany). Most respondents $(65.6 \%)$ reported reading more than 20 articles in the past year, but few (36.4\%) read more than 10 SRs/MAs. Participants reported that SRs and/or MAs are important in clinical practice: $83.8 \%$ giving a score of $>6 / 10$, and $23.0 \%$ reporting 10 . The large majority of the participants asked for more education in meta-research. In particular, there is need for educational courses for metaresearch in geriatric medicine, online or in person, organized by the EuGMS.Conclusion: Our survey shows that interest in SRs/MAs is high, and there is a demand for education on these topics in relation to geriatric medicine.

\section{Abstract \# 225}

Psychological consequences on Geriatric patients hospitalized in a non-Covid Unit during the containment period linked to the SARS-CoV-2 pandemic: observational study.

Bérengère Covens ${ }^{1}$, Tatiana Zuinen ${ }^{2}$, Vanessa Willems ${ }^{1}$, MarieNoelle Geurts ${ }^{1}$, Sandra Higuet ${ }^{1}$

${ }^{1} \mathrm{CHU}$ Charleroi, ${ }^{2} \mathrm{CHU}$ Charlerou

Introduction: During this period of the SARS-CoV-2 pandemic, each country has adopted measures such as containment. However, strict application of such measures has not been without risk for our vulnerable population, already affected by a context of loneliness. The goal of this approach is the search for rapid screening of people at risk of developing signs of anxiety or depression.

Methods: Prospective monocentric study of patients over 75 years old with an MMSE greater than 20/30 hospitalized in the geriatric wards of the ISPPC of Charleroi from March 15, 2020 until 15 May 2020, Agreement of the Ethics Committee. The demographic data, the Geriatric Evaluation and the HAD (Hospital Anxiety and Depression scale) as well as the shorter adaptation of the scale of emotions felt by patients in isolation were studied.

Results: 26 patients answered the questionnaires, 30\% of women, average age was 85 years old, $19 \%$ came from Nursing homes, mean AVQ was $14 / 24$. The emotions questionnaire revealed $46 \%$ of boredom, $61,5 \%$ isolation, but only $23 \%$ feeling of stigmatization and $88 \%$ felt safe in Hospital. Concerning HAD scale :only 1/26 was positive for anxiety and $3 / 26$ for depression. (score $>$ or $=11$ ). Limit scores (8-10) were observed in $26 \%$ for Anxiety and $30 \%$ for Depression.

Conclusion: We can see that our vulnerable elderly patients hospitalized during this unprecedented period achieved family isolation but was able to demonstrate the resources necessary to deal with this situation.By the way we had set up tablets allowing families to stay connected and this link was helpful. 


\section{Abstract \# 226 \\ Influence of COVID-19 concerns on psychological wellbeing in older patients approaching end-stage renal disease}

Carlijn GN Voorend ${ }^{1}$, Mathijs van Oevelen ${ }^{1}$, Margot Nieberg ${ }^{1}$, Noeleen C. Berkhout-Byrne ${ }^{1}$, Hanneke Joosten ${ }^{2}$, Casper F. M. Franssen ${ }^{3}$, Simon P. Mooijaart ${ }^{4}$, Alferso C Abrahams ${ }^{5}$, Willem Jan W Bos ${ }^{1}$, Marjolijn van Buren ${ }^{1}$

${ }^{1}$ Internal Medicine (Nephrology), Leiden University Medical Center, The Netherlands, ${ }^{2}$ Internal Medicine, Maastricht University Medical Center, The Netherlands, ${ }^{3}$ Nephrology, University Medical Center Groningen, The Netherlands, ${ }^{4}$ Gerontology and Geriatrics, Leiden University Medical Center, The Netherlands, ${ }^{5}$ Department of Neprhology and Hypertension, University Medical Center Utrecht, The Netherlands

Introduction: In February 2020 the first case of the coronavirus disease 2019 (COVID-19) pandemic was recognized in the Netherlands. To reduce infection rates, significant measures were taken by the Dutch government. Hospitals downscaled all non-acute nonCOVID-19 care. Feelings of both fear and depression increased after the emergence of the pandemic, particularly having an impact on (older) vulnerable persons who are at risk for COVID-19 and in need of routine hospital care. Our aim is to investigate the influence of COVID-19 concerns on psychological wellbeing in older patients nearing end-stage renal disease.

Methods: The Pathway for OLder patients reaching End stage Renal disease (POLDER) study enrolled patients of 70 years or older with an eGFR $<20 \mathrm{~mL} / \mathrm{min} / 1,73 \mathrm{~m}^{2}$ from October 2018 onward, to assess the feasibility of implementing a nephrology-tailored geriatric assessment. Before the COVID-19 outbreak, baseline data was collected on health-related quality of life (HRQoL), symptoms, optimism, and depression. In May 2020, we sent additional questionnaire to participants who were still alive and not lost to follow-up $(n=106)$. Response allows prospective comparison with baseline measurement on measures of HRQoL (using Short Form 12), symptoms (Dialysis Symptom Index), optimism (Life Orientation Test Revised), and depression (Geriatric Depression Scale 2/-15). In addition, fear (Hospital Anxiety Depression Scale) and stress (Perceived Stress Scale 10) during the COVID-19 pandemic will be assessed cross-sectionally.Results: First results are expected in July 2020.

Key conclusions: This study provides insights on the influence of the COVID-19 pandemic on the psychological wellbeing of older patients approaching end-stage renal disease.

\section{Abstract \# 227 \\ Effect of quarantine of COVID-19 pandemic on sleep quality, in elderly persons.}

Konstantinos Stolakis ${ }^{1}$, Stamatina Aggelakou-Vaitsi ${ }^{2}$, Konstantinos Fikas $^{3}$, Sofia Fika ${ }^{4}$, Nikolaos Vaitsis ${ }^{5}$

${ }^{1}$ Private Geriatric Medical Ambulatory, Kiato, Greece, ${ }^{2}$ Private Pain Management Medical Ambulatory, Farsala, Greece, ${ }^{3}$ Private Pharmacy, Farsala, Greece, ${ }^{4}$ Private Dentist Ambulatory, Volos, Greece, ${ }^{5}$ Health Data Analyst, Private Medical Ambulatory, Farsala, Greece

Introduction: To study the effect of quarantine of COVID-19 pandemic on sleep quality, in elderly persons.

Methods: In this repeated-measures study, a self-administered questionnaire consisting of demographics and Pittsburgh Sleep Quality Index (PSQI) was handed out to a convenience sample of elderly individuals aged $65-75$ years. The questionnaire was completed twice by the same individuals, 15 days after the start of quarantine and 15 days after its termination. McNemar test and correlation coefficients was selected for data analysis.

Results: The questionnaire was filled out by 102 persons. About $76.5 \%$ of our sample had a global PSQI score of more than 5 (cut off of poor sleep quality) during the quarantine. An exact McNemar's test determined that the poor sleep quality was associated with quarantine ( $p<0.01$. Regarding gender, it was found that men are more affected than women $(p<0.05)$. Regarding political beliefs, the quality of sleep was most affected by those who supported the government $(\mathrm{p}<$ 0.01 ).

Conclusions Our findings show that sleep quality is affected by quarantine, gender and political beliefs. Citizens who do not support the government seem to question the importance of precautionary measures against the COVID-19 pandemic. Political leaders, along with medical associations, need to make substantial reforms to change people's perceptions of the relationship between science and politics.

\section{Abstract \# 228}

Falls during covid-19 imposed social isolation

Franco Davies ${ }^{1}$, Victoria Massalha ${ }^{2}$

${ }^{1}$ Mater Dei Hospital, Malta, ${ }^{2}$ Physiotherapy Services, Ministry for Health

Mater Dei is the acute general hospital in Malta with the only fully equipped emergency department (ED) in the country. ED Physiotherapy services were established since 2016 and among the many conditions seen, falls in the elderly are a common occurrence. To facilitate management of the devastating consequences of this serious health problem, mechanisms of assessment and referral have been put in place to identify and treat frailty and frequent falls in Malta's aging population.With the onset of the Covid-19 pandemic with Malta registering its first case on March 7th 2020, a higher number of referrals to Physiotherapy of elderly frail patients sustaining falls in their homes was registered. The reasons for this rise may be multiple including possible social isolation due to the social distancing restrictions imposed that may have created a general disruption in elderly people's routine and support network and may have also accentuated any medical problems. It was also noted that falls were frequently missed during this crisis due to medical staff being concentration on the Covid-19 emergency.The Physiotherapist operating in the ED helped to raise this awareness with medical and nursing staff and to flag these falls patients by referring them to community teams to prevent further incidents.

\section{Abstract \# 229}

Influence of requests to refrain from going out and the suspension of exercise classes to prevent the spread of COVID-19 infection on participants in exercise classes.

Yuichi Onimura $^{1}$, Shoko Chijiki ${ }^{2}$, Akiko Tsukao $^{3}$, Takuya Tsuruzono $^{3}$, Masahiro Yamaguchi ${ }^{3}$, Shinya Kuno ${ }^{4}$

${ }^{1}$ University of Tsukuba, ${ }^{2}$ University of Tsukuba, Tsukuba Wellness Research, ${ }^{3}$ Tsukuba Wellness Research, ${ }^{4}$ University of Tsukuba, Tsukuba Wellness Research

Introduction: In order to prevent the spread of COVID-19 infection, a state of emergency was declared by the Japanese government on 16 April 2020 for all of Japan. Japanese people has required to refrain from nonessential outings. They were also required to suspend exercise facilities. The purpose of this study was to investigate the 
physical and mental effects of a state of emergency among participants of municipal exercise classes.

Methods: A questionnaire survey was conducted on 683 people (average 69.3 years old) who participated in a municipal exercise class in Mitsuke City, Niigata Prefecture. The participants were asked how refraining from going out and suspended exercise class affected their exercise habits, social participation and mental health.

Results: $72.2 \%$ of the participants felt that they were not getting enough exercise, and $63.4 \%$ reported that their communication with others had decreased. $11.4 \%$ of the participants reported that forgetfulness was a concern for them. Five-fold increase in the proportion of participants who reported that they felt their health had deteriorated compared to before the state of emergency was declared. Participants who reported reduced communication with others reported significantly lower mood, motivation, and interest in daily life compared to those who did not.

Key conclusions: The municipalities surveyed were in areas where there were no COVID-19 cases. Nevertheless, it was suggested that requests to refrain from going out or to suspend exercise facilities may affect motor function, mental health and cognitive function.

\section{Abstract \# 230 \\ Use of telemedicine in dementia drugs prescription: need and opportunity in the COVID-era}

Giulia Lucarelli ${ }^{1}$, Carlotta Mancini ${ }^{1}$, Marta Migliorini ${ }^{1}$, Giacomo Fortini $^{1}$, Giulia Pelagalli ${ }^{1}$, Maria Flora D'Andria ${ }^{1}$, Virginia Tortù ${ }^{1}$, Mauro Di Bari ${ }^{1}$, Stefano Fumagalli ${ }^{1}$, Raffaele Antonelli Incalzi ${ }^{2}$, Andrea Ungar ${ }^{1}$, Enrico Mossello ${ }^{1}$

${ }^{1}$ Division of Geriatric and Intensive Care Medicine, Careggi Hospital and University of Florence, Italy, ${ }^{2}$ Geriatric and Gerontology Department, Campus Bio-Medico University of Rome, Italy

Introduction: In March 2020, the Italian government adopted lockdown as a measure to contain the spread of COVID-19. Access to hospitals, including outpatient clinics, was limited to urgent cases, forcing health professionals to apply innovative approaches to managing patients with a variety of chronic diseases, including persons with dementia (PWDs).

Methods: A phone assessment of PWDs previously referred to the geriatric memory clinic of Careggi Hospital, Florence, Italy, was designed, mainly based on the application of checklists for prescription of cholinesterase inhibitors, memantine, and antipsychotics (APs). The tool was included in the national GeroCovid observational study. In selected cases, the caregiver was required to shoot and send via internet videos of the patient during daily life.

Results: From March 9 to April 30, 2020, 345 phone interviews and 222 checklists of drug prescription were completed. Videos were requested for 16 patients, mainly because of behavioural disorders and/or motor impairment, to evaluate the possibility of introducing or modulating the dosage of APs. A new AP was introduced, or the dose was up-titrated, in 8 out of 16 cases, whereas the drug was withdrew or witheld in 5 cases due to evidence of bradykinesia. Two cases of new-onset dyskinesias were detected.

Conclusion: Telemedicine may be an efficient way to monitor specific drug prescriptions in dementia care. This approach, which was mandatory during the epidemic period, might also be useful in the future for clinical monitoring of PWDs. Appropriate informatic platforms should be developed to improve data transmission and protection of personal data.

\section{Abstract \# 231}

Ramifications of COVID-19 Awareness: Psychology of Indian Geriatric population during nationwide lockdown.

Oshin Puri ${ }^{1}$, Arpit Singh ${ }^{1}$, Kavita Swami ${ }^{1}$, B. S. Vighnesh ${ }^{2}$, Monika Pathania $^{3}$, Jitendra Rohilla ${ }^{4}$

${ }^{1}$ MBBS Student, All India Institute of Medical Sciences, Rishikesh, India, ${ }^{2}$ Intern Doctor, All India Institute of Medical Sciences, Rishikesh, India, ${ }^{3}$ Associate Professor, General Medicine, All India Institute of Medical Sciences, Rishikesh, India

Introduction: According to $\mathrm{CDC}$, older adults [ $>$ 65years] are at higher risk of severe illness from COVID-19 and studies suggest a relation between anxiogenesis and immune response thus, understanding the cause of COVID associated anxiety among predisposed geriatric participants during the pandemic is necessary.

Methods: This cross sectional study was conducted with Indian geriatric participants aged $>$ 65years during lockdown excluding patients of COVID-19 or any psychiatric disorder. A questionnaire was administered using google forms through snowball sampling to quantify COVID awareness [self-designed, Likert validated scale], anxiety [GAD-7] and clinical phobia [DSM-5].

Results: Data of $\mathrm{N}=30$ participants [Age $=67.46 \pm 2.08,23$ males, 7 females] was collected. Anxiety and awareness were moderately correlated [Spearman's coefficient $=0.56$ ] and the anxious participants [GAD-7 score $>4$ ] were significantly more aware than nonanxious participants. [Mann Whitney U Z-factor $=3.05>1.96$ ]. Phobic participants were significantly more aware than non-phobic participants. [Mann Whitney U Z-factor $=2.50>1.96$ ]. Physical exercise, yoga or meditation practicing participants were less anxious although the difference was not significant.

Conclusions: Awareness about COVID deaths and cases renders the geriatric population anxious and, a smaller proportion phobic. Both these factors might influence the immune response of the participant and thus make him/her more susceptible to adverse outcomes in case of infection. Awareness is necessary but it's effects must be monitored by regular mental health follow ups to avoid its repercussions. Low sample size was a limitation due to avoidance of android devices but Indians geriatric population.

\section{Abstract \# 232}

Physical and psychological wellbeing during the COVID-19 Movement Restriction Order period among cognitively frail older persons with prior engagement in community-based multidomain intervention.

Resshaya Roobini Murukesu ${ }^{1}$, Devinder Kaur Ajit Singh ${ }^{1}$, Suzana Shahar $^{2}$, Ponnusamy Subramaniam ${ }^{3}$

${ }^{1}$ Physiotherapy Programme \& Centre for Healthy Ageing and Wellness, Faculty of Health Sciences, Universiti Kebangsaan Malaysia, Kuala Lumpur 50300, Malaysia, ${ }^{2}$ Dietetic Program and Centre for Healthy Ageing and Wellness, Faculty of Health Sciences, Universiti Kebangsaan Malaysia, Kuala Lumpur 50300, Malaysia, ${ }^{3}$ Health Psychology Programme \& Centre for Healthy Ageing and Wellness, Faculty of Health Sciences, Universiti Kebangsaan Malaysia, Kuala Lumpur 50300, Malaysia

Introduction: Movement Control Order (MCO) was implemented in Malaysia over a period of 12 weeks during the COVID-19 pandemic crisis. We compared physical and psychological wellbeing of two matched cognitively frail, community dwelling older persons groups, with prior engagement in a 6-month supervised community-based multi-domain intervention encompassing exercise and cognitive training (CBMDI) versus usual care (UC). 
Methods: Both CBMDI $(\mathrm{n}=21 ; 67.74 \pm 4.43$ years $)$ and $\mathrm{UC}(\mathrm{n}=21$; $70.79 \pm 7.03$ years) groups were interviewed via phone call. Cognitive frailty was identified using Clinical Dementia Rating Scale and Fried Frailty Index during previous study assessments. Physical and psychological wellness were assessed using International Physical Activity (IPAQ), Functional Activity (FAQ), General Health (GHQ) and Brief COPE Scale questionnaires. Data was compared using independent t-test.

Results: CBMDI group had significantly higher levels of estimated resting energy expenditure (MET) for 'walking' (CBMDI: $\mu=1723 \pm$ 780; UC: $\mu=537 \pm 581)(\mathrm{p}<0.01)$, 'moderate intensity' $(\mu=1422 \pm$ 1215; UC: $\mu=405 \pm 746)(\mathrm{p}<0.01)$ and lower 'sedentary' (CMDPI: $\mu=1723 \pm 780$; UC: $\mu=2130 \pm 697)(\mathrm{p}<0.05)$. activities compared to UC group. CBMDI group was also significantly more independent in functional activity $(\mu=1.76 \pm 1.73)$ compared to UC group $(\mu=5.57 \pm 8.31)(p<0.05)$. Regarding coping behaviour, CBMDI group relied significantly on the domains of religion $(\mathrm{CBMDI}: \mu=6.43 \pm 0.99$; UC: $\mu=6.09 \pm 1.09)(\mathrm{p}<0.05)$ and planning (CBMDI: $\mu=4.81 \pm 0.75$; UC: $\mu=4.04 \pm 1.28)(\mathrm{p}<0.05)$. In contrast, UC group relied on humour (UC: $\mu=3.14 \pm 1.19$; CBMDI: $\mu=2.38 \pm 0.74)(\mathrm{p}<0.05)$. However, there was no significant difference for GHQ scores between both groups (CBMDI: $\mu=$ $1.57 \pm 3.36$; UC: $\mu=1.24 \pm 3.28)(\mathrm{p}>0.05)$, indicating similar psychological wellbeing status.

Conclusion: Older persons with prior engagement in communitybased multi-domain intervention were more physically active, functionally independent and resorted to positive coping behavior using religion and planning strategies throughout the MCO. Communitybased multi-domain interventions should be advocated to promote resilience among older persons.

\section{Abstract \# 233 \\ Preferences of older adults for Covid-19 Tracking and Tracing apps}

Annemiek Linn ${ }^{1}$, Fam te Poel $^{1}$, Susanne Baumgartner ${ }^{1}$, Eline Smit ${ }^{1}$, Hande Sungur ${ }^{1}$, Liset van Dijk ${ }^{2}$

${ }^{1}$ Amsterdam School of Communication Research, ${ }^{2}$ Netherlands Institute for Health Services Research

Introduction: The COVID-19 pandemic is impacting the global population and especially older adults are facing the most serious threats. Governments are looking at tracking and tracing apps as a means to slow the spread of the virus. These apps have raised privacy concerns, as some of these apps need to store user data. Given these concerns, it is unclear if older adults are willing to use these. The aim of this study is to explore older adults' preferences for using Covid-19 tracking and tracing apps.

Methods: An online survey was distributed among panel members of a panel company in the Netherlands. The data were collected in April. A total of 1068 respondents completed the questionnaire. For this study, we only focus on participants aged 65 and older $(\mathrm{N}=259)$. The questionnaire included questions regarding media use and information needs during the Covid-19 pandemic.

Preliminary results: Most older adults $(96.5 \%)$ indicated that they heard of Covid-19 tracking and tracing apps. Just under a third indicated that they are willing to use such an app, while more than $40 \%$ of the respondents are not sure. Many of them believe that their privacy cannot be guaranteed (59.4\%). Besides, $20 \%$ mistakenly believes that the app will protect them against infection.

Key conclusions: If health organizations decide to start using a tracking and tracing app this app should be developed in collaboration with the users and based on existing guidelines for mHealth.

\section{Abstract \# 234}

General psychological health status and coping strategies during Lock-down due to COVID-19 outbreak among Malaysian Older Adults

Nurul Fatin Malek Rivan", Suzana Shahar ${ }^{2}$, Devinder Kaur Ajit Singh $^{3}$, Norhayati Ibrahim ${ }^{4}$, Arimi Fitri Mat Ludin', Hanis Mastura Yahya ${ }^{1}$, Noor Ibrahim Mohamed Sakian ${ }^{6}$, Hazlina Mahadzir $^{7}$, Ponnusamy Subramaniam ${ }^{4}$, Mohd Zul Amin Kamaruddin $^{8}$, Resshaya Murukesu ${ }^{3}$, Jing Wen Goh ${ }^{3}$, Muhammad Rabbani Abdul Malek ${ }^{2}$, Raazliza Rashid ${ }^{8}$

${ }^{1}$ Nutritional Sciences Programme and Centre for Healthy Ageing and Wellness (H-CARE), Faculty of Health Sciences, Universiti Kebangsaan Malaysia, Jalan Raja Muda Abdul Aziz, 50300 Kuala Lumpur, Malaysia, ${ }^{2}$ Dietetics Programme and Centre for Healthy Ageing and Wellness (H-CARE), Faculty of Health Sciences, Universiti Kebangsaan Malaysia, Jalan Raja Muda Abdul Aziz, 50300 Kuala Lumpur, Malaysia, ${ }^{3}$ Physiotherapy Programme \& Centre for Healthy Ageing and Wellness (H-CARE), Universiti Kebangsaan Malaysia, Jalan Raja Muda Abdul Aziz, 50300 Kuala Lumpur, Malaysia, ${ }^{4}$ Health Psychology Programme and \& Centre for Healthy Ageing and Wellness (H-CARE), Faculty of Health Sciences, Universiti Kebangsaan Malaysia, Jalan Raja Muda Abdul Aziz, 50300 Kuala Lumpur, Malaysia, ${ }^{5}$ Biomedical Science Programme, School of Diagnostic and Applied Health Sciences, Faculty of Health Sciences, Universiti Kebangsaan Malaysia, Jalan Raja Muda Abdul Aziz, 50300 Kuala Lumpur, Malaysia, ${ }^{6}$ Occupational Therapy Programme, Centre for Healthy Ageing and Wellness (H-CARE), Faculty of Health Sciences, Universiti Kebangsaan Malaysia, Jalan Raja Muda Abdul Aziz, 50300 Kuala Lumpur, Malaysia, ${ }^{7}$ Internal Medicine \& Geriatric Department, Pusat Perubatan Universiti Kebangsaan Malaysia, Jalan Yaacob Latif, Bandar Tun Razak, 56000 Batu 9 Cheras, Kuala Lumpur, Malaysia, ${ }^{8}$ Centre for Healthy Ageing and Wellness $(\mathrm{H}-$ CARE), Faculty of Health Sciences, Universiti Kebangsaan Malaysia, Jalan Raja Muda Abdul Aziz, 50300 Kuala Lumpur, Malaysia; Malaysia

Introduction: Older adults as the higher risk group for COVID-19 infection were known to face psychological and coping difficulties during this pandemic. Therefore, this study aimed to assess the general psychological health and coping strategies among Malaysian older adults during this pandemic crisis.

Method: A total of 340 of individuals aged above 60 years $(\mathrm{men}=$ 159 , women $=181)$, from the earlier cohort (LRGS TUA) and interventional studies from four selected states in Peninsular Malaysia were followed up during the lock-down period (MCO) due to COVID-19 outbreak (April-June 2020). Telephone interviews were conducted by trained interviewers from health sciences background to obtain participants' socio-demographic information, general psychological health, and coping strategies using Malay version of 12-items General Health (GHQ-12) and Brief COPE questionnaire. The analysis was performed using SPSS Statistics version 25.0 and presented as mean $\pm \mathrm{SD}$.

Result: The result revealed that the participants have a low mean score of GHQ-12 $(0.32 \pm 1.44)$. After adjusting for age, gender, ethnicity, years of education, household income, and smoking status, participants living with their family significantly have a greater impact on their psychological health $\left(R^{2}=0.326, p<0.05\right)$. Besides, the participants used adaptive coping strategies, in which the highest mean score was religion $(4.28 \pm 2.73)$, followed by positive reinterpretation $(3.59 \pm 2.09)$, acceptance $(3.42 \pm 1.90)$, planning (3.25 $\pm 1.84)$, active coping ( $3.20 \pm 1.72)$, use of instrumental $(2.63 \pm$ $1.04)$ and emotional support $(2.56 \pm 1.02)$.

Conclusion: This finding indicated that the Malaysian older adults had good psychological health status, especially when they are living 
together with their family and adapted positive coping mechanisms during this pandemic crisis.

\section{Abstract \# 235 \\ Investigation of leisure time activities and anxiety in elderly of Non-Covid living at home during COVID-19 period}

Sebahat Yaprak Cetin ${ }^{1}$, Alime Buyuk ${ }^{1}$, Gokce Yagmur Gunes Gencer $^{1}$

${ }^{1}$ Akdeniz University

Introduction: The COVID-19 are affected the elderly negatively around worldwide. In COVID-19 period in Turkey had a curfew for the elderly. The aim of our study was to examine leisure time activities (LTA) and anxiety levels in the elderly living at home.

Methods: 73 elderly adults ( 37 women, 36 men) with an average age of $67.41 \pm 5.60$ years living at home in a region of Antalya city participated in the study. LTA were determined by the elderly, such as reading, watching $\mathrm{TV}$, and doing exercise, and the frequency and duration of activities were recorded. Geriatric Anxiety Inventory (GAI) was used for anxiety assessment.

Results: It was found that the elderly attended LTA such as watching TV $(78.1 \%)$, meeting by phone $(69.9 \%)$, praying, cooking and reading respectively, at home for all day. GAI score was $10.41 \pm 5.23$. It was found that $97.3 \%$ of the elderly did not exercise at all. In addition, a positive correlation was found between GAI score and praying (r: 0.580 , p: 0.01).

Key conclusions: According to the results of this study, it was observed that the elderly do activities that they were not active during the period of COVID-19. In addition, the elderly had moderate anxiety. Also, as anxiety increases, the rate of praying increases. The results of the study support that LTA should be organized by health and community professionals in order to reduce the level of anxiety, ensure that they are more active and productive during the periods of obligation to stay at home.

\section{Abstract \# 236 \\ Impact of Covid19 restrictions on older patients attending an outpatient geriatric medicine service}

Ruth Daunt ${ }^{1}$, Alanna Keena ${ }^{1}$, StJohn Kelliher ${ }^{2}$, Alice O’Donoghue ${ }^{1}$, Colm Byrne ${ }^{1}$, Linda Brewer ${ }^{1}$

${ }^{1}$ Beaumont Hospital, ${ }^{2}$ University College Cork

Introduction: During the Covid19 pandemic older adults $(>70$ years) in Ireland were advised to stay at home and avoid unnecessary physical contact-termed 'cocooning'. During this time hospital outpatient appointments were conducted virtually (via telephone). This project aimed to assess patient's experience of Covid19 cocooning and the virtual outpatient service.

Methods: Patients attending an outpatient geriatric medicine service were invited to participate in this project following their routine virtual assessment. A proforma questionnaire was administered to participants contacted virtually within 1 week of consent. Data was anonymised, imported into excel and analysed using SPSS (chisquared test).

Results: 31 patients were included, median age was $83.52 \%$ were male, $25 \%$ lived alone and $52 \%$ had cognitive impairment. $75 \%$ had heard of 'cocooning', of which $67 \%$ correctly understood the term. Three-quarters stated that cocooning had negatively impacted their quality of life (QoL); anxiety, loneliness and depression were reported in $49 \%, 36 \%$ and $29 \%$ respectively. $39 \%$ strongly feared getting Covid19. Cognition and living status did not correlate significantly with the psychological impact of Covid19. 61\% did not use modern social media, its use did not impact QoL scores. $45 \%$ experienced a reduction in support services. $87 \%$ found the outpatient telephone service useful and $77 \%$ reported their needs were addressed. Threequarters favoured future virtual assessments.

Conclusion: One third of patients poorly understood 'cocooning'. Necessary Covid19 restrictions impacted negatively psychologically on this older cohort. Novel virtual clinics proved a positive experience and a useful future outpatient resource even amongst our oldest patients with dementia.

\section{Abstract \# 237}

What is the opinion of Brazilian and Portuguese elderly people about Covid19?

Manuel Barbosa ${ }^{1}$, Anna Fernandes ${ }^{2}$, Glaucia Martins ${ }^{2}$

${ }^{1}$ Universidade de Aveiro - Departamento de Educação e Psicologia, ${ }^{2}$ Escola de Artes, Ciências e Humanidades, EACH - USP

Introduction: The first case of a new coronavirus was identified in Wuhan, China, on December 1, 2019 [1,2] and was declared by the World Health Organization's World Pandemic on March 11, 2020. During this pandemic, the world has adopted measures of isolation and social distance.

Methods: This is a cross-sectional, quantitative and exploratory study, which included a sample collected online through social networks composed of 159 seniors aged between 60 and 65 years old, residing in Portugal and Brazil. According to the exclusion criteria described above, for the analysis of the results it was necessary to exclude 11 participants, 6 Portuguese and 5 Brazilian. The final sample included 96 Brazilian seniors and 52 Portuguese seniors who answered the questionnaire online, through Google Forms.

Results and main conclusions: Categorizing the responses of the participants, the results indicate that the majority of the elderly interviewed have feelings of fear and concern about the Coronavirus associating it with death. It is noticed that these manifestations are due to the condition of isolation necessary to contain the virus, in addition to the lack of general knowledge about the consequences of the same. It is necessary, however, in view of the reduced sample; expansion of the study, to better deepen the social issues that guide the opinions presented about the coronavirus.Keywords: COVID-19; Seniors; Brazilian and PortugueseBibliographic reference

[1] H. Lu, C.W. Stratton, Y.W. Tang - Outbreak of pneumonia of unknown etiology in Wuhan China: the mystery and the miracleJ Med Virol (2020 Jan 16), 10.1002/jmv.25678

[2] Q. Li, X. Guan, P. Wu, X. Wang, L. Zhou, Y. Tong, et al.Early transmission dynamics in Wuhan, China, of novel coronavirus-infected pneumoniaN Engl J Med (2020 Jan 29), 10.1056/ NEJMoa2001316

\section{Abstract \# 238}

Supporting older adults during the coronavirus outbreak: social initiatives of the LLM Care ecosystem and reference site

Evangelia Romanopoulou ${ }^{1}$, Vasiliki Zilidou ${ }^{1}$, Ionnna Dratsiou ${ }^{1}$, Maria Metaxa ${ }^{1}$, Aikaterini Katsouli ${ }^{1}$, Foteini Dolianiti ${ }^{1}$, Sotiria Gylou $^{1}$, Maria Karagianni ${ }^{1}$, Vasileia Petronikolou ${ }^{1}$, Panagiotis D. Bamidis ${ }^{1}$

${ }^{1}$ Lab of Medical Physics, School of Medicine, Aristotle University of Thessaloniki, Greece

Introduction: In the twenty-first century, the epidemics are spreading more rapidly than ever affecting ever-larger numbers of people [1]. 
The global pandemic of coronavirus seems to introduce a new crisis in different levels with severe consequences affecting the psychological condition of the older adults by raising degrees of stress, uncertainty or anxiety [2]. In the context of this new reality, the adoption of a productive and beneficial routine might be crucial for older adults. This study describes a (social care) initiative taken within the coronavirus outbreak and examines the potential benefits in older adults' quality of life.

Methods: For this purpose, the Integrated Healthcare System Long Lasting Memories Care (LLM Care) [3] was exploited, and more specifically the web-based cognitive training software [4] was used for 2 months. An online questionnaire, including consent form and assessments (SAST, IES-R-Gr, WHO-5, SUS) [5-8], was distributed to 20 older adults (mean age 69.54 years $\pm 4,61$ ) before and after the exploitation of cognitive training software aiming at evaluating the potential positive effect and usability of cognitive training software. Results: Overall, the study demonstrates interesting findings with regards to the degree of stress and anxiety, the quality of older adults' life, as well as the usability of the web-based cognitive training system.

Key conclusions: Consequently, the use of web-based applications is considered to play a significant role during this severe situation in improving older adults' health well-being. Acknowledgements: This research is supported by the business exploitation scheme of the ICTPSP funded project LLM, namely, LLM Care which is a self-funded initiative at the Aristotle University of Thessaloniki (www.1lmcare.gr). References:

[1] World Health Organization. (2018). Managing epidemics: key facts about major deadly diseases. World Health Organization.

[2] World Health Organization. (2020). Mental health and COVID19. Retrieved from http://www.euro.who.int/en/health-topics/ health-emergencies/coronavirus-covid-19/technical-guidance/mentalhealth-and-covid-19

[3] Long Lasting Memories Care. (2020). Retrieved from www.llmcare.gr

[4] BrainHQ. (2020). Retrieved from gr.brainhq.com

[5] Grammatikopoulos, I. A., Sinoff, G., Alegakis, A., Kounalakis, D., Antonopoulou, M., \& Lionis, C. (2010). The short anxiety screening test in Greek: Translation and validation. Annals of general psychiatry, $9(1), 1$.

[6] Mystakidou, K., Tsilika, E., Parpa, E., Galanos, A., \& Vlahos, L. (2007). Psychometric properties of the Impact of Event Scale in Greek cancer patients. Journal of Pain and Symptom Management, 33(4), 454-461

[7] Heun, R., Bonsignore, M., Barkow, K., \& Jessen, F. (2001). Validity of the five-item WHO Well-Being Index (WHO-5) in an elderly population. European archives of psychiatry and clinical neuroscience, 251(2), 27-31.

[8] Katsanos, C., Tselios, N., \& Xenos, M. (2012, October). Perceived usability evaluation of learning management systems: a first step towards standardization of the System Usability Scale in Greek. In 2012 16th Panhellenic Conference on Informatics (pp. 302-307). IEEE.

\footnotetext{
Abstract \# 239

The effects of virtual group and individual exercises on anxiety and depression among older persons during the COVID-19 pandemic

Amirah Fatin Ibrahim ${ }^{1}$, Maw Pin Tan ${ }^{2}$, Mei Chan Chong ${ }^{3}$

${ }^{1}$ Ageing and Age-Associated Disorders Research Group, University of Malaya, ${ }^{2}$ Department of Medicine, Faculty of Medicine,
}

University of Malaya, ${ }^{3}$ Department of Nursing, Faculty of Medicine, University of Malaya

Introduction: Social isolation has been found to increase the risk of poor health and death, and the risk has been said to be as high as cigarette smoking. Yet during the COVID-19 pandemic, governments internationally have to resort to social distancing. This study aims to overcome psychosocial repercussion of social distancing by determining the effects of virtual exercises for older persons on mental well-being and factors which influence their adherence to the physical exercises during the COVID-19 pandemic.

Methods: This is a quasi-experimental study involving participants of the Promoting Independence in Seniors with Arthritis (PISA) study which was carried out between 2015 and 2019. Mental well-being is measured using the Hospital Anxiety and Depression Scale (HADS) pre-intervention and post-intervention. HADS scores were also available from annual visits conducted during the study. The 4-week virtual exercise was tailor-designed for older persons. Daily $30 \mathrm{~min}$ exercise on Google Meet as the virtual platform is led by health care professionals. Participants are required to keep a diary of when they exercised to promote adherence.

Results: The original 251 PISA participants were approached using an online bulk text messaging application (Whatsapp) and 46 agreed to participate by replying to the text message. The results of their 4-week scores at baseline and 4-week follow-up will be available to report by mid of July.

Conclusions: Older persons are considered a vulnerable group during the COVID-19 pandemic. By conducting virtual exercises, the risk of COVID-19 through physical contact could be removed while maintaining social contact using modern telecommunications.

\section{Abstract \# 240}

Impact of the Covid-19 Pandemic on Physical Capacity and Activity of People over 60

\author{
Jessica Koschate ${ }^{1}$, Sandra Lau ${ }^{1}$, Michel Hackbarth ${ }^{1}$, Tania \\ Zieschang $^{1}$ \\ ${ }^{1}$ Carl von Ossietzky University Oldenburg, Geriatric Medicine
}

Introduction: The measures taken to flatten the infection rate during the Covid-19 pandemic were accompanied by enormous restrictions in social life and organized sports activities. Especially older people, a high-risk group for a severe course of the disease, severely restricted their range of action. However, especially in older adults, reduced physical activity (PA) can quickly lead to reductions in muscle mass. The aim of the study is to determine the extent to which seniors used alternative ways of maintaining physical fitness and to evaluate their efficacy.

Methods: Persons (60+) who trained in a standardized fitness circle which records individual training data, are examined. The participants are asked about their PA and mobility in daily life before, during, and after the pandemic-related restrictions. The primary endpoint of the study is the difference in the product of training weight and number of repetitions at the circle's leg extension compared between the three last trainings before and the first trainings after the restrictions.

Results: The training data are evaluated by means of analysis of variance. Level of significance will be set to $\alpha=5 \%$. Discussion: Based on the results of the data collection, the effects of the pandemic-related restrictions on the physical capacities of older people can be assessed. Based on these results, measures can be identified to maintain physical fitness of older adults when organized sports activities are not available and give recommendations for PA during a potential second wave of the pandemic. 


\section{Abstract \# 241}

“Online Society" - Elderly Groups

Athina Greka ${ }^{1}$

${ }^{1}$ Medecins Du Monde Greece

Abstract: In the context of the innovations required due to the unprecedented situation we experienced in Greece and worldwide, Medecins du monde, Greece, implemented a pilot for 4 months, a new application entitled "Internet companionship".

Introduction: Participants: Lonely elderly people of both sexes, over 65 years. The aim was to create virtual groups, in order to break the isolation of the lonely elderly people, for communication, under the guidance of a team of experts: Internist-Gerontologist, Nurse, Psychologist, Social worker . Program Coordinator Team members could interact with each other through simple activities. The functionality of new applications was tested, in relation to the perception and compliance of the elderly in them, in order to establish inventive communication solutions of this age group, which could be useful even after the end of the alert period due to the corona .

Method: The technology used was simple and suitable for the elderly. It was software built into a tablet with a simple and understandable form of use. At a predetermined time, the group as a whole participated in the online company.

Results: 1. Initially the elderly, presented fears and difficulties in understanding the new communication technology. 2. But finally they were looking forward to each meeting, in order to communicate with each other, but also with the program coordinator. 3. They also actively participated in their interactive activities on their screens (eg gymnastic exercises). 4. At the end of the program they were more familiar with the new communication.

\section{Abstract \# 242}

Feasibility of video-based physiotherapy for older people in the context of the COVID-19 pandemic in Germany

Lisa Happe ${ }^{1}$, Sandra Lau ${ }^{2}$, Rebecca Diekmann ${ }^{1}$, Tania Zieschang ${ }^{2}$, Andreas Hein ${ }^{1}$

${ }^{1}$ Assistance Systems and Medical Technology, Department of Health Services Research, Carl von Ossietzky University, Oldenburg, ${ }^{2}$ Geriatrics, Department of Health Services Research, Carl von Ossietzky University, Oldenburg

Introduction: Older people are a group at risk for a severe course of Covid-19 disease. The current measures to protect from infection are likely to cause adverse effects by reducing older peoples' physical activity. Currently, many older patients avoid attending physiotherapy although it can prevent a deterioration of functional abilities, especially in multimorbid patients. Real time video-based physiotherapy sessions could be a useful supplement to standard physiotherapy. Since this therapy option was introduced in Germany due to Covid-19 pandemic for the first time, physiotherapy practices are not equipped and empirical data do not exist, yet.This study aims to investigate the feasibility of video-based physiotherapy as an alternative therapy option for older patients ( $\geq 60$ years) and its influencing factors.

Method: Patients have been equipped with hardware and corresponding software for six weeks. Physiotherapy practices receive technical support. The number of video-based physiotherapy sessions are documented and individual experiences of physiotherapists and patients are reported via questionnaires. Therapists briefly document important aspects after each therapy session and for the whole treatment. Telephone interviews are used to collect demographic data, acceptance of technology and the use of technical communication devices from patients at the beginning and end of the study.
Results: Nine participants have been included so far (mean age 73.0 ( \pm 5.5 ) years). Video-based physiotherapy proves to be technically feasible. Acceptance and appraisal vary between individuals.

Conclusion: Barriers regarding the technical application of videobased physiotherapy exist on patients' side, but to a considerable extent also on the side of the physiotherapists.

\section{Abstract \# 243}

Nursing approaches and their effect on the health of the elderly with Covid-19

Fatimatüzzehra Genç ${ }^{1}$, Arzu Uslu ${ }^{1}$

${ }^{1} \mathrm{RN}, \mathrm{MSc}$

Fatımatüzzehra GENÇ1, Arzu USLU2 1, 2 Necmettin Erbakan University Faculty of Nursing, İnstructor, PhD student.

Introduction: Elderly individuals, considered as a part of the highrisk group, can be affected more severely by Coronavirus disease. This review aims to discuss the protection and appropriate nursing methods for managing COVID-19 in elderly.

Methods: This is a narrative review study.

Results: The increase in the elderly population worldwide and the higher prevalence of comorbid diseases in this group, the elderly are particularly at a high risk during this pandemic. Nurses are involved in follow-ups and care as well as in primary, secondary and tertiary health services for the elderly. The disease has adverse effects on the physical health of elder individuals who have a weak immune system, limited activity, and difficulty in taking adequate nutrition. Conditions such as an inability to cope with stress, weakness, and hopelessness adversely affect psychological health. Furthermore, conditions such as loneliness, being away from loved ones, stigmatization, and abuse adversely affect social health as well. Nursing theorists such as Nightingale, Orem, Rogers, Neuman, Roy, and Watson, who have significantly contributed to this discipline, have explained the relationship between the elderly and the environment, self-care, energy field, stress-coping methods, adaptation process, and spiritual care during a pandemic.

Conclusion: Nurses have a significant role and responsibility in preventing the transmission of the virus, treating and caring for the infected elderly, and providing post-discharge rehabilitation. Keywords: COVID-19, elderly, nursing, theory, protection

\section{Abstract \# 244}

Delirium incidence amongst older hip fracture patients in the time of COVID-19 pandemic.

Nur Atikah Mohd Asri ${ }^{1}$, Azrin Muslim ${ }^{1}$, Orlaith Shinners ${ }^{1}$, Lok Yi Tan ${ }^{1}$, Jude Ryan ${ }^{1}$

${ }^{1}$ University Hospital Limerick

NA Mohd Asri1, A Muslim1, Shinners O2, LY Tan2Tyrrell R2, Goh A2, Pamela H2, Finbarr C2, Ryan J11Department of Geriatrics Medicine, University Hospital Limerick, Ireland2Department of Trauma and Orthopaedic Surgery, University Hospital Limerick, Ireland.

Introduction: Opioid-induced delirium is very common amongst hip fracture patients. Introduction of Femoral Nerve Blocks (FNBs) offers a safe and effective method for improving pain in the setting of hip fractures leading to reduced opioid need. It is typically initiated in the emergency department in our institution prior to the COVID-19 pandemic. Due to the reconfiguration of services secondary to COVID-19, the majority of hip fracture patients are no longer receiving FNB which consequently leads to poor pain control and 
higher opioid needs. This paper aims to look at the relationship between delirium and FNB.

Method: Retrospective analysis was performed on all patients over 60 admitted with hip fracture $(n=359)$ in our hospital from June 2018 to June 2019. The data was collected from the Irish Hip Fracture Database (IHFD) and was correlated with the orthogeriatric database from the Electronic Patient Medical System (EPMS). Delirium was assessed using the 4AT tool. Data were analysed using SPSS for statistical analysis.

Result: Demographic information was obtained. The percentage of patients receiving FNB decreased during pandemic from $89.6 \%$ (69/ 77) to $52.5 \%$ (31/59) pre-pandemic. Delirium prevalence increased from $16.8 \%$ (13/77) pre-pandemic to $35.6 \%$ (21/59) during the pandemic. There is $111 \%$ increase in delirium incidence between the two-time points.

Conclusion: The number of patients not receiving FNB has a direct correlation with the incidence of delirium. Early FNB to control pain results in less opioid use and subsequently less incidence of delirium in hip fracture patients.

\section{Abstract \# 245 \\ Interstitial pneumonia in the elderly in the coronavirus era: covid-like or covid-free?}

Alessandra Pitocco ${ }^{1}$, Nelson Anzoletti ${ }^{2}$, Claudia Falcone ${ }^{1}$, Franco Colameco ${ }^{1}$, Rosa Scurti ${ }^{1}$

${ }^{1}$ UOC Geriatria - Pescara, ${ }^{2}$ UOC MALATTIE INFETTIVE PESCARA

An 81-year-old patient arrived in hospital on 4 May 2020 at the Geeiatric department with the diagnosis of interstitial pneumonia in a likely Sars-Cov2 infection. The patient went to the emergency room in the May 3 data for the reported persistence of fever for about three days complicated on the last day by acute dyspnea. She was a patient with advanced Alzheimer's disease complicated by lodging syndrome, Parkinson's disease, high blood pressure, severe dysphagia and hypothyroidism in replacement treatment. The patient takes the following drug therapy: cardioaspirin, lercadipine, levothyroxine, valsartan. In the emergency room, the patient had performed blood chemistry tests with detection of neutrophilic leukocytosis with partial lymphopenia, elevation of RCP, alteration of D-dimer and LDH, with substantial normality of the other laboratory indices, including procalcitonin. Gold-pharyngeal and nasopharyngeal swab negative for Sars-Cov2 research. The blood gas analysis documented acute hypoxemic-hypocapnic respiratory insufficience, mixed alkalosis with normale anion-gap and $\mathrm{P} / \mathrm{f}$ ratio equal to 169 . At the thorax the radiologist reported a "ground-glass" interstitial commitment framework compatible with a CO-RADS 4/5 classification (high probability of Sars-Cov2 infection). In light of the clinical, laboratory and instrumental picture, the infectious disease colleague placed the indication for hospitalization in the covid-area by our hospital. During the hospitalization, in consideration of the positive blood culture for Staphylococcus epidermidis, the patient was treated with antibiotics with a wide range and oxygen therapy, first with Venturi mask and $\mathrm{FiO} 231 \%$ passerà on the fifth day with nasal cannulas with low parameters . During hospitalization, the radiological checks documented a slow and incomplete resolution of the interstitial lung picture with constant negativity of the Sars-Cov2 research both on swab and on serological investigation. After 24 days of hospitalization, the patient was discharged with a complete resolution of the respiratory insufficiency and of the radiological and laboratory framework. In conclusion, the low sensitivity of the diagnostic means for COVID-19 in our possession, together with the great phenotypic variability of the clinical, laboratory and instrumental framework made the diagnosis of certainty and the subsequent therapeutic and management process of the elderly patient complex.

\section{Abstract \# 246 \\ Fighting physical inactivity and functional decline in high risk patients - Digital physiotherapy during COVID-19}

Sandra Lau ${ }^{1}$, Lisa Happe ${ }^{2}$, Jessica Koschate ${ }^{1}$, Andreas Hein ${ }^{2}$, Tania Zieschang ${ }^{1}$

${ }^{1}$ Carl von Ossietzky University Oldenburg, Geriatric Medicine, ${ }^{2}$ Carl von Ossietzky niversity Oldenburg, Assistance Systems and Medical Technology

Background: The COVID-19 pandemic requires innovative strategies in outpatient care. Local physiotherapy practices in northern Germany reported up to $30 \%$ cancelled interventions during the lockdown as older patients feared to be infected by SARS-CoV-2. Although federal restrictions aimed to protect this vulnerable group, the measures may also reduce physical activity and thus accelerate functional decline. Due to the pandemic, regulations were changed so that video-based physiotherapy can now be conducted in Germany. As this is a novel approach, we will investigate health related patient experiences regarding video-therapy.

Methods: Patients aged over 60 years and technically unequipped who had been prescribed physiotherapy were given the opportunity to attend video-therapy sessions. A semi-structured telephone interview was held at the beginning of the study and after 6 weeks questioning health status, physical activity, quality of life, participation, and evaluation of the video-based physiotherapy they received.

Results: Participants' age ranged from 64 to 82 years. Each subject received a maximum of twelve sessions of video-therapy. Patient reported outcomes and experiences are presented qualitatively. Patients' appraisal of video-therapy compared to conventional therapy varied. Further results will be presented in October.

Conclusion: Prospectively, video-therapy could be established during future pandemics, in patients in medically indicated isolation (e.g. in cancer with immunomodulating therapies), or in rural areas. Regarding the ongoing digitalization of the health care system, there is an urgent need to implement and evaluate alternatives to conventional therapy. Further research is needed on the effectiveness, identification of specific target groups, and clinical relevance of video-therapy.

\section{Abstract \# 247 \\ Impact of Anti-epidemic Measures on Non-COVID-19 Older Patients in Romania}

Gabriel-Ioan Prada ${ }^{1}$, Catalina Raluca Nuta ${ }^{2}$, Ioana Dana Alexa ${ }^{3}$, Ovidiu-Lucian Bajenaru ${ }^{1}$, Rozeta Draghici ${ }^{4}$, Alexandra Rusu ${ }^{4}$, Adina Ilie $^{3}$, Anca Pislaru ${ }^{3}$, Ioana Sandu ${ }^{3}$, Ramona Stefaniu ${ }^{3}$, Anna Marie Herghelegiu ${ }^{5}$

${ }^{1}$ University of Medicine and Pharmacy „Carol Davila”, Bucharest, Romania National Institute of Gerontology and Geriatrics „Ana Aslan”, Bucharest, Romania, ${ }^{2}$ University of Medicine and Pharmacy „Carol Davila”, Bucharest, Romania, ${ }^{3}$ University of Medicine and Pharmacy „Gr.T. Popa”, Iassi, Romania, ${ }^{4}$ National Institute of Gerontology and Geriatrics „Ana Aslan”, Bucharest, Romania, ${ }^{5}$ University of Medicine and Pharmacy „Carol Davila”, Bucharest, Romania, National Institute of Gerontology and Geriatrics „Ana Aslan”, Bucharest, Romania

New coronavirus infection labelled COVID-19 generated a significant pandemic despite various measures that have been implemented in 
order to reduce or stop the spread of this virus. One epidemiologic characteristic was that people with significant comorbidities presented the most severe forms of disease and the highest death rates. Since older people in general have a higher prevalence of chronic conditions they seemed to be at highest risk. Consequently, several measures for isolating this category of population have been implemented in many countries, including in Romania. This is a normal approach since so little was known about the specific behavior of this coronavirus infection. Nevertheless, not all older people are the same, they are a highly heterogenous group, as it is well known. Maybe for the future these protective measures need to be different when addressing robust older people and frail older people, tailored to their degree of frailty. This is important since strict home isolation of all older people, irrespective of their comorbid status, could have some consequences. These aspects, including psychological and pathological effects, are evaluated for two regions in Romania to help identify the impact of social isolation and strict home isolation of older people, especially those with little social support, including family support, comparing rural to urban communities.

\section{Abstract \# 248 \\ Teledermatology services for elderly people during COVID-19 pandemic}

Dóra Czintner $^{1}$, Antal Jobbágy ${ }^{1}$, Fanni Meznerics ${ }^{1}$, András Bánvölgyi ${ }^{1}$, Miklós Sárdy ${ }^{1}$

${ }^{1}$ Department of Dermatology, Venerology, and Dermatooncology of Semmelweis University

Introduction: The emergence and spread of COVID-19 have brought great challenges to public health services worldwide. In Hungary, like in many other countries, the amount of direct contact between doctors and patients was reduced according to the existing Hungarian legislation. In this extraordinary time at the Department of Dermatology, Venerology, and Dermatooncology of Semmelweis University, dermatologists have provided successful telemedicinal dermatology care with a store-and-forward system. Teledermatology allows dermatologists to evaluate, diagnose, and treat patients at a distance using telecommunication technology. Furthermore, teledermatology opened up the possibility in the healthcare system to provide remote dermatology care for a special group of elderly people (frailty, bedridden, disabled, etc.) as an alternative to traditional face-to-face visits.

Methods: We started the teledermatology healthcare service with a store-and-forward system on 28. March 2020. Patients or caregivers had to submit some personal data (name, date of birth, etc.), a short medical history, a list of the symptoms, a list of the regular medications, and upload at least five photos of the affected areas through a smartphone. Patients' pictures and medical data were stored on a secure platform. If further information was needed we performed a phone call. Every patient received a report and a treatment plan from a certified dermatologist. Nonemergency skin conditions were reviewed within three days, emergency conditions were reviewed within $24 \mathrm{~h}$.

Results: In 1 month, we have performed 3487 teledermatology consultation. The great majority of patients $(91 \%)$ were aged under 65 . The number of patients aged over 65 was $286(8,2 \%)$, consisting of 147 men (4,2\%), and 139 women (3.9\%), the mean age of men was 74.21 years, the mean age of women was 73.46 years. Only 56 patients aged over 65 took photos of themselves, 162 patients needed help in taking photos because of the localization of the skin conditions, and 68 patients were unable to take photos for various reasons (e.g., general condition, dementia, or insufficient technical skills). Further in-person examinations were needed in 55 cases. Only 11 patients had to be hospitalized. The majority of dermatological indications for telemedical consultations included various kinds of skin diseases, skin cancers, and wounds. The most common disorders in the elderly population were different forms of eczema $(n=62)$, including venous eczema $(n=19)$, psoriasis vulgaris $(n=10)$, leg ulcer $(\mathrm{n}=7)$. The most frequent infectious skin conditions were herpes zoster $(n=6)$, and scabies $(n=5)$. Among the findings, there were some rare skin conditions such as bullous pemphigoid $(\mathrm{n}=1)$, mycosis fungoides $(n=1)$, carcinoma erysipeloides $(n=1)$. Surgical procedures were needed in 11 cases. DiscussionNowadays telemedicine has become an important element of health care. Due to the visual character of the dermatological specialty, teledermatology may become a major tool in dermatological consultations, particularly for the elderly population. Teledermatology could be extremely useful for patients living with severe cognitive or physical disabilities, and for whom traveling even short distances can pose considerable practical and economical difficulties. Although our institute started teledermatology service in an emergency, it could be useful in normal circumstances to determine the emergency of a skin condition, establish obvious diagnoses, and perform follow-up visits for this fragile population. However, our data have higlighted an important aspect of teledermetology related to the underrepresentation of older patients among the cases which could be due to the little confidence of the elderly population in using electronic devices needed for a telemedical consultation. Therefore, caregivers may play an important role in teledermatology services for the elderly patients in the future.

\section{Abstract \# 249 \\ Influence the COVID-19 pandemic on the profile of patients in a palliative care unit.}

Yanira Aranda-Rubio ${ }^{1}$, Concepción Jiménez Rojas ${ }^{1}$, Javier Gómez Pavón ${ }^{1}$

${ }^{1}$ Hospital Central de la Cruz Roja, San José y Santa Adela.

The palliative care unit of Cruz Roja Hospital was inaugurated last December in 2019. In its first months of opening, the unit has 7 hospital beds. During the acute phase of the covid19 pandemic (March-May 2020), the unit continued to receive non-covid palliative patients. Through a descriptive observational study, we analyzed whether the pandemic influenced the profile and admission of the patients attended.

Demographic characteristics: Since the opening of the unit, a total of 90 patients (36 women) were admitted. Regarding age, $60 \%$ were older than 80 years (54 patients), most were referred from home by Home Care Support Teams.

Clinical features: $83.3 \%$ (75 patients) were cancer patients and the rest $(16.7 \%)$ were non-cancer. Of the latter group, most had degenerative neurological diseases (6 advanced mixed-profile dementias and one amyotrophic lateral sclerosis). The most common reason for admission was poor symptomatic control requiring continued hospital care.

Hospital income trajectory: After the start of the COVID pandemic in March, the average stay on the floor was significantly reduced: prior to it, the average stay was less than 4 days in $37 \%$ of patients, between 5 and 10 days in $34.2 \%$ and above ten days in $28.7 \%$.In patients admitted for advanced neurological disease, the average stay was 7 days. After the onset of the covid pandemic, the number of patients who died in a period of less than $48 \mathrm{~h}$ increased, and income from home was $100 \%$ of income. 


\section{Abstract \# 250}

Hallucination as a "porcupine" in the "stay at home" period because of the Covid-19 pandemic in a patient with Alzheimer's disease

Sumru Savas ${ }^{1}$

${ }^{1}$ Geriatrics Section, Internal Medicine Department, School of Medicine, Ege University, Izmir, Turkey

We are facing a pandemic caused by severe acute respiratory syndrome Corona virus 2 (SARS-CoV-2) widespread infection all around the world. During the pandemic, older patients represent an age group which is vulnerable to physical, psychological deteriorations because of the "lock down", loneliness, bad news coming from all over the world from social media and television which they were almost condemned to watch and read all they long with no other occupation, and communication. I report a patient in Izmir with Alzheimer's disease $(\mathrm{AD})$ dementia and hallucination as "porcupine" (the shape explained by the patient resembles SARS-CoV-2) which started 1 month after the first case of SARS-CoV-2 in Turkey on 10th March. The patient was 86 years old, hypertensive, diabetic, and had also coronary artery disease. He was a university graduate. AD diagnosis was present for 2 years, but no medication was able to be started because of intervening hypo-hyperglycemic, hypertensive attacks, and the unwillingness of his wife and the patient. Telephone calls and online video meetings were made with the patient and the caregiverwife, separately. The issue was not discussed with the patient, because the wife had mentioned that he did not want this situation to be shared with anybody. No medication was prescribed, and the caregiver's understanding and acceptance of the symptom was established by explaining. The hallucinations are fewer 2 weeks after moving to the "summer house" on 5th June, still following the precautions, but gardening, and taking little walks as lock down is over.

\section{Abstract \# 251}

How do older adults react to coronavirus disease 2019?

Ahmet Cigiloglu ${ }^{1}$, Zeynel Abidin Ozturk ${ }^{1}$, Eyyup Murat Efendioglu ${ }^{1}$

${ }^{1}$ Gaziantep University

Aim: Outbreaks have a wide spectrum of psychosocial impact on individuals. This study aimed to investigate the impact of the novel coronavirus disease on older adults in Turkey.

Methods: A survey was conducted on 104 adults who had to stay at home during the epidemic. Depressive symptoms, anxiety symptoms, nutrition status, health-related quality of life, and sleep quality of the participants were assessed.

Results: $37.5 \%$ of the older individuals had symptoms of depression and $29.8 \%$ anxiety. The frequency of depression was higher in women, aged 85 and over individuals and in those with lower education and monthly income. Anxiety disorders frequency was also higher in women and in those with lower education and monthly income. Quality of life and sleep were poorer in those with anxiety disorders and depression.

Conclusions: In our knowledge this is the first study in the medical literature investigating the effect of epidemic on mood, anxiety symptoms, nutrition status, quality of life and sleep of older adults during the epidemic period. Our findings indicate the importance of psychosocial support in older individuals during and after the epidemic period.

\section{Abstract \# 252}

The effect of reduced visiting $h$ on loneliness and mood of hospitalized elderly patients during the covid-19 pandemic

Maria Bonnici ${ }^{1}$, Clarissa-marie Zehlicke ${ }^{1}$, Rebecca Ceci Bonello ${ }^{2}$, Daniel Debattista ${ }^{1}$, Mohamed A. Salem ${ }^{1}$, Michelle Grech ${ }^{1}$, Claire Callus $^{1}$, John Cordina ${ }^{1}$

${ }^{1}$ Karin Grech Hospital, Malta, ${ }^{2}$ Mater Dei Hospital, Malta

Introduction: The covid-19 pandemic caused increased concern, anxiety, and mood changes amongst vulnerable groups, such as older adults [1]. The literature suggests that symptoms of mood disturbance tend to be more prevalent in hospitalized patients [2]. One of the measures taken to protect vulnerable patients in the local intermediate care hospital (Karin Grech Hospital), was to stop social visits. This could have contributed to the increased anxiety and low mood observed in our patients, attributable to loneliness. In this study we would like to investigate our patients' mood and their feelings of loneliness during this pandemic, and if there is a difference after reintroducing social visits.

Methods: This observational cohort study will be carried out in an intermediate care hospital in Malta (Karin Grech Hospital) [KGH], between the months of May and September 2020. All patients over 65 years of age will be included. Exclusion criteria include: the terminally ill; recent diagnosis of a psychiatric illness or any change in psychiatric medications in the previous 6 months; if no consent could be obtained from either patients and/or relative. The Montreal Cognitive Assessment test [3] will be done on each patient included in this study, together with the UCLA loneliness scale [4] and the Hospital Anxiety and Depression Scale (HADS) [5]. The Cornell scale [6] will be done instead of the UCLA and HADS scales, if the patient has severe dementia and is unable to answer the questionnaires. These tests will be repeated after a month of normal visiting $\mathrm{h}$ at $\mathrm{KGH}$.

Results: This study is a work in progress.

\section{References:}

1. World Health Organization (WHO) [internet]. Mental health and covid-19; 2020 [cited 2020 June 8]; Available from: http://www. euro.who.int/en/health-topics/health-emergencies/coronavirus-covid19/technical-guidance/mental-health-and-covid-192. Yohannes AM, Baldwin RC, \& Connolly MJ. Prevalence of depression and anxiety symptoms in elderly patients admitted in post-acute intermediate care. Int J Geriatr Psychiatry 2008; 23 (11):11413. Nasreddine ZS, Phillips NA, Béridian V, Charbonneau S, et al. The Montreal Cognitive Assessment, MoCA: A brief screening tool for mild cognitive impairment. J Am Geriatr Soc 2005; $53^{4}: 695-94$. Russell D, Peplau LA, \& Ferguson ML. Developing a measure of loneliness. Journal of Personality Assessment 1978; 42:290-2945. Zigmond AS, Snaith RP. The Hospital Anxiety and Depression Scale. Acta Psyhiatr Scand 1983; 67:361-3706. Alexopoulus GS. The Cornell Scale for Depression in Dementia: Administration and Scoring Guidelines. White Plains; NY: 2002, 2005

\section{Abstract \# 253 \\ Compliance elderly to the directions to stay at home during the covid 19 lockdown in Greek province}

Martha Andreou ${ }^{1}$, Naili Tersenidou ${ }^{1}$, Riola Tersenidou ${ }^{1}$, Marina Georgiadou ${ }^{1}$, Ioanna Aggelidou ${ }^{1}$, Eleni Paraskevopoulou ${ }^{1}$, Despoina Xatzipanagiotou $^{2}$

${ }^{1}$ Health Center of Avdira, NHS, ${ }^{2}$ Health Directorate of Xanthi Region

Introduction: The Prefecture of Xanthi is the second one in Greece in positive Covid 19 samples (over 200) and deaths (17). The present study recorded the compliance, of elderly patients in two Xanthi's 
Municipalities, to the instructions of EODY (National Public Health Organization) for quarantine from March 15 to May 31.

Methods: Using a structured questionnaire, we recorded age, gender, presence of partner and children, education, income, disease information, how to procure medicines and food, if they spent Easter/ Bayram holiday alone and consent to the strict measures taken.

Results: 265 elderly agreed to participate, of which $63.4 \%$ were women aged $73.4 \pm 5.9$ years, the majority were married $(71.7 \%)$, compulsory educated $(45.7 \%)$ with more than two children $(78.4 \%)$ and income $<15,000$ euros. Television was the main source of information $(89.8 \%$ ), while $85.7 \%$ spent Easter/Bayram alone. The supply of medicines $(77,8 \%)$ and food $(40 \%)$ was made to order. There was a strong correlation between children's presence and elderly compliance $(p=0.019)$. Almost all of them agreed with the strict measures taken $(99.2 \%)$.

Key conclusions: We found a high rate of compliance of the elderly in the lockdown period which agrees with almost zero positive people in the region ${ }^{3}$ in contrast to other Xanthi' $s$ Municipalities. The elderly should be informed to comply with personal protective measures by their family physicians as there is a large viral load in the area.

\section{Abstract \# 254 \\ A study comparing the frequency of falls amongst patients suffering from Dementia at Saint Vincent de Paul Long term facility (SVPR) in COVID-19 and non-COVID-19 eras.}

Lara Camilleri ${ }^{1}$, Doriella Galea ${ }^{1}$, Francesca Farrugia ${ }^{2}$, Julia Tua ${ }^{1}$, Melanie Debono ${ }^{2}$, Antoine Vella ${ }^{2}$

${ }^{1}$ Saint Vincent De Paul Long Term Care Facility, Luqa, Malta, ${ }^{2}$ Saint Vincent De Paul Long Term Facility, Luqa, Malta

Introduction: The COVID-19 pandemic has brought inevitable change in all institutions including SVPR which is a longterm facility in Malta. The aim of this study is to establish whether there was a change in fall frequency in people suffering from dementia at SVPR between COVID-19 and non COVID-19 periods. Method: An observational study including patients with dementia residing in closed wards at SVPR. Collected data included: number of patients who fell, total number of falls, mechanism and consequence of fall and physical restraints' use. We compared results of April 2019 (pre Covid-19) with April 2020 (COVID-19 era). Only patients residing in these wards during both years were included. Data was collected from patients' files.

Results: A total of 75 patients were included. 11 patients $(14.7 \%)$ sustained a fall in both April 2019 and April 2020. The total number of falls in 2019 was 13 compared with 16 falls in 2020. One patient had physical restraints in April 2019 compared to 3 patients in April 2020. The most common mechanism of injury was an unwitnessed fall in both years. No serious fall-related injuries occurred in 2019 but one patient sustained a hip fracture in 2020 .

Conclusion: There was no significant change in number of patients who fell and total number of falls in COVID-19 era. Study Limitations: small population size, short time-period and data collected from patient's notes is influenced by quality of documentation. The process of dementia itself has deteriorating consequences on gait and balance which might also affect falls' risk over 1 year.

\section{Abstract \# 255}

Feasibility, acceptability and usefulness of a telemedicine (TM) program in older people with dementia during COVID-19 pandemic

Barbara Senesi ${ }^{1}$, Camilla Prete ${ }^{1}$, Ekaterini Zigoura ${ }^{1}$, Alessandra Pinna ${ }^{1}$, Giacomo Siri ${ }^{1}$, Sabrina Zora ${ }^{1}$, Cristina Novello ${ }^{1}$, Vincenzo Solfrizzi $^{2}$, Carlo Custodero ${ }^{2}$, Alberto Cella ${ }^{1}$, Alberto Pilotto ${ }^{1}$

${ }^{1}$ Department of Geriatric Care, Orthogeriatrics and Rehabilitation, Galliera Hospital, Genova, Italy, ${ }^{2}$ 2) Department of Interdisciplinary Medicine, University of Bari, Italy

Aim: To investigate the feasibility, acceptability and usefulness of a telemedicine (TM) program in older people with dementia and their caregivers, during the COVID-19 pandemic isolation rules.

Methods: From March 9 to May 11, 2020, 140 older patients and their caregivers, followed-up by our Center for Cognitive Decline (CDCD), were contacted by telephone and interviewed by using a telephone-based survey. Of the 131 respondents, 119 participants accepted to receive a TM visit by using a dedicated connection (jiitsi meet/Galliera), including only the dyads with access to PC, tablet or smart phone for 2 way video interaction.

Results: Of the 131 respondents (mean age $80.6 \pm 6.1$ years; males $=$ $20 \%), 85(69.4 \%)$ lived accompanied, $40(30,5 \%)$ lived alone with caregiver supervision. During the lockdown period, the isolation measures forced $20 / 131$ respondents $(15,2 \%)$ to change their living arrangements. Grocery and pharmacy outings were performed by family members in $111 / 131$ participants $(84,7 \%)$. Health status was found to be stable in 79/131 respondents (60,3\%), with no COVID-19 symptoms; 1 patient/131 deceased for COVID-19 and 13 patients $(9,1 \%)$ were hospitalized for other health problems. 53 patients out of $131(41,9 \%)$ reported Behavioural and Psychological Symptoms of Dementia (BPSD) including sleep disorders $(26,7 \%)$, anxiety symptoms $(15,6 \%)$, delirium superimposted on dementia $(7 \%)$ with an increased burden of caregivers. 10 caregivers out of $131(7,6 \%)$ started a psychological support remote programme. Moreover, 63 patients $(48 \%)$ reported pain. Finally, in 39 patients $(29,7 \%)$ the COVID 19 emergency insight was present and 44 patients $(33,6 \%)$ without disease insight reported difficulties to respect the COVID-19 restrictive and confinement measures.

Conclusion: This TM program showed to be feasible and well accepted in most of patients $(93,6 \%)$. Monitoring BPSD and pain, tailoring actual therapies and delivering information and psychological care to patients and their caregivers were the most useful interventions in older patients with dementia and their caregivers.

\section{Abstract \# 256}

COVID-19: perceived stress and self-report mental efficiency in elderly individuals with mild cognitive impariment

Maria Devita ${ }^{1}$, Simone Paolini ${ }^{1}$, Alessandra Bordignon ${ }^{2}$, Giuseppe Sergi $^{3}$, Alessandra Coin ${ }^{4}$

${ }^{1}$ Department of General Psychology, ${ }^{2}$ Geriatrics Division, Department of Medicine - DIMED, ${ }^{3}$ Geriatrica Division, Department of Medicine, ${ }^{4}$ Geriatrics Division, Department of Medicine

Introduction: The detrimental effects of loneliness and social isolation have been extensively studied in the older population. The Covid-19 has thrown individuals into unexpected isolation and general deprivation, included older individuals with cognitive impairments, on which the literature on Covid-19 poorly focused so far. Are those individuals aware of the effects of the pandemic, to experience distress? How they evaluate their cognitive efficiency in a social and general deprivation condition? This study investigates the 
perceived stress and the self-report mental efficiency of individuals with mild cognitive impairment (MCI) and mild dementia, during and after the lockdown.

Methods: A total of 40 individuals, of which a deep neuropsychological evaluation was available immediately before the pandemic, were recruited. Participants answered to questionnaires investigating their perceived stress, and self-reported mental efficiency, by telephone. This evaluation was repeated three times, every 2 weeks, to monitor over time possible changes. A post-pandemic neuropsychological evaluation is currently ongoing.

Results: Although cognitive data are still lacking, data about perceived stress and self-reported mental efficiency suggest that people with MCI and mild dementia seem to not perceive stress related to Covid-19 and a detrimental effect of it on their cognitive functioning. Key conclusions: In people with MCI and mild dementia, the effects of a world-wide pandemic emergency seem to not affect their routine life, as well as their psychological and cognitive well-being. Data about neuropsychological actual status will be available, and will show if consistency between patients' self evaluation and objective consequences of Covid-19 on cognition exists.

\section{Abstract \# 257 \\ Telemedicine and it's role in a Geriatric Medicine Outpatients Department}

Mary Buckley ${ }^{1}$, Riaz Moola ${ }^{1}$, David Robinson ${ }^{1}$, Rory Nee ${ }^{1}$

${ }^{1}$ St. James's Hospital, Dublin, Ireland

Introduction: St James's is the largest hospital in Ireland with 6, 500 Geriatric Medicine outpatient attendances annually. COVID 19 resulted in transformation of the service necessitating a new way of working. This crisis has encouraged embracing telemedicine as an appropriate means of assessing our patient cohort. $79 \%$ of the $65-74$ year-old age group lack digital skills, therefore telephone consultations were chosen for our cohort.

Methods: St James's benefits from an Electronic Patient Record system. A remote working team was established including doctors in isolation and those redeployed from other services, who were given remote access. Secretarial staff informed patients of appointment cancellations and upcoming telephone consults. Telephone contact was made with both patient and next of kin, if required. Records were kept of consultations, and correspondence sent to the primary care physician. Results: Contact was made with 277/321 (86\%) patients. $24(16 \%)$ were discharged, $43(9 \%)$ warranted urgent reviews and $75 \%$ (210) were given further appointments. The reviews were facilitated in our day hospital on a next day basis. These reviews included - dementia with BPSD (7), Parkinson's disease review (3), medically unwell (3), falls (3), CCF (2), general decline (2), stroke (1), medication review (1), no details (2). Two of these were admitted. Feedback has been positive and evaluation is currently underway.

Conclusion: Telemedicine, despite substantial research undertaken in the late 1990s, has not gained mainstream support. It enabled rapid triage and further assessment in those who were perhaps avoiding hospitals otherwise. Feedback has been positive with formal evaluation underway.

\section{Abstract \# 258}

Fear and anxiety in gero-oncological patients in a pandemic

Carlo Cristini ${ }^{1}$, Alfredo Berruti ${ }^{2}$, Silvia Copeta ${ }^{3}$, Maria Angela Abrami $^{3}$, Michela Buglione ${ }^{2}$, Stefano M. Magrini ${ }^{2}$, Umberto Gelatti ${ }^{1}$

${ }^{1}$ University of Brescia, Italy, ${ }^{2}$ ATS, Spedali Civili, Brescia, Italy University of Brescia, Italy, ${ }^{3}$ ATS, Spedali Civili, Brescia, Italy
We often fear what is unknown, which appears uncertain and obscure, as can happen with the threat of a pandemic. Generally, we experience a state of anguish when we lose our stable references, there is uncertainty about the future, we foresee a condition of forced solitude and isolation, when our lives or those of our loved ones are affected by a challenging chronic or disabling disease, which are already the prevailing fears of the elderly. The most fragile among us, such as those who suffer from cancer, feel more exposed to events that can threaten their precarious state of health. The recent and current pandemic situation caused by Covid-19 can generate a particular state of anxiety, especially in elderly cancer patients. They are very frightened that they will be neglected, that they will be unable to complete the necessary treatment, the planned therapies, the periodic checks already scheduled for their cancer. Given their precarious health condition, cancer patients also fear that covid-19 may be more aggressive and dangerous for them than for other people. There is also the fear of being an easier source of contagion for their relatives. Fears tend to become more pronounced in the elderly men, most affected by the covid-19 infection.In this delicate period, elderly cancer patients, perhaps more than others, require specific psychological intervention, even more so if they are alone.In a medical service of North Italy, we examined several elderly cancer patients by a semi-structured interview, a specific questionnaire and DASS-21 scale.

\section{Abstract \# 259}

The impact of SARS-CoV-2 pandemic on older persons in Poland - a protocol and first progress report

Karolina Piotrowicz ${ }^{1}$, Agnieszka Kasiukiewicz ${ }^{2}$, Małgorzata FedykŁukasik $^{1}$, Ewa Klimek ${ }^{1}$, Zyta Beata Wojszel ${ }^{2}$, Barbara Gryglewska ${ }^{1}$, Tomasz Grodzicki ${ }^{1}$, Jerzy Gąsowski ${ }^{1}$

${ }^{1}$ Department of Internal Medicine and Gerontology, Faculty of Medicine, Jagiellonian University Medical College and University Hospital, Kraków, Poland, ${ }^{2}$ Department of Geriatrics, Medical University of Bialystok, Bialystok, Poland

Objective: To examine the impact of SARS-CoV-2 pandemic-related loneliness and anxiety on older community-living persons in Poland. To present the baseline data.

Methods: A prospective study of older who had a medical appointment cancelled due to the pandemic in Poland. The endpoints assessed 1 and 6 months after lifting the lockdown will include the self-reported: anxiety and depressiveness, cognitive impairment and emotional, perceptual and behavioural disturbances, malnutrition, sarcopenia, functional dependence, impaired physical performance, falls, incontinence, polypharmacy, impaired well-being. The comprehensive geriatric assessment will be done in the framework of regular care for older outpatients; the PTSD will be diagnosed according to the DSM-V criteria.

Results: The mean (SD) age of 138, COVID19-free, unquarantined participants (68.8\% women) was 78.4 (6.3), range 64-90 years. At baseline $39.0 \%$ lived alone, of them $56.6 \%$ received help from children, $11.3 \%$ from other relatives, and $5.7 \%$ from institutional caregivers. COVID19-related anxiety was reported by $65.9 \%$, including severe anxiety by $12.1 \%$ and depressive symptoms by $54.4 \%$. We found decreased every-day physical and mental activity in $43.5 \%$ and $27.5 \%$ participants, respectively. Falls since the beginning of the pandemic were reported by $8.7 \%$. The median $(\mathrm{Q} 1 ; \mathrm{Q} 3)$ quality of life scored $69.5(50 ; 80)$ points, with the well-being and physical deterioration reported by $44.2 \%$ and $28.3 \%$ participants, respectively. Key conclusions: At the beginning of the pandemic, the majority of older subjects felt anxiety and were depressed, with large numbers perceiving physical and cognitive deterioration. This is setting a stage 
for the post-pandemic recovery strategies to be centred on reassurance and physical exercise.

\section{Abstract \# 260 \\ Internet-telephone consultation services-a "Learning by Doing" project held for consultations of elderly during COVID-19 pandemic and healthcare lockdown \\ Neumann-Podczaska Agnieszka ${ }^{1}$, Seostianin Mikołaj ${ }^{2}$, Madejczyk Konrad $^{2}$, Wieczorowska-Tobis Katarzyna ${ }^{2}$ \\ ${ }^{1}$ Geriatrics Unit, Department of Palliative Medicine, Poznan University of Medical Sciences, ${ }^{2}$ Geriatrics Unit, Department of Palliative Medicine, Poznan University of Medical Sciences}

Background: Limited access to healthcare services during pandemic lockdown jeopardized controlling of chronic conditions in geriatric patients since numerous healthcare establishments remained closed or were turned monomial and were designated to tackle Coronavirus Disease 2019 (COVID-19) pandemic. Due to global university closure and lack of clinical rotations most of university classes were conducted online.

Method: In response to pandemic lockdown, medical and pharmacy students based in Poznań University of Medical Sciences in Poland have established remote consultation services under substantive supervision and guidance of tutors specialized in geriatrics and pharmacy. Data derived from consultations along with follow-up interviews of senior participants and consultants were collected and subjected to analysis.

Objective: The aim of this article is to present potential benefits of interdisciplinary, cooperative, "learning by doing" educational undertaking with the use of remote communication means towards improvement of care of elderly patients during pandemic lockdown.ResultsProper interventions were assigned to each of healthand pharmacotherapy-related problems reported by project's participants. Positive feedback results have shown that students' remote consultations proved to be beneficial for both elderly patients and medical and pharmacy students during pandemic lockdown and university closure.

Conclusions: Students and elderly participants can both benefit from remote consultations. Remote means are considered efficient in detection and counselling of health- and pharmacotherapy-related problems of Elderly (especially, during pandemic lockdown in regard to social distancing measures). "Learning by Doing" project has proved to be favourable method of learning during COVID-19 pandemic lockdown.

\section{Abstract \# 261}

Ensuring good quality care of elderly patients in LTC facilities during COVID19 pandemic: results of an interdisciplinary online consensus conference

Mihai-Viorel Zamfir ${ }^{1}$, Andreea G Marin ${ }^{2}$, Mihaela Zamfir (Grigorescu) $^{3}$, Ileana Ciobanu ${ }^{2}$

${ }^{1}$ „Carol Davila” University of Medicine and Pharmacy, Bucharest, Romania, ${ }^{2}$ Department of Neuro-Rehabilitation, „Elias” University Emergency Hospital, Bucharest, Romania, ${ }^{3}$ Department „Synthesis of Architectural Design”, Faculty of Architecture, „Ion Mincu” University of Architecture and Urbanism, Bucharest, Romania

Introduction: COVID19 pandemic represents a challenge for longterm care of older patients. Protocols for interdisciplinary patient care are needed.
Methods: In June 2020 we held a consensus online conference on the care of elderly patients in LTC facilities during COVID19 pandemic. The composition of the panel is diverse (MD geriatrics\&psychiatry, clinical psychologist, architect, researcher on dependent elderly care). We listed the problems related to care of elderly patients, then we discussed consequences of these problems on patients' QOL, finally we addressed solutions for increasing QOL of patients. Ideas were generated by brainstorming and were kept if all participants agreed upon.

Results: According to participants, physical and social distancing represent important factors that determine decrease in QOL of elderly patients. The major consequences are lack of activities and limitation of physical activity for patients, decrease in the degree of communication between residents and family members, staff members stress. These problems have impact on all dimensions of QOL. The solutions that the groups agreed upon are: using guidelines for safety measures against infections; implementing telehealth services; supporting physical activity for patients; finding alternatives for leisure activities for patients; ensuring communication of patients with family members; preserving as much as possible daily routine of residents; counseling and relaxation spaces for staff; public financing for LTC settings during pandemics.

Conclusions: COVID19 pandemic represents a new challenge for LTC settings. Ensuring good QOL of elderly residents requires the adoption of appropriate measures to serve the needs of elderly persons and staff.

\section{Abstract \# 262}

Pharmaceutical care service-remote consultations for elderly with polypharmacy during COVID-19 pandemic- the first service of its kind In Poland

\section{Neumann-Podczaska Agnieszka ${ }^{1}$, Wieczorowska-Tobis Katarzyna ${ }^{1}$ \\ ${ }^{1}$ Geriatrics Unit, Department of Palliative Medicine, Poznan University of Medical Sciences}

Background: Limited access to healthcare services during pandemic lockdown underlined the urgent need for pharmaceutical care service especially for elderly with multimorbidity and major polypharmacy since numerous healthcare establishments remained closed.

Method: The service is the first of its kind in Poland. It includes the detailed medical and pharmaceutical interviews of the pharmacological treatment undertaken by pharmacists trained in geriatrics. Thereafter recommendations are given for the elderly patients and-if necessary-also for their general practicioners.

Objective: The aim is to present the algorithm of the innovative Pharmaceutical Care Service financed by the Poznan City Hall. It is an effective, validated model of pharmaceutical care in geriatrics with the main beneficiary being the elderly with major polypharmacy.

Results: Remote consultations of seniors with major polypharmacy resulted in the improvement of the appropriateness of their pharmacotherapy, especially when over the counter drugs and herbal remedies were used in self-treatment. Additionally, the elderly could seek pharmaceutical advice in the time of health care system lockdown resulting in the improvement of their compliance to drug regimen.

Conclusions: This innovative project allows specially trained pharmacists to use validated, effective model of Pharmaceutical Care in geriatrics to detect, resolve and prevent medication-related problems in seniors also in the time of COVID-19 pandemic. Pharmaceutical Care Service is recommended to be included in a healthcare system in Poland. 


\section{Abstract \# 263}

Analysis of the impact of the 2019 coronavirus epidemic (CoViD 19) on the presentation and crowding of geriatric patients for minor emergencies at a Lombardy ED.

Gabriele Savioli ${ }^{1}$, Iride Francesca Ceresa ${ }^{2}$, Paolo Maggioni ${ }^{3}$, Francesca Grulli ${ }^{3}$, Elena Novara ${ }^{3}$, Tonia Persiano ${ }^{3}$, Viola Novelli ${ }^{4}$, Alba Muzzi ${ }^{4}$, Giovanni Ricevuti ${ }^{5}$, Enrico Oddone ${ }^{6}$, Maria Antonietta Bressan $^{7}$

${ }^{1}$ IRCCS Foundation Pliclinico San Matteo, ${ }^{2}$ IRCCS Policlinico San Matteo, ${ }^{3}$ università degli studi di pavia, ${ }^{4}$ Medical Direction, IRCCS Policlinico San Matteo, 27100 Pavia, Italy, ${ }^{5}$ University of Pavia, 27100 Pavia, Italy, ${ }^{6}$ Assistant Professor, Department of Public Health, Experimental and Forensic Medicine, University of Pavia, 27100 Pavia, Italy., ${ }^{7}$ Past Director Emergency Department, IRCCS Policlinico San Matteo, 27100 Pavia, Italy.

Background: The coronavirus 2019 (CoViD-19) epidemic in Italy originated in the south-west of Lombardy, on February 21, 2020. The Fondazione IRCCS Policlinico San Matteo di Pavia has been involved in the management of the outbreak since its beginning. All the hospitals involved have experienced a huge increase in crowding, while witnessing a reduction in the presentation of minor emergencies. These had been pointed out by some authors as one of the causes of crowding and included in the crowding input factors.Purpose: As part of a study that wanted to assess the impact of the CoViD pandemic on geriatric minor emergencies, we evaluated the geriatric population that went to the ER for minor signs and symptoms.

Materials and Methods: We evaluated all patients aged 75 years and older who were accessing our emergency room for minor medical emergencies from February 22 to May 1, 2020 and during the same period of the previous year.

Results and discussion: We enrolled 663 patients. There was a severe reduction in the total number of accesses for minor injuries: 208 in the CoViD period and 455 in the previous year. Age and vital parameters were overlapping. Patients in the CoViD pandemic tended to be more frequently carried by ambulance and 118 compared to the reference period ( $75 \%$ vs $53 \%$, the remaining half independent). The priority codes for the medical examination were not different $(71 \%$ minor emergencies, $7 \%$ non-urgency in the pandemic period vs 78 and 5\%). During the pandemic, they patient presented with higher discharge severity codes (yellow and red) with a slightly higher frequency compared to the reference period (25\% vs $13 \%)$ and more frequently need hospitalization ( $46 \%$ vs $28 \%$ ). Rarely this category of patients presents altered crowding indexes; Crowding input factors are however lower in the pandemic period: reduced patients (208 vs 455 ) and reduced average waiting times ( $86 \mathrm{~min}$ vs $118 \mathrm{~min}$ ). The percentage of patients who exceeded the waiting time target by priority code of medical examination was also reduced (13\% vs $19 \%$ ). Crowding throughput factors worsened only slightly: LOS (580 vs $425 \mathrm{~min})$. Crowding output factors also slightly worsened: the percentage of access block is higher during the pandemic (19\% vs 9\%). The Total Access Block Time is significantly higher during the CoViD period (36, $104 \mathrm{~min}$ vs $29,159 \mathrm{~min})$. The crowding for patients who came for all causes of our ED was higher during the pandemic period compared to the reference period $(\mathrm{OR}=3.95)$.

Conclusion: The epidemic led to a drastic reduction in access for minor signs and symptoms, with no effect on overall ED crowding. However, patients were more frequently in need of hospitalization and had more severe exit codes. Acknowledgements: we would like to thank all employees of the IRCCS Policlinico San Matteo Foundation for their extraordinary efforts during the pandemic.Keywords: COVID-19, minor emergencies, crowding, Time Dependent Pathologies, First Aid, Triage, Emergency Department.

\section{Abstract \# 264}

Lending ears to the patient can make a huge difference

Muhammad Shoaib Zaidi ${ }^{1}$

${ }^{1}$ university Diabetes Center, King Saud University, KSA

Introduction: The concerns of all patients esp., the elderly should be addressed, as these can be useful for their own diagnosis and management.In this era, Continuous blood glucose monitoring (CGM) is quite a helpful and accurate tool for glycemic regulation.

Methods: 75 yrs old gentleman with Type 2 diabetes (> $30 \mathrm{yrs}$ ), Hypertension, Primary Hypothyroidism, dyslipidemia, mixed polyneuropathy, Iron deficiency anemia, and benign prostatic hypertrophy.On followup in our center in March, 2020 his BP and self monitoring blood glucose readings were keeping high at home. He had a past history of subdural hematoma, intraventricular hemorrhage with hydrocephalus, with subsequent placement of shunt. The patient was on daily Inj.Glargine, 10 units, oral Gliclazide MR 120mg, Linagliptin 5mg, Amlodipine 5mg Valsartan160mg combination, Levothyroxine 100ug, Atorvastatin, Aspirin $81 \mathrm{mg}$, calcium carbonate $60 \mathrm{mg}$, along with twice daily iron fumarate $200 \mathrm{mg}$ and cholecalciferol 10,000 IU/week. He was fully conscious, well oriented and co-operative, of average built and height. BP 170/70 mmHg, pulse 93/m, RR 18/m, O2sat $100 \%$, afebrile, BMI $24.96 \mathrm{~kg} /$ $\mathrm{m}^{2}$. Fundoscopy normal. Systemic exam unremarkable, apart from dry feet and impaired monofilament and vibration testing.

Results: CBC-Hb\% 13.1g/dl (12.6 before), MCV 93.8fl, S.Ferritin $10.5 \mathrm{ug} / \mathrm{l}$ (30-400), Vit.B12270 pmol/1 (145-637), HbA1c 8\% (6.4 in Feb. 2020).His renal, liver and thyroid functions were intact. Albumin creatinine ratio $12.23 \mathrm{mg} / \mathrm{g}(0-30)$. Nerve conduction study-consistent with mixed polyneuropathy.He continued to follow-up physically even during the Covid crisis as he was very much concerned about the elevated SMBG and BP values. In June, 2020, the patient \& and his daughter were educated about the Libre (CGM) usage.His Gliclazide dose was optimized and Inj.Glargine, 10 units daily was started.The antihypertensives were also adjusted. On patient's follow-up in August, 2020, his time in range had improved to $80 \%$ (33\% in June, 2020), average glucose was $147 \mathrm{mg} / \mathrm{dl}$ (200 before), glucose variability was $27.8 \%$ (28.9), hypoglycemia (54-79 mg/dl) was $1 \%(0)$. Key conclusions: *Physicians should always pay heed to the patients' complaints, which can help in solving their problems.*Continuous blood glucose monitoring can be a more efficient method than glycosylated hemoglobin and self-monitoring of blood glucose, for detecting the correct glycemic status.

\section{Abstract \# 265}

Rehabilitation intervention for active elderly with balance disturbance after traumatic brain injury: a case report

Irma Ruslina Defi Irma Ruslina Defi ${ }^{1}$

${ }^{1}$ RS Hasan Sadikin Bandung

Abstract: Background: Gait and balance disturbance in older adults may lead to dysfunction and loss of independence, thereby reducing their quality of life. The aim of this case report is to describe the benefit of the rehabilitation program as an intervention for elderly patient with gait and balance disturbance after traumatic brain injury. Case presentation: This was a case report of a 75 years old woman with a balance disorder after a traumatic brain injury. The patient also fulfill the hakim's triad (gait disturbance, urinary frequency, mild cognitive impairment) for early stage of Normal Pressure Hydrocephalus (NPH). Eventhogh, Ventriculomegaly, multiple lacunar infarction on paraventricle and cerebral atrophy was found in CT scan of the head, these findings can not rule out the NPH for sure. 
Rehabilitation program that given to this patient include balance training, cardiopulmonary training, and family meeting to support environment modification.

Conclusion: This case showed the importance of rehabilitation program for gait and balance disturbance in elderly with instability. Rehabilitation medicine assessment and program should be conducted to elderly patients after traumatic brain injury to prevent falls and improve functional ability.

Keywords: balance, elderly, gait, traumatic brain injury

\section{Abstract \# 266}

Vet to vet maine companion program study: COVID-19 effect on increasing social isolation and loneliness

\author{
Marilyn R. Gugliucci ${ }^{1}$, Shirley A. Weaver ${ }^{2}$, Amy Lin ${ }^{1}$ \\ ${ }^{1}$ University of New England College of Osteopathic Medicine, ${ }^{2} \mathrm{Vet}$ \\ To Vet Maine
}

Background: Maine veterans represent $11.8 \%$ of Maine's population, twice that of the United States. Veterans are at risk for social isolation, loneliness and suicide. This pilot study assessed (1) the Vet2Vet Maine mission to reduce veterans' social isolation and loneliness; and (12 effects of the program on care-partners. Methods: Mixed-methods research spanned 6-months from 2019 to 2020. Trained older Veteran Volunteers-VV ( 8 males/2 females) were paired with older Veteran Friends-VF ( 8 males/2 females); also Veteran Friend's Care-PartnersCP (1 male/3 females) participated. VVs completed pre/post Veteran Rand 12-Item Health Survey (VR-12) and Patient Health Questionnaire-9 (PHQ-9). VFs completed pre/post VR-12, PHQ-9, and Late Onset Stress Symptomatology (LOSS) Short-Form. CPs completed pre/post Zarit Burden Interview-22. After 6-months, VV and VF qualitative interviews were conducted in pairs; CPs were interviewed individually. Data (pre/post assessments) were analyzed with the Wilcoxon-Sign-Test and NVivo 12 + Qualitative Software.

Results: COVID-19 was a confounding variable as Maine state stayat-home orders occurred at the study mid-point. Pre/post data failed to exhibit significance $(\mathrm{P}=0.05)$. VR-12 and LOSS scores exhibited increases in stress/poor-health. Four qualitative themes were selected: (1) Effects of COVID; (2) Veteran Volunteers and Veteran Friends; (3) Care-Partners; and (4) Three-Words. Despite differences in interests or military background, VV and VFs valued their commonality as veterans. CPs confirmed the importance and benefits of these relationships. COVID dramatically affected the number and nature of visits reducing contact hours by $72 \%$. Conclusions: The Vet2Vet Maine companion program relies on Veterans' face-to-face visits; COVID interfered with fostering companionship, mentoring, and supporting the Veterans' independence.

\section{Abstract \# 267 \\ Improving the diagnosis and management of lower urinary tract infections in older people \\ Haran Devakumar ${ }^{1}$ \\ ${ }^{1}$ North Middlesex Hospital Trust}

Introduction: In 2016, the National Point Prevalence Survey showed $62.5 \%$ of antibiotic prescriptions for Lower UTI were inappropriate. This has a significant impact on clinical effectiveness; it leads to increased admissions with recurrent Lower UTI and urosepsis, whilst also promoting antimicrobial resistance and healthcare associated infections such as C. Difficile.

Method: This resulted in a national CQUIN being created in April 2019 that focused on improving the management of Lower UTI in older people. The CQUIN set a mandate of achieving 90\% of antibiotic prescriptions for Lower UTI in older people meeting NICE guidance for Lower UTI and PHE diagnosis of UTI guidance in terms of diagnosis and treatment. Our baseline audit showed 29\% compliance in North Middlesex ED department. We created a focus group with ED staff and identified factors contributing to our primary outcome. We implemented changes targeted to these contributing factors using quality improvement methodology. We had a total of 3 iterative PDSA cycles and each cycle served as an audit-feedback loop. Implemented changes ranged from organising an educational programme for ED staff to applying behavioural change techniques using COM-B model.

Results: Our results showed dramatic improvement in each criteria over 3 quarters. Prescribed antibiotics are now compliant with NICE in $90 \%$ of cases compared with $44 \%$ in cycle 1 . Nitrofurantoin and Fosfomycin now make-up $83 \%$ of prescriptions, compared to $18 \%$ in cycle 1 . Whilst co-amoxiclav, which has resistance rates $>20 \%$, fell from $45 \%$ in cycle $1-9 \%$.

Conclusion: We used QI Methodology at the frontline to make significant and sustainable improvements in the diagnosis and management of Lower UTI in the over 65's. We were on target to reach the CQUIN mandate but failed to reach due to COVID-19.

\section{Abstract \# 268}

Assessment of health status and lifestyle habits of vulnerable, community-dwelling older adults during the covid-19 confinement

Mónica Machón ${ }^{1}$, Maider Mateo-Abad ${ }^{2}$, Kalliopi Vrotsou ${ }^{1}$, Naiara Peña ${ }^{3}$, Leonor Rico ${ }^{3}$, Itziar Vergara ${ }^{4}$

${ }^{1} 1$. Instituto de Investigación Sanitaria Biodonostia, Grupo de Atención Primaria, San Sebastián, Spain; 2. Red de Investigación en Servicios de Salud en Enfermedades Crónicas (REDISSEC),

Baracaldo, Spain; 3. Instituto de Investigación en Servicios de Salud Kronikgune, Baracaldo, Spain, ${ }^{2} 1$. Red de Investigación en Servicios de Salud en Enfermedades Crónicas (REDISSEC), Baracaldo, Spain; 2. Instituto de Investigación en Servicios de Salud Kronikgune, Baracaldo, Spain., ${ }^{3} 1$. Instituto de Investigación Sanitaria Biodonostia, San Sebastián, Spain., ${ }^{4} 1$. Instituto de Investigación Sanitaria Biodonostia, Grupo de Atención Primaria, San Sebastián, Spain; 2. Red de Investigación en Servicios de Salud en Enfermedades Crónicas (REDISSEC), Baracaldo, Spain; 3. Instituto de Investigación en Servicios de Salud Kronikgune, Baracaldo, Spain

Introduction: a state of alarm was declared in Spain to tackle the covid-19 pandemic. The population had permission to leave their homes only for essential activities. The objective of this study was to examine the health status and lifestyle habits of vulnerable, community-dwelling older adult during the confinement.

Methods: a social worker and a nurse performed a telephone interview at the end of confinement to a sample of people aged $>70$ years, living in Gipuzkoa (Spain), with a Barthel index score of $\geq 85$ points and frail or with high risk of falls. Besides, this assessment data was compared to a previous face-to-face interview carried out 9 months earlier.

Results: a total of 38 individuals were included (71\% women). During the confinement, a $63 \%$ showed difficulties in walking up 10 steps, $42 \%$ reported poor self-perceived health, $66 \%$ had sleep problems and $74 \%$ experienced pain. They were not anxious/depressed $(71 \%)$ and did not feel loneliness (60\%). Considering the previous assessment, a deterioration of basic and instrumental activities of daily living was observed. The body mass index decreased and a higher proportion of people at risk of malnutrition or malnourished was identified. Family support increased. 
Key conclusions: in a group of vulnerable older individuals, health status and lifestyle habits deteriorated during the covid-19 confinement. As the epidemic is still ongoing and new confinements are possible, it is necessary to be aware of the implications of this situation and implement specific measures to control these potential negative impacts.

\section{Abstract \# 269 \\ Utilisation of remote consultations significantly reduces exposure during the COVID-19 pandemic \\ Ealish Swift ${ }^{1}$ \\ ${ }^{1}$ University Hospitals Bristol and Weston NHS Foundation Trust}

Introduction: Exposure to the clinical environment during the COVID-19 pandemic has become a great source of anxiety and apprehension for the elderly and/or at-risk populations. However, many elderly patients are also poorly equipped to adapt to the technological advances designed to minimize risk, such as video consultations in GP practice. Proportion of remote consultations requiring 'conversion' (subsequent face-to-face consultation) was measured to determine if telephone and video consultations are sufficient to reduce footfall in the practice.

Methods: 2938 consultations were reviewed, comparing 'conversion' rates (CR) for the first 2 weeks of lockdown (March 2020) to the preceding 2 unrestricted weeks as a baseline. Footfall was calculated as 'converted' remote consultations plus primary face-to-face consultations.

Results: CR dropped from $20 \%$ at baseline to $2 \%$ in week 2 of lockdown, despite remote appointments being expanded in scope from minor triaged issues pre-lockdown, to any and all problems as remote consultation became the default. This decrease correlated with increasingly creative uses of technology, such as asking patients to email in photographs of skin lesions. There was also increased efficacy in stratifying patients to either self-help at home or referral to secondary care. Overall, footfall in the practice dropped by $91 \%$ as a result of these changes.

Key conclusions: Footfall and exposure to the clinical environment in a GP setting can be successfully reduced by creative and pragmatic utilization of video consultation technology. This is essential to protect the elderly and/or at-risk population during the COVID-19 pandemic.

\section{Abstract \# 270}

\section{Orthogeriatrics service in COVIDemia}

\author{
Madiha Arslan Hashmi ${ }^{1}$, Carmen Martin-Marero ${ }^{2}$ \\ ${ }^{1}$ West Middlesex; Chelsea and Westminster NHS trust, ${ }^{2}$ West \\ Middlesex; Chelsea and Westminster University Hospital
}

The purpose of this Quality Improvement Project [QIP] was to assess the effectiveness of dedicated Orthogeriatrics servicepathway during Covid Pandemia on the hip fracture service. Results wereevaluated against the Blue Book standards and recommendations made.

Method: prospective QIP, conducted in a district general hospital, London, UK, March -June 2020. All patients admitted with hip fracture wereincluded. Data gathered from medical notes and electronic patients'records was recorded.

Results: 71 patients, mean age 84 y.o., more females [66\% F: $34 \%$ M]. All patients were assessed by a Geriatrician/Registrar within 12-24 h of admission. A full MDT CGA was done in 3\% [March] and $4.2 \%$ [April] of cases. Improvement was noted for May $9.4 \%$ and June $19 \%$. The decline in COVID incidence appear to improve the service provision in May/June. Pre-fracture bone protection improved from $38 \%$ to $83 \%$ post fracture. None of the patients developed thromboembolic disease. Comorbidities and frailty syndromes were documented in $54 \%$ cases. The median length of stay was 30 days: postoperative delirium, sepsis and advance frailty were identifiable causes. April had $7 \%$ COVID induced delirium vs 3.5\% in May and $1.4 \%$ in June. 30 days cumulative mortality was $4.8 \%$ in March vs $7 \%$ in June. Advance frailty and postoperative complications can explain the surge. Readmissions improved consistently: March 30\%: June $0 \%$.

Conclusion: COVID had a negative impact across all the standards of care for hip fractures patients. This may guide future service development in the event of a second COVID pandemia.

\section{Abstract \# 271}

A primary care and british red cross partnership to support patients living with frailty during COVID-19

Helen Jones ${ }^{1}$, Katy Simpson ${ }^{2}$, Lorraine Dilworth ${ }^{3}$, James Mackerrow $^{4}$, Jamie Megaw ${ }^{2}$

${ }^{1}$ Royal Infirmary of Edinburgh, Edinburgh, UK, ${ }^{2}$ Midlothian Health and Social Care Partnership, Dalkeith, UK, ${ }^{3}$ British Red Cross, Midlothian, UK, ${ }^{4}$ Public Health Scotland, Edinburgh, UK

Introduction: The frail elderly are considered high risk for COVID19 but, for some, the stay at home policies intended to protect them have threatened their wellbeing and disrupted their support systems. Those without close family or friends are most vulnerable. Recognising this, we aimed to introduce a dedicated service to support the health and wellbeing of the frail elderly in Midlothian, Scotland. Methods Midlothian Health and Social Care Partnership, in partnership with British Red Cross (BRC), provided a proactive welfare calls service. Moderately and severely frail patients were identified using the electronic Frailty Index (a 'cumulative deficit' approach, using coding from primary care health records). BRC staff and volunteers contacted patients to offer advice, to ensure wellbeing and to identify needs requiring action.

Results The purpose of this Quality Improvement 10 of 12 GP Practices opted in. Of 83425 registered patients, 1929 (2.3\%) were identified as moderately frail and $780(9.3 \%)$ as severely frail. Between April and May 2020, 2630 (97\%) calls were made. Although most patients had support from family or friends, $209(8 \%)$ requested at least one intervention. These included groceries (159-76\%), social calls to mitigate loneliness (41-20\%) and provision of hearing aid batteries (27-13\%). Informal feedback from patients was positive.

Conclusions: This innovative relationship between GPs and BRC has enabled a proactive support service to be delivered to the frailest patients, who otherwise may not have received the help they needed during the pandemic. It has also benefitted GP Practices as certain social problems are now being addressed effectively elsewhere.

\section{Abstract \# 272 \\ Validation of telephone-administered version of the Multidimensional Prognostic Index (TELE-MPI) for remote monitoring of older adults during COVID-19 pandemic}

Carlo Custodero ${ }^{1}$, Barbara Senesi ${ }^{2}$, Alessandra Pinna ${ }^{2}$, Annarosa Floris $^{2}$, Martina Vigo ${ }^{2}$, Marcella Fama ${ }^{2}$, Carlo Sabbà ${ }^{1}$, Camilla Prete $^{2}$, Alberto Pilotto ${ }^{3}$

${ }^{1}$ Department of Interdisciplinary Medicine, University of Bari, Italy, ${ }^{2}$ Department of Geriatric Care, Orthogeriatrics and Rehabilitation, 
Galliera Hospital, Genova, Italy, Department of Interdisciplinary Medicine, University of Bari, Italy; Department of Geriatric Care, Orthogeriatrics and Rehabilitation, Galliera Hospital, Genova, Italy

Background: Between March and May 2020 Italian Health authorities imposed strict lock-down measures to contain the COVID-19 pandemic. Because of this, many vulnerable older patients with chronic conditions, normally followed in ambulatory setting, needed to be monitored and managed in alternative ways, including telemedicine.

Aim:The Multidimensional Prognostic Index (MPI) is a well-established CGA-based tool able to assess patient prognosis. In the framework of a telemedicine program, we aimed to validate a telephone-administered version of the MPI (TELE-MPI) among community-dwelling older outpatients.Methods. From March 9 to May 11, 2020, 131 older patients (mean age $82.1 \pm 6.6$ years; males $=$ $26 \%$ ), were contacted by telephone and interviewed by using a telephone-based survey to calculate the TELE-MPI. The TELE-MPI included the same scales of standard MPI to explore eight relevant domains, i.e. basal and instrumental activities of daily living, cognitive, nutrition, mobility, comorbidity, polypharmacy and cohabitation status. The standard MPI was performed by health professionals three months before or after the TELE-MPI. The BlandAltman methodology was used to measure the agreement between MPI and TELE-MPI

Results: The mean MPI and TELE-MPI values were $0.523( \pm 0.223)$ and $0.522( \pm 0.222)$, respectively. The mean difference was +0.001 (standard deviation $=0.063)$. Lower and upper 95\% limits of agreement were -0.122 and +0.124 , respectively, with only 6 of $131(4.6 \%)$ of observations outside the limits.

Conclusions: TELE-MPI showed a strong agreement with the standard MPI. TELE-MPI may represent an useful way to follow by remote the health status of older adults and to promptly identify changes in their prognosis.

\section{Abstract \# 273}

\section{Fear of falls of the elderly during the COVID-19 lockdown}

Konstantinos Stolakis ${ }^{1}$, Christos Marneras ${ }^{2}$, Theodoros Tosounidis ${ }^{3}$, Elias Panagiotopoulos 4

${ }^{1}$ University of Patras (U.P), Medicine School of Patras (M.S.P), Greece, ${ }^{2}$ University Hospital of Rio, Patras (U.H.P), Greece, ${ }^{3}$ Department of Orthopaedic Surgery, University Hospital of Heraklion (U.H.H), Crete, Greece, ${ }^{4}$ Rehabilitation Department, University of Patras (U.P), Rio-Patras, Greece

Introduction: Fear of falls is connected with negative outcomes, such as reduced quality of life, mobility and social contact. The aim of this study was to compare the fear of falls of the elderly before and during the emergency lockdown measures of COVID-19 in Patras. This research is co-financed by Greece and the European Union (European Social Fund-ESF) through the Operational Programme «Human Resources Development, Education and Lifelong Learning 2014-2020» in the context of the project "A Holistic Interdisciplinary Approach to Treating Patients with Fragility Fractures" (MIS 5047167).

Method: The cross-sectional study was conducted in January-March 2020, with random sampling from the list of members of an Open Protection Centre for the Elderly of Patras. The sample size was 64 people over 65 years, while the response rate of the repeat telephone interview was $85.93 \%(\mathrm{n}=55)$. The Falls Efficacy Scale International (FES-I) Greek questionnaire was used to investigate the fear of falls. Results: The largest percentage of the sample had a high fear of falls in both of FES-I measurements. Moreover, it was observed that in a short period of up to two months there was a significant reduction in fear of falls. Women had higher mean values of fear of falls compared to men. Finally, the Spearman's non-parametric $r$ control showed a positive correlation of the fear of falls before and during the lockdown $(r=0.744, p<0.05)$.Conclusions: The research showed a high fear of falls among the elderly, which was moderated during the pandemic.

\section{Abstract \# 274}

The Continuing Effect of the COVID-19 Pandemic on Admissions to an Acute General Hospital Stroke Unit

Siobhan Woods ${ }^{1}$

${ }^{1}$ Royal Bolton Hospital

Introduction: In England, lockdown due to the COVID-19 pandemic was announced on the 23rd March 2020. Some patient groups have been more reluctant to attend hospital during this time. Management of a stroke is time-sensitive and delay in presentation may affect initial management and long-term outcome.Methods:Statistical analysis of the Sentinel Stroke National Audit Programme (SSNAP) data and Bolton Hospital admission data, including time of symptoms on admission, tertiary centre management and length of stay (LOS). Data was compared from January TO March (pre-lockdown) to April-June (lockdown).

Results There was a significant decrease in stroke admissions, across all presentation time frames, throughout the United Kingdom (UK), chi squared $(4, \mathrm{~N}=39228)=14.07, \mathrm{p}<0.01$, with stroke admissions more than halving. In Bolton, stroke patients presented later in the months of April and May, than pre-lockdown. Patients were more likely to be initially managed in a tertiary centre pre-lockdown than during lockdown, chi squared $(2, \mathrm{~N}=126)=11.98, \mathrm{p}<0.01$. The overall length of stay of stroke patients did not differ significantly during lockdown (Mann-Whitney $\mathrm{U}=1913, \mathrm{Z}=-1.0526$, not significant at $\mathrm{p}<0.5$ ).

Key conclusions: Less stroke patients have presented to hospital during the COVID-19 lockdown in the UK. In Bolton there was a delay in presentations of a significant number of stroke patients, although this appears to be recovering since July. Proportionally less patients were transferred for initial management at a tertiary stroke centre, however no effect was seen on overall LOS in hospital.

\section{Abstract \# 275 \\ Effects of the home confinement due to the Covid-19 outbreak on wellbeing of former active elders}

Francesca Vitali ${ }^{1}$, Doriana Rudi ${ }^{1}$, Gregorio Paccagnella ${ }^{1}$, Federico Schena ${ }^{1}$

${ }^{1}$ University of Verona

Introduction: The home confinement in Italy due to the Covid-19 outbreak has exposed most individuals to unprecedented stressful situations. Staying home, while was a safe measure, have unintended negative consequences since such efforts to avoid human-to-human transmission of the virus may have led to reduce physical activity (PA). Recent studies [1] have underlined a strong health rationale for continuing PA while taking precautions.Methods. We investigated 112 elders $(\mathrm{M}=57.1 \%$; Mage: $70.25 \pm 15.15$ years $)$ who were regularly engaged in PA programs before the home confinement. During the lockdown period (about 10 weeks) they exercised at their home using video, leaflets, etc. Different scales were administered after the home confinement to measure PA, well-being, feelings, diet and sleep quality changes. In addition, several tests were performed to evaluate BMI, balance, strength and flexibility at the end of the 
lockdown and compared with the same measures collected before the home confinement.

Results: PA decreased during the home confinement, and well-being, feelings and sleep quality were affected significantly. BMI, balance, strength and flexibility do not change significantly between before and after home confinement.Key conclusions. Our data confirmed that a certain amount of PA in a safe home environment reduces the impact of prolonged quarantine functional abilities while it seems unable to avoid changes in well-being, feelings and sleep quality that were probably more related to psycho-social dimensions of exercise. References:

[1] Chen, P., Mao, L., Nassis, G.P., Harmer, P., Ainsworth, B.E., \& $\mathrm{Li}, \mathrm{F}$. (2020). Coronavirus disease (COVID-19): The need to maintain regular physical activity while taking precautions. Journal of Sport and Health Science, 9, 103-104.

\section{Abstract \# 276 \\ Ageism in times of pandemic: exploring age discrimination among health professionals \\ Agnieszka Bozanic ${ }^{1}$, Francisca Ortiz-Ruiz ${ }^{2}$, Andrés Aranda Ortega ${ }^{3}$ \\ ${ }^{1}$ Barcelona University, ${ }^{2}$ The University of Manchester, ${ }^{3}$ University of Chile}

The main objective of this presentation is to evaluate the level of ageism between health professionals, considering the presence of a Pandemic as the COVID-19. The data used has been produced in Chile by May of 2020, using a constructed survey to measure it. These are the first results about it applied in Chile, South America, and in the context of a pandemic (COVID-19). The analytical design used is the scale proposed by Becca Levy and Ellen Langer (1994), which explores the negative stereotypes of older people. It is tested and validated this scale in a context of two different aspects as the standard applications had been made: during a Pandemic and in a South American country. Until now, the survey has been applied during the entire month of May 2020, getting a total of 357 answers. In general, the results show that the image of older people has been associated with advice, family-oriented, capable, active, positive outlook, well-groomed, and walks slowly, wise and sick. Accordingly, the presentation gives some useful information about how to measure in a better way the level of ageism in a Spanish speaking country as Chile. It is concluded that there are some indicators of ageism between health professionals, which also depended on different attributes of the responder people. Identifying the ageist attitudes of healthcare professionals can help in the creation of a series of strategies oriented to reverse the trend of ageism and improve the quality of life of older people, especially in an unusual context as COVID pandemic.

\section{Abstract \# 277 \\ Pre-admission anti-coagulation does not improve all-cause mortality in geriatric COVID-19 patients \\ Flora Olcott ${ }^{1}$, Clare Hunt ${ }^{1}$, Terrence Chan ${ }^{1}$, George Williams ${ }^{1}$ \\ ${ }^{1}$ Dartford and Gravesham NHS Trust}

Introduction: Initial studies have shown that patients infected with SARS-CoV-2 are at high risk of venous-thromboembolic events (VTE) [1]. This retrospective observational study investigates whether pre-admission anticoagulation (with Warfarin or Direct Oral Anticoagulant (DOAC)) improves all-cause mortality for geriatric COVID-19 patients compared to those who are not anticoagulated preadmission.
Methods: A total of 309 patients aged 70 and over who were admitted to a district general hospital in South-East England between March 15th and May 31st 2020 were included. Infection with SARS-CoV-2 was confirmed with viral PCR testing during that admission. Preadmission anticoagulant therapy was ascertained by looking at the medications section of the most recent hospital discharge letter on electronic records.

Results: A total of $81(26.2 \%)$ were prescribed oral anticoagulation prior to admission, 69 were on a DOAC and 12 on Warfarin. Of those who were anticoagulated, $38(46.9 \%)$ died during admission, compared to $110(48.2 \%)$ amongst those without pre-admission anticoagulation. There was no statistically significant improvement in all-cause mortality for those patients who were anticoagulated preadmission on univariate analysis $(\mathrm{OR}=0.95(0.56-1.57), \mathrm{p}>$ $0.05)$.

Conclusion: Pre-admission anticoagulation therapy does not appear to improve all-cause mortality for elderly patients with COVID-19, suggesting that patients on anticoagulation therapy have the same mortality rate as those who are not taking these agents.

\section{References:}

[1] Middeldorp, S, Coppens, M, van Haaps, TF, et al. Incidence of venous thromboembolism in hospitalized patients with COVID-19. J Thromb Haemost. 2020; 18: 1995-2002. https://doi.org/10. $1111 /$ jth. 14888

\section{Abstract \# 278}

Basal geriatric state as a strong predictor of day 30 mortality in intensive care units: towards a decision aid tool for patients' triage.

Claire Falandry ${ }^{1}$

${ }^{1}$ Geriatrics Unit, Centre Hospitalier Lyon Sud, 165, Chemin du Grand Revoyet

Introduction: With the spread of COVID-19 pandemic there is an urgent need to define the best care courses of the patients, and notably the place of ICU admission, according an individualized benefit/risk ratio.

Methods: Senior-COVID-Rea is a multicentric survey designed to evaluate, on health data, in patients over 60 admitted in ICU for severe COVID-19 disease, the impact of age, geriatric parameters and paraclinical covariates on their mortality at 30 days of their admission. All patients over 60 admitted in ICU for severe COVID-19 infection (or their caregiver) were proposed to enter the study and to fulfill a questionnaire on their functional parameters prior (1 month before) COVID infection.

Results: From March to May 2020, 232 patients where included in 7 hospitals. Median age was $73,75 \%$ were male, $21 \%>2$ comorbidities grade 2 or higher according CIRS-G, $21 \%$ and $30 \%$ had ADL and IADL disability respectively, $23 \%$ had at least 3 Fried's criteria. In univariate analysis, each of these covariates were highly correlated with day 30 survival: age $>75(\mathrm{OR}=4.8, \mathrm{p}<0.001),>2$ grade 2 CIRSG comorbidities (OR $=2.5, \mathrm{p}<0.001)$, frailty according Fried $(\mathrm{OR}=3.6, \mathrm{p}=0.001)$, clinical frailty score 4 or higher $(\mathrm{OR}=3.5, \mathrm{p}<$ $0.001)$, ADL disability (any) $(\mathrm{OR}=3.8, \mathrm{p}<0.001)$, IADL disability (any) $(\mathrm{OR}=6.1, \mathrm{p}<0.001)$, fall in the last 6 months $(\mathrm{OR}=3.4, \mathrm{p}=$ $0.003)$.

Key conclusions: Geriatric state one month before COVID19 is a powerful predictor of day 30 mortality in ICU. A score is currently being developed to provide a triage tool to guide patients' orientation. 


\section{Abstract \# 279}

COVID-19 and older adults: a call to accelerate geriatrics differentiated healthcare services in Africa

Wuraola Akande-Sholabi ${ }^{1}$

${ }^{1}$ Department of Clinical Pharmacy and Pharmacy Administration, Faculty of Pharmacy, University of Ibadan

Introduction: COVID-19 pandemic is having a global impact on healthcare system around the world. Everyone is susceptible to COVID-19, but older adults aged 60 years and above due to physiological changes that come with ageing and possible underlying medical disorders are more susceptible [1]. The need to ensure access to healthcare services by the elderly during this pandemic is paramount. Nonetheless, it is essential that stakeholders continue to prioritize access to healthcare services and medicines among the older adults amid the fight against this global public health, especially in Africa where little attention is paid to geriatric care. Methods: This is a descriptive recommendation Abstract for countries in Africa based on tailored strategies to improve access to healthcare among the geriatric population during this pandemic and in the post-pandemic era

Discussion and key conclusion: When implementing interventions to reduce the spread of disease, such as partial or total lockdown, exceptional attention must be offered to older people. Remote consultations such as telemedicine have the potential to protect healthcare workers and older adults from unnecessary exposure to disease, while ensuring continuity in the delivery of care, and in addition, decreases resource utilization across the already stressed health-care infrastructure [2]. COVID-19 is a call to accelerate improved geriatrics differentiated healthcare services in Africa by leveraging on telemedicine and technology without necessarily exposing older adults to the risk of contracting diseases by visiting healthcare settings. During COVID-19 pandemic and post-pandemic telemedicine would have unlimited potential to provide effective, appropriate, and secure care in the context of highly transmissible disease epidemics, both for management, and for regular follow-up of chronic disease among older adults. The implementation of this technology should ensure that the future policy on telemedicine includes the end-users in the planning and implementation.

Keywords: Covid-19, telemedicine, Africa.

References:

1. Chen N, Zhou M, Dong X et al. Epidemiological and clinical characteristics of 99 cases of 2019 novel coronavirus pneumonia in Wuhan, China: a descriptive study. Lancet 2020; 395: 507-513.

2. Ohannessian R. Telemedicine: Potential applications in epidemic situations. Eur Res Telemed Rech Eur En Télémédecine 2015; 4:95-8.

\section{ABSTRACT \# 280}

End of life care during the Covid-19 pandemic: a retrospective comparison between care delivered by geriatricians and general medical consultants

Helen Bounds ${ }^{1}$, Robert Smith-Baker, Jessica Webster ${ }^{1}$, Hannah Cheston $^{1}$, Charlotte Ainscough ${ }^{2}$, Sadia Saber ${ }^{1}$, Cianan O'Sullivan ${ }^{1}$

${ }^{1}$ Homerton University Hospital NHS Foundation Trust, ${ }^{2}$ Homerton Universtiy Hospital NHS Foundation Trust

Background and aims: The Covid-19 pandemic saw patients present with severe respiratory distress and was associated with high inpatient mortality.Homerton University Hospital's crisis response saw medical wards become "Covid zones" where care was randomly allocated to general medical or geriatrician consultants. This retrospective analysis aims to compare the differences around end of life (EoL) care received when lead by geriatricians compared to other medical specialties.

Method: Parameters surrounding EoL Care were extracted from the electronic patient record of patients over the age of 65 admitted during the pandemic peak (16/03/2020-30/04/2020) who passed away.

Results: 251 patients greater than 65 years were admitted for which the mortality rate was $45 \%$. 90 patients who passed away were identified with a mean age of 82 years, male: female ratio $47: 43$. The median clinical frailly score was 6 . They have been subdivided into two groups: Group A care delivered by a geriatrician (38\%) and group B care delivered by general medical specialities. Group A: 82\% EoL pathway initiated, 94\% EoL medications prescribed, $87 \%$ EoL medications given, $33 \%$ on syringe driver, $85 \%$ families updated and $53 \%$ palliative care involvement. Group B: $74 \%$ EoL pathway initiated, $88 \%$ EoL medications prescribed, $82 \%$ EoL medications given, $22 \%$ on syringe driver, $76 \%$ families updated and $31 \%$ palliative care involvement.

Conclusion: Despite the challenges faced during the Covid pandemic, patients were well managed at the EoL with practice adapted accordingly. Standard of EoL care delivered was similar between non-geriatricians and geriatricians.

\section{Abstract \# 281}

Managing delirium in patients with COVID-19-lessons to learn

Omolade Okuwoga ${ }^{1}$, Sophie Picton ${ }^{1}$, Sadia Saber ${ }^{1}$, Cianan O’Sullivan ${ }^{1}$

\section{${ }^{1}$ Homerton University Hospital}

Introduction: Delirium is an acute confusional state that affects cognition and alertness. Older adults were disproportionately affected by the COVID-19 outbreak, in terms of susceptibly to the virus itself and to developing delirium as a result of the acute infection. This study evaluates the methods used to manage delirium in patients with COVID-19.

Methods: A retrospective analysis was conducted of electronic patient records of patients aged $>65$ who were admitted to Homerton University Hospital (London, UK) with coronavirus. Admissions during a 6-week period spanning the peak of the outbreak were included. Data was collected to establish what medical and psychosocial methods were used to manage delirium in patients with COVID-19.

Results: A total of 104 patients (mean age 84) with coronavirus were identified as having a concurrent diagnosis of delirium. $87.5 \%$ of patients received oxygen therapy. $58.7 \%$ of patients received injectable medications for symptomatic relief of agitation. $39 \%$ of all patients received psychosocial support- $27.9 \%$ had family visitors, $9.6 \%$ were able to communicate with family using telecommunication methods and 2 patients were referred for review by the Psychiatry team.

Conclusion: This study shows that the COVID-19 pandemic has had a considerable impact on the care of older adults. Particularly, in managing older adults with COVID-19 and delirium, the focus is easily drawn to medically treating the virus as a potential underlying cause of delirium. However, there is a need to consider the use of psychosocial methods and pharmacological treatment to actively manage the delirium. 


\section{Abstract \# 282 \\ Drug-induced prolong QT in older patients with SARs CoV-2 infection}

Blanca Garmendia Prieto ${ }^{1}$, Rocío Ayala Muñoz ${ }^{2}$, Maribel Quezada Feijoó $^{2}$, Isabel Lozano Montoya ${ }^{1}$, Javier Jaramillo Hidalgo ${ }^{1}$, Pamela Carrillo García ${ }^{1}$, Giovana Cristofori ${ }^{1}$, Eva Fernández de la Puente Rodríguez $^{1}$, Beatriz Neira Martínez ${ }^{1}$, Luisa Sánchez Osorio ${ }^{1}$, Mónica Ramos Sánchez ${ }^{2}$, Javier Gómez-Pavón ${ }^{1}$

${ }^{1}$ Geriatrics. Hospital Central de la Cruz Roja, San José y Santa Adela, ${ }^{2}$ Cardiology. Hospital Central de la Cruz Roja, San José y Santa Adela

Introduction: A prolonged QT interval in older patients seems to be associated with increased mortality. In the current SARS-CoV-2 pandemic, the combination of drugs such as hydroxychloroquine/ azithromycin and hydroxychloroquine/lopinavir have a QT interval prolonging effect. We analyze the electrocardiographic changes, arrhythmic profile and their association with mortality in older patient.

Methods: Unicenter ambispective cross-sectional study. Older adults $\geq 75$ admitted to an Acute Geriatric Unit diagnosed with SARS-CoV2 infection (confirmed by RT-PCR or suspected by clinical and imaging) from March and April 2020. Polypharmacy and cardiological comorbidities were collected. We selected all those patients with electrocardiogram (ECG) performed $48 \mathrm{~h}$ after starting medical treatment with any drug for SARS-CoV-2. Corrected QT was measured using the Bazett's formula. Prolonged QT was considered for women $>470 \mathrm{msc}$ and for men $>450 \mathrm{msc}$.

Results: The mean age was $86 \pm 6.5(68.2 \%$ women, $60.6 \%$ hypertension and $26 \%$ atrial fibrillation). We observed $43.9 \%$ of polypharmacy ( $\geq 5$ drugs) and $27.3 \%$ of extreme polypharmacy ( $\geq$ 10 drugs). The average QT was $430 \pm 47.43 \mathrm{msc}$. About SARS-CoV2 treatment: $87.5 \%$ received hydroxychloroquine, $60 \%$ azithromycin and $18.2 \%$ lopinavir being the most frequent combination hydroxychloroquine and azithromycin (48.5\%). In univariate binary regression analysis, only antidepressants were associated with a prolonged QT $(\mathrm{p}=0.09)$. In multivariate analysis, no drugs or cardiovascular morbidity was associated with a prolonged QT.

Conclusions: In our cohort only $24.6 \%$ of the population presented prolonged QT, there was no severe arrhythmia during treatment for SARS-CoV-2 and mortality wasn't associated with prolonged QT.

\section{Abstract \# 283}

COVID 19 outbreak: organisation of a geriatric assessment and coordination unit. A French example

Thomas Tannou ${ }^{1}$, Séverine Koeberle ${ }^{1}$, Régis Aubry ${ }^{1}$

${ }^{1} \mathrm{CHU}$ de Besançon, France

Introduction: During the COVID 19 outbreak, the assessment of the appropriate level of care for each older person and the organization of appropriate healthcare networks was essential.

Methods: We have created a centralized unit dedicated to evaluation, geriatric care coordination, including territorial organization and individual decision support. It is coordinated by a geriatrician and includes emergency physicians as well as a medical hygienist, a physician specializing in infectious diseases and a palliative care specialist.The individual level of care decision was made by the unit in two stages: an initial assessment according to the Clinical Frailty Scale (CFS), followed by a search for additional information to allow for collegial discussion if necessary, thus ensuring a systematic personalized approach to the level of care from "non-limiting intensive care" to "symptomatic management in the newly created 17-bed COVID + palliative unit“.
Results:The call platform received 235 calls in 16 days. Of these, $80 \%(\mathrm{~N}=189)$ concerned requests for assistance in determining the level of care. From them, 99 patients residing in nursing home. $11 \%$ of nursing home residents were transferred to hospital $(\mathrm{N}=11)$, including 2 patients for palliative unit management. $52 \%(\mathrm{~N}=38)$ of the patients residing at home were transferred to the hospital they were geographically dependent. The remaining 46 calls were about various issues: hygiene, drug supply, feedback on organizational problems.

Conclusion: This model allowed a reasoned and individualized ethics of access to care, allowing the avoidance of a form of ageism in pandemic situation.

\section{Abstract \# 284 \\ Clinical pharmacists activity in the initial coronavirus hospitalisation ward: 1.3 major pharmaceutical intervention per day}

Amaury Durand ${ }^{1}$, Catherine Chenailler ${ }^{1}$, Céline Guilloton ${ }^{1}$, Johanna Raymond $^{1}$, Cyril Gricourt ${ }^{1}$

\section{${ }^{1} \mathrm{CHU}$ Rouen}

Introduction: During the coronavirus outbreak, medication reconciliation (MR) and pharmaceutical analysis of prescription were reinforced in the ward housing patients awaiting the outcome of coronavirus PCR and/or imaging. The objective of this work was to assess the clinical impact of this activity.

Methods: Between March 23rd and April 27th 2020, three pharmacists have recorded alternately for each patient reconciled all data relative to pharmaceutical interventions (PIs) carried out. Clinical impact was assessed [1]

Results: 294 patients (mean age 78.2 years old) were admitted in the unit. 170 medication reconciliations (MRs) were performed $(58 \%$ of patients), notably $33 \%$ proactively; 143 unintended discrepancies (UDs) have been corrected (mean 1.3 UD by retroactive MR) and mainly concerned drugs from nervous system (27\%) and alimentary tract and metabolism (24\%). The most frequent UD were non-prescribed drug after transfer (57\%) and supra-therapeutic dosage (17\%). The clinical impact was major in $11 \%$ of cases. $79 \%$ of patients were transferred in another ward. Moreover, 113 PIs were done which $61 \%$ (69 PIs) were accepted. They concerned mainly drugs from cardiovascular system $(25 \%)$ and blood and forming organs $(23 \%)$. The most frequent inappropriate prescriptions were therapeutic drug monitoring requested $(22 \%)$ and supra-therapeutic dosage $(16 \%)$. The clinical impact was major in $13 \%$ of cases. Overall 25 PIs with a major clinical impact were accepted representing 1.3 per day and 0.1 per patient.

Key conclusion: The presence of pharmacists in the unit allowed correcting many inappropriate prescriptions and reduce the risk of adverse drug events, which was really appreciated by physicians. Bibliography:

[1] Vo TH, Charpiat B, Catoire C, Juste M, Roubille R, Rose F-X, et al. Développement de l'echelle multidimensionelle «Cléo » pour évaluer la pertinence d'une intervention pharmaceutique. Pharm Hosp Clin. 2014; 49(2).

\section{Abstract \# 285}

\section{Bilateral COVID-19 pneumonia-a case series}

Itxaso Marín-Epelde ${ }^{1}$, Agurne García-Baztán ${ }^{1}$, Isabel RodríguezSánchez ${ }^{2}$, Belén González-Glaría ${ }^{1}$, María Gonzalo-Lázaro ${ }^{1}$, César Cuevas-Lara ${ }^{3}$, Chenhui Chen ${ }^{1}$, Ana Sofía Pozo-Vico ${ }^{1}$, Álvaro CasasHerrero $^{1}$, Fabricio Zambom-Ferraresi ${ }^{4}$ 
${ }^{1}$ Complejo Hospitalario de Navarra, Pamplona, Spain, ${ }^{2}$ Hospital Clínico San Carlos, Madrid, Spain, Navarrabiomed, "Complejo Hospitalari de Navarra (CHN)", "Universidad Pública de Navarra (UPNA)", "IdiSNA", Pamplona, Navarre, Spain., " "Complejo Hospitalario de Navarra (CHN)", "Universidad Pública de Navarra (UPNA)", "IdiSNA", Pamplona, Navarre, Spain.

Introduction: Coronavirus disease 2019 (COVID-19) is a disease caused by the severe acute respiratory syndrome coronavirus 2 (SARS-CoV-2). Regarding the physiopathology of Acute Respiratory Distress Syndrome (ARDS), it seems largely related to a dysregulated macrophage response that causes a cytokine storm and subsequent abnormal inflammatory reaction.

Methods: We describe 10 patients (on average $71.8 \pm 12.2$ years old, Barthel Index $97 \pm 6.7$, Charlson Comorbidity Index $4 \pm 2.2$ ) admitted to our hospital (average-stay $16.9 \pm 5.4$ days) with severe bilateral COVID-19 pneumonia who received high-dose glucocorticoid therapy when they developed mild-to-moderate ARDS and their clinical evolutions (temperature) and radiological changes. We analyzed analytic parameters (D-Dimer). Furthermore, we compare glucocorticoids alone versus associated with Tocilizumab and we looked at differences between early or late administration of treatment in disease-evolution. They all received therapeutic-dose heparin.

Results and discussion After therapy, D-Dimer tended to go down, as did body temperature, and lymphocyte count went up. Patients who received glucocorticoid therapy in an early phase of disease evolution (50\%) presented a less severe D-Dimer elevation compared with those who received it in later phases. Combination therapy with glucocorticoids and Tocilizumab (60\%) was used when ARDS presented quicker and more severely, which coincides with a higher elevation of D-Dimer.

Conclusions: Although cause-effect relationships cannot be established due to the data being observational, there is a seemingly chronological relationship between glucocorticoid treatment and clinical, analytical and radiological improvement. These findings also point to the possibility of early anti-inflammatory treatment being justified in patients with early hypoxemia. Randomized Controlled Trials are urgently needed on this topic.

\section{Abstract \# 286 \\ Therapeutic exercise in adult post-COVID-19 patients in post- acute care}

Joan Ars ${ }^{1}$, Cristina Udina ${ }^{1}$, Alessandro Morandi ${ }^{1}$, Jordi Vilaró ${ }^{2}$, Josep de Bergua ${ }^{1}$, Marta Molins ${ }^{1}$, Maria Teresa Molins ${ }^{1}$, Clara Roig ${ }^{3}$, Ramón Giménez ${ }^{3}$, Leonor Sousa M. Abreu ${ }^{3}$, Mónica Zulueta ${ }^{4}$, Marco Inzitari $^{1}$

${ }^{1}$ REFiT Barcelona research group, Parc Sanitari Pere Virgili and Vall d'Hebron Institut de Recerca (VHIR), Barcelona, Spain, ${ }^{2}$ Blanquerna School of Health Sciences, Global Research on Wellbeing (GRoW), Universitat Ramon Llull. Barcelona, Spain, ${ }^{3}$ Department of Physical Medicine and Rehabilitation, Vall d'Hebron University Hospital, Barcelona, Spain, ${ }^{4}$ Department of Geriatric Medicine, Vall d'Hebron University Hospital, Barcelona, Spain

Introduction: COVID-19's impact increases with age. Besides mortality, patients experience relevant disability related to complications requiring Intensive Care Unit (ICU) admission or functional decline due to immobilization. Early and effective rehabilitation interventions are urgent. Aim: describe pre-post impact on physical performance of multi-component therapeutic exercise for postCOVID-19 rehabilitation.

Methods: Cohort study of post-COVID-19 patients in post-acute care. We collected demographical and clinical variables, cognitive status at admission, pre-COVID functional and frailty status. Intervention: 30-min 7-days/week multi-component therapeutic exercise. Outcomes: Short Physical Performance Battery (SPPB, gait speed, balance and chair-stand sub-tests Barthel Index for activities of daily living, ability to walk unassisted and single-podal balance. Descriptive statistics and comparison of pre-post outcome variables and postICU vs non-ICU groups.

Results: We included 33 patients $(66.2 \pm 12.8$ years, $57.6 \%$ women, $90.9 \%$ pneumonia, 60, 6\% ICU admission. Post-ICU patients were younger, with lower comorbidity, better pre-COVID-19 functional status and lower frailty. After the intervention (8.2 \pm 1.7 days), all physical performance measures improved significantly in the global sample $(\mathrm{p}<0.01)$. Compared to non-ICU, post-ICU patients experienced greater improvement in SPPB $(4.4 \pm 2.1$ vs $2.5 \pm 1.7, \mathrm{p}<$ $0.01)$ and gait speed $(0.4 \pm 0.2$ vs $0.2 \pm 0.1 \mathrm{~m} / \mathrm{s}, \mathrm{p}<0.01)$.

Conclusions: Our findings show that adults surviving COVID-19 seem to improve their functional status, despite ICU admission, through a short, individualized, multi-component therapeutic exercise intervention. Higher improvement in the post-ICU group might be due to their younger age and better functional and clinical status preCOVID-19. We need future research with larger study samples to better investigate and validate therapeutic exercise as a rehabilitation tool.

\section{Abstract \# 287 \\ QTe prolungation risk score and hydroxychroloquine in COVID} 19 patients: our experience

Alberto Castagna ${ }^{1}$, Raffaele Costa $^{2}$, Carmen Ruberto ${ }^{1}$, Michela Pugliese $^{3}$, Carlo Torchia ${ }^{1}$, Viviana Vespertini ${ }^{4}$, Lucio Cosco ${ }^{4}$, Giovanni Ruotolo ${ }^{5}$

${ }^{1}$ Center for Cognitive Disorders and Dementia, Azienda Sanitaria Provinciale di Catanzaro, Italy, ${ }^{2}$ Geriatric Unit, "Pugliese-Ciaccio" General Hospital, Catanzaro, Italy, ${ }^{3}$ Direttore Sanitario RSA/Recc San Vito Hospital /Associazione Vivere Insieme, ${ }^{4}$ Infectious Diseases Unit, "Pugliese-Ciaccio" General Hospital, Catanzaro, Italy, ${ }^{5}$ Geriatric Unit, "Pugliese-Ciaccio" General Hospital, Catanzaro, Italy.

Introduction: COVID-19 has been declared a global pandemic by WHO. Currently we have lacking therapeutic options. Some observational studies have suggested benefits of hydroxychloroquine (HCQ) for the treatment of Covid-19, whereas other treatment reports have described mixed results. As a result, these treatments are increasingly used off-label for patients with COVID-19. Tisdale et al found a valid method to quantify the risk of drug-induced QTc interval prolongation. In a retrospective study we assessed the usefulness of this method for the management of HCQ in relation to the prolongation of the QTc.

Methods: 58 hospitalized patients, positive for SARS-CoV-2 (PCR) treated with HCQ were examined. We calculated the risk score of prolongation of the QTc interval and examined its possible progression on the basal ECG (T0) and after 7 days from the insertion of the drug (T1).

Results: Of 58 patients $(\mathrm{M}=36,20 \%) 44,82 \%$ were $>65$ years of age (average age 76,10 $\pm 8,70$ years). The QTc interval was prolonged from 434,22 $\pm 26,08 \mathrm{~ms}$ (T0) to a value of 462,22 $\pm 37,24 \mathrm{~ms}$ (T1) (p $<0.000)$. The QTc were significantly prolungation in high risk group ( $\mathrm{n}=23$; score Tisdale $\geq 11$ points) as compare to low-moderate risk group $(\mathrm{n}=35$; Tisdale $<10$ points $)(477,33 \pm 39,7$ vs. $452,4 \pm 36,6$ ms; $\mathrm{p}<0,017)$.

Key conclusions: In our opinion the use of Hydroxychlorochinic in COVID -19 must be assessed case by case, by using QTc Interval 
Prolongation Risk Score, and paying attention to interaction with other drugs associated with QTc prolongation.

\author{
Abstract \# 288 \\ Antiplatelet therapy in patients with Covid-19: a retrospective \\ observational study \\ Josef Banik ${ }^{1}$, Christian Köhler ${ }^{1}$, Marco Schmidtmann ${ }^{1}$ \\ ${ }^{1}$ Department of Internal Medicine, Klinikum Fichtelgebirge, Selb, \\ Germany
}

Introduction: Covid-19 is associated with a high risk of venous thromboembolism. In addition, cases of arterial thromboembolism were also reported. We investigated the effect of antiplatelet therapy on the disease course.

Methods: We evaluated a cohort of inpatients with Covid-19 ( $\mathrm{n}=$ 152). We recorded the patient's demographic data, their comorbidities, medication use including the use of antiplatelets and anticoagulants, laboratory findings and data about ventilation. We then separated the patient's outcomes into either being „bad“ (dead or referral to higher level of care) or "good“ (discharge). Then we evaluated the factors that contributed to the patient needing ventilatory support and to showing typical radiological findings.

Results: In our cohort, 21 patients received ventilatory support whereas $131 \mathrm{did}$ not require the use of ventilators. 127 patients had good outcomes and 25 had bad outcomes. By using multivariate analysis, we found that the need for ventilatory support was the strongest predictor of a bad outcome. All patients who were on ventilators displayed typical radiological findings. The factors predicting the need for ventilatory support were LDH and CRP levels, the presence of cardiac conduction abnormalities as well as chronic lung conditions. Cardiac conduction abnormalities, LDH and CRP levels, and the use of antiplatelets, were factors that predicted typical radiological findings.

Conclusions: There was a higher incidence of typical radiological findings in patients on antiplatelet medication. However, it did not translate into changes in the ventilation requirement or in the outcome. The need for ventilation was the strongest predictor of a bad outcome.

\section{Abstract \# 289}

\section{Elderly patients dying with Covid-19: lessons learned}

Shan Liung Liew ${ }^{1}$, Muhammad Iqbal ${ }^{2}$, Atif Nasrullah ${ }^{2}$, Sherena Nair ${ }^{2}$

${ }^{1}$ Calderdale and Huddersfield NHS Trust, ${ }^{2}$ Leeds Teaching Hospitals NHS Trust

The start of the pandemic in the United Kingdom resulted in many elderly patients with Covid-19 dying in hospitals as rapid guidelines were being drafted to support the care of such patients with compassion and dignity [1]. Experience from caring for patients with Covid-19 found that the course of illness differed from diseases familiar to geriatricians which prompted further evaluation. A snapshot audit of 30 patients was conducted over a 2 week period on a geriatric medicine ward to assess the nature and progression of Covid19 and the challenges around care at the end of life. The unusual features found in these patients included a prolonged hypoactive delirium stage in $50 \%$ of patients with a mean time of 4 days from starting end-of-life care to death. Also, $30 \%$ of patients required increased doses of midazolam compared to average doses, prompting support from the specialist palliative care team to manage symptoms of agitation and breathlessness. In about $40 \%$ of patients, it was felt that death was caused by a slow deterioration in renal function due to hypoactive delirium and minimal oral intake, rather than a rapid progression of multi-organ failure; death seemed to be protracted in this group of patients. In conclusion, the progression of Covid-19 in older people represents an atypical trajectory to death. Hypoactive delirium seems to be a prominent feature and terminal agitation from presumed 'air hunger' described in Covid-19 patients seems to require higher doses of palliative drug doses towards the end of life.

\section{References:}

1. British Geriatrics Society. COVID-19: End of life care in older people.

https://www.bgs.org.uk/resources/covid-19-end-of-life-care-in-olderpeople (Accessed on 29th June, 2020).

\section{Abstract \# 290 \\ Mortality with different empiric treatments in covid-19 patients over 80 years old}

Elisabet Sanchez ${ }^{1}$, Eva Delgado Silveira ${ }^{1}$, Maria Muñoz Garcia ${ }^{2}$, Hilario Martinez Barros ${ }^{3}$, Ana Maria Alvarez Díaz ${ }^{2}$, Beatriz MonteroErrasquin $^{4}$, Alfonso Cruz-Jentoft ${ }^{4}$

${ }^{1}$ Hospital Universitario Ramón y Cajal, ${ }^{2}$ Hospital Ramon y Cajal, ${ }^{3}$ Hospital Ramón y Cajal, ${ }^{4}$ Geriatrics department. Hospital Ramon y Cajal

Aim: To explore mortality associated with different empiric treatments for severe COVID-19 in hospitalized patients over 80 years old.

Methods: We performed an observational retrospective study of all patients 10-20 years old hospitalized in a large hospital in Madrid with confirmed COVID-19 infection between 2nd March and 25th May. We assessed age, gender and hospital mortality by reviewing electronic medical records. Treatment was based on hospital protocols that changed along the study time. Drugs were used off-label, as no approved drug was available for COVID-19 at the time of the study.Results: 694 patients were included, mean age $86.2 \pm 4$ years (80-104), $52.6 \%$ men. In-hospital mortality was 39.3\% ( $\mathrm{n}=273)$. Those who died were older ( 86.7 vs 85.9 years, $p=0.019)$, and used a similar number of drugs usd for COVID-19 treatment (mean $1.85 \mathrm{vs}$ 1.89 drugs, $\mathrm{p}=0.6$ ). The most frequent drugs used were hidroxicloroquine $(81.6 \%)$, lopinavir/ritonavir $(49 \%)$ and azitromicine $(39 \%), 13.8 \%$ did not receive any drug. There were no differences in mortality based on the number of drugs used. Patients who died received less azitromicine $(32.6 \%$ vs $43,5 \%, p=0.04)$, less enoxaparin $(79.9 \%$ vs $90.3 \%, \mathrm{p}=0.001)$ and more corticoids pulses $(10.6 \%$ vs $5.2 \%, p=0.011)$. We didn't find differences in mortality related to the use of other drugs as hidroxocloroquine (79.55 vs $82.9 \%, \mathrm{p}=0.2)$, lopinavir/ritonavir $(52.4 \%$ vs $46.8 \%, \mathrm{p}=0.1)$, remdesivir $(9.5 \% \mathrm{vs} 10$, $2, \mathrm{p}=0.7)$ or tocilizumab $(0.7 \%$ vs $0.5 \%, \mathrm{p}=0.5)$.

Conclusion: Mortality during hospital admission in very old patients with severe COVID-19 was $39.3 \%$. Patients who died were older, received less azitromicine and heparin and more corticoids. These findings are only exploratory and should be confirmed in prospective, controled trials.

\section{Oral communications miscellaneous}

\section{Abstract \# 1}

Free 25-hydroxyvitamin D, but not free 1, 25-dihydroxyvitamin $\mathrm{D}$, predicts all-cause mortality in ageing men

Dejaeger Marian ${ }^{1}$, Antonio Leen ${ }^{2}$, Bouillon Roger ${ }^{2}$, Wu Frederick C. W. ${ }^{3}$, O'Neil Terence W. ${ }^{4}$, Pye Stephen R. ${ }^{4}$, Huhtaniemi Ilpo T. ${ }^{5}$, 
Rastrelli Giulia ${ }^{6}$, Casanueva Felipe F. ${ }^{7}$, Slowikowska-Hilczer Jolanta $^{8}$, Punab Margus 9 , Tournoy Jos ${ }^{1}$

${ }^{1}$ Department of Public Health and Primary Care, KU Leuven, Laboratory of Gerontology and Geriatrics, Leuven, Belgium, ${ }^{2}$ Department of Clinical and Experimental Medicine, KU Leuven, Laboratory of Clinical and Experimental Endocrinology, Leuven, Belgium, ${ }^{3}$ Andrology Research Unit, Centre for Endocrinology \& Diabetes, Institute of Human Development, Old St Mary's Building, The University of Manchester, Manchester, UK, ${ }^{4}$ Arthritis Research UK Epidemiology Unit, The University of Manchester, Manchester, UK, ${ }^{5}$ Institute of Reproductive and Developmental, Department of Metabolism, Digestion and Reproduction, Imperial College London, Hammersmith Campus, London, UK, ${ }^{6}$ Andrology Unit, Department of Clinical Physiopathology, University of Florence, Florence, Italy, ${ }^{7}$ Department of Medicine, Santiago de Compostela University, Complejo Hospitalario Universitario de Santiago (CHUS); CIBER de Fisiopatología Obesidad y Nutricion (CB06/03), Instituto Salud Carlos III; Santiago de Compostela, Spain, ${ }^{8}$ Department of Andrology and Reproductive Endocrinology, Medical University of Łódź, Łódź, Poland, ${ }^{9}$ Andrology Unit, United Laboratories of Tartu University Clinics, Tartu, Estonia

Introduction: Total 25-hydroxyvitamin D (25 (OH)D) and total 1, 25-dihydroxyvitamin $\mathrm{D}(1,25(\mathrm{OH}) 2 \mathrm{D})$ are associated with mortality. In line with the free hormone hypothesis some studies suggested that free $25(\mathrm{OH}) \mathrm{D}$ levels might be a better predictor for clinical outcomes. Here, the association between total and free $25(\mathrm{OH}) \mathrm{D}$ and 1 , $25(\mathrm{OH}) 2 \mathrm{D}$ with all-cause mortality in a prospective cohort of community-dwelling European men was examined.

Methods: 1970 men, aged 40-79, participated in the European Male Ageing Study between 2003-2005. In 5 of 8 centres, survival status was available until 1 April 2018. Free $25(\mathrm{OH}) \mathrm{D}$ and free 1,25 $(\mathrm{OH}) 2 \mathrm{D}$ were calculated. All measurements were divided into quintiles. Cox proportional hazard models were used, and results were expressed as hazard ratios (HR), adjusted for centre, BMI, smoking and self-reported health.

Results: 524 (26.6\%) men died during a mean follow-up of $12.3 \pm$ 3.4 years. Men in the lowest total $25(\mathrm{OH}) \mathrm{D}$ and total $1,25(\mathrm{OH}) 2 \mathrm{D}$ quintile (cutoff $<9.3 \mu \mathrm{g} / \mathrm{L}$ and $<46 \mathrm{ng} / \mathrm{L}$ respectively) had increased mortality risk (HR compared to highest quintile [HR 1.83 (95\% CI 1.34-2.50); $\mathrm{p}<0.001$ and 1.41 (1.04-1.90); $\mathrm{p}<0.05$ respectively]. Likewise, men in the lowest three free $25(\mathrm{OH}) \mathrm{D}$ quintiles (levels $<$ $4.43 \mathrm{ng} / \mathrm{L}$ ) had a higher mortality risk compared to the highest quintile [HR $1.91(1.34-.73) ; \mathrm{p}<0.001$ for the lowest quintile]. However, mortality risk was similar across all free 1, $25(\mathrm{OH}) 2 \mathrm{D}$ and DBP quintiles. Conclusions: Ageing men with low total $25(\mathrm{OH}) \mathrm{D}$, total 1, $25(\mathrm{OH}) 2 \mathrm{D}$ and free $25(\mathrm{OH}) \mathrm{D}$ levels but not low free 1,25 $(\mathrm{OH}) 2 \mathrm{D}$ have an increased future mortality risk. Hence, vitamin D deficiency is predictive for a higher mortality risk.

\footnotetext{
Abstract \# 2

The association between herpesvirus burden and mortality differs between older women and men

Timo Strandberg ${ }^{1}$, Pentti Tienari ${ }^{1}$, Kaisu Pitkälä ${ }^{1}$

${ }^{1}$ University of Helsinki and Helsinki University Hospital

Introduction: There are sex differences in immune responses which may contribute to different susceptibility to Covid-19 between sexes. We tested whether also the association between herpesviridae and mortality differs between sexes among home-dwelling older people. Methods: The DEBATE study consists of 400 women (65.2\%) and men aged 75-90 years and with a history cardiovascular disease. With other questionnaire, clinical and laboratory data (including education
}

and APOE genotype) at baseline, 383 (95.8\%) were assayed for serum anti-cytomegalovirus, anti $\mathrm{hV}-1$ and anti $\mathrm{hV}-2 \mathrm{IgG}$ antibody titers using enzyme immunoassay. Titers were summed up as herpesvirus burden (HVB) and divided in tertiles. Cox regression was used to assess 7-year mortality risk (hazard ratios [HR] with 95\% confidence interval $[\mathrm{CI}]$ ).

Results: HVB was significantly higher among women than men $(\mathrm{P}<$ 0.001 ). In the whole cohort (lowest HVB $=1.0$ ), age-adjusted HRs were $1.15(95 \%$ CI $0.77,1.72)$ and $1.60(1.05,2.45)$ in second and third HVB tertiles, respectively. There was a significant sex/HVB interaction $(P=0.01)$ and therefore sex-specific analyses were performed. Among women, mortality HRs for increasing HVB tertiles were 1.0 (ref.), $1.17(0.70,1.97)$, and $1.30(0.78,2.18)$, respectively; among men 1.0 (ref.), 1.27 (0.62, 2.60), and 2.14 (1.10, 4.18), respectively. The results prevailed after further adjusting for education and APOE4 genotype.

Conclusions: While being lower in older men as compared to women, higher herpesvirus burden was associated with increased mortality risk among men, not significantly among women. Differences in immune responses to common viruses may contribute to sex differences in longevity and mortality.

\section{Abstract \# 3 \\ TERC variants associated with short leukocyte telomeres: implication of higher early life leukocyte telomere attrition as assessed by the blood-and-muscle model}

Simon Toupance ${ }^{1}$, Maria G. Stathopoulou ${ }^{2}$, Alexandros M. Petrelis ${ }^{2}$, Vesna Gorenjak ${ }^{2}$, Carlos Labat ${ }^{1}$, Tsung-Po Lai ${ }^{3}$, Sophie VisvikisSiest $^{2}$, Athanase Benetos ${ }^{4}$

${ }^{1}$ Université de Lorraine, Inserm, DCAC, F-54000 Nancy, France, ${ }^{2}$ Université de Lorraine, IGE-PCV, F-54000 Nancy, France, ${ }^{3}$ Center of Human Development and Aging, Rutgers, The State University of New Jersey, New Jersey Medical School, Newark, NJ 07103, USA,

${ }^{4}$ Université de Lorraine, CHRU-Nancy, Pôle "Maladies du

Vieillissement, Gérontologie et Soins Palliatifs“, F-54000, Nancy, France

Introduction: Short leukocyte telomere length (LTL) is associated with atherosclerotic cardiovascular disease (ASCVD). Mendelian Randomization Studies, using single nucleotide polymorphisms (SNPs) associated with short LTL, infer a causal role of LTL in ASCVD. Recent results, using the blood-and-muscle model, indicate that higher early life LTL attrition, as estimated by the ratio between LTL and skeletal muscle telomere length (MTL), rather than short LTL at conception, as estimated by MTL, should be responsible of the ASCVD-LTL connection.

Methods: We combined LTL and MTL measurements by Southern blotting and SNPs profiling in 402 individuals to determine if 15 SNPs classically described as associated with short LTL at adult age were rather responsible for higher LTL attrition during early life than for shorter LTL at birth.Results: Two of these SNPs (rs12696304 and rs 10936599) were associated with LTL in our cohort ( $p=0.027$ and $p$ $=0.025$ respectively). These SNPs, both located on the TERC gene, were associated with the LTL/MTL ratio $(p=0.007$ and $p=0.037$ respectively) but not with MTL ( $p=0.78$ and $p=0.32$ respectively). Key conclusions: These results suggest that SNPs located on genes coding for telomere maintenance proteins may contribute to a higher LTL attrition during the highly replicative first years of life and have an impact later on the development of ASCVD. 


\section{Abstract \# 4}

Anticoagulant prescription and mortality in older patients with atrial fibrillation: the european study of older subjects with atrial fibrillation study (EUROSAF)

Nicola Veronese ${ }^{1}$, Alberto Cella ${ }^{2}$, Katerin Leslie Quispe Guerrero ${ }^{2}$, Alberto Ferri ${ }^{2}$, Stefania Maggi ${ }^{3}$, Maria Cristina Polidori ${ }^{4}$, Timo Strandberg ${ }^{5}$, Alberto Pilotto

${ }^{1}$ University of Palermo, ${ }^{2}$ Ente Ospedaliero Galliera, ${ }^{3}$ Consiglio Nazionale delle Ricerche, ${ }^{4}$ University of Cologne, ${ }^{5}$ University of Helsinki

Objectives: Some studies suggested that a different risk of mortality may influence the attitude of physicians in prescribing oral anticoagulants in older patients affected by atrial fibrillation (AF). The Multidimensional Prognostic Index (MPI) showed a high grade of accuracy, calibration and feasibility for predicting mortality in older people, but prognostic information calculated through the MPI class (i.e. MPI-1 low-risk, MPI-2 moderate risk, MPI-3 high risk of mortality) is not yet included in the decision algorithm of treatments in older patients affected by AF. The aim of this study was to evaluate the association between the use of oral anticoagulants and 1-year mortality in patients with different MPI score.

Methods: Older hospitalized patients (age $>65$ years) with nonvalvular $\mathrm{AF}$ were included. At baseline, functional and clinical information will be collected to calculate the MPI, the CHA2DS2VASC and the HAS-BLED scores.

Results: At 1st June 2020, 1708 older patients affected by AF from 22 European centers were included (mean age $=82.9 \pm 7.5$, range 65-104 years, women $=57.0 \%$ ). The mean HAS-BLED and CHA2DS2-VASC scores indicated patients at both higher risk of major bleedings and thromboembolic events. The mean MPI score was $0.50 \pm 0.21$ points, with $447(=26.2 \%)$ at low risk (MPI-1), 809 $(=47.4 \%)$ at intermediate risk (MPI-2) and $452(26.5 \%)$ at high risk of mortality (MPI-3). During 1 year of follow-up and after adjusting for MPI at discharge, age, gender, HAS-BLED, CHA2DS2VASC, the use of new oral anticoagulants (NOACs) significantly decreased the risk of mortality (hazard ratio, $\mathrm{HR}=0.49 ; 95 \% \mathrm{CI} 0.36-0.66$ ) and, less extensively, the use of vitamin $\mathrm{K}$ antagonists (VKAs) ( $\mathrm{HR}=0.74$; $95 \%$ CI $0.55-0.98$ ). The use of NOACs significantly decreased mortality risk independently from the MPI groups: MPI- $1 \mathrm{HR}=0.22$ $0.10-0.51, \mathrm{p}<0.0001$; MPI-2 HR $=0.52$ 0.33-0.82, $\mathrm{p}=0.005$; MPI-3 $\mathrm{HR}=0.550 .35-0.86, \mathrm{p}=0.009$.

Conclusions: In older patients with AF, the use of NOACs seems to decrease the risk of death compared to people not taking anticoagulants, independently from the MPI categories.

\section{Abstract \# 5 \\ Effect of nurse-coordinated transitional care in high risk older cardiac patients: the cardiac care bridge randomized clinical trial \\ Jepma Patricia ${ }^{1}$, Lotte Verweij ${ }^{2}$, Bianca Buurman ${ }^{3}$, Michel Terbraak $^{2}$, Sara Daliri ${ }^{4}$, Corine Latour ${ }^{2}$, Gerben ter Riet ${ }^{2}$, Fatma Karapinar- Carkit $^{4}$, Ron Peters ${ }^{5}$, Wilma Scholte op Reimer ${ }^{5}$ \\ ${ }^{1}$ Center of Expertise Urban Vitality, Faculty of Health, Amsterdam University of Applied Sciences, Amsterdam, the Netherlands, ${ }^{2}$ Center of Expertise Urban Vitality, Faculty of Health, Amsterdam University of Applied Sciences, Amsterdam, the Netherlands., ${ }^{3}$ Amsterdam UMC, University of Amsterdam, Department of Internal Medicine section of geriatrics, Amsterdam, the Netherlands., ${ }^{4}$ OLVG hospital, department of Clinical Pharmacy, Amsterdam, the Netherlands., \\ ${ }^{5}$ Amsterdam UMC, University of Amsterdam, Department of \\ Cardiology, Amsterdam, the Netherlands.}

Introduction: After hospitalization for cardiac disease, older patients are at high risk of readmission and death. Currently, the integration of cardiac and geriatric care is limited. The Cardiac Care Bridge transitional care program (CCB program) investigated if unplanned hospital readmission and mortality can be reduced by integrating case management, disease management and home-based cardiac rehabilitation (CR)

Methods: The CCB program was a Dutch single-blinded, multicenter randomized clinical trial. All cardiac patients $\geq 70$ years, admitted $\geq$ $48 \mathrm{~h}$ were eligible if they were at high risk of functional loss or if they experienced an unplanned hospital admission in the previous 6 months. The intervention included a comprehensive geriatric assessment and integrated care plan, a face-to-face handover with the community care registered nurse (CCRN) and four home visits. The CCRNs collaborated with physiotherapists who performed homebased CR and with a pharmacist in medication management. The primary composite outcome was first all-cause unplanned readmission or mortality within 6 months.

Results: In total, 306 patients were included. The mean age was 82 years, $57 \%$ had heart failure and $\geq 90 \%$ were acutely hospitalized. Nearly $50 \%$ have experienced a hospital admission or a fall in the previous 6 months. Furthermore, 31\% were cognitively impaired (MMSE < 24), 39\% had functional impairments (Katz-6) and 31\% were at risk of malnutrition (SNAQ). The main unblinded study outcomes will be presented during the online EUGMS conference.

Conclusions: This study provides new knowledge on the effectiveness of a nurse-coordinated, multidisciplinary transitional care program in high risk older cardiac patients (NTR6316, April 6, 2017).

\section{Abstract \# 6 \\ Short-term readmission and mortality after a pneumonia admission in older patients with dementia and use of benzodiazepines, opioids or anti-psychotics}

Susanne Boel Graversen ${ }^{1}$, Henrik Schou Pedersen ${ }^{2}$, Annelli Sandbaek ${ }^{3}$, Catherine Hauerslev Foss ${ }^{4}$, Victoria Palmer ${ }^{5}$, Anette Riisgaard Ribe ${ }^{2}$

${ }^{1}$ Research Unit for General Practice, Aarhus, Denmark. Department of Public Health, Aarhus University, Aarhus, Denmark., ${ }^{2}$ Research Unit for General Practice, Aarhus, Denmark., ${ }^{3}$ Steno Diabetes Center Aarhus, Aarhus, Denmark. Department of Public Health, Aarhus University, Aarhus, Denmark., ${ }^{4}$ Department of Geriatrics, Aarhus University Hospital, Aarhus, Denmark., ${ }^{5}$ Department of General Practice, Melbourne University, Melbourne, Australia.

Introduction: Pneumonia and dementia are commonly recognized as interlinked in the older population. Yet, despite this co-existence, little is known about the prognosis of people with dementia who survive their pneumonia admission, and whether the risk of an adverse event is modified by other factors, including medication often prescribed to people with dementia.

Methods: For people with pneumonia aged 65-99 years in 2000-2016, we used the Danish registries to estimate risk of 30-day mortality and readmission by calculating mortality rate ratios (MRRs) and incidence rate ratios (IRRs) for people with dementia compared to those without dementia. Further, we investigated whether there was an excess risk due to use of specific medications, and whether this risk was modified by age and time since discharge.

Results: We found 298,872 pneumonia admissions, hereof 25,948 $(8.7 \%)$ with dementia. Compared to pneumonia patients without dementia, risk of readmission was slightly higher (IRR $1.07,95 \%$ CI 1.04-1.10) for those with dementia, whereas risk of mortality was $129 \%$ higher (MRR 2.29, 95\% CI 2.21-2.37). For those with both dementia and use of anti-psychotics, there was a $16 \%(0.16,95 \%$ CI 
0.10-0.22) excess risk of dying, which was modified by age.Pneumonia patients with dementia had higher risk of dying throughout the 30 days follow-up regardless of medication use, whereas risk of readmission was only higher in the first three days after discharge. Conclusions: Knowledge about the prognosis following pneumonia for people with dementia is important in order to perform individualized advanced care planning at time of discharge.

\section{Abstract \# 7 \\ Effect of a multicomponent exercise programme (VIVIFRAIL) on cognitive function in Frail Community Elders With Cognitive Decline: Study Protocol for a Randomized Multicentre Control Trial}

Fernanda Ramón-Espinoza ${ }^{1}$, Iván Antón-Rodrigo ${ }^{1}$, Itxaso MarínEpelde $^{1}$, Juan Luis Sánchez-Sánchez ${ }^{1}$, Abel Cedeño-Veloz ${ }^{1}$, Marina Sánchez-Latorre $^{1}$, Chenhui Chen ${ }^{1}$, Fabricio Zambon-Ferraresi ${ }^{1}$, Alvaro Casas-Herrero ${ }^{1}$

${ }^{1} \mathrm{No}$

Introduction: Some studies have determined a bidirectional relationship between frailty and cognitive disorders. This relationship may be justified because both entities share common pathophysiological bases and adverse outcomes such as hospitalization, falls, fractures, disability, institutionalization, and mortality. This relationship could also be revealed through a possible response to common interventions.

Aims: Examine whether an innovative multicomponente exercise programme (VIVIFRAIL) has benefits for cognitive function and mood status in pre-frail or frail patients aged 75 years, with a Barthel Index $\geq 60$ and mild cognitive impairment or mild dementia (GDS $\leq$ 4). Methods: Multicentre randomized clinical trial conducted in the outpatient geriatrics clinics of three hospitals in Spain, with a Barthel Index $\geq 60$ and mild cognitive impairment or mild dementia, pre-frail or frail according to Fried criteria and having someone to help to supervise them when conducting the exercises, were randomly assigned to the intervention or control group.The VIVIFRAILis a multicomponent exercise intervention programme (www.vivifrail.com) designed to improve functional capacity and decrease risk of falls for patients over 70 years old.

Results: Preliminary sample 160 patients out of a total sample of 220 , mean age of $83.45( \pm 4.55)$ and Barthel 92.29 ( \pm 9.74.) Subjects assigned to the intervention group obtained improvements in cognitive function measured by MOCA test (pre $=14.9 \pm 5.97$, post $=$ $16.74 \pm 7.62, \mathrm{p}=0.035$ ) compared to the control group (pre $=10.98$ \pm 8.09 , post $=10.34 \pm 8.69, \mathrm{p}=0.035$ ). In contrast, physical exercise did not show statistically improved improvements in cognitive function measured by the MMSE $(p=0.941)$. Using Yesavege test significal improvements were found in mood status in the intervention group $(\mathrm{p}=0.047)$. Conclusion:If the preliminary results of this study are confirmed and the VIVIFRIAL exercise program is more effective than conventional care in improving cognitive function and mood, this project can help to change the current clinical care paradigm that is provided to frail patients with cognitive decline towards a more comprehensive management. Likewise, it is also foreseeable that it can help to generalize the prescription of physical exercise to this population group that is usually excluded from most research studies.

\section{Abstract \# 8}

Monetary value of informal caregiving in dementia from a societal perspective

Kaisu Pitkala ${ }^{1}$, Marja-Liisa Laakkonen ${ }^{2}$, Minna Raivio ${ }^{1}$, Eeva-Liisa Kallio $^{3}$, Hannu Kautiainen ${ }^{1}$, Reijo Tilvis ${ }^{4}$, Timo Strandberg ${ }^{4}$, Hanna Öhman $^{4}$

${ }^{1}$ University of Helsinki, ${ }^{2}$ Department of Social Services and Health Care, Helsinki Hospital, Geriatric Clinic, ${ }^{3}$ Clinical Neurosciences, Neuropsychology, University of Helsinki and Helsinki University Hospital, ${ }^{4}$ University of Helsinki, Clinicum, Department of Geriatrics

Background and aim: Dementia is one of the most expensive geriatric syndromes, with informal care being the most costly portion of the total costs. In previous studies, informal caregiving has been challenging to assess due to difficulties in estimating true time spent on caregiving work and how to value caregivers' time. The aim of this study was to compare the costs of dementia among patients living alone and among those living with a caregiver to show the monetary value of informal caregiving from a societal perspective.

Methods: Data from our four dementia trials using the same measures were combined, allowing 604 participants to be included. Participants were followed up for 2 years or until death for their use of health and social services. Use of all services was retrieved from medical/social records. We included also the costs of lost production of those caregivers who were not retired.

Results: The total mean cost of services and lost production was $22,068 € /$ person-year (pyrs). Participants living alone had a mean cost of $45,156 € /$ pyrs, whereas those living with a spouse had a mean cost of $16,416 € /$ pyrs (mean cost ratio $2.99,95 \%$ CI 2.64-3.39). Participants living alone and having $<15$ MMSE points had higher costs than the corresponding dementia patients in institutional care.Key conclusions: Detailed data of service use and characteristics of patients with dementia showed that from a societal perspective living alone is a very strong determinant of service use in dementia. Informal caregivers do invaluable work for society.

\section{Abstract \# 9 \\ Beat-to-beat blood pressure variability and cognitive function among participants of the Malaysian Elders Longitudinal Research (MELoR) Study}

Nur Fazidah Asmuje ${ }^{1}$, Sumaiyah $\mathrm{Mat}^{2}, \mathrm{Ai}-\mathrm{Vyrn} \mathrm{Chin}^{2}$, Shahrul B. Kamaruzzaman $^{2}$, Maw Pin Tan ${ }^{2}$

${ }^{1}$ Kolej Genius Insan, Universiti Sains Islam Malaysia; Department of Medicine, Faculty of Medicine, University of Malaya, Kuala Lumpur, Department of Medicine, Faculty of Medicine, University of Malaya, Kuala Lumpur

Introduction: Hypertension is considered an established risk factor for dementia is currently diagnosed using one-off measurements. Blood pressure, however, varies with each heartbeat. Blood pressure variability (BPV) has generated interest in the relation to stroke and cardiovascular events, but few studies have addressed BPV and cognitive performance. We, therefore, evaluated the relationship between BPV and cognitive performance in an urban multi-ethnic population.

Methods: Participants to the Malaysian Elders Longitudinal Research (MELoR) study comprised individuals aged $\geq 55$ years selected through simple random sampling from three local parliamentary constituencies stratified by ethnicity. Cognition was evaluated using the Montreal Cognitive Assessment (MoCA) test. Systolic (SBP) and Diastolic (DBP) beat-to-beat BPV was generated through time domain index; average real variability (ARV) and frequency domain 
indices; low frequency (LF); high frequency and ratio LF/HF for supine and standing positions were determined using the Taskforce monitor (CNSytems, Austria).

Results: Data on MoCA scores and BPV were available for 1246 participants. A significant difference between BPV was identified according to tertile MoCA scores for SBP-ARV, HF, ratio LF/HF and DBP LF/HF for the supine position $(\mathrm{p}<0.001)$ while standing $(\mathrm{p}<$ $0.005)$. Increased SBP-ARV index was associated with an increase of having the risk mild cognitive impairment (MCI) (odds ratio (OR) (95\%confidence interval, CI $)=1.475(1.166-1.855)$ and dementia $(\mathrm{OR}(95 \% \mathrm{CI})=2.073(1.633-2.631)$ compared to normal cognition. Increased the low frequency: high frequency ratio, using frequency domain was BPV analysis, associated lower risk of MCI (OR $(95 \%$ $\mathrm{CI})=0.935(0.900-0.972)$ or dementia $(\mathrm{OR}(95 \% \mathrm{CI})=0.814$ (0.769-0.863).

Conclusion: Increased time-domain BPV and increased sympathovagal balance with frequency-domain BPV were associated with increased risk of MCI and dementia. Further analysis will seek to determine potentially modifiable factors mediating these differences. Keywords: Blood pressure variability; cognition; Montreal Cognitive Assessment (MoCA); Malaysian; hypertension

\section{Abstract \# 10 \\ Diagnostic test accuracy of the 4AT for delirium detection: systematic review and meta-analysis}

Zoë Tieges ${ }^{1}$, Alasdair MacLullich ${ }^{1}$, Atul Anand ${ }^{2}$, Claire Brookes ${ }^{3}$, Maria Cassarino ${ }^{3}$, Margaret O'Connor ${ }^{4}$, Damien Ryan ${ }^{5}$, Thomas Saller $^{6}$, Rakesh Arora ${ }^{7}$, Yue Chang ${ }^{8}$, Kathryn Agarwal ${ }^{9}$, George Taffet $^{9}$, Terence Quinn ${ }^{10}$, Susan Shenkin ${ }^{1}$, Rose Galvin ${ }^{3}$

${ }^{1}$ Geriatric Medicine, Edinburgh Delirium Research Group, Usher Institute, University of Edinburgh, cotland, UK, ${ }^{2}$ Centre for Cardiovascular Science, University of Edinburgh, Scotland, UK, ${ }^{3}$ School of Allied Health, Faculty of Education and Health Sciences, Health Research Institute, University of Limerick, Ireland,

${ }^{4}$ Department of Ageing and Therapeutics, University Hospital Limerick, Dooradoyle, Limerick, Ireland, ${ }^{5}$ Retrieval, Emergency and Disaster Medicine Research and Development Unit (REDSPoT), Emergency Department, University Hospital Limerick, Dooradoyle, Limerick, Ireland, and Graduate Entry Medical School, University of Limerick, Ireland., ${ }^{6}$ Department of Anaesthesiology, University Hospital, LMU Munich, Munich, Germany, ${ }^{7}$ Department of Surgery, Section of Cardiac Surgery, Max Rady College of Medicine, University of Manitoba, Winnipeg, Manitoba, Canada; Cardiac Sciences Program, St. Boniface Hospital, Winnipeg, Manitoba, Canada, ${ }^{8}$ Department of Surgery, Section of Cardiac Surgery, Max Rady College of Medicine, University of Manitoba, Winnipeg, Manitoba, Canada, ${ }^{9}$ Section of Geriatrics, Baylor College of Medicine, Houston, Texas, USA; Houston Methodist Hospital, Houston, Texas, USA, ${ }^{10}$ Institute of Cardiovascular Medicine, University of Glasgow, Glasgow, Scotland, UK

Introduction: The 4 'A's Test (4AT; www.the4AT.com) is a short (< $2 \mathrm{~min}$ ) instrument for delirium detection that was purposely designed for use in clinical practice. We performed a systematic review and meta-analysis of diagnostic test accuracy of the 4AT for detecting delirium.

Methods: We searched the following electronic databases through Ovid: MEDLINE, Embase, and PsycINFO. Additional databases were searched: CINAHL (EBSCOhost), clinicaltrials.gov and Cochrane Central Register of Controlled Trials from 2011 (4AT publication) until 21 December 2019. Inclusion criteria: older adults ( $\geq 65)$ across any setting of care except critical care; validation study of the 4AT against a delirium reference standard (standard diagnostic criteria or validated tool). Two reviewers independently screened Abstracts and papers and performed the data extraction. Pooled estimates of sensitivity and specificity were generated from a bivariate random effects model.

Results: 17 studies $(\mathrm{n}=3721$ observations; total $\mathrm{n}$ with delirium $=$ 945) were included. Various settings including acute medicine, surgery and the emergency department were represented. The pooled sensitivity was 0.88 (95\% CI $0.80-0.93)$ and the pooled specificity was 0.88 (95\% CI $0.82-0.92)$. The methodological quality of studies was mostly good.

Key conclusions: The 4AT is now supported by a substantial evidence base comparable to other well-studied tools such as the Confusion Assessment Method (CAM). The strong pooled sensitivity and specificity findings for the 4AT in this meta-analysis along with its brevity and lack of need for specific training provide support for its use as an assessment tool for delirium.

\section{Abstract \# 11 \\ Palliative Care Training In Long-Term Settings To Improve residents' QOL and quality-of-dying}

Kaisu Pitkälä ${ }^{1}$, Pauli Lamppu ${ }^{1}$, Jouko Laurila ${ }^{2}$, Harriet Finne-Soveri ${ }^{3}$, Marja-Liisa Laakkonen ${ }^{4}$

${ }^{1}$ University of Helsinki, ${ }^{2}$ University of Oulu, ${ }^{3}$ National Institute of Health and Welfare, ${ }^{4}$ Department of Social Services and Health Care, Helsinki Hospital, Geriatric Clinic

Introduction: The aim of this trial was to examine the effects of palliative care staff training on nursing home $(\mathrm{NH})$ residents' healthrelated quality of life (HRQOL) and use of hospitals.

Methods: Single-blind, cluster-randomized, controlled trial (RCT) in 20 nursing home wards with 324 residents (with less than 12.-mo prognosis). The staff in the intervention wards received four smallgroup 4-h educational sessions on the principles of palliative care (advance care planning, the adverse effects of hospitalizations, symptom management, communication, giving support to proxies, challenging situations). The sessions were based on constructive learning theory, patient cases, roleplays and small-group discussions. Primary outcome measures were HRQOL by $15 \mathrm{D}$ assessed at baseline, 6 and 12 months and hospital days/person/years during 2-year follow-up. Secondary outcomes were symptoms by Edmonton symptom assessment system (ESAS) and hospitalizations.

Results: 15D declined in both groups and there was no difference in the changes between the groups ( $\mathrm{p}$ for time $<0.001$, group 0.75 , interaction 0.26). There was no difference in the use of hospital days between the groups (IRR 2.12 (95\% CI: 0.62-7.26, adjusted for age, sex, do-not-resuscitate orders and clustering). Total use of health care services was higher in the intervention thn in the control arms. There was no difference in the changes of ESAS or admissions to emergency department between the groups.

Conclusions: Our rigorous RCT on palliative care intervention in NHs showed no effects on HRQOL or use of hospitals. The reasons behind this and similar trials should be explored in future studies.

\section{Abstract \# 12 \\ Clinical clustering of eight orthostatic haemodynamic patterns in The Irish Longitudinal Study on Ageing (TILDA)}

David Moloney ${ }^{1}$, John O'Connor ${ }^{2}$, Louise Newman ${ }^{2}$, Siobhan Scarlett ${ }^{2}$, Belinda Hernandez ${ }^{2}$, Rose Anne Kenny ${ }^{1}$, Roman RomeroOrtuno $^{1}$ 
${ }^{1}$ Falls and Syncope Unit, Mercer's Institute for Successful Ageing, St James's Hospital, Dublin, Ireland, ${ }^{2}$ The Irish Longitudinal Study on Ageing (TILDA), Trinity College Dublin, Ireland

Background: Identifying orthostatic hypotension $(\mathrm{OH})$ has become complex due to implementation of non-invasive continuous beat-tobeat haemodynamic monitoring during active stand (AS) testing; this has resulted in large volumes of data outside the scope of the traditional $\mathrm{OH}$ definition. We explored clinical associations of different AS patterns in participants from Wave 1 of The Irish Longitudinal Study on Ageing (TILDA).

Methods: AS patterns were generated based on three sequential binary systolic blood pressure features: drop $\geq 40 \mathrm{mmHg}$ within $10 \mathrm{~s}$ post-stand ("immediate deficit"), failure to return to within $20 \mathrm{mmHg}$ of supine level at 40 after standing ("stabilisation deficit"), and drop $\geq 20 \mathrm{mmHg}$ at any time between $>40$ and 120 s post-stand ("late deficit"). Eight AS groups resulted from combining the presence/ absence of the three sequential features. The groups were cross-sectionally characterised, and their ability to independently predict orthostatic intolerance (OI) during AS, and falls or syncope in the past year, was evaluated using multivariate logistic regression models.

Results: 4899 participants were included (mean age 61), of which $3312(68 \%)$ had no deficits. There were significant differences in age, sex, co-morbidities and medication usage across groups. Regression models identified independent associations between OI and three immediate-deficit groups; those associations seemed stronger as more deficits were present. There was a significant association between falls history and the three-deficit group (OR 1.54, 95\% CI: 1.15-2.07, $\mathrm{p}=0.004)$.

Conclusions: More deficits seemed associated with higher risk of OI and history of falls. Understanding the underlying pathophysiology of these groups may help clinicians identify risk and personalise therapies.

\section{Abstract \# 13 \\ Trends in hip and distal femoral fracture rates in Italy from 2007 to 2017}

Brigid Unim ${ }^{1}$, Giada Minelli ${ }^{2}$, Roberto Da Cas ${ }^{3}$, Valerio Manno ${ }^{2}$, Francesco Trotta ${ }^{4}$, Luigi Palmieri ${ }^{1}$, Lucia Galluzzo ${ }^{1}$, Stefania Maggi ${ }^{5}$, Graziano Onder ${ }^{1}$

${ }^{1}$ Department of Cardiovascular, Endocrine-metabolic Diseases and Aging, Istituto Superiore di Sanità, Rome, Italy, ${ }^{2}$ Service of Statistics, Istituto Superiore di Sanità, Rome, Italy, ${ }^{3}$ National Centre for Drug Research and Evaluation, Istituto Superiore di Sanità, Rome, Italy, ${ }^{4}$ Italian Medicines Agency, Rome, Italy, ${ }^{5}$ Neuroscience Institute, Aging Branch, CNR, Padua, Italy

Introduction: Osteoporosis and associated fractures are a public health concern worldwide due to high societal and economic burden. The study assesses trends in incidence rates of hip and distal femoral fractures and in the use of anti-osteoporosis drugs in Italy between 2007 and 2017.

Methods: Patients with hip and distal femoral fractures (ICD-9-CM codes 820.x and 821.x) were identified in the Italian National Hospital Discharge Database. Anti-osteoporosis medication data were retrieved from the National Observatory on the Use of Medicines Database.

Results: Hospitalizations for femoral fractures were 991.059, of which $91.4 \%$ were hip fractures and $76.5 \%$ occurred in women. Agestandardized hip fractures rate per 100.000 person-years decreased both in women $(-8.7 \%$; 789.9 in 2007 to 721.5 in 2017) and in men $(-4.3 \%$; 423.9-405.6), while the rate of distal femoral fractures increased by $23.9 \%$ in women $(67.78-83.95)$ and $22.7 \%$ in men (27.76-34.06). These changes were associated with an increment in the use of anti-osteoporosis drugs from 2007 to 2011 (9.1-12.4 DDD/ 1000 inhabitants/day), followed by a plateau in the period 2012-2017. The use of bisphosphonates increased progressively from 2007 to 2010 (8.2-10.5 DDD/1000 inhabitants/day), followed by a plateau and then decreased from 2015 onwards.

Key conclusions: In the period 2007-2017, age-standardized hip fracture rates declined in Italy, while the rate of distal femoral fractures progressively increased. The use of anti-osteoporosis medications has varied in this period. Further research is needed to identify and implement interventions to prevent hip and distal femoral fractures.

\section{Abstract \# 14 \\ Several frailty parameters are highly prevalent in middle age (50- 65 Years) and are independent predictors of adverse events: the SUCCEED Cohort}

Segaux Lauriane ${ }^{1}$, Broussier Amaury ${ }^{1}$, Oubaya Nadia ${ }^{1}$, LeissingDesprez Claire ${ }^{1}$, Laurent Marie ${ }^{1}$, Naga Henri ${ }^{2}$, Fromentin Isabell ${ }^{2}$, David Jean-Philippe ${ }^{1}$, Bastuji-Garin Sylvie ${ }^{1}$

${ }^{1}$ Univ Paris Est Creteil, INSERM, IMRB, CEpiA Team (Clinical Epidemiology and Ageing), F-94010 Creteil, France, ${ }^{2}$ AP-HP, Hôpitaux Henri-Mondor, Departments of Geriatric Medicine, F-94010 Creteil and F-94450 Limeil-Brévannes, France

Introduction: Although frailty can arise in middle age (i.e. before 65), very few studies have investigated frailty in this age group. Our objectives were to assess the prevalence of frailty parameters in middle-aged individuals (aged 50-65) and probe the association with future adverse events.

Methods: We performed cross-sectional and longitudinal analyses of asymptomatic, community-dwelling individuals aged 50-65 $(\mathrm{n}=411$, median age: 59.0) having undergone a multidomain geriatric assessment (2010-2015) in an outpatient clinic in the greater Paris area of France (SUCCEED cohort). The primary outcome was a composite measure of adverse health events (non-accidental falls, fractures, unplanned hospitalizations, and death), recorded in 2016/2017. Multivariable logistic regression models were built to identify independent predictors, using multiple imputation for missing data. A sensitivity analysis in complete cases was performed.

Results: Nine frailty parameters were highly prevalent ( $>20 \%)$ : low physical activity (40.1\%), osteopenia (37.1\%), exhaustion (31.3\%), chronic pain $(29.6 \%)$, living alone $(28.5 \%)$, balance impairment (26.8\%), weakness $(26.7 \%)$, executive dysfunction $(23.2 \%)$, and urinary incontinence $(23.1 \%)$. Female sex (odds ratio: 2.67 [95\% confidence interval: 1.17-6.11], $\mathrm{p}=0.02)$, living alone $(2.39$ [1.32-4.33], $\mathrm{p}=0.004)$, balance impairment (2.09 [1.16-3.78], $\mathrm{p}=$ $0.02)$, executive dysfunction (2.61, [1.18-5.77], $\mathrm{p}=0.02)$, and exhaustion (2.98 [1.65-5.39], $\mathrm{p}<0.001)$ were independent predictors of adverse events in this middle-aged population. The sensitivity analysis in complete cases found similar results.

Conclusions: Many frailty parameters are already altered in middleaged individuals (50-65 years) and are predictive of adverse health events. Our findings highlight a need for frailty screening and preventive programs that target middle-aged individuals.

\section{Abstract \# 15 \\ Identification of five frailty profiles in community-dwelling individuals aged 50 to 75: a latent class analysis of the SUCCEED Survey Data}

Segaux Lauriane ${ }^{1}$, Oubaya Nadia ${ }^{1}$, Broussier Amaury ${ }^{1}$, Baude Marjolaine $^{2}$, Canoui-Poitrine Florence ${ }^{1}$, Naga Henri ${ }^{3}$, Laurent 
Marie $^{1}$, Leissing-desprez Claire ${ }^{1}$, Audureau Etienne ${ }^{1}$, Ferrat Emilie ${ }^{1}$, Fromentin Isabelle $^{3}$, David Jean-Philippe ${ }^{1}$, Bastuji-Garin Sylvie ${ }^{1}$

${ }^{1}$ Univ Paris Est Creteil, INSERM, IMRB, CEpiA Team (Clinical Epidemiology and Ageing), F-94010 Creteil, France, ${ }^{2}$ AP-HP,

Hôpital Henri-Mondor, Department of Public Health, Créteil, France, ${ }^{3}$ AP-HP, Hôpitaux Henri-Mondor, Departments of Geriatric Medicine, F-94010 Creteil and F-94450 Limeil-Brévannes, France

Introduction: Identification of frailty is recommended for people aged $\geq 75$. However, studies have shown that frailty parameters were already present in young seniors. We sought to identify frailty profiles in individuals aged $50-75$ by considering frailty as an unobservable latent variable in a latent class analysis (LCA).

Methods: Cohort of 589community-dwelling individuals aged 50-75 (median: 61.7 years) who had undergone a standardized, multidomain assessment in 2010-2015. Adverse outcomes (non-accidental falls, fractures, unplanned hospitalizations, death) that have occurred since the assessment were recorded in 2016-2017.The LCA used nine indicators (unintentional weight loss, relative slowness, weakness, impaired balance, osteoporosis, impaired cognitive functions, executive dysfunction, depression, and hearing impairment) and three covariates (age, gender, and consultation for health complaints). The resulting profiles were characterized by the Fried phenotype and adverse outcomes.

Results: We identified five profiles: "fit" (LC1, 29.7\% of the participants; median age: 59 years); "weight loss, relative slowness, and osteoporosis" (LC2, 33.2\%; 63 years); "weakness and osteopenia" (LC3, 21.9\%; 60 years); "impaired physical and executive functions" (LC4, 11\%; 67 years); and "impaired balance, cognitive functions, and depression" (LC5, 4.3\%; 70 years). Almost all members of LC3 and LC4 were female, were more likely to have a frail or pre-frail Fried phenotype. Non-accidental falls were more frequent in LC4. LC5 (almost all males) had the highest number of comorbidities and cardiovascular risk factors but none was frail.

Conclusions: Our data-driven approach identified five frailty profiles. With a view to tailoring interventions and prevention, frailty needs to be detected among young seniors.

\section{Abstract \# 16 \\ Frailty Trait Scale 5 (FTS5) longitudinal trajectories and mortality risk in a Spanish Cohort}

Alejandro Álvarez-Bustos ${ }^{1}$, Jose Antonio Carnicero ${ }^{2}$, Francisco José García-García $^{3}$, Cristina Alonso-Bouzón ${ }^{4}$, Leocadio RodriguezMañas ${ }^{4}$

${ }^{1} 1$. Fundación de Investigación Biomédica del Hospital Universitario de Getafe 2. Universidad Autónoma de Madrid, ${ }^{2} 1$. Fundación de Investigación Biomédica del Hospital Universitario de Getafe3. CIBER de Fragilidad y Envejecimiento Saludable (CIBERFES), ${ }^{3} 3$. CIBER de Fragilidad y Envejecimiento Saludable (CIBERFES) 4. Hospital Virgen del Valle, Toledo, España., ${ }^{4} 3$. CIBER de Fragilidad y Envejecimiento Saludable (CIBERFES) 5. Hospital Universitario de Getafe, Madrid, España

Introduction: Frailty have been associated with adverse outcomes. Frailty status could have different changes along the time raising trajectories [1,2]. However, few observational studies have examined how these different trajectories could have different effects on mortality risk and none of them have use the tool FTS5 [3]. Data driven methods allow us to group subjects according to similar FTS5 trajectories along baseline and follow-up score using the Toledo Study of Healthy Aging (TSHA) sample, a longitudinal cohort study. Our hypothesis is that decreasing frailty score could show lower death risk than those subjects that increase or maintain higher FTS5 scores.
Methods: Participants data were taken from the TSHA. Frailty was determined using FTS5, being 0 the lower (the most frail) and 50 the highest (the most robust) score. FTS5 score at baseline and follow-up at 5.04 median years later were used to construct frailty trajectories according growth mixture modelling. Deaths were ascertained through the Spanish National Death Index. Nested proportional hazard regression model was adjusted by age, sex, MiniMental State Examination, Charlson Comorbidity Index, Polypharmacy, Katz Index and Educational Level.

Results: 975 older adults (mean age $73.14 \pm 4.69 ; 43.38 \%$ men) were included. 5 FTS5 trajectories (FTS5T) were identified: Maintaining Non Frailty (MNF); Improving to Non Frailty (INF); Maintaining Frailty (MF); Developing Frailty (DF) and Augmenting Frailty (AF). Comparing with MNF (index group), except INF (HR $=1.11 ; 95 \% \mathrm{CI}$ 0.71-1.73), all trajectories had augmented their risk of mortality $($ FTS5T $=$ HR $(95 \%$ CI $):$ MF $=2.01(1.21-3.32) ;$ DF $=1.92$ $(1.18-3.12) ; \mathrm{AF}=2.67(1.48-4.81))$.

Key conclusions: Those who remain or become frail have a higher mortality risk, while those recovering from frailty to robustness have the same mortality as controls (robust at baseline). Efforts to improve frailty should be promoted to decreased the risk of death.

Keywords: Frailty, mortality.

References:

[1] Lang PO, Michel JP, Zekry D. Frailty syndrome: A transitional state in a dynamic process. Gerontology 2009; 55:539-49. https://doi.org/10.1159/000211949

[2] Kojima G, Taniguchi Y, Iliffe S, Jivraj S, Walters K. Transitions between frailty states among community-dwelling older people: A systematic review and meta-analysis. Ageing Res Rev 2019; 50:81-8. https://doi.org/10.1016/j. Arr.2019.01.010

[3] García-García FJ, Carnicero JA, Losa-Reyna J, Alfaro-Acha A, Castillo-Gallego C, Rosado-Artalejo C, et al. Frailty Trait Scale-Short Form: A Frailty Instrument for Clinical Practice. J Am Med Dir Assoc 2020. https://doi.org/10.1016/j.jamda.2019.12.008.

\section{Abstract \# 17}

Usefulness of the Multidimensional Prognostic Index to measure frailty progression. A 5-year longitudinal cohort study in community-dwelling older people.

Alberto Cella ${ }^{1}$, Nicola Veronese ${ }^{2}$, Giacomo Siri ${ }^{3}$, Katerin Leslie Quispe Guerrero ${ }^{4}$, Monica Pomata ${ }^{1}$, Barbara Senesi ${ }^{4}$, Ekaterini Zigoura $^{4}$, Federica Gandolfo ${ }^{4}$, Ilaria Indiano ${ }^{4}$, Rosetta Femia ${ }^{4}$, Francesca Tricerri ${ }^{4}$, Sara Garaboldi ${ }^{4}$, Romina Custureri ${ }^{4}$, Alberto Pilotto ${ }^{5}$

${ }^{1}$ PostAcute Care Unit, Department of Geriatric Care, Orthogeriatrics and Rehabilitation, E.O. Ospedali Galliera, Genova, Italy, ${ }^{2}$ Azienda ULSS 3 Serenissima, Primary Care Department, District 3, Venice, Italy, ${ }^{3}$ Scientific Directorate-Biostatistics, E.O. Ospedali Galliera, Genova, Italy, ${ }^{4}$ Geriatrics Unit, Department of Geriatric Care, Orthogeriatrics and Rehabilitation, E.O. Ospedali Galliera, Genova, Italy, ${ }^{5}$ Department of Interdisciplinary Medicine, University of Bari Aldo Moro, Bari, Italy \& Geriatrics Unit, Department of Geriatric Care, Orthogeriatrics and Rehabilitation, E.O. Ospedali Galliera, Genova, Italy

Introduction: Frailty progression is associated with lower quality of life, increased health care costs, and possibly mortality in older people. Aim of this study was to evaluate the usefulness of measuring the progression of frailty, as assessed by the time-changes in Multidimensional Prognostic Index (MPI), to predict mortality in community-dwelling older people over a 5-year follow-up period. Methods: This observational, longitudinal, cohort study included 407 community-dwelling older subjects. At baseline (V1), all subjects 
underwent a comprehensive geriatric assessment (CGA) to calculate MPI; moreover, an assessment of physical functions were carried-out by means of the Short Physical Performance Battery (SPPB), Timed Up\&Go test (TUGT), gait speed (GS) and handgrip strength test (HGS). A follow-up visit was scheduled 30 months later (V2) to repeat the same clinical and functional assessments. Mortality was then recorded over a 5-year period.

Results: The mean age of participants at V1 was $77.5 \pm 4.5$ years (females $=51.6 \%) .19$ subjects $(4.7 \%)$ died before V2; among the survivors, $261(67 \%)$ agreed to undergo the V2 evaluation. Basal MPI mean value was $0.23 \pm 0.13$. Compared to V1 values, the mean timechange of the MPI at V2 was $+0.06 \pm 0.08$. The overall mortality during the 5-year follow-up was $13 \%$ (53 subjects). In a multiple Cox model including the time-changes between V1 and V2, only MPI resulted significantly associated with death (HR 1.04, $\mathrm{p}<0.001)$, with an increase of $4 \%$ in 5-year mortality for each 0.1 -increase in MPI values. No significant associations were observed between 5-year mortality and physical function parameters, i.e. SPPB, TUGT, GS, HGS.

Conclusions: Frailty progression, as assessed by changes in the MPI score over time, is significantly associated with death in communitydwelling older people. The periodic re-assessment over-time of the MPI is useful for monitoring older people at risk of negative clinical outcomes.

\section{Abstract \# 18 \\ Using Point-of-care C-reactive protein to guide Antibiotic prescribing for Respiratory tract infections in Elderly nursing home residents (UPCARE)}

T. M. Boere ${ }^{1}$, L. W. van Buul ${ }^{1}$, R. M. Hopstaken ${ }^{2}$, M. W. van Tulder $^{3}$, T. J. M. Verheij ${ }^{4}$, C. M. P. M. Hertogh ${ }^{1}$

${ }^{1}$ Dept. of General Practice \& Elderly Care Medicine, Amsterdam Public Health research institute, Amsterdam University Medical Center, location VU University Medical Center, Amsterdam, the Netherlands, ${ }^{2}$ Star-shl diagnostic centers, Rotterdam/Etten-Leur, the Netherlands, ${ }^{3}$ Faculty of Behavioural and Movement Sciences, VU University, Amsterdam, ${ }^{4}$ Dept. of General Practice, Julius Centrum, University Medical Center Utrecht, Utrecht, the Netherlands

Background: C-reactive protein point-of-care testing (CRP POCT) might be a promising diagnostic tool to decrease diagnostic uncertainty around lower respiratory tract infections (LRTI) in elderly nursing home residents. Consequently, CRP POCT might reduce unnecessary antibiotic use. The overarching aim of the UPCARE study is to achieve better antibiotic stewardship by introducing a cheap, quick and easy-to-use diagnostic tool for the evaluation of LRTI in nursing homes: CRP POCT.

Methods: The design of the study is a cluster randomized controlled trial conducted in eleven nursing homes in the Netherlands (September 2018 until April 2020), with the nursing home as the unit of randomization. Nursing homes in the intervention group use CRP POCT, and nursing homes in the control group provide care as usual without CRP POCT. Nursing home residents with a new diagnosis 'suspected LRTI' fit the inclusion criterion for study participation. Patients are excluded if they reside at hospice/palliative wards, if they recently had a different type of infection, if they do not wish to be treated with antibiotics, or if they are already taking antibiotics.The main outcome is antibiotic prescribing for suspected LRTl at index consultation (multilevel logistic regression analysis). Secondary outcomes are cost-effectiveness and cost-benefit of the use of CRP POCT, associations between CRP POCT values and antibiotic treatment (logistic regression analysis) respectively LRTI signs/symptoms (prediction model, linear regression analysis).
Results and key conclusions: Results regarding the main outcome and part of secondary outcomes will be available and presented in October 2020.

\section{Abstract \# 19 \\ Survival after discharge from geriatrics vs internal medicine wards, by risk status and diagnosis}

Antonella Giordano ${ }^{1}$, Giulia Carrera ${ }^{2}$, Mauro Di Bari ${ }^{1}$, Luca Degli Esposti ${ }^{3}$, Paola Michelozzi ${ }^{4}$, Roberto Bernabei ${ }^{5}$, Niccolò Marchionni $^{6}$, Daniela Balzi ${ }^{7}$

${ }^{1}$ Research Unit of Medicine of Aging, Department of Experimental and Clinical Medicine, University of Florence, Florence, Italy; Unit of Geriatrics, Department of Medicine and Geriatrics, Azienda Ospedaliero-Universitaria Careggi, Florence, Italy, ${ }^{2}$ Research Unit of Medicine of Aging, Department of Experimental and Clinical Medicine, University of Florence, Florence, Italy, ${ }^{3}$ Clicon - Health, Economics \& Outcome Research, Ravenna, Italy, ${ }^{4}$ Department of Epidemiology of the Regional Health Service Lazio, Rome, Italy, 5 Fondazione Policlinico Agostino Gemelli IRCCS, Rome, Italy, ${ }^{6}$ Research Unit of Medicine of Aging, Department of Experimental and Clinical Medicine, University of Florence, Florence, Italy; Cardiothoracic and Vascular Department, Azienda OspedalieroUniversitaria Careggi, Florence, Italy, ${ }^{7}$ Department of Epidemiology, Azienda USL Toscana Centro, Florence, Italy

Introduction: In randomized clinical trials, compared to Internal Medicine, admission to Geriatrics improved clinical outcomes of frail older patients accessing the Emergency Department (ED). Whether this advantage is maintained also in the "real world" is uncertain. Methods: We compared long-term survival of patients admitted to Geriatrics or Internal Medicine wards after stratification for background risk and across a variety of discharge diagnoses. Data were derived from the "Silver Code National Project (SCNP)", an observational study of 180,079 unselected 75 + years old persons, admitted via the ED to Internal Medicine ( $\mathrm{n}=169,717,94.2 \%)$ or Geriatrics ( $\mathrm{n}$ $=10,362$ ) wards in Italy. The Dynamic Silver Code (DSC), based on administrative data, was applied to balance for background risk between participants admitted to Geriatrics or Internal Medicine.

Results: One-year mortality was $33.7 \%$, it was lower in participants discharged from Geriatrics than Internal Medicine (32.1 and 33.8\%, respectively; $p<0.001)$, and increased progressively across four DSC risk classes $(\mathrm{p}<0.001)$. Admission to Geriatrics was associated with survival benefit in DSC class II-IV participants, with HR (95\% CI) of $0.88(0.83-0.94), 0.86(0.80-0.92)$ and $0.92(0.86-0.97)$, respectively. Cerebrovascular diseases, cognitive disorders, and heart failure were the discharge diagnoses with the widest survival benefit from admission to Geriatrics, which was mostly observed in DSC class III. Conclusion: Admission to Geriatrics may provide long-term survival benefit in subjects who, based on the DSC, may be considered at an intermediate risk. Specific clinical conditions should be considered in the ED to improve selection of patients to be targeted for Geriatrics admission.

\section{Abstract \# 20}

Trajectories of physical functioning in late-life vary by the condition leading to death

Erwin Stolz ${ }^{1}$, Thomas Gill ${ }^{2}$, Hannes Mayerl ${ }^{1}$, Éva Rásky ${ }^{1}$, Wolfgang Freidl ${ }^{1}$

${ }^{1}$ Medical University of Graz, AUSTRIA, ${ }^{2}$ Yale University, USA 
Introduction: Previous research suggested that there might be distinct patterns of functional decline in the last years of life depending on the condition leading to death, but the validity of these results and hence the explanatory value of the condition leading to death for latelife disability are uncertain.

Methods: A total of 636 decedents from a cohort of 754 communityliving persons, $70+$ years of age (Yale PEP Study) provided 33,700 monthly observations of self-/proxy-reported disability during the last 5 years of life. Non-linear trajectories and short-term fluctuations of late-life disability by cause of death (cancer, organ failure, phenotypic frailty, severe dementia, sudden death, other) were estimated with flexible mixed spline regression models.

Results: Trajectories of functioning at the end of life varied distinctively by the condition leading to death. Estimated disability trajectories among cancer deaths increased gradually up until about 6 months before death, after which a steep terminal decline set in. Among those who died from organ failure, frailty and dementia disability was higher, increased more gradually, and there was no clearcut terminal phase. Adding the condition leading to death to other known risk factors increased the amount of explained between-person variance in late-life functioning from $\mathrm{R}^{2}=0.35$ to 0.49 . Short-term fluctuations were not specific for decedents with organ failure.

Conclusion: The condition leading to death is an important determinant of trajectories of functioning in late-life. These trajectories follow distinct patterns partially resembling a previously outlined theoretical typology.

\section{Abstract \# 21}

Comprehensive geriatric assessment using multidisciplinary meetings in care homes without nursing

Millicent Metz ${ }^{1}$, Emma Ladi ${ }^{1}$, Sharjeel Hasan ${ }^{2}$, Tania Kalsi ${ }^{3}$

${ }^{1}$ Kings College London, ${ }^{2}$ Quay Health Solutions Care Home Service, ${ }^{3}$ Dept of Ageing \& Health, Guys \& St Thomas' NHS Foundation Trust

Introduction: Comprehensive Geriatric Assessments (CGA) and multi-disciplinary meetings (MDMs) are effective in improving decision-making and outcomes but is less evaluated in the setting of care homes without nursing. This quality improvement project evaluates feasibility and effectiveness of delivering CGA using MDMs in care homes without nursing.

Methods: Retrospective review of clinical documentation using electronic patient records in London. Feasibility evaluated by professionals attendance, number of residents discussed and coverage of CGA domains. Effectiveness evaluated by number of intervention plans. Comparisons were made to CGA completed by a community geriatrician.

Results: 95 residents discussed between Jan and Oct 2019 across 7 MDMs in 2 residential homes. Mean age $83 \pm 8.3$ years (range $62-98$ ), mean 8.0 comorbidities. $100 \%$ of meetings held had a general practitioner, geriatrician and care home team leader present. A pharmacist was also present for $84.2 \%$, additional MDM members for $15.9 \%$. All but one CGA domain were discussed in $95.8-100 \%$ of residents including resuscitation and advance care planning. MDMs were less effective in discussing quality of life (63.2\%). Geriatriciandelivered CGA had previously been performed in $69(72.6 \%)$. The MDMs outperformed the liaison review for consistently reviewing all CGA domains. However, the liaison review out-performed the MDM in the number of intervention plans made $(4.5+/-2.4$ versus $3.3+/-1.7$ intervention plans, $\mathrm{p}=0.002$ ).

Conclusions: MDMs in care homes without nursing are feasible and comprehensive in delivering CGA with a small group of professionals. Geriatrician-liaison CGA appeared less comprehensive but more focused reflecting increased intervention plans. Likely the combination of both MDMs and focused geriatrician-liaison is required.

\section{Abstract \# 22 \\ Developing a data \& innovation platform for care homes in Scotland \\ Lucy Johnston $^{1}$, Jo Hockle ${ }^{2}$, David Henderson ${ }^{1}$ \\ ${ }^{1}$ Edinburgh Napier University, ${ }^{2}$ Department of Primary Palliative Care, Usher Institute, University of Edinburgh}

Introduction: The COVID-19 pandemic has increased the need for reliable, real time data on the care of older people. We aimed to identify current data availability, challenges, and priorities for future research and innovations in Care Homes.

Methods: Scoping fieldwork in six Lothian (Scotland) Care Homes included (a) 'audit' of all data items currently collected through paper or electronic care planning and (b) interviews with care home managers to discuss (i) availability /use of data; (ii) gaps; (iii) digital capabilities; and (iv) priorities for future research.

Results: All homes collected data routinely on dependency, nutrition, weight, falls, pressure sores and infections, and as required on wounds, frailty, bowels or fluid intake. Other data items are also recorded (e.g. pain, sleep, observations) that can identify changes in residents' condition in real time. There was significant variation between homes in recording processes and assessment tools. Data are often used as 'standalone' pieces of information rather than creating a holistic/longitudinal view of each resident. Priorities for future research included standardising documentation, recording non-care tasks, identifying change in residents' condition, and supporting staff. Key conclusions: Care homes can be data heavy, but there is significant heterogeneity in the data, and it is mostly used to direct immediate resident care. There is an opportunity for a National Care Home Data Platform, with strong foundations. This is essential to: provide a robust data source for policy and planning; enable real time digital connectivity between care homes and health \& social care providers; and inform future innovative technologies.

\section{Abstract \# 23}

\section{Nutritional status and loneliness among nursing home residents}

Pau Moreno-Martin ${ }^{1}$, Patricia Lizett Vilca-Salazar ${ }^{1}$, Meritxell BonetCosta $^{1}$, Pau Farrés-Godayol ${ }^{1}$, Míriam Molas-Tuneu ${ }^{1}$, Albert OliverasFabregas $^{1}$, Cristina Vaqué-Crusellas ${ }^{1}$, Javier Jerez-Roig ${ }^{1}$

${ }^{1}$ Research group on Methodology, Methods, Models and Outcomes of Health and Social Sciences (M3O). Faculty of Health Sciences and Welfare. Centre for Health and Social Care Research (CESS). University of Vic-Central University of Catalonia (UVic-UCC).

Introduction: Malnutrition is common in aging, usually associated with multiple factors, including psychological factors like depression. However, there is scarce evidence of its association with loneliness. This study aims to verify the prevalence of loneliness in nursing homes $(\mathrm{NH})$ residents and the risk of malnutrition and their possible correlation.

Methods: Cross-sectional study in $4 \mathrm{NH}$ in the Osona county of Catalonia (Spain). The Mini Nutritional Assessment long form (MNA-LF) was used to evaluate the nutritional risk and the De Jon Gierveld test (DJG) to measure loneliness. Exclusion criteria was; < 65 years, not living in $\mathrm{NH}$, refuse to participate and hospitalization. The Spearman test was used for bivariate analysis between MNA and DJG, quantitative variables are represented by mean \pm standard deviation with a confidence level of $95 \%$. 
Results: 145 individuals were initially recruited, of which 41 (28, $28 \%$ ) were excluded to no fulfil the study criteria. Of the 104 subjects included $(71.72 \%), 46(44.23 \%)$ were excluded for cognitive impairment, $4(3,5 \%)$ incomplete data on MNA-LF. The final sample was $54(37.2 \%), 44(81,5 \%)$ women, $10(18,5 \%)$ men, mean age of $83.8 \pm 7.3$. The prevalence of loneliness was $72.2 \%$ (CI $95 \%$ : $59.1-82.3)$ higher in women $(77.3 \%)$ than in men $(50.0 \%) ; 53,7 \%$ had risk of malnutrition, $42,6 \%$ normal status and 3,7\% malnutrition. A non-significative and negative correlation $(-0.133, \mathrm{p}=0.338)$ between MNA-LF and DJG was found.

Conclusions: The prevalence of loneliness was $72.2 \%$ and $3.7 \%$ and $53.7 \%$ had malnutrition and risk of malnutrition respectively. A nonsignificative and negative correlation was found between loneliness and nutrition status.

\section{Abstract \# 24 \\ Profiling COVID-19 infection in Irish nursing homes}

Aoife Fallon ${ }^{1}$, Adam Dyer ${ }^{2}$, Ruth Martin ${ }^{3}$, Siobhán Kennelly ${ }^{3}$, Alan Martin ${ }^{4}$, Desmond O'Neill ${ }^{1}$, Seán Kennelly ${ }^{1}$

${ }^{1}$ Tallaght University Hospital, ${ }^{2}$ St James Hospital, ${ }^{3}$ Connolly Hospital, Blanchardstown, ${ }^{4}$ Beaumont Hospital

Background: SARS-CoV-2 disproportionately affects nursing home $(\mathrm{NH})$ residents, who account for $24-82 \%$ of COVID-19-related deaths. In Ireland, the first NH case of COVID-19 occurred on 16/03/ 2020. A COVID-19 testing program of residents and staff took place from $18 / 04 / 2020$ to $05 / 05 / 2020$.

Aims: To examine characteristics of NHs across three Community Health Organisations (CHOs) in Ireland, proportion with COVID-19 outbreaks, staff and resident symptom profile and resident mortality rates.

Methods: Forty-five NHs across three CHOs were surveyed. Details were collected on number of beds, occupancy, COVID-19 outbreak, date of first laboratory-confirmed COVID-19 case (resident/staff), total symptomatic/asymptomatic cases, and outcomes for residents from $29 / 02 / 2020$ to $22 / 05 / 2020$.

Results: Surveys were returned from $62.2 \%$ (28/45) of NHs (2043 residents, 2303 beds). Three-quarters (21/28) had COVID-19 outbreaks (1741 residents, 1972 beds). Median time from first case of COVID-19 in Ireland to first case in included NHs was 27.0 days. COVID-19 incidence in residents was 43.9\% (764/1741): laboratoryconfirmed in $40.1 \%$ (710/1741), with $27.2 \%$ (193/710) asymptomatic, and clinically-suspected in $3.1 \%$ (54/1741). Resident case-fatality was $27.6 \%(211 / 764)$ for laboratory-confirmed/clinically-suspected COVID-19. Similar proportions of residents in NHs with "early" outbreaks (<28days) vs "late" outbreaks developed confirmed/suspected COVID-19. A lower proportion of residents in $\mathrm{NHs}$ with "early" outbreaks recovered compared to those with "late" outbreaks $\left(37.4 \%\right.$ vs $\left.61.7 \% ; \chi^{2}=56.9, \mathrm{p}<0.001\right) .675 \mathrm{NH}$ staff across twentyfour sites developed confirmed/suspected COVID-19; 23.6\% (159/ $675)$ were asymptomatic. There was a significant correlation between the proportion of staff with symptomatic COVID-19 and resident numbers with confirmed/suspected COVID-19 (Spearman's rho = $0.81, \mathrm{p}<0.001)$.

Conclusion: This study demonstrates the impact of COVID-19 on $\mathrm{NH}$ residents and staff. High infection rates lead to challenges in care provision.

\section{Abstract \# 25}

Adherence to the Mediterranean diet and frailty in communitydwelling older people

Alberto Cella ${ }^{1}$, Sabrina Zora ${ }^{1}$, Claudio Torrigiani ${ }^{2}$, Elena Zini ${ }^{2}$, Paola Giannoni $^{2}$, Valeria Pandolfini ${ }^{2}$, Ekaterini Zigoura ${ }^{1}$, Barbara Senesi ${ }^{1}$, Stefano Poli $^{2}$, Alberto Pilotto ${ }^{3}$

${ }^{1}$ Geriatrics Unit, Department of Geriatric Care, Orthogeriatrics and Rehabilitation, EO Galliera Hospital, Genova, Italy, ${ }^{2}$ Department of Education, University of Genoa, Genoa, Italy, ${ }^{3}$ Geriatrics Unit, Department of Geriatric Care, Orthogeriatrics and Rehabilitation, EO Galliera Hospital, Genova, Italy; Department of Interdisciplinary Medicine, University of Bari, Bari, Italy

Introduction: Close adherence to the so-called Mediterranean diet is reported to be an important preventive strategy in older people at risk of frailty. Recently, a self-administered version of the Multidimensional Prognostic Index (SELFY-MPI) for stratifying older subjects at risk of frailty was developed and validated in community and primary care settings. The aim of this study was to evaluate the association between the SELFY-MPI score and adherence to the Mediterranean diet in a large population of community-dwelling older people.

Methods: The MEDI-LITE score was used to assess adherence to the Mediterranean diet; the SELFY-MPI Short Form (SELFY-MPI-SF) was used to evaluate frailty by combining information on basic and instrumental activities of daily living, mobility (Barthel mobility), cognition (Test Your Memory-TYM Test), nutrition (Mini Nutritional Assessment-Short Form), comorbidity, medications and co-habitation status.

Results: A total of 1354 subjects (mean age $=77.3 \pm 7.6$ years, range $=56-107$ years; females $=55.8 \%$ ) were enrolled. The mean SELFYMPI-SF score was $0.20 \pm 0.15$ (range 0.0-0.88) and the mean MEDILITE score was $10.4 \pm 2.66$ (range 0.0-17.0). A significant correlation between age and SELFY-MPI-SF (Pearson coefficient $=0.441$, $\mathrm{p}<0.001)$ and a negative correlation between age and MEDI-LITE score (Pearson coefficient $=-0.089, \mathrm{p}=0.001$ ) were observed. Moreover, a significant negative correlation emerged between SELFY-MPI-SF and low MEDI-LITE scores (Pearson coefficient $=$ $0.152, \mathrm{p}<0.001$ ).

Conclusions: Older people at risk of frailty showed poor adherence to the Mediterranean diet. Screening older subjects by means of the SELFY-MPI-SF could be useful in order to identify those at risk of frailty, with a view to implementing strategies to enhance adherence to the Mediterranean diet.

\section{Abstract \# 26}

Prevalence of sarcopenic obesity and type 2 diabetes according to EWGSOP2 and FNIH sarcopenia definitions

Francesca Remelli ${ }^{1}$, Pasquale Abete $^{2}$, Antonio Cherubini ${ }^{3}$, Mauro Dibari $^{4}$, Mario $\mathrm{Bo}^{5}$, Marcello Maggio ${ }^{6}$, Francesco Landi ${ }^{7}$, Stefano Volpato $^{1}$

${ }^{1}$ Università di Ferrara, ${ }^{2}$ UNiversità Federico II Napoli, ${ }^{3}$ INRCA, Ancona, ${ }^{4}$ Univesità di Firenze, ${ }^{5}$ Uiversità di Torino, ${ }^{6}$ Università di Parma, ${ }^{7}$ Università Cattolica del Sacro Cuore, Roma

Background: Current EWGSOP2 and FNIH sarcopenia diagnostic criteria standardized skeletal muscle mass estimates using different methods. As consequence anthropometric characteristics of sarcopenic patients are substantially different according to the diagnostic criteria used. Objectives. To investigate the prevalence of sarcopenic obesity and type 2 diabetes in older persons with sarcopenia defined according to EWGSOP2 and FNIH criteria, evaluating the concordance between the two methods. 
Methods: Data are from the GLISTEN study, an observational multicenter study performed in 620 older patients admitted in 12 Italian hospitals in 2014. Data were collected through Comprehensive Geriatric Assessment; moreover, skeletal muscle mass (Bioelectrical Impedance Analysis) muscle strength (Hand Grip Strength) and physical performance (gait speed) were evaluated.

Results: Among sarcopenic patients, the prevalence of sarcopenic obesity was $30.8 \%$ according to FNIH criteria, while no patients were defined as sarcopenic using EWGSOP2 criteria. According to EWGSOP2 criteria, $23.7 \%$ of sarcopenic and $30.8 \%$ of non-sarcopenic patients had diabetes $(\mathrm{p}=0.11)$; whereas, using FNIH criteria, $36.3 \%$ of sarcopenic and $26.9 \%$ of non-sarcopenic patients were diabetic $(\mathrm{p}=0.04)$. In multivariable logistic model diabetes was significantly associated with the likelihood of sarcopenia according to FNIH (OR 1.67; 95\% CI 1.09-2.56) but not according to EWGSOP2 criteria ((OR 0.82; 95\% CI 0.51-1.30).

Conclusions: The EWGSOP2 and FNIH criteria have significantly different association with prevalence of sarcopenic obesity and type 2 diabetes, with EWGSOP2 criteria having very low sensitivity for intercepting patients with sarcopenic obesity.

\section{Abstract \# 27 \\ The Role of the somatotropic axis in geriatric patients with sarcopenia}

Uta Ferrari ${ }^{1}$, Ralf Schmidmaier ${ }^{1}$, Theresa Jung ${ }^{1}$, Sebastian Martini ${ }^{1}$, Martin Bildlingmaier ${ }^{1}$, Michael Drey ${ }^{1}$

${ }^{1}$ Department of Medicine IV; University Hospital, LMU Munich, Germany

Context: The pathogenesis of sarcopenia is still not fully understood. In particular, disturbances of the somatotropic axis in sarcopenic patients have not yet been adequately investigated. Patients with sarcopenia have lower serum levels of insulin like growth factor (IGF-I) than non-sarcopenic patients. For the biological effectiveness of IGF-I, the binding proteins IGFBP-3 (IGF-binding protein 3) and ALS (acid labile subunit), and for the effectiveness of growth hormone $(\mathrm{GH})$ on the liver, $\mathrm{GH}$ receptors are necessary.

Objective/Aim:Analysis of the association between the GH/IGF-I axis and muscle function to search for pathogenetic deficits in patients with sarcopenia.Methods. Cross-sectional study with assessment of sarcopenia status (EWGSOP2 criteria) determined from hand grip strength measurement and muscle mass (SMI, Skeletal Muscle Index; calculated by ALM/height ${ }^{2}$ ) measured by DXA. Measurement of serum levels of IGF-I, IGFBP-3, ALS, GH, GHBP (GH binding protein); assessment of BMI and routine laboratory parameters. For statistical analysis univariate and multiple regression models were performed.

Results: 130 patients ( 81 women) over 65 years with an average age of $83.3 \pm 7.7$ years and BMI of $25.34 \pm 5.02 \mathrm{~kg} / \mathrm{m}^{2}$ were included in the analysis. $26.7 \%(\mathrm{n}=35)$ of the patients had sarcopenia, $54.2 \%(\mathrm{n}$ $=71)$ and $45 \%(\mathrm{n}=59)$ had pathologically reduced hand grip strength or SMI. Compared to non-sarcopenic patients (IGF-I 83.07 \pm 50.08 $\mathrm{ng} / \mathrm{ml}$; IGFBP-3 $2028.33 \pm 1014.39 \mathrm{ng} / \mathrm{ml}$; ALS $321.37 \pm 219.38$ $\mathrm{mU} / \mathrm{ml}$; GHBP $921.30 \pm 451.11 \mathrm{ml} / \mathrm{min}$ ) patients with sarcopenia showed significantly $(\mathrm{p}<0.008)$ lower IGF-I $(59.31 \pm 29.13 \mathrm{ng} / \mathrm{ml})$, IGFBP-3 $(1531.06 \pm 793.92 \mathrm{ng} / \mathrm{ml})$, ALS $(210.40 \pm 129.98 \mathrm{mU} / \mathrm{ml})$ and GHBP (562.14 $\pm 313.67 \mathrm{pM})$ serum levels. ALS and IGF-I were constant statistically significant associated with sarcopenia status and pathological hand grip strength even after adjustment for age, sex, BMI, and albumin, but not for pathological SMI.

Summary/Conclusions: It was shown that not only IGF-I, but also IGFBP-3 and ALS are significantly decreased in patients with sarcopenia. These associations were not explained by age or BMI. The significantly decreased GHBP as parameter for GH receptor expression gives evidence for GH resistance as a cause of IGF-I/IFGBP-3/ ALS deficit. Further studies are needed to identify the role of ALS in its association to pathological hand grip strength in geriatric patients.

\section{Abstract \# 28}

Association between sarcopenia, epicardial and intra-abdominal fat in elderly: an observational study.

Klara Komici ${ }^{1}$, Maria Letizia Berloni ${ }^{2}$, Ornella Di Iuorio ${ }^{2}$, Maria Chiara Brunes ${ }^{2}$, Giorgio Giuliani ${ }^{2}$, Vito D'Agnano ${ }^{2}$, Fabio $\mathrm{D}^{\prime} \mathrm{Amico}^{2}$, Antonio Bianco ${ }^{2}$, Andrea Caiazzo ${ }^{2}$, Fabio Perrotta ${ }^{1}$, Germano Guerra ${ }^{1}$

${ }^{1}$ Department of Medicine and Health Sciences "Vincenzo Tiberio", University of Molise, 86100, Campobasso, Italy Sport Medicine Unit of "A. Cardarelli" Hospital, ASReM, 86100, Campobasso, Italy, ${ }^{2}$ Department of Medicine and Health Sciences "Vincenzo Tiberio", University of Molise, 86100, Campobasso, Italy

Sarcopenia is an age-related geriatric syndrome characterized by a progressive loss of muscle mass, strength and muscle function. Various pathophysiological mechanisms including chronic inflammation. Adipose tissue distribution and function change dramatically throughout life and is associated with modulation of inflammatory system. In this study, we evaluated the association between sarcopenia, intra-abdominal and epicardial fat in an elderly population. Methods: Elderly ambulatory patients were clinically evaluated. BIA, handgrip test, echocardiography and abdominal echography was performed.

Results: 92 patients were enrolled, and we identified 21 sarcopenic and 71 non-sarcopenic. Population mean age was $71.5 \pm 4.91$ years. We found a significantly higher thickness of intra-abdominal and epicardial fat in sarcopenic subjects compared to non-sarcopenic subjects: $6.22 \pm 1.14 \mathrm{~cm}$, vs $3.96 \pm 1.33 \mathrm{~cm} \mathrm{p}<0.0001,0.84 \pm 0.17$ $\mathrm{cm}$ vs $0.53 \pm 0.15 \mathrm{~cm} \mathrm{p}<0.0001$. The cardiac mass index was lower in sarcopenic patients $82.67 \pm 17.45 \mathrm{~g} / \mathrm{m}^{2}$, compared non-sarcopenic population $104.80 \pm 15.03 \mathrm{~g} / \mathrm{m}^{2}$. The linear regression analysis shows that reduced cardiac mass index, and increased epicardial and intraabdominal fat are associated with a reduction in muscle mass: $\mathrm{R}^{2}=$ $0.07 \mathrm{p}=0.01 ; \mathrm{R}^{2}=0.30 \mathrm{p}<0.001 ; \mathrm{R}^{2}=0.34 \mathrm{p}<0.001$.

Conclusions: In elderly population, sarcopenia is associated with a significant increase of epicardial fat, intra-abdominal fat and a reduction of cardiac mass index. These data can contribute to further studies focused on the effects of sarcopenia on adipose tissue distribution, and the effects of visceral fat on sarcopenia development.

\section{Abstract \# 29}

Prevalence and factors associated with poor performance in the 5chair stand test: findings from the Cognitive Function and Ageing Study II (CFAS II)

Richard M. Dodds ${ }^{1}$, James C. Murray ${ }^{1}$, Antoneta Granic ${ }^{1}$, Germaine Uwimpuhwe $^{2}$, Sarah Richardson ${ }^{1}$, Fiona E. Matthews ${ }^{3}$, Avan A. Sayer $^{1}$, MRC CFAS ${ }^{4}$

${ }^{1}$ AGE Research Group, Translational and Clinical Research Institute, Faculty of Medical Sciences, Newcastle University, Newcastle, UK, ${ }^{2}$ Durham Research Methods Centre, Durham University, ${ }^{3}$ Population Health Sciences Institute, Faculty of Medical Sciences, Newcastle University, Newcastle, UK, ${ }^{4}$ http://www.cfas. Ac.uk/

Introduction: Poor performance in the 5-chair stand test (5-CST) indicates reduced lower limb strength. The 5-CST has been recommended in the initial assessment of sarcopenia. Our aims were to 
describe the prevalence and factors associated with poor performance in the 5-CST, and to examine the relationship between 5-CST and gait speed.

Method: We used data from the Cognitive Function and Ageing Study II. Participants completed a comprehensive questionnaire, 5-CST and gait speed. We used multinomial logistic regression to assess associations between factors including the SARC-F questionnaire and 5-CST performance: fast $(<12 \mathrm{~s})$, intermediate $(12-15 \mathrm{~s})$, slow $(>15 \mathrm{~s})$ or unable, with the latter two classed as poor performance.ResultsA total of 7171 participants aged 65-95 were included ( $54.1 \%$ female). The proportion with poor performance in the 5-CST increased with age, from $34.3 \%$ at age $65-69$ to $89.3 \%$ at age $90-95$. Factors independently associated with poor performance included positive responses to SARC-F questions, physical inactivity, impaired cognition and multimorbidity. In a multivariable model, those with multimorbidity had a $23 \%(95 \%$ CI $8-40 \%)$ higher relative odds of slow 5-CST compared to those without, and a $26 \%$ (95\% CI 6-49\%) higher relative odds of being unable to perform the 5-CST. The majority of those with poor performance had slow gait speed or were unable to complete the gait test.

Key conclusions: Poor performance in the 5-CST is increasingly common with age and is associated with several risk factors. We recommend a low threshold for performing the 5-CST in clinical settings.

\section{Posters Miscellaneous}

\section{Abstract \# 1}

One-year mortality of hospitalized adult patients

J. Bowen ${ }^{1}$, A. Michael ${ }^{1}$, K. Hall ${ }^{1}$

${ }^{1}$ Russells Hall Hospital, Dudley, UK

Introduction: There is increased demand and intense pressure on NHS secondary care beds and the government has invested in primary care and community teams to avoid unnecessary hospital admissions. Subsequently patients who are admitted are markedly unwell with sever acute or acute on chronic illness. The mortality of these patients would be expected to be high. Many clinicians may be unaware of the prognosis and limited life expectancy of some patients, depriving them from planning and organizing end of life care. The aim of this work is to study the 1-year mortality of inpatients in different wards/ specialties and to explore the potential to plan end of life care for patients in their last year of life.

Methods: A prospective observational study; all adult inpatients in a certain day, in a UK teaching hospital, were identified. They were followed up 1 year later to see whether they were still alive.

Results: In total there were 716 inpatients on the specified day. A year later, 224 died $(31 \%)$. The risk is higher in elderly patients $(61 \%)$, oncology patients (55\%), High Dependency Unit patients $(50 \%)$, respiratory patients $(48 \%)$, rehabilitation patients $(43 \%)$, renal patients $(41 \%)$ and hip fracture patients $(41 \%)$.

Conclusions: Three in ten of adult inpatients would be expected to die within the subsequent 12 months of admissions. Early identification and recognition of those who are in the last 12 months of life will be helpful to deliver proactive care, advance care planning, better symptom control and prevention of unnecessary and avoidable admissions.

\section{Abstract \# 2}

Elevated NT-proBNP levels shows low correlation with heart failure presence in elderly hospitalised patients with suspected diagnosis of heart failure

Peter Olexa ${ }^{1}$, Štefan Tóth ${ }^{2}$, Ján Wohlfart ${ }^{2}$, Štefan Koval ${ }^{2}$, Monika Čerkalová $^{3}$

${ }^{1}$ Gerontology and Geriatric Clinic, University hospital of St.Michal, Medical Faculty, Košice, ${ }^{2}$ Gerontology and Geriatric Clinic, University hospital of St.Michal, Košice, Slovakia, ${ }^{3}$ Department of clinical biochemistry, University hospital of St. Michal, Košice, Slovakia

Background: The diagnosis of congestive heart failure (CHF) is often difficult and underestimated in geriatric population, especially when an echocardiographic evaluation is not available or feasible. NTproBNP assay is promoted as a key tool for the diferential diagnosis of CHF. But the diagnostic utility of NT-proBNP for systolic HF identification has been questioned in elderly, as NT-proBNP levels are influenced by age and several age-related comorbidities, such as anaemia, renal dysfunction, malnutrition. This study aimed to evaluate the diagnostic and prognostic value of NT-proBNP levels and its correlation with echocardiography in the group of elderly hospitalized patients initially not admitted with the diagnosis of HF.

Methods: We enrolled 517 consecutive elderly patients (age $>65$ years) admitted to the Geriatric Clinic during the year 2019 (1.1.31.12.2019). Admissions due to worsening of known HF were excluded. Patients with at least one sign or symptom compatible with CHF were tested for NT-proBNP (fatigue, exercise dyspnea at most). NT-proBNP values $<300 \mathrm{pg} / \mathrm{ml}$ were considered as an age-independent exclusion criterion for HF (high negative predictive value), while values above $1500 \mathrm{pg} / \mathrm{ml}$ were considered as a highly suspected diagnostic criterion for CHF. Main comorbidities and laboratory parameters were considered to adjust correlation analyses between NT-proBNP and LV systolic function. LV function was measured echocardiographically as ejection fraction $(\mathrm{EF})$ in all subjects with increased NT-proBNP values and correlation was calculated.Results:Mean age of the cohort was 81 years, mean NT-proBNP $2254.7 \mathrm{pg} / \mathrm{ml}$ and median value of $1659 \mathrm{pg} / \mathrm{ml}$. Circulating levels higher than $1000 \mathrm{pg} / \mathrm{ml}$ were reported in $45 \%$ of studied patients, $36 \%$ showed values $>1500 \mathrm{pg} / \mathrm{ml}$. Only $25 \%$ of all seniors admitted to hospital with non-cardiac symptoms presented NT-proBNP levels $<$ $300 \mathrm{pg} / \mathrm{ml}$. Only $11 \%$ of patients with elevated values higher than $1500 \mathrm{pg} / \mathrm{ml}$ proved echocardiographically low EF $<40 \%$. When performed univariate analysis, NT-proBNP values showed no significant correlation with EF, haemoglobin concentration, CRP, uricaemia and surprisingly age. Weak correlation was identified between NT-proBNP levels and value of eGFF and creatinaemia. No correlation with the LV ejection fraction was observed, even after considering of other parameters in multivariable regression analyses. ConclusionHighly elevated NT-proBNP values were present in most of the admitted non-cardiac studied seniors. Diseases different from CHF have appeared to affect NT-proBNP plasma levels significantly in the elderly population of patients and can falsely mislead to CHF diagnosis when relying the diagnostic process only on the biomarker assay. In contrast to younger

\author{
Abstract \# 3 \\ Age is an important clinical predictor of unsuccessful electric \\ cardioversion for atrial fibrillation -5 years experience \\ Peter Olexa ${ }^{1}$ \\ ${ }^{1}$ Gerontology and Geriatric Clinic, Univ. Hospital of St. Michal, \\ Košice, Slovakia
}


Background: Electric cardioversion is highly efficient therapy for patients with persistent atrial fibrillation and atrial flutter (AF). Despite its efficacy, there is still a group of patients, where even repeated application of electric energy does not lead to conversion to sinus rhythm (SR).

Aim: We aimed investigate retrospectively the incidence and predictive value of several clinical and echocardiographic parameters for an unsuccessful electric conversion to SR and short-term AF recurrence in patients who have experienced the electric cardioversion (ECV) for an episode of persistent AF.

Methods: We have examined data from 1380 patients who underwent ECV between Jun 2015 and Feb 2020 in our institution. Only those patients with failure to restore SR after an elective ECV or who experienced short -term recurrence of AF within $6 \mathrm{~h}$ after ECV were analyzed further. We assessed the predictive power of several potentially risk factors: senior age above $60 \mathrm{y}$., sex, obesity (BMI > $30 \mathrm{~kg} / \mathrm{m}^{2}$ ), presence of diabetes mellitus, clinically significant mitral regurgitation or form of coronary heart disease in the history of patient, echocardikographic parameters (LV ejection fraction, dilated left atrium (LA $>4,2 \mathrm{~cm})$, value of energy of the biphasic shock used for ECV, repeated ECV performed in past, type of antiarrhythmic medication and statins or ACE- inhibitors in the medication of the individual.

Results: The group of patients with unsuccessful ECV consisted of 53 individuals, $80 \%$ men. Mean age of the study group was 66 (median 66) years. Presence of recorded risk factors was reported in percentage. We could identify following markers of ECV failure: masculine gender ( $80 \%$ incidence), age $>60 \mathrm{y}$. (70\%), severe obesity $(68 \%)$, dilated LA $(82 \%)$. No pre-treatment with type I or III antiarrhythmics prior to ECV was indentified as powerfull risk factor for ECV failure (67\%) too. On the other side, presence of diabetes mellitus or CAD (only 12\%/15\% incidence), no ACEi (30\%), mitral regurgitation of II.grade and higher (58\%), low $\mathrm{EF}<40 \%(10 \%)$, "low" energy shock used $<150 \mathrm{~J}(10 \%)$, even the history of repeated ECV previously (40\%) were not identified as a significant risk factors for ECV failure in our group of patients. Amiodarone (60\%), but not propaphenone $(40 \%)$ when addeded to the therapy before repeated have increased the efficacy of the repeated ECV with the success rate $76 \%$ vs. $25 \%$. ConclussionThe combination of senior age, obesity, male gender, LA dilatation and no antiarrhythmic treatment (group I and III) could identify subjects with low success rate of ECV. Weight loss and adding amiodarone to medication prior ECV can significantly increrase the success rate of elective ECV of these high risk patients. Further data on larger populations of AF patients could prove pre presented concept.

\section{Abstract \# 4 \\ Seamless transition from hospital to home (H2H) in Singapore: a quality improvement activity (QIA) \\ Jing Wei Lim ${ }^{1}$, Ron $\mathrm{Ng}^{1}$, Shafia S. By Razali ${ }^{1}$, S. Kenneth Villain ${ }^{1}$, Chris Lien ${ }^{1}$, Surendra D. Varman ${ }^{1}$ \\ ${ }^{1}$ Changi General Hospital}

Background: $\mathrm{H} 2 \mathrm{H}$ aims to ensure continuity of care in patients discharged from hospital into the community. $\mathrm{H} 2 \mathrm{H}$ also requires elaborate care planning, knowledge of services, robust communication and handover processes. Geriatric Medicine (GRM) being main $\mathrm{H} 2 \mathrm{H}$ user, QIA was performed with primary aim to reduce re-admissions and with secondary aims to improve flow, enrollment along with user satisfaction.

Method: Current and future value states identified; Wastes in the workflow mapped upon Lean methodology, led to series of interventions over 6 months, focusing on workflow, education, referral and handover processes, including stakeholder's feedback. Data collected prospectively and descriptive analysis performed.

Results: Total referrals 6 months before and after interventions: 320 vs 396; acceptance rate: 95.9 vs $97.7 \%$; enrollment: 61.6 vs $75.3 \%$; timely enrollment: 63.5 vs $59.4 \%$; 30-day readmission $24.4 \%$ vs $17.1 \%$. Improvements in knowledge and user satisfaction amongst teams noted. Improved satisfaction rates with adequacy of verbal handovers were registered by $\mathrm{H} 2 \mathrm{H}$ team.

Conclusion: Better awareness, knowledge and communication seem to have improved referrals, acceptance and enrollment rates. Though we expected an increased volume resulting in higher re-admissions, our interventions have actually resulted in reduction in re-admissions along with improved satisfaction in both GRM and $\mathrm{H} 2 \mathrm{H}$ teams. Moving forward, we like to explore other factors in this complex cohort such as routine incorporation of advance care planning discussions; root cause analysis on re- admissions and usage of objective tools such as Care Transition Measure (CTM-3), to enhance $\mathrm{H} 2 \mathrm{H}$ flow across wider hospital spectrum in alignment with improving care of older people.

\section{Abstract \# 5}

Is re-admission a serious adverse event (SAE)?

Surendra D. Varman ${ }^{1}$, Samuel E. E. Cheng ${ }^{1}$, Rachael T. Tambokon ${ }^{1}$ ${ }^{1}$ Changi General Hospital

Aim: Older people are the major users of health and social care systems. The 30-day all-cause readmission rate in Geriatric medicine (GRM) is $20 \%$, a concern due to escalating costs in healthcare. This QIA with root-cause analysis (RCA) aims to identify the main causes of re-admissions; trends and propose possible solutions.

Methods: Patients readmitted to GRM wards within 30 days of index admission were identified. RCA and descriptive analysis was performed by a team of Geriatrician and Medical officers.

Results: Total readmitted 30; Male 43\%; Median age 88; Nursing Home $(\mathrm{NH})$ patients $10 \%$. Co-morbidities: Hypertension $80 \%$; polypharmacy $80 \%$; End organ diseases 53\%; Dementia 47\%; Diabetes Mellitus $40 \%$; Falls $17 \% .43 \%$ readmitted with same index diagnosis (infection, fluid overload, fall, delirium/dementia); Median to re-admission 10 days. Functional dependency $23 \%$ index admission vs $30 \%$ on readmission; End of care discussions (EOC) $30 \%$ vs $36 \%$; Social assessment $70 \%$ vs $85 \%$; Hospital to Home referrals $(\mathrm{H} 2 \mathrm{H}) 46$ $\%$ vs 0 ; NH placement $3 \%$ vs $15 \%$; Supported discharge $50 \%$ vs $27 \%$. Mortality on re-admissions: $7 \%(2 / 30)$.

Conclusion: Age, co-morbidity, poly-pharmacy, and poor functional status are the main risk factors whilst Infections, fluid overload, Fall and delirium/dementia, chief causes for re-admissions. Inadequate EOC and $\mathrm{H} 2 \mathrm{H}$ exploration noted as common theme. More readmitted patients discharged to $\mathrm{NH}$, stretching community services. Moving forward, we consider proposing readmission as SAE to increase awareness, accountability, to trigger RCA's, whilst using Comprehensive Geriatric Assessment, $\mathrm{H} 2 \mathrm{H}$, Advance care planning, and predictive tools as means to improve.

\section{Abstract \# 6 \\ Higher risk of haemodiynamic instability from major trauma in elderly compared to younger patients.}

Gabriele Savioli $^{1}$, Iride Francesca Ceresa ${ }^{2}$, Sarah Macedonio ${ }^{3}$, Sebastiano Gerosa ${ }^{3}$, Mirko Belliato ${ }^{4}$, Sabino Luzzi ${ }^{5}$, Federica Manzoni $^{6}$, Mattia Del Maestro ${ }^{5}$, Claudio Pavesi ${ }^{7}$, Guido Forini ${ }^{7}$, Matteo Ghiara ${ }^{7}$, Giorgio Antonio Iotti ${ }^{4}$, Maria Antonietta Bressan ${ }^{8}$, Giovanni Ricevuti ${ }^{3}$ 
${ }^{1}$ Emergency Department, IRCCS Policlinico San Matteo, Pavia, Italy,

${ }^{2}$ Emergency Department, Ospedale Civile, Vigevano, Italy, ${ }^{3}$ University of Pavia, Italy, ${ }^{4}$ Intensive Care Unit, IRCCS Policlinico San Matteo, Pavia, Italy, ${ }^{5}$ Neurosurgery, IRCCS Policlinico San Matteo, Pavia, Italy, ${ }^{6}$ Clinical Epidemiology And Biometry Unit, IRCCS Policlinico San Matteo, Pavia, Italy, ${ }^{7}$ Orthopaedia, IRCCS Policlinico San Matteo, Pavia, Italy, ${ }^{8}$ Past Director Emergency Department, IRCCS Policlinico San Matteo, Pavia, Italy

Premises and purpose of the study: in the last few years elder population exposed to MT significately increased. Elderly ie are often subjected to dynamics that, although still major, are lower than the ones of the younger patients. Methods: prospectic monocentric study of patients who accessed our Emergency Department for MT in 12 months: from 1st january 2018 to 31 december 2018. Results: We enrolled 450 patients in total, 45 over 75 years. Most frequent cause of MT is road accidents ( $75 \%$ of cases in the younger patients, $68 \%$ in the elderly); elderly appear to be more exposed to domestic accidents ( $25 \%$ vs $5 \%$ ) and to aggressions ( $2 \%$ vs $0.2 \%$ ). Regarding haemodynamic instability, vital signs' derived shock indices are more impaired in elderly comparedto young patients. In particular, Shock Index results $>1,4$ in $6 \%$ of elder patients compared to $1 \%$ in young ones; MSI results greater than 1.3 in $10 \%$ of elderly patients compared to $6 \%$ in young ones and age-shock index results greater than 65 in $16 \%$ of elderly compared to $1 \%$ of the young ones. Presence of haemodynamic instability as defined by the American College of Surgeon is $26.5 \%$ in eldrer population compared to $9.2 \%$ in the younger popiulation.Conclusions: Elderly, inside the same wide catchment area (around 400.000 inhabitants) of an italian second level trauma center, although in the face of an even greater trauma maybe wrongly considered minor, present more frequently haemodynamic instability and a greater impairment of the vital signs compared to the younger population.

\section{Abstract \# 7 \\ Reduced impact of major trauma coagulopathy and of haemorragy in case of major trauma in the older population compared to the younger one}

Gabriele Savioli $^{1}$, Iride Francesca Ceresa ${ }^{2}$, Sarah Macedonio ${ }^{3}$, Sebastiano Gerosa ${ }^{3}$, Viola Novelli ${ }^{4}$, Alba Muzzi ${ }^{4}$, Mirko Belliato ${ }^{5}$, Giorgio Antonio Iotti ${ }^{5}$, Sabino Luzzi ${ }^{6}$, Mattia Del Maestro ${ }^{6}$, Federica Manzoni ${ }^{7}$, Francesco Benazzo ${ }^{8}$, Guido Forini ${ }^{8}$, Mario Mosconi ${ }^{8}$, Matteo Ghiara ${ }^{8}$, Fabrizio Cuzzocrea ${ }^{8}$, Claudio Pavesi ${ }^{8}$, Giovanni Ricevuti $^{3}$, Maria Antonietta Bressan ${ }^{9}$

${ }^{1}$ Emergency Department, IRCCS Policlinico San Matteo, Pavia, Italy, ${ }^{2}$ Emergency Department, Ospedale Civile, Vigevano, Italy,

${ }^{3}$ University of Pavia, Italy, ${ }^{4}$ Direzione Medica di Presidio, IRCCS Policlinico San Matteo, Pavia, Italy, ${ }^{5}$ Intensive Care Unit, IRCCS Policlinico San Matteo, Pavia, Italy, ${ }^{6}$ Neurosurgery, IRCCS Policlinico San Matteo, Pavia, Italy, ${ }^{7}$ Clinical Epidemiology And Biometry Unit, IRCCS Policlinico San Matteo, Pavia, Italy, ${ }^{8}$ Orthopaedia, IRCCS Policlinico San Matteo, Pavia, Italy, ${ }^{9} 10$ Past Director Emergency Department, IRCCS Policlinico San Matteo, Pavia, Italy

Premises and purpose of the study: One of the main causes of avoidable deaths in the young patients is bleeding, often due to trauma-related coagulopathy. The purpose of the study is to analyze if this data are valid also in elder population.

Methods: prospectic monocentric observational study on patients who accessed our Department of Fondazione for MT in 12 months: from 1st january 2018 to 31 december 2018.

Results: We enrolled 450 patients in total. 45 over 75 years old, (64\% male); 404 under $75 y$, (73\% male). In our population, the presence of haemodynamic instability as defined by American College of Surgeons is greater in the elder population, being $26,5 \%$ against $9,2 \%$ in the younger population. Also mortality rate is greater in the elder patients (4\% vs 1,3\%). Evaluating the coagulopathy presence, it is similar in the two subpopulations $(36.2 \%$ in the younger and $38 \%$ in the elder patients), whilst the haemotrasfusion need results greater in the younger patients (3.8\% against $2 \%$ ), in a statistically significant way with $\mathrm{p}<0.05$. Acid $\mathrm{pH}$, precipitating factor of trauma coagulopathy, results to be more elevated in the younger patients $(8.6 \%)$ and never present in our elderly case study.

Conclusions: Elderly, inside the same catchment area (aroun 400,000 inhabitants) of a second level italian trauma center, present less haemotransfusional need and the same coagulopathy rate compared to the younger population; although they present a greater mortality rate. Elderly's mortality could therefore be linked to other factors rather than bleeding.

\section{Abstract \# 8 \\ Head trauma and coma: major risk for elder patients in case of major trauma}

Gabriele Savioli ${ }^{1}$, Iride Francesca Ceresa ${ }^{2}$, Roberta Guarnone ${ }^{3}$, Sarah Macedonio ${ }^{4}$, Sebastiano Gerosa ${ }^{4}$, Mirko Belliato ${ }^{5}$, Giorgio Antonio Iotti ${ }^{5}$, Sabino Luzzi ${ }^{6}$, Mattia Del Maestro ${ }^{6}$, Federica Manzoni ${ }^{7}$, Sara Cutti ${ }^{8}$, Elvis Lafe ${ }^{9}$, Anna Simoncelli ${ }^{9}$, Giovanni Ricevuti ${ }^{4}$, Maria Antonietta Bressan ${ }^{10}$

${ }^{1}$ Emergency Department, IRCCS Policlinico San Matteo, Pavia, Italy, ${ }^{2}$ Emergency Department, Ospedale Civile, Vigevano, Italy., ${ }^{3}$ Emergency Department, IRCCS Policlinico San Matteo, Pavia, Italy, ${ }^{4}$ University of Pavia, Italy, ${ }^{5}$ Intensive Care Unit, IRCCS Policlinico San Matteo, Pavia, Italy, ${ }^{6}$ Neurosurgery, IRCCS Policlinico San Matteo, Pavia, Italy, ${ }^{7}$ Clinical Epidemiology And Biometry Unit, IRCCS Policlinico San Matteo, Pavia, Italy, ${ }^{8}$ Direzione Medica di Presidio, IRCCS Policlinico San Matteo, Pavia, Italy, ${ }^{9}$ Neuro Radiodiagnostic, IRCCS Policlinico San Matteo, Pavia, Italy, ${ }^{10}$ Past Director Emergency Department, IRCCS Policlinico San Matteo, Pavia, Italy

Introduction: Elderly, due to many co-factors (unstable walking, reduced sight, blance and reflexes), are exposed to a greater risk of falling and head trauma. We therefore analyzed if this statement is also valid in our Major Trauma (MT) population and if this could lead to worse outcomes.

Methods: We made a prospectic monocentric observational study of all the patients who accessed in our Emergency and Acceptance Department for MT in 12 months.

Results: We enrolled 501 patients in total; 51 over 75 years old (64\% male). Regarding the body districts affected, in the elder population head trauma is the most frequently affected (in $62 \%$ of cases), followed by chest $(52 \%)$, lower limb $(24 \%)$, abdominal (22\%), upper limb $(14 \%)$, pelvis $(26 \%)$, spine $(36 \%)$. In the younger population chest is the most affected district (in $45 \%$ of cases), followed, in order of frequency, by head trauma (44\%), spine trauma (34\%), lower limb trauma (26\%), upper limb trauma (25\%), abdominal trauma (15\%), pelvis trauma (13\%). Head trauma is more frequent in the elderly in a statistially significant way $(p=0.016)$. This is further confirmed by the fact that in the elder population there is a greater request for maxillo-facial surgeon advice (10\% against $2 \%$ in younger patients) and neurosurgeon advice (38\% against 26\%). Consequently also GCS is more impaired in elder subjects, in fact $12 \%$ of them had CGS $<9$ against $3 \%$ in the younger population.Key Conclusions: Elderly with MT present more frequently head trauma compared to the younger population, needing more specialistic surgeon visits (maxillofacial and neurosurgical) and presenting more severe sensorial impairment. 


\section{Abstract \# 9}

\section{Elderly and urinary tract infection in emergency department}

Gabriele Savioli ${ }^{1}$, Iride Francesca Ceresa ${ }^{2}$, Roberta Guarnone ${ }^{1}$, Sarah Macedonio $^{3}$, Luca Ciceri ${ }^{3}$, Valentina Angeli ${ }^{3}$, Sebastiano Gerosa ${ }^{3}$, Antonello Malfitano ${ }^{4}$, Alba Muzz ${ }^{5}$, Sara Cutti ${ }^{5}$, Viola Novelli ${ }^{5}$, Elisa Bonadeo $^{5}$, Carlo Marena ${ }^{5}$, Giuseppina Grugnetti ${ }^{6}$, Maria Antonietta Bressan $^{7}$, Giovanni Ricevuti ${ }^{3}$

${ }^{1}$ Emergency Department, IRCCS Policlinico San Matteo, Pavia, Italy, ${ }^{2}$ Emergency Department, Ospedale Civile, Vigevano, Italy,

${ }^{3}$ University of Pavia, Italy, ${ }^{4}$ Malattie Infettive IRCCS Policlinico San Matteo, Pavia, Italy, ${ }^{5}$ Direzione Medica di Presidio IRCCS

Policlinico San Matteo, Pavia, Italy, ${ }^{6}$ SITRA IRCCS Policlinico San Matteo, Pavia, Italy, ${ }^{7}$ Past Director Emergency Department, IRCCS Policlinico San Matteo, Pavia, Italy

Introduction: Describe and typify the elderly population that accesses our emergency room for signs or symptoms which then make the main diagnosis of urinary tract infections.

Methods: We retrospectively analyzed all patients who received diagnosis of urinary tract infection at our emergency room in 10 consecutive months (January 1, 2018-October 31, 2018).

Results: Of the 664 patients admitted for urinary tract infection, 275 were elderly. Of those $61 \%$ received low priority codes of medical examination and allocation at the low intensity of treatments, 39\% instead a code of high priority to the medical examination, and more precisely $24 \%$ was attributed to the low-intensity wing and $15 \%$ to the medium-high intensity wing. The medical severity code at discharge in elderly patients was elevated in $17 \%$ of cases, compared to only $9 \%$ of cases in the general population. In addition, the elderly population required access to the intensive short observation to stabilize the clinical picture in $10 \%$ of cases compared to only $5 \%$ of the general population. While $\mathrm{n}$ the general population $75 \%$ of patients are discharged, and only $24 \%$ hospitalized; in the elderly population as many as $39 \%$ need hospitalization.

Key conclusions: Elderly patients who come to the emergency room for urinary tract infection are the most fragile category of the general population. They more often they need higher priority codes to the medical examination, more frequently they require medium-high intensity of care. they also more frequently need access to a stabilization area such as short intensive observation and more frequently they need hospitalization.

\section{Abstract \# 10 \\ Pneumonia in the elderly: when pulmonary infections determine emergency room access}

Gabriele Savioli $^{1}$, Iride Francesca Ceresa ${ }^{2}$, Roberta Guarnone ${ }^{1}$, Sarah Macedonio $^{3}$, Sebastiano Gerosa ${ }^{3}$, Valentina Angeli ${ }^{3}$, Antonello Malfitano ${ }^{4}$, Alba Muzzi ${ }^{5}$, Viola Novelli ${ }^{5}$, Elisa Bonadeo ${ }^{5}$, Sara Cutti ${ }^{5}$, Carlo Marena ${ }^{5}$, Maria Antonietta Bressan ${ }^{6}$

${ }^{1}$ Emergency Department, IRCCS Policlinico San Matteo, Pavia, Italy, ${ }^{2}$ Emergency Department, Ospedale Civile, Vigevano, Italy,

${ }^{3}$ University of Pavia, Italy, ${ }^{4}$ Malattie Ifettive IRCCS Policlinico San Matteo, Pavia, Italy, ${ }^{5}$ Direzione Medica di Presidio IRCCS Policlinico San Matteo, Pavia, Italy, ${ }^{6}$ Past Director Emergency Department, IRCCS Policlinico San Matteo, Pavia, Italy

Aim: Describe and typify the population that accesses our emergency room for signs or symptoms that then lead to the diagnosis of pneumonia.

Methods: We retrospectively analyzed all patients who received pneumonia diagnosis in our emergency room in 10 consecutive months (1 January 2018-31 October 2018). Results: of the 565 patients with pneumonia, 443 were elderly. Among elderly 33\% received low priority codes of medical examination and allocation at the low intensity of care, $67 \%$ instead a code of high priority to medical examination, of this $5 \%$ was attributed to the low intensity of care wing and the remaining $95 \%$ to the medium-high intensity care wing.. The medical severity code at discharge was green in $53 \%$ of cases, $43 \%$ yellow and $4 \%$ of cases red. $53 \%$ required access to the intensive brief observation for stabilization of the clinical picture. $66 \%$ of patients were hospitalized, $6 \%$ transferred to a lower-care facility and only the remaining $28 \%$ discharged.

Key conclusions: patients who come to the emergency room for pneumonia are more frequently elderly. These are complex patients, in large part they require a medium-high intensity of care for the administration of aerosol, oxygen and trp cadenced, multiparametric monitoring and oximetry. An intensive care organization with short intensive observation with a dedicated team therefore allowed immediate allocation to the most correct treatment area and excellent waiting and process issues.

\section{Abstract \# 11}

Hospitalisation rate and length of hospital stay in case of major trauma: a comparison between older and younger population

Gabriele Savioli $^{1}$, Iride Francesca Ceresa ${ }^{2}$, Roberta Guarnone ${ }^{1}$, Sarah Macedonio $^{3}$, Sebastiano Gerosa ${ }^{3}$, Mirko Belliato ${ }^{4}$, Giorgio Antonio Iotti $^{4}$, Federica Manzoni ${ }^{5}$, Sabino Luzzi ${ }^{6}$, Mattia Del Maestro ${ }^{6}$, Viola Novelli ${ }^{7}$, Giovanni Ricevuti ${ }^{8}$, Maria Antonietta Bressan ${ }^{9}$

${ }^{1}$ Emergency Department, IRCCS Policlinico San Matteo, Pavia, Italy, ${ }^{2}$ Emergency Department, Ospedale Civile, Vigevano, Italy, ${ }^{3}$

University of Pavia, Italy, ${ }^{4}$ Intensive Care Unit, IRCCS Policlinico San Matteo, Pavia, Italy, ${ }^{5}$ Clinical Epidemiology And Biometry Unit, IRCCS Policlinico San Matteo, Pavia, Italy, ${ }^{6}$ Neurosurgery, IRCCS Policlinico San Matteo, Pavia, Italy, ${ }^{7}$ Direzione Medica di Presidio IRCCS Policlinico San Matteo, ${ }^{8}$ University of Pavia, ${ }^{9}$ Past Director Emergency Department, IRCCS Policlinico San Matteo, Pavia, Italy

Introduction: Elderly have been, in the last few decades, more and more exposed to Major Trauma (MT) and the rate of elderly who reported a MT has been progressively raising. The old patient is universally considered a frail subject and therefore present the risk of worse outcomes.

Methods: AWe did a prospectic monocentric observational study of all the patients who accessed to the Emergency and Acceptance Department of Fondazione Policlinico IRCCS S. Matteo in Pavia for MT in 12 months: from 1st january 2018 to 31 december 2018. Results: We enrolled 501 patients in total. Between the patients over 75 , we enrolled 51 patients, $64 \%$ of which were male. 449 were, instead, under 75 , and $73 \%$ of them were male. Hospitalisation rate resulted comparable in the two subpopulation, being $72 \%$ in the elder patients and $74 \%$ in the younger ones. The ICU hospitalisation rate was $16 \%$ in the elder subjects and $12 \%$ in younger subjects, a non statistically significant difference (p 0, 3736). Although the mean duration of the ICU stay was greater in older patients (18.75 days against 9.45 in younger patients), it does not reach statistical significativity. The latter statement is valid also for the need of operation theatre during hospitalisation (18\% against $12.88 \%$ ).

Conclusions: Elderly with MT do not present a greater rate of hospitalisation, or ICU stay. They do not present a statistically significant increase in the duration of ICU stay neither in the operation theater need. This, in the face of a greater statistically significant mortality that emerged in our population. 


\section{Abstract \# 12 \\ Major trauma mortality: the risk increases in the elderly population}

Gabriele Savioli ${ }^{1}$, Iride Francesca Ceresa ${ }^{2}$, Roberta Guarnone ${ }^{1}$, Sarah Macedonio $^{3}$, Sebastiano Gerosa ${ }^{3}$, Fabio Sciutti ${ }^{4}$, Mirko Belliato ${ }^{4}$, Giorgio Antonio Iotti ${ }^{4}$, Sabino Luzzi ${ }^{5}$, Mattia Del Maestro ${ }^{5}$, Anna Simoncelli ${ }^{6}$, Federica Manzoni ${ }^{7}$, Leandro Gentile ${ }^{8}$, Giovanni Ricevuti ${ }^{3}$, Maria Antonietta Bressan ${ }^{9}$

${ }^{1}$ Emergency Department, IRCCS Policlinico San Matteo, Pavia, Italy, ${ }^{2}$ Emergency Department, Ospedale Civile, Vigevano, Italy, ${ }^{3}$ University of Pavia, Italy, ${ }^{4}$ Intensive Care Unit, IRCCS Policlinico San Matteo, Pavia, Italy, ${ }^{5}$ Neurosurgery, IRCCS Policlinico San Matteo, Pavia, Italy, ${ }^{6}$ Neuro Radiodiagnostic, IRCCS Policlinico San Matteo, Pavia, Italy, ${ }^{7}$ Clinical Epidemiology And Biometry Unit, IRCCS Policlinico San Matteo, Pavia, Italy, ${ }^{8}$ Direzione Medica di Presidio, IRCCS Policlinico San Matteo, Pavia, Italy, ${ }^{9}$ Past Director Emergency Department, IRCCS Policlinico San Matteo, Pavia, Italy

Introduction: Major Trauma (MT) is the main cause of mortality in the world for under 40 years patients. Although, especially in Western Countries, the elder population with a greater risk has significally increased.

Methods: We made a prospectic monocentric observational study on all the patients who accessed to the Emergency Department for MT in 13 months: from 1 st of january 2018 to 30th of january 2019.

Results: We enrolled 501 patient in total. Between over 75 patients we enrolled 51 patients, representing $10 \%$ of total; $36 \%$ are women and $64 \%$ are men. $89,8 \%$ presented MT accordinc to dynamic criteria, $18,4 \%$ anatomical criteria and $26,5 \%$ clinical criteria. 449 were under 75 years of age, $90 \%$ of the total; between them $27 \%$ were women and $73 \%$ men. $92 \%$ had MT according to dynamic criteria, $15 \%$ to anatomical criteria, $9 \%$ to clinical criteria. The more frequent cause of MT were road accidents, more frequent in the younger population ( $75 \%$ vs $68 \%$ ). In older subpopulation there was greater incidence of domestic accidents (26\% vs 6\%). Total Body CT resulted more frequently positive in younger subjects $(74.7 \%$ vs $68 \%$ of the cases). Mortality, instead, was statistically significantly higher in older patients ( $4 \%$ vs $1.33 \%$ in young patients; $p=0.052$ ).

Conclusions: Elderly statistically significant higher mortality compared to younger patients, although they do not present a non superior trauma dynamic compared to the younger population and although lesion shown by $\mathrm{CT}$ result less frequent (but more severe).

\section{Abstract \# 13}

\section{Major trauma and elderly: the risk of underestimating}

Gabriele Savioli ${ }^{1}$, Iride Francesca Ceresa ${ }^{2}$, Roberta Guarnone ${ }^{1}$, Sarah Macedonio $^{3}$, Sebastiano Gerosa ${ }^{3}$, Mirko Belliato ${ }^{4}$, Giorgio Antonio Iotti $^{4}$, Sabino Luzzi ${ }^{5}$, Mattia Del Maestro ${ }^{5}$, Elvis Lafe ${ }^{6}$, Federica Manzoni $^{7}$, Viola Novelli ${ }^{8}$, Alba Muzzi ${ }^{8}$, Elisa Bonadeo ${ }^{8}$, Maria Antonietta Bressan ${ }^{9}$

${ }^{1}$ Emergency Department, IRCCS Policlinico San Matteo, Pavia, Italy, ${ }^{2}$ Emergency Department, Ospedale Civile, Vigevano, Italy, ${ }^{3}$ University of Pavia, Italy, Intensive Care Unit, IRCCS Policlinico San Matteo, Pavia, Italy, ${ }^{5}$ Neurosurgery, IRCCS Policlinico San Matteo, Pavia, Italy, ${ }^{6}$ Neuro Radiodiagnostic, IRCCS Policlinico San Matteo, Pavia, Italy, ${ }^{7}$ Clinical Epidemiology And Biometry Unit, IRCCS Policlinico San Matteo, Pavia, Italy, ${ }^{8}$ Direzione Medica di Presidio, IRCCS Policlinico San Matteo, Pavia, Italy, ${ }^{9}$ Past Director Emergency Department, IRCCS Policlinico San Matteo, Pavia, Italy

Introduction: Elderly, due to many co-factors (unstable walking, reduced sight, blance and reflexes), are exposed to greater risk of falling and trauma. Often falling are victims of major trauma. We want to find out if Major Trauma (MT) is more often underestimated. Methods: prospectic monocentric observational study of all patients who accessed Emergency Department for MT in 12 months: from 1st january 2018 to 31 december 2018.

Results: We enrolled 450 patients in total. 45 over 75 years. in older subpopulation, only $76 \%$ of the population received high priority code wait for the visit (red code), compared to $85 \%$ in younger. Also Trauma Team activation was lower (48\% against $56 \%$ ) in the elderly. The elder population presented more often haemodynamic instability (26\% in the elderly vs $9 \%$ in the younger partients); they also present a more often impaired anatomic severity index like ISS $>15$ (44\% of the elder population against $32 \%$ of the younger population). Undertriage, calculated by using modified Cribari formula is $35 \%$ in the elder population and in $23 \%$ of the younger population. Overtriage was greatere in ounger patients $(45 \%)$ compared to older patients $(34 \%)$.

Conclusions: Elderly present a more elevated underestimation. This is due to many causes: vital signs in elderly are often indipendently more compromised, so their impairment is sometimes attributed to preexisting causes. Furthermore, elderly more often present MT causes usually wrongly identified as low-risk like domesic accidents and other-s violence (in our elderly $26 \%$ and $2 \%$ compared to $6 \%$ and $0.2 \%$ in younger).

\section{Abstract \# 14}

Acute stroke and the deathly hallows: the risk factors in a large coorte of elderly patients undergoing thrombolysis

Gabriele Savioli ${ }^{1}$, Iride Francesca Ceresa ${ }^{2}$, Anna Cavallin ${ }^{3}$, Alessandra Persico ${ }^{3}$, Francesca Grulli ${ }^{4}$, Elena Novara ${ }^{4}$, Alessandra Martignoni $^{5}$, Elvis Lafe ${ }^{6}$, Anna Simoncelli ${ }^{6}$, Viola Novelli ${ }^{7}$, Sara Cutti $^{7}$, Giorgio Antonio Iotti ${ }^{8}$, Chiara Gagliardone ${ }^{6}$, Andrew Wimer ${ }^{9}$, Maria Antonietta Bressan ${ }^{10}$, Francesco Geraci ${ }^{11}$

${ }^{1} 1$. Emergency Department IRCCS Policlinico San Matteo Pavia Italy, ${ }^{2} 2$. Emergency Department Ospedale Civile Vigevano Italy, ${ }^{3} 3$. Stroke Unit IRCCS Policlinico San Matteo Pavia Italy, ${ }^{4}$ University of Pavia, Italy, ${ }^{5}$ internal Medicine IRCCS Policlinico San Matteo Pavia Italy, ${ }^{6}$ IRCCS Policlinico San Matteo Pavia Italy, ${ }^{7}$ Direzione Medica di Presidio IRCCS Policlinico San Matteo Pavia Italy, ${ }^{8}$ Emergency Department IRCCS Policlinico San Matteo Pavia Italy, ${ }^{9}$ Università degli Studi di Pavia, Italy, ${ }^{10}$ Past Director Emergency Room IRCCS Policlinico San Matteo Pavia Italy, ${ }^{11}$ ASST Pavia Italy

Aim: Analyze among patients aged 75 and over who have acute stroke and have thrombolysis the distribution of risk factors in the global population and depending on the presentation symptomatic framework.

Methods: We conducted a prospective and monocentric observational study of all patients with acute stroke referred to the Department of Emergency and Acceptance from May 1, 2017 to May 1, 2018.

Results: Elderly patients diagnosed with strokes in our emergency room and subjected to thrombolysis for a total of 49 patients were analyzed. This population has a high incidence of risk factors: $94 \%$ had at least one risk factor, $63 \%$ of the study population had at least two risk factors, and $30 \%$ had 3 or more. By far the most represented risk factor in this population is high blood pressure in $73.5 \%$ of cases. The prevalence of atheromasia superortic trunks present in $32.6 \%$ of patients, ischemic heart disease $26.5 \%$, atrial fibrillation $26.5 \%$ and diabetes mellitus $20.4 \%$ follow with substantially overlapping prevalence. with slightly lower percentage of ical disease $22.4 \%$; while cigarette smoking habit has a lower incidence in this population (4\%). We then divided the population of thrombolysis patients into clinical syndromes: motor, sensory, language and nonspecific 
symptoms. Conclusions: in elderly patients who are candidates for thrombolysis, high blood pressure is the widely represented risk factor, while cigarette smoking is poorly represented. Particular attention should be given to all the other risk factors under consideration. Patients with atypical symptomatic cadres, on the other hand, have a lower prevalence of FA, suggesting that in these cases the rate of heart disease pathogenic stroke is reduced

\section{Abstract \# 15 \\ Invisibily cloak: recognition delay of atypical stroke symptoms in patients with more than $\mathbf{7 5}$ years}

Gabriele Savioli ${ }^{1}$, Iride Francesca Ceresa ${ }^{2}$, Roberta Guarnone ${ }^{1}$, Francesca Grulli ${ }^{3}$, Elena Novara ${ }^{3}$, Giovanni Ricevuti ${ }^{3}$, Anna Cavallin $^{4}$, Alessandra Persico ${ }^{4}$, Alessandra Martignoni ${ }^{5}$, Elvis Lafe ${ }^{5}$, Francesco Geraci ${ }^{6}$, Chiara Gagliardone ${ }^{5}$, Andrew Wimer ${ }^{3}$, Maria Antonietta Bressan

${ }^{1}$ 1. Emergency Department IRCCS Policlinico San Matteo Pavia Italy, ${ }^{2} 2$. Emergency Department Ospedale Civile Vigevano Italy, ${ }^{3}$ University of Pavia, Italy, ${ }^{4}$ Stroke Unit IRCCS Policlinico San Matteo Pavia Italy, ${ }^{5}$ IRCCS Policlinico San Matteo Pavia Italy, ${ }^{6}$ ASST 118 Pavia Italy, ${ }^{7}$ Past Director Emergency Room IRCCS Policlinico San Matteo Pavia Italy

Introduction: According to different symptomatological spectrum at presentation, the purpose of this study is to ana-lyze times needed for recognition and management of patients over 75 years affected by stroke.

Methods: We conducted a prospective and monocentric observational study of all patients with acute stroke referred to the Department of Emergency and Acceptance in Pavia from May 1, 2017 to May 1, 2018.

Results: We enrolled a total of 430 patients with more than 75 years who received diagnosis of stroke at our Emer-gency Department from May 1st 2017 to May 1st 2018. The average age was 83.8 y and women were in slight majority (female 60\%). At presentation, symptomatological spectrum was so divided: $56.7 \%$ motor symptoms, $15.7 \%$ sensory symptoms, $46.8 \%$ speech disorder and $36.5 \%$ atypical symptoms (including dizziness, confusion and syncope). While the first three groups with typical symptoms accurately presented an high priority code for medical examination (about $90 \%$ had yellow code or higher), only $74 \%$ of patients belonging to the forth group (atypical symptoms) had a high pri-ority code. This resulted in different waiting times: average of $38 \mathrm{~min}(\mathrm{~min})$ for the first three categories, average of $55 \mathrm{~min}$ for patients with atypical symptoms. This time delay in the fourth group was primarily dependent on recogni-tion at door, while subsequent times (request for imaging, neurological consultation and process time) were normalized.

Conclusions As our results demonstrated, patients presenting atypical symptoms of stroke were more difficult to recognize at door as neurological acuities, leading to increased waiting times.

\section{Abstract \# 16 \\ Toc toc: who is it? elderly with acute stroke knocks on the door of the emergency room. the characterization of the elderly population with acute stroke in real life}

Gabriele Savioli ${ }^{1}$, Iride Francesca Ceresa ${ }^{2}$, Roberta Guarnone ${ }^{1}$, Anna Cavallin $^{3}$, Alessandra Martignoni ${ }^{4}$, Anna Simoncelli ${ }^{4}$, Francesco Geraci $^{5}$, Chiara Gagliardone ${ }^{4}$, Andrew Wimer ${ }^{6}$, Francesca Grulli ${ }^{6}$, Elena Novara ${ }^{6}$, Maria Antonietta Bressan ${ }^{7}$
${ }^{1}$ Emergency Department IRCCS Policlinico San Matteo Pavia Italy, ${ }^{2}$ Emergency Department Ospedale Civile Vigevano Italy, ${ }^{3}$ Stroke Unit IRCCS Policlinico San Matteo Pavia Italy, ${ }^{4}$ IRCCS Policlinico San Matteo Pavia Italy, ${ }^{5}$ ASST 118 Pavia Italy, ${ }^{6}$ Università degli Studi di Pavia, Italy, ${ }^{7}$ Past Director Emergency Room IRCCS Policlinico San Matteo Pavia Italy

Aim: Provide a detailed photograph of the patient $>75 \mathrm{y}$ who shows up in the emergency room for acute stroke in a HUB center for stroke. Methods: We conducted prospective and monocentric observational study from May 1, 2017 to May 1, 2018.

Results: Were analyzed 430 patients. (average age 83 y). $85 \%$ of patients are given a priority code for high visit with a yellow or red code. More than $90 \%$ needed hospitalization. $77 \%$ were admitted to our DEA and $14 \%$ transferred to a low-intensity neurological institution. $75 \%$ of patients had high process-term severity code. 52 patients were proven to be candidates for thrombolysis, systemic or mechanical or both. Looking at risk factors such as diabetes mellitus, high blood pressure, cigarette smoking habit, previous ictal disease, ischemic heart disease, superortic athemtic athesia and atrial fibrillation is seen as $64 \%$ of the study population had at least two risk factors, and $35 \% 3$ or more. Only $9 \%$ did not take drugs at home. The most represented drugs were antipertensive, anticlusters, anticoagulants, polypomizers, beta blockers, oral antidiabetics, PPPs, antidepressants. $41 \%$ were already in antiaggregating trp and $16 \%$ were already anticoagulant trp.

Conclusions: elderly who report acute stroke are highly complex population as demonstrated by high prevalence of risk factors and other concomitant pathologies, high percentage of hospitalization, complexity of home therapy and from high percentage of high severity codes to discharge. They have high cardiovascular risk and home drug polytherapy. It is often already antiaggregated or anticoagulated. It has high hospitalization rates.

\section{Abstract \# 17}

Waiting time and fibrinolysis in the elderly with acute stroke in the emergency room in real life: the time machine

Gabriele Savioli $^{1}$, Iride Francesca Ceresa ${ }^{2}$, Roberta Guarnone ${ }^{1}$, Anna Cavallin $^{3}$, Alessandra Persico ${ }^{3}$, Francesca Grulli ${ }^{4}$, Elena Novara ${ }^{4}$, Alessandra Martignoni ${ }^{5}$, Anna Simoncelli ${ }^{5}$, Alba Muzzi ${ }^{5}$, Viola Novelli $^{5}$, Federica Manzoni ${ }^{6}$, Francesco Geraci ${ }^{7}$, Chiara Gagliardone ${ }^{5}$, Andrew Wimer ${ }^{4}$, Giovanni Ricevuti ${ }^{4}$, Maria Antonietta Bressan ${ }^{8}$

${ }^{1}$ Emergency Department IRCCS Policlinico San Matteo Pavia Italy, ${ }^{2}$ Emergency Department Ospedale Civile Vigevano Italy, ${ }^{3}$ Stroke Unit IRCCS Policlinico San Matteo Pavia Italy, ${ }^{4}$ Università degli Studi di Pavia, Italy, ${ }^{5}$ IRCCS Policlinico San Matteo Pavia Italy, ${ }^{6}$ Clinical Epidemiology and Biometry Unit, Scientific Direction, San Matteo Hospital Foundation, Pavia, Italy, ${ }^{7}$ ASST 118 Pavia Italy, ${ }^{8}$ Past Director Emergency Room IRCCS Policlinico San Matteo Pavia Italy

Aim: Analyze the waiting time, process of neurological evaluation and CT in a large cohort of elderly patients with acute stroke and treated with.

Methods: We conducted a prospective and monocentric observational study rom May 1, 2017 to May 1, 2018.

Results: A total of 49 patients were enrolled in the study of the age of 75 or older diagnosed with acute stroke in our emergency room and treated with thrombolysis. These patients had average age of 81.8 Y.O. with equal distribution of gender. Waiting time was mean of 12 min with median of $8 \mathrm{~min}$. Mean wait was $2 \mathrm{~h}$ and $54 \mathrm{~min}$ with median waiting time of $2 \mathrm{~h}$ and $40 \mathrm{~min}$; The mean stay was $3 \mathrm{~h}$ and 6 min with a median time of stay of $3 \mathrm{~h}$ and $7 \mathrm{~min}$. Median times of the 
neurological examinations were $31 \mathrm{~min}$; those requiring CT scan (execution and reporting) with intracranial and extracranial circulation evaluation had median of $1 \mathrm{~h}$ and $32 \mathrm{~min}$. All of the patients were admitted to the stroke unit after an initial observation in the emergency room. Population that underwent thrombolysis was taking multiple medications at home. $83 \%$ was taking at least one prescription medication, $67 \%$ was taking at least $348.98 \%$ was taking at least $516.4 \%$ at least $8.36 .7 \%$ was taking antiplatelet therapy and $20.5 \%$ taking anticoagulants.

Conclusion: Elderly population who underwent thrombolysis is represented by patients with many pathologies, at high cardiovascular risk and taking cardiovascular medications at home.

\section{Abstract \# 18 \\ Risk factors for acute stroke within aging populations: friends or enemie? Alias the hateful eight}

Gabriele Savioli ${ }^{1}$, Iride Francesca Ceresa ${ }^{2}$, Roberta Guarnone ${ }^{1}$, Anna Cavallin $^{3}$, Alessandra Persico ${ }^{3}$, Francesca Grulli ${ }^{4}$, Elena Novara ${ }^{4}$, Andrew Wimer ${ }^{4}$, Elvis Lafe ${ }^{5}$, Alessandra Martignoni ${ }^{5}$, Francesco Geraci $^{6}$, Chiara Gagliardone ${ }^{5}$, Giovanni Ricevuti ${ }^{4}$, Maria Antonietta Bressan $^{7}$

${ }^{1}$ Emergency Department IRCCS Policlinico San Matteo Pavia Italy, ${ }^{2}$ Emergency Department Ospedale Civile Vigevano Italy, ${ }^{3}$ Stroke Unit IRCCS Policlinico San Matteo Pavia Italy, ${ }^{4}$ Università degli Studi di Pavia, Italy, ${ }^{5}$ IRCCS Policlinico San Matteo Pavia Italy, ${ }^{6}$ ASST 118 Pavia Italy, ${ }^{7}$ Past Director Emergency Room IRCCS Policlinico San Matteo Pavia Italy

Introduction: We analyze the risk factors in a large cohort of patients who have been diagnosed with acute stroke in our emergency room. In particular: diabetes mellitus, arterial hypertension, smoking, previous stroke, ischemic cardiopathy, atheroma of supra-aortic vessels and atrial fibrillation in various patients over 75 years of age.

Materials and Methods: We conducted a prospective and monocentric observational study of all patients with acute stroke from May 1, 2017 to May 1, 2018.

Results: 430 patients were enrolled in the study. These patients presented the average age of 83.8 Y.O. with a predominance for the female sex $(60 \% \mathrm{~F})$. The general population evaluated demonstrated a high incidence of risk factors: over $91.3 \%$ presented at least one risk factor, $63.7 \%$ of the study population presented at least two risk factors, and $34.6 \%$ presented three or more. The most common risk factor represented was arterial hypertension, which was present in $73 \%$ of the cases. Following: Smoking in $5.1 \%$, previous stroke in $27.6 \%$, ischemic cardiopathy in $23.8 \%$, atheroma of supra-aortic vessels in $30 \%$, atrial fibrillation in $25.5 \%$ and diabetes mellitus in 23 . Conclusions: It can be deduced from the careful analysis of the risk factors together with the collection of signs and symptoms that an improvement in early recognition can be brought to the attention to patients with acute neurological problems and as a consequence the entire diagnosis and treatment process in elderly patients can be facilitated when recent medical history can be correctly be collect.

\section{Abstract \# 19 \\ Risk factors in various clinical frameworks of acute stroke within aging populations}

Gabriele Savioli ${ }^{1}$, Iride Francesca Ceresa ${ }^{2}$, Roberta Guarnone ${ }^{1}$, Anna Cavallin $^{3}$, Francesca Grulli ${ }^{4}$, Elena Novara ${ }^{4}$, Alessandra Martignoni ${ }^{5}$, Elvis Lafe ${ }^{5}$, Andrew Wimer ${ }^{4}$, Francesco Geraci ${ }^{6}$, Chiara Gagliardone $^{4}$, Giovanni Ricevuti ${ }^{4}$, Maria Antonietta Bressan ${ }^{7}$
${ }^{1}$ Emergency Department IRCCS Policlinico San Matteo Pavia Italy, ${ }^{2}$ Emergency Department Ospedale Civile Vigevano Italy, ${ }^{3}$ Stroke Unit IRCCS Policlinico San Matteo Pavia Italy, ${ }^{4}$ Università degli Studi di Pavia, Italy, ${ }^{5}$ IRCCS Policlinico San Matteo Pavia Italy, ${ }^{6}$ ASST 118 Pavia Italy, ${ }^{7}$ Past Director Emergency Room IRCCS Policlinico San Matteo Pavia Italy

Introduction: we analyze the risk factors in a large cohort of patients over 75 years who have been diagnosed with acute stroke. We have therefore divided the population according to the presentation of the following symptoms: motor, sensory, language deficit and atypical (dizziness, confusion and syncope). Methods: prospective and monocentric observational study of all patients with acute stroke from May 1, 2017 to May 1, 2018.

Results: 430 patients were enrolled in the study. The 4 symptom based groups are results are superimposable for age, distribution of sex and outcome o the hospitalization. The symptom based group with motor disturbances, language deficit and non-specific have been substantially demonstrated to be superimposable for the number and distribution of risk factors. In particular: arterial hypertension $71-74 \%$ with a peak in $83 \%$ of patients with sensory symptoms; cigarette smoking in $5-6 \%$, previous stroke in $23-27 \%$, ischemic cardiopathy in 22-29\%, atheroma of supra-aortic vessels in 29-33\%, atrial fibrillation in $23-27 \%$ and diabetes mellitus in $15-20 \%$ with a peak in $26 \%$ in patients with atypical symptomatology.

Conclusions: It can be deduced from the careful analysis of the risk factors together with the collection of signs and symptoms that an improvement in early recognition can be brought to the attention to patients with acute neurological problems and as a consequence the entire diagnosis and treatment process in elderly patients can be facilitated when recent medical history can be correctly be collect.

\section{Abstract \# 20}

\section{Sepsi in the elderly: a window in the emergency department}

Gabriele Savioli ${ }^{1}$, Iride Francesca Ceresa ${ }^{2}$, Roberta Guarnone ${ }^{1}$, Sarah Macedonio $^{3}$, Valentina Angeli ${ }^{3}$, Luca Ciceri ${ }^{3}$, Antonello Malfitano ${ }^{4}$, Giovanni Ricevuti ${ }^{3}$, Maria Antonietta Bressan ${ }^{5}$

${ }^{1}$ Emergency Department, IRCCS Policlinico San Matteo, Pavia, Italy, ${ }^{2}$ Emergency Department, Ospedale Civile, Vigevano, Italy,

${ }^{3}$ University of Pavia, Italy, ${ }^{4}$ Malattie Infettive IRCCS Policlinico San Matteo, Pavia, Italy, ${ }^{5}$ Past Director Emergency Department, IRCCS Policlinico San Matteo, Pavia, Italy

Purpose: Describe and analize signs and syntoms of population who go our Emergency Department with the aim to identify early the sepsis.

Methods: Survey is on all inpatients of the ED that are received the sepsis diagnosis in 10 months (from 1st of January to the 31th of October of 2018). Results: 336 inpatients are enrolled in study, of which 275 are elderly, with male prevalence $(55 \%)$. The average age is 79 . Only $22 \%$ of the elderly went spontaneously to the ED, the $79 \%$ was accompanied by the ambulance. $20 \%$ of the elderly inpatients received a high gravity level after the medical visit, $25 \%$ was assigned to the minor intensity care area and the last $75 \%$ to the medium-high intensity level of care. The average waiting time to the medical examination was $29 \mathrm{~min} .22 \%$ of these inpatients needed to recover in Short Observation Intensity for an average time of $18 \mathrm{~h}$ to stabilize the clinic state. Nearly all ED inpatient were recovered in and only the $1.1 \%$ were transferred to an hospital with a minor intensity level of care.

Conclusions: The ED inpatients with sepsis belongs to the elderly population. They are vulnerable and they need a medium-high level of intensity of care to take the drugs, to be monitored and to be subject of invasive procedures. A dedicated team and a care organization of 
the elderly inpatients with the sepsis determine the allocation of the more correct area of care and good waiting time and the process.

\section{Abstract \# 21 \\ The elderly inpatients of the emergency department for infective disease. Overview of a vulnerable population from the triage to the end of the process in an emergency departmetn organized by different level of intensity of care}

Gabriele Savioli ${ }^{1}$, Iride Francesc Ceresa ${ }^{2}$, Roberta Guarnone ${ }^{1}$, Sarah Macedonio $^{3}$, Valentina Angeli ${ }^{3}$, Luca Ciceri ${ }^{3}$, Antonello Malfitano ${ }^{4}$, Giuseppina Grugnetti ${ }^{5}$, Giovanni Ricevuti ${ }^{3}$, Maria Antonietta Bressan $^{6}$

${ }^{1}$ Emergency Department, IRCCS Policlinico San Matteo, Pavia, Italy, ${ }^{2}$ Emergency Department, Ospedale Civile, Vigevano, Italy,

${ }^{3}$ University of Pavia, Italy, ${ }^{4}$ Malattie Infettive IRCCS Policlinico San Matteo, Pavia, Italy, ${ }^{5}$ SITRA IRCCS Policlinico San Matteo, Pavia, Italy, ${ }^{6}$ Past Director Emergency Room, IRCCS Policlinico San Matteo, Pavia, Italy

Purpose: describe and analyze elderly population who go to the Emergency Department Organized By Different Level Of Intensity Of Care for fever caused by infectious.

Methods: we enrolled all patients wiage $>75$ years who went to the Emergency Department for fever caused by an infectious disease in 10 consecutive months (from 1st of January to the 31 th of October of 2018).

Results: 1636 patients were admitted for fever, of which 1034 were elderly. From all admissions the $4 \%$ was for fever. Only $30 \%$ of the elderly received the allocation to a low intensity level of care area, while $70 \%$ needed to be care in a medium-high intensity level of care. The pneumoniae and the sepsis determine a more high rage of the admission in the elderly, respectively (43\% vs $59 \%$ for the pneumoniae and $27 \%$ vs $20 \&$ for the sepsis). While the admissions for Urinary infectious disease are less frequent $(26 \%$ vs $40 \%$ of the general population). The admission for colecistite is $4 \%$ vs $5 \%$ of the general population. Frequently the elderly is recovered (77\% vs $55 \%)$. $70 \%$ of the hospital wards received a in patient from the ED, to do emocoltures. The admissions in ICU was less then $5 \%$.

Conclusions: The elderly with fever are frequently serious and they need a medium-high level of intensitive of care to take drugs, to multiparameters monitor and an observation. Frequently they are affected by sepsis or pneumoniae, determining a high admission rage.

\section{Abstract \# 22 \\ The effect of the elderly in-patients on the planning and the caryying out of the peimaf (intrahospital emergency plan for maxi-afflux of casualties). An Italian Second Level Dea Experience.}

Iride Francesca Ceresa ${ }^{1}$, Gabriele Savioli ${ }^{2}$, Valentina Angeli ${ }^{3}$, Viola Novelli ${ }^{4}$, Carlo Marena ${ }^{4}$, Alba Muzzi ${ }^{4}$, Giuseppina Grugnetti ${ }^{5}$, Giovanni Ricevuti ${ }^{3}$, Maria Antonietta Bressan ${ }^{6}$

${ }^{1}$ Emergency Department, Ospedale Civile, Vigevano, ${ }^{2}$ Emergency Department, IRCCS Policlinico San Matteo, Pavia, Italy, ${ }^{3}$ University of Pavia, Italy, ${ }^{4}$ Direzione Medica di Presidio IRCCS Policlinico San Matteo, Pavia, Italy, ${ }^{5}$ SITRA IRCCS Policlinico San Matteo, Pavia, ${ }^{6}$ Past Director Emergency Room, IRCCS Policlinico San Matteo, Pavia, Italy

Introduction: we want evaluate the effect of the vulnerable populations, in particular the elderly, on the Bed Surge Capacity IN Peimaf (Intrahospital Emergency Plan For Maxi-Afflux Of Casualties).
Methods: collect data about the numbers of the available/readily available beds in 18 wards of an Italian General Hospital with a II level DEA (Emergency-Acceptance Department) at 2 and $24 \mathrm{~h}-$ respectively, T2 and T24 -after an hypothetically Major Incident. The study consists of 4 simulations ( 3 for the Phase 1 or Study Phase and 1 for the Phase 2 or Operative Phase), both of which has 2 surveys (T2 and T4), detected on working days and on holidays.

Results: In 6 surveys 2326 inpatients are considered, with a media of 388 inpatients. Of which $52 \%$ are over 70 years old. Possibility to discharge/to transfer inpatients is influenced by: admission diagnosis, intensity level of care and age. Phase 1 reveals that $32,5 \%$ beds occupied by inpatients under 70 years are readily available versus only $23 \%$ occupied by under 70 . This data can be explained considering phisiopathology of elder population. Multimorbilities, disabilities, health instability and social factors of this vulnerable population determin the need to evaluate old inpatient using a multidimensional approach, considering both the diagnosis phase and the therapeutic process. All these factors determine elderly a vulnerable population to be need of an accurately hospital evaluation.Conclusions: The results highlight the nedd to consider the vulnerable population, as the elderly and the multipathological inpatients, in the planning of the PEIMAF.

\section{Abstract \# 23}

Elderly with pulmonary embolism presenting to the emergency department: phenomenology of the clinic and difficulties of atypical symptoms

Gabriele Savioli ${ }^{1}$, Iride Francesca Ceresa ${ }^{2}$, Michele Pagani ${ }^{3}$, Mirko Belliato, Giorgio Antonio Iotti ${ }^{3}$, Paolo Maggioni ${ }^{4}$, Massimiliano Lava $^{5}$, Federica Manzoni ${ }^{6}$, Giovanni Ricevuti ${ }^{4}$, Maria Antonietta Bressan $^{7}$

${ }^{1}$ Emergency Department, IRCCS POLICLINICO SAN MATTEO, Pavia, ITALY, ${ }^{2}$ Emergency Department, Ospedale Civile, Vigevano, ITALY, ${ }^{3}$ INTENSIVE CARE UNIT, IRCCS POLICLINICO SAN MATTEO, PAVIA, ITALY, ${ }^{4}$ University of Pavia, PAVIA, ITALY, ${ }^{5}$ RADIODIAGNOSTIC, IRCCS POLICLINICO SAN MATTEO, PAVIA, ITALY, ${ }^{6}$ Clinical Epidemiology and Biometry Unit, IRCCS POLICLINICO SAN MATTEO, PAVIA, ITALY, ${ }^{7}$ Past Director emergency room, IRCCS POLICLINICO SAN MATTEO, Pavia, ITALY

Introduction: we want Analyze patient over 65 years of age, affected by acute pulmonary embolism, and comparing those presenting typical symptoms with those presenting with atypical symptomatology. Methods: monocentric retrospective observational study on a group of elderly patients that includes all whom arrived at our emergency department and that received a diagnosis of pulmonary embolism (01/ 01/2017-31/12/2018).

Results: 71 patients were recruited, of which 56 with typical symptomatology and 15 with atypical symptoms. The two groups are comparable for mean age (respectively 78,77 years). Typical patients group showed a slight female majority (59\%), while the atypical group showed a slight masculine majority (56\%). Regarding vital parameters, patients with atypical symptomatology had desaturation $<90 \%$ (25\% vs $13.7 \%)$ and tachycardia with HR $>110$ bpm (25\% vs $10.3 \%)$. Despite this data, the patients with atypical symptomatology were underestimated at the Triage and more often it was given them a low priority code for the medical visit (35\% vs $23 \%$ ). Concerning outcomes, the patients with atypical symptomatology presented a moderate increase of prevalence of an augmented short-term mortality risk, calculated with sPESI index ( $47 \%$ vs $44 \%$ ), while no differences are present amongst the two groups for the length of stay and the need of hospitalization in the Intensive Care unit. 
Conclusions: the results show that the subpopulation of patients with atypical symptoms is not negligible (21\%) and is often underrecognized at the Triage, while it doesn't differ in a significative way for the severity of pulmonary embolism and for its outcomes.

\section{ABSTRACT \# 24 \\ Impact in the elderly of severity of pulmonary embolism as massive pulmonary embolism on the early recognition in the emergency department}

Gabriele Savioli ${ }^{1}$, Iride Francesca Ceresa ${ }^{2}$, Mirko Belliato ${ }^{3}$, Michele Pagani $^{3}$, Silvia Mongodi ${ }^{3}$, Giorgio Antonio Iotti ${ }^{3}$, Paolo Maggioni ${ }^{4}$, Massimiliano Lava ${ }^{5}$, Federica Manzoni ${ }^{6}$, Alba Muzzi ${ }^{7}$, Viola Novelli ${ }^{7}$, Giovanni Ricevuti ${ }^{4}$, Maria Antonietta Bressan ${ }^{8}$

${ }^{1}$ Emergency Department, IRCCS POLICLINICO SAN MATTEO, Pavia, ITALY, ${ }^{2}$ Emergency Department, ospedale civile, Vigevano, ITALY, ${ }^{3}$ INTENSIVE CARE UNIT, IRCCS POLICLINICO SAN MATTEO, PAVIA, ITALY, ${ }^{4}$ University of Pavia, PAVIA, ITALY, ${ }^{5}$ RADIODIAGNOSTIC, IRCCS POLICLINICO SAN MATTEO, PAVIA, ITALY, ${ }^{6}$ Clinical Epidemiology and Biometry Unit, IRCCS POLICLINICO SAN MATTEO, PAVIA, ITALY, ${ }^{7}$ Direzione Medica di Presidio IRCCS Policlinico San Matteo, Pavia, Italy, ${ }^{8}$ Past Director emergency room, IRCCS POLICLINICO SAN MATTEO, Pavia, ITALY

Aim: analyzing clinical manifestations and assess the earliness of identification from the Triage, in an Emergency Department divided in differentiated areas of care intensity, amongst elderly patients (age $>65$ years) arrived with symptoms that suggested the diagnosis of pulmonary embolism, comparing those who presented massive pulmonary embolism to those presenting a peripheral manifestation.

Methods: monocentric retrospective observational study from 01/01/ 2017 to $31 / 12 / 2018$.

Results: 72 patients were recruited, of which 41 with massive pulmonary embolism and 31 with peripheral manifestation. The two groups are superimposable according to age (mean age 78 years) and for a minimal female prevalence (58\% vs 54\%).Concerning assignment of priority code for the medical examination, the patients with massive pulmonary embolism presented a slight increase in the rate of high priority codes, however without reaching statistical significance. In particular, they had a rate of white codes of $0 \%$; green codes $25 \%$; yellow codes-low intensity care of $0 \%$; yellow codes-high intensity care of $75 \%$; red codes of $0 \%$. Patients presenting with peripheral pulmonary embolism presented a rate of white codes of $4 \%$; green codes of $33 \%$; yellow codes-low intensity care of $4 \%$; yellow codeshigh intensity care of $55 \%$; red codes of $4 \%$. Concerning allocation towards the medium intensity care unit, there is a higher allocation of patient with massive pulmonary embolism compared to patients with peripheral $(75 \%$ vs $59 \%)$. The rate of under-triage is superior in patients with peripheral pulmonary embolism (25\% massive vs $41 \%$ peripheral).

Conclusions: subpopulation with peripheral pulmonary embolism more frequently undergoes to under-triage.

\author{
Abstract \# 25 \\ Impact on elderly population of the presence of organ damage \\ in pulmonary embolism on the early recognition in the emergency \\ department from the admission \\ Gabriele Savioli ${ }^{1}$, Iride Francesca Ceresa ${ }^{2}$, Michele Pagani ${ }^{3}$, Mirko \\ Belliato $^{3}$, Silvia Mongodi ${ }^{3}$, Paolo Maggioni ${ }^{4}$, Massimiliano Lava, \\ Federica Manzoni ${ }^{6}$, Viola Novelli ${ }^{7}$, Alba Muzzi ${ }^{7}$, Giovanni Ricevuti ${ }^{4}$, \\ Giorgio Antonio Iotti ${ }^{3}$, Maria Antonietta Bressan ${ }^{8}$
}

${ }^{1}$ Emergency Department, IRCCS POLICLINICO SAN MATTEO, Pavia, ITALY, ${ }^{2}$ Emergency Department, Ospedale Civile, Vigevano, ITALY ${ }^{3}$ INTENSIVE CARE UNIT, IRCCS POLICLINICO SAN MATTEO, PAVIA, ITALY, ${ }^{4}$ University of Pavia, PAVIA, ITALY, ${ }^{5}$ RADIODIAGNOSTIC, IRCCS POLICLINICO SAN MATTEO, PAVIA, ITALY, ${ }^{6}$ Clinical Epidemiology and Biometry Unit, IRCCS POLICLINICO SAN MATTEO, PAVIA, ITALY, ${ }^{7}$ Direzione Medica di Presidio IRCCS Policlinico San Matteo, Pavia, Italy, ${ }^{8}$ Past Director emergency room, IRCCS POLICLINICO SAN MATTEO, Pavia, ITALY

Aim: analyzing the clinical manifestations and assess the earliness of detection from the Triage, in an Emergency Department divided in different areas of care intensity, in elderly patients (age $>65$ years) with acute pulmonary embolism, comparing those presenting with organ damage to those not presenting it.

Methods: monocentric retrospective observational study from 01/01/ 2017 to 31/12/2018. RESULTS: 72 patients were recruited, of which 30 with organ damage and 42 without it. The two groups were comparable for the age (mean age of 78 years). The group with organ damage showed a female prevalence $(65 \%)$, while the group without organ damage didn't show a specific gender prevalence (female/male $50 \%$ ). Concerning attribution of the priority code for the medical examination, the patients with organ damage were given a white code in $0 \%$ of cases; green code in $19 \%$ of cases; yellow code-low intensity of care in $3 \%$ of cases; yellow code-high intensity of care in $68 \%$ of cases; red code in $3 \%$ of cases. Patients not presenting organ damage were given a white code in $3 \%$ of cases; green code in $33 \%$; yellow code-low intensity of care in 3\% of cases; yellow code-high intensity in $61 \%$ of cases. Red code in $0 \%$ of cases. Concerning the allocation towards the medium intensity care, patients with organ damage are more directed to the medium intensity care compared to the patients without it (71\% vs $61 \%$ ), however without reaching statistical significance. The rate of under-triage is higher in patient with pulmonary embolism without organ damage (29\% with organ damage vs $39 \%$ without it).

Conclusions: subpopulation of elderly patients with pulmonary embolism without organ damage is more frequently subjected to under-triage.

\section{Abstract \# 26}

Shock indexes derived from vital parameters: their role in stratification of risk in the elderly populations with acute pulmonary embolism. markers of greater severity and worse outcomes

Gabriele Savioli ${ }^{1}$, Iride Francesca Ceresa ${ }^{2}$, Michele Pagani ${ }^{3}$, Mirko Belliato $^{3}$, Giorgio Antonio Iotti ${ }^{3}$, Silvia Mongodi ${ }^{3}$, Paolo Maggioni ${ }^{4}$, Massimiliano Lava ${ }^{5}$, Federica Manzoni ${ }^{6}$, Giovanni Ricevuti ${ }^{4}$, Maria Antonietta Bressan ${ }^{7}$

${ }^{1}$ Emergency Department, IRCCS POLICLINICO SAN MATTEO, Pavia, ITALY, ${ }^{2}$ Emergency Department, Ospedale Civile, Vigevano, ITALY, ${ }^{3}$ INTENSIVE CARE UNIT, IRCCS POLICLINICO SAN MATTEO, PAVIA, ITALY, ${ }^{4}$ University of Pavia, PAVIA, ITALY, ${ }^{5}$ RADIODIAGNOSTIC, IRCCS POLICLINICO SAN MATTEO, PAVIA, ITALY, ${ }^{6}$ Clinical Epidemiology and Biometry Unit, IRCCS POLICLINICO SAN MATTEO, PAVIA, ITALY, ${ }^{7}$ Past Director emergency room, IRCCS POLICLINICO SAN MATTEO, Pavia, ITALY

Aim: Assess the ability of shock indexes in a population of elderly to evaluete the severity grade of pulmonary embolism intended as presence of massive pulmonary embolism and increase of short-term mortality score (sPESI). 
Materials and methods: Monocentric, retrospective observational study on a group of patients older than 65 years $(01 / 01 / 2017-31 / 12 /$ 2018).

Results: 72 patients were recruited. The shock indexes considered: shock index (SI), modified shock index (MSI) and age-shock index (age-SI). SI was altered in $30 \%$ of patients with massive pulmonary embolism and in $16 \%$ of patients with peripheral one. Similar data for MSI (altered in $12 \%$ in massive, compared to $6 \%$ of peripheral). More interesting data concerning age-SI, altered in $62 \%$ of cases of massive pulmonary embolism and only in $24 \%$ of peripheral. The values of shock indexes considered were also averagely higher in patients with high short-term mortality index (SPESI), comparing classes with sPESI equal to 0 and 1 to the class with sPESI $>1$. In particular SI was altered in $31 \%$ of patients with elevated sPESI and in $18 \%$ of non-elevated sPESI. Similar data for MSI (altered in 24\% compared to $3 \%$ ). More interesting data of age-SI, altered in $58 \%$ of patients with elevated sPESI and only in $35 \%$ of patients with non-elevated sPESI.

Conclusions: shock indexes are easily available from the start, being obtained by the vital parameters and the patients' personal data. They allow an immediate identification of the most severe patients that present massive pulmonary embolism, higher short-term mortality risk and organ damage.

\section{Abstract \# 27 \\ Role of Intensive Brief Observation (IBO) unit in the management of patients with acute heart failure: real life example}

Gabriele Savioli ${ }^{1}$, Iride Francesca Ceresa ${ }^{2}$, Francesco Geraci ${ }^{3}$, Chiara Gagliardone $^{4}$, Maria Antonietta Bressan ${ }^{5}$

${ }^{1}$ Emergency Department IRCCS Policlinico San Matteo Pavia Italy, ${ }^{2}$ Emergency Department Ospedale Civile Vigevano Italy, ${ }^{3}$ ASST 118 Pavia, Italy, ${ }^{4}$ IRCCS Policlinico San Matteo Pavia Italy, ${ }^{5}$ Past Director Emergency Room IRCCS Policlinico San Matteo Pavia Ital

Purpose: Analyze impact that Intensive Brief Observation Unit (IBO, an area dedicated to observe unstable patients and stabilize them supervised by a team of chosen and dedicated doctors) can have in management patients over 75 years affected by AHF.

Method: longitudinal study from January 1st to December 31st 2018. Results: We enrolled 694 patients. Of these, 54\% were transferred to our IBO. The IBO population seemed to be more complex and unstable as demonstrated by higher percentage among these patients of high admission color codes (IBO $81 \%$ vs no-IBO $71 \%$ ), tachycardia (heart rate $>110 \mathrm{bpm}: 12 \%$ vs $10 \%$ ) and arterial hypertension (systolic blood pressure $>190 \mathrm{mmHg}$ : $3 \%$ vs $2.6 \%$; diastolic blood pressure $>120 \mathrm{mmHg}: 3.2 \%$ vs $2.9 \%$ ). In IBO stabilization rate was higher as indicated by reduction of color code upon discharge (IBO $44 \%$ vs no-IBO $30 \%$ ). Therefore, patients stabilized in IBO had a higher transfer rate to centers of less intensive care (10\% vs $5 \%)$ and a lower return rate at 7, 14 and 30 days (IBO patients: respectively $2 \%$, $5 \%, 13 \%$; no-IBO patients: respectively $3 \%, 7 \%, 15 \%$ ).

Conclusions: the Intensive Brief Observation Unit has gradually allowed improving in management patients with acute heart failure in Emergency Department, with particular attention to elderly and more fragile patients. Indeed, IBO Unit has the aim of correctly assess patients before potential hospitalization, instead hospitalize them for making diagnosis and treatment. This results in better management of patients with AHF, reduce their hospitalization rate and, consequently, optimize healthcare resources.

\section{Abstract \# 28}

Intracranial Haemorrhage (ICH) in Minor Head Injury (MHI): The Role Of Old Age

Gabriele Savioli ${ }^{1}$, Iride Francesca Ceresa ${ }^{2}$, Mirko Belliato ${ }^{3}$, Fabio Sciutti $^{3}$, Luca Ciceri ${ }^{4}$, Federica Manzoni ${ }^{5}$, Giovanni Ricevuti ${ }^{4}$, Sabino Luzzi ${ }^{6}$, Mattia Del Maestro ${ }^{6}$, Maria Antonietta Bressan

${ }^{1}$ Emergency Department IRCCS Policlinico San Matteo Pavia Italy, ${ }^{2}$ Emergency Department Ospedale Civile Vigevano Italy,

${ }^{3}$ INTENSIVE CARE UNIT, IRCCS POLICLINICO SAN MATTEO, PAVIA, ITALY, ${ }^{4}$ University of Pavia, PAVIA, ITALY, ${ }^{5}$ Clinical Epidemiology and Biometry Unit, IRCCS POLICLINICO SAN MATTEO, PAVIA, ITALY, ${ }^{6}$ Neurosurgery, IRCCS Policlinico San Matteo, Pavia, Italy, ${ }^{7}$ Past Director Emergency Room, IRCCS Policlinico San Matteo, Pavia, Italy

Background: Minor head injury (MHI) represents one of the most common causes of presentation to Emergency Departments.

Methods: evaluate all patients accessed for MHI during 2016 and 2017.

Results: We enrolled 2163 patients who reported MHI, of which 1088 aged over 69 years Elder population had higher ICH rates ( $12.1 \%$ vs $5.1 \%)$, higher hospitalization rates ( $11.7 \%$ vs $5.5 \%)$, higher rates of readmission at 30 days $(6.8 \%$ vs. $3.2 \%)$. Elder people took more often anti-platelet or anticoagulant therapy: in Younger Population $92 \%$ did not take drugs, $6 \%$ was in antiplatelet therapy, $1 \%$ in VKA and $1 \%$ in NOAC while in EP $41 \%$ did not take drugs, $39 \%$ was in antiplatelet therapy, $13 \%$ in VKA, $7 \%$ in NOAC. A major increase in the rate of intracranial haemorrhage has been noted in the EP in which the risk of bleeding increases by $180 \%(\mathrm{OR}=2.82, \mathrm{p}<0.001)$ compared to the YP. Age was an independent risk factor for bleeding.Conclusion \& perspectives: age is an important risk factor for intracranial haemorrhage. In particular the age of 70 entails an increase in the risk of bleeding by $180 \%$. While age represents a risk factor for $\mathrm{ICH}$, on the other hand the elder is a fragile subject more prone to falls, resulting in increased risk of MHI. Elder is also at risk for changes in his homeostasis and for taking more drugs. Elderly is more exposed to falls with MHI. Outcomes are worse in terms of ICH, hospitalization and returns.

\section{Abstract \# 29}

Experiences with and attitudes towards screening for geriatric vulnerability among older Emergency Department patients: a qualitative study

Laura C. Blomaard ${ }^{1}$, Mareline E. Olthof ${ }^{1}$, Yvette Meuleman ${ }^{1}$, Bas de Groot $^{1}$, Jacobijn Gussekloo ${ }^{1}$, Simon P. Mooijaart ${ }^{1}$

\section{${ }^{1}$ Leiden University Medical Center}

Background: Screening tools assessing geriatric vulnerability in older Emergency Department (ED) patients are seldom put to use in routine care. This study explores experiences with and attitudes towards routine screening for geriatric vulnerability among older ED patients.

Methods: Individual face-to-face semi-structured interviews were conducted at home in older patients ( $\geq 70$ years) within one month after they completed the 'Acutely Presenting Older Patient' (APOP) screener at the ED of Leiden University Medical Center. Purposive convenience sampling was used to select a heterogeneous sample of participants regarding age, disease severity and APOP-screening result. Interviews were audio recorded and transcribed verbatim. Transcriptions were analyzed inductively using thematic analysis with Atlas.ti software. (Preliminary) results. After 13 interviews (6 men, 7 women, median age 81 years), data saturation was reached. Most 
participants considered the vulnerability screening to be part of routine care and could not remember the particular screening questions, except for the cognition test. Their overall attitude towards the concept of screening was positive. Participants believed that vulnerability screening could help to identify the patient as a whole and adapt care to the patient's needs, which they considered important for all patients, regardless of age. Participants who did not consider themselves to be vulnerable, did not personally experience an added value of the screening but they did believed in the added value for others. Conclusion: From an ED-patients' perspective, screening for geriatric vulnerability was experienced as a part of routine ED care and was considered to be of added value for optimal ED care.

\section{Abstract \# 30 \\ Characteristics and outcomes of older patients visiting the Emergency Department with a fall}

Laura C. Blomaard ${ }^{1}$, Leonie J. van Meer ${ }^{1}$, Julia Leander ${ }^{1}$, Jacinta A. Lucke $^{2}$, Jelle de Gelder ${ }^{1}$, Sander Anten ${ }^{3}$, Jacobijn Gussekloo ${ }^{1}$, Bas de Groot $^{1}$, Simon P. Mooijaart ${ }^{1}$

${ }^{1}$ Leiden University Medical Center, ${ }^{2}$ Spaarne Hospital, ${ }^{3}$ Alrijne Hospital

Background: Older patients frequently visit the Emergency Department (ED) with falls. It is often unknown which of these falls represent frailty of the patient, although this might be important information for ED management. This study explores patient- and fall characteristics (causes and circumstances) of older patients with fallrelated ED visits and assesses if these characteristics are associated with adverse outcomes.

Methods: This was a secondary analysis of the observational multicenter Acutely Presenting Older Patient (APOP) study, in which ED patients aged $\geq 70$ years presenting with a fall were prospectively included. Fall characteristics were retrospectively collected from patient records. We compared adverse outcomes (functional decline and mortality 3 and 12 months after the ED visit), between patients with different patient- and fall characteristics.

Results: We included 393 patients with fall-related ED visits $(61.8 \%$ females, median age 80 (IQR 76-86) years). The cause of falls was extrinsic in $49.6 \%(n=195)$, intrinsic in $29.3 \%(n=115)$, unexplained in $6.4 \%(n=25)$ and missing in $14.8 \%(n=58)$ of patients. Patients had a higher risk on 3 months adverse outcomes if the cause of the fall was intrinsic compared to extrinsic (OR 2.14 (95\% CI 1.32$3.47)$ ), if they fell indoors (OR $3.03(1.80-5.11)$, if they had a hip fracture (OR $3.00(1.66-5.42)$ ), and if they were frail according to the APOP-screener (OR 3.72 (2.28-6.07)).

Conclusion: In older patients with fall-related ED visits, patient- and fall characteristics were associated with adverse outcomes. Both should be taken into account in the ED, and could guide follow-up geriatric assessment and interventions.

\section{Abstract \# 31}

\section{Falls in hospital: a case-control study}

G. Cathrine L. de Groot ${ }^{1}$, Ahmad Al-Fattal ${ }^{1}$, Irene Sandven ${ }^{2}$

${ }^{1}$ Department of Medicine, Telemark Hospital, Norway, ${ }^{2}$ Oslo Biostatistics and Epidemiology, Oslo University Hospital, Norway

Introduction: Falls among inpatients are common. This study was aimed at investigating major risk factors for falls in the hospital setting.

Methods: This was a retrospective case-control study. A total of 842 patients from three wards were included, whereof 172 cases had experienced one or more fall (s) during hospitalization and 670 random controls had not fallen. Data were analysed according to a pragmatic strategy.

Results: Compared to patients who did not fall, patients who fell were 21 times more likely to have poor balance $(\mathrm{OR}=21.50,95 \% \mathrm{CI} 10.26$ $-45.04)$ and 19 times more likely to have very poor balance $(\mathrm{OR}=$ $19.62,95 \%$ CI $9.55-40.27)$, twice as likely to be men $(\mathrm{OR}=1.82$, $95 \%$ CI $1.24-2.68)$, and $50 \%$ increased probability of fall with every 10 year increase of age $(\mathrm{OR}=1.51,95 \%$ CI 1.34-1.69). Furthermore, the patients who fell were more likely to use antidepressant drugs (OR $=3.85,95 \%$ CI $1.09-13.63)$, antipsychotic drugs $(\mathrm{OR}=3.27,95 \% \mathrm{CI}$ 1.94-5.51), anxiolytic/hypnotic drugs $(\mathrm{OR}=1.80,95 \% \mathrm{CI}$ 1.22-2.67), and antiepileptic drugs $(\mathrm{OR}=1.13,95 \% \mathrm{CI}: 1.11-4.06)$ than patients who did not fall.

Conclusions: During hospital stay, patients who fell had a higher risk profile than patients who did not fall. Clinicians should work to reduce the risk of falls by accurately assessing balance and mobility as a form of primary prevention. We recommend that a review of the patient medications should be conducted upon falling, as a form of a secondary preventive strategy against falls.

\section{Abstract \# 32 \\ Educating clinical technicians on hospital acquired pressure injury \\ Melissa Smith Eichenlaub ${ }^{1}$ \\ ${ }^{1}$ Columbus State University}

Hospital-acquired skin injury (HAPI) is described as preventable events, but in many instances prevention is relative to knowledge deficit. HAPI is not ole the responsibility of the nurse, but inclusive of the entire healthcare team. Acute care facilities continue to see an increase in the number of HAPI resulting in increased mortality rates annually. In order to preserve and reduce the current rates, development of education specific to the clinical technician (CT) will promote a comprehensive approach for this level of care provider. Prevention of HAPI is of great concern, therefore ensuring that the knowledge deficit is not the cause, a proactive approach to education for the CT is vital.

\section{Abstract \# 33 \\ Importance of previous hospital stays on the risk of hospital readmission in older adults: a real-life analysis of the PAERPA study population}

Fabien Visade ${ }^{1}$, Genia Babykina ${ }^{2}$, Antoine Lamer $^{3}$, Marguerite-Marie Defebvre $^{4}$, David Verloop ${ }^{4}$, Grégoire Ficheur ${ }^{5}$, Mickael Genin ${ }^{5}$, François Puisieux ${ }^{5}$, Jean-Baptiste Beuscart ${ }^{5}$

${ }^{1}$ Lille catholic hospitals, Geriatrics Department, Lille, France.4- Univ. Lille, CHU Lille, ULR 2694-METRICS: Évaluation des Technologies de Santé et des Pratiques Médicales, F-59000 Lille, France, ${ }^{2} 4$ - Univ. Lille, CHU Lille, ULR 2694-METRICS: Évaluation des Technologies de Santé et des Pratiques Médicales, F-59000 Lille, France, ${ }^{3} 1$ - Univ. Lille, CHU Lille, ULR 2694-METRICS: Évaluation des Technologies de Santé et des Pratiques Médicales, F-59000 Lille, France, ${ }^{4}$ ARS Hauts de France, 556 Avenue Willy Brandt, F-59777 EuraLille, France., ${ }^{5}$ Univ. Lille, EA2694 - Evaluation des Technologies de Santé et des Pratiques Médicales, F-59000 Lille, France

Background: Consideration of the first hospital readmission only, and failure to take account of previous hospital stays, are two significant limitations when studying risk factors for hospital readmission.The objective of the study was to use appropriate 
statistical models to analyze the impact of previous hospital stays on the risk of hospital readmission among older patients.

Method: An exhaustive analysis of hospital discharge and health insurance data for a cohort of patients participating in the PAERPA ("Care Pathways for Elderly People at Risk of Loss of Personal Independence") project in the Hauts de France region of France. All patients aged 75 or over were included. All data on hospital readmissions via the emergency department were extracted. The risk of unplanned hospital readmission was estimated by applying a semiparametric frailty model, the risk of death by applying a timedependent semiparametric Cox regression model.

Results: A total of 24,500 patients (median [interquartile range] age: 81 [77-85]) were included between January 1st, 2015, and December 31 st, 2017. In a multivariate analysis, the relative risk [95\% confidence interval $(\mathrm{CI})]$ of hospital readmission rose progressively from 1.8 [1.7-1.9] after one previous hospital stay to 3.0 [2.6-3.5] after five previous hospital stays. The relative risk [95\% CI ] of death rose slowly from 1.1 [1.07-1.11] after one previous hospital stay to 1.3 [1.1-1.5] after five previous hospital stays.

Conclusion: Analyses of the risk of hospital readmission in older adults must take account of the number of previous hospital stays. The risk of death should also be analyzed.

\section{Abstract \# 34}

Urinary catheterization in the hospitalized elderly patients

Maria Elena Castro Vilela ${ }^{1}$, Miguel Sanchez Ortiz ${ }^{1}$, Elisa García Simón ${ }^{1}$, Ana Mateo Abad ${ }^{1}$, Maria Del Mar Soguero Perez ${ }^{1}$

${ }^{1}$ Hospital San Jose-Teruel-Spain

Introduction: To investigate prevalence and factors associated with the use of urinary catheters in hospitalized elderly patients.

Method: The retrospective study enrolled patients admitted, between January and December 2019 in the geriatric service of the Hospital San Jose with urinary catheterization during their hospital admission. Characteristics of patients and catheter use were collected.

Results: 970 patients were admitted during the period of the study, of whom $99(10.20 \%)$ used urinary catheters: 23 , permanent and 76 (76.7\%) temporarily. Males: $60.6 \%$. Average age: 86.5 years. Most common admission diagnoses: respiratory infection (23.2\%) and urinary tract infection (16.2\%). Average length of stay: 17 days. Totally dependent: $49 \%$. Polymedication: $87.7 \%$. Delirium:49\%. Comorbidity: Dementia (42\%), diabetes $(35 \%)$, congestive heart failure $(46.5 \%)$, chronic kidney disease $(44.4 \%)$, urinary incontinence $(79 \%)$, benign prostatic hyperplasia ( $70 \%$ of males).Regarding temporary urinary catheters: $26.3 \%$ were placed in the emergency room, $28.9 \%$ in another medical service and $43.4 \%$ in the geriatric service. The median of days with the catheter was 7.5 days. The most common reason to use urinary catheter was acute urinary retention (AUR) $(67.7 \%)$. After catheterization, a urine culture was needed in $55.6 \%$ of patients and among these, $64.3 \%$ were positive. At hospital discharge $28.3 \%$ of patients needed to continue with the urinary catheter.

Conclusions: In our study, the most common cause of urinary catheterization during the hospitalization is AUR.-The average urinary catheterization time is high.-It is necessary to improve the prescribing habits of urinary catheterization and its early withdrawal through specific educational efforts.

\section{Abstract \# 35}

Prospective determination of the incidence and severity of hyponatremia in elderly hospitalized patients with acute urinary tract obstruction

Dvorah Sara Shapiro $^{1}$, Irina Alexandrovich ${ }^{2}$, Moshe Sonnenblick ${ }^{1}$, Linda Shavit ${ }^{3}$, Gavriel Munter ${ }^{4}$, Reuven Friedmann ${ }^{5}$

${ }^{1}$ Shaare Zedek Medical Center Jerusalem Israel Department of Geriatrics, ${ }^{2}$ Shaare Zedek Medical Center Jerusalem Israel Department of Geriatrics Department of Geriatrics, ${ }^{3}$ Shaare Zedek Medical Center Jerusalem Israel Adult Nephrology Unit, ${ }^{4}$ Shaare Zedek Medical Center, Medicine C and Endocrine Unit, ${ }^{5}$ Shaare Zedek Medical Center Department of Geriatrics

Abstract: Introduction: Acute urinary tract obstruction (aUTO) is a common finding in hospitalized elderly patients. Anecdotal reports from our hospital and elsewhere have drawn attention to hyponatremia in patients with aUTO, which subsides rapidly with relief of the obstruction. The aim of this study was to determine the incidence, severity and subsequent correction of hyponatremia in patients with aUTO.

Methods: Prospective assessment of serum sodium of 104 patients diagnosed with aUTO during 4 years, as compared with a control group of 50 patients with urinary bladder catheterization without obstruction during the same period.Results: There were no statistically significant differences between the groups in age, major comorbidities, chronic medical treatment and causes of hospitalization. $29(28 \%)$ patients in the urinary retention group developed hyponatremia (mean $127.7 \mathrm{meq} / \mathrm{L}$ ) as compared to $9(18 \%)$ patients in the control group (mean $130.2 \mathrm{meq} / \mathrm{L}$ ). Seven of the patients with aUTO presented with severe hyponatremia $(<125 \mathrm{meq} / \mathrm{L}$, mean $119.6 \mathrm{meq} /$ L) compared to none of the control group. Serum sodium levels in these seven patients increased to $126.4 \mathrm{meq} / \mathrm{L}$ within $24 \mathrm{~h}$ of catheter insertion ( $p=0.04)$. In 4 of these 7 patients, catheter insertion was the only change in management preceding a rise in the serum sodium level.

Key conclusion: Hyponatremia is a common finding in patients with aUTO. Insertion of a urinary catheter is often sufficient in the correction of hyponatremia. It is unclear whether urinary retention is a cause or consequence of hyponatremia.

\section{ABSTRACT \# 36}

Respiratory syncytial virus (RSV) vs Influenza virus: mortality and complications comparison in a geriatric population.

Caryn Recto $^{1}$, Cristiano Donadio ${ }^{2}$, Maurizio Magri ${ }^{3}$, Lorenza di Rosario $^{4}$, Antonio Rainone ${ }^{3}$, Bruno Oquendo ${ }^{5}$, Christian de Tymowski ${ }^{6}$, Christian Kassasseya ${ }^{7}$

${ }^{1}$ Sorbonne Université, Paris FRANCE, ${ }^{2} 1$ University Hospital PitiéSalpêtrière- Charles Foix, Geriatric's Department, AP-HP, Sorbonne University, Paris, France., ${ }^{3}$ University Hospital Pitié-SalpêtrièreCharles Foix, Geriatric's Department, AP-HP, Sorbonne University, Paris, France., ${ }^{4}$ Geriatric's Department « Centre Intercommunal De Créteil » Hospital, Creteil, France., ${ }^{5}$ University Hospital PitiéSalpêtrière - Charles Foix, Geriatrics Departement La Triade, Sorbonne Université, Paris- France., ${ }^{6}$ Anesthesia \& Intensive Care Department, AP-HP, CHU Bichat-Claude Bernard, Paris Nord Val de Seine University, Paris, France., ${ }^{7}$ Emergency Department, APHP, CHU Henri Mondor, Creteil's Paris-Est University, Creteil, France.

Introduction: RSV infection is underestimated in adults as they tend to have more variable and less distinctive clinical findings than children, being the viral infection often unsuspected and symptoms attributed to underlying conditions. 
Methods: Over the 1225 polymerase chain reaction performed in the $>75$ years old patients consulting in the Emergency Room of Herni Mondor's University Hospital, at Creteil, France, in 4 consecutive winters, 60 tested positive to RSV and 292 to influenza virus. We compared these groups in terms of mortality and complications: secondary bacterial pneumonia, underlying condition's decompensation, in-hospital admission or intensive care unit (ICU) admission criteria.

Results: RSV and Influenza groups were comparable in terms of age, comorbidities and autonomy. The average age was 84,8 years (75-102.4), patients older than 80 years representing $77 \%$ of the total. The mortality rate was comparable between groups at 28 (8.3 vs $8.2 \%, \mathrm{p} 0.57$ ) and 365 days ( 15 vs $14 \%, \mathrm{p} 0.49)$, as it was the inhospital length of stay ( 7 days vs 5 days, p 0.16 ) and ICU admission criteria (10\% vs $7.2 \%$, p 0.43). In-hospital admission was more frequent in the RSV group (83.3\% vs $70.9 \%$, p 0.031), as it were decompensation of an underlying condition (50\% vs $31.7 \%$, p 0.006), and confirmed pneumonia (30\% vs $17.1 \%$, p 0.020 ).

Key conclusions: RSV infection is comparable to influenza-virus infection in terms of mortality, being non-lethal complications statistically more frequent among RSV-infected patients.

\section{Abstract \# 37 \\ Assessing the impact of serious game-based interventions in hospitalized older patients: a systematic review.}

César Cuevas Lara ${ }^{1}$, Mikel Izquierdo ${ }^{2}$, Mikel L Sáez de Asteasu ${ }^{1}$, Robinson Ramírez-Vélez ${ }^{1}$, Fabiola Zambom-Ferraresi ${ }^{1}$, Fabricio Zambom-Ferraresi ${ }^{1}$, Nicolás Martínez-Velilla ${ }^{3}$

${ }^{1}$ Navarrabiomed, Hospital Complex of Navarra (CHN) - Public University of Navarra (UPNA), Navarra Health Research Institute (IdisNa), Pamplona, C/Irunlarrea 3, 31008 Pamplona, Navarra, Spain., ${ }^{2}$ Health Science Department, Public University of Navarra, C/Irunlarrea s/n, 31008 Pamplona, Navarra, Spain, ${ }^{3}$ Geriatric Department, Hospital Complex of Navarra (CHN), C/Irunlarrea 3, 31008 Pamplona, Navarra, Spain.

Objectives: To assess the effectiveness of game-based interventions on health-related outcomes in acutely hospitalized older patients. Materials and methods: Randomized controlled trials (RCT) and non-randomized trials for assessing the impact of games interventions on health-related outcomes (i.e. functional capacity, quality of life) in older adults (aged 65 years or older) admitted to an Acute Care for Elderly unit were selected.

Results: 4 RCTs were included in the review. The interventions were based on the implementation of serious games programs using Nintendo Wii in acute medical patients. Across the included studies, no significant differences were observed between groups on functional capacity and health-related quality of life. Significant differences were found between groups on the adherence to treatment (in favor of the control group), but no differences were obtained in other outcomes such as enjoyment and motivation.

Conclusions: The findings suggest that the existing evidence is very limited and insufficient to state that game-based interventions are effective for improving health-related outcomes in acutely hospitalized older adults.

\section{Abstract \# 38}

Are patients having their capacity to agree to remain in hospital assessed and a deprivation of liberty safeguard put in place?

Soumia Lakehal ${ }^{1}$, Richard Gentry ${ }^{1}$, Samin Chowdhury ${ }^{1}$, Arturo Vilches-Moraga ${ }^{1}$
${ }^{1}$ Ageing and Complex Medicine Department, Salford Royal NHS Foundation Trust

Introduction: The Deprivation of Liberty Safeguards (DoLS) is a legal framework designed to protect the liberty of incapacitated patients admitted to hospital in England and Wales. The initial purpose of this Quality Improvement project was to determine whether cognitively impaired patients had their capacity to consent to remain in hospital assessed on admission to acute geriatric wards and if a DoLS urgent requests were completed when appropriate. As the project evolved, we also tested the impact of providing training sessions for doctors and nurses aimed at improving engagement with mental capacity assessment (MCA) and DoLS.

Method: We completed 7 PDSA cycles between September 2018 and March 2020. At each cycle, we prospectively reviewed the Electronic Patient Records of all patients discharged from 2 geriatric wards and we recorded documentation of MCA to consent to remain in hospital and DoLS urgent requests. During this time we completed 4 teaching interventions involving educating medical students, junior doctors, consultants and nurses on how to assess capacity, the principles of DoLS, when one should be applied for and how patients benefit.

Results: A total of 387 cases were reviewed. Pre-intervention, $22 \%$ of patients had MCA documented and 0\% had an urgent DoLS request made. Post-interventions $80 \%$ of patients had assessment MCA documented and 32\% had an urgent DoLS request

Key conclusions: Although we achieved significant improvement in February 2020 through staff education and engagement, the number of urgent DoLS requests declined significantly in March 2020 amid COVID-19 pandemic and changes to medical/nursing ward cover.

\section{Abstract \# 39}

\section{Geriatric care in Belgium during COVID pandemic}

Johan Flamaing $^{1}$, Nicolas Berg ${ }^{1}$

${ }^{1}$ Belgian Society of Gerontology and Geriatrics

Older people are among the most affected by the COVID-19 epidemic. However, little specific attention has been paid to the management of the epidemic and the scientific literature is scarcely dedicated to older persons. Through an online questionnaire, we collected the experience of Belgian geriatricians and their hospital teams in the management of the geriatric COVID-19 patients.The survey started on 18/5/2020 and includes 40 questions on geriatric practice, on the multidisciplinary approach, on consultation on the intensity of care, on collaboration with nursing homes, and the impact on standard geriatric care. The subjective perception of the geriatricians was also noted.The survey has not yet been completed; we currently have 57 responses, on approximately 300 active Belgian geriatricians. The first results show an involvement of geriatricians in all CoviD units, whether geriatric or not. Ambulatory activities have ceased. Geriatricians have often been involved in the triage of patients in the emergency and intensive care units (ICU), but the age limit rarely appears to be a hindrance, except in cases of saturation of ICU beds, where the age limit of 80 years is the most frequent. A sense of value, recognition and solidarity are the positive elements; stress, isolation, and the media's misinterpretation of the involvement of gerritricians in COVID-management in nursing homes are the negative elements. The COVID pandemic has profoundly changed geriatric practices, but the involvement of geriatricians has been important at all levels. Sharing the Belgian experience is an initiative of the Belgian Society of Gerontology and Geriatrics to recognize and value this involvement. 


\section{Abstract \# 40}

Two-way negative relationship between clinical frailty/functional status and hospitalization of older patients with infection in a nongeriatric department

Efterpi Mougakou ${ }^{1}$, Evridiki Kravvariti ${ }^{2}$, Michail Ampeliotis ${ }^{3}$, Dionysios Mantzos ${ }^{3}$, Stavroula Fotiadou ${ }^{3}$, Vassiliki Tzavara ${ }^{3}$

${ }^{1}$ First Department of Internal Medicine, "Erythros Stavros" Hospital, Athens, Greece/Postgraduate Medical Studies in the Physiology of Aging and Geriatric Syndromes, School of Medicine, National and Kapodistrian University of Athens, Postgraduate Medical Studies in the Physiology of Aging and Geriatric Syndromes, School of Medicine, National and Kapodistrian University of Athens, ${ }^{3}$ First Department of Internal Medicine, "Erythros Stavros" Hospital, Athens, Greece

Introduction: Physical frailty is an identified risk factor for in-hospital mortality. In the Greek health system, due to lack of specialty of geriatrics, older people are hospitalized in Internal Medicine Departments with no recordings of frailty status or the interplay between functional status and clinical outcomes.

Methods: Patients aged $\geq 65$, admitted to the general medical ward of a tertiary hospital due to acute infection were recorded over a 1 -year period (2019). Pre-admission and discharge frailty and functional status were evaluated using the Clinical Frailty Scale (CFS) and the modified Rankin Scale (mRS).

Results: Among 322 eligible patients, 314 were included (51\% females, median age 82 years). Urinary (48\%) and low respiratory tract $(43 \%)$ were the most common sites of infection, combined in $7 \%$ of patients. Mean pre-admission mRS score was 3.5 ( \pm 1.44 ): $55 \%$ of patients had loss of autonomy (mRS $\geq 4)$. Mean CFS was 5.6 ( \pm 1.99): $21 \%$ with CFS1-3 (non-frail). Discharge mRS increased into $35 \%$ of patients and CFS into 29\%. Among patients with initially preserved autonomy (mRS0-3), 20\% lost autonomy (mRS4-6). Among patients characterized initially as non-frail (CFS1-3), 31\% became frail (CFS4-9). In-hospital mortality was 16\%: patients with preadmission mRS4-6 had a 6-fold increased risk than those with mRS0-3 (95\% CI 2.78-14.71, p-value < 0.05).

Conclusions: Preadmission physical frailty is highly prevalent in Greek older general medical ward inpatients and functional dependence, as evaluated by the mRS, is strongly associated with inhospital mortality. Additional measures are required to prevent further loss of function during hospitalization, both among frail and non-frail inpatients.

\section{Abstract \# 41 \\ Investigation of opposition to diagnostic or therapeutic procedures in older people hospitalised in acute geriatric services: the OPTAH study. \\ Thomas Tannou ${ }^{1}$, Hélène Trimaille ${ }^{1}$, Florence Mathieu-Nicot ${ }^{1}$, Séverine Koeberle ${ }^{1}$, Régis Aubry ${ }^{1}$, Aurélie Godard-Marceau ${ }^{1}$ \\ ${ }^{1} \mathrm{CHU}$ de Besançon - France}

Introduction: Opposition and refusal of care are key concepts in geriatric research. Even if these concepts are frequently used, they are not yet well understood in the medical literature, and there is a lack of consensus on their definition. We therefore explored those concepts by defining opposition to diagnostic or therapeutic proposals in geriatrics acute care in a reproducible framework.

Method: Our study is based on a mixed methodology (epidemiological and qualitative research). Opposition and refusal as defined were quantified, and semi-structured interviews were conducted with patients, their relatives and referring carers to explore their core elements. Multidisciplinary meetings that were associated with these situations were also analysed.

Results: Thirty-three situations were included in the epidemiological component, of which 5 situations were included in the exploratory qualitative component leading to 15 interviews. Of the 33 situations, only 6 corresponded to an informed rejection of the proposed acute medical management. After multidisciplinary meetings, 12 situations were actively managed despite opposition. Persistent opposition situations usually occurred on the 9th day of hospitalization Interviews show that good symptom control and anosognosia modify the patient's adherence to medical strategies that he does not understand because he no longer feels sick. This favours the rupture of therapeutics before their term or etiological investigations and preventive strategies.

Conclusion: The consequences of these situations are major in terms of public health with, in addition to the increase in the length of hospital stays, excess mortality and early re-hospitalisation. They also have consequences on the daily management of care.

\section{Abstract \# 42}

\section{Developing an integrated comprehensive geriatric unit}

Christopher Sin Chan $^{1}$, Malin Farnsworth, Rachel Emery ${ }^{1}$, Chirag Patel $^{1}$, Blessing Mazanzi ${ }^{1}$, Cath Merrick ${ }^{1}$

${ }^{1}$ Epsom General Hospital \& Surrey Downs Health and Care

Introduction: Surrey Downs Health and Care (SDHC) is an innovative partnership consisting of the acute trust, community provider, three local GP federations and local authority. Together they deliver integrated health and care services for the Surrey Downs population. .In April 2019, SDHC formally took over the management of an acute escalation ward at Epsom General Hospital. The aim was to redesign the model of care to offer a more integrated approach towards the management of patients with frailty.

Method: A change in leadership with interface frailty consultants developing an integrated multidisciplinary team (MDT) with reassignment of community staff. All members of the MDT had an equal voice and this helped develop the one team ethos.There were many developments along the way, but key changes included the agreement that a patients' time is the most valuable currency and that we should be changing conversations from "what is the matter with you?" to "what matters most to you?".

Results: 1. A $100 \%$ increase in average daily discharges 2. An increase to $70 \%$ being discharged to their own home, versus $20 \%$ previously 3 . A reduction from an average length of stay of 40-13 days compared to the same time last year 4 . Reduced 30-day readmissions at $15 \%$ versus previous average of $25 \%$.

Conclusion: By blurring boundaries between the acute and community, allowed a frictionless pathway for patients. This has led to improvement in patient care and outcomes for the patient and system as a whole.

\section{Abstract \# 43}

Gastrointestinal bleeding in the elderly and contribution of the ultrasound

Vito Carrieri ${ }^{1}$, Antonella Bray $^{2}$, Giovanni Argentieri ${ }^{2}$, Gloria Mazelli $^{2}$, Vincenzo Paternò ${ }^{2}$, Luca Di Lena ${ }^{2}$

${ }^{1}$ Geriatrics Department Hospital ”A. Perrino“ Brindisi Italy, ${ }^{2}$ Geriatrics Department Hospital ”A. Perrino“ Brindisi Italy

Introduction: The objective of the study is to verify in a population of 300 patients over the age of 65 , admitted to Geriatrics with the 
diagnosis of gastrointestinal bleeding, melena, hematemesis, enterorrhagia, rectorrhagia, bleeding from esophageal varices or from hemorrhoids, if the ultrasound (US) performed before the endoscopy allows to modify the diagnostic procedure, increasing the appropriateness of the diagnostic and therapeutic prescriptions.

Materials and methods: 300 patients were included in the study, over the age of 65 (170 women, 130 men, from 65 to 95 years) hospitalized in Geriatrics. All patients underwent US. Subsequently, in all cases, an esophagogastroduodenoscopy (EGDS) and/or colonoscopy and/or rectoscopy was performed as an emergency. US diagnoses were correlated with clinical data and endoscopic diagnosis.

Results: The diseases detected with US were: 95 liver cirrhosis, 35 hepatocarcinomas, 20 pancreatic neoplasms, 10 gallbladder neoplasms, 22 suspected abdominal neoplastic pathologies, 30 abdominal ascites, 10 masses from gastroduodenal neoplasia, 10 masses from colon cancer, 18 pelvic masses from likely uteroovaric neoplasia. In 50 patients the US was normal. Endoscopic diagnoses were then analyzed in these 300 patients. Esophageal, gastric or duodenal or rectal bleeding was present in 60 cases of cirrhosis and in $20 \mathrm{HCC}$. In all other cases, it was necessary to prescribe CT or angioCT or arteriography, which showed bleeding of varying amounts in correspondence with the neoplastic masses suspected by the US. In all cases, at the same time as the request for second level diagnostic tests, specialist advice (surgical, urological, gynecological, etc.) was requested in relation to the clinical situation and the diagnostic suspicion formulated by the US. In addition, the urgent therapeutic decisions, always completed by the request for blood transfusion, were set up taking into account the US reports and endoscopy, pending the report of the second level investigations required for further investigation.

Conclusions: In conclusion, the US, even in the presence of gastrointestinal bleeding, can be considered as the most appropriate method to be performed in emergency, before endoscopy. The US abdominal examination in fact in most cases allows to detect unknow diseases of the elderly patient, to prescribe appropriated diagnostic procedures and the therapy. In the elderly patient with numerous chronic diseases, in fact, often gastrointestinal bleeding can be caused by drugs or other pre-existing diseases that are associated with neoplastic pathologies that endoscopy alone cannot detected. Therefore, before prescribing invasive investigations, the US allows easily to study the abdomen and often the unknown neoplastic pathologies that are the cause of progressive increase of anemia in the elderly, especially if associated with iatrogenic causes, can be quickly detected.

\section{Abstract \# 44 \\ Seasonal incidence of community-acquired pneumonia: a retrospective study in a Tertiary Care Hospital in Kathmandu, Nepal \\ Dr. Mohammed Ainnul Haque ${ }^{1}$ \\ ${ }^{1}$ Patan hospital Nepal}

Introduction: Community-acquired pneumonia (CAP) is the major cause of death in adult and elderly persons with a variety of presentations. Seasonal variation in the incidence of the disease is essential for clinicians and epidemiologists who deal with such diseases. The study was aimed at analysing the clinical profile and outcomes of community-acquired pneumonia during different seasons of the year in a tertiary care hospital, Manmohan Memorial Teaching Hospital (MMTH), of Kathmandu, Nepal.

Method: The aetiology and clinical profile of 378 patients with CAP who were admitted to MMTH over a period of 1 year were taken into account in this retrospective cross-sectional hospital-based study.
Data were retrieved from the hospital medical records section and the Department of Pathology. All patients with a primary diagnosis of CAP admitted to the hospital were included in the study. Monthly and seasonal trends, aetiology, comorbidities, and mortality rates were analysed.

Results: Of 378 patients with CAP, 160 patients (42.3\%) had associated chronic obstructive pulmonary disease (COPD), 92 patients (24.3\%) had hypertension (HTN), 59 patients (15.6\%) had diabetes, 12 patients $(3.1 \%)$ had active pulmonary tuberculosis, seven patients $(1.85 \%)$ had kidney disease, and the remaining 48 patients $(12.6 \%)$ had only CAP. Seasonal variation of CAP was noted in 131 patients $(35 \%)$ in the winter, $98(26 \%)$ in autumn, $86(23 \%)$ in spring, and 63 patients $(16 \%)$ in summer seasons. None of the patients were vaccinated against influenza and pneumococcus. The most common organism isolated in CAP was Acinetobacter calcoaceticus baumannii (ACB) complex (4.7\%), which was more distinguished in the winter season. The second most isolated organism was Pseudomonas aeruginosa $(2.6 \%)$. The most common clinical presentation was fever $(63 \%)$, followed by cough $(47 \%)$ and shortness of breath $(47 \%)$. Sputum culture was found to be positive in 51 cases (13.4\%). Among 378 patients, 78 patients $(20.6 \%)$ received treatment in the Intensive Care Unit (ICU) and the rest of the patients received treatment in the general medical ward. The mortality rate was found to be $6.6 \%$.

Conclusion: An incidence of sputum-positive CAP was found in 51 cases $(13.4 \%)$. The most common organism was ACB complex, followed by Pseudomonas aeruginosa, which were sensitive to polymyxins. Both of them were predominant in the winter and spring.

\section{Abstract \# 45}

Not all fever indicates Coronavirus (COVID- 19) illness at the pandemic time. The case report of a Perforated Appendicitis in a 79- years old patient.

\section{Oksana Borodin $^{1}$, Tal Orenstein ${ }^{2}$, Yael Orion ${ }^{2}$}

${ }^{1} \mathrm{MD}$, Tel Aviv Sourasky Medical Center (Ichilov), Internal Geriatric Department, Tel Aviv, Israel.Tel Aviv University, Sackler Faculty of Medicine, Tel Aviv, Israel., ${ }^{2} \mathrm{MD}$

The patient, a 79-year-old male, was admitted to the hospital with a fever after exposure to a patient with Covid-19. He had no abdominal pain, vomiting, dysuria, cough or dyspnea. He had no abdominal pain and physical examination was normal, but he looked ill. Patient's blood tests demonstrated leukocytosis and elevated CRP while COVID- 19 PCR was negative.The patient has been treated with empiric antibiotic therapy. Due to a lack of clinical response to the treatment, $\mathrm{CT}$ of chest and abdomen was performed where perforation of the appendix was diagnosed. Acute perforation of the appendix is one of the complications of appendicitis that is associated with increased morbidity and mortality. The common risk factors for perforated appendicitis include extremes of age, male sex, immunosuppression, comorbid medical conditions and previous abdominal surgery. Another risk factor late arrival for medical treatment. Among the key reasons for this are living alone, fear of hospitals.Less than half of the elderly patients with acute appendicitis present with the classical symptoms including nausea, loss of appetite, abdominal pain and localized tenderness.Time duration from onset of symptoms to ED arrival $>24 \mathrm{~h}$, heart rate $\geq 90$ beats/min, respiratory rate $\geq 20$ breaths/min and generalized guarding are the significant factors associated with perforated acute appendicitis in geriatric patients.

Conclusion: Elderly patients have atypical clinical features. A high degree of suspicion and early use of CT scan can shorten the time to the appropriate treatment and improve outcomes. 


\section{Abstract \# 46 \\ Study of patients aged 90 and over admitted to a geriatric ward for COVID 19}

Mariam Rodriguez-Piñera ${ }^{1}$, Mónica Fernández ${ }^{1}$, Laura Pellitero ${ }^{1}$, Beatriz García ${ }^{1}$, Carlos Martinez ${ }^{1}$, Jesús Canteli ${ }^{1}$, Luis Hevia ${ }^{1}$, Francisco Suárez ${ }^{1}$

${ }^{1}$ Hospital Cruz Roja Gijón

Introduction: During the SARS-COV2 pandemic, elderly people were the most affected. We believe it is relevant to learn the characteristics of patients aged 90 and over who required hospitalization for this reason, and whether the deceased showed some distinctive features.

Materials and method: Sample group: People aged 90 and over hospitalized by COVID 19 between 15/03/2020 and 15/05/2020. Not ICU candidates. Where: Geriatric ward at Hospital Cruz Roja Gijón (Asturias, Spain).Variables: (a) Sociodemographic (age, gender and place of residence); (b) Functional (Barthel index and mobility); (c) Pathology (Hypertension, Diabetes, COPD, Heart failure, AFib, previous stroke, kidney failure, dementia; (d) Drugs (ACE inhibitors/ ARBs) and more than 8 drugs a day; (e) Status on admission (CURB 65 , oxygen saturation, radiology, blood test); (f) Specific pharmacological treatment.

Results: There were 37 patients hospitalized. Average stay: 14.5 Average age: 93.2 (90-100), 70\% women, $70 \%$ in care institutions. Average Barthel index 55.6, and $19 \%$ were highly dependent. Most frequent pathologies were hypertension $(73 \%)$, dementia $(46 \%)$ and diabetes $(32 \%)$. Average oxygen saturation on admission was $90 \%$. $42 \%$ were treated with hydroxychloroquine, $55 \%$ with azithromizine and $8 \%$ with lopinavir or corticosteroids. Twelve patients $(32 \%)$ died in hospital. The bivariate analysis shows no relationship between mortality and the other variables.

Conclusions: Within our sample group, no association could be established between age, type of treatment or previous pathology and the risk of death. Given the small sample of this study, more research is needed to look at the variables of poor prognoses in such elderly people.

\section{Abstract \# 47 \\ Effects of a gamification program on mobility in hospitalized older patients: a pilot study}

César Cuevas-Lara ${ }^{1}$, Mikel L. Sáez de Asteasu ${ }^{2}$, Fabiola ZambomFerraresi $^{2}$, Cristina Antoñanzas-Valencia ${ }^{2}$, Fabricio ZambomFerraresi ${ }^{2}$, Isabel Rodríguez-Sánchez ${ }^{3}$, Juan García-Martínez ${ }^{3}$, Itxaso Marín-Epelde ${ }^{4}$, Ana Sofia Pozo-Vico ${ }^{4}$, Chenhui Chenhuichen ${ }^{4}$, Imanol Reparaz-Escudero ${ }^{2}$, Álvaro Casas-Herrero ${ }^{4}$, Mikel Izquierdo ${ }^{5}$, Nicolás Martínez-Velilla ${ }^{6}$

${ }^{1}$ Navarrabiomed, Hospital Complex of Navarra (CHN) - Public University of Navarra (UPNA), Navarra Health Research Institute (IdisNa), Pamplona, C/Irunlarrea 3, 31008 Pamplona, Navarra, Spain.), ${ }^{2}$ Navarrabiomed, Hospital Complex of Navarra (CHN) Public University of Navarra (UPNA), Navarra Health Research Institute (IdisNa), ${ }^{3}$ (Geriatric Department, Hospital Complex of Navarra (CHN), ${ }^{4}$ Geriatric Department, Hospital Complex of Navarra (CHN), ${ }^{5}$ Health Science Department, Public University of Navarra, ${ }^{6}$ Geriatric Department, Hospital Complex of Navarra

Introduction: The iatrogenic nosocomial disability usually generated by hospital stay in older people as a consequence of prolonged bedrest episodes. Low-mobility behaviour during hospitalization and the reduced functional and physiological reserve of older medical patients result in many adverse effects including loss of functional autonomy in activities of daily living.

Aims: To assess the effectiveness of a gamification program on mobility in acutely hospitalized older patients. Methods: Non-randomised controlled trial of older adults (aged 65 years or older) admitted to Acute Care for Elderly of Complejo Hospitalario de Navarra, Spain. Patients in the intervention group performed a gamification program. The gamification program contained different routes of multicomponent activities (physical and cognitive games). Patients in the control group received the hospital habitual care. The main outcome was the change in physical activity levels of fiscal activity assessed by accelerometery.

Results: 43 patients were included in the study $(\mathrm{n}=21$ intervention group, $\mathrm{n}=22$ in control group). Of these 43 patients, 27 were man (63\%); mean (standard deviation) age was 87.1 (3.9) years. Mean steps per days was 5485.6 (436.7) for the intervention and 4227.8 (2372.3) for the control group, respectively $(\mathrm{p}=0.0 .68)$.

Conclusions: The gamification program did not provide significant benefits on physical activity levels over hospital usual care. However, gamification programs could be a useful and complementary strategy for changing sedentary behaviour during hospitalization.

\section{Abstract \# 48}

Manifestation of urinary tract infections in acute geriatric unit Benediktas Radavičius ${ }^{1}$, Jurgita Knašienè ${ }^{2}$

${ }^{1}$ Regional Hospital of Telšiai, Lithuania, ${ }^{2}$ Clinical Department of Geriatrics, Lithuanian University of Health Sciences, Kaunas Clinical Hospital, Lithuania

Introduction: Urinary tract infections (UTIs) are the most common infections in the Acute Geriatric Unit. This study investigated clinical findings, causative microorganisms and antimicrobial susceptibility in geriatric patients diagnosed with UTIs.

Methods: 207 cases of hospitalised patients with UTIs were analysed within two years between February 2018 and January 2020 at the Kaunas Clinical Hospital Geriatric Unit. Cases were chosen when there was a positive urine culture result.

Results: Saprophytic infections accounted for $79.3 \%$ cases, hospital infections $-20.7 \%$. Polyinfection was detected in $21.7 \%$ cases. The most common cause of UTI is Escherichia coli. The second most common pathogen is Klebsiella pneumoniae. Among the older patients with UTIs, neutrophilia and CRP elevations were the most common. Elevated levels of nitrites and leukocytes are most commonly detected in BTI, leukocytes and 4 points of bacteria in microscopy. Leukocytosis, neutrophilia and CRB elevations were more common in men with UTI than in women $(\mathrm{p}=0.005, \mathrm{p}=0.01, \mathrm{p}$ $=0.001$ ). In UTI caused by E. coli or Corynebacterium urealyticum, leukocyte counts are usually normal in the blood, and neutrophilia is most commonly observed in E. coli-induced UTI $(p=0.039) .46 \%$ of pathogens were found to be susceptible to cefuroxime and $48.7 \%$ to ciprofloxacin.

Conclusions: Keep in mind that in the geriatric patients often occurs atypical UTIs course and laboratory blood tests shouldn't be blindly trusted for the diagnosis of UTIs. A thorough history of UTIs should be collected before prescribing empirical antibiotic therapy. Important to pay attention to treatment of concomitant diseases that worsen the course of UTIs, shortening of catheterization time, treatment of urinary incontinence or double incontinence. 


\section{Abstract \# 49}

Multidimensional prognostic index (MPI) predicts in-hospital mortality in older adults with acute respiratory failure (ARF).

Federica Gandolfo ${ }^{1}$, Carlo Custodero ${ }^{2}$, Alberto Cella ${ }^{1}$, Lisa Cammalleri ${ }^{1}$, Romina Custureri ${ }^{1}$, Simone Dini ${ }^{1}$, Rosetta Femia ${ }^{1}$, Sara Garaboldi $^{1}$, Ilaria Indiano ${ }^{1}$, Clarissa Musacchio ${ }^{1}$, Silvia Podestà ${ }^{1}$, Francesca Tricerri ${ }^{1}$, Vincenzo Solfrizzi ${ }^{2}$, Alberto Pilotto ${ }^{3}$

${ }^{1}$ Department of Geriatric Care, Orthogeriatrics and Rehabilitation, E.O. Galliera Hospital, Genova, Italy, ${ }^{2}$ Department of Interdisciplinary Medicine, University of Bari, Italy, ${ }^{3}$ Department of Geriatric Care, Orthogeriatrics and Rehabilitation, E.O. Galliera Hospital, Genova, Italy; Department of Interdisciplinary Medicine, University of Bari, Italy

Background: Acute respiratory failure (ARF) is a very common complication among hospitalized older adults with pulmonary diseases. Its incidence dramatically increases with age and presence of comorbidities. Non-invasive ventilation (NIV) may avoid admission to intensive care units, intubation and their related complication. Besides the need of NIV, comprehensive geriatric assessment (CGA) could have a role in defining the short-term prognosis of older adults with ARF.

Aim: In this study we aimed to investigate whether the Multidimensional Prognostic Index (MPI), a CGA-based assessment tool of patients' prognosis, may help to identifying older adults with ARF more at risk of in-hospital mortality.

Methods: This is a prospective observational study which consecutively enrolled patients older than 70 years, admitted to the Acute Geriatric Unit of Galliera Hospital (Genoa, Italy) for pneumonia or exacerbation of chronic obstructive pulmonary disease (COPD) whit ARF $(\mathrm{PaO} 2 / \mathrm{FiO} 2$ ratio $<300)$. A standardized $\mathrm{CGA}$ was used to calculate the MPI at admission. Multivariate regression analysis was conducted to test if MPI score could predict in-hospital mortality. Receiver operator curve (ROC) analysis was used to identify the best MPI cut-off to predict mortality in this population.

Results: We enrolled 232 patients (47\% females, mean age $88.2 \pm$ 5.9 years). Mean MPI at admission was $0.76 \pm 0.16$ ). 61 patients $(26.3 \%)$ received NIV during hospitalization and did not significantly differ for age, gender and MPI score compared to those who did not receive NIV. Median length of stay was 13 days (IQR: 10) and mortality rate was $33.7 \%$. In multivariable analysis, we found that the only significant predictor of in-hospital death in this population was the MPI at admission $(\beta=6.33, p<0.0001)$, such that higher MPI scores predicted worst survival independently by age, gender and NIV use. The best MPI cut-off predicting in-hospital mortality was 0.78 (HR: $3.83,95 \%$ CI 2.04-7.18).

Conclusions: MPI at admission might be a useful tool to early detect patients more at risk of in-hospital death among older adults with ARF due to pulmonary disease.

\section{Abstract \# 50}

\section{Readmission to secondary care following COVID-19}

Mark Holloway ${ }^{1}$, Frances Rickard ${ }^{1}$, Emma Mitchell ${ }^{1}$, Kathryn McCarthy $^{1}$, James Hesford ${ }^{1}$, Philip Braude ${ }^{1}$

${ }^{1}$ Southmead Hospital, North Bristol Trust, NHS, UK

Introduction: Guidance for COVID-19 admissions has focussed on criteria for hospital discharge but not on readmission risk. English readmission rate in $2017 / 18$ was $13.8 \%$ [1]. This observational cohort study examined patients readmitted to hospital following COVID-19. Methods: These data were collected as part of the COPE study (COVID-19 in Older People; HRA 20/HRA/1898). Patients included were admitted to Southmead Hospital between 28-Jan and 24-April 2020 with confirmed and/or clinically diagnosed COVID-19. Medical records were analysed prospectively on the nature of the readmission and length of hospital stay (LOS).

Results: 141 patients were included; initial COVID-19 admission median LOS was nine-days $(\min 1, \max 62, \mathrm{IQR} 15)$. Nineteen $(13.4 \%)$ were readmitted with an eight-day median time to readmission (min: 1, max: 23, IQR 8). Of those readmitted, median LOS was 2 days ( $\min 1$, $\max 45$, IQR 6.5). Ten readmissions were attributable to COVID-19, with half having a $<1$ day LOS. Readmission presenting complaints consisted: chest pain, breathlessness, pyrexia, cough and diarrhoea and vomiting. New diagnoses: unilateral pneumonia $\mathrm{n}=3$, bilateral pneumonia $\mathrm{n}=2$, musculoskeletal chest pain $\mathrm{n}=2$, COPD exacerbation $\mathrm{n}=1$, cough secondary to COVID-19 $\mathrm{n}=1$, diarrhoea and vomiting secondary to COVID-19 $\mathrm{n}=1$, lowerlimb DVT $\mathrm{n}=1$.

Key conclusions: Readmission rate following COVID-19 was no more frequent than the background readmission rate. Over half of readmissions were directly related to COVID-19 symptoms with respiratory diagnoses predominating. Those that were readmitted had a shorter index hospital stay than the median.[1] NHS Outcomes Framework.

\section{Abstract \# 51}

The front-door geriatrician: impact on polypharmacy and deprescribing in Barnet Hospital

Carmel Bergbaum ${ }^{1}$, Megan Knight ${ }^{1}$, Tunay Hussein ${ }^{1}$, Deborah Bertfield $^{1}$, Mark James Rawle ${ }^{1}$, Naomi Newman ${ }^{1}$

${ }^{1}$ Barnet General Hospital, Royal Free London NHS Foundation Trust

Introduction: Polypharmacy is a growing problem among older adults. More than one-fifth of all adults aged 80 years or over are prescribed ten or more medications in the United Kingdom (UK) [1]. Polypharmacy is associated with negative clinical outcomes, including increased risk of falls and adverse drug reactions [2] and reduced cognitive and physical capability [3]. Acute hospital admissions via specialist geriatric medicine services provide opportunities for medication review and de-prescribing. We evaluated the impact of daily geriatrician input on polypharmacy in older adults admitted to the acute Clinical Decisions Unit (CDU) of Barnet Hospital, a UK district general hospital.

Method: Daily geriatrician reviews were introduced for older adults admitted to CDU in November 2019. Prior to this, reviews were performed by general medicine consultants. Data on polypharmacy were retrospectively collected using electronic records from patients aged over 70 years who were referred to medicine over 2-week periods pre (October 2019, $\mathrm{n}=40$ ) and post (November 2019, $\mathrm{n}=44$ ) intervention.

Results: Following introduction of daily geriatrician review, more patients had one or more medications deprescribed during their admission $(38.6 \%$ versus $15 \%, \mathrm{p}<0.05)$. Of those who had medications deprescribed, there was an increased total number of medications stopped ( $\mu 2.4$ versus 1.5 ). There was also increased documented recognition of polypharmacy ( $21 \%$ versus $10 \%)$.

Key conclusions: Introduction of a front-door geriatrician reduced polypharmacy and improved de-prescribing, likely through Comprehensive Geriatric Assessment. This could lead to reduced adverse clinical outcomes and associated costs, although further work is needed to delineate this.

\section{References:}

1. Guthrie B, Makubate B, Hernandez-Santiago V, et al. The rising tide of polypharmacy and drug-drug interactions: Population database analysis 1995-2010. BMC Med 2015; 13: 742. Fried TR, O’Leary J, 
Towle V, et al. Health outcomes associated with polypharmacy in community-dwelling older adults: A systematic review. J Am Geriatr Soc 2014; 62: 2261-22723. Rawle MJ, Cooper R, Kuh D, et al. Associations between polypharmacy and cognitive and physical capability: a British birth cohort Study. J Am Geriatr Soc 2018; 66: 916-923.

\section{Abstract \# 52 \\ An evaluation of an older person assessment and liason service in an acute hospital \\ Sarah Peppard ${ }^{1}$, Grace Corcoran ${ }^{1}$, Ivan Clancy ${ }^{1}$, Anne D’Arcy ${ }^{1}$, Aifric Conway ${ }^{1}$, Margaret O'Donoghue ${ }^{1}$, Naoise Waldron ${ }^{1}$, Carol Lyons $^{1}$, Elaine Bradley ${ }^{1}$, Siobhan Magner ${ }^{1}$ \\ ${ }^{1}$ Beaumont Hospital, Dublin, Ireland}

Introduction: Comprehensive Geriatric Assessment (CGA) can lead to improved outcomes (1) and reduce hospital stay and re-admission rates in non-electively admitted older people. The number of older people admitted to hospital exceeds Specialist Geriatric Ward (SGW) bed capacity; therefore an alternative model of care is required. A skilled multidisciplinary Older Person Assessment and Liaison team, (OPAL), was formed to carry out CGA and provide interventions for frail older patients on acute medical wards.

Methods: 1. Team formation• Therapy manager, physiotherapists (x2), occupational therapist, medical social worker, dietician, speech and language therapist, advanced nurse practitioner and patient flow representative 2. Referral process $\bullet$ Patients admitted to an alternative acute ward due to lack of capacity on SGW3. Operational - Daily team huddles and intervention by OPAL team• Data collection of patient demographics, outcomes and key performance indicators. 4. Service user feedback.

Results: Over a 4 week period, 51 patients admitted under the care of 21 consultants on 11 different wards were accepted by the service. Average age was 84 years with a mean Clinical Frailty Scale score of 6. Discharge outcome included home (76\%), off-site rehab (12\%), long-term care $(8 \%)$, RIP (4\%). Re-admission rate at 30 days post discharge was $19 \%$. Average length of stay was 11 days. Service user feedback was positive.

Key conclusions: A high volume of frail older adults are admitted under general medical consultants. This pilot indicates that a specialist MDT OPAL team could improve patient outcome and reduce length of stay in the absence of additional SGW beds to meet demand. References:

1. Ellis, $\mathrm{G}$ et al. Comprehensive geriatric assessment for older adults admitted to hospital. Cochrane Database of Systematic Reviews 2017, Issue 9. Art. No.: CD006211. https://doi.org/10.1002/14651858. CD006211.pub3.

\section{Abstract \# 53 \\ The maiden voyage of the corrib ward: shining a spotlight on older adult care during a pandemic: an Irish perspective \\ Dr. Karen Dennehy ${ }^{1}$, Caoimhe Hanrahan ${ }^{1}$, Gearoid Coughlan ${ }^{1}$, Catriona Reddin $^{1}$, Edel Mannion ${ }^{1}$, Clodagh McDermott ${ }^{1}$, Dr. Michelle Canavan ${ }^{1}$, Dr. Ruairi Waters ${ }^{1}$ \\ ${ }^{1}$ Galway University Hospital, Ireland}

Introduction: During the COVID 19 pandemic a higher case-fatality ratio, has been observed in the older patient ( $>80$ years) in both Chinese $(14.8 \%)$ and Italian populations $(21 \%)(1,2)$. As geriatricians we knew we had the ability to dramatically impact clinical decision making during this pandemic. One of the cornerstones of caring for the elderly is identifying patients maximally benefiting from goal orientated multidisciplinary care (3).

Methods: We introduced a geriatric COVID 19 service.This team consisted of geriatric consultants, trainees, multi-disciplinary team and advanced nurse practitioners. We admitted, treated and rehabilitated all query and confirmed COVID 19 geriatric patients over a 12 weeks period in our tertiary referral centre. All patients were admitted and clinical frailty scale score assigned. An early and honest discussion around goals of care were conducted in collaboration with patients and their families.

Results: Initially,we analysed the first 100 patients through our service for this abstract. Comprehensive geriatric assessments were completed on everyone. Seventy-five percent of patients were admitted from home, $21 \%$ from nursing homes and $4 \%$ from respite care.The average age of our patient cohort was 82years.Average CFS was 5 . Seven patients were palliated with end of life care implemented by our team, their average CFS was higher (8).

Conclusions: We feel our care positively impacted on the care of older patients during this pandemic. Fatality number were reduced in this cohort. Older adults care is optimized when treated by the physicians that have experience with their unique needs.

\section{Abstract \# 54}

Delirium bay introduction improving care for our older patients: an Irish experience

Karen Dennehy ${ }^{1}$, Aisling Whelan ${ }^{2}$, Gearoid Coughlan ${ }^{1}$, Ruari Waters ${ }^{1}$

${ }^{1}$ Galway University Hospital, Ireland, ${ }^{2}$ Galway University Hospital

Background: The acute care for elderly patient models (ACE) have shown significant benefit in patient outcomes. A focus on patient centred care,with consistent care provided in a specially designed environment, results in an improvement in adverse patient events including falls, delirium, functional decline, shorter hospital stay, institutionalization, readmission and reduction in cost.

Methods: A dedicated delirium unit was introduced into our institution in March 2019. This unit comprised of 4 beds with a dedicated health care assistant and nurse. There was a focus on early input from our multi-disciplinary team as well as restful recovery. Continuity of care was central to medical and nursing care with a dedicated team. Results: We captured 152 patient episodes over a 6 month period. Key aspects to a successful older patients hospitalization were recorded with favourable results, these included continence, nutrition, sleep, mobility, falls, behaviour and mobility.Presence of delirium significantly improved (29\% vs $14 \%$ ) as well as sleep quality ( $74 \%$ vs $38 \%$ ). Majority of patients were dressed in their day clothes (79\%), with regular bowel motions (89\%) and adequate nutritional intake (100\%). Discharge destinations have also been optimized with $66 \%$ of these vulnerable patients being discharged home, $28 \%$ to nursing homes and $7 \%$ passing away.

Conclusion: Our data reflects positively on this new initiative, which was introduced with minimal cost and without the requirement of additional staff members. This dedicated unit has optimized patient outcomes in our institution.

\section{Abstract \# 55 \\ Comparison of the prevalence of CKD in elderly people using three different equations: preliminary results from PrHoDi Study (Protected Hospital Discharges Study)}

Alberto Castagna ${ }^{1}$, Carmen Ruberto ${ }^{1}$, Raffaele Costa ${ }^{2}$, Laura Greco ${ }^{2}$, Rosa Paola Cerra ${ }^{2}$, Giuseppe Coppolino ${ }^{3}$, Giovanni Ruotolo ${ }^{2}$ 
${ }^{1}$ Center for Cognitive Disorders and Dementia, Azienda Sanitaria Provinciale di Catanzaro, Italy, ${ }^{2}$ Geriatric Unit, "Pugliese-Ciaccio" General Hospital, Catanzaro Italy, ${ }^{3}$ Renal Unit, Department of Health Sciences, "Magna Graecia“ University, Catanzaro, Italy

Introduction: Chronic kidney disease(CKD) is an independent cardiovascular risk factor. Glomerular filtration rate (GFR) is generally accepted as the best indicator of kidney function. As direct measurement of GFR is complex, GFR is commonly estimated based on serum creatinine concentration. The MDRD equation is widely recognized equations to estimate GFR in adults; the BIS1 equation was designed for older adults and the more recent FAS equation for estimating the glomerular filtration rate that can be used across the full age spectrum. The aim of our study was to measure the difference in prevalence of CKD . by three differente GFR equations in elderly people.

Methods: The population screened comprised 488 patients consecutively enrolled after Hospital Discharge from Geriatric Unit. GFR was estimated using the MDRD, BIS1 and FAS equations.

Results: Mean age was $81,54 \pm 6,91$ years. GFR by MDRD, BIS and FAS, were $51,55 \pm 22,58 ; 47,11 \pm 17,19$ and $46,56 \pm 19,44 \mathrm{~mL} / \mathrm{min} /$ $1.73 \mathrm{~m}^{2}$ respectively $(F=9,262 ; p 0,000)$. The prevalence estimates of CKD stages by MDRD,BIS and FAS, were: 5,33\%, 1,02\% and 2,46 $\%$, respectively for stage $1 ; 24,59 \%, 21,93 \%$ and $21,72 \%$ for stage 2 ; $28,28 \%, 27,87 \%$ and $25 \%$ for stage $3 \mathrm{a}, 22,95 \%, 30,74 \%$ and $28,48 \%$ for stage $3 b ; 18,85 \%, 18,44 \%$ and $21,93 \%$ for stage 4 ; and $0 \%, 0 \%$ and $0,41 \%$ for stage 5 .

Key conclusions: Our study show that MDRD, BIS1 and FAS equations cannot be considered interchangeable to assess eGFR in elderly people. The prevalence of stage $3 \mathrm{~b}$ and $4 \mathrm{CKD}$ varies strongly following the method used for estimating GFR. Such discrepancies must be a important impact on our therapeutic approach.

\section{Abstract \# 56 \\ Severe dementia: factors affecting in-hospital death in a large Canadian health region: a retrospective cohort study \\ Irina Adamova ${ }^{1}$, Zahra Goodarzi ${ }^{2}$, Aynharan Sinnarajah ${ }^{2}$ \\ ${ }^{1}$ University of Calgary, Cumming School of Medicine, ${ }^{2}$ University of Calgary, Alberta Health Services}

Introduction: Many patients with severe dementia are subjected to aggressive medical interventions and burdensome transitions between health care facilities at the end of life; a high percentage die in acute health care facilities. The study aims to analyze the factors associated with in-hospital death.

Methods: A population-based retrospective cohort study in the Calgary Zone, Alberta Health Services, Canada of patients 65 years and older, with documented severe dementia (Cognitive Performance Score $(\mathrm{CPS})>5$ ), from different places of residence, who died between January 1, 2011, and December 31, 2015. Acute care utilization during the last year of life (emergency department (ED) visits, hospital admissions), and palliative care (PC) involvement was analyzed using a multivariate logistic regression model. Variables included: a history of ED visits, place of residence (long term care, home, other), PC involvement (including timing), the intensity of hospital admissions, cause of death, gender, Charlson comorbidity score, and age.

Results: In-hospital death is strongly associated with at least one ED visit, residence outside LTC, and a number of hospital admissions during the last year of life. Cause of death, Charlson comorbidity index, younger age, male sex, and lack of early PC involvement were also strongly associated with in-hospital death.

Conclusions: Community-dwelling individuals with severe dementia are at higher risk of dying in acute care facilities. Early PC involvement is associated with decreased chances of in-hospital death. To further improve the quality of end-of-life care, PC should be involved in patients with severe dementia.

\section{Abstract \# 57 \\ Strategic planning of number of geriatric acute care units (GACU) beds and geriatricians needed in Spain based on health resources: projections 2018-2025}

Ainhoa Esteve Arrien ${ }^{1}$, Pedro Ortiz Nuñez ${ }^{2}$, Concepción Jiménez Rojas $^{3}$, Barbara Perez Pena ${ }^{4}$, Jesús Lopez Arrieta ${ }^{5}$, Cristina Alonso Bouzon $^{6}$

${ }^{1}$ Hospital Universitario Infanta Leonor, Madrid, Spain, ${ }^{2}$ Independent, ${ }^{3}$ Hospital Central Cruz Roja, Madrid, Spain, ${ }^{4}$ Clínica Psicogeriátrica Josefina Arregui, Navarra; Spain, ${ }^{5}$ Hospital Universitario La PazCantoblanco, ${ }^{6}$ Hospital Universitario de Getafe, Madrid, Spain

Introduction: Spain's National Summary of Hospital Morbidity2018 (ENMH) was published February-2020. Based on Health resourcesuse, the aim of this study was to calculate the number of geriatricians needed to cover $25-60 \%$ of GACU health resources needed regarding hospital beds and number of geriatricians in Spain. Methods: Hospital discharges of people aged over 75 year 2012-2018 were registered in 17 Communities-Health-Systems and yearly projections were made until 2025 . Average length of stay(ALOS) was calculated multiplying A-LOS in each age group by number of admissions in each group. Hospital-GACU beds were calculated with Bridgman's formula under different assumptions. Considering $60 \%$ of acute admissions to be candidates to GACUadmission and considering a minimum recommended by Spanish Geriatric Medicine Society (SEMEG) of 25\% admissions of over 75yr-old. Ratios of 10-8 beds/geriatrician were considered.

Results: During 2018 ENMH publishes 1.442 .935 hospital acute-care admissions in people over 75 years with A-LOS of 11.08 days. Depending on the standard considered, between 12889 GACU-beds (1289-1611 geriatricians) and 30993 GACU-beds (3093-3867geriatricians) are needed considering 2018 admissions. When hospital admissions projections based on previous years are considered until $2025,1.684 .613$ admissions are projected with an A-LOS of 13,51 days. Between 13575 and 32579 GACU-beds and ranges of 1357-1697 and 3258-4072 geriatricians will be needed.

Conclusions: Strategic planning of care needs may be useful to take actions to plan both health care resources, continuing care needs and young geriatricians training needs.

\section{Abstract \# 58}

Abstract 2. Strategic planning of number of geriatric acute care units (GACU) beds and geriatricians needed in spain based on population standards

\section{Ainhoa Esteve Arrien ${ }^{1}$}

\section{${ }^{1}$ Hospital Universitario Infanta Leonor, Madrid, Spain}

Introduction: Based on quality standards regarding GACU calculated on population needs by Spanish Geriatric Medicine Societies (SEMEG and SEGG) the aim of this study was to calculate the number of geriatricians needed to cover Geriatric-Acute-Care-Units (GACU) needs regarding hospital beds and number of geriatricians in Spain.

Methods: National and Communities Census-2019 (NC-2019) of people aged over 75 from 2012-2018 were reviewed in 17 Communities-Health-Systems. Hospital AGCU beds were calculated with standards published by Spanish Geriatric Medicine Societies 
(SEMEG/SEGG) under different assumptions: 1.5-4.3 GACU beds per $1000>75$ years old (SEMEG) and 5,2 beds/1000 >65 years (SEGG). Ratios of 10-8 GACU-beds per geriatrician were considered. Results: National Census-2019 published 2020 reveals 9.047.193 people over 65 and 4482558 people over 75 out of 47026208 general census in Spain-2019. Based on first assumption, 6724 GACU-beds and 672-840 geriatricians were needed and based on second assumption 47097 GACU-beds and 4710-5887 geriatricians were needed. Considering Communities populations, Andalousia and Catalonia account for the greatest needs, both with at least 1020 and 1063 GACU-beds needed in the lowest assumption, followed by Madrid with 864 GACU-beds needed. Currently there are no GACU-beds nor geriatricians in Andalousia.

Conclusions: Strategic planning of care needs may be useful to take actions to plan both health care resources, continuing care needs and young geriatricians training needs.

\section{Abstract \# 59}

Introduction of a new initiative for nursing home residents in the acute hospital to improve patient care

Irfan Rasool Malik ${ }^{1}$, Melissa Ryan ${ }^{1}$, Siobhan Kennelly ${ }^{2}$, Ruth Martin ${ }^{2}$

${ }^{1}$ Connolly Hospital and RCSI, ${ }^{2}$ Connolly hospital and RCSI

Introduction: Nursing home $(\mathrm{NH})$ residents are vulnerable population,often with multi-morbidity and advanced frailty. Their use of acute hospital services is more than twice that of community dwellers. Hospital admissions have been found to be protracted with detrimental outcomes for these residents. To improve care provided to $\mathrm{NH}$ residents in the acute hospital, a dedicated geriatrician-led team was introduced

Methods: A pre/post intervention study was conducted to analyse outcomes for NH residents in Connolly hospital,a teaching hospital in Dublin. The intervention was a geriatrician-led team managing the care and early supported discharge of these residents, with community follow-up. The aim was to assess the benefits of this initiative to $\mathrm{NH}$ residents. Data was collected from the hospital electronic patient record with comparison for six months pre and post commencement of the initiative in July 2019, with a focus on length of stay (LOS) and total bed-days.

Results: There were $381 \mathrm{NH}$ residents admitted to Connolly hospital 6 months pre-intervention compared with 368 admissions in the six months post. The average LOS was 9.25 days pre-intervention,compared with 6.6 days post ( $p$ value 0.014 ). There was a decrease in total bed -days from 3449 to 2416 , a saving of 1033 days ( $p$ value 0.04 ). Key conclusions: The study proved the effectiveness of a dedicated geriatric team in reducing hospital LOS for $\mathrm{NH}$ residents, due to specialist care and follow-up. With the saving of 1033 bed-days over six months,patient flow within the hospital was significantly improved.This small study highlights the big potential improvements for this frail cohort with specialist care.

\author{
Abstract \# 60 \\ Potential efficacy of pragmatic exercise program (sprint) \\ during hospitalization in older adults on health care and physical \\ performance: a pilot study \\ Eva Peyrusqué ${ }^{1}$, Fanny Buckinx ${ }^{1}$, Aline Bolduc ${ }^{1}$, Caroline Law ${ }^{1}$, \\ Marie-Jeanne Kergoat ${ }^{1}$, Mylène Aubertin-Leheudre ${ }^{1}$ \\ ${ }^{1}$ Centre de Recherche, Institut Universitaire de Gériatrie de Montréal \\ (IUGM), CIUSSS du Centre-Sud-de-l'Île-de-Montréal, Montreal, \\ Canada
}

Introduction: Immobilization contribute to iatrogenic decline in hospitalized older adult. Implementing physical activity (PA) seems to be one of the best solution. However, PA are poorly integrated into usual care and those available are either non-specific, supervised or requested materials. Thus, we aimed to assess the effect of a pragmatic, unsupervised, and specific PA program (SPRINT) on health care practice and functional capacities in hospitalized older patients. Methods: Single arm interventional pragmatic pilot study. Of the 37 patients ( $>70$ years) hospitalized and eligible, 19 agreed to participate (AP) and 18 declined (N-AP). One of the 4 PA programs, developed by our team, was allocated according to mobility profile. Individual functional capacities (balance, walking speed, functional mobility profile (PFMP)), active time (METS $>1.5$ : min), length of hospitalization (LOS), discharge orientation were assessed during the hospitalization.

Results: At admission, the groups were comparable. At discharge, the AP group improved more on walking speed $(0.57 \pm 0.21$ vs. $0.64 \pm 0.19 ; p=0.013)$, balance $(41.8 \pm 13.7$ vs. $45.1 \pm 9.7 ; \mathrm{p}=0.017)$ and PFMP $(54.0 \pm 7.1$ vs $55.1 \pm 5.5 ; p=0.042)$ than the N-AP group. The LOS was significantly shorter in AP group compared to the N-AP group ( 25 vs. 36 days; $p=0.026$ ) and more subjects in the AP group were oriented at home without health or social services $(89.5$ vs. $60 \%$; $p=0.065$ ).

Conclusion: SPRINT appears effective to counteract iatrogenic decline and decreased the LOS. Moreover, this simple pragmatic PA tool seems to improve the life trajectory and healthcare practice in aging population. Further research are needed to confirm these promising pragmatic results.

\section{Abstract \# 61 \\ Strategic planning of number of geriatric functional recovery units (GFRU) beds and geriatricians needed in spain based on population standards}

Ainhoa Esteve Arríen ${ }^{1}$, Pedro Ortiz-Nuñez ${ }^{2}$, Concepción Jimenez Rojas ${ }^{3}$, Barbara Pérez Pena ${ }^{4}$, Jesús Lopez Arrieta ${ }^{5}$

${ }^{1}$ Hospital universitario Infanta Leonor, Madrid, Spain, ${ }^{2}$ IndependentMadrid-Spain, ${ }^{3}$ Hospital Central de la Cruz Roja, Madrid, Spain, ${ }^{4}$ Clínica Psicogeriátrica Josefina Arregui, Navarra; Spain, ${ }^{5}$ Hospital Universitario La Paz-Cantoblanco, Madrid, Spain

Introduction: Based on quality standards regarding GFRU calculated on population needs by Spanish Geriatric Medicine Societies (SEMEG and SEGG) the aim of this study was to calculate the number of geriatricians needed to cover GFRU needs regarding hospital beds and number of geriatricians in Spain.

Methods: National Census-2019 (NC-2019) of people aged over 75 were reviewed in 17 Communities-Health-Systems. Hospital GFRU beds were calculated with standards published by Spanish Geriatric Medicine Societies (SEMEG/SEGG) under different assumptions: 1.3-3 GFRU beds per $1000>75$ years old (SEMEG) and 4,6 beds/ $1000>65$ years (SEGG). Ratios of 20 GFRU-beds per geriatrician were considered.

Results: National Census-2019 published 2020 reveals 9.047 .193 people over 65 and 4482558 people over 75 out of 47026208 general census in Spain-2019. Based on first assumption, 5827-13448 GFRUbeds and 291-672 geriatricians were needed and based on second assumption 41663 GFRU-beds and 2083 geriatricians were needed. The autonomous communities of Catalonia and Andalousia accounting for the greatest needs with 922-2127 GFRU-beds (46-106 geriatricians) for Catalonia and 884-2040 GFRU-beds (44-102 geriatricians) for the first assumption and 6637 beds/332 geriatricians for Catalonia and 6624beds/331geriatricians for Andalousia under the second assumption. Followed by Madrid with $749-1728$ beds (37-86 
geriatricians) for the first assumption and 5433 beds and 272 geriatricians under the second assumption.

Conclusions: Strategic planning of care needs may be useful to take actions to plan both health care resources, continuing care needs and young geriatricians training needs

\section{Abstract \# 62 \\ Geriatric follow-up reduces readmission of older patients discharged to temporary care at a skilled nursing facility \\ Katja Thomsen ${ }^{1}$, Anders Fournaise ${ }^{2}$, Lars Erik Matzen ${ }^{3}$, Karen Andersen-Ranberg ${ }^{4}$, Jesper Ryg ${ }^{4}$ \\ ${ }^{1}$ Odense Universitets Hospital, ${ }^{2}$ Region of Southern Denmark, Odense University Hospital, University of Southern Denmark, ${ }^{3}$ Odense University Hospital, ${ }^{4}$ Odense University Hospital, University of Southern Denmark}

Introduction: Hospital readmission is a burden to patients, relatives, and society. Especially frail older patients are at higher risk of readmission and its negative outcomes. We aimed at examining whether follow-up visits by an outgoing multidisciplinary (nurse and doctor) geriatric team (OGT) can reduce unplanned hospital readmission in older patients discharged to temporary care at a skilled nursing facility.

Methods: The study population included all initially acutely hospitalized patients discharged from a geriatric department to a skilled nursing facility during Jan 1 st 2016-Feb 25th 2020. Intervention by OGT was implemented Mar 12th 2018. Patients acutely readmitted to hospital (within 4 hours-30 days) before (-OGT group) and after (+OGT group) the implementation were identified and compared using Chi2-test. A multivariable analysis using Cox regression was carried out to adjust for age, sex, Barthel-Index, length of stay of index admission, and 30-day mortality.

Results: A total of 847 patients were included (440 -OGT; 407 +OGT). The proportion of patients readmitted was significantly lower in +OGT compared to-OGT, $15 \%$ (95\% CI 12-19) and 26\% (95\% CI $22-30)$, respectively $(p<0.001)$. No difference was found between the two groups in regard to age, sex, Barthel-Index, and 30-day mortality. Compared to the-OGT, the adjusted risk for readmission in the +OGT was HR=0.57 (CI 95\% 0.42-0.79).

Conclusion: Follow-up visits by an OGT to patients discharged to temporary care at a skilled nursing facility significantly reduced 30-day readmission in frail older patients. We propose a scheduled follow-up in these patients.

\section{Abstract \# 63}

Acute abdomen in elderly patients: role of ultrasonography

Vito Carrieri ${ }^{1}$, Antonella Bray ${ }^{1}$, Giovanni Argentieri ${ }^{2}$, Vincenzo Paternò $^{2}$

${ }^{1}$ Geriatrics Department Hospital ”A. Perrino “ Brindisi Italy,

${ }^{2}$ Geriatrics Department Hospital ”A. Perrino“ Brindisi Italy

Introduction: Abdominal acute diseases are increasing in patients older than 65 years. The aim of the study is to evaluate the diagnostic contribution of ultrasonography in the elderly patient with suspected Acute Abdomen.

Methods: The Ultrasonography was performed within $6 \mathrm{~h}$ from hospitalization both in the Emergency Room (ER) and in Geriatrics Department (GD). 100 patients older than 65 years with a suspected diagnosis of Acute Abdomen were examined with Ultrasonography: 30 in the ER and 30 in GD.
Results: The Ultrasonography made in Emergency Room identified some causes of Acute Abdomen for which was required immediate hospitalization for emergency surgery. The Ultrasonography made in Geriatrics Department identified several conditions that required careful monitoring in the $70 \%$ of the elderly patients and in $30 \%$ of patients identified serious diseases that required a transfer in surgical department.

Conclusion: The use of Ultrasonography in Emergency Room prevents inappropriate hospital admissions and unnecessary specialist consultations and identifies immediately the elderly patient who requires emergency surgery. The use of Ultrasonography in Geriatrics Department within 6 hours from hospitalization certainly reduces the risk to the elderly patient with suspected Acute Abdomen and other serious diseases and helps to apply very quickly the appropriate diagnostic and therapeutic program and increases the effectiveness and efficiency of care in Geriatrics.

\section{Abstract \# 64}

Are we improving outcome for haemorrhagic stroke patients at RCHT?

Kyaw Lin Saw ${ }^{1}$, Katja Adie ${ }^{1}$, Richard Barrett ${ }^{1}$

${ }^{1}$ Royal Cornwall Hospitals NHS Trust, Truro, Cornwall, TR1 3LJ

Intracerebral haemorrhage was the cause of $14 \%$ of all strokes presenting to Royal Cornwall Hospital in 2017 and was associated with poor outcome. In-hospital mortality was $39 \%, 30$ day mortality was $41 \%$ and $39 \%$ were left with significant disability (Rankin 3+). We audited the management of blood pressure control, anticoagulant reversal, treatment escalation plans, involvement of neurosurgery and intensive care and compared the mortality of haemorrhagic stroke of 2017 to that of 2018. We have seen a reduction in mortality for haemorrhagic stroke in comparison to last year's audit (33\% vs 39\%). Patients who were discharged with no to minimal symptoms received appropriate BP management, anticoagulant reversal and discharged within 2 weeks. Better BP control, timely anticoagulant reversal and full resus status was associated with lower mortality. Neurosurgery was lifesaving, but did not improve the quality of life.

\section{Abstract \# 65}

Determinants of discharge at home: a proposal.

Liliana Mazza ${ }^{1}$, Riccardo Ievoli $^{2}$, Daniela Pinto ${ }^{1}$, Pietro Calogero ${ }^{1}$

${ }^{1}$ Acute Geriatric Unit, Sant'Orsola-Malpighi University Hospital, Bologna, Italy, ${ }^{2}$ Department of Economics and Management, University of Ferrara, Italy

Introduction: Discharge planning is a procedure which needs to be started as soon as the patient is admitted in hospital in order to facilitate the return to home [1]. Clinicians need to identify precociously the risk of difficult discharge with the aim to provide resolutive measures for the transition of care.

Material and methods: We collected 612 patients, acutely ill, who were admitted to our Geriatric Unit in six months (April-September 2019). Mean age was 84 (SD = 7.7), 54\% were females. In order to avoid multicollinearity and endogeneity, among the disposable variables we selected Blaylock Risk Assessment Score (BRASS) (assuming values between 0 and 40), gender and length of stay (in days) as determinants of home discharge.

Results: Firstly, Mann-Whitney test confirms a statistically significant difference in BRASS between patients discharged at home and those who were discharged elsewhere. Surprisingly, BRASS reveals to be uncorrelated with the length of stay $(p$ value $=0.27)$. In the 
multivariate logistic model, length of stay and BRASS appear statistically significant $(p$ value $=0.005$ and $p$ value $<0.0001$, respectively), showing a negative impact on home discharge (OR = 0.96 and $\mathrm{OR}=0.92$, respectively). The in-sample evaluation of the model presents a high specificity (93\%), an acceptable accuracy (71\%) but a poor sensitivity (26\%).

Key conclusions: Although BRASS is confirmed as a predictor of discharge in elderly patients, poor results in terms of sensitivity need serious improvements of the model. Further researches are ongoing to find proper additional covariates to understand profiles of non-home discharged patients.

References: [1] Shepperd Sasha et al. Discharge planning from hospital to home. Cochrane database of systematic reviews 1 (2013).

\section{Abstract \# 66 \\ Malnutrition in inpatients with ESPEN, GLIM, and MNA-SF in relation to muscle strength \\ Sumru Savas ${ }^{1}$, Asli Kilavuz ${ }^{2}$, Fehmi Akcicek ${ }^{1}$ \\ ${ }^{1}$ Section of Geriatrics, Department of Internal Medicine, School of Medicine, Ege University, ${ }^{2}$ Section of Geriatrics, Department of Internal Medicine,School of Medicine, Ege University}

Purpose: The aim of this study was to investigate malnutrition with conventional and recently proposed tools at the hospital.

Material and methods: All of the patients hospitalized between January 1st and February 22nd 2020 were included in the study. Exclusion criteria were as follows; patients not able to communicate, and $<60$ years of age. Malnutrition was identified with Mini Nutritional Assessment-Short Form (MNA-SF), European Society of Clinical Nutrition and Metabolism (ESPEN) and the Global Leadership Initiative on Malnutrition (GLIM) criteria. Hand grip strength (HGS) was performed with Takei digital dynamometer. The other variables were taken retrospectively from hospital records.

Findings: A total of 48 patients were enrolled in the study, with $23 \%$ women, and $25 \%$ men. The number of patients with normal nutritional status was 10 (20.8\% of all). By MNA-SF, malnutrated patients were $41.7 \%$, and at risk of malnutrition $37.5 \%$. The prevalence of malnutrition with ESPEN and GLIM were $16.7 \%$, and $52.1 \%$. The agreements between the tools were poor to fair (MNA-SF vs. ESPEN: $\kappa=-0.036, \mathrm{P}=0.112$; MNA-SF vs. GLIM: $\kappa=-0.122, \mathrm{P}<0.001$; ESPEN vs. GLIM: $\kappa=0.230, P=0.028$ ), and the agreement between low HGS and tools ESPEN, MNA-SF, and GLIM were $\kappa=0.72, \mathrm{P}=$ $0.014 ; \kappa=-0.094, \mathrm{P}=0.220 ; \kappa=0.084, \mathrm{P}=0.081$, respectively.

Conclusion: As malnutrition prevalence varies with different screening tools and assessments, standardization for malnutrition screening and assessment is essential. With the aim of comparing latest two malnutrition diagnostic assessment methods with MNA-SF, we report that malnutrition screened by those tools was substantially variable. PS: Will be also presented at AGC 2020.

\section{Abstract \# 67 \\ ENTEROBACTAGE: empiric antibiotherapy for enterobacteriaceaes bloodstream infections in older patients \\ Albane Roseau-Vincenti ${ }^{1}$, Emmanuel Forestier ${ }^{2}$, Marie-Christine Carret $^{2}$, Pauline Caraux-Paz ${ }^{3}$, Jean-Philippe Lanoix ${ }^{4}$, Marc Paccalin ${ }^{5}$, Gaetan Gavazzi ${ }^{6}$, Claire Roubaud-Baudron ${ }^{1}$ \\ ${ }^{1}$ Geriatric department, CHU de Bordeaux, Bordeaux, France, \\ ${ }^{2}$ Infectious disease department, $\mathrm{CH}$ de Chambéry, Chambéry, France,}

${ }^{3}$ Infectious disease department, $\mathrm{CH}$ de Villeneuve Saint Georges, ${ }^{4}$ Infectious disease department, CHU d'Amiens, Amiens, ${ }^{5}$ Geriatric department CHU de Poitiers, ${ }^{6}$ Geriatric department CHU de Grenoble

Introduction: Enterobacteriaceaes (EB) bloodstream infection (BSI) are frequent and serious in older patients who are at risk for multidrug resistance (MDR). Physicians are faced with the dilemma of prescribing adequate antibiotic (AB) taking into account MDR risk factors and sparing broad-spectrum $A B$ prescription. Objective: To determine whether the empiric $\mathrm{AB}$ introduced to treat $\mathrm{EB} \mathrm{BSI}$ is adequate in older patients.

Material and methods: 47 French and Belgian centers enrolled retrospectively up to 10 patients aged 75 and over recently hospitalized with EB bacteremia.

Results: The 505 enrolled patients (mean age $86+/-5,9$ ) were mostly dependent (ADL score median: 4,5 (IQR: $2.5 ; 6.0$ )); 70\% had at least one risk factor of being infected by EB-ESBL. E. coli was the most frequent pathogen $(n=353,70 \%) ; 11 \%$ were resistant to 3 rd generation cephalosporins (3GC) concordant with a majority of UTI as source of infection $(70,1 \%)$. Infection was severe in $30 \%$ of cases. $434(86 \%)$ patients received an empirical $\mathrm{AB}$ (32\% of dual therapy) which was adequate in $87 \%$ of cases $(n=377)$. 3GC prescriptions $(n=$ 326, 64\%) were most common. Prescription of penems as first line was not frequent $(n=14,3 \%)$. In-hospital mortality rate was $17 \%(n=$ 85). Prescription of an inappropriate $A B$ (i.e. no $A B$ prescription before BSI positivity or inadequate $\mathrm{AB}$ ) was not associated with mortality. At discharge, 233 (46\%) patients experienced a functional decline.

Conclusion: This study confirmed that EB BSI is a severe event for older patients. Empirical antimicrobial treatment was adequate in most cases sparing penems even with a high prevalence of MDR risk factors.

\section{Abstract \# 68 \\ Efficacy of respiratory rehabilitation in people with cystic fibrosis: systematic literature review}

Rita Melo ${ }^{1}$, Sandra Amado ${ }^{1}$, Carlos Albuquerque ${ }^{2}$, Hugo Neves ${ }^{3}$, Vítor Parola ${ }^{4}$

${ }^{1}$ Centro Hospitalar e Universitário de Coimbra, ${ }^{2}$ Escola Superior de Saúde de Viseu, ${ }^{3}$ Health Sciences Research Unit: Nursing,

${ }^{4}$ Universidade Fernando Pessoa

Background: Cystic Fibrosis (CF) is a hereditary, autosomal, recessive pathology. Management of CF through Respiratory Rehabilitation Programs (RRP) appears to help control the progression of the disease, minimizing disabilities, and preventing complications.Objectives: To evaluate the effectiveness of RRP in people with CF.

Methods: A Systematic Literature Review was carried out, using the following databases: CINAHL Complete; MEDLINE Complete; Nursing and Allied Health Collection; MedicLatina. Studies published between 2005 and 2019 were included. A total of 186 articles were identified, which were independently evaluated by two researchers, according to the established criteria. The review corpus consisted of six articles, with only two meeting methodological criteria for meta-analysis through RevMan 5.2.8 software.

Results: Studies included evidence improvement in the quality of life of patients with CF enrolled in RRP. When personalized and individualized, these programs lead to increased activity tolerance. Low to moderate adherence to RRP evidence worse radiological findings.Within RRP, Positive Expiratory Pressure (PEP) appears to be the most relevant technique, leading to half as many pulmonary exacerbations, and fewer pharmacological measures. Also, it is easier to adapt, thus favoring autonomy. However, due to sample sizes and 
methodological quality of the included studies, meta-analysis statistics suggest no significant differences between RRP and other techniques.

Conclusions: Despite the studies' low quality, RRP evidence promising results in the management of patients with $\mathrm{CF}$. As part of nursing rehabilitation practice, structured and individualized RRP constitute a therapeutic resource with positive effects on the quality of life and health of patients with CF.

\section{Abstract \# 69}

Esophagogastroduodenoscopy is safe in the nonagenarian population but is more appropriate as a means of treatment than as a diagnostic test

Raphael Ellis ${ }^{1}$, Dvorah Shapiro ${ }^{1}$

${ }^{1}$ Shaare Zedek Medical Center

Introduction: Gastrointestinal (GI) procedures are increasingly performed on much older patients. Data regarding safety and performance of esophagogastroduodenoscopy (EGD) in this population is scarce. We compared the indications, safety and benefits of EGD for nonagenarians compared to octogenarians.

Methods: Case-control study of patients aged 90-99 $(\mathrm{n}=231)$ compared to patients aged $80-89(\mathrm{n}=241)$ who underwent EGD from 2013 to 2018 in an academic medical center. Indication, comorbidities, sedation, safety, findings and treatment implications were compared.

Results: Nonagenarians were more likely to undergo inpatient procedures $(64.9 \%$ vs. $33.6 \%, \mathrm{p}<0.001)$, more commonly for bleeding $(55 \%$ vs. $43 \%, p=0.009)$, less due to dyspepsia ( $7 \%$ vs. $18 \%, p=$ $0.003)$. Sedation, including a three sedative combination, was given to $45 \%$ of octogenarians compared to $12 \%$ of nonagenarians, $\mathrm{p}<0.001$. Significant findings, including active GI bleeding and GI cancer, were more common amongst nonagenarians $(41 \%$ vs. $32 \%, 7.3 \%$ vs. $3.3 \%$, $\mathrm{p}=0.04, \mathrm{p}=0.05)$. Endoscopic therapy was performed more commonly in nonagenarians $(24 \%$ vs. $8 \%, \mathrm{p}=0.001)$ but treatment modifications were less common $(56 \%$ vs. $72 \%, \mathrm{p}<0.001)$. Only $1 \%$ of the nonagenarian population undergoing EGD was treated for newly diagnosed upper GI cancer. Early and late complications were very rare (5 early, 9 late) evenly distributed between groups.

Conclusions: EGD performed in the nonagenarian population has a good safety profile; is frequently diagnostically significant and important for local treatment. However, its efficacy in altering a 90 -year-old patient's management plan, when used as an investigative test, remains equivocal.

\section{Abstract \# 70 \\ Nursing records on oral hygiene of hospitalized elderly patients \\ Elaine de Oliveira Souza Fonseca ${ }^{1}$, Larissa Chaves Pedreira ${ }^{1}$, Roberta Pereira Góes ${ }^{1}$, João Tavares ${ }^{2}$ \\ ${ }^{1}$ Federal University of Bahia. Nursing school. Postgraduate Nursing and Health Program. Brazil, Bahia., ${ }^{2}$ University of Aveiro. Department of Health Sciences, Aveiro, Portugal.}

Introduction: In the aging process, preventing diseases is intended, and among them are those related to the care of the oral cavity. Nowadays, with the coronavirus pandemic, this care must be improved as it reduces the microbial load in the mouth and the complications associated with COVID-19. The aim of this study was to analyze the nursing care for oral health of hospitalized elderly through their records.
Methods: Descriptive and qualitative research, parts of the master's dissertation. Data collection occurred through interviews, between September and October 2018, with 35 professionals from the nursing team of the intensive care unit and the ward of a Brazilian university hospital in Salvador, Bahia. The data were analyzed by Thematic Content Analysis. The study followed all ethical standards.

Results: Participants reported insufficient records on care for the oral cavity, especially nursing technicians. The personnel is limited to checking the time of the care, without materials or hygiene technique descriptions and primarily lacking records about aspects of the oral cavity. The main justification of the team for this conduct is the excessive demand in the units. Flawing to register interferes in evaluation of care results, preventing improvements in care and careful analysis of the oral cavity.

Key conclusions: Personnel sizing and depreciation of assistance to the oral cavity impact on nursing care and, thereafter, on the related records.

\section{ABSTRACT \# 71}

\section{Old frequent users in a tertiary hospital: Portuguese reality} at a glance

Andre Martins ${ }^{1}$, Sara Encarnação Encarnação ${ }^{2}$, Margarida Tojo ${ }^{3}$, Cristina Amaral ${ }^{4}$, Inês Rego Figueiredo ${ }^{5}$, Heidi Gruner ${ }^{5}$, Teresa Fevereiro $^{6}$

${ }^{1}$ Serviço de Urgência, Hospital de São José , Centro Hospitalar Universitário Lisboa Central, ${ }^{2}$ Serviço de Urgência, Hospital de São José , Centro Hospitalar Universitário Lisboa Central, ${ }^{3}$ Serviço de Urgência, Hospital de São José , Centro Hospitalar Universitário Lisboa, ${ }^{4}$ Serviço de Urgência-H São José, Centro Hospitalar Universitário Lisboa Central, ${ }^{5}$ Consulta Multidisciplinar de Geriatria do Centro Hospitalar Lisboa Central, ${ }^{6}$ Serviço de Urgência, Hospital São José, Centro Hospitalar Universitário Central

Introduction: Portugal is the third country with the oldest population in the world, but still with only few geriatricians. We face a huge challenge especially in the Emergency Departments (ER) where the elderly are prevalent and account for the largest group of frequent users (defined as more than 4 visits a year). It is essential to evaluate these patients in order to create policies directed to avoid extensive and unnecessary use of the available health resources and on the other hand to create adequate health offers to meet the needs.

Methods: Retrospective descriptive study of patients older than 75 years admitted to the ER of a tertiary hospital, during 2018. The analyzed data were obtained from the electronic file namely demographic data (gender and age), priority score determined in the triage (red- immediate resuscitation, orange-very urgent, yellow- emergent, green-non urgent, blue), admittance with an ambulance that meant that there was probably a previous telephonic contact with the National Institute of Emergency Medicine (INEM), diagnostic tests ordered, medical specialties that evaluated the patient, time spent in the ER and destination.

Results: There were 1913 patients included, with an average age of 81,29 (of which 639 had over 85years), 1115 (58,3\%) females. There were 406 patients with more than 6 visits and 116 with more than 9 visits, accounting for a total of 10705 episodes.A sample of 2082 episodes was evaluated, the priority score showed $11(0,5 \%)$ red, 196 $(11,4 \%)$ were orange and the majority $1104(53 \%)$ were considered yellow patients, there were $771(37.7 \%)$ of non-emergent episodes. Patients arrived with an ambulance in $579(27,8 \%)$ episodes. The average length of stay was 9:12 hours (0:13 minutes to 10 days). Concerning diagnostic tests, blood test was performed in 1303 $(62,58 \%)$, ECG in 767 (36,9\%), radiographs in $1046(50,24 \%)$ and CT-scans in $290(13,8 \%)$. The patients admitted to the medical and 
chirurgical wards were $357(17,2 \%)$, discharged to the health care center or outpatient clinic or home were $1371(65,9 \%)$, only 1 died. Discussion: Elders are more vulnerable due to multi morbidities and polypharmacy and the clinical presentation of acute disease tends to differ from the young adult, being frequently vague, this assumes importance in the ED that is at the core of the healthcare for acutely ill . There is a tendency and a necessity for the use and abuse of diagnostic tests and medical specialties, that increases both human and monetary costs.

\section{Abstract \# 72 \\ Infectious diseases and its characteristic features in a geriatrics service}

Nora Molina Torres ${ }^{1}$, Carmen Cánovas Pareja ${ }^{1}$, Elena Rebollar Torres ${ }^{1}$, Ruth Jorge García ${ }^{1}$

${ }^{1}$ Hospital Nuestra Señora de Gracia

Introduction: We aimed to define the antibiotic use and microbiological profile of a geriatrics service in a secondary hospital between the years 2018-2019, with an antibiotic stewardship program PROA (Programa de optimización de antibióticos). The information was collected from the program's database.

Results: Our hospital accounts for 151 beds. 54 of them belong to our service. Regarding bacteriemias, $51,36 \%$ of the hospital's belonged to geriatric patients: $45 \%$ of S. coagulase negative, $53,84 \%$ streptococcus spp, 55,38\% of E. coli (including BLEE) and $45 \%$ of S. aureus. The proportion of bacteriemias with origin in the community rose significantly (from 30,20 to 48,70\%; p = 0,017).From 2018 to 2019, the consumption of all antibiotics rose. However, the use of quinolones was reduced by $56,26 \%$. Wide spectrum antibiotics use became higher, but not statistically signitficant. $(r=0,3 ; p=0,2)$.Concerning multiresistant bacteria, they originated mostly in nursing homes ( $\mathrm{p}=$ $0,001)$. The bacteria cultured in our service, in proportion with the rest of the hospital, were: SAMR 50,90\%, E. coli BLEE 59,49\%, K. pneumoniae BLEE $48,78 \%$, enterobacteria spp $50 \%$ and acinetobacter spp 33,33\%. The most frequent multiresistant bacteria in our patients were SARM y E. coli.

Conclusions: Infectious diseases have specific characteristics in a Geriatrics Service. The Geriatrics Service accounted for most of the hospital's bacteriemias. Infections due to multiresistant bacteria belonged mostly from nursing homes. In our service the use of quinolones was reduced, although the use of wide spectrum antibiotics rose slightly.

\section{Abstract \# 73 \\ Call me maybe? Differences in "next of kin" and "contact in emergency" contacts in the acute hospital setting \\ Robert P Murphy ${ }^{1}$, Christina D Campbell ${ }^{1}$, Grainne O'Malley ${ }^{1}$, Paula Hickey $^{1}$, Fiona O'Sullivan ${ }^{1}$ \\ ${ }^{1}$ Sligo University Hospital}

Background: A large number of hospitals in Ireland use the same patient information management system. This interface gives patients the option of inputting both "next of kin" (NOK) and "contact in emergency" contact options. This can regularly lead to confusion when communicating with families, and there is often misunderstanding among staff and patients about the significance of these terms.

Methods: We conducted a cross sectional observational cohort study of all hospitalised inpatients to examine how many patients have the same designated NOK and "contact in emergency" person.
Results: 152 patients were included. 103 patients $(67.8 \%)$ had a different person nominated as NOK and contact in emergency. 35 patients $(23 \%)$ had the same designated person for each, and 13 $(8.6 \%)$ had only of these two options filled in. The most common discrepancy was where a spouse was listed as NOK, and a child listed as contact in emergency, followed by two separate children in each role, and then two separate siblings. Subgroup analysis showed no significant difference in mean age $(72.8$ vs 74 years, $p=0.36)$ or mean length of stay in days (11.6 vs 7.9, p 0.11) in patients who designated a different contact person for NOK and contact in emergency versus those who did not. There was no difference in gender ( $p$ $=0.23$ ) between the two groups.

Discussion: We found large discrepancies in the contact details for NOK and contact in emergency. False beliefs exist that the NOK term confers additional powers to those nominated to that role, and we would recommend a re-organisation of the IT administration systems to consolidate these two confusing labels into one emergency contact.

\section{Abstract \# 74}

Clinical semiology of anemia in patients over 75 years: a prospective study conducted in France by the SiFMI group

Abrar-Ahmad Zulfiqar ${ }^{1}$, Noël Lorenzo-Villalba ${ }^{1}$, Hassanine Ben Malek $^{1}$, Thomas Vogel ${ }^{2}$, Emmanuel Andres ${ }^{1}$

${ }^{1}$ University Hospital of Strasbourg, Department of Internal Medicine, Strasbourg, France, ${ }^{2}$ University Hospital of Strasbourg, Department of Geriatrics, Strasbourg, France

Introduction: Anemia remains one of the main concerns of the daytoday life of Internist since it ranks third among the pathologies seen in Internal Medicine Departments. It seemed appropriate for the SiFMI Group (Common Situations in Internal Medicine of the SNFMI) to carry out a study of anemia evaluating the contribution to the diagnosis of the «classical» semiology of the anemic syndrome. This work reports the results from the study of 204 patients aged 75 years and more.

Method: A prospective, non interventional multicenter study was carried out in Internal and Geriatric Departments in the period September 2015-September 2017. Clinical and biological variables were collected.

Results: 204 patients from 10 Internal or Geriatric Departments were included in the period September 2015-September 2017. They were assigned into two groups with and without anemia. According to this study, it seems that the following parameters: asthenia, dyspnea (NYHA stages III and IV), palpitations, chest pain, tachycardia, hypotension as well as confusion, falls, depression and MMSE showed no significant statistical difference between both groups in contrast to pallor, edema, albumin and ECG.

Conclusion: Clinical diagnosis of anemia in elderly individuals remains a challenge. It is difficult to establish a diagnostic protocol in this population.

\section{Abstract \# 75}

Explaining he sex difference in gait speed among older adults: differential sensitivity for and exposure to lifestyle, social, health and sociodemographic factors

Lena D. Sialino ${ }^{1}$, Laura A. Schaap ${ }^{1}$, Sandra H. van Oostrom ${ }^{2}$, Hendrika S.J. Picavet ${ }^{2}$, Johannes W.R. Twisk ${ }^{3}$, Monique W. M. Verschuren ${ }^{4}$, Marjolein Visser ${ }^{1}$, Hanneke A.H. Wijnhoven ${ }^{1}$

${ }^{1}$ Department of Health Sciences, Faculty of Science, Amsterdam Public Health research institute, VU University Amsterdam, 
Amsterdam, the Netherlands, ${ }^{2}$ Centre for Nutrition, Prevention and Health Services, National Institute for Public Health and the Environment, Bilthoven, the Netherlands, ${ }^{3}$ Department of Clinical Epidemiology and Biostatistics, VU University Amsterdam, the Netherlands, ${ }^{4} 2$ Centre for Nutrition, Prevention and Health Services, National Institute for Public Health and the Environment, Bilthoven, the Netherlands

Introduction: Older women have a lower gait speed compared to older men (i.e. female disadvantage), which the current study aims to explain.

Methods: Data from the Longitudinal Aging Study Amsterdam (LASA) were used. In total, 4109 participants were included in 1992/2002 (aged 55-81 years, with 25/15 years follow-up, every 3-4 years). Gait speed and various lifestyle, social, health and socio-demographic factors were assessed. Mixed model multivariate association analysis was used to investigate sex differences in (1) sensitivity for factors (interaction term with sex) and (2) in exposure to factors (percentage change of the sex difference) associated with gait speed.

Results: Older women had a lower gait speed compared to men ($0.047 \mathrm{~m} / \mathrm{s} 95 \%$ CI -0.064 to -0.029 , height and age adjusted). Women had a stronger association of higher BMI, higher waist circumference, pain and lower physical activity with lower gait speed (i.e. sensitivity), where higher BMI and especially pain were also more prevalent among women (i.e. exposure). In addition, low education and chronic diseases were also more prevalent among women. In contrast, two factors associated with higher gait speed were more often found among women: larger personal network size and higher physical activity.

Key conclusions: The female disadvantage in gait speed represents a combination of (1) a higher exposure to most risk factors and (2) a higher sensitivity to some risk factors, of older women compared to men. From a prevention point of view, the female disadvantage could be bridged by intervening at the modifiable factors pain, BMI, and physical activity.

\section{Abstract \# 76 \\ Short telomeres length in leukocytes is the key risk factor of both the atherosclerosis and arteriosclerosis}

Irina Strazhesko ${ }^{1}$, Anna Akopyan ${ }^{2}$, Olga Tkacheva ${ }^{3}$, Ekaterina Dudinskaya $^{3}$, Anna Kruglikova ${ }^{3}$, Nataliya Sharashkina ${ }^{3}$, Nataliya Brailova $^{3}$, Olesya Isaykina ${ }^{4}$, Maria Pokrovskaya ${ }^{4}$, Vladimir Vygodin $^{4}$, Dmitry Skvortsov ${ }^{1}$

${ }^{1}$ 1- Lomonosov Moscow State University, Moscow, Russian Federation, ${ }^{2} 1$ - Lomonosov Moscow State University, Moscow, Russian Federation 2- Russian Clinical Research Center for Gerontology, Moscow, Russian Federation, ${ }^{3} 2$ - Russian Clinical Research Center for Gerontology, Moscow, Russian Federation, ${ }^{4} 3$ National Medical Research Center for Preventive Medicine, Moscow, Russian Federation Russian

Introduction: Increased arterial stiffness, atherosclerotic plaques presence are important aspects of vascular aging, which may be considered as morphological and functional basis for cardiovascular diseases (CVD). These two processes can occur in isolation or simultaneously in the arterial wall. Peripferal blood leukocytes telomeres length (TL) is cellular senescence and vascular aging biomarker, which has been proposed as an independent predictor of cardiovascular diseases. But presently there is no detailed information regarding the interactions of TL and conventional cardiovascular risk factors (CVRF) in vascular aging. The aim of this study was to determine the role of TL in their interaction with CVRF in vascular aging process.
Methods: The study group included 303 ambulatory participants (104 males and 199 females) mean age $51.8 \pm 13.3$ years, free of known cardiovascular diseases, diabetes mellitus, antihypertensive and lipid lowering medications, but with one or more CVRF (age, smoking, arterial hypertension, obesity, dyslipidemia, fasting hyperglycemia, insulin resistance-HOMA IR $>2,5$ ). The study sample was divided into the two groups according to the age of participants: "younger" (m $<45$ years, $\mathrm{f}<55$ years) and "older" ( $\mathrm{m}>45$ years, $\mathrm{f}>55$ years). Plaques presence was determined by ultrasonography in both left and right carotid arteries. Arterial stiffness was appreciated by carotidfemoral pulse wave velocity (c-f PWV) measuring with the help of SphygmoCor (AtCor Medical). It was increased in case of c-f PWV > $10 \mathrm{~m} / \mathrm{s}$. TL was determined by quantitative polymerase chain reaction. TL $<9.25$ (the shortest telomeres) corresponded to the first quartile of the distribution (Q1)

Results: The results of multiple logistic regression analysis (with adjustment for CVRF) in the two age groups are represented in the table. "Younger" group. c-f PWV $>10 \mathrm{~m} / \mathrm{sPredictor} \beta \pm \mathrm{SE} \chi^{2}$ Wald statistics, p OR 95\% CI Q1 TL 2,372 $\pm 0,700 \quad 11,485 \quad 0,001 \quad 10,72$ 2,72-42,27 "Younger" group. Plaques $>0 \mathrm{Q} 1$ TL 2,832 $\pm 1,0667,059$ 0,008 16,98 2,10-137,01"Older" group. c-f PWV > $10 \mathrm{~m} / \mathrm{sQ1} \mathrm{TL}$ $0,965 \pm 0,3916,1040,0142,631,22-5,64$. There was no independent significant association of TL and PP in "older" group.

Conclusions: TL shows more stable relationship with increased stiffness of arteries than with atherosclerosis. TL $<9.25$ is risk factor of increased arterial stiffness in both age groups, and is a risk factor of atherosclerosis only in the "younger" age group. The relationship of TL with arterial stiffness is stronger in the "younger" group.

\section{Abstract \# 77 \\ Clinical implication of obesity in esophageal cancer in the elderly: a nationwide population-based cohort study of South Korea}

Jea Ho Cho ${ }^{1}$, Eun Ji Lee ${ }^{1}$, Dong Ho Lee ${ }^{1}$, Cheol Min Shin ${ }^{1}$, Young Soo Park ${ }^{1}$, Nayoung Kim ${ }^{1}$

${ }^{1}$ Department of Internal Medicine, Seoul National University Bundang Hospital

Background: Esophageal cancer (EC), which is composed of squamous cell carcinoma (SCC) in around $90 \%$ in South Korea, is a disease occurring in the older adults. Recently, it has been reported that obesity is associated with EC. However, there are limited data on the relationship between obesity and EC in the elderly. Thus, we investigated this relationship in a nationwide population-based cohort. Methods: A retrospective cohort study of 3,102,063 individuals who were over 65 years old and had regular health check-ups provided by the National Health Insurance Corporation between 2009 and 2012 in South Korea was conducted. Overall obesity was assessed as body mass index (BMI), and abdominal obesity was defined as a waist circumference $>90 \mathrm{~cm}$ for men and $>85 \mathrm{~cm}$ for women. We estimated hazard ratios (HRs) and $95 \%$ confidence intervals (CIs) using $\chi^{2}$-test and Cox proportional hazard model adjusted for confounding factors. The primary outcome was newly developed EC.

Result: During a median follow-up period of 6.4 years, 6,098 individuals $(0.19 \%)$ developed EC. BMI was negatively associated with EC risk (HR 1.61, 95\% CI 1.45-1.79; underweight $\left[<18.5 \mathrm{~kg} / \mathrm{m}^{2}\right]$ versus normal BMI [18.5-22.9 kg/m2]). However, after adjusting for BMI, abdominal obesity increased the risk of EC (HR 1.27, 95\% CI 1.18-1.36). Among overweight (BMI 23-24.9 kg/m2) and obese I (BMI $25-29.9 \mathrm{~kg} / \mathrm{m} 2$ ) elderly individuals, abdominal obesity was a risk factor for EC (HR 1.15, 95\% CI 1.02-1.30; HR 1.27, 95\% CI $1.13-1.43$, respectively). 
Discussion: Increasing abdominal obesity may be associated with an increased risk for esophageal SCC in the older adults. Further studies are warranted to confirm the relationship.

\section{Abstract \# 78 \\ DNA damage accumulation and decreased DNA repair capacity in the elderly \\ Panagiotis Ntouros $^{1}$, Evrydiki Kravvariti ${ }^{2}$, Vassilis L Souliotis ${ }^{3}$, Petros P Sfikakis ${ }^{2}$ \\ ${ }^{1}$ First Department of Propaedeutic Internal Medicine and Joint Rheumatology Program, School of Medicine, NKUA, Athens, Greece, ${ }^{2}$ Postgraduate Medical Studies in Geriatric Syndromes and Physiology of Aging, School of Medicine, NKUA, Athens, Greece, ${ }^{3}$ Institute of Chemical Biology, National Hellenic Research Foundation, Athens, Greece}

Introduction: Increased endogenous DNA damage endangers cellular health, since it may lead to genomic instability, cellular senescence and apoptosis. In this study, we tested the hypothesis that deregulated DNA damage Response and Repair (DDR/R) network is implicated in the aging process through the accumulation of DNA damage over time.

Methods: Peripheral blood mononuclear cells (PBMCs) were isolated from 40 older adults (mean age \pm SD: $78 \pm 10$ ) and 50 apparently healthy younger controls (HC mean age \pm SD: $36 \pm 12$ ). The older individuals were characterized as: 15 non-frail [Clinical Frailty Scale (CFS):1-3], 10 prefrail (CFS: 4-5) and 15 frail persons (CFS: 6-9). Endogenous DNA damage using alkaline Comet assay, DNA damage formation by measuring oxidative stress (GSSG to GSH ratio) and abasic DNA sites as well as two main mechanisms of DNA damage repair, namely double-strand break repair (DSB-R) using confocal microscopy and nucleotide excision repair (NER) following UVC irradiation were evaluated.

Results: Increased endogenous DNA damage in PBMCs was observed in the older subjects [Olive Tail Moment (OTM): $7.88 \pm$ 3.44] compared to HC (OTM: $4.55 \pm 1.63, \mathrm{p}<0.001)$. This increase versus HC remained significant, when analyzing each subset separately (non-frail: $7.09 \pm 2.75, \mathrm{p}<0.01$; pre-frail: $8.36 \pm 3.27, \mathrm{p}<$ 0.01 ; frail: $9.45 \pm 5.16, \mathrm{p}<0.01)$. Significantly higher oxidative stress and abasic DNA sites were evident in the older subjects compared to controls. While NER capacity was preserved, DSB-R capacity was deficient in all three frailty subsets.

Conclusion: Older adults exhibit augmented endogenous DNA damage compared to younger individuals, which could be attributed to the increased formation of DNA damage and deficient DSB-R capacity.

\footnotetext{
Abstract \# 79

Quantification of peripheral blood angiogneic and senescent $T$ cell subsets in non-frail and frail older adults: Preliminary analysis

Thomas Byrne ${ }^{1}$, Pádraig Bambrick ${ }^{2}$, Edel McNeela ${ }^{1}$, John Cooke ${ }^{2}$, Michael Harrison ${ }^{1}$

${ }^{1}$ WIT, Waterford, Ireland, ${ }^{2} \mathrm{UHW}$, Waterford, Ireland

There is an important vascular dimension to age-related frailty, including associations with increased cardiovascular morbidity and mortality and vascular cognitive impairment. Vascular biomarkers offer the potential to monitor response to treatment in frail populations, with respect to the vascular risk element of the condition. CD3+ $\mathrm{T}$ cells expressing the CD31 adhesion molecule (PECAM-1), have been shown to play a role in vascular homeostasis and repair and are
}

termed angiogenic $\mathrm{T}$ cells (TANG). T cells that no longer express CD28 (CD28NULL) are regarded as senescent $\mathrm{T}$ cells and have a proinflammatory phenotype. These $\mathrm{T}$ cell subsets are appealing biomarkers for the vascular dimensions of frailty due to their biological associations with vascular repair, inflammation, and immunosenescence. Using flow cytometry, we have quantified TANG and CD28NULL T cell subsets in young adults $(\mathrm{n}=21)$, non-frail $(\mathrm{n}=$ 16) and frail $(n=12)$ older adults. The $\%$ frequency of TANG in the young, non-frail older and frail older groups were $44.2 \pm 2.4 \%, 20.8$ $\pm 2.4 \%$ and $27.3 \pm 2.2 \%$ respectively with the frequency lower $(\mathrm{p}<$ 0.001 ) in both older cohorts compared to young and the difference between frail and non-frail approaching significance $(\mathrm{p}=0.068)$. The $\%$ frequency of CD28NULL $\mathrm{T}$ cells in the respective groups were $13.3 \pm 3.3 \%, 15.3 \pm 3.4 \%$ and $30.6 \pm 6.1 \%$ with the frequency lower $(\mathrm{p}<0.05)$ in the young and non-frail compared to the frail and no difference between the young and the non-frail $(\mathrm{p}=0.691)$. There is some evidence in our preliminary data that $\mathrm{T}$ cell subsets with biological associations to vascular health can distinguish frail and nonfrail older adult groups.

\section{Abstract \# 80}

Restrictive cardiomyopathy due to cardiac amyloidosis

Atef Michael ${ }^{1}$

${ }^{1}$ Russells Hall Hospital, Dudley, UK

Case report: An 80-year-old male was admitted with dyspnea and increasing pedal oedema. His medical history included hypertension, $\mathrm{AF}, \mathrm{CCF}$ and carpel tunnel syndrome. He was non-smoker. Examination was unremarkable apart from ankle oedema up to the thighs. FBC, LFTs and Ca were normal and eGFR 41. Serum protein electrophoresis showed no paraproteins and urine protein electrophoresis revealed no monoclonal free light chains. ECG showed AF, LBBB and poor $\mathrm{R}$ wave progression. Echocardiography revealed restrictive cardiomyopathy with mirror image hypertrophy of both ventricles suggestive of Amyloid infiltration. There was moderate bi-atrial dilatation. He underwent Coronary angiogram and myocardial biopsy. Histopathology showed features consistent with cardiac amyloidosis. Discussion: Cardiac amyloidosis (CA) has to be considered if the patient has other manifestations suggestive of amyloidosis or heart failure with preserved left ventricular function or heart failure without cardiomegaly or if he has left ventricular hypertrophy without identifiable cause. ECG can be normal or show voltage criteria of LVH or low-voltage complexes and pseudo-infarction patterns. Echocardiography may reveal thick walled ventricles and apical sparing is typical in CA. Thickening of both the LV and RV is an important echocardiographic clue that can differentiate $\mathrm{CA}$ from other diseases. Cardiac MRI is a more accurate imaging tool; late gadolinium enhancement is characteristic. CA is almost always ATTR or AL type. There is less cardiac amyloid deposit in patients with ATTR compared to patients with AL CA and ATTR has a better prognosis than AL. Up to $25 \%$ of patients 85 years and older have ATTRwt amyloid deposits on autopsy studies. $13 \%$ of patients 60 years and older hospitalized with heart failure with preserved ejection fraction had scintigraphy features consistent with ATTR-CA Endomyocardial biopsy is the gold standard to diagnose CA however in patients with normal serum free light chain and normal immunofixation, a strongly positive $99 \mathrm{~m}$ Tc PYP scan is diagnostic of ATTR CA (without endomyocardial biopsy).Amyloid patients are sensitive to digitalis, and may be intolerant to beta-blockers or angiotensin converting enzyme inhibitors. Cardiac involvement is the cause of death in approximately $50 \%$ of patients with AL Amyloidosis. 


\section{Abstract \# 81 \\ Ageism deteriorates the adeherence for anticoagulation therapy guidelines in seniors with atrial fibrillation and increases mortality of the cohort - current clinical experience from outpatient clinics \\ Peter Olexa ${ }^{1}$ \\ ${ }^{1}$ Gerontology and Geriatric Clinic, Univ. Hospital of St.Michal, Košice, Slovakia}

Background: Atrial fibrillation (AF) is a problem of growing prevalence as a consequence of the ageing population, is associated with high morbidity, mortality, and healthcare costs. The risk is significantly reduced by oral anticoagulation. Adherence to guidelines may lower the risks for both all cause and cardiovascular (CV) deaths. Methods: Our objective was to evaluate the type and adherence of prescribed antithrombotic treatment according to the 2012 and 2016 European Society of Cardiology (ESC) guidelines in studied group of consecutive patients managed in private cardiology office in years 2012 and 2017. Only patients with diagnosis of AF were analysed. We aimed to prove, if nonadherence is associated with higher rate of adverse outcomes. Data were obtained from consecutive patients managed in private cardiology office in years 2012 and 2017. Only patients with diagnosis of AF older than 65 years were analysed.

Results: Among 2850 patients examined in 2012, 213 (8\%) were discharged with a diagnosis of AF. In 2017 we examined 4389 patients, 401 (9\%) diagnosed with $\mathrm{AF}$, among them 350 older than 65 $(87.5 \%)$. Median age in both groups was 76ys. Significant differences in the frequency and type of anticoagulation therapy were observed between 2012 and 2016-warfarin was used in 102 (47\%) vs. 110 (31\%), NOACs in $25(12 \%)$ vs. $189(54 \%)$, ASA or clopidogrel in 68 $(322 \%)$ vs. $41(12 \%)$. None antitrombotics were used in $20(10 \%)$ vs. $10(3 \%)$. Based on ESC guidelines, both groups were divided into two subgroups according to the guideline adherence to proper anticoagulation therapy. The quality of medical treatment increased significantly from 2012 to 2017 . $61 \%$ of patients were on guidelineadherent thromboprophylaxis, $39 \%$ were undertreated in 2012, while in 2017 the guidelines were followed much better - 14\% were undertreated vs. $86 \%$ treated properly. We examined statistically the importance of age, frailty, history of ischaemic stroke, minor heamorhage, and values of CHADS2 and HASBLED indexes for the nonadherence to Guideline aproved antitrombotic management. Series of binary logistic analyses showed that increasing age $(\mathrm{p}=0.05)$, and concomitant history of minor bleeding $(\mathrm{p}=0.003)$ were associated with undertreatment in 2012, while in 2017 HASBLED score (p $<0.051$ ), nor other studied factor led to non-adherence to Guideline aproved therapy. AF patients who were guideline adherent had a lower rate of all-cause death $(p=0.007)$ compared to those nonadherent. Binary logistic regresion analysis showed that guidelinenonadherent patients had a higher risk for all-cause mortality ( $\mathrm{p}=$ $0.003)$.

Conclusions: Non-adherence to guidelines is currently less prevalent among elderly AF patients compared to clinical treatment in previous years. Proper Guideline-adherent treatment is being independently associated with lower risk of all-cause mortality. Efforts to improve guideline adherence would lead to better outcomes for elderly and frail AF patients.

\section{Abstract \# 82}

Interest of ambulatory management of patients with chronic heart failure by protocolized follow-up and therapeutic education: results of the USICAR experiment.

Anne Jenneve $^{1}$, Guy Courdier ${ }^{1}$, Samy Talha ${ }^{2}$, Noël LorenzoVillalba $^{3}$, François Severac ${ }^{4}$, Abrar-Ahmad Zulfiqar ${ }^{3}$, Patrick Arnold $^{1}$, Philippe Lang ${ }^{1}$, Gérald Roul ${ }^{5}$, Emmanuel Andres ${ }^{3}$

${ }^{1}$ Unité de Suivi des Patients Insuffisants Cardiaques, Clinique du Diaconat, Mulhouse, France, ${ }^{2}$ Equipe de recherche EA 3072

"Mitochondrie, Stress oxydant et Protection musculaire", Faculté de Médecine de Strasbourg, Université de Strasbourg), Strasbourg, France, ${ }^{3}$ Service de Médecine Interne, Diabète et Maladies Métaboliques, Clinique Médicale B, Hôpitaux Universitaires de Strasbourg, Strasbourg, France, ${ }^{4}$ Département de Santé Publique / DIM et Biostatistiques, Hôpitaux Universitaires de Strasbourg, Strasbourg, France, ${ }^{5}$ Unité fonctionnelle dédiée à l'insuffisance cardiaque, Pôle médicale et chirurgicale des maladies cardiovasculaires, Hôpitaux Universitaires de Strasbourg, Strasbourg, France

Introduction: The objective of this study is to determine whether the implementation of regular and structured follow-up of patients with chronic heart failure, combined with therapeutic education, led to better management of these patients.

Patients and method: This was a monocentric, retrospective study on a cohort of patients with a proven chronic heart failure, followed in the Mulhouse region (France), between January 2016 and December 2017, by the Unit for Monitoring Heart Failure Patients (USICAR). These patients benefited from a regular protocolized follow-up and a therapeutic education program for a period of 2 years. The main criterion of this study was: the number of days of hospitalization for Heart Failure per year and per patient. The secondary endpoints were: the number of days of hospitalization for cardiac causes other than Heart Failure and the number of hospital stays for Heart Failure per patient. These criteria were collected over the one-year period before inclusion, at one year follow-up, and at two years follow-up.

Results: 159 patients with a mean age of 72.9 years were included in this study. They all had a chronic heart failure, mainly stage I-II NYHA $(88.7 \%)$, of predominantly ischemic origin $(50.9 \%)$, with altered left ventricular ejection fraction in $69.2 \%$ of cases. The primary endpoint averaged 8.33 days $(6.84-10.13)$ in the year prior to inclusion, 2.6 days (1.51-4.47) in the first year of follow-up, and 2.82 days (1.30-6.11) ( $\mathrm{p}<0.01$ for both comparisons). The mean number of days of hospitalization for other cardiac causes other than Heart Failure to patient numbers was: 1.73 days (1.16-2.6), 1.81 days (1.04-3.16), and 1.32 days $(0.57-3.08)(\mathrm{p}=\mathrm{ns})$. The percentage of hospitalization for Heart Failure for each patient was: $69.5 \%$ (60.2-77.4) before inclusion, $16.2 \%$ (10-25.2) during the first year of follow-up and $19.3 \%$ (11-31.8) during the second ( $p<0.001$ for both comparisons).

Conclusion: This study demonstrates the value of a protocolized follow-up associated with a therapeutic education program to improve the management of ambulatory chronic heart failure patients, particularly for moderate chronic heart failure.

\section{Abstract \# 83}

The quality of life in elderly patients with metabolic syndrome and chronic heart failure with intermediate ejection fraction

Elena Gosteva (1), Olga Osipova (2)

${ }^{1} \mathrm{~A},{ }^{2} \mathrm{O}$

Introduction: In recent years, the number of elderly patients with metabolic syndrome (MS) and heart failure with intermediate ejection 
fraction (HFmrEF) had a tendency to increase. Hyperstimulation of the hypothalamic-pituitary-adrenal system in insulin resistance, which leads to increased cortisol excretion, is considered to be one of the possible reasons for the decline of their quality of life, and may be associated with the development of depression as geriatric syndrome. Methods: We perforned opened, randomized study in which were involved 65 patients with HFmrEF MS, mean age $66.7 \pm 4.3$ years, control group - 30 patients without HFmrEF MS, and 20 patients without cardiovascular diseases. The W. Zung scale (1980) was used to assess anxiety and the degree of depressive disorder. Quantitative data were presented as the median (Me) and upper and lower quartiles (25\%; 75\%), significant differences were considered at $\mathrm{p}<0.05$.

Results: In the control group, anxiety was measured in 18.1 (16.0; 19.5) points, in the HFmrEF group without MS 35.9 (30.8; 43.0), in the studied HFmrEF group with MS - $60.2(52.6 ; 67.9)$ points $(\mathrm{p}<$ 0.001 compared to patients without MS and healthy. In the control group, self-assessment of depression was $27.3(22.9 ; 31.1)$ points, in HFmrEF without MS-53.2 (44.8; 59.6) points, in the studied group of HFmrEF in the presence of MS-78.1 $(61.9 ; 85.3)$ points $(\mathrm{p}<0.001$ compared with patients without MS and healthy).

Conclusion: the results of the study indicate that elderly patients with HFmrEF had increased levels of anxiety and depression. The combination of HFmrEF with metabolic syndrome led to a further increase in anxiety-depressive disorders as geriatric syndromes.

\section{Abstract \# 84 \\ The cut-off value of serum homocysteine in relation to increase of coronary artery calcification \\ Nam-Seok Joo ${ }^{1}$, Susie Jung ${ }^{2}$ \\ ${ }^{1}$ Ajou University School of Medicine, ${ }^{2}$ Ajou University Hospital}

Purpose: Recent study reported that coronary artery calcification and serum homocysteine were well associated, however, no report is available for the cut-off value of serum homocysteine according to increase of coronary artery calcification volume score.

Materials and methods: The data of 469 subjects out of 777 subjects in one health promotion center located in Seoul were selected after exclusion of the missing data of serum homocysteine and coronary artery calcification volume score (CVS). CVS was categorized into two groups; CVS $=0$, and CVS $>1$. Serum homocysteine according to the CVS groups was compared and the cut-off value of serum homocysteine according to the increase of CVS $(>1)$ was calculated using ROC curve.

Results: Mea age was 54.5 years and proportion of female was 22.2\%. Mean serum homocysteine concentration and CVS was 11.2 $\mathrm{uMol} / \mathrm{L}$ and 50.4, respectively. After adjustments with age and sex, serum homocysteine was associated with CVS $(r=0.167, p=0.001)$ and $\log$ (Homocysteine) also showed significantly difference according to the CVS groups. The cut-off value of serum homocysteine according to the increased of CVS $(>1)$ was $9.45 \mathrm{uMol} / \mathrm{L}$ [AUC $=0.569$ (95\% CI 0.512-0.625), $\mathrm{p}=0.015$ ].

Conclusion: The cut-off value of serum homocysteine was 9.45 $\mathrm{uMol} / \mathrm{L}$ according to increase of coronary artery calcification volume score.

\section{Abstract \# 85}

Evaluation of the French national program on home return of patients with chronic heart failure (PRADO-IC): pilot study of 91 patients during its deployment in the Bas Rhin area

Radreau Mylène ${ }^{1}$, Samy Talha ${ }^{2}$, Jean-Jacques Von Hunolstein ${ }^{3}$, Noël Lorenzo-Villalba ${ }^{1}$, Michel Hanssen ${ }^{4}$, Anne Koenig ${ }^{3}$, Philippe Couppie $^{4}$, Bernard Gény ${ }^{2}$, Gérald Roul ${ }^{3}$, Emmanuel Andrès ${ }^{1}$

${ }^{1}$ Service de Médecine Interne, Diabète et Maladies Métaboliques, Clinique Médicale B, Hôpitaux Universitaires de Strasbourg, Strasbourg, France, ${ }^{2}$ Service de Physiologie et Laboratoire d'Explorations Fonctionnelles, Hôpitaux Universitaires de Strasbourg, Strasbourg, France, ${ }^{3}$ Service de Cardiologie, Unité Fonctionnelle dédiée à l'insuffisance cardiaque, Pôle Médicochirurgical de Cardiovasculaire, Hôpitaux Universitaires de Strasbourg, Strasbourg, France, ${ }^{4}$ Service de Cardiologie, Centre Hospitalier de Haguenau, France

Objective: The main objective of this study was to evaluate the impact of the French national program on home return of chronic heart failure patients (PRADO-IC) in terms of re-hospitalizations for heart failure (HF) during its deployment in the Bas-Rhin (France).

Patients and methods: This was a pilot, descriptive, quantitative, retrospective, and bi-centric study (University Hospitals of Strasbourg and Haguenau' Hospital Center, France). It included all patients included in the PRADO-IC program from these centers between January 1, 2015 and December 31, 2015. The primary endpoint of our study was the evaluation of the number of 30-day, 6-month and 1-year re-admissions to the hospital in relation to an acute HF episode, before and after the inclusion of patients in the PRADO-IC program. The secondary endpoints were the number of overall re-hospitalizations (all-cause); the number of days of hospitalization for HF; the time to first re-hospitalization and the average length of hospital stay, before and after inclusion in PRADO-IC; and the overall and cardiovascular mortality rates.

Results: 91 patients with a mean age of 79.2 years (67-94) were included. They all had chronic HF, essentially class II-III NYHA $(90.1 \%)$, mostly of ischemic origin $(41.9 \%)$, with altered left ventricular ejection fraction in $71.4 \%$ of cases. A reduction in the mean number of hospitalizations for HF per patient at 30 days, 6 months and 1 year was observed, respectively, from $0.18 \pm 0.42$ per patient before inclusion to $0.15 \pm 0.36$ after inclusion $(p=0.56) ; 0.98 \pm 1.04$ hospitalizations to $0.53 \pm 0.81$ at 6 months $(\mathrm{p}<0.01)$; and $1.64 \pm$ 1.14 hospitalizations $1.04 \pm 1.05$ at 1 year $(\mathrm{p}<0.001)$. Patients were hospitalized less overall after inclusion in the PRADO-IC program. The number of days of hospitalization for HF was reduced after inclusion of patients from: $18.02 \pm 7.78$ days before inclusion to $14.28 \pm 11.57$ days for the 6-month follow-up ( $\mathrm{p}=0.006$ ); and 22.07 \pm 10.33 days before inclusion to $16.39 \pm 15.94$ days for the 1 -year follow-up ( $\mathrm{p}<0.001)$. In contrast, inclusion in PRADO-IC statistically increased the mean time to first re-hospitalization for HF from mean $99.36 \pm 72.39$ days before inclusion to $148.11 \pm 112.77$ days after inclusion $(\mathrm{p}<0.001)$.

Conclusion: This study demonstrates the value of the PRADO-IC program in improving the management of chronic HF patients in ambulatory care, particularly with respect to HF re-hospitalization.

\author{
Abstract \# 86 \\ Pre-capillary pulmonary hypertension in elderly patients: \\ a descriptive study \\ Claire Caby $^{1}$, Tommy Courtel ${ }^{1}$, Noël Lorenzo-Villalba ${ }^{2}$, Amélie \\ Servettaz ${ }^{3}$, François Metz ${ }^{4}$, Abrar-Ahmad Zulfiqar ${ }^{5}$
}


${ }^{1}$ Department of General Medicine, University Hospital of Reims, 51000 Reims, Reims, France, ${ }^{2}$ Department of Internal Medicine, University Hospital of Strasbourg, 67000 Strasbourg, France, ${ }^{3}$ Department of Internal Medicine, University Hospital of Reims, 51000 Reims, Reims, France, ${ }^{4}$ Department of Cardiology, University Hospital of Reims, 51000 Reims, Reims, France, ${ }^{5}$ Department of Internal Medicine, University Hospital of Strasbourg, 67000 Strasbourg, Strasbourg, France

Introduction: Pulmonary hypertension $(\mathrm{PH})$ is a serious medical condition subsequently complicating many other common diseases. $\mathrm{PH}$ seems to be increasing in the elderly; however, it has been poorly studied in this group. The aim of this study was to determine the diagnostic and prognosis features as well as management of precapillary PH in the elderly compared to young subjects.

Methods: We performed a single center retrospective study in the Regional Hospital of Reims, France. Patients with precapillary PH diagnosed by right cardiac catheterization between January 2008 and December 2016 were included. Elderly patients (aged $\geq 65$ years at diagnosis) were compared to younger patients (18-64 years). Patients with Post-capillary PH were excluded.

Results: A total of 146 patients were included, 82 patients were aged 65 years or more (median age 74 years $(68-78), 56 \%$ women) and 64 non-elderly (median age 54 years $(48-61,5), 52 \%$ women). In the elderly group, $31.7 \%$ had pulmonary arterial hypertension (PAH): $15 \%$ idiopathic $\mathrm{PAH}, 36.6 \% \mathrm{WHO}$ group $3 \mathrm{PH}$ and $28 \% \mathrm{WHO}$ group $4 \mathrm{PH}$, with no significant differences with younger patients. In the group of 56 patients with PAH, 26 were elderly (46\%). Frequency of comorbidities was similar in both groups, except for arterial hypertension more prevalent in the elderly $(52 \%$ vs. $34 \%, \mathrm{p}=0.029)$. Older patients often had lower extremities oedema $(53.3 \%$ vs $36.2 \%, \mathrm{p}=$ $0.045)$ and reduced 6-minutes walking distance (189 $\mathrm{m}$ vs $289, \mathrm{p}=$ $0.004)$. Younger patients had lower FEV1 (58\% vs 75\%, p = 0.003) and higher right atrial pressure (12 vs $9, \mathrm{p}=0.023)$. Oxygenotherapy and diurectis were more often used in elderly subjects $(58.5 \%$ vs. $34.4 \%, \mathrm{p}=0.004)$ and $(82.9 \%$ vs. $67.2 \%, \mathrm{p}=0.027)$ respectively. Three-year and five-year survival were lower in elderly patients $(53 \%$ and $24 \%$, vs $86 \%$ and $63 \%, p=0.003$ and $p=0,004$ respectively). Conclusion: $\mathrm{PH}$ mainly affects individuals aged 65 years and more. Post-capillary $\mathrm{PH}$ is more prevalent but precapillary $\mathrm{PH}$ could be encountered. Clinical and paraclinical features seem to be similar in both groups. This entity has a worse impact on general health in elderly patient and the prognosis is poor

\section{Abstract \# 87 \\ Prevalence and predictors of hypotension in nursing home residents undergoing ambulatory blood pressure monitoring}

Giulia Rivasi ${ }^{1}$, Virginia Tortù ${ }^{1}$, Maria Flora D’Andria ${ }^{1}$, Giada Turrin $^{1}$, Evelina Giuliani ${ }^{1}$, Ludovica Ceolin ${ }^{1}$, Martina Rafanelli ${ }^{1}$, Enrico Mossello ${ }^{1}$, Andrea Ungar ${ }^{1}$

${ }^{1}$ Referral Centre for Hypertension Management in Older Adults, Division of Geriatric and Intensive Care Medicine, Careggi Hospital and University of Florence, Italy, ${ }^{2}$ Referral Centre for Hypertension Management in Older Adults, Division of Geriatric and Intensive Care Medicine, Careggi Hospital and University of Florence, Italy.

Introduction: In nursing home $(\mathrm{NH})$ residents, hypotension may predispose to adverse events including falls, fractures and mortality. Yet, evidence on hypotension detected by ambulatory blood pressure monitoring (ABPM) is scarce in this setting. This study aimed to determine the prevalence and the clinical predictors of ambulatory hypotension in NH residents and to analyze its related ABPM profile. Methods: We retrospectively analysed data from a sample of $100 \mathrm{NH}$ residents undergoing a comprehensive geriatric assessment and
ABPM. Ambulatory hypotension was defined as a systolic blood pressure (SBP) drop $\geq 20 \mathrm{mmHg}$ between two consecutive measurements to a minimum SBP $<100 \mathrm{mmHg}$. Participants with nocturnal hypotension were excluded from the analysis. Predictors of ambulatory hypotension were investigated using multivariate logistic regression adjusted for comorbidities, functional status and hypotensive medications.

Results: Among 91 residents (mean age 83, 56\% female), 50 showed ambulatory hypotension. They had a higher prevalence of heart failure $(\mathrm{p}=0.02)$ and atrial fibrillation $(\mathrm{p}=0.007)$ and more commonly received digoxin $(p=0.001)$ and benzodiazepines $(p=0.015)$. At ABPM they had lower $24 \mathrm{~h}$, daytime and nighttime SBP $(\mathrm{p}<$ $0.001)$ and more frequently showed a white coat effect $(\mathrm{p}<0.001)$ and a reverse-dipping profile $(\mathrm{p}=0.013)$. At multivariate analysis, benzodiazepines (OR 4.040), digoxin (OR 19.987) and creatinine $<1$ $\mathrm{mg} / \mathrm{dl}$ (OR 3.939) were independently associated with ambulatory hypotension.

Key conclusions: Hypotension is common in $\mathrm{NH}$ residents undergoing ABPM and more frequently occurs in subjects with lower ambulatory SBP, a reverse dipping profile and a white coat effect. Benzodiazepines, digoxin and low creatinine are predictors of ambulatory hypotension in $\mathrm{NH}$ residents.

\section{Abstract \# 88 \\ Intracranial artery calcifications: risk factors and association with cardiovascular disease and cognitive function.}

Nienke Golüke ${ }^{1}$, Esther de Brouwer ${ }^{1}$, Annemarieke de Jonghe ${ }^{2}$, Jules Claus $^{2}$, Salka Staekenborg ${ }^{2}$, Mariëlle Emmelot-Vonk ${ }^{1}$, Pim de Jong ${ }^{1}$, Huiberdina Koek ${ }^{1}$

${ }^{1}$ UMC Utrecht, ${ }^{2}$ Tergooi Hospital

Background and aims: We know little about clinical outcomes of arterial calcifications. This study investigates the risk factors of intracranial artery calcifications and the association with cardiovascular disease and cognitive function.

Methods: Patients were recruited from a Dutch memory clinic, between April 2009 and April 2015. The intracranial internal carotid artery (iICA) and basilar artery were analysed on the presence of calcifications. Calcifications in the iICA were also assessed on severity and location in the tunica intima or tunica media. Using logistic regression, risk factors of intracranial artery calcifications were analysed, as well as the association of these calcifications with cardiovascular disease, cognitive function and type of cognitive disorder (including subjective cognitive impairment, mild cognitive impairment and dementia). Cognitive function was assessed with the Cambridge Cognitive Examination.

Results: 1992 patients were included with a median age of 78.2 years. Around $40 \%$ of patients was male. The majority of patients had calcifications in the iICA. Basilar artery calcifications were less prevalent. Risk factors for cerebral intracranial calcifications were age, diabetes mellitus (medial iICA), hypertension (intimal iICA and basilar artery) and smoking (intimal iICA and basilar artery). iICA calcifications were associated with stroke and intimal calcifications also with myocardial infarction. Intracranial artery calcifications were not associated with cognitive function or type of cognitive disorder. Conclusion: The majority of memory clinic patients had intracranial artery calcifications. Cardiovascular risk factors are differentially related to medial or intimal iICA calcifications. iICA calcifications were associated with myocardial infarction and stroke, but not with cognitive outcomes. 


\section{Abstract \# 89}

Clinical predictors of frailty in elderly patients with cardiovascular diseases

Natalya Vorobyeva ${ }^{1}$, Irina Tsareva ${ }^{1}$, Galina Semochkina ${ }^{1}$, Yulia Kotovskaya $^{1}$, Olga Tkacheva ${ }^{1}$

${ }^{1}$ Pirogov Russian National Research Medical University of Ministry of Healthcare of the Russian Federation, Russian Gerontology Clinical Research Center

Background: Cardiovascular diseases (CVD) and frailty are age-related conditions, however the associations between them have not yet been studied. Predictors of frailty among CVD in elderly patients still undefined.

Purpose: To evaluate the associations between CVD and frailty and determine clinical predictors of frailty in elderly CVD patients.

Methods: Four hundred and fifty-six CVD in-patients (18\% male) aged 75-100 (mean $81 \pm 4$ ) years were included in the study. Among CVD, we evaluated the presence of hypertension, stable angina, myocardial infarction, myocardial revascularization, chronic heart failure (CHF), atrial fibrillation (AF), stroke, valvular heart disease, peripheral arterial disease (PAD), and pacemaker. Frailty was diagnosed by Short Physical Performance Battery (SPPB) tests.

Results: Median of SPPB score was 6 (interquartile range 3-8) points. Frailty rate was $69 \%$. Univariate analysis showed that these CVD increased the frailty risk: stable angina (OR 1,64; $95 \% \mathrm{CI}$ $1,06-2,54 ; \mathrm{p}=0,026)$, CHF (OR 1,58; 95\% CI 1,04-2,39; $\mathrm{p}=0,034)$, AF (OR 1,88; 95\% CI 1,12-3,16; p = 0,017), PAD (OR 12,46; 95\% CI $1,67-92,78 ; \mathrm{p}=0,014)$, stroke (OR 2,33; 95\% CI 1,28-4,24; $\mathrm{p}=$ $0,006)$. Multivariate analysis showed that age (OR 1,57; 95\% CI $1,19-2,09 ; \mathrm{p}=0,002)$, sex (female) (OR 1,87; 95\% CI 1,10-3,18; $\mathrm{p}=$ $0,021)$, the CVD number $\geq 3$ (OR 1,$67 ; 95 \%$ CI $1,09-2,55 ; p=0,019)$, and PAD (OR 10,84; 95\% CI 1,42-82,99; $\mathrm{p}=0,022$ ) were independent predictors of frailty in elderly CVD patients.

Conclusion: In elderly CVD patients, frailty rate was $69 \%$. Among CVD, stroke, CHF, AF, PAD, stable angina, and the CVD number $\geq 3$ were associated with a 1,6-12,5-fold increase in the frailty risk.

\section{Abstract \# 90}

Interleukin-6 in very elderly patients with coronary artery disease

Svetlana Topolyanskaya ${ }^{1}$, Tatyana Eliseeva ${ }^{2}$, Olga Vakulenko ${ }^{2}$, Leonid Dvoretski ${ }^{1}$

${ }^{1}$ I.M. Sechenov First Moscow State Medical University (Sechenov University), ${ }^{2}$ War Veterans Hospital N3

Introduction: Limited data are available on interleukin-6 (IL-6) in very elderly patients with coronary artery disease (CAD), therefore, we evaluated IL-6 levels and their relationship with various disorders. Methods: 50 patients were enrolled in cross-sectional study: 34 with CAD — in study group, 16 without CAD - in control group. Patient's age varied from 78 to 98 years; $53.1 \%$ of patients were $>90$ years. Serum IL- 6 concentrations were determined by enzyme-linked immunosorbent assay.

Results: Elevated IL-6 levels were found in $49 \%$ of patients. Mean IL-6 concentration was $7.96+5.1(1.5-30.6) \mathrm{pg} / \mathrm{ml}(\mathrm{N}<7.0)$. In patients aged $<90$ years mean IL-6 level was $6.7+3.2 \mathrm{pg} / \mathrm{ml}$, in longlivers-9.1 $+6.2 \mathrm{pg} / \mathrm{ml}(\mathrm{p}=0.09)$. In patients with CAD mean IL-6 concentration reached $10.9 \mathrm{pg} / \mathrm{ml}$, in control group $-5.9 \mathrm{pg} / \mathrm{ml}(\mathrm{p}=$ 0.02). Higher IL-6 levels were found in patients with heart failure ( 13.3 and $7.6 \mathrm{pg} / \mathrm{ml}, \mathrm{p}=0.04)$. Relative risk of high IL-6 levels in patients with heart failure was increased by 4.4. times $(R R=4.4 ; p=$ $0.02)$. Among patients with hyperuricemia mean IL-6 values were $10.5+3.1 \mathrm{pg} / \mathrm{ml}$, with normal uric acid $-7.1+3.1 \mathrm{pg} / \mathrm{ml}(\mathrm{p}=0.001)$.
Relative risk of high IL-6 levels in patients with hyperuricemia was increased by 9.7 times $(R R=9.7 ; p=0.003)$. Positive correlations were found between IL- 6 and tumor necrosis factor- $\alpha(\mathrm{R}=0.34 ; \mathrm{p}=$ $0.01)$, creatinine $(\mathrm{R}=0.35 ; \mathrm{p}=0.01)$ and urea $(\mathrm{R}=0.28 ; \mathrm{p}=0.05)$ concentrations. Regression analysis demonstrated that the most important factor influencing IL-6 levels was hyperuricemia ( $\beta=0.7$, $=0.00003$ ).

Key conclusions: Study results demonstrated frequent IL-6 increase in very elderly patients with CAD along with relationships between IL-6, heart failure and hyperuricemia.

\section{Abstract \# 91 \\ Tumor necrosis factor-alfa in very elderly patients with coronary artery disease}

Svetlana Topolyanskaya ${ }^{1}$, Tatyana Eliseeva ${ }^{2}$, Olga Vakulenko ${ }^{2}$, Leonid Dvoretski ${ }^{1}$

${ }^{1}$ I.M. Sechenov First Moscow State Medical University (Sechenov University), ${ }^{2}$ War veterans hospital N3

Introduction: Limited data are available on tumor necrosis factor- $\alpha$ $(\mathrm{TNF}-\alpha)$ in very elderly patients with coronary artery disease (CAD), therefore, we evaluated TNF- $\alpha$ levels and their relationship with various disorders.

Methods: 130 patients were enrolled in cross-sectional study: 102 patients with CAD - in study group, 28 without CAD-in control group. Patient's age varied from 77 to 101 years; $56.2 \%$ of patients were over 90 years old. Serum TNF- $\alpha$ levels $(\mathrm{N}<8.1 \mathrm{pg} / \mathrm{ml})$ were determined by enzyme-linked immunosorbent assay.

Results: Mean TNF- $\alpha$ concentration was 9.2+4.7 (3.9-31.9) pg/ml. Increased TNF- $\alpha$ levels were found in $54.6 \%$ of patients. In patients with CAD mean TNF- $\alpha$ concentration reached $10.0+4.9 \mathrm{pg} / \mathrm{ml}$, in control group $-6.1+1.8 \mathrm{pg} / \mathrm{ml}(\mathrm{p}=0.000001)$. TNF- $\alpha$ levels were higher in patients with heart failure $(\mathrm{p}=0.002)$. Significant correlations were revealed between TNF- $\alpha$ concentration and left atrium diameter $(\mathrm{R}=0.3 ; \mathrm{p}=0.003)$, and right ventricle size $(\mathrm{R}=0.3 ; \mathrm{p}=$ $0.001)$. In patients with hyperuricemia mean TNF- $\alpha$ concentration was $10.9+5.3 \mathrm{pg} / \mathrm{ml}$, in patients with normal uric acid $-7.5+2.5 \mathrm{pg} /$ $\mathrm{ml}(\mathrm{p}=0.000006)$. Positive correlations were found between TNF- $\alpha$ levels and uric acid $(\mathrm{R}=0.45 ; \mathrm{p}<0.000001)$, creatinine $(\mathrm{R}=0.26 ; \mathrm{p}=$ $0.002)$, urea $(\mathrm{R}=0.37 ; \mathrm{p}=0.00004), \beta$-Cross Laps $(\mathrm{R}=0.53$; $\mathrm{p}=0.0001)$ and interleukin $-6(\mathrm{R}=0,34, \mathrm{p}=0,01)$ concentrations. Negative correlations were registered between TNF- $\alpha$ and HDLcholesterol ( $\mathrm{R}=-0.38 ; \mathrm{p}=0.00005)$, and leptin $(\mathrm{R}=-0.26 ; \mathrm{p}=$ $0.01)$ levels. In patients younger than 90 years mean TNF- $\alpha$ values reached $10.5 \mathrm{pg} / \mathrm{ml}$, in long-livers $-8.1 \mathrm{pg} / \mathrm{ml}(\mathrm{p}=0.003)$.

Key conclusions: Study results demonstrated frequent TNF- $\alpha$ increase in very elderly patients with CAD. High TNF- $\alpha$ levels are associated with chronic heart failure and hyperuricemia.

\section{Abstract \# 92}

Management of post-hospitalization heart failure in elderly patients through the PRADO program: patients point of view and satisfaction

Maurizio Magri ${ }^{1}$, Lorenza De Rosario ${ }^{2}$, Caryn Recto ${ }^{1}$, Antonio Rainone $^{1}$, Cristiano Donadio ${ }^{1}$, Joel Belmin ${ }^{1}$

\section{${ }^{1}$ Hopital Charles-Foix, ${ }^{2} \mathrm{CHIC}$ Créteil}

Background: Heart failure is responsible for around 100,000 hospitalizations per year in France and it is the main cause of hospitalization for people over 65 years old. The re-hospitalization rate is particularly high after a first hospitalization for heart failure, 
around $40 \%$ within 1 year. The "PRADO" program was launched by national health system in 2013 to help and anticipate the needs of patients after hospitalization for heart failure, improve their quality of life and to strengthen the quality of care for the generalist, by seeking to reduce the rate of re-hospitalization.

Methods: The aim of this study was to collect testimonials (through a questionnaire) from elderly patients ( $>$ or $=75$ years) after hospitalization for heart failure in order to evaluate their satisfaction and their possible improvement in quality of life in a follow-up with the PRADO program.

Results: They feel more secure and protected (20 out of 32 patients have positive feedback on the PRADO program).

Conclusions: Thanks to the follow-up with PRADO program (visiting nurses at home and organized appointments), patients suffering from heart failure get to know their illness and recognize the main symptoms of a worsening of their health. Identifying early warning signs can positively affect the percentage of readmissions. The impact of PRADO program could therefore be significant because the patients in this study declare that they have learned more about heart failure.

\section{Abstract \# 93 \\ Periprocedural edoxaban management in routine clinical practice in the elderly is associated with low bleeding risk: Outcomes from the EMIT AF/VTE and the ETNA Korea/Taiwan studies}

Martin Unverdorben ${ }^{1}$, Christian Von Heymann ${ }^{2}$, Amparo Santamaria $^{3}$, Manish Saxena ${ }^{4}$, Thomas Vanassche ${ }^{5}$, James Jin ${ }^{1}$, Robert Wilkins ${ }^{6}$, Cathy Chen ${ }^{1}$, Paolo Colonna (7)

${ }^{1}$ Daiichi Sankyo, Global Medical Affairs, Basking Ridge, New Jersey, United States of America, ${ }^{2}$ Vivantes Klinikum im Friedrichshain, Department of Anesthesia \& Intensive Care Medicine, Emergency Medicine, and Pain Therapy, Berlin, Germany, ${ }^{3}$ University Hospital Vall d'Hebron, Service of Haematology, Haemostasis and Thrombosis Unit, Barcelona, Spain, ${ }^{4}$ Barts Health NHS Trust, NIHR CV BRC, London, United Kingdom, ${ }^{5}$ University Hospitals (UZ) Leuven, Department of Cardiovascular Sciences, Leuven, Belgium; ${ }^{6}$ QPS Consulting, LLC, Ashburn, Virginia, United States of America, ${ }^{7}$ Polyclinic Hospital of Bari, Department of Cardiology, Bari, Italy

Introduction: Annually $>10 \%$ of patients on oral anticoagulation as part of the management of atrial fibrillation (AF) undergo diagnostic or therapeutic procedures. Periprocedural management of their anticoagulation therapy is still a matter of debate, especially in patients aged 75 years or more.

Methods: Data was collected from 228 centres in 9 countries, describing the management (from 5 days pre to 30 days post procedure) of edoxaban treated patients. Procedures from 1,442 patients were evaluated. Peri-procedural edoxaban management was determined by the attending physician.

Results: Of the 1,442 patients, $280(19 \%)$ were < 65, $550(38 \%)$ were $65-74,514(36 \%) 75-84$, and $98(7 \%)$ were 85 years old or older. Comorbidities and risk scores increased with age. Any bleeding complications were uncommon and not different across age groups, ranging from $3.9 \%$ in patients $<65$ to $4.1 \%$ in those 85 years or older; major bleeding rates in any age group were $\leq 0.6 \%$. Thromboembolic events were rare but more common in the elderly, with all nine events occurring in those $>65$, and seven in patients aged $>75$ years.

Conclusions: Despite increased bleeding risk factors in the elderly, bleeding rates were low and similar across all age groups. However, there was a trend toward more thromboembolic complications with advancing age.

\section{Abstract \# 94}

Complete AV block during pulmonary embolism and atypical (o reverse) Tako Tsubo: a complex case of cardiac syncope

Virginia Tortu' ${ }^{1}$, Giulia Rivasi ${ }^{1}$, Maria Flora D'Andria ${ }^{1}$, Giada Turrin $^{1}$, Evelina Giuliani ${ }^{1}$, Andrea Ungar ${ }^{1}$

${ }^{1}$ University of Florence

Introduction: Cardiac syncope in the elderly is very common. The mechanism involved can be arrhythmic or structural, such as high degree AV block or pulmonary embolism respectively. Tako Tsubo syndrome can be a cause of cardiac syncope as well. In literature is reported that pulmonary embolism end high degree AV block can both represent a trigger for Tako Tsubo cardiomyopathy, and each of these disease can lead to cardiac syncope. So it might be difficult determine what is the effective mechanism responsible of syncope when more disease are contemporary present.

Methods: We describe a complex case of cardiac syncope in patient with pulmonary embolism, atypical Tako Tsubo and subsequent complete AV block.

Results: A 82-year old patient was hospitalized in 2019 for syncope without prodroms during physical activity (cyclette). At ECG a first degree AV block I (PR $210 \mathrm{~ms}$ ) and V3-V5 diphasic negative T wave, were recorded. Laboratory test and EGA demonstrated increase in hscTn (volevo dire che proprio fa la curva $22.7 \mathrm{pg} / \mathrm{ml} 223 \mathrm{pg} / \mathrm{mL} 180$ $\mathrm{pg} / \mathrm{mL}$ ),NT proBNP $1607 \mathrm{pg} / \mathrm{ml}$, mild hypossia and hypocapnia. Echocardiogram showed normal biventricular kinesis and EF, mild MI, VDAD $40 \mathrm{mmHg}$. CTCA was negative but revealed bilateral segmental and subsegmental PE, not suspected at admission; it was confirmed at pulmonary artery Angio-CT. hscTn movement was thought to be not a consequence of PE in this patient, so a coronary angiography was performed: no significant coronary artery lesions were shown, but ventricolography evidenced an atypical Tako Tsubo syndrome (reverse pattern). Syncope recurred during a transient third degree AV block and escapement junctional rhythm, with rapid recovery. A PM was implanted. A pre-dimission ecochardiography confirmed Tako Tsubo revers pattern.

Conclusions: This is the first case report of cardiac syncope during concomitant pulmonary embolism, Tako Tsubo Syndrome and complete AV block. The relationship between these ree entities in unclear. Syncope is likely a consequence of third degree AV block, but it is not clear if Tako Tsubo syndrome has been triggered by pulmonary embolism or by complete AV block; both mechanism are described in literature.

\section{Abstract \# 95}

Premature aging assessment in patients with arterial hypertension and subclinical hypothyroidism

Anastasiia Radchenko ${ }^{1}$, Olena Kolesnikova ${ }^{2}$

${ }^{1}$ GI "L.T.Malaya Therapy National Institute of the National Academy of Medical Sciences of Ukraine", ${ }^{2}$ GI "L.T. Malaya Therapy

National Institute of the National Academy of Medical Sciences of Ukraine"

Introduction: Biological age (BA) assessment can predict deterioration rates of human health better than chronological age (CA). The study aimed to examine the relationship between premature aging and routine biomarkers in patients with arterial hypertension $(\mathrm{AH})$ and subclinical hypothyroidism (SH).

Methods: 87 patients (55\% female; mean age 43.9 years, $\mathrm{SD}=10.4$, range 25-59) were included in the study and divided into group 1 (controls, $n=22)$ and group $2(n=65)$ composed of patients with grade $1 \mathrm{AH}$ and $\mathrm{SH}$. Standard clinical and laboratory parameters were 
evaluated in all patients. BA was estimated using CA and laboratory parameters according to the method of M. Levine et al. (2018).

Results: The difference between BA and CA was less in group 2 compared to controls ( -1.38 vs 0.3 years). In controls, significant associations with BA were observed between monocytes $(\mathrm{p}=0.000)$, granulocytes $(\mathrm{p}=0.001)$, triglycerides $(\mathrm{p}=0.003)$, total cholesterol $(\mathrm{p}$ $=0.001)$, LDL-cholesterol $(\mathrm{p}=0.031)$, VLDL-cholesterol $(\mathrm{p}=0.002)$ and glomerular filtration rate $(\mathrm{p}=0.03)$. Whereas in group 2 such associations were not detected and BA correlated with hemoglobin $(\mathrm{p}=0.007)$, platelet count $(\mathrm{p}=0.000)$, erythrocyte sedimentation rate $(p=0.000)$, all bilirubin fractions $(p \leq 0.005)$, urea $(p=0.000)$, as well as with waist circumference $(\mathrm{p}=0.023)$ and BMI $(\mathrm{p}=0.01)$.

Conclusions: Premature aging rates are slowed down in patients with $\mathrm{AH}$ and $\mathrm{SH}$ compared to controls and associated with blood rheological properties, liver tests and anthropological parameters rather than with lipid profile and renal function. One of the probable reason for this may be standard drug therapy of $\mathrm{AH}$, which may reduce the rate of the underlying aging mechanisms.

\section{Abstract \# 96}

Characterization of a home-bound population with heart failure Cloie June Chiong ${ }^{1}$, Alan Miller ${ }^{2}$, Cassidy June Chiong ${ }^{3}$

${ }^{1}$ University of North Carolina, Chapel Hill, ${ }^{2}$ University of FloridaShands Jacksonville, ${ }^{3}$ MedEx Health Network, Inc.

Introduction: Home-bound patients are characterized as those seen at home or at a non-medical facility. These patients have a multitude of comorbidities associated with aging, with heart failure being the leading diagnosis. Therefore, it is important to examine this group to evaluate the correlations between demographics and comorbidities, as well as potential interactions of guideline-directed treatments for their conditions.

Methods: A total of 471 home-bound, heart failure patients seen from January 2016-March were reviewed for age, sex, Body Mass Index (BMI), race, blood pressure, diagnoses, and outcomes. Data was extracted via electronic medical record.

Results: Among the home-bound cohort, the mean age was $78.9 \pm 11.6$ and the mean BMI was $26.5 \pm 6.3$ (overweight). A majority of the group consisted of females $(60.3 \%)$ and the most prominent race was White $(79.2 \%)$, followed by Asian (11.3\%), then Black (8.7\%). The average blood pressure was $131 / 78 \mathrm{mmHg}$ and four leading diagnoses were present: Hypertension (75.8\%), Diabetes Mellitus (36.7\%), Chronic Obstructive Pulmonary Disease (20.8\%), and Chronic Kidney Disease (13.4\%). 39.9\% of the population passed away during the study period.Conclusion: It is important to focus on interactions between cardiac illness, diabetes, and respiratory disease when determining treatments for the home-bound population.

\section{Abstract \# 97 \\ Revisiting the LDL-C (low-density lipoprotein cholesterol) paradox in the elderly: an updated systematic review \\ Sofia A Oliveira ${ }^{1}$, Inês Antunes ${ }^{2}$ \\ ${ }^{1}$ UCSP de Alvalade, ACeS Lisboa Norte and Faculdade de Medicina, Universidade de Lisboa, Portugal; ${ }^{2}$ USF Travessa da Saúde, ACeS Loures-Odivelas, Portugal}

Objective: A controversial systematic review in 2016* revealed that in 68,094 individuals $>60$ years from 19 cohort studies, LDL-C levels were inversely associated with overall and with cardiovascular mortality or had no association. Conversely, 2019 European guidelines on dyslipidemia management in the elderly recommend statin use in primary prevention based on a reduction in major vascular events in clinical trials. Therefore, our aim was to revisit the association between LDL-C and mortality in the general geriatric population in light of the latest cohort studies.

Methods: The authors independently searched PubMed for cohort studies with the following keywords: lipoprotein AND (old OR elderly) AND mortality NOT animal NOT trial. We excluded nonEnglish reports, studies in high cardiovascular risk groups, and papers with electronic publication date before $18 / 12 / 2015$ or after $1 / 5 / 2020$. Results: We identified 5 papers including 72,036 elderly individuals from 6 cohorts where all-cause mortality was recorded, two of which also analysed cardiovascular mortality $(\mathrm{N}=70,132)$. Statistically significant inverse association between all-cause mortality and LDL$\mathrm{C}$ was seen in 4 cohorts representing $98 \%$ of the total number of participants. In 2 cohorts, no association was found. Regarding cardiovascular mortality, females with the lowest LDL-C quartile had significantly higher cardiovascular mortality in one cohort (no association found in males), while in the second cohort no association was found

Conclusions: Recent cohort studies reinforce the LDL reverse epidemiology in the elderly. Even though statins appear to be safe, in older people higher LDL levels may be protective for non-cardiovascular diseases such as cancer, respiratory/digestive disease, and injuries. *Ravnskov et al. BMJ Open 2016;6(6):e010401.

\section{Abstract \# 98}

Increased number of cases of deep venous thrombosis in context of prolonged immobilization in an outpatient clinic

Izabela Corina Platon ${ }^{1}$

\section{${ }^{1}$ S.C. Antiage Care srl}

DVT and EP are two manifestation of the same disease, with fatal EP being the most fearful complication of the disease. During lockdown we noticed an increased number of cases of DVT and superficial thrombophlebitis in elderly patients, which could be explained by the prolonged immobilization in the past months, and necessitated anticoagulant treatment.

\section{Abstract \# 99 \\ Role of body mass index on cardiac adrenergic derangement in elderly patients with heart failure}

Leonardo Bencivenga ${ }^{1}$, Klara Komici ${ }^{2}$, Dino Franco Vitale ${ }^{3}$, Roberto Formisano $^{4}$, Brunella Puzone ${ }^{5}$, Antonio Cittadini ${ }^{5}$, Alberto Cuocolo ${ }^{5}$, Pasquale Perrone FIlardi ${ }^{5}$, Nicola Ferrara ${ }^{6}$, Giuseppe Rengo ${ }^{7}$

${ }^{1}$ Department of Translational Medical Sciences, "Federico II", Naples, Italy Department of Advanced Biomedical Sciences, University of Naples "Federico II", Naples, Italy, ${ }^{2}$ Department of Medicine and Health Sciences, University of Molise, Campobasso, Italy, ${ }^{3}$ Clinica San Michele, Via Appia 187, 81024 Maddaloni, CE, Italy, ${ }^{4}$ Istituti Clinici Scientifici Maugeri SpA Società Benefit (ICS Maugeri SpA SB), Telese Terme, BN, Italy, ${ }^{5}$ Department of Translational Medical Sciences, University of Naples "Federico II", Naples, Italy, ${ }^{6}$ Department of Translational Medical Sciences,

University of Naples "Federico II", Naples, Italy, ${ }^{7}$ Department of Translational Medical Sciences, University of Naples "Federico II", Naples, Italy, Istituti Clinici Scientifici Maugeri SpA Società Benefit (ICS Maugeri SpA SB), Telese Terme, BN, Italy

Introduction: Overweight and obesity represent relevant public health issues and augmented sympathetic tone has been described in 
patients with increased Body Mass Index (BMI). An extensive literature indicates that heart failure (HF)-related cardiac denervation, measured through iodine-123 metaiodobenzylguanidine (123I-mIBG) imaging, constitutes an independent predictor of cardiovascular outcomes and mortality. Moreover, it has been also demonstrated that aging independently affects cardiac sympathetic innervation, which is also influenced by BMI in HF patients. However, the impact of BMI on cardiac 123I-mIBG uptake in elderly HF patients has not been specifically investigated.

Methods: We studied 161 patients with systolic HF aged 65 years or older, collecting demographic, clinical, echocardiographic data and 123I-mIBG imaging parameters. The propose of the study was to assess the impact of BMI on cardiac adrenergic derangement, measured by $123 \mathrm{I}-\mathrm{mIBG}$, in elderly HF patients.

Results: The study population presented a mean age of $73.32 \pm 5.72$ years and mean left ventricular ejection fraction (LVEF) of $31.16 \% \pm$ 7.23. Forty-seven patients $(29.2 \%)$ presented BMI $\geq 30 \mathrm{~kg} / \mathrm{m} 2$ and obese patients showed a significant reduction in early heart to mediastinum $(\mathrm{H} / \mathrm{M})$ ratio $(1.623 \pm 0.219$ vs. $1.724 \pm 0.256 ; \mathrm{p}=$ $0.013)$ compared to patients with BMI $<30 \mathrm{~kg} / \mathrm{m} 2$. Multiple regression analysis revealed that $\mathrm{BMI}$ and LVEF were correlated with early $\mathrm{H} / \mathrm{M}$ ratio, whereas late $\mathrm{H} / \mathrm{M}$ ratio resulted to be significantly related to LVEF.

Conclusions: BMI, together with LVEF, is independently correlated with cardiac 123I-mIBG uptake in elderly HF patients.

\section{Abstract \# 100}

\section{Aortic stenosis and its impact in the quality of life}

Carmen Elías de Molins Peña ${ }^{1}$, Jorge Eduardo Corrales Cardenal ${ }^{1}$, María Dolores Domingo Sanchez ${ }^{1}$, Oscar Perez Berasategui ${ }^{1}$

${ }^{1}$ Hospital Nuestra Señora de Gracia, Zaragoza, Spain

Aortic stenosis (AoE) is the most common heart valve disease in developed countries, causing with time a series of symptoms that limits the patients and diminishes it's quality of life.

Objectives: Characterize and describe the quality of life perceived by the outpatient that comes to a geriatric assesment previous a transcatheter aortic valve replacement.

Method: Descriptive, retrospective and transversal study. All the outpatients that comes to a geriatric assesment previous a transcatheter aortic valve replacement since August 2016 to February 2020. VariablesSocio-demographic characteristics. Barthel's index. Lawton and Brody Index. Pfeiffer's test. Mini-nutritional assesment. Quality of life: EuroQOL.

Results: 71 outpatients. Mean age: 81.77 years old. 50.7\% men. 95.8\% lived at home. Mean 9.68 drugs. Barthel's Index 88.52. Lawton and Brody Index: 5.3. Pfeiffer's Test: 1.4 mistakes. MiniMNA: 10.76. Quality of life-EuroQOL:-Question 1: $60.6 \%$ answer 1. $39.4 \%$ answer 2. -Question 2: $71.8 \%$ answer 1. 28.2\% answer 2. Question 3: $52.1 \%$ answer 1. $43.7 \%$ answer 2. $4.2 \%$ answer 3. Question 4: $47.9 \%$ answer 1. $43.7 \%$ answer 2. 8.5\% answer 3. Question 5: 46.5\% answer 1. 46.5\% answer 2. 7\% answer 3.

Conclusions: Patient profile: octogenarian, men, lives at home, polypharmacy, low dependency to basic and instrumented life activities, no cognitive impairment and in risk of malnutrition.Severe aortic stenosis presents a worsening in quality of life, more than half presented problems doing usual activities, feel pain or discomfort and felt moderate anxiety/depression.

\section{Abstract \# 101}

The association of inguinal hernia with abdominal aortic aneurysm in geriatric patients: a retrospective study

Konstantinos Paschos ${ }^{1}$, Michalis Kirmanidis ${ }^{1}$, Anestis Chatzigeorgiadis ${ }^{1}$

\section{${ }^{1}$ General surgery, Hospital of Drama, Greece}

Several studies in the medical bibliography documented a high incidence of inguinal hernia in patients with abdominal aortic aneurysm (AAA), which is attributed to collagen synthesis disturbance and increased enzymatic activity of elastase and collagenase. However, the reverse association among the two is an ambiguous matter.

Aim: To reveal the incidence of AAA in geriatric patients with diagnosed inguinal hernia.

Methods: We studied 134 geriatric patients, only 4 women, who were admitted to the Surgical Department during a period of 3 years for the surgical repair of inguinal hernia. Their mean age was 72.6 years (70-92). 26 presented bilateral hernia. Smokers were 77/134 and hypertensive $89 / 134$ patients. All were subjected to an abdominal clinical examination and ultrasound scan (US) of the abdominal aorta searching for an AAA. An AAA was defined as a abdominal aorta diameter greater than $3 \mathrm{~cm}$.

Results: 28 patients (21\%) had AAA which was diagnosed via a US as a random finding, with a mean diameter 4.4 (3.3-10.6). Their mean age was 79 years. 11 had a bilateral inguinal hernia, while smokers were $23 / 28(82 \%)$.

Conclusions: Patients with an inguinal hernia present high incidence of AAA, especially those who smoke. In our department $21 \%$ of the patients who were admitted for surgical repair of an inguinal hernia presented AAA. Frequent US control of patients with inguinal hernia is necessary for early AAA diagnosis.

\section{Abstract \# 102}

Real world data from a small outpatient registry on tolerability and titration of Sacubitril/Valsartan in elderly affected from heart failure with reduced ejection fraction.

Andrea Herbst ${ }^{1}$, Francesco Orso ${ }^{1}$, Giulia Lucarelli ${ }^{1}$, Alessandra Pratesi $^{2}$, Marta Migliorini ${ }^{3}$, Simona Virciglio ${ }^{3}$, Viola Camartini ${ }^{3}$, Giacomo Fortini $^{3}$, Silvia Tognelli ${ }^{3}$, Camilla Ghiara ${ }^{3}$, Andrea Ungar ${ }^{3}$, Francesco Fattirolli ${ }^{4}$, Samuele Baldasseroni ${ }^{3}$

${ }^{1}$ Heart Failure Clinic, Division of Geriatric Medicine and Intensive Care Unit, Azienda Ospedaliero-Universitaria Careggi, Florence, Italy, ${ }^{2}$ Department of Experimental and Clinical Medicine, University of Florence, ${ }^{3}$ Heart Failure Clinic, Division of Geriatric Medicine and Intensive Care Unit, Azienda Ospedaliero-Universitaria Careggi, Florence, Italy, ${ }^{4}$ Department of Cardiothoracovascular Medicine, Azienda Ospedaliero-Universitaria Careggi, Italy.

Introduction: In last years new drugs able to change prognosis in Heart Failure (HF) with reduced ejection fraction (HFrEF), like Sacubitril/Valsartan $(\mathrm{Sa} / \mathrm{Va})$, are emerged. However, evidence of their use in elderly are poor. So, our aim is to described tolerability and titration of $\mathrm{Sa} / \mathrm{Va}$ in elderly patients affected by HFrEF.

Methods: We enrolled patients with HFrEF followed by our outpatient clinic for HF in elderly from November 2016 to January 2020 undergoing treatment with $\mathrm{Sa} / \mathrm{Va}$, evaluating their suspension and titration 3 months after beginning of therapy.

Results: We enrolled 95 patients with mean age 73 years; $45 \%$ were over 75 years and $19 \%$ woman. After 3 months $87 \%$ of patients were still being treated with $\mathrm{Sa} / \mathrm{Va}$ : $55 \%$ at initial dosage, $28 \%$ at intermediate dosage and $17 \%$ at maximum dosage. Only $15 \%$ of patients underwent on titration; the main causes of non-titration were 
symptomatic hypotension (41\%) but also worsening renal function $(13 \%)$ and hyperkalaemia (11\%). $11 \%$ of patients suspended it: in $2 \%$ of cases because of severe hypotension, in $1 \%$ for worsening renal function and hyperkalaemia, respectively, and in 3\% for drug-related allergic phenomena. In multivariate analysis, corrected for age and gender, advanced age ( $\mathrm{p}=0.010$, CI $0.783-0.968)$ and low initial systolic blood pressure $(\mathrm{SPB})$ values $(\mathrm{p}=0.002$, CI 1.032-1.115) resulted predictors of non-tritration of $\mathrm{Sa} / \mathrm{Va}$.

Key conclusion: $\mathrm{Sa} / \mathrm{Va}$ titration in elderly was influenced principally by orthostatic hypotension but also by hyperkalaemia and worsening renal function. Low initial SBP values and advanced age represented non-titration predictors.

\section{Abstract \# 103 \\ ARNI \& SGLT2i: an intriguing and promising association to be used with caution-a clinical case report.}

Andrea Herbst ${ }^{1}$, Marta Migliorini ${ }^{1}$, Francesco Orso ${ }^{1}$, Alessandrsa Pratesi $^{2}$, Simona Virciglio ${ }^{1}$, Viola Camartini ${ }^{1}$, Giacomo Fortini ${ }^{1}$, Silvia Tognelli ${ }^{1}$, Camilla Ghiara ${ }^{1}$, Giulia Lucarelli ${ }^{1}$, Andrea Ungar ${ }^{1}$, Francesco Fattirolli ${ }^{3}$, Samuele Baldasseroni ${ }^{1}$

${ }^{1}$ Heart Failure Clinic, Division of Geriatric Medicine and Intensive Care Unit, Azienda Ospedaliero-Universitaria Careggi, Florence, Italy, ${ }^{2}$ Department of Experimental and Clinical Medicine, University of Florence, ${ }^{3}$ Department of Cardiothoracovascular Medicine, Azienda Ospedaliero-Universitaria Careggi, Italy

Introduction: Heart Failure (HF) is a very common disease in elderly. In last years new drags were emerged to treat $\mathrm{HF}$ with reduced ejection fraction (HFrEF), like Sacubitril/Valsartan ( $\mathrm{Sa} / \mathrm{Va})$ and Sodium-Glucose Cotransporter 2 inhibitors (SGLT2i). Although recent evidence underscores safety of these therapies, also in the elderly, few is known about safety of their combination in real world. With this clinical case we want to offer a hint for thought on this.

Case: We report the case of a 68-year-old male outpatient, affected by HFrEF and type 2 diabetes, treated with high dose of $\mathrm{Sa} / \mathrm{Va}$ in whom we decided to introduce empagliflozin for poor glycemic control with metformin alone during summer. He had a good renal function and a good pressure control and was already on therapy with high dosage of beta-blockers, a mineral-corticosteroids receptor antagonist and low dosage of furosemide. Despite having reduced diuretic therapy he rapidly developed hypovolaemia, weight loss, acute kidney failure and hyperkaliaemic metabolic acidosis, needing hospitalisation.

Conclusion: Physicians aiming to undertake this promising association should consider timing, diuretic treatment, volemic status and renal function reassessment, patient's education on weight selfmonitoring and climate variations.

\section{Abstract \# 104 \\ Evaluation of the estimated Glomerular Filtration Rate with CKD-EPI equation of older adults aged 90 years and over with cardiovascular diseases}

Liudmila Merkusheva ${ }^{1}$, Nadezda Runichina ${ }^{1}$, Sergey Lysenkov ${ }^{2}$, Irina Strazhesko ${ }^{1}$, Yulia Kotovskaya ${ }^{1}$, Natalia Sharashkina ${ }^{1}$, Anastasia Shvedko ${ }^{1}$, Olga Tkacheva ${ }^{1}$

${ }^{1}$ Pirogov Russian National Medical University, The Russian Clinical Research Center for Gerontology, Moscow, Russia, ${ }^{2}$ Lomonosov Moscow State University, Biological Faculty, Moscow, Russia
Aim of the study: To investigate kidney function in older adults $>90$ years with cardiovascular disease (CVD) using the methods of calculation of the estimated glomerular filtration rate(eGFR).

Materials and methods: 457older adults aged $>90$ years were analysed. Patients with critical and terminal conditions were not included in this study. The following equations were used: CKDEPICreatinine(2009), CKD-EPI CystatinC (2012) and CKDEPICreatinine-CystatinC (2012). The Bland-Altman method was used to compare two measurement techniques for the same variable. Results: A total of $370(92,5 \pm 2,4$ SDyears $)$ patients were analysed, $362(79.2 \%)$ were female. All patients had comorbidity, including CVD 282(61,7\%)- the main group and control group - 175 (38.3\%) without CVD. Main groups differ from the control one in age $(92.4 \pm$ 2.3 vs $92.6 \pm 2.6, \mathrm{p}=0.23)$ and has less women $(75.5 \%$ vs $85.1 \%, \mathrm{p}=$ 0.017).All patients demonstrated low levels of eGFR. Values of eGFR in the main gr. vs control gr. by formulas: CKD-EPICreatinine: $56,6 \pm 13,1$ vs $59 \pm 14 \mathrm{ml} / \mathrm{min} / 1.73 \mathrm{~m}^{2}$ ( $\left.\mathrm{p}=0.08\right)$, CKD-EPICystatinC: $32 \pm 7,7$ vs $33 \pm 9 \mathrm{ml} / \mathrm{min} / 1.73 \mathrm{~m}^{2}(\mathrm{p}=0.09)$,CKD-EPICreatinineCystatinC: $41,8 \pm 9,4 \mathrm{vs} 43,7 \pm 10,6 \mathrm{ml} / \mathrm{min} / 1.73 \mathrm{~m}^{2}(\mathrm{p}=0.052)$ respectively.Differences between values are decreasing along with the decrease in means of eGFR. However, even using minimal values, one of the equation overestimates values versus using other equations. The correlation between eGFR and CVD wasn't obtained. It was found, that eGFR in older adults aged $>90$ years varied depending on the method of calculation with up to two-fold difference.

Conclusion: The kidney function in older adults aged $>90$ years was decreased and didn't depend on the CVD. For the lowest values of eGFR CKD-EPI CystatinC equation overestimate means versus using other equations. For estimation of the degree of decrease of kidney function more research is needed bearing in mind the significant difference in estimated values.

\section{Abstract \# 105}

Role of PDW and MPV in stratification of heart failure severity in older adults

Diana Lelli ${ }^{1}$, Veronica Adiletta ${ }^{1}$, Gaetano Maddalena ${ }^{1}$, Ilaria Bisconti ${ }^{1}$, Raffaele Antonelli Incalzi ${ }^{1}$, Claudio Pedone ${ }^{1}$

${ }^{1}$ Area di Geriatria -Università Campus Bio-Medico di Roma

Introduction: Severity stratification of older adults affected by heart failure (HF) is difficult. Mean platelet volume (MPV) and platelet distribution width (PDW), as platelet activation and thus inflammation markers, might be used for this purpose. The objective of the study was to analyse the association between MPV/PDW and HF severity indicators in older adults.

Methods: retrospective cross-sectional study enrolling 415 patients aged $\geq 65$ years admitted to an Acute Geriatric Ward for acute HF. Participants were stratified according diagnosis of HF with preserved ejection fraction (HFpEF) (N: 250) or not (HFmrEF/HFrEF) (N: 165). The association with ejection fraction (EF), NT-proBNP, right ventricular function (TAPSE), and diastolic function was analysed using linear or multinomial regression models, as appropriate, adjusted for potential confounders.

Results: mean age was 83.1 years (SD 7), $41.2 \%$ were male. MPV was positively associated with NT-proBNP logarithm in the total sample (Adjusted OR: 0.16, $\mathrm{P}=0.008$ ) and in the HFmrEF/HFrEF group (Adjusted OR: $0.215, \mathrm{P}=0.015$ ), but not in the HFpEF; no association was found with $\mathrm{EF}$ (analysed only in $\mathrm{HFmrEF} / \mathrm{HFrEF}$ ) or TAPSE. Using type I diastolic disfunction as reference, increasing MPV values were associated with higher risk of monophasic pattern (Adj. OR:1.44, 95\% CI 1.08-1.92) and III grade disfunction (Adj.OR $1.54,95 \%$ CI 1.01-2.35) in the total sample, data documented only in the HFmrEF/HFrEF group (Monophasic Adj. OR 2.51, 95\% CI 
1.33-4.74; III grade Adj. OR 4.77, 95\% CI 2.05-11.1). Similar results were found analyzing PDW.

Conclusions: MPV and PDW are unexpensive potential markers for severity stratification of older adults with $\mathrm{HFmrEF} / \mathrm{HFrEF}$, but not with HFpEF. Further studies are needed to confirm these results.

\section{Abstract \# 106 \\ Impact of MPI on short-term outcome in elderly patients with acute myocardial infarction}

Klara Komici ${ }^{1}$, Leonardo Bencivenga ${ }^{2}$, Pierangela Nocella ${ }^{3}$, Brunella Puzone $^{3}$, Angela Mancini ${ }^{3}$, Fabrizio Vincenzo Grieco ${ }^{3}$, Dino Franco Vitale $^{4}$, Graziamaria Corbi ${ }^{5}$, Carmine Morisco ${ }^{6}$, Nicola Ferrara ${ }^{7}$, Giuseppe Rengo ${ }^{7}$

${ }^{1}$ Department of Medicine and Health Sciences, University of Molise, Italy., ${ }^{2}$ Department of Translational Medical Sciences, University of Naples "Federico II", Italy; Department of Advanced Biomedical Sciences, University of Naples "Federico II", Italy., ${ }^{3}$ Department of Translational Medical Sciences, University of Naples "Federico II", Italy, ${ }^{4}$ Clinica San Michele, Via Appia 187, 81024, Maddaloni, CE, Italy., ${ }^{5}$ Department of Medicine and Health Sciences, University of Molise, Campobasso, Italy, ${ }^{6}$ Department of Advanced Biomedical Sciences, University of Naples "Federico II", Italy., ${ }^{7}$ Department of Translational Medical Sciences, University of Naples "Federico II", Italy

Background: Assessing the prognosis of elderly patients with acute myocardial infarction (AMI) is particularly challenging since clinical evolution and prognosis of these patients is the result of a complex combination of functional, biological, environmental and psychological factors. The Multidimentional Prognostic Index (MPI) is a prognostic tool able to predict the outcome in different clinical settings. However, the prognostic role of MPI in elderly patients with acute myocardial infarction has not yet been explored. Therefore, the aim of the present study was to evaluate the role of MPI on short term survival in elderly patients with AMI.

Methods: 302 elderly patients hospitalized with a diagnosis of IMA and undergoing coronary angiography and angioplasty have been studied. A comprehensive geriatric assessment was performed after the clinical stabilization of the patient through the MPI. It was also calculated the predictive index Grace Score.

Results: The study population presented a mean age of $73.0 \pm 6.8$ years. During a 6-month follow-up, 29 non-survivors were registered. The logistic regression analysis revealed the GRACE Score and the MPI to be significantly and independently predictive for mortality: OR 1.04 (95\% CI 1.02-1.07) and 12.55 (95\% CI 4.68-33.62), respectively. From the analysis of ROC curves, it emerged that a predictive model including both Grace Score and MPI is better than the ones taking the two score separately (AUC 0.91).

Conclusions: MPI is an independent predictor of short-term outcome in elderly patients with AMI. MPI predictive value is comparable to Grace Score and application of both models together improves the efficacy of model.

\section{Abstract \# 107 \\ Cardiovascular regulation as an indicator of aerobic fitness: comparison between healthy older people and patients with type 2 diabetes \\ Jessica Koschate ${ }^{1}$, Tania Zieschang ${ }^{1}$, Uwe Hoffmann ${ }^{2}$ \\ ${ }^{1}$ Carl von Ossietzky University Oldenburg, Geriatric Medicine, \\ ${ }^{2}$ German Sport University, Exercise Physiology}

Introduction: The kinetics of the cardiovascular system (CVS) describe the dynamic adaptation to work rate (WR) changes and is, unlike the gold standard for assessing aerobic fitness (maximum oxygen uptake [V'O2max]), measurable in moderate WR intensities. This offers advantages, especially for vulnerable patient groups. The aim is therefore to describe potential differences in aerobic fitness in the moderate and maximum WR range in healthy, physically inactive older adults (HPIOA) and type 2 diabetes patients (T2D).

Methods: 27 HPIOA ( $73 \pm 3$ years) and 27 inactive T2D patients $(60$ \pm 8 years) were tested on a cycle ergometer with pseudo-randomized WR changes of $30 \mathrm{~W}$ and $80 \mathrm{~W}$, followed by the assessment of V'O2max. Heart rate (HR) and oxygen uptake (V'O2) data were measured and kinetics information was calculated using cross-correlation-functions (CCF) between WR and the respective parameter. Higher maxima of CCFs indicate faster adjustments in the CVS. V'O2max was determined over the last 30s of the test.

Results: V'O2max did not differ significantly $(\mathrm{P}=0.675)$ between the groups (HPIOA: $22.7 \pm 5.2 \mathrm{ml} \bullet \mathrm{min}-1 \bullet \mathrm{kg}-1$ vs. T2D: $22.1 \pm 5.8$ ml•min- $1 \bullet \mathrm{kg}-1)$. The CCFmax $(\mathrm{HR})$ of T2D $(0.25 \pm 0.05)$ was significantly $(\mathrm{P}=0.018)$ lower than that of HPIOA $(0.30 \pm 0.09)$, but not the CCFmax (V'O2) (T2D: $0.31 \pm 0.04$ vs. HPIOA: $0.31 \pm 0.07 ; \mathrm{P}=$ 0.684).

Discussion: Despite their younger age, HR regulation in T2D patients seems to be slower. This was not evident by the exclusive analysis of V'O2max. HR regulation is of great importance for the adaptation to changes in WR in everyday life and should be assessed in different patient groups for early identification of changes in the CVS.

\section{Abstract \# 108}

Age-related differences in association between telomere length and cardiovascular risk

Olena Kolesnikova ${ }^{1}$

${ }^{1}$ GI "L.T.Malaya Therapy National Institute of the NAMS of Ukraine", Kharkiv, Ukraine

Introduction: Available data allow us to judge the relationship of shortened telomere length (TL) with cardiovascular risk (CVR), early vascular ageing, atherosclerosis. Aim: to determine age differences in the association between TL and CVR.

Methods: 60 patients aged 31-75 years with moderate CVR without clinical symptoms were included in the main group and 63 control individuals aged $30-75$ years - in the control group. Study subjects were categorized into 3 groups: $30-44$ years (young group, $n=20$ ), 45-60 years (middle group, $\mathrm{n}=20$ ) and over 60 years (older group, $\mathrm{n}$ $=20$ ). There were no significant differences in the sex between the groups.

Results: According to the data of correlation analysis we did not find relationship TL and Telomerase Activity (TA) with any factors in control groups of young and mild ages. Though there was an association between TA and TL $(r=0.71, p<0.05)$ in the old control group. Analyses the data of the young CVR group indicated, that TL was associated with age $(r=0.67, p<0.05)$, TA with blood pressure (BP) $(r=0.79, p<0.05)$. The data of mild CVR group did not indicate the association of TA and TL with any factors, but was a significant relationship between body mass index (BMI) and BP $(r=$ $0.62, p<0.05)$, HOMA index $(r=0.68, p<0.05)$. TA in the old main group was strongly associated with HOMA $(\mathrm{r}=0.79, \mathrm{p}<0.05)$, creatinine $(r=0.77, \mathrm{p}<0.05)$.

Conclusions: The established relationship TA and TL with factors cardiovascular disease may indicate the important role of TA/TL in the cardiovascular changes in patients with CVR. 


\section{Abstract \# 109}

\section{Metabolic syndrome in hospitalized geriatric patients}

Patricia Mateo Martín ${ }^{1}$, Nuria Cristina Herrera Fernández ${ }^{2}$, Javier Alonso Ramìrez ${ }^{2}$, Ortzi Barrasa Bermejo ${ }^{1}$, Francisco Javier Balea Fernández ${ }^{1}$, Dácil Cabezas Jaén ${ }^{2}$, Rolf Christian Sander Zúñiga ${ }^{2}$, Ruth Paz Maya ${ }^{2}$

${ }^{1}$ Training in physician of geriatrician at Lanzarote Insular Hospital, ${ }^{2}$ Geriatrician physician at Lanzarote Insular Hospital.

Introduction: metabolic syndrome (MetS) increases with age. This assumes a public health problem by encompassing factors that promote greater cardiovascular morbidity and mortality. We aimed to establish the prevalence and variables related to MetS in pa

\section{Abstract \# 110 \\ Frailty and calcium homeostasis in elderly patients with severe aortic stenosis \\ Pablo Enrique Solla Suárez ${ }^{1}$, Javier Montero Muñoz ${ }^{2}$, Marta Fernández Fernández ${ }^{2}$, Jorge Eduardo Herrera Parra ${ }^{2}$, Eva María López Álvarez ${ }^{2}$, José Gutiérrez Rodríguez ${ }^{1}$ \\ ${ }^{1}$ Área de Gestión Clínica de Geriatría Hospital Monte Naranco, Oviedo, Asturias, Spain. Instituto de Investigación Sanitaria del Principado de Asturias, Oviedo, Asturias, Spain, ${ }^{2}$ Área de Gestión Clínica de Geriatría Hospital Monte Naranco, Oviedo, Asturias, Spain}

Introduction: Severe aortic stenosis (SAS) is the most common valve disease in the elderly. SAS pathophysiology is not well understood. Recent studies have suggested a possible association between AS and calcium balance.Objectives.Frailty detection and description of calcium metabolism in patients over 75 years of age with AS included in a Geriatric Assessment Program.

Methods: Prospective study, including patients over 75 years of age with SAS referred from cardiology to a specialized geriatric consult. Variables:-Baseline characteristics: age, sex, functionality (Lawton and Barthel indices), nutritional status [Mini Nutritional Assessment Short-Form, (MNA-SF)], cognitive function [Mini Mental State Examination (MMSE)]. -Frailty: Short Physical Performance Battery (SPPB). -Biological parameters: GFR-CKD-EPI $(\mathrm{mL} / \mathrm{min} / 1.73 \mathrm{~m} 2)$. Serum Albumin $(\mathrm{g} / \mathrm{L})$, calcium $(\mathrm{mmol} / \mathrm{L})$, phosphorus $(\mathrm{mmol} / \mathrm{L})$, 25-OH-vitaminD (ng/mL), iPTH $(\mathrm{pg} / \mathrm{ml})$, corrected Calcium for serum albumin $(\mathrm{mmol} / \mathrm{L})$.

Results: $\mathrm{n}=50$, age $83.66 \pm 3.86$, female: $54.0 \%$. Lawton Male: 4.48 \pm 0.84 and Female: $6.67 \pm 1.79$ (independent: $56.0 \%$ ), Barthel 94.80 \pm 7.42 (independent: $76.0 \%$ ), MNA-SF $12.52 \pm 1.50$ (normal: $80.0 \%$ ), MMSE $27.84 \pm 2.30$ (cognitive impairment: $6 \%$ ). SPPB: $8.64 \pm 1.74$, frail (0-6): $18.0 \%$. GFR-CKD-EPI $6.95 \pm 17.26(<$ $60 \mathrm{~mL} / \mathrm{min} / 1.73 \mathrm{~m}^{2}: 58 \%$ ). Albumin $44.36 \pm 2.52$ (hypoalbuminemia: $0 \%)$, calcium $2.37 \pm 0.10(<2.20 \mathrm{mmol} / \mathrm{L}: 4.0 \% ;>2.55 \mathrm{mmol} /$ L:6.0\%), phosphorus $1.02 \pm 0.18(<0.87 \mathrm{mmol} / \mathrm{L}: 25.0 \%$; $>$ $1.45 \mathrm{mmol} / \mathrm{L}: 2.1 \%), \quad 25-\mathrm{OH}$-vitaminD $\quad 12.36 \pm 5.18 \quad(<10 \quad \mathrm{ng} /$ $\mathrm{mL}: 40.0 \%$; $<20 \mathrm{ng} / \mathrm{mL}: 92 \%)$, iPTH $76.82 \pm 42.61$ ( $>65 \mathrm{pg} /$ $\mathrm{ml}: 40 \%)$, corrected Calcium for serum albumin $2.26 \pm 0.85$ (< $2.20 \mathrm{mmol} / \mathrm{L}: 18.0 \%$; > $2.55 \mathrm{mmol} / \mathrm{L}: 0.0 \%$ ).

Key conclusions: Elderly patients with SAS included in our study were mostly in good functional, cognitive and nutritional status; and $18 \%$ were frail. Alterations in calcium metabolism were frequently detected: hypocalcemia (18\%), hypophosphatemia $(25 \%)$ and high levels of iPTH (40\%). Vitamin D deficiency was present in most patients (92\%), being severe in almost half of the cases.

\section{Abstract \# 111}

Number and replating capacity of endothelial colony forming cells are telomere length dependent: implication for human atherogenesis

Simon Toupance ${ }^{1}$, Stéphanie Simoncini ${ }^{2}$, Carlos Labat ${ }^{1}$, Chloé $^{2}$ Dumoulin $^{2}$, Tsung-Po Lai ${ }^{3}$, Oualid Ayad ${ }^{1}$, Cécile Lakomy ${ }^{1}$, Véronique Regnault ${ }^{1}$, Patrick Lacolley ${ }^{1}$, Françoise Dignat-George ${ }^{2}$, Florence Sabatier ${ }^{2}$, Abraham Aviv ${ }^{3}$, Athanase Benetos ${ }^{4}$

${ }^{1}$ Université de Lorraine, Inserm, DCAC, F-54000 Nancy, France, ${ }^{2}$ Aix Marseille University, Inserm, INRA, C2VN, F-13005 Marseille, France, ${ }^{3}$ Center of Human Development and Aging, Rutgers, The State University of New Jersey, New Jersey Medical School, Newark, NJ 07103, USA, ${ }^{4}$ Université de Lorraine, CHRU-Nancy, Pôle

"Maladies du Vieillissement, Gérontologie et Soins Palliatifs", F-54000, Nancy, France

Introduction: Short leukocyte telomere length (TL) is associated with atherosclerotic cardiovascular disease (ASCVD). Given that TL is equivalent across somatic cells within the individual, patients with ASCVD might have short telomeres in circulating cells other than leukocytes. These include endothelial colony forming cells (ECFCs), which behave as progenitor cells displaying endothelial repair activity. To explore a potential role of short TL in atherogenesis, we examined associations of TL with proliferative dynamics of ECFCs. Methods: To isolate ECFCs, we performed a clonogenic assay on blood samples donated by 116 participants (aged 24-94) in the TELARTA study. We detected no ECFC in 29 blood samples (Group 1) but detected clones with no replating capacity in other 29 samples (Group 2). In additional 58 samples, we isolated clones with replating capacity (Group 3). TL was measured by Southern blotting in leukocytes (LTL) and ECFCs (ECFC-TL).

Results: Age- and sex-adjusted LTL was the shortest in Group 1 $(6.51 \pm 0.13 \mathrm{~kb})$, longer in Group $2(6.69 \pm 0.13 \mathrm{~kb})$ and the longest in Group 3 (6.78 $\pm 0.09 \mathrm{~kb} ; \mathrm{p}<0.05)$. In group 3, ECFC-TL was associated with the number of generated clones $(\mathrm{p}<0.01)$. ECFC-TL was strongly correlated with LTL $(r=0.82 ; \mathrm{p}<0.0001)$ but was always longer (7.98 $\pm 0.13 \mathrm{~kb}$ vs. $6.74 \pm 0.12 \mathrm{~kb} ; \mathrm{p}<0.0001)$.

Conclusions: Blood samples with a longer LTL yield a higher number of self-renewing ECFCs. These results suggest that individuals with a longer LTL might have a better endothelial repair capacity.

\section{Abstract \# 112}

Characterization and prognosis of very elderly patients, with anemia, cardio-cerebrovascular disease and iron deficiency in 4 Portuguese centres, in 2017

Margarida Madeira ${ }^{1}$, Eugeniu Gisca ${ }^{2}$, Daniela Brigas ${ }^{1}$, Ana Rita Rego $^{3}$, Ana Vigário ${ }^{3}$, Arsénio Barbosa ${ }^{4}$, Sílvia Pereira ${ }^{2}$, Marta Soares ${ }^{4}$, Ricardo Racha-Pacheco ${ }^{5}$, Ana Macedo ${ }^{5}$

${ }^{1}$ CHS-HSB, Portugal, ${ }^{2} \mathrm{GO}$, Portugal, ${ }^{3} \mathrm{CHP}-\mathrm{HSA}$, Portugal, ${ }^{4} \mathrm{CHSJ}$, Portugal, ${ }^{5}$ Keypoint, Portugal

Introduction: Vascular diseases are the main death cause in Portugal, associated with cardio and cerebrovascular diseases (CVD). Anemia and iron deficiency (ID) are vascular risk factors common in the elderly, associated with worse clinical outcomes. Given the lack of national data concerning on this matter, our study aimed at characterizing and evaluating prognosis of very elderly patients (VEP), with anemia and CVD, with and without ID.

Methods: Data was obtained through local data of the participating centres of patients admitted to Internal Medicine wards in 2017 and 
fulfilling our inclusion criteria, at admission: VEP ( $\geq 80$ years old); anemia; CVD.

Results: In the participating centres 16,446 patients were admitted in 2017, of which 514 fulfilled inclusion criteria. Median age was 86 years, $64.4 \%$ were female, and $81.5 \%$ dwelled at home. Median hospitalization was 8.5 days, mortality rate 18 -months after discharge was $39.9 \%$, and 30 -day readmission rate was $16.5 \%$. Median haemoglobin was $10.7 \mathrm{~g} / \mathrm{dl}$, serum iron was $34 \mathrm{mcg} / \mathrm{dl}$, ferritin was 117.8 $\mathrm{mcg} / \mathrm{dl}$, and transferrin saturation was $12 \%$. Iron parameters were only available for $59 \%$ of patients and only $21 \%$ were on iron supplements. Mortality was associated with: duration of hospitalisation (OR 1.057, 95\% CI 1.030-1.084, p = 0.001), age at admission (OR $1.063,95 \%$ CI $1.015-1.112, \mathrm{p}=0.009)$, and haemoglobin levels $(\mathrm{OR}$ $0.874,95 \%$ CI $0.769-0.994)$.

Key conclusions: Iron parameters are not available for a large proportion of very elderly patients hospitalised with anemia and CVD. Higher age, longer hospitalisations, and lower haemoglobin levels were associated with higher mortality rates 18 -months after discharge.

\section{Abstract \# 113 \\ Elderly heart failure patient's self-care in a nursing heart failure clinic \\ Joana Pereira Sousa ${ }^{1}$, Célia Costa $^{1}$, Lina Melo ${ }^{1}$, Ana Fresco ${ }^{1}$, Margarida Felício ${ }^{1}$, Paula Dias ${ }^{1}$, Hugo Neves ${ }^{2}$, Jorge Canais ${ }^{1}$ \\ ${ }^{1}$ Centro Hospitalar e Universitário de Coimbra, ${ }^{2}$ Health Sciences Research Unit: Nursing}

Introduction: Heart failure is a chronic disease where self-care must be performed as a continuum, to avoid decompensation and maintain patient stability. Elderly people, with multiple comorbidities, show difficulty in performing self-care behaviours.

Methods: In a descriptive-correlational study, in a nursing heart failure consultation, at a cardiology department, it was analysed selfcare in heart failure patients through the European Heart Failure SelfCare Behaviour Scale (EHFScB).

Results: In a total of 121 patients assessed, with an average age of 73.95 (7.17) years, first assessment of the EHFScB evidenced an average of 33.42 (8.90) points. Positive and significant relation was observed between age and EHFScB scores $(p<0.01)$, indicating that older people had worse self-care behaviours $(\mathrm{ScB})$. Although illiterate individuals displayed, in average, worse $\mathrm{ScB}$, no statistically significant difference was observed concerning education ( $\mathrm{p}>0.05$ ). Concerning marital status, although divorced individuals displayed worse $\mathrm{ScB}$ with an average score of 43.00 (4.24), specially in comparison with married individuals, no significantly statistical difference was observed ( $p>0.05)$. The presence of devices (e.g. pacemakers) displayed very similar averages, and with no statistically significant differences between the type of device ( $p>0.05$ ).

Key conclusion: After application of the program, a total of 59 patients were assessed in a 6-month's follow-up nursing consultation. Overall, there was an average decrease of -1.52 (2.87) points in EHFScB scores, indicating that individuals assessed displayed better $\mathrm{ScB}$ when in comparison to the first moment of assessment. No other variable included in the study evidenced impact in $\mathrm{ScB}$ differences between assessments.

\section{Abstract \# 114 \\ Quality of Life improvement in elderly heart failure patients engaged in a nursing consultation}

Joana Pereira Sousa ${ }^{1}$, Célia Costa ${ }^{1}$, Lina Melo ${ }^{1}$, Ana Fresco ${ }^{1}$, Margarida Felício ${ }^{1}$, Paula Dias ${ }^{1}$, Hugo Neves ${ }^{2}$, Jorge Canais ${ }^{1}$
${ }^{1}$ Centro Hospitalar e Universitário de Coimbra, ${ }^{2}$ Health Sciences Research Unit: Nursing

Introduction: Due to physiological and psychological changes, aged persons might have difficulty performing self-care, which influences quality of life.

Methods: In a descriptive-correlational study, in a nursing heart failure consultation, it was analyzed the quality of life in elderly heart failure patients through the Kansas City Cardiomyopathy Questionnaire (KCCQ).

Results: In a total of 81 patients assessed, with an average age of 73.23 (7.35) years, the first assessment of the KCCQ evidenced higher scores for the dimensions Symptoms $(78.27 \pm 13.76)$ and Quality of Life (62.45 \pm 18.34$)$, and worse scores in Self-care Efficacy (51.08 \pm 16.23). Overall, in the sample, we found higher scores in the Functional Summary dimensions in contrast with the Clinical Summary dimension. No correlation was found between age and the multiple dimensions of the KCCQ. Except for the dimensions Symptoms and Functional Summary $(p>0.05)$, education appears to play a major role in the health status of heart failure patients $(\mathrm{p}<0.05)$, with higher levels of education positively influencing health status in these dimensions. As for the presence of devices, with the exception of the dimensions Symptoms and Functional Summary $(p>0.05)$, there was evidence that CRT patients scored worse health status in all dimensions compared to patients with pacemakers or no device $(\mathrm{p}<0.05)$. Key conclusion: After the application of KCCQ at first time nursing consultation, a total of 81 patients were assessed. It was observed that elderly patients with higher education and CRT perceived their health status as worse, and therefore with the worst quality of life perception.

\section{Abstract \# 115}

Acute-on-chronic heart failure secondary to severe mitral valve regurgitation due to chordae tendineae rupture: a case report

Cristiana Canelas Mendes ${ }^{1}$, Leila Duarte ${ }^{1}$, Mariana Dias ${ }^{1}$, João Madeira Lopes ${ }^{1}$, Lurdes Alvoeiro ${ }^{1}$

\section{${ }^{1}$ Serviço de Medicina 2, Centro Hospitalar Universitário Lisboa Norte}

Mitral regurgitation (MR) is an abnormal backflow of blood into the left atrium during cardiac systole, caused by an apparatus dysfunction with potential for hemodynamic compromise. MR is a strong predictor of cardiac outcome in patients with ischemic heart disease and is associated with higher mortality. A 85 -year-old man with chronic heart failure with preserved ejection fraction of multiple-factor aetiology, previously submitted to coronary artery bypass grafting and coronary angiography with stent placement, maintaining severe two vessel disease, mild mitral and tricuspid regurgitation, permanent atrial fibrillation, essential hypertension, type 2 diabetes and chronic kidney disease, admitted due to progressive worsening of dyspnoea and bilateral oedema of the lower limbs. Physical examination: jugular vein distension, pansystolic murmur best heard over the apex, abolish murmur in the lower half of the right hemithorax and bilateral oedema of the lower limbs until the inguinal zone. Laboratory investigation: elevated Nt-proBNP. X-ray: increased cardiothoracic ratio, right pleural effusion. EKG: atrial fibrillation, nonspecific ST-T wave abnormalities. Transthoracic and transoesophageal echocardiograms: dilated left atrium and ventricle with preserved ejection fraction, severe MR due to chordal rupture with A2 flail and annulus dilatation, severe tricuspid regurgitation and high probability of pulmonary hypertension. After multidisciplinary discussion percutaneous mitral valve repair (PMVR) was performed with improvement of symptomatology. PMVR is an important procedure for patients at high risk of surgical repair, which has successfully reduced patient symptoms, disease morbidity, improved quality of life, and facilitated reverse remodelling with potential for a survival advantage. 


\section{Abstract \# 116}

\section{Study of dementia in elderly patient in neurology consultation}

Marta Mercedes Gonzalez Eizaguirre ${ }^{1}$, Maria Jesus Vicente Romero ${ }^{1}$, Carmen Oquendo Marmaneu ${ }^{1}$, Miguel Sanchez Ortiz ${ }^{1}$, Ana Mateo Abad $^{1}$, Elisa Garcia Simon ${ }^{1}$

${ }^{1}$ Hospital San José de Teruel

Introduction: The study of the major neurocognitive disorder in elderly patients can be carried out by geriatricians, neurologists and sometimes psychiatrists. Primary care doctors are the people in charge of making referrals to the different specialists.

Method: A retrospective descriptive study of all patients over 69 years of age is performed at the Neurology office for dementia study from November 1, 2019 to January 31, 2020. Subsequently, patients were classified according to the health center. where the consultations for the study of patients came from and if the primary care doctors performed any screening test before the consultation.

Result: 20 patients come to the Neurology office to study dementia. Of the total number of consultations, in 15 of them the primary care physician had not performed any tests for the study of prior cognitive impairment, and in 5 of them there was a previous study. Of the 15 patients $(75 \%)$ who did not present a study, $9(60 \%)$ came from a village health center and $6(40 \%)$ from a health center in the capital. The 5 doctors $(25 \%)$ who did a study test, $4(80 \%)$ came from a town health center and $1(20 \%)$ from the capital's health center.

Conclusions: The primary care doctors who are in the health centers of villages are those who usually perform screening tests to make referrals for dementia study and those of health centers in the capital, but the general tonic is to make consultations only because of the history of patients' relatives.

\section{Abstract \# 117}

Codex and MMSE: what to choose?

Abrar-Ahmad Zulfiqar ${ }^{1}$, Arnaud Thomasset de Longuemarre ${ }^{2}$

${ }^{1}$ University Hospital of Strasbourg, Department of Internal Medicine, Strasbourg, France, ${ }^{2}$ University Hospital of Rouen, Department of General Medicine, Rouen, France

Introduction: The objective of this study was to compare in general practice, patients aged 65 years and over: the MMSE, screening tools for cognitive disorder recommended by the HAS and the Codex.

Method: 53 patients were included between June and October 2017 at La Charmille general practice in Limpiville in rural areas during an observational study. Patients over 65 years of age were included following a consultation with their general practitioner. The MMSE and the Codex were compared on their results, intrinsic variables, the time of handover. Geriatric comorbidity factors are compared between groups of patients "with" and "without" cognitive disorders detected.

Results: Of the 53 patients, 94\% CI 95\% [4.10; 20.25] had cognitive impairment, at an average age of 74.15 years. The predominance is female with 30 patients (57\%). The passage time of the Codex is on average $1 \mathrm{~min} 43 \mathrm{~s}$, significantly faster $(\mathrm{p}=4,29 * 10-26)$ than the time of passing the MMSE $4 \mathrm{~min} 51 \mathrm{~s}$ on average. There is a better satisfaction of the Codex compared to the MMSE $\left(\mathrm{p}=1.675^{*} 10-6\right)$ with an average digital scale score of 7.3 against 6.3 respectively. There is no significant difference between population with cognitive and noncognitive impairment and geriatric comorbidity factor due to selection bias.

Conclusion: Codex appears to be a rapid, reliable, reproducible cognitive impairment screening tool adapted to the general practice consultation format. An important axis to work, awareness, education of doctors at the university and continuing education on the screening and management of dementia syndromes and fragility.

\section{Abstract \# 118}

\section{Adipokines in Alzheimer's disease}

Hana Vankova ${ }^{1}$, Eva Jarolimova ${ }^{1}$, Iva Holmerova ${ }^{2}$, Robert Rusina ${ }^{3}$, Marketa Vankova

${ }^{1}$ Third Faculty of Medicine, Charles University, Czech Republic,

${ }^{2}$ Third Faculty of Medicine, Charles University, Czech Republic,

${ }^{3}$ Thomayer Hospital, Czech Republic, ${ }^{4}$ Institute of Endocrinology, Czech Republic

Introduction: Impaired insulin signalling and insulin resistance in the brain cells has been found to be of importance for $\mathrm{AD}$ pathogenesis $[1,2]$. However, it seems that not only insulin resistance but also other factors can link neurodegenerative diseases with metabolic ones [3]. Methods: Our study examined plasma levels of adipokines in patients with Alzheimer's disease. Patients with AD are defined on the basis of international NINCDS-ADRA criteria. Peripheral levels of adipokines, including resistin and visfatin, are measured using Bio-Plex ProHuman Diabetes 10-Plex Assay and Bio-Plex ProHuman Diabetes Adipsin and Adiponectin Assays. The results are compared in patients with Alzheimer's disease and in controls of the same age.

Results: According to preliminary results, significant differencies between the group of Alzheimer's disease patients and healthy controls of the same age in adipokines levels in plasma have been found. Conclusions: Future research should focus on changes in adipokine levels in patients with Alzheimer's disease and study their role in pathogenesis. Findings of our study are promising, especially in context of studies investigating potential protective effects of resistin against amyloid- $\beta$ neurotoxicity in animal models, suggesting that resistin prevents apoptosis signals [4]. Clarification of the role of adipokines in pathogenesis of $\mathrm{AD}$ may contribute to finding new targets for AD treatment.Supported by the grant NV18-01-00399 of the Ministry of Health of the Czech Republic

References: 1. Arnold, S. E., et al. Brain insulin resistance in type 2 diabetes and Alzheimer disease: concepts and conundrums. Nature Reviews Neurology, 2018, 14.3: 168. 2. M De La Monte, S.. Brain insulin resistance and deficiency as therapeutic targets in Alzheimer's disease. Current Alzheimer Research, 2012, 9.1: 35-66. 3. Vankova M. et al. Preliminary evidence of altered steroidogenesis in women with Alzheimer's disease: Have the patients "OLDER" adrenal zona reticularis?. The Journal of steroid biochemistry and molecular biology, 2016, 158: 157-177. 4. Liu, J., et al. Resistin protection against endogenous $A \beta$ neuronal cytotoxicity from mitochondrial pathway. Brain research, 2013, 1523: 77-84.

\section{Abstract \# 119}

Use of antipsychotic drugs for patients with dementia in general practice (GP) in UK

\section{Milad Golsharifi ${ }^{1}$}

${ }^{1}$ East Sussex Healthcare Trust

SmDiagnosis and management of dementia requires a structured and comprehensive assessment tailored to each patient's needs. Throughout the natural progression of this disorder patients may exhibit a variety behavioural and psychological patterns. For a minority of patients, the use of antipsychotics are acceptable and in line with UK's National Institute of Clinical Excellence's (NICE) published clinical guidance. However, there remain cases where antipsychotics are prescribed inappropriately to people with dementia, 
often due to lack of adequate assessment or prescribing errors. This is important since studies have shown a positive correlation between use of antipsychotics in patients with dementia and increased risk of stroke, as high as 9 folds, in just four weeks from initiation of antipsychotic drugs [1]. The aim of this quality improvement project was to establish whether current practice in prescription of antipsychotics meets national guidance on this topic provided by NICE in a UK GP surgery and improve any substandard domain in this topic.A retrospective database search was conducted with inclusion criterias of "dementia and "antipsychotics", after which 30 patients were identified and their documentation and drug chart were audited against current standards. Followed by a prospective search of same inclusion criterias 3 months after introduction of interventions to improve standards. There was a 50\% improvement in documentation regarding discussion between prescribing healthcare professional and patient/carer about the risk/benefits of antipsychotic medication. There was $40 \%$ increase in use of non pharmacological interventions prior to initiating antipsychotics. There were marked improvements in all set standards and overall an increased awareness regarding prescribing in dementia related care

References: 1. National Institute for Health and Care Excellence. Dementia: assessment, management and support for people living with dementia and their carers. Available at: https://www.nice. org.uk/guidance/ng97

\section{Abstract \# 120}

Restless leg syndrome and association with cognitive impairment

Panagiota Voskou ${ }^{1}$, Andreas Kyrozis ${ }^{2}$

${ }^{1}$ Dr, ${ }^{2}$ Assoc.Prof

Introduction: There are studies supporting the existence of impaired attention, frontal executive and mental flexibility functions in Restless leg syndrome (RLS), whereas others describe no differences between RLS patients and controls or even better performance in RLS. Aim of the present study was to review the association between RLS and cognitive decline.

Methods: The literature (database Pubmed) was reviewed.

Results: Factors which could be associated with cognitive dysfunction in RLS are vascular risk factors, decreased attention and comorbid psychiatric conditions, while findings about the impact of sleep disruption and depression are conflicting. Chronic sleep loss might produce deficits in pre-frontal cortical functions like those related to acute sleep deprivation. Working memory deficits have been found in severe RLS and repeated RLS symptomatic attacks are related to cerebral cortical dysfunction. Executive and visual-spatial functions are impaired in RLS, whereas memory and overall cognitive function are mostly preserved. RLS is assumed to lead to an increased risk for developing dementia due to a brain damage associated with sleep deprivation, while concomitant dopamine deficiency in RLS may be associated with cognitive decline and progression to dementia long term.

Conclusions: Future studies should examine the effects of RLS in more than one cognitive domains or the risk of developing dementia, ideally under larger samples, the mechanisms underlying the cognitive impairment due to RLS, while functional neuroimaging may be helpful. Electrophysiological studies could investigate cognitive functions in RLS possibly related to impairment of attention and/or arousal state.

\section{Abstract \# 121}

Dementia as economic point of view in romanian population: pilot study

Sorina Maria Aurelian ${ }^{1}$, Andreea Zamfirescu ${ }^{1}$, Ruxandra Mihalache ${ }^{1}$, Justin Aurelian ${ }^{1}$, Vlad Onetiu ${ }^{2}$, Ana Capisizu ${ }^{1}$

${ }^{1}$ University of Medicine and Pharmacy "Carol Davila", ${ }^{2}$ University Sheffield

Introduction: Urinary tract infections (UTI) are among the most prevailing infectious diseases with considerable morbidity and mortality with a substantial financial burden on society. The elderly persons are the most susceptible to UTI that frequently requires successive hospitalization.

Methods: We conducted a one-year retrospective study (2018) in Chronic Diseases Hospital Sf. Luca, on 1791 patients with a meanage73 $\pm 6,23$ years. Data for the study were Urinalysis and antibiograms performed by AutomatMicroscan. We analyzed the demographic profile, the incidence of the favoring factors and the frequency of the symptoms encountered. Isolated microorganisms and their sensitivity to antibiotics were the basis of a comparative analysis using SPSS software.

Results: There were 221 Urinalysis 23 of which were asymptomatic bacteria. $82.32 \%$ of patients are women; $51 \%$ are from rural areas; age group (75-84 years) are the most numerous (46.6\%). Constipation and insufficient hydration are the most frequent favoring factors $(50 \%)$; drug abuse is very rare in Romanian elderly $(0.5 \%)$. The most common symptoms (67\%) are pollakiuria and dysuria.The distribution ofUrine culture showed growth of Escherichia Colli $(58.4 \%)$, Klebsiella (17.7\%); Enterococcus and Pseudomonas in approx. equal to $(4.52 \%)$. Escherichia Coli were sensitive to quinolones $(73 \%)$, cephalosporins (54\%) and carbapenem (90.9\%). The most effective antibiotics were Amikacinum (80\% sensitivity) for Proteus and Pseudomonas; Carbapenem (90\% sensitivity) for Escherichia Coli and Proteus. The uropathogens that were the most resistant to antibiotics: Klebsiella (60\%); Enterococcus (62\%), Staphylococcus Aureus (68\%).

Key conclusions: Our outcomes show that isolated uropathogens have a low sensitivity to antibiotics. This increasing resistance to broad-spectrum antibiotics is particularly troublesome at elderly, a frail populational group.

\section{Abstract \# 122}

\section{Hypnosis for couples affected by Alzheimer's disease}

Wawrziczny emilie ${ }^{1}$, Buquet Amandine ${ }^{2}$, Picard Sandrine ${ }^{1}$, Huvent Dominique $^{3}$, Puisieux Francois ${ }^{3}$, Pasquier Florence ${ }^{4}$, Doba Karyn ${ }^{1}$

${ }^{1}$ University of lille, ${ }^{2}$ University of Lille, ${ }^{3}$ Hôpital Gériatrique Les Bateliers, Lille, ${ }^{4}$ Memory Resource and Research Center, Lille

Introduction: Alzheimer's Disease causes changes in the couple life which may cause negative effects on the quality of life and entraine a high level of distress of both partners. Hypnosis was evaluated as being effective in a number of healthcare fields for the control of symptoms and distress. Nevertheless, literature on the use of hypnosis in the field of Alzheimer's Disease is extremely sparse and show contradictory results.

Method: We treated two couples which one of the partners has Alzheimer's disease and and living together at home. The two couples took part in weekly sessions together at home over a course of 8 weeks. Interviews, led before and after the intervention, were analysed and provided access to the couple's subjective experience in terms of the emotions they feel, the quality of the relationship and the benefits of the treatment. 
Results: Hypnotic intervention appeared to ameliorate both psychological difficulties and the quality of life for both partners. They described a state of well-being and relaxation after each session. They feel less upset and more focused on the important things in their lives. In terms of the couple relationship, they feel more affectivity, tenderness and closeness between them.

Key conclusions: Systematic research into the effectiveness of hypnosis with Alzheimer's disease needs to be undertaken in the future

\section{Abstract \# 123 \\ Improvement of cognitive deficit by curcumin in scopolamine- induced Alzheimer's disease models}

Guzin Cakmak ${ }^{1}$, Davut Sinan Kaplan², Caner Yildirim², Hasan Ulusal $^{3}$, Mehmet Tarakcioglu ${ }^{4}$, Zeynel Abidin Ozturk ${ }^{1}$

${ }^{1}$ Gaziantep University, Faculty of Medicine, Department of Internal Medicine, Division of Geriatrics, ${ }^{2}$ Gaziantep University, Faculty of Medicine, Department of Physiology, ${ }^{3}$ Gaziantep university, Faculty of Medicine, Department of Biochemistry, ${ }^{5}$ Gaziantep University, Faculty of Medicine, Department of Biochemistry

Introduction: Curcumin is suggested for the treatment of Alzheimer's Disease (AD) because of its' protein modifying and antioxidative features. In this study, anti-dementia effects of curcumin on scopolamine-induced AD rat models were evaluated.

Methods: Twenty-one male Wistar Albino rats of $300 \pm 25 \mathrm{grams}$, 1 -year-old were introduced to study. They were divided into 3 groups (n:7 in each group); untreated control, exposed to scopolamine and treated by curcumin, and then exposed to scopolamine. Animals were evaluated for behavioral tasks with the Morris Water Maze test. After behavioral tests; interleukin-6 (IL-6) and tumor necrosis factor-alpha (TNF-alpha) were measured in hippocampal tissues. SPSS version 26.0 was used for data analysis.

Results: In this study, we found that distance to reach the escape platform (DTZ) was highest in the scopolamine group, lowest in the curcumin group $(\mathrm{p}=0.004)$. Time to reach the platform was longest in the scopolamine group, shortest in the curcumin group $(\mathrm{p}=0.025)$. Spatial learning seemed to be conserved in the curcumin group. DTZ was highest in the scopolamine group, lowest in the control group in the probe test $(p=0.035)$. Reference memory seemed to be deteriorated in the scopolamine group. IL-6 levels were higher in the scopolamine group than the curcumin group $(p=0.017)$ and the control group $(\mathrm{p}=0.005)$. TNF-alpha levels were highest in the scopolamine group, there was no statistical significance.

Key conclusions: We revealed that curcumin provides a protective effect on scopolamine-induced dementia. The anti-inflammatory feature of curcumin was shown too. Curcumin could be neuroprotective for people at risk of becoming $\mathrm{AD}$.

\section{Abstract \# 124 \\ Comparative study of stigma and burnout among professional and family caregivers in dementia care \\ Marianna Mantzorou ${ }^{1}$, Dimos Mastrogiannis ${ }^{2}$, Evmorfia Koukia ${ }^{3}$ \\ ${ }^{1}$ University of West Attica, ${ }^{2}$ University of Thessaly, ${ }^{3}$ National and Kapodistrian University of Athens}

Introduction: Dementia is a part of life of millions of people worldwide but poor understanding contributes towards the stigma surrounding the disease with negative consequences for the wellbeing of their caregivers. This study was conducted to explore the relationship between stigma and burnout comparing professional and family caregivers.
Material and methods: A cross-sectional study was carried out in a convenience sample of 171 geriatric nurses and non-graduate caregivers working in 16 nursing homes and 153 pairs of patients and family caregivers who attended 3 day-care centers. The research tools used were questionnaires regarding sociodemographic and clinical data, the Maslach Burnout Inventory (MBI) and the Family Stigma in Alzheimer's Disease Scale (FS-ADS).

Results: The study highlighted the presence of low to moderate levels of stigma, with family caregivers showing higher Caregiver Stigma scores compared to professional ones $(\mathrm{U}=9066.5, \mathrm{p}=0.001)$. Professional caregivers displayed moderate while family caregivers moderate to low levels of burnout. Multivariate regression analysis showed that cognitive, emotional and behavioral attributions of stigma, were independent predictive factors of the dimensions of burnout, showing that stigma contributes towards their burnout. Family caregivers presented lower depersonalization levels $(\mathrm{U}=$ 10599.5, $\mathrm{p}=0.003)$ and higher levels of personal achievements $(\mathrm{U}=$ $8866.5, \mathrm{p}=0.001)$ possibly due to their close familial relationship and emotional involvement while nurses may often experience a sense of low status and social recognition of their profession.

Key conclusions: Conclusions drawn with regard to the contribution of stigma towards burnout of professional and family caregivers, can contribute to a better educational preparation of geriatric nurses as well as carefully planned strategies to increase public awareness and destigmatize dementia.

\section{Abstract \# 125}

The influence of neuropsychiatric symptoms of elderly with dementia, on stigma and burnout of family caregivers

Marianna Mantzorou ${ }^{1}$, Dimos Mastrogiannis ${ }^{2}$, Evmorfia Koukia ${ }^{3}$

${ }^{1}$ University of West Attica, ${ }^{2}$ University of Thessaly, ${ }^{3}$ National and Kapodistrian University of Athens

Introduction: The neuropsychiatric symptoms of elderly persons with dementia contribute towards the burnout of family caregivers. Limited research data documents a correlation of stigma with burden of caregivers but there has been no research examining the correlations of neuropsychiatric symptoms with stigma and burnout in caregivers.

Materials and methodology: 153 pairs of patients and family caregivers who attended 3 day-care centers in Attica were administered questionnaires regarding sociodemographic and clinical data, the Neuropsychiatric Inventory Questionnaire (NPI), the Maslach Burnout Inventory (MBI) and the Family Stigma in Alzheimer's Disease Scale (FS-ADS).

Results: The patients' most aggravating symptoms for family caregivers were found to be (a) apathy/indifference, (b) depression, (c) agitation/aggression and (d) anxiety. The multivariate linear regression analysis showed that family caregivers who feel the greatest psychological stress from their patient's neuropsychiatric symptoms stigmatize him more intensely and feel more emotionally exhausted. It was also found that the higher the number, severity and frequency of patients' neuropsychiatric symptoms, the greater the stigma that, according to caregivers, people in society place on their relatives $(\mathrm{rs}=0,267, \mathrm{p}=0,001)$ as well the stigma they deposit on their relatives ( $r s=0,320, p=0,001)$ and the sense of shame and pity for the patients ( $r s=0,214, p=0,008)$. The sense of personal achievement of the family caregiver $\sigma$ decreased $(r s=-0.203, p=0.012)$ and depersonalization increased ( $\mathrm{rs}=0.278, \mathrm{p}=0.001$ ) as the severity of patients' neuropsychiatric symptoms increased.

Key conclusion: Positive correlations found between severity of neuropsychiatric symptoms, stigma and burnout of family caregivers call for development of educational programs that will help caregivers 
manage the symptoms of their relatives and reduce the stigma surrounding the disease.

\section{Abstract \# 126 \\ Hypercog: cognitive screening in older adults with hypertension: a pilot study}

Maria Flora D'Andria ${ }^{1}$, Giulia Rivasi ${ }^{1}$, Virginia Tortu' ${ }^{1}$, Giada Turrin $^{1}$, Daniele Falzone ${ }^{1}$, Antonella Giordano ${ }^{1}$, Andrea Ungar ${ }^{1}$

${ }^{1}$ Hypertension Centre, Head Division of Geriatrics and Intensive Care Unit, University of Florence, Careggi Hospital, Florence, Italy

Hypertension is a recognized risk factor for cognitive impairment. In older hypertensive adults cognitive screening tests should be included in the assessment of hypertension-related organ damage, as the 2018 $\mathrm{ESH} / \mathrm{ESC}$ guidelines recommend. The present study was carried out in a sample of older hypertensive adults without dementia and compare the diagnostic accuracy of cognitive screening tests, including the MiniCog Test, the Montreal Cognitive Assessment (MoCA), the Mini Mental State Examination (MMSE) and the Clock Drawing Test (CDT). The study was carried out in the Centre for Hypertension Management in Older Adults, Careggi Hospital, Florence, Italy, between February 2017 and May 2019. Subjects aged 65 or older without a prior diagnosis of cognitive impairment underwent a cognitive screening including the MMSE, the MoCa, the Mini-Cog and the CDT, followed by an extensive neuropsychological evaluation. Sensitivity and specificity of each screening test were assessed, using the ROC curves. Among 94 participants, 35 (37.2\%) had a diagnosis of mild cognitive impairment or dementia at the neuropsychological evaluation. According to the ROC curves, the MoCA and the MMSE achieved the best detection of cognitive impairment with a cut-off score of 24 (AUC 0.746) and 27.5 (AUC 0.689), respectively. The MoCA had the highest diagnostic accuracy, providing a $80 \%$ sensitivity and a $59 \%$ specificity. Among the available screening tests, the MoCA seems to have a good diagnostic accuracy in this population and could be included in the assessment of hypertension-related organ damage, to screen for cognitive impairment.

\section{Abstract \# 127}

\section{Aromatherapy for dementia: cochrane review}

Emily Ball ${ }^{1}$, Bethan Owen-Booth ${ }^{2}$, Amy Gray $^{3}$, Susan Shenkin ${ }^{4}$, Jonathan Hewitt ${ }^{5}$, Jenny McCleery ${ }^{6}$

${ }^{1}$ Centre for Clinical Brain Sciences, University of Edinburgh, UK, ${ }^{2}$ Occupational Therapy, Glyndwr University, UK, ${ }^{3}$ Medicine of the Elderly, Western General Hospital, UK, ${ }^{4}$ Geriatric Medicine, Usher Institute, University of Edinburgh UK, ${ }^{5}$ Cardiff University, UK, ${ }^{6}$ Oxford Health NHS Foundation Trust, UK

Introduction: Licensed anti-dementia medications are ineffective against distressed behaviours in dementia. Caregivers often look to complementary therapies, including aromatherapy, as alternatives. The aim of this Cochrane review is to assess the efficacy and safety of aromatherapy for people with dementia.

Methods: We searched electronic databases on 5 May 2020. We included randomised controlled trials which compared an aromatherapy intervention for dementia with placebo aromatherapy or with usual care. We conducted no meta-analyses because of heterogeneity between studies, but presented a narrative synthesis of results. Results: We included 13 studies involving 708 participants with dementia, all resident in care homes. Our primary outcomes were agitation, overall behavioural and psychological symptoms (BPSD), and adverse events. Ten studies assessed agitation and eight assessed BPSD. Five and three studies respectively reported no usable data or we had very low confidence in their results. We had moderate or low confidence in the results of the remaining studies. For agitation, four of these studies reported no significant effect of aromatherapy on agitation, one study reported a significant benefit. For BPSD, four studies reported a significant benefit from aromatherapy, one study reported no significant effect. Adverse events were poorly reported or not reported in most studies.

Key conclusions: We found no convincing evidence that aromatherapy is beneficial for people with dementia although there are many limitations to the data. In order for clear conclusions to be drawn, better design and reporting and consistent outcome measurement would be needed in future trials.

\section{Abstract \# 128}

A phase II, randomized, double-blind, placebo-controlled trial to investigate the efficacy and safety of avidekel oil for the treatment of patients with agitation related to dementia Vered Hermush ${ }^{1}$

${ }^{1}$ Technion Medical School

Introduction: The treatment options for patients with severe dementia are limited and lead to severe side effects. Clinical studies on adults show that cannabinoids (CBD) could have a beneficial effect on behavioral disorders in general, and in dementia-related disorders in particular. The aim of this randomized clinical trial (RCT) was to evaluate the safety and efficacy of CBD rich oil for the treatment of subjects with agitation related to dementia.

Methods: 64 patients were randomly assigned in a 2:1 ratio to receive drops of Avidekel Oil or placebo respectively. Over the course of sixteen weeks, ten visits were conducted. In each visit data on vital signs, behavioral disorders (based on the Cohen-Mansfield Agitation Inventory (CMAI), Neuropsychiatric Inventory (NPI-NH), Clinical Global Impression Severity-Agitation/Aggression), Mini-Mental State Examination (MMSE), concomitant medications, adverse events, and safety tests were collected.

Results: 64 eligible patients were screened and recruited. Their average age was 79 with $37.5 \%$ men. Four patients withdrew before randomization, 60 patients began cannabis/placebo treatment, 40 in the active treatment group and 20 in the control group. Baseline CMAI scores in the treatment and the placebo groups were 57.28 and 58.5 , respectively $(p>0.83)$. About $72 \%$ of the patients in the treatment group achieved the primary outcome of CMAI reduction of 4 points, versus $30 \%$ in the control group $(\mathrm{p}<0.003)$, with a mean change of 13.3 and 2.3 in the treatment and placebo groups, respectively $(\mathrm{p}<0.002)$. No differences were found in the baseline NPI-NH agitation/aggression sub score between the two groups $(p>0.95)$. However, the mean change in NPI-NH was 3.3 and 1.1 points in the treatment and the placebo groups, respectively $(\mathrm{p}<0.02)$.

Conclusions: Neuropsychiatric symptoms are very common among patients with dementia. CBD rich oil can reduce agitation and improve the behavioral symptoms in dementia.

\section{Abstract \# 129}

The risk and incidence of neurocognitive disorders in patients with chronic kidney disease and diabetes in the North Wales Denbighshire cohort, United Kingdom.

Peter Hobson ${ }^{1}$

${ }^{1} \mathrm{Dr}$

Background and aims: Cognitive impairment is commonly reported in chronic kidney disease (CKD). This study will calculate the risk 
and the incidence for neurocognitive disorders (NCD) in a $\mathrm{CKD}$ cohort with diabetes.

Method: Cognitive function was assessed in 92 CKD patients (mean age $75.8+9.1 ; 49$ males: 43 female), at baseline and at 36-months. Cognitive impairment diagnosis was based upon patient, informant, case note review, neuropsychological assessment and application of Diagnostic and Statistical Manual of Mental disorders version 5 (DSM-5) for an NCD. An age and gender matched sample of 143 subjects without $\mathrm{CKD}$, diabetes, or cognitive impairment acted as a control group.

Results: Follow-up neuropsychological assessment and application of DSM-5 criteria revealed that 25/92 (27\%) of the CDK cohort had developed an NCD (mild $n=19$; dementia $n=6$ ). The CKD patients had a twofold increased for the development of an NCD compared to the controls adjusted for age and sex. The incidence rate for an NCD in the CKD cohort was 10.5 and 5.1 in the controls. No association was observed with the stage of CKD and cognitive function. The strongest neuropsychological predictors for developing an NCD were impairments in memory, fluency and executive function.

Conclusion: This study found that CKD patients are at a twofold increased risk for the development of an NCD compared to those without the condition. The higher risk and incidence for cognitive function reported here suggest that routine neuropsychological assessment should be incorporated and screened in this population as part of normal clinical practice.

\section{Abstract \# 130 \\ Unrecognized cognitive impairment in cardiac rehabilitation patients \\ Bojana Potic ${ }^{1}$, Nebojsa Despotovic ${ }^{1}$, Predrag Erceg ${ }^{1}$, Slavica Vasilic $^{1}$, Sanja Zdravkovic ${ }^{2}$, Snezana Tomic ${ }^{3}$ \\ ${ }^{1}$ Department for Geriatrics,CHC Zvezdara, Belgrade, Serbia, ${ }^{2}$ Department for Geriatrics, Belgrade, Serbia, ${ }^{3}$ Department for Geriatrics, CHC Zvezdara, Belgrade}

Introduction: To determine the prevalence of unrecognized brain dysfunction accompaning chronic severe cardiac disease, we examined 20 clinically stable consecutive admissions to a cardiac rehabilitation service who were free of known stroke or dementia.

Methods: Age range was 65-85 years,thre were 50\% male and 50\% female.They suffered from different types of Cardiomyopatihies (50\% ishaemic, 30\% dilatative). They also had arterial hypertension $70 \%$, lung disease $30 \%$, gastritis $30 \%$ and different anemias $20 \%$.

Results: Multiple cognitive deficits including significant memory impairment and disorientation were present in eight patients (40\%), seven of this eight patients were unable to administer their own medications reliably.An additional six patients (30\%) showed milder impairments.One patient was found to be normal after neurological examination ,four showed evidence of a single brain lesion and 15 of $20(75 \%)$ had multipleneurological abnormalities suggesting multifocal brain disease.

Conclusion: The mechanism of cognitive deficits in cardiac patients is unclear and it may be related to multiple infarcts or acute or chronic hypoxic damage secondary to arrhythmias,cardiac failure or small vessel disease of the brain.Patients with cardiac disease should unergo cognitive screening, as early identification of patients at risk of progressive intelectual loss may allow early use of peventive therapy.

\section{Abstract \# 131}

Systematic review and without meta-analysis of clinical practice guidelines (CPGs) relating to cognition testing in older patients

Clayton Micallef ${ }^{1}$, Terry Quinn ${ }^{1}$

${ }^{1}$ University of Glasgow

Introduction: Most people with dementia admitted to hospital will have a primary medical diagnosis(es) and the importance of their dementia is overlooked despite a growing number of over $65 \mathrm{~s}$ presenting as unscheduled care. We set out to analyze current CPGs relating to cognitive assessment in these patients.

Methods: We generated a robust search strategy on the 12/02/2019 encompassing the Medline, Embase, and Cinahl and PsycInfo databases concerning dementia and delirium. This was developed in an iterative process to further refine and improve the search of the relevant databases.

Results: In all 8 eligible CPGs were found, with 5 rated as high quality covering both dementia and delirium. 2 were of moderate quality, and 1 of low quality. There is a paucity of primary evidence for most recommendations. SIGN uniquely recommends the 4AT tool for delirium, although they almost unanimously recommend an informant history to aid diagnosis. A normal cognitive screen doesn't rule out dementia, and changes in presentation should prompt a further cognitive screen. All dementia guidelines recommend a cognitive and functional assessment as workup.

Conclusions: Primary gold standard studies are lacking, and most CPG recommendations are based on expert consensus. Both delirium and dementia CPGs almost unanimously recognize the importance of taking an informant history to assess a patient's baseline, and the utility of assessing for delirium at the initial presentation. The use of validated tools to detect delirium and performing an initial screen on presentation agreed upon by half delirium CPGs. All dementia guidelines recommend a cognitive and functional assessment as part of diagnostic workup.

\section{Abstract \# 132}

Does late-life depression counteract the beneficial effect of physical activity on cognitive decline? Results from the NESDO study

Marieke Henstra ${ }^{1}$, Erik Giltay ${ }^{2}$, Roos van der Mast ${ }^{2}$, Didi Rhebergen $^{2}$, Nathalie van der Velde ${ }^{1}$, Nathaly Rius Ottenheim ${ }^{2}$

${ }^{1}$ Amsterdam UMC, location AMC, ${ }^{2}$ LUMC

Objectives: Physical activity (PA) may postpone cognitive decline in older persons. It is unknown whether depression, which affects both $\mathrm{PA}$ and cognitive functioning, counteracts this association. We therefore investigated the association between baseline PA and cognitive functioning over time, stratified for depression.

Design: Longitudinal.

Setting and participants: We used data of the Netherlands Study of Depression in Older persons (NESDO), a multi-site cohort study with 6-years follow-up. 297 depressed and 97 non-depressed older participants with complete data on baseline PA and cognitive functioning were included.

Measures: Linear mixed models, stratified for depression, were used to assess the association of self-reported baseline PA with five standardized outcomes of cognitive functioning (Mini Mental State Examination, interference-control, verbal memory, working memory, and their compound-score) at baseline, and after 2 and 6 years. We also assessed the association between time and cognitive functioning stratified for depression. 
Results: Mean age was 70.1 years (SD 7.1), and $63 \%$ were women. No significant associations between baseline PA and decline of any cognitive outcome were found, irrespective of the presence of depression. In both groups, also no significant interactions of PA with time were found, indicating that the extent of the rate of cognitive decline over time was largely unaffected by PA. Only decline of working memory was significantly worse in the depressed group compared to the non-depressed group.

Conclusions and implications: At older age, the level of PA may not impact long-term cognitive functioning, irrespective of the presence of depression. Depression appeared to worsen age-related decline of working memory only. However, selective attrition may have biased our results. As a higher PA-level at older age has a positive effect on a multitude of other health outcomes, PA should still be encouraged in this population.

\section{Abstract \# 133 \\ Sedative load in community-dwelling older adults with mild- moderate Alzheimer's disease: longitudinal relationships with adverse events, delirium and falls \\ Adam Dyer $^{1}$, Claire Murphy ${ }^{2}$, Brian Lawlor ${ }^{3}$, Sean Kennelly ${ }^{3}$ \\ ${ }^{1}$ Trinity College Dublin, ${ }^{2}$ Age-Related Heathcare, Tallaght University Hospital, ${ }^{3}$ Global Brain Health Institute}

Background: Older adults are frequently prescribed medications with sedative effects, which are associated with numerous adverse consequences. However, the prevalence and longitudinal associations of sedative medication use in community-dwelling older adults with mild-moderate Alzheimer Disease (AD), has not been explored to date.

Methods: The association between Sedative Load (SL) and adverse events, unscheduled healthcare utilisation, delirium and falls over 18 months was assessed in older adults with mild-moderate AD from 9 Countries. Additionally, the impact of SL on dementia progression was evaluated.

Results: Over half $(52.4 \% ; 267 / 510)$ of those with mild-moderate AD $(72.8 \pm 8.3$ years, $61.9 \%)$ were prescribed a regular medication with sedation as a primary effect or prominent side effect with $17.7 \%$ (90/ $510)$ having a high SL $(\geq 3)$. The most common medications contributing to SL were antidepressants, antipsychotics, anxiolytics and hypnotics. Over 18 months, increasing SL was associated with adverse events (IRR 1.18, 1.14-1.23, p <0.001)/serious adverse events (IRR 1.32, 1.18-1.49, $\mathrm{p}<0.001$ ) and unscheduled GP visits (IRR 1.26, 1.15-1.38, $\mathrm{p}<0.001$ ). Further, increasing SL was associated with greater likelihood of incident delirium (IRR 1.47, $1.25-1.73, \mathrm{p}<0.001$ ) and falls (IRR 1.25, 1.06-1.48, p = 0.007) which persisted after covariate adjustment. SL was not associated with accelerated cognitive decline or AD progression.

Conclusions: Most adults with mild-moderate AD are prescribed at least one drug with a sedative effect and a significant minority have a high SL. Increasing SL was associated with a greater likelihood of adverse events, delirium and falls, highlighting the need for optimal prescribing in this potentially vulnerable cohort.

\section{Abstract \# 134 \\ Long-term antipsychotic use, orthostatic hypotension and falls in mild-to-moderate Alzheimer's disease}

Adam Dyer ${ }^{1}$, Claire Murphy ${ }^{1}$, Brian Lawlor ${ }^{2}$, Sean Kennelly ${ }^{2}$

${ }^{1}$ Trinity College Dublin, ${ }^{2}$ Global Brain Health Institute
Background: Antipsychotics are frequently used in older adults with Alzheimer's disease (AD) and are associated with numerous adverse events and increased mortality. Whilst frequently listed as causing Orthostatic Hypotension $(\mathrm{OH})$, the relationship between antipsychotic use and $\mathrm{OH}$ has never been explored in mild-to-moderate $\mathrm{AD}$, a group who may be particularly vulnerable to their adverse effects.

Methods: We analysed longitudinal data from community-dwelling older adults with mild-to-moderate AD in 9 European Countries. We assessed the effect of antipsychotic use on orthostatic blood pressure (BP) measured on eight occasions over 18-months, in addition to the association between antipsychotic use and incident falls.

Results: Of 509 older adults (aged $72.9 \pm 8.3$ years, $61.9 \%$ female) with mild-to-moderate $\mathrm{AD}, 10.6 \%(\mathrm{n}=54)$ were prescribed a longterm antipsychotic. Ongoing antipsychotic use was associated with a greater likelihood of developing sit-to-stand $\mathrm{OH}$ at both 1 minute (OR $1.15,1.01-1.32, \mathrm{p}=0.04)$ and 5 minutes (OR 1.32, 1.07-1.42, $\mathrm{p}=$ $0.004)$ in addition to a greater drop in systolic BP at 5 minutes $(\beta$ : $0.53,1.10-0.96, p=0.02$ ) over the 18 month study duration. Further, ongoing antipsychotic use was associated with incident falls (IRR $1.80,1.11-2.92, \mathrm{p}=0.02)$. Findings persisted after covariate adjustment.

Conclusions: Even in mild-to-moderate $\mathrm{AD}$, ongoing antipsychotic use was associated with sit-to-stand $\mathrm{OH}$ and incident falls over 18 months. Further attention to optimal prescribing interventions in this cohort is warranted and may involve screening antipsychotic users for orthostatic symptoms.

\section{Abstract \# 135}

Is ongoing anticholinergic burden associated with greater cognitive decline and dementia severity in mild to moderate Alzheimer's disease?

Adam Dyer ${ }^{1}$, Claire Muprhy ${ }^{1}$, Brian Lawlor $^{2}$, Sean Kennelly ${ }^{2}$

${ }^{1}$ Trinity College Dublin, ${ }^{2}$ Global Brain Health Institute

Background: Use of anticholinergic medication is associated with an increased risk of cognitive impairment and/or dementia. Despite this, the impact of continuing medication with anticholinergic properties in those diagnosed with Alzheimer's Disease (AD) is not clear.

Methods: Analysis of data from NILVAD, an 18-month randomized controlled trial of Nilvadipine in AD. Effects of ongoing Anticholinergic Cognitive Burden (ACB) on cognition (ADAS-Cog: Alzheimer's Disease Cog Subsection) and dementia severity (CDRsb: Clinical Dementia Rating-Sum of Boxes/DAD: Disability Assessment for Dementia) over 18 months was evaluated adjusting for important clinical covariates.

Results: Just over one-quarter $(27.90 \%, n=142 / 510)$ of patients with mild to moderate $\mathrm{AD}$ were prescribed a potential/definite anticholinergic. While ACB score was not associated with greater progression on the ADAS-Cog/CDR-sb over time, a higher total ACB predicted greater dementia severity on the DAD, which persisted after robust covariate adjustment $(\beta$ Coef $-1.53,95 \%$ CI -2.83 to $-0.23, p=0.021)$. There was a significant interaction between APOE $\varepsilon 4$ status and ACB score, with carriers experiencing greater progression on both the CDR-Sb ( $\beta$ Coef: $0.36,95 \%$ CI $0.05-0.67, p=$ 0.021 ) and DAD ( $\beta$ Coef: $-3.84,95 \% \mathrm{CI}-7.65$ to $0.03, \mathrm{p}=0.049$ ). Conclusions: Ongoing use of anticholinergic medication was associated with greater dementia progression on the DAD, but not the CDR-sb. APOE \& 4 carriers may be particularly vulnerable to the effect of ongoing anticholinergic medication on dementia severity, with significant APOE $\varepsilon 4 \times$ ACB score interactions demonstrated on both the DAD and CDR-sb. 


\section{Abstract \# 136}

Towards the promotion of cognitive health among older people aged 65+ years: an exploratory study In Luxembourg.

Mathilde Barbier ${ }^{1}$, Elisabeth Bourkel ${ }^{2}$, Jean-Paul Steinmetz ${ }^{2}$, Carine Federspiel $^{2}$

${ }^{1}$ Aix Marseille University, France, ${ }^{2}$ ZithaSenior S.A., Luxembourg

Between 2018 and 2050, the proportion of people over 65 years of age will increase about $10 \%$ in the EU-28. Thus, the promotion of $\operatorname{cog}$ nitive health among older people will gain further importance in the EU countries in the coming years. The present small-scale, exploratory study focuses on the perception of cognitive health amongst health care providers and older people. We conducted semi-structured interviews with two physicians, and two persons without cognitive impairment aged 65 and 68 years. The interviews were analysed using thematic analysis. The results show two types of knowledge of cognitive health: a scientific one, held by physicians, socially and culturally transmitted and disseminated; and a reified one, popularised, and specifically anchored among older adults. Additionally, our results suggest several perceived barriers and levers regarding the promotion of cognitive health. Lack of motivation can be one of these barriers. Furthermore, barriers include public policies and the health care system (e.g., lack of provision, or unsuitable settings), or can be linked to communication. Several levers directly related to these barriers have been identified. We found the need for better communication between health care providers and older adults related to the promotion of cognitive health. Our results also suggest using economic levers, such as insurance coverage or vouchers to improve the participation in the promotion of cognitive health amongst older adults. Our findings provide foundation for refining and expanding the offerings dedicated to the promotion of cognitive health among older people in Luxembourg.

\section{Abstract \# 137 \\ Activities of daily living at hospital admission associated with mortality in geriatric patients with dementia: a Danish nationwide population-based cohort study}

Thomas Veedfald $^{1}$, Karen Andersen-Ranberg ${ }^{2}$, Frans Waldorff ${ }^{3}$, Pavithra Laxsen Anru ${ }^{4}$, Tahir Masud ${ }^{5}$, Jesper Ryg ${ }^{2}$

${ }^{1}$ Department of Geriatric Medicine, Odense University Hospital, Svendborg, Denmark, ${ }^{2}$ Department of Geriatric Medicin, Odense University Hospital, Odense, Denmark, ${ }^{3}$ Research Unit for General Practice and Sectino of General Practice, Department of Public Health, University of Copenhagen, Copenhagen, Denmark, ${ }^{4}$ Center for Clinical Epidemiology, Odense University Hospital, Odense, Denmark, ${ }^{5}$ Geriatric Department, Nottingham University Hospital, Nottingham, UK

Introduction: Patients suffering from dementia may need advanced care planning when affected by acute illness and hospitalization, and prognostic assessment tools are relevant. We aimed at studying the association between basic activities of daily living at hospital admission and mortality among older patients with dementia.

Methods: All patients aged $\geq 65$ years with diagnosed dementia in the population-based National Danish Geriatric Database from 2005 to 2014 were included and followed until death, emigration, or study termination (31.12.2015). Data on Barthel-Index-100 (BI) was used to assess ADL. Patients were categorized into four predefined standard BI subcategories according to the national Danish version of the statistical classification of diseases $(\mathrm{BI}=0-24$ (very low ADL), BI = 25-49 (low ADL), BI = 50-79 (moderate reduced ADL), and BI = 80-100 (independent ADL)). Association with mortality was assessed using multivariable Cox regression analysis adjusting for age, marital status, Charlson Comorbidity Index, BMI, prior hospitalizations, year of admission and polypharmacy.

Results: In total, 6,543 patients (women $62 \%$ ) were included, median (IQR) age 84 (79-88) years and BI 37 (13-63). Mortality increased significantly with decreasing $\mathrm{BI}$ in both the crude and multivariable analysis. In subcategories $\mathrm{BI}=(80-100)$ and $\mathrm{BI}=(0-24)$ survival time (median $(95 \%)$ ) was $3.6(3.4-3.9)$ years and $0.8(0.7-0.9)$ years, respectively. Also, in patients with $\mathrm{BI}=(0-24)$, the overall mortality risk (HR $(95 \% \mathrm{CI})$ ) was $2.4(2.2-2.7), 30$-day risk $11.8(5.8-23.9)$, and 1 -year risk $4.4(3.5-5.5)$ when using $\mathrm{BI}=(80-100)$ as reference. Conclusion: BI is independently associated with all-cause mortality among older patients with dementia admitted to hospital. BI may be helpful when discussing future advanced care planning with patients and their families prior to discharge from hospital.

\section{Abstract \# 138}

Physical activity and exercise in dementia: an umbrella review of intervention and observational studies

Jacopo Demurtas ${ }^{1}$, Daniel Schoene ${ }^{2}$, Gabriel Torbahn ${ }^{2}$, Alessandra Marengoni $^{3}$, Giulia Grande ${ }^{4}$, Mirko Petrovic ${ }^{5}$, Stefania Maggi 6 , Matteo Cesari ${ }^{7}$, Sarah Lamb ${ }^{8}$, Pinar Soysal ${ }^{9}$, Cornel Sieber ${ }^{10}$, Christoph Mueller ${ }^{11}$, Susan Shenkin ${ }^{12}$, Lukas Schwingshackl ${ }^{13}$, Lee Smith $^{14}$, Nicola Veronese ${ }^{15}$

${ }^{1}$ ASL Grosseto, ${ }^{2}$ Friedrich-Alexander University Erlangen-Nürnberg, ${ }^{3}$ University of Brescia, ${ }^{4}$ Karolinska Institute, ${ }^{5}$ University of Ghent, ${ }^{6}$ Consiglio Nazionale delle Ricerche, ${ }^{7}$ Univesity of Milan, ${ }^{8}$ University of Exeter, ${ }^{9}$ Bezmialem Vakif University, ${ }^{10}$ Friedrich-AlexanderUniversität Erlangen-Nürnberg, ${ }^{11}$ King's College London,

${ }^{12}$ University of Edinburgh, ${ }^{13}$ University of Freiburg, ${ }^{14}$ Anglia Ruskin University, ${ }^{15}$ University of Palermo

Background: Dementia is a common condition in older people. Among the potential risk factors, increasing attention has been focused on sedentary behaviour. However, synthesizing literature exploring whether physical activity/exercise can affect health outcomes in people with dementia or with mild cognitive impairment (MCI) is still limited. Therefore, the aim of this umbrella review, promoted by the European Geriatric Medicine Society (EuGMS), is to understand the importance of physical activity/exercise for improving cognitive and non-cognitive outcomes in people with dementia/MCI. Methods: Umbrella review of systematic reviews (SR) (with or without meta-analyses) of randomized controlled trials (RCTs) and observational (prospective and case-control in people with $\mathrm{MCI}$ ) studies based on a systematic literature search in several databases. The certainty of evidence of statistically significant outcomes attributable to physical activity/exercise interventions was evaluated using Grading of Recommendations Assessment, Development and Evaluation (GRADE) approach.

Results: Among 1,160 articles initially evaluated, 27 systematic reviews (4 without meta-analysis) for a total of 28,205 participants with dementia/MCI were included. No observational study on physical activity/exercise in MCI for preventing dementia was included. In SRs with MAs, physical activity/exercise was effective in improving global cognition in Alzheimer's disease and in all types of dementia (very low/low certainty of evidence). Moreover, physical activity/ exercise significantly improved global cognition, attention, executive function, and memory in MCI, with a certainty of evidence varying from low to moderate. Finally, physical activity/exercise improved non-cognitive outcomes in people with dementia including falls and neuropsychiatric symptoms. SRs, without meta-analysis, corroborated these results. 
Conclusions: Supported by very low to moderate certainty of evidence, physical activity/exercise has a positive effect on several cognitive and non-cognitive outcomes in people with dementia and MCI, but RCTs, with low risk of bias/confounding, are still needed to confirm these findings.

\section{Abstract \# 139 \\ Associations between neurogeriatric disorders in Moscow nonagenarians}

Ruslan Isaev ${ }^{1}$, Elen Mkhitaryan ${ }^{1}$, Natalya Vorobyeva ${ }^{1}$, Irina Strazhesko $^{1}$, Olga Tkacheva ${ }^{1}$, Nikolay Yakhno ${ }^{2}$

${ }^{1}$ Pirogov Russian National Research Medical University, Russian Clinical and Research Center of Gerontology, ${ }^{2}$ Federal State Autonomous Educational Institution of Higher Education I.M. Sechenov First Moscow State Medical University of the Ministry of Health of the Russian Federation (Sechenov University), Research department of neurology of Biomedicine Technopark

Introduction: Neurogeriatric disorders are a common problem in the elderly but associations between them has been poorly studied in nonagenarians. The purpose of this study was to estimate the associations between dementia, depression, and insomnia in patients aged $\geq 90$ years.

Methods: One hundred and eighty-eight outpatients (23\% male) aged 90-101 (mean $93 \pm 2$ ) years were included in the study. We evaluated cognitive function using the Mini-Mental State Examination (MMSE) and the Frontal Assessment Battery (FAB), mood-the Geriatric Depression Scale (GDS-15), and insomnia-the Insomnia Severity Index (ISI).

Results: The prevalence of dementia (MMSE $\leq 23$ ) was $46,8 \%$, insomnia (ISI $\geq 8$ ) $-54,3 \%$, and depression (GDS-15 $\geq 5$ ) $-69,7 \%$. Median of MMSE was 24 (IQR 20-27). Patients with dementia (n = 88 ) had the higher frequency of depression $(79,5 \%$ vs $61 \% ; p=0,006)$ and frontal lobe dysfunction $(\mathrm{FAB} \leq 15)(97,7 \%$ vs $83 \% ; \mathrm{p}=0,001)$ than those without $(n=100)$. MMSE score was correlated with FAB $(r$ $=0,552 ; \mathrm{p}<0,001)$ and GDS-15 $(\mathrm{r}=-0,299 ; \mathrm{p}<0,001)$ score. Logistic regression showed that dementia was associated with female sex (OR 2,16; 95\% CI 1,06-4,42; $\mathrm{p}=0,035)$, frontal lobe dysfunction (OR 8,81; 95\% CI 1,97-39,31; $\mathrm{p}=0,004)$, and depression (OR 2,49; $95 \%$ CI $1,29-4,79 ; p=0,006)$. Insomnia and low educational level did not associate with dementia, but high educational level had a protective effect (OR 0,48; 95\% CI 0,26-0,89; $\mathrm{p}=0,019)$.

Conclusion: In Moscow nonagenarians, the dementia prevalence was $46,8 \%$. Dementia was associated with female sex, frontal lobe dysfunction, and depression.

\section{Abstract \# 140}

Timed up-and-go dual-task test: age- and gender-specific reference values and test-retest reliability in healthy controls

Hanna Bozkurt Åhman ${ }^{1}$, Caroline Lundberg ${ }^{2}$, Ylva Cedervall ${ }^{1}$, Maja Blomberg $^{3}$, Agnes Widenfalk ${ }^{3}$, Vilmantas Giedraitis ${ }^{1}$, Erik Rosendahl ${ }^{4}$, Lars Berglund ${ }^{1}$, Anna Cristina Åberg ${ }^{5}$

${ }^{1}$ Department of Public Health and Caring Sciences, Geriatrics, Uppsala University, Sweden, ${ }^{2}$ Department of Nursing, Åland University of Applied Sciences, Finland, ${ }^{3}$ Department of Statistics, Uppsala University, Sweden, ${ }^{4}$ Department of Community Medicine and Rehabilitation, Physiotherapy, Umeå University, Sweden, ${ }^{5}$ Department of Public Health and Caring Sciences, Geriatrics, Uppsala University, Sweden and School of Education, Health and Social Studies, Dalarna University, Sweden
Introduction: We study dual-task test performance with Timed Upand-Go (TUG) [1] combined with naming animals (TUG dual-task, TUGdt) [2-4], where number of animals/10s can discriminate between patients with dementia, mild cognitive impairment, subjective cognitive impairment, and healthy controls [4]. For TUGdt, reference values and reliability estimates have not previously been presented.

Aim: To present reference values for TUGdt and to establish testretest reliability in healthy controls. Methods Study population: 166 healthy controls (age 50-91 years) for reference values and 43 (age $50-91$ years) for reliability.

Test procedures: One movement sequence starting from sitting position, standing up and walking three meters, turning, walking back and sitting down, combined with the simultaneous task of naming animals. For reliability, tests were repeated in $10 \pm 3$ days.

Statistical analyses: Reference values were calculated with quantile regression [5] in age and gender groups. Intra-class correlation coefficients (ICC) were used for reliability analyses.

Results: For men in age groups 50-59, 60-69, 70-79, and 80- years, reference values (2.5th percentile) were 5.2, 4.0, 2.8 and 1.8, and for women $5.4,4.7,3.9$ and 3.3 animals/10s, respectively. The test-retest reliability was fair-good for number of animals named $(\mathrm{ICC}=0.57$ ), fair-good for number of animals/10s (ICC $=0.58$ ), excellent for TUGdt time (ICC $=0.86$ ), and poor for TUGdt cost (defined as the relative time difference between TUG and TUGdt) $(\mathrm{ICC}=0.34)$.

Key conclusions: Reference values allow for comparisons between patients' TUGdt performances with age- and gender-specific values from cognitively unimpaired individuals. The TUGdt parameters' reliability vary, which should be considered when using the test.

References: 1 . Podsiadlo D, Richardson S: The timed "Up \& Go": a test of basic functional mobility for fraielderly persons. J Am Geriatr Soc 1991, 39(2):142-148. 2. Cedervall Y, Stenberg AM, Åhman HB, Giedraitis V, Tinmark F, Berglund B, Halvorsen K, Rosendah R, Åberg AC: Timed Up-and-Go Dual-Task Testing in the Assessment of Cognitive Function: A mixed methods observational study for development of the UDDGait Protocol. J Environ Res Public Health 2020, 17(5). 3. Åhman HB, Giedraitis V, Cedervall Y, Lennhed B, Berglund L, McKee K, Kilander L, Rosendahl E, Ingelsson M, Aberg AC: Dual-Task Performance and Neurodegeneration: Correlations Between Timed Up-and-Go Dual-Task Test Outcomes and Alzheimer's Disease Cerebrospinal Fluid Biomarkers. J Alzheimers Dis 2019. 4. Åhman HB, Cedervall Y, Kilander L, Giedraitis V, Berglund L, McKee K, Rosendahl K, Ingelsson M, Åberg AC: Dual-task tests discriminate between dementia, mild cognitive impairment, subjective cognitive impairment, and healthy controls - a cross-sectional cohort. 5. Yu, K., Lu, Z., and Stander, J. (2003). "Quantile Regression: Application and Current Research Areas.” Journal of the Royal Statistical Society, Series D 52:331-350.

\section{Abstract \# 141}

Dual-task tests improve capacity of models for predicting dementia incidence: a prospective cohort study

Hanna Bozkurt Åhman ${ }^{1}$, Ylva Cedervall ${ }^{1}$, Lars Berglund ${ }^{1}$, Lena Kilander ${ }^{1}$, Vilmantas Giedraitis ${ }^{1}$, Kevin $\mathrm{McKee}^{2}$, Martin Ingelsson ${ }^{1}$, Erik Rosendahl $^{3}$, Anna Cristina Åberg ${ }^{4}$

${ }^{1}$ Department of Public Health and Caring Sciences, Geriatrics, Uppsala University, Sweden, ${ }^{2}$ School of Education, Health and Social Studies, Dalarna University, Sweden, ${ }^{3}$ Department of Community Medicine and Rehabilitation, Physiotherapy, Umeå University, Sweden, ${ }^{4}$ Department of Public Health and Caring Sciences, Geriatrics, Uppsala University, Sweden and School of Education, Health and Social Studies, Dalarna University, Sweden 
Introduction: Identifying dementia disorders at early stages is essential for initiating interventions. At such stages, patients may present with either subjective cognitive impairment (SCI) or mild cognitive impairment (MCI).

Methods: Participants: In total 172 patients (age range 39-91 years, 78 women) with SCI or MCI were included, of whom 51 developed dementia within 2.5 years.

Data collection: A dual-task test involving Timed Up-and-Go [1] simultaneous with naming animals (TUGdt) [2] was performed at baseline. Time score, number of animals named, and number of animals/10s $[3,4]$ were calculated. Data on demographic characteristics and results of the cognitive tests Mini-Mental State Examination (MMSE) and Clock Drawing were collected. Diagnoses were identified after 2.5 years.

Statistical analyses: Logistic regression was used to predict dementia incidence. C-statistics were calculated for predictive capacity. Analyses were stratified by age.ResultsThe TUGdt variable "animals/10s" showed the highest association with dementia incidence in the total sample (OR per 1 std decrease 4.06, 95\% CI 2.28-7.23, $\mathrm{p}<0.001$ ). Among younger patients (under median age, 72 years), the corresponding OR was 19.4, 95\% CI 3.53-106.17, p $<0.001$, and "animals/10 s" added predictive capacity to a model with age, gender, educational level, MMSE, and Clock Drawing test, with an increase of c-statistics from 0.88 to 0.94 . In the total sample, the increase in c-statistics was negligible (0.85 to 0.86$)$.Key conclusionsAmong younger patients with SCI or MCI, "animals/10s" adds value to demographic characteristics and standard cognitive tests regarding predicting dementia disorders. Hence, TUGdt may become a clinically useful screening tool. References: 1 . Podsiadlo D, Richardson S: The timed "Up \& Go": a test of basic functional mobility for frail elderly persons. Journal of the American Geriatrics Society 1991, 39(2):142-148. 2. Cedervall Y, Stenberg AM, Ahman HB, Giedraitis V, Tinmark F, Berglund L, Halvorsen K, Ingelsson M, Rosendahl E, Aberg AC: Timed Up-and-Go Dual-Task Testing in the Assessment of Cognitive Function: A Mixed Methods Observational Study for Development of the UDDGait Protocol. Int J Environ Res Public Health 2020, 17(5). 3. Ahman HB, Giedraitis V, Cedervall Y, Lennhed B, Berglund L, McKee K, Kilander L, Rosendahl E, Ingelsson M, Aberg AC: Dual-Task Performance and Neurodegeneration: Correlations Between Timed Up-and-Go Dual-Task Test Outcomes and Alzheimer's Disease Cerebrospinal Fluid Biomarkers. Journal of Alzheimer's disease 2019, 71:75-83. 4. Åhman HB, Cedervall Y, Kilander K, Giedraitis V, Berglund L, McKee K, Rosendahl E, Ingelsson M, Åberg AC: Dual-task tests discriminate between dementia, mild cognitive impairment, subjective cognitive impairment, and healthy controls - a cross-sectional cohort study. Under review 2020

\section{Abstract \# 142}

The development of a post diagnostic support service for people with dementia and initial evaluation and audit findings

Claire Rooney ${ }^{1}$, Dr. Roisin Purcell ${ }^{1}$

${ }^{1}$ Our Lady's Hospice \& Care Services

Introduction: Cognitive Stimulation Therapy (CST) has been shown to improve memory and quality of life for people with dementia. NICE guidance on assessment, management and support for people living with dementia and their carers (2018) recommend that group CST is offered to people living with mild to moderate dementia. Despite this, there is limited availability of CST in Ireland. The aim of the out-patient CST service was to provide post diagnostic support to people with dementia and offer support to their families/carers.
Methods: In 2019, a post diagnostic support service was established at Our Lady's Hospice and Care Services, where the Occupational Therapy Department delivered CST adapted from the "Making a Difference" evidence-based group programme. Twenty patients took part in a 10-week programme that was facilitated three times over the year. The programme was facilitated once a week over an 8-week period with a pre and post session (week 1 and week 10 respectively). Outcome measures were used pre and post CST. This abstract reports on MMSE scores and qualitative feedback completed by the participant and carer separately.

Results: Audit findings indicate that mean cognitive function measured higher at the end of the CST programme for participants. Results from qualitative feedback indicate that the service was positively received and there were trends towards improved mood and communication and interaction skills. This finding reflects both participant and carer feedback.

Key conclusions: Clinical audit of the service suggests compliance with international standards set out by NICE and the Memory Services National Accreditation Programme.

\section{Abstract \# 143}

THE effect of acetylcholinesterase inhibitors and cognitive training on individuals with mild dementia

Maria Devita ${ }^{1}$, Fabio Masina ${ }^{2}$, Daniela Mapelli ${ }^{1}$, Pasquale Anselmi ${ }^{3}$, Giuseppe Sergi ${ }^{4}$, Alessandra Coin ${ }^{4}$

${ }^{1}$ Department of General Psychology, University of Padua, Italy, ${ }^{2}$ IRCCS San Camillo Hospital, Venice, Italy, ${ }^{3}$ Department of Philosophy, Sociology, Education and Applied Psychology FISSPA, University of Padua, Italy, ${ }^{4}$ Geriatric Division, Department of Medicine - DIMED, University of Padua, Italy.

Introduction: Acetylcholinesterase inhibitors (AChEI) and Cognitive Training (CT) are the gold-standard pharmacological and non-pharmacological treatments for mild to moderate dementia [1]. The present study aims to investigate the effect of each treatment, alone or combined, on neuropsychological profile of patients with mild dementia.

Methods: Forty participants, enrolled on clinical (geriatric assessment) and morphological (CT/RMN) assessments, were assigned to three groups receiving, respectively, only the pharmacological treatment (AChEI, $n=14)$, both the treatments (AChEI + CT, $n=15)$, or only the cognitive training $(\mathrm{CT}, \mathrm{n}=11)$. Cognitive profile was evaluated, at the baseline and after three months, by the MMSE [3], and an extended neuropsychological battery of tests, the Brief Neuropsychological Examination-2 [4]. To evidence differences among the treatments, in terms of changes in the patients' neuropsychological profile, linear mixed models were conducted.

Results: A general improvement at the MMSE emerged only in patients which received $\mathrm{AChEI}+\mathrm{CT}$, and $\mathrm{CT}$, compared to the group that received only AChEI. Interestingly, after the intervention, individuals receiving the $\mathrm{CT}$ showed a significant improvement in immediate memory recall with respect to the AChEI group. In addition, only the group receiving both the treatments (AChEI + CT) showed an improvement in delayed recall after the intervention, compared to the other groups.

Key conclusions: The combination of AChEI and CT seems to bring the greatest benefit for persons with mild dementia. More interestingly, CT is more effective than AChEI alone even in improving memory, which classically is considered as the "lost" cognitive domain.

References: [1] Calabria, M., Geroldi, C., Lussignoli, G., Sabbatini, F., \& Zanetti, O. (2009). Efficacy of acetyl-cholinesterase-inhibitor (ACHEI) treatment in Alzheimer's disease: A 21-month follow-up 
"real world" study. Archives of gerontology and geriatrics, 49(1), e6e11. [2] Dementia, N. I. C. E. (2018). disability and frailty in later life: mid-life approaches to delay or prevent onset. Guidance and guidelines. [3] Folstein, M. F., Folstein, S. E., \& McHugh, P. R. (1975). "Mini-mental state": a practical method for grading the cognitive state of patients for the clinician. Journal of psychiatric research, 12(3), 189-198. [4] Mondini, S., Mapelli, D., Vestri, A., Arcara, G., \& Bisiacchi, P. S. (2011). L'Esame Neuropsicologico Breve-2 (Brief Neuropsychological Examination-2). Milano, Italy: Raffaello Cortina Editore.

\section{Abstract \# 144 \\ The role of age in the NIA-AA ATN scheme in patients of a German university memory clinic \\ Blasius Carolin ${ }^{1}$, Becker Ingrid ${ }^{2}$, Pickert Lena ${ }^{1}$, Maier Franziska ${ }^{3}$, Meiberth Dix ${ }^{4}$, Tscheuschler Maike ${ }^{3}$, Schild Ann-Katrin ${ }^{3}$, Rostamzadeh $\mathrm{Ayda}^{3}$, Sannemann Lena ${ }^{3}$, Müller Theresa ${ }^{3}$, Onur Özgür $^{5}$, Jessen Frank ${ }^{4}$, Polidori Maria Cristina ${ }^{6}$}

${ }^{1}$ Ageing Clinical research, Dpt. II of Internal Medicine and Center of Molecular Medicine, Medical Faculty, University of Cologne, Cologne, Germany, ${ }^{2}$ Department of Medical Statistics, Medical Faculty, University of Cologne, Cologne, Germany, ${ }^{3}$ Department of Psychiatry, Medical Faculty, University of Cologne, Cologne, Germany, ${ }^{4}$ Department of Psychiatry, Medical Faculty, University of Cologne, Cologne, Germany; German Center for Neurodegenerative Diseases (DZNE), Cologne, Germany, ${ }^{5}$ Department of Neurology,

Medical Faculty, University of Cologne, Cologne, Germany, ${ }^{6}$ Ageing Clinical research, Dpt. II of Internal Medicine and Center of Molecular Medicine, Medical Faculty, University of Cologne, Cologne, Germany; Cologne Excellence Cluster on cellular stress respondes in Aging-associated Diseases

The aging society with age-related pathologies, in particular dementia, challenges the traditional healthcare paradigms. Both aging and age-related cognitive decline are heterogeneous, multifactorial processes with a high demand for studies aimed at better characterizing the interface between dementia and Alzheimer's disease (AD). In this study, the ATN classification system was applied to the records of 223 patients with a diagnosis of mild cognitive impairment (MCI) and dementia admitted to the Memory Clinic of the University Hospital of Cologne. Patients were divided into two age groups: $<70$ years $(\mathrm{n}=$ $98,44 \%)$ and $\geq 70$ years $(n=125,56 \%)$. In the 223 participants with full data sets including cerebrospinal fluid, neuropsychological testing, diagnosis and age, $\mathrm{A}+\mathrm{T}+\mathrm{N}+$ was the most common group (52\%). Significant differences were found between the two age groups in AD continuum groups (ie. all groups with pathological amyloid) and nonAD continuum groups $(\mathrm{p}=0.018)$, whereby the older group belongs to $\mathrm{AD}$ groups to a larger extent compared with the younger one. A relationship between amyloid pathology and cognition (Mini-Mental State Examination) was found $(p=0.003)$, but only the younger participants showed significant differences of cognition in amyloid stages $(p<0.001)$ and not the older ones $(p=0.639)$. The latter observation is in agreement with recent studies from other groups. Our results show that the overlap between aging and dementia needs to be considered in every interpretation of diagnostics. Age-related cut-off values might be a better fit. Aging and pathologies other than amyloid may play a substantial role in cognitive decline of older patients. Diagnostic examinations of oldest-old patients might have to be considered more comprehensively than those of young-old persons.

\section{Abstract \# 145}

\section{Dementia in home geriatric care program}

Rajkumar Chinnadurai ${ }^{1}$, Francisco Javier Balea Fernandez ${ }^{1}$, Cristina Cazorla Guillen ${ }^{2}$, Antonia Jesús Solano Benítez ${ }^{1}$

${ }^{1}$ Hopital Insular de Lanzarote, ${ }^{2}$ Nutritionist in Centro Hebe

Introduction: Life expectancy in Spain is the highest in the European Union, standing at 83.4 years (European average 80.9 years). Data published by the WHO shows that there are 50 million people worldwide with dementia. Alzheimer's Type Dementia (ATD) is the most common form common dementia, representing 60-70\% of all dementias. Interventions with caregivers and training in symptom management, both cognitive and behavioral, have shown more efficient than pharmacological treatment. Families assume $85 \%$ of the cost of their care.

Objective: To analyze the prevalence of dementia and the profile of caregivers in the Home Geriatric Care Program (AGD) of the Insular Hospital of Lanzarote.

Method: Descriptive cross-sectional observational study. Patients treated at AGD in 2018.

Results: The sample consists of 214 patients, with a mean age of 84.73 years ( $\mathrm{Sx}$ 7.37). Regarding sex, the sample corresponds to $73.4 \%$ (157) women and 26.6\% (57) men. Barthel index score was 41.5 (Sx 30.12), GDS of 3.58 (Sx 1.85) and a Frailty index of 0.47 (Sx 0,12). Polypharmacy ( $>5$ drugs) was $75.7 \%$. The reason for consultation was mainly due to immobility $(32.7 \%)$ and conduct disorder (26.2\%). The caregiver profile was the couple (10.3\%); siblings $(47.6 \%)$, paid caregiver $(35.5 \%) .90 .2 \%$ of sample were living with their family.

Conclusions: We are visiting people with advanced age and high level of dependence. Families assume most of the cost of care. The main reasons for home care are dementia, immobility and conduct disorders. The profile of the caregiver are family members.

\section{Abstract \# 146 \\ Audiological evaluation as a component of a memory clinic assessment: the patient experience}

Anna McDonough ${ }^{1}$, Joshi Dookhy ${ }^{1}$, Cathy McHale ${ }^{1}$, Jennifer Sharkey ${ }^{1}$, Siobhan Fox ${ }^{2}$, Sean Kennelly ${ }^{1}$

${ }^{1}$ Tallaght University Hospital, ${ }^{2}$ University College Cork

Introduction: With the evolving knowledge on hearing as a mid-life risk factor for dementia, identification of patients who are at risk becomes increasingly important. Our memory clinic is based in a tertiary referral centre and refers patients with mild cognitive impairment (MCI) for audiological evaluation based on cognitive diagnosis, rather than self-reported hearing deficits. This study sought to examine the patient experience and understanding of this process. Methods: All patients with MCI who were referred for audiology review were contacted. A patient survey was delivered over the phone. Outpatient letters and the memory clinic database were reviewed.

Results: 30 patients were contacted, 9 (30\%) could not recall their audiology assessment. 20 patients were surveyed. $8(40 \%)$ had selfreported hearing loss. $9(45 \%)$ had mild-moderate hearing loss and 8 $(40 \%)$ had moderate-severe. 6 patients $(30 \%)$ could recall having the rationale behind having a hearing test as part of their memory assessment explained to them. The majority (75\%) felt a hearing test was an important part of their memory assessment, but just 7 patients $(35 \%)$ identified a link between hearing loss and memory problems. All patients who provided feedback on the service itself made positive 
comments, although $20 \%$ felt they did not get adequate information about the results.

Conclusions: Patients are satisfied with incorporating audiological evaluation into a memory clinic assessment and a significant amount of hearing impairment can be detected. We need to improve communication around the relevance of this, as well as the results, to improve compliance with hearing aid recommendations and optimise a holistic management plan for their cognitive disorder.

\section{Abstract \# 147 \\ A comparison of electronic and paper versions of the Chinese version of the quick mild cognitive impairment screen}

Yangfan $\mathrm{Xu}^{1}$, Xiaocong $\mathrm{Mo}^{1}$, Suimin $\mathrm{Guo}^{2}$, Dan Zhao ${ }^{3}$, Lan Zhou ${ }^{4}$, D. William Molloy ${ }^{5}$

${ }^{1}$ The Sixth Affiliated Hospital, Sun Yat-Sen University, Guangzhou, China, ${ }^{2}$ Sports rehabilitation, Guangzhou Sport University, Guangzhou, China, ${ }^{3}$ Puyang Traditional Chinese Medicine Hospital, Puyang, China, ${ }^{4}$ Rehabilitation College, Gannan Medical College, Ganzhou, China, ${ }^{5}$ Centre for Gerontology and Rehabilitation, University College Cork, St Finbarr's Hospital, Cork, Ireland

Introduction: The Quick Mild Cognitive Impairment (Qmci) screen is a short cognitive screening instrument designed to separate normal cognition from mild cognitive impairment and early dementia. An electronic version of the Qmci screen is now available online, though it is not known how it compares to the traditional paper version. We compared the Chinese versions of the paper and electronic Qmci (pQmci-CN and eQmci-CN, respectively).

Methods: We performed an observational study using a repeated measures design. A convenience sample of 48 community-dwellers aged $>=18$ years were randomly divided into two groups and completed the eQmci-CN and pQmci-CN in random order allowing $48 \mathrm{~h}$ of 'washout time' between versions. A survey of user experience and preferences was conducted. Total and sub-scale scores and administration times were recorded.

Results: A strong statistically significant correlation $(\mathrm{r}=0.77)$ was found between the eQmci-CN and pQmci-CN. Examining scores according to which modality was administered showed no statistically significant difference in total scores $(\mathrm{p}>0.05)$; however, there was a statistically significant difference for the verbal fluency subtest $(\mathrm{p}<$ 0.05). For this subscale, participants scored lower on the eQmci-CN than pQmci-CN. There was no statistically significant difference in the self-reported comfort level or willingness to use either modality again in the future $(\mathrm{p}>0.05)$.

Key conclusions: The eQmci-CN showed strong correlation with the $\mathrm{pQmci-CN}$ and received positive feedback from users. This suggests that it is an acceptable alternative to pencil-and-paper testing, although larger studies in different healthcare settings and other languages are now required to confirm these findings.

\section{Abstract \# 148 \\ Influence of a 12-month structured exercise program on the micronutrient-cognitive fitness-physical fitness association profiles in mild cognitive impairment}

Weigert Hannah ${ }^{1}$, Stuckenschneider Tim ${ }^{2}$, Rossi Andrea ${ }^{3}$, Pickert Lena $^{1}$, Meyer Anna Maria ${ }^{1}$, Nelles Gereon ${ }^{4}$, Schulz Ralf-Joachim ${ }^{5}$, Stahl Wilhelm ${ }^{6}$, Schneider Stefan ${ }^{2}$, Polidori Maria Cristina ${ }^{7}$

${ }^{1}$ Ageing Clinical Research, Department II of Internal Medicine and Center for Molecular Medicine Cologne, University of Cologne, Faculty of Medicine and University Hospital Cologne, Cologne,
Germany; ${ }^{2}$ Institute of Movement and Neurosciences, German Sport University, Cologne, Germany; VasoActive Research Group, School of Health and Sport Sciences, University of the Sunshine Coast, QLD, Australia;, ${ }^{3}$ Department of Emergency and Urgency Hospital Center, Bolzano, Italy;, ${ }^{4}$ Outpatient Clinic NeuroMedCampus Hohenlind, Cologne, Germany;, ${ }^{5}$ Department of Geriatrics, St. Marienhospital, Cologne, Germany;, ${ }^{6}$ Institute of Biochemistry and Molecular Biology I, Heinrich-Heine University Düsseldorf, Germany; ${ }^{7}$ Ageing Clinical Research, Department II of Internal Medicine and Center for Molecular Medicine Cologne, University of Cologne, Faculty of Medicine and University Hospital Cologne, Cologne, Germany; Cologne Excellence Cluster on Cellular Stress- Responses in AgingAssociated Diseases (CECAD), University of Cologne, Faculty of Medicine and University Hospital Cologne, Cologne, Germany.

Background and aim: Preventive lifestyle strategies have been proven useful against age-related cognitive decline, but there is still a lack of evidence on the reciprocal relationships between nutrition biomarkers and measures of both cognitive and physical performance. To fill this gap of knowledge, the relationship of plasma levels of the robust nutrition- and antioxidant defense-related biomarkers carotenoids and tocopherols with both indicators of cognitive and physical performance-within the established field of nutritional cognitive neuroscience-was investigated in a group of persons with mild cognitive impairment (MCI) participating in a structured exercise program at the German Sport University in Cologne, Germany. Participants and methods: At baseline and after one year of either aerobic (AE) or stretching and toning (S\&T) exercise, 73 participants (36 F, $37 \mathrm{M}, 72.3 \pm 5.6$ years) underwent cognitive and motoric performance tests as well as blood withdrawal. Carotenoids including lutein, zeaxanthin, $\beta$-cryptoxanthin, lycopene, and $\alpha$ - and $\beta$-carotene were analysed by HPLC with UV/vis detection at $450 \mathrm{~nm}$. A second UV/vis detector was connected in series and set at 325 and $292 \mathrm{~nm}$ for quantitation of retinol (vitamin A), and $\alpha$ - and $\gamma$-tocopherol (vitamin E), respectively.

Results: With respect to the associations found at baseline and published in Gerger et al., Nutrients 2019, when specifically looking at the physical performance outcome measures significant correlations at FU were found, independently of fruit and vegetable intake, between plasma levels of lutein and number of steps per day (S\&T group) $(\mathrm{p}<0.05)$, lutein and chair-rising test $(\mathrm{S} \& \mathrm{~T}$ group) $(\mathrm{p}=$ $0.002)$, zeaxanthin and chair-rising test (S\&T group) $(\mathrm{p}=0.003), \beta-$ cryptoxanthin and Timed Up\&Go test $(\mathrm{AE})(\mathrm{p}<0.02), \beta$-carotene and chair-rising test (S\&T group) $(\mathrm{p}=0.001), \alpha$-tocopherol and number of steps per day (S\&T group) $(\mathrm{p}<0.05)$. Correlation analyses with cognitive measures are ongoing.

Discussion: In light of the increasing attention towards the nutritional cognitive neuroscience of carotenoids, physical fitness measures might be further implemented in future studies investigating the effects of lifestyle interventions against cognitive and physical impairment.

\section{Abstract \# 149}

\section{Long distance cognitive rehabilitation during COVID19} pandemic: a study report

Andreea G Marin ${ }^{1}$, Ileana Ciobanu ${ }^{1}$, Mihai-Viorel Zamfir ${ }^{2}$, Mihaela Zamfir (Grigorescu) ${ }^{3}$, Mihai Berteanu ${ }^{4}$

${ }^{1}$ Department of Neuro-Rehabilitation, „Elias” University Emergency Hospital, Bucharest, Romania, ${ }^{2}$ Physiology II-Neurosciences Division, Faculty of Medicine, „Carol Davila” University of Medicine and Pharmacy, Bucharest, Romania, ${ }^{3}$ Department „Synthesis of Architectural Design”, Faculty of Architecture, „Ion Mincu" University of Architecture and Urbanism, Bucharest, 
Romania, " Carol Davila" University of Medicine and Pharmacy, Bucharest, Romania

Introduction: Due to limited access at healthcare services for elderly patients during COVID19 pandemic, significant disruptions occurred regarding long term therapeutic interventions. Considering the absolutely necessary social distancing measures, reorganizing and finding the proper working techniques, equipment and materials to be applied in cognitive rehabilitation programs represent a challenge for the rehabilitation clinician.

Methods: We present a study report of a 78 years old male with minor cognitive deficit and minor aphasia due to stroke (Nov.2019). In order to evaluate the efficiency of remote approach, we proceeded to following the therapeutic protocol that involves evaluation, initiation of therapy and post-intervention evaluation. We applied MMSE for cognitive screening and Speech Therapy Evaluation Sheet to assess the language indices.

Results: We identified communication platforms able to ensure the continuity of therapeutic process and we got access to the digital instruments compatible with the remote mode. We used the Wordwall software to create our own interactive resources, using custom templates, accessible on any web device. We also used cognitive training computerized applications. The intervention was performed for 8 weeks, 2 times/week, 60 minutes/session. We noticed increase in cognitive performance indices and improvement of language indices. The patient described good adherence to the sessions.

Conclusions: The use of communication platforms and of digital tools to provide optimal cognitive training in remote mode has an essential role in situations requiring social distancing. This type of approach is a valuable alternative solution in COVID19 pandemic to ensure patient safety and also the quality health care.

\section{Abstract \# 150 \\ Evaluation of quality of life in patients with major neurocognitive disorder \\ Nuria Cristina Herrera Fernández ${ }^{1}$, Rolf Christian Sander ${ }^{1}$, Carmen Dolores Hernandez Hernandez ${ }^{1}$, Patricia Mateo Martín ${ }^{1}$, Ortzi Barrasa Bermejo $^{1}$, Francisco Javier Balea Fernández ${ }^{1}$ \\ ${ }^{1}$ Hospital Insular de Lanzarote}

Introduction: Dementia is a syndrome in which there is an increase in dependency and a decrease in quality and life expectancy. Quality of life is a multidimensional concept whose evaluation is complex, but very useful in the therapeutic approach. The objective was to assess the quality of life in institutionalized elderly diagnosed with dementia.

Material and methods: Descriptive cross-sectional observational study carried out on institutionalized patients in Lanzarote (Spain) during February 2020. The variables recorded were: sociodemographic (age and sex), baseline situation (Barthel index -IB-, global deterioration scale-functional assessment staging -GDS-FAST- and social resources scale -OARS-) and quality of life determined by the specific QUALID scale (Quality of life in late-stage dementia), whose score ranges from 11-55 points (inversely proportional results). The statistical analysis was carried out in the statistical program SPSSV26.

Results: 35 patients were included, with a mean age of $83.91 \pm 7.66$ years. In relation to the comprehensive geriatric assessment: $97.14 \%$ had severe functional dependence (mean IB $12.71 \pm 15.69$ ), the mean GDS-FAST was $6.00 \pm 1.08$ and the mean OARS was $2.51 \pm 0.74$. The mean score obtained in the scale was $26.09 \pm 11.14$. If we analyze the items separately, smiling and physical contact obtain the highest scores $(3.03 \pm 1.72$ and $2.97 \pm 1.10$, respectively). In contrast, crying and irritability obtained the lowest scores $(1.31 \pm$ 0.76 and $1.91 \pm 1.44$, respectively).

Key conclusions: The determination of quality of life could be useful to decide the therapeutic attitude in a patient diagnosed with dementia.

\section{Abstract \# 151}

\section{Neurogeriatrics: an emerging task}

Polidori Maria Cristina ${ }^{1}$, Prell Tino ${ }^{2}$, Klucken Jochen ${ }^{3}$, Jacobs Andreas ${ }^{4}$, Dodel Richard ${ }^{5}$, Warnecke Tobias ${ }^{6}$, Bartsch Thorsten ${ }^{7}$, von Arnim Christine ${ }^{8}$, Jahn Klaus ${ }^{9}$, Maetzler Walter ${ }^{7}$

${ }^{1}$ Ageing Clinical Research, Department II of Internal Medicine and Center for Molecular Medicine Cologne, University of Cologne, Faculty of Medicine and University Hospital Cologne, Cologne, Germany; Cologne Excellence Cluster on Cellular Stress- Responses in Aging- Associated Diseases (CECAD), University of Cologne, Faculty of Medicine and University Hospital Cologne, Cologne, Germany, ${ }^{2}$ Department for Neurology, Jena University Hospital, Jena, Germany, ${ }^{3}$ Department of Molecular Neurology, University Hospital Erlangen, Friedrich-Alexander University (FAU) Erlangen-Nürnberg, Germany; Research Group Digital Health Pathways, Fraunhofer IIS, Erlangen, Germany, ${ }^{4}$ Klinik für Geriatrie mit Neurologie, Johanniter Krankenhaus, Bonn und European Institute for Molecular Imaging (EIMI), Westfälische Wilhelms Universität (WWU) Münster, Germany;, ${ }^{5}$ Chair of Geriatrics, University Hospital Essen and Geriatriezentrum Haus Berge, Contilia Group, Germany, ${ }^{6}$ Department of Neurology, University of Münster, Germany, ${ }^{7}$ Department of Neurology, University Hospital Schleswig-Holstein Campus Kiel, Kiel University, Germany, ${ }^{8}$ Department of Geriatrics, University of Göttingen, Germany, ${ }^{9}$ Schoen Klinik Bad Aibling, Germany; German Center for Vertigo and Balance Disorders (DSGZ), LudwigMaximilians-University of Munich, Germany

Background: Neurologic symptoms, cognitive impairment, motor dysfunction, disorders of mood and behaviour, chronic pain occur frequently in older adults. These conditions not only have a strong negative impact on intrinsic capacity and mantainance of autonomy; they also increasingly coexist, with advancing age, with non-neurologic comorbidities and geriatric syndromes as well as their therapeutic management. In this complex figure, the ability to properly address presence, severity and treatment of an underlying or concomitant neurologic condition is essential to prevent disability and/or enhance recovery after an acute illness. If on one hand the Comprehensive Geriatric Assessment (CGA) is the tool of choice to uncover neurocognitive and neuropsychiatric deficits among others, there is a paucity of data on the method and degree of implementation of neurological know-how systematically used by or to be integrated in geriatric medicine to improve the management of neurogeriatric patients.

Methods: To fill this gap of knowledge, recently a Task Force for Neurogeriatrics was established in Germany. Physicians specialized both in neurology and geriatrics as well as geriatricians with expertise in cognitive impairment compose the group.

Results: The Task Force is currently (1) conducting a nation-wide survey to retrieve information on assessments, performance and functions tests conducted in geriatric units to examine neurologic status; (2) completing ethical issues clearance to prospectively collect data on assessments, performance and functions tests harmonized across German geriatric units to examine homogeneously neurologic status in the near future. Additionally, one of the main priorities of the Task Force is to foster neurogeriatric research and training concepts as well as to provide innovative care strategies for geriatric patients with leading neurological conditions and disabilities. 


\section{Abstract \# 152}

Assessment and prevention of venous thromboembolism in orthogeriatrics inpatients

Benjamin Blackburn ${ }^{1}$, James Ferryman ${ }^{1}$

${ }^{1}$ East and North Hertfordshire Trust

Introduction: Venous thromboembolism is estimated to cause between 24000 and 32000 deaths annually in hospital inpatients with a proportionally large incidence in the elderly population (approximately 8 per 1000 in the population over the age of 85 ). Appropriate use of thromboprophylaxis is shown to reduce the incidence of VTE and death from PE. The rationale for its use is both scientifically plausible and evidence based.

Methods: The adherence of VTE assessment and prescribing to Trust guidelines was reviewed over a 2 week period on Orthogeriatrics wards in all inpatients fulfilling the Trust requirements for formal VTE assessment. Data was initially gathered on 71 patients from $18 / 2 / 19$ to $2 / 3 / 19$. Following implementation of change data was gathered on 70 patients from $9 / 10 / 19$ to $24 / 10 / 19$. Primary outcomes were appropriateness of initial VTE assessment on admission and appropriateness of re-assessment within 24 hours of admission. Changes implemented were a ward round checklist and informational ward posters informing doctors of the indications for VTE assessment and re-assessment.

Results: Data gathered initially from $18 / 2 / 19$ to $2 / 3 / 19$ showed $40 / 71$ $(56 \%)$ of patients received appropriate initial VTE assessment on admission and 2/71 (3\%) were correctly re-assessed in 24 hours after admission. Data gathered after the interventions were implemented from $9 / 10 / 19$ to $24 / 10 / 19$ showed $41 / 70(58.5 \%)$ of patients received appropriate initial VTE assessment on admission and 22/70 (31.4\%) were correctly re-assessed within 24 hours of admission.

Conclusions: Implementation of ward round checklists and posters resulted in a tenfold increase in proportions of patients being appropriately re-assessed within $24 \mathrm{~h}$ of admission. This improvement following the provision of easily accessible information on the Trust guidelines may reflect a lack of awareness of this particular guideline amongst junior staff.

\section{Abstract \# 153 \\ Importance given by elderly people to the components of a Comprehensive Geriatric Assessment in terms of the influence on health: VALINTAN tool.}

Iñaki Martin Lesende ${ }^{1}$, Irati Merino Pardo ${ }^{2}$, Luis Ignacio Mendibil Crespo $^{3}$, Ainara Hernández del Blanco ${ }^{4}$, Amaia Berrizbeitia González ${ }^{5}$, Iker Llamosas Luengo ${ }^{5}$

${ }^{1}$ San Ignacio Primary Care Health Centre, Bilbao-Basurto Integrated Healthcare Organisation (IHO), Basque Health Service (Osakidetza), Spain, ${ }^{2}$ Basque Health Service (Osakidetza), Bizkaia. Spain, ${ }^{3}$ Bizkaia Unit for Multiprofessional Training in Family and Community Care, Spain, ${ }^{4}$ San Ignacio Primary Care Health Centre, Bilbao-Basurto IHO. Unit for Multiprofessional Training in Family and Community Care, Spain, ${ }^{5}$ Basque Health Service (Osakidetza). Bizkaia Unit for Multiprofessional Training in Family and Community Care, Spain

Introduction: It is important to consider involve patients in the health strategies/interventions that affect them.

Objective: Considering perceived importance in health of the components of a comprehensive geriatric assessment (CGA), by people aged $\geq 70$.

Methods: Cross-sectional study in 109 people in primary care; those with cognitive impairment, at the end of life, with sensory or mental/ psychiatric illness that made collaboration difficult, were
excluded.The main variables: 23 items of a standard CGA (physi$\mathrm{cal} /$ clinical, functional, mental, social/family areas). Others: age, sex, VIDA questionnaire of instrumental activities of daily living (iADL), medication, Charlson index.

Results: Median age 78 years (75P 84), 64.2\% (70) women, without statistically significant differences in age (women 78 with $75 \mathrm{P} 84$, men 77 and 84, p.0.79), nor in VIDA questionnaire (global median 37 points, IQR 35-38), nor in medicines number (global median 5, IQR $3-8)$. Four people $(3.7 \%)$ had alteration in VIDA questionnaire $(<32$ points) and 98 comorbidity absence considering Charlson index. Most valued aspects grouping scores $4-5$ on the Likert scale: taking drugs properly (102 people, $93.6 \%, 95 \%$ CI $87.3-96.8)$, mouth/teeth state (101, 92.7\%, 95\% CI 86.2-96.2), $\operatorname{mood}(100,91.7 \%, 95 \%$ CI 85.1-95.6) and capacity for ADL (100, 91.7\%, 95\% IC 85.1-95.6). Smoking and memory according to analysis using centralization statistics.

Conclusions: Aspects considered most influential in health: smoking, mouth/teeth state, proper taking of medication, ADL, memory; included in the CGA VALINTAN tool, except mouth/teeth state.Discrepancies with other scare similar studies, lead new ways to go deeper into the design of a CGA also focused on patient preferences.

\section{Abstract \# 154}

Comparison and concordance between CGA using the VALINTAN computer tool and that performed by geriatricians: pilot study.

Iñaki Martin Lesende ${ }^{1}$, Luis Ignacio Mendibil Crespo ${ }^{2}$, Iker Llamosas Luengo $^{3}$, Itziar Palazuelos Hernández ${ }^{4}$, Jon Saiz Baceta ${ }^{5}$, Nerea Aguirre Basaras ${ }^{6}$

${ }^{1}$ San Ignacio Primary Care Health Centre, Bilbao-Basurto Integrated Healthcare Organisation (IHO), Basque Health Service (Osakidetza), Spain, ${ }^{2}$ Bizkaia Unit for Multiprofessional Training in Family and Community Care, Spain, ${ }^{3}$ Gazteleku Primary Care Health Centre. Bilbao-Basurto IHO, Osakidetza, Spain, ${ }^{4}$ San Adrian Primary Care Health Centre. Bilbao-Basurto IHO, Osakidetza, Spain, ${ }^{5}$ Bombero Etxaniz Primary Care Health Centre. Bilbao-Basurto IHO, Osakidetza, Spain, ${ }^{6}$ San Ignacio Primary Care Health Centre, BilbaoBasurto IHO. Bizkaia Unit for Multiprofessional Training in Family and Community Care. Basque Health Service (Osakidetza), Spain

Funding: Funding from the Bilbao-Basurto IHO (OSIBB19/009), and from the Health Department of the Basque Country (file 2018111088) Introduction: VALINTAN www.valintan.com, is a computerized comprehensive geriatric assessment (CGA) tool, for primary care (PC) professionals; focused on predefined diagnoses and effective facilitated interventions, emphasizing functionality/frailty.Objective. To compare/analyze the concordance in diagnoses/interventions between the VALINTAN applied by PC physicians/nurses and a CGA by geriatricians, in 12 patients aged $\geq 70$.

Methods: Descriptive pilot study. Twelve patients with different degrees of functional impairment (Barthel index) were assessed through VALINTAN by 3 doctors/3 nurses in PC, and the day before/ after through a standard CGA by 3 geriatricians. Diagnoses/interventions, duration, and patient/PC professional satisfaction (survey) were collected.

Results: There were 109 DIAGNOSES (88 VALINTAN/86 CGA), $60.6 \%$ matching. Added in CGA: visual impairment, immobility, insomnia. More prevalent: comorbidity (12 VALINTAN, 12 CGA), polypharmacy $(12,10)$, inappropriate prescription $(9,9)$, fall risk $(9$, $6)$, affective alteration $(8,8)$. With none/1 case: smoking, malnutrition, caregiver overload. There were 87 INTERVENTIONS (82 VALINTAN / 72 CGA), 74.7 matching. Most frequents: pathologies 
management $(12,12)$, medication adequacy $(12,10)$, exercise $(12$, 8),VALINTAN DURATION median 30 min (IQR 25-35), CGA 57.7 (45-60), $p<0.001$.Every item (utility, duration, repeating) of patient SATISFACTION with median of 5 (maximum score) in both assessments. In PC professional satisfaction median 4 in overall, duration 4 , advantages 4 , recommendation 4.5 , content 4.5 , registry 5 . Conclusions: High diagnoses/interventions matching between both assessments, mainly in interventions that are finally the most decisive. Higher efficiency of VALINTAN in terms of time, and high degree of patient/professional satisfaction. Useful to setting changes in VALINTAN content.

\section{Abstract \# 155}

The effect of age on the development of cardiometabolic changes in patients with non-alcoholic fatty liver disease in combination with subclinical hypothyroidism

Olena Kolesnikova ${ }^{1}$, Anna Potapenko ${ }^{1}$, Kira Vovk ${ }^{2}$, Vilena Chupina ${ }^{3}$

${ }^{1}$ SI “ L.T. Malaya Therapy National Institute, NAMS of Ukraine,

${ }^{2}$ Kharkiv National University named after V.N. Karasin", ${ }^{3}$ Kharkiv National Medical University

The combination of non-alcoholic fatty liver disease (NAFLD) and subclinical hypothyroidism ( $\mathrm{SH})$ attracts attention due to general metabolic changes that can make an independent contribution to the formation of cardiovascular diseases (CVD) depending on age.The purpose of the study was evaluation of the cardiometabolic changes formation features in patients with NAFLD in combination with SH depending on age.

Materials and methods: We examined 74 patients with verified diagnosis of NAFLD and SH. Patients were divided into 2 groups (I-n $=31$, under 50 years old; II-n $=43$, over 50 years old).

Results: In patients of first group, a significant change in the main cardiometabolic indicators, indicators of endothelial dysfunction (ED) and systemic inflammation (SI) was already noted: moderate fasting hyperglycemia $(\mathrm{p}=0.010)$, increased HbA1c level $(\mathrm{p}<0.001)$, increased LDL cholesterol ( $p=0.030)$, HDL cholesterol $(p=0.038)$, gamma-glutamyl transpeptidase (GGTP) $(\mathrm{p}=0.016)$, circulating desquamated endothelial cells $-\mathrm{CDEC}(\mathrm{p}=0.006)$; vasculoendothelial growth factor-VEGF $(p=0.001)$, C-reactive protein-CRP $(p=$ $0.001)$; tumor necrotic factor-TNF- $\alpha(\mathrm{p}=0.001)$, which depended on the level of TSH. Moreover, in patients of second group, along with dyslipidemia and hyperinsulinemia $(\mathrm{p}<0.001)$, alkaline phosphatase ( $p=0.025)$ was increased. The indicators of ED and SI in II group depended on the thickness of the intima media complex.

Conclusion: In patients with NAFLD in combination with SH under the age of 50 years old, the early formation of cardiometabolic changes create the prerequisites for premature vascular aging.

\section{Abstract \# 156 \\ Design of a consensus-based geriatric assessment tailored for older patients approaching end stage kidney disease}

Carlijn Voorend ${ }^{1}$, Hanneke Joosten ${ }^{2}$, Noeleen C Berkhout-Byrne ${ }^{1}$, Adry Diepenbroek $^{3}$, Casper FM Franssen ${ }^{3}$, Willem Jan W Bos ${ }^{1}$, Simon P Mooijaart ${ }^{4}$, Marjolijn van Buren ${ }^{5}$

${ }^{1}$ Internal Medicine (Nephrology), Leiden University Medical Center, The Netherlands, ${ }^{2}$ Internal Medicine, Maastricht University Medical Centre, The Netherlands, ${ }^{3}$ Nephrology, University Medical Centre Groningen, The Netherlands, ${ }^{4}$ Gerontology and Geriatrics, Leiden University Medical Center, The Netherlands, ${ }^{5}$ Gerontology and Geriatrics, Leiden University Medical CenterGerontology and
Geriatrics, Leiden University Medical Center, Gerontology and Geriatrics, Leiden University Medical Center, The NetherlandsThe Netherlands, The Netherlands

Introduction: Routine geriatric evaluation in older patients approaching end stage kidney disease (ESKD), benefits disclosure of highly prevalent unidentified functional and cognitive impairments. Although recommended in guidelines, a suitable standardized geriatric test set is lacking. We aim to propose a consensus-based test set for geriatric assessment useful in both routine care and research in older ( $\geq 65$ year) patients approaching ESKD.

Methods: A multidisciplinary expert panel of physicians, nurses and supportive disciplines with clinical and/or scientific experience in geriatric nephrology was assembled. Preconditions and selectioncriteria for the selection of potential measures resulted from general geriatric principles, critical appraisal of literature, inventory of conventional instruments, and focus group meetings with patients, carers and health professionals. Older patients (aged $\geq 65$ years) approaching ESKD $\left(e G F R<20 \mathrm{ml} / \mathrm{min} / 1.73 \mathrm{~m}^{2}\right)$ were selected as the target population. An expert panel meeting and subsequent online round of comments led to agreement on the best suitable test set.

Results: The final consensus set contains instruments in functional, cognitive, phycological, and somatic domains, and patient preferences, nutritional status and fall risk. The set comprises a patient questionnaire (six instruments) and a professional-administered test set (including ten instruments). Estimated time for administration in pilot testing was 20 and 40 minutes respectively.

Key conclusions: We propose a consensus-based nephrology-tailored geriatric assessment, to benefit clinical care for older (pre-)ESKD patients and enhance research comparability. Future research should investigate effectiveness, feasibility of implementation, patients satisfaction and the value for treatment decision making and outcome improvement.

\section{Abstract \# 157}

The SELFY-MPI short-form: a self-administered frailty screening tool in primary care

Sabrina Zora ${ }^{1}$, Alberto Cella ${ }^{1}$, Alberto Ferrari ${ }^{2}$, Giuseppe Rengo ${ }^{3}$, Vincenzo Solfrizzi ${ }^{4}$, Nicola Veronese ${ }^{5}$, Alberto Pilotto ${ }^{6}$, on behalf of the SELFY-MPI SIGOT Investigators

${ }^{1}$ Geriatrics Unit, Department of Geriatric Care, Orthogeriatrics and Rehabilitation, EO Galliera Hospital, Genova, Italy, ${ }^{2}$ Geriatrics Unit, Arcispedale S. Maria Nuova, AUSL Reggio Emilia, Reggio Emilia, Italy, ${ }^{3}$ Department of Translational Medical Sciences, Division of Geriatrics, Federico II University, Napoli, Italy, ${ }^{4}$ Department of Interdisciplinary Medicine, University of Bari, Bari, Italy, ${ }^{5}$ Primary Care Department, Azienda ULSS 3 Serenissima, Venice, Italy, ${ }^{6}$ Geriatrics Unit, Department of Geriatric Care, Orthogeriatrics and Rehabilitation, EO Galliera Hospital, Genova, Italy; Department of Interdisciplinary Medicine, University of Bari, Bari, Italy, ${ }^{7}$ SELFYMPI SIGOT

Introduction: In primary care a reliable screening test to identify older subjects at risk of frailty to adopt intervention and prevention strategies are needed. The Multidimensional Prognostic Index (MPI) is a validate prognostic tool to predict negative health outcomes in older people. Recently, a self-administered version of the MPI (SELFY-MPI-SF) has been developed and validated in communitydwelling older people. Aim of the study was to evaluate feasibility and accuracy of the SELFY-MPI-SF in primary care setting.

Methods: A training course on methods and clinical value of the MPI was held by 24 geriatricians to about 300 General Practitioners (GPs) throughout in Italy. All participant GPs recruited older patients who fullfilled the SELFY-MPI-SF questionnaire to explore mobility, basal 
and instrumental activities of daily living (Barthel mobility, ADL IADL), cognition (Test Your Memory-TYM Test), nutrition (Mini Nutritional Assessment-Short Form-MNA-SF), comorbidity, medications, and co-habitation.

Results: 178 eligible patients were enrolled, mean age $=79 \pm 7.06$ years, range $66-97$ years, male/female ratio $=0.81 .73 .6 \%$ of patients were from North Italy, 22.5\% from South Italy and 3.9\% from Central Italy. The mean value of the SELFY-MPI-SF was 0.27 (range $0-0.75$ ). The large majority of patients was categorized in MPI 1 (low-risk) category $(=73.6 \%)$, followed by MPI $2(18.0 \%)$ and MPI 3 $(8.4 \%)$. A significant correlation between MPI and age was observed (Pearson coefficient $=0.55, \mathrm{p}<0.0001$ ).

Key conclusions: The SELFY-MPI-SF (Short Form) is a feasible and accurate multidimensional tool for the screening of older patients at risk of frailty, easy to integrate into routine clinical practice in primary care.

\section{Abstract \# 158 \\ Implementation Of The SELFY_MPI to stratify vulnerable community-dwelling people: the EU-cofunded EFFICHRONIC project}

Sabrina Zora ${ }^{1}$, Nicola Veronese ${ }^{2}$, Alberto Cella ${ }^{1}$, An L.D. Boone ${ }^{3}$, Marta Pisano Gonzalez ${ }^{4}$, Yves-Marie Pers ${ }^{5}$, Hein Raat ${ }^{6}$, Graham Baker $^{7}$, Alberto Ferri ${ }^{1}$, Alberto Pilotto ${ }^{8}$, on behalf of the EFFICHRONIC Consortium ${ }^{9}$

${ }^{1}$ Geriatrics Unit, Department of Geriatric Care, Orthogeriatrics and Rehabilitation, EO Galliera Hospital, Genova, Italy, ${ }^{2}$ Primary Care Department, Azienda ULSS 3 Serenissima, Venice, Italy, ${ }^{3}$ FICYT Foundation for Applied Scientific Research and Technology in Asturias, Oviedo, Spain, ${ }^{4}$ SESPA, Health Service of the Principality of Asturias, Oviedo, Spain, ${ }^{5}$ Clinical Immunology and Osteoarticular Diseases Therapeutic Unit, Rheumatology Department, Lapeyronie University Hospital, Montpellier, France, ${ }^{6}$ Erasmus University M edical Center, Rott er dam, The Netherlands, ${ }^{7}$ Qismet, Portsmouth, United Kingdom, ${ }^{8}$ Geriatrics Unit, Department of Geriatric Care, Orthogeriatrics and Rehabilitation, EO Galliera Hospital, Genova, Italy; Department of Interdisciplinary Medicine, University of Bari, Bari, Italy, ${ }^{9}$ EFFICHRONIC

Introduction: EFFICHRONIC is a EU co-funded project aimed to enhance health systems' sustainability by providing effective interventions of self-management for patients with chronic conditions in five European Countries (France, Italy, Spain, The Netherlands, United Kingdom). In this context, a self-administered version of the Multidimensional Prognostic Index (SELFY-MPI) was developed and validated to stratify community-dwelling people with different risk of physical and cognitive impairments and /or socioeconomic vulnerability. Aim of this study was to describe data of the implementation of the SELFY_MPI questionnaire in community-dwelling people who underwent a self-management program to improve management of their chronic diseases.

Methods: The SELFY-MPI is a multidimensional tool that explores mobility, basal and instrumental activities of daily living (Barthel mobility, ADL IADL), cognition (Test Your Memory-TYM Test), nutrition (Mini Nutritional Assessment-Short Form-MNA-SF), comorbidity, medications, and socio-economic status (social-familiar evaluation scale-SFES). Subjects were stratified according to SELFYMPI score in low risk (0-0.33), moderate risk (0.34-0.66) and high risk $(0.67-1)$.

Results: A total of 1273 of subjects from five European Countries (mean age $61.8 \pm 14.7$ years, range $20-94$ years; females $=63 \%$ ) filled the SELFY-MPI. The mean value of the SELFY-MPI was 0.22 \pm 0.15 (range $0.06-0.88$ ). Most of participants were in the low-risk group (83.9\%): $14 \%$ and $2.1 \%$ were in the moderate-risk and highrisk groups, respectively. A significant correlation between SELFYMPI and age was observed $(r=0.125, \mathrm{p}<0.0001)$.

Key conclusions: This ad-interim analysis demonstrated a good implementation of the SELFY-MPI to stratify community-dwelling people of different ages at risk for physical and cognitive impairments and/or socioeconomic vulnerability.

\section{Abstract \# 159}

A retrospective study of geriatric outpatient referrals in Malta

Philip Sciberras ${ }^{1}$, Ebony Giusti ${ }^{1}$, Nicole Sciberras ${ }^{1}$, Peter Ferry ${ }^{2}$

${ }^{1}$ Mater Dei Hospital, ${ }^{2}$ Karin Grech Hospital

Introduction: Geriatric outpatient clinics in Malta take place at Karin Grech Hospital and referrals are made from an acute hospital setting, medical/surgical outpatients and on a GP basis.Aim: To identify the commonest reasons for referral to Geriatric Outpatient Clinics reviewed in 2019, as well as geriatric patient demographics.

Methods: All new geriatric case referrals reviewed in 2019 were analysed and the data extracted was as follows: age, gender, source of referral and nature of complaint. Data was anonymised for data protection.

Results and conclusion: In 2019, a total of 934 patients were reviewed at geriatric outpatients. The patients reviewed at the geriatric outpatient department during 2019 had mostly been referred in the same year. The patients showed a female predominance with an average age of 78.4 and Mater Dei Hospital was the commonest referral source. The most frequent complaints were cognitive and mobility issues, specifically cognitive impairment and falls respectively.

\section{Abstract \# 160}

Multidimensional prognosis index (MPI) and mortality: a year follow up

Jorge Eduardo Corrales Cardenal ${ }^{1}$, Claudia Patricia Murillo Erazo ${ }^{1}$, Beatriz Gamboa Huarte ${ }^{1}$, María Dolores Domingo Sanchez ${ }^{1}$

${ }^{1}$ Hospital Nuestra Señora de Gracia, Zaragoza, Spain

Objectives: Assess the relationship between one-year mortality and the application of MPI based on a comprehensive geriatric assessment (CGA).

Method: Prospective, longitudinal study. Inpatients in an acute care geriatric unit from August 2018 to January 2019. Review of medical history one, three, six months and one year after assessment. Variables: socio-demographics, severity of MPI, mortality one, three, six months and one year.

Results: 144 patients. 66,7\% women. Mean age: 88 years. $93,1 \%$ admitted trough emergency room. Average hospital stay 11 days. Pfeiffer's test: 5,6 mistakes. Exton-Smith scale 14 points. Katz index 3. Lawton and Brody's index 2. Cumulative illness rating scale 3. Mini nutritional assessment 17 points. $54,5 \%$ lived at home accompanied. 36,6\% institutionalized and $8,3 \%$ lived alone. Grade of severity-MPI: $43,8 \%$ moderate. $41 \%$ severe. $15,3 \%$ mild. Mortality at the end of the follow up $42,8 \%$. One month: $8,3 \%$. Three months: $13,1 \%$. Six months: $3,4 \%$. One year: $9,7 \%$. Bivariate study: Moderate-Severe/mild MPI and mortality after a year follow up $\mathrm{p}=0,059$. Severe/other MPI and mortality after a year follow up $\mathrm{p}=0,009$.

Conclusions: Women, octogenarian, admitted trough emergency department, moderate cognitive impairment that lives at home. Moderate or severe MPI. In our study after a year follow up there is a clear association between mortality and severe MPI category and a 
light tendency of association of mortality and moderate MPI category. MPI based on a CGA may be used as a useful tool for decision making in the routine clinical practice in an acute geriatric unit.

\section{Abstract \# 161 \\ Dysphagia prevalence in a Greek sample of elderly patients with neurological diseases}

Sophia Demeneopoulou ${ }^{1}$, Marianna Mantzorou ${ }^{2}$, Nikoletta Margari ${ }^{2}$, Petra Mandysova ${ }^{3}$, Eleni Dokoutsidou ${ }^{2}$, Aggeliki Tsiligianni ${ }^{4}$, Theodoula Adamakidou ${ }^{2}$

1 “Help at Home", Municipality of Saronikos, Greece, ${ }^{2}$ Nursing Department, University of West Attica, Greece, ${ }^{3}$ Department of Nursing, University of Pardubice, Czech Republic, ${ }^{4}$ General University Hospital of Larisa, Greece

Introduction: Dysphagia is a common problem in hospitalized, nursing home residents as well as community dwelling elderly. Risk factors are associated with advanced age, clinical history and physical frailty. The aim of this study was to examine possible correlations between dysphagia and patients' clinical characteristics.

Methods: A cross-sectional study was conducted in a convenience sample including 80 neurological patients in a general hospital and a rehabilitation center in Athens. A trained nurse assessed each for the eight (8) parameters of the Brief Bedside Dysphagia Screening TestRevised (BBST-R) tool and completed a demographic and clinical characteristics questionnaire. Results were compared to the medical diagnosis in patients' files. SPSS v. 22.0 was used for data analysis. Results: $91.4 \%$ of patients examined for dysphagia were over the age of 61 years old while $53.8 \%$ of them were over the age of 81 years old, $53.8 \%$ were females, $65 \%$ had a stroke, $41.3 \%$ were widowers and their primary caregiver was a paid non-relative caregiver $(31.3 \%)$. The prevalence of dysphagia was $62.5 \%$ (95\% CI 50-72\%). No statistical relationship was found between dysphagia and demographic and clinical characteristics of the sample. Cultural adaptation of the BBST-R tool showed that it is a reliable tool for the assessment of dysphagia.

Key conclusions: The study confirms the high prevalence of dysphagia in neurological patients. Reliable and valid tools as well as protocols must be used by nurses in every day clinical practice for dysphagia assessment in order to minimize the time that patients remain undiagnosed and the subsequent complications.

\section{Abstract \# 162 \\ Comprehensive geriatric assessment at the front-door pilot: improving older adults care outcomes in Barnet Hospital}

Megan Knight ${ }^{1}$, Carmel Bergbaum ${ }^{1}$, Tunay Hussein ${ }^{1}$, Naomi Newman $^{1}$, Deborah Bertfield ${ }^{1}$, Mark James Rawle ${ }^{1}$

${ }^{1}$ Barnet Hospital, Royal Free London NHS Trust

Introduction: Total hospital admissions among older adults in the United Kingdom (UK) are rising [1], and delayed discharges of complex older adults are increasing in frequency [2]. Early comprehensive geriatric assessment (CGA) has been shown to increase older patients' likelihood of being alive and in their own homes after acute admission [3]. We evaluated the impact of the CGA via daily geriatrician input on an acute Clinical Decisions Unit (CDU) on outcomes for older inpatients in Barnet District General Hospital in the UK.

Method: We collected data for patients aged 70 or over admitted to CDU over two 2-week periods in October $2019(\mathrm{n}=40)$, and November $2019(\mathrm{n}=44)$. Between these time periods, we implemented daily geriatrician review, and introduced a multidisciplinary board round.

Results: Our intervention reduced mean length of stay on CDU ( 2.15 versus 6.4 days, $\mathrm{p}<0.05)$ and increased referrals to outpatient services $(25 \%$ versus $7.5 \%, \mathrm{p}<0.05)$. We noted improvements in numbers of discharges directly from CDU (56.8\% versus $42.5 \%)$, total length of hospital stay (5.8 versus 6.7 days) and discharges within five-days $(66 \%$ versus $50 \%)$ or to patients' own homes $(85 \%$ versus $71 \%$ ); however data from a larger sample is required to confirm these trends. Thirty-day mortality and readmission rates were unaffected.

Key conclusions: Geriatrician led-care in the acute setting improved length of stay on CDU, and use of specialist outpatient facilities. This could potentially enhance patient satisfaction and reduce costs associated with prolonged inpatient care, supporting the role of a dedicated front-door geriatrician.

References: [1] The King's Fund. 2020. How Hospital Activity In The NHS In England Has Changed Over Time. [online] Available at: $<\mathrm{https} / / /$ www.kingsfund.org.uk/publications/hospital-activity-fundingchanges $>$ [Accessed 9 June 2020]. [2] The King's Fund. 2020. NHS Hospital Bed Numbers. [online] Available at: <https: //www.kingsfund.org.uk/publications/nhs-hospital-bed-numbers $>$ [Accessed 9 June 2020]. [3] Ellis, G., Jamieson, C., Alcorn, M. and Devlin, V., 2012. An Acute Care for Elders (ACE) unit in the emergency department. European Geriatric Medicine, 3(4), pp. 261-263.

\section{Abstract \# 163 \\ Social network, depressive symptoms and loneliness among institutionalized older people}

Miriam Molas-Tuneu ${ }^{1}$, Pau Farrés-Godayol ${ }^{1}$, Anna Escribà-Salvans ${ }^{1}$, Sandra Rierola-Fochs ${ }^{1}$, Pau Moreno-Martin ${ }^{1}$, Dyego Leandro Bezerra de Souza ${ }^{2}$, Montse Masó-Aguado ${ }^{1}$, Javier Jerez-Roig ${ }^{1}$, Jordi NaudóMolist $^{3}$

${ }^{1}$ Research group on Methodology, Methods, Models and Outcomes of Health and Social Sciences (M3O). Faculty of Health Sciences and Welfare. Centre for Health and Social Care Research (CESS). University of Vic-Central University of Catalonia (UVic-UCC)., ${ }^{2}$ Research group on Methodology, Methods, Models and Outcomes of Health and Social Sciences (M3O). Faculty of Health Sciences and Welfare. Centre for Health and Social Care Research (CESS). University of Vic-Central University of Catalonia (UVic-UCC) and 2Federal University of Rio Grande do Norte, Department of Collective Health, Graduate Programme in Collective Health, Natal, Brazil., ${ }^{3}$ Research group Mental Health and Social Innovation (SAMIS). Faculty of Health Sciences and Welfare. Centre for Health and Social Care Research (CESS). University of Vic-Central University of Catalonia (UVic-UCC).

Introduction: The presence of depressive symptoms, loneliness and changes in social network are issues that impact the quality of life of older adults. We aim to verify the prevalence of depressive symptoms, loneliness and social isolation of older people living in nursing homes $(\mathrm{NH})$.

Methodology: A cross-sectional study was conducted in $4 \mathrm{NH}$ in the Osona (Barcelona, Spain) region between January and March 2020 (Clinical Trials registration number NCT04297904). The main variables were depressive symptoms according to the 15 -item Geriatric Depression Scale (GDS-15), perceived loneliness with the 6-item De Jong Gierveld Loneliness Scale (GLS-6) and social network with the Lubben Social Network Social Scale (LSNS-6). Absolute and relative frequencies were indicated for descriptive analysis. 
Results: 145 individuals were initially recruited, $33(61.1 \%)$ were excluded because legal guardians refusing to participate, $5(3.4 \%)$ were less than 65 years old, $4(2.7 \%)$ were hospitalized or not living permanently in the residence, $64(62.1 \%)$ were excluded due to cognitive impairment and $2(1.9 \%)$ presented incomplete data. Among the 37 included participants, mean age was $81.5 \pm 8.1), 26$ $(70.3 \%)$ were women and $11(29.7 \%)$ were men. The prevalence of depressive symptoms was $86.5 \%$ (CI 95\% 72.0-94.1), loneliness 70.3\% (CI 95\% 54.2-82.5) and 46\% (CI 95\% 31.0-61.6) high risk of social isolation.

Conclusions: The results indicate that most of the institutionalized people suffered depressive symptoms and feelings of loneliness, and approximately half of them were at high risk of social isolation.

\section{Abstract \# 164}

\section{Outpatient geriatrics consultation: a 3 years result}

Rita Tourais Martins ${ }^{1}$, Eduardo Doutel Haghighi ${ }^{2}$, José Augusto Barata $^{3}$

${ }^{1}$ Medical Doctor, Geriatrics consultation colaborator, Hospital de Vila Franca de Xira, Lisbon, Portugal, ${ }^{2}$ Internal Medicine Assistant, Coordinator of the Geriatrics Consultation at Hospital de Vila Franca de Xira, Lisbon, Portugal, ${ }^{3}$ Internal Medicine Service Director, Hospital de Vila Franca de Xira, Lisbon, Portugal

Introduction: Geriatrics outpatients consultations are becoming increasingly more common in Portugal. Gradually the benefit of these consultations is being seen by the portuguese medical community. In this work, we intent to examine 3 years casuistics by looking at the progress the patients made.

Methods: We examined the last Comprehensive Geriatric Assessment made to the evaluated patients and compared it to the first one. Analysed scales included Katz Index of Independence in Daily Activities (K), Lawton Instrumental Activities of Daily Living scale (L), Mini Nutritional Assessment ${ }^{\circledR}$ (MNA), Mini Mental State Examination (MMSE) and Yesavage Depression scale (Y). Average, 1st, 2nd and 3rd quartiles here compared. Statistic significance was investigated using Wilcoxon test. Version 24 of the SPSS software was used. The number of diagnosis was also examined as well as their distribution between different categories.

Results: During 3 years period, 117 patients were examined. The average number of diagnosis was 2,8 per patient. Of the total number of patients $65.8 \%$ were reexamined $(n=77)$. Average time between first and last consultations were 4 months. Motives for non reavaluation were discharge and death of patient. Progress was observed in all scales (specially in the first quartile) but the $\mathrm{Y}$, with statistical significance in the $\mathrm{L}(\mathrm{p}<0,03)$ and MNA scale $(\mathrm{p}<0,01)$.

Key conclusions: This casuistics clearly demonstrates the benefit of a Geriatrics outpatient consultation. Such a consultation should existi in every hospital in order to prevent funtional and cognitive decline and to better control polypharmacy.

\section{Abstract \# 165 \\ Prevalence of potentially inappropriate prescribing in older adults in a rural area of central Greece}

Maria Tampaki ${ }^{1}$, Alexandra Livada ${ }^{2}$, Maria- Niki Fourka ${ }^{2}$, Elli Lazaridou $^{1}$, Marina Kotsani ${ }^{3}$, Athanasios Benetos ${ }^{3}$, Petros P Sfikakis $^{4}$, Evrydiki Kravvariti ${ }^{4}$

${ }^{1}$ Primary medical care unit of Marmari, S. Evia, General Hospital of Karystos, Greece, ${ }^{2}$ Department of Statistics, Athens University of Economics and Business, Greece, ${ }^{3}$ Université de Lorraine, CHRU-
Nancy, Pôle « Maladies du Vieillissement, Gérontologie et Soins Palliatifs », F-54000, Nancy, France, ${ }^{4}$ Postgraduate Medical Studies in Geriatric Syndromes and Physiology of Aging, School of Medicine, NKUA, Athens, Greece

Introduction: A systematic approach to identify and avoid inappropriate medications is needed in geriatric primary care. Our aim was to evaluate the usefulness of the Screening Tool of Older Persons' Prescriptions (STOPP) and the Screening Tool to Alert doctors to the Right Treatment (START) in identifying clinically relevant potentially inappropriate prescribing (PIP) among older rural primary health care users.

Methods: A cohort of consenting outpatients aged $\geq 65$ years in a rural Greek primary care center was assessed for potentially inappropriate medication (PIM) and potential prescribing omissions (PPO) using the START/STOPP version 2 criteria [1]. Medications, comorbidities, functional status, and laboratory investigations were recorded by the treating provider during the medical visit, and complimented by the greek universal electronic prescribing system. The 6-month incidence of hospitalization and death were prospectively recorded.

Results: Among 104 participants (median age 78 years, 49.1\% women), 61\% were prescribed PIM and $78 \%$ had PPO. The PIM number was associated with the patient's total medication number, 6 -month incident hospitalization $(\mathrm{p}=0.032)$ and death $(=0.041)$. The most commonly met PIM criteria were prolonged prescription $(56 \%)$, lack of evidence-based indication (46\%), and duplicate drug class prescription (14\%). All prescriptions for benzodiazepines and xanthine-oxidase-inhibitors were classified as inappropriate. Most of PPOs $(84 \%)$ regarded vaccinations, and $31 \%$ regarded guidelinesupported drugs such statins in atherosclerotic disease, b-blockers for coronary artery disease, or treatment for osteoporosis.

Conclusions: The revised STOPP/ START criteria may contribute to identifying inappropriate medications, guiding de-prescribing and potentially improving clinical outcomes in rural community-dwelling older adults.

References: 1. Denis O' Mahoni et. al., STOPP/START Criteria for Potentially Inappropriate Prescribing in Older People: Version 2, Age Ageing, 2015 Mar;44(2):213-8

\section{Abstract \# 166}

A snapshot from the older patients' emergency department visit: six months after discharge

Dominik Rudol ${ }^{1}$, Marcel Rarek ${ }^{1}$, AM Meyer ${ }^{1}$, L Pickert $^{2}$, A Pilotto ${ }^{3}$, T Benzing ${ }^{4}, \mathrm{~V}$ Burst $^{4}$, MC Polidori ${ }^{5}$

${ }^{1}$ 1Ageing Clinical Research, Dpt. II for Internal Medicine, University Hospital of Cologne, Cologne, Germany; 2Nephrology, Rheumatology, Diabetology and Internal Medicine, Dpt. II for Internal Medicine, University Hospital of Cologne, Cologne, Germany; 3Emergency Department, University Hospital of Cologne, Cologne, Germany, ${ }^{2}$ Ageing Clinical Research, Dpt. II for Internal Medicine, University Hospital of Cologne, Cologne, Germany; 2Nephrology, Rheumatology, Diabetology and Internal Medicine,, ${ }^{3}$ Department of Geriatric Care, Orthogeriatrics and Rehabilitation, Frailty Area, E.O. Galliera, Genova, Italy, ${ }^{4} 2 \mathrm{Nephrology}$, Rheumatology, Diabetology and Internal Medicine, Dpt. II for Internal Medicine, University Hospital of Cologne, Cologne, Germany; 3Emergency Department, University Hospital of Cologne, Cologne, ${ }^{5}$ Ageing Clinical Research, Dpt. II for Internal Medicine, University Hospital of Cologne, Cologne, Germany; 2Nephrology, Rheumatology, Diabetology and Internal Medicine, Dpt. II for Internal Medicine, University Hospital of Cologne, Cologne, Germany 
Background: The first contact of older patients in the hospital system is usually in the emergency department (ED). At this early stage of treatment, a comprehensive geriatric assessment (CGA) can identify their individual treatment needs and can guide clinical decisions. As a result, the duration of hospitalization can be shortened and the number of rehospitalizations reduced, which increases the quality of life (QoL). However, there is a paucity of information on patients' trajectories and quality of life after hospitalization in advanced age. Methods: The inclusion criterion for the study was an age of the patients of at least 75 years (75+), as well as the treatment in the ED of the University Hospital Cologne. Only patients who had given their informed consent were admitted to the study. On admission, patients underwent physical and instrumental examination as well as CGAbased calculation of the Multidimensional Prognostic Index (MPI). Geriatric syndromes and resources were also collected. Healthcarerelated indicators were collected at baseline as well as at a phonefollow up interview conducted every six months up to 2 years after discharge. The follow-up is ongoing.

Results: Of a total of 287 patients with complete datasets and meeting inclusion criteria, $51.6 \%$ belonged to MPI-1 (low risk), $41.1 \%$ to MPI-2 (medium risk) and $7.3 \%$ to MPI-3 (high risk) groups. The mean values for the number of geriatric syndromes were 3.2 for the MPI-1, 4.9 for the MPI-2 and 6.1 for the MPI-3. Accordingly, $81 \%$ of the MPI-1, $96 \%$ of the MPI- 2 and $100 \%$ of the MPI-3 patients was defined multimorbid. The Kendall-Tau-b correlation coefficient with the MPI risk group was 0.4 for the number of geriatric syndromes ( $\mathrm{p}=$ 0.01 ), 0.3 for multimorbidity ( $\mathrm{p}=0.01$ ). On the other side, the MPI- 1 group hat 7.3 resources on average, MPI-2 5.9 and MPI-3 5.0 resources. The Tau-b correlation coefficient between MPI and GR was $-0.37(\mathrm{p}=0.01)$. At six months after ED visit, $64(20.3 \%)$ patients were lost to follow-up; of the remaining 251 patients, 48 (19.1\%) had died, 75 (38.1\%) were re-hospitalized (of these, $36 \%$ belonged to the MPI-1-, 47\% to the MPI-2-, and 33.\% to the MPI-3group). The length of stay was 9.6, 18.3 and 32 days in the MPI-1, MPI-2 and in the MPI-3 groups, respectively (Tau-b-correlation coefficient $0.34, \mathrm{p}=0.01$ ).

Conclusions: The MPI could be a valid tool to predict patient trajectories already at the ED contact and it can be used to effectively identify older patients with complex needs and accordingly ensure targeted, tailored interventions.

\section{Abstract \# 167 \\ Validity and reliability of the social autonomy scale (Social- SMAF): Turkish Version \\ Ayten Küçük ${ }^{1}$, Oya Nuran Emiroğlu ${ }^{2}$ \\ ${ }^{1}$ Afyonkarahisar University Of Health Science Atatürk Vocational School Of Health Services, ${ }^{3}$ Hacettepe University Nursing Faculty}

Background and aim: The aim of this study was to evaluate validity and reliability of "Social Autonomy Scale", which was developed to evaluate social functions affecting autonomy and health as well as physical functions of older individuals in Turkish society.

Materials and methods: It was carried out on 92 older individuals who were living in 3 nursing homes located in Afyonkarahisar and who did not have any psychological and mental disorders.

Results: At the end of analysis, it was found that social autonomy scale including 6 items was assembled under one dimension (factor) explaining $70.186 \%$ of total variance with an eigenvalue of 3.768 . Sampling adequacy was revealed for the applicability of factor analysis (Bartlett's Test $=211.779 ; \mathrm{p}<0.001$ and $\mathrm{KMO}=0.770$ ). Factor loads of all items were found to be above 0.40. Cronbach's Alpha coefficient for internal consistency of social autonomy scale was calculated as 0.80 . At the end of confirmatory factor analysis, a suitable model including one factor was produced for social autonomy scale.

Conclusion: "Social Autonomy Scale" which was adapted to Turkish, was found to be valid and reliable for measuring social independencies of the individuals who were aged 60 years and older. Keywords: Autonomy and self-efficacy, social functions, quality of life, nursing home

\section{Abstract \# 168 \\ Characteristics of geriatric syndromes and geriatric ressources of older, multimorbid patients in $\mathbf{4}$ different settings}

Pickert Lena ${ }^{1}$, Meyer Anna Maria ${ }^{1}$, Becker Ingrid ${ }^{2}$, Heeß Annika ${ }^{1}$, Rarek Marcel ${ }^{1}$, Rudol Dominik ${ }^{1}$, Betz Thomas ${ }^{3}$, Kochanek Matthias ${ }^{4}$, Burst Volker ${ }^{1}$, Benzing Thomas ${ }^{1}$, Polidori Maria Cristina ${ }^{1}$

${ }^{1}$ Ageing Clinical Research, Department II of Internal Medicine and Center for Molecular Medicine Cologne, University of Cologne, Faculty of Medicine and University Hospital Cologne, Cologne, Germany, ${ }^{2}$ Institute of Medical Statistics and Computational Biology, Faculty of Medicine and University Hospital of Cologne, Cologne, Germany, ${ }^{3}$ Outpatient Clinic Dr. Thomas Betz, Much, Germany, ${ }^{4}$ Intensive and Intermediate Care Units, Department I of Internal Medicine, University of Cologne, Germany;

Characteristics of geriatric syndromes and geriatric ressources of older, multimorbid patients in 4 different settingsPickert L1*, Meyer AM1*, Becker I2, Heeß A1, Rarek M1, Rudol D1, Betz T4, Kochanek, M5, Burst V1, Benzing T1,5, Polidori MC1,51Ageing Clinical Research, Department II of Internal Medicine and Center for Molecular Medicine Cologne, University of Cologne, Faculty of Medicine and University Hospital Cologne, Cologne, Germany; 2Institute of Medical Statistics and Computational Biology, Faculty of Medicine and University Hospital of Cologne, Cologne, Germany; 3Outpatient Clinic Dr. Thomas Betz, Much, Germany; 4Intensive and Intermediate Care Units, Department I of Internal Medicine, University of Cologne, Germany; 5Cologne Excellence Cluster on Cellular Stress- Responses in Aging- Associated Diseases (CECAD), University of Cologne, Faculty of Medicine and University Hospital Cologne, Cologne, Germany; *equal contributors.

Background: Geriatric syndromes (GS) are very common in older patients and are disclosed through the Comprehensive Geriatric Assessment (CGA). The grouping of complexes geriatric multifactorial conditions under the conceptual framing of GS allows the standardization and thus simplification of the clinical treatment of older patients. Geriatric resources (GR), on the other hand, represent a similar construct in terms of patients' capacities. Neither GS nor GR, however, are currently collected as a standard in everyday clinical practice. Therefore, the actual prevalence of these two entities is largely unknown across settings.

Patients and methods: All patients in this study were prospectively enrolled in 4 different studies and received a CGA at admission and discharge, as well as GS and GR recording at admission. Patients were all over 65 years of age and had at least two chronic conditions. The studies were performed at an internal medicine ward (IM, 499 patients), in the emergency room (A\&E, 270 patients), at a general practice (GP, 125 patients), and in the intensive care unit (ICU, 56 patients). The GS recorded were polypharmacy, instability, incontinence, inanition, immobility, irritability/depression, cognitive impairment, insomnia, impoverishment, swallowing disorder, chronic pain, sensorial impairment, irritable colon, iatrogenic disease, social isolation, fluid/electrolyte problems, incoherence/delirium. The GR recorded were physical, good living conditions, social, economic, competence-related, intellectual, spiritual, motivational, emotional and mnestic resources. The syndromes and resources were collected 
either by direct questioning the patient or relatives, by researching the medical history or by CGA.

Results: The mean age of the patients was $77.7( \pm 5.8, \mathrm{IM}), 80.7( \pm$ $4.7, \mathrm{~A} \& \mathrm{E}), 79.2( \pm 6.5, \mathrm{GP})$ and $74.8( \pm 6.5, \mathrm{ICU})$ years $(\mathrm{p}<0.001)$, the median length of hospital stay was 9 (5-17, IM), 4 (1-10, A\&E), zero (GP) and $18(8-36$, ICU) days $(\mathrm{p}<0.001)$. The most frequent admission diagnoses were kidney failure $(38.7 \%$, IM), cardiovascular diseases $(23.7 \%$ at A\&E and $17.6 \%$ at GP) and acute infections $(19.6 \%$, ICU; $p<0.001)$. Both the number of GS per setting and the number of GR per setting were statistically significant $(\mathrm{p}<0.001)$. The highest number of GS were in patients admitted to IM with a median of 6 GS (4-7), while GR were mostly present in patients admitted to the A\&E and the ICU, with a median of 7 (6-8 and 5-8). Conversely, GP patients displayed the lowest GS number (median 4, IQR 3-5), patients at the A\&E had a median amount of 4 (IQR 3-6) GS and at the ICU a median amount of 5 (IQR 3-8) was counted. The lowest amount of GR occurred in patients admitted to IM and at the GP (median for both 6, IQR 4-7). The distribution of the various GS and GR was, with a few exceptions (for GS "impoverishment" and for GR "living conditions", "social" "motivational" and "competence-related" were not significant), also statistically significant.

Discussion: It is possible to collect GS and GR in many different settings where older, multimorbid patients are typically managed and treated. Additionally, there is a different distribution of GS and GR between these settings, which is likely to play an important role in patients' trajectories but has not been adequately addressed yet. How these findings can influence clinical decision making and what conclusions can be drawn for physicians and nurses needs to be investigated in further studies.

\section{Abstract \# 169}

GPS analysis of activity spaces and walking behavior in frail older person over 70 years: a pilot study

Sandra Lau ${ }^{1}$, Frerk Müller-zu Aschwege ${ }^{2}$, Lena Elgert ${ }^{3}$, Tania Zieschang $^{1}$, Jürgen M. Bauer ${ }^{4}$, Andreas Hein ${ }^{5}$, Rebecca Diekmann ${ }^{5}$

${ }^{1}$ Carl von Ossietzky University Oldenburg, Geriatric Medicine, ${ }^{2}$ OFFIS - Institute for Informatics, Oldenburg, ${ }^{3}$ Peter L. Reichertz Institute, TU Braunschweig and Hannover Medical School, Medical Informatics, ${ }^{4}$ University of Heidelberg, Geriatric Center Agaplesion Bethanien Hospital, ${ }^{5}$ Carl von Ossietzky University Oldenburg, Assistance Systems and Medical Technology

Introduction: Frailty is common in older, multimorbid patients and has a negative impact on physical activity. Walking as a main functional ability to participate in activities of daily living is of key importance. Established questionnaires assessing physical activity are based on self-report and are strongly biased. In this study, we focused on GPS data to detect and compare real time walks and individual mobility characteristics in frail older persons.

Methods: Community-dwelling adults $\geq 70$ years with (pre-)frailty received a GPS Logger for 10 months. Walking speed and distances were analyzed by customized software. In parallel, Short Physical Performance Battery (SPPB) including gait speed (GS) and Timedup-and-Go Test (TUG) were conducted monthly.

Results: GPS data of 20 participants $(84.5( \pm 5.2)$ years, $85 \%$ women, mean frailty index $1.9(70 \% \geq 2)$ points $)$ were analyzed and revealed three types of walkers: Type A presented only a few, short walks nearby the home with significantly reduced means in GS $(0.45( \pm 0.1)$ $\mathrm{m} / \mathrm{s})$, SPPB $(3.8( \pm 0.8)$ points) and TUG performance $(23.4 \mathrm{~s}$ ( \pm 4.9)). Type B showed the largest activity space, exceeding walking distances and highest mobility scores $(0.82( \pm 0.1) \mathrm{m} / \mathrm{s}(\mathrm{GS}), 7.0( \pm$ 1.3) points (SPPB), 13.2( \pm 1.4$)$ s (TUG)). Type C performed constantly high numbers, durations and distances of walks with an average of $0.63( \pm 0.2) \mathrm{m} / \mathrm{s} \quad(\mathrm{GS}), 6.5( \pm 2.4)$ points (SPPB), 17.1( \pm 4.4$)$ s (TUG).

Conclusion: Walks and individual activity spaces can be mapped via GPS. Heterogeneous walking behaviors and a wide range of functional performance were observed. GPS data can make a valuable contribution to the assessment of physical activity under everyday conditions in frail older people.

\section{Abstract \# 170 \\ Brief older people's quality of life (OPQOL-b) questionnaire and frailty in a Geriatrics Clinic}

Ines Figueiredo ${ }^{1}$, Marilia Fernandes ${ }^{1}$, Maria Saldanha ${ }^{1}$, Carla Barbosa $^{1}$, Manuela Barão ${ }^{1}$, Ricardo Silva ${ }^{1}$, Joana Guerreiro ${ }^{1}$, Catarina Santos ${ }^{1}$, Heidi Gruner ${ }^{1}$

${ }^{1}$ Consulta Multidisciplinar de Geriatria, Hospital Curry Cabral, Centro Hospitalar Universitário Lisboa Central

Background: Policies related to older people include interventions directed towards health, independence, activity, social and economic participation. Assessment of quality of life (QoL) is an endpoint used to evaluate the efficacy of these. The OPOQOL 35 questionnaire addresses gaps from previous questionnaires.

Methods: Cross-sectional study in the Geriatric Clinic outpatients. Patients fulfilled the OPQOL-brief questionnaire with the nurse, as well as the EQ-5D-3L questionnaire. Clinical data was collected. Statistical analysis was performed using STATA, a p value $<5$ was considered significant.

Results: A total of 40 patients were included, 28 (70\%) female, the median age of 80 (76-88). Considering global geriatric evaluation, a median of 5 (4-7) on the Lawton \& Brody scale, 6 on the Katz scale, and $2(2-3,5)$ on PRISMA7. The OPQOL-brief questionnaire answers had a median score of $56(48,5-59)$, the visual analogue scale (VAS) of quality of life had a median 60 (50-75), and QALYs median was $0,44(0,35-0,55)$. OPQOL-brief correlated with QALYs (correlation coefficient 0,57 , p-value $=0,0001$ ), and with geriatric scores: Lawton\&Brody (correlation coefficient 0,5 , p-value $=0,002$ ), Katz (correlation coefficient $0,48, p$-value $=0,005$ ) and PRISMA7 (correlation coefficient $-0,5$, p-value $=0,006$ ).

Discussion/conclusion: OPQOL-brief varies between 13 and 65, with higher scores standing for better QoL. Our sample had a median of 56, which reveals an overall good QoL. Most importantly, it seemed that the quality of life was tightly related to the geriatric scores associated with dependency and frailty, and there was no relation to demographic factors.

\section{Abstract \# 171}

\section{Clinical relevance of jump parameters in older adults}

Sandra Lau ${ }^{1}$, Andrea Heinks ${ }^{2}$, Rebecca Diekmann ${ }^{3}$, Christian Werner $^{1}$,Tania Zieschang ${ }^{4}$, Jürgen M. Bauer ${ }^{2}$

${ }^{1}$ University of Heidelberg, Geriatric Center, Agaplesion Bethanien HospitalMedicine, ${ }^{2}$ University of Heidelberg, Geriatric Center, Agaplesion Bethanien Hospital, ${ }^{3}$ Carl von Ossietzky University Oldenburg, Assistance Systems and Medical Technology, ${ }^{4}$ Carl von Ossietzky University Oldenburg, Geriatric Medicine

Introduction: Muscle mass and muscle power are known to be sensitive predictors for functional decline in older people. Objective and quick to perform assessments of muscle power are still missing. Jumps seem to be a promising approach to calculate complex muscle power during a highly demanding task, but little is known about jumps as a predictor of functional decline. The purpose of this 
systematic review is to summarize and evaluate different jump testing approaches and their clinical relevance in the field of geriatric medicine.

Methods: To determine existing technical and non-technical approaches to assess and measure vertical jumps in an older adult cohort including 60-year-old persons up to the oldest old, multiple databases are screened independently by two authors. We will include all published trials regardless of study design. We aim not to restrict study eligibility by publication status, but we will exclude studies that are not published in German or English. If applicable, we will also report on jumping procedures, normative values and main findings associated with jumps. All findings of the data search will be provided and presented according to the high-quality standards for systematic reviews. The review will be registered in PROSPERO.

Results: Basic information will be collected and described qualitatively for each study concerning methods (e.g. study design, sample size), participants (e.g. selection criteria and diagnoses, age, gender), type of jump test and used technical devices for measurement. Calculated parameters and additional outcome variables will be provided additionally. Results will be available by October.

\section{Abstract \# 172 \\ The relation between delirium and frailty in hospitalized geriatric patients : delirium day datas \\ Asli Tufan Çinçin ${ }^{1}$, Rabia Gökçen Umurca ${ }^{2}$ \\ ${ }^{1}$ Marmara University Medical Faculty, ${ }^{2}$ Marmara University Medical Faculty}

Introduction and objective: As a result of aging world population, health problems of the elderly are encountered more frequently in daily practice. Delirium which is an acute confusional state with fluctuating course during the day is frequently observed in elderly patients experiencing acute stress such as hospitalization. Various strategies are being developed to detect and prevent this syndrome which causes serious morbidity and mortality worldwide. In our study, which we conducted on the delirium awareness day, we determined the point prevalence of delirium in geriatric inpatients in our country by using the Confusion Assessment Method (CAM), and revealed its relationship with frailty, a geriatric syndrome which is a common cause of serious morbidity.

Materials and method: Sixty-two patients aged 60 years or older who were hospitalized in the Internal Medicine Clinic of Marmara University Pendik Training and Research Hospital on March 13, 2019 were included in the study. The demographic information, comorbidities and medical history of the patients were obtained from the patient or first degree relatives, and patients' medications were obtained from the patient files. Mini Nutritional Assessment (MNA) to assess nutritional status of patients, FRAIL frailty scale (Fatigue, Resistance, Ambulation, Illnesses, Loss of weight) to assess frailty status, Katz Index of Independence Activities of Daily Living (ADL), Lawton-Brody Instrumental Activities of Daily Living (IADL) to assess functional status, delirium risk factors and delirium evaluation test (CAM) were applied. If there was mortality during follow up, the date was stated.

Results: The mean age of the patients was $71,9+8,21$. Twenty six patients $(41.9 \%)$ were female and $36(58.1 \%)$ were male. According to CAM, delirium was detected in 18 (29\%) of the patients. According to the FRAIL scale, $21(33.9 \%)$ patients were prefrail and $41(66.1 \%)$ were frail. All $18(100 \%)$ cases developing delirium were found to be frail. In multivariate logistic regression analysis, being older than 70 years, low Katz ADL score and low MNA score were found to be independent risk factors for delirium development.
Conclusion: Delirium and frailty are common in hospitalized elderly patients, and frailty is a risk factor for delirium. Health care workers should be aware of these geriatric syndromes, recognize them early, and develop delirium prevention strategies.

\section{Abstract \# 173}

Delirium is associated with increased mortality in older COVID19 patients

Sophie Picton ${ }^{1}$, Omolade Okuwoga ${ }^{1}$, Sadia Saber ${ }^{1}$, Cianan O'Sullivan ${ }^{1}$

${ }^{1}$ Homerton University Hospital, Hackney, London, UK

Abstract title: Delirium is associated with increased mortality in older COVID-19 patientsAuthors: S. Picton, O. Okuwoga, S. Saber, C. O'Sullivan; Homerton University Hospital, London, United Kingdom

Introduction: The COVID-19 outbreak is a worldwide pandemic that has severely affected patients aged $>65$ years. This study investigates the outcomes of 104 older COVID-19 positive patients (mean age 84) diagnosed with delirium on admission to Homerton University Hospital, London, UK. It was conducted as a retrospective analysis of electronic patient records of patients admitted during the peak of the coronavirus outbreak. The aims of the study are twofold: To assess how delirium is diagnosed in COVID-19 patients. To evaluate the outcomes of older COVID-19 patients diagnosed with delirium.

Methods: Data was collected from electronic patient records to determine, firstly, how delirium was diagnosed. Confusion was diagnosed by 2 methods: History documented in the notes, or formal use of an assessment tool-the Abbreviated Mental Test Score (AMTS).Outcomes of these patients were subcategorised: Still an inpatient, discharged from hospital, deceased.

Results: 243 older COVID-19 positive patients were admitted during the study period, 104 were diagnosed with delirium. 45 were diagnosed using the AMTS. 52 were diagnosed based upon clinical history. Of the 104 diagnosed with delirium 36 were discharged from hospital. 64 patients died. 4 remained as an inpatient.

Conclusion: Delirium is a common presentation of COVID-19 in older adults. It is a poor prognostic indicator, associated with high mortality. The results of this study demonstrate that older adults often present atypically, with acute delirium rather than cough and fever. Recognition of this association and the risk of increased mortality will help inform clinical decision making and improve patient care.

\section{Abstract \# 174}

Multidimensional Prognostic Index (MPI) predicts delirium in older patients with hip fracture who underwent surgical intervention

Clarissa Musacchio $^{1}$, Carlo Custodero ${ }^{2}$, Monica Razzano ${ }^{1}$, Rita Raiteri ${ }^{1}$, Andrea Del Rio ${ }^{1}$, Domenico Torriglia ${ }^{1}$, Marco Stella ${ }^{1}$, Vincenzo Solfrizzi $^{2}$, Antonella Barone ${ }^{1}$, Alberto Pilotto ${ }^{3}$

${ }^{1}$ Department of Geriatric care, Orthogeriatrics and Rehabilitation, E.O. Galliera Hospital, Genova, Italy, ${ }^{2}$ Department of Interdisciplinary Medicine, University of Bari, Italy, ${ }^{3}$ Department of Geriatric care, Orthogeriatrics and Rehabilitation, E.O. Galliera Hospital, Genova, Italy; Department of Interdisciplinary Medicine, University of Bari, Italy

Background: Delirium is a severe clinical complication in acute older patients hospitalized for hip fracture who need surgical intervention. The onset of delirium increases the risk of delay in surgical intervention, length of in-hospital stay, morbidity and mortality. 
Unfortunately, no tools have been yet validated to identify the patients at risk of delirium in the acute hospital setting.

Aim: In this retrospective observational cohort study, we evaluated whether the Multidimensional Prognostic Index (MPI) could predict the delirium onset in older individuals admitted to the hospital for hip fracture who underwent surgical intervention.

Methods: Consecutive older patients admitted to the hospital for hip fracture underwent a comprehensive geriatric assessment (CGA) to calculate the Multidimensional Prognostic Index (MPI) from information on functional, cognitive, mobility and nutritional status, comorbidity, number of medications and co-habitation. According to previous cut-off, MPI was expressed in three grades, i.e. MPI-1 (lowrisk), MPI-2 (moderate-risk) and MPI-3 (high risk of mortality). Delirium was assessed during hospitalization using 4 AT test.

Results: 244 older patients (mean age $85 \pm 6.9$ years; females $=$ $84.2 \%$ ) who underwent surgery for hip fracture were included. 104 subjects $(43 \%)$ received a diagnosis of delirium. Patients with delirium showed higher pre-operative cognitive impairment $(\mathrm{p}=0.0001)$, lower baseline functional status $(\mathrm{p}=0.001)$ and were older than patients who did not experience delirium. Logistic regression analysis demonstrated a significant association between MPI grade and MPI score and the onset of delirium during hospitalization $(\mathrm{p}<0.05$ and $\mathrm{p}$ $<0.0001$, respectively). Overall, the incidence of delirium during hospitalization was significantly higher in patients with more severe MPI score.

Conclusions: MPI may be an useful tool to identify older patients at risk of delirium when admitted to hospital for hip fractures and have to undergo surgical intervention.

\section{Abstract \# 175 \\ Training improves identification of delirium for primary care providers'}

João Tavares ${ }^{1}$, Lígia Passos ${ }^{2}$, Daniela Figueiredo ${ }^{3}$, Larissa Pedreira ${ }^{4}$

${ }^{1}$ School of Health Sciences, University of Aveiro, Center for Health Technology and Services Research; The Health Sciences Research Unit: Nursing, ${ }^{2}$ Department of Education and Psychology, University of Aveiro, ${ }^{3}$ School of Health Sciences, Center for Health Technology and Services Research (CINTESIS.UA), ${ }^{4}$ Federal University of Bahia

Introduction: The evaluation of delirium is a central domain in caring for older adults. Studies on delirium evaluation focus mainly on the hospital context. However, the identification of delirium in primary health care is essential, especially because of complaints of changes in mental status [1]. This study aims to assess the effects of a short educational intervention on the knowledge of health care clinicians (HCC) in primary care (HCC-PC) to identify delirium in older adults in the community.

Methods: The pretest-posttest design followed a single group of HCC-PC. To assess knowledge, a standardised case vignette of delirium was used [2]. Two hours of education and training were provided in a single session. The data were analysed with a Wilcoxon test, accepting a p-value $<0.05$ as statistically significant.

Results: Fourteen clinicians participated in the training (nine nurses and five physicians). Eleven of the participants were female. The average age was $46.3 \pm 15.5$ years; the average professional time was $27.8 \pm 11.2$ years. Eleven reported no previous education or training in delirium. In the pretest, eight did not recognize the acute change in mental state and twelve did not recognise delirium and the subtype. In the posttest, twelve and eleven recognised the acute change in mental state and delirium, respectively; all participants identified the subtype. Posttest recognition of acute mental status $(p=0.014)$, recognition of delirium $(p<0.01)$ and identification of subtype $(p<0.01)$ significantly improved from the pretest.
Key conclusions: Implementing dynamic and interactive methodological training with analyses of vignettes was effective for recognition of delirium by HCC-PC.

References: 1. Inouye SK, Westendorp RGJ, Saczynski JS (2014) Delirium in elderly people. Lancet 383:911-922. 2. Fick DM, Kolanowski AM, Hill NL, Yevchak A, DiMeglio B, Mulhall PM (2013) Using Standardized Case Vignettes to Evaluate Nursing Home Staff Recognition of Delirium and Delirium Superimposed on Dementia. Ann Longterm Care 21:34-38

\section{Abstract \# 176}

Candiduria by Candida Tropicallis: an unusual cause of delirium

Leonor P.Silva ${ }^{1}$, Rafael Silva Teixeira ${ }^{2}$, Raquel Dias Moura ${ }^{1}$, Mariana Estrela Santos ${ }^{1}$, Joana Mascaranhas ${ }^{1}$, Rafaela Verissimo ${ }^{1}$

${ }^{1}$ Internal Medicine Service, Centro Hospitalar V.N.Gaia, ${ }^{2}$ Cardiology Service, Centro Hospitalar V.N.Gaia

Introduction: Delirium is common, particularly in hospitalized elderly patients (up to $30 \%$ ) (1). The identification of underlying causes is crucial to avoid adverse outcomes.

Case report: An 80-year-old-woman was admitted to the emergency department with a convulsive crisis. She presented a Glasgow coma scale of 9 , little reactive isochoric pupils, febrile $\left(38,5{ }^{\circ} \mathrm{C}\right)$ and normotensive. There were no other symptoms suggesting infection, the remaining physical examination was normal and glycaemia was $508 \mathrm{mg} / \mathrm{dL}$. She had clinical record of incipient dementia, diabetes, hypertension, epilepsy and nonadherence to medication. She had no previous hospitalizations or genitourinary tract manipulation. The lab results demonstrated leukocytosis $(15.19 \times 103)$ with neutrophilia and normal Reactive Protein C. Urine analyse was normal, with no drugs identified; Sars-Cov2 and influenza swabs were negative. Cranio-encephalic computed tomography (CT) had no signs of ischemic or haemorrhagic events. Abdominal ultrasound and cerebrospinal fluid had no signs of infection. The hyperglycaemia was corrected and was medicated with valproate bolus and ceftriaxone, without improvement during hospitalization. Endocarditis, HIV, Hepatitis B or Hepatitis C were excluded and Thoraco-abdominalpelvic CT was normal. The blood cultures were negative and the urine culture identified a Candida tropicalis; she started fluoconazole, presenting gradual improvement of consciousness and resolution of fever.

Conclusion: Candiduria by Candida tropicallis is rare (12,7\%) (2), especially without risk factors such as genito-urinary tract manipulation, immunosuppression, surgery or recent antibiotherapy. In this case, the only risk factors identified were uncontrolled diabetes and age. As candiduria may present only as delirium in elderly, it is pivotal an early detection and treatment. 1. Martins S, Fernandes L. Delirium in elderly people: A review. Front Neurol. 2012; Jun(June):1-12. 2. Negri M, Silva S, Henriques M, Oliveira R. Insights into Candida tropicalis nosocomial infections and virulence factors. Eur J Clin Microbiol Infect Dis. 2012;31(7):1399-412.

\section{Abstract \# 177 \\ Implementation of the 4AT delirium detection tool in clinical practice: data from 69,462 acute medical admissions in two hospitals}

Alasdair MacLullich ${ }^{1}$, Atul Anand ${ }^{2}$, Zoë Tieges ${ }^{3}$, Miriam $^{2}$ Veenhuizen $^{4}$, April Covington ${ }^{4}$, Alasdair MacLullich ${ }^{3}$

${ }^{1}$ Geriatric Medicine, University of Edinburgh, ${ }^{2}$ Centre for Cardiovascular Science, University of Edinburgh, Scotland, 
${ }^{3}$ Edinburgh Delirium Research Group, Department of Geriatric Medicine, University of Edinburgh, Scotland, ${ }^{4}$ Edinburgh Medical School, University of Edinburgh, Scotland

Introduction: There are multiple delirium assessment tools. Yet validation data have mostly been generated under study conditions. There are few studies providing information in full clinical populations on (a) tool completion rates and (b) proportions of scores positive for delirium. The 4AT (www.the4AT.com) is a globally-used clinical tool for delirium detection. We examined completion and detection rates of the 4AT as implemented in two university hospitals. Methods: We analysed electronic records on all consecutive patients aged $\geq 50$ years admitted to acute medical wards between Apr 2016 and May 2019. The 4AT is scored from 0 to 12. Scoring: 0, negative; 1-3, cognitive impairment but no delirium; 4-12, possible delirium. We assessed completion rates, percentages with scores in the three scoring bands, and length of stay.

Results: Of the 69,462 consecutive admissions aged $\geq 50$ years old, $51,238(74 \%)$ had a completed 4AT. $77 \%$ of patients aged $\geq 65$ had a completed 4AT. In the 51,238 with completed 4AT, 33,028 (64\%) had a score of $0,9,499(19 \%)$ had a score of $1-3$, and $8,711(17 \%)$ had a score of 4-12. Median length of stay in days in relation to scoring categories was 0: 3.6 days (IQR 1.3-8.6); 1-3: 8.9 days (IQR 2.8-26.1); 4-12: 9.2 (IQR 3.3-26.8).

Key conclusions: These findings suggest that the 4AT is (a) implementable in clinical practice, and that (b) when performed it detects clinically realistic levels of delirium. Further studies in full clinical populations will enhance knowledge of the implementability of delirium detection tools.

\section{Abstract \# 178}

Potential risk predictor in type $\mathbf{2}$ diabetes mellitus patients

Simona Opris ${ }^{1}$, Gianina Ioana Constantin ${ }^{1}$

${ }^{1}$ National Institute of Gerontology and Geriatrics “Ana Aslan”

${ }^{1}$ Type 2 diabetes mellitus is associated with lipid abnormalities and an increase in plasma atherogenicity that can be measured by atherogenic index (AI). The objective of the study was to determine if $\mathrm{AI}$ is a good risk predictor in diabetes mellitus patients.

Material and method: Present work included 199 older patients (over 65 years): 91 diabetes mellitus and 108 healthy control patients. Findings: Study revealed an increase of triglycerides $(\mathrm{p}<0.001)$, increase of $\mathrm{AI}(2.99 \pm 1.5$ vs. $3.58 \pm 1.77 ; \mathrm{p}<0.01)$ and a decrease of HDL-cholesterol $(\mathrm{p}<0.0001)$ at diabetes mellitus patients vs. control. Diagnostic test evaluation indicated that AI test has $90.1 \%$ sensitivity, $31.48 \%$ specificity, and relative risk estimate $=2.51$. Multivariate logistic regression analysis showed that, compared to low risk, patients with high AI had 4.18-fold risk for developing diabetes mellitus [OR 4.18, 95\% CI 1.88-9.30; $p=0.0004$ ].

Conclusion: Our results suggest that the higher is AI, the higher are the chances to develop diabetes mellitus, and therefore, prevention strategies and treatment guidelines should include AI as good risk predictor.

\section{Abstract \# 179 \\ Hypoglycaemia in frail or disabled older patients with diabetes over 75 years old treated by basal insulin}

Marie Danet-Lamasou ${ }^{1}$, Rémi Sitta ${ }^{2}$, Fara Ratsimbazafy ${ }^{1}$, Jessica Durrieu $^{1}$, Romain Fossaert ${ }^{1}$, Laura Richert ${ }^{3}$, Isabelle BourdelMarchasson $^{4}$
${ }^{1}$ Pôle Gérontologie Clinique, CHU Bordeaux, Bordeaux, France, ${ }^{2}$ Pôle Santé Publique, CHU Bordeaux, Bordeaux, France, ${ }^{3}$ Université Bordeaux, Inserm, Bordeaux Population Health Research Center + Pöle Santé Publique, CHU Bordeaux, Bordeaux, France, ${ }^{4}$ UMR 5536 Résonance Magnétique des systèmes Biologiques, CNRS/Université de Bordeaux, Bordeaux, France + Pôle Gérontologie Clinique, CHU Bordeaux, Bordeaux, France

Introduction: Basal insulin is widely used in older patients with Type 2 Diabetes (T2D). Long-acting insulin analogues (LIA) induce less nocturnal hypoglycemia than NPH insulin because of their flat action. However, older patients with diabetes have rarely insulin resistance : their risk of nocturnal hypoglycemia may be higher.Aim : to assess the number of hypoglycemia episodes (HE) according to basal insulin type, cognitive and nutritional status in frail older people with T2D. Methods: Prospective, exploratory single center study. Inclusions stratified on type of insulin: NPH/LIA. Frail or dependent patients ( $>$ 75 years) with T2D treated only with basal insulin in geriatric wards or nursing homes used a continuous glucose monitoring system during 120 hours.

Results: 25 patients were enrolled: 9 treated with NPH, 16 with LIA. Four HE were found: 1 nocturnal in NPH-group (11\%); 3 in LIAgroup (19\%), 2 nocturnal, 1 diurnal.Among 12 patients with MMS > $=21$ no HE was noted, while 3 of 10 patients with MMS 11-20 and 1 of 2 patients with MMS $<=10$ had HE. No HE was noted among 6 patients with normal nutritional status according MNA. Diurnal HE was noted in 1 of 9 malnourished patients, also obese. Nocturnal HE were found in 2 of 9 malnourished patients and 1 at risk of malnutrition. Their BMI were in normal range or overweight.

Key conclusions: HE was frequent in patients with MMS $<20$ or with malnutrition or a risk of malnutrition according to MNA. HE also occurred in obese patients.

\section{Abstract \# 180 \\ Effectiveness of the diabetic and oral care program for SENIOR (DOCS) IN older people with type 2 diabetes mellitus (T2DM) in Muang district, NAKHON Ratchasima Province, Thailand}

Namon Phetnin ${ }^{1}$

${ }^{1}$ Faculty of Dentistry, Mahidol University

The aim of this study was to assess the effectiveness of the Diabetic and Oral Care Program for Seniors (DOCS) in terms of improving oral health perception, behaviours, oral hygiene and reduced glycaemic status in older patients with type 2 diabetes mellitus (T2DM). A double-blind randomised controlled study was conducted in the two health centres located in Muang district, Nakhon Ratchasima province, between July 2019 and January 2020. Control and intervention groups were created and thirty-five T2DM older patients with chronic periodontitis who attended the centres were randomly recruited into each group. The intervention group received an oral health education program based on the Health Belief Model (HBM), individual oral hygiene instruction, scaling and root planning at one month, and individual oral hygiene instruction at three months. The patients in the control group received the routine program. Outcomes were assessed using a questionnaire, a simplified oral hygiene index, and glycaemic status (HbA1c). These measures were applied at baseline, three months and six months. Data were analysed using a Chi-square test, a $\mathrm{t}$-test, and repeated measure ANOVA at p-value $=0.05$. The results at three and six months showed that the intervention group significantly improved their HBM and oral health behaviour scores. Furthermore, their OHI-S scores and HbAlc levels $(p<0.05)$ decreased, while there was no significant difference in the control group. The findings suggested that the proposed program was effective among diabetic older people who have periodontitis in terms of improving oral health 
perception, behaviours, oral hygiene, and glycaemic status as indicated in evaluations occurring three and six months after the initiation of the study.

\section{Abstract \# 181 \\ Effect of antidiabetic medications on major adverse cardiovascular events in the elderly: meta-analysis of cardiovascular outcome trials}

Thomas Karagiannis ${ }^{1}$, Eleni Athanasiadou ${ }^{1}$, Apostolos Tsapas ${ }^{1}$, Eleni Bekiari $^{1}$

${ }^{1}$ Clinical research and Evidence-Based Medicine Unit, Aristotle University of Thessaloniki, Thessaloniki, Greece

Introduction: The effects of antidiabetic drugs on cardiovascular outcomes in the elderly is unclear.

Methods: We conducted a meta-analysis of cardiovascular outcome trials of glucagon-like peptide-1 (GLP-1) receptor agonists, sodium glucose cotransporter 2 (SGLT2) inhibitors and dipeptidyl peptidase4 (DPP-4) inhibitors, based on age-specific subgroups $(<65, \geq 65$, and $\geq 75$ years). We calculated hazard ratios (HRs) and 95\% confidence intervals (CIs) for the composite outcome of major adverse cardiovascular events (MACE).

Results: We included 13 cardiovascular outcome trials. For GLP-1 receptor agonists, in patients $<65$ years the HR was $0.94(95 \%$ CI $0.87-1.02)$ and in patients $\geq 65$ years and $\geq 75$ years it was 0.86 $(0.80-0.92, \mathrm{p}$ for interaction $=0.101)$ and $0.75(0.59-0.94,0.056)$, respectively. For SGLT2 inhibitors, in patients $<65$ years the HR was $0.95(0.86-1.05)$ and in patients $\geq 65$ years and $\geq 75$ years it was 0.84 $(0.76-0.92,0.085)$ and $0.77(0.60-0.99,0.159)$, respectively. DPP-4 inhibitors were similar to placebo for all age categories.

Key conclusions: Elderly patients with type 2 diabetes, especially those $\geq 75$ years, are more likely to benefit from the effects of GLP-1 receptor agonists and SGLT2 inhibitors on cardiovascular outcomes. Funding: This research is co-financed by Greece and the European Union (European Social Fund- ESF) through the Operational Programme «Human Resources Development, Education and Lifelong Learning 2014-2020» in the context of the project "Methodological framework for the development and updating of individualized and evidence-based guidelines. A case study on type 2 diabetes mellitus" (MIS 5047909).

\section{Abstract \# 182 \\ Glucose metabolism and lipid profile in centenarians: what can they predict? \\ Lubov Machekhina ${ }^{1}$, Ksenia Eruslanova $^{1}$, Ekaterina Dudinskaya ${ }^{2}$, Olga Tkacheva ${ }^{2}$ \\ ${ }^{1}$ Pirogov Russian National Medical University, ${ }^{2}$ Pirogov RNMU}

Aim of the study: to assess the most important metabolic factors in centenarians and their impact on mortality and prognosis.

Materials and methods: It was a longitudinal study, including 82 centenarians (95 years and older), who live in Moscow. Complex geriatric assessment and blood tests were performed. Complex geriatric assessment included past medical history, FRAIL, IADL-C, MNA, GDS-15 and MOCA scores. We also analyzed levels of HbA1c, cholesterol, LDL, HDL. QoL questionnaires were used as well. In one year after the investigations we contacted patients' relatives or social workers to find out about patients' status. The statistical analysis was performed using IBM SPSS Statistics package Version 26. Statistically significant were differences with $p<0,05$.
Results: Mean age of the patients was 98,3 [ $\pm 1,9]$ years, while $87,8 \%$ of the cohort were women. Analyzing functional status we found out that $34,4 \%$ of the patients were frail, and the number of prefrail patients was $56,2 \%$. Cognitive impairments of different severity were presented in $84,4 \%$ of the patients. The median lipids values were as follows: total cholesterol-4,8 $[4,2 ; 5,8]$, triglycerides-0.97 [0,8; 1.2], HDL-1.3 [4.2; 5,8), LDL-3,1 [2,6;3,7], $\mathrm{HbAlc}-5,8[5,6 ; 6,1]$. In $59 \%$ of the patients $\mathrm{HbA} 1 \mathrm{c}$ was below $6 \%$; $33 \%$ had concentrations between $6 \%$ and $6,4 \%$, and only in $8 \%$ we found $\mathrm{HbA} 1 \mathrm{c}$ higher than $6,5 \%$. None of the patients received any sugar-lowering therapy and only $2(3,2 \%)$ were previously diagnosed with diabetes. No correlation was also found between $\mathrm{HbA} 1 \mathrm{c}$ values and lipids profile. After dividing patients into subgroups depending on stage of carbohydrate metabolism disorders we did not notice any statistically significant differences in lipid profile in them $(p=0,005)$. Comparing survivors and non-survivors groups we did not find any significant differences in total cholesterol, LDL, HDL and HbAlc (p $<0,005)$. While comparing functional status and QoL with metabolic profile we discovered positive correlation $(r=0.834)$ between total cholesterol and index of instrumental activity, and between LDL and IADL as well as MNA score ( $r=0.732$ and 0.634 respectively).

Conclusion: In centenarians usual prognostic factors such as $\mathrm{HbA} 1 \mathrm{c}$ and lipids do not have any impact on survival but they influence functional status and QoL. Further investigations of metabolic status in super old persons are needed to personalize their lifestyle and treatment goals.

\section{Abstract \# 183}

Foot care knowledge and practices amongst Maltese individuals with diabetes: trends and determinants.

Clarice Cilia ${ }^{1}$

${ }^{1}$ University of Malta

Background: Diabetes Mellitus (DM) is one of the emergent global health emergencies of the 21st century. In 2019, The International Diabetes Federation estimated that 463 million people had DM and this number was predicted to reach 578 million by 2030 , and 700 million by 2045 . Between 2014 and 2016, the prevalence of individuals with Type 2 DM aged between 25 and 64 years in Malta was found to be $10.39 \%$ (95\% CI 9.47-11.38) (Cuschieri, Vassallo, Calleja, Pace, \& Mamo, 2016). DM not only lessens the quality of life and life expectancy but also leads to several microvascular and macrovascular complications, including those affecting with the lower limb (LL). The burden of DM-associated complications worldwide is a chief healthcare problem. Patient self-management is crucial to effectively manage DM and prevent its complications, including those of the LL. Some of the risk factors can be minimised if individuals have satisfactory knowledge of foot-care and are able and motivated to transfer that knowledge into practice (Chiwanga \& Njelekela, 2015). International studies have shown inequalities in foot-care knowledge and practices across the globe. Various potential determinants have been identified, with cultural issues being amongst those factors found to possible influence foot care. Although small scale studies on foot-care were conducted in Malta, these failed to effectively address determinants of foot-care knowledge and practice. Hence, this study was aimed to investigate the trends and determinants of foot-care knowledge and practice amongst individuals with Type 2 DM living in Malta.

Methods: A cross-sectional telephone-based quantitative study was conducted between April 2019 and January 2020. The survey tool investigated socio-demographic characteristics, foot-care knowledge and foot-care practice of the participants. Clinical data was collected via the participant's medical history record. Questionnaires were 
tested for validity and reliability. A pilot study was also conducted. 400 participants were conveniently recruited from 6 podiatry clinics across Malta. Data collected was first weighted to enhance representativeness and then analysed using univariate and multivariate logistic regression analysis.

Results: 384 individuals with Type 2 DM were interviewed, giving a response rate of $96 \%$. After weighting, data analysis was performed on 376 participants. Participants were found to have good foot-care knowledge (mean score: 12.80 CI 12.61, 13.00; total possible score: 16) and practice (mean score: 4.32 CI: $53.63,55.01$; total possible score: 75). Satisfactory knowledge was identified regarding LL complications, foot hygiene and drawbacks of barefoot walking and flip-flop use. Lack of knowledge was mainly reported about daily soaking of feet. Good foot-care practice was noted with regards to foot care hygiene, care of toenails, use of socks, corn remedies and use of external sources of heat. On the other hand, most participants did not apply a daily emollient and choice of footwear was unsatisfactory at times. Age, gender, history of ulceration and/or amputation and previous foot care education were found to determine foot-care knowledge whilst history of ulceration and/or amputation, current DM treatment and previous foot-care education were found to determine foot-care practice.

Conclusion: Findings of this study have shed light on the prevalence and determinants of foot care. The latter seem to be shaped by individuals' psychological responses to their condition, by sociocultural factors and current approaches to health service delivery. Recommendations were developed accordingly, and included amongst other, the development of a screening algorithm which will help guide the delivery of individualised foot care education during podiatry consultations. The development of formal, culturally sensitive foot-care education programme and other research around this area of practice were also recommended.

\section{Abstract \# 184 \\ Diseases flocking together}

Muhammad Shoaib Zaidi ${ }^{1}$

${ }^{1}$ King Saud University

Introduction: The autoimmune polyglandular syndromes(APS),(Types1-4), are the most common polyendocrine adenopathies. These occur as a consequence of autoimmune destruction of self-antigens in endocrine glands and other tissues.

Methodology: A case of polyglandular syndrome,most likely PGA3 ,is being described. She had Type 1 diabetes,Hashimoto's thyroiditis, vitiligo and pernicious anemia.

Result: 65 years old post-menopausal lady with Type $1 \mathrm{DM}>15$ years, Primary Hypothyroidism, Dyslipidemia, Vitiligo and Minimal 5NPDR. She was worked up previously for evolving cognitive defects e.g. memory lapses and was found to have Vit.B12 deficiency (B12$69 \mathrm{pmol} / \mathrm{l}, \mathrm{S}$. folate NA). Was treated e Inj.B12 replacement and got somewhat improved. Family Hx insignificant. Systemic review: NAD (No GI symptoms,candidiasis). Drug Hx: Metformin $1 \mathrm{gBD}$, Novorapid (10-10-6 units),Lantus, 16 units PM, Levothyroxine100 mcg/d, Aspirin, $81 \mathrm{mg} / \mathrm{d}$, Inj. Methyl Cobalamin-1mg/monthly, Ferrousfolic-once daily, Refresh eye drops. Exam: Vitally stable e no postural instability. Wt 62.6 (62 kgs in 2017), BMI-27.85kg/m ${ }^{2}$ Vitiligo+ mouth, hands and feet. No hyperpigmentation. Otherwise General and Systemic exams unremarkable. Labs: HbA1c 9.8\% (was $8.5 \%$ before),CBC-Hb\% 11.8g/l, MCV 82.2fl,ACR $7.68 \mathrm{mg} / \mathrm{gm}$, Lipids (T. Cholesterol-5.91 mmols/l, LDL-3.41 mmols/l, TGs $0.95 \mathrm{mmols} / 1$, HDL $2.07 \mathrm{mmols} / \mathrm{l}$ ), Corrected Ca++ $2.43 \mathrm{mmols} / \mathrm{l}$. TFTs (TSH 3.7
mIU/l, FT4 19.1 pmol/l). Rest WNL. Other Investigations: CT scan brain-normal, Short Synecthen Test negative (ACTH 4.8pmol/l), Anti-gastric parietal cell and Anti-Intrinsic factor Antibodies strongly positive(Anti-parietal ;47.4 units-normal: 020),Anti-Intrinsic factor Abs-69.2 units-normal 0-1.1 units). ECG \& CXR-unremarkable. Anti-Islet cell Abs-Negative,Anti-GAD-65 Abs Highly Positive5504.1 U/ml (0.0-5.0), S.Gastrin-30 pg/ml (0115). Anti-thyroid Abs: Anti TPO-1761 units $(0-100)$,Anti TG-278.34 units $(<0.6$ unitsnegative). Elective surveillance upper GI endoscopy: unremarkable apart from the finding of a $3 \mathrm{mms}$, whitish nodule in the gastric antrum. Gastric histopathology: (4 specimens).Specimen from the gastric nodule revealed mild to moderate inactive gastritis, gastric atrophy and intestinal metaplasia. Antral Bx-positive for H.pylori like organisms. Otherwise biopsies from incisura \& body of stomach showed mild to moderate inactive gastritis. Gastroenterology followup was still awaited.

Conclusion: Autoimmune disorders can co-exist and can present in close succession.With Type 1 diabetes and Hashimoto's thyroiditis, other autoimmune diseases e.g. adrenal insufficiency,vitiligo,hypoparathyroidism,candidiasis,pernicious anemia etc. can appear later on, as part of one of the polyglandular syndromes.

\section{Abstract \# 185 \\ Retinopathy detection program in diabetic patients by teleophthalmology in primary care}

Leticia Sierra-Martínez ${ }^{1}$, Rosario Martínez-Fuerte ${ }^{2}$, Francisco Javier Posadas-Alonso ${ }^{2}$, Gonzalo Calderón-Calvo ${ }^{2}$, Natalia Sanz-González ${ }^{3}$

${ }^{1}$ Valladolid Este Primary Assistance Gerency, Valladolid, Spain, ${ }^{2}$ Pilarica Health Center, Valladolid Este Primary Assistance, Valladolid, Spain, ${ }^{3}$ Parquesol Senior Center, JCyL Social Services Gerency, Valladolid, Spain

Background and aim: Carry out systematic screening of the entire diabetic population for the early detection of ocular pathology caused by Diabetes Mellitus.

Method design: Screening test based on teleophthalmology. The patient is recruited in primary care consultations and is included in the detection program with a procedure based on the capture of images by non-medical personnel, the images are sent electronically to the reading center, where they are evaluated by a team of opticians and ophthalmologists who are responsible for checking if there is any pathology; and if so, its severity, the frequency of its follow-up or, if necessary, the referral to the ophthalmologist at your hospital for a more complete study or the establishment of treatment are established.Setting: Urban health area, en Castilla y León (Spain) Population and Sample:Diabetic patients $(n=3738)$.Time period: From November 2, 2017 to October 31, 2019.

Results: Patients with diabetic retinopathy (DR) lesions: 471 $(12,60 \%)$ Patients with other pathologies: 361 (9,65\%)Patients referred to the Ophthalmology Service of the Hospital for DR: 63 $(1,7 \%)$ Patients urgently referred to the Hospital Ophthalmology Service for other pathologies: $2(0,05 \%)$

Conclusions: Teleophthalmology allows the early detection of one of the most important and frequent complications of diabetes: retinopathy associated with poor glycemic control. Examining patients remotely makes it possible to optimize the work of professionals as a result of better coordination between the different levels of care.In addition, it allows reducing referrals to the specialist's office and, consequently, reducing waiting lists, avoiding unnecessary trips for patients residing in rural areas. 


\section{Abstract \# 186}

The role of oxidative stress in type 2 diabetes to older patients G.I. Constantin ${ }^{1}$, C.M. Pena ${ }^{1}$, S. Opris ${ }^{1}$

${ }^{1}$ National Institute of Gerontology and Geriatrics "Ana Aslan", Bucharest, Romania

Introduction: Diabetes mellitus has been recognized as a chronic metabolic disease which includes a variety of syndromes with different etiologies characterized mainly by hyperglycemia. Most of the studies have suggested that oxidative stress plays a key role in the pathogenesis of diabetes and its complications. The mechanisms by which oxidative stress interfere in the appearance of vascular lesions in diabetic patients are: intensification of low density lipoproteins oxidation (oxLDL), endothelial dysfunction, vascular inflammation, activation of thrombosis processes.

Methods: The purpose of this study was to assess the LDL susceptibility to lipid peroxidation in two study groups of older patients (aged $72 \pm 6$ years): a control group and a group with type 2 diabetes mellitus (NIDDM). The LDL susceptibility to "in vitro" induced lipid peroxidation was evaluated following its incubation with a prooxidant system.

Results: The results obtained in patients with NIDDM showed a significant increase in the susceptibility of LDL to lipid peroxidation compared to the control group $(8.25 \pm 3.79$ vs $5.02 \pm 2.51 \mathrm{mmol}$ MDA / dL serum), indicating that the oxidative process increases in diabetes leading to increased LDLox levels.

Key conclusions: In conclusion, our results suggests that lipid alteration and lipoprotein oxidizability may contribute to oxidative stress in diabetes mellitus. Thus, the involvement of oxidative stress in diabetes is justified by the essential role it plays in the occurrence of specific cellular, tissue or organ lesions called oxidative lesions.

\section{Abstract \# 187 \\ The impact of physical exercise in daily living of older people with type II diabetes}

Ioannis Savvakis ${ }^{1}$, Antonia Aravantinou-Karlatou ${ }^{1}$, Eirini Stratidaki ${ }^{1}$, Athina Patelarou ${ }^{1}$, Theodoula Adamakidou ${ }^{2}$, Christos Kleisiaris ${ }^{1}$

${ }^{1}$ Department of Nursing, Faculty of Health Sciences, Hellenic Mediterranean University, Heraklion, Crete, Greece, ${ }^{2}$ Department of Nursing, Faculty of Health Sciences, University of West Attica, Athens, Crete, Greece

Introduction: Diabetes mellitus (type II) is an expected to be the seventh leading cause of death in the world by 2030 . Evidence have shown that fluctuations on glucose levels may not significantly influenced by increasing age, but mostly due to obesity and lack of physical activity. Therefore, the aim of this review is to present an overview of the effect of physical exercise on older people with type II diabetes.

Methods: A literature review was conducted in biomedical database (PubMed) using MeSH terms such as "insulin resistance", "exercise", "aerobic exercise" etc, related to physical activity improvement. Articles in language other than English were excluded. Results: Evidence has shown that Well-designed progressively increasing intense of exercise programs improves cardiorespiratory function and consequently blood glucose levels. Also improves body composition, reduces fat mass, cholesterol and triglycerides and can reduce medication and insulin administration and even inhibit it. Resistance exercise is even more effective when it is combined with diet in overweight individuals.

Conclusion: A physical activity of any kind can significantly improve the quality of life of older people with type II diabetes. Therefore, it is necessary for people with diabetes type II to include exercise and all forms of physical activity in daily living.

\section{Abstract \# 188 \\ Impact of SGLT2 inhibitors on renal function in elderly with type 2 diabetes in the first-year treatment}

Leonor P. Silva ${ }^{1}$, Catarina Almeida ${ }^{2}$, Tiago Costa $^{1}$, Elena Suarez ${ }^{1}$, Rute Lopes Caçola $^{1}$, Pedro Caiano Gil ${ }^{1}$, Luis Andrade ${ }^{1}$, Rafaela Verissimo $^{1}$

${ }^{1}$ Internal Medicine Service, Centro Hospitalar V.N.Gaia, ${ }^{2}$ Nephrology Service, Centro Hospitalar V.N.Gaia

Introduction: SGLT2 inhibitors(iSGLT2) treatment was associated with a decrease in albuminuria and has reno-protective effect in diabetic kidney disease. This study aims to evaluate the effect of iSGLT2 on glomerular filtration rate(eGFR) and albuminuria in elderly inpatients during the first year of treatment.

Methods: Retrospective cross-sectional study including elderly patients $(>65$ years) with type 2 diabetes treated with iSGLT2. Data were collected at the iSGLT2 introduction and 3, 6, 9 and 12 months of treatment.

Results: Were Included 115 elderly, 48.7\% male, mean age $72.4 \pm$ 5,2 years, median $\mathrm{HbA} 1 \mathrm{c} 8.4 \pm 1,7 \%$ and median DM2 duration of 17 \pm 12 years. Dapagliflozin was initiated in $60.9 \%$ and empagliflozin in $39.1 \%$.An eGFR of $30-60 \mathrm{ml} / \mathrm{min} / 1.73 \mathrm{~m}^{2}$ was observed in $21.7 \%$ with moderate albuminuria in $12.2 \%$ and severe albuminuria in $4.3 \%$. Throughout the first year, there was a significant reduction in $\mathrm{HbAlc}$ ( $\mathrm{p}<0.038)$. Regarding eGFR, no significant differences at the beginning and after 1 year were observed, nonetheless, a non-significant reduction was observed in the first semester, followed by a significant increase in eGFR $\left(71.37-84.93 \mathrm{ml} / \mathrm{min} / 1.73 \mathrm{~m}^{2} ; \mathrm{p}<0.006\right)$ in the second semester. As to the variation of eGFR yearlong, there were no significant differences between dapagliflozin and empagliflozin, although in the first semester, empagliflozin presented a greater variation in $\mathrm{eGFR}(\mathrm{p}=0.021)$. There was no significant reduction in albuminuria.

Conclusion: In our sample of elderly patients, there was no variation in eGFR after 1-year treatment with iSGLT2, although it reduced in the first semester, particularly with empagliflozin, followed by an improvement in the next semester. Against evidence, Albuminuria did not ameliorate during the first year of treatment.

\section{Abstract \# 189}

Evaluation of the estimated glomerular filtration rate with CKDEPI equation of older adults aged 90 years and over with diabetes

Liudmila Merkusheva ${ }^{1}$, Nadezda Runichina ${ }^{1}$, Sergey Lysenkov ${ }^{2}$, Irina Strazhesko ${ }^{1}$, Yulia Kotovskaya ${ }^{1}$, Anastasia Shvedko ${ }^{1}$, Natalia Sharashkina ${ }^{1}$, Olga Tkacheva ${ }^{1}$

${ }^{1}$ Pirogov Russian National Medical University, The Russian Clinical Research Center for Gerontology, Moscow, Russia, ${ }^{2}$ Lomonosov Moscow State University, Biological Faculty, Moscow, Russia

Aim of the study: To investigate kidney function in older adults $>90$ years using the methods of calculation of the estimated glomerular filtration rate(eGFR).

Materials and methods: 453 older adults aged $>90$ years were analysed. Patients with critical and terminal conditions were excluded. The following equations were used: CKD-EPI Creatinine(2009), CKD-EPI Cystatin C (2012) and CKD-EPI Creatinine-Cystatin C (2012). The Bland-Altman method was used to compare two measurement techniques for the same variable. 
Results: Patients were 453 (mean 92,5 $\pm 2,5$ SD years), $79.2 \%$ were female. The main group included $64(14,1 \%)$ patients with diabetes and $359(79.2 \%)$ control group without diabetes. Main group didn't differ from the control one in age $(92.0 \pm 2.3$ vs $92.5 \pm 2.4, \mathrm{p}=0.12)$ and percent of women $(85.9 \%$ vs $78.2 \%, \mathrm{p}=0.18)$. All patients demonstrated low levels of eGFR. Values of eGFR in main group vs control group by formulas: CKD-EPI Creatinine: $57,2 \pm 12,8$ vs 57,7 $\pm 13,1 \mathrm{ml} / \mathrm{min} / 1.73 \mathrm{~m}^{2}(\mathrm{p}=0.79)$, CKD-EPI CystatinC: $31,4 \pm 7,5 \mathrm{vs}$ $32,6 \pm 8,4 \mathrm{ml} / \mathrm{min} / 1.73 \mathrm{~m}^{2}(\mathrm{p}=0.31)$,CKD-EPI Creatinine-CystatinC: $41,7 \pm 9,6$ vs $42,7 \pm 9,9 \mathrm{ml} / \mathrm{min} / 1.73 \mathrm{~m}^{2}(\mathrm{p}=0.47$ ) respectively. Differences between values were decreasing along with the diabetes in means of eGFR, however, even using minimal values, one of the equation overestimates values versus another equation. It was found, that eGFR in older adults aged 90 years and over varied depending on the method of calculation with up to two-fold difference.

Conclusion: The kidney function in older adults aged $>90$ is decreased and not depends on the diabetes. For the lowest values of eGFR CKD-EPI CystatinC equation overestimates values over another equation. For estimation of the degree of decrease of eGFR more research is needed bearing in mind the significant difference in estimated values.

\section{Abstract \# 190}

\section{Ocular manifestations of diabetes in the elderly}

Alexandra Trivli ${ }^{1}$, Evridiki Patelarou ${ }^{2}$, Athina Patelarou ${ }^{2}$

${ }^{1}$ Ophthalmologist, Athens, Greece, ${ }^{2}$ Department of Nursing, Faculty of Health Sciences, Hellenic Mediterranean University, Heraklion, Crete, Greece

Introduction: Diabetes is a growing public health concern, with a prevalence two times higher among elderly people compared to middle and younger ages and an estimate of $1 / 3$ of geriatric population affected [1]. Complications of diabetes develop rapidly or over time and are the result of microvasulopathy affecting several systems and leading to events such as heart attack, stroke, kidney and ocular damage [2].

Methods: A PubMed search was conducted in English language for the last 6 years, in order to investigate and highlight various ocular complications associated with Diabetes in elderly population.

Results: The hallmark of diabetes regarding the eye is diabetic retinopathy (DR). Loss of pericytes leads to capillary damage and eventual break in the inner blood-retinal barrier. DR may be proliferative or non-proliferative and the main risk factors are longer duration of diabetes and poor metabolic control. Concomitant diseases, commonly encountered in elder patients such as hypertension, hyperlipidemia, smoking and obesity may have an adverse effect on DR. Macular edema, ischemic maculopathy, vitreous hemorrhage and retinal detachment are the main sequelae of DR [2]. Visual impairment in the diabetic patient may also be attributed to corneal pathology such as punctate or neurotrophic keratitis, tear film abnormalities and endothelial changes. Finally, glaucoma risk is higher in diabetic patients as they are more susceptible to the development of Primary Open Angle Glaucoma and its progression or they can develop neovascular glaucoma secondary to proliferative DR [3].

Key conclusions: Untreated diabetes can result in visual impairment which can further negatively impact the quality of life. Routine eye examination is essential, since $90 \%$ of vision loss from diabetes can be prevented.

\section{Abstract \# 191}

Management of community dwelling older patients in a general diabetes clinic

Christine Eileen Mc Carthy ${ }^{1}$, Sean Donohue ${ }^{2}$, Aisling O'Connor ${ }^{2}$, Marcia Bell ${ }^{2}$

${ }^{1}$ National University of Ireland Galway, ${ }^{2}$ University Hospital Galway

Introduction: Management of type 2 diabetes mellitus (T2DM) in the geriatric population is both complex and nuanced. The American Diabetic Association has dedicated recommendations in this area. Without a systematic approach, and within the time restraints of a busy clinic, areas considered in these guidelines are not always addressed. We sought to audit our practice in Galway University Hospital (GUH) in relation to these guidelines.

Method: Consecutive entries in the diabetic electronic patient record were reviewed. Entries were considered for inclusion in the audit, if the patient was born between January 1934 and January 1944, was community dwelling, and had been reviewed in the previous year for T2DM. Areas reviewed were: Assessment of geriatric domains, meeting $\mathrm{HbA} 1 \mathrm{C}$ targets, seeking hypoglycaemic episodes and considering deintensification of treatment if a patient meets criteria.

Results: 200 records were reviewed. The mean patient age was 79.7 years $(\mathrm{SD}=2.7) .56 \%$ were male $(\mathrm{n}=111)$. Assessment of geriatric domains was considered in only $22 \%$ of cases $(n=44)$. The mean $\mathrm{HbA} 1 \mathrm{c}$ was $61.8 \mathrm{mmol} / \mathrm{mol}(\mathrm{SD}=15)$, with $67.5 \%(\mathrm{n}=135)$ either at or below target. Hypoglycaemia was sought in $75 \%$ of cases $(\mathrm{n}=$ 150 ), but regime deintensification was considered in only $17.4 \%$ of cases $(\mathrm{n}=32)$.

Conclusion: Certain areas that may impact quality of life and outcomes appear to be neglected in our current model of busy undifferentiated T2DM clinics. Exploring education, a dedicated diabetic geriatric assessment form, or dedicated diabetic geriatric clinics may be options to explore.

\section{Abstract \# 192}

Attitudes and knowledge towards sun light exposure with regard to vitamin $D$ deficiency: a survey of Maltese medical doctors

Maria Bonnici ${ }^{1}$, Sacha Buttigieg ${ }^{2}$, Stefania Abdilla ${ }^{2}$, Peter Ferry ${ }^{1}$

${ }^{1}$ Karin Grech Rehabilitation Hospital, Malta, ${ }^{2}$ Primary Health Care, Malta

Background: Vitamin D deficiency is increasing worldwide. Despite high ultraviolet irradiation in southern European countries, vitamin D levels were found to be low in older adults. Doctors are a crucial source of information to their patients and therefore this study attempts to assess knowledge and attitudes of Maltese doctors on sunlight exposure with regard to vitamin D deficiency.

Methods: A cross-sectional survey of all Maltese doctors was carried out, excluding doctors that do not come into frequent contact with older adults and doctors who were not currently practicing/residing in Malta. In order to reach a $95 \%$ confidence interval with a margin of error of $5 \%$ a total of 847 medical practitioners were included. The survey was disseminated via an online link sent by e-mail and a hard copy via post. Descriptive statistics were used for data analysis.

Results: The final population included 138 participants. $82.6 \%$ believe that exposure to outdoor sunlight is the main source of vitamin D in Malta. 44.2\% managed vitamin D deficiency by giving advice to receive more natural sunlight. In summer $73.9 \%$ advice their patients to use sun protection at all times during the peak UV index. During the winter, $25.4 \%$ still suggest to use sun protection at all times during peak UV index, with $19.6 \%$ either giving the advice to use sun protection most of the time during peak UV index, but to 
receive some direct sunlight, or to not use sun protection outside peak UV times and receive direct sunlight during this time.

Conclusions: A national clinical guidance on sun exposure with regard to vitamin D deficiency is recommended to be available for all Maltese doctors.

\section{Abstract \# 193 \\ How to successfully promote adherence to geriatric interventions in socialization \\ Pascale Dinan ${ }^{1}$, Sylvie Bonin-Guillaume ${ }^{2}$, Helene Petit-Bhoyroo ${ }^{3}$ \\ ${ }^{1}$ Geriatrician, Member of EAMA and SFGG, Mauritian Republic, ${ }^{2}$ Geriatrician, Marseilles CHU, France, ${ }^{3}$ Rheumatologist, Member Of Mauritian And French Rheumatologist Society and the GRIO}

Loneliness is a prevalent problem for adult populations and yields negative feelings of depression, psychological stress and pain (1). Is is also linked with multiple chronic conditions such as metabolic and cardiovascular disease, overall morbidity and mortality. It is important to create social connectedness through which one has constructive relationships with individuals and groups. Many systematic reviews confirm that the most effective interventions are group interventions aimed at increasing skills, strengthening social support, increasing social interactions and socio cognitive training. They are more effective when their programs are individualised, adapted culturally to the participants, when they aim to promote a healthy active lifestyle and when done on a regular routinely basis $(2$, $3)$. As an example we can refer to the multi-ethnic and multi-cultural ageing society of the Mauritian Republic, ranked highest in the African region (42th position) in the Global Age Watch Index 2015 measuring social and economic wellbeing of elderly $(4,5)$. The seniors can become engaged in geriatric interventions through free service non governmental associations (International Federation of Associations for elderly FIAPA and Alzheimer Association Mauritius, Third Age Mauritius University) and parastatal bodies (Senior Citizen Council of Mauritius comprising of 850 senior groups working for their well-being). The geriatric interventions in socialization include recreational and physical activites, various activities in retirement homes, life long learning and education, geriatric talks, cognitive stimulation in day care centers for Alzheimer patients. By promoting adherence to socialization a society of geriatrics and gerontology is thus emerging in the Indian Ocean region where health care professionals can share knowledge, organise congresses. Hence, it has a facilitating role on geriatric interventions in terms of training of care partners and continuous medical education, in raising awareness on geriatric topics towards different sectors of the community and in ensuring that government policy is on the right track (6).

\section{Abstract \# 194 \\ A cross-professional qualification tool to optimized pharmaceutical care for geriatric patients (OAV)}

Alina Katharina Lente ${ }^{1}$, Frank-Christian Hanke ${ }^{2}$, Hans Jürgen Heppner $^{1}$

${ }^{1}$ Department of Geriatrics, Witten/Herdecke University, ${ }^{2}$ Gero PharmCare Ltd.

Introduction: The progress of modern medicine makes a longer life possible. At the same time, the combination of drugs can trigger serious diseases, so called adverse drug events (ADEs). Especially in the case of elderly patients with multiple morbidities and correspondingly extensive medication plans the dimensions of these events are greatly underestimated. In nursing care, 60 to 80 percent of these events are due to a lack of monitoring of therapy among other things [1]. The aim of the project OAV is to prevent or reduce ADEs through interprofessional team-partnership. Through interprofessional collaboration it is possible to increase medication safety in the long term [2]. This means that the various disciplines must expand their usual action patterns and cooperate. For this purpose, a cross-professional qualification tool was developed that builds on the previous experiences and resources of all actors involved (nursing staff, physicians and pharmacists) and sustainably increases the competence of the actors to guarantee treatment safety.

Method: To determine the contents of the qualification tool, a document analysis was carried out. Existing curricula and corresponding professional articles/books served as a basis. The results were evaluated in several discussion rounds and finally condensed into a curriculum. The qualification tool is finally composed of six modules. One module comprises four chapters in which the technical and social competences are built up step by step.

Results: It is expected that the qualification tool will improve attention to drug therapy safety and thus reduce drug-induced risk situations for elderly patients. 1. Gurwitz JH, Field TS, Harrold LR et al. (2003) The incidence of adverse drug events in two large academic long-term care facilities. The American journal of medicine 118:251-258. 2. Köberlein-Neu J, Mennemann H, Hamacher S et al. (2016) Interprofessional Medication Management in Patients With Multiple Morbidities. Deutsches Arzteblatt international 113:741-748.

\section{Abstract \# 195}

Perception and motivation of residents in elderly care medicine concerning evidence-based medicine education

Wing Tong ${ }^{1}$, Belinda Ommering ${ }^{2}$, Jenny van der Steen ${ }^{1}$

${ }^{1}$ Department of Public Health and Primary Care, Leiden University Medical Center, Leiden, The Netherlands, ${ }^{2}$ Center for Innovation in Medical Education, Leiden University Medical Center, Leiden, The Netherlands

Introduction: Evidence-based medicine courses are part of a resident internship of the elderly care physician training programs in the Netherlands. Residents of Leiden University Medical Center (LUMC) also perform mandatory healthcare research (termed "WLO"). Recently, LUMC-researchers found that in undergraduate medical students, intrinsic motivation influences research involvement. The aim of our study is to examine perception and motivation of residents in elderly care medicine to conduct research and to evaluate attitudes around the mandatory WLO study.

Methods: An educational ethics committee approved this mixedmethods study (NVMO: 2009.7.7). Residents of three groups that started March 2018, March 2019, and September 2019 were asked to participate. A questionnaire included validated perception and motivation scales with response options ranging 1 to 7 . Qualitative interviews which also examined attitude regarding the WLO study were underpinned by interpretative phenomenological analysis.

Results: Of 38 residents, 23 questionnaires were obtained (15 from female residents). The mean score on perceptions of research was 4.1 (SD 0.8), intrinsic motivation 4.8 (SD 1.0), and extrinsic motivation 4.3 with a higher SD of 1.4. There was also diversity in attitudes concerning research and the WLO study, related to any previous experiences in conducting research. Eight interviews indicated that doing research was experienced as difficult, in particular statistical analyses of the WLO data.

Key conclusions: Residents show diversity of attitudes, and we found low motivation to conduct medical research compared with 
undergraduate students. This knowledge will be used to improve the mandatory WLO study.

\section{Abstract \# 196 \\ Educational policies and legislation at European level for LGBT+ inclusive education: building a MOOC}

Antonio López-Villegas ${ }^{1}$, Remedios López-Liria ${ }^{2}$, Patricia Rocamora-Pérez ${ }^{2}$, Alfonso Pezzella ${ }^{3}$

${ }^{1}$ CTS-609 Research Team, Poniente Hospital, El Ejido, Spain. ${ }^{2}$ Hum498 Research Team, Health Research Centre, University of Almería, Almería, Spain., ${ }^{3}$ Research Centre for Transcultural Studies in Health, Middlesex University, London, United Kingdom

Introduction: The European project IENE9 "Developing a culturally competent and compassionate LGBT+ curriculum in health and social care education " $\neg$ aims to enhance the skills regarding LGBT+ issues and develop teaching tools to support their inclusion.

Methods: Internet mapping and systematic documentation to develop a list of policy recommendations towards improving and safeguarding LGBT+ people. Seven countries (UK, Spain, Italy, Cyprus, Romania, Denmark, Germany) co-work to create an easy to navigate resource with information about legislations/guidance/policies. Finally, development of the training courses with tools and methodologies to strengthen theoretical and practical knowledge and skills on LGBT+ issues, and to affect attitudes and prejudices in the relationship with LGBT+ patients/users who ask for psychological support or health care.

Results: There is a growing awareness of the need for LGBT+ competency training to ensure that the health and social care services offered to LGBT+ people are suitable and gender sensitive. Emerging lines of inquiry to improve health include populations such as LGBT+ veterans, reproductive health and parenting, intimate partner violence, sexual minority persons living with chronic illness, disability, etc.

Key conclusions: In some cases, healthcare professionals do not know how to approach and provide treatment to LGBT+ persons and admit their need for specific training. The program aims to improve this environment, with observance of human rights and free from all forms of discrimination and violence. A new culturally competent and compassionate LGBT+ curriculum will be developed and delivered through a MOOC for health and social care teachers/workers and learners worldwide.

\section{Abstract \# 197 \\ Interdisciplinary strategy for promoting mobility in hospitalized elderly patients \\ Maria Elena Castro Vilela ${ }^{1}$, Miguel Sanchez Ortiz ${ }^{2}$, Purificación Montero Cercos ${ }^{3}$, Carlos Gala Serra ${ }^{3}$, Inma Ponz Flor ${ }^{3}$, Mercedes Forcano Garcia ${ }^{3}$ \\ ${ }^{1}$ Hospital San Jose, Teruel, Spain, ${ }^{2}$ Hospital San Jose, Teruel, Spain, ${ }^{3}$ Hospital San Jose, Teruel, Spain}

Introduction: To know the prevalence of functional decline in hospitalized elderly patients and implement an early mobilization strategy, with the participation of patients, caregivers and health workers.

Method: Interdisciplinary team intervention. The prospective study enrolled patients admitted to the acute geriatric unit, between April and June 2018. Patients excluded: those in palliative care, with severe dementia and with Barthel Index: $<20$. Patients were divided into three levels: Level A: able to walk, B: able to transfer to a chair, C: not able to stand. A complete evaluation of mobility was conducted, during admission, hospitalization and discharge. Clinical and epidemiological characteristics were collected.

Results: Creation of posters to identify mobility level, multicomponent exercise protocols and mobilization activities for each level, design of mobility promotion posters and brochures. 32 patients out of 102 admitted to the study period were included. Average age 86.6. Average length of stay: 9.8 days, Barthel index median at the admission: 62.2 and at the discharge: 57.6, Mini Nutritional Assessment Short Form: 9.22. Mobility Level Registration: 90\%. Patients and/or family members who received brochure:80\%. Functional decline associated with hospitalization: $31.2 \%$ (70\% male, $50 \%$ older than 90 years old, $70 \%$ institutionalized, $50 \%$ moderate dependence, $80 \%$ dementia, $60 \%$ malnourished, $90 \%$ delirium)

Conclusions: The establishment of an early mobilization strategy is possible with the involvement of the health workers, patients, and caregivers. $\bullet$ Functional decline among hospitalized elderly patients is common but with an early intervention, this can be reduced.

\section{Abstract \# 198}

Does age and sex matter among the relationship between history of physical activity level and functional capacities and body composition in elderly people?

Buckinx Fanny ${ }^{1}$, Peyrusque Eva ${ }^{1}$, Granet Jordan ${ }^{1}$, Aubertin-Leheudre Mylène ${ }^{1}$

1(1) Department of Exercise Science, Groupe de recherche en activité physique adapté (GRAPA), Université du Québec à Montréal, Montréal (Qc), Canada (2) Centre de Recherche de l'Institut Universitaire de Gériatrie de Montréal, Montréal (Qc), Canada

Objective: Being active and history of physical activity (PA) habits are nowadays recognized as important predictor to prevent physical aging. However, it is unclear if sex or age influence this relationship and if a sub-type of voluntary PA is more efficient to maintain healthy aging. Thus, we aim to assess the impact of the last 5-years of PA level and functional capacities and body composition among elderly people.

Material and methods: Functional capacities using different validated tests (i.e. grip strength, Timed Up and Go, sit-to-stand, muscle power, alternate step test, leg extension, $\mathrm{VO} 2$ max), body composition (fat \& fat-free masses) using DXA were assessed. Last 5-years of global (total) and specific (aerobic, resistance or body and mind) PA levels (duration) were obtained through a questionnaire. Multiple regressions, adjusted on age, sex and BMI, were performed to assess the relationship between past PA level and body composition or functional capacities. Sub-group analysis, according to the sex and age ( $<65$ year vs. $\geq 65$ year) were also performed using Pearson Correlations.

Results: 525 subjects $(61.7 \pm 8.1$ year; women: $68.9 \%$; $\mathrm{BMI}=26.4$ $\pm 4.8 \mathrm{~kg} / \mathrm{m}^{2}$ ) were enrolled. After adjustment on confounding factors, past level of PA has no impact on functional capacities and body composition, regardless of sex. Among people under 65 years, there is no relationship between time spent on total physical activity and functional capacities or body composition. However, our study found a significant correlation between total physical activity and balance $(\mathrm{r}$ $=0.19 ; \mathrm{P}=0.01)$, alternate-step test $(\mathrm{r}=0.24 ; \mathrm{P}=0.02)$ and $\mathrm{VO} 2 \max$ $(\mathrm{r}=0.19 ; \mathrm{P}=0.02)$ in people aged 65 and over. More precisely, the time spent on cardio and resistance activities influence balance $(\mathrm{r}=$ $0.16 ; \mathrm{P}=0.03$ and $\mathrm{r}=0.15 ; \mathrm{P}=0.04$, respectively) in this age category.

Conclusion: Our results highlights that PA history has little or no influence on functional capacities and body composition in healthy aging population. 


\section{Abstract \# 199 \\ Nurses' evidence-based practice competence as essential element of high-quality healthcare}

Stefania Schetaki ${ }^{1}$, Christos Kleisiaris ${ }^{2}$, Antonia AravantinouKarlatou $^{2}$, Symeon Panagiotakis ${ }^{3}$, Evridiki Patelarou ${ }^{2}$, Athina Patelarou $^{2}$

${ }^{1} 1$. Department of Nursing, Faculty of Health Sciences, Hellenic Mediterranean University, Heraklion, Crete, Greece. 2. Annousakeio Therapeutic Center Impatient Recovery and Rehabilitation Center "Agios Spyridon", Kisamos, Crete, Greece, ${ }^{2}$ 1. Department of Nursing, Faculty of Health Sciences, Hellenic Mediterranean University, Heraklion, Crete, Greece, ${ }^{3} 3$. Geriatrics Clinic, University General Hospital of Heraklion, Heraklion, Greece

Introduction: Evidence-based practice (EBP) has been defined as "the integration of the best research evidence with clinical expertise and patient values" [1]. Research highlights that EBP improves healthcare quality, reliability and patient outcomes as well as reduces variation in care and integrates cost-effective methods [2]. Nevertheless, individual and organizational barriers can hamper the implementation of EBP in healthcare system such as, inadequate EBP knowledge, skills and attitude, lack of education, limited leadership support and no access to EBP mentors [3, 4].

Methods: The authors carried out a bibliographic search using Medline database for scientific papers published over the last decade. Search terms used were chosen from the USNLM Institutes of Health list of MeSH (Medical Subject Headings).

Results: Even with these tremendous benefits the implementation of EBP is still not embraced in many health care systems across the globe because of health professionals' lack of EBP competence [5]. Nurses represent the largest group of health professionals, so it is essential for health care system nursing personnel to engage with EBP. Specific characteristics determine and define the key competences toward EBP $[4,5]$. Particularly, EBP application into clinical practice requires nurses' positive EBP-attitude in combination with high level of EBP-knowledge, skills and utilization [4, 5]. The first step to this direction is the evaluation of nurses' level of EBP competence and the assessment of their educational needs.

Key conclusions: The high level of nurses' competence can bridge the gap between EBP and clinical practice by supporting system in healthcare institution and clinical education [6]. Ensuring that nurses are well-educated and have achieved high level of EBP competence will lead to EBP implementation into clinical practice and quality improvement promotion $[4,5]$.

References: 1. Sackett, D. L., Strauss, S. E., Richardson, W. S., Rosenberg, W., \& Haynes, R. B. Evidence based medicine: how to practice and teach EBM. 2000; Edinburgh: Churchill Livingstone. 2. Yildiz, E., \& Güngörmüs, Z. The validity and reliability study of the Turkish version of the evidence-based practice evaluation competence questionnaire. Nurse education today, 2016; 45, 91-95. https://doi.org/10.1016/j.nedt.2016.05.030. 3. Melnyk BM, Gallagher-Ford L, Long LE, \& Fineout-Overholt E. The establishment of evidence-based practice competencies for practicing registered nurses and advanced practice nurses in real-world clinical settings: Proficiencies to improve healthcare quality, reliability, patient outcomes, and costs. Worldviews on Evidence-Based Nursing. 2014;11(1), 5-15. https://doi.org/10.1111/wvn.12021. 4. Patelarou AE, Laliotis A, Brokalaki H, Petrakis J, Dafermos V, Koukia E. Readiness for and predictors of evidence base practice in Greek healthcare settings. Appl Nurs Res. 2017;35:64-70. https://doi.org/10.1016/ j.apnr.2017.02.003. 5. Melnyk BM, Gallagher-Ford L, Zellefrow C, Tucker S, Thomas B, Sinnott LT, \& Tan A. The first US study on nurses' evidence-based practice competencies indicates major deficits that threaten healthcare quality, safety, and patient outcomes. Worldviews on Evidence-Based Nursing. 2018; 15(1), 16-25. https://doi.org/10.1111/wvn.12269. 6. Stevens K R. Essential competencies for evidence-based practice in nursing. Academic Center for Evidence-Based Practice, University of Texas Health Science Center at San Antonio. 2009; https://doi.org/10.3928/01484834-20090515-06

\section{Abstract \# 200}

\section{Are there any barriers on the way to evidence-based nursing} practice adoption?

Stefania Schetaki ${ }^{1}$, Christos Kleisiaris ${ }^{2}$, Antonia AravantinouKarlatou $^{2}$, Eirini Stratidaki ${ }^{2}$, Evridiki Patelarou ${ }^{2}$, Athina Patelarou ${ }^{2}$

${ }^{1} 1$. Department of Nursing, Faculty of Health Sciences, Hellenic Mediterranean University, Heraklion, Crete, Greece 2. Annousakeio Therapeutic Center Inpatient Recovery and Rehabilitation Center “Agios Spyridon", Crete, Greece, ${ }^{2}$ 1. Department of Nursing, Faculty of Health Sciences, Hellenic Mediterranean University, Heraklion, Crete, Greece

Introduction: The implementation of evidence-based practice (EBP) has tremendous benefits in health care systems including the quality improvement, reliability, and patients' best outcomes [1]. As nurses' play a crucial role in the delivery of health care, they need to embrace new and innovative techniques to provide effective and best possible treatment to their patient. Nevertheless, it was found that several institutional and personal barriers were hindering nurses' for adopting EBP [2]

Methods: The authors carried out a bibliographic search using Medline database for scientific papers published over the last decade. Search terms used were chosen from the USNLM Institutes of Health list of MeSH (Medical Subject Headings).

Results: The international literature highlights that the majority of nursing personnel holds a positive attitude towards EBP, but individual and organizational barriers can hamper the implementation of EBP [3]. According to experts, the top barrier is the lack of nursing competencies toward EBP in terms of knowledge, skills and attitude. Furthermore, lack of time, low self-confidence, absence of EBP mentors and limited leadership can influence the quality of nursing practice [4]. Research has found that other common barriers to EBP embracement in the clinical setting are the lack of informational resources, poor understanding of research findings, appraising and synthesizing the evidence and resistance to change [4].

Key conclusions: Multiple barriers influence implementation EBP in clinical practice. Most frequently cited barriers included limited EBP competence and lack of training and education programs. Disinterest in implementing a new way of practice on the part of the individual or organization and lack of availability of materials and resources for training are potential barriers to implement evidence-based practice in clinical practice.

References: 1. Melnyk BM, Gallagher-Ford L, Zellefrow C, Tucker S, Thomas B, Sinnott LT, \& Tan A. The first US study on nurses' evidence-based practice competencies indicates major deficits that threaten healthcare quality, safety, and patient outcomes. Worldviews on Evidence-Based Nursing. 2018; 15(1), 16-25. https:// doi.org/10.1111/wvn.12269. 2. Majid, S., Foo, S., Luyt, B., Zhang, X., Theng, Y. L., Chang, Y. K., \& Mokhtar, I. A. (2011). Adopting evidence-based practice in clinical decision making: nurses' perceptions, knowledge, and barriers. Journal of the Medical Library Association: JMLA, 2011; 99(3), 229. https://dx.doi.org/ 10.3163\%2F1536-5050.99.3.010. 3. Patelarou AE, Laliotis A, Brokalaki H, Petrakis J, Dafermos V, Koukia E. Readiness for and predictors of evidence base practice in Greek healthcare settings. ApplNurs Res. 2017;35:64-70. https://doi.org/10.1016/j.apnr. 2017.02.003. 4. Labrague, L. J., McEnroe-Pettite, D., Tsaras, K., D'Souza, M. S., Fronda, D. C., Mirafuentes, E. C., ... \& Graham, M. 
M. Predictors of evidence-based practice knowledge, skills, and attitudes among nursing students. In Nursing forum 2019; (Vol. 54, No. 2, pp. 238-245). https://doi.org/10.1111/nuf.12323

\section{Abstract \# 201 \\ Designing a holistic interdisciplinary community model of care to improve compliance of patients with fragility fractures through educational and strengthening processes}

Konstantinos Stolakis ${ }^{1}$, Christos Marneras ${ }^{2}$, Theodoros Tosounidis ${ }^{3}$, Elias Panagiotopoulos ${ }^{4}$

${ }^{1}$ University of Patras (U.P), Medicine School of Patras (M.S.P), Greece, ${ }^{2}$ University Hospital of Rio, Patras (U.H.P), Greece, ${ }^{3}$ Department of Orthopaedic Surgery, University Hospital of Heraklion (U.H.H), Crete, Greece, ${ }^{4}$ Rehabilitation Department, University of Patras (U.P), Rio-Patras, Greece

Introduction: The aim of the study was to develop an innovative model for the holistic interdisciplinary approach of elderly over 65 who had a fragility fracture in order to improve their quality of life. Method: Through literature review, good practices abroad were studied, as well as Greek efforts made to deal with fragility fractures. It was found that most programs are based on patient mobilization with a focus on exercise, nutrition and pharmaceutical interventions, in a dual effort for the treatment of polypharmacy and osteoporosis. Results: A key finding for the Greek reality was that the main problem in supporting efforts for elderly patients with fragility fractures outside the hospital was the low compliance to the guidelines. Based on this finding, the idea of a Community model of care was developed with the aim of empowering elderly patients with fractures and improving the level of their compliance, in order to avoid a second fracture. A six-month multilevel, digital intervention program, entitled "School of Healthy Aging", was designed, including: Individual physical exercise, Nutritional education, Falls Prevention Training, Search of spirituality, Development of an experiential relationship with interdisciplinary team members.

Conclusions: This model is in the process of implementation, as it will be applied to a group of twenty (20) elderly patients in the second semester of 2020 and its results will be published.

\section{Abstract \# 202 \\ Overview of Junior geriatricians' profile: training, activities and risk of burnout: results of a French national survey}

Tannou Thomas ${ }^{1}$, Jobard Nathalie ${ }^{2}$, Boisnet Amélie ${ }^{3}$, Leroy Victoire $^{4}$, Piccoli Mathieu ${ }^{5}$

${ }^{1}$ Gériatrie, CHU de Besançon, France, ${ }^{2}$ Gériatrie, Hopital Lyon Sud, France, ${ }^{3}$ Association des jeunes Gériatres, ${ }^{4}$ Gériatrie, CHU de Lille, France, ${ }^{5}$ Geriatrics Department, APHP, Centre University of Paris, site Broca, France

Introduction: Geriatrics is a professional field that has evolved rapidly throughout the world, particularly in recent years. Thus, the individual profiles leading to the practice of our speciality are varied. The French Association of Young Geriatricians aimed, , through its 2020 national survey, to provide a picture of the profile and training of geriatricians in their early carreer (specialist geriatricians with less than 8 years experience).

Methods: The survey was carried out in the form of an online questionnaire sent to members throughout France. It consisted of a three-part structured form:(1) description of academic training, (2) description of the mode of practice and (3) description of personal background. Finally, the wishes for future practice and the feeling of burnout risk were collected.

Results: One hundred and fifteen participants responded to the survey. Their responses confirm the eclecticism of geriatrician profiles. Nevertheless, certain trends emerge. In particular, it can be noted that initial training is mainly a qualification in general medicine. Most of the practice is full-time, with multiple activities (predominance of regular activity in acute care combined with consultation activity) and participation in permanent care. The practice assignments are mainly fixed-term contracts. More than half of those surveyed feel that they are or have been in an episode of potential burnout. Finally, most respondents are female, in relationships, having young children.

Conclusions: This first national survey describing the profiles of geriatric practices could serve as a support to support the evolution of our profession.

\section{Abstract \# 203}

Geriatric ultrasound training program for general practitioners

Vito Carrieri ${ }^{1}$, Antonella Bray ${ }^{1}$, Giovanni Argentieri ${ }^{1}$, Vincenzo Paternò $^{1}$, Gloria Mazelli ${ }^{1}$, Luca Di Lena ${ }^{1}$

${ }^{1}$ Geriatrics Department Hospital “A.Perrino” Brindisi Italy

Introduction: The professional activity of General Practitioners(GPs)is prevalent with commitment of time and resources for patients over 65 years. Objectives of the work are to illustrate a Geriatric Ultrasound training program for GPs,according to practical clinical ultrasound objectives and starting from real clinical cases,and to evaluate the impact on the approach of GPs to the problems of elderly patients.

Materials and methods: A 5 day Geriatric Ultrasound traininig program was carried out addressed to the Gps,with theoretical and practical activities in the department of Geriatrics.10 groups of GPs were set up each of 3 people, 10 tutors were used,who carried out teaching activities. Each of the 30 GPs has personally run at least 30 US. Every day 2 hours of interactive lessons with presentation of clinical cases and 3 hours of practical activity were performed. Some clinical situations have been examined,both from an ultrasound and from a clinical point of view:chronic heart failure, liver cirrhosis, chronic renal failure, urinary retention, acute renal failure, jaundice, weight loss, lymphadenomegaly and fever, severe anemia, digestive haemorrhage, abdominal pain, intestinal obstruction, digestive haemorrhage, hematuria, abdominal volume increase, pulsating abdominal mass, neck mass, pelvic mass, rectorrhagia, metrorrhagia, hydrocele.

Results: After the 5 day training program,all GPs were much more motivated in dealing with acute and chronic practical problems of geriatric patients.The US performed in selected patients in relation to the clinical problem and symptoms frequently encountered in clinical practice, has allowed the correlation of symptoms, diagnostic hypotheses and ultrasound findings. The Gps easily formulated diagnostic conclusions,prescribed the further diagnostic procedure or subsequent therapy with high appropriateness. A questionnaire proposed to GPs 6 months after the ultrasound training program, allows to confirm that the approach to the clinical problems of the elderly patient, examined with ultrasound in the training program,allows to observe a better outpatient management.The numbers of specialist consultations and of hospitalizations were reduced by $30 \%$ compared to the usual management methods before attending the Geriatric Ultrasound training program.

Conclusions: The results obtained demonstrated the usefulness of ultrasound competence for GPs in patients over 65 years.The ultrasound examination also has allowed to detected unknown diseases of the elderly patients. The Gps can learn the easy management of the 
elderly patient, without invasive diagnostic methods.The increase in the prescribing appropriateness of GPs confirms that ultrasound is an indispensable method for daily clinical practice of Gps in the management of geriatric patients.

\section{Abstract \# 204 \\ How clinically vulnerable doctors can contribute during a pandemic - lessons from COVID-19}

Marianne Phillips ${ }^{1}$, Catriona Davidson ${ }^{1}$, Harriet Mark ${ }^{1}$, Parth Patel ${ }^{1}$, Stefanos Kateroglou ${ }^{1}$

${ }^{1}$ Addenbrookes, Cambridge University Hospital

Introduction: In March 2020, in response to the COVID-19 pandemic, the UK Government classified approximately 1.5 million individuals as extremely vulnerable and advised them to "shield" [1]. Shielding comprised advice to stay indoors and avoid contact with the general population. This included a minority of UK healthcare workers who faced a complete change in working, at a time when the pressure upon both hospitals and colleagues was set to increase. The feeling of responsibility permeates all aspects of a doctor's life and hence doctors cannot simply leave their working identity at the hospital [2]. We describe our experience as a small cohort of "shielded" doctors from a large Teaching Hospital. Whilst working from home, under the supervision of a Consultant Geriatrician, we instigated a number of projects to support patients and colleagues.

Methods: A policy on remote working for self-isolating medical staff was ratified in April 2020. The team comprised of 1 Consultant Geriatrician and 5 trainees. Clinicians undertook three strands of working practice: clinical work, education and research.

Results: Clinical work: a telemedicine service was established to proactively manage patients with COVID-19 at home. Educational work: a medical emergencies handbook was developed and disseminated throughout the region to support re-deployed clinicians. In addition, online lectures for medical students and doctors were provided to replace face-to-face teaching. Research: involvement in a number of projects including data collection for a multi-centre study. Conclusion: Despite being confined to home we feel that we have made a significant contribution to the efforts in tackling COVID-19. References: (1) Guidance on shielding and protecting extremely vulnerable persons from COVID-19: https://www.gov.uk/ government/publications/guidance-on-shielding-and-protectingextremely-vulnerable-persons-from-covid-19/guidance-on-shieldingand-protecting-extremely-vulnerable-persons-from-covid-19 [23/05/ 2020]. (2) Wessely, A. \& Gerada, C. When doctors need treatment: an anthropological approach to why doctors make bad patients. BMJ 347, (2013).

\section{Abstract \# 205}

\section{What does COVID-19 mean for inter-professional education?}

Louise Thornhill $^{1}$, Gillian Shepherd ${ }^{1}$, Sanja Thompson ${ }^{1}$

${ }^{1}$ Oxford University Hospital Trust

Geriatric medicine could be considered the epitome of multidisciplinary patient management. It requires the co-operative input of physicians, nurses, speech and language therapists, physiotherapists, occupational therapists and more. Their effective collaboration is vital to improve overall health outcomes in the geriatric population. The underlying concept of inter-professional education (IPE) is that a workforce trained in an inter-professional environment will be better equipped to work within the multidisciplinary team (MDT), leading to improved outcomes and care of older patients. During recent years, the Geratology department of Oxford University Hospital, in conjunction with the Oxford Medical School, and Oxford Brooks University have been establishing a curriculum of IPE and teaching using case-based discussions involving students from a multitude of training programs. The COVID-19 pandemic and its social distancing requirements have significantly disrupted student teaching. With little hope of resuming normal face-to-face teaching programs in the near future, we have been evaluating how to safely deliver IPE in the current climate. Methods of distanced IPE teaching used by other institutions include online seminars, presentations, teaching modules, discussion forums for case studies, virtual clinic and video conferencing and live grand rounds. In the upcoming months we aim to restart Geratology IPE teaching using a modified teaching curriculum. We intend to use strategies such as prerecorded communication skills scenarios with professional actors and online presentations, with live student participation. This is with the understanding that flexibility in plans will be required. The outcome of our socially distanced teaching will be evaluated by students and educators.

\section{Abstract \# 206}

Reduction of family caregivers' burden by an innovative videobased remote coaching system with a multimodal comprehensive care methodology for dementia: a feasibility study

Masaki Kobayashi ${ }^{1}$, Saki Une ${ }^{1}$, Sumio Yasutake $^{2}$, Miwako Honda ${ }^{1}$

${ }^{1}$ Department of Geriatric Medicine, National Hospital Organization Tokyo Medical Center, ${ }^{2}$ Haradoi Hospital

Background: Family caregivers experience psychological, physical, and social burdens in caring for patients with dementia. This study was conducted to assess the feasibility of a video-based remote coaching system with multimodal comprehensive care methodology training to reduce the care burden of family caregivers of people with dementia at home.

Methods: A pre-post prospective study was designed. The participants were six family caregivers. First, they received on-site training for a multimodal comprehensive care methodology. Subsequently, participants recorded their care at home on an iPad and sent to the coach online. The video was evaluated by the innovative system which recorded the comments of the coach, and simultaneously drew recommended movements of caregivers with arrows which was recorded on the same video. The participants reviewed the video and practised at home. The duration of coaching was three months. Feasibility was analysed using the Zarit Burden Interview (ZBI) score, semistructured interviews, and questionnaires.

Results: Four participants completed the three-months follow-up. The reason for the dropout was a difficulty in using an iPad. The ZBIscore decreased $(41.3-40.0, \mathrm{P}=0.95)$, but was not statistically significant. The four participants were satisfied with the programme as revealed in interviews.

Conclusions: This feasibility study of an innovative remote coaching system with multimodal care methodology can reduce family caregiver burden for patients with dementia. An expanded study needs to be followed.

\section{Abstract \# 207}

Analysis of sedentary behaviour patterns in nursing home residents

Pau Farrés-Godayol ${ }^{1}$, Sandra Rierola-Fochs ${ }^{1}$, Anna Escribà-Salvans ${ }^{1}$, Miriam Molas-Tuneu ${ }^{2}$, Pau Moreno-Martin², Vinícius R. de Oliveira $^{3}$, Meltem Yildirim ${ }^{2}$, Albert Oliveras-Fabregas ${ }^{2}$, Dyego Leandro Bezerra de Souza ${ }^{4}$, Javier Jerez-Roig ${ }^{2}$, Maria Giné-Garriga ${ }^{5}$ 
${ }^{1}$ Research group on Methodology, Methods, Models and Outcomes of Health and Social Sciences (M3O). Faculty of Health Sciences and Welfare. Centre for Health and Social Care Research (CESS). University of Vic-Central University of Catalonia (UVic-UCC)., ${ }^{2}$ Research group on Methodology, Methods, Models and Outcomes of Health and Social Sciences (M3O). Faculty of Health Sciences and Welfare. Centre for Health and Social Care Research (CESS). University of Vic-Central University of Catalonia (UVic-UCC), ${ }^{3}$ Research group on Methodology, Methods, Models and Outcomes of Health and Social Sciences (M3O). Faculty of Health Sciences and Welfare. Centre for Health and Social Care Research (CESS).

University of Vic-Central University of Catalonia (UVic-UCC) AND Department of Physical Therapy. EUSES University School. University of Barcelona-University of Girona (UB-UdG), ${ }^{4}$ Research group on Methodology, Methods, Models and Outcomes of Health and Social Sciences (M3O). Faculty of Health Sciences and Welfare. Centre for Health and Social Care Research (CESS). University of Vic-Central University of Catalonia (UVic-UCC) AND Federal University of Rio Grande do Norte, Department of Collective Health, Graduate Programme in Collective Health, Natal, Brazil, ${ }^{5}$ Blanquerna Faculty of Psychology, Education and Sport Sciences, Ramon Llull University, Barcelona, Spain. AND Blanquerna Faculty of Health Sciences, Ramon Llull University, Barcelona, Spain.

Introduction: Nursing home $(\mathrm{NH})$ residents are the least active segment of the population. This study aims to describe the sedentary behaviour (SB) patterns in $\mathrm{NH}$ residents.

Methods: A cross-sectional observational study (Clinical Trials NCT04297904) was conducted in 4 NHs in the Osona county (Barcelona, Spain). The dependent variable was SB evaluated with ActivPAL3 (AP) during 7 days including: number of steps, total SB time in hours (h), total upright time in $\mathrm{h}$, number of sit to stand transitions (STST), total awake $\mathrm{h}$ and \% of SB in daytime (\%SB). Results: From 145 recruited subjects, 53 (36.6\%) were excluded: 43 $(29.6 \%)$ because individuals or their legal guardians refused to participate, $5(3.4 \%)$ were $<65,, 3(2.1 \%)$ hospitalized, $1(0.7 \%)$ did not live permanently in $\mathrm{NH}$ and $1(0.7 \%)$ no AP available. Of the 92 subjects $(63.6 \%)$ who met the inclusion criteria, $23(15.8 \%)$ did not complete the 7 consecutive days wearing AP. The final sample was 69 (47.6\%); $54(78.2 \%)$ women and $15(10.3 \%)$ men, mean age of 85.1 \pm 8.1 . Mean number of daily steps was $1345.1 \pm 2417.4$, mean total $\mathrm{SB}$ time was $9.0 \mathrm{~h} \pm 1.6$, mean total upright time $1.6 \mathrm{~h} \pm 1.9$, mean STST $18.2 \pm 18.2$, mean total awake hours $10.7 \pm 1.1$ and mean $\%$ SB $84.2 \% \pm 16.8 \%$.

Conclusions: This sample of NH residents from Osona spent most of their daily time in SB (82\%, i.e. 9 hours per day) and less than 2 hours standing or walking during their awaking time of $11 \mathrm{~h}$.

\section{Abstract \# 208 \\ Using a combination of teaching interventions and continuous monthly feedback to sustain improvement in the quality of discharge communication for older patients (SHOP 75+)}

Hanni Cross ${ }^{1}$, Amanda Pederson ${ }^{2}$, Jo Evans ${ }^{2}$, Rebecca Robey ${ }^{2}$, David Carey $^{2}$, Arturo Vilches-Moraga ${ }^{2}$, Olivier Gaillemin ${ }^{2}$

${ }^{1}$ Salford Royal NHS Foundation Trust (SRFT), UK, ${ }^{2}$ SRFT, UK

Introduction: Discharge summaries (DS) are an important part of communication between secondary and primary care. Poor quality DS are associated with poor outcomes [1, 2, 3]. There is guidance from the National Institute for Health and Care Excellence and the Academy of Medical Royal Colleges on writing DS [4, 5].

Method: DS for patients aged $>75$ from several wards were reviewed prospectively in August 2017, and thereafter monthly with a mean of 61 DS per sample. In February 2018 a live-feedback system was introduced whereby wards received monthly feedback on their results with specific recommendations to improve the quality of their DS. From September 2018 teaching interventions were also delivered at junior doctor inductions. Delivery of monthly feedback to wards paused between April 2019 and September 2019.

Results: The aggregate results of the four main components of DS (follow-up plans, medications, clinical summary, and functional assessment), scored "good" in $13 \%$ of DS at baseline, $57 \%$ in August 2018, 22\% in September 2018, 40\% in March 2019, 20\% in July 2019 and $31 \%$ in October 2019.A temporary decrease in quality in September 2018 coincided with change-over of junior doctors in August. A further fall in quality registered following the suspension of monthly feedback in 2019. Quality improved once regular feedback resumed in September 2019.

Conclusion: A combination of teaching interventions and monthly feedback are needed to improve and sustain a better quality of DS. Teaching interventions bring up the baseline quality of DS but without monthly feedback standards slipped within a few months.

References: 1. Witherington EM, Pirzada OM, Avery AJ. Communication gaps and readmissions to hospital for patients aged 75 years and older: observational study. Qual Saf Health Care. 2008 Feb;17(1):71-5. 2. Al-Damluji MS, Dzara K, Hodshon B, Punnanithinont N, Krumholz HM, Chaudhry SI, Horwitz LI. Association of Discharge Summary Quality with Readmission Risk for Patients Hospitalized with Heart Failure Exacerbation. Circ Cardiovasc Qual Outcomes. 2015 Jan;8(1):109-111. 3. Hesselink G, Schoonhoven L, Barach P, Spijker A, Gademan P, Kalkman C, Liefers J, VernooijDassen M, Wollersheim H. Improving patient handovers from hospital to primary care: a systematic review. Ann Intern Med. 2012 Sep18;157(6):417-428. 4. NICE (National Institute for Health and Care Excellence). Transition between inpatient hospital settings and community or care home settings for adults with social care needs. 2015. Retrieved from https://www.nice.org.uk/guidance/ng27. 5. Health and Social Care Information Centre, Academy of Medical Royal Colleges. Standards for the clinical structure and content of patient records. 2013. Retrieved from https://www.aomrc. org.uk/wp-content/uploads/2016

\section{Abstract \# 209}

Evaluating online undergraduate geratology teaching at the University of Oxford Medical School during the Covid 19 pandemic

Antonio Cosimetti ${ }^{1}$, Sanja Thompson ${ }^{1}$

${ }^{1} \mathrm{OUH}, \mathrm{UK}$

Introduction: The Covid 19 pandemic led to the cessation of face to face teaching at the University of Oxford Medical School as it did amongst many universities globally. The use of online teaching in undergraduate medical education (including online lectures) has been shown to achieve similar outcomes compared with face to face delivery[1]. In response, the geratology department delivered its series of 4th year undergraduate teaching sessions online through prerecorded lectures via the software Canvas for individual student use. Here we evaluate the novel use of online material to deliver the undergraduate geratology curriculum at Oxford University Medical School.

Method: Feedback for face to face and online lectures was evaluated and compared using feedback forms recording learner comments and multi-domain learner rated scores. A focus group consisting of lecturers and students was also conducted. Comments were evaluated using thematic analysis and feedback scores compared. 
Results: Online delivery enabled more content to be taught and also encouraged individual learning styles through self directed student exploration of lecture resources whilst maintaining social distancing. Conclusion: The Covid 19 pandemic has meant adapting undergraduate medical education to ensure high quality, consistent and safe teaching standards. We believe that delivering online tutorials can achieve this. Further analysis including comparisons of end of module assessments pre and post implementation and improving content and delivery are areas of interest to develop online teaching.

Reference: 1. Pei L, Wu H. Does online learning work better than offline learning in undergraduate medical education? A systematic review and meta-analysis. Med Educ Online. 2019; 24(1):1666538<tel:1666538>. $\quad$ https://doi.org/10.1080/10872981. 2019.166653

\section{Abstract \# 210}

Staff training interventions to improve end-of-life care among nursing home residents: a systematic review

\section{Kaisu Pitkala ${ }^{1}$, Pauli Lamppu ${ }^{1}$ \\ ${ }^{1}$ University of Helsinki}

Objectives: The aim was to review evidence from all randomized, controlled trials using palliative care education or staff training as an intervention to improve nursing home residents' quality-of-life (QOL) or quality of dying (QOD) or to reduce burdensome hospitalizations. Design: A systematic review with a narrative summary.Setting and participants: Residents in nursing homes and other long-term care facilities.

Methods: We searched PubMed, CINAHL, PsycINFO, Cochrane, Scopus, and Google Scholar, references of known articles, previous reviews and recent volumes of key journals. Randomized controlled trials (RCT) were included for the review. Methodological quality was assessed.

Results: The search yielded 932 articles after removing the duplicates. Of them, 16 cluster RCTs fulfilled inclusion criteria for analyses. There was a great variety in the interventions in respect to learning methods, intensity, complexity and length of staff training. Most interventions featured other elements besides staff training. In the six high quality trials only one showed reduction in hospitalizations, whereas among six moderate quality trials two suggested reduction in hospitalizations. None of the high quality trials showed effects on residents' QOL or QOD. Staff reported improved QOD in one moderate quality trial.

Conclusion and implications: Irrespective of means of staff training, there were surprisingly little effects of education on residents' QOL, QOD or burdensome hospitalizations. Further studies are needed to explore the reasons behind these findings

\section{Abstract \# 211 \\ Perceptions of frailty and desirable components of a training programme to support primary healthcare professionals in the identification and management of frailty}

Christina Avgerinou ${ }^{1}$, Marina Kotsani ${ }^{2}$, Martha Andreou ${ }^{3}$, DimitraIosifina Papageorgiou ${ }^{4}$, Violeta Roka ${ }^{5}$, Magda Gavana ${ }^{4}$, George Soulis $^{6}$, Despoina Symintiridou ${ }^{7}$, Chrysanthi Manolaki $^{8}$, Emmanouil Smyrnakis ${ }^{9}$

${ }^{1}$ Department of Primary Care and Population Health, University College London, London, UK, ${ }^{2}$ Université de Lorraine, CHRUNancy, Pôle « Maladies du Vieillissement, Gérontologie et Soins Palliatifs », F-54000, Nancy, France, ${ }^{3}$ Avdira Health Center,
Prefecture of Xanthi, Greece, ${ }^{4}$ Laboratory of Primary Health Care, General Practice and Health Services Research, Aristotle University of Thessaloniki, Greece, ${ }^{5}$ Farkadona Health Center, Prefecture of Trikala, Greece, ${ }^{6}$ Outpatient Geriatric Assessment Unit, Henry Dunant Hospital Center, Athens, Greece, ${ }^{7} 1$ st TOMY Serron,

Prefecture of Serres, Greece, ${ }^{8}$ Rodolivos Health Center, Prefecture of Serres, Greece, ${ }^{9}$ Laboratory of Primary Health Care, General Practice and Health Services Research, Aristotle University of Thessaloniki, Greece. On behalf of the Aristotle University of Thessaloniki Primary Health Care Research Network

Introduction: Frailty has the potential to be delayed or reversed by appropriate interventions, however these are often not available in countries lacking formal geriatric education/infrastructure. The aim of this study was to: (a) explore ideas, perceptions and attitudes of primary health care (PHC) professionals towards frailty in a country where geriatrics is not recognised as a specialty; (b) explore PHC professionals' training needs in frailty; and (c) define components of a frailty educational programme in PHC.

Methods: Qualitative study. Two focus groups with PHC professionals were conducted in Thessaloniki, Greece in November 2019. Focus groups were audio recorded, transcribed, and data was thematically analysed.

Results: 31 PHC professionals participated in the study (17 physicians, 12 nurses, and 2 health visitors). Four main themes were identified: (1) Perceptions and understanding of frailty; (2) Facilitators and barriers to frailty identification and management; (3) Motivation to participate in a frailty training programme; (4) Education and training. The main barriers for the identification and management of frailty were associated with the healthcare system (e.g. duration of appointments, focus on prescribing, staffing of allied health professionals, multidisciplinary team not available). A gap in education on frailty was highlighted, with scarce training opportunities, based on personal incentive. Professionals were receptive to training in frailty with a focus on peer learning and practical skills with direct clinical application.

Key conclusions: Training in frailty for PHC professionals and a shift in culture towards interdisciplinary collaboration are necessary to provide high standards of care for community-dwelling frail older people.

\section{Abstract \# 212}

Geriatric medicine, undergraduate medical students and the COVID-19 pandemic: experiences of transitioning from clinical placement to the online setting

Clodagh McDermott ${ }^{1}$, Maria Costello ${ }^{2}$, Caoimhe Hanrahan ${ }^{1}$, Gearoid Coughlan ${ }^{1}$, Martin O’Donnell ${ }^{1}$, Michelle Canavan ${ }^{1}$

${ }^{1}$ Department of Geriatric Medicine, University Hospital Galway, Saolta University Health Care Group, Galway, Ireland, ${ }^{1}$ School of Medicine, College of Medicine, Nursing and Health Sciences, National University of Ireland, Galway

Introduction: The emergence of severe acute respiratory syndrome coronavirus 2 (SARS-CoV-2) has impacted healthcare institutions worldwide leading to uncertainty and disruption among medical schools for students on clinical placement. Many universities, including our institution, paused all clerkships for several reasons; for safety of students and hospital patients given the contagious nature of SARS-CoV-2, cancellation of procedures, active transition to telehealth services and limited stocks of personal protective equipment (PPE). In lieu of the clinical geriatric placement model, all teaching was transitioned to case-based collaborative, online sessions. Our aim was to determine the efficacy of this model and its acceptability to students when compared with the traditional model. 
Methods: All third-year medical students attending the online collaborative sessions were invited to anonymously complete an electronic feedback survey.

Results: 60/200 students completed the survey. Of these, $65 \%$ found the online format straightforward to navigate and $96 \%$ found it easy to ask questions with $80 \%$ finding it easier to ask questions than in person setting. $43 \%$ had completed a clinical geriatric medicine placement. Themes which emerged from students' reflections on the new model included lack of face to face communication with older persons, the reality of managing multi-morbidity and polypharmacy in the hospital setting, practising gait and delirium assessments and missing on-site interaction with the multidisciplinary team.

Key conclusions: Students demonstrated insight into core principles of management of the complex older person often learned during clinical immersion. Medical educators must be cognisant to incorporate these competencies in adapted curricula such as hi-fidelity simulation.

\section{Abstract \# 213 \\ Converting a traditional undergraduate geratology course in year 5 into an online learning module in the COVID-19 era \\ Christopher Horton ${ }^{1}$, Ellen Brunet ${ }^{2}$, Charlotte Willis ${ }^{2}$, Sanja Thompson ${ }^{1}$ \\ ${ }^{1}$ University of Oxford, Oxford University Hospitals NHS Trust, ${ }^{2}$ Oxford University Hospitals NHS Trust}

Geratology teaching for University of Oxford fifth year medical students is delivered using traditional formats, including lectures, tutorials and quiz-based consolidation sessions. Following the COVID-19 outbreak, traditional face-to-face geratology teaching was halted. Lecturers were therefore required to develop and deliver remote teaching sessions through alternative methods. We aimed to compare students' opinions on the novel, online geratology teaching provision with existing traditional teaching methods. Prior to COVID19 , feedback on geratology teaching was collected using both paper and online forms. Following the cessation of face-to-face teaching, sessions were converted to virtual formats: lectures were recorded, small group teaching transferred to online platforms and quizzes adapted into online assessments. Feedback was collected using online forms and mixed method comparisons were made. The comparison will include student attendance and the ability to achieve the desired learning outcomes. We know from the students that, although they appreciated the flexibility allowed by online learning, some reported reduced ability to maintain attention to topic matters in comparison to in-class lectures. We will also analyse the impact on the students' ability to ask questions and for teachers to provide immediate feedback, which may not be easily replicated using pre-recorded, online platforms. We now aim to incorporate a combination of pre-recorded and live online teaching sessions to meet all desired learning outcomes, as well as to ensure students can engage in thoughtful discussions and critically assess their academic progress. We also aim to provide pre-recorded sessions with geratology patients in place of face-to-face bedside teaching sessions.

\section{Abstract \# 214 \\ Feasibility and efficiency of a short educational program on frailty destined to primary health care professionals with no prior geriatric background}

Marina Kotsani ${ }^{1}$, Christina Avgerinou ${ }^{2}$, Anna- Bettina Haidich ${ }^{3}$, Magda Gavana ${ }^{4}$, Martha Andreou ${ }^{5}$, Iosifina-Dimitra Papageorgiou ${ }^{6}$,
Stamatia Kokkali ${ }^{7}$, Doukas Zeimbekis ${ }^{8}$, Despoina Symintiridou ${ }^{9}$, George Soulis ${ }^{10}$, Emmanouil Smyrnakis ${ }^{11}$, Violeta Roka ${ }^{12}$

${ }^{1}$ Université de Lorraine, CHRU-Nancy, Pôle « Maladies du Vieillissement, Gérontologie et Soins Palliatifs », F-54000, Nancy, France, ${ }^{2}$ Department of Primary Care and Population Health, University College London, London, UK, ${ }^{3}$ Laboratory of Hygiene, Social-Preventive Medicine and Medical Statistics School of Medicine, Medical School, Aristotle University of Thessaloniki, Greece, ${ }^{4}$ AUTH Medical School, Nea Michaniona Health Center, Prefecture of Thessaloniki, Greece, ${ }^{5}$ Health Center of Avdira, Prefecture of Xanthi, Greece, ${ }^{6}$ Laboratory of Primary Health Care, General Practice and Health Services Research, Aristotle University of Thessaloniki, Greece, ${ }^{7}$ Nea Kallikrateia Heatlh Center, Prefecture of Chalkidiki, Greece, ${ }^{8}$ Kalloni Health Center, Prefecture of Lesvos, Greece, ${ }^{9} 1$ st TOMY Serron, Prefecture of Serres, Greece,

${ }^{10}$ Outpatient Geriatric Assessment Unit, Henry Dunant Hospital Center, Athens, Greece, ${ }^{11}$ Laboratory of Primary Health Care, General Practice and Health Services Research - Medical School, Aristotle University of Thessaloniki, Greece.On behalf of the Aristotle University of Thessaloniki Primary Health Care Research Network, ${ }^{12}$ Health Center of Farcadona, Prefecture of Trikala, Greece

Introduction: Educational opportunities on practical aspects of management of frailty are scarce in countries where geriatric medicine is under development. We aimed to evaluate the feasibility and efficiency of a short educational program for primary health care (PHC) professionals on the detection, assessment and management of frailty.

Methods: A single-day workshop on frailty was organized by the Aristotle University of Thessaloniki PHC Research Network, in November 2019 in Thessaloniki, Greece, facilitated by three physicians trained in geriatrics abroad. Self-administered anonymous questionnaires were delivered before, upon completion and 3 months after the workshop to evaluate attitudes, knowledge and everyday practices regarding frailty, using Likert-type scales.

Results: Out of 31 participants (17 physicians, 12 nurses, 2 health visitors), 31 (100\%) filled in the first, 30 (97\%) the second, and 25 $(81 \%)$ the third questionnaire. Significant improvements were reported in familiarization with the frailty syndrome $(p=0.041)$ and in self-perception of knowledge and skills to detect $(\mathrm{p}<0.001)$ and manage $(\mathrm{p}<0.001)$ frailty, that were also sustained 3 months afterwards ( $p=0.001$ and $p=0.003$ respectively). Significant change was observed in the attitude that frailty is an inevitable consequence of aging $(p=0.007)$ and in the reported frequency of application of screening (but not management) strategies, 3 months following the workshop compared to baseline $(\mathrm{p}=0.014)$. Level of agreement that systematic screening for frailty was unfeasible in their daily practice was $2.4 / 5$ three months after versus $1.6 / 5$ before the workshop ( $\mathrm{p}=$ 0.006).

Conclusions: A short skill-oriented educational program can significantly and sustainably improve PHC professionals' attitudes and practices regarding frailty.

\section{Abstract \# 215}

Spaces for the prevention of staff burn-out syndrome in elderly care institutions

Mihaela Zamfir (Grigorescu) ${ }^{1}$, Ileana Ciobanu ${ }^{2}$, Andreea G Marin², Dragoş-Cristian Bogdan ${ }^{3}$, Mihai-Viorel Zamfir ${ }^{4}$

${ }^{1}$ Department „Synthesis of Architectural Design”, Faculty of Architecture, „Ion Mincu” University of Architecture and Urbanism, Bucharest, Romania, ${ }^{2}$ Department of Neuro-Rehabilitation, „Elias” University Emergency Hospital, Bucharest, Romania, ${ }^{3}$ Valdomedica, Bucharest, Romania, ${ }^{4}$ Physiology II-Neurosciences Division, Faculty 
of Medicine, „Carol Davila” University of Medicine and Pharmacy, Bucharest, Romania

Introduction: According to ICD11, burn-out is a syndrome conceptualized as resulting from chronic workplace stress that has not been successfully managed. Our research aims to identify the qualities of an anti-burn-out space within the elderly care institutions.

Methods: We used focus group method, 4 participants working in elderly care institutions (MD geriatrician \& psychiatrist, psychologist, scientific researcher biologist \& physical therapy assistant, physical therapist) and one facilitator (architect). During 1 hour 17 relevant questions were asked on the issue of burn-out which concerned: work volume, needs of breaks/relaxation for each professional, preferences in terms of relaxation activities and the architectural qualities of a relaxation space at work

Results: The professionals responded that they work between 5 and 12 hours per day and fatigue occurs from 7 hours onwards. All professionals considered that activity with the patient is greatly influenced by the quality of the space in which it takes place. Professionals have opted for both passive and active relaxation. At none of participants' workplaces exist spaces dedicated to staff relaxation. Natural light, colours, natural soundcapes are preferred. A relaxation space is intended to be different from the one professionals work in, sometimes tonic, cheerful, sometimes quiet and comfortable, with areas of interaction but also with the possibility of being alone, connected to internet or practising mindfulness techniques on individual basis.

Key conclusions: The research clearly revealed the need for spaces specifically dedicated for staff relaxation although the claims proved to be quite small. These suggest insufficient education about space potential in preventing burn-out among healthcare professionals.

\section{Abstract \# 216 \\ Teaching communication skills: feedback from students and actors \\ David Molinas Zocche ${ }^{1}$, Sanja Thompson ${ }^{1}$ \\ ${ }^{1}$ Oxford University Hospitals NHS}

Introduction: Many healthcare professionals lack confidence and skills in communicating with patients suffering from dementia, especially if patients also suffer with behaviours that challenge. The communication skills course for medical students addresses these issues.

Methods: The course was delivered with the help of professional actors and focused on non-verbal communication; managing a triadic interview with a patient, relative/carer, and clinician; and managing behaviour that challenges. 89 students attended the course in January and February 2020. Both students and actors evaluated the course via anonymous written feedback. Quantitative and qualitative data were analysed.

Results: All students provided feedback. Average response regarding usefulness was $4.78 / 5$. $78 \%$ of students ranked 5 points considering the session very useful. As for "how well was teaching conducted", the average score was $4.87 / 5$ with $86 \%$ students scoring 5 points. Students indicated addressing carers' concerns and learning deescalating techniques as main learning points. Students gave no negative comments and suggested more time for scenarios, individual feedback and debriefing. The actors identified more areas for improvement. They were concerned about responsibility allocated to them when working with less experienced facilitators, about short time allocation for each scenario and for reflection after scenarios. Actors suggested encouraging students to express their thoughts in between role-plays and querying about previous experience with dementia. The lack of discussion of broad range of approaches being discussed was also identified.

Conclusion: Although feedback in education usually focuses on students and facilitators, exploring actors' perspective may highlight further areas for improvement of future teaching sessions.

\section{Abstract \# 217}

Strategies supporting residential homes in the geriatric palliative care: close cooperation between acute geriatric care in hospital and residential homes

Dana Hrnciarikova $^{1}$, Stanislav Filip ${ }^{2}$, Pavel Vyroubal ${ }^{1}$, Vladimir Blaha $^{1}$

${ }^{1}$ Department of Gerontology and Metabolic Care, University Hospital in Hradec Kralove, Czech Republic, ${ }^{2}$ Palliative team, University Hospital in Hradec Kralove, Czech Republic

Introduction: A lot of geriatric patients admitted to the hospital suffer from one or more chronic progredient diseases (oncological and also non-oncological) and need except for treatment also some palliative care (early, middle or late). Approximately fourth part of admitted geriatric patients is from residential homes. Especially in Czech residential homes is not the palliative care fully developped. We were searching for strategies how to support residential homes in the palliative care.

Methods: Geriatric patients admitted to the hospital especially from residential homes are examined not only with Comprehensive geriatric assessment, but also with NECPAL CCOMS-ICO 2 tool, Palliative Prognostic Score (PPS) and Palliative Prognostic Index (PPI). Patients with the need of palliative care and their relatives are discussed on diagnosis, prognosis, advance care planing and their needs.

Results: We started close cooperation with 14 residential homes and prepared for them individual palliative care plans for their residents who were admitted to the hospital. We started educate nurses and general practitioners in these residential homes in the palliative care. As a first result of our work was significant decrease of readmission of dying patients because of better terminal care in residential homes.

Key conclusion: Close cooperation between acute geriatric care in hospital and residential homes in the field of geriatric palliative care and education could be the way of increasing the quality of life of the palliative geriatric patients.

\section{Abstract \# 218}

Paramedics' perceptions on advance care plans and end-of-life in the community: results of a survey

Alvin Shrestha ${ }^{1}$

${ }^{1}$ Croydon University Hospital

Introduction: Advance care plans (ACP) establish what treatments patients may or may not want and form an important part of care patients with frailty and co-morbidity. Paramedics are often the frontline staff encountering such patients at a time of crisis and must decide what treatment to offer and ultimately whether to convey to hospital or not. This study was designed to find out their perceptions in managing such patients, including at end of life, through advance care plans.

Method: A survey was created and copies left in Croydon University Hospital's Emergency Department for paramedics to voluntarily fill, with a total of 52 completed surveys collected.

Results: Paramedics find ACPs helpful but only if they are detailed enough- only $27 \%$ of paramedics thought they were good enough 
"most of the time". Paramedics value, specifically, advice on how to manage expected symptoms, baseline observations, baseline behaviour, prognosis and contact details of services involved in patients' care. Hospice advice in-hours and community rapid response were favoured as the best services in managing end of life at home, whilst hospice out-of-hours and General Practice (GP) out-of-hours ranked the worst. This was reflected in which services required the most improvement, with hospice out-of-hours and GP out-of-hours coming out as the highest in need for improvement.

Conclusion: ACPs need to be detailed and specific to be of most use. There may be a need for improvement to both hospice and GP out-ofhours services to help end-of-life care in the community.

\section{Abstract \# 219}

\section{Palliative thoracentesis and pleurodesis at home}

Panagiotis Zotos ${ }^{1}$, John Poularas ${ }^{2}$

${ }^{1}$ Director of ICU in "Ippokrateio Institution of Agrinio", ${ }^{2} \mathrm{MD}, \mathrm{ICU}$ clinic, General State Hospital of Athens "G,Gennimatas"

Introduction: Malignant pleural effusion (MPE) is a very distressing condition for the patient, generally requiring symptomatic relief. However, most of these patients are long-suffering, at a terminal stage of their disease and negative to any transportation outside home. Ultrasound-guided thoracentesis could be a key palliative intervention for the management of this vulnerable population at home.

Methods: Two patients (69 and 79 years old) with metastatic breast cancer, suffering from severe, recurrent, symptomatic MPEs, required thoracentesis, but refused to leave their house. After written consent, an indwelling pulmonary catheter (IPC) (Pleuracan-B. Braun) was inserted at home under portable ultrasound guidance (Chison ECO 5). Results: In one patient the IPC remained for 10 days and chemical pleurodesis with sterile talc powder (4 grams of graded talc) was attempted. Subsequently, she remained free of respiratory symptoms (dyspnea, chest discomfort or heaviness, cough) for the last 2 months of her life. In the second patient, the IPC remained for 15 days. After removal of the IPC, no new thoracentesis was required thereafter, probably due to autopleurodesis. The patient did not experience any severe respiratory distress until the end of her life 70 days later. In both patients, after IPC removal, lung sliding could not be detected with thoracic ultrasonography.

Conclusion: Palliative thoracentesis and pleurodesis under ultrasound guidance could be safely and successfully performed at home from a skilled staff without need for transportation of the patient.

\section{Abstract \# 220 \\ Is secure mailing communication possible for elderly patients hospitalized in a French teaching hospital? \\ Durand Amaury ${ }^{1}$, Chenailler Catherine ${ }^{1}$, Varin Rémi ${ }^{1}$ \\ ${ }^{1} \mathrm{CHU}$ Rouen}

Introduction: In France, the General Data Protection Regulation (GDPR) established in 2018 strengthens the patients' rights and empowers actors dealing with critical data such as health data. The hospital report and associated documents should be provided at discharge to community professionals by secure messaging. We wanted to know whether this approach was feasible for patients leaving acute geriatrics or rehabilitation care from our French teaching hospital. Methods: During a 5 months period (November 2019-March 2020), for each patient who benefited from a medication reconciliation, were recorded the general practitioner and community pharmacist identities and the existence of a secure messaging. Then, we were able to calculate the percentage of professionals who could benefit from a secure information transmission at discharge.

Results: 493 patients benefited from a medication reconciliation. The general practitioner and community pharmacist identities were filled respectively for $414(83.9 \%)$ and $366(74.2 \%)$ patients. $267(64.5 \%)$ general practitioners and $222(60.7 \%)$ pharmacists had secure messaging.

Key conclusions: According to an extraction from ASIP Health data, $12.2 \%$ of French physicians and $52.1 \%$ of French pharmacists, all specialties combined, had secure messaging in December 2019. The results of our study testify that the secure messaging deployment is good in our region, in particular through the training sessions organization with hospital and community health managers since 2017. However, it remains perfectible in order to allow secure and fast transmission of information at discharge.

\section{Abstract \# 221 \\ Experiences of advance care planning in Parkinson's disease and atypical Parkinsonian disorders from the perspective of patients, carers and healthcare professionals: a systematic review}

Danielle Nimmons ${ }^{1}$, Lee Hatter ${ }^{1}$, Nathan Davies ${ }^{2}$, Elizabeth L Sampson $^{3}$, Kate Walters ${ }^{1}$, Anette Schrag ${ }^{4}$

${ }^{1}$ Centre for Ageing Population Studies, Research Department of Primary Care and Population Health, UCL, U.K, ${ }^{2}$ Centre for Ageing Population Studies, Research Department of Primary Care and Population Health, UCL, U.K and Centre for Dementia Palliative Care Research, Marie Curie Palliative Care Research Department, Division of Psychiatry, UCL, U.K, ${ }^{3}$ Centre for Dementia Palliative Care Research, Marie Curie Palliative Care Research Department, Division of Psychiatry, UCL, U.K, ${ }^{4}$ Department of Neurology, Institute of Neurology, UCL, U.K

Introduction: Advance care planning (ACP) allows people to plan for their future care needs. It is important but underutilised in noncancer conditions, such as Parkinson's disease. We aimed to explore the experiences of ACP for people with Parkinson's disease, atypical Parkinsonian disorders, their family carers and healthcare professionals.

Methods: We conducted a systematic search of online databases in April 2019; using a narrative synthesis approach, thematic analysis and tabulation to synthesise the findings.

Results: We identified 507 articles and 27 were included. There were five themes: (1) What is involved in ACP discussions? Discussions included a wide range of topics, but coverage was patchy. (2) When and how are ACP discussions initiated? It was unclear when it should be initiated, some patients wanted early discussions, but others did not. It depends on patients' readiness and professional's willingness to discuss the topic; which often resulted in discussions first taking place in response to a crisis, such as a hospital admission. (3) Barriers to ACP. This included a lack of information and knowledge related to palliative care by both patients and professionals, and practical barriers such as time in consultations. (4) The role of healthcare professionals. Access and collaboration with palliative care services and multi-disciplinary teams led to clear plans. (5) The role of the family carer. Carers were a facilitator but could be a barrier if emotionally burdened.

Key conclusions: This evidence was used to highlight eight effective components to support optimal ACP in Parkinsonian disorders, which can inform best practice. 


\section{Abstract \# 222 \\ Home palliative consultation for geriatric patients, a retrospective analysis \\ Ricardo Fernandes ${ }^{1}$, Márcia Oliveira ${ }^{1}$, Cátia Macedo ${ }^{1}$, Ludovina Paredes $^{1}$, Ana Paula Costa ${ }^{1}$ \\ ${ }^{1}$ Palliative Care Team, Centro Hospitalar de Vila Nova de Gaia- Espinho}

Introduction: Palliative care is multi and interdisciplinary, specialized care focused on improving quality of life for patients with serious illness(both oncologic and non-oncologic) and their families, since the diagnosis to the bereavement. The PalliativSe Care Team (PCT) is a multidisciplinary team that has many possibilities of evaluating the patients-outpatient clinic, inpatient evaluation, home consultation and telephonic follow-up.

Methods: Review of the patients $\geq 65$ years old followed in the Home Palliative Care Unit since June 2015 to May 2020.

Results: We followed 645 patients, $549(85,25 \%)$ had $\geq 65$ years. The average age was 78,9 years old. Most had oncological disease 464 versus 85 non-oncological. The most frequent diagnosis was colon and rectum cancer $(\mathrm{N}=81)$, gastric $(\mathrm{N}=69)$; lung $(\mathrm{N}=55)$; pancreas $(\mathrm{N}=17)$. Sympton control was the main reason for assistance $(\mathrm{N}=378 ; 58,7 \%)$, followed by care organization $(\mathrm{N}=165$; $30,0 \%)$; decision making $(\mathrm{N}=4 ; 0,72 \%)$. Most patients were referred from the hospital wards [HW] $(\mathrm{N}=92)$, palliative care outpatient clinic $(\mathrm{N}=380 ; 69,2 \%)$, primary care $(\mathrm{N}=8 ; 1,45 \%)$, other palliative care teams $(\mathrm{N}=19 ; 3,46 \%)$. They were cared by their children $(\mathrm{N}=$ $275)$, spouse $(\mathrm{N}=165)$, formal caregiver $(\mathrm{N}=9)$, siblings $(\mathrm{N}=26)$ and 17 in geriatric residences [GR]. In total 481 died, 359 in their houses, 50 in the HW, 41 in GR, 25 in the emergency department. Conclusion: Although the preferred place of death is at home, it is often not possible for this wish to be fulfilled, whether due to the impossibility of the patient's management by the caregivers or due to the current insufficient response by the PCT (community and hospital). This close follow-up, promoting dignity and comfort is well received with gratitude.

\section{Abstract \# 223}

Performance of the socio-health assessment unit in traumatology

Marta Mercedes González Eizaguirre ${ }^{1}$, Maria Jesús Vicente Romero ${ }^{1}$, Carmen Oquendo Marmaneu ${ }^{1}$, Miguel Sanchez Ortiz ${ }^{1}$, Ana Mateo Abad $^{1}$, Elisa García Simón ${ }^{1}$, Magdalena Linge Martin ${ }^{1}$

${ }^{1}$ Hospital San José de Teruel

Introduction: The Unit of Social and Health Assessment constitutes a transversal resource within the Health System that provides collaboration to professionals of all levels of care, being responsible for the comprehensive assessment and in-hospital management of the target population and to facilitate the coordination and continuation of the care of this population once discharged.

Method: A retrospective descriptive study of patients over 69 years old seen by the unit of assessment admitted to the trauma plant due to a hip fracture from January 1, 2019 to November 30, 2019 is carried out. Demographic variables are extracted.

Results: 76 patients. 70 of the 75 patients $(93.3 \%)$ were admitted from the Emergency Department. 5 patients $(6.6 \%)$ came from another hospital. 2 patients died on admission and subsequently 2 more died in another hospital. 1 of the fractures was tumor pathological. 19 patients were men and 56 women. The average hospital stay was 5.64 days. 11 were referred to a convalescence unit. Of the total fractures, 42 belong to the lower right limb (56\%) and 33 to the lower left limb (44\%). Of total hip fractures, these can be classified according to the location of the lesion in the femur by finding: 27 pertrochanteric fractures $(36 \%), 15$ subtrochanteric fractures $(20 \%)$, 33 intracapsular fractures. (44\%).

Conclusions: The profile of the patient with a hip fracture is similar to that of other territories, being an elderly patient with a right intracapsular fracture, whose stay does not generally exceed 6 days.

\section{Abstract \# 224}

\section{Elderly-femoral fractures-prevention}

Tiziana Candiani ${ }^{1}$, Domenico Prestamburgo ${ }^{2}$, Antonino Mazzone ${ }^{3}$

${ }^{1}$ UOS Geriatrics H Legnano ASST Ovest Milanese, ${ }^{2}$ UOC Ortopedia $\mathrm{H}$ Legnano ASST Ovest Milanese, ${ }^{3}$ UOC Internal Medicine H Legnano ASST Ovest Milanese -Italy

In the geriatric field, a large chapter is dedicated to falls in elderly subjects, also great interest is aimed at fractures of the femur deriving from these falls. Today we are increasingly talking about prevention, but perhaps we are not talking about health education in the home.We know that most femoral fractures occur in the home environment (inadequate slippers, slipping, stumbling block, rapid movements etc ...) and that most individuals are female.In light of the above, from 1 November 2019 to the present day, we wanted to check with an appropriate medical history and considering the functional status A.D.L (Katz scale), I.A.D.L. (Lawton scale), M.M.S.E. (MF. Folstain), how many fractures of the femur of patients over the age of 75 were due to home falls, implying hospitalization for a surgical procedure at the Orthopedics Department of the Legnano hospital.30 individuals, 6 men and 24 women, mean age 83.5 years, $60 \%$ of them live alone, mean $\mathrm{ADL}=4.5 / 6, \mathrm{IADL}=4.3 / 8, \mathrm{MMSE}=22.8 / 30$. Twenty-six fell exactly in the domestic environment, two down the outside steps, one on the sidewalk, one, car accident. The data confirmed what previously stated and also demonstrate that the patients in this study still show good functional activity and memory, considering their average age. In conclusion, we believe that a good disclosure could prevent acute events thus avoiding the loss of daily functional and instrumental activities

\section{Abstract \# 225}

Comprehensive hospitalist care for elderly patients with vertebral compression fracture

Takahiko Tsutsumi ${ }^{1}$

${ }^{1}$ Takatsuki General Hospital

Introduction: Vertebral compression fracture (VCF) is traditionally seen only by orthopedic physicians in Japan. Although common in geriatric patients, it requires specific care; as well as acute management of VCFs, maintenance of quality of life may also require comprehensive geriatric assessment and prevention of additional fractures. Currently, such a multi-faceted approach to VCF in the elderly is thought to be uncommon in Japan. We investigate the impact of co-management of VCF by hospitalists compared with orthopedic treatment alone.

Methods: In a retrospective single-center Japanese observational study, we assessed patients over 65 years old who were admitted with VCF. Usual orthopedic care (UOC) was provided between January and August in 2017 and 2018, and between January and August in 2019, patients received comprehensive hospitalist care (CHC). We compared patients in CHC group with patients in UOC group. Variability of functional independence measure (FIM) was evaluated with FIM on the admission day as covariate. We also evaluated cost, length 
of stay, initiation rate of treatments for osteoporosis during hospitalization, and choice of analgesics.

Results: In CHC group, initiation rate of treatments for osteoporosis was higher $(56.7 \%$ vs. $0 \% ; \mathrm{P}<0.001)$ and fewer NSAIDs were used $(2.9 \%$ vs. $50 \% ; \mathrm{P}<0.001)$ than in OGC group. Moreover, although there was no statistical significance, there was a trend of lower cost and shorter hospitalization than in the OGC group. Variability of FIM did not differ significantly between two groups.

Key conclusions: Internal medicine hospitalists may provide better preventive management while maintaining treatment standards for VCF in elderly patients.

\section{Abstract \# 226 \\ Concurrent validity of the GAITRite walkway compared to functional balance test in geriatric outpatients}

Johannes Riis ${ }^{1}$, Stephanie Byrgesen ${ }^{1}$, Kristian Kragholm ${ }^{1}$, Marianne Mørch $^{2}$, Dorte Melgaard ${ }^{1}$

${ }^{1}$ Center for Clinical Research, North Denmark Regional Hospital, Hjørring, Denmark, ${ }^{2}$ Department og Geriatric Medicine, North Denmark Regional Hospital, Hjørring, Denmark

Introduction: The GAITRite walkway allows for quantitative gait assessment and is used in falls risk assessment. However, few studies have examined the validity for falls risk assessment. This study aimed to examine the concurrent validity between the quantitative gait parameters and functional balance test commonly used in falls risk assessment.

Methods: Consecutive sampling from one geriatric outpatient clinic. One physiotherapist evaluated the patients on the GAITRite walkway with three repetitions. Patients were further evaluated with Bergs Balance scale (BBS), Dynamic Gait index (DGI), Timed Up and Go (TUG), and Sit To Stand test (STS). Correlations between quantitative gait parameters and functional balance test were analyzed with Spearman's Rank correlations. Correlations strength were considered as follows; negligible $<0.1$, weak $0.10-0.39$, moderate $0.40-0.69$, and strong $\geq 0.70$.

Results: We included 24 geriatric outpatients in the study with a mean age of 80.6 years (SD: 5.9). Patients received 8 (SD: 4.5) different medications on average and $7(29.2 \%)$ patients used walkers during ambulation.Correlations between quantitative gait parameters and functional balance test ranged from weak to moderate. The strongest correlations were observed for DGI (mean rho: 0.51 [range 0.41-0.64]), TUG (mean rho: 0.55 [range 0.41-0.67]), and BBS (mean rho: 0.50 [range $0.36-0.55]$ ), while STS showed weaker correlations (mean rho: 0.29 [range $0.17-0.38$ ]). For outpatients analyzed on the GAITRite while using walkers, correlations showed no clear pattern across parameters with large variation within balance tests.

Key conclusion: The GAITRite parameters were moderately correlated with most functional balance tests. The GAITRite walkway may not be valid for use with walkers.

\section{Abstract \# 227 \\ The majority of men referred to a Falls Clinic have low or very low testosterone levels \\ Linda Katharina Karlsson ${ }^{1}$, Mette Midttun ${ }^{1}$ \\ ${ }^{1}$ Department of Internal Medicine, Geriatrics, University Hospital Copenhagen, Herlev, Denmark}

Introduction: Testosterone levels decline gradually with age, and more rapidly in the elderly. Low testosterone concentrations are one of the key factors related to sarcopenia in men; and thus frailty, subsequently increasing the risk of falls. Previous research indicates that testosterone treatment may have a positive impact on muscle strength and physical performance in frail men. The aim of this abstract is to evaluate the level of total testosterone in men above 70 years old who were referred to a Falls Clinic.

Methods: The 77 patients referred to the Geriatric Falls Clinic at Gentofte Hospital, Copenhagen, were screened for total plasma testosterone levels in addition to standard blood samples at their first visit. Low testosterone levels were defined as below $13 \mathrm{nmol} / \mathrm{l}$ in the present study, whilst $10 \mathrm{nmol} / \mathrm{l}$ or thereunder was considered very low.

Results: The included patients were between 70 and 94 years old, with a mean age of 81.5 years, and mean testosterone levels of 13.8 $\mathrm{nmol} / \mathrm{l}$. Testosterone levels below $13 \mathrm{nmol} / \mathrm{l}(0.2-13.0 \mathrm{nmol} / \mathrm{l})$ were found in $34 / 77 \mathrm{men}$, whereas $20 / 77$ had levels below $10 \mathrm{nmol} / \mathrm{l}$ $(0.2-9.5 \mathrm{nmol} / \mathrm{l})$.

Key conclusions: Nearly half of the patients referred to the Falls Clinic had low or very low testosterone levels. We therefore call for randomized trials, investigating whether testosterone treatment in combination with physical exercise can prevent falls and improve physical function in elderly men with hypogonadism, as a supplement to standard treatment in Falls Clinics.

\section{Abstract \# 228}

\section{Sarcopenia as a risk factor for falls in older adults}

Guzin Cakmak $^{1}$, Ercument Ozturk ${ }^{2}$, Zeynel Abidin Ozturk ${ }^{2}$

${ }^{1}$ Gaziantep University Faculty of Medicine, Department of Internal Medicine, Division of Geriatrics, ${ }^{2}$ Gaziantep University, Faculty of Medicine, Department of Internal Medicine, Division of Geriatrics

Introduction: Postural and gait balance are important indicators of health in the elderly. Sarcopenia is a geriatric syndrome defined as the loss of muscle strength, mass, and function. In this study we evaluated the relationship between sarcopenia and risk of falls.

Methods: This is a cross-sectional study based on the data from patients who admitted to outpatient polyclinic of geriatrics. Evaluation for sarcopenia was done according to EWGSOP2 criteria. The risk of falls was evaluated with the Tinetti balance and gait assessment and Timed Up and Go Test (TUG). The IBM SPSS Statistics version 22 was used to analyze data.

Results: The study population was composed of 57 women and 43 men, of mean age $72.8 \pm 7.4$ years. SARC-F score was negatively related to the Tinetti balance score, gait score, and total Tinetti score. Handgrip strength was positively related to the Tinetti balance score, gait score, and total Tinetti score. Handgrip strength was negatively related to the TUG score. The skeletal muscle mass index was negatively related to the TUG score. Gait speed was positively correlated with the Tinetti balance score, gait score, total Tinetti score, and negatively correlated with the TUG score. In linear regression analysis, the total Tinetti score was related to SARC-F, skeletal muscle mass index, and gait speed $\left(\mathrm{r}^{2}=0.312, \mathrm{p}=0.028 ; \mathrm{p}<0.001 ; \mathrm{p}=\right.$ $0.023)$.

Key conclusions: In this study, we revealed that sarcopenia was related to imbalance. Thus, sarcopenia could be handled as a risk factor for falls and fractures. Prospective studies could be better to evaluate the effect of sarcopenia prevention and treatment strategies on falls and fractures. 


\section{Abstract \# 229}

Developing a falls pathway for patients discharged from the emergency department in a tertiary hospital

Donal Fitzpatrick $^{1}$, Kara McLoughlin ${ }^{1}$, Louise Martin ${ }^{1}$, Raja Muhammad Shahid Ali ${ }^{1}$, Denys Shchetkovsky ${ }^{1}$, Pat Barry ${ }^{1}$

${ }^{1}$ Cork University Hospital

Introduction: Falls are a major source of harm in older adults in Ireland and internationally. Patients admitted to hospital have defined pathways of care. However, we have noted gaps in services for those discharged in our hospital's catchment area.

Method: We retrospectively reviewed ED notes of patients aged $>65$ discharged from ED with a fall in November 2019 in a university teaching hospital.

Results: Of 123 older patients discharged after a fall, $27(21.9 \%)$ were seen by FITT (Frailty Intervention Team); 18 patients (14.6\%) were referred to community falls services. There were 52 patients $(42 \%)$ discharged out of hours. Patients discharged out of hours are not seen by FITT. Fractures were sustained in 29 patients (23.6\%). Only 3 patients with fractures $(10.3 \%)$ were referred to services. Services for early identification and intervention in patients suffering osteoporotic fractures are lacking. Community falls services are urban centric with $44 \%$ being outside the catchment area.

Conclusion: FITT functions as the cornerstone of multidisciplinary integrated care in our hospital. We believe this is an effective model of care. However, services and staffing must be commensurate with the needs of the increasing number of patients with falls. Patients discharged out of hours and patients living outside of catchment areas need improved services. Falls pathways must employ early identification and community intervention for patients suffering osteoporotic fractures. As the population in Ireland and in Europe ages, such effective pathways of integrated care become even more important.

\section{Abstract \# 230 \\ Fracture of the left proximal humerus, after accidentally falling from its own height. \\ Iker Sierra-Martínez ${ }^{1}$, Leticia Sierra-Martínez ${ }^{2}$, Rosario Martínez- Fuerte $^{3}$, Natalia Sanz-González ${ }^{4}$ \\ ${ }^{1}$ Traumatology Department, Hospital of Medina de Campo (Valladolid), Spain, ${ }^{2}$ Valladolid Este Primary Assistance Gerency, Valladolid, Spain, ${ }^{3}$ Pilarica Health Center, Valladolid Este Primary Assistance, Valladolid, Spain, ${ }^{4}$ Parquesol Senior Center, JCyL Social Services Gerency, Valladolid, Spain}

85-year-old woman, who presented pain and functional impotence in the left shoulder after accidentally falling from her own height forward. It denies the loss of sensitivity in said member. No traumatic brain injury or previous dizziness. Physical exam: conscious and oriented, good general condition, normohydrated, eupneic. Left shoulder: hematoma and pain in the left shoulder, in the lateral anterior and lateral zone, mobility limited by pain, good mobility of the elbow, good pulse and distal perfusion. Shoulder X-ray: Left proximal humerus fracture.Therapeutic plan: arm with the position of immobilization in a sling (placed outside the clothing). Local ice. Mobilization of the elbow and fingers. Paracetamol $1 \mathrm{~g}$ every 8 hours.Evolution: revision at 17 days, immobilized with a sling. She is now a caregiver dependent for activities of daily living, previously the fracture was independent. You have performed scheduled exercises. RX control: no bone displacement. Physical examination: normal flexion, abduction of $80^{\circ}$, internal rotation of $45^{\circ}$. Therapeutic plan, immobilization with a sling for one more week. It is included in an early mobilization protocol and is referred to your family doctor for
control.Final comment: The importance of this case for Primary Care is the mechanism of fracture production: from the patient's own height, who apparently does not suffer from underlying pathologies that make her vulnerable. However, he has already presented a fracture due to a mechanism that should not cause it, so bone fragility should be suspected. At this point, it is essential to confirm the existence of osteoporosis in this patient, and to establish preventive and therapeutic measures to improve bone status and prevent future fractures or other complications.

\section{Abstract \# 231}

\section{Complicated hip fracture}

Iker Sierra-Martínez ${ }^{1}$, Leticia Sierra-Martínez ${ }^{2}$, Rosario MartínezFuerte $^{3}$, Natalia Sanz-González ${ }^{4}$

${ }^{1}$ Traumatology Department, Hospital of Medina de Campo (Valladolid), Spain, ${ }^{2}$ Valladolid Este Primary Assistance Gerency, Valladolid, Spain, ${ }^{3}$ Pilarica Health Center, Valladolid Este Primary Assistance, Valladolid, Spain, ${ }^{4}$ Parquesol Senior Center, JCyL Social Services Gerency, Valladolid, Spain

Introduction: Hip fracture is a frequent process in the elderly population in which it is necessary to make an early diagnosis and adequate and coordinated treatment between orthopedists, rehabilitators, geriatricians and specialists in primary care.

Description of the case: 70 -year-old patient who goes to the emergency department after suffering a fall at home. He reports pain in the lower right limb at the level of the upper femur. He was admitted with the diagnosis of right pertrochanteric fragility fracture to perform surgery.

Personal history: allergy to pyrazolones and NSAIDs, surgery for a hysterectomy with double adnexectomy, high blood pressure, repeated psychotic episodes, mild mental retardation, generalized tonic crisis a year ago. He underwent surgery, performing reduction and osteosynthesis with an endomedullary nail of the proximal femur. In the review of the four-month consultation, it was observed that the nail had broken, so the operation was performed again, the anterior nail was extracted, grafting to the femoral head and reosteosynthesis with an endomedullary nail. long. With evolution without incident. Recommendations: A hip fracture and/or an endomedullary nail fracture complication is a serious injury with life-threatening complications. It is important that before all the patients with osteoporosis and/or multiple pathologies in the follow-up in the primary care consultation, an evaluation of the risk of falls is carried out, through a physical and emotional evaluation, which allows us to individualize a prevention and implantation. intervention with each patient in the care plan to reduce their incidence and complications.

\section{Abstract \# 232}

\section{Hip fracture and the urinary bladder}

A Michael ${ }^{1}$, Arlinda Lago ${ }^{1}$

${ }^{1}$ Russells Hall Hospital

Introduction: Some older people have falls, resulting in hip fracture, while going to the bathroom to urinate, or coming back. The aim of this study is to analyse the "phenomenon" of hip fracture related to the urinary bladder.

Methods: Prospective observational study of consecutive patients admitted to a UK teaching hospital with hip fracture in an eight months period. Patients, notes and electronic records were reviewed; data were downloaded on excel sheet and analysed using descriptive statistics. 
Results: 377 hip fracture patients were admitted in the study period, of whom 51 patients $(14 \%)$ had falls related to the bathroom. Of these $20 \%(10 / 51)$ male and $80 \%(41 / 51)$ female with average age of 81.6 and 82.8 years respectively. $51 \%(26 / 51)$ had urinary incontinence. $51 \%(26 / 51)$ had hypertension and 31\% (16/51) had osteoarthritis. $67 \%(34 / 51)$ were on $>4$ drugs, $55 \%(28 / 51)$ were on antihypertensives, $22 \%(11 / 51)$ were on diuretics and $31 \%(16 / 51)$ were on an antidepressant. Falls were multifactorial; frailty in $41 \%(21 / 51)$ and postural hypotension in $24 \%$ (12/51) were considered the most contributing factors.

Discussion: The number of patients who had fractures related to the urinary bladder could be underestimated as some older people with dementia were found on the floor with no reliable history; many of them could have been going to the bathroom. There are many reasons why elderly people might fall while going to the bathroom including postural hypotension, rushing to avoid urge incontinent, hypnotic or extrapyramidal side effects of medications, not using the mobility aids and poor lighting conditions.

Conclusion: In this study $14 \%$ of falls resulting in hip fracture was attributed to the urinary bladder. Urinary bladder symptoms are a frequently forgotten risk factor for falls and fractures. To reduce the risk in this group of patients, urinary tract symptoms need to be explored, diagnosed and treated. Medications; specially antihypertensives and CNS active drugs should be rationalized and diuretics are to be avoided if possible.

\section{Abstract \# 233 \\ Influence of the frailty in patients with hip fracture treated with blood transfusion and/or intravenous iron infusion, measuring haemoglobin levels pre and post-surgical intervention \\ Genesis Estefania Olaya Loor ${ }^{1}$, María Loreto Alvarez Nebreda ${ }^{1}$, Alfonso Jose Cruz Jentoft ${ }^{1}$, Erick Arturo Rodriguez Espeso ${ }^{1}$, Celia Corral Tuesta ${ }^{1}$, Alvaro Rodriguez Diaz-Pavon ${ }^{1}$, Ana Lopez Iglesias ${ }^{1}$ \\ ${ }^{1}$ Geriatric Department of the Hospital Universitario Ramón y Cajal (IRyCIS)}

Objectives: to describe the impact of the frailty on hip fracture patients following blood transfusion and/or intravenous iron infusion treatments, during the hospitalization period and three months after the surgery.

Methods: Descriptive study conducted in the Orthogeriatrics Unit from the University Hospital Ramon y Cajal, February-April 2019. Demographic and preinjury characteristics of the patients in each of the FRAIL categories were described.

Results: In a cohort of 127 patients (mean $87 \pm 6$ years), $73 \%$ were females and $78 \%$ lived in dwelling houses. The pertrochanteric was the most frequent fracture. Evaluation of the patient using FRAIL Questionnaire showed that 50\% were frail and 37\% were prefrail. Most of the frail patients were dependent; showed malnutrition, major comorbidities, polypharmacy and frequent alterations in the iron kinetics profile. Blood transfusion was used in $35 \%$ of these patients, and the haemoglobin levels were comparable between the admission and after three months $(12,4 \pm 1,8$ to $12,6 \pm 1,5 \mathrm{~g} / \mathrm{dL})$. Alternatively, intravenous iron infusion was the prevalent treatment given to $30 \%$ of the prefrail patients. Similarly, haemoglobin levels did not change significantly $(12,6 \pm 1,8$ to $12,3 \pm 2,2 \mathrm{~g} / \mathrm{dL})$. Linear regression models adjusted by frailty category, were used to examine the association between the chosen treatment and the haemoglobin levels, measured during the study.

Conclusion: in this particular cohort of patients, the prevalence of frailty is high and blood transfusion were preferably prescribed. However, intravenous iron infusion was frequently used in the prefrail group. The analysis performed concludes that haemoglobin levels do not change regardless the frailty status, independently of the chosen treatment.

\section{Abstract \# 234 \\ Disruption of proprioception increases the risk of falling in elderly patients undergoing hemodialysis}

\section{Ozkan Ulutas ${ }^{1}$}

${ }^{1}$ Inonu University Faculty of Medicine, Department of Nephrology

Backround: Patients undergoing HD experience more accidental falls compared to the senior population not on HD, causing increased morbidity and mortality in this patient group. In the present study, we hypothesized that ankle proprioceptive sensation may have been impaired in HD patients with a history of falling.

Materials and methods: Sixty-three patients on hemodialysis aged 65 or more included to the present study. Patients who have experienced 1or more falls last year considered as fallers and others as nonfallers. Demographic data, laboratory values, timed up and go test duratians and inclinometric deviation of ankle joint were compared between two groups. Proprioceptive sensation of the ankle was measured using a simple digital inclinometer instrument on the dominant side. While the patients were in supine position, and their eyes were closed, their ankles were brought to 10 degrees of dorsiflexion by the help of study nurse. Afterwards, the patients were asked to come back to the starting point, and actively return to the set angle 3 times without help. All attempts of the patients measured and the error angle deviated from the set angle was used as the result value. The average of 3 results was accepted as the test result.

Results: Of 63 patients 25 (39.7\%) were fallers. Mean inclinometric deviation of the study population was $4.1 \pm 1.9$ degrees, which was significantly higher $(5.5 \pm 1.9, \mathrm{p}=0.000)$ among patients in the faller group when compared with patients in the non-faller $(3.1 \pm 1.2)$ group. Age, serum albumin levels, PTH, TUG test duration, inclinometric deviation of patients have been included in the logistic regression analysis. Of these parameters, only inclinometric deviation $(\mathrm{OR}=0.393, \mathrm{p}=0.004)$ was determined as an independent predictor of falls.

Conclusion: Falls are common in elderly hemodialysis patients, and disruption of ankle proprioception is evident in HD patients who fell.

\section{Abstract \# 235}

\section{Trauma of the hand, fracture of the proximal phalanx}

Iker Sierra-Martínez ${ }^{1}$, Leticia Sierra-Martínez ${ }^{2}$, Rosario MartínezFuerte $^{3}$, Natalia Sanz-González ${ }^{4}$

${ }^{1}$ Traumatology Department, Hospital Medina del Campo (Valladolid), Spain, ${ }^{2}$ Valladolid Este Primary Assistance Gerency, Valladolid, Spain, ${ }^{3}$ Pilarica Health Center, Valladolid Este Primary Assistance, Valladolid, Spain, ${ }^{4}$ Parquesol Senior Center, JCyL Social Services Gerency, Valladolid, Spain

Introduction: The initial care of the patient with the traumatized hand largely determines the final result. It is the doctor who attends the urgent demand who has the most difficult and responsible mission because he is the one who determines the orientation to arrive at an accurate diagnosis and treatment.

Description of the case: 69 -year-old male patient who comes to the consultation after falling from his height 24 hours ago with inflammation and pain in the right hand.

Physical examination: Great inflammation of the right hand, great hematoma, pain on palpation of the proximal phalanx of the 3rd and 4th fingers. Good mobility and sensitivity of the fingers. Neuro- 
vascular distal preserved.X-ray:nondisplaced fracture of the proximal phalanx 3rd and 4th fingers of the right hand. He was referred to Traumatology for urgent evaluation, immobilization with a plaster dorsal splint. Plan:-Raised hand-Local ice-Paracetamol 1g/8h if pain.Follow-up in the hand unit of the trauma service.

Recommendations: Hand injuries are a common presentation problem in urgent demand in Primary Care. It is important to assess, diagnose, and treat quickly and accurately to prevent short or long term dysfunction. In the event of any suspicion of fracture or dislocation of elements of the hand, radiographs (always at least two projections) should be made centered on the suspicious area (to try to obtain maximum detail) and not of the entire complete hand in a systematic way, taking Keep in mind that the most important thing is the previous exploration of the hand.

\section{Abstract \# 236 \\ Bone mineral density in long-living patients with coronary artery disease \\ Svetlana Topolyanskaya ${ }^{1}$, Tatyana Eliseeva ${ }^{2}$, Olga Vakulenko ${ }^{2}$, Leonid Dvoretski ${ }^{1}$ \\ ${ }^{1}$ I.M. Sechenov First Moscow State Medical University (Sechenov University), ${ }^{2}$ War veterans hospital N3}

Introduction: Limited data are available on osteoporosis in longliving patients. Therefore, we evaluated bone mineral density in longliving patients with coronary artery disease (CAD).

Methods: 202 patients hospitalized with CAD were enrolled in this cross-sectional study. The patients' age ranged from 90 to 101 years. The majority of study participants $(64.4 \%)$ were women. Bone mineral density (BMD) was measured by dual-energy X-ray absorptiometry.

Results: Normal lumbar spine BMD was observed in $40.9 \%$, osteoporosis-in $26.9 \%$, osteopenia-in $32.2 \%$ of patients. Normal proximal femur BMD values was observed in $21.3 \%$, osteoporosisin $39.9 \%$, and osteopenia-in $38.8 \%$ of patients. Normal femoral neck BMD was registered only in $10.4 \%$ of patients, osteoporosis was observed in $60.4 \%$, osteopenia-in $29.2 \%$. Significant positive correlation was found between all BMD values and body mass index of patients $(\mathrm{p}<0.001)$. Positive correlation was registered between $\mathrm{BMD}$ values and serum uric acid $(\mathrm{p}=0.0005)$. Positive correlation between triglycerides levels and T-score was revealed $(\mathrm{p}=0.02)$. Negative correlation between frailty severity and BMD values ( $\mathrm{p}=$ 0.01 ) was found. Positive correlation was found between BMD values and functional abilities of patients assessed using Barthel index ( $\mathrm{p}=$ $0.000002)$ and IADL scale $(\mathrm{p}=0.00008)$. Fractures in history were observed in $27.6 \%$ of patients.

Key conclusions: The study results indicate some features of BMD in long-livers. In the study group significant relationships were found between bone mineral density on the one hand and patients' functional abilities on the other. It is advisable to further study the state of bone tissue in long-livers involving a large sample of patients.

\section{Abstract \# 237 \\ Falls in patients with and without diabetes: a consequence of neuropathy?}

Lubov Machekhina ${ }^{1}$, Ekaterina Dudinskaya ${ }^{1}$, Neil Reeves ${ }^{2}$, Edward Jude $^{3}$

${ }^{1}$ Russian National Research Medical University, ${ }^{2}$ Manchester Metropolitan University, ${ }^{3}$ University of Manchester, Manchester Metropolitan University
Falls Background-objective: Falls are a common reason for hospitalization. However, limited data exists regarding differences in people with and without diabetes mellitus (DM) with falls. The aim of this study was to examine for differences in demographic, clinical, and biochemical characteristics in subjects with and without DM who were hospitalized after falls.

Methods: This is a retrospective non-interventional study that examined all falls hospitalized in two teaching hospital from June 2017 until December 2019. Data of 458 patients (with DM: $n=109$; age $75.8 \pm 9.6 ; 22$ men and 87 women; and non-DM: $n=349$; mean age $76.0 \pm 8.9$ years; 72 men and 277 women and) who were admitted after falls were reported and analyzed.

Results: In the total cohort, falls were much more common in women than in men ( 79.5 vs $20.5 \%$, respectively), while no gender differences were found between patients with and without DM $(\mathrm{p}=0.569)$. Neuropathy was more common in the DM than the non-DM group ( 30.3 vs $15.2 \%$, respectively, $\mathrm{p}=0.001$ ). No significant differences were found between DM and non-DM patients regarding prevalence of foot deformities, amputations, and those wearing or not shoes during falls. Common comorbidities and acute diseases reported during admission (DM vs non-DM) were: hypertension (82.6 vs $83.1 \%, \mathrm{p}=0.882$ ), visual impairment (65.1 vs $57.0 \%, \mathrm{p}=0.133$ ), chronic kidney disease (57.8 vs $62.8 \%, \mathrm{p}=0.353)$, heart disease $(38.5$ vs $45.8, \mathrm{p}=0.151$ ), dizziness ( 37.6 vs $39.8 \%, \mathrm{p}=0.680)$, fatigue $(37.6$ vs $38.4 \%, \mathrm{p}=0.884)$, cognitive impairment (19.3 vs $18.9 \%, \mathrm{p}=$ $0.934)$, palpitation (13.8 vs $17.2 \%, \mathrm{p}=0.398)$, osteoporosis (10.1 vs $14.6 \%, \mathrm{p}=0.341)$, head trauma ( 11.9 vs $12.0 \%, \mathrm{p}=0.968)$, neck pain (11.0 vs $9.2 \%, \mathrm{p}=0.569)$, chronic obstructive pulmonary disease $(9.2$ vs $11.7 \%, p=0.456)$, hemorrhage $(10.1$ vs $8.0 \%, p=0.499)$, muscle weakness (11.9 vs $10.3 \%, \mathrm{p}=0.635)$, alcohol consumption (10.1 vs $6.3 \%, \mathrm{p}=0.182)$ and reduced consciousness $(7.3$ vs $6.9 \%, \mathrm{p}=0.869)$. Low back pain was more in patients with than in those without DM ( 33.9 vs $23.2 \%, p=0.025$ ). There was a trend of stroke to be more common in DM vs non-DM group (17.4 vs 10.6, $\mathrm{p}=0.057)$. The values of pulse rate, hemoglobin, serum glucose, sodium, potassium, triglycerides, urea and creatinine at admission were not different between patients with and without DM. Significantly more patients with DM used b-blockers in comparison with those without DM (50.5 vs $15.2 \%, \mathrm{p}<0.001$ ), while no differences were found in the use of calcium channel blockers, diuretics, non-steroidal anti-inflammatory medications, antipsychotics or proton pump inhibitors. The median value (interquartile range) of hospital stay was similar between patients with [8 (6.25-9.0)] and without DM [8 (6.0-9.0) days, p = 0.938).

Conclusion: In the total cohort, falls were almost four times more common in women than in men. Neuropathy was more frequently found in patients with DM hospitalized for falls. No differences were found in other comorbidities, clinical, demographic and biochemical characteristics between patients with and without DM. Common comorbidities reported by patients who fell were hypertension, visual impairment, chronic kidney disease, heart diseases, dizziness and fatigue.

\section{Abstract \# 238}

Hip fracture and the urinary bladder

Atef Michael ${ }^{1}$, Arlinda Lago ${ }^{1}$

${ }^{1}$ Russells Hall Hospital, Dudley, UK

Introduction: Some older people have falls, resulting in hip fracture, while going to the bathroom to urinate, or coming back. The aim of this study is to analyse the "phenomenon" of hip fracture related to the urinary bladder. 
Methods: Prospective observational study of consecutive patients admitted to a UK teaching hospital with hip fracture in an eight months period. Patients, notes and electronic records were reviewed; data were downloaded on excel sheet and analysed using descriptive statistics.

Results: 377 hip fracture patients were admitted in the study period, of whom 51 patients $(14 \%)$ had falls related to the bathroom. Of these $20 \%(10 / 51)$ male and $80 \%$ (41/51) female with average age of 81.6 and 82.8 years respectively. $51 \%(26 / 51)$ had urinary incontinence. $51 \%(26 / 51)$ had hypertension and 31\% (16/51) had osteoarthritis. $67 \%(34 / 51)$ were on $>4$ drugs, $55 \%(28 / 51)$ were on antihypertensives, $22 \%(11 / 51)$ were on diuretics and $31 \%(16 / 51)$ were on an antidepressant. Falls were multifactorial; frailty in $41 \%$ (21/51) and postural hypotension in $24 \%$ (12/51) were considered the most contributing factors.

Discussion: The number of patients who had fractures related to the urinary bladder could be underestimated as some older people with dementia were found on the floor with no reliable history; many of them could have been going to the bathroom. There are many reasons why elderly people might fall while going to the bathroom including postural hypotension, rushing to avoid urge incontinence, hypnotic or extrapyramidal side effects of medications, not using the mobility aids and poor lighting conditions.

Conclusion: In this study $14 \%$ of falls resulting in hip fracture was attributed to the urinary bladder. Urinary bladder symptoms are a frequently forgotten risk factor for falls and fractures. To reduce the risk in this group of patients, urinary tract symptoms need to be explored, diagnosed and treated. Medications; specially antihypertensives and CNS active drugs should be rationalized and diuretics are to be avoided if possible.

\section{Abstract \# 239}

\section{Gait, balance and fall risk in the elderly}

Viktorija Sevcenko $^{1}$, Asta Mastaviciute ${ }^{1}$, Julius Griskevicius ${ }^{2}$, Kristina Daunoraviciene ${ }^{2}$, Liudvikas Petrauskas ${ }^{2}$, Andrius Apsega ${ }^{1}$, Dovydas Vitkus ${ }^{1}$, Marija Tamulaitiene ${ }^{1}$, Vidmantas Alekna ${ }^{1}$

${ }^{1}$ Vilnius University, Faculty of Medicine Vilnius, Lithuania, ${ }^{2}$ Vilnius Gediminas Technical University, Faculty of Mechanics Vilnius, Lithuania

Introduction: Reduced gait velocity and poor balance are associated with higher risk of falls in the elderly. Objective: The aim of this study was to evaluate relationship between gait, balance and risk of falls in the elderly.

Materials and methods: In this cross-sectional study 60 years and older man and women participated. Sociodemographic data, MiniMental State Examination, Berg Balance scale (BERG), Dynamic Gait Index (DGI) and Tinetti test were performed. Gait parameters such as gait velocity and time were analysed using 6 inertial sensors. Subjects were divided to age groups: 60-69 years. consisted of 28 participants, 70-79 years.- 56 subjects, and 80+ years. - 29 participants.

Results: The data of 113 subjects (32 men and 81 women) which average age was $74.9 \pm 8.33$, were analysed. In $80+$ age group BERG balance score $(37.79 \pm 8.00)$ was statistically significant lower compared to $60-69(45.68 \pm 6.70)$ and $70-79(42.66 \pm 7.15)$ groups (respectively, $\mathrm{p}=0.000$ and $\mathrm{p}=0.013$ ). DGI result was higher in $60-69(18.36 \pm 3.05)$ compared to $80+(14.59 \pm 4.33)$ group $(p=$ $0.001)$. It was found that increasing age was related with higher risk of falls. Age correlated with gait velocity $(\mathrm{r}=-0.299, \mathrm{p}=0.001)$, gait time $(\mathrm{r}=0.320, \mathrm{p}=0.001)$, BERG score $(\mathrm{r}=-0.353, \mathrm{p}=0.000)$, DGI score $(r=-0.323, p=0.000)$ and Tinetti test score $(r=-0.270$, $\mathrm{p}=0.004)$.
Key conclusions: The study results revealed that gait and balance were statistically significant different between age groups. It was found negative relationship between different age groups and Tinetti score, BERG scale, DGI and gait velocity. Gait time showed a positive relationship with age groups.

\section{Abstract \# 240}

\section{Snapshot of a pioneer swiss orthogeriatric unit}

Coutaz Martial $^{1}$, Weiss Lucien ${ }^{1}$

${ }^{1}$ Service de geriatrie, Hopital du Valais

Introduction: Proximal femur fractures are a frequent and lifechanging event among elderly patients. In order to optimize care, the Valais Hospital in Switzerland created a pilot orthogeriatric unit in 2018. This innovative unit, managed by a geriatrician who functions within the orthopedic department, allows timely, standardized management of geriatric syndromes.

Methods: Prospective cohort study from January 1, 2018 through March 1, 2020. Inclusion criteria were hip fracture among patients $\geq$ 70 years, or any elective orthopedic surgery $\geq 75$ years. Primary outcome measures were postoperative complications and mortality.

Results: We enrolled 356 patients, mean age 84 years, 77\% female. The majority were classified as "vulnerable" health status, and 1/3 were "fit". Forty percent had cognitive impairment. Twenty-six percent had osteoporosis, only $1 / 5$ of whom were treated. Malnutrition was prevalent (NRS 5: 22\%). We treated 287 hip fractures $(45 \%$ neck, $48 \%$ intertrochanteric, $7 \%$ periprosthetic). Mean time to operation was 33.5 hours. The most common orthopedic complication was screw displacement $(8.8 \%)$. The most frequent geriatric complications were delirium (17.7\%), urinary retention (13.3\%) and obstipation (6.6\%), along with medical complications that included acute renal failure $(23 \%)$, urinary and pulmonary infections $(21.9 \%)$ and heart failure (13.7\%). Postoperative mortality rate was 5.9\% (17/287).

Key conclusions: In a vulnerable population, our approach showed a low postoperative mortality rate (5.9\%) and a short pre-operative time (33.5 hours). Perioperative complications are frequent, and are often due to non-orthopedic problems, thereby justifying early geriatrician involvement.

\section{Abstract \# 241 \\ Acceptance and effectiveness of the recurrent use of a fall prevention app}

Sophie Rabe ${ }^{1}$, Swantje Müller ${ }^{1}$, Arash Azhand ${ }^{1}$, Wolfgang Pommer ${ }^{2}$, Anika Steinert ${ }^{3}$

${ }^{1}$ Lindera GmbH, ${ }^{2}$ Hochschulmedizin Freie Universität - Charité Berlin/Kuratorium für Dialyse und Nierentransplantation, ${ }^{3}$ Charité Universitätsmedizin Berlin, corporate member of Freie Universität Berlin, Humboldt-Universität zu Berlin, and Berlin Institute of Health; Geriatrics Research Group

Background: A fall prevention app was developed to support care takers in performing a multifactorial fall risk assessment according to required standards [1]. The app generates an individual fall risk score (FRS) [2] with recommendations for fall prevention. The study aimed to evaluate the acceptance of the app as well as its effectiveness in reducing individual FRSs.

Methods: To address these questions, we investigated data from 113 $(83.7 \pm 9.9$ years, $79.5 \%$ female $)$ care recipients participating in a pilot project supported by the AOK Nordost who had completed the fall risk assessment twice (73 \pm 43 days between assessments). Effectiveness of fall risk prevention was evaluated by comparing the 
FRS at baseline and follow-up assessment. Acceptance was analysed through a standardized questionnaire that was completed by 42 geriartric nurses that had used the app.

Results: Mean FRS decreased significantly from 27.2 points at baseline to 24.3 points at follow-up ( $\mathrm{p}=0.002$ ). Regarding acceptance, $81 \%$ of nurses indicated that the use of the app supports them in planning prevention measures, $71 \%$ stated that the use of the application simplifies documentation and $74 \%$ perceived an ease-of-use of the app.

Conclusions: Results showed that the recurrent use of the fall prevention app was associated with a significant decrease in FRS. High acceptance among nurses was reported. The fall risk assessment app has the potential to support care takers in implementing required fall prevention measures. Furthermore, it enables nurses to measure the effectiveness of fall prevention.

References: [1] Schmidt S. Expertenstandard Sturzprophylaxe in der Pflege. In: Schmidt S, ed. Expertenstandards in der Pflege - eine Gebrauchsanleitung. Berlin, Heidelberg, s.l.: Springer Berlin Heidelberg, 2016: 85-106. [2] Rabe, Sophie; Azhand, Arash; Pommer, Wolfgang; Müller, Swantje; Steinert, Anika (2020): Descriptive Evaluation and Accuracy of a Mobile App to Assess Fall Risk in Seniors: Retrospective Case-Control Study. In: JMIR aging 3 (1), e16131. https://doi.org/10.2196/16131.

\section{Abstract \# 242}

\section{Fear of falls in elderly with a fracture history}

Konstantinos Stolakis ${ }^{1}$, Christos Marneras ${ }^{2}$, Theodoros Tosounidis ${ }^{3}$, Elias Panagiotopoulos ${ }^{4}$

${ }^{1}$ University of Patras (U.P), Medicine School of Patras (M.S.P), Greece, ${ }^{2}$ University Hospital of Rio, Patras (U.H.P), Greece, ${ }^{3}$ Department of Orthopaedic Surgery, University Hospital of Heraklion (U.H.H), Crete, Greece, ${ }^{4}$ Rehabilitation Department, University of Patras (U.P), Rio-Patras, Greece

Introduction: The aim of the study was to compare the fear of falls and relevant characteristics between elderly people who had a fracture and those who had a reported fall.

Method: The cross-sectional study was conducted in March 2020, with random sampling from the list of members of an Open Protection Centre for the Elderly of Patras (194 active members). The sample size was 64 people over 65 years (50 women). The Falls Efficacy Scale International (FES-I) Greek questionnaire was used to investigate the fear of falls, while the Cronbach's Alpha reliability test showed very high reliability $(\alpha=0.952)$.

Results: The mean age of the sample was 72.15 years $( \pm 6.04)$. $32.8 \%$ of the sample reported a fall (during 2019) and 37.5\% reported a fracture in the last 3 years. The Kruskal-Wallis non-parametric test showed a statistically significant difference between men and women concerning fractures $(\mathrm{X} 2=9.585, \mathrm{DF}=1, \mathrm{p}=0.002<0.01)$. The non-parametric Mann-Whitney test showed a statistical difference in FES-I, indicating that women are more afraid of a possible fall $(\mathrm{U}=$ $227.00, p=0.045, \dot{X}=29.90, S D= \pm 11.15$ ). Finally, the Spearman's non-parametric $\mathrm{r}$ control showed a positive correlation between FES-I with sex $(\mathrm{r}=0.252, \mathrm{p}<0.05)$ and number of falls $(\mathrm{r}=0.306, \mathrm{p}<$ 0.05 ), while a negative correlation was found with self-perception of health $(\mathrm{r}=-0.300, \mathrm{p}<0.05)$, falls $(\mathrm{r}=-0.270), \mathrm{p}<0.05)$ and vision problems $(\mathrm{r}=-0.419, \mathrm{p}<0.01)$.

Conclusions: The study found important risk factors of fear of falls, indicating the need of the systematic education of the elderly to prevent it.

\section{Abstract \# 243}

Frailty and the rate of fractures in patients initiated on antihypertensive medication

Marc Osterdahl $^{1}$, Angel Wong ${ }^{1}$, Ian Douglas ${ }^{1}$, Sarah-Jo Sinnott ${ }^{1}$, Liam Smeeth $^{1}$, Elizabeth Williamson ${ }^{1}$, Laurie Tomlinson ${ }^{1}$

${ }^{1}$ London School of Hygiene \& Tropical Medicine, London, UK

Introduction: There is concern regarding adverse effects of antihypertensive treatment, including falls and subsequent fractures, especially hip fractures. As frailty is increasingly recognised as an important risk factor for adverse outcomes, we examined its relationship to fracture rates in older patients after starting antihypertensives.

Methods: Using the Clinical Practice Research Datalink (CPRD), we identified participants over 65 -years old starting a first-line antihypertensive medication. Using deficits identified in CPRD we classified patient-level frailty as 'Fit', 'Mild', 'Moderate' or 'Severe' using the Electronic Frailty Index. We calculated the rate of fractures by frailty level and fracture site, and determined the rate ratio (RR) of first fracture by frailty level, adjusting for confounding. We conducted sensitivity analyses to additionally adjust for ethnicity, deprivation, and bisphosphonate use.

Results: $44 \%$ of participants were classified as mildly frail or greater, but frail participants experienced $58 \%$ of all fractures, and $63 \%$ of hip fractures. The whole cohort showed a crude rate of 14.1 fractures/ 1000 person-years , with 4.5 hip fractures $/ 1000$ person-years. In severe frailty, this rises to 51.0 fractures/1000 person-years, and 17.7 hip fractures/1000 person-years. After adjustment for confounding, increasing frailty was associated with greater rate of any fracture, reaching RR 2.85 (95\% confidence interval 2.43-3.33) for severe frailty versus fit. Results were unchanged in sensitivity analyses.

Key conclusions: Frailty and fracture are both common in older participants who start antihypertensive medications. Increasing frailty was positively associated with increased rates of fracture. Clinicians need awareness of this relationship to consider fracture risk assessment and prevention in these patients.

\section{Abstract \# 244}

The effect of vitamins Cyanocobalamin (B12) and 25-OH-vitamin D3 (D3) and anticholinergic drugs on balance and falls. Results from a geriatric patients sample in the clinic for fall prevention, osteoporosis and sarcopenia (C.F.P.O.S) of the General Univer

Konstantinos Stolakis ${ }^{1}$, Zinon Kokkalis ${ }^{2}$, Eleftheria Antoniadou ${ }^{3}$, Xristina Kalivioti ${ }^{3}$, Amalia Koloniari ${ }^{3}$, Manolis Mentis ${ }^{4}$, Panagiotis Megas $^{2}$, Minos Tyllianakis ${ }^{2}$, Elias Panagiotopoulos ${ }^{5}$

${ }^{1}$ School of Medicine, University of Patras (U.P), Greece, ${ }^{2}$ Orthopedic Department, General University Hospital of Patras (G.U.H.P), Greece, ${ }^{3}$ Rehabilitation Clinic, General University Hospital of Patras (G.U.H.P), Greece, ${ }^{4}$ Department of Education and Social Work, University of Patras (U.P) Greece, ${ }^{5}$ Rehabilitation Clinic, General University Hospital of Patras (G.U.H.P), Greece. Orthopedic Department, General University Hospital of Patras (G.U.H.P), Greece.

Introduction: Balance disorder and falls are common in the geriatric population with multifactorial etiology. The aim of the present study was to evaluate the influence of vitamin D3, vitamin B12 and drugs with anticholinergic action on falls, balance and fractures.

Methods: The level of vitamin D3, vitamin B12 and Hand Grip strength (HGA) were evaluated in 96 patients of both sexes and age $\geq$ 65, from January 2018 to December 2019 during their first visit to the C.F.P.O.S. The number of falls and fragility fractures of the last year 
was also recorded and the ANTICHOLINERGIC-COGNITIVEBURDEN Scale score (ACBscore) was calculated. The balance was evaluated with the Mini-Balance-Evaluation-Systems-Test (MiniBESTest).

Results: Linear Bivariate Correlation did not indicate a statistical strong relationship between vitamin B12 level, Mini BESTest score and the number of falls and fractures. A statistical negative correlation was found between blood level of vitamin D3 and the number of falls and fractures ( $r s(93)=-0.274, p=0.008<0.05$ ). Multiple Linear Regression analysis, using the Enter method, indicate that from the studied factors, vitamin D3 can predict with statistical significance the number of falls and fractures $(p=0.028<0.05)$ and that ACBscore can predict with statistical significance the Hand Grip values levels $(\mathrm{p}=0.022<0.05)$.

Conclusion: In the present study vitamin D3 appears to influence falls and fractures in contrast to vitamin B12. The use of anticholinergic drugs affects muscle strength negatively, but not falls and fractures. A larger sample of participants and further studies are needed to confirm our findings.

\section{Abstract \# 245}

Verbal working memory is associated with bone mineral density in women assessed for bone fragility

Antonino Catalano ${ }^{1}$, Alberto Sardella ${ }^{1}$, Federica Bellone ${ }^{1}$, Carmen Lasco $^{1}$, Gabriella Martino ${ }^{1}$, Morabito Nunziata ${ }^{1}$, Francesco Corica ${ }^{1}$

${ }^{1}$ Department of Clinical and Experimental Medicine, University Hospital of Messina, Messina, Italy

Introduction: Bone loss and related fractures have been previously associated with cognitive and psychological features. The aim of our study was to explore the association between executive functions with fracture risk in a cohort of postmenopausal women.

Methods: We performed cognitive tests assessing executive functions (Trial Making Test-B, Digit Span Backward, Digit Span Forward), questionnaires measuring psychological features (Hamilton Anxiety Scale and Back Depression Inventory), physical performance evaluation (Short Physical Performance Battery and handgrip strength), evaluation of 10-year probability of major and hip fractures (Fracture Risk Assessment tool - FRAX), bone mineral density (BMD) measurement (Dual-Energy X-ray Absorptiometry at lumbar spine and femoral neck).

Results: Participants $(n=60$, mean age $66 \pm 7.99$ year) showed a Mini Mental State Examination (MMSE) score $\geq 24$. Trial Making Test B score $(r=-0.25)$ and Digit Span Backward $(r=-0.34)$ were associated with FRAX score for major fractures; the FRAX score for hip fracture was associated with handgrip strength $(\mathrm{r}=$ - 0.39). BMD at lumbar spine was associated with Digit Span Backward $(r=-0.32)$ and with depression $(r=-0.33)$. After adjustment for age, BMI, MMSE score, handgrip strength, Digit Span Forward score, Verbal Fluency score, Trial Making Test-B score, a multiple regression analysis showed BMI $(\beta=0.09$, SE $0.03, p=$ $0.013)$, Beck Depression Inventory score $(\beta=-0.09$, SE $0.06, p=$ $0.04)$ and Digit Span Backward score $(B=0.55$, SE 0.17, p = 0.002) independently predicted lumbar BMD.

Key conclusions: Verbal working memory, as assessed by Digit Span Test, was associated with BMD and could contribute to fracture risk prediction in postmenopausal women.

\section{Abstract \# 246}

Factors associated with blood transfusion during hospital treatment of hip fractures

Noelia CC Salazar ${ }^{1}$, Luana Sandoval Castillo ${ }^{2}$, Julie Braüner Christensen ${ }^{3}$, Martin Aasbrenn ${ }^{3}$, Anette Addy Ekmann ${ }^{2}$, Thomas Giver Jensen ${ }^{4}$, Troels Haxholdt Lunn ${ }^{5}$, Henrik Palm ${ }^{4}$, Charlotte Suetta $^{2}$

${ }^{1}$ Hospital General de Zona \#2, Instituto Mexicano del Seguro Social, Hermosillo, Mexico, ${ }^{2}$ Department of Geriatric Medicine, Bispebjerg and Frederiksberg Hospital, Copenhagen, Denmark, ${ }^{3}$ CopenAge, Copenhagen University, Denmark, ${ }^{4}$ Department of Orthopedic surgery, Bispebjerg and Frederiksberg Hospital, Copenhagen, Denmark, ${ }^{5}$ Department of Anesthesia, Bispebjerg and Frederiksberg Hospital, Copenhagen, Denmark

Introduction: The purpose of the present study was to investigate the factors associated with perioperative blood transfusions in patients with hip fractures.

Methods: Patients above 65 years admitted to the department of orthopedic surgery at Bispebjerg and Frederiksberg Hospital, Copenhagen, Denmark with a hip fracture in 2018 were included in the study. Characteristics of the surgery and the anesthesia, demographics, comorbidities, medication, blood pressure values and blood tests were registered. A multivariable analysis was used to determine risk factors, data are presented as odds ratios (OR) with $95 \%$ confidence intervals (CI).

Results: In total, 300 patients with a mean age of $83 \pm 9$ years were included. $27 \%$ were men. $54 \%$ had femoral neck fractures, $42 \%$ pertrochanteric fractures and $4 \%$ subtrochanteric fractures. $31 \%$ of the patients had at least one blood transfusion. The mean preoperative haemoglobin $(\mathrm{Hb})$ was $12.4 \pm 1.9 \mathrm{~g} / \mathrm{dL}$, the mean postoperative $\mathrm{Hb}$ was $10.3 \pm 1.8 \mathrm{mmol} / \mathrm{L}$. Predictors for transfusion included cardiac failure (OR 1.9, 95\% CI 1.1-3.5), operation with intramedullary hip screw (IMHS) (OR 4.8, 95\% CI 2.7-8.7), lowest intraoperative diastolic blood pressure under $50 \mathrm{mmHg}$ (OR 10.6, 95\% CI 1.8-62.7), subtrochanteric fracture (OR 9.3, 95\% CI 1.1-79.8) and surgical bleeding above $300 \mathrm{ml}$ (OR 3.2, 95\% CI 1.5-6.7).

Conclusions: In the present cohort, cardiac failure, subtrochanteric fractures, IMHS surgery, intraoperative diastolic blood pressure under $50 \mathrm{mmHg}$ and surgical bleeding above $300 \mathrm{ml}$ were factors associated with blood transfusion. Careful preoperative planning and multidisciplinary management efforts are crucial to reduce the use of postoperative transfusions.

\section{Abstract \# 247}

Vertebral-fracture treatment gap in hospitalized old frail patients Ekaterini Zigoura $^{1}$, Alberto Cella ${ }^{2}$, Annarosa Floris ${ }^{1}$, Alberto Pilotto ${ }^{3}$

${ }^{1}$ Bone Disease Center, Department of Geriatrics, Orthogeriatrics and Rehabilitation, Galliera Hospital, Genova, Italy, ${ }^{2}$ Post-Acute Care Unit, Department of Geriatrics, Orthogeriatrics and Rehabilitation, Galliera Hospital, Genova, Italy, ${ }^{3}$ Department of Geriatrics, Orthogeriatrics and Rehabilitation, Galliera Hospital, Genova, Italy and Department of Internal Medicine of Bari University, Bari, Italy

Introduction: The prevalence of osteoporosis and its consequences are increasing worldwide due to global population aging. Despite numerous campaigns, osteoporosis is not considered to be a serious disease in the elderly and many patients with a fragility fracture do not receive a prescription for diagnostic tests or anti-osteoporotic therapy. In this study, we examined whether old patients hospitalized for a medical condition with evidence of vertebral fracture, due to fragility, received a pharmacological treatment for osteoporosis. 
Methods: 200 medical records of patients who received acute hospital geriatric care between February and March 2020 have been examined. Patients hospitalized for COVID-19 were excluded. Radiologic exams performed for low back pain or other purposes have been considered.

Results: 29 patients presented vertebral fractures revealed by radiologic exams. The mean age was 86.8 years old and the range of the fragility index measured by MPI (multi-prognostic-index) was between 0,56 and 0.88 . In some cases vitamin $\mathrm{D}$, calcium supplementation and a neurosurgeon consultancy have been indicated but, no one received an anti-fracture therapy.

Key conclusions: Vertebral fractures are often misdiagnosticated, but even when diagnosticated are under-treated. There is currently sufficient evidence to initiate appropriate treatment in the elderly with osteoporosis and osteoporotic fractures. A fracture liaison service could be the bridge between the patient and the bone specialist, enabling the initiation of effective programs of secondary prevention.

\section{Abstract \# 248 \\ Development of prediction models for falls and recurrent falls in community-dwelling older adults}

Bob van de Loo ${ }^{1}$, Lotta Seppala ${ }^{2}$, Natasja van Schoor $^{3}$, Martijn Heymans ${ }^{3}$, Stephanie Medlock ${ }^{4}$, Dietrich Rothenbacher ${ }^{5}$, Michael Denkinger $^{6}$, Ameen Abu-Hanna ${ }^{4}$, Nathalie van der Velde ${ }^{7}$

${ }^{1}$ Department of Internal Medicine section of Geriatrics - location AMC, Department of Epidemiology and Biostatistics - location VUmc, Amsterdam UMC, UvA, APH, Amsterdam, The Netherlands, ${ }^{2}$ Department of Internal Medicine section of Geriatrics - location AMC, Amsterdam UMC, UvA, APH, Amsterdam, The Netherlands, ${ }^{3}$ Department of Epidemiology and Biostatistics - location VUmc, Amsterdam UMC, VU Amsterdam, APH, Amsterdam, The Netherlands, ${ }^{4}$ Medical Informatics Department - location AMC, UvA, Amsterdam UMC, Amsterdam, The Netherlands, ${ }^{5}$ Institute of Epidemiology and Medical Biometry, Ulm University, Ulm,

Germany, ${ }^{6}$ Agaplesion Bethesda Clinic, Geriatric Research Unit Ulm University, Ulm, Germany, ${ }^{7}$ Department of Geriatric Medicine location AMC, Amsterdam UMC, UvA, APH, Amsterdam, The Netherlands

Introduction: Effective fall prevention strategies require an early detection of high-risk patients. However, previous tools for predicting falls have generally shown poor predictive performance, which may be partly due to a limited number of considered candidate predictors. Our goal is to create better performing prediction models for falls by combining several cohort studies and taking a wide variety of variables in consideration.

Methods: Harmonized data from three cohort studies of adults aged 65 years and older (LASA, ActiFE-Ulm, and B-Proof) were used to fit two logistic regression models: one for predicting any fall and another for predicting recurrent falls ( $\geq 2$ falls) over a period of 12 months. Candidate predictors were selected based on previously established associations with falls. Backward selection was performed. We conducted internal-external cross-validation to assess generalisability of the models.

Results: A total of 5882 participants were included in the analyses, of whom $1868(31.8 \%)$ endured at least one fall and $702(11.9 \%)$ endured two or more falls. The final models for any fall and recurrent falls included 13 and 11 variables, respectively. Predictors present in both models were: BMI, functional limitations, history of falls, smoking status, and educational level. The models for any fall and recurrent falls showed good calibration and yielded C-statistics of 0.66 and 0.71 , respectively. Internal-external cross-validation showed limited performance for both models in ActiFE-Ulm.
Conclusions: We developed two prediction models for falls using pooled data of cohort studies. Our models compare favourably to previous tools for predicting (recurrent) falls.

\section{Abstract \# 249}

Effectiveness of interventions for preventing falls and fall-related fractures in community- dwelling older adults: a systematic review and network meta-analysis

Lauren Dautzenberg ${ }^{1}$, Shanthi Beglinger ${ }^{2}$, Sofia Tsokani ${ }^{3}$, Stella Zevgiti $^{3}$, Renee Raijmann ${ }^{1}$, Nicolas Rodondi ${ }^{2}$, Rob Scholten ${ }^{4}$, Anne Rutjes $^{5}$, Marcello Di Nisio ${ }^{6}$, Marielle Emmelot-Vonk ${ }^{1}$, Andrea Tricco $^{7}$, Sharon Straus ${ }^{8}$, Sonia Thomas ${ }^{9}$, Lisa Bretagne ${ }^{2}$, Wilma $\mathrm{Knol}^{1}$, Dimitris Mavridis ${ }^{10}$, Dineke Koek ${ }^{1}$

${ }^{1}$ Department of Geriatric Medicine, University Medical Centre Utrecht, Utrecht University, Utrecht, the Netherlands, ${ }^{2} 1$ Department of General Internal Medicine, Inselspital, Bern University Hospital, University of Bern, Bern, Switzerland. 2 Institute of Primary Health Care (BIHAM), University of Bern, Bern, Switzerland., ${ }^{3}$ Department of Primary Education, School of Education, University of Ioannina, Ioannina, Greece, ${ }^{4}$ Cochrane Netherlands / Julius Centre for Health Sciences and Primary Care, University Medical Centre Utrecht, Utrecht University, Utrecht, The Netherlands, ${ }^{5} 1$ Institute of Primary Health Care (BIHAM), University of Bern, Bern, Switzerland 2. Institute of Social and Preventive Medicine (ISPM), University of Bern, Bern, Switzerland, ${ }^{6}$ Department of Medicine and Ageing Sciences, University G. D’Annunzio, Chieti, Italy, ${ }^{7} 1 \mathrm{Li}$ Ka Shing Knowledge Institute, St. Michael's Hospital, 209 Victoria Street, East Building, Toronto, Ontario, M5B 1W8, Canada. 2. Epidemiology Division, Dalla Lana School of Public Health and Institute for Health, Management, and Evaluation, University of Toronto, Toronto, Ontario, Canada, ${ }^{8} 1$ Li Ka Shing Knowledge Institute, St. Michael's Hospital, 209 Victoria Street, East Building, Toronto, Ontario, M5B 1W8, Canada. 2. Epidemiology Division, Dalla Lana School of Public Health and Institute for Health, Management, and Evaluation, University of Toronto, Toronto, Ontario, Canada 3. Department of Geriatric Medicine, University of Toronto, Toronto, Ontario, Canada, ${ }^{9}$ Li Ka Shing Knowledge Institute, St. Michael's Hospital, 209 Victoria Street, East Building, Toronto, Ontario, M5B 1W8, Canada, ${ }^{10} 1$ Department of Primary Education, School of Education, University of Ioannina, Ioannina, Greece. 2. Paris Descartes University, Sorbonne Paris Cité, Faculté de Médecine, Paris, France.

Objective: To compare the effectiveness of single, multiple and multifactorial interventions to prevent falls in community-dwelling older persons, with a focus on multimorbidity.

Design: Systematic review, network meta-analysis (NMA).Data sources: MEDLINE, Embase and Cochrane Central Register of Controlled Trials until February 27, 2019.

Eligibility criteria: Randomised controlled trials evaluating the effectiveness of fall prevention interventions in community-dwelling adults aged $\geq 65$ years. Main outcome measures: number of fallers, number of fall-related fractures.

Results: After screening 3005 studies for eligibility, 220 studies with 104,638 participants were included. NMA ( $\mathrm{N}=190$ studies) revealed a reduction in number of fallers, compared to usual care, for single interventions exercise (risk ratio (RR) 0.83 ; $95 \%$ confidence interval (CI) 0.77-0.90) and quality improvement strategies (RR 0.90; 95\% CI 0.83-0.99). Most effective multiple interventions were assistive technology in combination with basic falls risk assessment (RR 0.52; 95\% CI 0.30-0.90) and assistive technology in combination with quality improvement strategies (RR 0.58 ; 95\% CI 0.41-0.81). Relatively few studies $(\mathrm{N}=14)$ were available specifically for multimorbid participants and the NMA revealed no statistically 
significant changes in number of fallers compared to usual care. The number of fall-related fractures was reduced by the single interventions basic falls risk assessment (RR $0.60 ; 95 \%$ CI $0.39-0.94$ ) and exercise (RR 0.62; 95\% CI 0.42-0.90).

Conclusion: Several single and multiple fall prevention interventions lead to fewer falls. Basic falls risk assessment and exercise lead to fewer fall-related fractures. Few studies have investigated the effect of fall prevention interventions in multimorbid older people, a topic which is of high importance for future research.

\section{Abstract \# 250 \\ Association of nonselective beta-blocker eyedrops and injurious falls: a Danish nationwide active comparator, new user propensity score matched cohort study \\ Sidsel Arnspang Pedersen ${ }^{1}$, Mette Reilev ${ }^{1}$, Lars Christian Lund ${ }^{1}$, Daniel Henriksen ${ }^{2}$, Bjarke Viberg ${ }^{3}$, Jesper Ryg ${ }^{4}$ \\ ${ }^{1}$ University of Southern Denmark, ${ }^{2}$ Department of Clinical Pharmacology, Odense University Hospital, ${ }^{3}$ Department of Orthopedic Surgery, Odense University Hospital, ${ }^{4}$ Department of Geriatric Medicine Odense Universitetshospital}

Background: Non-selective beta-blocking eye drops such as timolol potentially increase the risk of falls due to systemic absorption. Our aim was to investigate the association between initiation of timolol eye drops and injurious falls in older patients.

Methods: We conducted an active comparator, new user cohort study using information on redeemed prescriptions and hospital discharge diagnoses from the Danish nationwide registries. We identified all patients aged $\geq 65$ years, who initiated timolol eye drops or topical prostaglandin analogues and matched them in a 1:1 ratio, based on a high-dimensional propensity score. Primary outcome was injurious falls (contusion, wounds, fractures other than hand/feet), within 90 days of first prescription. Secondary outcome was fall-related fractures. We obtained incidence rate ratios (IRR) and incidence rate differences (IRD).

Results: We identified 40,420 users of timolol and 55,754 users of prostaglandin analogues; $43 \%$ were male and age (median [IQR]) was 75 [70-80] years. For the unmatched cohort, the IRR of injurious falls was 1.20 (95\% CI 0.98-1.49) and IRD was 4 (95\% CI 0-8) cases/ 1000 person years for treatment with timolol compared to prostaglandin analogues. In the propensity score matched cohort, we included 28,431 users of timolol and prostaglandin analogues, respectively. The IRR of injurious falls was 0.96 (95\% CI $0.73-1.27$ ) and IRD was- 1 (95\% CI -6 to 5 ) cases/1000 person years. For fallrelated fractures, the IRR was 1.30 (95\% CI 0.96-1.76) and IRD was 4.0 (95\% CI -0.5 to 8.4$)$ cases $/ 1000$ person years.

Conclusions: We found no association between initiation of timolol eye drop treatment and injuries falls in older people.

\section{Abstract \# 251}

\section{Falls in patients with the frailty}

Anton Naumov ${ }^{1}$, Natalia Khovasova ${ }^{1}$, Viktoriya Moroz ${ }^{2}$

${ }^{1}$ Department of aging diseases of Pirogov Russian National Research Medical University, Russian Clinical and Research Center of Gerontology, ${ }^{1}$ Department of aging diseases of Pirogov Russian National Research Medical University, Russian Clinical and Research Center of Gerontology

Introduction: Falls and the frailty are geriatric syndromes having common risk factors and adverse health outcomes. Falls weigh the course of the frailty, which leads to an increase in the human dependence on others. To maintain the existing level of autonomy, early detection of risk factors and prevention of falls in the group of the elderly patients with the frailty is necessary.

Aim: To evaluate the incidence of falls, their characteristics and functional status in patients with the frailty.

Materials and methods: 170 patients with the frailty $(78.6 \pm 7.8$ years, 136 women, $80 \%$ ) were divided into 2 groups: 1 group - 117 $(68.8 \%)$ patients with falls, 2 group $-53(31.2 \%)$ patients without falls. All patients underwent a general clinical examination and a comprehensive geriatric assessment. Charlson Comorbidity Index did not differ significantly in both groups.

Results: The incidence of falls was $68.8 \%$. The number of falls is $2.29 \pm 1.9 ; 2$ or more falls occurred in $65(55.6 \%)$ people. $97(82.9 \%)$ people had falls earlier, $54.7 \%$ of them developed a fear of falls. Fractures were diagnosed in $13.7 \%$. Common risk factors for falls in patients with the frailty were: vitamin D deficiency $(86.1 \%)$, chronic pain $(84.7 \%)$, sensory deficiency $(69.9 \%)$, imbalance $(60.2 \%)$, inactivity $(56.1 \%)$ and cognitive impairment (44.9\%). In patients with falls, compared with patients without them, the following were lower: muscle strength $(16.04 \pm 7.5$ and $22.4 \pm 6.1, \mathrm{p}<0.05)$, walking speed $(0.44 \pm 0.34$ and $0.52 \pm 0.22, \mathrm{p}<0.05)$ and SPPB score $(3.98$ \pm 2.1 and $4.9 \pm 2.12, \mathrm{p}<0.05)$. Patients in 1 group were 2 times more likely to have a pronounced dependence (Bartel index). The number of geriatric syndromes was higher in patients of 1 group $(8.4 \pm 2.4$ and $6.2 \pm 3.5, \mathrm{p}<0.05)$.

Conclusion: Every second patient with the frailty has a fall, which worsens the functional status of the patient and leads to a decrease in autonomy. Falls are prevented in most cases by screening for risk factors and correcting them.

\section{Abstract \# 252}

Cross-country comparison between asian and caucasian older adults on the association between obesity and falls

Sheng Hui Kioh ${ }^{1}$, Sumaiyah Mat ${ }^{1}$, Phyo Kyaw Myint ${ }^{2}$, Wolgang Kemmler $^{3}$, Ellen Freiberger ${ }^{4}$, Maw Pin Tan ${ }^{5}$

${ }^{1}$ Ageing and Age-Associated Disorders Research Group, University of Malaya, 50603, Kuala Lumpur, Malaysia, ${ }^{2}$ Ageing Clinical \& Experimental Research (ACER) Group, Institute of Applied Health Sciences, University of Aberdeen, United Kingdom, ${ }^{3}$ Institute of Medical Physics, University of Erlangen-Numberg, Germany, ${ }^{5}$ Institute of Biomedicine of Aging, University of Erlangen-Numberg, Germany, ${ }^{5}$ Department of Medicine, Faculty of Medicine, University of Malaya, Malaysia

Introduction: Previous studies on obesity or increased BMI and falls in older adults have not evaluated potential regional references. This study, therefore, aimed to identify potential differences in the relationship between increased body mass index (BMI) and falls between an Asian and two Caucasian populations.

Methods: Baseline screening data from the MELoR (Malaysia), FoRMOSA (Germany) and EPIC-NORFOLK (UK) studies were utilized. Basic demographic characteristics, medical history, anthropometric parameters, physical assessments and 12-month falls history were extracted from the existing datasets. Only data for women aged 65 years and over and women aged 70 years and above from the MELoR and EPIC-NORFOLK to allow for direct comparisons with FORMOSA. Comparisons were conducted using logistic regression with MELoR as the reference group.

Results: Data from 3536 (1751 EPIC-NORFOLK, 1319 FoRMOSA and 466 MELoR) older women were included. A significant association was observed between BMI and falls in the previous 12 months for both FoRMOSA and MELoR study but not EPIC-NORFOLK. The relationship between BMI and falls was attenuated for MELoR 
women after additional adjustment for \%body fat $(\mathrm{aOR}=1.03$, $95 \% \mathrm{CI}=0.98-1.10)$, but the relationship remained unchanged for FoRMOSA.

Conclusion: Notable differences in country patterns for the relationship between BMI and falls were identified, which could be attributed to body composition and lifestyle differences observed between the populations. Our findings will inform cross-cultural translation of existing evidence for fall prevention in older persons with increased adiposity, while increased body fat could be a modifiable risk factor for falls in the Malaysian, but not German or UK settings.

\section{Abstract \# 253 \\ Potentially inappropriate prescribing (PIP) in a real-world falls registry \\ Rosalind Chiam ${ }^{1}$, Tan Maw Pin ${ }^{1}$ \\ ${ }^{1}$ University of Malaya}

Introduction: Polypharmacy is common in older adults, with increased risk of adverse events, which increases with potentially inappropriate prescribing (PIP). This study applied the STOPPSTART criteria to a real-world registry and to determine the burden of PIP among older adults attending a falls and syncope clinic.

Methods: The falls and syncope registry at the University of Malaya Medical Centre comprised a dataset containing individuals $\geq 65$ years evaluated for dizziness, syncope and falls since it was started in 2014. Information on symptoms, medical history, medications and diagnoses were recorded. The STOPP-START criteria were applied to individual prescriptions by one researcher and cross-checked by a second researcher.

Results: At least one PIP was identified in $275(65.6 \%)$ accounting for $85 \%$ of the prescriptions. Patients with two or more comorbidities were more likely to be exposed to two or $\geq 2$ PIP. Of those with polypharmacy ( $\geq 5$ medications), 144 (81.4\%) were exposed to $\geq 2$ PIMs. 361 (54.8\%) PIP were within the STOPP K criteria which were "drugs that predictably increase the risk of falls in older adults".

Conclusions: Vasodilators were the PIP identified in the real-world falls registry. The STOPP \& START criteria is a useful medication review tool for PIP in older patients within a fall and syncope clinic setting and identifies potential omissions as well as inclusion.

\section{Abstract \# 254}

\section{Hospital falls and implicated drugs in older patients}

Ana Andrade Oliveira ${ }^{1}$, Sílvia Oliveira ${ }^{1}$, Paulo Gouveia ${ }^{1}$

\section{${ }^{1}$ Hospital de Braga}

Introduction: Hospital falls are prevalent among the elderly and connected to increased morbidity and mortality. Several drugs are associated with increased risk of falls, mainly the ones working on brain and circulation. We analyzed the high-risk drugs administered 24 hours before the falls among hospitalized patients older than 74 years old.

Methods: We performed a retrospective study covering the hospital falls recorded in 2018. Data were collected through clinical files. Statistical analysis was executed with SPSS.

Results: In 2018 were recorded 270 hospital falls, in 246 patients; $48 \%$ of them happened in patients older than 74 years old. In the 24 hours before the fall, each patient received a medium of $8.8 \pm 3.1$ drugs. The most prescribed medicines were psychotropic drugs $(68 \%)$, being benzodiazepines the most prescribed psychotropics (70\%). The second most prescribed drugs were anti-hypertensives
(42\%), with a preference for angiotensin-converting-enzyme inhibitors (ACE inhibitors) (71\%). Opiate analgesics were administered in $26 \%$ of the patients.

Discussion: The risk of falls rises with the number of drugs prescribed, nevertheless older patients continue under an excessive number of drugs during hospitalization, as we demonstrate. Although psychotropic drugs are known to increase falls risk, with literature advising against their use in the elderly, we continue to see more than half of these patients under them. Drugs are a modifiable risk factor for falls, a periodical drug review on high-risk medications should be made in elderly patients.

\section{Abstract \# 255 \\ Sarcopenia evaluation with computed tomography in trauma patients}

Funda Datlı Yakaryılmaz ${ }^{1}$, Mehtap Ilgar ${ }^{2}$

${ }^{1}$ Malatya Education and Research Hospital, Department of Geriatrics, ${ }^{2}$ Malatya Education and Research Hospital, Department of Radiology

Aim: Sarcopenia is characterized by a decline in muscle mass, muscle strength, and physical activity(1). Recent studies have shown that sarcopenia is associated with impaired physical capability, poor quality of life, adverse metabolic effects, falls, disability, mortality, and hospitalization (2). The sectional area of skeletal muscle taken from the abdomen is strongly related to the whole body muscle distribution, and it is known that the cross-sectional skeletal muscle index (SMI, $\mathrm{cm}^{2}$ ) at L4 level is highly associated with the total body skeletal muscle mass (2). It also provides the ability to detect muscle radiation attenuation (SMRA, Hounsfield Units (HU)), a measure of muscle quality that is inversely related to muscle fat content (3). In this study, we aimed to show the change of muscle area and density measured by cross-sectional CT by age.

Methods: In January-March 2020, 265 patients who were admitted to the emergency room of our hospital due to trauma, who had underwentabdominal computed tomography (CT) scan including L3 and umbilical levels for measuring SMI and transverse psoas muscle thickness, respectively, were included. Sarcopenia was defined as a L3 skeletal muscle index of $49 \mathrm{~cm}^{2} / \mathrm{m}^{2}$ for men and $31 \mathrm{~cm}^{2} / \mathrm{m}^{2}$ for women using Korean-specific cutoffs (4).

Results: 265 patients were included. The average age was $62.8 \pm 12.4$ years, and 133 patients $(50.2 \%)$ were men. One hundred seven (40.4\%) patients met the criteria for SMI-sarcopenia. The mean SMRA level was significantly lower in the sarcopenia group than the non-sarcopenia group $(p=0.008)$. There was a statistically significant negative correlation between age and SMI, SMRA $(\mathrm{p}=0.005, \mathrm{p}=$ 0.008 , respectively).

Conclusions: The high diagnosis of sarcopenia with CT taken in patients admitted to the hospital after trauma indicates that sarcopenic individuals are more susceptible to trauma.Therefore, early diagnosis and treatment of sarcopenia in older people will be effective in reducing hospital applications.

Referances: 1. Delmonico MJ, Harris TB, Visser M, Park SW, Conroy MB, VelasquezMieyer P, et al. Longitudinal study of muscle strength, quality, and adipose tissue infiltration. Am J Clin Nutr 2009;90:1579-1585. 2. Tanimoto Y, Watanabe M, Sun W, Sugiura Y, Tsuda Y, Kimura M, et al. Association between sarcopenia and higher-level functional capacity in daily living in community-dwelling elderly subjects in Japan. Arch Gerontol Geriatr 2012;55:e9-e13. 3. Di Sebastiano KM, Mourtzakis M. A critical evaluation of body composition modalities used to assess adipose and skeletal muscle tissue in cancer. Appl Physiol Nutr Metab. 2012;37:811-21. 4. Kim YS, Lee Y, Chung YS, Lee DJ, Joo NS, Hong D, et al. Prevalence of sarcopenia and sarcopenic obesity in the Korean population based on 
the Fourth Korean National Health and Nutritional Examination Surveys. J Gerontol A Biol Sci Med Sci. 2012; 67(10):1107-13. Epub 2012/03/21. https://doi.org/10.1093/gerona/gls071 PMID: 22431554.

\section{Abstract \# 256 \\ Nocebo effects increase with advancing age in clinical trials of bisphosphonate therapy for osteoporotic vertebral compression fracture prevention \\ Evrydiki Kravvariti ${ }^{1}$, Pelagia Mouratidou ${ }^{1}$, Petros P Sfikakis ${ }^{1}$ \\ ${ }^{1}$ Postgraduate Medical Studies in Geriatric Syndromes and Physiology of Aging, School of Medicine, NKUA, Athens, Greece}

Introduction: Adverse reactions to a medical intervention due to negative treatment expectations, i.e. nocebo effects, can be quantified as the rate of dropouts from the placebo arm of a double-blind randomized controlled trials (RCTs) due to reported "treatment-related" adverse effects. Nocebo effects have been identified as a potential cause of medication non-adherence, but their role in bisphosphonate intolerance and their association with advancing age has not been clarified.

Methods: We searched MEDLINE, EMBASE, CENTRAL, and Web of Science electronic databases for placebo-controlled double-blind RCTs evaluating the effectiveness of oral bisphosphonates versus placebo for secondary fracture prevention in patients with osteoporotic vertebral compression fractures (OVCFs) that reported dropouts due to adverse effects in the placebo arms.

Results: We identified 8 RCTs collectively involving 4122 older participants in the placebo arms. Mean participant age ranged between 64 and 72 years. Dropouts due to adverse events in the placebo arms (i.e., nocebo-related dropouts) ranged between $6 \%$ and $17 \%$, a notably high rate for a preventive intervention, and was similar to the rate observed in the bisphosphonate arm for most studies. We found a statistically significant correlation between these nocebo-related dropouts and mean participant age (Spearman's rho $=$ $0.76, \mathrm{p}=0.029)$. The most commonly stated reasons for withdrawal were gastrointestinal complains, echoing expected adverse events as referenced in trial consent forms.

Conclusions: Nocebo effects associated with bisphosphonate use in patients with OVCFs may be more common among participants of advanced age, potentially mediating non-adherence to first line antiosteoporotic treatment.

\section{Abstract \# 257 \\ Mediating effect of knee pain in the association between diabetes and falls: cross sectional analysis from Malaysian Elders Longitudinal Research (MELoR) study \\ Sumaiyah Mat ${ }^{1}$, Maw Pin $\operatorname{Tan}^{2}$ \\ ${ }^{1}$ Ageing and Age Associated Disorder Research Group, Faculty of Medicine, University of Malaya, ${ }^{2}$ Geriatric Division, Department of Medicine, Faculty of Medicine, University of Malaya}

Introduction: Diabetes mellitus (DM) is a highly prevalent noncommunicable disease affecting $20-30 \%$ of worldwide population with a rapidly rising prevalence in lower- and middle-income countries. While knee pain due osteoarthritis is associated DM, few studies have evaluated mediating role of knee pain in the associations between DM and falls.

Methods: A cross-sectional analysis was conducted using the Malaysian Elders Longitudinal Study (MELoR) first wave data. The MELoR study recruited Klang Valley residents aged 55 years and over stratified by the three main ethnic groups and age deciles.
Diabetes mellitus was defined as fasting hyperglycaemia (serum fasting glucose $>6.66 \mathrm{mmol} / \mathrm{l})$ or $\mathrm{HbAlc}>42 \mathrm{mmol} / \mathrm{mol}(6.1 \%)$ or self-reported diabetes by participants or consumption of hypoglycaemic medications. Both falls and knee pain were identified by asking about the presence of fall in the preceding 12 months and whether the respondent often experienced pain, and whether this was in the knee during home-based interviews.

Results: Of 1211 participants, DM was present in $622(51.4 \%)$ and knee pain was present in $402(33.17 \%)$ individuals, while 276 $(22.8 \%)$ had $\geq 1$ fall in the past 12 months. The presence of DM was associated with knee pain [odds ratio, OR( $95 \%$ confidence interval, CI): 1.51 (1.19-1.92)] and increased risk of falls [OR (95\% CI) 1.41 (1.07-1.85)] respectively. There was significant indirect effect of DM on falls through knee pain ( $\mathrm{p}$-value $=0.013$ ) following the adjustment of age and sex.

Conclusion: Knee pain mediated the relationship between DM and falls. This finding is important in directing future population-based strategies in addressing falls in a population with an increasing diabetes incidence. Future studies should establish whether prevention or treatment of knee pain will effectively reduce falls in DM.

\section{Abstract \# 258}

Characteristics and outcomes of older adults requiring prolonged admission at the time of low trauma wrist fracture: a prospective cohort study

Maire Rafferty ${ }^{1}$, J Bernard Walsh ${ }^{1}$, Kevin McCarroll ${ }^{1}$

${ }^{1}$ St James's Hospital

Introduction: We aimed to examine the characteristics of patients admitted to hospital for more than 10 days following a wrist fracture versus those not admitted or briefly admitted for surgery ( $<3$ days). We also aimed to assess for differences in clinical outcomes in both patient groups in the 12 month period post their fracture.

Methods: Patients aged $>50$ who had a new X-ray diagnosis of low trauma wrist fracture were recruited between January and October 2018. Patient assessments were completed at the time of the fracture and at 6 and 12 months. Outcome data included mortality, re-admission to hospital, falls, further fractures and disability as assessed by patient self report using the Disability of the Arm, Shoulder and Hand (DASH) questionnaire.

Results: 134 patients were recruited and 113 attended follow up. Two patients were inpatients at the time of fracture. Overall, $27(20.1 \%)$ patients were admitted for $<3$ days for surgery. 18 (13.4\%) were admitted for $>10$ days and had a median length of stay of 48 days (IQR 12.5-98). Those admitted for $>10$ days were older (80.7 vs 67.6, $\mathrm{p}<0.001)$ and frailer $(\mathrm{p}<0.001)$, had a higher Charlson comorbidity index $(\mathrm{p}=0.001)$ and were pre-morbidly more likely to require help with ADLs $(50 \% \mathrm{v} 11.4 \%, \mathrm{p}=0.000)$ and take more medications $(\mathrm{p}<0.001)$. They were also more likely to have a lower BMD at the femoral neck $(\mathrm{p}=0.013)$, total hip $(\mathrm{p}=0.007)$ and forearm $(\mathrm{p}=0.026)$ and have a previous vertebral fracture $(\mathrm{p}=0.042)$ though had higher $25(\mathrm{OH}) \mathrm{D}(\mathrm{p}=0.001)$. Comparing outcomes, they had a higher readmission rate $(31.3 \%$ vs $10.9 \%, \mathrm{p}=0.025)$ and worse performance on DASH at the time of fracture $(\mathrm{p}=0.021)$ and at 6 months $(p<0.001)$ and 12 months $(p=0.026)$ There was no difference in mortality with one death in each group.

Conclusion: Wrist fracture in older, frail adults is associated with much longer hospital admissions, three times the readmission rate and greater disability over a one year follow up period. Findings highlight the importance of identifying these at risk patients and ensuring robust pathways to optimise care for this vulnerable group. 


\section{Abstract \# 259}

ADFICE_IT: the development of a clinical decision support system and patient portal: how to support joint medication management for preventing falls

Kelly Kim de Wildt ${ }^{1}$, Sara Groos ${ }^{2}$, Kimberley Ploegmakers ${ }^{1}$, Annemiek Linn ${ }^{2}$, Julia van Weert ${ }^{2}$, Natasja van Schoor ${ }^{1}$, Nathalie van der Velde

${ }^{1}$ Amsterdam UMC, ${ }^{2}$ University of Amsterdam

Introduction: Recent meta-analyses have confirmed the association between increased fall-risk in older adults, and fall-risk increasing drugs (FRIDs). Together with the patient, physicians need to carefully assess how these FRIDs should be withdrawn. A personalized risk estimate for drug-related falls and information on alternatives could help patients and physicians in FRID withdrawal. Digital tools such as a Patient Portal and Clinical Decision Support System (CDSS) have become increasingly useful in assisting patients and physicians in this process. The aim of this study is to describe the development of a theoretical and evidence-based Portal and CDSS to prevent falls in older adults.

Methods: The Portal and CDSS development was guided by the first step of the Medical Research Council framework, i.e. based on a systematic literature review, focus groups with patients and physicians, a European survey among patients and physicians, and a Delphi study. Previously developed fall-risk prediction models were modified prior to implementation in the CDSS and Portal.

Results: The Portal includes (1) a (printable) personalized risk visualized in traffic-light colors, (2) a Question Prompt List, and (3) Generic Fall-related information. The CDSS includes (1) a (printable) personalized risk visualized by percentages and traffic-light colors, (2) information about the model validity, (3) interactive features, and (4) a list of other risk factors.

Key conclusions: Applying the framework enabled a systematic description of developing a ground-breaking approach to improve joint medication management between older adults and physicians. Practically, this study could serve as a guide for the development of other interventions.

\section{Abstract \# 260 \\ Risk factor profile of attendees to community falls risk assessment clinics \\ Eoin Duggan ${ }^{1}$, Agnes Jonsson ${ }^{1}$, Kieran O'Connor ${ }^{1}$, Patrick Barry ${ }^{2}$, Spencer Turvey ${ }^{3}$, Finola Cronin ${ }^{3}$, Eileen Moriarty ${ }^{3}$, Sheena McHugh ${ }^{4}$ \\ ${ }^{1}$ Mercy University Hospital, Cork, Ireland, ${ }^{2}$ Cork University Hospital, Cork, Ireland, ${ }^{3}$ Cork Kerry Community Healthcare, Health Service Executive, Ireland, ${ }^{4}$ School of Public Health, University College Cork, Ireland}

Background: An Integrated Falls Prevention Pathway was established in 2015 in our Community Health Organisation. The services included in the pathway are the specialist falls clinics, community rehabilitation teams and community-based multifactorial falls risk assessment clinics (FRACs). We examined the demographics and risk factor profiles of those attending the FRACs over a 3-month period. Methods: Those who attended the five FRACs between January and March 2018 were identified from the falls service administrative database. Information on 11 risk factors for falls were extracted from Quickscreen ${ }^{\circledR}$ summary reports. Quickscreen ${ }^{\circledR}$ involves taking a falls history, medication review, and it assesses vision, cognition, sensation, strength, balance and reaction time using formal screening tools. Fracture risk was assessed with the Fracture Risk Assessment Tool (FRAX $\left.{ }^{\circledR}\right)$.
Results: Overall 82 people attended the FRACs during the study period. The average age was 78 years (range 54-95) and 65 (79\%) were female. The average number of falls risk factors was 6 (range 1-9). $74(90 \%)$ attendees had a history of falls, $69(84 \%)$ were on four+ medications. Visual impairment was present in $47(58 \%)$ of attendees and $61(74 \%)$ had impairments of strength, reaction times or balance.

Conclusion: In this group of attendees at FRACs the average number of risk factors was 6 making them a high-risk group for falls. There were high levels of polypharmacy, visual and cognitive impairment and issues with balance and sensation. This is a complex group that needs multifactorial interventions to reduce their risk of falling.

\section{Abstract \# 261 \\ Prevalence of hyperparathyroidism in older patients presenting with hip fracture}

Lok Yi Tan ${ }^{1}$, Nur Atikah Mohd Asri ${ }^{2}$, Azrin Muslim², Orlaith Shinners ${ }^{1}$, Richard Tyrrell ${ }^{1}$, Alicia Goh ${ }^{1}$, Pamela Hickey ${ }^{1}$, Finbarr Condon $^{1}$, Jude Ryan ${ }^{2}$

${ }^{1}$ Department of Trauma and Orthopaedic Surgery, University Hospital Limerick, Ireland, ${ }^{2}$ Department of Geriatrics Medicine, University Hospital Limerick, Ireland

Introduction: Hyperparathyroidism is a risk factor of osteoporosis. Excess parathyroid hormone (PTH) accelerates bone loss via bone reabsorption and increases the risk of fragility fracture. Fragility fractures are associated with serious consequences in older patients [1]. This study aimed to look at the prevalence of hyperparathyroidism and vitamin D deficiency in older patients (age $>60$ ) who presented with hip fractures in an Irish tertiary hospital.

Methods: Data was collected from Irish Hip Fracture Database (IHFD) and the Ortho-geriatric Electronic Patient Medical System (EPMS) between July 2019 and April $2020(\mathrm{n}=207)$. Demographic data and bone health profile (PTH, Vitamin D, and Calcium) were analysed using SPSS for statistical analysis.

Results: The median age of patients was 81 years (SD 8.7), and $68.3 \%$ of patients were female. Median 10-year probability of major osteoporotic and hip fracture risk was $22.5 \%$ and $12.2 \%$, respectively. $29.9 \%$ were diagnosed with hyperparathyroidism with prevalence rates of primary and secondary hyperparathyroidism of $6.7 \%$ and $23.1 \%$, respectively. The majority of these $(87.3 \%)$ were normocalcaemic hyperparathyroidism. $50.7 \%$ were vitamin $\mathrm{D}$ deficient, with $11.5 \%$ being severely deficient (Vitamin $\mathrm{D}<20 \mathrm{nmol} / \mathrm{L}$ ).

Conclusion: The prevalence of hyperparathyroidism is higher in the hip fracture group than in the general population [2]. Vitamin D deficiency is also prevalent in this group. FRAX score indicated significant proportion of patients had high major osteoporosis and hip fracture risks. Routine bone health profile checks in this high-risk group allow early identification and management of hyperparathyroidism and vitamin $\mathrm{D}$ depletion, which can potentially reduce the risk of future fracture.

Reference: 1. Bukata SV, DiGiovanni BF, Friedman SM, Hoyen H, Kates A, Kates SL, Mears SC, Mendelson DA, Serna Jr FH, Sieber FE, Tyler WK. A guide to improving the care of patients with fragility fractures. Geriatric Orthopaedic Surgery \& Rehabilitation. 2011 Jan;2(1):5-37. 2. Press DM, Siperstein AE, Berber E, Shin JJ, Metzger R, Jin J, Monteiro R, Mino J, Swagel W, Mitchell JC. The prevalence of undiagnosed and unrecognized primary hyperparathyroidism: a population-based analysis from the electronic medical record. Surgery. 2013 Dec 1;154(6):1232-8 


\section{Abstract \# 262}

Characteristics of patients attending specialist bone health clinic following vertebral fracture.

Maire Rafferty ${ }^{1}$, Nessa Fallon ${ }^{1}$, Georgina Steen ${ }^{1}$, Niamh Maher ${ }^{1}$, J Bernard Walsh ${ }^{1}$, Kevin McCarroll ${ }^{1}$

${ }^{1}$ St James's Hospital

Introduction: Vertebral fractures are the most common osteoporotic fracture with considerable consequences yet this group is less extensively studied that those with other major osteoporotic fractures. We aimed to investigate the characteristics of patients attending a specialist bone health clinic, in particular looking at the prevalence of osteoporosis by DXA and the predictors of BMD. We also aimed to investigate how these characteristics differed between genders.

Methods: Data, including demographic, clinical and bone health variables, regarding all patients attending the bone health clinic following vertebral fracture were recorded prospectively as the patients were seen. Statistical analysis was performed using SPSSv26.

Results: Data were available for 1779 patients (mean age $71.7 \pm$ 12.4, 76.4\% female) seen between 2003 and 2018. Other fractures were prevalent: $23.9 \%$ wrist and $19.7 \%$ hip. $57.9 \%$ of the group had osteoporosis by DXA criteria and 32.5\% osteopaenia. Women $(\mathrm{p}<$ $0.001)$ older patients $(\mathrm{p}<0.001)$, patients with a lower BMI $(\mathrm{p}<$ $0.001)$ and those with other fractures $(\mathrm{p}<0.001)$ were more likely to have osteoporosis. $46 \%$ were taking osteoporosis medication. Age, gender and BMI were shown to predict between 16 and $31 \%$ of BMD at different sites. Men who had had a vertebral fracture were more likely to be younger $(67.4 \mathrm{v} 73, \mathrm{p}<0.001)$ have higher BMD at all sites ( $\mathrm{p}<0.017$ at all sites) and were less likely to be on osteoporosis treatment $(35 \%$ v $49 \%, \mathrm{p}<0.001)$

Conclusion: Awareness of the patient characteristics described in this study allows design of targeted fracture prevention strategies. We describe the significance, in particular, of age, gender and BMI in predicting lower BMD and therefore future fracture risk. We explore the difference between the profile of male versus female patients sustaining the fracture highlighting the importance of being aware of the risk of this fracture in younger men with relatively higher BMD as well as the lower rate of prescribing of osteoporosis treatment in this group.

\section{Abstract \# 263}

\section{Prescription review in frail patients with femur fracture}

Felix-Jorge Morel-Corona ${ }^{1}$, Maricelis Cruz-Grullon ${ }^{1}$, Santiago Castejon-Hernandez ${ }^{1}$, Nuria Molist-Brunet ${ }^{2}$, Mariona EspaulellaFerrer $^{2}$, Daniel Sevilla-Sanchez ${ }^{3}$, Joan Espaulella-Panicot ${ }^{2}$

${ }^{1}$ Geriatrics Department, Hospital Universitari de Vic, Vic, Barcelona, Spain, ${ }^{2}$ Geriatrics Department, Hospital Universitari de la Santa Creu, Vic, Barcelona, Spain, ${ }^{3}$ Pharmacy Department, Hospital Universitari de Vic, Vic, Barcelona, Spain

Introduction: Femur fracture is considered a potentially serious entity for the frail patients for its high morbidity and mortality. Anticholinergic drugs are frequently used, increasing the risk of adverse reactions, such as falls [1].

Aim: to review the prescription in frail patients with femur fracture. Methods: Retrospective and observational study. Analyzing patients $\geq 85$-years-old or younger than 85 years-old with cognitive impairment with femur fracture admitted to Geriatric Acute Unit shared with Traumatology Unit between April to October 2019. Variables: Sociodemographic, cognitive, functional, pharmacological (polypharmacy (global: $\geq 5$ drugs and severe: $\geq 10$ drugs), preventive drugs for osteoporosis, inappropriate prescription (IP) identified through the application of patient-centered prescription model [2], and prescription optimization, falls and anticholinergic burden with Drug Burden Index (DBI) 0: null, $<1$ : low, 1-2: moderate, $>2$ high. Frail-VIG Index (IF-VIG) was calculated: $<0.20$ non-frail, $0.20-0.35$ mild-frail, 0.36-0.50 moderate-frail, IF $>0.50$ severe-frail [3].

Results: 67 patients included (67\% women, median age 90 -years-old (79-101); cognitive impairment: 65.7\%). Median of previous Barthel Index 60/100. IF-VIG $22.4 \%$ non-frail, $29.4 \%$ mild-frail, $38.8 \%$ moderate-frail, $10.4 \%$ severe-frail. $43.2 \%$ previous falls. $73 \%$ had polypharmacy, $25.3 \%$ of them severe. $72 \%$ had no preventive drugs for osteoporosis before the fracture. $80.6 \%$ had anticholinergic burden, $38.8 \%$ low, $32.8 \%$ moderate and $9 \%$ high, being null in $19.4 \%$. IP identified in $70 \%$. Main reason: overtreatment $(62 \%)$. Proposed discontinuation of drugs: $47.7 \%$ and dose reduction: $22.3 \%$.

Conclusions: Patients with femur fracture have high polypharmacy, IP and anticholinergic burden. The prescription optimization is one of the modifiable factors in the prevention of falls.

References: 1. Collamati A, Martone AM, Poscia A, et al. Anticholinergic drugs and negative outcomes in the older population: from biological plausibility to clinical evidence. Aging Clin Exp Res. 2016;28(1): 25-35. 2. Molist Brunet N, Espaulella Panicot J, SevillaSánchez D, Amblàs Novellas J, Codina-Jané C, Altimiras-Roset J, et al. A patient-centered prescription model assessing the appropriateness of chronic drug therapy in older patients at the end of life. Eur Geriatr Med. 2015;6:565-9. 3. Amblàs-Novellas J, et al. Índice frágilVIG: diseño y evaluación de un índice de fragilidad basado en la Valoración Integral Geriátrica. Rev Esp Geriatr Gerontol. 2016.

\section{Abstract \# 264}

Comparison of prescription in patients with and without femur fracture

Felix-Jorge Morel-Corona ${ }^{1}$, Maricelis Cruz-Grullon ${ }^{1}$, Santiago Castejon-Hernandez ${ }^{1}$, Nuria Molist-Brunet ${ }^{2}$, Mariona EspaulellaFerrer $^{2}$, Daniel Sevilla-Sanchez ${ }^{3}$, Joan Espaulella-Panicot ${ }^{2}$

${ }^{1}$ Geriatrics Department, Hospital Universitari de Vic, Vic, Barcelona, Spain, ${ }^{2}$ Geriatrics Department, Hospital Universitari de la Santa Creu, Vic, Barcelona, Spain, ${ }^{3}$ Pharmacy Department, Hospital Universitari de Vic, Vic, Barcelona, Spain

Introduction: Femur fractures are common in elderly patients. Several factors have been identified as fall-risk increasing such as pharmacological treatment. Aim: to analyze the association between polypharmacy, inappropriate prescription (IP), anticholinergic burden and falls, comparing patients with and without femur fracture.

Methods: A case-control study in patients older than 85-years or younger with cognitive impairment admitted to Geriatric Acute Unit with femur fracture and history of falls compared with control group. Variables: Sociodemographic, cognitive, functional, pharmacological (polypharmacy (global: $\geq 5$ drugs), IP using patient-centered prescription model [1] and prescription optimization and anticholinergic burden with Drug Burden Index (DBI): 0: null, $<1$ : low, 1-2: moderate, $>2$ high) and falls. Frail-VIG Index (IF-VIG) was calculated: $<0.20$ non-frail, $0.20-0.35$ mild-frail, $0.36-0.50$ moderate-frail, IF $>$ 0.50 severe-frail [2].

Results: 134 patients included, $50 \%$ presented falls and femur fracture. Median age 88 years-old (76-101); cognitive impairment $62 \%$. Median of previous Barthel Index 60/100. IF-VIG 15.7\% non-frail, $46.3 \%$ mild-frail, $29 \%$ moderate-frail, $9 \%$ severe-frail. Patients with femur fracture presented $(\mathrm{p}<0.05)$ : higher global polypharmacy (73\% versus $49.2 \%)$ than non-femur fractures. Also higher IP $(70 \%$ versus $43 \%)$, the most frequent reason being overtreatment $(63 \%$ versus $35.8 \%$ ) and higher DBI: (null 19.4\%, low 38.8\%, moderate $32.8 \%$, high $9 \%$ versus non-femur fracture: null $52.2 \%$, low $19.4 \%$, 
moderate $22.4 \%$, high $6 \%$ ). $63 \%$ of patients with a femur fracture required prescription optimization (non-femur fracture $43 \%$ ).

Conclusions: Patients with femur fracture present higher polypharmacy, IP, anticholinergic burden and falls, preventable adverse events that suggest the importance of individualized prescription optimization.

References: 1. Molist Brunet N, Espaulella Panicot J, Sevilla-Sánchez D, Amblàs Novellas J, Codina-Jané C, Altimiras-Roset J, et al. A patient-centered prescription model assessing the appropriateness of chronic drug therapy in older patients at the end of life. Eur Geriatr Med. 2015;6:565-9. 2. Amblàs-Novellas J, et al. Índice frágil-VIG: diseño y evaluación de un índice de fragilidad basado en la Valoración Integral Geriátrica. Rev Esp Geriatr Gerontol. 2016.

\section{Abstract \# 265 \\ Falls risk profile of community dwelling geriatric patients in Athens area}

George Soulis ${ }^{1}$, Helen Zigkiri ${ }^{2}$, Alexandra Tsantili ${ }^{3}$, Zikos Kentros ${ }^{3}$, Efrosini Chatzaki ${ }^{4}$, Anastasia Koutsouri ${ }^{4}$

${ }^{1}$ Outpatient Geriatric Assessment Unit Henry Dunant Hospital Center, ${ }^{2}$ Outpatient Geriatric Assessment Unit and Physica Medicine and Rehabilitation Department, Henry Dunant Hospital Center, ${ }^{3}$ Physical Medicine and Rehabilitation Department, Henry Dunant Hospital Center, ${ }^{4}$ Outpatient Geriatric Assessment Unit, Henry Dunant Hospital Center

Introduction: Greece has little experience in geriatric medicine which is not recognized at all as a distinct field. Henry Dunant's Outpatient Geriatric Assessment Unit aspires to fill this gap by providing up to date geriatric services of high quality and by promoting geriatric research.Aim. Evaluate physical status variables related to falls risk of older people examined at the outpatient Geriatric Assessment Unit of Henry Dunant Hospital Center.

Methods: We analyzed data collected during Geriatric Assessment routine visit. We used the following cut-off values. For timed up and go test $>14 \mathrm{sec}$ for increased fall risk, 5 times sit to stand test $>12$ $\mathrm{sec}$ for high fall risk and also hand grip strength values $>26 \mathrm{~kg}$, as normal for male subjects and $>18 \mathrm{~kg}$ as normal for female subjects. Results: We evaluated 60 patients between 1 January 2019 and 31 December 2019. Females and people $>85$ represent the majority of geriatric consultations (70 \% and $72,4 \%$ respectively). Demographic data demonstrate that people that visited our Unit is a highly selected sample since $48,8 \%$ are people with higher education attainment. 33 subjects have been evaluated for falls risk by using Timed Up and Go test (TUG). Only 9,1\% were found with score over 14 seconds. 33 patients where evaluated by using the Five Times Sit to Stand test (\%SST test) and 57,6\% of them were measured with times greater than 12 seconds. We also used hand grip strength to evaluate general physical status in 7 male and 24 female subjects. Only $14,3 \%$ of males had grip strength over 26 kilograms while $62,5 \%$ of the females had values over 18 kilograms.

Conclusion: This is the first attempt to use comprehensive geriatric assessment in an organized outpatient geriatric unit in Greece. We observed that among the tools that can be used to evaluate falls risk there can be differences that have to be taken into account when providing the final personalized plan for every geriatric patient.

\section{Abstract \# 266}

Preoperative pain management in patients with and without neurocognitive disorders and Hip fracture

Mathilde Ruel ${ }^{1}$, Bastien Boussat ${ }^{2}$, Medhi Boudissa ${ }^{3}$, Virginie Garnier $^{4}$, Catherine Bioteau ${ }^{3}$, Jerome Tonetti ${ }^{3}$, Regis Paihle ${ }^{5}$, Gaëtan Gavazzi $^{6}$

${ }^{1}$ Geriatric Department, CHU Grenoble-Alpes, ${ }^{2}$ Public Health Department, Geriatric Department, University hospital of GrenobleAlpes, ${ }^{3}$ Ortho-Geriatric Department, University hospital of GrenobleAlpes, ${ }^{4}$ Geriatric department, Geriatric Department, University hospital of Grenoble-Alpes, ${ }^{5}$ Orthopedic Department, University hospital of Grenoble-Alpes, ${ }^{6}$ Geriatric Department, University hospital of Grenoble-Alpes

Introduction: Patients with neurocognitive disorders (NCD) have a 3 -fold higher risk of Acute Hip fracture (AHF) than a control population of the same age. A poorly controlled preoperative pain is a risk factor for confusion, poor functional prognosis, and chronic pain. Patients with NCD receive $20-60 \%$ less pain medication than patients without NCD

Methods: The primary objective of our retrospective descriptive monocentric orthogeriatric descriptive study of a cohort of patients 75 years of age and older, hospitalized for AHF, was to compare the amount of strong opioids administered as morphine equivalent in milligrams per day prior to AHF surgery, in patients with and without NCDs. The group of patients with NCDs was defined as patients with moderate to severe NCDs diagnosed pre-fracturia (MMS $\leq 20 / 30$ ).

Results: Our population of 69 patients had a median age of 90 years and $46 \%$ had NCD. In the preoperative period, the same amount of strong opioids was administered in both groups $(13.1 \mathrm{mg} / \mathrm{d}$ versus $10.8 \mathrm{mg} / \mathrm{d}(\mathrm{p}=0.38)$ ). Patients with NCD had more frequent inhospital confusion $(\mathrm{p}<0.01)$ and received more psychotropic drugs post-operatively $(\mathrm{p}=0.025)$.

Conclusions: In contrast to usual literature and because of a strong co-management between Orthopedics, Anesthesiologist and Geriatricians patients 75 years of age and older received a comparable mean daily amount of strong opioids in preoperative hip fractures, regardless of cognitive status.

\section{Abstract \# 267}

Frax assement and osteoporotic prevention in a portuguese orthogeriatric unit

Mariana Santos ${ }^{1}$, Raquel Moura ${ }^{1}$, Luciana Silva ${ }^{1}$, Leonor Silva ${ }^{1}$, Agripino Oliveira $^{1}$, Rafaela Veríssimo ${ }^{1}$

${ }^{1}$ Centro Hospitalar Vila Nova de Gaia/Espinho

Introduction: Osteoporosis and osteoporotic fractures are prevalent in countries like Portugal and are an important cause of hospitalization among the elderly population, so should be early identified. The Fracture Risk Assessment Tool (FRAX) give us a ten-year risk of hip fracture (FRAX1) or a 10-year risk of a major osteoporosis-related fracture (FRAX2). Patients with FRAX1 $\geq 3 \%$ or a FRAX2 $\geq 11 \%$ should be considered for treatment.

Methods: This study includes all geriatric patients admitted in an orthogeriatric ward in one year. FRAX tool and KATZ scale was applied in all patients and descriptive analysis was performed. The main goal was to evaluate who should be considered for osteoporotic treatment and who were doing such treatment before fracture occurrence.

Results: 112 patients were admitted and $80.4 \%$ were female. The mean age was 84 years and $31.3 \%$ of patients were KATZ A at admission.FRAX tool was applied in 69 patients and 50\% (60 
patients) of them had FRAX1 $\geq 3 \%$ and $40.2 \%$ (45 patients) had a FRAX $2 \geq 11 \% .76 .7 \%$ of the patients with a FRAX $1 \geq 3 \%$ and $75.6 \%$ of the patients with FRAX2 $\geq 11 \%$ were not doing any osteoporotic treatment.KEY

Conclusions: In this Portuguese orthogeriatric sample an important percentage of patients were at high risk of osteoporotic fractures and didn't do any type of treatment previously to orthogeriatric unit. It is extremely important to actively access this risk so it could be preventable, treating them adequately avoiding fractures.

\section{Abstract \# 268 \\ Prevention of frailty in the elderly through physical activity and nutrition}

Brunilda Elezi ${ }^{1}$, Erjona Abazaj ${ }^{2}$, Erjola Bolleke ${ }^{3}$, Marsida Kasa ${ }^{4}$

${ }^{1}$ Lecturer,PhD*(Corresponding author) "Aleksander Xhuvani" University, Faculty of Technical Medical Sciences, Elbasan, Albania, ${ }^{2} \mathrm{PhDInstitute}$ of Public Health, Department of Epidemiology and Control of Infectious Diseases, Tirana, Albania, ${ }^{4} \mathrm{PhDNephrology}$ and Dialysis Unit, UHC Mother Theresa, Tirana, Albania, ${ }^{4}$ Trauma University Hospital, Tirana, Albania

Introduction: Frailty is considered often as a physical impairment in the elderly and its characteristics are weakness, weight loss, exhaustion, a decrease of the physical activity accumulating also co morbidity. It is considered the geriatric syndrome related to the aging process, declining of biological reserve that results in an increase of vulnerability for negative results including disability, hospitalization, and death. Frailty was defined as "a state of increased vulnerability on the stress factors due to the decline of the intramuscular, metabolic, physiologic reserve and immune systems.

Aim: To evaluate the role of physical activity and nutrition in the frail older adults, early interventions to slow down the process of fragility and disability in everyday life.

Methodology: Comprehensive research was performed in literature Medline, PubMed, Research gate, The Journal of frailty and aging to realize the aim of this study. The selected criteria of the literature were the published articles during 2007-2018 containing specific keywords such as elderly, fragility, nutrition, physical activity, co morbidity. Included were the studies where the fragility syndrome in the elderly is defined.

Conclusion: Regular studies came to the same conclusion that physical activity has preventive effects in frail elderly even though uncertainty exists in optimal exercise programs regarding the frequency, kind of nutrition and duration. To prevent the fragility, necessary measurements need to be taken with nutrition e proteins, vitamin D and other alimentary, but this information is not yet public domain. The benefits of physical exercises in frailty elderly include increased movement, increased performance in activities of daily living, improved walk, elimination of falls, improvement of mineral bone density and the increase of overall health. However, systems of health care actually are not organized to offer integrated care on daily life, but to identify and treat acute diseases.

Recommendation: Physical activity and adequate nutrition, especially regarding vitamin $\mathrm{D}$, calcium and proteins are fundamental in improving at the same time bones, muscles, and the functional results in elder population, if they are individually adapted and determined. Preventive methods through physical activity in frailty elder should be described with an individual progressive plan as in the other medical treatments.

Keywords: Physical activity, frailty, elder, prevention, nutrition.

\section{Abstract \# 269}

The minimal clinically important difference of six-minute walk in Asian older adults

Kaysar Mamun ${ }^{1}$

${ }^{1}$ Singapore General Hospital

Introduction: Rehabilitation interventions promote functional recovery among frail older adults and little is known about the clinical significance of physical outcome measure changes. The purpose of our study is to examine the minimal clinically important difference (MCID) for the 6-minute walk distance (6MWD) among frail Asian older adults.

Methods: Data from the "Evaluation of the Frails' Fall Efficacy by Comparing Treatments" study were analyzed. Distribution-based and anchor-based methods were used to estimate the MCID of the 6MWD. Participants who completed the trial rated their perceived change of overall health on the Global Rating of Change (GROC) scale. The receiver operating characteristic curve (ROC) was used to analyze the sensitivity and specificity of the cut-off values of 6MWD (in meters) for GROC rating of "a little bit better" $(+2)$, based on feedback from participants.

Results: The mean (SD) change in 6MWD was 37.3(46.2) $\mathrm{m}$ among those who perceived a change (GROC $\geq 2$ ), while those who did not was 9.3(18.2) $\mathrm{m}$ post-intervention $(\mathrm{P}=0.011)$. From the anchor-based method, the MCID value for the 6MWD was $17.8 \mathrm{~m}$ (sensitivity $56.7 \%$ and specificity $83.3 \%$ ) while distribution-based method estimated $12.9 \mathrm{~m}$.

Key conclusion: The MCID estimate for 6 MWD was $17.8 \mathrm{~m}$ in the moderately frail Asian older adults with a fear of falling. The results will aid the clinicians in goal setting for this patient population.

\section{Abstract \# 270}

The results of complex geriatric assessment in frail elderly patients from the Republic of Moldova

\section{Ana Popescu ${ }^{1}$}

${ }^{1}$ Gabriela Soric

Introduction: The process of human aging is complex and multifactorial, it is accompanied by the diminution of functional capacities of the human body.Changes occur in all organs and organ systems.The evaluation of the elderly patient focuses on an overall interpretation.

Material and methods: The study included 224 patients, men $21.42 \%$ and women $78.54 \%$, aged between 65 and 89 years, the average $73.44 \pm$ ES 0.38, admitted to Geriatric and Internal diseases departments, January-June 2019. The complex geriatric evaluation include cognitive ability (MMSE), locomotor capacity (Tinetti Test), psychological capacity (depressive symptoms), medication (more than 5 drugs) and autonomy (ADL, IADL).The fragility syndrome was established according to Fried (2001) criteria.The data obtained were analyzed in the StatSoft database Statistics 7.

Results: The frailty syndrome was established in $51.78 \%$ of patients.Fragility was diagnosed more frequently in women $-81.03 \%$ cases.Patients with fragility had a higher cognitive deficit compared to prefragile patients - MMSE $23.48 \pm 0.14$ vs. $24.0 \pm 0.19 ; \mathrm{p}=0.03$. Locomotor capacity, mean Tinetti test score $18.81 \pm 0.47$ vs.23.06 \pm $0.31, \mathrm{p}<0.001$, concomitant diseases were determined $-5.05 \pm 0.17$ vs $4.37 \pm 0.18, \mathrm{p}=0.01$, and the polypharmacy is higher in the fragile patients $-13.79 \%$ vs $4.59 \%, p=0.029$. The autonomy disorder of the frail elderly was determined - ADL $10.17 \pm 0.19$ vs $11.44 \pm 0.07$, p $<0.001$; IADL $11.87 \pm 0.24$ vs. $14.39 \pm 0.16, \mathrm{p}<0.001$. 
Conclusion: The frailty syndrome represents a state of increased vulnerability commonly encountered in hospitalized elderly persons, affecting all life areas.

\section{Abstract \# 271 \\ Frailty syndrome: evaluation of its knowledge and screening tools by general medicine interns}

Zulfiqar Abrar-Ahmad ${ }^{1}$, Lorenzo-Villalba Noël ${ }^{1}$, Piedallu JeanBaptiste $^{2}$, Andres Emmanuel ${ }^{1}$

${ }^{1}$ University Hospital of Strasbourg, Department of Internal Medicine, Strasbourg, ${ }^{2}$ University Hospital of Rouen, Department of General Medicine, Rouen

Introduction: This study aims to describe and evaluate the knowledge about frailty, its screening tests and management in a population of general practice interns.

Method: A prospective, descriptive and mono-centric study using an online survey was conducted. The 2018's promotion of general practice interns in Rouen's University participated. Evaluation of the general knowledge (e.g diagnostic criteria) and specific knowledge (e.g ranking different conditions such as: undernourishment leading to asthenia) and management algorithm (e.g to address the patient for geriatric evaluation).

Results: 90 questionnaires were analyzed. Ten interns (11\%) stated to know at least one screening test or to know a set of 4 major frailty criteria. Thirty four interns knew at least 4 frailty criteria (major or minor) and 46 interns had partial knowledge. Fried's phenotype was the most known. Seventy-five interns $(83 \%)$ stated to know the frailty syndrome. However, $88 \%$ of the interns had little knowledge about its pathophysiology. Concerning frailty management, $78 \%$ (70 interns) stated to know how to screen frailty and prescribe first line treatments. Thirty-one among them knew how to reassess initial treatment if failure. In 59 interns (65\%), identifying frailty would have led to a geriatric consultation. Seventy-nine interns $(87 \%)$ were favorable to go through further training on frailty.

Conclusion: These general practice interns were able to identify frailty criterion but, in contrast, global understanding of the syndrome was missing. Further training about this topic seems to be necessary.

\section{Abstract \# 272 \\ Validation of the Zulfiqar Frailty Scale (ZFS): a new tool for frailty screening in general medicine.}

Abrar-Ahmad Zulfiqar ${ }^{1}$, Noël Lorenzo-Villalba ${ }^{1}$, André Gillibert ${ }^{2}$, Alexandre Sebaux ${ }^{3}$, Phuc-Thien Le ${ }^{3}$, Emmanuel Andres ${ }^{1}$

${ }^{1}$ University Hospital of Strasbourg, Department of Internal Medicine, Strasbourg, France, ${ }^{2}$ University Hospital of Rouen, Department of Biostatistics-Epidemiology, Rouen, France, ${ }^{3}$ General Practitioner, Plancoët, France

Introduction: Very few frailty scales are used by general practitioners as they are time consuming and cumbersome. We have designed a new scale for rapid detection of frailty.

Methods: We developed a frailty screening tool for use in General Practice referred to as the Zulfiqar Frailty Scale (ZFS). This scale was tested in a general medicine office for 6 months in Plancoët. Only patients over 75 years of age with Activities of Daily Living (ADL) $>=4$ were included. The objective of this research was (1) to validate the scale and evaluate its performance; and (2) to compare this screening tool with other scales such as the Fried Scale, Gerontopole Frailty Screening Tool (GFST), Short Emergency Geriatric Assessment (SEGA) Grid A, and the standardized gerontological evaluation.
Results: One hundred two patients were included, with a mean age of $82,65 \pm 4,79 ; 55$ were women and 47 were men. The proportion was $74.5 \%$ for our scale, 69\% the SEGA (Short Emergency Geriatric Assessment) grid A scale, $75.5 \%$ for the GFST (Gerontopole Frailty Scale Tool) scale, $60.8 \%$ for the FRIED scale. After a comprehensive geriatric assessment, frailty syndrome was found for 57 patients $(55.9 \%)$. In general, both scales showed good performance and differences between them in the sample is minimal. Because the EGS showed a prevalence of frailty of $55.9 \%$, a similar prevalence threshold for the ZFS, i.e. $64 \%$ at the threshold $\geq 3$ could be assessed. The completion time of our scale was less than 2 minutes and staff required no training beforehand. Its sensitivity was $83.9 \%$ and its specificity was $67.5 \%$. Its positive predictive value was $80 \%$, and its negative predictive value was $73 \%$. The Pearson correlations between the geriatric scores were all strong and roughly equivalent to each other.

\section{Abstract \# 273}

Screening of frailty in elderly subjects admitted to an emergency department: an exploratory investigation

Abrar-Ahmad Zulfiqar ${ }^{1}$, Marphy Kerif ${ }^{2}$, Noël Lorenzo-Villalba ${ }^{1}$, Emmanuel Andres ${ }^{1}$

${ }^{1}$ University Hospital of Strasbourg, Department of Internal Medicine, Strasbourg, France, ${ }^{2}$ Hospital of Troyes, Emergency Unit, Troyes, France

Introduction: It is currently considered that screening for frailty in elderly subjects is a major public health issue.

Methods: a cross sectional study involving elderly subjects (over 75 years of age) admitted at the Emergency Department of the hospital of Troyes in the period August 24th to August 30th , 2017 was conducted. Patients were screened for frailty using the SEGAm (part A) grid, correlated with the subjective opinion of the triage nurse and the senior physician/interns. This study was aiming at evaluating the screening of frailty at the emergency department.

Results: 100 patients were included during the study period, mean age was 84.34 years (range $75-97), 56$ patients $(56 \%)$ were female, the average CHARLSON score was 4.28 (range 0-11). The patient's previous medical history was remarkable for cardiovascular diseases. The main reason of hospital admission was fall (26 subjects, 26\%). Hospitalization was suitable in 52 subjects $(52 \%)$. The average SEGAm score was $6.3 \pm 3.59$. The completion time for the SEGAm (part A) score was about 5 minutes. According to Cohen's kappa, the concordance between the subjective opinion of the triage nurse and the SEGA grid was average, while the concordance between the subjective opinions of the senior physicians/interns was good. Conclusion: The SEGAm score appears to be well-suited and useful in the Emergency Department. It is easy to use, allows an overall evaluation of the patient and is not time- consuming.

\section{Abstract \# 274 \\ Frailty changes predict mortality in four longitudinal studies of aging \\ Erwin Stolz ${ }^{1}$, Emiel Hoogendijk ${ }^{2}$, Hannes Mayerl ${ }^{1}$, Wolfgang Freidl ${ }^{1}$ \\ ${ }^{1}$ Medical University of Graz, AUSTRIA, ${ }^{2}$ VU University Medical Centre Amsterdam, Netherlands}

Introduction: Baseline frailty index (FI) values have been shown to predict mortality among older adults, but little is known about the effects of changes in FI on mortality. 
Methods: In a coordinated approach, we analyzed data from four population-based cohorts: the Health and Retirement Study (HRS), the Survey of Health, Ageing and Retirement in Europe (SHARE), the English Longitudinal Survey of Ageing (ELSA) and the Longitudinal Aging Study Amsterdam (LASA), comprising a total of 26,241 respondents $(65+), 100,449$ observations, up to 9 repeated FI assessments, and up to 23 years of mortality follow-up. The effect of time-varying FI on mortality was modelled with joint regression models for longitudinal and time-to-event data.

Results: Baseline FI differences were consistently associated with mortality (hazard ratio $(\mathrm{HR})=1.04(95 \%$ confidence interval $(\mathrm{CI})=$ 1.03-1.05) per 0.01 FI) across studies. More importantly, individuals with steeper FI growth also had a higher mortality risk: an increase in annual FI growth by 0.01 was associated with an increased mortality risk $(\mathrm{HR})$ in $\mathrm{HRS}=1.42(95 \% \mathrm{CI}=1.38-1.47), \mathrm{SHARE}=1.47(95 \% \mathrm{CI}$ $=1.39-321.55), \mathrm{ELSA}=1.39(95 \% \mathrm{CI}=1.26-1.54)$, and LASA $=$ $1.62(95 \% \mathrm{CI}=1.43-1.85)$.

Conclusion: FI changes consistently predicted mortality independently of baseline FI differences. Repeated assessment of frailty and individual's frailty trajectory could provide a means to anticipate further health deterioration and mortality, and could thus support clinical decision making.

\section{Abstract \# 275 \\ Assessment of relationship between sarcopenia, frailty and malnutrition by exploiting new criteria \\ Güzin Çakmak $^{1}$, Eyyüp Murat Efendioglu ${ }^{1}$ \\ ${ }^{1}$ Gaziantep University Faculty of Medicine, Department of Internal Medicine, Division of Geriatrics}

Introduction: Frailty, sarcopenia, and malnutrition are common geriatric syndromes and associated with reduced life expectancy. In this study, we aimed to define the relationship between frailty, sarcopenia, and malnutrition.

Methods: This cross-sectional study was conducted between September 2019 and December 2019. Malnutrition was assessed by the Mini Nutritional Assessment-Short Form (MNA-SF), Long Form (MNA-LF), and Global Leadership Initiative on Malnutrition (GLIM) criteria. Independence in Activities of Daily Living (ADL) and Instrumental Activities of Daily Living Scale (IADL) was assessed for disability. Frailty was evaluated by Fried's criteria and Study of Osteoporotic Fractures (SOF) criteria. EWGSOP 2 criteria were used to diagnose sarcopenia.

Results: 57 women and 43 men, of mean age $72.8 \pm 7.4$ years, were applied to the study. According to SOF criteria 35 patients were prefrail and 45 patients were frail. According to Fried's criteria 49 patients were pre-frail, 46 were frail. Gait speed and TUG were related to frailty according to both Fried's and SOF criteria. Handgrip strength was related to SOF and Frieds' criteria in linear regression analysis $\left(r^{2}=0.222, p=0.003 ; r^{2}=0.200, p<0.001\right)$. GLIM criteria were the only malnutrition measure independently related to SOF criteria $\left(r^{2}=0.414, p=0.01\right)$. Fried criteria were related with MNASF, MNA-LF and GLIM criteria $\left(\mathrm{r}^{2}=0.474 ; \mathrm{p}=0.007, \mathrm{p}=0.017, \mathrm{p}=\right.$ $0.01)$. SOF criteria were related to IADL $\left(\mathrm{r}^{2}=0.117, \mathrm{p}=0.011\right)$.

Key conclusions: Frailty and sarcopenia are important geriatric syndromes. New screening and diagnosing methods are under development. In this study, SOF criteria and GLIM criteria were shown to be better when the relationship with other syndromes considered.

\section{Abstract \# 276}

Which frailty measurement tool is more effective in polypharmacy studies?

\section{Guzin Cakmak ${ }^{1}$, Zeynel Abidin Ozturk ${ }^{2}$}

${ }^{1}$ Gaziantep University, Faculty of Medicine, Department of Internal Medicine, Division of Geriatrics, ${ }^{2}$ Gaziantep University Faculty of Medicine, Department of Internal Medicine, Division of Geriatrics

Introduction: Frailty is a common geriatric syndrome that carries an increased risk for poor health outcomes. The aim of this study is to investigate the relationship between frailty and polypharmacy in the elderly.

Methods: This cross-sectional study was conducted for a period of 4 months from September 2019 to January 2020. Frailty was assessed by using Fried and SOF (Study of Osteoporotic Fractures) criteria. Patients were evaluated for inappropriate medication use by TIME (Turkish Inappropriate Medication Use in the Elderly) criteria. Data analysis was done by using SPSS version 22 .

Results: The study population was composed of 57 women and 43 men and the mean age was $72.8 \pm 7.4$ years. The frequency of polypharmacy was $\% 14$ in this population. Polypharmacy was related with both Fried and SOF scores $(\mathrm{p}=0.005, \mathrm{r}=0.288 ; \mathrm{p}=0.011, \mathrm{r}=$ $0.260)$. We found that Frieds' score was associated with polypharmacy in regression analysis $\left(r^{2}=0.166, p=0.031\right)$. We showed a cutoff value of 5.5 of drug number for being frail according to Frieds' scale in the ROC curve. The frequency of inappropriate medicine use was $38 \%$.

Key conclusions: In this study, we revealed that frailty was related to polypharmacy. Our study is the first that the relationship between polypharmacy and frailty was assessed with SOF criteria. We designated that only Fried criteria influenced polypharmacy independently. That made us thought that Fried criteria were more useful to define the relationship between frailty and polypharmacy. Prospective studies to evaluate the effect of decreasing the number of used drugs on frailty could be useful.

\section{Abstract \# 277}

The role of frailty and social support in hospitalized older patients Enrica Patrizio ${ }^{1}$, Paolo Dionigi Rossi ${ }^{2}$, David Rigamonti ${ }^{3}$, Matteo Cesari $^{4}$

${ }^{1}$ Azienda di Servizi alla Persona Istituti Milanesi Martinitt e Stelline e Pio Albergo Trivulzio, Milan, Italy, ${ }^{2}$ Geriatric Unit, Foundation IRCCS Ca' Granda Ospedale Maggiore Policlinico, Milan, Italy, ${ }^{3}$ School of Medicine, University of Milan, Milan, Italy, ${ }^{4}$ Department of Clinical Sciences and Community Health, University of Milan, Milan, Italy

Background: Environmental and social conditions play a major role in the development and progression of negative health-related outcomes. Nevertheless, they still often remain overlooked because priority is given to the clinical manifestations. The aim of the study was to explore the importance of frailty and social support in the definition of health-related outcomes.

Methods: A retrospective analysis was conducted on medical records of 87 patients (mean age 87.5 years old, $\mathrm{SD}=6.3$, women $75.9 \%$ ) admitted to a geriatric acute care unit. A 42-item Frailty Index (FI) was computed from clinical variables. Logistic regression models were performed to study the influence of frailty and social support towards mortality, length of hospital stay, and risk of institutionalization.

Results: During the hospital stay (median length 12 days; IQR = 7-20), $6(6.9 \%)$ patients died. 27 patients (31\%) were discharged 
towards other institutions. 38 patients $(43.7 \%)$ had social support. The mean FI was 0.39 ( $\mathrm{SD}=0.12$ ). The FI showed a suggestive association with mortality $(\mathrm{OR}=1.07,95 \% \mathrm{CI}=0.99-1.15, \mathrm{p}=0.09)$, and was predictive of longer length of stay $(\mathrm{OR}=1.05,95 \% \mathrm{CI}=$ $1.00-1.09, \mathrm{p}=0.05)$, after adjustment for potential confounders. The presence of a caregiver was the only factor significantly associated with the discharge at home $(\mathrm{OR}=0.23,95 \% \mathrm{CI}=0.08-0.68, \mathrm{p}=$ 0.01 ).

Conclusions: Social and environmental factors play a critical role in determining the future of hospitalized older persons, and should be considered in clinical decisions and care plans of frail patients. Health systems should be organized according to an integrated model of care to adequately address the complex health needs of older people.

\section{Abstract \# 278 \\ Trauma of the shoulder, rupture of the supraspinatus muscle tendon}

Iker Sierra-Martínez ${ }^{1}$, Leticia Sierra-Martínez ${ }^{2}$, Rosario MartínezFuerte $^{3}$, Natalia Sanz-González ${ }^{4}$

${ }^{1}$ Traumatology Department, Hospital of Medina de Campo (Valladolid), Spain, ${ }^{2}$ Valladolid Este Primary Assistance Gerency, Valladolid, Spain, ${ }^{3}$ Pilarica Health Center, Valladolid Este Primary Assistance, Valladolid, Spain, ${ }^{4}$ Parquesol Senior Center, JCyL Social Services Gerency, Valladolid, Spain

Introduction: The shoulder is the most mobile joint in the human body, it is also the one with the most injuries it can have. Rotator cuff injuries can occur primarily due to two causes, trauma or sudden falls in the arm and degeneration of the arm.

Description of the case: A 69-year-old male patient came to the clinic for presenting sudden onset pain in his left shoulder after an accidental fall due to stumbling over the extension arm.Examination: inability to actively abduct, positive fall sign, abducts the upper limb up to 90 degrees and when it stops holding it, it falls abruptly and positive painful arc. X-ray and ultrasound: confirms the rupture of the supraspinatus muscle tendon. Treatment: rest and Aines and is referred to the trauma service. They indicate surgery to repair the tendon. After arthroscopic surgery, the process evolved favorably.

Recommendations: The painful shoulder is a very frequent pathology of the locomotor system. The time factor and the levels of uncertainty in Primary Care consultations make it essential to follow a clear differential etiopathogenic scheme if we want to carry out a correct approach for its diagnosis and treatment. This scheme includes multiple pathologies, frequent, to always take into account in our clinical reasoning and others that, due to their lower frequency, are not always part of the first diagnostic hypothesis, but which should not be forgotten when the former do not explain the clinical picture.

\section{Abstract \# 279 \\ Introducing an exercise intervention to reverse frailty and build resilience in primary care consultations \\ John Travers ${ }^{1}$, Roman Romero-Ortuno ${ }^{2}$, Marie-Therese Cooney ${ }^{3}$ \\ ${ }^{1}$ University College Dublin and Trinity College Dublin, ${ }^{2}$ Trinity College Dublin and St James's Hospital Dublin, ${ }^{3}$ St Vincent's University Hospital Dublin and University College Dublin}

Introduction: Resistance exercises have been shown to reverse frailty yet common practice appears low. GPs are in a unique position to discuss frailty and resilience and offer interventions. We wished to assess uptake of an exercise regime; rating of its ease and subjective health after participation; and how a follow-up telephone-call might affect compliance.

Methods: Patients aged $65+$, meeting inclusion criteria, who presented to a GP over 4 months, were invited to participate. They were offered a pictorial leaflet with 11 resistance-based exercises and informative discussion. Demographics, health indicators, SHARE-FI and Rockwood frailty scores were recorded. Half the randomised participants were telephoned after 1 month and all after two.

Results: 57 of 63 eligible patients $(90 \%)$ participated (average age 75 , 36 female $(63 \%)$ ). All females enrolled while $19 \%$ males declined. Only $18 \%$ had previously considered resistance exercises. At one month, $60 \%$ called were exercising regularly ( $67 \%$ of females, $50 \%$ of males). At two months, compliance among those previously called was higher: $74 \%$ ( $77 \%$ of females, $67 \%$ of males). $35 \%$ described exercises as 'very easy', 35\% 'somewhat easy', 6\% 'neither easy nor hard', 24\% 'somewhat hard', $0 \%$ 'very hard'. $6 \%$ felt 'much better', $50 \%$ 'slightly better', $44 \%$ 'about the same', $0 \%$ 'slightly worse'/ 'much worse'. Many described how the home-based exercises helped with staying active and reducing anxiety while housebound during the Covid-19 pandemic.

Conclusions: There was positive engagement in discussion on frailty and resilience and exercise uptake was high. A single telephone call appeared to help increase participation by $>20 \%$.

\section{Abstract \# 280}

Frailty index changes in late-life: acceleration in health deficit accumulation rate indicates the presence of frailty

Erwin Stolz ${ }^{1}$, Hannes Mayerl ${ }^{1}$, Emiel O. Hoogendijk ${ }^{2}$, Joshua J. Armstrong $^{3}$, Regina Roller-Wirnsberger ${ }^{1}$, Wolfgang Freidl ${ }^{1}$

${ }^{1}$ Medical University of Graz, ${ }^{2}$ VU University Amsterdam, ${ }^{3}$ Lakehead University

Background: Frailty has implications for clinical practice and public health but it is unclear which cut-off in the established frailty index (FI) indicates the presence of frailty. In this study, we assess the onset of accelerated health deficit accumulation within individuals against time-to-death (TTD) as an indicator of when frailty is present.

Methods: 23,393 repeated observations from (up to) the last 21 years of life of 5,713 deceased participants of the AHEAD cohort $(91 \%$ complete mortality) in the Survey of Health and Retirement (HRS) were assessed. A FI with 32 health deficits was calculated for (up to) 10 successive biannual assessments (1995-2014), and FI changes according to TTD were analyzed with a piecewise linear mixed model with random change points stratified by sex.

Results: The average normal (pre-terminal) health deficit accumulation rate in both men and women was 0.01 FI per year. 2.5 (men) respectively 3.9 (women) years before death, this increased to 0.04 (women) respectively 0.06 FI (men) per year. This accelerated (terminal) rate of health deficit accumulation started at a FI value of 0.27 (men) respectively 0.30 (women).

Conclusion: The substantial acceleration in health deficit accumulation in the very last years of life that follows from declining physiological reserves and failing repair mechanisms suggests a conceptually meaningful and empirically derived cut-off value close to $0.30 \mathrm{FI}$ for the presence of frailty in the continuous FI. 


\section{Abstract \# 281}

Effect of frailty and multi-comorbidities on quality of life of older adults receiving home care

Antonia Aravantinou Karlatou ${ }^{1}$, Eirini Stratidaki ${ }^{1}$, Ioannis Savvakis ${ }^{1}$, Labrini Tasioudi ${ }^{2}$, Theodoula Adamakidou ${ }^{3}$, Evridiki Patelarou ${ }^{1}$, Chariklia Tziraki ${ }^{4}$, Christos Kleisiaris ${ }^{1}$

${ }^{1}$ Department of Nursing, Faculty of Health Sciences, Hellenic Mediterranean University, Heraklion, Crete, Greece, ${ }^{2}$ Social Worker, Home Care Program, Municipality of Chania, Crete, Greece, ${ }^{3}$ Department of Nursing, Faculty of Health Sciences, University of West Attica, Athens, Greece, ${ }^{4}$ MELABEV-Community Club of Elders, and Hebrew University, Jerusalem Israel

Introduction: The impact frailty on the quality of life (QoL) has not fully examined especially on homebound older adults with cognitive decline, depression, and multi-comorbidities. Therefore, this study explores the effect of frailty adjusting for possible confounding effects on QoL of elderly people aged 65 years old receiving home care.

Methods: A per-screening for frailty was conducted in "help at home" programs during (March-May 2019) using validated screening tools. Particularly, QoL was assessed using the WHOQoL-brief, frailty using SHARE-Frail Instrument, cognitive decline using Montreal Cognitive Assessment (MoCA), disability using the Barthel Activities of Daily Living and depression using the Geriatric Depression Scale (GDS)

Results: The mean age of the 301 participants $(63.1 \%$ women) was 78.04 years, $38.5 \%$ of them identified as frail and $13.6 \%$ with severe depression. Using QoL as a dependent variable, multiple linear regression revealed that frail individuals had significantly lower QoL $\left(\beta^{\prime}=-2.65, p<0.001\right)$ compared to non-frail ones. Also, individuals identified with severe depression had significantly lower QoL ( $\beta^{\prime}=-$ $5.71, \mathrm{p}<0.001)$ compared to patients with no depressive symptoms, while this relationship was not significant in individuals with dementia $\left(\beta^{\prime}=-2.25, \mathrm{p}=0.159\right)$ after adjusting for the independent variables (age, gender, comorbidity, homebound status, etc.).

Key conclusions: Our findings suggest that both frailty and depression are important risk factors for "poor" QoL among older adults receiving home care. Thus, preventive actions offering an opportunity for significant enhancement of functional status and quality of life of older people are crucially important.

\section{Abstract \# 282}

The Impact of socioeconomic factors and age-related syndromes on frailty among older adults receiving home care

Antonia Aravantinou Karlatou ${ }^{1}$, Ioannis Savvakis ${ }^{1}$, Athina Patelarou ${ }^{1}$, Stefania Schetaki ${ }^{1}$, Georgios Markakis ${ }^{2}$, Simeon Panagiotakis ${ }^{3}$, Christos Kleisiaris ${ }^{1}$

${ }^{1}$ Department of Nursing, Faculty of Health Sciences, Hellenic Mediterranean University, Heraklion, Crete, Greece, ${ }^{2}$ Department of Social Work, Faculty of Health Sciences, Hellenic Mediterranean University, Heraklion, Crete, Greece, ${ }^{3}$ Geriatric Clinic, University General Hospital of Heraklion "PAGNI", Greece

Introduction: Socioeconomic factors including age-related syndromes (dementia, depression) play an important role in the development of frailty. Therefore, we sought to investigate their impact on frailty.

Methods: A pre-screening for frailty program was conducted enrolling registered members of "Help at Home" programs in the Reference Region of Crete (March-May 2019) using validated screening tools. Frailty was assessed with the SHARE-Frailty
Instrument, dementia with the Montreal Cognitive Assessment (MoCA), depression using the Geriatric Depression Scale (GDS), Physical functionality was measured with the Scale (Barthel-Activities of Daily Living) and comorbidity with Charlson Comorbidity Index.

Results: The mean age of the 301 participants $(63.1 \%$ female) was $78.45( \pm 7.87)$ years old. The proportion of $38.5 \%$ was identified as frail, $45.5 \%$ as pre-frail and $16.1 \%$ as non-frail. Logistic regression analysis revealed that elders with dementia were significantly more likely to be frail $(\mathrm{OR}=3.06$, CI $1.21-7.69, \mathrm{p}=0.017)$ compared to elders without dementia. Multivariate analysis showed that only patients with mild depression were significantly associated with an increased risk of having frailty $(\mathrm{OR}=2.62$, CI $1.33-5.17, \mathrm{p}=0.005)$ in comparison to those with normal depression. More importantly, elders with an increased annual individual income ( $>4500$ euros) were less likely to be frail $(\mathrm{OR}=0.45$, CI $0.25-0.83, \mathrm{p}=0.011)$ compare to those with lower annual individual income $(<4500$ euro per year).

Key conclusions: Our results suggest that mild depression (and not severe) is an important risk factor for frailty development in older adults receiving home care. Therefore, health professionals especially caring older adults have to focus on early recognition of depression.

\section{Abstract \# 283}

Frailty and older age are the chief predictors of mortality in COVID-19 patients: a single centre cohort study

Rajkumar Chinnadurai $^{1}$, Onesi Ogedengbe ${ }^{1}$, Priya Agarwal ${ }^{1}$, Sally Money-Coomes ${ }^{1}$, Ahmad Z Abdurrahman ${ }^{1}$, Sajeel Mohammed ${ }^{1}$, Philip A Kalra ${ }^{2}$, Nicola Rothwell ${ }^{1}$, Sweta Pradhan ${ }^{1}$

${ }^{1}$ Acute Medical Unit, Fairfield General Hospital, ${ }^{2}$ Department of Renal Medicine, Salford Royal NHS Foundation Trust

Background: There is a need for more observational studies across different clinical settings to better understand the epidemiology of the novel COVID-19 infection in Western populations.

Methods: We describe the clinical characteristics of all consecutive COVID-19 positive patients $(n=215)$ admitted to the acute medical unit at Fairfield General Hospital between 23 March 2020 and 30 April 2020 based on the outcome at discharge (alive or deceased). We investigated the risk factors that were associated with mortality using binary logistic regression analysis. Kaplan-Meir (KM) charts were generated by following the outcome in all patients until 12 May 2020 . Results: The median age of our cohort was 74 years with a predominance of Caucasians $(87.4 \%)$ and males $(62 \%)$. Of the 215 patients, $86(40 \%)$ died. A higher proportion of patients who died were frail (63 vs 37\%, p < 0.001), with a higher prevalence of cardiovascular disease $(50$ vs $33 \%, \mathrm{p}<0.001)$ and respiratory diseases ( 38 vs $25 \%, \mathrm{p}=0.034$ ). In the multivariate logistic regression models, older age (odds ratio (OR) 1.03; $\mathrm{p}=0.03$ ), frailty (OR 5.1; $\mathrm{p}$ $<0.001)$ and lower estimated glomerular filtration rate (eGFR) on admission (OR 0.98; $\mathrm{p}=0.01$ ) were strong and independent predictors of inpatient mortality. KM charts showed a clear difference in survival outcome in the frail older patients.

Conclusion: Older age and frailty are strong and independent risk factors associated with mortality in COVID-19 patients hospitalised to an acute medical unit at secondary care level. A holistic approach by incorporating these factors is warranted in the management of patients with COVID-19 infection. 


\section{Abstract \# 284}

The effect of task-oriented activities in the pre-frail older people

Ioannis Savvakis ${ }^{1}$, Eirini Stratidaki ${ }^{1}$, Antonia Aravantinou-Karlatou ${ }^{1}$, Athina Patelarou ${ }^{1}$, Theodoula Adamakidou ${ }^{2}$, Simeon Panagiotakis ${ }^{3}$, Christos Kleisiaris ${ }^{1}$

${ }^{1}$ Department of Nursing, Faculty of Health Sciences, Hellenic Mediterranean University, Heraklion, Crete, Greece, ${ }^{2}$ Department of Nursing, Faculty of Health Sciences, University of West Attica, Athens, Crete, Greece, ${ }^{3}$ Geriatric Clinic, University General Hospital of Heraklion "PAGNI", Crete, Greece

Introduction: It is widely accepted that physical activity has an independent effect on functional capacity of old people at risk for frailty, but the time often devoted is not based on the retraining of a particular skill. On the other hand, task-oriented exercises and/or training might improve physical independence of older adults and reduce the risk of frailty.

Methods: An advanced (And-Or-Not) research was applied using specific keywords "pre-frail", exercise", "functional capacity" etc, and filters "last 5 years", "age 65+", "pre-frail NOT frail" in biomedical database PubMed. Articles with no reference to the title at least one of the nominated terms were finally excluded.

Results: Our critical article reviewing showed that utilizing a form of training requires a variety of exercises, with specialized skills and facilities tailored to the patient's goals and capabilities. Also, multiple activities improve balance and reduce falls better than any other approach. Interestingly, there are important indications for the effectiveness of task-oriented activity training in reducing the risk o frailty and improving the autonomy of older people. Also, important, rehabilitation should be based on the practice of daily functional activities through a person-centred approach focused on parallel on his environment.

Conclusion: A well-coordinated and Task-Oriented rehabilitation program may reduce the risk for frailty and improve functional capacity thus independent living of older people.

\section{Abstract \# 285 \\ Characteristics of people having lower limb osteoarthritis with persistent severe pain showing resilience}

Natasja M. van Schoor ${ }^{1}$, Erik J. Timmermans ${ }^{1}$, Martijn Huisman ${ }^{1}$, Willem Lems ${ }^{1}$, Elaine M. Dennison ${ }^{2}$, Maria Victoria Castell ${ }^{3}$, Michael D. Denkinger ${ }^{4}$, Nancy L. Pedersen ${ }^{5}$, Stefania Maggi ${ }^{6}$, Dorly J.H. Deeg ${ }^{1}$

${ }^{1}$ Amsterdam UMC, The Netherlands, ${ }^{2}$ Southampton General Hospital, Southampton, United Kingdom, ${ }^{3}$ Universidad Autonoma de Madrid, Spain, ${ }^{4}$ University of Ulm, Germany, ${ }^{5}$ Karolinska Institute, Sweden, ${ }^{6}$ National Research Council, Italy

Introduction: Most previous research in osteoarthritis have been problem-based in nature focusing on risk factors for disease. The current study focuses on resilience in people with clinical lower limb osteoarthritis (LLOA), which is defined as functioning well despite having persistent severe pain. The objectives are: (1) to identify what proportion of older people with LLOA and persistent severe pain show good functioning; and (2) to examine characteristics of individuals showing resilience.

Methods: Data from the European Project on OSteoArthritis Study (EPOSA) were used. Clinical LLOA (i.e. knee and/or hip osteoarthritis) is defined according to the American College of Rheumatology clinical classification criteria. Persistent severe pain is defined as the highest tertile of the pain subscale of the Western
Ontario and McMaster Universities OA Index (WOMAC) both at baseline and follow-up.

Results: In total, 95 (14.9\%) out of 638 individuals having LLOA had persistent severe pain. Of these, $10(11.0 \%)$ had good physical functioning, $54(57.4 \%)$ had good mental functioning, and $49(53.8 \%)$ had good social functioning. Consumption of alcohol, higher physical activity levels, no chronic disease, higher mastery, lower age, being male and having more network contacts with friends, were significantly associated with resilience in one or more domains.

Key conclusions: Only few people having LLOA with persistent severe pain showed good physical functioning and about half of participants showed good mental or social functioning, indicating that pain severely impacts functioning. Characteristics of being resilient differed between domains of functioning, and might provide new insights for prevention.

\section{Abstract \# 286 \\ Fatigue resistance measured with pneumatic and hydraulic handgrip systems are not interchangeable}

De Dobbeleer Liza ${ }^{1}$, Beckwée David ${ }^{2}$, Arnold Pauline ${ }^{3}$, Baudry Stéphane $^{4}$, Beyer Ingo ${ }^{1}$, Demarteau Jeroen ${ }^{3}$, Lieten Siddhartha ${ }^{1}$, Punie Ynes ${ }^{5}$, Bautmans Ivan ${ }^{6}$

${ }^{1}$ a. Gerontology Department, Faculty of Medicine and Pharmacy, Vrije Universiteit Brussel, Laarbeeklaan 103, 1090 Brussels, Belgium b. Frailty in Aging Research (FRIA) Group, Faculty of Medicine and Pharmacy, Vrije Universiteit Brussel, Laarbeeklaan 103, 1090 Brussels, Belgium c. Department of Geriatrics, Universitair Ziekenhuis Brussel, Laarbeeklaan 101, 1090 Brussels, Belgium, ${ }^{2}$ a. Gerontology Department, Faculty of Medicine and Pharmacy, Vrije Universiteit Brussel, Laarbeeklaan 103, 1090 Brussels, Belgium b. Frailty in Aging Research (FRIA) Group, Faculty of Medicine and Pharmacy, Vrije Universiteit Brussel, Laarbeeklaan 103, 1090 Brussels, Belgium d. Department of Rehabilitation Sciences and Physiotherapy, Faculty of Medicine and Health Sciences, University of Antwerp, Campus Drie Eiken, Universiteitsplein 1, 2610 Wilrijk, Belgium e. SOMT University of Physiotherapy, Softwareweg 5, 3821 Amersfoort, The Netherlands f. Rehabilitation Sciences Research Department, Vrije Universiteit Brussel, Laarbeeklaan 103, B-1090 Brussels, Belgium, ${ }^{3}$ a. Gerontology Department, Faculty of Medicine and Pharmacy, Vrije Universiteit Brussel, Laarbeeklaan 103, 1090 Brussels, Belgium b. Frailty in Aging Research (FRIA) Group, Faculty of Medicine and Pharmacy, Vrije Universiteit Brussel, Laarbeeklaan 103, 1090 Brussels, Belgium e. SOMT University of Physiotherapy, Softwareweg 5, 3821 Amersfoort, The Netherlands, ${ }^{4} \mathrm{~g}$. Laboratory of Applied Biology, Research Unit in Applied Neurophysiology (LAB Neuro), Faculté des Sciences de la Motricité, Université Libre de Bruxelles, Lenniksebaan 808, 1070 Brussel, Belgium, ${ }^{5}$ e. SOMT University of Physiotherapy, Softwareweg 5, 3821 Amersfoort, The Netherlands, ${ }^{6}$ a. Gerontology Department, Faculty of Medicine and Pharmacy, Vrije Universiteit Brussel, Laarbeeklaan 103, 1090 Brussels, Belgium b. Frailty in Aging Research (FRIA) Group, Faculty of Medicine and Pharmacy, Vrije Universiteit Brussel, Laarbeeklaan 103, 1090 Brussels, Belgium c. Department of Geriatrics, Universitair Ziekenhuis Brussel,

Laarbeeklaan 101, 1090 Brussels, Belgium e. SOMT University of Physiotherapy, Softwareweg 5, 3821 Amersfoort, The Netherlands

Introduction: Fatigue resistance (FR), as can be measured with the FORTO mobile tooling, here defined as the time (in seconds) during which grip strength drops to $50 \%$ of its maximum during sustained contraction, can detect early loss of intrinsic capacity. But different grip strength systems exist and their relationship to frailty remains 
unclear. Therefore we compared FR obtained by using a pneumatic (Pneu) and hydraulic (Hydr) system.

Methods: In a large sample ( $\mathrm{n}=1381,18-100$ years) of (no-)frail: young healthy, middle-aged $\&$ older community dwelling persons, and hospitalized patients FR was measured with both systems. 53 community-dwelling women $(63-100 \mathrm{y})$ of the sample were categorized according to tertiles on the Frailty Index score (FI) as: low-frail (FI $<0.19$ ), intermediate-frail (FI 0.19-0.36), and high-frail (FI>0.36)[1].

Results: Overall, independent of age category and frailty category, FR measured by Pneu $(54.9 \pm 34.8 \mathrm{~s})$ was higher compared to Hydr $(33.7 \pm 18.2 \mathrm{~s}, \mathrm{p}<0.001)$. FR, when measured with Pneu was approximately double in low-frail $(44.3 \pm 24.6 \mathrm{~s})$ compared to highfrail participants $(23.9 \pm 12.7 \mathrm{~s}, \mathrm{p}=0.011)$, whereas FR was similar across frailty groups when measured with Hydr. There was a proportional difference in FR measured with both systems $\left(R^{2}=0.36, p\right.$ $<0.001$ ), highlighting the longer participants could sustain the maximal effort, the higher the difference in FR measured with both systems.

Discussion: Our results suggest that Pneu is a more appropriate handgrip system compared with Hydr in a (no-)frail sample. Moreover, Hydr is less able to identify community-dwelling women with higher levels of FR. Pneu assessed FR might be a good additional intrinsic capacity marker to include in frailty tools.1. Theou, O., et al., A comparison of the relationship of 14 performance-based measures with frailty in older women. Applied Physiology, Nutrition \& Metabolism, 2011. 36(6): p. 928-938.

\section{Abstract \# 287 \\ Cross-sectoral inter-rater reliability of the clinical frailty scale: a Danish translation and validation study}

Anders Fournaise ${ }^{1}$, Soeren Kabell Nissen ${ }^{2}$, Jørgen T. Lauridsen ${ }^{3}$, Jesper Ryg ${ }^{4}$, Christian H. Nickel ${ }^{5}$, Claire Gudex ${ }^{6}$, Mikkel Brabrand ${ }^{7}$, Karen Andersen-Ranberg ${ }^{8}$

${ }^{1}$ Department of Cross-sectoral Collaboration, Region of Southern Denmark (RSD); Department of Geriatric Medicine, Odense University Hospital (OUH); Epidemiology, Biostatistics and Biodemography, Department of Public Health, University of Southern Denmark (SDU), ${ }^{2}$ Institute of Regional Health Research (IRS) Centre South West Jutland, SDU; Department of Emergency Medicine, Hospital of South West Jutland (SVS), ${ }^{3}$ Department of Business and Economics, SDU, ${ }^{4}$ Department of Geriatric Medicine, OUH; Department of Clinical Research, SDU, ${ }^{5}$ IRS Centre South West Jutland, SDU; Emergency Department, University Hospital Basel, University of Basel, ${ }^{6}$ Department of Clinical Research, SDU; Open Patient data Explorative Network (OPEN), RSD, ${ }^{7}$ IRS Centre South West Jutland, SDU; Department of Emergency Medicine, SVS; Department of Clinical Research, SDU, ${ }^{8}$ Department of Geriatric Medicine, OUH; Epidemiology, Biostatistics and Biodemography, Department of Public Health, SDU; Department of Clinical Research, SDU

Introduction: The increasing focus on frailty status when designing care plans within and across primary and secondary health care sectors necessitates a common measure of frailty. The aim of this study was to translate the Clinical Frailty Scale (CFS) into Danish (CFSDK) and to test inter-rater reliability for key health care professionals in the primary and secondary sectors using CFS-DK.

Methods: The CFS was translated into Danish using the ISPOR principles for translation and cultural adaptation, including a review by the instrument developer. For the validation exercise, 40 raters were asked to rate 15 clinical case vignettes using the CFS-DK. Raters were recruited from four professional groups: primary care physicians $(n=10)$, community nurses $(n=10)$, and hospital doctors from internal medicine $(\mathrm{n}=10)$ and intensive care $(\mathrm{n}=10)$. Inter-rater reliability was assessed using intraclass correlation coefficients (ICC), and sensitivity analysis was performed using multilevel random effects linear regression.

Results: The CFS was translated and culturally adapted into Danish. The cross-sectoral ICC was 0.98 . For individual ratings within groups, ICC ranged from 0.81 to 0.90 . Sensitivity analysis showed no significant impact of profession or clinical experience. Raters considered the CFS-DK relevant for cross-sectoral collaboration.

Key conclusions: The CFS was successfully translated and culturally adapted into Danish. CFS-DK ratings showed high inter-rater reliability for key health care professionals in primary and secondary health care sectors. The CFS appears suitable as a common reference tool for frailty in cross-sectoral collaborations treating and rehabilitating older patients.

\section{Abstract \# 288 \\ Neuroimaging markers of persistent fatigue in older adults: a secondary analysis from the MAPT trial}

Davide Angioni ${ }^{1}$, Philipe de Souto Barreto ${ }^{2}$, Jeremy Raffin ${ }^{3}$, Kelly Virecoulon Giudici ${ }^{3}$, Yves Rolland ${ }^{3}$, Matteo Cesari ${ }^{3}$, Bruno Vellas ${ }^{3}$

${ }^{1} \mathrm{CHU}$ Toulouse, La Grave Hospital, ${ }^{2}$ Institut of Ageing, Toulouse, ${ }^{3}$ Institut of Ageing - Toulouse

Introduction: The brain imaging signature of fatigue in older people is not well-known. The objective of this study was to examine the cross-sectional associations between persistent fatigue and brain imaging markers in a population of community-dwelling older adults. Methods: We performed a cross-sectional analysis using data from the The Multidomain Alzheimer Preventive Trial (MAPT) trial. We included 433 subjects with data on both persistent fatigue and brain imaging. Persistent fatigue was defined as indicated in the Frailty Phenotype, with the symptom reported at two consecutive clinical visits 6 months apart between the study baseline and 12-month follow-up visit. The following brain imaging markers were assessed by magnetic resonance imaging (MRI): global cortical thickness, hippocampus and white matter hyperintensity volumes. The associations between persistent fatigue and the brain imaging markers were explored using adjusted multiple linear regressions.

Results: The mean age of the participants was approximately 75 years old and around $37 \%$ of the subjects were women. Forty-four participants $(10 \%)$ presented persistent fatigue. People with persistent fatigue were older compared to subjects without persistent fatigue. We do not find significant associations between persistent fatigue and any of the MRI markers in fully adjusted analysis.

Conclusion: Persistent fatigue is not cross-sectionally associated with brain imaging markers. A longitudinal study examining the temporal association between persistent fatigue and neuroimaging markers would be useful, in particular when using an assessment tool specifically designed and validated for measuring fatigue.

\section{Abstract \# 289 \\ Impact of cognitive and emotional impairment on frailty in old persons with multiple diagnoses}

Ali Vahedi ${ }^{1}$, Maria Eriksdotter ${ }^{2}$, Torgeir Bruun Wyller ${ }^{3}$, Hege IhleHansen $^{4}$, Pernilla Bengtsson ${ }^{5}$, Brynjar Fure ${ }^{6}$

${ }^{1}$ M.D., Department of Internal Medicine, Central Hospital Karlstad, ${ }^{2}$ Professor M.D. Ph.D Geriatric Medicine, division of Clinical geriatrics, Department of Neurobiology, Care Sciences and Society, 
Karolinska Institutet, Huddinge, Sweden and Theme Aging, Karolinska University Hospital, Huddinge, Sweden, ${ }^{3}$ Professor M.D. $\mathrm{Ph} . \mathrm{D}$, Institute of Clinical Medicine, University of Oslo and Dept. of Geriatric Medicine, Oslo University Hospital, ${ }^{4}$ Hege Ihle-Hansen, M.D. PhD, Stroke Unit, Department of Neurology, Oslo University Hospital, ${ }^{5}$ Registered Nurse, Region Värmland, Faculty of Health, Science and Technology, Department of Health Sciences, Karlstad University, ${ }^{6}$ Associate Professor M.D. PhD, School of Medical Sciences Örebro University and Department of Internal Medicine Central Hospital Karlstad

Background: The concept of frailty, according to the Fried phenotype, is largely based on reduced physical functioning. Still, there seems to be a connection between frailty and cognitive impairment. Previously, various mechanisms have been proposed as explanations for these links. However, results from different studies have been inconsistent, and the associations between MCI or dementia and frailty remain unexplained.

Aim: To examine the impact of emotional and cognitive impairment on frailty in old persons with multiple diagnoses.

Methods: We will include 150 patients $>80$ years, hospitalized in the acute geriatric unit, Central Hospital Karlstad, Sweden. Comprehensive geriatric assessment will be performed at baseline and three months after discharge with classification of mild cognitive impairment or dementia including etiological subclassification as neurodegenerative, vascular or mixed. We will assess any associations between domains of frailty and cognitive impairment, anxiety and depression, and assess the relationship to cerebrovascular pathology and radiological markers on cerebral CT. The following screenings and investigations will be performed:Frailty Index, Clinical Frailty Scale, Montreal Cognitive Assessment, Trail Making test A and B, Clinical Dementia Rating, 4AT, Confusion Assessment Method, Hospital Anxiety and Depression Scale, Timed Up and Go, hand grip strength, Katz ADL index, Mini Nutritional Assessment, Informant Questionnaire on Cognitive Decline in the Elderly, cerebral CT, cerebrospinal fluid (dementia markers).

Time aspect: Inclusion starts in 2020 and proceeds in approximately one year or until 150 patients have been included. Data analysis and writing of articles will go on through 2021-2024. This study is a PhDproject.

\section{Abstract \# 290 \\ Correlation between frailty levels and sedentary behavior patterns in nursing home residents}

Vinicius Rosa de Oliveira ${ }^{1}$, Pau Farrés-Godayol ${ }^{2}$, Pau MorenoMartin $^{2}$, Pol Gràcia-Micó ${ }^{2}$, Sandra Rierola-Fochs ${ }^{2}$, Eduard MinobesMolina $^{2}$, Javier Jerez-Roig ${ }^{2}$

${ }^{1} 1$. Research group on Methodology, Methods, Models and Outcomes of Health and Social Sciences (M3O). Faculty of Health Sciences and Welfare. Centre for Health and Social Care Research (CESS).

University of Vic-Central University of Catalonia (UVic-UCC); 2.Department of Physical Therapy. EUSES University School. University of Barcelona-University of Girona (UB-UdG)., ${ }^{2}$ Research group on Methodology, Methods, Models and Outcomes of Health and Social Sciences (M3O). Faculty of Health Sciences and Welfare. Centre for Health and Social Care Research (CESS). University of Vic-Central University of Catalonia (UVic-UCC).

Introduction: Nursing home $(\mathrm{NH})$ residents spend most of their time inactive. We aimed to analyze the correlation between frailty levels and sedentary behavior (SB) patterns of $\mathrm{NH}$ residents.
Methods: Cross-sectional study in 4 nursing homes from Osona (Barcelona) conducted between January and March 2020. Subjects who agreed to participate were men and women aged 65 years-old or more and permanently institutionalized. Frailty levels were measured using the Clinical Frailty Scale (CFS). SB total time and number of daily steps (NDS) were evaluated by ActivPAL device during 7 days. Quantitative variables are represented by mean \pm standard deviation and correlations by Spearman correlation coefficients.

Results: Initially, 145 subjects were recruited, being $53(36.5 \%)$ of them excluded because 33 (62.2\%) legal guardians refused to participate, $10(18.8 \%)$ subjects declined participation, $5(9.4 \%)$ were $<$ 65 years old and $5(9.4 \%)$ for other reasons. From the remaining 92 $(63.4 \%)$ subjects, $23(15.8 \%)$ did not accomplish the 7 consecutive days wearing ActivPAL device. Therefore, the final sample consisted of $69(47.6 \%)$ subjects; mean aged $85.1 \pm 8.1$ years old, whom 54 $(78.2 \%)$ were female. Those scored $9.0 \pm 1.6$ hours in SB, $1345.23 \pm$ 2417.3 NDS, and their frailty levels were: 24 (34.8\%) no frail, 18 (26.1\%) moderately frail, $16(23.2 \%)$ severely frail and $11(15.9 \%)$ mildly frail subjects. A positive low correlation (0.359) was found between frailty and time in SB $(\mathrm{p}<0.01)$, and a negative moderatehigh correlation $(-0.665)$ for frailty and NDS $(\mathrm{p}<0.01)$.

Conclusions: Frailty correlates positively with time in SB and negatively with NDS in NH residents.

\section{Abstract \# 291 \\ Frailty and functional recovery related factors in a geriatric day hospital}

Jorge Eduardo Corrales Cardenal ${ }^{1}$, Carmen Elías de Molins Peña ${ }^{1}$, María Dolores Domingo Sanchez ${ }^{1}$, Oscar Perez Berasategui ${ }^{1}$

${ }^{1}$ Hospital Nuestra Señora de Gracia, Zaragoza, Spain

Objectives: Know the most common illness and it's characteristics at admission in a GHD. Describe several factors related with frailty that can influence in functional recovery after the GHD intervention.

Method: Descriptive, transversal study. Patients that have completed the functional recovery process in GHD, that have been assessed since October 2019. Exclusion criteria: Not being a candidate to the GHD program, not completed the process, discharged from the GHD for any other cause. VariablesAll scales were made at admission and discharge. -Scales: SARC-F, Grip strength (GS), Short portable performance battery (SPPB). Barthel's index (BI). Pfeiffer's test (PT). Mini-Nutritional Assesment short form (MiniMNA).

Results: 87 outpatients. Admitted: $71.3 \%$. 64.5\% women. Mean age: 84.6 years. Referred by: Social health assessment unit (SHAU) $38.7 \%$. Inpatients $37.1 \%$. Geriatrics external consult $12.9 \%$. Primary health care $11.3 \%$. Admission illness: Hip fracture 51.6\%. Cerebrovascular accident: $14.5 \%$. Others: $12.9 \%$. Altered gait $8.1 \%$. Functional impairment in elderly inpatients $2.9 \%$. Completed functional recovery process: 14 (16\%).57.1\% men. Mean age: 86.14 years. Admission illness: hip fracture 50\%. At admission: BI 40. PT: 3.4 mistakes. MiniMNA: 8.43. SARC-F: 7.29. SPPB: 1.79. GS: women: $9.87 \mathrm{~kg}$; men: $13.67 \mathrm{~kg}$.At discharge: BI: 70. PT: 3.4 mistakes. MiniMNA: 8.86. SARC-F:6.664. SPPB: 4.29. GS: women: $10 \mathrm{~kg}$; men: $17.22 \mathrm{~kg}$.

Conclusions: Most patients admitted at the GHD have suffered a hip fracture, referred by the SHAU, with a great loss of functionality, achieving at discharge a functional recovery almost complete. Specific interventions during the GHD program, related with frailty contributes at the functional recovery in our patients. 


\section{Abstract \# 292}

Acceptability of a community-based exercise and nutritional intervention in frail older adults

Arveen Jeyaseelan ${ }^{1}$, Patrick O'Donoghue ${ }^{1}$, Padraig Bambrick ${ }^{1}$, Thomas Byrne ${ }^{2}$, Riona Mulcahy ${ }^{1}$, Michael Harrison ${ }^{3}$, George Pope ${ }^{1}$, Niamh O'Regan ${ }^{1}$, John Cooke ${ }^{1}$

${ }^{1} \mathrm{UHW},{ }^{2} \mathrm{PMBRC}$, WIT, ${ }^{3} \mathrm{WIT}$

Introduction: Diet and Exercise in frailty (DEFRAIL) was a study performed to evaluate the effect of exercise and protein supplementation on frailty. Subjects participated in an hour-long session prioritising resistance exercise three times per week for eight weeks. They also consumed $500 \mathrm{~mL}$ of a protein-enriched milk beverage each day. This study was performed to evaluate participants' acceptance of the intervention.

Methods: A standardised telephone survey was administered to 21/28 individuals who had participated in DEFRAIL. The remaining 7 patients could not be contacted or were unable to participate.

Results: Of the participants surveyed, 19/21 enjoyed themselves during the exercise program. 15 participants found the exercises fun. 18 would return to a similar program in the future. 19 found the program beneficial overall. The median time to perceived benefit from the exercise programme was 2 weeks. 13 felt that they were more active after the program. All participants felt the instructors were sensitive to their needs as older adults and would recommend the program to someone in their age group. 5 participants developed muscles aches which affected day to day activities. 10/21 felt that exercise was the most important part of the intervention. 8 felt that the social aspect was most important. 2 felt that venturing outside of their home was most important to them.

Conclusion: This novel exercise program has generated positive feedback overall and we intend to proceed with a definitive intervention incorporating the participant feedback summarised here.

\section{Abstract \# 293 \\ Frailty and limited functional capacity of the elderly combined with social and medical factors may cause serious injuries and traumas in daily activities}

Konstantinos Paschos ${ }^{1}$, Michalis Kirmanidis ${ }^{1}$, Anestis Chatzigeorgiadis ${ }^{1}$

${ }^{1}$ General Surgery, Hospital of Drama, Greece

Introduction: Old people present an exacerbating functional decline, which makes them vulnerable to serious injuries and traumas during their daily activities. Moreover, the use of easily accessible household, transport or agricultural machinery may further aggravate unfavorable results of potential accidents.

Methods: This study attempted to assess old patients who were admitted at the Surgical Department through the Emergency Department (ED), due to serious injuries and traumas, following daily activities and aimed to identify factors associated with functional decline.

Results: The sample included 85 participants aged between 70 and 98 years old, who were transported at the ED the last 5 years. The medium age was 82.4 years and women predominated $(n=67 ; 79 \%)$. The majority were married $(\mathrm{n}=49 ; 57.6 \%)$, followed by widowers ( $\mathrm{n}$ $=24 ; 28 \%)$ and lived in the rural periphery $(n=62,73 \%)$. Their lifestyle and healthcare analysis revealed that $82 \%$ reported healthy diet, $77 \%$ had a good sleep quality, although $68 \%$ used sleeping medication, and $45 \%$ had no physical exercise (no one was bedridden). Moreover, $58 \%$ received 4 or more medications daily and $82 \%$ presented comorbidities such as, cardiovascular diseases, diabetes mellitus, neurological diseases, urinary, renal dysfunction, etc. The modes of injury were slip and fall, fall from height, pedestrian knock down by automobiles, car and motrocycle driving accidents, agricultural machinery handling. The region of body involved included the head (76\%), face (48\%), thorax (39\%), abdomen and pelvis, extremities and spine, vascular.

Conclusions: With aging the elderly witness physical limitations that negatively influence their intellectual, social and physical functioning. Multiple comorbidities, chronic diseases, regimen with multiple medications and lack of exercise appear to further accentuate these problems causing serious injuries and traumas in everyday activities. Preventive strategies of physical frailty, training and special precautions in daily activities of the elderly should be implemented to reduce high morbidity.

\section{Abstract \# 294}

\section{Prevalence of frailty in older adults at primary care services}

João Tavares ${ }^{1}$, Daniela Figueiredo ${ }^{2}$, Lígia Passos ${ }^{3}$, Larissa Pedreira ${ }^{4}$

${ }^{1}$ School of Health Sciences, University of Aveiro, Center for Health Technology and Services Research; The Health Sciences Research Unit: Nursing, ${ }^{2}$ School of Health Sciences, Center for Health Technology and Services Research (CINTESIS.UA), ${ }^{3}$ Department of Education and Psychology, University of Aveiro, ${ }^{4}$ Federal University of Bahia

Introduction: Frailty is a prevalent and critical geriatric syndrome among older adults often associated with adverse outcomes [1]. This study examines the prevalence of frailty among older adults in the community using a Frailty Index (FI).

Methods: A cross-sectional study was performed from June to September 2019, with older adults who were users of primary care. EASYcare was used for data collection. The FI construction was based on data provided from EASYcare and 44 variables were scored ' 1 ' if the value fell outside the normal range and ' 0 ' otherwise. For example, an individual with 20 of a possible 44 deficits would have an FI score of $20 / 44=0.45$. The cut-off point of $\geq 0.25$ indicates frailty [2].

Results: 100 older adults have participated (mean age: $77.99 \pm 7.24$ years; $61 \%$ female) Most of the participants had lower levels of education (one to three years) (67\%). The EASYcare summarising indexes showed: the independence score $(0-100)$ was $17.28 \pm 18.2$; the risk of a breakdown in care (0-7) was $2.6 \pm 1.9$; the risk of falls ( $\geq 3$ in total of 8 ) was $41 \%$. The mean FI was $0.29 \pm 0.13$. More than half $(55 \%)$ of the participants were frail.

Key conclusions: In this study, the prevalence of frailty was high among older adults in the community; however, these results could be affected by the FI construction. These data show the importance of implementing frailty prevention intervention in the community.

References: 1. Chang S-F, Wen G-M (2016) Association of frail index and quality of life among community-dwelling older adults. J Clin Nurs 25:2305-2316. 2. Rockwood K, Andrew M, Mitnitski A (2007) A Comparison of Two Approaches to Measuring Frailty in Elderly People. Journals Gerontol Ser A Biol Sci Med Sci 62:738-743

\section{Abstract \# 295}

Multiple-case study design to investigate the implementation of a structured and multidimensional nurse domiciliary visittions program to frail older adults

João Tavares ${ }^{1}$, Larissa Pedreira ${ }^{2}$, Lígia Passos ${ }^{3}$, Daniela Figueiredo ${ }^{4}$

${ }^{1}$ School of Health Sciences, University of Aveiro, Center for Health Technology and Services Research; The Health Sciences Research 
Unit: Nursing, ${ }^{2}$ Federal University of Bahia, ${ }^{3}$ Department of Education and Psychology, University of Aveiro, ${ }^{4}$ School of Health Sciences, Center for Health Technology and Services Research (CINTESIS.UA)

Introduction: Intervention to prevent the progression of frailty is crucial to promote the quality of life among older adults (OA) [1]. This study analyses the impact of a structured and multidimensional nurse domiciliary visitions program (NDVP) in the management of frail OA.

Methods: Multiple-case studies were performed with three frail OAs (age $85.7 \pm 2.7$ years). In the NDVP, five visits were made for three months. The NDVP included comprehensive assessment, nutritional intervention, home environment and therapeutic regime assessments and management, home exercise program, motivation interview and no structured reminiscence. After the first visit, an interdisciplinary plan was performed. Outcomes were gait speed (GS) $(\geq 0.8 \mathrm{~m} / \mathrm{s}, 4$ metres), nutritional status (Mini Nutritional Assessment), symptoms of depression (Geriatric Depression Scale) and pain (numeric scale). Results: Two OAs lived alone, and one lived with a caregiver. During the NDVP, two cases needed home environment adaptations. All OAs were referred to a primary care physician for therapeutic reconciliation, vitamin $\mathrm{D}$ supplementation and health surveillance. At the end of the NDVP, two OAs improved GS $(0.17-0.2 \mathrm{~m} / \mathrm{s}$ and $0.77-0.84 \mathrm{~m} / \mathrm{s})$, one of which represented an improvement in fragility. One OA had worsened GS $(0.21-0.16 \mathrm{~m} / \mathrm{s})$. Two OAs reported pain relief. All OAs improved their nutritional status, especially mid-arm and calf circumference, and had fewer symptoms of depression.

Key conclusions: These case studies demonstrated the effectiveness and efficiency of structured and multidimensional NDVPs in the management of frail OAs. However, experimental studies are needed to confirm these results.

References: 1. Apóstolo J, Cooke R, Bobrowicz-Campos E, Santana S, Marcucci M, Cano A, Vollenbroek-Hutten M, Germini F, D'Avanzo B, Gwyther H, Holland C (2018) Effectiveness of interventions to prevent pre-frailty and frailty progression in older adults. JBI Database Syst Rev Implement Reports 16:140-232

\section{Abstract \# 296 \\ The trials and tribulations of a geriatric COVID-19 service \\ Caoimhe Hanrahan ${ }^{1}$, Karen Dennehy ${ }^{1}$, Clodagh M Flannery Mc Dermott $^{1}$, Catriona Reddin ${ }^{1}$, Edel Mannion ${ }^{1}$, Ruairi Waters ${ }^{1}$, Michelle Canavan ${ }^{1}$ \\ ${ }^{1}$ Department of Geriatric Medicine, Galway University Hospital}

Introduction: Older adults have higher risk for severe infection and have greater mortality rates due to Covid-19 and as such, require an individualised approach to treatment (1).

Methods: A dedicated Geriatric service was initiated in our Tertiary referral centre in order to meet the needs of older adults admitted via the Covid-19 pathway. Targetted interventions were provided including education. Data was collected from the patient cohort over a one month period.

Results: 133 patients were captured in this review. Rockwood Clincal Frailty Scale (2) showed high levels of dependency (average score $6)$. High levels of acuity were noted. There were marked levels of both delirium $(31 \%)$ and incontinence $(50 \%)$. Implementation of the Covid Geriatric service posed a unique set of challenges. There were barriers to communication due to wearing of personal protective equipment and the reduced duration and frequency of patient interactions. There was delayed time to presentation to the acute setting which necessitated liaison with palliative care services. Patients were more vulnerable to prolonged and complicated delerium. Constraints on availability of investigations and delayed swab results led to increased patient deconditioning and length of stay. Interventions were wide ranging and included nurse education, management of delerium and the use of a multi-disciplinary approach.

Conclusion: Through the introduction of a dedicated Covid Geriatric Team, we aimed to reduce common adverse effects of hospital admission on frail older adults by delivering focussed and individualised care.

References: 1. Lithander, F.E., Neumann, S., Tenison, E., Lloyd, K., Welsh, T.J., Rodrigues, J.C.L., Higgins, J.P.T., Scourfield, L., Christensen, H., Haunton, V.J., Henderson, E.J., COVID-19 in older people: a rapid clinical review, Age and Ageing, afaa093. 2. Rockwood $\mathrm{K}$ et al. A global clinical measure of fitness and frailty in elderly people. CMAJ. 2005;173(5):489-95.

\section{Abstract \# 297 \\ The use of a SIM pendant alarm in reducing hospital length of stay}

Amal Samsudeen ${ }^{1}$, Lauren Wentworth ${ }^{1}$, Natalie Geist ${ }^{2}$, Sarah Millican $^{3}$, Lindsey Mallory ${ }^{4}$

${ }^{1}$ Manchester Foundation Trust, ${ }^{2}$ British Red Cross, ${ }^{3}$ Trafford Housing Trust, ${ }^{4}$ Trafford Metropolitan Borough Council

Background: Trafford Hospital (TGH) works collaboratively with the Red Cross to support early discharge and prevent future readmissions in those who are socially isolated and frail. This service has already been shown to reduce future ED attendances and readmissions (EUGMS 2018). In December the Homesafe project (SIM pendant alarm) funded by Trafford Council was launched in partnership with Trafford Housing Trust, TGH and Red Cross. These devices are able to be installed immediately, excluding the need for referral to Telecare services. Traditionally a pendant alarm takes 3-5 days from referral to installation.

Methods: The aim of this study was to evaluate the impact of the Homesafe project on length of stay from the patient being recorded as safe for discharge, future hospital attendances and admissions, comparing data from 2 months before and after implementation.

Results: Following implementation there were no requests for Telecare on the AMU.The median number of admissions prior to implementation was 1 , and post was 0 . The median length of stay was 2 days after being deemed medically safe for discharge. Service User and NOK feedback was positive.

Conclusion: The homesafe project has been shown to reduce length of stay on AMU with subsequent reduction in reattendance and admission rates with good service user feedback. It demonstrates the importance of collaborative working across sectors to support those patients who are socially isolated and vulnerable. It should be considered across other AMUs to support patient flow and patient experience.

\section{Abstract \# 298 \\ Frail older adults report high approval for an exercise programme integrated within a sports centre for young and elite athletes}

Arveen Jeyaseelan ${ }^{1}$, Patrick O’Donoghue ${ }^{1}$, Padraig Bambrick ${ }^{1}$, Thomas Byrne $^{2}$, Riona Mulcahy ${ }^{1}$, Michael Harrison ${ }^{3}$, George Pope ${ }^{1}$, Niamh O'Regan ${ }^{1}$, John Cooke ${ }^{1}$

${ }^{1} \mathrm{UHW},{ }^{2} \mathrm{PMBRC}$, WIT, ${ }^{3}$ WIT

Introduction: DEFRAIL (Diet and Exercise for Frailty) was a study performed to evaluate the impact of a formal exercise programme and dietary protein supplementation on frailty. Subjects participated in an 
hour-long session prioritising resistance exercise in a sports centre affiliated with a local higher education institution three times per week for 8 weeks. This allowed frail older adults to integrate with a community of young and elite athletes. They also consumed $500 \mathrm{~mL}$ of a protein-enriched milk beverage each day. The purpose of this study was to evaluate participants' views of the integrated environment.

Methods: A telephone survey was administered to 21 out of 28 subjects who participated in the intervention and results were recorded anonymously via an online survey application.

Results: Of the participants surveyed, 19 out of 21 disagreed with the statement "The sports centre is only suitable for younger generations". Participants were also asked about previous involvement in sports in their youth. 10 considered themselves to be active participants in sport in their youth while 8 did not.

Conclusion: Our findings indicate that frail older adults can successfully complete a dedicated exercise programme in an integrated environment with younger and elite athletes. Their perception of the integrated environment was not biased by their past sporting participation. This suggests that exercise programmes in the frail older adult should not be restricted to geriatric day ward/rehabilitation settings but should be promoted in community sports centres and gymnasia. This integrated environment offers numerous societal benefits to all involved.

\section{Abstract \# 299 \\ Evaluation of the estimated Glomerular Filtration Rate with CKD-EPI equation of older adults aged 90 years and over with frailty}

Liudmila Merkusheva ${ }^{1}$, Nadezda Runichina ${ }^{1}$, Sergey Lysenkov ${ }^{2}$, Irina Strazhesko ${ }^{1}$, Yulia Kotovskaya ${ }^{1}$, Anastasia Shvedko ${ }^{1}$, Natalia Sharashkina ${ }^{1}$, Olga Tkacheva ${ }^{1}$

${ }^{1}$ Pirogov Russian National Medical University, The Russian Clinical Research Center for Gerontology, Moscow, Russia, ${ }^{2}$ Lomonosov

Moscow State University, Biological Faculty, Moscow, Russia

Aim of the study: To investigate kidney function in older adults $>90$ years with frailty using the methods of calculation of the estimated glomerular filtration rate(eGFR).

Materials and methods: 398 older adults aged $>90$ years were analysed. Patients with critical and terminal conditions were excluded. CKD-EPI Creatinine(2009), CKD-EPI CystatinC(2012) and CKD-EPI Creatinine-CystatinC(2012) were used. The Bland-Altman method was used to compare two measurement techniques for the same variable.

Results: Mean age of the patients was $92,6 \pm 2,5$ SD years, 370 (77.1 $\%$ ) were women. The main group included $335(84.2 \%)$ patients with frailty and63(15.8\%) patients without frailty were control group. Main group has more women $(80.3 \%$ vs $60.3 \%, \mathrm{p}=0.001)$, consists of slightly older patients $(92.7 \pm 2.5$ vs $91.9 \pm 1.9 \mathrm{y}, \mathrm{p}=0.016)$ All patients showed low levels of eGFR. Values of eGFR in the main vs control group were calculated using formulas(mean $\pm \mathrm{SD})$ :CKD-EPI Creatinine: $56,8 \pm 13,6$ vs $56,3 \pm 10,7 \mathrm{ml} / \mathrm{min} / 1.73 \mathrm{~m}^{2}(\mathrm{p}=0.79)$, CKD-EPI CystatinC: $32,3 \pm 8,3$ vs $32,3 \pm 6,9 \mathrm{ml} / \mathrm{min} / 1.73 \mathrm{~m}^{2}$ (p $=$ 0.99),CKD-EPI Creatinine-CystatinC: $42,1 \pm 10$ vs $42,1 \pm 7,9 \mathrm{ml} /$ $\min / 1.73 \mathrm{~m}^{2}(\mathrm{p}=0.97)$ respectively. Result showed that differences between values are decreasing along with the decrease in means of eGFR. However, even using minimal values, one of the equation overestimates values versus using other equations. The correlation between eGFR in frail and not frail patients wasn't obtained. Values of eGFR in older adults aged $>90$ years varied depending on the method of calculation with up to two-fold difference.
Conclusion: The kidney function in older adults aged $>90$ years was decreased and didn't depend on frailty. For the lowest values of eGFR CKD-EPI CystatinC equation overestimate means versus using other equations. For estimation of the degree of decrease of eGFR, more research is needed bearing in mind the significant difference in estimated values.

\section{Abstract \# 300}

Evaluating the need for a frailty in-reach service in the CTCCU (Cardiothoracic Critical Care Unit at Wythenshawe Hospital)

Marina Boulton ${ }^{1}$, Lauren Wentworth ${ }^{2}$

${ }^{1}$ Medical Student, University of Manchester, ${ }^{2}$ Consultant Geriatrician, Wythenshawe Hospital, Manchester

Introduction: Inadequate frailty assessment and a consequent lack of anticipatory care planning and management can have impacts on surgical decisions and post-operative outcomes. All Elective admissions over 65 years old at Wythenshawe Hospital should be screened for frailty using the CFS and referred for a CGA where appropriate as per Trust guidelines.

Method: A prospective review of 31 patients was undertaken on the Cardiothoracic Coronary Care Unit looking specifically at frailty scores, cognitive functioning and patient outcomes in those patients undergoing elective surgery.

Results: No patient had a CFS or CGA prior to surgical intervention. Together with an inconsistency in care responsibility and clinical decision making between the cardiothoracic surgeons and anaesthetists impacted on post-operative care planning. Illustrated by a near-complete absence of resuscitation decisions and a prolonged length of stay.A significant proportion of patients suffered episodes of delirium with subsequent new prescriptions of antipsychotics which are known to worsen existing cognitive impact and negatively impact recovery.

Key conclusions: More extensive anticipatory frailty assessment is due, including objective assessments (see Essential Frailty Toolset) and supplementary testing of cognitive function. Assessment should be done before decision-to-treat, by a frailty in-reach service such as those already established and proven successful in ortho-geriatrics, oncology and general surgery.Ultimately, further research into length of stay and surgical outcomes in this frail patient group, and the impact of a frailty in-reach service on this, is necessary to facilitate patients to make more informed decisions regarding whether to undertake such invasive cardiothoracic procedures.

\section{Abstract \# 301}

Prevalence of frailty among hospitalized patients in geriatric unit and correlation with several commonly used serum biomarkers

\section{Biljana Petreska-Zovic ${ }^{1}$}

${ }^{1} \mathrm{PHI}$ Specialized Hospital for Geriatric and Palliative Medicine 13 November, Skopje

Introduction: Frailty is defined as a clinically recognizable state of increased vulnerability resulting from aging-associated decline in function, with increased risk for poor health outcomes (falls, disability, hospitalization and mortality). The diagnosis of frailty is usually clinical, based on specific criteria, which are sometimes inconsistent. A notable emerging area for frailty screening is the use of biomarkers to identify frail older adults.

Methods: A cross-sectional study of the prevalence of frailty among patients over 65 years admitted in Geriatric Unit, from November 2019 till April 2020. Inclusion criteria: age 65+, Mini-Mental State 
Examination score $>20$. Exclusion criteria: malignancy, acute illness, severe vision, hearing, speech difficulties. Edmonton's Frailty Scale (EFS) and data for routinely performed biochemical parameters: Hemoglobin $(\mathrm{g} / \mathrm{dL})$, Albumin $(\mathrm{g} / \mathrm{L})$ and HbA1c $(\%)$ and cGFR $(\mathrm{ml} /$ $\min / 1,73 \mathrm{~m}^{2}$ ), were used.

Results: 133 patients age $65+$ were enrolled, 63 male, 70 female, with mean age $79.72 \pm 7.35$ years. EFS score and the values for the biomarkers were: Vulnerable $15.7 \%$ ( $10.53 \%$ male, $5.2 \%$ female), Hgb $12.83 \pm 1.33$, Albumin 40.58 \pm 4.19 , HbA1c $5.37 \pm 0.91$, GFR $67.09 \pm 19.85$, Mild frailty $25.5 \%$ ( $15.04 \%$ male, $10.53 \%$ female), Hgb $13.22 \pm 1.13$, Albumin $40.57 \pm 3.82$, HbA1c $5.50 \pm 0.96$, GFR $80.4 \pm 15.58$. Moderate frailty $26.3 \%$ ( $10.53 \%$ male, $15.79 \%$ female), $\mathrm{Hgb} 12.31 \pm 1.35$, Albumin $37.46 \pm 4.58$, HbA1c $5.41 \pm 1.08$, GFR $73.14 \pm 17.01$. Severe frailty $32.5 \%$ ( $11.28 \%$ male, $21.5 \%$ female $)$, Hgb $12.05 \pm 1.32$, Albumin 35.43 \pm 3.76 , HbA1c $5.24 \pm 0.81$, GFR $73.00 \pm 16.27$. Statistically significant association were revealed between frailty and albumin level.

Key conclusion: The prevalence of moderate and severe frailty was $58.8 \%$. Higher age, female gender and lower level of the serum albumin were associated with severity of frailty.

\section{Abstract \# 302 \\ DEFRAIL (diet and exercise for frailty): the effect of a novel multi-component group exercise program and protein supplementation on frailty in older adults}

Pádraig Bambrick ${ }^{1}$, Thomas Byrne ${ }^{2}$, Niamh Phelan ${ }^{3}$, Emma Grant $^{3}$, Ríona Mulcahy ${ }^{1}$, Michael Harrison ${ }^{2}$, John Cooke ${ }^{1}$

${ }^{1}$ University Hospital Waterford, Royal College of Surgeons in Ireland, ${ }^{2}$ Waterford Institute of Technology, ${ }^{3}$ University Hospital Waterford

Introduction: Resistance exercise and protein supplementation can delay or reverse frailty in older adults. However, they have not yet been widely implemented, due to challenges with translation into practice and limited capacity within health services.

Methods: The DEFRAIL (Diet and Exercise for Frailty) intervention was developed by consensus using a modified Delphi process. It involves an eight-week multicomponent group exercise program, suitable for community delivery, with protein supplementation. Participants recruited from Geriatric Medicine clinics were assessed at baseline, after eight weeks of regular activity (Control period), and following the eight-week program (Intervention period). The primary outcome measure was the Fried frailty score. Secondary outcome measures included measures of physical performance, body composition, cognition, mood, pain and frailty biomarkers.

Results: 28 participants ( 22 female, 6 male) commenced the program, of which 22 (16 female, 6 male) completed follow-up assessments. There were no significant changes in outcome measures during the 8 -week control period. However, the median Fried frailty score improved from 4 (IQR 3-4) to 2 (IQR 1 to2) $(\mathrm{Z}=-4.102, \mathrm{p}<0.001)$ over the eight-week intervention, while the median "Timed Up \& Go" improved from 18.09 seconds (IQR 13.34-22.20) to $15.56 \mathrm{sec}-$ onds (IQR 12.45-18.69) $(\mathrm{Z}=-2.45, \mathrm{p}=0.014)$, and the median 30 -second sit-to-stand improved from 6 (IQR 4.5-8.0) to 8 (IQR 3.75-10) $(\mathrm{Z}=-2.58, \mathrm{p}=0.010)$.

Key conclusions: The DEFRAIL intervention may provide an effective community-based means of reversing frailty and its associated adverse outcomes. To demonstrate this, a larger longitudinal study is required.

\section{Abstract \# 303}

Cost analysis of a community-based exercise and nutritional intervention in frail older adults

Joseph Walsh ${ }^{1}$, Padraig Bambrick ${ }^{1}$, Michael Harrison ${ }^{2}$, Thomas Byrne $^{3}$, George Pope ${ }^{1}$, Niamh O'Regan ${ }^{1}$, Riona Mulcahy ${ }^{4}$, John Cooke $^{4}$

${ }^{1} \mathrm{UHW}$, Waterford, Ireland, ${ }^{2}$ WIT, Waterford, Ireland, ${ }^{3} \mathrm{PMBRC}$, WIT, Ireland, ${ }^{4} \mathrm{UHW} / \mathrm{RCSI}$, Ireland

Introduction: Diet and Exercise in FRAILty (DEFRAIL) was a study performed to evaluate the effect of exercise and protein supplementation on frailty. The aim of this cost analysis is to use published data to estimate a potential monetary saving that the DEFRAIL intervention could provide to the Irish healthcare system.

Methods: DEFRAIL uses the Fried Frailty Index (FFI) to identify and track changes in frailty. A total of 22 patients completed the 8 week intervention. Median FFI pre-intervention and post intervention were 4 and 2 respectively. Components of the service costs required to deliver DEFRAIL were the exercise facility, dietary supplementation, instructors and equipment. The cost of these components were evaluated retrospectively. Potential cost savings due to DEFRAIL were calculated for each patient individually using data from a prospective 3 month analysis on the association of FFI with healthcare costs in Germany.

Results: The cost of DEFRAIL was €341.62 per participant and $€ 7,515.66$ in total. At baseline prior to DEFRAIL the total projected 3 month healthcare cost of all 22 patients corresponding to their FFI was $€ 29,911.41$. After DEFRAIL, projected 3 month healthcare costs aligned to the re-calculated FFI, had significantly reduced to $€ 4,650.02$ potentially saving an average of $€ 1148.25$ per patient or $€ 25,261.39$ in total anticipated healthcare related costs.

Conclusion: This analysis using published data indicates that there may be a significant cost saving in terms of healthcare resource use post DEFRAIL. It warrants further cost-effectiveness analysis using a definitive intervention trial.

\section{Abstract \# 304}

Frailty in the elderly patient with symptomatic severe aortic stenosis: prevalence and associated risk factors

Pablo Enrique Solla Suárez ${ }^{1}$, Javier Montero Muñoz ${ }^{2}$, Jorge Eduardo Herrera Parra ${ }^{2}$, Marta Fernández Fernández², Eva María López Álvarez ${ }^{2}$, José Gutiérrez Rodríguez ${ }^{1}$

${ }^{1}$ Área de Gestión Clínica de Geriatría Hospital Monte Naranco, Oviedo, Asturias, Spain. Instituto de Investigación Sanitaria del Principado de Asturias, Oviedo, Asturias, Spain, ${ }^{2}$ Área de Gestión Clínica de Geriatría Hospital Monte Naranco, Oviedo, Asturias, Spain

Introduction: Frailty is associated with adverse health outcomes in older patients with symptomatic severe aortic stenosis (SAS) undergoing valve replacement, making its determination of great relevance in clinical practice.

Objectives: This study aims to determine its prevalence and analyze its association with demographic, clinical, cardiological, laboratory, nutritional, functional and mental variables.

Material and methods: Prospective longitudinal epidemiological study that included all subjects over the age of 75 with symptomatic SAS who could benefit from undergoing valve replacement, attended by the Heart Team between May 2018 and February 2020.

Results: 286 patients with a mean age of $83.99 \pm 4.01$ years, most of them women $(61.2 \%)$ without high associated comorbidity (abbreviated Charlson $1.44 \pm 1.29$ ) were included in the study; with good nutritional (MNA $11.61 \pm 1.80$ ), functional (Lawton males $4.09 \pm$ 
1.29 and females $6.25 \pm 2.20$, Barthel $92.90 \pm 11.52$ ) and mental (MMSE $26.68 \pm 3.39$ ) status. A frailty prevalence of $19.6 \%$ and a mean SPPB score of $8.45 \pm 2.39$ were found. The highest causal association was observed with Atrial Fibrillation $(\mathrm{OR}=2.42$ [1,226-4,983]), comorbidity assessed by abbreviated Charlson $(\mathrm{OR}=1,302[1,004-1,688])$, age $(\mathrm{OR}=1,139[1,040-1,248])$ and Barthel $(\mathrm{OR}=0.909$ [0.877-0.942]).

Key conclusions: Functionality, age and comorbidity (especially Atrial Fibrillation) could be risk factors for frailty; and ultimately determining factors for adverse results in these patients. Attempting to improve the functional situation through a "pre-rehabilitation program", based on low-intensity, multi-component physical exercise prior to valve replacement, could have a beneficial impact on the elderly with symptomatic SAS and improve their health results.

\section{Abstract \# 305 \\ Association of multidimensional frailty and physical frailty with mortality in community-dwelling older people: a five-year longitudinal follow-up cohort study.}

Alberto Cella ${ }^{1}$, Nicola Veronese ${ }^{2}$, Giacomo Siri ${ }^{3}$, Monica Pomata ${ }^{1}$, Katerin Leslie Quispe Guerrero ${ }^{4}$, Clarissa Musacchio ${ }^{4}$, Alberto Pilotto ${ }^{5}$

${ }^{1}$ PostAcute Care Unit, Department of Geriatric Care, Orthogeriatrics and Rehabilitation, E.O. Ospedali Galliera, Genova, Italy, ${ }^{2}$ Azienda ULSS 3 Serenissima, Primary Care Department, District 3, Venice, Italy, ${ }^{3}$ Scientific Directorate-Biostatistics, E.O. Ospedali Galliera, Genova, Italy, ${ }^{4}$ Geriatrics Unit, Department of Geriatric Care, Orthogeriatrics and Rehabilitation, E.O. Ospedali Galliera, Genova, Italy, ${ }^{5}$ Department of Interdisciplinary Medicine, University of Bari Aldo Moro, Bari, Italy \& Geriatrics Unit, Department of Geriatric Care, Orthogeriatrics and Rehabilitation, E.O. Ospedali Galliera, Genova, Italy

Introduction: Frailty is a common syndrome in older people that carries an increased risk for poor health outcomes including mortality. Two main models previously described frailty as a loss of physical functions or as accumulation of multiple deficits. Recently, a novel conceptual model of multidimensional frailty has emerged based on the loss of harmonic interaction between multiple domains (biological, functional, cognitive, psycho-social and economic) that ultimately lead to homeostatic instability. Aim of our study was to compare the physical frailty index developed in the Cardiovascular Health Study (CHS) with a multidimensional frailty tool, the Multidimensional Prognostic Index (MPI), in predicting death in community-dwelling older subjects.

Methods: In this observational longitudinal cohort study, 407 community-dwelling older subjects were enrolled. Each subject underwent a comprehensive geriatric assessment (CGA) with the calculation of the MPI and CHS. Mortality was then recorded over the following 5 years. Cox-PH models were used to assess the effect of CHS and MPI, respectively: the difference between the observed $\mathrm{C}$-indexes were adopted to compare the performance of mortality risk prediction.

Results: In the sample as a whole (mean age $77.5 \pm 4.5$ years; $51.6 \%$ female), at the baseline the prevalence of physical frailty, according to the CHS index, was $9.3 \%$, and of pre-frailty $26.5 \%$. According to the MPI score, $2 \%$ of subjects were in the high-risk category (MPI-3) and $18 \%$ in the moderate risk category (MPI-2). During the 5-year follow-up period 53 subjects (13\%) died. Both MPI and CHS index were able to predict mortality. However, the MPI was significantly more accurate than CHS to predict mortality $(\mathrm{C}$-index $=0.69$ and 0.59 , respectively), with a statistically significant difference of $10 \%$ (95\% CI 0.02-0.18, $\mathrm{p}=0.013)$.
Conclusions: Multidimensional frailty, assessed by MPI, predicts five-year mortality in community-dwelling older people better than physical frailty, as assessed by the CHS index.

\section{Abstract \# 306 \\ Neuroleptic malignant-like syndrome in Parkinson's disease; combination of antipsychotic drugs and withdrawal of dopaminergic medications is a potential precipitant}

Sophie Wallace (1)

(1) Russells Hall Hospital, Dudley, UK

Case report: A 76-year-old woman was hospitalised because she gradually became unresponsive. Her medical history included Parkinson's disease and PD related dementia. She was refusing her co-careldopa (25/100 mg TDS) in the days prior to becoming unresponsive. She was also on rivastigmine $1.5 \mathrm{mg} \mathrm{BD}$ and quetiapine $25 \mathrm{mg}$ OD On examination her Glasgow Coma Scale (GCS) was 7 and she was mildly hypertensive. Heart, chest and abdominal exam was normal. She had cogwheel rigidity bilaterally. FBC and CRP were normal. Urea and Creatinine were elevated at $13.9 \mathrm{mmol} / \mathrm{L}$ and $114 \mu \mathrm{mol} / \mathrm{L}$ respectively. Creatine kinase (CK) was elevated at 2444 IU/L. Chest X-ray and CT head showed no acute pathology. Neuroleptic malignant-like syndrome was diagnosed. The patient was started on a rotigotine patch $8 \mathrm{mg} / 24 \mathrm{~h}$ and intravenous fluids. A nasogastric tube was inserted but pulled by the patient on several occasions. She remained afebrile, initially renal function improved, however level of consciousness continued to fluctuate reaching a maximum GCS of 12 and rigidity persisted. Subsequently the patient developed acute kidney injury (AKI) stage 3, deteriorated and was managed palliatively.

Discussion: Neuroleptic malignant syndrome (NMS) is a rare lifethreatening disorder, which is most often due to an adverse reaction to an anti-psychotic drug. First generation anti-psychotics were the usual culprits but every class has been implicated in reported cases. NMS can also occur in PD patients if their dopaminergic drug therapy is abruptly stopped or reduced, as in this case, and has been referred to in the literature as neuroleptic malignant-like syndrome or malignant syndrome in PD. NMS is defined by the tetrad of hyperthermia, mental status change, muscle rigidity and autonomic dysfunction. Elevated CK levels, usually $>1000 \mathrm{IU}$, increase the suspicion of NMS; the level of elevation correlates with disease severity and prognosis. Leucocytosis, elevation of liver transaminases, hypocalcaemia, hypomagnesaemia and hyperkalaemia are common but not specific. Our patient remained afebrile throughout hospitalisation. Although hyperthermia is a classic feature of NMS, there have been reported cases where fever has not been present. The exact pathogenesis of NMS is unknown. Central blockade of the hypothalamic and nigrostriatal dopamine receptors interferes with dopamine's role. If NMS is suspected, the precipitating drug should be discontinued. In PD patients, if dopaminergic therapy had been abruptly stopped, it should be re-started. Temperature and blood pressure should be monitored. Dehydration is a known precipitant of NMS and hydration is vital. Rhabdomyolysis and AKI are the two most common complications in NMS and AKI is a significant predicator of mortality. In this case, despite the patient being adequately fluid resuscitated she developed profound AKI, which proved fatal. NMS mortality could be as high as $50 \%$ The best treatment is prevention. Dantrolene, Bromocriptine, or amantadine have been tried in NMS; however, the evidence supporting their use is limited.

Conclusion: Physicians need to be aware of the potential of developing NMS in PD patients when a dopaminergic medication is suddenly withdrawn or reduced especially if the patient was on an antipsychotic or an antipsychotic was started. In this case, withdrawal 
of L Dopa on a background of use of an antipsychotic drug precipitated NMS, and age, development of AKI and rhabdomyolysis and having a single kidney contributed to the poor outcome.

\section{Abstract \# 307 \\ Integrating a front door frailty service in the emergency department: results of a pilot study \\ Liam Dunnell ${ }^{1}$, Alvin Shrestha ${ }^{1}$, Elaine $\mathrm{Li}^{1}$, Zahra Khan ${ }^{1}$, Nima Hashemi $^{1}$ \\ ${ }^{1}$ Croydon University Hospital}

Introduction: Increasing old age and frailty is putting pressure on health services with 5-10\% of patients attending the emergency department (ED) and $30 \%$ of patients in acute medical units classified as older and frail. National Health Service improvement mandates that by 2020 hospital trusts with type one EDs provide at least 70 hours of acute frailty service each week.

Methodology: A 2-week pilot (Monday-Friday $8 \mathrm{am}-5 \mathrm{pm}$ ) was undertaken, with a "Front Door Frailty Team" comprising a consultant, junior doctor, specialist nurse and pharmacist, with therapy input from the existing ED team. They were based in the ED seeing patients on arrival, referrals from the ED team and patients in the ED observation ward - opposed to the usual pathway of referral from the ED team to medical team. Data was captured using "Cerner" electronic healthcare records. A plan, do, study, act methodology was used throughout with daily debrief and huddle sessions.

Results: 95 patients were seen over two weeks. In the over $65 \mathrm{~s}$, average time to be seen was 50 minutes quicker than the ED team over the same period, with reduced admission rate $(25.7 \%$ vs $46.5 \%)$. The wait between decision to admit and departure was shortened by 119 minutes. Overall, this led to patients spending on average 133 minutes less in the ED. 64 patients were discharged, of which 44 had community follow-up (including 37.5\% of 64 referred to acute elderly clinic and $25 \%$ to rapid response). 47 medications were stopped across 25 patients.

Conclusion: The pilot shows that introduction of an early comprehensive geriatric assessment in the ED can lead to patients being seen sooner, with more timely decisions over their care and reduction in hospital admissions. It allowed for greater provision of acute clinics and community services as well as prompt medication review and real time medication changes.

\footnotetext{
Abstract \# 308

High caregiver burden among acutely presenting older patients in the emergency department

Tessel Zaalberg ${ }^{1}$, Roel Kurvers ${ }^{2}$, Dennis Barten ${ }^{3}$, Caroline van Heugten $^{4}$, Petra Klijnsma ${ }^{5}$, Lieve Knarren ${ }^{6}$, Ytje Hiemstra ${ }^{7}$, Anita Lekx $^{8}$, Simon Mooijaart ${ }^{9}$, Maryska Janssen-Heijnen ${ }^{10}$

${ }^{1}$ VieCuri Medical Center, Venlo, ${ }^{2}$ Department of Pediatrics, Maastricht University Medical Centre, Maastricht, the Netherlands., ${ }^{3}$ Department of Emergency Medicine, VieCuri Medical Center, Venlo, The Netherlands., ${ }^{4}$ Department of Neuropsychology and Psychopharmacology, Maastricht University, ${ }^{5}$ Department of Geriatric Medicine, VieCuri Medical Center, Venlo, The Netherlands., ${ }^{6}$ Department of Internal Medicine, VieCuri Medical Center, Venlo, The Netherlands., ${ }^{7}$ Caregiver representative, MantelzorgNL, Zeist, The Netherlands., ${ }^{8}$ Department of Emergency Medicine, VieCuri Medical Center, Venlo, ${ }^{9}$ Department of Gerontology and Geriatrics, Leiden University Medical Center,
}

Leiden, ${ }^{10}$ Department of Epidemiology, VieCuri Medical Center, Venlo

Background: In many developed countries, the number of older individuals that live independently at home is on the rise. These older individuals often rely on caregivers who typically have a similar age and health status. Therefore, caregivers may experience a high burden. The aim of this study was to determine the caregiver burden, to investigate potential risk factors and to assess the burden persisted four weeks after ED presentation.

Methods: Prospective observational cohort study of 200 primary caregivers of patients aged $\geq 70$ years visiting the ED. Caregiver burden was measured by the caregiver strain index (CSI). Additionally, data form medical records were used. Univariate and multivariate regression analyses were performed to identify independent determinants for burden.

Results: The mean age of patients was 82 (SD 6.7) years, $50.5 \%$ was female. The mean age of a caregiver was 66 (SD 12.4) years, $67.7 \%$ was female. Seventy-eight caregivers (39\%) experienced a high burden. Multivariate analysis showed a significant association between high caregiver burden and patients with cognitive impairment or dependency for instrumental activities of daily living (IADL) and a higher number of self-reported hours caring per day.

Conclusion: Almost $40 \%$ of caregivers of acutely presenting older patients experience a high burden. This burden is significantly associated with cognitive impairment and IADL dependency of the patient as well as a high number of self-reported hours caring per day by the caregiver. Clinicians should be aware of this problem and address possible burden when communicating with caregivers in the ED.

\section{Abstract \# 309}

The "GERI-toolbox". Initial outcomes of an e-Health instrument for the prevention of acute hospital admission of frail older adults.

Karen Andersen-Ranberg ${ }^{1}$, Janni Thygesen ${ }^{2}$, Anders Fournaise ${ }^{3}$, Jesper Ryg ${ }^{4}$

${ }^{1}$ Danish Aging Research Centre, and Dept. of Clinical Research, University of Southern Denmark and Odense University Hospital, ${ }^{2}$ Dept. of Public Health, University of Southern Denmark, ${ }^{3}$ Dept. of Cross-sectoral Collaboration, Region of Southern Denmark, and Dept. of Public Health, University of Southern Denmark, ${ }^{4}$ Dept. of Geriatric Medicine, Odense University Hospital and Dept. of Clinical Research, University of Southern Denmark

Introduction: In frail older adults late diagnostics of acute disease may increase disease severity and risk of complications from comorbid conditions, eventually leading to acute hospitalization. Early diagnostics and treatment is necessary, but challenging due to weak/atypical symptoms, and lack of specific geriatric knowledge among carers. Our study aimed at observing and describing the effect of a composite initiative consisting of (1) increasing knowledge of early disease symptoms among frontline homecare staff, (2) clear communication of symptoms to homecare nurses, and (3) to assess the use of a basic clinical objective toolbox and cross-sectoral communication.

Material and methods: An 18 months (01.03.2018-31.08.2019) observational study in the home care setting of four Danish municipalities and frail home care receiving citizens. e-Learn program to all home care employees on early recognition of emerging disease, systematic clinical assessment (Barthel ADL, vital status (ABCDE), "GERI-toolbox" containing point-of-care-testing (POCT) for basic blood analyses), and a digital cross-sectoral communication platform. Results: In total, 1850 cases (1559 individuals) (median age $83 \mathrm{y}$; males $43 \%$ ) were registered with at least one "GERI-toolbox" assessment. Nurses experienced good support in their clinical 
decision-making. Two-thirds of general practitioners (GPs) $(n=49$; $45 \%$ of eligible) believed that the "GERI-toolbox" reduced acute hospital admissions. Interviewed citizens $(n=63 ; 100 \%)$ felt safe by the acute nurse assessment using the "GERI-toolbox" (98\%), and $51 \%$ found the service equivalent to a GP's house call.

Conclusions: The GERI-toolbox is perceived useful in preventing acute admissions. Quantitative analysis of admission data is pending; results will be presented at the congress.

\section{Abstract \# 310 \\ Spontaneous rectus steath hematoma, a rare incident of anticoagulant therapy in an older adult}

Ana Maria Turcu ${ }^{1}$, Adina Carmen Ilie ${ }^{2}$, Anca Iuliana Pislaru ${ }^{2}$, Ramona Stefaniu ${ }^{2}$, Ioana Alexandra Sandu ${ }^{2}$, Anna Marie Herghelegiu $^{3}$, Catalina Raluca Nuta ${ }^{3}$, Ovidiu Lucian Bajenaru ${ }^{3}$, Gabriel Ioan Prada ${ }^{3}$, Ioana Dana Alexa ${ }^{2}$

${ }^{1}$ C.I.Parhon Hospital Iasi, ${ }^{2}$ University of Medicine an Pharmacy Iasi, ${ }^{3}$ University of Medicine an Pharmacy Bucharest

Rectus sheath hematoma is a rare entity that is most often associated with abdominal wall trauma or anticoagulation. It accounts for only about $1-2 \%$ of all causes of acute abdominal pain. Its diagnosis can be difficult given due to the associated symptoms that can mimic a variety of intra-abdominal pathologies. We will present the case of a 78-year-old female patient to emphasis the unspecific symptomatology and the importance of early diagnose. The patient is hospitalized for instable angina with a new left branch block. Biochemical data revealed mild myocardial cytolysis, with a slow downward trend. The cardiologist recommended conservative treatment which included antiplatelet and anticoagulant. The patient developed an acute abdominal pain on the 6th day of hospitalization. A new abdominal wall tumor was found. Lab tests revealed an $8 \mathrm{~g} / \mathrm{dl}$ drop of hemoglobin level and increase in creatinine level. The abdominal ultrasound revealed a rectus steath tumor and aortic aneurysm. The differential for the acute abdominal pain associated with hemoglobin drop was between spontaneous rectus steath hematoma and aortic aneurysm rupture, two conditions with to completely different approach and prognosis. The computer tomography (CT) and angiographic CT confirmed the diagnosis of rectus steath hematoma. General and vascular surgeons recommended conservative treatment. The evolution was favorable; abdominal pain disappeared, hemoglobin level increased and the abdominal tumor decreased. Anticoagulant incidents are more frequent in older adults. The rectus stealth hematoma is a rare condition, but we must consider it. Undiagnosed, rectus sheath hematoma associated with anticoagulant therapy may be a life threatening condition.

\section{Abstract \# 311}

\section{Phytobezoar diagnosis and treatment in geriatric patients}

\author{
Konstantinos Paschos ${ }^{1}$, Michalis Kirmanidis ${ }^{1}$, Anestis \\ Chatzigeorgiadis ${ }^{1}$

\section{${ }^{1}$ General Surgery, Hospital of Drama, Greece}

Phytobezoars constitute conglomerates of indigestable food materials which may cause pathological conditions in the digestive system. They may appear in any part of the gastrointestinal tract, although more frequently in the stomach.

Methods: This study included geriatric patients with phytobezoars surgically treated for ileus in the last decade in our hospital. The analysis involved demographic characteristics, pre- and postoperative findings, imaging findings, surgical or endoscopic treatment and the postoperative course.

Results: 7 patients were studied, from 68 to 83 years old. They all visited the Emergency Department (ED) with abdominal pain and vomiting and underwent an abdominal CT scan. One patient had a double phytobezoar in the stomach and small intestine, while the others had intestinal masses. Three had a gastric operation in the past, one had neurological disease and three had mastication problems due to artificial dentures. They all were subjected to an exploratory laparotomy and the intestinal phytobezoars were removed through enterotomy. In the case with a double mass, the gastric one caused a temporary jaundice due to duodenal blockage and was removed endoscopically. All patients had an uneventful postoperative course. Conclusions: Phytobezoars may usually cause obstruction of the stomach or small bowel. The double mass and jaundice are extremely rare. The disease should be suspected in patients with mastication problems, high fiber consumption, past gastric surgery or neurological disease that affects gastric emptying.

\section{Abstract \# 312 \\ Intestinal obstruction in a spigelian hernia with a concomitant jejunal diverticulum}

Konstantinos Paschos ${ }^{1}$, Michalis Kirmanidis ${ }^{1}$, Anestis Chatzigeorgiadis ${ }^{1}$

${ }^{1}$ General Surgery, Hospital of Drama, Greece

The spigelian hernia ( $\mathrm{SH}$ ) is a rare type of hernia, usually of limited diameter, which commonly presents complications, including incarceration and strangulation of the major omentum or/and the small or large bowel.

Case presentation: a 70 year-old woman was admitted at the Emergency Department (ED) due to abdominal pain in the right side of the abdomen and vomiting. Her medical history included hypertension and atrial fibrillation. At the ED she presented normal arterial pressure and pulses, while her blood tests were within normal limits. She underwent an ultrasound tomography and a computerized tomography (CT) scan, which revealed small bowel obstruction within the sac of a $\mathrm{SH}$ in the right abdominal wall. The patient underwent a surgical operation where the $\mathrm{SH}$ sac was removed, while the incarcerated intestinal part was viable and was returned in the abdomen. The abdominal control also revealed a jejunal diverticulum which was removed through a wedge resection. The postoperative course was normal; the patient had no problems 1 year postoperatively.

Conclusions: Although rare, SH may cause complications and emergency surgery in more than $2 \%$ of the patients with hernia. The medical personnel should know the special features of this hernia and ask for the proper imaging exams when someone presents pain and a palpable mass in the lower abdomen.

\section{Abstract \# 313}

Improving the quality of telephone triage for older persons seeking unplanned care: a Delphi study protocol

Farah Islam $^{1}$, Nele Aerssens ${ }^{2}$, Marc Sabbe ${ }^{3}$, Koen Milisen ${ }^{4}$

${ }^{1} \mathrm{KU}$ Leuven, Department of Public Health and Primary Care, ${ }^{2} \mathrm{KU}$ Leuven, Faculty of Medicine, ${ }^{3} \mathrm{KU}$ Leuven, Department of Public Health and Primary Care; University Hospitals Leuven, Department of Emergency Medicine, Belgium, ${ }^{4} \mathrm{KU}$ Leuven, Department of Public Health and Primary Care; University Hospitals Leuven, Department of Geriatric Medicine 
Intro: The safety of telephone triage (TT) services for delivering outof-hours $(\mathrm{OOH})$ care to older persons has been widely debated. However, few efforts have been made to establish clear criteria for managing calls addressing unplanned needs of older persons. Currently, there are no standardized methods outlining how to obtain group consensus regarding such issues. We therefore aim to establish a clear protocol for a Delphi process seeking to establish expert consensus on the management of TT calls related to older persons (over 65 years of age) seeking $\mathrm{OOH}$ unplanned care.

Methods: As part of the TRANS-SENIOR international training and research network, a three-part Delphi consensus will be conducted. A sample of 12-15 experts with at least 2 years of prior professional experience relevant to unplanned care for older persons, and/or telephone triage will be recruited. The first two parts of the study will consist of online surveys where experts will rate and provide feedback regarding the use of current TT protocols for managing calls related to older persons. During the third part of the study, a face-to-face round table meeting will be held to achieve further agreement on unresolved protocol details.

Data analyses: Descriptive and thematic analyses will be used to aggregate responses. All responses will be quantitively and qualitatively analyzed over several rounds for a maximum of two additional rounds per survey. Final consensus of protocol details will be attained during the planned meeting.

Conclusion: A clear protocol describing our planned study will ensure scientific transparency and may potentially guide best practice methods for implementing a Delphi process.

\section{Abstract \# 314 \\ Age-related in-hospital mortality in patients aged $\geq 65$ years admitted to the emergency department with suspected sepsis \\ Marius Myrstad $^{1}$, Kanika Kuwelker ${ }^{1}$, Marit Stordal Bakken ${ }^{2}$ \\ ${ }^{1}$ Department of Internal Medicine, Bærum Hospital Vestre Viken Hospital Trust, ${ }^{2}$ Haraldsplass Deaconess Hospital}

Introduction: Sepsis is a critical organ dysfunction caused by dysregulated host responses to infection. We aimed to characterize patients with sepsis and to study in-hospital mortality by age group in patients aged $\geq 65$ years admitted to the Emergency Department (ED) with suspected sepsis.

Methods: Patients aged $\geq 65$ years admitted to the EDs of two Norwegian non-university hospitals from October 2017 to September 2018 were included in the study. Sepsis was defined accoring to the most recent guidelines, as an evident infection with acute change in total Sequential Organ Failure Assessment score of $\geq 2$ points consequent to the infection.

Results: A total of 221 patients aged $\geq 65$ years were included in the study. After exclusion of 25 patients without evident infection, 196 patients (mean age 81.1 years) were eligible for this analysis. Out of these, 100 patients were diagnosed with sepsis. Pneumonia was the most common diagnosis $(41 \%)$ in patients with sepsis, followed by urinary tract infections (25\%). Patients with sepsis were more likely to be men, have anemia and renal failure than patients without sepsis. In-hospital mortality was $9 \%$ in patients with sepsis and $1 \%$ in patients without sepsis $(\mathrm{p}<0.05)$. In-hospital mortality was $14 \%$ in sepsis patients aged $\geq 80$ years and $2 \%$ in sepsis patients aged $65-79$ years $(\mathrm{p}<0.05)$.

Key conclusions: Patients aged $\geq 65$ years admitted to the ED with sepsis had increased in-hospital mortality. The in-hospital mortality was seven times higher in sepsis patients aged $\geq 80$ years compared to sepsis patients aged $65-79$ years.

\section{Abstract \# 315}

Transfers of older people living in nursing homes to emergency departments: the multicentre descriptive caregency study

F. Javier Afonso Argilés ${ }^{1}$, Xavier Ichart Tomás ${ }^{2}$, Teresa Pujadas Lafarga $^{3}$, Alba Sinfreu Pujol ${ }^{4}$, Marta Blázquez Andión ${ }^{5}$, Miguel Alberto Rizzi Bordigoni ${ }^{5}$, Héctor Villanueva Sánchez ${ }^{6}$, Isabel Tejero $\mathrm{Cano}^{7}$, J. María Gómez Roldán ${ }^{3}$, Mercè Comas Serrano ${ }^{8}$, Anna Renom Guiteras ${ }^{9}$, Mireia Puig Campmany ${ }^{10}$, Dolors García Pérez ${ }^{10}$, Isabel Cirera Lorenzo ${ }^{10}$, Ana Belén Vena Martínez ${ }^{10}$

${ }^{1}$ Department of Geriatric Medicine and Palliative Care. Blauclinic (CBC) Putget Dolors Aleu. Barcelona. Spain, ${ }^{2}$ Emergency Department. Hospital Universitario Arnau de Vilanova. Lleida. Spain., ${ }^{3}$ Department of Geriatric Medicine and Palliative Care. Badalona Serveis Assistencials. Badalona. Spain, ${ }^{4}$ Emergency Department. Fundació Althaia. Xarxa Assistencial Universitaria de Manresa. Manresa. Spain, ${ }^{5}$ Emergency Department. Hospital de la Santa Creu i Sant Pau. Barcelona. Spain, ${ }^{6}$ Emergency Department. Servicio de Urgencias. Parc de Salut Mar. Barcelona. Spain, ${ }^{7}$ Department of Geriatric Medicine. Parc de Salut Mar. Barcelona. Spain, ${ }^{8}$ Epidemiology and Evaluation Department. Parc de Salut Mar. Member of the Health Services Chronic Patient Research Network (REDISSEC), Spain, ${ }^{9}$ Department of Geriatric Medicine. Parc de Salut Mar. Member of the Health Services Chronic Patient Research Network (REDISSEC). Barcelona. Spain, ${ }^{10}$ on behalf of the Caregency Group

Introduction: Transfers of older people living in Nursing Homes (NH) to Emergency Departments (ED) have not been sufficiently evaluated in Catalonia. The aims of this study were: to describe the demographic and clinical characteristics of residents, and the outcomes of their hospitalisation processes; and to analyse the factors associated with 30-day mortality.

Methods: A multicentre retrospective observational study was carried out. We identified the residents ( $\geq 65$ years) transferred from $\mathrm{NH}$ to the ED throughout 2017 of five university public hospitals located in Catalonia, and we selected a randomized subsample of residents, for whom data were extracted from the electronic admission records and from the Minimum Basic ED Data Set. We gathered socio-demographic and clinical data, and 30-day readmission and mortality. Descriptive analyses were conducted, in addition to a multivariate analysis to evaluate the factors associated with mortality.

Results: Out of 12,580 visits, a randomized subsample of 2,444 visits was evaluated (2,059 participants), with mean age 85.9 (standard deviation [SD] $7.1 ; 67.7 \%$ women), and mean Charlson index adjusted to age 7.13 (SD 1.85). A $77.9 \%$ had cognitive impairment and $44.3 \%$ had established severe functional dependence. A $50.5 \%$ had a Triage Score III and $42.9 \%$ required hospitalisation. Main diagnoses were: infections $(33.5 \%)$, falls $(13.5 \%)$ and heart disease $(8.5 \%)$. The 30 -day readmission and mortality rates were $21.2 \%$ and $15.5 \%$, respectively. Results on the factors associated with mortality will be presented in the EUGMS Conference 2020.

Key conclusions: This study will inform about the residents' patterns of ED utilization, which can help us design better interventions to address the complex needs of this population, including end-of-life care.

\section{Abstract \# 316 \\ Quantifying the burden of coexisting acute medical problems in patients with a hip fracture in the emergency department \\ Ruth Daunt ${ }^{1}$, Michael Willoughby ${ }^{1}$, Mina Al Emam ${ }^{1}$, StJohn Kelliher $^{2}$, Michael Quirke ${ }^{1}$, Neil Burke ${ }^{1}$, Linda Brewer ${ }^{1}$ \\ ${ }^{1}$ Beaumont Hospital, ${ }^{2}$ University College Cork}


Introduction: Hip fractures are a major cause of morbidity and mortality in older adults. Orthopaedic ward admission within 4 hours is an important target to improve clinical outcome. In our hospital most patients do not meet this target. This audit aimed to quantify the burden (within this cohort) of coexisting acute medical issues on presentation, as possible obstacles to safely fast-track to the ward.

Methods: All patients presenting with hip fracture over six months were included. A data collection proforma was used to record medical, laboratory and radiological details. Data was anonymised, imported into excel and analysed using SPSS.

Results: 108 patients were included, 63\% were female, median age was $79 y$. $49 \%$ were prescribed $>5$ medications. $27 \%$ had $>4$ comorbidities. $10 \%$ had a coexistent fracture. Airspace or interstitial abnormalities were seen on only $8 \%$ of chest radiographs. $18 \%$ had brain imaging, acute haemorrhage seen in one. Altered mental status, syncope/seizure or acute coronary syndrome was documented in only $5.5 \%, 2 \%$ and $0 \%$ respectively. $11 \%$ required review (but not admission) by the medical team. Documented heart rate, oxygen saturations, blood pressure and temperature were normal in $79 \%$, 93\%, $76 \%$ and $98 \%$ respectively. Laboratory results were significantly abnormal in less than $10 \%$.

Conclusion: Despite multi-morbidity, most of our hip fracture cohort did not present with acute coexisting medical issues. Radiological and laboratory investigations and vital signs were normal for most patients and did not impact on acute management. Fast-tracking to the ward is safe and should remain the focus in these patients.

\section{Abstract \# 317 \\ Deep venous thrombosis associated with Plummer-Vinson syndrome in elderly : a case report \\ Mrouki Maroua $^{1}$, Derbal Samar ${ }^{1}$, Ben Dahmen Fatma ${ }^{1}$, Abdallah Maya $^{1}$ \\ ${ }^{1}$ Regional hospital of Ben Arous}

Introduction: Plummer-Vinson syndrome refers to the association of iron-deficiency anemia with dysphagia secondary to a post-cricoid web. We report a case of deep venous thrombosis (DVT) revealing Plummer-Vinson syndrome.

Case report: A 75-year-old woman was admitted in our department of Internal Medicine with edema of the left lower limb, poor appetite, weight loss and dysphagia. Microcytic anaemia were identified. An ultrasound duplex showed a deep venous thrombosis of the left superficial femoral vein. On presentation, she had a smooth tongue, angular cheilitis and koilonychia. Investigations revealed an anaemia with a haemoglobin level of $9 \mathrm{~g} / \mathrm{dl}$, mean cell volume $71.2 \mathrm{fl}$ and irondeficiency. A barium swallow revealed a post-cricoid web. It was thereafter ascertained on pharyngo-esophageal endoscopy. Then, our patient was diagnosed of Plummer-Vinson syndrome associated with DVT. However, no biopsy was taken. Given that our patient was elderly and DVT may be paraneoplastic, a thoraco-abdominal scan, gastric endoscopy and colonoscopy did no show any abnormality. She was treated with iron therapy and anti-coagulant drugs. She has been followed up for a period of 24 months with improvement in her dysphagia and general nutritional status with no recurrence of DVT. Conclusion: Plummer-Vinson syndrome is very rare and usually diagnosed in young adults. This case highlights that, although rare, Plummer-Vinson syndrome can occur in elderly. When it is associated with DVT, a neoplasia must be searched. Thereby, early diagnosis, treatment and close follow-up is of paramount importance in elderly.

\section{Abstract \# 318}

Comparison of the variable indicative of placement risk to short frailty screens in the emergency department

Jane McGauran ${ }^{1}$, Megan Alcock ${ }^{1}$, Mark R O’Donovan ${ }^{1}$, Elizabeth Moloney $^{2}$, Ciara Walsh' ${ }^{3}$, Anne O'Hea ${ }^{3}$, Mary Hayes ${ }^{3}$, Kieran $\mathrm{O}^{\prime}$ Connor ${ }^{1}$, Rónán $\mathrm{O}^{\prime} \mathrm{Caoimh}^{1}$

${ }^{1}$ Department of Geriatric Medicine, Mercy University Hospital, Cork, Ireland, ${ }^{1}$ Department of Geriatric Medicine, Cork University Hospital, Cork, Ireland, ${ }^{3}$ Frailty Intervention Therapy Team, Mercy University Hospital, Cork, Ireland

Introduction: The number of frail older adults presenting to Emergency Departments (ED) is increasing. Prompt recognition of frailty is important to target Comprehensive Geriatric Assessment (CGA). The Variable Indicative of Placement risk (VIP) is a quick and easyto-use frailty screen but it has not been compared to other instruments in ED. This diagnostic accuracy study examines the ability of the VIP to identify frailty against more-widely validated screens in ED.

Methods: Consecutive adults aged $\geq 70$ years were screened for frailty in a University Hospital ED by trained nurses, with the VIP-3, VIP-4, Clinical Frailty Scale (CFS) and PRISMA-7, performed in random order. An independent CGA using a battery of assessments determined their frailty status. Accuracy was measured from the area under the ROC curve (AUC).

Results: In all, 198 patients were included. Their median age was 79 $( \pm 10)$ years; $46 \%$ were female. The median VIP-3 score was $1 / 3$, VIP-4 score $1 / 4$, CFS score $4 / 9$, and median PRISMA-7 score was 4/7. Based upon the CGA, $49 \%$ were identified as frail. The most accurate instruments for separating frail from non-frail patients were the CFS (AUC 0.91) and PRISMA-7 (AUC 0.91), followed by the VIP-4 (AUC 0.85) and VIP-3 (AUC 0.84). The CFS was significantly more accurate than the VIP-3 ( $\mathrm{p}=0.03)$ or VIP-4 $(\mathrm{p}=0.048)$. There was no significant difference between the CFS and PRISMA-7 ( $p$ $=0.96$ ).

Key conclusions: These results suggest that the CFS and PRISMA-7 are more accurate and should be used in preference to the VIP to screen for frailty in EDs.

\section{Abstract \# 319}

\section{Balance physiotherapy}

Walz Isabelle ${ }^{1}$, Genna Gregory ${ }^{2}$, Liston Matthew ${ }^{3}$, Kikididis Dimitris $^{4}$, Nikitas Christos ${ }^{4}$, Bamiou Doris-Eva ${ }^{2}$, Pavlou Marousa ${ }^{5}$, Maurer Christoph ${ }^{1}$

${ }^{1}$ Department of Neurology and Neuroscience, Faculty of Medicine, Medical Center, University of Freiburg, Freiburg, Germany, ${ }^{2}$ The Ear Institute, Faculty of Brain Sciences, University College London, United Kingdom, ${ }^{3}$ Centre for Human \& Applied Physiological Sciences, King's College London, United Kingdom, ${ }^{4}$ Division of ENT, National and Kapodistrian University of Athens, Greek, ${ }^{5}$ Centre for Human \& Applied Physiological Sciences, King's College London, United Kingdom

Background: Balance disorder due to age-related progressive loss of functioning are considered a global epidemic according to the World Health Organization. Generally, balance disorders contribute to a falling rate of approximately $40 \%$ in the older population $(>65$ years). An evidence-based prevention- and rehabilitation strategy of falling should include balance training. However, for clinical routine, no standardized exercise procedures exist, even if practice guidelines provide recommendations for the management of older adults at risk for falls. As part of the EU-project HoloBalance, a systematic review of the previous guidelines on balance physiotherapy was carried out 
under the leadership of the University College London working group.

Methods: A systematic approach was adopted using MEDLINE (PUBMED), Psych Info, EMBASE, and CINAHL to review guidelines on balance physiotherapy (2008-2018).

Results: The systematic analysis included 19 guideline papers leading to the following preliminary key recommendations for balance physiotherapy: (1) Exercising should include both balance training and strength training on an individualised basis. (2) The training period should exceed $50 \mathrm{~h}$ of exercising with a frequency of two hours per week. (3) Training must be progressive over time.

Conclusion: The consensus view from the reviewed guidelines is that strength and balance training should be provided to older adults who fall. However, less than half of the guidelines provided detailed recommendations on what balance training should consist of, leaving opportunities for inconsistences in approach and variations in practice. Clearer guidance and key requirements for interventions may provide more focused and effective interventions in the future.

\section{Abstract \# 320 \\ Influence of physical activity on seniors life quality in social facilities}

Frantisek Nemeth ${ }^{1}$, Luboslava Kundracikova ${ }^{2}$, Lucia Kendrova ${ }^{3}$, Tatiana Santova ${ }^{2}$, Andrea Sulicova ${ }^{2}$, Lubica Derniarova $^{3}$, Jakub $_{\text {Cuj }}{ }^{2}$

${ }^{1}$ Profesor of Medecine, ${ }^{2}$ Master of Health, ${ }^{3}$ Pedagogical doctor

Introduction: Healthy aging is one of the main challenges of WHO. Prolonging of the age is positively influenced by improving the quality of health, while active aging means aging in health and being independent in everyday life. The main purpose of this thesis is to point out the importance of involving regular physical activity in the life of seniors that are living in social facilities. The theoretical part relates to senior's mobility, define factors that affect quality of their life. It also consists of recommendations to sustain mobile, aspects that affect it and definition of physical and mobile activity practiced by seniors and its positive effects on their organism, including prevention of complications.

Methodology: We used experiments to demonstrate that seniors' lives over 60 years old are improving due to their physical activity. The thesis includes subjective perception of life quality evaluated by WHO questionnaires, as well as the objective measurements and evaluation of physical activity performed by seniors, obtained using accelerometer GT3X.

Results: On the basis of the results we evaluated, we statistically demonstrated an improvement in the quality of life of seniors due to the positive impact of physical activity. Intense physical activity can positively act as a prevention of age-related decrease in the level of cognitive function, limiting the development and progression of chronic diseases.

Discussion: To motivate and encourage seniors for regular exercise it is also important for the seniors in social facilities to involve competent staff. With the right approach, motivation and the appropriate creation of a physical program, we can effectively work with the social group of seniors and develop their physical activity.

Conclusion: Based on the results of the study, we concluded that the involvement of regular physical activity into senior's life and their maintenance has a positive impact on the quality of their life. On the basis of objective results, it is possible to recommend the adaptation of the lifestyle for each senior with the accent on his / her individual needs.

Keywords: Quality of life, senior, Motion activity, Nursing, Social facilities

\section{Abstract \# 321}

Physical activity, life satisfaction and activities of daily living associations in the elderly

Ernestas Stundža ${ }^{1}$, Asta Mastavičiūte ${ }^{1}$

${ }^{1}$ Department of Rehabilitation, Physical and Sport Medicine, Health Science Institute, Faculty of Medicine, Vilnius University

Introduction: Number of older adults is increasing and therefore it is important to identify the factors that affect healthy ageing. Aim of the study. To investigate the associations between physical activity, life satisfaction and activities of daily living in the elderly.

Methods: In this cross-sectional study subjects 60 years and older with Mini-Mental State Examination (MMSE) score $\geq 21$ were included. Exclusion criteria were as following speech problems and use of compensatory aids. Cognitive functions evaluated by Montreal cognitive assessment and MMSE. Daily activities were assessed by Activity of Daily Living Scale, Instrumental Activities of Daily Living Scale. Physical activity was evaluated by Physical Activity Scale for the Elderly. Life satisfaction was assessed by Life Satisfaction Scale. Emotions were evaluated by Geriatric Depression Scale Short Form.

Results: Study included 100 subjects: $\mathrm{n}=20$ were men and $\mathrm{n}=80$ were women. Mean age was $68.8 \pm 6.1$ years. Study results showed that physical activity was weakly positively associated with life satisfaction $(r=0.27, p=0.007)$. Physical activity was weakly negatively associated with depression $(r=-0.31, p=0.002)$. It was found that life satisfaction was moderately negatively associated with depression $(\mathrm{r}=-0.45, \mathrm{p}<0.001)$.

Conclusions: Study results showed that higher physical activity level associated with higher life satisfaction.

\section{Abstract \# 322}

The survival benefit after acute stroke in elderly patients managed in a new dedicated Stroke Pathway dedicated to the Elderly (SPEI) compared to usual management

Oquendo Bruno $^{1}$, Jarzebowski Witold ${ }^{2}$, Oasi Christel ${ }^{3}$, Leger Anne ${ }^{4}$, Samson Yves ${ }^{4}$, Nouhaud Charlotte ${ }^{5}$, Recto Caryn ${ }^{6}$, Donadio Cristiano $^{3}$, Lafuente Carmelo ${ }^{3}$, Therry Charlotte ${ }^{3}$, Belmin Joel ${ }^{3}$

${ }^{1}$ Paris Sorbonne - Pitie Salpetriere-Charles-Foix, ${ }^{2}$ Geriatrics Department, Bastia Hospital, Bastia, France, ${ }^{3}$ Geriatrics Department, Sorbonne Université, Paris, France, ${ }^{4}$ Urgences Cérébro-Vasculaires, APHP, Hôpital Pitié-Salpêtrière, Paris, France, ${ }^{5}$ Geriatrics Department, Louis Mourier hospital, Colombes, France, ${ }^{6}$ Paris Sorbonne Université

Background: A Stroke Pathway dedicated to the Elderly (SPEl) patients with acute stroke was created in 2013 at the CHU PitiéSalpêtrière-Charles Foix (Paris, France). It is characterized by a neurovacular unit and a post stroke geriatric unit (PSGU) including rehabilitation and management of geriatric syndromes. The aim was to study the survival in the SPEl compared to other rehabilitation care in patients over 70 years of age after stroke.

Methods: Retrospective study including all consecutive patients over 70 years of age admitted to the neurovascular emergency department for acute stroke. Patients transferred to the PGSU (composing the SPEl) were compared to patients transferred to other departments for rehabilitation. The primary endpoint was the two-year survival of patients. The results were adjusted for confounding factors using a Cox model. A propensity score was used.

Results: Of the 262 patients included, those in the SPEl were older, had a higher Charlson comorbidity score and a higher NIHSS initial severity score. In multivariate analysis, hospitalization in the SPEl 
improved the two-year survival of these patients: $\mathrm{HR}=0.53(95 \% \mathrm{CI}$ $=[0.297 ; 0.946], \mathrm{p}=0.032)$. A propensity score was calculated, hospitalization in the SPEl improved the two-year survival of these patients too: $\mathrm{HR}=0.43(95 \% \mathrm{CI}=[0.191 ; 0.982], \mathrm{p}=0.05)$.

Conclusion: The Stroke Pathway dedicated to the Elderly (SPEl) with the creation of a post-stroke geriatric unit (PSGU) has a positive impact in survival of elderly patients after stroke.

\section{Abstract \# 323 \\ The physical activity intervention for reducing loneliness in older adults: randomised controlled feasibility trial}

Alexander Dubov ${ }^{1}$, Ludmila Merkusheva ${ }^{1}$, Anastasia Shvedko ${ }^{1}$, Irina Strazhesko $^{1}$, Yulia Kotovskaya ${ }^{1}$, Olga Tkacheva ${ }^{1}$

${ }^{1}$ Pirogov Russian National Medical University, The Russian Clinical Research Center of Gerontology, Moscow, Russian Federation

Abstract: The value of maintaining social connections through the life is important for mental health and well-being of older adults [3]. The isolation regime during coronavirus disease 2019 (COVID-19) showed that it can exacerbate the negative health decline associated with loneliness in older adults, which is closely linked to the increased morbidity [1] and mortality [2]. In this regard, the focus of health specialists was placed on implementation of effective health strategies of delivering physical activity to decrease loneliness in older adults [4].

Methods: Participants were healthy, inactive, community-dwelling older adults at risk for loneliness, aged 60-92 years. The intervention included: once weekly group walking and health education workshops with a wait listed control group. The feasibility outcomes were to estimate recruitment, retention and adherence rates. Secondary outcome measures (not blinded assessment) were body mass index, blood pressure, physical activity, and psychosocial variables.

Results: Forty-eight participants were recruited over 4 months with a recruitment rate of $25 \%$ (48/195); $52 \%$ (25/48) met the inclusion criteria and $100 \%(25 / 25)$ were randomised into the intervention $(\mathrm{N}=$ 12) and WL control groups $(\mathrm{N}=13)$. Participants were 25 older adults (mean (SD) 68.5(8.05) years), $14(56 \%)$ female, and $18(72 \%)$ white. At 12 weeks, 10/12 (83.3\%) intervention and 10/13 (76.9\%) control participants completed the final assessments. The average attendance rate was $58.3 \%$ for the intervention group (range 33.0-75.0\%) and $42.3 \%$ (range $23.1-69.2 \%$ ) among controls. The a priori recruitment and retention criteria for progression were not met. No serious adverse events occurred.

Conclusion: Research showed that physical activity can be effective for loneliness reduction in older adults, however there is a need for improvement of adherence, which is likely to decrease over time.

References: 1. Cacioppo, J. T., Hawkley, L. C., Crawford, L. E., Ernst, J. M., Burleson, M. H., Kowalewski, R. B., ... \& Berntson, G. G. (2002). Loneliness and health: Potential mechanisms. Psychosomatic medicine, 64(3), 407-417. 2.Holt-Lunstad, J., Smith, T.B. and Layton, J.B., 2010. Social relationships and mortality risk: a metaanalytic review. PLoS med, 7(7), p.e1000316. 3. Shvedko, A., Whittaker, A. C., Thompson, J. L., \& Greig, C. A. (2018). Physical activity interventions for treatment of social isolation, loneliness or low social support in older adults: A systematic review and metaanalysis of randomised controlled trials. Psychology of Sport and Exercise, 34, 128-137. 4. Shvedko, A. V., Thompson, J. L., Greig, C. A., \& Whittaker, A. C. (2020). Physical Activity Intervention for Loneliness (PAIL) in community-dwelling older adults: a randomised feasibility study. Pilot and Feasibility Studies, 6(1), 1-15.

\section{Abstract \# 324}

Validity and reliability of the Utrecht's Scale for Evaluation of Rehabilitation (USER) in geriatric rehabilitation

Michael Jansen ${ }^{1}$

${ }^{1}$ Amsterdam UMC

Validity and reliability of the Utrecht's Scale for Evaluation of Rehabilitation (USER) in geriatric rehabilitation.Authors: Jansen, M; Doornebos, AJ; Waal, de MWM; Wattel, L; Visser,D; Spek, B; Smit, EB

Introduction: The USER is a multi-domain instrument meant to measure physical and cognitive functioning and subjective complaints (pain, fatigue and emotions). Objective of this study was to determine reliability, measurement error, content and structural validity of the observational (physical and cognitive) scales of the USER in geriatric rehabilitation (GR).

Methods: The study consisted of three independent studies. Study 1 aimed to determine the content validity of the USER by means of a consensus-meeting with GR-professionals focussing on relevance, comprehensiveness and comprehensibility. Study 2 was a cross-sectional study where a pair of GR-nurses individually scored USERs for all patients on the GR-ward. This study determined inter-rater reliability (IRR), standardized-error-of-measurement (SEM) and smallestdetectable-change (SDC). Finally, study 3, with routine care data, was set up to evaluate the structural validity with a confirmatory factor analysis (CFA) and internal consistency by Cronbach's Alpha.

Results: The content validity was sufficient for both scales. SEM was 5 points for both scales, with an SDC of 14 for the physical and 13 for the cognitive scale. CFA showed acceptable structural fit for both scales with a second order unidimensional model. The internal consistency was good (Cronbach's alpha;0.94).

Conclusions: The USER is a valid and reliable instrument for measuring physical and cognitive functioning in geriatric rehabilitation. Future research should determine the responsiveness and interpretability of the USER.

\section{Abstract \# 325}

A pharmacist's unique opportunity within a multidisciplinary team to review drug-related problems in older adults in an intermediate care setting

Amy Byrne ${ }^{1}$, Sharon Byrne ${ }^{1}$, Kieran Dalton ${ }^{2}$

${ }^{1}$ Pharmacy department, Our Lady's Hospice \& Care Services (OLH\&CS), Harold's Cross, Dublin 6W, Ireland, ${ }^{2}$ School of Pharmacy, University College Cork, Cork, Ireland

Introduction: There is a paucity of research describing the pharmacist's role in the multidisciplinary care of older adults in intermediate care settings. The study aimed to determine the types of drug-related problems (DRPs) in older patients, to evaluate the implementation rate of pharmacist recommendations, the factors affecting implementation, and to assess the clinical significance of recommendations.

Methods: A prospective interventional study was conducted over a 12 -week period for all patients $\geq 65$ years admitted to an intermediate care unit. Data was collected on one pharmacist's recommendations to reduce clinically relevant DRPs. The clinical significance of recommendations was judged by four independent assessors using a validated tool. Statistical significance was predetermined as $\mathrm{p}<0.05$. Results: Of 494 clinically relevant DRPs identified in 91 patients, 406 recommendations were communicated to the medical team, and $89.2 \%$ were implemented. Overall, $48.5 \%$ were communicated verbally, but no difference was found between the implementation rates 
of verbal and written recommendations $(87.8 \%$ versus $90.4 \%$; $=$ $0.4)$. Recommendations judged to be of 'moderate significance' (66.8\% of total) were implemented more often than those of 'minor significance' $(93.2 \%$ versus $81.6 \%$; $p<0.001)$. The consultant was provided with a significantly higher proportion of recommendations of 'moderate significance' when compared to the junior doctor (79.6\% versus $63.3 \% ; \mathrm{p}=0.02)$, but implemented significantly fewer recommendations $(69.4 \%$ versus $91.9 \% ; \mathrm{p}<0.0001)$.

Conclusion: The high implementation rate shows the importance of pharmacist involvement to reduce DRPs in older adults in this setting. Future research should focus on the impact of pharmacist interventions on older patient outcomes and the associated cost effectiveness in this setting.

\begin{abstract}
\# 326
"Patients come with two garbage bags full of problems and we have to sort these." Experiences of professionals on patient care in short-term residential care and implications for organization and further development of short-term residential care
\end{abstract}

Judith van den Besselaar ${ }^{1}$, Linda Hartel ${ }^{1}$, Janet MacNeil- Vroomen ${ }^{1}$, Joost Wammes ${ }^{1}$, Bianca Buurman ${ }^{1}$

\section{${ }^{1}$ Amsterdam UMC}

Objectives: Short-term residential care facilities (STRC) were recently implemented in the Netherlands for community-dwelling older adults with low complex health problems with the aim to provide short-term care and return home. In practice $40 \%$ is discharged to long-term care. The objective of this study is to identify the needs of patients admitted to an STRC and to describe their care pathway using interviews with STRC staff.

Design: Qualitative research using semi-structured focus groups Setting and Participants: Eight nursing homes and three hospitals providing STRC. In total 28 participants working in an STRC were interviewed.

Analysis: Thematic analysis of semi-structured group interviews. Pseudonymized patient cases of 39 discharged patients were discussed from admission to discharge. Results: The patients were admitted with multiple complex problems that were not identified in the handover by the GP or hospital. These problems made returning to home difficult. Main themes contained: (1) the policy of low complex problems and returning home does not fit with practice; which results in STRC in a (2) mismatch between patient needs and care delivered by short-term residential care facilities. Therefore (3) planning appropriate care before and after discharge are important, such as advance care planning, social care and home adaptations are important.

Conclusions and implications: Short-term residential care is used by a patient population with an increasing complexity of health problems and often a longer existing functional decline. The provision of better targeted and evidence-based care is warranted. Adequate staffing and resources is necessary. Also, the problems of STRC should be seen in the larger context: a the environmental context of the patient need to be addressed to enable older adults to live longer independently at home.

\author{
Abstract \# 327 \\ Socio-demographic and clinical associated factors of healthcare \\ services utilisation in older persons with knee osteoarthritis: \\ a retrospective study. \\ Devinder Kaur Ajit Singh ${ }^{1}$, Maw Pin Tan ${ }^{2}$ \\ ${ }^{1}$ Physiotherapy Program \& Centre for Healthy Ageing \& Wellness, \\ Faculty of Health Sciences, Universiti Kebangsaan Malaysia, Kuala
}

Lumpur, Malaysia., ${ }^{2}$ Division of Geriatric Medicine, Department of Medicine, Faculty of Medicine, University of Malaya, Kuala Lumpur, Malaysia

Healthcare utilisation among individuals with knee osteoarthritis (OA) is exceeding globally. However, there is limited information regarding socio-demographic and clinical associated factors with utilisation of healthcare services among Malaysian older persons. The objective of this study was to determine the socio-demographic and clinical associated factors of healthcare services utilisation in older persons with knee OA. Data were obtained retrospectively from the Malaysian Elders Longitudinal Research (MELoR) study. Cases were selected based on pre-determined criteria. Socio-demographic and clinical characteristics were examined as associated factors for healthcare utilisation. The results showed that healthcare utilisation among older adults with knee OA was significantly higher compared to those without knee OA $(\mathrm{p}<0.01)$ with outpatient care services significantly used by this population $(\mathrm{p}<0.01)$. Being married, female gender and having an income were associated with outpatient care (OR 11.136, 95\% CI 1.73-52.82, p < 0.01), inpatient care (OR: $0.126,95 \%$ CI $0.021-0.746, \mathrm{p}<0.05$ ) and medication (OR 10.439, $95 \%$ CI 1.187-91.812, $\mathrm{p}<0.05)$ respectively. Older persons who are married, women and having an income were more likely to utilise healthcare services and medication to manage knee OA. There may be a need to provide self-management education for knee OA to this group of older persons.

\section{Abstract \# 328 \\ Investigation of the effect of pelvic floor muscle training on incontinence and constipation in elderly: randomized controlled trial}

Sebahat Yaprak Cetin ${ }^{1}$, Alime Buyuk ${ }^{1}$

\section{${ }^{1}$ Akdeniz University}

Introduction: The first aim of the study was to investigation of the effect of pelvic floor muscle (PFMT) and behavioral training (BT) on incontinence and constipation in elderly. The second aim was to examine whether PFMT were superior to BT.

Methods: 84 elderly adults with an average age of $66.34 \pm 6.88$ (66 women,18 men) years participated in the study. The elderly were randomly divided into two groups. Pelvic floor muscle training (PFMT) group and behavioral training (BT) group received 1 hour of training 2 days a week for 8 week. International Consultation Incontinence Questionnaire-Short form (ICIQ-SF), Urogenital Distress Inventory (UDI - 6), Incontinence Impact Questionnaire (IIQ-7) was used for incontinence assessment, Patient Assessment of Constipation Quality of Life Questionnaire (PAC-QOL) and Constipation Severity Instrument (CSI) was used for constipation assessment.

Results: There was a significant difference in all parameters of incontinence and constipation after PFMT (except of pain sub-parameter, Z: -4.20- -1.27, p: 0.04-0.00) and BT (except of CSI score, $\mathrm{Z}:-4.87--2.23$, p:0.04-0.01). After training, PFMT group was found to be superior in ICIQ-SF, UDI-6 and IIQ-7 scores compared to the BT group (Z: -2.68- -1.28, p:0.01-0.00).

Key conclusions: According to the results of the study, both PFMT and BT had a positive effect on incontinence and constipation in the elderly. In addition, PFMT had more positive effects in terms of incontinence than BT. These results support that both exercise and training should be given in order to increase the quality of life in terms of incontinence and constipation in the elderly. 


\section{Abstract \# 329}

History of falls in the previous year, physical function, and dualtasks tests in older patients of the hospital ward

Łukasz Magnuszewski (1), Zyta Beata Wojszel (1), Agnieszka Kasiukiewicz (1)

\section{(1) Medical University of Bialystok}

Introduction: Falls are a major public health concern with at least one-third of people aged 65 years and over falling at least once per year. This study aimed to verify if recurrent falls reported over the past year correlated with physical function and motor-cognitive dualtask tests in older people.

Material and methods: A cross-sectional study of 250 patients consecutively admitted to the geriatric ward at the turn of 2019 and 2020. Comprehensive geriatric assessment results were analyzed and additionally, the physical function was measured by Short Physical Performance Battery (SPPB), Barthel Index (BI), Performance-Oriented Mobility Assessment (POMA), Timed Up and Go Test (TUG), TUG-Cognitive (TUG-C) The TUG-Manual (TUG-M) and Clinical Frailty Scale (CFS).

Results: The average age of participants was 81 (6.65) years, and $73.2 \%$ were women. $42 \%$ of them reported falls in the last 12 months. Fallers were significantly older than non-fallers $(81.76 y$ (6.60) versus 80.6y (6.68), $\mathrm{P}<0.017)$. A statistically significant difference was detected in the duration of TUG test $(20.45(15.11-31.0)$ versus 15.33 (12.0-24.16) $\mathrm{P}<0.001)$ and in CFS (4.0 (3.0-6.0) versus 4.0 (3.0-5.0) $\mathrm{P}=0.031)$. Fallers had worse result of SPPB test $(3.0$ $(1.0-7.0)$ versus $5.0(1.5-8.0) \mathrm{P}=0.047)$. There were no significant differences in dual tasks TUG-C, TUG-M. Functional status measured with BI was worse in patients with a fall in last year (80.0 (60.0-95.0) versus 90.0 (70.0-95.0) $\mathrm{P}=0.062$ )

Conclusions: As we expected, comparing to non-fallers, patients who reported falls in the previous year had significantly reduced functional efficiency in SPPB test and gait speed but they did not differ in other dual tasks and BI functional status characteristics.

\section{Abstract \# 330}

Epidemiological analysis of COVID-19 at short-term rehabilitation facility for elderly in Örebro Municipality, Sweden

Ann-Sofi Eriksson ${ }^{1}$, Britt-Marie Hennerdal ${ }^{1}$

${ }^{1}$ Orebro University

Ann-Sofi Eriksson1 M.D.Britt-Marie Hennerdal2 M.D.Adrian D. Meehan3 M.A.(Hons), P.G.C.E., M.D., Ph.D.DisclosureASE, BMH and ADM have nothing to declare. The authors have judged that there is no conflict of interest that could be perceived as prejudicing the impartiality of the research reported. CorrespondenceDr. Ann-Sofi ErikssonÄngens Health Care Centre, Faculty of Medicine and Health, Örebro University, Örebro, Sweden.Tel: +46 (0)19 XXX XXXX. Fax: +46 (0)19 XXXXXXEmail: ann-sofi.eriksson@ regionorebrolan.se

Funding: This research project did not receive any other specific grant from any funding agency in the public, commercial or non-profit sector.

Abstract introduction: General transmission of Covid-19 in Sweden was declared on 6 March 2020. Studies show that the elderly, particularly those with pre-existing comorbidities, are at risk of developing severe illness. Residents at care-homes have an increased degree of frailty making them especially vulnerable to the outcomes of a Covid outbreak.

Methods: Tullhuset is an intermediary rehabilitation short-term carehome caring for 64 residents and is staffed cooperatively by Örebro
Municipality and from the Regional Örebro County health authority. Most patients are admitted from the University Hospital. Responding to the general outbreak, a ward was assigned on 26 March for potentially frail (identified through Clinical Frailty Scale instrument) elderly individuals who needed additional care provision. An outbreak occurred at the facility and an investigation was initiated by monitoring patients and furthermore with a questionnaire to staff with confirmed infection.

Results: During April 2020, a total of 17 patients (7 women; median age 83) and 38 staff members (34 women; median age 49) were diagnosed with Covid-19. All individuals, apart from two, were thought to have been infected at the care facility. Patients' symptomatology was discrete and differed significantly from the staff ( $\mathrm{p}$ value $=0.04) .3$ patients died, thus giving a case fatality of $17 \%$ (3 of 17). Intensive educative initiatives lead to a complete reduction of infectivity.

Key conclusions: Structured daily monitoring of frail patients is vital in detecting symptoms related to Covid-19. Simultaneous and continuous education of staff concerning infectivity is important for the optimal care of vulnerable patients and to minimise re-introduction of infection.

Keywords: Covid-19, frailty, infection, care-home

\section{Abstract \# 331}

The effects of single and dual task training on balance and functional mobility in the elderly: a randomized controlled study

Emel Taşvuran Horata ${ }^{1}$, Sebahat Yaprak Çetin ${ }^{2}$, Suat Erel $^{3}$

${ }^{1}$ Afyonkarahisar Health Science University, ${ }^{2}$ Akdeniz University,

${ }^{3}$ Pamukkale University

Aim: Dual task training improves the balance skills and mobility performance of the elderly. For the beneficial effects of dual task training, it should include a certain level of exercise load and task specificity. According to this informations, the aim of current study was to evaluate the effects of single and dual task training on balance and functional mobility in healthy elderly individuals.

Methods: The randomized controlled trial included 32 healthy elderly individuals, aged 60-75 years. Subjects were randomly separated into two groups as the single $(\mathrm{n}=16,64.56 \pm 3.34$ years $)$ and dual task training group $(\mathrm{n}=16,65.62 \pm 2.57)$ according to Timed Up and Go Test. The Tinetti Balance and Gait Assessment, Functional Reach Test and Single Limb Balance Test and The Timed Up and Go Test was used for assessment. All evaluations were performed at baseline and at the end of the 6th week.

Results: Both groups showed an improvement in balance and functional mobility values $(\mathrm{p}<0.05)$ after the trainings, with the exception of gait score of the single task training group ( $p>0.05$ ). Only the dual task training group showed an improvement in cognitive parameters $(\mathrm{p}<0.05)$. When the mean changes between groups were compared, dual task training group was superior to single task training group in all parameters except in the SLBT scores $(\mathrm{p}>0.05)$. No side effects were observed during the exercises.

Conclusions: The current study concluded that standardized individual-based dual task training is more useful than single task training to improve the balance of healthy elderly individuals and to improve their functional mobility. 


\section{Abstract \# 332 \\ Effect of COVID19 infection on rehabilitation processes and outcomes in a specialist geriatric rehabilitation unit}

Michelle Fitzgerald ${ }^{1}$, Eimear Flood ${ }^{1}$, Louise McCarron ${ }^{1}$, Claire Griffin $^{1}$, Ciara Lynch ${ }^{1}$, Elaine Guinan ${ }^{1}$, Elaine Cribbin ${ }^{1}$, Mark Kelly ${ }^{1}$, Patrick Doyle ${ }^{1}$, Dan Ryan ${ }^{2}$, Desmond O'Neill ${ }^{2}$

${ }^{2}$ Peamount Healthcare, Dublin, Ireland, ${ }^{3}$ Peamount Healthcare and TCD, Dublin, Ireland

Introduction: Significant rehabilitation provision is necessary following infection with COVID-19 but no data exists on impact of intercurrent COVID-19 infection on current rehabilitation processes. This study profiled older rehabilitation patients during the pandemic, including interventions required and discharge outcomes in a specialist geriatric rehabilitation unit.

Method: Observational cohort study of current inpatients who contracted COVID-19 during their stay via healthcare record review including rehabilitation input and outcomes.

Results: The mean age ( \pm SD) of 16 patients (57\% women) who acquired COVID-19 was $78.4( \pm 7.7)$ years: four died, two were discharged home and three transferred to acute hospital. The 7 patients included in this study had a planned discharge date (PDD) prior to COVID19 diagnosis. There was a mean delay of $15.6( \pm 1.7)$ days in receiving active rehabilitation post diagnosis, reducing to 3 $(+1.4)$ days 2 weeks post initial diagnosis, with $57 \%$ newly oxygen dependent, $57 \%$ experiencing swallow function deterioration and $86 \%$ experiencing a mean weight loss of $3.3 \pm 0.9 \%$. Mean modified Barthel deteriorated from $42.9(+26.9)$ to $25.2(+20.8)$. Two months post initial COVID19 diagnosis, it was not possible to calculate any PDD and mean length of stay was $91.9( \pm 52.4)$ days.

Key conclusions: COVID-19 has not only a significant mortality among older rehabilitation patients but also a major impact on the rehabilitation journey, resulting in transfers to the acute hospital and altered therapy schedules, culminating in poorer functional outcomes and delayed discharge. Rehabilitation strategies must be adapted to the altered and more complex needs of older people infected with COVID-19.

\section{Abstract \# 333 \\ Age-dependent differences in reactive balance control during perturbation-treadmill walking \\ Michel Hackbarth ${ }^{1}$, Jessica Koschate ${ }^{1}$, Sandra Lau ${ }^{1}$, Tania Zieschang ${ }^{1}$ \\ ${ }^{1}$ Carl von Ossietzky University of Oldenburg}

Introduction: Despite its high relevance in everyday life, reactive balance is not focused on as a fall risk factor in clinical practice. Mediolateral contralateral perturbations lead to particularly pronounced instability in the gait pattern. The aim of this study is to compare reactive balance between younger and older people with and without a history of falls at individually adjusted walking speed and perturbation intensity and to simultaneously conduct an in-depth gait analysis.

Methodology: The reactive balance of healthy young adults (18-30 years), healthy robust older people, as well as older people with a history of falls ( $\geq 70$ years) is tested on a perturbation-treadmill (BalanceTutor $\left.{ }^{\circledR}\right)$. Fall history and functional performance (MiniBalance-Evaluation-Systems-Test) are assessed. The intensities of the perturbations are initially determined in a static position in each individual and carried out dynamically at habitual walking speed. Gait parameters are recorded during the dynamic test using coupled inertial-sensors and depth-cameras with bodytracking-capabilities.
Results: We will be able to present data on changes in gait parameters, such as stride-length, track- width and their variability, as well as step-threshold in comparison of the groups.

Discussion: This is the first study comparing younger people with older healthy people and older people with a history of falls using an individually tolerated perturbation intensity protocol. Furthermore, a gait analysis with inertial sensors validated by camera visualization is applied during perturbations. The results may provide new starting points for targeted interventions to prevent falls in people at risk of falling.

\section{Abstract \# 334 \\ Rehabilitation programs for the elderly in the pre-operative period after spine surgery: scoping review protocol \\ Andreia Santos ${ }^{1}$, Joana Alvané ${ }^{2}$, Hugo Neves ${ }^{3}$ \\ ${ }^{1}$ Spine Center, Hospital da Luz Coimbra, ${ }^{2}$ Unidade Cuidados Continuados de Longa Duração e Manutenção da Santa Casa da Misericórdia de Pinhel, ${ }^{3}$ Health Sciences Research Unit: Nursing}

Introduction: After spine surgery, the elderly are, consistently subject to numerous critical moments of stress and vulnerability. Nursing rehabilitation plays an essential role in the capacitation of the elderly before and after surgery. First stage interventions of the rehabilitation nurse require education/training with the purpose of maximizing the elderly's autonomy and independence in the activities of daily living. Objective: Map the rehabilitation programs for the elderly submitted to spine surgery.

Method: A scoping review, based on JBI's recommendations will be carried out, using the following databases: CINAHL Complete; MEDLINE Complete; Medline, Cochrane, and PEDro. Studies focused on the elderly submitted to spine surgery (Population), subject to a motor and respiratory rehabilitation program (Concept) in the pre-operative period (Context). No time frame will be considered, with studies published in English and Portuguese to be included.

Results: Extraction tables will allow for the mapping of the characteristics of the rehabilitation programs, allowing for a better understanding of the settings requirements and potential adherence of these patients to these programs. Also, the knowledge that arises, will help develop a nursing rehabilitation intervention in a reference spine center in Portugal.

Key conclusions: Nursing rehabilitation plays an important role in the adaption of the elderly to the implications of spine surgery. This scoping review will allow us to develop a better approach in the preoperative period.

\section{Abstract \# 335}

Mapping the knowledge centered on the proprioceptive rehabilitation programs in the institutionalized elderly

Joana Alvané ${ }^{1}$, Andreia Santos ${ }^{2}$, Hugo Neves ${ }^{3}$

${ }^{1}$ Unidade Cuidados Continuados de Longa Duração e Manutenção da Santa Casa da Misericórdia de Pinhel, ${ }^{2}$ Spine Center - Hospital da Luz Coimbra, ${ }^{3}$ Health Sciences Research Unit: Nursing

Introduction: The aging process brings serious physiological changes in the human being. Balance, muscle strength, and articular movement are some of the functions that may suffer some type of impairment because of this process. The decline in the neurological components that are responsible for these functions will lead to a decrease in autonomy and independence, particularly in the performance of daily-living activities, increasing the rate of falls, with an impact on the well-being and health experience of the elderly. 
Objective: Map the proprioceptive rehabilitation programs for the institutionalized elderly with balance impairment.Method: A scoping review, based on JBI's recommendations will be carried out, using the following databases: CINAHL Complete; MEDLINE Complete; Medline, Cochrane, and PEDro. Studies focused on the elderly with balance impairment (Population), subjected to a proprioceptive rehabilitation program (Concept) in nursing homes (Context). No time frame will be considered, with studies published in English and Portuguese to be included.

Results: Extraction tables will allow for the mapping of the characteristics of proprioceptive rehabilitation programs, allowing for a better understanding of which programs have a better potential to adapt to the Portuguese reality. Also, the knowledge that arises will help develop a nursing rehabilitation intervention in a nursing home in central Portugal, that will be assessed for dissemination.

Conclusions: This research will help develop evidence-based proprioceptive programs to the institutionalized elderly potentially reducing the rate of falls, demonstrating the importance of the rehabilitation nurse in these contexts.

\section{Abstract \# 336}

Effectiveness of the diabetic and oral care program for senior in older people with type 2 diabetes mellitus in Muang District, Nakhon Ratchasima Province, Thailand

Namon Phetnin ${ }^{1}$

${ }^{1}$ Mahidol University

The aim of this study was to assess the effectiveness of the Diabetic and Oral Care Program in terms of improving oral health perception, behaviours, oral hygiene and reduced glycaemic status in type 2 diabetes mellitus older patients. A double-blind randomised controlled study was conducted in the two health centres between July 2019 and January 2020. Control and intervention groups were created and thirtyfive T2DM older patients with chronic periodontitis who attended the centres were randomly recruited into each group. The intervention group received an oral health education program based on the Health Belief Model (HBM), individual oral hygiene instruction, scaling and root planning at one month, and individual oral hygiene instruction at three months. The patients in the control group received the routine program. Outcomes were assessed using a questionnaire, a simplified oral hygiene index, and glycaemic status. These measures were applied at baseline, three months and six months. Data were analysed using a Chi-square test, a t-test, and repeated measure ANOVA at p-value = 0.05 . The results at three and six months showed that the intervention group significantly improved their HBM and oral health behaviour scores. Furthermore, their OHI-S scores and HbA1c levels $(\mathrm{p}<0.05)$ decreased, while there was no significant difference in the control group. The findings suggested that the program was effective among diabetic older people who have periodontitis in terms of improving oral health perception, behaviours, oral hygiene, and glycaemic status as indicated in evaluations occurring three and six months.

\section{Abstract \# 337 \\ Frailty sindrome in prostate cancer patients under antiandrogen therapy \\ Omar Cauli ${ }^{1}$, Rut Navarro-Martínez ${ }^{2}$, Cristina Buigues ${ }^{2}$, Maria Serrano-Carrascosa ${ }^{3}$, Julio Fernández-Garrido ${ }^{4}$, José Rubio-Briones ${ }^{5}$, Vanessa Sánchez-Martínez \\ ${ }^{1}$ University of Valencia, Nursing Department,Valencia, Spain, \\ ${ }^{2}$ University of Valencia, Nursing Department,c/Jaume Roig s/n,}

${ }^{3}$ Department of Urology, Fundación IVO, Valencia, Spain, ${ }^{4}$ Department of Nursing, University of Valencia, Valencia, Spain, ${ }^{5}$ Department of Urology, Fundación IVO, Valencia, Spain., ${ }^{6}$ Department of Nursing, University of Valencia, Valencia

Introduction: Androgen deprivation therapy (ADT) can accelerate the progression of frailty syndrome in older men with prostate cancer (PCa). The diagnosis of this syndrome and related biomarkers can help clinicians to identify most vulnerable patients and to add therapeutic strategies to limit these adverse effects.

Methods: A total of 46 men (51-92 years old) with PCa and receiving ADT were classified as frail, prefrail or robust according to the Fried scale. A geriatric assessment was performed, based on the Minimental State Examination for cognitive function, the Barthel index for basic activities of daily living, the Yesavage scale for geriatric depression, and the Athens insomnia scale. In addition, blood samples were collected to assess peripheral inflammation biomarkers including proinflammatory cytokines, fibrinogen, CRP and leukocyte differential count, as well as other biochemical and hematological parameters.

Results: A significant negative correlation between the severity of frailty syndrome and lymphocyte count was observed $(\mathrm{P}<0.01)$. The concentration of IL-6 $(\mathrm{P}<0.05)$, CRP $(\mathrm{P}<0.05)$, and fibrinogen $(\mathrm{P}<$ 0.01 ) were significantly associated with frailty syndrome, but not of TNF- $\alpha$, IL-beta, or IL- 8 . The severity of frailty syndrome was not dependent upon the clinical disease stage at diagnosis, the time elapsed since $\mathrm{CaP}$ diagnosis, the presence of metastases, or prostatectomy

Conclusions: Further research into the role of peripheral inflammation and the associated adverse outcomes in PCa patients under ADT is warranted in order to tailor interventions aimed at reducing symptoms of frailty syndrome, such as loss of muscle strength and low physical activity.

\section{Abstract \# 338}

\section{Rib trauma and lung carcinoma}

Iker Sierra-Martínez ${ }^{1}$, Leticia Sierra-Martínez ${ }^{2}$, Rosario MartínezFuerte $^{3}$, Natalia Sanz-González ${ }^{4}$

${ }^{1}$ Traumatology Department, Hospital of Medina de Campo (Valladolid), Spain, ${ }^{2}$ Valladolid Este Primary Assistance Gerency, Valladolid, Spain, ${ }^{3}$ Pilarica Health Center, Valladolid Este Primary Assistance, Valladolid, Spain, ${ }^{4}$ Parquesol Senior Center, JCyL Social Services Gerency, Valladolid, Spain

Introduction: Lung cancer is a major health problem. It is the leading cause of death from cancer in the world. It is the second neoplasm in men and the third in women. The median age of diagnosis is 65 years. Case summary: 69-year-old patient who went to the emergency department after an accidental fall 8 days ago, hitting himself in the right rib region. After the examination, an X-ray of the right rib and chest is requested. On the rib radiograph there are no acute bone lesions and on the chest radiograph there is condensation in the left hemitorax (we have no previous radiographs). After performing an anamnesis again, she comments on two episodes of colds in the last months, without fever, without expectoration, or chest pain, without dysthermia or thermometric fever. pak yeer (27), units of alcohol per day (4). The patient does not report weight loss. The patient is referred to Pneumology to complete the study of the pulmonary mass of accidental findings. Lung cancer is diagnosed and evaluated in oncology, where appropriate treatment is started.

Recommendations: Tobacco causes $90 \%$ of lung cancers, the cessation of this habit is vital to decrease their mortality. It is important to promote comprehensive care for the smoker from the health system. And do not forget that before a smoker with the most frequent 
presenting symptoms are cough, dyspnea, pain and weight loss. The appearance of persistent cough in a smoker should make us suspicious. The first on-demand imaging test is a chest $\mathrm{x}$-ray.

\section{Abstract \# 339 \\ Pathologic fracture by metastasis in adenocarcinoma of straight}

Iker Sierra-Martínez ${ }^{1}$, Leticia Sierra-Martínez ${ }^{2}$, Rosario MartínezFuerte $^{3}$, Natalia Sanz-González ${ }^{4}$

${ }^{1}$ Traumatology Department, Hospital of Medina de Campo (Valladolid), Spain, ${ }^{2}$ Valladolid Este Primary Assistance Gerency, Valladolid, Spain, ${ }^{3}$ Pilarica Health Center, Valladolid Este Primary Assistance, Valladolid, Spain, ${ }^{4}$ Parquesol Senior Center, JCyL Social Services Gerency, Valladolid, Spain

Introduction: The high incidence of bone metastases secondary to carcinoma and the serious functional repercussion they cause are reasons for studying evaluation, diagnosis and treatment methods. Pain is the most frequent presenting symptom, although sometimes the beginning is a pathological fracture.

Description of the case: A 67-year-old female patient with a history of hypertension, nephrectomy for nephrocalcinosis, adenocarcinoma of the rectum at an advanced stage for which she had a discharge colostomy, and also presented bone metastases at the level of the right hip. The patient without previous trauma presents intense pain at the level of the right inguinal region, with total inability to wander. Therefore, he was admitted to the hospital with the diagnosis of a subcapital fracture of the right femur. She undergoes surgery by performing a partial hip arthroplasty. It presents good evolution, reason why it is discharged from the hospital with indication of control by the family doctor and the oncologist.

Recommendations: Colorectal cancer (CRC) bone metastases are rare. When present, they generally correspond to a late manifestation of the disease. CRC meets the necessary conditions to consider it as a neoplasm capable of primary, secondary and tertiary prevention. In the field of primary prophylaxis, the intervention of the family doctor is very important, through education, aimed at promoting diet modifications and genetic counseling. On the other hand, the detection of preneoplastic lesions are the basis of the screening programs carried out in Primary Care for an early diagnosis and to avoid metastasis.

\section{Abstract \# 340 \\ Not only a matter of breast surgery: geriatrician can have its say for endometrial and ovarian cancer surgery too! Results from a pilot study \\ Filippo Cioli Puviani ${ }^{1}$, Pierandrea De Benedetti ${ }^{1}$, Pierandrea De $\mathrm{Iaco}^{2}$, Anna Myriam Perrone ${ }^{2}$, Giulia Dondi ${ }^{2}$, Safia Boussedra ${ }^{2}$, Giampaolo Bianchi ${ }^{2}$, Caterina Galetti ${ }^{3}$ \\ ${ }^{1}$ Bologna University, ${ }^{2}$ Bologna University Hospital - Bologna University, ${ }^{3}$ Bologna University Hospital}

Introduction: Growing evidences support safety and feasibility of elective breast surgery in elderly patients, while surgical treatment of other gynecological cancers seems to be a neglected issue.

Methods: All over 65 y.o. patients referred for CGA before endometrial or ovarian cancer surgery from $02 / 15$ to $02 / 20$ at Bologna University Hospital were prospectively included. For each patient we recorded: oncological and surgical variables, G8 score, CGA test results, 30-day postoperative complications and hospital length of stay. Logistic regression identified predictive variables for complications.
Results: 65 patients were included (median age 73); 53,7\% had a FIGO stage $\geq 3$ cancer. Although $60.3 \%$ was ASA $\geq 3$, the majority were independent for ADL and IADL, had no cognitive impairement, no CIRS severe comorbidities, good physical performance and normal nutritional status. At least one complication was recorded for 26 patients $(40 \%)$, but only $5(7.7 \%)$ were Clavien Dindo $\geq 3$. Independent predictors of complications are number of CIRS-G categories endorsed $(\mathrm{OR}=1,488 ; \mathrm{P}=0.019)$ and the $\mathrm{G} 8(\mathrm{OR}=0.785 ; \mathrm{P}=0.048)$, which is the only independent predictor for major complications (OR $=0.560 ; \mathrm{P}=0.012)$; surgical variables do not reach statistical significance. Exclusive laparoscopy $(\mathrm{p}<0.001)$ and SLNB $(\mathrm{P}=0.012)$ seem to be associated with a shorter length of stay, robotic surgery with longer operating time $(\mathrm{P}=0.009)$, without changes in outcome. Key conclusions: In this setting, surgery could be safe, CGA can be more complex and appropriate than anesthesiologist's judgement, G8 could be a complication predictor and minimally invasive techniques may be feasible, but further studies are needed to assess their real benefit

\section{Abstract \# 341}

Hyperglycaemia in older people receiving chemotherapy \& the development of a clinical care pathway

Pavun Basra ${ }^{1}$, Rupa Ali $^{1}$, Cliona Flanagan ${ }^{2}$, Mamta Joshi ${ }^{3}$, Rosarie Atkinson $^{3}$, Anna Brackenridge ${ }^{3}$, Gordana Babic-Illman ${ }^{2}$, Danielle Harari $^{4}$, Tania Kalsi ${ }^{5}$

${ }^{1}$ Kings College London, ${ }^{2}$ GOLD Team, Guys \& St Thomas' NHS Foundation Trust, ${ }^{3}$ Dept of Diabetes and Endocrine, Guys \& St Thomas' NHS Foundation Trust, ${ }^{4}$ GOLD Team, Guys \& St Thomas NHS Foundation Trust and Kings College London, ${ }^{5}$ GOLD Team, Guys \& St Thomas' NHS Foundation Trust and Kings College London

Introduction: $20 \%$ of cancer patients have pre-existing diabetes. Corticosteroids are an important part of supportive treatment with chemotherapy but risks hyperglycaemia during chemotherapy.

Objectives: 1 . To evaluate how glycaemia is assessed and monitored in older cancer patients undergoing chemotherapy with steroids. 2 . Develop a clinical pathway to improve care.

Methods: A retrospective case note review of older patients (aged $70+)$ in a London hospital receiving chemotherapy with dexamethasone for any cancer from October 2018 TO March 2019. Hyper/ hypoglycaemia and hospital admissions within 6 months of the 1st dose of chemotherapy were evaluated. Data analysed with SPSS.

Results: 336 patients received chemotherapy. Mean age $75.4+/-4.3$ years (range 70-89). 15.2\% (51/336) had pre-existing diabetes. 45/336 $(13.4 \%)$ of patients and 22/51 $(43.1 \%)$ of people with diabetes had a baseline HbA1C. Random capillary blood glucose (CBG) was measured in 103/336 (30.7\%). CBG $>11.1 \mathrm{mmol} / \mathrm{l}$ occurred in $21.4 \%$ of patients. Hyperglycaemia occurred in $100 \%$ of those already on insulin or with baseline $\mathrm{HbA} 1 \mathrm{C} \geq 60 \mathrm{mmol} / \mathrm{mol}$. Hospital admission $\geq 1$ occurred in $41.4 \%$ (139/336). This was associated with raised CBG ( $\mathrm{p}=0.025$ ) but not with a pre-existing diabetes diagnosis $(\mathrm{p}=0.370)$.

Conclusion: Most patients do not have measures of glycaemia during chemotherapy, even those with diabetes. Hyperglycaemia appears common and is associated with hospital admission. A standardised glycaemic control clinical care pathway has been developed and will be evaluated. 


\section{Abstract \# 342}

Most patients with cancer referred to comprehensive geriatric assessment are in need of geriatric intervention

Ann-Kristine Weber Giger ${ }^{1}$, Sambavy Nadaraja ${ }^{2}$, Stefan Starup Jeppesen $^{2}$, Katja Thomsen ${ }^{1}$, Lars Erik Matzen ${ }^{3}$

${ }^{1}$ Department of Geriatrics, Odense University Hospital, Denmark, ${ }^{2}$ Department of Oncology, Odense University Hospital, Denmark, ${ }^{3}$ Department of Geriatrics, Odense University Hospital, Denmark, and OPEN - Odense Patient Data Explorative Network, Odense

University Hospital and Department of Clinical Research, University of Southern Denmark

Introduction: The aim of this study was to describe patient characteristics and applied interventions in patients referred to the Geriatric Comorbidity Outpatient Clinic (GCOC).

Methods: Patients referred to GCOC 2014-2019, from cancer treating departments before start/change in cancer treatment, were included. Patients were offered a multi-domain assessment with interventions as needed. We registered data including cancer type, Charlson Comorbidity Score (CCI), Barthel-20, Orientation-Memory-Cognition test (OMC), Chair Stand Test (CST), Hand Grip Strength (HGST), Body Mass Index (BMI), medications within ATC-groups, interventions by geriatricians and nurses, referrals for physical/occupational therapists, and number of outpatients visits (OV).

Results: The study included 99 patients (males: $n=65$ ). The medians [Inter Quantile Range (IQR)] were: Age: 73.5 [52.5-87.5], Barthel20: 20 [18-20], CCI score: 2 [1-3], OMC: 24 [22-28], CST: 9 [7-11], right HGST: $25 \mathrm{~kg}$ [20-33]. 72.2\% (95\% CI 63.4-81.0\%) had BMI $>23,50 \%$ (95\% CI 39.9-60.1\%) had weight loss of 1-3 kg. Cancer site was most frequently lungs $(n=30)$, urological $(n=30)$ and gastrointestinal $(n=28)$ organs.93\% (95\% CI 88-98\%) received interventions from geriatricians, $33 \%$ (95\% CI 23.7-42.2\%) received interventions from geriatricians and at least one more health care professional, $7 \%$ (95\% CI 2-12\%) received no interventions. $72 \%$ (95\% CI $62-81 \%$ ) received at least one drug intervention. Most changes were within the ATC- groups diuretics, opioids, and laxatives. $33.3 \%$ had more than one visit to the GCOC.

Key conclusions: The functional level of patients referred for CGA by the cancer treating departments was high. However, most patients needed some kind of health intervention.

\section{Abstract \# 343 \\ Medical complications of hormone therapy in older patients with prostate cancer \\ Patrick Britto $^{1}$, Amelia Barber ${ }^{2}$, Louisa Fleure ${ }^{2}$, Muhammad Khan ${ }^{2}$, Cliona Flanagan $^{3}$, Deborah Enting ${ }^{2}$, Gordana Babic-Illman ${ }^{3}$, Danielle Harari $^{4}$, Tania Kalsi ${ }^{4}$ \\ ${ }^{1}$ Kings Collge London, ${ }^{2}$ Dept of Oncology, Guys \& St Thomas' NHS Foundation Trust, ${ }^{3}$ GOLD Team, Guys \& St Thomas' NHS Foundation Trust, ${ }^{4}$ GOLD Team, Guys \& St Thomas' NHS Foundation Trust \& Kings College London}

Introduction: Prostate cancer is common in older men. Androgen Deprivation Therapy (ADT) is an effective treatment but is associated with long-term medical complications including osteoporosis, hypertension, type 2 diabetes, hypercholesterolaemia, obesity and ischaemic heart disease. Despite this, there are no national/international guidelines on how to systematically monitor and manage these risks in patients receiving ADT. We evaluate current clinical practice in formally assessing relevant comorbidities at the start of ADT with a view to developing a clinical pathway to improve care.
Methods: Consecutive patients attending the oncology first hormone injection clinic between 07/08/2018 and 23/04/2019 are included. Data was retrospectively collected from patients notes accessed via electronic patient records.

Results: 190 patients were included. Median age was 70 (age range 53-94). Most common pre-existing comorbidities were hypertension $(60.5 \%)$, hypercholesterolaemia (38.3\%) and diabetes (19.2\%). Although parameters such as BP were often taken, explicit baseline assessment of comorbidity control was low. $22.1 \%$ had an explicit baseline assessment of blood pressure control, $20.6 \%$ diabetes control, cholesterol $4.2 \%$, DEXA $11.1 \%$ and vitamin D $20.0 \%$. This was not more likely even if the comorbidity was already present. The need for monitoring these comorbidities was not often explicitly communicated to the general practitioner (GP). As a result, a new protocolised baseline comorbidities assessment and communication plan was developed.

Key conclusion: Relevant comorbidities are not systematically assessed at the start of ADT suggesting the urgent need for national guidelines. A new protocolised assessment and communication aid will be evaluated.

\section{Abstract \# 344 \\ Preoperative risk factors for non-surgical postoperative complications in older colorectal cancer patients stratified by prehabilitation}

H.C. van der Hulst ${ }^{1}$, E. Bastiaannet ${ }^{2}$, J.E.A. Portielje ${ }^{3}$, J.M. van der $\mathrm{Bol}^{4}$, J.W.T. Dekker ${ }^{1}$

${ }^{1}$ Department of Surgery, Reinier de Graaf Hospital, Delft, the Netherlands, ${ }^{2}$ Department of Surgery, Leiden University Medical Center, Leiden, the Netherlands, ${ }^{3}$ Department of Medical Oncology, Leiden University Medical Center, Leiden, the Netherlands, ${ }^{4}$ Department of Geriatrics, Reinier de Graaf Hospital, Delft, the Netherlands

Introduction: Postoperative complications in older colorectal cancer (CRC) patients are common and associated with worse outcome. Prehabilitation might improve the outcome. The aim is to evaluate postoperative non-surgical complications and determine risk factors in older CRC patients, stratified by prehabilitation.

Methods: This observational cohort included $\geq 70$ years non-metastatic CRC patients who had elective surgery from 2014-2019 in Reinier de Graaf Hospital, Delft. Patients were divided into prehabilitation and non-prehabilitation group. The primary outcome was non-surgical complications and the secondary outcome surgical complications.

Results: In total, we included 357 patients. The 86 patients $(24.0 \%)$ in the prehabilitation group presented with higher comorbidity scores and walking-aid use than the non-prehabilitation group. In the prehabilitation group $26.7 \%$ experienced non-surgical and $23.3 \%$ surgical complications. In the non-prehabilitation group $21.8 \%$ had non-surgical and $13.7 \%$ surgical complications (p-value 0.339 and 0.034 , respectively). Multivariate analyses showed that $\geq 2$ comorbidities (OR 3.1 95\% CI 1.6-5.7), ASA score $\geq$ III (OR $2.795 \%$ CI 1.5-4.9), walking-aid use (OR $2.295 \%$ CI 1.1-4.4) and positive Geriatric-8 score (OR 2.7 95\% CI 1.1-6.9) were associated with nonsurgical complications in all patients. These risk factors remained significant in the non-prehabilitation group, but had no association in the prehabilitation group.

Conclusions: Despite differences in baseline characteristics, we observed similar non-surgical complication rates between prehabilitation and non-prehabilitation group. Furthermore, risk factors for non-surgical complications had no association in the prehabilitation group. This suggests that prehabilitation might compensate 
preoperative risk factors for non-surgical complications. Further research is needed to confirm this hypothesis and define tailored prehabilitation.

\section{Abstract \# 345 \\ Small bowel neuroendocrine tumors with acute abdomen presentation-report of two geriatric cases}

Konstantinos Paschos ${ }^{1}$, Michalis Kirmanidis ${ }^{1}$, Anestis Chatzigeorgiadis ${ }^{1}$

${ }^{1}$ General Surgery, Hospital of Drama, Greece

Introduction: Neuroendocrine tumors (NETs) arise from specialized cells dispersed throughout the body. Small bowel neuroendocrine tumors (SBNETs) have been increasing in frequency over the past decades and constitute now the most common type of intestinal tumors.

Methods: We present two geriatric male patients with acute abdomen due to SBNETs, who were successfully treated surgically.

Case presentation: The first was a 68-year-old man who was admitted at the Emergency Department (ED) due to abdominal pain, nausea and anorexia. He had a positive Mc Burney sign. The computerized tomography (CT) scan showed acute appendicitis and he underwent an appendectomy. The histology report (HR) described a NET at the periphery of the appendiceal mesentery. He underwent a new operation (right colectomy) and the new HR described an SBNET of low grade, located at the ileocecal region, infiltrating the intestinal wall, the surrounding fatty tissue and 4 out of 13 lymph nodes $(\ln )$ of the surgical specimen. The postoperative course was uneventful. The second was an 81-year-old man admitted at the ED due to abdominal pain and vomiting. His past medical history included arterial hypertension and atrial fibrillation. He underwent a CT scan which revealed a mesentery mass with intestinal ischemia and had an intestinal resection with ln removal around the mass. The HR described an SBNET with infiltration of $2 \mathrm{ln}$.

Conclusions: SBNETs usually have slow development which makes difficult early diagnosis. They may appear through a carcinoid syndrome, ileus or abdominal pain, frequently associated with distant metastases.

\section{Abstract \# 346 \\ Nonsteroidal anti-inflammatory drugs (NSAIDs) in the elderly: a forbidden Drug ?? \\ Ana Valadares ${ }^{1}$, Heidi Gruner ${ }^{2}$ \\ ${ }^{1}$ UCSP Sete Rios, ACES Lisboa Norte;Consulta Multidisciplinar de Geriatria Hospital Curry Cabral, Centro Hospitalar Universitário Lisboa Central, ${ }^{2}$ Consulta Multidisciplinar de Geriatria Hospital Curry Cabral, Centro Hospitalar Universitário Lisboa Central}

Introduction: In geriatric care, multimorbidity and the associated polypharmacy are a challenge and subsequent iatrogeny and the prescription cascade a reality. The risk increases when new medication is introduced, due to acute disease such as pain. Pain is a common complaint in the elderly (25-50\% of community-dwelling elderly) whose prevalence increases with frailty and chronic diseases, arthritis, bone and joint disorders and cancer and still difficult to treat. Options include opioid and non-opioid analgesics, NSAIDs should be avoided. Case report: A 70 years old, autonomous, married and retired engineer, presented with an overwhelming mechanical right knee pain, that made walking impossible. He had a history of hypertension, dyslipidemia, ischemic heart disease, benign prostate hypertrophy and previous herniorrhaphy, spine-cell carcinoma, actinic keratosis and surgery to eye cataract, medicated with alprazolam, simvastatin, dutasteride, tamsulosin, trazodone, acetylsalicylic acid, verapamil. Physiotherapy, glucosamine and piketoprofen cream were prescribed, and he started to autonomously take naproxen. The X-ray and magnetic resonance showed right internal meniscus fracture of the posterior horn, gonarthrosis and tendinitis. After 3 month he complained of heartburn and epigastralgia, iatrogeny was assumed and motivated the use of pantoprazole. Due to persistence of complaints, an upper digestive endoscopy was made, showing upper gastrointestinal pulsatile bleeding from a giant gastric ulcer with irregular borders in the transition body- antrum. The haemostatic therapy attempt was impossible due to hemodynamic instability and cardiac disease. The patient underwent urgent subtotal gastrectomy with Roux-en-Y gastrojejunostomy. Anatomopathological examination showed poorly differentiated gastric adenocarcinoma with mixed pattern and the thoraco-abdominal-pelvic CT scan had no signs of metastasis (pT3N0). The patient did adjuvant chemo and radiotherapy. At 1year follow up gastric cancer and the knee pain are controlled.

Conclusions: Nowadays there is no role for the use of NSAIDs in geriatric patients' treatment according to BEERs and START and STOP criteria, but over the counter use is still very frequent. In this lucky case report, the upper gastrointestinal bleeding due to silent gastric cancer (still the fifth most common cancer but declining) was enhanced by the side effects of the overlap of acetylsalicylic acid and other NSAIDs. Glucosamine was not an innocent bystander but might contribute to epigastralgia.

\section{Abstract \# 347}

Group-based reminiscence interventions in older adults' with dementia assisted by technological innovations: a scoping review

Isabel Gil $^{1}$, Paulo Santos-Costa ${ }^{1}$, Elzbieta Bobrowicz-Campos ${ }^{2}$, Eduardo Santos ${ }^{3}$, Rosa Carla Silva ${ }^{4}$, Maria de Lurdes Almeida ${ }^{1}$, João Apóstolo ${ }^{1}$

${ }^{1}$ Health Sciences Research Unit: Nursing (UICISA: E), Nursing School of Coimbra (ESEnfC), ${ }^{2}$ Faculty of Psychology and Education Sciences of the University of Coimbra, ${ }^{3} 3$ Rheumatology Department, Centro Hospitalar e Universitário de Coimbra (CHUC),

${ }^{4}$ Universidade Católica Portuguesa, School of Nursing

Introduction: Among the non-pharmacological interventions frequently implemented in older adults with dementia, group-based Reminiscence therapy (RT) emerges in the literature due to its efficacy in enhancing older adults' cognition and quality of life, while decreasing the incidence of depressive symptomatology. In parallel, there is a growing interest on the part of international researchers and healthcare professionals in the inclusion of technological and digital resources during the implementation of non-pharmacological interventions. Therefore, this study aimed to map the available evidence on group-based RT sessions/activities aided by technological innovations, and critically discussed the potentialities and weaknesses of its use.

Methods: Following the recommendations of the Joanna Briggs Institute for scoping reviews, the review question and inclusion/exclusion criteria were defined according to the Participants, Concept, and Context (PCC) mnemonic. A three-phased search strategy was conducted in order to develop a comprehensive list of keywords and indexation terms. Two independent reviewers analyzed the relevance of the studies, extracted and synthesized data.

Results: Of the 318 potentially relevant studies found, seven were included in this review. Overall, while the use of technological innovations seems to support the interaction and communication 
between the older adults and their relatives/healthcare personnel, recurrent issues related to ergonomics, connectivity, and intention of use were highlighted.

Conclusion: Although technological innovations seem to potentiate group-based RT, there is still a wide gap in the literature regarding the involvement of older adults' with dementia in the development of such resources.

\section{Abstract \# 348 \\ Tele-call service (TCS) and no shows: Falls Assessment Clinic (SOC)—an evaluation}

Surendra D Varman ${ }^{1}$, Kiat Sern Goh ${ }^{1}$, Agc Alexis ${ }^{1}$, Diraviyam Balasubramanian ${ }^{1}$

${ }^{1}$ Changi General Hospital

Background: Audits on our Falls SOC over the past few years have mandated us in implementing few interventions to improve attendance. TCS was implemented using available resources in Geriatric medicine (GRM) in 2016. From 2016 until 2018, calls were made at two weeks prior to appointment by two executives and empty slots identified for rapid access flow. However in 2019 due to attrition and stretched out resources, existing service was modified with TCS at week prior for 3 months (April-June), followed by no TCS, JulySeptember.

Aim: To evaluate TCS, to assess its overall effectiveness in improving attendance; impact of service modification and justification for continuity of service and need for additional funding, the data of 2019 was analyzed.

Methods: A retrospective descriptive analysis was performed on the data of first nine months in 2019, from January till September.

Results: Available slots in 2019: 32 per month. Attendance: TCS at two weeks prior, from Jan tO March $2019,45 \%$; at one week prior, April-June 2019, 45\%; no prior TCS, July-Sep 2019 , 30\%.

Conclusion: TCS seems to have made improvement in attendances, similar to previous trends; and clear impact compared with no TCS. The timing at one week or two weeks prior, however seemed to have made no impact in attendances. Hence, moving forward, we recommend continuation of TCS, at two weeks prior, giving ample time for the unconfirmed slots for Rapid access flow. However for future continuation of TCS, depends upon designated funding from GRM.

\section{Abstract \# 349 \\ Developing a bedside robot with monitoring and communicative functions for older people in residential care homes \\ Kazuko Obayashi ${ }^{1}$, Naonori Kodate ${ }^{2}$, Takahiro Nonoda ${ }^{3}$, Yoshimi Okamoto $^{4}$, Yoko Ishii ${ }^{5}$, Shigeru Masuyama ${ }^{6}$ \\ ${ }^{1}$ Faculty of Healthcare Management, Nihon Fukushi University, ${ }^{2}$ School of Social Policy, Social Work and Social Justice, University College Dublin, ${ }^{3}$ Universal Accessibility Ageing Research Centre, \\ ${ }^{4}$ Social Welfare Corporation Tokyo Seishin-kai, ${ }^{5}$ Universal Accessibility Evaluation Organization Japan, ${ }^{6}$ Tokyo Medical University}

Introduction: It has been reported that monitoring of older people's safety can improve the quality of their life in nursing homes and reduce the burden on care professionals at nighttime. The aim of this paper is to report the progress in our efforts to develop a bedside robot which functions as an Input/Output device between a monitoring system and older people.

Methods: Development of the robot was based on the PDCA cycle, which is a method of continuous improvement. The research team, composed of system developers, software engineers, care professionals and researchers, followed four processes in parallel: basic design (definition and design of functions including shape and system); user interface A (design) and B (system improvement); and detailed system design, including design of the communication function for the robot.

Results: Improvements were observed in each PDCA cycle, with a shape and sound quality that is easy for older people to use at the bedside (size: $33 \mathrm{~cm}$ x $27 \mathrm{~cm}$ x $26 \mathrm{~cm}, 285 \mathrm{~g}$ ). The communication robot can hold basic conversations, respond to signals from the infrared monitoring device and vital sensors, and send alerts to staff and older people. The quality of life also improved for both parties.

Key conclusions: The value and usefulness of a communication robot, combined with monitoring and alert functions, can be enhanced if the system is carefully designed and developed while reflecting the wishes of the users. This study was in part supported by the Japan Keirin Autorace Foundation.

\section{Abstract \# 350}

Quality of life at long-term of patients with remote monitoring of pacemakers

Antonio Lopez-Villegas ${ }^{1}$, Remedios Lopez-Liria ${ }^{2}$, Cesar Leal-Costa ${ }^{3}$, Salvador Peiro ${ }^{4}$, Emilio Robles-Musso ${ }^{5}$, Rafael Jesus Bautista-Mesa ${ }^{6}$, Knut Tore Lappegard ${ }^{7}$, Patricia Rocamora-Perez ${ }^{8}$, Daniel CatalanMatamoros 9

${ }^{1}$ Social Involvement of Critical and Emergency Medicine, CTS-609 Research Team, Hospital de Poniente, Almeria, Spain., ${ }^{2}$ Department of Nursing Science, Physiotherapy and Medicine, Hum-498 Research Team, Health Research Centre, University of Almería, Almería, Spain., ${ }^{3}$ Nursing Department, University of Murcia, Murcia, Spain., ${ }^{4}$ Health Services Research Unit, FISABIO-PUBLIC HEALTH, Valencia, Spain, ${ }^{5}$ Intensive Care Unit, Hospital de Poniente, El EjidoAlmería, Spain., ${ }^{6}$ Management Unit, Hospital de Poniente, Almeria, Spain., ${ }^{7}$ Division of Medicine, Nordland Hospital, Bod $\varnothing$, Norway., ${ }^{8}$ Department of Nursing Science, Physiotherapy and Medicine, Hum498 Research Team, Health Research Centre, University of Almería, Almería, Spain.., ${ }^{9}$ Department of Communication Studies, University Carlos III of Madrid, Madrid, Spain.

Introduction: health-related quality of life (HRQoL) and functional capacity values at short and medium term after pacemaker implantation have been analized previously; however, not much has been known about its long-term effects. The main objective of this study was to compare the health-related quality of life at long-term of users with remote monitoring of pacemakers (RM) versus conventional monitoring $(\mathrm{CM})$ at hospital.

Methods: this is a single-centre, controlled, non-randomised, nonblinded clinical trial. Data were collected pre-implantation and after 60 months. The patients in the PONIENTE study were assigned to two different groups: RM and CM. The EuroQol-5D (EQ-5D) questionnaire was used to assess HRQoL and Duke Activity Status Index was used to assess the functional capacity.

Results: After 5 years, 55 patients completed the study $(\mathrm{RM}=21$; $\mathrm{CM}=34$ ). EuroQol-5D and functional capacity values were improved; however, significant differences were observed only in the EQ5D visual analogue scale $(\mathrm{p}<0.001)$.

Conclusions: remote monitoring was equally feasible, reliable, safe, and clinically useful as CM. The frequencies of rehospitalisations and emergency visits did not differ between the groups. RM was found to be safe and effective in early detection and treatment of medical- and device-related events and in reducing hospital visits. Improved HRQoL was described not only immediately after PM implantation but also extended over a long time. 


\section{Abstract \# 351 \\ Cost-utility analysis at long-term of patients with remote monitoring of pacemakers}

Antonio Lopez-Villegas ${ }^{1}$, Rafael Jesus Bautista-Mesa ${ }^{2}$, Salvador Peiro $^{3}$, Daniel Catalan-Matamoros ${ }^{4}$, Emilio Robles-Musso ${ }^{5}$, Remedios Lopez-Liria ${ }^{6}$, Cesar Leal-Costa ${ }^{7}$

${ }^{1}$ Social Involvement of Critical and Emergency Medicine, CTS-609 Research Group, Hospital de Poniente, Almeria, Spain., ${ }^{2}$ Management Unit, Hospital de Poniente, Almeria, Spain., ${ }^{3}$ Health Services Research Unit, FISABIO-PUBLIC HEALTH, Valencia, Spain., ${ }^{4}$ Department of Communication Studies, University Carlos III of Madrid, Madrid, Spain., ${ }^{5}$ Pacemaker Unit, Intensive Care Unit, Hospital de Poniente, El Ejido-Almería, Spain., ${ }^{6}$ Department of Nursing Science, Physiotherapy and Medicine, Hum-498 Research Team, Health Research Centre, University of Almería, Almería, Spain., ${ }^{7}$ Nursing Department, University of Murcia, Spain.

Introduction: Cost-effectiveness studies on pacemakers have increased in the last years. However the number of long-term costutility studies is limited. The objective of this study was to perform a cost-utility analysis comparing remote monitoring (RM) versus conventional monitoring $(\mathrm{CM})$ in hospital of patients with pacemakers, 5 years after implant.

Methods: This is a controlled, not randomized nor masked clinical trial. Initially, 82 patients with pacemakers were selected $(\mathrm{CM}=52$; $\mathrm{RM}=30$ ). Patients were monitored during 5 years, after what a total 34 for CM in hospital and 21 for RM finalised the study. It was performed a cost-utility analysis to assess the RM of patients with pacemakers in terms of costs per gained quality-adjusted life years (QALYs). Costs from National Health Service (NHS) and patient perspectives were considered.

Results: After follow-up period, total costs per patient were $23.02 \%$ higher in the CM than in the RM group, corresponding to a $€ 82.10$ cost saving per patient ( $\mathrm{p}=0.033$ ). However, the reduction of in-hospital visits derived from RM does not impact significantly costs from the NHS perspective, although there was a cost saving of $15.04 \%$ per patient $(\mathrm{p}=0.144)$. Patients in the CM group showed a slightly better QALY at the end of the study (3.579) than RM group (3.306). Costs/ QALYs obtained by the CM group were lower than the RM group $(\mathrm{p}=0.773)$. The incremental cost-effectiveness ratio of $\mathrm{CM}$ in comparison to RM becomes positive (€301.16).

Conclusions: This study confirms that total costs per patient were $23.02 \%$ higher in the $\mathrm{CM}$ than in the $\mathrm{RM}$ group $(\mathrm{p}=0.033)$.

\section{Abstract \# 352 \\ Digital versus conventional rehabilitation after total knee arthroplasty: a long-term follow-up}

Ellen C. H. Pereira Nery ${ }^{1}$, Fernando Correia ${ }^{2}$, Maria Molinos ${ }^{3}$, José Tulha $^{4}$, Rosmaninho Seabra ${ }^{4}$, Virgilio Bento ${ }^{5}$, Rui Neves ${ }^{1}$

${ }^{1}$ Mestrado em Gerontologia Aplicada, Universidade de Aveiro, ${ }^{2}$ Serviço de Neurologia, Centro Hospitalar do Porto, ${ }^{3}$ SWORD Health, AS, ${ }^{4}$ Serviço de Ortopedia, Hospital da Prelada, ${ }^{5}$ SWORD Health, AS; Instituto Universitário da Maia

Older population has increased in the last decade linked with an increase of chronic diseases, as Osteoarthritis. Arthroplasty is a properly-established solution to this pathology witch Total Knee Arthroplasty (TKA) is one of the most common in the world. Concurrently, technological advancement improved in many areas, including digital rehabilitation. Most of the studies assess rehabilitation outcomes only in a short time period, and when compared in a long-time, it does not include a digital rehabilitation program. This study aimed to present follow-up results comparing two different home-based programs after TKA in older people: (1) conventional face-to-face sessions (CRT; $\mathrm{n}=15$ ) and (2) digital intervention (DRT; $\mathrm{n}=16$ ) performed through an artificial intelligence-powered biofeedback system under remote clinical monitoring (Sword Health); and verify if DRT it is a good possibility to this population after a longterm follow up. Patients were assessed in baseline and follow up (22.6 \pm 3.4 months after TKA) through Timed Up and Go test (TUG) and Knee Injury and Osteoarthritis Outcome Score (KOOS). The results showed that both groups attained clinically relevant improvements from baseline to long-term follow-up $(\mathrm{p}<0.05)$. KOOS subscale scores and TUG were similar in both groups $(\mathrm{p}>0.05)$. Therefore, this study demonstrated that digital intervention rehabilitation after TKA is feasible and engaging. Furthermore, it is a promising alternative by demanding fewer human resources in the current paradigm imposed by the pandemic situation through Covid-19.

\section{Abstract \# 353}

Older adult engagement in user-centered, innovative digital solutions for active and healthy ageing

Maria Metaxa ${ }^{1}$, Ioanna Dratsiou ${ }^{1}$, Evangelia Romanopoulou ${ }^{1}$, Panagiotis D. Bamidis ${ }^{1}$

${ }^{1}$ Lab of Medical Physics, School of Medicine, Faculty of Health Sciences, Aristotle University of Thessaloniki, Greece

Introduction: Health is considered to be a major determinant of quality of life, well-being, social participation and accessibility to healthcare [1]. Assistive technology (AT) is promoted as a means of retaining autonomy and quality of life for older adults [2]. Thus, the evolution of digitisation of services and eHealth technologies will be key to the restructuring of care delivery and help to personalise and customise individual care [3]. In this context, the SHAPES project [4] aims to create the first European open Ecosystem enabling the largescale deployment of a broad range of digital solutions for supporting and extending healthy and independent living for older adults who are facing permanently or temporarily reduced functionality and capabilities.

Methods: The SHAPES Pan-European Pilot Campaign aims at engaging +2000 older adults across 11 European countries and bringing forward technology to improve their health, well-being and autonomy. A user-centered interoperable SHAPES Technological Platform will be developed, including smart digital solutions tailored to health and care delivery. Among others, the Integrated Healthcare System Long Lasting Memories Care (LLM Care) [5], a web-based platform that combines state-of-the-art mental exercises against cognitive deterioration with physical activity [6-8], will be exploited by older adults with or without neurodegenerative diseases.

Key conclusions: This venture will lay the ground for the expansion of LLM Care's Ecosystem and its wider deployment across Europe aiming at improving health, well-being and autonomy of older adults. Acknowledgements: This project has received funding from the European Union's Horizon 2020 research and innovation programme under Grant Agreement No 857159.

References: [1] Sustainable development in the European Union: Monitoring Report On Progress Towards The SDGs In An EU Context, 2018 edition, Eurostat, European Union, September 2018, p. 63, https://ec.europa.eu/eurostat/documents/3217494/9237449/KS-01-18656-EN-N.pdf/2b2a096b-3bd6-4939-8ef3-11cfc14b9329. [2] Zwijsen, S. A., Niemeijer, A. R., \& Hertogh, C. M. (2011). Ethics of using assistive technology in the care for community-dwelling elderly people: An overview of the literature. Aging \& mental health, 15(4), 419-427. [3] European Commission. (2018). Communication on enabling the digital transformation of health and care in the Digital 
Single Market; empowering citizens and building a healthier society. [4] SHAPES H2020. (2020). Retrieved from https://shapes2020.eu. [5] Long Lasting Memories Care. (2020). Retrieved from www.llmcare.gr. [6] Bamidis, P. D., Fissler, P., Papageorgiou, S. G., Zilidou, V., Konstantinidis, E. I., Billis, A. S., ... \& Tsilikopoulou, G. (2015). Gains in cognition through combined cognitive and physical training: the role of training dosage and severity of neurocognitive disorder. Frontiers in Aging Neuroscience, 7, 152. [7] Romanopoulou, E. D., Zilidou, V. I., \& Bamidis, P. D. (2017). Creating and sustaining a social health care ecosystem: the case of LLM care services in Greece. HELLENIC JOURNAL OF NUCLEAR MEDICINE, 20(2), 40-48. [8] Romanopoulou, E. D., Zilidou, V. I., \& Antoniou, P. E. (2015). Spinning off gerontechnology business activities: the LLM care best practice paradigm. In Handbook of Research on Innovations in the Diagnosis and Treatment of Dementia (pp. 426-436). IGI Global.

\section{Abstract \# 354 \\ 'E-faturas' service's [E-invoice service] usability evaluation by senior citizens of the Viseu Municipality \\ Manuel Barbosa ${ }^{1}$, Ana Isabel Veloso ${ }^{2}$ \\ ${ }^{1}$ Universidade de Aveiro - Departamento de Educação e Psicologia ${ }^{2}$ Universidade de Aveiro -Departamento de Comunicação e Arte / DigiMedia}

Introduction: The use of Information and Communication Technologies (ICT) among the senior population despite rising, there is still some distance (Barbosa, M., \& Veloso, A. 2020). The current information society presents an increase of ICT services to replace traditional presential services. For this reason, this research aims to "understand the difficulties that senior citizens experience when using the "e-faturas'service [e-invoice service]".

Methods: A mixed methodology was used, with a sample for convenience, of 50 seniors 29 were men and 21 women, aged between 65 and 80 years old $(M=70.80 ; S D=4.70)$ residing in nine parishes in the municipality of Viseu, in Portugal, a pilot study was carried out with 12 seniors in order to validate the data collection instrument used in this study.

Results and key conclusions: Through the identification of obstacles that senior citizens encounter in using the service, and also in assessing the usability of the service, it is clear that the 'e- faturas' service of the Portuguese Finance Portal is not a senior friendly service reinforcing results of other investigations (Macedo, Veloso, A. \& Costa, 2019). Although three usage patterns have been identified according to the degree of independence with which each senior performs each task, the usability problems related to the 'e- faturas' service are highlighted, which are related to the lack of contrast between the background and the various graphic and textual elements. Keyword: Senior citizen; Digital inclusion; 'e-faturas' service [einvoice service];

References bibliographical: Barbosa, M., \& Veloso, A. (2020). AVALIAÇÃO DA USABILIDADE DO SERVIÇO 'E-FATURA' ENTRE CIDADÃOS SENIORES DO CONCELHO DE VISEU. Páginas A\&b, s.3(2020), 175-187 https://doi.org/https://doi.org/ 10.21747/21836671/pag2020ed. Macedo, T. G. F., Veloso, A. I., \& Costa, L. V. (2019). Estudo exploratório da usabilidade do serviço «efatura» com o cidadão sénior. Páginas A\&b Arquivos \& Bibliotecas, (Especial), 39-47. https://doi.org/10.21747/21836671/pagespa4

\section{Abstract \# 355}

Using immersive virtual reality as a cognitive assessment tool for the elderly: a systematic literature review

Laurent Zikos (1), Bruno Lenne (2), Florence Pasquier (3)

(1) Université de Lille, Inserm 1172, France, (2) Université Catholique de Lille, France, (3) Université de Lille, Inserm 1172, CH, Labex Distalz, LiCEND, Lille, France

Introduction: Virtual reality (VR) tasks for cognitive assessment are increasingly being developed. VR can provide different levels of immersion and fully immersive tasks are said to be the more promising as they allow more ecologically valid measures. The aim of this study was to conduct a systematic literature review of immersive VR tasks for cognitive evaluation in the elderly in order to assess the scope of existing research in this field.

Methods: Using Preferred Reporting Items for Systematic Reviews and Meta-Analyses guidelines, a systematic review of the published literature on immersive VR tasks used on the elderly for cognitive assessment was conducted.

Results: A total of 22 studies were included in this review out of 2838 screened articles. Immersive VR tasks provided measures for assessing memory $(n=4)$, navigation and neglect $(n=7)$, executive functions and daily activities $(n=6)$, and global cognitive efficiency $(n=5)$, which were mostly correlated with existing pencil and paper tasks. Furthermore, immersive VR tasks did not raise any major concern on feasibility aspects as subjects were able to go through them without any evidence of significant anxiety, cybersickness or usability difficulty.

Conclusions: Immersive VR tasks are promising cognitive assessment tools for the elderly as they can serve both a classical diagnostic purpose and provide more ecological data. Further studies are needed in order to confirm these results as current literature suffers from methodological limitations.

\section{Abstract \# 356}

Wearable-sensors based personalized assessment (WeSPA) of frailty: preliminary findings

Domenico Minici ${ }^{1}$, Antonella Giordano ${ }^{2}$, Guglielmo Cola $^{3}$, Silvana Antoci $^{4}$, Elena Girardi ${ }^{4}$, Mauro Di Bari ${ }^{2}$, Marco Avvenuti ${ }^{2}$

${ }^{1}$ Dept. of Information Engineering, University of Pisa, Pisa, Italy, ${ }^{2}$ Research Unit of Medicine of Aging, Department of Clinical and Experimental Medicine, University of Florence, Firenze, Italy; Unit of Geriatrics - Geriatrics Intensive Care Unit, Department of Medicine and Geriatrics, Careggi Hospital, Firenze, Italy, ${ }^{3}$ Institute of Informatics and Telematics, IIT-CNR, Pisa, Italy, ${ }^{4}$ Research Unit of Medicine of Aging, Department of Clinical and Experimental Medicine, University of Florence, Firenze, Italy

Introduction: Wearable sensors allow monitoring older subject's physical activity in daily life and, therefore, are particularly appealing in the evaluation of older persons in their environment, to capture early signs of frailty- and mobility-related problems. Aim of the WeSPA study is to explore the use of body-worn accelerometers for automated frailty assessment.

Methods: After screening by geriatricians for the presence of frailty (Fried's criteria), 34 persons aged $70+$ years were asked to walk $20 \mathrm{~m}$ at their preferred speed while wearing two accelerometers, one positioned on the lower back and the other on the wrist. Sensorderived signals were then analyzed independently to compare the ability of the two signals (wrist vs back) in the identification of frailty status. A gait-cycle detection technique was applied to divide each signal into segments made of four gait cycles, which were then used 
as input to a feature-extraction phase. Features in the time domain and the time-frequency domain (i.e., Wavelet Transform) were computed on these segments, and eventually a subset of 18 features was selected. Finally, different machine learning models were trained to classify subjects as robust or non-robust (prefrail/frail).

Results: The wrist sensor identified non-robust subjects with $100 \%$ sensitivity and $73 \%$ specificity, compared to only $87 \%$ sensitivity and $73 \%$ specificity of the back sensor.

Conclusions: A wrist-worn accelerometer signal provides valuable information for the recognition of frail and pre-frail older subjects and could be a valid tool for an automated assessment of frailty in the elderly.

\section{Abstract \# 357}

\section{Acute hospital in-reach service}

Dr Ashraf Nasim ${ }^{1}$, Dr Fady Zakharious ${ }^{2}$, Maria Clarke ${ }^{1}$, Sonia Durroni ${ }^{1}$, Sam Adby ${ }^{3}$

${ }^{1}$ Berkshire Healthcare NHS Foundation Trust, ${ }^{2}$ Berkshire Healthcare NHS Foundation trust, ${ }^{3}$ Berkshire Healthcare NHS foundation trust

Admissions to NHS hospitals are at all time high especially for elderly patients and is rising. So called 'baby boomers' account for largest age group admissions with age between 65 and 74 years. Avoidable hospital admissions for the elderly are at record levels, with people over 65 seeing over a hundred per cent rise, according to an interim report by Age UK. Acute Hospital in-reach service' is a unique service designed to support acute hospital with their bed flow, seamless transfer of care from an acute bed to a community ward, admission avoidance for stable patients/ social admissions, help reduce DTOC by proactively selecting $\&$ transferring pts from acute hospital to a community bedand develop local pathways for direct admissions from community. Data Collected for past 11 months: January 2019-November 2019. Referrals received from 3 surrounding acute hospitals including local community. Referral criteria were based on locally developed acute admissions avoidance pathways: 1 . Sub-Acute Community Bed Admission Pathway2. Discharge to Access Pathway3. In Patient Rehabilitation admission Pathway4. Admission Avoidance PathwayBHFT Community Team Acute Hospital In-reach service was able to pro-actively identify, prevent avoidable acute hospital admission \& support same day discharges from emergency department consistently over past 11 months. Of a total of $998(83.3 \%)$ out of 1198 referrals from acute hospital over a period from Jan to Nov 2019 were discharged or transferred to a suitable community bed. Total Bed Days Saved: 6790/ 11 months. Same day 'admission avoidance pathway' have saved local commissioning group around $£ 64,860 /$ - over past 11 months by discharging around $19.3 \%$ of all referrals on the same day. Cost saving for local commissioning group by preventing 970 acute hospital admission episodes: $£ 269,790 /-$ over 11 months. With National Avg. LOS of 7 days: total cost saving for NHS England: $£ 2.342$ Millions/ 11 months period. BHFT Community acute hospital inreach service' have so far delivered an outstanding result by preventing acute hospital admissions consistently over the past 16 months. Service is one of its kind in the UK that is pro-active, consistent and cost effective for both the local CCG and Acute Hospitals.

\section{Abstract \# 358}

Creation of an orthogeriatria consultation: a new project, a reality

Marta Mercedes Gonzalez Eizaguirre ${ }^{1}$, Maria Jesús Vicente Romero ${ }^{1}$, Miguel Sanchez Ortiz ${ }^{1}$, Carmen Oquendo Marmaneu ${ }^{1}$, Elisa García Simón ${ }^{1}$, Ana Mateo Abad ${ }^{1}$, Magdalena Linge Martin ${ }^{1}$

${ }^{1}$ Hospital San José de Teruel

Objective: The objective of the project is to improve the attention of the hip process by following up Traumatologists and geriatricians in external orthopedics consultations one month after the hip fracture intervention, at 3 months, at 6 and 1 year.

Methods: A review was performed during the first month after hip fracture, at 6 months and one year with a radiography of the hip and subsequent observation by both specialists, review of post-surgical complications in both wound and in limb, review of medical complications after surgery,reinforcement in the explanation of exercises for the rehabilitation of these patients with family involvement. Patient data was collected and segmented.

Results: From January 1, 2019 to November 30, 2019, a total of 76 patients were admitted to the Traumatology plant of the Hospital with a hip fracture process. $93.9 \%$ of patients leave with their appointment for first month orthogeriatrics consultation. At discharge, 62 patients $(87.32 \%)$ took some treatment to avoid osteoporosis (calcium, vitamin D, antiresorptive or osteoforming). $100 \%$ of patients who go to orthogeriatrics consultation a month, leave with a review appointment at 6 months. Of the total of patients who should have attended a review visit of 6 , that is 23,20 of them come to the clinic, that is, $86.95 \%$.

Conclusions: It has been possible to implement a joint consultation of trauamtology and geriatrics, obtaining that a minimum of $80 \%$ of the patients go to revision consultations and be assessed in a multidisciplinary way. The user satisfaction survey gives a score higher than 9 in this query.

\section{Abstract \# 359 \\ IoT-based home monitoring: supporting practitioners' assessment by behavioral analysis \\ Enrico Montanari ${ }^{1}$, Stefano Nunziata ${ }^{2}$, Francesca Cocchi ${ }^{2}$, Enrico Montanari $^{3}$ \\ ${ }^{1}$ Neurologist LHA Parma, ${ }^{2}$ Informatic Engineer, ${ }^{3}$ Neurologist LHA Parma}

This abstract presents methodologies and results for the ACTIVAGE project [14], funded by the European Union Horizon 2020 programme; more specifically, the Italian Deployment Site (DS-RER) [15] is presented, based in the Emilia-Romagna Region (RER), aiming at introducing ICT-enabled services into the current practice of regional Health Service (AUSL, Local Health Authority). General practitioners, formal caregivers, care and case-managers are involved in the experimentation, creating an IoT-augmented care continuum network. The DS-RER approach is actually suitable for a wide range of use cases; however, in the pilot at hand, the target population has been narrowed to older adults $(65+)$ recovering from a stroke event, and still suffering from its after-effects. Such a use-case is, indeed, numerically relevant: in Italy, stroke is the third cause of death (approximately, 10-12\% of all deaths per year [16], after cardiovascular diseases and neoplasms); furthermore, about 7000 stroke adverse events are recorded each year in the Emilia-Romagna region [17]. The DS-RER approach aims at empowering care professional with additional information that is not commonly provided in current care practice, namely their behavioral routines. Such information can not 
be directly mapped to conventional clinical markers, for which telemedicine solutions already exist and are commonly used (e.g., blood pressure monitors, pulse oximeters and weight scales, among others); nonetheless, changes in behavioral habits may reflect changes in overall wellbeing of a person (e.g., sleep routines, toilet usage changes).

\section{Abstract \# 360 \\ Mood state of vigor-activity of the older people in wheelchairs living in long term care}

Chen-Yuan $\mathrm{Hsu}^{1}$

${ }^{1}$ Department of Nursing, Dayeh University

Introduction: The proportion of the Taiwanese older people who living in long- term care (LTC) facilities has increased as the population rapidly, however, it is also resulted to this population using wheelchair increasingly [1]. The purpose of this study is to understand the mood states of the older people in wheelchairs living in LTC.

Methods: This study uses a cross-section survey for research, based on the population of the LTC facilities in Changhua and using a convenient access to the study sample, expected to have the number of samples 150. This study uses a structured questionnaire; data collection includes demographic information and Profile of Mood States Short Form (POMS) [2]. Data analysis will use SPSS 12.0 for Window 2000, frequency distribution table, percentage, chi-square test, $\mathrm{t}$ test, and ANOVA analysis to answer questions.

Results: The study found that the basic information of older people in wheelchairs who was different between male and female, such as: "age", "education", "marriage" and "Mini-Mental State Examination; MMSE" were all significantly different $(\mathrm{P}<0.05)$. Those people were no significant difference in the overall mood states (Total Mood Disturbances, TMD), which showed that the male and female were in the same mood states (POMS). Those people have significant differences in their positive mood state with "vigor-activity" and "time period of living in long-term care institutions" $(\mathrm{P}<0.05)$.

Key conclusion: The results will enable to the nursing staff to understand the mood states of the older people in wheelchairs living in LTC, and to expecting by this study provides the research outcomes for nursing staff to have a real knowledge, to pay attention for those people on the mental health promotion program in the future, and resulted to improving the mental health and well-being for older people in wheelchairs.

References: 1 . Hsu CY, Moyle W, Cooke M, Jones C. Seated T'ai Chi in Older Taiwanese People Using Wheelchairs: A Randomized Controlled Trial Investigating Mood States and Self-Efficacy. Journal of alternative and complementary medicine (New York, NY). 2016;22(12):990-6. 2. McNair, D. M.; Lorr, M.; Droppleman, L. F. Profile of Mood States (POMS) Manual; Educational and Industrial Testing Service: San Diego, CA, 1992.

\section{Abstract \# 361}

Self-reliance care mode to support elderly in the physical health Chen-Yuan $\mathrm{Hsu}^{1}$

${ }^{1}$ Department of Nursing, Dayeh University

Introduction: The percentage of the Taiwanese elderly is increasing and related to living in the long-term care, and also losing the physical activities of self-reliance due to the care mode of the facility, therefore, a health preservation for those people is an important.

Methods: This study was based on the population of a long-term care in the middle of Taiwan. There was a following research on the self- reliance care mode of daily life self-ability with the number of samples 40 collected by this study, comparing the self-reliance care mode group $(n=20)$ and general care mode group $(n=20)$ and used a structured questionnaire as the Physical Health Dimension of Quality of Life [1], as data collection included demographic information and the dimensions of physical health. Data analysis used SPSS 22.0 for Window 2000, frequency distribution table, percentage and t-test to answer questions.

Results: The results of this study, which examined the Physical Health Dimension of Quality of Life for Taiwanese elderly. A Chinese version of the Physical Health Dimension of Quality of Life including 7 items, has been used for elderly from the long term-care in Taiwan with good reliability, Cronbach's alpha 0.77 [2-3]. For the sex distribution, there were 22 males $(55 \%)$ and 18 female subjects (45\%); there were 35 subjects aged 65-85 years and five subjects aged above 85 years; there were 25 subjects speaking both Mandarin and Taiwanese languages, 12 subjects speaking Taiwanese language, and 3 subjects speaking Mandarin. Finding shows the result of t-test analysis. Increasing physical health mostly showed a positive correlation with quality of life, the result suggested that there was a higher level of physical health dimension of quality of life on the self-reliance care mode group (25.5 \pm 3.62$)$ comparing with the general care mode group (18.45 \pm 4.16$)$. Finding shows the comparison of physical health dimension of quality of life significant differences with two groups $\mathrm{p}=.000(\mathrm{p}<0.05)$.

Key conclusion: This study indicates that Taiwanese elderly living in the long-term care, suggesting a trend, that choosing the self-reliance care mode group was higher than that general care mode group on the physical health dimension of quality of life. The results can also provide the reference for community, to encourage Taiwanese elderly living in the long-term care to participate in self-reliance care mode for daily life self-ability in the future, and therefore improving their physical health dimension of quality of life.

References: 1. WHOQOL group (1998). Development of the World Health Organization WHOQOL-BREF quality of life assessment. Psychol Med, 28, 551-558. 2. Hsu CY. The effects of Tai Chi on Quality of Life of Older People Living in the Long-Term Care and Using Wheelchairs for Mobility: A Randomized Controlled Trial. Doctoral dissertation, Griffith University, Dissertation Abstracts International, 2012. 3. Hsu CY, Moyle W, Cooke M, Jones C. Seated Tai Chi versus usual activities in older people using wheelchairs: A randomized controlled trial. Complementary Therapies in Medicine. 2016; 24:1-6.

\section{Abstract \# 362 \\ Risk factors associated with dialysis catheter-related bloodstream infections (DCRBI): a retrospective analysis in a regional dialysis unit}

Emmanouil Menegakis ${ }^{1}$, Dimitrios Xydakis ${ }^{2}$, Stefania Schetaki ${ }^{3}$, Evridiki Patelarou $^{4}$, Michail Zografakis-Sfakianakis ${ }^{4}$, Athina Patelarou $^{4}$

${ }^{1} 1$. Department of Nursing, Faculty of Health Sciences, Hellenic Mediterranean University, Heraklion, Crete, Greece 2. Nephrology Department of Venizeleio Hospital of Heraklion, Heraklion, Crete, Greece, ${ }^{2}$ 2. Nephrology Department of Venizeleio Hospital of Heraklion, Heraklion, Crete, Greece, ${ }^{3} 1$. Department of Nursing, Faculty of Health Sciences, Hellenic Mediterranean University, Heraklion, Crete, Greece 3. Annousakeio Therapeutic Center Inpatient Recovery and Rehabilitation Center "Agios Spyridon", Kissamos, Crete, Greece, ${ }^{4} 1$. Department of Nursing, Faculty of Health Sciences, Hellenic Mediterranean University, Heraklion, Crete, Greece 
Abstract introduction: Although the use of central venous catheters (CVC) is widespread and contributes to patient survival, hematogenous infections associated with their application are a major cause of morbidity and mortality. The treatment of these infections varies depending on the type of catheter and the micro-organism [1-3]. The contribution of nursing care is catalytic at all stages of the implementation of the CVC. Risk minimization is linked with the prevention of hematogenous infections through the implementation of evidence-based best practices promoting in this way the quality of care and patients' safety [3-5]. The aim of the study was the investigation of the risk factors for hemodialysis catheter-related bloodstream infections.

Methods: A retrospective study was performed on 208 patients with acute or chronic renal failure who were admitted to the Nephrology Department of Venizeleio Hospital of Heraklion from February 2013 to December 2018. The data were collected from the database of the Nephrology Department's electronic file and the file of the Microbiology Laboratory of Venizeleio Hospital of Heraklion. We assessed dialysis DCRBI risk factors including demographics, comorbidities, and duration and frequency of hemodialysis and we investigated the microbiologic etiology of these infections. For the analysis, the statistical pack IBM SPSS STATISTICS 24.0 was used, while $\mathrm{p}<0.05$ was considered a level of statistical significance.

Results: Age groups 70-79 and 80+ had the highest frequency in our sample with $38,0 \%(n=79)$ and $31,7 \%(n=66)$, respectively. Women had a higher incidence of microbiaemia $(62,3 \%)$, whereas S. epidermidis was the most common pathogen in all cases, with $37.9 \%$. It is worth noting that the results revealed a high incidence of co-morbidity with $92.8 \%$ hypertension, $53.8 \%$ diabetes mellitus, and $53.8 \%$ coronary artery disease. Duration of catheterization of over 10 days was OR $3.16(2.02-4.94)$. The OR was higher in the case of catheter placement [OR: $2.92(2.12-4.03)]$ compared to fistula and graft [OR: $0.14(0.09-0.22)]$. The most common pathogens were Gram + germs ,associated with almost $75 \%$ of the cases.

Key conclusions: Vascular access to hemodialysis patients is a primary parameter in the treatment of the disease. Nurses' continuing education and further training in combination with patients' involvement through holistic care and information can lead to the prevention of vascular access complications by aiming in reducing duration of CVC and compliance to hygiene measures.

References: 1. Falagas, M.E., \& Kompoti, M.K. (2006). Obesity and infection. The Lancet. Infectious diseases, 6 7, 438-46. 2. Farid, M. Chaudhary, H. Nazir, I. Sirwal, I. (2018). Haemodialysis; Haemodialysis Catheter Related Infections. https://doi.org/ 10.29309/TPMJ/18.4878. 3. Soi, V., Moore, C. L., Kumbar, L., \& Yee, J. (2016). Prevention of catheter-related bloodstream infections in patients on hemodialysis: challenges and management strategies. International journal of nephrology and renovascular disease, 9, 95-103. https://doi.org/10.2147/IJNRD.S76826. 4. Fysaraki, M., Samonis, G., Valachis, A., Daphnis, E., Karageorgopoulos, D. E., Falagas, M. E., Kofteridis, D. P. (2013). Incidence, clinical, microbiological features and outcome of bloodstream infections in patients undergoing hemodialysis. International journal of medical sciences, 10(12), 1632-1638. https://doi.org/10.7150/ijms.6710. 5. Sahli, F. Feidjel, R. Laalaoui, R. (2017). Hemodialysis catheter-related infection: rates, risk factors and pathogens. Journal of Infection and Public Health, Volume 10, Issue 4, 2017, Pages 403-408.

\section{Abstract \# 363}

Facilitators and barriers of the implementation of a personalised integrated stepped care approach to BPSD (STIP method)

Verstraeten Helma W.M.F. ${ }^{1}$, van Maar Corine ${ }^{2}$, Ziylan Canan ${ }^{2}$ Achterberg Wilco P. ${ }^{3}$, Gerritsen Debby L. ${ }^{4}$, Huijsman Robbert ${ }^{5}$, de Lange Jacomine ${ }^{1}$, Smalbrugge Martin ${ }^{6}$, van der Steen Jenny T. ${ }^{3}$, Zuidema Sytse U.7 , Bakker Ton J.E.M. ${ }^{1}$

${ }^{1}$ Rotterdam University of Applied Sciences, Rotterdam, Netherlands, ${ }^{2}$ Van Maar Advies, Vleuten, Netherlands, ${ }^{2}$ Leiden University Medical Center, Leiden, Netherlands, ${ }^{4}$ Radboud University Medical Centre, Nijmegen, Netherlands, ${ }^{5}$ Erasmus University Rotterdam, Rotterdam, Netherlands, ${ }^{6}$ Amsterdam University Medical Center, Amsterdam, Netherlands, ${ }^{7}$ University Medical Center Groningen, Groningen, Netherlands

Introduction: Most of people with dementia in nursing homes show one or more symptoms of behavioural and psychological symptoms of dementia (BPSD). Two consecutive Dutch Health inspectorates in nursing homes showed a late, inadequate or incorrect response to BPSD. Three proven effective approaches have joined forces to develop one combined intervention, the STIP method: the personalised integrated stepped care approach to BPSD (Dutch guideline 2018). We are implementing STIP, supported with the BPSD Care web application in two nursing homes $(\mathrm{N}=200)$ with the aim to investigate facilitators and barriers to perform STIP.

Methods: The implementation of STIP is executed by means of sounding board groups including informal carers, care professionals and managers. Qualitative data on facilitators and barriers were extracted from 24 transcripts. Quantitative data about the extent of implementation were extracted from patient records $(n=40)$.

Results (preliminary): Qualitative analysis points towards the following facilitators: acknowledgement of difficulties in dealing with and treating of BPSD; acknowledgement for the need of a more integrated approach; leadership; ownership and collaboration. Barriers include a lack of notion about the costs and benefits of STIP; insufficient support from managers and a less-methodological workflow. Quantitative analysis shows multiple deficits in clinical reasoning e.g. the absence of an integral interdisciplinary goal attainment plan.

Key conclusions: Both qualitative and quantitative data underline specific difficulties in the implementation of STIP in nursing homes. Insight in barriers and facilitators is a prerequisite for nursing homes to pinpoint the bottlenecks and subsequently to effectively counteract them.

\section{Abstract \# 364}

\section{Demand and development of multifunctional intelligent bed} for the elderly patients

Chen-Yuan $\mathrm{Hsu}^{1}$, Feng-Min Lai ${ }^{2}$

${ }^{1}$ Department of Nursing, Dayeh University, ${ }^{2}$ Design and Materials for Medical Equipment and Devices, Dayeh University

Introduction: Traditionally, elderly patients with long-term care must stay in bed for a long time, in order to avoid long-term lying and pressure sores, nursing staff need to turn over for those patients for two to four hours, which may cause occupational injuries to nursing staff and affect medical quality. Furthermore, in clinical practice, the choice of the bed frame of the hospital bed has a structure that can raise the head of the bed and the end of the bed, but in order to clean and avoid those patients from developing bed sores due to prolonged lying, those patients still needs to turn over. The bed frame is still unable to meet the needs of making those patients easy to turn over. 
Therefore, Dayeh University established the Long-Term Care and Intelligent Assistive Device Laboratory in 2017 to design and develop multifunctional intelligent bed, and expected it can meet with the demands for elderly patients.

Objectives: The demands of the multifunctional intelligent bed will be established and analyzed by the reference for the future revisions.MethodsThis creation was jointly developed by Taichung Veterans General Hospital and Daye University to carry out creative research and development, adding the function of bed end assisted getting out of bed, left and right turning functions, the bed posture control adds eye tracker and mobile phone APP control methods, and the patient can look at the eye tracker screen or mobile phone operation to adjust the posture through the eyeball, to avoid the problem of bedsores, so this smart bed expects to be able to reduce the stress and physical load of care providers.

Results: The multifunctional intelligent bed was designed and developed with the Taiwan patent certificate (No 1652055) at the current stage. This study assumes that the demand of multifunctional intelligent bed for the elderly is to help those patients turn over, thereby reducing the burden of the patients' families and reducing the chance of nursing staff working hours and occupational injuries, and the research is still processing as well.

Conclusions: The research results will provide medical supplies development of the Long-Term Care and Intelligent Assistive Device Laboratory in the future and to understand the demands of the elderly for multifunctional intelligent bed, and to avoid the problem of bedsores, then therefore to reduce the stress and physical load of care providers.

\section{Abstract \# 365 \\ Feeding practices among people with dementia in residential care facilities: an exploratory study \\ Lígia Passos $^{1}$, João Tavares ${ }^{2}$, Daniela Figueiredo ${ }^{2}$ \\ ${ }^{1}$ Department of Education and Psychology, University of Aveiro, 3810-193 Aveiro, Portugal, ${ }^{2}$ Center for Health Technology and Services Research (CINTESIS.UA), School of Health Sciences, University of Aveiro, 3010-193 Aveiro, Portugal}

Introduction: In advanced stages of dementia, people with this condition may have feeding difficulties, usually manifested by oropharyngeal dysphagia and/or refusal to eat. More than a nutritional act, mealtimes play a social, religious, biological, and symbolic role in most cultures. It has been increasingly recommended that feeding difficulties can be reduced by applying a social-ecological approach to care, such as the C3P Model - Change the Person, Change the People, Change the Place[1]. This study aimed to explore feeding practices in institutionalized people with dementia (PwD) according to the dimensions of the C3P Model.

Methods: Twenty-three nursing assistants (NA) were included in this exploratory-descriptive study. Their skills while offering lunch to institutionalized PwD were assessed through the Feeding Skills Checklist [2].

Results: All participants were female, with an average age of 44.73 years $(\mathrm{SD}=10.42)$ and with 10.28 years of professional practice. Of the 41 meals observed, only $37.7 \%$ of good practices were observed. The worst performance was in the PwD dimension, which had only $21.2 \%$ of good practices performed. There is not a meal routine and no oral hygiene after the meal was performed. A significant positive correlation was found for time of profession practice and the FSC environment dimension ( $\mathrm{rs}=0.435, \mathrm{p}=0.038$ ).

Key conclusions: There is a weakness in the interpersonal relationship between NA and PwD, and better performance in the environmental (and institutional) dimensions. It is necessary to train
NA on the social-ecological factors that influence an adequate personcentred mealtime.

References: 1. Amella EJ, Batchelor-Aselage MB. Facilitating ADLs by caregivers of persons with dementia: The C3P model. Occup Ther Heal Care. 2014;28(1):51-61. 2. Batchelor-Murphy M, Amella EJ, Zapka J, Mueller M, Beck C. Feasibility of a web-based dementia feeding skills training program for nursing home staff. Geriatr Nurs (Minneap). 2015;36(3):212-8. Available from: http://dx.doi.org/10.1016/j.gerinurse.2015.02.003

\section{Abstract \# 366}

Cost-related medication non-adherence of older people

Ilia Kazantzaki ${ }^{1}$, Theodoula Adamakidou ${ }^{2}$

${ }^{1}$ Health Center of Thivas, Greece, ${ }^{2}$ Nursing Department, University of West Attica, Greece

Introduction: Medication non-adherence is a complex behavior with multidimensional causes. WHO refers to financial strain as one of the most important factors for it. This literature review describes the factors associated with cost-related medication non-adherence (CRMN).

Methodology: A literature review was conducted at PubMed database using the keywords "adherence", "compliance", "financial strain", "older adults", "cost-related medication nonadherence" and "community nurse".

Results: In developed countries, CRMN rated $60 \%$ for people over 60 years old, which was commonly expressed as unfilled or delayed prescriptions and decreased frequency and doses. Elderlies have multiple chronic conditions and multiple medication use is needed. Frequently, they cannot afford them due to out-of-pocket payments and inadequate coverage by insurance. Evidence has shown that socioeconomic characteristics such as female sex, being uninsured, and living in poverty are associated with a higher likelihood of CRMN. There are also health-related factors (multimorbidity, mental disease, functional limitations and poor health) and other factors such as older people's relationship with healthcare providers and healthcare system which are associated with a higher likelihood of CRMN. Heterogeneity exists between disease groups and cultural backgrounds. The consequences of that behavior are complications in patient's condition and consequently higher costs for healthcare systems.

Key conclusions: Community nurses encounter and manage first these types of patients' behaviors and should be appropriately prepared to manage them. On the other hand, reliable healthcare systems and political decisions must guarantee equity and equality of healthcare provisions for all.

\section{Abstract \# 367 \\ General practitioners' appreciation of the return home program of elderly patients hospitalized for heart failure PRADO}

Lorenza De Rosario ${ }^{1}$, Giorgia Querin ${ }^{2}$, Maurizio Magri ${ }^{3}$, Caryn Recto $^{3}$, Antonio Rainone ${ }^{3}$, Cristiano Donadio ${ }^{3}$

${ }^{1}$ CHIC Hospital, Paris 6 University, ${ }^{2}$ Pitié-Salpêtrière Hospital, Paris, ${ }^{3}$ Charles-Foix Hospital, Paris

Background: The ageing of the population and medical progress are leading to an increase in chronic pathologies such as chronic heart failure to which the problems of follow-up, therapeutic and social education are added. The PRADO-IC programme (Return Home Companionship Program - Heart Failure) was deployed by the French 
health system in 2013 to reduce the risk of re- hospitalization after a decompensating heart failure in older people.

Methods: Our aim was to understand the degree of satisfaction, the difficulties and expectations of general practitioners concerning the PRADO-IC programme through self-administered questionnaires, carried out from March 2019 to October 2019 to 60 GPs following patients hospitalised between 01/01/17 and 03/03/19 aged 70 or over and included at the hospital discharge in this program. A descriptive analysis of the sample and a statistical analysis according to age and department of work of the practitioners have been performed.

Results: The response rate was $41.1 \%$. This survey showed us that the program is well appreciated overall, especially by older doctors. General practitioners believe in the use of the program and think it can improve the treatment of heart failure in elderly patients. However, some complain of poor involvement in scheduling appointments.

Conclusion: The PRADO-IC allows an efficient organization of the return home with a link between several care actors. Our study shows fairly positive feedback from general practitioners, but highlights some problems, such as the need for greater participation by the general practitioner in organizing patient follow-up.

\section{Abstract \# 368 \\ Correlation between number of visits and Lubben Scale to evaluate the social network in Nursing Home Residents}

Miriam Molas-Tuneu ${ }^{1}$, Pau Farrés-Godayol ${ }^{1}$, Anna Escribà-Salvans ${ }^{1}$, Sandra Rierola-Fochs ${ }^{1}$, Pau Moreno-Martin ${ }^{1}$, Meltem Yldirim ${ }^{1}$, Jordi Naudó-Molist ${ }^{2}$, Javier Jerez-Roig ${ }^{1}$

${ }^{1}$ Research group on Methodology, Methods, Models and Outcomes of Health and Social Sciences (M3O). Faculty of Health Sciences and Welfare. Centre for Health and Social Care Research (CESS). University of Vic-Central University of Catalonia (UVic-UCC)., ${ }^{2}$ Research group Mental Health and Social Innovation (SAMIS). Faculty of Health Sciences and Welfare. Centre for Health and Social Care Research (CESS). University of Vic-Central University of Catalonia (UVic-UCC)

Introduction: Most Nursing Home (NH) residents have difficulties in responding to questionnaires due to cognitive impairment. Assessing their social network through a health professional (proxy) may be useful. We aimed to analyze the correlation between the number of visits (by proxy respondents) and the Lubben Social Network Scale of 6 items (LSNS-6, by older people) to evaluate the social network of $\mathrm{NH}$ residents.

Methods: Cross-sectional study in $4 \mathrm{NH}$ from Osona (Barcelona), between January and March 2020. The Spearman test was used for bivariate analysis between LSNS-6 and number of visits ("how many visits per month does the resident receive?", with a confidence level of $95 \%$.

Results: Among the recruited 145 subjects, 33 (22.7\%) were excluded due to individuals or their legal guardians refused to participate, 5 $(3.4 \%)$ were less than 65 years old, $4(2.7 \%)$ were hospitalized or other reason, $64(62.1 \%)$ for cognitive impairment and $6(5.8 \%)$ presented incomplete data on the two main variables. Final sample was $33(22.7 \%)$, mean age of 82 years $( \pm 8.05)$, being $23(69.7 \%)$ women. Mean number of monthly visits was $12.5 \pm 10.99 ; 13(39.4 \%)$ had low risk of social isolation and $20(60.6 \%)$ high risk. A significative negative correlation $(-0.50, p=0.003)$ between LSNS- 6 and number of visits was found.

Conclusions: Number of monthly visits are significatively and negatively correlated with high risk of social isolation. It is necessary to achieve a larger sample to elucidate if the number of visits is a reliable measure to assess social network in $\mathrm{NH}$ residents.

\section{Abstract \# 369}

Review of community Intermediate care team providing hospital at home service in Liverpool

Aaseem Farid $^{1}$, Manoj Selvaraj ${ }^{1}$

${ }^{1} \mathrm{NHS}$

Introduction: Intensive community care team is an intermediate care service where patients are managed in their own homes. It is a multidisciplinary team consisting of GPs,Nurses and other professionals. It relies on hospital based approach to deal with community problems. It involves visits by different professionals recorded on computer system and use of different scores like pain scores, NEWS scores (RCP London, 2017), MUST scores (Bapen.org.uk, 2011) to monitor patients. Also using regular Multi-disciplinary meetings to stimulate a virtual ward round.

Methodology: This review looked at all referrals into the team during the month of October 2019.Data was collected on 15/11/19 and 15/1/ 20. It involved collecting data which are recorded routinely and are available on the computer system. The parameters studied include the NEWS score, Pain score, duration of admission into the service, source of admission and place of discharge.

Results: The total number of patients admitted into the service was sixty. Out of this, there were 36.67 percent of males and 66.33 percent females.Patients were referred into it from different sources. Half of them were referred by the GPS.Eight patients were referred from the Hospitals.One fifth of the referrals came from the social services and another one fifth came from district nurses.Ambulance service referrals were 5 in number which accounted for a sixth of the referrals.6.67\% of referrals came from the IV team.In the review, 46 out of 55 patients who had NEWS done had either had better NEWS scores or remained the same. Seventeen patients had better final NEWS scores in comparison with initial NEWS scores. Also nine patients had worse NEWS scores on the final score in comparison to the first NEWS score.Pain score is measured with numerical rating scale. This is based on patient's self assessment (Childs et al, 2005). Twelve patients were better with the pain scores on the final discharge when compared with eight who had worse scores. Among the places of discharge, forty four patients were in their normal places of residence. One patient who was palliative ,died at home. Fifteen patients were admitted in hospital. The total number of days the patients were under the service was 716 days. This divided by the total number of patients which is 60 gives a mean stay of 11.93 days.

Conclusions: The results of the review were positive overall with good outcomes reached for patients. This is reflected in the NEWS scores and the pain scales. Also the number of days the patient is within the service is within the parameters of the National Institute of Clinical Excellence.

References: Bapen.org.uk. (2011). [online] Available at: https://www.bapen.org.uk/pdfs/must/must_full.pdf [Accessed 12 Jan. 2020]. Childs JD, Piva SR, Fritz JM. Responsiveness of the numeric pain rating scale in patients with low back pain. Spine 2005;30:1331-4. RCP London. (2017). National Early Warning Score (NEWS) 2. [online] Available at: https://www.rcplondon.ac.uk/ projects/outputs/national-early-warning-score-news-2 [Accessed 10 Jan. 2020]. 


\section{Abstract \# 370 \\ Quality of life of caregivers of patients with Alzheimer's disease in Greece}

Aggeliki Maritsa ${ }^{1}$, Dimitra Psilou $^{1}$, Sotiris Plakas ${ }^{2}$, Chrysoula Tsiou $^{3}$, Theodoula Adamakidou ${ }^{3}$, Eleni Dokoutsidou ${ }^{3}$

${ }^{1}$ General Hospital 'Sotiria" Athens, Greece, ${ }^{2}$ Nursing Department, University of West Attica, Creece, ${ }^{3}$ Nursing Department, University of West Attica, Greece

Introduction: Caring for people with Alzheimer's disease causes physical and psychological problems in caregivers and diminished quality of life (QoL). This study aimed to assess of caregivers of Alzheimer's disease patients and determine factors affecting it.

Method: A descriptive cross-sectional survey was carried out with 70 patients and their family caregivers in Athens, Greece between May and July 2019. The SF-36 General Health Questionnaire and a sociodemographic and relevant medical conditions questionnaire were used for data collection.

Results: The mean age of the caregivers was $61.4 \pm 12.9$. Of them $61.4 \%$ were women, $84,3 \%$ were first degree relatives and for $48.6 \%$ of them their relation were spouse. The average number of years caring for the patient was $4.7 \pm 3.0$ and the mean daily time care was $10.9 \pm 7.9$ hours. The "physical composite score" was $52,1 \pm 8,2$ and the "mental composite score" $37,7 \pm 10,3$, which were significantly lower from the normative means of the general population. It was found that in caregivers of unable to walk patients, there was a significant correlation with lower scores in "physical functioning" $(p=0,031)$, "general health" $(p=0,001)$ "vitality" $(p=0,028)$ and "mental health" $(\mathrm{p}=0,04)$. Duration of caregiving and the age of caregivers were correlated with lower scores in "role-emotional" $(\mathrm{p}=0,024)$ and "physical health composite" $(\mathrm{p}=0,021)$ respectively.

Key conclusions: This study has shown that in a society where caregivers for Alzheimer's disease patients are mostly family members appropriate interventions and supportive systems are needed to improve caregiver's health-related quality of life.

\section{Abstract \# 371}

Are all pressure sores just pressure sores?

Jean Marc Mizzi ${ }^{1}$, Stephen Mangion ${ }^{2}$, Antoine Vella ${ }^{2}$

${ }^{1}$ Mater Dei Hospital of Malta, Medicine, Msida Malta, ${ }^{2}$ St Vincent De Paul Long-Term Care Facility, Geriatric Medicine, Luqa Malta

Case presentation: A 73 y/o male who is a resident at a long-term care facility and a known case of advanced dementia, diabetes mellitus and hypertension presented with a 1-week history of multiple rapidly enlarging pressure sores and reduced oral intake. Due to the rapidly progressive nature of the pressure sores, a potential dermatological cause was sought. The patient was reviewed and noted to have had multiple oral ulcerating lesions and on the patient's arms he was noted to have multiple small (few mms in size) ulcerating lesions and healed excoriation marks. A collateral history was obtained from the patient's wife which yielded that he was previously diagnosed with a dermatological condition years prior. On the national database of investigations, he was noted to have been tested for Prickle Cell Desmosome Antibodies and the results showed $>1: 40$. A provisional diagnosis of Pemphigus Vulgaris induced pressure sores was made. After treatment with high dose intravenous steroids the patient's pressure sores have started to resolve and the treatment has proved to be promising.

Learning points: This case illustrates the fact that pressure sores may have complex aetiological factors involved, apart from the classical causes of decreased nutrition, frailty and decreased mobility. Due to the Covid-19 crisis, this patient was not able to undergo a skin biopsy due to reduction into dermatological services offered. This case also shows the importance of making clinical decisions with the tools available to the geriatrician at the time. Poster to Include Images of Lesions and Discussion Section.

\section{Abstract \# 372 \\ Positive anti-smooth muscle antibodies and interstitial pneumonitis in an old patient with multiple system atrophy \\ Derbal Samar ${ }^{1}$, Ben Dahmen Fatma ${ }^{1}$, Abdallah Maya ${ }^{1}$ \\ ${ }^{1}$ Regional hospital of Ben Arous}

Introduction: Multiple system atrophy (MSA) is a progressive neurodegenerative disorder among synucleinopathies. The aetiology of MSA remains unknown, but it can involve the extrapyramidal and pyramidal system, the autonomic nerves and the cerebellum. The main clinical manifestations are Parkinson's symptoms, cerebellar ataxia, pyramidal tract signs and autonomic nervous system disorders. Several conditions can be associated. We report a case of MSA associated with interstitial pneumonitis and positive anti-muscle antibodies.

Case report: A 79-year-old patient with a medical history of arterial hypertension was followed in the our department of internal medicine for progressive balance disorders, diffuse myalgia, frequent falls, orthostatic hypotension and parkinsonism. The patient was diagnosed with probable MSA and a cerebral and spinal magnetic resonance imaging was not consistent with Parkinson's disease.The clinical examination showed a cerebellar syndrome, a pyramidal syndrome, a parkinsonian syndrome and autonomic dysfunction: urinary incontinence and constipation. Pulmonary auscultation revealed crackling rales. The thoracoabdominal $\mathrm{CT}$ objectified lesions of nonspecific interstitial pneumonitis. Hepatic, renal, metabolic and inflammatory assessments were normal. Hepatitis B, C and HIV serologies were negative. The immunoassay revealed positive anti-smooth muscle antibodies. Anti-nuclear antibodies, anti-extractable nuclear antigens and anti-neuronal antibodies were negative. The patient was treated with levodopa $500 \mathrm{mg}$ per day combined with corticosteroid therapy $0.5 \mathrm{mg} / \mathrm{kg} / \mathrm{day}$, motor rehabilitation and education of his family. Corticosteroids were tapered gradually. The patient is currently followed the the outpatient department with no further signs.

Conclusion: MSA is a rare but serious condition. Its atypical clinical presentation can lead to other diagnosis in elderly. We found no previous reports in literature, of coexistence of MSA and positive anti-smooth muscle antibodies and interstitial pneumonitis.

\section{Abstract \# 373}

Toxic megacolon by clostridium as a cause of death in oncohematological patient

Yanira Aranda ${ }^{1}$, Cristina Carrasco ${ }^{2}$, Beatriz Neira ${ }^{3}$, Arís Somoano ${ }^{3}$, Carmen Alcaraz-L ${ }^{4}$

${ }^{1}$ Hospital Central de la Cruz Roja., ${ }^{2}$ Hospital Gregorio Marañón, ${ }^{3}$ Hospital Central de la Cruz Roja, ${ }^{4}$ Hospital Virgen del Rosell

A male (85-year-old) with myelodysplastic syndrome type AREB-1 diagnosed in 2016.In 2019, the patient went to the emergency department for extreme malaise with diarrhea. We showed hemoglobin $7.5 \mathrm{~g} / \mathrm{dL}$, severe thrombopenia and worsening renal function. Coproculture was collected and the result was Clostridium difficile toxin positive, so antibiotic treatment with metronidazole was started with torpid evolution due to persistence of fever and diarrhoeal stools, with the need to associate vancomycin. Despite the prescribed 
treatment, the patient presented fever of $38.7^{\circ}$, hypotension and intense abdominal pain with distension. Abdominal radiography revealing a dilation at the level of the colon of $8.19 \mathrm{~cm}$ : diagnosis of toxic megacolon. Due to the terminal condition after the progression of his hematological disease, it was decided to prioritize comfort and symptomatic control, and the patient died after 48 hours.Toxic megacolon is a rare and potentially fatal complication. It is a nonobstructive dilation of the colon, which is usually associated with systemic toxicity. SIt is often associated with inflammatory bowel disease, especially ulcerative colitis, however, any condition that causes inflammation of the colon may be its etiology. In recent times, epidemiology has shifted towards infectious causes, specifically due to an increase in Clostridium difficile-associated colitis, possibly due to the extensive use of broad-spectrum antibiotics. As responsible physicians, we must justify as much as possible any initiation of antibiotic treatment and initiate sequential therapy with antibiotics oriented to compatible antibiograms, reserving broad-spectrum treatments to selected cases and not in a generalized way.

\section{Abstract \# 374 \\ Quality of life of the elderly patients with chronic cardiac pathologies and associated co-morbidities \\ Gabriela Soric ${ }^{1}$ \\ ${ }^{1}$ Ana Popescu}

Introduction: Aging has a major impact on the health of the cardiovascular system, so cardiovascular pathology is becoming more common among the elderly population.

Material and methods: The study included 1158 patients - men $41.45 \%$ and women $58.54 \%$, aged between 65 and 96, mean $71.74 \pm$ ES 0.17, admitted to the Geriatrics and Internal Medicine departments, from 2015-2017. The obtained data were analyzed in the StatSoft database Statistics 7.

Results: Evaluation of the weight of chronic cardiac pathology in elderly patients revealed-high blood pressure- $88.63 \%$ cases, angina pectoris-45.44\%, rhythm disorders-33.49 \% cases. Chronic heart failure was diagnosed in $92.6 \%$ of cases. The types of rhythm disorders in elderly patients were-atrial fibrillation, extrasystole, sinus tachycardia.The assessment of cardiovascular risk factors in elderly patients showed a high level of dyslipidemia- $49.43 \%$, aggravated hereditary anamnesis- $16.47 \%$, glucose tolerance disorder- $10.22 \%$, alcohol consumption-9.09\%, smoking- $6.25 \%$ cases, overweight, with a mean BMI of $29.96 \pm 0.48$. Among the diagnosed co-morbidities the most frequent were osteoarticular pathology- $98.0 \%$ cases, cerebrovascular disease- $86.36 \%$, digestive pathology-79.49\%, urogenital pathology-77, $82 \%$ and Diabetes- $24.42 \%$ cases. The impact of heart disease and co-morbidities on the quality of life of elderly patients was negative, the most affected item of quality of life was energy, with the mean value of $59.59 \pm 3.02$, followed by of pain- $53.67 \pm 2.30$, physical ability-48.29 \pm 2.018 , sleep quality-47.7 \pm 2.60 , emotional reaction- $35.20 \pm 2.16$ and social isolation- $27.78 \pm 221$.

Conclusion: The quality of life of the elderly patients was affected in both groups, it was stablished that geriatric syndromes and chronic cardiac pathology had a negative impact on the quality of life, affecting more often the emotional reaction, affecting all life fields.

\section{Abstract \# 375 \\ Geriatric syndromes in consultation of neurology}

Marta Mercedes González Eizaguirre ${ }^{1}$, Maria Jesus Vicente Romero ${ }^{1}$, Ana Mateo $\mathrm{Abad}^{1}$, Elisa García Sancho ${ }^{1}$, Carmen Oquendo Marmaneu $^{1}$, Mercedes Forcano García ${ }^{1}$
${ }^{1}$ Hospital San José de Teruel

Introduction: Today, one in six people has a neurological disorder, equivalent to approximately $15 \%$ of the population. In this group of diseases are Alzheimer's, migraine, stroke (stroke), Parkinson's, epileptic seizures or multiple sclerosis. Very prevalent pathologies in the elderly patient, some of them being geriatric syndromes. Sometimes these pathologies are treated by neurologists and not by geriatricians.

Method: A retrospective descriptive study of all patients over 69 years of age is carried out at the Neurology office from November 1, 2019 to January 31, 2020. Subsequently, the patients are classified by the pathology by which they are derived from primary care.

Result: 126 patients come to the consultation. The main reason for consultation is for the study of cognitive impairment with 20 consultations, then 14 consultations for clopidogrel renewal, 14 for the onset of tremor, 13 repercussion after cerebral infarction, 9 for performing a dementia medication visa, 8 for headache, 7 for Parkinson's control, 7 for neuropathy and paraesthesia, 5 for control of epilepsy, 4 for control of trigeminal neuralgia, 3 for mitochondrial myopathy, 3 for agitation, 2 for brain neoplasia 1 for renewal of anticoagulation visa, and 9 that are a miscellaneous.

Conclusions: Geriatric syndrome is the term used for the symptom or set of complex symptoms with high prevalence in older adults resulting from multiple diseases and risk factors. These syndromes are not only assessed by the geriatrician, sometimes it is the neurologist who receives the patients and treats these pathologies.

\section{Abstract \# 376}

Ankylosing spondylitis and anterior uveitis

Iker Sierra-Martínez ${ }^{1}$, Leticia Sierra-Martínez ${ }^{2}$, Rosario MartínezFuerte $^{3}$, Natalia Sanz-González ${ }^{4}$

${ }^{1}$ Traumatology Department, Hospital of Medina de Campo (Valladolid), Spain, ${ }^{2}$ Valladolid Este Primary Assistance Gerency, Valladolid, Spain, ${ }^{3}$ Pilarica Health Center, Valladolid Este Primary Assistance, Valladolid, Spain, ${ }^{4}$ Parquesol Senior Center, JCyL Social Services Gerency, Valladolid, Spain

Ankylosing spondylitis (AS) is a chronic inflammatory disease with joint and extra-articular manifestations, such as anterior uveitis, aortic incompetence, cardiac conduction defects, pulmonary fibrosis, neurological involvement, and renal amyloidosis.

Description of the case: 67-year-old male patient with a history of hypovitaminosis D. He has a daughter and sisters diagnosed with axial and peripheral spondylitis. In 1985 he started with inflammatory low back pain and ankle arthritis. All three sections of the vertebral column ended up being affected and bilateral coxarthrosis also appeared. Several tests were performed, detection of HLA-B27+, with a diagnosis of spondylitis. Later bilateral uveitis appeared. It had an outbreak in 2013 resistant to salazopyrine and NSAIDs and methotrexate $20 \mathrm{mg} /$ day was started. The pains persisted, with high CRP maintained despite uveitis control, so biological treatment was proposed and Cemidon was started, but was poorly tolerated due to the appearance of severe asthenia. A year ago it was the last review with Ophthalmology, in the area of Ocular Immunology, where the dose of methotrexate was reduced due to being well controlled.

Recommendations: Ocular manifestations are frequent in ankylosing spondylitis. These can occur before the articular manifestations of AS. Family history and laboratory and physical examination studies are essential to avoid a delay in diagnosis and treatment. With early treatment, most uveitis responds favorably; however, without treatment or with a late approach, serious complications can occur that can lead to amaurosis. 


\section{Abstract \# 377}

Nursing personnel in the era of precision health and geriatric care

Athina Patelarou ${ }^{1}$, Marios Spanakis ${ }^{2}$, Stefania Schetaki ${ }^{3}$, Evridiki Patelarou $^{1}$

${ }^{1} 1$. Department of Nursing, Faculty of Health Sciences, Hellenic Mediterranean University, Heraklion, Crete, Greece, ${ }^{2}$ 1. Department of Nursing, Faculty of Health Sciences, Hellenic Mediterranean

University, Heraklion, Crete, Greece 2. Computational BioMedicine Laboratory, Institute of Computer Science, Foundation for Research and Technology -Hellas (FORTH), Heraklion, Crete, Greece, ${ }^{3} 1$. Department of Nursing, Faculty of Health Sciences, Hellenic Mediterranean University, Heraklion, Crete, Greece. 3. Annousakeio Therapeutic Center Impatient Recovery and Rehabilitation Center "Agios Spyridon"

Abstract introduction: Elderly's people health status is characterized by changes in physiology as well as, multimorbidity due to the presence of chronic diseases and frailty. Moreover, they represent a special population group that receive the largest number of drug prescriptions and excessive needs for healthcare [1].

Methods: The authors carried out a bibliographic search using Medline database for the last decade. Search terms used were chosen from the USNLM Institutes of Health list of MeSH (Medical Subject Headings) and free text key terms were used as well.

Results: Personalized nursing care (PNC) for elder, in the context of precision health, involves the interpretation and clinical use of -omics information as they derive from system medicine approaches (biology, pharmacology) towards patient advocacy and support throughout testing, treatment adherence and compliance as well as, ongoing chronic monitoring and support for patient decision making [2]. Nurses, representing the largest group of professionals in healthcare, should adopt in the challenges posed from this new era that shifts medicine from the reactive or one-size-fits-all scenario towards participatory, preventive, predictive and personalized healthcare ( $\mathrm{P} 4$ medicine) [3]. PNC, entailing the uniqueness of an elder individual, not only should be able to adopt this knowledge but also consider the special characteristics of elder population such as polypharmacy or pathophysiological changes that result in different pharmacological response and clinical outcome [4].

Key conclusions: It is essential for nursing profession to stay up to date with the changes generated from the realization in clinical practice of precision medicine and integrate relative content for continuous education [5]. This will allow them to utilize state-of-theart approaches to the bedside, making contributions in the areas of symptom assessment, prevention, management, and treatment towards optimal healthcare in elder patients [4].

References: 1. Yarnall AJ, Sayer AA, Clegg A, Rockwood K, Parker $\mathrm{S}$, Hindle JV. New horizons in multimorbidity in older adults. Age Ageing. 2017;46(6):882-888. https://doi.org/10.1093/ageing/afx150. 2. Han CJ. A Concept Analysis of Personalized Health Care in Nursing. Nurs Forum. 2016;51(1):32-39. https://doi.org/10.1111/nuf. 12117. 3. Flores M, Glusman G, Brogaard K, Price ND, Hood L. P4 medicine: how systems medicine will transform the healthcare sector and society. Per Med. 2013;10(6):565-576. https://doi.org/ 10.2217/pme.13.57. 4. Martha SR, Auld JP, Hash JB, Hong H. Precision Health in Aging and Nursing Practice. J Gerontol Nurs. 2020;46(3):3-6. https://doi.org/10.3928/00989134-20200129-03. 5. Fu MR, Kurnat-Thoma E, Starkweather A, et al. Precision health: A nursing perspective. Int J Nurs Sci. 2019;7(1):5-12. https://doi.org/ 10.1016/j.ijnss.2019.12.008

\section{Abstract \# 378}

\section{Assessing the quality of life in elderly glaucoma patients}

Alexandra Trivli ${ }^{1}$, Evridiki Patelarou ${ }^{2}$, Athina Patelarou ${ }^{2}$

${ }^{1}$ Ophthalmologist , Athens, Greece, ${ }^{2}$ Department of Nursing, Faculty of Health Sciences, Hellenic Mediterranean University, Heraklion, Crete, Greece

Introduction: Glaucoma is a major cause of irreversible blindness worldwide which appears mainly in people over the age of 60 and results in progressive damage to the optic nerve [1]. Nowadays, more than 64 million people are supposed to have glaucoma, with this estimate increasing over the years [1,2]. Vision impairment is an important public health issue that influences the quality of life of elderly people $[3,4]$.

Methods: We carried out a bibliographic search using Medline database (English language) for the last decade, in order to investigate the effects of glaucoma on social and emotional wellbeing.

Results: Because of the relatively strong association with older age, patients may suffer from multiple co morbidities. Advanced age, family history, race, ocular pathologies and injury and other pathologies such as diabetes and hypertension represent major risks for developing glaucoma.According to the current literature, there are various instruments available to measure quality of life. Many studies are carried out by the developed two instruments for measuring quality of life by WHO, revealing that several aspects of the disease may impair quality of life for glaucoma patients. Visual impairment is the major determining factor for a worse quality of life in such cases and may disturb the patient's daily habits such as reading, walking, driving and recognizing people and objects. Furthermore, fear of going blind and social isolation, side effects of conservative therapy and treatment costs may result in psychological disturbance such as anxiety, fear and depression.

Key conclusions: Close monitoring and trusting healthcare providerpatient relationship remain essential for such patients, in order to maintain and improve their quality of life by using appropriate and individualized medical therapy.

References: 1 . Tham YC, Li X, Wong TY, et al. Global prevalence of glaucoma and projections of glaucoma burden through 2040: a systematic review and meta-analysis. Ophthalmology. 2014;121:2081-2090. 2. Quigley HA. Glaucoma. Lancet. 2011;377:1367-1377. 3. Quaranta L, Riva I, Gerardi C, Oddone F, Floriani I, Konstas AG. Quality of Life in Glaucoma: A Review of the Literature [published correction appears in Adv Ther. 2016 Jun;33(6):982]. Adv Ther. 2016;33(6):959-981. 4. Sleath B, Sayner R, Vitko M, et al. Glaucoma patient-provider communication about vision quality-of-life. Patient Educ Couns. 2017;100(4):703-709.

\section{Abstract \# 379}

\section{Dry eye in women over 50 years old}

Alexandra Trivli ${ }^{1}$, Evridiki Patelarou ${ }^{2}$, Athina Patelarou ${ }^{2}$

${ }^{1}$ Ophthalmologist, Athens, Greece, ${ }^{2}$ Department of Nursing, Faculty of Health Sciences, Hellenic Mediterranean University, Heraklion,

Crete, Greece

Introduction: Dry eye disease (DED) is a multifactorial chronic condition of the ocular surface and tears film. The prevalence in older population is $15 \%$ in patients $70-80$ years old and $20 \%$ in those older than 80 years [1]. Advanced age results in a rise of its prevalence, especially in post-menopausal woman. Typically categorized into aqueous deficient and evaporative type, the main mechanism is tear film instability and subsequent ocular surface inflammation, resulting 
in eye discomfort, decreased and fluctuating vision and compromised quality of life.

Methods: A Medline database search was conducted based on English language publications over a 10-year period in order to review the published literature in this field.

Results: Large epidemiological studies have observed that the rate of DED in women is higher than in men over 50 years old $(7 \%$ and $4 \%$, respectively). Even though the pathophysiologic mechanisms are not well understood, several menopausal and/or postmenopausal patients suffer from dry eye symptoms [2]. Furthermore, hormonal disturbance can influence a wide range of bodily functions with the endocrine system playing a prominent role in the regulation of the ocular surface, the occurrence and treatment of aqueous-deficient and evaporative dry eye disease (DED) [3]. More in depth, both estrogen, progesterone and androgen imbalances affect the anatomy and physiology of the lacrimal gland and tear secretion.

Key conclusions: Variation of sex hormones represents a major factor in the pathophysiology of DED in menopause. The healthcare provider should keep in mind that various conditions predispose older patients to dry eye, such as higher rates of medication use, lid laxity, chronic diseases and oxidative stress.

References: 1. Anushree Sharma, Holly B. Hindman. Aging: A Predisposition to Dry Eyes. J Ophthalmol. 2014; 2014: 781683. Published online 2014 Aug 14. 2. Peck T, Olsakovsky L, Aggarwal S. Dry Eye Syndrome in Menopause and Perimenopausal Age Group. J Midlife Health. 2017;8(2):51-54. 3. Matossian C, McDonald M, Donaldson KE, Nichols KK, MacIver S, Gupta PK. Dry Eye Disease: Consideration for Women's Health. J Womens Health (Larchmt). 2019;28(4):502-514.

\section{Abstract \# 380 \\ Choosing wisely in geriatrics: results of the first French national campaign}

Tannou Thomas ${ }^{1}$, Menand Emilie ${ }^{2}$, Laigle Francine ${ }^{3}$, Berthou Julie ${ }^{4}$, Daucourt Valentin ${ }^{5}$, Jarno Pascal ${ }^{6}$, Somme Dominique ${ }^{7}$, Corvol Aline $^{7}$

${ }^{1} \mathrm{CHU}$ de Besançon - FRANCE, ${ }^{2}$ Geriatrie - CHU Rennes - FRANCE, ${ }^{3}$ France Assos Santé - Rennes, ${ }^{4} \mathrm{CHU}$ Besançon - FRANCE, ${ }^{5}$ REQUA - Besançon - FRANCE, ${ }^{6}$ CAPPS Bretagne - Rennes - FRANCE,

${ }^{7}$ Geriatrie - CHU de Rennes - FRANCE

Introduction: International Choosing Wisely campaign aims to improve the appropriateness of care, by raising awareness among physicians and end-users of the risks in over-medicalisation. In France, the Société Française de Gériatrie et Gérontologie (SFGG) join by implementing recommendation to improve the appropriateness of care provided to older inpatients or those living in nursing homes.

Method: The recommendations were drawn up according to an iterative model of co-construction with end-users and physicians with preliminary experiment in one region. Analysis and feedback of endusers lead to 5 final recommendations. These refer to the use of antibiotics in urinary tract infections, anxiolytics for a extended period of time, neuroleptics in psycho-behavioural disorders, statins for primary prevention and, finally, the proper collection of preferences concerning level of care of the end-users. The professional practice assessment was carried out using a one day data collection approach on medical records.

Results: A total of 5428 practice screenings were conducted among patient-residents, and 1139 patients were included in the practice assessment. These revealed a total of 280 patient-resident situations corresponding to acknowledged practices (i.e. $25 \%$ ) versus 859 situations (i.e. $75 \%$ ) whose practice is questionable considering the literature. These situations of practices to be optimised mainly concerned the use of benzodiazepines, neuroleptics and antibiotics in case of urinary colonisation.

Conclusion: The Choosing Wisely campaign in geriatrics in France shows a strong involvement of health actors in this type of evaluation, and very heterogeneous practices, particularly in the field of deprescription practices related to psychotropic drug, which must be specifically supported.

\section{Abstract \# 381}

Gallbladder disease's in elderly patients with metabolic syndrome:role of ultrasonography

Vito Carrieri ${ }^{1}$, Antonella Bray ${ }^{2}$, Giovanni Argentieri ${ }^{2}$, Vincenzo Paternò $^{2}$, Luca Di Lena ${ }^{3}$, Gloria Mazelli ${ }^{2}$

${ }^{1}$ Geriatrics Department Hospital”A.Perrino Brindisi Italy, ${ }^{2}$ Geriatrics Department Hospital “A. Perrino" Brindisi Italy, ${ }^{3}$ Geriatrics Department Hospital "A.Perrino" Brindisi Italy

Introduction: The aim of the study is to illustrate the role of Ultrasonography (US) in the management of the elderly patients with Metabolic Syndrome (MS) and with suspected gallbladder's diseases. Material and methods: 500 elderly patients ( 65-95 years old, 300 women and 200 men) with Metabolic Syndrome were selected. Four groups of patients were identified: 1.100 with diabetes mellitus and dyslipidemia; 2. 200 with diabetes, dyslipidemia, hypertension; 3.300 with diabetes, dyslipidemia, hypertension, obesity; 4. 100 with diabetes ,hypertension and obesity. Ultrasonography was performed in all elderly patients, with and without symptoms of suspected gallbladder's diseases.

Results: Ultrasonography detected in 300 patients ( 210 women , 90 men ) the following diseases of gallbladder: 150 gallbladders with stones, 50 gallbladders with sludge, 5 gallbladder neoplasms, 20 gallbladders with cholesterolosis, 50 gallbladders with increased volume, 25 cholecystitis. In the group of patients with diabetes and dyslipidemia only in the $20 \%$ of patients US detected gallbladder diseases; in the group with diabetes,dyslipidemia and hypertension the US revealed gallbladder's diseases in $25 \%$ of patients; in the group with diabetes, dyslipidemia, hypertension, obesity the US diagnosed gallbladder's diseases in $60 \%$ of patients; in the group with diabetes, hypertension, obesity the US detected gallbladder's diseases in $50 \%$ of patients. In the 200 elderly patients without gallbladder's diseases US revealed liver steatosis in $50 \%$ of patients and the prevalence was of the $90 \%$ in the groups with obesity.

Discussion and conclusions: Metabolic Syndrome in the elderly patients is often associated with gallbladder's diseases. The diabetes with neuropathy causes gallbladder hypotonia with biliary stasis and often in the elderly patients with gallbladder sludge we observe dyspeptic syndrome and reduced gastric motility. In the elderly patients with diabetes and obesity, US detected early and easily gallblader's diseases and the prevalence was very high. In patients with the association between diabetes and dyslipidemia or hypertension was observed a low prevalence of gallbladder's disease. In conclusion, the data of our study show that in the elderly patients with metabolic syndrome is the obesity the most important risk factor for gallbladder's diseases, while the diabetes plays a fundamental role , also related to neuropathy. The high blood pressure is not a risk factor for gallbladder's disease. The dyslipidemia slightly increases the prevalence of gallbladder's diseases. The early U S in elderly patients is very important to detect the serious inflammatory complications of gallbladder: in fact cholecystitis, associated with fever and abdominal pain is often observed in diabetic elderly patients. We suggest that US should be performed in all elderly patients with metabolic syndrome, also without symptoms of gsllbladder's diseases. US 
allows to apply very quickly the appropriate diagnostic and therapeutic program and increases the effectiveness and efficiency of care in geriatrics.

\section{Abstract \# 382 \\ Demographic and clinical characteristics of home bound patients with chronic heart failure}

Cassidy June Chiong ${ }^{1}$, Alan B. Miller ${ }^{2}$

${ }^{1}$ MedEx Health Network, Inc, ${ }^{2}$ University of Florida

Introduction: Chronic heart failure (CHF) results in physical disability and decline in functional state resulting in loss of independence leading to home bound state. Physician home visits are increasing and generally patients are older and sicker. MedEx Health Network was originally established in 2011 to serve homebound CHF patients with the visiting cardiologist also serving as the primary care physician. This study aimed to explore characteristics of homebound elderly $(>65)$ with $\mathrm{CHF}$ from the first cardiology home visit program. Methods: We analysed 3 year data from MedEx Cardiology Home program (MedEx) on CHF home visits and compared to American College of Cardiology's National Cardiovascular Data Registry (NCDR) Practice Innovation and Clinical Excellence (PINNACLE) outpatient Registry ${ }^{\circledR}$.

Results: From March 2016 to March 2020, 423 patients were visited. When compared to the PINNACLE $(n=697,542)$, MedEx patients $(n=423)$ are much older ( $81.3 \pm 9.1$ yo vs. $69.1 \pm 13.5$ yo $)$, more women ( $62.9 \%$ vs. $43.4 \%)$, non smokers ( $83 \%$ vs $43 \%$ ), higher systolic blood pressure (SBP) $(130.3 \pm 21.8 \mathrm{mmHg}$ vs. $128.1 \pm 18.8 \mathrm{mmHg})$ and heart rate (HR) $(76.1 \pm 12.9 \mathrm{bpm}$ vs. $73.1 \pm 13.6 \mathrm{bpm})$, higher ejection fraction (EF) $(60.5 \pm 8.7$ vs. $48.5 \pm 16.0)$ with more preserved ejection fraction $(69.3 \%$ vs. $46.5 \%)$, stroke $(13.2 \%$ vs. $9.7 \%)$, and diabetics (37.4\% vs. $26.1 \%)$. MedEx patients have lower body mass index (BMI) $(26.1 \pm 5.5$ vs. $29.9 \pm 6.5)$, fewer hypertension history $(74.5 \%$ vs. $75.6 \%)$ and atrial fibrillation/flutter (30\% vs. $34.4 \%)$.In addition, $30.5 \%$ of MedEx patients have dementia, $21.5 \%$ have COPD, $97 \%$ have any form of vascular disease including coronary artery, peripheral vascular, carotid, aortic disease in any vascular imaging and $41.8 \%$ of MedEx patients expired during the follow up.

Conclusions: In comparison with NCDR-PINNACLE registry, MedEx patients are a distinct group of CHF population. More research is needed to demonstrate the unique needs and effectiveness of cardiology home visiting practices among these frail home bound chronic heart failure population.

References: 1. Sairenji T, Jetty A, Peterson LE. Shifting Patterns of Physician Home Visits. J Prim Care Community Health. 2016 Apr;7(2):71-5. Epub 2015 Nov 16. PMID: 26574565. 2. Ibrahim NE, Song Y, Cannon CP, Doros G, Russo P, Ponirakis A, Alexanian C, Januzzi JL Jr. Heart failure with mid-range ejection fraction: characterization of patients from the PINNACLE Registry ${ }^{\circledR}$. ESC Heart Fail. 2019 Aug;6(4):784-792. Epub 2019 Jul 3.

\section{Abstract \# 383}

Liver cirrhosis in the elderly: clinical and ecographic correlations

Vito Carrieri ${ }^{1}$, Antonella Bray ${ }^{1}$, Giovanni Argentieri ${ }^{2}$, Gloria Mazelli ${ }^{1}$, Luca Di Lena ${ }^{1}$, Vincenzo Paternò ${ }^{3}$

${ }^{1}$ Geriatrics Department Hospital "A.Perrino" Brindisi Italy, ${ }^{2}$ Geriatrics Department Hospital “A. Perrino” Brindisi Italy, ${ }^{3}$ Geriatrics department Hospital "A. Perrino” Brindisi Italy

Introduction: The aim of the study is to verify the clinical and ultrasound correlations in a series of 200 hepatocellular carcinomas
(HCC) complicating liver cirrhosis in patients over the age of 65, hospitalized in the Geriatrics department.

Materials and methods: 200 patients observed over the course of 8 years, aged 65 to 95 years, 119 men and 81 women,were included in the clinical ultrasound evaluation. All patients had liver cirrhosis (129 related $\mathrm{HCV}$ and 46 related $\mathrm{HBV}, 25$ non-viral) for over 10 years. All patients underwent ultrasound (US) every 6 months and were included in the study when only one solid intrahepatic lesion of up to $2 \mathrm{~cm}$ in diameter was found. The US diagnosis of HCC has been confirmed with at least one of the following methods (US with contrast medium, CT, MRI, Biopsy). The echogenicity of HCC has been correlated with AFP levels, patient age, cirrhosis etiology and Child Pugh classification.

Results: 140 hypoechoic nodules (diameter between 10 and $20 \mathrm{~mm}$ ), 10 isoechogenic nodules (diameter between 17 and 20mm), 50 hyperechoic nodules (diameter between 10 and $20 \mathrm{~mm}$ ) were highlighted with US. AFP values were higher than normal in $30 \%$ of hypoechoic nodules, in $20 \%$ of isoechoic nodules, in $10 \%$ of hyperechoic nodules. In patients aged 85 to 95 all nodules were hypoechoic. Hypoechoic nodules were observed in 15 patients with non-viral cirrhosis out of a total of 25 patients (including 10 with alcoholic cirrhosis, 13 with cryptogenic cirrhosis, 2 with hemochromatosis cirrhosis), in 89 patients with HCV-related cirrhosis and in 36 patients with cirrhosis HBV related. Isoechoic nodules have been observed in 10 patients with cryptogenetic cirrhosis; hyperechoic nodules were found in 20 patients with HCV cirrhosis, in 20 patients with HBV cirrhosis and in 10 patients with non-viral cirrhosis. In relation to the classification of Child Pugh, 45 patients were observed in class A, 5 of which with isoechoic HCC, 15 hyperechoic and 25 hypoechoic; 80 patients with 67 hypoechoic and 13 hyperechoic HCC are included in class B and 75 patients with $61 \mathrm{HCC}$ hypoechogens and 14 hyperechogenic $\mathrm{HCC}$ are present in class C.

Conclusions: The analysis of the data allows to affirm that in the elderly patients with cirrhosis, above all of age $>85$ years, the small $\mathrm{HCC}$ is hypoechoic to the US. In most cases, the cirrhosis is HCV related; the dosage of AFP does not allow adequate surveillance of the elderly with cirrhosis but periodic control with US is essential. According to the Child Pugh classification, people in class B and C are prevalent in the case studies, with the prevalence of the hypoechoic aspects of HCC in both classes; the isoechogenic aspects of HCC have been highlighted only in class A. In conclusion, our study confirms that US is the most appropriate method for surveillance of the elderly patient with cirrhosis and for the early diagnosis of the small HCC. The most frequent ultrasound aspects are those of the unique hypoechoic nodule, without correlations with the etiology of cirrhosis and the classification of Child Pugh.

\section{Abstract \# 384 \\ Anticholinergic burden scales as predictors of functional impairment in elderly multimorbidity patients}

Angela Tristancho-Perez ${ }^{1}$, Maria Dolores Santos-Rubio ${ }^{2}$, Bernardo Santos-Ramos ${ }^{1}$, Angela Villalba-Moreno ${ }^{3}$, Eva Alfaro-Lara ${ }^{4}$, Susana Sanchez-Fidalgo 5

${ }^{1}$ Pharmacy Service. University Hospital Virgen del Rocio. Seville, Spain, ${ }^{2}$ Pharmacy Service .University Hospital Juan Ramón Jiménez. Huelva, Spain, ${ }^{3}$ Pharmacy Service. Riotinto Hospital, Huelva, Spain, ${ }^{5}$ Pharmacy Service. Virgen del Rocio University Hospital, Seville, Spain, ${ }^{5}$ Department of Preventive Medicine and Public Health, University of Seville. Seville, Spain

Introduction: There are several scales that assess the anticholinergic risk (AR), but is unknown about their predictive value to functional 
impairment (FI) in elderly multimorbidity patients (EMP). Objective: to evaluate the ability of the different scales to predict FI in EMP. Methods: Retrospective, observational and multicenter study. EMP with at least 1 drug with anticholinergic burden $(\mathrm{AB})$ in anyone scale. $\mathrm{AB}$ and $\mathrm{AR}$ were assessed with the Anticholinergic Burden Calculator, which contains ten scales. FI was considered when Barthel index decreased 20 points (3-15 months). Association (chi-square test), positive and negative predictive values (PPV, PNV) and ROC plot were calculated.

Results: 473 patients (84 years (IQR=10), 60.3\% women) were included and $40 \%$ suffered FI. Median of medications with $\mathrm{AB}$ was 2 $(\mathrm{IQR}=2)$. Among patients with FI the most prevalent pathology was cardiac disease $(45.5 \%)$ and the most prescribed anticholinergic drug was furosemide (94 prescriptions). Among this cohort, those with high AR according to each scale were: 113 patients in the ABC scale (59.8\%), 48 in DBI (25.4\%), 32 in DURAN (16.9\%), 29 in ACB (15.3\%), 24 in ALS (12.7\%), 22 on ADS (11.6\%), 18 in CrAS $(9.5 \%)$, 17 on CHEW (9\%), 12 in AAS (6.3\%) and 6 on ARS (3.2\%). Significant association was only observed with $\mathrm{ABC}$ in patients with a time between barthel index determinations between 3 and 6 months $(\mathrm{p}=0.019)$, showing an $\mathrm{AUC}=0.63[0.52-0.74](\mathrm{p}=0.032)$. The cut-off point in $\mathrm{ABC}$ score was $\mathrm{AB}=2.5$ (sensitivity $=70 \%$, specificity $=54 \%$ ), $\mathrm{PPV}=54 \%$ and $\mathrm{PNV}=70 \%$.

Key conclusions: In EMP, only $\mathrm{ABC}$ showed association with FI, being its sensitivity and PPV moderate.

\section{Abstract \# 385 \\ Discovery of systemic scleroderma in the context of a pulmonary arterial hypertension assessment in an 83-year-old patient.}

Antonio Rainone ${ }^{1}$, Bruno Oquendo ${ }^{1}$, Cristiano Donadio ${ }^{1}$, Maurizio Magri $^{1}$, Lorenza De Rosario ${ }^{2}$, Caryn Recto ${ }^{3}$, Lorenzo Tommasi ${ }^{1}$, Livia Labon ${ }^{4}$, Fatima Meksem Mellaz ${ }^{5}$, Adeline Gouronnec ${ }^{1}$

${ }^{1}$ Hôpital Charles-Foix AP-HP, ${ }^{2} \mathrm{CHI}$ Creteil, ${ }^{2}$ Hôpital Henri-Mondor AP-HP, ${ }^{4}$ Hôpital Emile-Roux AP-HP, ${ }^{5}$ Hôpital Jean-Verdier AP-HP

Introduction: Systemic Scleroderma (SSc) is an autoimmune disease affecting connective tissue, arterioles and microvessels. It is considered incurable. Its course can be controlled. Approximately 8000 patients are affected by SSc per year in France. They are mostly women between 50 and 60 years old. We present the case of an 83 -year-old patient with a diagnosis of SSc.

Case: An 83 year old patient with no particular history (cognitive disorders and Hashimoto's thyroiditis) was hospitalized for assessment of altered general state and loss of autonomy for 3 months, exertional dyspnea for several months with blockpnea, Raynaud's syndrome. Trans-thoracic ultrasound revealed a pericardial effusion at $12 \mathrm{~mm}$ circumferential diameter with right heart failure, pulmonary arterial hypertension $(\mathrm{PH})$ at $100 \mathrm{mmHg}$ and dilatation of the right cavities. Antinuclear factor antibodies and centromere antibodies returned positive. Right catheterization revealed pre-capillary $\mathrm{PAH}$ associated with limited systemic cutaneous scleroderma.

Discussion: $\mathrm{PH}$ affects around 10\% of patients with SSc and is marked by a high mortality rate of $30 \%$. PH associated with SSc remains a feared and little-known complication with a poorer response to treatment and a poorer prognosis than in idiopathic $\mathrm{PH}$. Conclusion: In a $\mathrm{PH}$ assessment in the elderly people, the hypothesis of SS should be systematically evoked.

\section{Abstract \# 386}

\section{Sinus tachycardia and Parkinson's disease}

Derbal Samar ${ }^{1}$, Cherif Yosra $^{1}$, Ben Dahmen Fatma ${ }^{1}$, Abdallah Meya ${ }^{1}$

${ }^{1}$ Regional Hospital of Ben Arous

Introduction: Parkinson's disease (PD) is a neurodegenerative condition that involves motor and non-motor symptoms such as autonomic dysfunction which include cardiac system. Neurogenic orthostatic hypotension is the most common cardiac manifestation. Another cardiac effect in PD, however, is changing in heart rate.

Case report: A 55-year-old woman with high blood pressure, diabetes and kidney failure related to diabetic kidney disease was admitted for hyperglycemia. She reported a deep fatigue. At the electrocardiography, she had sinus tachycardia at 120 beats per minute without any other abnormalities. The cardiovascular examination was normal. Moreover, she had a motor slowness (bradykinesia), a stiffness of the two wrists and tremors of the distal extremities. The biological tests, including the cardiac enzymes were normal. The cerebral computed tomography didn't show any abnormalities. After having ruled out all the cardiac and extra-cardiac causes of sinus tachycardia, the diagnosis of PD with autonomic dysfunction was retained.

Conclusion: In PD the autonomic control of the cardiac system is impaired affecting even heart rate. Heart rate variability is more pronounces in patients who developed PD than those who did not. Cardiac autonomic dysfunction can be an early non-motor symptom of PD.

\section{Abstract \# 387 \\ Benefits of computed tomography of the thorax, abdomen and pelvis in inflammatory syndromes of unknown origin in older people}

Roubaud-Baudron Claire ${ }^{1}$

\section{${ }^{1}$ Bordeaux}

Introduction: The atypical clinical presentation as well as the high frequency of an increased plasmatic $\mathrm{C}$ reactive protein (CRP) of unknown origin are frequent in older patients and make diagnosis difficult to be raised. CT TAP scan might be helpful in those situations.

Objective: To determine the proportion of CT TAP scans performed for an increased CRP of unknown origin that contribute to the diagnosis. Material and methods: We conducted an observational prospective study at Bordeaux University Hospital between January 2019 and June 2020. All consecutive hospitalized patients aged $\geq 75$ years that underwent a CT TAP scan for an inflammatory syndrome of unknown origin (CRP $>5 \mathrm{mg} / \mathrm{L}$ ) were enrolled. Clinical data were collected the day of the $\mathrm{CT}$ scan request. Factors associated with a helpful CT scan and the prevalence of incidentalomas were reported. Results: Among the 100 enrolled patients (mean age $86 \pm 5,7$ years, $48 \%$ female), the CT TAP scan allow the physician to raise the diagnosis up for 56 patients, mainly pulmonary infections $(n=24)$ and gastrointestinal infections $(n=7)$. No factor associated with helpful exams was identified except a high level of CRP. The most frequent incidentalomas reported were Pleural and pericardial effusion $(\mathrm{n}=$ $35)$, cysts $(n=29)$ and old bone fractures $(n=27)$.

Conclusion: In this pilot study, CT scan seems to be a useful tool to explore unexplained inflammatory syndrome in older patients hospitalized in acute geriatric units.

Keywords: older population, unexplained inflammatory syndrome, CT TAP, outcome, diagnostic and useful exam 


\section{Abstract \# 388 \\ Peripheral neuropathy revealing systemic Amyloidosis in elderly: case report}

Derbal Samar ${ }^{1}$, Ben Dahmen Fatma ${ }^{1}$, Abdallah Maya ${ }^{1}$

${ }^{1}$ Regional hospital of Ben Arous

Introduction: Amyloid light chain (AL) amyloidosis due to deposition of monoclonal immunoglobulin light chains, is a rare and heterogeneous hematologic disease. We report a case of chronic polyradiculoneuropathy revealing $\mathrm{AL}$ amyloidosis in an old patient. Case report: A 65-year-old man admitted for edema of the lower limbs, weight loss, and progressive motor slowdown affecting the 4 limbs evolving for 3 months. Physical examination showed a proximal and distal symmetrical sensory and motor deficiency with abolished ankle reflexes. The rest of the examination was normal apart from an infiltrated purpura of the 2 lower limbs. Viral serologies were negative. The bioassay eliminates a phosphocalcic disorder, a hemostasis disorder, diabetes and vitamin deficiency. Brain CT was normal. The first electromyography (EMG) demonstrated a severe sensory and motor neuropathy. Negative serology for hepatitis B, hepatitis $\mathrm{C}$, rheumatoid factor, anti-CCP antibodies, and antinuclear, anti-dsDNA, anti-neuronal, and anti-neutrophil cytoplasmic antibodies confirmed the absence of underlying auto-immune diseases. Skin biopsy confirmed amyloidosis. The immunohistochemical investigation was positive for AL deposits. Serum protein electrophoresis did not show a monoclonal component. Immunofixation of serum proteins revealed normal kappa/lambda ratio. The bone marrow cytology showed $5 \%$ plasma cell infiltration with no other signs multiple myeloma. There were no bone lesions on radiographs of the complete skeleton. The patient was diagnosed with peripheral neuropathy revealing $\mathrm{AL}$ amyloidosis. There was also no evidence of cardiac or renal involvement. He was treated with high-dose corticosteroids with no improvement of the motor deficiency after 48 months.

Conclusion: Peripheral neuropathy is described in $15-20 \%$ of cases of AL amyloidosis. This involvement may mark the beginning of the disease and may present with progressive sensorimotor polyneuropathy, focal neuropathy, autonomic neuropathy, as well as other unusual clinical presentations. Diagnosis is often delayed. It is prudent that physicians consider the diagnosis of $\mathrm{AL}$ amyloidosis in patients with neuropathy, so that these patients may be treated earlier.

\section{Abstract \# 389}

\section{Telephone support for complex chronic patients}

Francisco Javier Balea Fernandez ${ }^{1}$, Cristina Cazorla Guillen ${ }^{1}$, Antonia Jesús Solano Benítez ${ }^{1}$

${ }^{1}$ Hospital Insular de Lanzarote, ${ }^{2}$ Nutritionist in Centro Hebe

Medical activity in geriatric units extends beyond mere routine clinical practice and it is essential for a real, easy and direct healthcare continuity. However, in the XXI century access to medical specialists in hospitals remain a Penrose Stair for geriatric patients. The particularities of this population require that accessibility to medical resources become more fluid and direct. At this point, why something so basic and cheap as a phone consultation in the age of telecommunications is something so unusual? Perhaps by the overloading of clinicians, lack of time and support that allows registration or because its has been doing in an informal way for long without any registration. The benefits of continuity of care in this population are well documented today. Phone support reduce hospitalizations and emergency departments conultations $1,3,6$, premature readmissions, improve the health status of chronic patients 2,3 , improves prognosis of patients with heart failure 5, and terapeutic adherence 7 . It has also been incorporated into the comprehensive managment in palliative care services with improvements in quality of care perceived by patients 10 . Within the framework of state programs chronicity justifies the need to develop and document this innate care activity in most geriatric units since its inception and is now extrapolating and incorporating other services. The aim of our study is to enhance the recording of telephone consultations in the geriatric unit which for years has been carrying out this activity and analyze the user profile to provide an efficient, actual non-contact continuous and specialist care and focused on the particularities of our population

\section{Abstract \# 390 \\ Cardio-metabolic risk in elderly patients in the context of the COVID-19 pandemic}

Dragos Munteanu ${ }^{1}$, Leonard Iosif Pertea ${ }^{1}$, Alexandru Vlase ${ }^{1}$, Andrei Cernomaz $^{1}$

\section{${ }^{1} \mathrm{UMF}$ Iasi}

Pre-existing cardiovascular and metabolic diseases could be a predisposing factor in itself for COVID-19 induced cardiac impairment, as elderly patients with heart injuries frequently have a history of coronary heart disease and hypertension, and metabolic pathologies (diabetes mellitus). Thus, in the report published in JAMA (april 2020), $25 \%$ and respectively $58.3 \%$ of patients with critical COVID19 had a history of cardiovascular and metabolic diseases. Although there is currently little evidence to establish a direct link between acute cardiopulmonary lesions associated with COVID-19 and cardio metabolic comorbidities, it can be assumed that patients with chronic cardio metabolic disorders are susceptible to acute cardiac injury. Once these patients are infected and develop severe SARS-COV-2 pneumonia, myocardial ischemia, cardiac dysfunction, or pulmonary thromboembolism are more likely to eventually lead to acute hemodynamic impairment.

Material and results: we further present the case of a 72-year-old woman patient, hospitalized in the Internal Medicine and GeriatricsGerontology Clinic Iasi, Romania, between may 21-31, 2020. The patient was transferred from the Infectious Diseases Hospital in the context of SARS-COV-2 (positive test April 2020) for continued treatment and monitoring of SARS-COV-2 infection. Hospitalization diagnosis: SARS-COV-2 infection (lung damage), SARS-COV-2 pneumonia, essential hypertension grade III additional high risk, type 2 diabetes. Among the reasons for hospitalization are dyspnea and moderate neurasthenia. The patient's evolution was stationary until May 30, when the general condition suddenly deteriorated, with loss of consciousness, decreased blood pressure, bradycardia, agonizing breathing, and generalized edema. Cardiopulmonary resuscitation maneuvers were instituted, without clinical symptoms, and the patient's death was finally declared.

Discussions and conclusions: Chronic cardiopulmonary pathology in an elderly patient with severe form SARS-VOC-2 infection (viral pneumonia) represent an high risk factor in these conditions and can cause sudden hemodynamic and respiratory decompensation with irreversible cardiopulmonary arrest. Thus, severe lung damage, caused by pneumonia, can lead to severe respiratory distress syndrome (ARDS), evolving into septic shock, which cause the death of the patient.

Keywords: ELDERLY, SARS-COV-2, CARDIOMETABOLIC DISORDERS.All the authors are quality as first author 


\section{Abstract \# 391}

\section{Healthy range of $\mathrm{BMI}$ in old adults}

Olof Gudny Geirsdottir ${ }^{1}$, Alfons Ramel ${ }^{1}$, Milan Chang ${ }^{1}$, Palmi V Jonsson $^{1}$, Inga Thorsdottir ${ }^{1}$

${ }^{1}$ University of Iceland, ${ }^{2}$ University Hospital of Iceland

The World Health Organization defines a healthy body weight range for adults as a body mass index (BMI) between 18.5 and 24.9. Healthy body weight rage is based primarily on studies in younger adults, for the risks of non-communicable diseases and mortality associated with increased body weight. Examine association in BMI and geriatric health parameters in the Age, Gene/Environment Susceptibility-Reykjavik study (AGES- Reykjavík study) participants.Population of 5696 participants with mean age of $77 y$ in AGES-Reykjavik study in the years of 2002-2006. Geriatric health parameters and mortality were analysed according to BMI tenth tiles.BMI was divided in tenth tiles with lowest tenth tiles with mean BMI $20.0 \pm 1.5$ and highest tenth tiles with mean BMI $35.8 \pm 3.0$, the mean BMI of the population was $27.0 \pm 4.4$. Cox-regression showed significant higher risk of hip-fractur in lowest BMI tenth tiles $(\mathrm{P}<$ $0.001)$ and logistic regression showed significant higher mortality rate $(\mathrm{p}<0.001)$ in lowest BMI tenth tiles corrected for age and gender. Higher BMI was not associated with worse geriatric health parameters in general like serum cholesterol or blood pressure. Low BMI within the healthy range of BMI (18.5-24.9) is associated with higher frequency of hip-fracture and higher mortality rate in Icelandic old adults.

\section{Abstract \# 392 \\ Is prognostic nutritional index useful for evaluation of the nutritional status in elderly patients?}

Guzin Cakmak $^{1}$, Ahmet Cigiloglu ${ }^{1}$, Zeynel Abidin Ozturk ${ }^{1}$

${ }^{1}$ Gaziantep University, Faculty of Medicine, Department of Interal Medicine, Division of Geriatrics

Introduction: Malnutrition is an important geriatric syndrome that can be prevented and reversed. In this study we aimed to evaluate the efficacy of prognostic nutritional index (PNI) in the elderly. We compared PNI with geriatric nutritional risk index (GNRI) according to their relationship with other nutritional tests and frailty indices.

Methods: This cross-sectional study was conducted between September 2019 and December 2019. Malnutrition was assessed by mini nutritional assessment short-form (MNA-SF), long-form (MNALF), Global Leadership Initiative on Malnutrition criteria (GLIM), PNI, and GNRI. Frailty was assessed by using Fried and SOF (Study of Osteoporotic Fractures) criteria. Data analysis was done by SPSS version 22

Results: The study population was composed of 43 women and 35 men, of mean age $72.6 \pm 7.4$ years. According to PNI 8 of them were severely malnourished and 11 of them mildly malnourished. According to GNRI 6 of them had high risk, 6 of them had moderate risk and 5 of them had a low risk for malnutrition. PNI and GNRI were related to each other $(\mathrm{p}<0.001, \mathrm{r}=0.714)$. PNI and GNRI were positively related to MNA-SF, MNA-LF, and negatively related to GLIM. SOF scores were negatively related to PNI and GNRI. Fried scores were only related to GNRI. In linear regression analysis, only GNRI were independently related to MNA-SF and MNA-LF $\left(\mathrm{r}^{2}=\right.$ $0.214, \mathrm{p}=0.007 ; \mathrm{r}^{2}=0.121, \mathrm{p}=0.018$ ).

Key conclusions: The results of this study made us thought that PNI was not as useful as GNRI in the geriatric population. More studies in larger populations could be better.

\section{Abstract \# 393}

Which malnutrition test is more useful in polypharmacy studies? Guzin Cakmak $^{1}$, Zeynel Abidin Ozturk ${ }^{1}$

${ }^{1}$ Gaziantep University Faculty of Medicine, Department of Internal Medicine, Division of Geriatrics

Introduction: Malnutrition is prevalent among older adults and associated with morbidity and mortality. The aim of this study was to investigate the relationship between malnutrition and polypharmacy in the elderly.

Methods: This cross-sectional study was conducted for a period of 4 months between September 2019 and January 2020. Malnutrition was assessed by mini nutritional assessment short-form (MNA-SF), longform (MNA-LF), Global Leadership Initiative on Malnutrition criteria (GLIM), and geriatric nutrition risk index (GNRI). Patients were evaluated for inappropriate medication use by TIME (Turkish Inappropriate Medication Use in the Elderly) criteria. Data analysis was done by SPSS version 22 .

Results: The study population was composed of 57 women and 43 men, of mean age $72.8+/-7.4$ years. Polypharmacy was seen in $14 \%$ of the study population. MNA-SF, MNA-LF and GLIM were correlated with polypharmacy $(\mathrm{p}=0.05 ; 0.023 ; 0.047)$. MNA-LF was associated with polypharmacy in linear regression analysis $\left(\mathrm{r}^{2}=0.079\right.$, $\mathrm{p}=0.005$ ). We demonstrated a cut-off value of 5.5 of drug numbers for carrying the risk of malnutrition according to MNA in the ROC curve (sensitivity $76 \%$; specificity $52 \%$; $\mathrm{CI}=95 \%, \mathrm{p}=0.031$ ). The frequency of inappropriate medicine use was $38 \%$.

Key conclusions: In this study, we revealed that malnutrition was related to polypharmacy. Our study is the first that the relationship between polypharmacy and malnutrition was assessed by GLIM criteria. We designated that MNA-LF influenced polypharmacy independently. That made us thought that MNA-LF was more useful for polypharmacy studies. Prospective studies for evaluating the effect of the number of drugs used on malnutrition could be useful.

\section{Abstract \# 394}

\section{New nutritional index for sarcopenia and sarcopenic obesity}

Guzin Cakmak $^{1}$, Zeynel Abidin Ozturk ${ }^{1}$

${ }^{1}$ Gaziantep University, Faculty of Medicine, Department of Internal Medicine, Division of Geriatrics

Introduction: Geriatric nutritional risk index (GNRI) is an easy-applied tool to assess nutrition. In this study, we aimed to evaluate the efficiency of GNRI for predicting sarcopenia and sarcopenic obesity. We also proposed a new index to predict nutritional and muscle status.

Methods: Patients admitted to polyclinic between January 2018 and January 2019 were evaluated retrospectively. Patients who had a creatinine clearance of less than $60 \mathrm{ml} / \mathrm{min} / 1.73 \mathrm{~m} 2$ were excluded. EWGSOP 2 criteria were used to diagnose sarcopenia. Malnutrition was evaluated by GNRI $[(1.489 \times$ serum albumin $(\mathrm{g} / \mathrm{L}))+(41.7 \times$ (current body weight/ideal weight). We designed a new index, "creatinine x1000/GNRI". We analyzed the correlation of these indices with sarcopenia and sarcopenic obesity. Data analysis was done SPSS version 22 .

Results: The study population was composed of 97 women and 80 men, of mean age $70.3 \pm 6.7$ years. GNRI was negatively correlated with skeletal muscle mass index (SMMI). The new index was positively correlated with handgrip strength, SMM, and SMMI. Fat-mass, fat percent, hip and waist circumference, and body mass index were correlated positively with GNRI, negatively with the new index. In linear regression analysis, GNRI was related to fat-mass, fat percent, 
and BMI $\left(\mathrm{r}^{2}=0.774 ; \mathrm{p}=0.038, \mathrm{p}=0.013, \mathrm{p}<0.001\right)$. New index was related with SMM and SMMI $\left(\mathrm{r}^{2}=0.447 ; \mathrm{p}=0.01, \mathrm{p}<0.001\right)$.

Key conclusions: We revealed that GNRI was correlated with obesity and diminished muscle mass. Contrarily, a new index was inversely related to sarcopenia and sarcopenic obesity. The new index could predict good nutritional and muscle status more efficiently.

\section{Abstract \# 395 \\ Changes in nutrition intake between 2007 and 2017 among older long-term care residents in Helsinki \\ Karoliina Salminen (1) \\ (1) University of Helsinki, Department of General Practice and Primary Health Care}

Introduction: The aim of this study was to explore the changes in nutrition, especially energy and protein intake between 2007-2017 in two cross-sectional cohorts of older long-term care residents in Helsinki. We also studied how the residents' disability and stage of dementia modifies the association between cohort year and protein intake $(\mathrm{g} / \mathrm{kg})$.

Methods: In this cross-sectional study residents' nutrient intake was determined by one- or two days food record in $2007(\mathrm{n}=350)$ and 2017 ( $\mathrm{n}=476)$. Residents' disability was determined by Clinical Dementia Rating (CDR) scale "personal care" question and stage of dementia was determined by CDR.

Results: There was no significant difference in energy intake between the cohort years. Carbohydrate, total protein and protein $\mathrm{g} / \mathrm{kg}$ intakes were significantly lower in 2017 than in 2007. Fat intake was higher in 2017 than in 2007. In 2017 the intake of some vitamins and minerals was reduced (tiamin, calcium), but some increased (vit. A, $\mathrm{D}, \mathrm{C}$, E) compared to 2007. Residents' disability ( $\mathrm{p}=0.049)$ and cohort year $(\mathrm{p}=0.037)$ were significantly associated with protein intake $(\mathrm{g} / \mathrm{kg})$, but the interaction was not significant $(\mathrm{p}=0.35)$. Stage of dementia was not associated with protein intake $(\mathrm{p}=0.22)$, but the cohort year was $(p<0.001)$. The interaction was not significant $(\mathrm{p}=0.30)$.

Conclusions: There have been some improvements in long-term care residents' nutrition during the decade. However, the intake of energy, protein and some vitamins are even lower. As long-term care residents become more disabled in the future, more attention should be paid to nutrition.

\section{Abstract \# 396}

\section{Assessing nutritional status in geriatric patients}

Gyte Damulevičiene ${ }^{1}$, Sandra Starkovaite ${ }^{2}$, Vita Lesauskaité ${ }^{2}$, Jurgita Knašiené ${ }^{2}$

${ }^{1}$ Lithuanian University of Health Sciences (LSMU), ${ }^{2}$ LSMU

Introduction: The aim was to assess the nutritional status of geriatric patients and to determine the suitability of used different malnutrition screening measures.

Methods: 111 geriatric in-patients of Kaunas Clinical Hospital Geriatric Department were enrolled. The MNA, MNA-SF, and NRS 2002 questionnaires were used. The diagnosis and stage of malnutrition were determined based on Global Leadership Initiative on Malnutrition (GLIM) Criteria. Pearson coefficient, t-test, and MannWhitney U test were used.

Results: $67.6 \%$ of patients were women, mean age was $81.3 \pm 7.3$ years. Using the MNA, malnutrition and the risk of malnutrition were found to be 8.7 and $61.2 \%$ respectively. The average score was $21.8 \pm 3.9$. Using the MNA-SF, malnutrition was found to be $25.5 \%$, and the risk of malnutrition was found to be $46.9 \%$, with an average score of $9.8 \pm 2.9$. Malnutrition was found in $46.8 \%$ of patients using the NRS 2002 scale, with a mean score of $2.5 \pm 0.7$. Moderate malnutrition based on phenotypic and etiological diagnostic GLIM criteria was found in $20.0 \%$, severe malnutrition-in $11.4 \%$ of subjects. The sensitivity of the MNA and MNA-SF questionnaires was $97 \%$, and the specificity was 42 and $46 \%$ respectively. The sensitivity of the NRS 2002 questionnaire was $85 \%$, and the specificity was $71 \%$. Conclusions: The prevalence of malnutrition among geriatric patients was determined using different malnutrition screening questionnaires - the results differed significantly. Based on GLIM criteria, malnutrition was found in $29.7 \%$ of geriatric in-patients. The MNA and MNA-SF were the most sensitive in diagnosing malnutrition, but NRS 2002 was the most specific.

\section{Abstract \# 397}

Evaluation of oropharyngeal dysphagia in geriatric in-patients

Gyte Damulevičiene $\dot{1}^{1}$, Evelina Pikčiūnaite ${ }^{2}$, Raimonda Putnaite $\dot{e}^{2}$, Mantas Juozaitis ${ }^{2}$, Vita Lesauskaite ${ }^{2}$

${ }^{1}$ Lithuanian University of Health Sciences (LSMU), ${ }^{2}$ LSMU

Introduction: There is no data on the prevalence of oropharyngeal dysphagia (OD) in Lithuania. The usual diagnostic method is a water drinking sample taken at the patient's bedside. The worldwide questionnaire EAT-10 is still rarely used in Lithuania. The aim of this study was to evaluate the prevalence of OD in geriatric in-patients and to determine the sensitivity and specificity of the swallowing disorder screening tool EAT-10.

Methods: 353 geriatric in-patients of Kaunas Clinical Hospital Geriatric Department were enrolled. The Lithuanian version of the EAT-10 was used. The EAT-10 score $\geq 3$ was defined as suspected dysphagia. A clinical water drinking sample was performed in order to justify the diagnosis of OD. Data was analysed with ANOVA, Pearson coefficient, and Student's t-test.

Results: $71.1 \%$ of patients were women, mean age was $82.0 \pm 7.4$ years. The mean EAT-10 score was $2.6 \pm 4.3$. Cronbach's alpha for the EAT-10 questionnaire was 0.888 ; as patient age increased, the reliability of the questionnaire decreased to 0.882 . EAT- 10 score $\geq 3$ was observed in $32.9 \%$ of patients. Bedside water drinking test was positive in $10.2 \%$ of patients. No statistically significant differences were found in EAT-10 and bedside test among age and sex groups. The EAT-10 tool had a sensitivity value of $94.4 \%$, and a specificity value of $74.1 \%$.

Conclusions: One third of geriatric in-patients were suspected of having dysphagia. However, the prevalence of OD based on the water drinking test was only $10.2 \%$. A high EAT-10 value of sensitivity and an insufficient value of specificity were determined.

\section{Abstract \# 398 \\ EuGMS-2020 The anticholinergic burden is associated with malnutrition risk in older adults: a cut-off point for the nutritional management}

Bilal Katipoglu ${ }^{1}$, Ilker Tasci ${ }^{2}$, Mehmet Ilkin Naharci ${ }^{1}$

${ }^{1}$ Division of Geriatrics, University of Health Sciences, Gulhane Faculty of Medicine and Gulhane Training and Research Hospital, Ankara, Turkey., ${ }^{2}$ Department of Internal Medicine, University of Health Sciences, Gulhane Faculty of Medicine and Gulhane Training and Research Hospital, Ankara, Turkey.

Introduction: Drugs with anticholinergic properties use are common in the elderly despite growing evidence of their adverse outcomes. 
Information is scarce concerning the impact of anticholinergic exposure on nutritional status in this population. Therefore, we evaluated the association between anticholinergic burden and the malnutrition risk in community-dwelling older adults.

Methods: We enrolled a total of 470 subjects who had undergone comprehensive geriatric assessment (CGA) between 2017 and 2019 years in this cross-sectional study. Nutritional status was checked by the Mini Nutritional Assessment-Short Form (MNA-SF) (The malnutrition risk: a score of $11 / 14$ or lower) [1]. Each participant's anticholinergic burden was evaluated using the Anticholinergic Cognitive Burden (ACB) scale [2].

Results: The mean age of the patients was $77.6 \pm 6.7$ years, and $64.9 \%$ (n: 305) were female. The prevalence of malnutrition risk and no malnutrition were 20\% (n: 94) and 80\% (n: 376). An ACB score of 1 or greater was higher in the malnutrition risk group than controls ( $46.8 \%$ vs. $31.6 \%, p=0.006)$. Multivariate analysis showed a 1.207 fold increase in the malnutrition risk among adults taking one or more anticholinergics independent of age, gender, and comorbidities (OR: 1.207 95\%, CI:1.006-1.448; p=0.043). Analysis of the ROC curves reveal that, optimal cut-off value for ACB score was found 1 for malnutrition (AUC:0.703 \pm 0.114 , sensitivity:0.625, specificity:0.658; $\mathrm{p}=0.049$ ).

Conclusions: Drugs with anticholinergic properties identified by the ACB scale are associated with the risk of malnutrition, which could lead to many adverse outcomes in older adults. Further studies are necessary to address the causality relationship between anticholinergics and impaired nutrition.FundingThis study was supported by the department of the Scientific Research Projects of University of Health Sciences, Turkiye (Project No. 2017/007).

References: 1. Rubenstein, Laurence Z., et al. "Screening for undernutrition in geriatric practice: developing the short-form mininutritional assessment (MNA-SF)." The Journals of Gerontology Series A: Biological Sciences and Medical Sciences 56.6 (2001): M366-M372. 2. Carnahan, R.M., Lund, B.C., Perry, P.J., Pollock, B.G., Culp, K.R., 2006. The anticholinergic drug scale as a measure of drug-related anticholinergic burden: associations with serum anticholinergic activity. The Journal of Clinical Pharmacology 46, $1481-1486$.

\section{Abstract \# 399 \\ How do older people perceive appetite and its loss? A qualitative study \\ Natalie J Cox ${ }^{1}$, Kinda Ibrahim ${ }^{1}$, Leanne Morrison ${ }^{1}$, Sian M Robinson $^{2}$, Helen C Roberts ${ }^{3}$ \\ ${ }^{1}$ University of Southampton, UK, ${ }^{2}$ Newcastle University, UK, ${ }^{3}$ University of Southampton}

Background: Appetite loss in older people is common and associated with malnutrition, sarcopenia, frailty and mortality. Effective management of appetite loss may prevent these major health burdens, but no current guidance exists. This is in part due to a lack of understanding of how older individuals experience appetite loss.Aim: To understand older individual's perceptions of appetite and appetite loss.

Methods: Qualitative research with men and women aged $\geq 65$ years, in their own home. Semi-structured interviews were audiorecorded and transcribed. Reflexive thematic analysis, with inductive coding, generated themes with data examples.

Results: 13 participants ( 8 female, 4 living alone). Perceptions of appetite and appetite loss were described in two ways and valued differently by individuals. 'Appetite as an experience' encompassed escalating positive thoughts and feelings, driving a desire to eat. Then, appetite loss as struggling to initiate eating due to negative thoughts and feelings. 'Appetite as a physical function' encompassed physical descriptions of bodily need, without thought or feeling. Then, appetite loss as a continued ability to initiate eating but with reduced capacity from fullness.Perceptions of appetite and appetite loss shaped individual's adaptations to poor appetite. Either seeking out desirable food (appetite as an experience) or having smaller meals (appetite as a physical function).

Conclusions: Appetite and appetite loss are perceived by older individuals in distinct ways. Importantly these viewpoints affect individuals' adaptation to poor appetite. This highlights a need for novel person-centred interventions for appetite loss, based on understanding of the individual's experience.

\section{Abstract \# 400 \\ Post-stroke dysphagia: swallowing and nutritional status after hospital discharge}

Eirini Stratidaki ${ }^{1}$

${ }^{1}$ Hellenic Mediterranean University

Eirini Stratidaki1, Stefania Schetaki1, Antonia Aravantinou-Karlatou1, Evridiki Patelarou1, Ioannis Savvakis1, Theodula Adamakidou2, Athina Patelarou1, Christos Kleisiaris1,1 Department of Nursing, Faculty of Health Sciences, Hellenic Mediterranean University, Heraklion, Crete, Greece 2Department of Nursing, Faculty of Health Sciences, University of West Attica, Athens, Crete, Greece

Introduction: It is widely recognized that malnutrition is one of the most common complications in stroke survivals post-stroke dysphagia due to dysphagia. However, the long-term effect on the rehabilitation of these patients has not fully investigated[1,2]

Methods: In this prospective study we enrolled (vascular) stroke patients hospitalized at two Hospitals in Heraklion Crete, Greece. Patients were clinically monitored for a month (hospital discharge [baseline] to a monthly follow-up). The National Institutes of Health Stroke Scale (NIHSS) was used to assess the neurological status of patients and, the Mann Assessment of Swallowing Ability-MASA (Mann) scale for the assessment of dysphagia. Also, the Eating Assessment Tool (EAT-10) was used to assess their ability to eat and drink for 1 month.

Results: We studied 60 stroke patients $(61,7 \%$ female). Between baseline measure and a month later (follow-up) it was observed significant improvements in neurological status -NIHSS (1st 10,6 $\pm 8,1$ vs. 2nd $6,3 \pm 8,1, \mathrm{p}<0,001)$. On the contrary, stroke patients were presented with significantly decreased homebound status during their rehabilitation ( 1 st $2,2 \pm 1,3$ vs. 2 nd $1,0 \pm 1,2, \mathrm{p}<0,001$ ). Also, stroke survivors presented with significantly less dysphagia and/or improved swallowing ability - MMASA (1st $75.9 \pm 24.4$ vs. 2nd $84.8 \pm 20.2$, $\mathrm{p}<0,001)$. Similarly, our study sample was presented also with fewer dysphagia problems such as feeding/rehydration - EAT scale (1st $20.7 \pm 17.2$ vs. 2 nd $16.4 \pm 16.3$, $\mathrm{p}<0,002$ ).

Key conclusion: Our data confirm the existing implications of stroke mainly the dysphagia problem as an important risk factor for malnutrition in our sample. It is obvious therefore that health professionals must focus on comprehensive clinical assessment as best practice for post-acute rehabilitation.

References: 1. Meshia, JF, Bushnell, C., Boden-Albala, B., Braun, LT, Bravata, DM, Chaturvedi, S., Creager, MA, Eckel, RH, Elkind, MSV, Fornage, (2014) Guidelines for the Primary Prevention of Stroke: A Statement for Healthcare Professionals From the $\tau \eta v$ American Heart Association /American Stroke Association. Stroke 45. 2. Guyomard V, Fulcher RA, Redmayne O, Metcalf AK, Potter JF, Myint PK, (2009) Effect of dysphasia and dysphagia on inpatient 
mortality and hospital length of stay: a database study. J Am Geriatr Soc. (57), 2101-6

\section{Abstract \# 401 \\ Post-stroke dysphagia: swallowing and nutritional status after hospital discharge}

Eirini Stratidaki ${ }^{1}$, Stefania Schetaki ${ }^{1}$, Antonia Aravantinou-Karlatou (1), Evridiki Patelarou (1), Ioannis Savvakis (1), Theodula Adamakidou (1), Athina Patelarou (1), Christos Kleisiaris (1)

(1) Hellenic Mediterranean University

Eirini Stratidaki1, Stefania Schetaki1, Antonia Aravantinou-Karlatou1, Evridiki Patelarou1, Ioannis Savvakis1, Theodula Adamakidou2, Athina Patelarou1, Christos Kleisiaris1,1 Department of Nursing, Faculty of Health Sciences, Hellenic Mediterranean University, Heraklion, Crete, Greece 2Department of Nursing, Faculty of Health Sciences, University of West Attica, Athens, Crete, Greece

Introduction: It is widely recognized that malnutrition is one of the most common complications in stroke survivals post-stroke dysphagia due to dysphagia. However, the long-term effect on the rehabilitation of these patients has not fully investigated $[1,2]$.

Methods: In this prospective study we enrolled (vascular) stroke patients hospitalized at two Hospitals in Heraklion Crete, Greece. Patients were clinically monitored for a month (hospital discharge [baseline] to a monthly follow-up). The National Institutes of Health Stroke Scale (NIHSS) was used to assess the neurological status of patients and, the Mann Assessment of Swallowing Ability-MASA (Mann) scale for the assessment of dysphagia. Also, the Eating Assessment Tool (EAT-10) was used to assess their ability to eat and drink for 1 month.

Results: We studied 60 stroke patients $(61,7 \%$ female). Between baseline measure and a month later (follow-up) it was observed significant improvements in neurological status -NIHSS (1st 10,6 $\pm 8,1$ vs. 2nd $6,3 \pm 8,1, \mathrm{p}<0,001)$. On the contrary, stroke patients were presented with significantly decreased homebound status during their rehabilitation ( 1 st $2,2 \pm 1,3$ vs. 2 nd $1,0 \pm 1,2, \mathrm{p}<0,001$ ). Also, stroke survivors presented with significantly less dysphagia and/or improved swallowing ability - MMASA (1st $75.9 \pm 24.4$ vs. 2nd $84.8 \pm 20.2$, $\mathrm{p}<0,001)$. Similarly, our study sample was presented also with fewer dysphagia problems such as feeding/rehydration - EAT scale (1st $20.7 \pm 17.2$ vs. 2 nd $16.4 \pm 16.3, \mathrm{p}<0,002$ ).

Key conclusion: Our data confirm the existing implications of stroke mainly the dysphagia problem as an important risk factor for malnutrition in our sample. It is obvious therefore that health professionals must focus on comprehensive clinical assessment as best practice for post-acute rehabilitation.

References: 1. Meshia, JF, Bushnell, C., Boden-Albala, B., Braun, LT, Bravata, DM, Chaturvedi, S., Creager, MA, Eckel, RH, Elkind, MSV, Fornage, (2014) Guidelines for the Primary Prevention of Stroke: A Statement for Healthcare Professionals From the $\tau \eta v$ American Heart Association /American Stroke Association. Stroke 45. 2. Guyomard V, Fulcher RA, Redmayne O, Metcalf AK, Potter JF, Myint PK, (2009) Effect of dysphasia and dysphagia on inpatient mortality and hospital length of stay: a database study. J Am Geriatr Soc. (57), 2101-6

\section{Abstract \# 402}

Multiple electrolyte deficiencies are more common in the elderly

Antonino Catalano ${ }^{1}$, Diego Chilà ${ }^{1}$, Federica Bellone ${ }^{1}$, Loddo Saverio $^{1}$, Nunziata Morabito ${ }^{1}$, Giorgio Basile ${ }^{1}$, Salvatore Benvenga ${ }^{1}$, Francesco Corica ${ }^{1}$

${ }^{1}$ Department of Clinical and Experimental Medicine, University Hospital of Messina, Messina, Italy

Introduction: Electrolites disturbances are often observed in older people. The aim of this study was to recognize the prevalence of combined low serum concentrations of calcium, magnesium and potassium in the real life.

Methods: This retrospective study was based on admission data from out-patients and in-patients whose serum levels of magnesium, calcium and potassium were simultaneously measured from a single centralized laboratory at the University Hospital of Messina, Messina (Italy), between January 1, 2015 and December 31, 2017, according to standard methods. Age and gender as well as estimated kidney function calculated by CKD-EPI (Chronic Kidney Disease Epidemiology Collaboration) formula were considered.

Results: Out of 8,108 patients, hypocalcemia, hypomagnesemia and hypokalemia accounted in 377,305 and 108 subjects, respectively. Combined deficits raged from 0.29 to $0.65 \%$. Hypomagnesemia and hypocalcemia simultaneously appeared in $n=53$ patients; of these, $\mathrm{n}=18$ ( $\mathrm{n}=9$ males, $\mathrm{n}=9$ females) were under 65 -year-old and $\mathrm{n}=35$ $(\mathrm{n}=13$ males; $\mathrm{n}=22$ females, $\mathrm{p}=0.05)$ over 65-year-old $(\mathrm{p}=0.001)$. Hypomagnesemia and hypokalemia were found simultaneously in $\mathrm{n}=24$ patients; of these, $\mathrm{n}=11$ ( $\mathrm{n}=9$ males, $\mathrm{n}=2$ females; $\mathrm{p}=0.008)$ were under 65 -year-old, while $n=13$ ( $n=5$ male; $n=8$ females) over 65 -yearold. Association between hypocalcemia and hypokalemia was found in $n=51$ patients, of which $n=20 \quad(n=9$ males; $n=11$ females $)$ were under 65-year-old and $n=31$ ( $n=16$ males; $n=15$ females) over 65 -year-old ( $\mathrm{p}=0.04)$, Kidney failure has been found in most cases of multiple electrolyte deficiencies.

Key conclusions: Low concentrations of serum electrolytes are more often combined in the elderly. Kidney failure is a condition associated with multiple electrolyte deficiency.

\section{Abstract \# 403 \\ No nutritional intervention despite malnutrition; no malnutrition and yet nutritional intervention? An exploration in the nursing home setting}

Franz Großhauser ${ }^{1}$, Eva Kiesswetter ${ }^{1}$, Gabriel Torbahn ${ }^{1}$, Cornel C. Sieber $^{1}$, Dorothee Volkert ${ }^{1}$

${ }^{1}$ Institute for Biomedicine of Aging, Friedrich-Alexander-Universität Erlangen-Nürnberg, Nuremberg, Germany

Rationale: Little is known about the selection of nursing home residents receiving nutritional interventions (NI). This study investigated the use of NI and corresponding main reasons in residents with or without malnutrition.Methods: 246 residents from 7 German nursing homes were examined within the framework of nutrition Day. Malnutrition was defined as BMI $<20 \mathrm{~kg} / \mathrm{m}^{2}$ or weight loss $\geq 5 \%$ in last three months or low dietary intake (plate diagram $<1 / 2$ ). The use of NI (enriched meals and/or oral nutritional supplements [ONS]) and corresponding reasons (open questions) were elicited in interviews with caregivers.

Results: Of 80 malnourished residents 44 received NI. Enriched meals were offered for the following reasons: low intake $14 x$, weight loss (WL) $5 \mathrm{x}$, low BMI 4x, poor nutritional status $2 \mathrm{x}$, tumour $2 \mathrm{x}$. ONS were provided due to low intake $1 \mathrm{x}$, poor nutritional status $1 \mathrm{x}$. Both NI were offered on account of low intake 7x, WL 7x, tumour 1x. 
Causes against NI in 36 malnourished residents were: awaiting further weight trends $10 x$, satisfactory weight $6 x$, adequate BMI $6 x$, not judged as malnourished $5 \mathrm{x}$, a living will $5 \mathrm{x}$, refusal by legal guardian $1 \mathrm{x}$, sufficient appetite $1 \mathrm{x}$, terminal situation $1 \mathrm{x}$, awaiting results of dietary records 1 x. 29 residents without malnutrition were given enriched meals $23 \mathrm{x}$, ONS $1 \mathrm{x}$, or both $5 \mathrm{x}$ for the following reasons: unsatisfactory intake $15 x$, BMI $>20<235 x$, WL $4 x$, no explanation $3 \mathrm{x}$, tumour $2 \mathrm{x}$.

Conclusion: A huge discrepancy between residents being malnourished and those receiving NI was revealed, which can partially - but not always - be explained by valid reasons.

\section{Abstract \# 404 \\ The association between nutritional status and functional status of community-dwelling older adults}

Teresa Madeira ${ }^{1}$, Carla Lopes ${ }^{1}$, João Gorjão Clara ${ }^{1}$

${ }^{1}$ Instituto de Medicina Preventiva e Saúde Pública, Faculdade de Medicina, Universidade de Lisboa; Instituto de Saúde Ambiental, Faculdade de Medicina, Universidade de Lisboa. Lisbon, Portugal, ${ }^{2}$ EPIUnit - Instituto de Saúde Pública da Universidade do Porto; Departamento de Ciências da Saúde Pública e Forenses e Educação Médica, Unidade de Epidemiologia da Faculdade de Medicina da Universidade do Porto. Porto, Portugal, ${ }^{3}$ Instituto de Medicina Preventiva e Saúde Pública, Faculdade de Medicina, Universidade de Lisboa; Instituto de Saúde Ambiental, Faculdade de Medicina, Universidade de Lisboa. Lisbon, Portugal.

Introduction: A good functional status is a key factor for healthy ageing, and nutrition has an impact on functionality. The aim of this study was to examine the association between nutritional status and instrumental functional status in community-dwelling older adults $(\geq$ 65 years old).

Methods: This study $(\mathrm{n}=928)$ analysed data from a wider project, the PEN-3S, that included a nationally representative sample of Portuguese older adults. Nutritional status was assessed by the 18 -item Mini Nutritional Assessment (MNA $\left.{ }^{\circledR}\right)$, body mass index (BMI), and waist circumference. Functional status for instrumental activities of daily living (IADLs) was assessed with the Lawton scale (no limitations $>7$ points). Logistic regression models using complex samples analysis (SPSS $\left.{ }^{\circledR} 24.0\right)$ were performed.

Results: The estimated prevalence of functional limitations was $19.9 \%$ (95\% CI 15.9, 24.5) for the ' $<75$ years' group, $35.6 \%$ (IC $95 \%$ $27.9,44.1$ ) for the ' $75-84$ ' age group, and $45.2 \%$ (95\% CI 19.1, 74.1) in the oldest group. Waist circumference $(\mathrm{OR}=1.03,95 \% \mathrm{CI} 1.00$, $1.05)$ and MNA score $(\mathrm{OR}=0.83,95 \% \mathrm{CI} 0.76,0.90)$, but not BMI, were significantly associated with functional status after adjusting for sex, age, and education. When models were further adjusted for selfreported health, only the association with $\mathrm{MNA}(\mathrm{OR}=0.90,95 \% \mathrm{CI}$ $0.82,0.98)$ remained statistically significant.

Key conclusions: A better nutritional status as assessed by MNA, but not BMI or waist circumference alone, is associated with less functional impairment. Therefore, MNA seems to be more clinically relevant than BMI for older adults' functional status.

\section{Abstract \# 405 \\ Assessment of disease related malnutricion(drm) in geriatric patients: GLIM criteria.}

Rocio Onieva ${ }^{1}$, Julia Castillo ${ }^{1}$, Carmen Cánovas ${ }^{1}$, Sofía Solsona ${ }^{1}$, Beatriz Monterde ${ }^{1}$, Genoveva Labari ${ }^{1}$, Eduina Pérez ${ }^{1}$

${ }^{1}$ Hospital Nuestra Señora de Gracia, Zaragoza
Introduction: Hospital malnutrition in geriatric patients is a prevalent problem that needs to be approached in a priority manner. In this assessment, the new GLIM criteria have been acknowledged, endorsed for the general population.

Objectives: To know the prevalence of malnutrition in our patients. To identify whether GLIM criteria are an effective tool in our patients.METHODOLOGY: Cross-sectional descriptive study in hospitalized patients in two days in January/February 2020. Personal history, biochemical, anthropometric data, intake, MNA and GLIM were collected. SPSS.

Results: 73 patients, $73.2 \%$ women, average age: 88 years (SD 6.15). Barthel.I $<20$ : $29.7 \%$. Cholesterol $<150: 45.5 \%$. Albumin $<3: 18.5 \%$. According to GLIM criteria, 58.6\% malnourished. According to MNA, $43.37 \%$ malnourished and at risk $42 \% .52 .4 \%$ suffered chronic disease malnutrition. Phenotypic criteria: 52\% did not know their usual weight. For this reason, only the involuntary weight loss could be calculated. BMI $<22: 31.5 \%$. Size/Length elbow-wrist: $69.86 \%$. Brachial circumference $(\mathrm{BC})<21 \mathrm{~cm}: 15 \%$, calf circumference $(\mathrm{CC})$ $<31 \mathrm{~cm}: 64.38 \%$. Etiological criteria: Possible reduction of the usual intake: $33.8 \%$. Inflammatory load in the hospital little appraisable: $56 \%$.

Key conclusions: GLIM is a less useful tool in the dependent geriatric patient, taking into account the absence of real recording of criteria. Biochemical markers are not valid as nutritional parameters during hospitalization as they are known to be modified in acute processes. BC in postmenopausal women underestimates the loss of muscle mass by greater accumulation of adipose tissue. The use of CC is prefered. MNA provides our patients a better evaluation and more complete nutritional, functional, dietetic and anthropometric information.

\section{Abstract \# 406 \\ Simplified Nutritional Assessment Questionnaire to screen for anorexia: optimal cutoff and association with outcomes in non-frail community dwelling older adults}

Wee Shiong Lim ${ }^{1}$, Sabrina Lau ${ }^{2}$, Justin Chew ${ }^{2}$, Jun Pei Lim², Kalene Pek $^{1}$, Noor Hafizah ${ }^{3}$, Yew Yoong Ding ${ }^{2}$

${ }^{1}$ Institute of Geriatrics and Active Aging, Tan Tock Seng Hospital, ${ }^{2}$ Department of Geriatric Medicine, Tan Tock Seng Hospital, ${ }^{3}$ Department of Continuing and Community Care, Tan Tock Seng Hospital

Introduction: Anorexia is commonly reported by older people and portends malnutrition and adverse outcomes. The 4-item Simplified Nutritional Appetite Questionnaire (SNAQ) predicted weight loss and adverse outcomes in outpatient and inpatient settings at cutoff of $\leq 14$. We aimed to establish optimal cutoff of SNAQ and association with outcomes in non-frail community-dwelling older adults.

Methods: This is a cross-sectional study of 230 participants (mean age 67.2 years; $72.6 \%$ females) who were non-frail by FRAIL criteria. We performed receiver operating characteristic curve analysis for optimal cutoff of malnutrition risk via Mini-Nutritional Assessment. Using linear regression adjusted for age and gender, we determined the association of anorexia with International Physical Activity Questionnaire (IPAQ) and Frenchay Activities Index (FAI); Life Space Assessment (LSA); 15-item Geriatric Depression Scale (GDS); Social Frailty Scale (SFS-8); and modified Fried criteria for physical frailty.

Results: The optimal cutoff for malnutrition risk was 15 (Area under curve $=0.71, \quad 95 \%$ CI: $0.56-0.85, \quad$ Sensitivity $=69.2 \%$, Specificity $=61.8 \%)$. SNAQ cutoffs of $\leq 15$ and $\leq 14$ identified $93(40.4 \%)$ and $42(18.3 \%)$ participants respectively with anorexia. SNAQ cutoff $\leq 15$ was associated with physical activity measured using IPAQ 
(beta=-.183, p=.005) and FAI (beta= -.140, p=.025); life space mobility (beta $=-1.93, \mathrm{p}=.003$ ); depressive symptoms (GDS, beta $=.219, \mathrm{p}=.001$ ); social frailty (SFS-8, beta=.209, $\mathrm{p}=.001$ ); and physical frailty (beta=.132, $\mathrm{p}=.033$ ), compared with life space mobility (beta $=-.165, \mathrm{p}=.012$ ) and GDS (beta=.189, $\mathrm{p}=.004$ ) only for $\leq 14$.

Conclusion: Our results support the use of SNAQ to screen for anorexia amongst non-frail community-dwelling older adults. Using a higher cutoff improves detection and is associated with relevant outcomes.

\section{Abstract \# 407 \\ Correlation between minerals and weight change in patients older than 65 \\ Athina Greka ${ }^{1}$ \\ ${ }^{1}$ EEMEG}

Abstract: In the present study, the weight loss ratio of people over 65 years of age was studied in relation to their muscle mass.Screening test: laboratory profiles a targeted diet exercise program in conjunction with their medication.

Introduction: Over a three months period, 30 patients aged over 65 were examined. (None with pacemaker) All the patients followed. Diet based: mix 50\% carbohydrates, $30 \%$ protein and $20 \%$ fat. Proper hydration: more than 2 liters of water daily. A light exercise program for torso and main body and walking for a period of 40 minutes/ daily. Solar exposure for more than 20 minutes per day in average Method: BIA body consistency analysis DSM- BIA Method (Direct Segmental Multi-frequency - Bioelectrical Impedance Analysis) Measurement data: Body Composition Analysis: Body weight is the sum of Total Body Water, Protein, Minerals and Body Fat MassAfterwards the patient's situation was reassessed monthly and the progress was put down on progress reports which included the measurement according to date for height, weight, body mass, minerals and fat percentage.

Results: Weight change $67 \%$ reduced their weight and increased minerals $33 \%$ increased their weight.

Conclusion: Minerals do not have an increasing tendency in old age. However, weight loss with a studied diet, exposure to sunlight and exercise, help the elderly to increase the percentages of inorganic components and slow down the reduction of bone metabolism.

Keys: Minerals, Weight, BIA

\section{Abstract \# 408}

\section{Nutritional assessment in a Portuguese orthogeriatric unit}

Luciana Silva $^{1}$, Mariana Santos ${ }^{1}$, Raquel Moura ${ }^{1}$, Agripino Oliveira ${ }^{1}$, Rafaela Veríssimo ${ }^{1}$

\section{${ }^{1}$ Centro Hospitalar de Vila Nova de Gaia e Espinho}

Introdution: Malnutrition is a well-known syndrome in geriatric patients, associated with serious health problems and poor outcome. Studies suggest that $20-65 \%$ patients suffer from nutritional deficiencies and it's very prevalent in geriatric patients with hip fracture. Nevertheless, its importance is not fully recognized [1-3].

Methods: This study includes all patients with neck femur fractures admitted in an orthogeriatric unit in one year. The data included were: age, frailty score(FS), KATZ scale, comorbidities, nutritional assessment, length of stay and mortality. Were excluded all patients who didn't have any nutritional assessment.The nutritional assessment was made using the Nutritional Risk Screening 2002(NRS) which identifies patients at risk of malnutrition.[4]
Results: Were admitted 114 patients, $58.8 \%(n=67)$ submitted to a nutritional evaluation. The majority were female, $77.6 \%(n=52)$, with an average age of 84 years. The FS more prevalent at admission was 6 $(34.3 \% \mathrm{n}=23)$ and KATZ level was A, 31.3\% $(\mathrm{n}=21)$. The mean length of stay was 19.7 days. The BMI adjusted to age $<20.5$ prevalence was $22.4 \%(n=15)$ and $46.3 \%(n=31)$ had a NRS $>3$. In patients with FS $\leq 5$ the prevalence of NRS $>3$ was $40 \%$ compare to $55.5 \%$ in FS $>5$.

Key conclusions: This study reveals that almost half of the patients are at risk of malnutrition, should initiate a personalized diet and nutrition supplements. The FS and malnutrition seem to be correlated and could lead to a worst outcome. If we miss a nutritional assessment, it could mean that a significant number of patients will lose treatment strategies and have a higher risk of worst outcome.[1,5-6] References: 1. Wells J, Dumbrell A. Nutrition and aging: assessment and treatment of compromised nutritional status in frail elderly patients. Clinical Interventions in Aging. 2006;1(1):67-79. 2. Elmståhl S, Persson M, Andren M, Blabolil V. Malnutrition in geriatric patients: a neglected problem?. Journal of Advanced Nursing. 1997;26(5):851-855. 3. Malafarina V, Reginster J, Cabrerizo S, Bruyère $\mathrm{O}$, Kanis $\mathrm{J}$, Martinez $\mathrm{J}$ et al. Nutritional Status and Nutritional Treatment Are Related to Outcomes and Mortality in Older Adults with Hip Fracture. Nutrients. 2018;10(5):555. 4. Hersberger L, Bargetzi L, Bargetzi A, Tribolet P, Fehr R, Baechli V et al. Nutritional risk screening (NRS 2002) is a strong and modifiable predictor risk score for short-term and long-term clinical outcomes: secondary analysis of a prospective randomised trial. Clinical Nutrition. 2019; 5. Wei K, Nyunt M, Gao Q, Wee S, Yap K, Ng T. Association of Frailty and Malnutrition With Long-term Functional and Mortality Outcomes Among Community-Dwelling Older Adults. JAMA Network Open. 2018;1(3):e180650. 6. Volkert D, Saeglitz C, Gueldenzoph H, Sieber $\mathrm{C}$, Stehle P. Undiagnosed malnutrition and nutrition-related problems in geriatric patients. The journal of nutrition, health \& aging. 2010;14(5):387-392.

\section{Abstract \# 409 \\ Using GLIM to compare the impact of malnutrition in an Internal Medicine Ward by age group}

Ines Rego Figueiredo ${ }^{1}$, Marilia Fernandes ${ }^{1}$, Anna Taulaigo ${ }^{2}$, Maria Saldanha $^{3}$, Carolina Baltazar ${ }^{4}$, Emanuel Noivo ${ }^{4}$

${ }^{1}$ Consulta Multidisciplinar de Geriatria, Hospital Curry Cabral, Centro Hospitalar Universitário Lisboa Central, ${ }^{2}$ Serviço de Medicina Interna 7.2, Hospital Curry Cabral, Centro Hospitalar Universitário Lisboa Central, ${ }^{3}$ Unidade de Nutrição, H Curry Cabral, Centro Hospitalar Universitário Lisboa Central), (4) NOVA Medical School, Universidade Nova de Lisboa

Background: The Global Leadership Initiative on Malnutrition (GLIM) proposed in 2019 a consensus for diagnosing malnutrition in adults in clinical settings. First screening "at risk" status using a validated screening tool, second diagnosing and assessing the severity of malnutrition. The new consensus was used to assess the impact of malnutrition in length of stay, readmission and mortality in an acute medicine ward.

Methods: Prospective study of elderly patients admitted to an internal medicine ward, for 6 months, willing to participate (signed informed consent). Demographic and clinical data were obtained from the electronic file, and antropometric data (height, weight, midarm diameter and nutritional history) from the patient. Nutritional data were analyzed with the GLIM Consensus and MUST for "at risk" screening, and patients divided by diagnosis of malnutrition. The assessed outcomes were length of stay, readmission at 1 month and mortality. Statistical analysis was performed using STATA (StataCorp. Stata statistical software: release 14. College Station, TX: 
StataCorp LP). A p value of $<0.05$ was considered significant. Parametric data were expressed as mean (standard error of the mean, SEM), non-parametric data as median (interquartile range, IQR). Statistical tests for univariate analysis were T-student and chi square and for multivariate analysis logistic regression.

Results: A total of 30 patients were included, of whom 10 (33\%), had a diagnosis of malnutrition using the GLIM criteria. Age (79 $\pm 8,5$ vs $82 \pm 7,3$, p-value $=0,35)$ and female gender predominance $(50 \%$, p-value $=1$ ) was similar in both groups. Malnourished patients exhibited a slight tendency to infection as diagnosis at admission (40 vs $15 \%$, p-value $=0.07$ ), not statistically significant due to similarity in other diagnosis. Both groups had similar levels of comorbidity burden by the Charlson index $(4 \pm 2,4$ vs $5,3 \pm 3,8$, p-value $=0.3)$, dependence by the Lawton\&Brody scale $(4,7 \pm 2,3$ vs $4.3 \pm 3,3$, p-value $=0.6)$ and frailty by PRISMA7 $(3,2 \pm 1,8$ vs $2,8 \pm 2$, p-value $=0,6)$. Considering the outcomes, there was only a difference in mortality at 6 months $(10$ vs $40 \%$ for the malnourished, p-value $=0.05$ ), with similar length of stay $(10,7 \pm 5,7$ vs $29 \pm 85$, p-value $=0.09)$, readmission ( 40 vs $20 \%$, $\mathrm{p}$-value $=0.2)$ and in-admission mortality $(10$ vs $20 \%$, p-value $=0.4)$.

Discussion: Despite the small size sample, we observed 1/3 of malnourished patients, that did not seem to differ from the other in terms of demographic and geriatric features. The higher impact appears to be on medium-term mortality.

\section{Abstract \# 410 \\ GLIM used to estimate the prevalence of malnutrition in an Internal Medicine Ward}

Ines Rego Figueiredo ${ }^{1}$, Marilia Fernandes ${ }^{1}$, Anna Taulaigo ${ }^{2}$, Maria Saldanha $^{3}$, Carolina Baltazar ${ }^{4}$, Emanuel Noivo ${ }^{4}$, Heidi Gruner ${ }^{1}$

${ }^{1}$ Consulta Multidisciplinar de Geriatria, Hospital Curry Cabral, Centro Hospitalar Universitário Lisboa Central, ${ }^{2}$ Serviço de Medicina Interna 7.2, Hospital Curry Cabral, Centro Hospitalar Universitário Lisboa Central, ${ }^{3}$ UNidade de Nutrição Centro Hospitalar Universitário Lisboa Central, ${ }^{4}$ NOVA Medical School, Universidade Nova de Lisboa

Background: Malnutrition is an often unrecognized and untreated cause and consequence of disease in adults. It has a substantial impact on health across all care settings, including the Hospital setting.The Global Leadership Initiative on Malnutrition (GLIM) recently proposed a consensus scheme for diagnosing malnutrition in adults in clinical settings on a global scale. It is a two-step approach, first screening to identify "at risk" status using a validated screening tool, and second, assessment for diagnosis and grading of the severity of malnutrition. Our aim is to use this new consensus to estimate the prevalence of malnutrition in the elderly in an acute medicine ward. Methods: Cross sectional study performed at an internal medicine ward, throughout 4 months, to patients willing to participate following signed informed consent. Demographic and clinical data gathered from the electronic file, and antropometric data (height, weight, midarm diameter and nutritional history) collected from the patient. Nutritional data was analysed according to the GLIM consensus together with MUST for at risk screening. Patients were divided into 2 age groups ( $<65$ and $>65$ years old). The outcome was prevalence of malnutrition. Statistical analysis was performed using STATA (StataCorp. Stata statistical software: release 14. College Station, TX: StataCorp LP). A $p$ value of $<0.05$ was considered significant. Parametric data were expressed as mean (standard error of the mean, SEM), non-parametric data as median (interquartile range, IQR). Statistical tests for univariate analysis were T-student and chi square and for multivariate analysis logistic regression.

Results: A total of 44 patients were included, of those $30(68 \%)$ were elderly, with a prevalence of the female gender (64 and 50\%, under and over 65 respectively). The main causes for admission were similar between young and elderly: cardiovascular (29 vs 39\%), infectious (14 vs $23 \%$ ) and respiratory (21 vs $7 \%$ ). As expected, elderly patients had a higher comorbidity burden, with a higher Charlson index $(2,4 \pm 1,4$ vs $4,5 \pm 2,9$, p-value $=0.01)$, were more dependent according to Lawton\&Brody scale $(6,3 \pm 1,8$ vs $4,5 \pm 2$, $\mathrm{p}$-value $=0.007)$, and were more frail by PRISMA7 $(1,4 \pm 1,7$ vs 3 $\pm 1,8$, p-value $=0.008$ ). All patients were on an oral diet, and a majority was not taking any multivitamin supplements (100\% vs 90\%). Concerning nutritional status, average BMI was similar, although slightly lower in the elderly $(29 \pm 8,5$ vs $26 \pm 7$, p-value $=0.2)$. Using MUST as a screening tool, $46 \%$ of the young and $40 \%$ of the elderly were at risk for malnutrition ( $\mathrm{p}$-value $=0.7$ ). Using the GLIM criteria, this number decreased slightly to $43 \%$ in the young and $33 \%$ in the elderly (p-value $=0.5$ ).

Discussion: We observed a high percentage of patients at risk for malnutrition and with a diagnosis of malnutrition using MUST as a screening tool and the GLIM criteria as diagnostic. This was observed both in patients under and over 65 . Surprisingly, despite increased comorbidity burden, dependence and frailty, the elderly patients exhibited a lower percentage of malnutrition, despite not statistically significant.

\section{Abstract \# 411}

\section{EuGMS-2020: gait speed predicts depression in mild cognitive} impairment

Mehmet Ilkin Naharci ${ }^{1}$, Bilal Katipoglu ${ }^{1}$, Betül Gülsüm Yavuz Veizi ${ }^{1}$, Ilker Tasci ${ }^{2}$

${ }^{1}$ Division of Geriatrics, University of Health Sciences, Gulhane Faculty of Medicine and Gulhane Training and Research Hospital, Ankara, Turkey., ${ }^{2}$ Department of Internal Medicine, University of Health Sciences, Gulhane Faculty of Medicine and Gulhane Training and Research Hospital, Ankara, Turkey.

Objective: Slowed gait speed, an established measure of reduced functional performance, is considered a sensitive predictor of worsening cognitive status. Depression symptoms are known to influence gait speed in cognitively healthy adults. In this study, we determined the relationship between slow gait speed and depression symptoms in a sample of patients with mild cognitive impairment.

Method: We measured 15-feet gait speed and depression symptoms in community-dwelling older people ( $\geq 65$ years) with mild cognitive impairment recruited from an outpatient setting of a tertiary geriatric care unit. The subjects were classified as slow and normal gait speed based on the Fried criteria.

Results: We enrolled a total of 152 subjects with mild cognitive impairment between 2017 and 2019 years (mean age 80.05 years; $75.6 \%$ female). The prevalence of slow gait speed was $39.5 \%(n=60)$. Compared to normal speed group $(n=92)$, these patients had more clinically significant depression (geriatric depression scale score $>5$ ) $(\mathrm{p}<0.001)$, cardiovascular disease diagnosis $(\mathrm{p}=0.004)$, recent falls and anticholinergic burden $(\mathrm{p}=0.001)$. Multivariate models yielded associations between age $(\mathrm{OR}=1.201 ; \mathrm{CI}: 1.101-1.311 ; \mathrm{p}<0.001)$, female gender (OR=3.891; CI:1.266-11.955; $\mathrm{p}=0.018)$, depression $(\mathrm{OR}=3.744$; CI:1.475-9.500; $\mathrm{p}=0.005)$, fall history $(\mathrm{OR}=2.751$; CI $1.100-6.880 ; \mathrm{p}=0.030)$, and cardiovascular disease $(\mathrm{OR}=3.422 ; \mathrm{CI}$ 1.265-9.256; $\mathrm{p}=0.015$ ) and slow gait speed.

Conclusion: Depression is significantly and independently associated with slow gait speed in older people with mild cognitive impairment. Hence, slow gait speed in these patients may not always indicate worsening cognitive status. Future studies are required to test whether the treatment of depression can improve functional performance in mild cognitive impairment.Funding:This study was supported by the 
department of the Scientific Research Projects of Universityof Health Sciences, Turkiye (Project No. 2017/007).

\section{Abstract \# 412 \\ Depression is associated with dementia and insomnia in Moscow nonagenarians \\ Ruslan Isaev ${ }^{1}$, Elen Mkhitaryan ${ }^{1}$, Natalya Vorobyeva ${ }^{1}$, Irina Strazhesko $^{1}$, Olga Tkacheva ${ }^{1}$, Nikolay Yakhno ${ }^{2}$ \\ ${ }^{1}$ Pirogov Russian National Research Medical University, Russian Clinical and Research Center of Gerontology, ${ }^{2}$ Federal State Autonomous Educational Institution of Higher Education I.M Sechenov First Moscow State Medical University of the Ministry of Health of the Russian Federation (Sechenov University), Research department of neurology of the Biomedicine Technopark}

Introduction: In many studies old people had high prevalence of depression. Associations between depression dementia and insomnia has been poorly studied in nonagenarians. The aim of our study was to investigate the prevalence of depression and its associations with dementia and insomnia in patients aged $\geq 90$ years.

Methods: One hundred and eighty-eight outpatients (23\% male) aged 90-101 (mean 93 \pm 2 ) years were included in the study. We evaluated mood using the Geriatric Depression Scale (GDS-15), cognitive functions - the Mini-Mental State Examination (MMSE) and the Frontal Assessment Battery (FAB), and insomnia - the Insomnia Severity Index (ISI).

Results: The prevalence of depression (GDS-15 $\geq 5$ ) was $69,7 \%$, insomnia (ISI $\geq 8$ ) $-54,3 \%$, and dementia (MMSE $\leq 23$ ) $-46,8 \%$. Median of GDS-15 was 7 (IQR 4 to 10). Patients with depression $(n=131)$ had the higher frequency of dementia $(53,4 \%$ vs $31,6 \%$; $\mathrm{p}=0,006)$ and insomnia $(60,3 \%$ vs $40,4 \% ; \mathrm{p}=0,012)$ than those without $(\mathrm{n}=57)$. GDS-15 score was correlated with FAB $(\mathrm{r}=-0,335 ; \mathrm{p}<0,001)$, MMSE $(\mathrm{r}=-0,299 ; \mathrm{p}<0,001)$, and ISI $(\mathrm{r}=0,339 ; \mathrm{p}<0,001)$ score. Logistic regression showed that depression was associated with dementia (OR 2,49; 95\% CI 1,29-4,79; $\mathrm{p}=0,006$ ) and insomnia (OR $2,25 ; 95 \%$ CI 1,19-4,24; $\mathrm{p}=0,012)$. FAB score as a rank variable was associated with depression. (OR 1,86; 95\% CI 1,21-2,86; $\mathrm{p}=0,005$ ). Conclusion: We observed a high prevalence $(69,7 \%)$ of depression in Moscow nonagenarians. Depression was associated with dementia and insomnia.

\section{Abstract \# 413 \\ Social Isolation Scale: Portuguese version: a content validity study}

João Tavares ${ }^{1}$, Sofia Silva ${ }^{2}$, Filipa Marques ${ }^{2}$

${ }^{1}$ School of Health Sciences, University of Aveiro, Center for Health Technology and Services Research; The Health Sciences Research Unit: Nursing, ${ }^{2}$ Coimbra Education School, Polytechnic Institute of Coimbra

Introduction: Social isolation (SI) has severe consequences for health and quality of life, especially to older adults (OAs). Identification of the OA at risk of SI is crucial to implement strategies that mitigate the negative impact of SI. This study aims to (1) translate, adapt, and validate the Social Isolation Scale (SIS) for the Portuguese population and (2) evaluate the content validity index (CVI).

Methods: The principles of good practice for the translation and cultural adaptation were used [1]. The content validity criteria were CVI, probability of a chance occurrence (pc) and modified Kappa (MK) [2]. In this procedure, we appealed to nine experts with doctoral degrees related to gerontology.
Results: At the end of the translation process, a preliminary version of the Social Isolation Scale - PT (14 items) was obtained. After the first round, six items showed a CVI $<0.8$. After review, the second evaluation had seven items that showed a $C V I \geq 0.89$ and the remaining items had a $\mathrm{CVI}=1$. The average value of the CVI was 0.95 , the universal agreement of the CVI was 0.5 and the pc values ranged between 0.018 and 1 . MK had excellent values that ranged between 0.89 and 1 .

Key conclusions: The SIS demonstrated good psychometric properties. The study showed high levels of CVI, pc and MK in the Portuguese version of this scale, which supports the validity of the instrument for recognising SI. Additional criteria and construct validation studies are needed to explore the SIS in Portuguese.

References: 1. Wild D, Grove A, Martin M, Eremenco S, McElroy S, Verjee-Lorenz A, Erikson P (2005) Principles of Good Practice for the Translation and Cultural Adaptation Process for Patient-Reported Outcomes (PRO) Measures: report of the ISPOR Task Force for Translation and Cultural Adaptation. Value Heal 8:94-104. 2. Polit DF, Beck CT (2006) The Content Validity Index: Are You Sure You Know What's Being Reported? Critique and Recommendations. Res Nurs Health 29:489-497

\section{Abstract \# 414}

Age and intensity of psychopathological symptoms in COVID-19 survivals

Natalia Kotovskaya ${ }^{1}$, Alexander Rozanov ${ }^{2}$, Tatiana Manevich ${ }^{2}$, Valentina Ostapenko ${ }^{2}$, Anna Schedrina ${ }^{2}$, Andrey Izyumov ${ }^{2}$, Ksenia Eruslanova $^{2}$, Madina Balaeva ${ }^{2}$, Natalia Deryugina ${ }^{3}$, Yulia Kotovskaya $^{2}$, Olga Tkacheva ${ }^{2}$, Igor Belokrylov ${ }^{1}$

${ }^{1}$ RUDN University, Moscow, Russia, ${ }^{2}$ Pirogov RNMU - RCRCG, Moscow, Russia, ${ }^{3}$ MSU, Moscow, Russia

Introduction: The aim of the study was to investigate the severity of psychopathological symptoms in previously healthy patients of different age groups survived moderate-to-severe COVID-19 pneumonia.

Methods: Before the discharge from the hospital patients who gave informed consent filled in the Symptom Checklist-90-R designed to assess the 11 symptoms dimensions: somatization (SOM), obsessivecompulsive (O-S), interpersonal sensitivity (INT), depression (DEP), anxiety (ANX), hostility (HOS), phobic anxiety (PHOB), paranoid ideation (PAR), psychoticism (PSY). Cognitively impaired patients were not included into the study.

Results: The study involved 106 patients aged 26 to 84 years: 44 (24 men, 20 women) $<65$ years (median age 55) and 62 (34 men, 28 women) patients $>65$ years (median age 78.5). In both groups, symptoms were detected on the scales of SOM, DEP, ANX, HOS, PAR, and no symptoms on PSY and PHOB scales were reported. Despite a similar pattern of symptoms, most of them tended to be were pronounced in $65+$ patients vs $<65$ years. Older vs younger patients had insignificantly lower score on SOM (0.96 (IQR 0.44) vs 0.89 (IQR 0.25) and PAR 0.22 (IQR 0, 11) vs 0.24 (IQR 0.17), and higher score on DEP 0,53 (IQR 0.26) vs 0.45 (IQR 0.35) and ANX 0.52 (IQR 0,25 ) vs 0.39 (IQR 0.26 ), and significantly higher scores on HOS 0.58 (IQR 0.55$)$ vs $0.31(\mathrm{IQR}=0.25), \mathrm{p}=0.02$, and $\mathrm{O}-\mathrm{S} 0.60$ (IQR 0.35) vs 0,33 (IQR 0.21), $\mathrm{p}=0.01$.

Conclusions: The results suggest that survivors after moderate-tosevere COVID-19 pneumonia potentially need further psychological rehabilitation and psychiatric assessment. 


\section{Abstract \# 415 \\ Evaluating perioperative shared decision making}

Vittoria Vergani ${ }^{1}$, Jamie Mawhinney ${ }^{1}$ Theodore Chevallier ${ }^{1}$, Dina Radenkovic $^{1}$, Ramai Santhirapala ${ }^{2}$, Rajni Lal ${ }^{1}$, Fionna Martin ${ }^{1}$

${ }^{1}$ Perioperative Medicine for Older People undergoing Surgery, Guy's and St Thomas' NHS Foundation Trust, London, UK, ${ }^{2}$ Department of Theatres, Anaesthesia and Perioperative Care, Guy's and St Thomas' NHS Foundation Trust, London, UK

Introduction: Shared decision making (SDM) describes the process by which clinicians and patients jointly consider the best available evidence and the patient's personal values in order to make judgements about healthcare. This is advocated by a number of organisations including NICE. Perioperative Care of Older People undergoing Surgery (POPS) is an assessment and management service for multi-morbid and frail patients within which SDM should be routinely practiced. This quality improvement project aims to evaluate satisfaction in SDM amongst clinicians and patients in the POPS preoperative clinic.

Methods: POPS preoperative clinics in September/October 2019 (first cycle) and November 2019 (second cycle) were selected for analysis. Following each consultation, clinicians and patients completed paired SDM-Q9 questionnaires to evaluate SDM satisfaction. This tool has been previously validated in this population. Results were standardised and compared using paired t-tests. Group discussions were used to identify strategies for improvement.

Results: 68 (52\%) questionnaires were initially returned completed. SDM satisfaction was $89.2 \%$ for patients and $78.9 \%$ for clinicians $(\mathrm{p}<0.001)$ and further analysis suggested that junior clinicians found SDM particularly challenging. Following this, a teaching programme led by senior clinicians was instigated. In the second cycle, $31(57 \%)$ questionnaires were returned completed and there was a significant increase in patient satisfaction in SDM in the junior doctors group $(\mathrm{p}=0.045)$.

Conclusions: Although SDM is of generally high quality in the POPS preoperative clinic, junior clinicians found this more challenging. Patient satisfaction in SDM was significantly improved following the commencement of a dedicated teaching programme.

\section{Abstract \# 416 \\ Enhanced recovery after surgery in patients $\geq 70$ years undergoing elective colorectal surgery}

Fagard Katleen ${ }^{1}$, Wolthuis Albert ${ }^{2}$, Verhaegen Marleen ${ }^{3}$, Flamaing Johan $^{1}$, Deschodt Mieke ${ }^{4}$

${ }^{1}$ Dpt of Geriatric Medicine, University Hospitals Leuven, Leuven, Belgium, ${ }^{2}$ Dpt of Abdominal Surgery, University Hospitals Leuven, Leuven, Belgium, ${ }^{3}$ Dpt of Anaesthesiology, University Hospitals Leuven, Leuven, Belgium, ${ }^{4}$ Dpt of Public Health and Primary Care, KU Leuven, Leuven, Belgium

Background: Enhanced recovery programs (ERPs) in colorectal surgery have demonstrated beneficial effects on postoperative complications, return of bowel function, length of stay, and costs, without increasing readmissions or mortality. However, ERPs were not specifically designed for older patients and feasibility in older patients has been questioned.

Aim: The aim of this study was to assess ERP adherence and outcomes in older patients and to identify risk factors for postoperative complications and prolonged length of stay.

Method: Retrospective analysis of consecutive patients ( $\geq 70$ years) undergoing elective colorectal resection in a tertiary referral hospital in 2017.
Results: Ninety-six patients were included. Adherence rates were above $80 \%$ in 18 of 21 ERP interventions considered. The lowest adherence rates were noted for preoperative carbohydrate loading and cessation of intravenous fluids. Postoperative complications (ClavienDindo $\geq 2$ ) and prolonged postoperative length of stay ( $>75$ th percentile) were observed in $39.6 \%$ and $26.3 \%$, respectively. Median length of stay was 7 days. The 30-day mortality, readmission and reoperation rates were $2.1 \%, 12.6 \%$ and $8.3 \%$, respectively. Multivariable analysis indicated that polypharmacy and site of surgery were independent risk factors for postoperative complications, while higher age, American Society of Anesthesiologists class and preoperative radiotherapy were independent risk factors for prolonged postoperative length of stay.

Conclusion: ERP adherence in older patients undergoing colorectal resection is high and ERP is therefore considered feasible. Postoperative complications and prolonged postoperative length of stay are common, so at risk patients should be targeted with tailored geriatric interventions.

\section{Abstract \# 417 \\ Perioperative management of antithrombotic therapy in hip fracture patients}

Chris Reidy ${ }^{1}$, Saied Ali ${ }^{1}$, Joseph Walsh ${ }^{2}$, David Lorrigan ${ }^{3}$, Lorraine Smith $^{4}$, Terence Murphy ${ }^{5}$, May Cleary ${ }^{5}$, Sudhir Immanni ${ }^{6}$, John Cooke $^{7}$, Riona Mulcahy ${ }^{7}$, George Pope ${ }^{7}$, Niamh O'Regan ${ }^{7}$

${ }^{1}$ Senior House Officer, Department of General Medicine, University Hospital Waterford, Ireland, ${ }^{2}$ Specialist Registrar, Department of Medicine for the Elderly, University Hospital Waterford, Ireland, ${ }^{3}$ Specialist Registrar, Department of Anaesthesiology and Intensive Care University Hospital Waterford, Ireland, ${ }^{4}$ Trauma Coordinator, Department of Orthopaedics, University Hospital Waterford, Ireland, ${ }^{5}$ Consultant Orthopaedic Surgeon, Department of Orthopaedics, University Hospital Waterford, Ireland, ${ }^{6}$ Consultant Anaesthetist, Department of Anaesthesiology and Intensive Care, University Hospital Waterford, Ireland, ${ }^{7}$ Consultant Geriatrician, Department of Medicine for the Elderly, University Hospital Waterford, Ireland

Background: Early operative management of fragility hip fractures improves outcomes, hence surgery within 48 hours of presentation remains an important metric in standard of care. One reason for delay to surgery is anti-thrombotic medication use. University Hospital Waterford (UHW), a busy Irish trauma unit (c. 450 hip fractures per year) has local guidance on peri-operative anti-thrombotic management. We aimed to assess application of these guidelines to hip fracture patients, and explore the contribution of anti-thrombotic medication use to delays to surgery.

Methods: A convenience sample of 50 charts were reviewed (Dec 2019-April 2020).

Results: Of 50 patients, 19 had an anti-thrombotic reason to delay surgery (anticoagulation/clopidogrel). Neither reason for delay to surgery, nor time of surgery was clearly documented, hence we calculated duration to surgery in hours from admission until 09:00 on morning of surgery. The mean duration from presentation to surgery for those on anticoagulation/clopidogrel was 73.8 hours (range 32-159), with surgery delayed by more than 48 hours in two-thirds. Conversely, all but one patient on aspirin alone $(n=13)$ had surgery within 48 hours.

Conclusions: Over one-quarter of this convenience sample had surgery delayed greater than 48 hours due to anti-thrombotic therapy. Peri-operative guidelines for anti-thrombotic management is based largely on studies in elective surgery, and may not serve hip fracture patients well given the cumulative risk of mortality with each day surgery is delayed. Further studies assessing the risk/benefit of earlier surgery in this cohort on anti-thrombotics are required. 


\section{Abstract \# 418 \\ The association between frailty and severity of cognitive impairment in older inpatients with postoperative delirium}

Hui Min Khor ${ }^{1}$, Jun Leong Cheong ${ }^{1}$, Mahmoud Danaee ${ }^{1}$, Ina Ismiarti Shariffuddin ${ }^{1}$, Soon Chen Teang ${ }^{1}$, Pui San Loh ${ }^{1}$

${ }^{1}$ University of Malaya

Introduction: Frailty and cognitive impairment are risk factors for postoperative delirium and predictors of negative health outcomes. The objective of the study is to determine the association of frailty and severity of cognitive impairment in older inpatients with postoperative delirium.

Methods: Consecutive patients aged $\geq 65$ years old admitted to a tertiary teaching hospital for semi-urgent and elective surgery were recruited over 6 months period. Frailty status was identified using Fried Frailty Index. All patients had cognitive assessment using Montreal Cognitive Assessment(MOCA) and delirium was diagnosed using the Cognitive Assessment Method and 4AT. Patients were then divided into category $\mathrm{A}$ (dementia with frailty), category $\mathrm{B}$ (dementia alone), category $\mathrm{C}$ (mild cognitive impairment(MCI) with frailty, also termed cognitive frailty), category $\mathrm{D}(\mathrm{MCI}$ alone), category $\mathrm{E}$ (frailty alone) and category $\mathrm{F}$ (normal cognition with no frailty).

Results: A total of 50/447(11.2\%) had postoperative delirium with mean age of 76.8 (SD7.5) years. Patients with postoperative delirium had significantly lower MOCA score on admission [21.1(SD5.3) vs 14.9(SD7.8)] compared to those who had no delirium. The average Fried Frailty score for all patients was 1.3 (SD 1.4) with $14.8 \%$ categorised as non-frail, $59.3 \%$ pre-frail and $26 \%$ frail. The relationship between frailty and cognitive impairment with postoperative delirium after adjustment with age was found to have the following order of significance; Category A [OR=12.33 (95\% CI: 4.27-35.65), $\mathrm{p}<0.01]$, Category B $[\mathrm{OR}=5.0$ (95\% CI:1.9-13.6), $\mathrm{p}<0.01]$, Category $\mathrm{C}$ $[\mathrm{OR}=3.5(95 \% \mathrm{CI} 1.1-10.7), \mathrm{p}=0.03]$, Category D [OR=1.0 (95\% CI 0.3-3.2), $\mathrm{p}=0.94]$, Category $\mathrm{E}[\mathrm{OR}=4.2$ (95\% CI: 1.0-18.4), $\mathrm{p}=0.05]$, and Category $\mathrm{F}$ as reference group.

Conclusion: Frailty syndrome and cognitive impairment are common in older surgical inpatients. Patients with dementia and mild cognitive impairment are associated with the development of postoperative delirium and the significance is stronger when combined with the presence of frailty.

\section{Abstract \# 419 \\ Self-care positioning for the elderly in post-operative period after neurosurgery \\ Ana Filipa Frutuoso ${ }^{1}$, Ana Sofia Ferreira ${ }^{1}$, Hugo Neves ${ }^{2}$ \\ ${ }^{1}$ Escola Superior de Enfermagem de Coimbra, ${ }^{2}$ Health Sciences Research Unit: Nursing}

Background: According to Orem's Self-Care Deficit Nursing Theory, nurses assume an essential role in preventing complications in the postoperative neurosurgical period in the elderly, specifically concerning the focus of attention of positioning.

Objective: Map the elderly's postures/positions that enhance security and reduce complications in the postoperative neurosurgical period. Methods: A scoping review based on Joanna Briggs Institute's recommendations was carried out through the following databases: Medline (PubMed) and CINAHL (EBSCO). Studies in English, Spanish and Portuguese, with no time limitation, were included in the search. Two independent reviewers assessed studies for title and abstract in the first stage, and full text in the second stage, with a third reviewer assessing in case of discordance.
Results: A total of thirteen articles were included in the analysis. From these studies, eight evidenced reduction of Intracranial Pressure (ICP) by raising the head-of-bed by 30 degrees, while keeping Cerebral Perfusion Pressure $(\mathrm{CPP})$ stable $(\mathrm{p}<0.05)$. When raising head-of-bed to 45 degrees, a slight increase in ICP and a decrease in CPP was observed $(\mathrm{p}<0.05)$. Regarding Chronic Subdural Hematoma $(\mathrm{CSDH})$, although not significant, three articles evidenced the presence of complications when raising head-of-bed $(p<0.05)$, with another article evidencing a positive impact in the length of stay. Conclusion: Raising the head-of-bed by $30^{\circ}$ degrees in the postoperative period contributes to a quicker recovery, through optimization of ICP and CPP, with subsequent improvement of venous return. Incorporating this evidence into nursing care will help reduce complications and length of stay in the elderly submitted to a neurosurgical procedure.

\section{Abstract \# 420}

Neuroprotective effect of rapalogs in a pharmacological model of Parkinson's disease

María José Hormazábal ${ }^{1}$

${ }^{1}$ Universidad de Chile

Introduction: Parkinson's Disease (PD) is a neurodegenerative disorder characterized by a progressive loss of dopaminergic neurons into substantia nigra (SN). Rapamycin, used in transplanted patients as immunosuppresor, has shown a therapeutic potential in pre-clinical model of PD through the neuroprotection of dopaminergic neurons in SN. Moreover Rapamycin has been associated to glucose metabolic disorders. Previous studies suggested that analogs of Rapamycin (Rapalogs) have similar neuroprotective effects by signaling for mTOR pathway. We investigated if Rapalogs called TAM-1 and TAM-3 has a neuroprotective effect and avoids metabolic alterations in a pharmacological PD model in comparison with Rapamycin effects (performance).

Material and methods: Adult male C57BL/6 mice were injected unilaterally into the $\mathrm{SN}$ with the neurotoxin 6-OHDA, validated PD model. Animals were pretreated with Rapamycin, TAM-1, TAM-3 or vehicle ( 2 injections administered intraperitoneally). After 7 days, we evaluated the motor performance by Cylinder test and determine neurodegeneration process by histopathological analysis using antityrosine hydrolase antibody. Moreover, we determine metabolic parameters as glucose, insulin, and leptin levels in serum samples of these mice.

Results: Rapamycin, TAM-1 and TAM-3 prevent the loss of dopaminergic neurons of the nigrostriatal pathway. Additionally, TAM-3 ameliorate the motor impartment and TAM-1 did not trigger glucose resistance in PD model.

Discussion: These results suggest that TAM-1 and TAM-3 exert neuroprotective effect of nigrostriatal circuits and alleviate the motor impairment, similar to Rapamycin in the pharmacological model of PD by signaling the mTOR pathway.

\section{Abstract \# 421}

Chronic kidney disease and appropriate prescribing in hospitalized older adults: an observational study

Silvia Menale ${ }^{1}$, A. Giordano ${ }^{1}$, L. Lambertucci ${ }^{1}$, G. Rivasi ${ }^{1}$, A. Corsonello $^{2}$, A. Ungar ${ }^{1}$

${ }^{1}$ Division of Geriatrics and Geriatric Intensive Care Medicine, Careggi Hospital and University of Florence, Italy. ${ }^{2}$ National Institute for Geriatric Hospitalization and Care V.E.II (INRCA), Cosenza, Italy. 
Introduction: Chronic kidney disease (CKD) is highly prevalent in older adults, but is frequently omitted in medical records, which may lead to inappropriate prescriptions. This study aimed to assess the under-reporting of CKD in discharge summaries of older hospitalized patients and to evaluate appropriate prescribing according to renal function.

Methods: Patients aged 65+ were enrolled in a geriatric acute care setting between January and May 2018, if creatinine was available within 3 days before discharge. Renal function was assessed according to the following formulas: Cockroft-Gault (CG), Chronic Kidney Disease- Epidemiology Collaboration (CKD-EPI), Modification of Diet in Renal Disease (MDRD), Berlin Initiative Study 1 (BIS1) and Full Age Spectrum (FAS). Prescriptions were evaluated according to the Screening Tool of Older Persons' Prescriptions (STOPP) and Screening Tool to Alert to Right Treatment (START) 2013 criteria.

Results: 192 patients were enrolled (mean age 85). In discharge summaries, CKD was omitted in the $54 \%$ of patients, $25 \%$ of whom showing severe CKD. According to the CG formula, STOPP criteria were not applied in the majority of patients taking thrombin inhibitors $(50 \%)$, Xa inhibitors $(64 \%)$ and colchicine $(100 \%)$, since patients' weight was missing. These prescriptions were instead appropriate according to CKD-EPI. None of the patients with CKD showing $\mathrm{Hb} \leq$ $11 \mathrm{mg} / \mathrm{dl}$ were prescribed erythropoietin.

Conclusions: CKD is frequently omitted in discharge summaries. The under-reporting of CKD and difficulties in the use of CG formula limit the use of STOPP and START criteria. Accurate reporting of $\mathrm{CKD}$ in medical records may favor appropriate prescribing in these patients.

\section{Abstract \# 422 \\ Prevalence of crushing medication in people aged 65 years or more, living at home in Martinique \\ Cécilia Cofais ${ }^{1}$, Lidvine Godaert ${ }^{2}$, Moustapha Drame ${ }^{3}$ \\ ${ }^{1} \mathrm{CHU}$ Rennes, ${ }^{2} \mathrm{CH}$ Valenciennes, ${ }^{3} \mathrm{CHU}$ Martinique}

Introduction: The safety of medicine management is a public health issue. Crushingmedication (defined as modifications of the solid oral drug products (chewing,splitting, crushing...) has been identified as a common medical error. Several studiesconducted in long-term care facilities for the elderly have shown that the frequency ofcrushing medication varies between $7 \%$ and $35 \%$. To the best of our knowledge, nodata exists about how often community-dwelling older people crush drugs to facilitateingestion. The aim of this study was to estimate the prevalence of crushingmedication in people aged 65 years or older living at home.

Method: A cross-sectional, observational, epidemiological study was carried out. Inclusion criteria were: age 65 years or more, living at home, and to have taken atleast one oral medication for two weeks or more. The subjects included wererecruited from different geriatric departments in Martinique. The primary outcome ofthis study was the prevalence of crushing medication.

Results: In total, 209 patients were included; $17.7 \%$ were found to crush theirmedication. We observed that family carers were more likely to crush medicationthan nurses or the patients themselves. There was no significant difference betweennurses and patients in the frequency of crushing medication.

Discussion: Our findings show that crushing medication is frequent amongcommunity-dwelling older people. The iatrogenic risk incurred by this practice isunknown to caregivers, carers, and patients. Campaigns are warranted to raiseawareness among the general population and caregivers of the risk of this practice.

\section{Abstract \# 423}

LESS-PHARMA-CHRON: electronic platform for the optimization of the pharmacotherapy in chronic patients Aitana Rodríguez- Pérez ${ }^{1}$, Ángela Villalba-Moreno ${ }^{2}$, María Dolores Toscano-Guzmán ${ }^{1}$, Mercedes Galván-Banqueri ${ }^{3}$, Bernardo SantosRamos $^{1}$, Eva Rocío Alfaro-Lara ${ }^{1}$

${ }^{1}$ Pharmacy Service. University Hospital Virgen del Rocío, ${ }^{2}$ Pharmacy Service. Hospital of Riotinto, ${ }^{3}$ Pharmacy Service. University Hospital Virgen de Valme

Introduction: Anticholinergic burden, excessive therapeutic burden and preventable adverse drug events usually jeopardies the success of chronic patients' treatment. Three important tools have been developed to counteract these problems: ABC-Calculator, Less-Chron and Trigger-Chron. They have demonstrated clinical benefits in some papers. The objective is to reveal to the scientific community an electronic platform that integrates and makes a much easier clinical use of these three important tools.

Methods: (1) Concept, plan and coordination (2) Technological development (3) Validation (4) Diffusion plan.

Results: A free access, friendly, comprehensive, integrated web platform and app (English/Spanish). The main features are:- Easy free registration, access, description and bibliographic evidence of the tools. Continuous updates. Once the treatment and patient are introduced all tools calculus may be automatically done, selecting the tool to apply.- Patients: 1. ABC: calculates anticholinergic burden and estimates the risk of developing anticholinergic side effects summarizing 10 scales in a numerical and colored graphical display. 2. LESS-CHRON: generates deprescribing recommendations starting at patient clinical status and drug profile. A time/clinical parameter pack to evaluate the success with a proposed review date automatically generated (agenda), are also provided. 3. TRIGGER-CHRON: informs about the previous patient risk on drug adverse eventsPatient history/Doctor's file: anonymized patient database plus all patients file from each individual user that may be exported.

Key conclusions: LESS-PHARMA-CHRON manages to integrate into a single portal three useful tools for the optimization of the pharmacotherapy of chronic patients, thus supporting their applicability in clinical practice.

\section{Abstract \# 424}

Factors associated with potentially inappropriate medications in patients taking anti-dementia drugs: a secondary analysis of a nationwide survey of prescribing pharmacies

Yusuke Suzuki ${ }^{1}$, Mikio Sakakibara ${ }^{2}$, Nariaki Shiraishi ${ }^{2}$, Hitoshi Komiya $^{1}$, Masahiro Akishita ${ }^{4}$, Masafumi Kuzuya ${ }^{5}$

${ }^{1}$ Nagoya University Hospital, ${ }^{2}$ Sugi Pharmacy, ${ }^{3}$ Nihon Fukushi University, ${ }^{4}$ The University of Tokyo Hospital, ${ }^{5}$ Nagoya University

Background: As the number of older people with dementia (PWD) increases, safe pharmacotherapy in this population has attracted attention in recent years.

Aim: The aim of this study was to clarify the prescribing patterns in older patients who were prescribed anti-dementia drugs and to investigate the association of potentially inappropriate medications (PIMs) with the use of anti-dementia drugs.

Methods: Adults aged $\geq 65$ years, who were prescribed anti-dementia drugs at 585 pharmacies across Japan $(\mathrm{N}=7953)$, were surveyed. The percentage of prescriptions for anti-dementia drugs and the effect of those prescriptions on PIMs were investigated.

Results: Prescriptions for anti-dementia drugs were found in $4.4 \%$ of the entire study population. A logistic regression analysis revealed 
that the use of anti-dementia drugs reduced the risk of prescribing psychotropic drugs, which represented PIMs, and that a combination of anti-dementia drugs (e.g., choline esterase inhibitor with memantine) may reduce the risk of prescribing PIMs compared with monotherapy.

Conclusions: Anti-dementia drugs were under-prescribed relative to the possible prevalence of dementia, and the use of anti-dementia drugs or the combination of available drugs may reduce the risk of prescribing PIMs.

\section{Abstract \# 425 \\ Relationship between polypharmacy, anticholinergic burden, therapeutic complexity and inappropriate prescription with frailty}

Núria Molist-Brunet ${ }^{1}$, Daniel Sevilla-Sánchez ${ }^{2}$, Emma PuigoriolJuvanteny $^{2}$, Lorena Bajo-Peña ${ }^{1}$, Jordi Amblàs-Novellas ${ }^{1}$, Joan Espaulella-Panicot ${ }^{1}$

${ }^{1}$ Hospital Universitari de la Santa Creu de Vic, ${ }^{2}$ Hospital Universitari de Vic

Introduction: Polypharmacy, anticholinergic burden, therapeutic complexity and inappropriate medication are related with adverse outcomes, but there is not an established relationship with frailty index based on comprehensive geriatric assessment.

Methods: Cross-sectional study. Community-dwelling patients are included (both people living in their own homes and nursing homes) if they showed frailty (measured by Frail-VIG Index) $\geq 0.20$. Data were collected from June to December 2019. Each patient's pharmacotherapeutic plan was reviewed by an interdisciplinary healthcare team (including primary care physician, nurse, pharmacist and a consultant team with geriatrician and clinical pharmacist) applying a patient centred prescription model. ANOVA test was applied to associate frailty with polypharmacy, anticholinergic burden (measured by DBI), therapeutic complexity (measured by MRCI) and inappropriate prescription (IP).

Results: We included 148 patients (mean age 83.3, SD 7.7; 63.9\% female; $61.2 \%$ nursing home residents). Mean use drug per patient was 8.7 (SD 4.0), mean MRCI was 32.12 (SD 17.15) and median was DBI 1.85 (IQR 0.66 - 1.99). 91.2\% had at least 1 o more PI. Median Frail-VIG index was 0.40 (IQR $0.32-0.48)$. Polypharmacy $(\mathrm{p}=0.239)$ and therapeutic complexity $(\mathrm{p}=0.701)$ did not show significance relationship with frailty. However, inappropriate prescription $(\mathrm{p}=0.017)$ and anticholinergic burden $(\mathrm{p}=0.008)$ showed relationship with frailty.

Key conclusions: Total number of drugs and therapeutic complexity in the prescription is mainly the same, independently of the frailty degree. This lack of tailoring may well explain the highest inappropriate prescription detected in the frailest patients.

\section{Abstract \# 426 \\ Impact of a patient centred prescription model on pharmacogical outcomes in a community-dwelling cohort \\ Daniel Sevilla-Sanchez ${ }^{1}$, Núria Molist-Brunet ${ }^{2}$, Emma Puigoriol- Juvanteny $^{3}$, Mariona Espaulella-Ferrer ${ }^{4}$, Javier Gonzalez-Bueno ${ }^{5}$, Joan Espaulella-Panicot ${ }^{6}$ \\ ${ }^{1}$ Pharmacy Department - Consortium Vic Hospital / Santa Cre's Vic Hospital - Central Catalonia Chronicity Research Group (C3RG), ${ }^{2}$ Geriatrics Department - Santa Creu's Vic Hospital - Central Catalonia Chronicity Research Group (C3RG), ${ }^{3}$ Epidemiology Department - Consortium Vic Hospital, ${ }^{4}$ Geriatrics Department -}

Santa Creu's Vic Hospital / Consortium Vic Hospital, ${ }^{5}$ Pharmacy Department - Consortium Vic Hospital, ${ }^{7}$ Geriatrics Department Santa Creu's Vic Hospital / Consortium Vic Hospital - Central Catalonia Chronicity Research Group (C3RG)

Introduction: It is known that polypharmacy, anticholinergic burden, therapeutic complexity and inappropriate medication are related to adverse effects. Our purpose is to assess if application of patient centred prescription (PCP) model (based on a shared decision-making process including patient, physicians and clinical pharmacist) reduces these outcomes.

Methods: Quasi-experimental study (pre-post study, assessment at 3 months). Community-dwelling patients are included (nursing homes and regular homes) from June to December 2019. Each patient's pharmacotherapeutic plan was reviewed by an interdisciplinary healthcare team (including primary care physician, nurse, pharmacist and consultant team with geriatrician and clinical pharmacist) applying a PCP model. Pharmacological outcomes were recorded: polypharmacy, medication complexity (by MRCI) and anticholinergic burden (by DBI). Fisher-test, Wilcoxon-test or McNemar-Bowker-test were used to compare both periods.

Results: We included 157 patients (mean age 83.4, SD 7.94; 66.4\% female; 58.4\% nursing home; median Index Barthel 50/100; 73.7\% cognitive impairment $12.7 \%$; mortality at 3 months).Pre-intervention mean drug use per patient was 9.02 (SD 4.05) and decreased to 7.99 (SD 3.46) at 3 months $(\mathrm{p}<0.001)$. Medication complexity was 34.00 (SD 17.55) at the beginning and decreased down to 26.05 (SD 16.61) at follow-up $(\mathrm{p}<0.001)$. Anticholinergic burden decreased from 1.39 (IQR 0.66-2.11) to 1.16 (IQR 0.63-1.77) at 3 months $(\mathrm{p}<0.001)$. Percentage of patients with high level of polypharmacy ( $\geq 10$ drugs), MRCI ( $\geq 40)$ and DBI $(\geq 2)$ decreased from $41.6 \%$ to $30.7 \%$, from $33.6 \%$ to $16.7 \%$ and from $27.0 \%$ to $16.11 \%$ respectively $(\mathrm{p}<0.001)$. Key conclusions: PCP model is successful in decreasing polypharmacy, medication complexity and anticholinergic burden.

\section{Abstract \# 427}

\section{Pharmacologic mediated unilateral fixed mydriasis}

Julie McCarthy $^{1}$, Atef Michael ${ }^{1}$

${ }^{1}$ Mary Stevens Hospice, UK, ${ }^{2}$ Russells Hall Hospital, Dudley, UK

Case report: A 79-year-old lady was admitted with shortness of breath. The patient had a diagnosis of adenocarcinoma of the lung on a background of COPD. She was started on nebulised salbutamol $2.5 \mathrm{mg}$ and ipratropium bromide $500 \mathrm{mcg}$. On Day 5 , it was observed that the patient had a fixed $6 \mathrm{~mm}$ dilated left pupils and the right pupil was $3 \mathrm{~mm}$. In the previous day the pupils were non-dilated, equal and reactive bilaterally. The patient was not on mydriatic eye drops. On examination there were no other lateralising neurological signs; cranial nerves as well as motor exam of the upper and lower limbs were normal, apart from the dilated fixed left pupil. After clinically excluding an acute neurological event, the mydriatic effect of ipratropium anti-muscarinic action was considered to be a potential cause for the fixed dilated pupil, however this did not explain the unilateral involvement. The patient was observed while using the nebuliser and it was noticed that she could not tolerate the mask affixed to her face to form an adequate seal, rather, she was holding it aloft and favouring her right side, such that the mist dispersed more readily to the left; this explained the unilateral manifestations. The assumption was subsequently proven upon discontinuing the ipratropium, when the pupils size, symmetry and reactivity returned to normal after around 24 hours, in keeping with timescales documented in previous case reports.

Discussion: Ipratropium bromide, as an antagonist of muscarinic acetylcholine receptors, causes mydriasis due to unopposed action of 
the dilator pupillae muscle, and cycloplegia due to ciliary muscle relaxation. The ocular complications of ipratropium bromide are due to the local effect of the medication; i.e. when the aerosolised ipratropium comes into contact with the eyes. The incidence of ipratropium induced ocular complications is between $1 \%$ to $<1$ per 1000 patients. Whilst there have been some case reports of ipratropium causing mydriasis, it remains in practice, a poorly recognised adverse drug reaction (ADR). Patients must be instructed in the correct administration of ipratropium nebuliser to avoid its release into the eye. Clinicians need to be aware of the uncommon mydriatic side effect of ipratropium to avoid unnecessary imaging and other investigations.

\section{Abstract \# 428 \\ Massive weight loss from rivastigmine patch in an older patient: a rare case \\ Mehmet Ilkin Naharci ${ }^{1}$, Betül Gülsüm Yavuz Veizi ${ }^{1}$, Bilal Katipoglu ${ }^{1}$, Ece Akcan ${ }^{2}$, Fatma Yoruk ${ }^{3}$, Ilker Tasci ${ }^{3}$ \\ ${ }^{1}$ Division of Geriatrics, University of Health Sciences, Gulhane Faculty of Medicine and Gulhane Training and Research Hospital, Ankara, Turkey., ${ }^{2}$ Department of Internal Medicine, University of Health Sciences, Gulhane Faculty of Medicine and Gulhane Training and Research Hospital, Ankara, Turkey, ${ }^{3}$ Department of Internal Medicine, University of Health Sciences, Gulhane Faculty of Medicine and Gulhane Training and Research Hospital, Ankara, Turkey.}

Here, we report a case of an older woman with a history of AD who lost weight unintentionally due to the rivastigmine patch. "Ms. G" was a 75-year-old woman presented to the outpatient service of geriatrics clinic in September 2018 with a history of loss of appetite, fatigue, a feeling of hopelessness, and loss of interest in daily activities. She lost 42-lb (19 kg) gradually during this period. She started the rivastigmine patch prescribed by her neurologist for $\mathrm{AD}$ since November 2017, and the dosage increased to $13.3 \mathrm{mg} / \mathrm{d}\left(15 \mathrm{~cm}_{2}\right)$ in March 2018. Also, her relatives reported that she was brought to an emergency room for nausea and fatigue a couple of times after the dosage raise of rivastigmine patch to $13.3 \mathrm{mg} / \mathrm{d}\left(15 \mathrm{~cm}_{2}\right)$. Her baseline weight and body mass index obtained from medical records were $137 \mathrm{lb}(62 \mathrm{~kg})$ and $27.9 \mathrm{~kg} / \mathrm{cm}_{2}$. Her relatives shared information on emesis and anorexia occurred at one month of donepezil use. The detailed physical examination and laboratory studies did not consider a malignancy or malabsorption. Massive weight loss was attributed to the rivastigmine patch and associated exacerbated depression. The rivastigmine patch was discontinued, and the nutrition counseling recommendations were received by the dietitian who was involved in the multidisciplinary geriatric team. Two months later, we saw a marked improvement in mood and cognition, and her weight increased to $112 \mathrm{lb}(51 \mathrm{~kg})$. Nutritional status and weight of patients should be closely monitored after initiating therapy and each dose increment.

\author{
Abstract \# 429 \\ Effectiveness of pharmacy students in an acute geriatrics \\ department: association of the analysis of prescriptions \\ to the medication reconciliation \\ Durand Amaury ${ }^{1}$, Lenglet Aurélie ${ }^{1}$, Bloch Frédéric ${ }^{1}$, Belhout \\ Mohamed $^{1}$ \\ ${ }^{1}$ CHU Amiens Picardie
}

Introduction: Medication reconciliation and pharmaceutical analysis of prescriptions helps to avoid potentially inappropriate medications and drug-related problems. In an acute geriatrics unit, pharmacy students do not analyse prescriptions during medication reconciliation at admission. For training purposes, they have been asked to do so. Methods: Between November 2018 and March 2019, pharmacy students were instructed to obtain a medication history, reconcile this list with the medical chart and identify drug related problems. These pharmaceutical interventions were then exposed to a pharmacy resident and to the prescriber to assess the appropriateness of these interventions. At the end of the training period, the students were asked to give their opinion on this practice in an open manner.

Results: Pharmacy students identified 600 discrepancies in 88 patients, $11.5 \%$ of which were rated as unintentional. Geriatricians confirmed $93.8 \%$ of the discrepancies and admitted that $8.3 \%$ of the discrepancies were unintentional. Moreover, students performed 30 pharmaceutical interventions, $60 \%$ of which were accepted by prescribers. Students appreciated this mission, which enabled them to better integrate into the unit and to understand the comprehensive geriatric assessment.

Key conclusions: The acceptance rate of pharmaceutical interventions performed by students was not significantly different from the acceptance rate of those performed by the resident during this period, although this is partly related to the low number of pharmaceutical interventions completed. Pharmacy students provided a valuable service, improved the quality of patient care by identifying drugrelated problems.

\section{Abstract \# 430}

Efficiency of the pharmacist in geriatrics: should we favored acute or rehabilitation units?

Durand Amaury ${ }^{1}$, Chenailler Catherine ${ }^{1}$, Varin Rémi ${ }^{1}$

${ }^{1}$ CHU Rouen

Introduction: In a teaching hospital, a pharmacist analyze the prescriptions in two acute geriatric units and in four geriatric rehabilitation units. The objective of this work is to assess the efficiency of this practice.

Methods: The pharmacist integrated in en excel file all the pharmaceutical interventions (PIs) carried out between November 2019 and March 2020 with the type of unit, the number of prescriptions analyzed, the time spent, the number of PIs and their clinical impact according to the CLEO scale [1] and the acceptance rate.

Results: In acute geriatrics, 550 PIs were performed for 541 patients (1.0 PI per patient) of which $390(70.9 \%)$ were accepted $(0.7$ per patient). The clinical impact of PIs was major for $26.0 \%$ of cases. The average time required was 8.7 minutes per analysis, 8.6 minutes for 1 PI, 12.1 minutes for 1 accepted PI and 43.8 minutes for 1 accepted PI with a major clinical impact.In rehabilitation units, 239 PIs were performed for 388 patients benefited ( 0.6 PI per patient) of which 128 (53.6\%) were accepted ( 0.3 per patient). The clinical impact of PIs was major for $25.0 \%$ of patients. The average time required was 7.1 minutes per analysis, 11.6 minutes for 1 PI, 21.6 minutes for 1 accepted PI and 74.7 minutes for 1 accepted PI with a major clinical impact.

Key conclusion: Actually the pharmacist is more efficient in acute geriatrics which invites to improve the working method in rehabilitation units or to reorganize the activity.

Bibliography: 1. Vo TH, Charpiat B, Catoire C, Juste M, Roubille R, Rose F-X, et al. Développement de l'echelle multidimensionelle «Cléo » pour évaluer la pertinence d'une intervention pharmaceutique. Pharm Hosp Clin. 2014;49(2). 


\section{Abstract \# 431}

Medication-related hospital admission in geriatrics, pharmacist's impact in managing therapeutic

Durand Amaury ${ }^{1}$, Leguillon Romain ${ }^{1}$, Chenailler Catherine ${ }^{1}$, Varin Rémi $^{1}$

${ }^{1} \mathrm{CHU}$ Rouen

Introduction: Within a teaching hospital, a pharmacist and a pharmacy resident analyze prescriptions and make medication reconciliation among two acute geriatric units (60 beds) and four geriatric rehabilitation wards (140 beds). Objectives were to assess the percentage of medication related hospital admission (MRA), to highlight the number of pharmaceutical interventions (PIs) performed and to observe 7-day re-admission is these units.

Methods: Drug assessment in patients admitted between November 2019 and March 2020 was determined by Kempen's tool, based on hospitalization report. All PIs performed were recorded to assess the targeted drugs, the clinical impact and the acceptance rate. Sevendays re-admission was audited on the computerized patient record.

Results: 493 patients received a medication reconciliation. Hospitalization was possibly related to an adverse drug reaction (ADR) in $19.7 \%$ of cases (97 patients). 19 patients $(19.6 \%)$ benefited from a reconciliation at discharge. A PI was performed upon entry for 28 patients $(28.9 \%)$, during the stay for 37 patients $(38.1 \%)$ and at discharge for 7 patients $(36.8 \%)$. A total of 147 PIs were performed, involving mainly ATC classes N (43 PIs, 29.3\%) and A (37 PIs, $25.2 \%$ ), had a major clinical impact in $21.8 \%$ of cases (32 PIs) and were accepted in $81.0 \%$ of cases (119 PIs). One patient was re-hospitalized less than 7 days after discharge, for the same ADR.

Key conclusion: Pharmacists can improve the drug management in geriatrics wards where medication-related hospital admission is frequent, up to $40 \%$ in a recent study even though very few patients are readmitted soon.

Bibliography: 1. Direction générale de l'offre de soins, Agence technique de l'information sur l'hospitalisation. Le taux de réhospitalisation dans un délai de 1 à 7 jours en MCO : Outil pour accompagner la mise à disposition d'un indicateur hospitalier de vigilance pour alerter, analyser et agir. 2017. 2. Kempen TGH, Hedström M, Olsson H, Johansson A, Ottosson S, Al-Sammak Y, et al. Assessment tool for hospital admissions related to medications: development and validation in older patients. Int $\mathrm{J}$ Clin Pharm. 2019;41(1):198-206. 3. Thevelin S, Mounaouar LE, Marien S, Boland B, Henrard S, Dalleur O. Potentially Inappropriate Prescribing and Related Hospital Admissions in Geriatric Patients: A Comparative Analysis between the STOPP and START Criteria Versions 1 and 2. Drugs Aging. 2019;36(5):453-9.

\section{Abstract \# 432}

Securing pharmacological management of the elderly patients by a pharmacist

Durand Amaury ${ }^{1}$, Chenailler Catherine ${ }^{1}$, Varin Rémi ${ }^{1}$

${ }^{1} \mathrm{CHU}$ Rouen

Introduction: Medication reconciliation (MR) and analysis of prescriptions (AP) allow to secure medication management. In two geriatrics units (60 beds) and 4 geriatrics rehabilitations units (140 beds), these activities are performed since November 2019 by a pharmacist and three pharmacy students.

Methods: During 5 months, the pharmacist noted on an Excel spreadsheet:-The number of pharmaceutical interventions (PI) performed, the problems posed, the type of interventions, the ATC classes, the clinical impact of each PI according to the CLEO scale of the French Society of Clinical Pharmacy and the rate of PI accepted. The number of medication reconciliations (MR) performed, the number of unintended discrepancies (UD), the ATC classes and the clinical impact.

Results: 789 PI were performed for 929 patients mainly for ATC classes: digestive tract and metabolism A (29\%), blood and hematopoietic organs B (21\%), cardiovascular system C (13\%) and nervous system $\mathrm{N}(13 \%)$. The most frequent inappropriate prescriptions were therapeutic absence for validated medical indication (18\%), supra-therapeutic dosage (14\%) and non-prescribed posttransfer drug (14\%). The clinical impact was major in $24 \%$ of cases. At admission and at discharge, 493 and 107 MR were performed for 507 patients, respectively; 235 and 62 UD were found, respectively and concerned ATC classes N (22\%), C (18\%) and A (17\%). The most frequent UD was non-prescribed post-transfer drug (57\%). The clinical impact was major for $13 \%$ of UD.

Key conclusion: The pharmaceutical presence in the unit allowed to correct many inappropriate prescriptions and reduce the risk of adverse drug events.

Bibliography: 1. Journal Officiel de la République Française. Arrêté du 6 avril 2011 relatif au management de la qualité de la prise en charge médicamenteuse et aux médicaments dans les établissements de santé. 2011; 2. World Health Organization. The High 5s project Standard operating protocol - Assuring medication accuracy at transitions in care: medication reconciliation. 2014. 3. Haute Autorité de Santé. Comment améliorer la qualité et la sécurité des prescriptions de médicaments chez la personne agée? 2014. 4. Vo TH, Charpiat B, Catoire C, Juste M, Roubille R, Rose F-X, et al. Développement de l'echelle multidimensionelle «Cléo » pour évaluer la pertinence d'une intervention pharmaceutique. Pharm Hosp Clin. 2014;49(2).

\section{Abstract \# 433}

\section{Quinolones. Health alert. Responsible use}

Carmen Elías de Molins Peña ${ }^{1}$, Carmen Canovas Pareja ${ }^{2}$, Elena Rebollar Torres ${ }^{3}$, Jorge Eduardo Corrales Cardenal ${ }^{4}$, Elisa Lasala Hernandez ${ }^{3}$

${ }^{1}$ Dra, ${ }^{2}$ Dra., ${ }^{3}$ Sra., ${ }^{4}$ Dr.

Introduction: Fluoroquinolone $(\mathrm{FQ})$ is a broad spectrum antibiotic.There are negative aspects considering its safety: therapeutic group with the highest ecological impact, bacterial resistance and adverse effect (risk factors and the elderly). Scientific societies have evaluated said impact and alerted about its rational use; so as not to compromise the therapeutic value, it should be preserved and not used as a first line of treatment.

Objectives: -Prevalence. -Prescription adequacy according to the established criteria.-Record of adverse effects.Method:Prospective, descriptive study. Inpatients with infectious disease(ID) treated with antibiotic from March-December 2019. Variables: Prescription and posology. Type of ID. Beta-lactam allergy(BL), history of risk factors and tolerance.

Results: 637 inpatients with antibiotic therapy:108(16.9\%) used FQ:levofloxacin:79\%, ciprofloxacin:21\%.Mean age 87 years old. Women:68\% Type of infection: respiratory:64\%, urinary tract:16\%, dermatological: $15 \%$, abdominal:5\%, Osteoarticular: $1 \%$. Not severe infection:68\%. Mean days of prescription:7 days. BL allergy:33\%. Alternative to use FQ:77\%. 60\% sample culture (blood:30\%, urine:20\%, dermic: $15 \%$, sputum:10\%). Change after result:25\%. Sequential oral therapy:62\%. History of risk factors:aortic aneurysm(1), giant cell arteritis(1), Atherosclerosis:65\%, Atrial fibrillation:35\%. Adverse effects: digestive:7.8\% (abdominal pain: $2.2 \%$, diarrhea:5.6\%). Exanthema:2.2\%. Hyperglycaemia: $4.5 \%$. Delirium: $8 \%$. No adverse effects: $79 \%$. 
Conclusion: The use of FQ represents a low percentage in comparison other antibiotics, however, there are alternatives, for non-severe infections and absence of BL allergy. It's used more in respiratory diseases (presence of bronchiectasis and pseudomonas superinfection) with BL allergy and less in urinary tract infection (high resistance rate). In general, are well tolerated, even though in our patients (high comorbidities and polypharmacy) it's hard to attribute some adverse effects.

\section{Abstract \# 434}

Trends in benzodiazepine receptor agonists use and associated factors in belgian older adults: analysis of the belgian health interview survey data

\section{Catherine Pétein ${ }^{1}$, Anne Spinewine ${ }^{1}$, Séverine Henrard ${ }^{2}$}

${ }^{1}$ UCLouvain, Louvain Drug Research Institute, Clinical Pharmacy Research Group, Brussels, Belgium, ${ }^{12}$ UCLouvain, Louvain Drug Research Institute, Clinical Pharmacy Research Group/ UCLouvain, Institute of Health and Society (IRSS), Brussels, Belgium

Introduction: Benzodiazepine receptor agonists (BZRA), that include benzodiazepines and z-drugs, are commonly prescribed for insomnia and anxiety in older adults and often used long-term. Yet, due to an unfavorable benefit-risk ratio, recommendations suggest avoidance or a maximal treatment duration of 4 weeks. We aimed to describe trends of BZRA use in older adults ( $\geq 65$ years) over a 10 -year period and associated factors in Belgium.

Methods: BZRA use prevalence rates, standardized for age, sex and region, were calculated using Belgian Health Interview Survey data in 2004, 2008 and 2013. Multivariable logistic regression was performed to assess factors associated with BZRA use in 2013 ( $\mathrm{N}=1286)$ and included socio-demographic factors, geriatric factors, comorbidities, medication use, healthcare services use, and subjective, mental and social health indicators.

Results: Overall, standardized prevalence rate of BZRA use decreased significantly from $22 \%$ to $18 \%$ between 2004 and 2013 (rate difference $(95 \% \mathrm{CI})$ : $-4.0 \%\left(-6.8 ;-{ }^{1} 1.3\right)$ ). However, no decline was observed in z-drugs use nor in multiple BZRA users. In multivariable analysis, sleeping disorder was associated with BZRA use $(\mathrm{OR}(95 \% \mathrm{CI})=1.86(1.31 ; 2.64))$ but not with anxiety disorder. Other variables significantly associated to BZRA use included female gender, poor mental health, polypharmacy, trazodone and other antidepressant use.

Key conclusions: An encouraging decline in BZRA use was observed from 2004 to 2013, but it remained highly prevalent in Belgian older adults. Promotion of alternatives to BZRA for sleeping problems remains essential. High-risk subgroups such as multiple BZRA users or concomitant users of BZRA and antidepressant should be targeted in deprescribing interventions.

\section{Abstract \# 435 \\ Deprescribing tool for STOPPFall (screening tool of older persons prescriptions in older adults with high fall risk) items}

L.J. Seppala ${ }^{1}$, M. Petrovic ${ }^{2}$, J. Ryg ${ }^{3}$, G. Bahat ${ }^{4}$, E. Topinkova ${ }^{5}$, K. Szczerbińska $^{6}$, T.J.M. van der Cammen ${ }^{7}$, S. Hartikainen ${ }^{8}$, B. Ilhan ${ }^{9}$, F. Landi ${ }^{10}$, Y. Morrissey ${ }^{11}$, A. Mair ${ }^{12}$, M. Gutiérrez-Valencia ${ }^{13}$, M.H. Emmelot-Vonk $^{14}$, M.A. Caballero Mora ${ }^{15}$, M. Denkinger ${ }^{16}, \mathrm{P}$ Crome $^{17}$, S.H.D. Jackson ${ }^{18}$, A. Correa-Pérez ${ }^{19}$, W. Knol ${ }^{20}$, G. Soulis $^{21}$, A. Gudmundsson ${ }^{22}$, G. Ziere ${ }^{23}$, M. Wehling ${ }^{24}$, D. $\mathrm{O}^{\prime}$ Mahony ${ }^{25}$, A. Cherubini ${ }^{26}$, N. van der Velde ${ }^{27}$
${ }^{1}$ Department of Internal Medicine, Section of Geriatric Medicine, Amsterdam Public Health Research Institute, Amsterdam UMC, University of Amsterdam, Meibergdreef 9, 1105AZ Amsterdam, The Netherlands, ${ }^{2}$ Department of Internal Medicine and Paediatrics (section of Geriatrics), Ghent University, Ghent, Belgium, ${ }^{3}$ Department of Geriatric Medicine, Odense University Hospital, Odense, Denmark and Geriatric Research Unit, Department of Clinical Research, University of Southern Denmark, Odense, Denmark, ${ }^{4}$ Istanbul Medical School, Department of Internal Medicine, Division of Geriatrics, Istanbul University, Capa, 34093 Istanbul, Turkey, ${ }^{5}$ Department of Geriatrics and Gerontology, 1st Faculty of Medicine, Charles University, Prague, Czech Republic and Faculty of Health and Social Sciences, South Bohemian University, Česke Budějovice, Czech Republic, ${ }^{6}$ Laboratory for Research on Aging Society, Department of Sociology of Medicine, Epidemiology and Preventive Medicine Chair, Faculty of Medicine, Jagiellonian University Medical College, Krakow, Poland, ${ }^{7}$ Faculty of Industrial Design Engineering, Delft University of Technology, Delft, The Netherlands, ${ }^{8}$ School of Pharmacy, University of Eastern Finland, Kuopio, Finland, ${ }^{9}$ Division of Geriatrics, Department of Internal Medicine, Şişli Hamidiye Etfal Training and Research Hospital, University of Medical Sciences, Istanbul, Turkey., ${ }^{10}$ Department of Gerontology, Neuroscience and Orthopedics, Catholic University of the Sacred Heart, Rome, Italy, ${ }^{11}$ Health Care of Older People, East Kent Hospitals University NHS Foundation Trust, Canterbury, Kent, UK., ${ }^{12}$ Effective Prescribing and Therapeutics, Health and Social Care Directorate, Scottish Government, Edinburgh, Scotland, UK, ${ }^{13}$ Unit of Innovation and Organization, Navarre Health Service, Pamplona, Spain, ${ }^{14}$ Department of Geriatrics, University Medical Center Utrecht, Utrecht University, Utrecht, The Netherlands, ${ }^{15}$ Servicio de Geriatría, Hospital General Universitario de Ciudad Real and CIBER de Fragilidad y Envejecimiento Saludable, Spain,

${ }^{16}$ Agaplesion Bethesda Clinic, Geriatric Research Unit Ulm University and Geriatric Centre Ulm, Ulm, Germany., ${ }^{17}$ Research Department of Primary Care and Population Health, University College London, London, UK., ${ }^{18}$ Department of Clinical Gerontology, King's College, London, England, UK., ${ }^{19}$ Servicio de Geriatría, Hospital Universitario Ramón y Cajal (IRYCIS), Madrid, Spain, ${ }^{20}$ Department of Geriatric Medicine and Expertise Centre Pharmacotherapy in Old Persons, University Medical Center Utrecht, Utrecht University, Utrecht, The Netherlands., ${ }^{21}$ Outpatient Geriatric Assessment Unit, Henry Dunant Hospital Center, Athens, Greece,

${ }^{22}$ Landspitali University Hospital, Reykjavik, Iceland and Faculty of Medicine, University of Iceland, Reykjavik, Iceland, ${ }^{23}$ Department of Internal Medicine, Erasmus MC, University Medical Centre Rotterdam, Rotterdam, The Netherlands and Department of Epidemiology, Erasmus MC, University Medical Centre Rotterdam, Rotterdam, The Netherlands, ${ }^{24}$ Institute for Clinical Pharmacology, Medical Faculty Mannheim, Ruprecht-Karls-University Heidelberg, Germany, ${ }^{25}$ Department of Geriatric Medicine, Cork University Hospital, Cork, Ireland and Department of Medicine, University College Cork, Cork, Ireland, ${ }^{26}$ Geriatria, Accettazione Geriatrica e Centro di ricerca per l'invecchiamento, IRCCS INRCA, Ancona, Italy.

Background: Health care professionals are often reluctant to deprescribe fall-risk-increasing drugs (FRIDs). Lack of knowledge and skills form a significant barrier. To support clinicians in the management of FRIDs and to facilitate the deprescribing process, a deprescribing tool was developed by a European expert group for STOPPFall (Screening Tool of Older Persons Prescriptions in older adults with high fall risk) items.

Methods: STOPPFall was created using an expert Delphi consensus process in 2019 and in 2020, 24 panellists from EuGMS SIG on Pharmacology and Task and Finish on FRIDs completed deprescribing tool questionnaire. To develop the questionnaire, a Medline ${ }^{\circledR}$ 
literature search was performed. The panellists were asked to indicate for every medication class a possible need for stepwise withdrawal and strategy for withdrawal. They were asked in which situations withdrawal should be performed. Furthermore, panellists were requested to indicate those symptoms patients should be monitored for after deprescribing and a possible need for follow-ups.

Results: Practical deprescribing guidance was developed for STOPPFall medication classes. For each medication class, a decision tree algorithm was developed including steps from medication review to symptom monitoring after medication withdrawal.

Conclusion: STOPPFall was combined with a practical deprescribing tool designed to optimize medication review. This practical guide can help overcome current reluctance towards deprescribing in clinical practice by providing an up-to-date and straightforward source of expert knowledge.

\section{Abstract \# 436 \\ Medication review strategies to reduce hospital readmissions in the elderly: a systematic review and network meta-analysis}

Dautzenberg Lauren ${ }^{1}$, Lisa Bretagne ${ }^{2}$, Dineke Koek ${ }^{1}$, Sofia Tsokani ${ }^{3}$, Stella Zevgiti ${ }^{3}$, Nicolas Rodondi ${ }^{2}$, Rob Scholten ${ }^{4}$, Anne Rutjes ${ }^{5}$, Marcello Di Nisio ${ }^{6}$, Renee Raijmann ${ }^{1}$, Marielle Emmelot-Vonk ${ }^{1}$, Dimitris Mavridis ${ }^{7}$, Wilma Knol ${ }^{1}$

${ }^{1}$ Department of Geriatric Medicine, University Medical Center Utrecht, Utrecht University, Utrecht, The Netherlands, ${ }^{2} 1$ Department of General Internal Medicine, Inselspital, Bern University Hospital, University of Bern, Bern, Switzerland 2. Institute of Primary Health Care (BIHAM), University of Bern, Bern, Switzerland., ${ }^{3}$ Department of Primary Education, School of Education, University of Ioannina, Ioannina, Greece, ${ }^{4}$ Cochrane Netherlands / Julius Center for Health Sciences and Primary Care, University Medical Center Utrecht, Utrecht University, Utrecht, The Netherlands., ${ }^{5}$ Institute of Primary Health Care (BIHAM), University of Bern, Bern, Switzerland. 2. Institute of Social and Preventive Medicine (ISPM), University of Bern, Bern, Switzerland, ${ }^{6}$ Department of Medicine and Ageing Sciences, University G. D'Annunzio, Chieti, Italy, ${ }^{7} 1$ Department of Primary Education, School of Education, University of Ioannina, Ioannina, Greece. 2 Paris Descartes University, Sorbonne Paris Cité, Faculté de Médecine, Paris, France

Objective: To assess the effectiveness of medication review interventions for preventing hospital readmissions in older adults.Design: Systematic review, network meta-analysis (NMA).

Data sources: Ovid MEDLINE, Embase, The Cochrane Central Register of Controlled Trials and CINAHL until September 13, 2019. Eligibility criteria: Randomised controlled trials evaluating the effectiveness of medication review interventions with or without cointerventions to prevent hospital readmissions in hospitalised or recently discharged adults aged 65 and older.

Main outcome: Persons experiencing at least one all cause hospital readmission.

Results: After screening 3,257 studies for eligibility, 25 studies were finally included. For the outcome persons experiencing at least one all cause hospital readmission within 30 days, a NMA including 11 studies (7,318 participants) was conducted. Medication review in combination with medication reconciliation and patient education (risk ratio (RR) 0.45 ; 95\% confidence interval (CI) $0.26-0.80$ ) and medication review in combination with medication reconciliation, patient education, professional education and transitional care (RR 0.64 ; 95\% CI 0.49-0.84) were associated with a lower risk of hospital readmissions compared to usual care. There was no statistically significant effect for medication review without co-interventions. For at least one all cause hospital readmission without any time constraints, the NMA included 24 studies (11,677 participants). Medication review in combination with medication reconciliation, patient education, professional education and transitional care resulted in a lower risk of hospital readmissions (RR 0.82;95\%CI 0.74-0.91) compared to usual care.

Conclusion: Medication review with co-interventions reduced the risk of hospital readmissions compared to usual care. Medication review without co-interventions is not effective.

\section{ABSTRACT \# 437}

\section{Linezolid induced pancytopenia}

Jorge Eduardo Corrales Cardenal ${ }^{1}$, Carmen Elías de Molins Peña ${ }^{1}$, Beatriz Gamboa Huarte

${ }^{1}$ Hospital Nuestra Señora de Gracia, Zaragoza, Spain

Case report: 80 -year-old. Personal history: Type 2 diabetes, high blood pressure, atrial fibrillation, bladder cancer. Starts with sudden lumbago, treated in 3 occasions with nonsteroidal anti-inflammatory drugs without recovery, associates fever and it's referred to emergency room. It's diagnosed with urinary sepsis and is admitted in the geriatric ward. Starts with broad spectrum antibiotic (imipenem). Due to persistency of abdominal and back pain is performed an abdominal CT that show L1-L2 and L3-L4 spondylodiscitis + bilateral psoas muscle abscess. Blood and urine culture were negative. Abscess puncture is not performed. Conservative treatment with broad-spectrum antibiotic is initiated empirically with vancomycin, tigecycline and gentamicin. Periodic blood work is done without abnormalities. After 2 weeks of parenteral treatment, oral treatment is initiated with linezolid, fosfomycin and doxycycline and is discharged. Re-admitted a few days later with sickness, vomiting and atrial fibrillation. Blood analysis: anemia + thrombocytopenia + hyperlactatemia. Blood tests show an evolution towards a non-regenerative pancytopenia. Antibiotic treatment is suspended due to suspicion of a serious adverse reaction to linezolid. Ruling out the presence of deficiency factors. Is treated with support measures and analytical follow-up control. The symptoms disappear without sequels one month later.

Conclusions: Pancytopenia is one of the most serious adverse reactions associated with the use of Linezolid and usually results in treatment termination. Routine analytical monitoring is considered advisable in the use of linezolid in geriatric patients.

\section{Abstract \# 438}

Potentially inappropriate medication use in older adults with mild-moderate Alzheimer's disease: prevalence and associations with adverse events

\section{Claire Murphy ${ }^{1}$, Adam Dyer ${ }^{1}$, Sean Kennelly ${ }^{2}$, Brian Lawlor ${ }^{3}$}

${ }^{1}$ Department of Age-Related Healthcare, Tallaght University Hospital, ${ }^{2}$ Department of Age-Related Healthcare, Tallaght University Hospital. Department of Medical Gerontology, School of Medicine, Trinity College Dublin, ${ }^{3}$ Department of Medical Gerontology, School of Medicine, Trinity College Dublin. Mercer's Institute for Research on Ageing, St James's Hospital, Dublin, Ireland

Introduction: Potentially Inappropriate Medication (PIM) use is prevalent in older adults and is associated with adverse events, hospitalisation and mortality. We assessed patterns and associations of PIM use in older adults with mild-to-moderate Alzheimer's Disease (AD), who may represent a particularly vulnerable group.

Methods: Analysis of data from NILVAD, an 18-month Randomised Control Trial of Nilvadapine in mild-to-moderate AD. 448 older adults with mild-to-moderate $\mathrm{AD}$ from 23 centres in 9 European 
countries were included. The v2 STOPP criteria were applied in duplicate to identify PIM use. Associations between PIM use and adverse events/unscheduled healthcare visits in addition to the associations between PIM use and AD progression were evaluated.

Results: Of 448 participants (mean age: $72.56 \pm 8.19$ years), over half $(55.8 \%)$ were prescribed a PIM with $30.1 \%$ being prescribed $2+$ PIMs. The most frequent PIMs were (i) long term benzodiazepines $(11.6 \% \mathrm{~N}=52 / 448)$, (ii) SSRIs (Selective Serotonin Reuptake Inhibitors) without appropriate indication $(11.1 \% \mathrm{~N}=50 / 448)$ and (iii) Proton-Pump Inhibitors (PPI) without appropriate indication $(10.7 \%$ $\mathrm{N}=48 / 448$ ). Increasing number of PIMs was associated with a greater risk of adverse events (IRR 1.17, 1.13-1.19, $\mathrm{p}<0.001$ ), serious adverse events (IRR 1.27; 1.17-1.37, $\mathrm{p}<0.001$ ), unscheduled hospitalisations (IRR 1.16, 1.03-1.30, p = 0.016) and GP visits (IRR 1.22, $1.15-1.28, \mathrm{p}<0.001)$. PIM use was not associated with dementia progression.

Conclusions: PIM use is highly prevalent in mild-to-moderate $\mathrm{AD}$ and is associated with adverse events and unscheduled healthcare utilisation. Further attention to de-prescribing in this vulnerable group is warranted.

\section{Abstract \# 439}

\section{Cause of syncope in an old adult}

Raluca Brodocianu ${ }^{1}$, Adina Carmen Ilie $^{2}$, Anca Iuliana Pislaru ${ }^{2}$, Ioana Alexandra Sandu ${ }^{2}$, Ramona Stefaniu ${ }^{2}$, Anna Marie Herghelegiu ${ }^{3}$, Catalina Raluca Nuta ${ }^{3}$, Ovidiu Lucian Bajenaru ${ }^{3}$, Gabriel Ioan Prada ${ }^{3}$, Ioana Dana Alexa ${ }^{2}$

${ }^{1}$ CI Parhon Hospital Iasi, ${ }^{2}$ University of Medicine and Pharmacy Iasi, ${ }^{3}$ University of Medicine and Pharmacy Bucharest

Ivabradine is a selective inhibitor of the sinus node I(f) channel often prescribed in patients with heart failure, but also in patients with chronic coronary disease. This medication is documented to be well tolerated by the older patients. It is one of the few cardiac medications without orthostatic hypotension as side effect. Syncope is a frequent emergency in the old adult. To emphasis a less common side effect of the ivabradine, we present a clinical case of an 84-year-old patient, addressed for a syncope. He associated other symptoms such as severe vertigo, physical asthenia and fatigue. Emergency cranial CT scan ruled out a possible neurological etiology. His personal hystory included: chronic coronary syndrome, orthostatic hypotension and benign prostatic hyperplasia. Its chronic treatment included: ivabradine due to its intolerance to beta-blocker, nitrates and calciumblockers, aspirine and tamsulosin. Clinical examination revealed orthostatic hypotension, considered secondary to dehydration, chronic venous insufficiency, and tamsulosine side effects. The ECG Holter revealed intermittent second-degree atrioventricular block. We considered the second-degree atrioventricular block as iatrogenic, secondary to ivabradine treatment. The ivabradine treatment was stopped and after the proper wash-out period another ECG Holter mounted. The ECG Holter was normal during all monitored period. The patient did not experience another syncope or associated symptomatology. Atrioventricular block of second degree is a very rare, less than $0.01 \%$, side effect of ivabradine. In an older patient, ivabradine treatment is well tolerated, despite his multiple co-morbidities and polypharmacy. The occurrence of atrioventricular block and syncope in patients treated with ivabradine is relatively low in the older patients, nevertheless we must consider it.

\section{Abstract \# 440}

From simple painkiller to an TNF-alpha inhibitor and back again or Doing less is always more in geriatrics

Leners Jean-Claude ${ }^{1} \backslash$

\section{${ }^{1} \mathrm{LTCF}$}

A male patient aged 81 years now, is known to us for years with a primary diagnosis of learning disability. (LD) Over years he complained on back-pain due to osteopenia and cervico-dorsolombarthrosis and treated by simple pain-killers.During a break in our medical follow-up (longer humanitarian mission), the patient was addressed to a rheumatologist and treated for rheumatoid disease with prednisone and TNF alpha inhibitor. Pain level never changed (Eular:2 pts) but patient's communication was poor and probably the major reason for medical misunderstanding .After 6 months appeared: aphthous lesions, high temperature, herpetic vesicles, diarrhoea and pneumonia. The blood sample showed a pancytopenia with leukopenia $1.3 \mathrm{giga} / \mathrm{l}$; neutrophils at $0.38 \mathrm{giga} / \mathrm{l} ; \mathrm{Hb}$ at $9.8 \mathrm{~g} / \mathrm{dl}$ and thrombopenia at $39 \mathrm{giga} / \mathrm{lDouble}$ antibiotic treatment, isolation and immediate stop of etanercept cured the patient and even changed to a thrombocytosis $(600$ giga/l). We excluded a possible myeloproliferative syndrome (search for mutation in gene Jak2 : negative).Since one year now, blood analysis are normal, few symptoms present and Rx of the vertebra still shows simple degenerative signs and a diffuse idiopathic skeletal hyperostosis ( DISH) .In conclusion, we faced severe side-effects of TNF alpha inhibitor (mostly rare), which fortunately regressed after stopping the drug. The most important message from this case study is: (1) make a clear and plain communication to elderly with LD ; (2) trust in simple and standardized evaluation tools and (3) go slowly in the introduction of new biotherapeutics.

\section{Abstract \# 441}

Association between number of medications and mortality in geriatric inpatients: a Danish nationwide register-based cohort study

Kristoffer K. Brockhattingen ${ }^{1}$, Pavithra Laxsen Anru ${ }^{2}$, Tahir Masud ${ }^{3}$, Mirko Petrovic ${ }^{4}$, Jesper Ryg ${ }^{5}$

$\left.{ }^{1} 1\right)$ Department of Geriatrics, Odense University Hospital Svendborg Hospital, Denmark; 2) Geriatric Research Unit, Department of Clinical Research, University of Southern Denmark, Odense, Denmark, ${ }^{2} 3$ ) Research Unit of Clinical Epidemiology, Department of Clinical Research, University of Southern Denmark, Odense, Denmark; 4) Center for Clinical Epidemiology, Odense University Hospital, Odense, Denmark, ${ }^{3}$ ) Geriatric Research Unit, Department of Clinical Research, University of Southern Denmark, Odense, Denmark; 5) Geriatric Department, Nottingham University Hospital, Nottingham, UK; 6) Department of Geriatric Medicine, Odense University Hospital, Odense, Denmark, ${ }^{4} 7$ ) Department of Geriatrics, Ghent University Hospital, Ghent, Belgium, ${ }^{4}$ 2) Geriatric Research Unit, Department of Clinical Research, University of Southern Denmark, Odense, Denmark; 6) Department of Geriatric Medicine, Odense University Hospital, Odense, Denmark

Purpose: To explore the association between the number of medications and mortality in geriatric inpatients taking activities of daily living and comorbidities into account.

Methods: A nationwide population-based cohort study was performed including all patients aged $\geq 65$ years admitted to geriatric departments in Denmark during 2005-2014. The outcome of interest was mortality. Activities of daily living using Barthel-Index (BI) were measured at admission. National health registers were used to link 
data on an individual level extracting data on medications, and hospital diseases. Patients were followed to the end of study (31.12.2015), death, or emigration, which ever occurred first. KaplanMeier survival curves were used to estimate crude survival proportions. Univariable and multivariable analyses were performed using Cox regression. The multivariable analysis adjusted for age, marital status, period of hospital admission, BMI, and BI (model 1), and further adding either number of diseases (model 2) or Charlson comorbidity index (model 3).

Results: We included 74603 patients $(62.8 \%$ women), with a median age of 83 (interquartile range [IQR] 77-88) years. Patients used a median of 6 (IQR 4-9) medications. Increasing number of medications was associated with increased overall, 30-days, and 1-year mortality in all 3 multivariable models for both men and women. For each extra medication the mortality increased by $3 \%$ in women and $4 \%$ in men in the fully adjusted model.

Conclusion: Increasing number of medications was associated with mortality in this nationwide cohort of geriatric inpatients. Our findings highlight the importance of polypharmacy in older patients with comorbidities.

\section{Abstract \# 442 \\ Prevalence and predictors of adverse drug reactions in older hypertensive adults}

Giada Turrin ${ }^{1}$, Antonio Coscarelli ${ }^{2}$, Maria Flora D'Andria ${ }^{2}$, Virginia Tortu $^{2}$, Evelina Giuliani ${ }^{2}$, Andrea Ungar ${ }^{2}$, Giulia Rivasi ${ }^{2}$

${ }^{1}$ Referral Centre for Hypertension Management in Older Adults, Division of Geriatric and Intensive Care Medicine, Careggi Hospital and University of Florence, Italy., ${ }^{2}$ Referral Centre for Hypertension Management in Older Adults, Division of Geriatric and Intensive Care Medicine, Careggi Hospital and University of Florence, Italy

Introduction: Polipharmacy is common in older people, thus increasing the risk of adverse drug reactions. This study aimed to analyse prevalence and predictors of the most relevant adverse drug reactions in older hypertensive adults.

Methods: We retrospectively analysed data on antihypertensive- and statin-related adverse drug reactions in adults aged 65 or older evaluated at the Hypertension Clinic of Careggi Hospital, Florence, Italy, between January 2018 and December 2019. Predictors were investigated using multivariate logistic regression.

Results: Of 262 participants (mean age 75.9 years, $55.3 \%$ female), the $19 \%$ of the patients taking ACE-inhibitors reported cough while $33.6 \%$ of the patients taking calcium channel blockers (CCB) reported peripheral edema, occuring more frequently of amlodipine. The prevalence of statin-related myalgia was $23.5 \%$. At multivariate analysis SNRI antidepressants and gastroesophageal reflux disease (GERD) were predictive of ACE-inhibitors induced cough (OR 16.220, 95\% CI 1.243 - 211.621; OR 4.207, 95\% CI 1.249 - 4.171, respectively) and angiotensin-receptor blockers were associated with an increased risk of peripheral edema on CCB (OR 2.500, 95\% CI 1.095 - 5.710). GERD was predictive for statin-induced myalgia (OR $6.737,95 \%$ CI 1.530 - 29.662).

Conclusion: ACE inhibitor-induced cough, CCB-related peripheral edema and statin-induced myalgia are common in older hypertensive adults. SNRI antidepressants and GERD are independently associated with ACE inhibitor-induced cough, while angiotensin-receptor blockers are predictive of CCB-related peripheral edema. GERD is also associated with statin myalgia.

\section{Abstract \# 443}

Statin use in older adults with Alzheimer disease: associations with dementia progression and adverse events

Claire Murphy ${ }^{1}$, Adam Dyer ${ }^{1}$, Sean Kennelly ${ }^{2}$, Brian Lawlor ${ }^{3}$

${ }^{1}$ Department of Age-Related Healthcare, Tallaght University

Hospital, ${ }^{2}$ Department of Age-Related Healthcare, Tallaght

University Hospital. Department of Medical Gerontology, School of

Medicine, Trinity College Dublin, ${ }^{3}$ Department of Medical

Gerontology, School of Medicine, Trinity College Dublin. Mercer's Institute for Research on Ageing, St James's Hospital, Dublin, Ireland

Background: Whilst statin use is recommended for secondary prevention of cardiovascular disease, increasing attention has been paid the potential benefits of these drugs in delaying the onset or course of dementia. Whilst some reports have generated concerns over statin safety in vulnerable groups, there is a paucity of data assessing their potential benefit and harm in older adults with dementia.

Method: We analysed data from NILVAD, a randomised control trial of Nilvadapine in mild-moderate Alzheimer's Dementia (AD). We analysed the effect of statin use on cognitive function and dementia severity on three validated tools over 18 months using mixed-effects linear models in addition to the association between statin use and adverse events using poisson regression.

Results: Of the NILVAD cohort (mean age: 77.9 years; $61.9 \%$ female) , $34.9 \%$ (178) were prescribed a statin for the 18 month duration. Statin use was not associated with the rate of cognitive decline or dementia progression under either unadjusted/adjusted models. Similarly, statin use was not associated with an increase in adverse events (IRR 0.93, 0.85-1.02; $\mathrm{p}=0.13$ ), serious adverse events (IRR 0.77, $0.57-1.03$; p 0.08), unscheduled GP visits (IRR 1.01, $0.80-1.27, \mathrm{p}=0.93$ ) or unscheduled hospitalisation (IRR $0.81, \mathrm{CI}$ $95 \% 0.51-1.29$ ).

Conclusion: Whilst statin use was not associated with attenuated cognitive decline or dementia progression in $\mathrm{AD}$, they were not associated with any increase risk of adverse events or unscheduled healthcare utilisation. Our findings support the safety of statin use in older adults with $\mathrm{AD}$ for the ongoing prevention of cardiovascular disease.

\section{Abstract \# 444}

Improving documentation of direct oral anticoagulant prescribing in stroke patients

Ruth Daunt ${ }^{1}$, Owen Feely ${ }^{1}$, David Williams ${ }^{1}$, Karl Boyle ${ }^{1}$, Barry Moynihan ${ }^{1}$

${ }^{1}$ Beaumont Hospital

Introduction: Direct Oral Anticoagulants (DOACs) are effective treatments for stroke prevention in atrial fibrillation (SPAF). The ESC (European Society of Cardiology) guidelines describe specific quality indicators for initiating DOACs. This quality improvement project aimed to review current documentation practises concerning DOAC initiation and to develop a strategy to improve documentation.

Method: Medical charts and discharge letters were reviewed on patients admitted under the stroke team in Beaumont Hospital over three months. Data collected included, documentation of initiation of a DOAC, patient information and information provided to their general practitioner regarding new DOAC prescription.

Results: 10 patients were commenced on a DOAC for SPAF during their hospital admission. $80 \%$ had the name of the DOAC initiated documented in their medical chart. The dose was documented in the medical chart in $30 \%, 10 \%$ documented the weight and renal function. Review of other prescribed medications was documented in 
$30 \%$. $60 \%$ of patients received verbal information but none were documented to have received written information. $80 \%$ of discharge letters documented new prescription of a DOAC, $10 \%$ documented all the recommendations outlined in the ESC guidelines.

Conclusions: This project highlighted potential areas for improvement. Group discussion following audit presentation led to a design of a DOAC sticker to meet ESC guidelines. The sticker includes prompts for correct prescribing, education of patients and details to highlight in the discharge letter. Due to COVID 19 the introduction of this sticker was delayed until June 2020 but this sticker approach could help other centres improve DOAC documentation.

\section{Abstract \# 445 \\ Prevalence of potentially inappropriate prescriptions (STOPPfrail criteria) in patients with end-stage dementia}

Noelia Perez-Abascal $^{1}$, Eva Delgado Silveira ${ }^{2}$, María Muñoz García ${ }^{2}$, Beatriz Esteban Cartelle ${ }^{2}$, Jesús Mateos-Nozal ${ }^{1}$, Lorena GarcíaCabrera $^{3}$, Lourdes Rexach Cano ${ }^{4}$, Alfonso José Cruz Jentoft ${ }^{5}$

${ }^{1}$ Servicio de Geriatría. Hospital Universitario Ramón y Cajal (IRYCIS). Madrid. Spain, ${ }^{2}$ Servicio de Farmacia. Hospital Universitario Ramón y Cajal (IRYCIS). Madrid. Spain, ${ }^{3}$ Unidad de Cuidados Paliativos. Hospital Universitario Ramón y Cajal (IRYCIS).Madrid.Spain, ${ }^{4}$ Unidad de Cuidados Paliativos. Hospital Universitario Ramón y Cajal (IRYCIS). Madrid. Spain, ${ }^{5}$ Servicio de Geriatría. Hospital Universitario Ramón y Cajal. Madrid. Spain

Introduction: STOPPFrail is a screening tool to detect potentially inappropriate medication (PIM) use in frail older adults with limited life expectancy. The objective of this study was to estimate the prevalence of chronic use of PIM in patients with end-stage dementia using the STOPPFrail criteria.

Methods: Retrospective study of patients with end-stage dementia (Global Deterioration Scale 7) who died during 2017-2019 in an Acute Geriatric Unit (AGU). We collected sociodemographic variables, Charlson comorbidity index, geriatric syndromes, number of admissions and emergency department (ED) visits in the last year of life and previous admissions to the AGU. Chronic use of PIM on admission and along the last year of life were recorded.

Results: $\mathrm{n}=99$. Mean age $92.6 \pm 4.6,77 \%$ female. $44 \%$ lived in nursing homes. Mean Charlson index $3.2 \pm 1.5$. $76 \%$ on polypharmacy (mean $6.8 \pm 3.1$ drugs). $67 \%$ had malnutrition, $44 \%$ pressure ulcers, $84 \%$ dysphagia and $89 \%$ delirium. They had a mean of 2.7 ED visits and 1.7 admissions in the last year of life. 57\% met at least one STOPPFrail criteria, mean $0.9 \pm 1.0(0-5)$. The $36 \%$ that had previous admissions to AGU had fewer PIM (40\% vs $67 \%, \mathrm{p}=0.011)$. The most frequent PIM were: proton pump inhibitors (PPI) (34.3\%), antipsychotis $(17.2 \%)$, calcium supplements $(12 \%)$, statins $(8.1 \%)$, memantine $(8.1 \%)$ and antiplatelet drugs $(7.1 \%)$.

Key conclusions: More than half of the patients with end-stage of dementia admitted to an AGU use PIM at the end of life. Former admission to the AGU was associated with a reduced use of PIM.

\section{Abstract \# 446 \\ Usual geriatric management reduces Benzodiazepine receptor agonists (BZRA) load}

Sibille François-Xavier ${ }^{1}$, Maljean Laurentine ${ }^{2}$, Dehanne Fabian ${ }^{3}$, Schoevaerdts Didier ${ }^{1}$, Spinewine Anne ${ }^{1}$, de Saint-Hubert Marie ${ }^{1}$

${ }^{1}$ CHU UCL Namur/ UCLouvain (Belgium), ${ }^{2}$ Université GrenobleAlpes (France), ${ }^{3} \mathrm{CHU}$ UCL Namur (Belgium)
Introduction: Benzodiazepine receptor agonists (BZRA) are associated with several adverse events (AE) such as falls, fractures, or cognitive decline, especially in older multimorbid patients and in association with other psychotropic drugs. However, the prevalence of BZRA use is high in this population. Hospitalizations in geriatric wards can be an opportunity for deprescription. We aimed to describe BZRA use among a cohort of older multimorbid patients receiving usual care during their hospital stay in a geriatric ward and to analyze factors associated with BZRA deprescription during their hospitalization.

Methods: A retrospective study within 3 Belgian geriatric wards between January and December 2018. Data were collected from medical records of 1277 consecutively admitted patients discharged alive. BZRA load was measured by converting dosages into equivalent-lorazepam and deprescription was defined as cessation or dose decrease for BZRA users at admission. Descriptive statistics and univariate analyses were performed.

Results: BZRA use was highly prevalent $(49.7 \%$ at admission and $48.6 \%$ at discharge, $\mathrm{P}=0.240$ ) and often associated with at least another psychotropic drug use (71.1\% and $69.2 \%$, respectively). Global BZRA load was significantly reduced (0.92 equivalent-lorazepam and 0.76 respectively, $\mathrm{P}<0.001$. Among BZRA users at admission, the deprescription rate was $38.6 \%$. Factors associated with a higher deprescription rate were depression $(\mathrm{P}=0.035)$ and delirium $(\mathrm{P}=0.044)$.

Conclusions: Prevalence of BZRA use was high at admission and discharge, often associated with other psychotropic drugs. Usual geriatric management significantly reduced BZRA load. Further research is needed to better understand factors related to long-term adherence and to improve deprescription rate during hospitalization.

\section{abstract \# 447}

Deprescription of benzodiazepines and benzodiazepine-like drugs in orthogeriatric patients

C.G. Gouveia ${ }^{1}$, T. Duarte $^{1}$, A.F. Carvalho ${ }^{2}$, B. Figueiredo ${ }^{3}$, B.

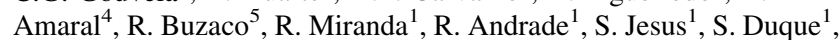
A. Martins ${ }^{1}$, C. Fonseca ${ }^{1}$, L. Campos ${ }^{1}$

${ }^{1}$ Centro Hospitalar Lisboa Ocidental - HSFX, ${ }^{2}$ USF Novo Mirante ACES Loures-Odivelas, ${ }^{3}$ USF Benfica Jardim - ACES Lisboa Norte, ${ }^{4}$ Hospital Divino Espirito Santo, Ponta Delgada, (5) USF Mosteiro ACES Loures-Odivelas

Introduction: It is established that benzodiazepines and benzodiazepine-like drugs (BZD) are associated with a higher risk of cognitive decline, falls and fractures in geriatric patients. However, they are still overprescribed. Our objective was to determine the percentage of patients admitted to our Orthogeriatric Unit (OGU) with proximal femur fracture that were chronically taking BZD, and how many underwent deprescription after comprehensive geriatric assessment.

Methods: Retrospective study of patients discharged from the OGU during a 2-year period through electronic clinical record analysis. We Compared the preadmission and discharge chronic medication according to the discharge report. Patients taking BZD before the hospital admission were classified into 5 categories according to the intervention at discharge: maintenance of the previous prescription; switching to lower risk BZD; dosage reduction; tapering schedule; discontinuation.

Results: We included 384 patients, $68.92 \%$ women, average age 84 years-old, Barthel score 81.29, Lawton score 4.01, Global deterioration scale 3.10, "Cumulative Illness Rating Scale-Geriatric" 8.9. $38.54 \%(\mathrm{~N}=148)$ were taking BZD before hospitalization. $88.51 \%$ were taking one BZD, $11.49 \%$ were taking more than one. The most 
prescribed were: bromazepam (20.86\%), lorazepam (19.63\%), alprazolam (18.40\%), zolpidem (12.27\%), diazepam (6.75\%). At discharge $58.12 \%$ were submitted to deprescription either by drug discontinuation $(37.84 \%)$, dosage reduction $(10.14 \%)$, tapering plan $(4.73 \%)$ or switching from a long acting to an intermediate or short acting benzodiazepine $(5.41 \%)$.

Conclusion: A significant percentage of patients were submitted to the deprescription of BZD, increasing the chronic medication safety profile, reducing the risk of adverse events and potentially improving older patients quality of life.

\section{Abstract \# 448}

Proton pump inhibitors: when will the paradigm shift?

T. Duarte ${ }^{1}$, C.G. Gouveia ${ }^{1}$, A.F. Carvalho ${ }^{2}$, S. Duque ${ }^{1}$, S.B.

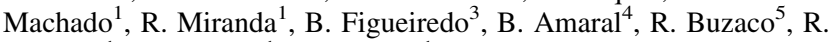
Andrade $^{1}$, L. Campos ${ }^{1}$, A. Martins ${ }^{1}$

${ }^{1}$ Hospital São Francisco Xavier, CHLO, ${ }^{2}$ USF Novo Mirante - ACES Loures-Odivelas, ${ }^{3}$ USF Benfica Jardim - ACES Lisboa Norte, ${ }^{4}$ Hospital Divino Espírito Santo, Ponta Delgada, ${ }^{5}$ USF Mosteiro ACES Loures-Odivelas

Background: The overuse of proton pump inhibitors (PPI) in the older patients remains a current practice despite its adverse effects and risk of drug interactions, considering the multimorbidity and polypharmacy in this group.

Aim: To assess the adequacy of PPI prescription prior to admission to an Orthogeriatric ward.Methods: We conducted a retrospective crosssectional study in an Orthogeriatric Unit. All patients over 64 years of age with femoral fracture admitted in this ward, between June 2016 and June 2018, were included $(n=399)$. Demographic and clinical data was collected from clinical records, namely preadmission chronic medication and diagnosis that could justify the use of PPI, according Food and Drug Administration and National Institute for Clinical Excellence recommendations and prescription criteria for older patients (STOPP and START and Beers). Statistical analysis was performed using IBM SPSS Statistics $26{ }^{\circledR}$.

Results: A total of 399 patients were included, 255 (63.9\%) were women, with an average age of $85.6 \pm 7.73$ years, Barthel score 81.4 and "Cumulative Illness Rating Scale-Geriatric" 9.1. At admission, 179 patients $(44.9 \%)$ were on PPI, 84 patients $(46.9 \%)$ of which had no indication for it. Of the patients who had an indication for PPI therapy $(n=95,53.1 \%)$, the majority were in the context of gastrointestinal bleeding prophylaxis, in high-risk patients $(n=50$, $52.6 \%)$.

Conclusions: Despite well-established recommendations, the misuse of PPI in older patients remains an important problem to address, considering the high risk of potentially fatal consequences associated.

\section{Abstract \# 449 \\ The evaluation of a geriatric discharge medication and its link to rehospitalisations and days spent at home: first results of the prospective AGITATE trial}

Mathias Freitag $^{1}$, Tanja Bülow ${ }^{2}$, Olaf Krause $^{3}$, Albrecht Eisert ${ }^{4}$, Cornelius Bollheimer ${ }^{5}$, Thea Laurentius ${ }^{5}$

${ }^{1}$ Geriatric Medicine (Med. Clinic VI) and Hospital Pharmacy, Uniklinik RWTH Aachen, Germany, ${ }^{2}$ Department of Medical Statistics, Uniklinik RWTH Aachen, Germany, ${ }^{3}$ Centre for Medicine of the Elderly, DIAKOVERE Henriettenstift, Hannover, Germany; Institute for General Practice, Hannover Medical School, Hannover, Germany, ${ }^{4}$ Hospital Pharmacy and Institute of Clinical
Pharmacology, Uniklinik RWTH Aachen, Germany, ${ }^{5}$ Geriatric Medicine (Med. Clinic VI), Uniklinik RWTH Aachen, Germany

Introduction: The transition from secondary to primary care is a critical process, often accompanied by information loss and insufficient communication, especially in geriatric patients with accumulated comorbidities resulting in polypharmacy. In these patients, discharge medication might be discontinued or changed by the general practitioner (GP) or a rehabilitation clinic (RC) with possible negative effects.

Methods: The objective of this first part's evaluation of the AGITATE study (clinicaltrials NCT03412903) was to determine if a negative GP's or RC's medication evaluation upon discharge from a specialized geriatric clinic has an impact on the number of rehospitalisations and days spent at home as well as the time until rehospitalisation, documented at 1, 3 and 6 months after discharge and adjusted for comorbidity and functional status. Negative medication evaluation was defined as changes in the hospital medication during rehabilitation or GP's first contact with the patient, resulting in two groups based on a positive or negative evaluation.

Results: 85 patients participated in the first phase and the results of 74 probands can be used in the still ongoing analysis.

Key conclusions: The results of this study could give insight into the question if immediately changing the specialized geriatric clinic's discharge medication has a negative impact on the patient. This is part of the discussion if a hospital admission in geriatric patients should be seen as an isolated event or rather as a part of the process of functional impairment requiring long-term adjustments in pharmacotherapy.

\section{Abstract \# 450}

\section{Portuguese older patients' attitudes toward deprescribing}

Anabela Pereira $^{1}$, Óscar Ribeiro ${ }^{2}$, Manuel Veríissimo ${ }^{3}$

${ }^{1}$ University of Aveiro, CINTESIS, Portugal, ${ }^{2}$ University of Aveiro, AgeingC CINTESIS, Portugal, ${ }^{3}$ University of Coimbra, faculty of medicine, Portugal

Introduction: Deprescribing is the process of withdrawal of an inappropriate medication, supervised by a health care professional to manage polypharmacy and improve outcomes. It is a complex process influenced by multiple factors, facing several barriers to its implementation. Patients' attitudes and perspectives towards deprescribing is one of the most common obstacles encountered. In Portugal, polypharmacy prevalence in the elderly is among the highest in Europe $(36.9 \%)$. Successful deprescribing strategy implementation needs to know the Portuguese reality in this area.

Methods: Translation and cultural validation to Portugal of the revised version of the Patients Attitudes Toward Deprescribing questionnaire (rPATD). Quantitative cross-sectional study, with patients aged 65 or older with at least one medication, using the rPATD Portuguese version, sociodemographic characteristics and clinical data collection. The main outcome is older patients' willingness to deprescribe inappropriate medications. The cross-sectional study is in progress.

Results (preliminary): 25 older patients, 16 females $(64 \%)$ and nine males $(36 \%)$ completed the study. The majority $(80 \%)$ are overall satisfied with their current medicines. Still, at the same time, they would be willing to stop one or more of the regular medication if their doctor said it was possible (11.1\% strongly agreed, $62.9 \%$ agreed.) Key conclusion: These are preliminary results, but they aligned with other studies using this instrument. The rPATD helps to identify if patients willing to have medicines deprescribed, which can guide Portuguese doctors in their clinical practice selecting patients most likely to succeed in this process. 


\section{Abstract \# 451 \\ Relationship between pneumonia and sarcopenia in old people-a cross-sectional study}

Takae Ebihara $^{1}$, Tatsuma Okazaki ${ }^{2}$, Kumiko Nagai ${ }^{3}$, Shinichi Izumi ${ }^{2}$, Koichi Kozaki ${ }^{1}$

${ }^{1}$ Kyorin Univ. School of Med., ${ }^{2}$ Tohoku Univ.School of Med., ${ }^{3}$ Kyorin Univ.School of Med.

Background: Respiratory muscle (RM) strength plays important roles in coughing and ventilating. Whereas sarcopenia is an aging related loss of muscle mass and function (strength or performance) whose worsening is associated with malnutrition. Nobody has investigated the relationship between RM weakness and malnutrition on pneumonia in old people.

Objective: Determine effects of RM weakness in correlation with malnutrition on incidence of pneumonia.Design A cross-sectional study Setting Geriatric ward at 2 hospitals in Japan Participants Inpatients with incidence of pneumonia $(\mathrm{n}=47)$ and controls without pneumonia $(\mathrm{n}=35)$ aged 70 years and older between April 2018 to March 2019. Measurements RM strength (maximal inspiratory pressure (PIMAX) and maximal expiratory pressure), the tongue muscle (TM) strength and sarcopenia, according to the European Working Group on Sarcopenia in Older People were evaluated. Serum-albumin levels at admission and somatic-fat volume were determined as index of malnutrition of the study. All parameters excluding serum-albumin were measured at the time of recovery from pneumonia or other disease.

Results: The RM and the TM strengths, body-trunk muscle mass, serum-albumin level, and somatic-fat were significantly lower in pneumonia patients than in controls. A logistic analysis rendered the depressed PIMAX strengths, the low body-trunk muscle mass and the low serum-albumin level in patients with healthcare-associated pneumonia (odds Ratio: 6.85, 6.86 and 5.46, respectively).

Conclusion: Functional and quantitative decrease in RM in patients with pneumonia may force old people susceptible to develop pneumonia and intractable to be cured. Furthermore, malnutrition may involve it.

\section{Abstract \# 452 \\ Spanish translation, adaptation and validation of the SarQoL@: a health-related quality of life specific for sarcopenia}

Beatriz Montero-Errasquín ${ }^{1}, \mathrm{M}^{\mathrm{a}}$ Nieves Vaquero-Pinto ${ }^{2}$, Vicente Sánchez-Cadenas ${ }^{2}$, Jesús Mateos-Nozal ${ }^{2}$, Elisabet Sánchez-García ${ }^{2}$, Luisa Alejandra Hernández-Sánchez ${ }^{2}$, Alfonso José Cruz-Jentoft ${ }^{2}$

${ }^{1}$ Hospital Universitario Ramón y Cajal, IRYCIS. Madrid, Spain., ${ }^{2}$ Hospital Universitario Ramón y Cajal, IRYCIS. Madrid, Spain

Introduction: Sarcopenia is a progressive and generalized disorder of skeletal muscle morphology and function. It's associated with an increased risk of adverse health outcomes and impaired quality of life (QoL). In 2015, a questionnaire intended to measure the QoL in patients with sarcopenia, SarQoL ${ }^{\circledR}$, was developed and validated in French.

Objectives: Translation, cultural adaptation and validation of the psychometric properties of the original version of SarQoL ${ }^{\circledR}$ into Spanish.

Material and methods: cross-sectional study, 86 participants. Two direct translations, a synthesized version of the direct translations, two reverse translations, a pre-final version, pre-test and final version were developed. Discrimination power, construct validity, internal consistency, test-retest reliability and ceiling/floor effects were analyzed and compared with the original psychometric properties.
Results: The Spanish version showed no discriminating power between sarcopenic and non-sarcopenic subjects (EWGSOP and FNIH diagnostic criteria of sarcopenia). It did show discriminating power between patients with decreased vs normal muscle strength (54.9 vs. $62.6, \mathrm{p}=0.009$ ) and low vs. normal physical performance ( 57.3 vs. $70.2 ; \mathrm{p}=0.005$ ). The Spanish questionnaire presented a good construct validity (high correlation with comparable domains of the SF-36: physical function, limitation by physical problems, vitality, mobility and ADLs), high internal consistency (Cronbach's alpha coefficient:0.84 ) and excellent test-retest reliability (intraclass correlation coefficient:0.967, 95\% CI $0.950-0.967)$. No ceiling or floor effect was found.

Conclusions: the Spanish version of SarQoL® does not discriminate between sarcopenic and non-sarcopenic patients according to the EWGSOP or FNIH criteria although it does with subjects with low muscle strength and low physical performance. The rest of the psychometric properties are similar to those of the original instrument.

\section{Abstract \# 453}

Milk Intervention Muscle AgeiNg (MIlkMAN) pilot study: quantifying the internal training response to resistance exercise in older adults at risk of sarcopenia

Christopher Hurst ${ }^{1}$, Lorelle Dismore ${ }^{1}$, Avan A Sayer ${ }^{1}$, Emma Stevenson ${ }^{2}$, Terry Aspray ${ }^{1}$, Antoneta Granic ${ }^{1}$

${ }^{1}$ AGE Research Group, Translational and Clinical Research Institute, Newcastle University, UK, ${ }^{2}$ Population Health Sciences Institute, Newcastle University, UK

Introduction: Resistance exercise (RE) represents an effective strategy for counteracting age-associated declines in muscle strength and physical performance. However, as exercise induced adaptation is primarily driven by the physiological stress imposed (i.e. the internal training response), eliciting a consistent and standardised training dose is necessary to evaluate potential intervention effects.

Methods: Thirty community-dwelling older adults $(71.7 \pm 3.6$ years; 12 female) naïve to RE, performed 6 weeks of twice weekly, machine-based progressive RE involving a combination of upper- and lower-body exercises. Internal training response was quantified using ratings of perceived exertion (RPE). Following each RE session, participants provided an overall rating of perceived exertion (sRPE) and differential ratings of perceived exertion for upper-body muscle exertion (sRPE-U) and lower-body muscle exertion (sRPE-L) using the CR100 scale (range 0-100 arbitrary units [AU]; 12, easy; 22, moderate; 35 , somewhat hard; 50, hard; 100 , maximal).

Results: Twenty-nine participants completed the intervention $(97 \%$ attendance) with mean RE session intensity rated as between 'somewhat hard' and 'hard' (sRPE: $38 \pm 19$, sRPE-U: $41 \pm 21$, sRPE-L: $35 \pm 19$ ). There was no change in mean RPE throughout the intervention (sRPE, mean slope: $-2 ; 95 \%$ confidence interval $[\mathrm{CI}]-9$ to 4, sRPE-U: $-2[-9,5]$, sRPE-L: $-3[-10,3])$, despite substantial variability between participants (sRPE, slope SD: 15 ; 95\% CI [10, 21], sRPE-U 14 [9, 21], sRPE-L 15 [11, 21]).

Key conclusions: Ratings of perceived exertion remained consistent throughout the intervention. These metrics should be validated against objective measures of RE response such as improved muscle function. 


\section{Abstract \# 454}

Milk Intervention Muscle AgeiNg (MIlkMAN) pilot study: feasibility and acceptability of a milk and resistance exercise intervention

Antoneta Granic ${ }^{1}$, Christopher Hurst ${ }^{1}$, Lorelle Dismore ${ }^{1}$, Emma Stevenson ${ }^{2}$, Avan A Sayer ${ }^{1}$, Terry Aspray ${ }^{1}$

${ }^{1}$ AGE Research Group, Translational and Clinical Research Institute, Newcastle University, ${ }^{2}$ Population Health Sciences Institute, Newcastle University

Introduction: Intervention studies have shown that adequate protein intake combined with resistance exercise (RE) can counteract declines in muscle mass and function (sarcopenia). However, the role of whole foods rich in protein, such as milk, in sarcopenia in older adults is less understood. We aimed to examine the feasibility and acceptability of milk $+\mathrm{RE}$ as an intervention for sarcopenia in communitydwelling older adults, and to collect exploratory pilot data for future substantive research.

Methods: Thirty community-dwelling older adults $(71.7 \pm 3.6$ years; $40 \%$ women) were randomised into three groups: WM (whole milk $3.6 \%$ fat) + RE, SM (skimmed milk $0.3 \%$ fat)+RE, and C (control (isocaloric carbohydrate drink)+RE. A 6-week intervention was delivered in a local gym twice weekly. After a structured RE programme for upper- and lower-body strength exercises (2-3 sets, 8-10 repetitions), participants consumed $500 \mathrm{ml}$ of milk ( $\sim 20 \mathrm{~g}$ protein) or control drink within 45 minutes post-exercise and another $500 \mathrm{ml}$ within the following 5-6 hours.

Results: The groups had similar sociodemographic, health and lifestyle characteristics. Twenty-nine participants completed the intervention with an overall attendance of $97.1 \% ; 89.7 \%$ rated their overall study experience as 'excellent'/'very good'. Drinking compliance was $97.1 \%(\mathrm{WM}), 98.3 \%$ (SM), and 95\% (C); 93.1\% rated their drink intake as 'easy'/'very easy'. RE compliance was $97.5 \%$ (WM), 98.3\% (SM), and 95.8\% (C) with overall resistance training intensity rated as 'somewhat hard' and 'hard'. No adverse effects were reported. Collection of exploratory pilot data was successful.

Conclusion: The milk $+\mathrm{RE}$ intervention conducted in a community gym setting was feasible and acceptable to older adults.

\section{Abstract \# 455 \\ Milk Intervention Muscle AgeiNg (MIlkMAN) pilot study: a content analysis of motivators and barriers to engagement \\ Lorelle Dismore $^{1}$, Christopher Hurst ${ }^{1}$, Avan A Sayer ${ }^{1}$, Emma Stevenson $^{2}$, Terry Aspray ${ }^{1}$, Antoneta Granic ${ }^{1}$ \\ ${ }^{1}$ AGE Research Group, Translational and Clinical Research Institute, Newcastle University, UK, ${ }^{2}$ Population Health Sciences Institute, Newcastle University, UK}

Introduction: Resistance training (RT) combined with adequate protein intake are recognised as preventative measures of sarcopenia, yet few older adults meet recommended levels. Additionally, limited number of studies have investigated the role of whole foods rich in protein in combination with RT in sarcopenia. To promote healthier lifestyles in older adults, an understanding of the acceptability of interventions and motivations and barriers to continued engagement is needed.

Methods: Thirty community-dwelling older adults (aged 65.8-80.1 years) took part in a 6-week intervention consuming either whole milk, skimmed milk or an isocaloric control drink, in combination with RT. A health psychologist conducted interviews with 29 participants using standardised open-ended questions about their experiences in the study, acceptability of the intervention, and motivations and barriers to continued engagement. Data were analysed using content analysis.

Results: The analysis revealed positive experiences and outcomes of taking part in the intervention, including: self-perceived improved health and physical fitness; knowledge acquisition in nutrition and exercise; positive reported outcomes (healthier lifestyle choices, increased energy and reduced pain), and social wellbeing. Social bonds and peer encouragement were motivations to continued engagement in widowed women. Having a personal instructor and being in a fun environment were highly valued by most. Drink consumption was viewed as a reward without negative effects on appetite. Barriers included affordability, environmental factors and concerns over negative health outcomes.

Conclusion: To increase engagement, muscle health promoting strategies should focus on education and interventions should be enjoyable and offer social opportunities to promote and maintain health behaviours.

\section{Abstract \# 456}

The prevalence of sarcopenia in older people north-west region of the Russian Federation

Eugeniy Zotkin ${ }^{1}$

${ }^{1}$ Co-author

Objectives: assessment of functional status and analysis of the prevalence of sarcopenia in people over 65 living in community.

Material and methods: 230 people were included in the cross-sectional study (average age $74 \pm 6.5$ years; $96.5 \%$ women). EWGSOP2 criteria were adopted. Skeletal muscle strength was determined by grip strength and Chair stand test (5-times sit-to-stand). Skeletal muscle mass was calculated by Appendicular skeletal muscle mass (ASMM) by Dual-energy X-ray absorptiometry (DXA). Muscle function was evaluated by Short physical performance battery (SPPB).

Results: Muscle strength in sarcopenics is lower than in non-sarcopenics $(\mathrm{p}<0.001)$. The overall score for SPPB tests was reduced in both groups, but the sarcopenic level was lower than the non-sarcopenic level $(p=0.035)$. The prevalence of sarcopenia is $30 \%$ for older people living in the community, aged $65-74$ years at $21.4 \%$, $85+$ years at $52.9 \%$. Men have a risk factor for sarcopenia of 2.64 (95\% CI: $1.68-4.15, \mathrm{p}<0.0001$ ). Sarcopenia was statistically significantly less in patients with obesity $3.0 \%$ (95\% CI: $0.4-10.4, p$ $<0.0001)$ and metabolic syndrome $7.0 \%$ (95\% CI: 2.6-14, 6, p $<0.0001)$. Sarcopenia is greater in patients with chronic anemia $(95 \%$ CI: $2.3-14.6, p=0.015)$. The risk of sarcopenia is 14.30 (95\% CI: 6.39-31.98) times higher in patients with a body mass index (BMI) of less than $25 \mathrm{~kg} / \mathrm{m}^{2}$.

Conclusions: Sarcopenia is a common problem in older people, especially men. Low BMI, chronic anemia increased the risk of sarcopenia

\section{Abstract \# 457}

Effects of 12-week mixed training on muscle function adaptations in middle-aged and older HIV-positive men: does HIV duration matter?

Peyrusqué Eva ${ }^{1}$, Aubertin-Leheudre Mylène ${ }^{1}$, Granet Jordan ${ }^{1}$, Bass $\mathrm{Alec}^{2}$, Kaur Navaldeep ${ }^{3}$, Mayo Nancy ${ }^{3}$, Brouillette Marie-Josée ${ }^{3}$, Fellows Lesley $\mathrm{K}^{3}$, Buckinx Fanny ${ }^{4}$

${ }^{1}$ UQAM-CRIUGM, ${ }^{2} \mathrm{U}$ MONTREAL - CRIR, ${ }^{3}$ McGill University, ${ }^{4}$ UQAM - CRIUGM 
Introduction: Muscle function decline with age. HIV seems accelerate aging process. Exercise reduces aging effects. However, how HIV duration impacts exercise adaptations is unknown. We investigated if HIV duration influence muscle function adaptations following a mixed training.

Methods: Twenty-five HIV-positive men, who completed mixed training, were divided a-posteriori in 2 groups according to HIV

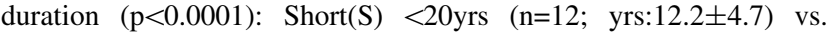
$\operatorname{Long}(\mathrm{L}) \geq 20 \mathrm{yrs}(\mathrm{n}=13$; yrs:24.7 \pm 6.3 ). Intervention (12-weeks, 3times/week): High Intensity Interval Training (15 min/session; cycle: 30s at Borg $>17+1$ min 30 of Borg: 12-15) on elliptical device + Mixed Power Training (25min/session; 2 sets; 12 repetitions; workload: $80 \%$; tempo:1-0-2-1) in major muscles. Grip strength $(\mathrm{Kg}$; Jamar(C), leg power (W; leg power rig (C), body composition (Fat (FM; \%) and Fat-free masses; DXA), muscle composition (qPCT), performance on 4-m (normal and fast speed) and 6-min (distance) walking tests were assessed pre and post intervention.

Results: No difference in chronological age (S: $51.6 \pm 6.4$ vs. L: $54.9 \pm 5.3$ yrs). S-group have lower grip strength (S: $34.4 \pm 6.9$ vs. L: $41.1 \pm 8.1, \mathrm{p}=0.039$ ) and leg power (S: $169 \pm 65$ vs. L: $239 \pm 93$, $\mathrm{p}=0.04$ ) but also higher appendicular ( $\mathrm{S}: 53 \pm 19$ vs. L: $35 \pm 22$, $\mathrm{p}=0.038$ ) and gynoid (S: $34 \pm 10$ vs. $\mathrm{L}: 22 \pm 14, \mathrm{p}=0.035) \mathrm{FM}$ than L-group. Following the intervention, all parameters improved in both groups. No change difference between groups was observed.

Conclusion: HIV therapy rather than duration may be a more important aging factor. Exercise appears to be effective in HIV-positive men whatever HIV duration. Large randomized controlled trials are needed with drug therapy stratification (era of diagnosis).

\section{Abstract \# 458 \\ Sarcopenia among nursing home residents in Catalonia: prevalence according to the new criteria of the European Working Group on Sarcopenia in Older People (EWGSOP2)}

Anna Escribà Salvans ${ }^{1}$, Sandra Rierola Fochs ${ }^{2}$, Pau Farrés Godayol ${ }^{3}$, Miriam Molas Tuneu ${ }^{2}$, Javier Jerez Roig ${ }^{3}$, Eduard Minobes Molina ${ }^{3}$, Dyego Leandro Bezerra De Souza ${ }^{3}$, Patricia Lizett Vilca Salazar ${ }^{2}$, Pau Moreno Martín ${ }^{3}$

${ }^{1}$ Autor Principal, ${ }^{2}$ Autora, ${ }^{3}$ Autor

Introduction: Given the need to establish new criteria, the EWGSOP2 defines the sarcopenia as the loss of strength and muscle mass and determines the parameters by diagnosis. The objective was to identify the prevalence of sarcopenia in institutionalised older people who live in Catalonian nursing homes $(\mathrm{NH})$, according to the new criteria of the EWGSOP2.

Methods: A cross-sectional study in $4 \mathrm{NH}$ of the Osona region (Barcelona) was conducted from January to March 2020. Residents with sarcopenia were identified using the algorithm developed and suggested by the EWGSOP2 for sarcopenia case finding and screening in practice. The main variables in the diagnosis of sarcopenia were strength, assessed by a hand grip dynamometer, muscle mass, measured by bioelectrical impedance analysis and physical performance, evaluated by the Four Meter Walking Test.

Results: 145 individuals were initially included, of which 64 were excluded to no fulfil criteria of the main study (Clinical Trials register number NCT04297904) or for not being able to perform the three assessments. 81 participants $(55.8 \%)$, mean age of 84.6 years $(\mathrm{SD}= \pm$ 7.8), $67(82.7 \%)$ women, were finally included. $6(7.4 \%)$ had no sarcopenia, $55(67.9 \%)$ were diagnosed of probable sarcopenia, 12 $(14.81 \%)$ of confirmed sarcopenia and $8(9.87 \%)$ of severe sarcopenia.

Conclusions: The study indicates that the $92.6 \%$ of the institutionalised older people in Central Catalonia is suffering of probable, confirmed or severe sarcopenia, according to the new criteria of the EWGSOP2.

\author{
Abstract \# 459 \\ Myostatin as a biomarker of muscle wasting and other \\ pathologies: state of the art and knowledge gaps \\ Jan Bączek ${ }^{1}$, Marta Silkiewicz ${ }^{2}$, Zyta Beata Wojszel ${ }^{3}$ \\ ${ }^{1}$ Doctoral School, Medical University of Bialystok, ${ }^{2}$ Medical \\ University of Białystok, ${ }^{3}$ Department of Geriatrics, Medical \\ University of Białystok
}

Sarcopenia is a geriatric syndrome with significant impact on older patients' quality of life, morbidity and mortality. Despite the available new criteria of sarcopenia developed by European Working Group on Sarcopenia in Older People (EWGSOP2 algorithm), its early diagnosis remains difficult, highlighting the necessity of looking for a valid muscle wasting biomarker. Myostatin, a muscle mass negative regulator, is one of the potential candidates. The aim of this work is to point out various factors affecting the potential of myostatin as a biomarker of muscle wasting. Based on the literature review, we can say that although myostatin raised high hopes as a candidate for sarcopenia biomarker, recent studies produced conflicting results and revealed numerous potential confounding factors influencing its use in sarcopenia diagnosing. These factors include physiological variables affecting serum myostatin abundance (such as age, sex, physical exercise and activity of various agents inhibiting and promoting myostatin pathway), as well as a variety of disorders (including heart failure, obesity and insulin resistance, kidney failure, chronic obstructive pulmonary disease and inflammatory diseases) and differences in laboratory measurement methodology.Our final conclusion is that although serum myostatin alone might not prove to be a feasible singular biomarker, it could become an important part of a recently proposed panel of muscle wasting biomarkers. However, a thorough understanding of the interrelationship of these markers for muscle mass and function, as well as establishing valid measurement methodology for myostatin and revising current research data in the light of new criteria of sarcopenia is needed.

\section{Abstract \# 460 \\ Impact of sporadic high intensity interval training on muscle and functional capacities in healthy older adults: an unplan 24 month follow up study}

Granet Jordan ${ }^{1}$, Buckinx Fanny ${ }^{2}$, Blanchard Valentin ${ }^{2}$, AubertinLeheudre Mylène ${ }^{2}$

${ }^{1}$ ((1) Department of Exercise Science, Groupe de recherche en activité physique adapté (GRAPA), Université du Québec à Montréal, Montréal (Qc), Canada (2) Centre de Recherche de l'Institut Universitaire de Gériatrie de Montréal, Montréal (Qc), Canada), ${ }^{2}(1)$ Department of Exercise Science, Groupe de recherche en activité physique adapté (GRAPA), Université du Québec à Montréal, Montréal (Qc), Canada (2) Centre de Recherche de l'Institut Universitaire de Gériatrie de Montréal, Montréal (Qc), Canada)

Introduction: Aging is characterized by a decline in functional capacities and muscle function (i.e. muscle mass, strength and quality) as well by an increase in fat mass (around 1-5\%/year). A sedentary lifestyle is associated with accelerated aging while physical activity (PA) improves or maintain general health, even in elders. However, it is unknow if sporadic supervised intervention, and more specifically High Intensity Interval Training (HIIT); can counter the effects of aging over time. Thus, we aimed to evaluate if a sporadic 
HIIT intervention can counter 2 years aging process effects in previously sedentary older adults.

Methods: Older ( $>65$ years old: 21 men +23 women) sedentary (steps $<7500 /$ d) adults who completed a 12 week HIIT intervention were reevaluated 2 years later (T2) (unplanned follow-up). Body composition (DXA), muscle function (upper and lower muscle strength, power and muscle quality), functional capacities (TUG, sitto-stand and 6min walking tests) and physical activity (PA) level were compared between baseline and T2 (SPSS; $\mathrm{p}<0.05$ : significant).

Results: Through time, PA level did not changed. We observed a decrease in total lean body mass $(>5 \%)$ whereas muscle strength and quality are maintained. Finally, muscle power and functional capacities such gait speed (TUG) still improved.

Key conclusion: A sporadic HIIT Intervention seems to slow down the aging physical process over the time. These results could have a clinical impact but need to be confirmed by comparison with a control population (e.g. active or frail) or with other type of PA training.

\section{Abstract \# 461 \\ Relationship between sarcopenia and ACTN3 R577X gene in older adults}

Mustafa Kahraman ${ }^{1}$, Banu Özulu Türkmen ${ }^{2}$, Gülistan Bahat Öztürk ${ }^{2}$, Ayla Şahin ${ }^{3}$, Aynur Dağlar ${ }^{3}$, Şükrü Öztürk ${ }^{3}$, Ali Sarper Diler ${ }^{1}$, Mehmet Akif Karan ${ }^{2}$

${ }^{1}$ İ.Ü., İstanbul Tip Fakültesi, Fizyoloji AD, ${ }^{2}$ İ.Ü., İstanbul Tip Fakültesi, İç Hastalıkları AD Geriatri Bilim Dalı, ${ }^{3}$ I.Ü̈., İstanbul Tıp Fakültesi, İç Hastalıkları AD Tıbbi Genetik Bilim Dalı

Purpose: We aimed to evaluate whether there is a relationship between ACTN3 R577X gene polymorphism and low muscle mass or sarcopenia in older Turkish adults.

Methods: The study included patients $>65$ years admitted to the geriatric outpatient clinic. Muscle mass was assessed with bioimpedance analysis (BIA). Muscle strength was evaluated by hand grip strength with a Jamar hydraulic hand dynamometer. Sarcopenia was diagnosed according to EWGSOP2 criteria. Skeletal muscle mass index (SMMI) was adjusted with two alternative methods: SMMI (height) and SMMI(BMI).

Results: 197 participants were included aged between 65 and 99 years (76.2 $\pm 6,1$ years old). 151 patients $(76.6 \%)$ were female. RR, RX and XX genotype of ACTN3 were present in $31 \%, 45.2 \%$, and $23.9 \%$, respectively. No significant difference was observed in mean age, height, weight, body mass index, timed-up-and-go-test, chair stand, gait speed across the genotypes. 50.8\% had low SMMI (BMI), whereas all participants except one case had normal SMMI (height). Alleles were not different between SMMI normal and low groups $(\mathrm{p}=0.81) .44 .6 \%$ of the participants had probable sarcopenia, $29.4 \%$ had confirmed sarcopenia, and $19.2 \%$ had severe sarcopenia. In univariate analyses, there was no significant difference in ACTN3 polymorphism between older people with probable/confirmed/severe sarcopenia, and control group. In regression analyses, neither low muscle mass nor sarcopenia was associated with ACTN3 R577X genotypes.

Conclusion: Our study suggests that there is no significant relationship between muscle mass or sarcopenia parameters and ACTN3 R577X gene polymorphism in community dwelling older adults in Turkey.

\section{Abstract \# 462}

Cutoff values to identify low muscle mass by computed tomography at 13 vertebra level

Gulistan Bahat ${ }^{1}$, Banu Ozulu Turkmen ${ }^{1}$, Samil Aliyev ${ }^{2}$, Nezahat Muge Catikkas ${ }^{1}$, Baris Bakir ${ }^{2}$, Mehmet Akif Karan ${ }^{1}$

${ }^{1}$ Istanbul University, Istanbul Medical School, Department of Internal Medicine, Division of Geriatrics, Istanbul, Turkey., ${ }^{2}$ Istanbul

University, Istanbul Medical School, Department of Radiology, Istanbul, Turkey.

Introduction: Computed tomography (CT) is considered as gold standard for evaluation of total skeletal muscle quantity. Skeletal muscle assessments at L3 vertebra level were revealed to be significantly correlated with whole body muscle measurements. Herein, we aimed to provide gender specific cutoff sfor psoas muscle mass index (PMI) and skeletal muscle mass index (SMI) at the third lumbar vertebra level in Turkish population.

Material and method: Preoperative plain CT images of living adult liver donors admitted our hospital transplantation center between June 2010- April 2018 were evaluated to determine PMI, skeletal muscle area (SMA) and SMI at the level of the third lumbar vertebra. Cutoff values using both 5th percentile and two standard deviations were considered to define low muscle mass in total study population, and in younger population aged 18-40.

Results: 601 patients (age 18-59; 326 male, 275 female) were evaluated; 482 (\%80) were young (age 18-40; 268 male, 214 female). In patients aged 20-40 gender specific SMI by using 5th percentile were calculated as $41.42 \mathrm{~cm}^{2} / \mathrm{m}^{2}$ for males and $30.7 \mathrm{~cm}^{2} / \mathrm{m}^{2}$ for females respectively. In the same age group, gender specific SMI by using two standard deviations were estimated as $38.67 \mathrm{~cm}^{2} / \mathrm{m}^{2}$ for males and $27.8 \mathrm{~cm}^{2} / \mathrm{m}^{2}$ for females respectively. In whole study population, gender specific SMI by using 5th percentile were calculated as 41.33 $\mathrm{cm}^{2} / \mathrm{m}^{2}$ for males and $31.4 \mathrm{~cm}^{2} / \mathrm{m}^{2}$ for females respectively. In the same age group, gender specific SMI by using two standard deviations were estimated as $37.84 \mathrm{~cm}^{2} / \mathrm{m}^{2}$ for males and $27.82 \mathrm{~cm}^{2} / \mathrm{m}^{2}$ for females respectively. In our study, cutoff values for SMI were comparable with the other cutoffs reported in so far studied populations. Conclusion: Our study provides cutoff values for PMI and SMI at the third lumbar vertebra level for Turkish population with different calculation methods.

\section{Abstract \# 463}

Physical activity, muscle strength and quantity in an innovative life course cohort: preliminary findings

from the MASS_Lifecourse Study

Katherine Bowden-Brown ${ }^{1}$, James Murray ${ }^{2}$, Christopher Hurst ${ }^{2}$, Antoneta Granic ${ }^{2}$, Avan Aihie Sayer ${ }^{2}$, Richard Matthew Dodds ${ }^{2}$

${ }^{1}$ AGE Research Group, Translational and Clinical Research Institute, Faculty of Medical Sciences, Newcastle University, Newcastle, UK, ${ }^{2}$ AGE Research Group, Translational and Clinical Research Institute, Faculty of Medical Sciences, Newcastle University, Newcastle, UK.

Introduction: We established the MASS_Lifecourse study to investigate changes in skeletal muscle between ages 45-85. Reduced physical activity (PA) is a key factor linked to the development of sarcopenia. Our aim was the describe the amount and patterns of PA and sedentary behaviour (SB), and relationships with muscle strength and quantity, among MASS_Lifecourse participants.

Method: Participants wore a triaxial accelerometer on their dominant wrist for seven days. Recordings were analysed to calculate time in moderate-vigorous PA (MVPA) with time not in MVPA classified as SB based on wrist position. Muscle strength was measured with a 
Jamar grip dynamometer and the 5 chair-stand test. Muscle quantity was assessed using appendicular lean muscle mass from dual-energy $\mathrm{X}$-ray absorptiometry.

Results: 68 participants (31 male) aged 47-84 (mean 65) participated in the present study. Median daily MVPA was 19.1 (IQR 7.1, 36.6) minutes and was lower with age. The pattern of MVPA across the day changed with age: the oldest group (75-84 years) achieved a lower morning activity peak followed by an earlier decline. The participants were more active than population reference data from the Active Lives Survey. Time spent in MVPA was positively associated with muscle strength, whereas SB was negatively associated. Muscle quantity was not associated with PA or SB.

Key conclusions: Participants in the MASS_Lifecourse study are more active than the general population but still show age-related declines in physical activity and strength. Future work in the cohort aims to elucidate mechanisms underlying the age-related loss of muscle strength and quantity.

\section{Abstract \# 464 \\ Comparison of EWGSOP 1 and EWGSOP 2 criteria and its use for diagnosing of sarcopenic obesity in over 70 years old patients \\ Tereza Vágnerová ${ }^{1}$, Helena Michálková ${ }^{2}$, Eva Topinková ${ }^{3}$ \\ ${ }^{1}$ Charles University, 1st Faculty of Medicine, Department of Gerontology \& Geriatrics, Prague, Czech Republic; Charles University, 1st Faculty of Medicine, Institute of Hygiene and Epidemiology, Prague, Czech Republic, ${ }^{2}$ Charles University, 1st Faculty of Medicine, Department of Gerontology \& Geriatrics, Prague, Czech Republic; Uniersity of South Bohemia, Faculty of Health and Social Sciences, Institute of Nursing, Midwifery and Emergency Care, České Budějovice, Czech Republic, ${ }^{3}$ Charles University, 1st Faculty of Medicine, Department of Gerontology \& Geriatrics, Prague, Czech Republic}

Background and aim: Combination of obesity and sarcopenia, recently defined as sarcopenic obesity (SO), is a relatively new nosological unit with negative health outcomes and impaired quality of life.This study aim is to validate the new diagnostic algorithm of sarcopenia EWGSOP-2 (2019) and compare it to the EWGSOP-1 (2010); further to describe prevalence of sarcopenia and SO in patients screened for SPRINTT study (The "Sarcopenia and Physical Frailty in Older People: Multicomponent Treatment Strategies").

Methods: 126 outpatients were included, $20.6 \%(\mathrm{n}=26)$ were men and $79.4 \%(\mathrm{n}=100)$ woman, mean age of $81 \pm 6,3$ years in tertiary hospital, Prague, Czech Republic.Testing of physical performance (hand-grip test, $400 \mathrm{~m}$ walk test, chair stand test, gait speed), anthropometric measurement and SARC-F, ADL, IADL, MNA-SF, SPPB were used for determination of physical, functional and nutritional status, while muscle mass and fat mass were measured by DXA-scans.

Results: The prevalence of sarcopenia (ALM $<19.75$ for men, $<14.02$ for women) was $26.9 \%(\mathrm{n}=34)$, SO in $13.5 \%(\mathrm{n}=17) .50 \%$ of all sarcopenic participants were diagnosed with SO (FM $>27 \%$ for men and $>38 \%$ for women; waist circumference $>90 \mathrm{~cm}$ for men and $>85$ $\mathrm{cm}$ for women). EWGSOP-1 criteria for diagnosing sarcopenia showed better sensitivity of $85.3 \%$ than the EWGSOP-2 $44.1 \%$, while specificity reached $100 \%$ in both criteria used.

Conclusion: Our results suggests that EWGSOP-1 (2010) criteria identified patients with SO better than EWGSOP-2 (2019). Using the newer criteria may leve significant proportion of sarcopenig obese patients undetected despite their physical and functional deficit. High prevalence of obesity among elderly people and rather low sensitivity of current diagnostic criteria for SO call for intensified research and broad international consensus in this field.

\section{Abstract \# 465}

Mechanism of action of the specific nutrient combination of actisyn to support muscle building in sarcopenia

Janneke van Wijngaarden ${ }^{1}$, Francina Dijk ${ }^{1}$, Miriam van Dijk ${ }^{1}$, Lisette ${\text { de } \text { Groot }^{2} \text {, Yves Boirie }}^{3}$, Yvette Luiking ${ }^{1}$

${ }^{1}$ Danone Nutricia Research, Utrecht, The Netherlands, ${ }^{2}$ Division of Human Nutrition and Health, Wageningen University \& Research, Wageningen, the Netherlands, ${ }^{3}$ University of Clermont Auvergne, INRA, Human Nutrition Unit, Centre for Research in Human Nutrition Auvergne, Clermont-Ferrand, France, University Hospital Clermont-Ferrand, Clinical Nutrition Unit, Clermont-Ferrand, France

Introduction: Sarcopenia is the loss of muscle, accumulating across the lifespan. Multiple factors cause or exacerbate sarcopenia, with aging as the primary factor and malnutrition, inactivity and diseases as secondary factors. We aimed to design a nutritional strategy to manage sarcopenia.

Methods: Our research program investigated: nutrient deficiencies in sarcopenic older adults, muscle protein synthesis (MPS) response in preclinical models, and effects of a specific nutrient combination (whey protein, leucine and vitamin D - ActiSyn), present in the medical nutrition product FortiFit, on MPS in older adults.

Results: We observed lower protein and vitamin D intake in sarcopenic versus healthy older adults $(\mathrm{p}<0.05)$ [1], and higher prevalence of sarcopenia among those with lower blood levels of leucine, essential amino acids (EAA) and vitamin $\mathrm{D}(\mathrm{p}<0.05)$ [2]. Intake of a leucine-enriched whey supplement in healthy older adults resulted in higher blood levels of leucine and EAA compared with casein-based supplements $(\mathrm{p}<0.001)[3,4]$. vitamin D supplementation activated MPS in vitamin D deprived $\mathrm{C} 2 \mathrm{C} 12$ myotubes [5], as did leucine-enriched whey protein in aged mice $(p<0.05)$ [6]. Intake of a leucine-enriched whey supplement stimulated MPS in healthy $(\mathrm{p}<0.05)[7,8,9]$ and sarcopenic older adults $(\mathrm{p}<0.01)$ [10].

Conclusion: The specific combination of whey, leucine and vitamin D (ActiSyn) provides the right strategy for muscle building in sarcopenia, an environment where these nutrients are often deficient. This combination acts through a proven anabolic mode of action with optimal nutrient bioavailability for the muscle to stimulate MPS.FortiFit and ActiSyn are trademarks of N.V. Nutricia.

References: [1] Verlaan et al., Clin Nutr 2017;36(1):267-74. [2] Ter Borg et al., JNHA 2019;23(1):27-34. [3] Luiking et al., Clin Nutr 2016;35(1):48-58. [4] van Wijngaarden et al., Clin Nutr. 2017;36:S158-S9. [5] Dijk et al., Clin Nutr ESPEN 2018;24:127-33. [6] Salles et al., Mol Nutr Food Res 2013;57(12):2137-46. [7] Kramer et al., JCEM. 2015;100(11):4124-32. [8] Luiking et al., Nutr J. 2014;13:9. [9] Chanet et al., J Nutr. 2017;147(12):2262-71. [10] Kramer et al., Clin Nutr. 2017;36(5):1440-9.

\section{Abstract \# 466}

The FortiFit clinical trial program demonstrates effective muscle building in sarcopenia

Janneke van Wijngaarden ${ }^{1}$, Johan de Vogel- van den Bosch ${ }^{1}$, Yves Boirie $^{2}$, Jürgen Bauer ${ }^{3}$, Peter Weijs ${ }^{4}$, Yvette Luiking ${ }^{1}$

${ }^{1}$ Danone Nutricia Research, Utrecht, The Netherlands, ${ }^{2}$ University of Clermont Auvergne, INRA, Human Nutrition Unit, Centre for Research in Human Nutrition, Auvergne, Clermont-Ferrand, France, University Hospital Clermont-Ferrand, Clinical Nutrition Unit, Clermont-Ferrand, France, ${ }^{3}$ Center for Geriatric Medicine, and Network Aging Research, University Heidelberg, Heidelberg, Germany, ${ }^{4}$ Department of Nutrition and Dietetics, Faculty of Sports and Nutrition, Amsterdam University of Applied Sciences,

Amsterdam, the Netherlands, Department of Nutrition and Dietetics, 
Amsterdam University Medical Centers, Vrije Universiteit, Amsterdam, the Netherlands

Introduction: The medical nutrition product FortiFit, containing ActiSyn (whey protein, leucine and vitamin D), is designed to support muscle building in patients with muscle loss. ActiSyn provides high bioavailability of leucine and other essential amino acids for stimulation of muscle protein synthesis. We aimed to demonstrate the effects of FortiFit on muscle building in healthy and sarcopenic older adults and on muscle preservation in obese (type 2 diabetic) older adults during a weight-loss lifestyle intervention.

Methods: We performed 4 randomized-controlled trials with an intervention duration of 6 to 13 weeks; significance was tested vs control.

Results: Appendicular muscle mass significantly increased in healthy older adults $(\mathrm{p}<0.05$ vs non-caloric control, $\mathrm{N}=24)$ [1] and sarcopenic older adults ( $\mathrm{p}=0.045$ vs iso-caloric control, $\mathrm{N}=380$ ) [2]. During a 13 -week lifestyle intervention with energy restriction and exercise training in obese older adults with $(\mathrm{N}=123)$ or without $(\mathrm{N}=60)$ type 2 diabetes, FortiFit preserved appendicular lean mass ( $\mathrm{p}<0.05$ vs isocaloric control) $[3,4]$. Chair-stand time significantly improved after 13 -week intervention in sarcopenic older adults $(\mathrm{p}=0.018$ vs isocaloric control, $\mathrm{N}=380$ ), but improvements in handgrip strength and SPPB (primary outcomes) were only significant versus baseline $(\mathrm{p}<0.05)$ and not versus control [2].

Conclusion: FortiFit effectively supports muscle building in healthy, sarcopenic and obese older adults. The improvement in chair-stand time in sarcopenic older adults is clinically relevant.FortiFit and ActiSyn are trademarks of Nutricia. [1] Chanet et al., J Nutr. 2017;147(12):2262-71. [2] Bauer et al., JAMDA 2015;16(9):740-7. [3] Memelink et al., Clin Nutr. 2018;37:S216-S217. [4] Verreijen et al., AJCN 2015;101(2):279-86.

\section{Abstract \# 467 \\ Correlation between muscle mass and weight change in patients older than 65}

Athina Greka

${ }^{1}$ EEMEG

Abstract: In the present study, the weight loss ratio of people over 65 years of age was studied in relation to their muscle mass.Screening test: laboratory profiles a targeted diet exercise program in conjunction with their medication.

Introduction: Over a three months period, 30 patients aged over 65 were examined. (None with pacemaker) All the patients followed $\bullet$ Diet based: mix $50 \%$ carbohydrates, $30 \%$ protein and $20 \%$ fat • Proper hydration: more than 2 liters of water daily $\bullet$ A light exercise program for torso and main body and walking for a period of 40 minutes/ daily $\bullet$ Solar exposure for more than 20 minutes per day in average

Method: BIA body consistency analysis DSM- BIA Method (Direct Segmental Multi-frequency - Bioelectrical Impedance Analysis)Afterwards the patient's situation was reassessed monthly and the progress was put down on progress reports which included the measurement according to date for height, weight, body mass and fat percentage.

Results: Of the 30 patients, $77 \%$ (about 23.1 patients) had weight loss $23 \%$ had increased weight Weight and muscle mass ratioOf the 30 patients, $77 \%$ (about 23.1 patients) had weight lossAt a rate of $77 \%$ of patients who lost weight - Percentage $18 \%$ increase muscle mass $>$ $2 \%$ • Percentage $46 \%$ increase muscle mass $1-2 \% \bullet$ Percentage $36 \%$ increase muscle mass $<1 \%$
Conclusion: In conclusion, diligent and intensive guidance on nutrition and exercise especially in geriatric patients in the doctor's office is as important as the instructions for their medication.

Keys: Muscle mass, BIA, Weight loss

\section{Abstract \# 468}

Outcomes in sensitivity of uropathogens in Romanian elderly population:-pilot study

Ana Capisizu ${ }^{1}$, Justin Aurelian ${ }^{2}$, Andreea Zamfirescu ${ }^{2}$, Ruxandra Mihalache $^{2}$, Sorina Maria Aurelian ${ }^{2}$

${ }^{1}$ University of Medicine and Pharmacy "Carol Davila" Bucharest, ${ }^{2}$ University of Medicine and Pharmacy "Carol Davila", Bucharest

Introduction: Urinary tract infections (UTI) are among the most prevailing infectious diseases with considerable morbidity and mortality with a substantial financial burden on society. The elderly persons are the most susceptible to UTI that frequently requires successive hospitalization.

Methods: We conducted a one-year retrospective study (2019) in Hospital Sf. Luca, on 1791 patients with a mean-age $73 \pm 6,23$ years. Data for the study were Urinalysis and antibiograms performed by AutomatMicroscan. We analyzed the demographic profile, the incidence of the favoring factors and the frequency of the symptoms encountered. Isolated microorganisms and their sensitivity to antibiotics were the basis of a comparative analysis using SPSS software. Results: There were 221 Urinalysis 23 of which were asymptomatic bacteria. $82.32 \%$ of patients are women; $51 \%$ are from rural areas; age group (75-84y) are the most numerous (46.6\%). Constipation and insufficient hydration are the most frequent favoring factors $(50 \%)$; drug abuse is very rare in Romanian elderly $(0.5 \%)$. The most common symptoms $(67 \%)$ are pollakiuria and dysuria.The distribution of Urine culture showed growth of Escherichia Colli(58.4\%), Klebsiella(17.7\%); Enterococcus and Pseudomonas in approx. equal to (4.52\%). Escherichia Coli were sensitive to quinolones $(73 \%)$, cephalosporins(54\%) and carbapenem(90.9\%). The most effective antibiotics were Amikacinum (80\% sensitivity) for Proteus and Pseudomonas; Carbapenem(90\%sensitivity) for Escherichia Coli and Proteus. The uropathogens that were the most resistant to antibiotics: Klebsiella (60\%); Enterococcus(62\%), Staphylococcus Aureus(68\%). Key conclusions: Our outcomes show that isolated uropathogens have a low sensitivity to antibiotics. This increasing resistance to broad-spectrum antibiotics is particularly troublesome at elderly, a frail populational group.

\section{Abstract \# 469}

Misuse of treatments for benign prostatic hyperplasia in the elderly population

Renoncourt Thomas ${ }^{1}$, Bloch Frederic ${ }^{2}$, Saint Fabien ${ }^{2}$

${ }^{1}$ Chu Amiens Picardie, ${ }^{2} \mathrm{Chu}$ Amiens Picardie

Introduction: The benign prostatic hyperplasia (BPH) is one of the most frequent pathology in elderly patients, with a prevalence of $80.7 \%$ at 60 years old [1]. the main complications are renal insufficiency, urinary retention (acute or chronic) [2], and repeated falls. This study aims to define the misuse of drugs for HBP and to assess if this misuse is associated with significant side effects.

Methods: A 14 days cross-sectional study was realized between February 25th - March 3rd, and March 25th - March 31th, from a request on the prescriptions of all treatments for BPH on our hospital. It included 117 male patients aged 75 or more and treated either by $\alpha$ adrenergic blockers or by $5 \alpha$-reductase inhibitors. 
Results: In our sample, we had 84 patients $(71.8 \%)$ in the misuse group. Patients in this group had more often a follow-up by an urologist with more lab and radiologic investigations $(\mathrm{p}<0.026)$. This group had more $\alpha$-adrenergic blockers $(\mathrm{p}<0.001)$ prescribed compared to the group without misuse. The misuse group also had less drugs, but more orthostatic hypotension ( $80 \%$ of tests performed) and were more often falling patients $(p<0.043)$ than the non-misuse group, without difference in the use of antihypertensive drugs between these two groups. A contraindication was present for $12.8 \%$ of the patients (orthostatic hypotension). In the misuse group, the risk of falling was increased with an OR of 3.22 ( $p<0.039$; CI [1.1-10.2]). Conclusion: According to this study, the misuse of treatments for BPH increases the risk of falls with an OR of 3.22 (p $<0.039$; CI [1.110.2]). The prescription of these drugs int this elderly population must be personalized according to the comorbidities, associated treatments and the patient's quality of life. 1. Irwin DE, Milsom I, Kopp Z, Abrams P, Artibani W, Herschorn S. Prevalence, severity, and symptom bother of lower urinary tract symptoms among men in the EPIC study: impact of ove- ractive bladder. Eur Urol 2009;56(1):1420. 2. FLAM T., DEBRE B. : Hypertrophie prostatique bénigne: symptômes qui motivent la consultation. In: L'hypertrophie bénigne de la prostate en questions. Mise au point. Edited by S. Khoury, C. Chatelain, L. Denis, F. Debruyne, et G. Murphy. France : SCI, 1991 ; $5: 125-127$

\section{Abstract \# 470 \\ A single-centered prospective study to investigate effect of fesoterodin treatment of urinary incontinence on quality of life, anxiety and depression}

\section{Arif Aksak ${ }^{1}$, Guzin Cakmak ${ }^{2}$, Zeynel Abidin Ozturk ${ }^{2}$}

${ }^{1}$ Gaziantep University, Faculty of Medicine, Department of Internal Medicine, ${ }^{2}$ Gaziantep University, Faculty of Medicine, Department of Internal Medicine, Division of Geriatric Medicine

Introduction: Urinary incontinence (UI) is an important health problem of elderly women. UI is thought to be related with depression and quality of life (QOL). In this study, we aimed to evaluate effect of UI treatment on QOL, depression, anxiety, and disability.

Methods: This prospective study was conducted for a period of 6 months from August 2019 to February 2020. Women applied to outpatient clinic with urge incontinence were included to the study. Patients were evaluated for QOL, anxiety, depression, disability and geriatric syndromes before and after treatment. QOL was assessed by International Consultation on Incontinence Questionnaire-Short Form (ICIQ-SF) and Incontinence of Quality of Life Questionnaire (IQOL). Anxiety and depression was assessed by Hospital Anxiety and Depression scale (HADs). SPSS versiyon 22 was used for data analysis.

Results: The study population was composed of 42 women; aged $69.7 \pm 4.3$ years. QOL, anxiety and depression symptoms, and daily activities of living (ADL) were revealed to be improved after treatment. ADL and I-QOL scores were positively, ICIQ-SF and HAD scores were negatively correlated to treatment. ICIQ-SF, I-QOL and HADs scores were associated with UI treatment when evaluated with one-way MANOVA $(\mathrm{F}[4,79]=3.25, \mathrm{p}=0.00$, Wilk's $\Lambda=0.859$, partial $\left.\eta^{2}=0.14\right)$.

Key conclusions: This study revealed that fesoterodin improved QOL and psychological symptoms. Those findings demonstrated that proper treatment of UI is critical for healty ageing. Our study is unique because there was no previous study about the effect of anticholinergic treatment on life quality, anxiety and depression in patients with UI.

\section{Abstract \# 471}

Association between urinary incontinence and sedentary behaviour in nursing home residents

Pau Farrés-Godayol ${ }^{1}$, Meltem Yildirim ${ }^{1}$, Vinícius R. de Oliveira ${ }^{2}$, Sandra Rierola-Fochs ${ }^{1}$, Anna Escribà-Salvans ${ }^{1}$, Miriam MolasTuneu $^{3}$, Pau Moreno-Martin ${ }^{3}$, Albert Oliveras-Fabregas ${ }^{1}$, Eduard Minobes-Molina ${ }^{1}$, Jo Booth ${ }^{4}$, Maria Giné-Garriga ${ }^{5}$, Javier JerezRoig $^{3}$, Dyego Leandro Bezerra de Souza ${ }^{6}$

${ }^{1}$ Research group on Methodology, Methods, Models and Outcomes of Health and Social Sciences (M3O). Faculty of Health Sciences and Welfare. Centre for Health and Social Care Research (CESS). University of Vic-Central University of Catalonia (UVic-UCC)., ${ }^{2}$ Research group on Methodology, Methods, Models and Outcomes of Health and Social Sciences (M3O). Faculty of Health Sciences and Welfare. Centre for Health and Social Care Research (CESS).

University of Vic-Central University of Catalonia (UVic-UCC) AND 2Department of Physical Therapy. EUSES University School. University of Barcelona-University of Girona (UB-UdG), ${ }^{3}$ Research group on Methodology, Methods, Models and Outcomes of Health and Social Sciences (M3O). Faculty of Health Sciences and Welfare. Centre for Health and Social Care Research (CESS). University of Vic-Central University of Catalonia (UVic-UCC), ${ }^{4}$ School of Health \& Life Sciences, Glasgow Caledonian University, UK., ${ }^{5}$ Blanquerna Faculty of Psychology, Education and Sport Sciences, Ramon Llull University, Barcelona, Spain AND Blanquerna Faculty of Health Sciences, Ramon Llull University, Barcelona, Spain., ${ }^{6}$ Research group on Methodology, Methods, Models and Outcomes of Health and Social Sciences (M3O). Faculty of Health Sciences and Welfare. Centre for Health and Social Care Research (CESS). University of Vic-Central University of Catalonia (UVic-UCC) AND Federal University of Rio Grande do Norte, Department of Collective Health, Graduate Programme in Collective Health, Natal, Brazil

Introduction: There is no evidence supporting the relationship between sedentary behaviour (SB) and urinary incontinence (UI) in nursing homes $(\mathrm{NH})$ residents. This study aims to determine this potential association.

Methods: A cross-sectional observational study (Clinical Trials NCT04297904) in 4 NH was conducted in the Osona county (Barcelona, Spain). Dependent variable was UI presence (by Minimum Data Set 3.0 version), and independent variables were $\%$ of SB in daytime hours (\%SB) and total upright time (TUT) in hours (standing and walking) by ActivPAL3 device (AP) during 7 consecutive days. Exclusion criteria were $<65$ years, transient $\mathrm{NH}$ residence, refusal to participate, hospitalization and palliative care. Data was analysed with a confidence level of $95 \%$ through the Mann-Whitney U test with the mean \pm standard deviation.

Results: The final sample consisted of 67 subjects, $53(79.1 \%)$ women and $15(10.3 \%)$ men, mean age $85.3 \pm 8.0$. Mean $\%$ SB was $69.6 \% \pm 12.7 \%$ and mean TUT $2.8 \mathrm{~h} \pm 1.3 \mathrm{~h}$ in the continent group $(\mathrm{n}=14,20.8 \%)$. Mean $\% \mathrm{SB}$ was $88.0 \% \pm 15.9 \%$ and mean TUT $1 \mathrm{~h} \pm 1.4 \mathrm{~h}$ in the incontinent group $(\mathrm{n}=53,79.1 \%)$. The \%SB in the UI group was significantly higher $(\mathrm{p}<0.001)$, whereas TUT was lower in the incontinent group $(\mathrm{p}<0.001)$.

Conclusions: NH residents with UI spent significantly more time in SB than in TUT compared to continent residents. Longitudinal studies are necessary to confirm whether SB is a risk factor for UI in the $\mathrm{NH}$ population. 


\section{Abstract \# 472}

Urinary incontinence in nursing home residents

Pau Moreno-Martin ${ }^{1}$, Meltem Yildirim ${ }^{1}$, Montse Romero-Mas ${ }^{1}$, Míriam Torres-Moreno ${ }^{1}$, Míriam Molas-Tuneu ${ }^{1}$, Anna EscribàSalvans ${ }^{1}$, Laura Coll-Planas ${ }^{1}$, Pau Farrés-Godayol ${ }^{1}$, Eduard MinobesMolina ${ }^{1}$, Javier Jerez-Roig ${ }^{1}$

${ }^{1}$ Research group on Methodology, Methods, Models and Outcomes of Health and Social Sciences (M3O). Faculty of Health Sciences and Welfare. Centre for Health and Social Care Research (CESS). University of Vic-Central University of Catalonia (UVic-UCC)

Introduction: Urinary Incontinence (UI) is a geriatric syndrome with important social, emotional and economic impact. We aim to verify the prevalence and types of UI and the UI control measures applied in nursing homes $(\mathrm{NH})$.

Methodology: A cross-sectional study in $4 \mathrm{NHs}$ from Osona county (Barcelona, Spain) was conducted between January and March 2020 (Clinical Trials NCT04297904). UI was evaluated using the Minimum Data Set (MDS) version 3.0 answered by their caregivers.

Results: 145 individuals were initially recruited, of whom 41 (26.9\%) were excluded for not meeting the inclusion criteria. Among the 104 (73.1\%) included individuals, $2(1.9 \%)$ were not classifiable for having a bladder catheter, $81(77.9 \%, 95 \%$ CI $69.0-84.8)$ presented UI; $39(37.5 \%)$ were occasionally incontinent, 19 (18.3\%) were frequently incontinent and $23(22.1 \%)$ were always incontinent. Regarding the type of UI; $50(48.1 \%)$ individuals had UI due to cognitive deterioration, $39(32.7 \%)$ due to walking difficulties, 20 $(19.2 \%)$ had urgency incontinence and $1(1.0 \%)$ presented stress incontinence. According to caregivers, scheduled toileting every 2 hours was applied to $31(29.8 \%) \mathrm{NH}$ residents, $24(77.4 \%)$ improved their UI, of whom 9 (29.0\%) achieved continence.

Conclusions: The prevalence of UI in institutionalized older people was found high $(77.9 \%)$ with the most common type being functional incontinence $(80.8 \%)$ due to cognitive deterioration $(48.1 \%)$ or walking difficulties (32.7\%). Most individuals (77\%) who followed scheduled toileting improved their incontinence of whom some $(29 \%)$ achieved continence.

\section{Abstract \# 473 \\ Discussing the rational use of diapers and bladder catheters to preserve urinary continence in hospitalized elderly}

Roberta Pereira Góes ${ }^{1}$, Larissa Chaves Pedreira ${ }^{2}$, João Paulo Almeida Tavares $^{3}$, Elaine de Oliveira Souza Fonseca ${ }^{2}$, Pedro Henrique Costa Silva $^{2}$, Fernanda Cajuhy dos Santos ${ }^{2}$

${ }^{1}$ Federal University of Bahia, ${ }^{2}$ Federal University of Bahia, ${ }^{3}$ University of Aveiro

Introduction: Devices such as diapers and bladder catheters, when installed, represent a change in the usual urination strategies, which may affect the state of continence. This study aimed to identify reasons for the indiscriminate use of diapers and bladder catheters in hospitalized elderly.

Method: Qualitative study carried out with ten professionals from the nursing team of a Hospital in northeastern Brazil, using the focus group as the collection technique. Situations related to the investigated outcome were discussed in the focus group workshops, which were recorded, transcribed and had the content of the statements analyzed.

Results: Many situations related to the indiscriminate use of diapers have been reported, especially in elderly women with impaired mobility, despite the possibility of using the urinal. Factors such as shame (modesty), fear of getting wet and the greater need for help from the team for support at this time, influenced this practice. The majority of the team doesn't observe previous states of continence when it is not related to the reason for the admission of the elderly. There was little association of the participants related to the use of the bladder catheter and the risk of urinary incontinence.

Main conclusions: The nursing team routinely uses diapers and a bladder catheter in hospitalized elderly people, even if they have maintained continence status, mainly with impaired mobility as a justification. It warns of the need for instruments to guide this care practice, establishing criteria for the use of diapers and bladder catheters, in order to manage the risk of urinary incontinence.

\section{Abstract \# 474 \\ Urinary incontinence and quality of life: a systematic review and meta-analysis}

Nicola Veronese ${ }^{1}$, Damiano Pizzol ${ }^{2}$, Stefano Celotto ${ }^{3}$, Gabriele Angiolelli ${ }^{1}$, Jacopo Demurtas ${ }^{4}$, Stefania Maggi ${ }^{5}$

${ }^{1}$ ULSS 3 Serenissima, ${ }^{2}$ Agenzia Cooperazione Internazionale, ${ }^{3}$ USL Medio-Collinare, Friuli, ${ }^{4}$ ASL Grosseto, ${ }^{5}$ Consiglio Nazionale delle Ricerche

Background: Urinary incontinence (UI) and low quality of life (QoL) are two common conditions in older adults. Some recent literature proposed that these two entities can be associated. However, no attempt has been made to collate this literature. Therefore, the aim of this study was to conduct a systematic review and meta-analysis of existing data to estimate the strength of the association between UI and QoL.

Methods: An electronic search of major databases up to 18th April 2020 was carried out. Meta-analysis of cross-sectional and casecontrol studies comparing QoL tools between patients with UI and controls was performed, reporting random-effects standardized mean differences (SMDs) $\pm 95 \%$ confidence intervals (CIs) as the effect size. Heterogeneity was assessed with the I2.

Results: Out of 7,981 articles initially screened, 23 were finally included for a total of 24,983 participants $(8,723$ with UI; 16,260 controls), mainly women. UI was significantly associated with poor QoL as assessed by the short form 36 (SF-36) total score, $(n=6$ studies; UI: 473 vs. 2971 controls; SMD $=-0.89$; $95 \%$ CI: -1.3 to $-0.42 ; \mathrm{I}^{2}=93.5$ ) and by the sub-scales of SF-36 and $5 / 8$ of the domains included in the SF-36. Similar results were found using other QoL tools. The risk of bias of the studies included was generally high. Conclusions: UI is associated with a poor QoL, with a strong level of certainty. This work, however, mainly based on cross-sectional and case-control studies, highlights the necessity of future longitudinal studies for better understanding the importance of UI on QoL.

\section{Abstract \# 475}

The awareness of medical specialists about severe complications of Herpes zoster in Lithuania

Laura Račkauskaite $\dot{1}^{1}$, Karolina Minikavičiūte ${ }^{1}$, Jurgita Knašienè Knašienè ${ }^{2}$

${ }^{1}$ Lithuanian University of Health Sciences, Faculty of Medicine, Kaunas, Lithuania, ${ }^{2}$ Department of Geriatrics, Lithuanian University of Health Sciences, Kaunas Clinical Hospital, Lithuania

Introduction: Postherpetic neuralgia (PHN) is the most common complication of acute herpes zoster (AHZ). An increased incidence of PHN is in older population [1].

Methods: An internet survey was conducted in 2019, December in Lithuania. In total, 331 medical specialists filled in the anonymous 
questionnaire. Statistical analysis was performed using IBM SPSS Statistics 24.0 software.

Results: $26.6 \%$ of respondents were family medicine physicians, $53.8 \%$ - specific physicians/residents, $12.7 \%$ - medical students. The majority of medical professionals $(63.5 \%)$ confirmed that they very rarely treat patients for PHN. We asked medical specialists "What is the most common complication of HZ?". The vast majority of respondents $(96.4 \%)$ chose the correct answer of PHN. But when we asked "When is PHN diagnosed?", the opinions differed. Only $41.7 \%$ of respondents chose the correct answer - "pain lasts over 90 days". What concerns rare but serious $\mathrm{HZ}$ complication, $77.9 \%$ of physicians picked the correct answer "granulomatous arteritis".

Conclusions: The medical professionals of Lithuania rarely consult patients with PHN. Most physicians know that the main complication of $\mathrm{HZ}$ is PHN but there is still a lack of knowledge, when this complication is detected. Only part of the doctors know that the pain must last over 90 days, then it is a typical neuropathic postherpetic pain. It is also noteworthy that not all physicians are aware that a rare but very complicated complication is granulomatous arteritis. We consider that medical professionals facing AHZ should always collect the anamnesis of pain and follow patients for serious complications. References: 1. Kawai K, Gebremeskel BG, Acosta CJ. Systematic review of incidence and complications of herpes zoster: towards a global perspective. BMJ Open. 2014 Jun 10;4(6):e004833.

\section{Abstract \# 476 \\ Associations between pneumococcal vaccination and reduction in mortality and hospital admissions among nursing home residents: an observational study}

Charlotte Havreng Théry ${ }^{1}$, Joël Belmin ${ }^{2}$, François Bertin-Hugault ${ }^{3}$, Nathavy Um Din ${ }^{4}$, Cristiano Donadio ${ }^{4}$, Quoc Duy Nghiem ${ }^{4}$, Bruno Oquendo $^{4}$, Linda Benattar ${ }^{3}$, Carmelo Lafuente-Lafuente ${ }^{5}$

${ }^{1}$ ORPEA SA, ${ }^{2}$ Sorbonne Université, ${ }^{3}$ ORPEA, ${ }^{4}$ Hôpital Charles Foix, ${ }^{5}$ Sorbonne Universite, Paris, France

Background: Residents of nursing homes are a population at-risk for pneumococcal infection; however, the efficacy of pneumococcal vaccine is still the subject of debates in these settings.

Objectives: To investigate associations between pneumococcal polysaccharide vaccination and mortality and hospital admissions in a large population of frail and very old people living in French nursing homes.

Design: Observational cohort study.

Setting: 254 French nursing homes.

Participants: Population-based sample of 35,998 residents admitted after January 1, 2014 and followed until the end of 2018. Follow-up was complete. Exposure: 23-valent or 13-valent pneumococcal vaccination prior or during nursing home stay.Main outcome measures: We extracted from electronic medical records information about pneumococcal vaccination status, dependency level, diseases and drug use, and calculated their Charlson comorbidity score. Times to mortality and first hospitalization were analyzed among vaccinated and unvaccinated residents of the propensity score-matched cohort using Cox proportional hazards regression and the Kaplan-Meier method.

Results: Among the 35,998 residents of the cohort (mean age $87.0 \pm 7.1$ years, females $66.2 \%), 2684(7.46 \%)$ had pneumococcal vaccination. The mortality rate was significantly lower among vaccinated residents compared with unvaccinated residents (hazard ratio, 0.438 ; $95 \%$ CI: 0.410 to $0.468 ; \mathrm{p}<10-4)$. The probability of first hospitalization was significantly reduced among vaccinated residents than among unvaccinated residents (hazard ratio, 0.721; 95\% CI: 0.685 to $0.758 ; \mathrm{p}<10-4)$
Conclusions: Our findings suggest that pneumococcal vaccination is beneficial among nursing home residents.

\section{Abstract \# 477 \\ Influenza and pneumococcus immunization status of older adults in typical rural community in Greece amidst the emerging COVID-19 pandemic}

Maria Tampaki ${ }^{1}$, Alexandra Livada ${ }^{2}$, Maria- Niki Fourka ${ }^{2}$, Elli Lazaridou $^{2}$, Marina Kotsani ${ }^{3}$, Athanasios Benetos ${ }^{3}$, Petros P Sfikakis $^{4}$, Evrydiki Kravvariti ${ }^{4}$

${ }^{1}$ Primary medical care unit of Marmari, S. Evia, General Hospital of Karystos, Greece, ${ }^{2}$ Department of Statistics, Athens University of Economics and Business, Greece, ${ }^{3}$ Université de Lorraine, CHRUNancy, Pôle « Maladies du Vieillissement, Gérontologie et Soins Palliatifs », F-54000, Nancy, France, ${ }^{4}$ Postgraduate Medical Studies in Geriatric Syndromes and Physiology of Aging, School of Medicine, NKUA, Athens, Greece

Introduction: Immunosenescence and high risk of complicated COVID- 19 infection in older persons mandates intensification of efforts to prevent co-infections, by improving compliance with existing preventive strategies of public health via immunization [1-4]. Our aim was to evaluate influenza and pneumococcal vaccination rates among older primary care outpatients in the period immediately preceding the outbreak of 2019 novel coronavirus (COVID-19) infection.

Methods: A cross-sectional study including people $\geq 65$ was conducted in February 2020 in a primary health care unit of rural Greece. Vaccination status of each participant was recorded according to self report and Greece's electronic universal prescribing system.

Results: The study sample included 104 participants (median age $=78$ years, $49.1 \%$ women) from a population base of approximately 300 older adults in the community. The majority of the population was vaccinated with quadrivalent influenza vaccine for the current and previous influenza season (59.6\%), whereas only $40.4 \%$ had been immunized against pneumococcus with either conjugate or polysaccharide vaccines after turning 65 years old. In total, $37.5 \%$ of the participants had not been immunized for either infection. In more detail, among 65 participants with inadequate vaccinations $61.8 \%$ lacked both vaccines, $30.9 \%$ lacked only pneumococcal immunization and $7.4 \%$ lacked only influenza immunization.

Conclusions: Our findings highlight the unmet need for adequate influenza and pneumococcus immunization in rural communitydwelling older adults in the context of the evolving COVID-19 pandemic.

References: 1. Jung Yeon Heo et. al., Effects of Influenza Immunization on Pneumonia in the Elderly, Hum Vaccin Immunother., 2018 Mar 4;14(3):744-749. 2. Jan Smetana et. al., Influenza Vaccination in the Elderly, Hum Vaccin Immunother., 2018 Mar 4;14(3):540-549. 3. Evelyna Derhovanessian et. al., Vaccination in the Elderly, Microb Biotechnol. 2012 Mar;5(2):226-32. 4. Ding Q et. al., The clinical characteristics of pneumonia patients co-infected with 2019 novel coronavirus and influenza virus in Wuhan, China. J Med Virol. 2020 Mar

\section{Abstract \# 478 \\ Is flu vaccination decrease severity and complications of influenza in elderly hospitalized populations}

Patrick Bavelele ${ }^{1}$, Marc Paccalin ${ }^{2}$, Benoit De Wazieres ${ }^{3}$, Claire Roubaud $^{4}$, Louis Bernard ${ }^{5}$, Thibault Fraisse ${ }^{6}$, Laurence Degout ${ }^{7}$, Jean 
Pierre Aquino ${ }^{8}$, Olivier Guérin ${ }^{9}$, Patrice François ${ }^{10}$, Emmanuel Forestier $^{11}$

${ }^{1}$ Geriatric Department, University hospital of Grenoble-Alpes, ${ }^{2} \mathrm{CHU}$ Poitiers, France, ${ }^{3} \mathrm{CHU}$ Nîmes, France, ${ }^{4} \mathrm{CHU}$ Bordeaux, France, ${ }^{5} \mathrm{CHU}$ Tours, France, ${ }^{6} \mathrm{CH}$ Alès, France, ${ }^{7} \mathrm{CH}$ Saint Julien, France, ${ }^{8}$ Societe Française de Gériatrie et Gérontologie, ${ }^{9} \mathrm{CHU}$ Nice, France, ${ }^{10} \mathrm{CHU}$ Grenoble-Alpes, ${ }^{11} \mathrm{CH}$ Chambéry, France

Background: Flu is one of the most prevalent winter-season infection, leading to poor prognosis in older populations (mortality, complex morbidity and functional decline). Vaccines is variably be efficient to prevent influenza but the question of a potential role of vaccines on severity of influenza episode is not well addressed. We aim to study this role using a cohort study of Older populations with influenza admitted to Hospital and performed in winter 2017.

Materials/methods: We achieved a comparative analysis of vaccinated and non vaccinated patients who experimented a flu episode ( $>$ 80 years) included in a national retrospective cohort over the
2016-2017 influenza outbreak in France (15 122016 to 3003 2017). Demographic and baseline Data , all type of acute and middle complications (Mortality and functional status after 3 months) according use of antibiotic and oseltamivir have been analysed.

Results: out of 515 patients with positive PCR, vaccine status wasn't known for 272 (52,3\%), 159 (30.8\%) were vaccinated and 84 (16.3\%) non vaccinated. Mean Charlson index was the only variable statisically different between both goups at baseline. Mortality rate, acute organ failures, nosocomial influenza, length of stay, nursing home admissions were not different but functional decline at 3 month was lower in the vaccine group.

Conclusions: Proven influenza hospitalized patients who were previously vaccinated exhibit a higher rate of comorbidities, but have a better functional outcome 3 months after a flu episode as compared with whose who were not vaccinated. No other outcome differences have been found. A prospective study measuring the the impact of influenza vaccine on influenza associated functional decline is warranted. 


\section{Author index}

Ángela, Óscar P128

Álvarez Díaz, Ana María P140

Öûztürk, Zehra Kosuva OC50

Şahin, Sevnaz OC47

Aasbrenn, Martin PM246

Abad, Ana Mateo P125, PM116, PM223, PM358, PM375

Abad, Ana Mateo Abas PM34

Abazaj, Erjona PM268

Abdilla, Stefania PM192

Abdullah, A. P11

Abdullah, Aimy P14, P17, P80, P90

Abdurrahman, Ahmad Z PM283

Abel, Cedeño Veloz Bernardo P108

Åberg, Anna Cristina PM140, PM141

Abete, Pasquale OCM26

Abrahams, Alferso P226

Abrami, Maria Angela P258

Abrar-Ahmad, Zulfiqar PM271

Abreu, Leonor Sousa M. P286

Abu-Hanna, Ameen PM248

Achterberg, Wilco P89

Adamakidou, Theodoula PM161, PM187, PM281, PM284, PM366, PM370

Adamakidou, Theodula PM401

Adamova, Irina PM56

Adby, Sam PM357

Addlestone, Jamie P74, P75, P76

Adie, Katja PM64

Adiletta, Veronica PM105

Aerssens, Nele PM313

Agacfidan, Ali P207

Agarwal, Kathryn OCM10

Agarwal, Priya PM283

Aggelakou-Vaitsi, Stamatina P227

Aggelidou, Ioanna P187, P253

Agnieszka, Neumann-Podczaska P260, P262

Agozzino, Bruno OC34, P8, P9

Ahern, Emer P52

Åhman, Hanna Bozkurt PM140, PM141

Ahmed, Mohammed OC13

Ahrenfeldt, Linda Juel P210

Aini, Ainoriza M OC39

Ainscough, Charlotte P280

Airaghi, Victoria Garay P85

Akçiçek, Fehmi OC47, OC50

Akande-Sholabi, Wuraola P279

Akbarian-Tefaghi, Ladan P139

Akcan, Ece PM428

Akcicek, Fehmi PM66

Akintimehin, Abisoye O. P105

Akishita, Masahiro PM424

Akopyan, Anna PM76

Aksak, Arif PM470

Al Emam, Mina PM316

Al-Fattal, Ahmad PM31

Al-Ramadhani, Bahar P168

Al-Saad, Salwan P45

Al-Salihi, Alaa P41

Albanese, Paolo P12, P13

Albert, Wolthuis PM416

Alberto, Tifanie P29

Albornoz, Luis P163

Albuquerque, Carlos PM68
Alcañiz, Ester Hoyos P106

Alcaraz-L, Carmen P51, PM373

Alcock, Megan PM318

Aldcroft, Daniel P213

Alec, Bass PM457

Alekna, Vidmantas PM239

Alenskaya, Tatsiana P222

Alexa, Ioana Dana P21, P100, P148, P247, PM310, PM439

Alexandrovich, Irina PM35

Alexis, Agc PM348

Alfaro, Victoria Sáínchez-Flor OC3

Alfaro-Lara, Eva P53, PM384

Alfaro-Lara, Eva Rocío PM423

Ali, Rupa PM341

Ali, Saied PM417

Aliberti, Máírlon OC43, P185, P211

Aline, Corvol PM380

Aliyev, Samil PM462

Allen, Carley P122

Alme, Katinka P49

Alme, Katinka Nordheim P119

Almeida, Raquel P198

Almeida, Catarina PM188

Alonso-Bouzón, Cristina OCM16

Alvané, Joana PM334, PM335

Álvarez, Eva María López PM110, PM304

Álvarez-Bustos, Alejandro OCM16

Alves, Maurício P128

Alves, Rita P184

Alvoeiro, Lurdes PM115

Amado, Sandra PM68

Amandine, Buquet PM122

Amaral, Andreia P216

Amaral, Cristina PM71

Amaral, B. PM447, PM448

Amaury, Broussier OCM14, OCM15

Amaury, Durand PM220, PM429, PM430, PM431, PM432

Amblàs-Novellas, Jordi PM425

Amélie, Boisnet PM202

Amouyel, Philipper P176

Ampeliotis, Michail PM40

Anaick, Besozzi P153

Anand, Atul OCM10, PM177

Andersen-Ranberg, Karen P210, PM62, PM137, PM287, PM309

Andión, Marta Blázquez PM315

Andrade, Luis PM188

Andrade, R. PM447, PM448

Andrea, Rossi PM148

Andreas, Jacobs PM151

Andreou, Martha P144, P187, P253, PM211, PM214

Andres, Emmanuel PM74, PM82, PM272, PM273

Andrès, Emmanuel PM85

Angeli, Valentina PM9, PM10, PM20, PM21, PM22

Angiolelli, Gabriele PM474

Angioni, Davide PM288

Ann-Katrin, Schild PM144

Anne, Leger PM322

Anne, Spinewine PM446

Annika, Heeß PM168

Annke, Nelde P66

Anru, Pavithra Laxsen PM137, PM441

Anselmi, Pasquale PM143

Anten, Sander PM30

Anthony, Rebecca P93

Antoci, Silvana PM356

Antognoli, Rachele P141 
Antón-Rodrigo, Iván OCM7

Antoñanzas-Valencia, Cristina PM47

Antoniadou, Eleftheria PM244

Antonio, Giorgio PM25

Antonova, Elena P31

Antunes, Inês PM97

Anzoletti, Nelson P245

Aojula, Anuriti P139

Aparicio-Mollá, S. P156

Apóstol, João PM347

Apsega, Andrius PM239

Aquino, Jean Pierre PM478

Araújo, José Lomelino OC48, P198

Arai, Hidenori OC38

Arain, Resham P67

Aranda, Yanira PM373

Aranda-Marteniz, Agustin P121

Aranda-Rubio, Yanira P249

Aras, Sevgi P178

Aravantinou-Karlatou, Antonia PM187, PM199, PM200, PM284, PM401

Aravindhan, Kiirtaara OC40

Arenas, M.C. P156

Arends, Lesley P120

Argentieri, Giovanni PM43, PM63, PM203, PM381, PM383

Argilés, F. Javier Afonso PM315

Arits, Jolijn P39

Arman, Pinar P132

Armas, Laura Gómez OC4, P33

Armellini, Andrea P175

Armstrong, Joshua J. PM280

Arnau-Barrés, Isabel OC29

Arnold, Patrick PM82

Arnott, Rosemary P138

Arora, Rakesh OCM10

Arrien, Ainhoa Esteve OC24, PM57, PM58

Arríen, Ainhoa Esteve PM61

Arrieta, Jesús Lopez OC24

Arrieta, Jesús Lopez PM57, PM61

Ars, Joan P286

Aschwege, Frerk Müller-zu PM169

Asmuje, Nur Fazidah OCM9

Aspray, Terry PM453, PM454, PM455

Asri, Nur Atikah Mohd P94, P244, PM261

Asunción, Carla Gamez P96

Asuncion, Carla Gamez P85, P98, P183

Athanasiadou, Eleni PM181

Athanasopoulou, Katerina P223

Atkins, Janice OC33

Atkinson, Rosarie PM341

Atmis, Volkan P178

Atouguia, Domingas OC7

Aubertin-Leheudre, Mylène PM60

Aubry, Régis P283, PM41

Aurélie, Abraham P153

Aurelian, Sorina Maria PM121, PM468

Aurelian, Justin PM121, PM468

Aurélie, Lenglet PM429

Avelino-Silva, Thiago P185, P211

Avgerinou, Christina PM211, PM214

Avila-Funes, José Alberto P221

Aviv, Abraham PM111

Avvenuti, Marco PM356

Ayad, Oualid PM111

Ayda, Rostamzadeh PM144

Aykar, Fisun Şenuzun OC47
Azevedo, Luisa P216

Azhand, Arash PM241

Azhar, Mahrukh P23

Aziminia, Nikoo OC19, P64, P69, P70

Azzini, Margherita P189

Büla, Chistophe OC27

Bánvölgyi, András P248

Babic-Illman, Gordana PM341, PM343

Babykina, Genia PM33

Baceta, Jon Saiz PM154

Baciarello, Marco OC15

Bączek, Jan PM459

Baeyens, Hilde P10, P55, P188

Baeyens, Jean-Pierre P10

Baeyens, J. P. P188

Bahat, Gulistan P50, P207, PM462

Bahat, G. PM435

Bailey, Laura P101

Bailey, Morgan P104

Bajenaru, Ovidiu Lucian P21, P148, PM310, PM439

Bajenaru, Ovidiu-Lucian P100, P247

Bajo-Peña, Lorena PM425

Baker, Graham PM158

Bakhai, Ameet OC19, P64, P69, P70

Bakir, Baris PM462

Bakken, Marit Stordal P49, PM314

Balaeva, Madina PM414

Balasegaram, Sooria P212

Balasubramanian, Diraviyam PM348

Balcaen, Christine P188

Baldasseroni, Samuele PM102, PM103

Balhana, Sílvia OC48

Ball, Emily PM127

Ballesteros, Cristina Gómez OC23

Balseven, özgül P137

Baltazar, Carolina PM409, PM410

Balzi, Daniela OCM19

Bambrick, Pádraig PM79, PM302

Bambrick, Padraig PM292, PM298, PM303

Bamidis, Panagiotis D. P238, PM353

Banik, Josef P288

Bannay, Aurelie P117

Barak, Orly P61

Barão, Manuela PM170

Barata, José Augusto PM164

Barber, Amelia PM343

Barbier, Mathilde PM136

Barbosa, Manuel P237, PM354

Barbosa, Arsénio PM112

Barbosa, Carla PM170

Barneto-Soto, Matilde OC25

Barone, Antonella PM174

Barradas, Ana P44

Barrett, Richard PM64

Barros, Hilario Martínez P140

Barros, Hilario Martinez P290

Barry, Rachel P52

Barry, Pat PM229

Barry, Patrick PM260

Barten, Dennis PM308

Barth, Nathalie OC52

Bartoli-Abdou, John P130

Basaran, Seniha P50

Basaras, Nerea Aguirre PM154

Basile, Giorgio PM402 
Basra, Pavun PM341

Basri, Sazali P14

Bastiaannet, E. PM344

Bastiani, Sophie OC1

Batres, Carlos P163

Baudouin, Édouard OC1

Bauer, Jürgen M. PM169, PM171

Bauer, Jürgen PM466

Baumgartner, Susanne P233

Bautista-Mesa, Rafael Jesus PM350, PM351

Bavelele, Patrick PM478

Baybaş, Buğçe OC47

Bayrakdar, Sena P50, P207

Beatty, Sharon P23

Beaudart, Charlotte P224

Beaumont, Lara OC12

Beck, Sanne Have P72

Becker, I. OC36

Beckett, Nigel P173

Beglinger, Shanthi PM249

Beindorf, Andreas P175

Bekiari, Eleni PM181

Bell, Marcia PM191

Belliato, Mirko PM6, PM7, PM8, PM11, PM12, PM13, PM23, PM24, PM25, PM26, PM28

Bellone, Federica PM245, PM402

Belmin, Joël OC6, OC26, P25, P84

Belmin, Joel PM92

Belmin, Joël PM476

Belokrylov, Igor OC5, PM414

Benattar, Linda PM476

Benazzo, Francesco PM7

Bencivenga, Leonardo PM99, PM106

Benetos, Athanase P117, P135, OCM3, PM111

Benetos, Athanasios PM165, PM477

Bengtsson, Pernilla PM289

Benítez, Antonia Jesús Solano PM145

Benítez, Antonia Jesús Solano PM389

Bennett, Siofra P40

Benoit, Dominique P55

Bentley, Nicholas OC12

Bento, Virgilio PM352

Benvenga, Salvatore PM402

Benzing, T. OC36, PM166

Benzing, Thomas P48

Berasategui, Oscar Perez PM100, PM291

Berbecila, Daniela P138

Berg, Nicolas PM39

Bergbaum, Carmel PM51, PM162

Berge, Trygve P170

Berglund, Lars PM140, PM141

Berkhout-Byrne, Noeleen C. P226, PM156

Berloni, Maria Letizia OCM28

Bermúdez, Juana María Vicario P154

Bermejo, C. P156

Bermejo, Ortzi Barrasa PM109, PM150

Bernabei, Roberto P175, OCM19

Bernard, Louis PM478

Bernardo, David Caldevilla OC3

Bernardo, Janice P129

Berruti, Alfredo P258

Bershedas, Kalpesh P65

Berteanu, Mihai PM149

Bertfield, Deborah Lee P112

Bertfield, Deborah PM51, PM162

Bertin-Hugault, François PM476
Besozzi, Anaïck P126, P127

Beuscart, Jean-Baptiste P99, PM33

Beyens, Hilde P36

Bezerra, Dyego Leandro PM207

Bezerra de Souza, Dyego Leandro PM163, PM458, PM471

Bianchetti, Angelo P15, P16

Bianchi, Camilla P141

Bianchi, Giampaolo PM340

Bianco, Céline OC1

Bianco, Antonio OCM28

Bielawski, Celia P145

Bieri-Brüning, Gabriela P142

Bignami, Elena Giovanna OC15

Bildlingmaier, Martin OCM27

Billon, Cecile P117

Bioteau, Catherine PM266

Bisconti, Ilaria PM105

Blackburn, Benjamin PM152

Blaha, Vladimir PM217

Blomaard, Laura C. PM30

Blomberg, Maja PM140

Bo, Mario OCM26

Bobrowicz-Campos, Elzbieta PM347

Boddaert, Jacques OC1

Boere, T. M. OCM18

Bogdan, Drago-Cristian PM215

Boirie, Yves PM465, PM466

Boland, Benoit P35

Bolduc, Aline PM60

Bolleke, Erjola PM268

Bollheimer, Cornelius PM449

Bolzetta, Francesco P12, P13

Bonadeo, Elisa PM9, PM10, PM13

Bondevik, Gunnar Tschudi P119

Bonello, Rebecca Ceci P63, P252

Bonet-Costa, Meritxell OCM23

Bongue, Bienvenu OC52

Bonin-Guillaume, Sylvie PM193

Bonnardeaux, Pedro López-Dóriga P26

Bonnici, Maria P63, P252, PM192

Boone, An L.D. PM158

Booth, Jo PM471

Bordignon, Alessandra P256

Bordigoni, Miguel Alberto Rizzi PM315

Borekci, Sermin P132

Borges, Diogo Drummond P184, P216

Borodin, Oksana PM45

Bos, Willem Jan W. P226, PM156

Bosshard, Wanda OC27

Botbol, Sasha P157, P192

Botteri, Emanuele P175

Bottone, Stefania OC36

Boudissa, Medhi PM266

Boulanger, Eric P99, P176

Boully, Clémence OC1

Boulton, Marina PM300

Bounds, Helen P124, P280

Bouras, Panagiotis P146, P149

Bourdel-Marchasson, Isabelle PM179

Bourdonnec, Laurianne OC1

Bourkel, Elisabeth PM136

Boussat, Bastien PM266

Boussedra, Safia PM340

Bouvard, Eric OC1

Bouzon, Cristina Alonso OC24, PM57

Bowden-Brown, Katherine PM463 
Bowen, J. PM1

Bowyer, Ruth C. E. OC18

Bowyer, Ruth C.E. P166

Boyle, Gerard P208, P209

Boyle, Karl PM444

Bozanic, Agnieszka P276

Brabrand, Mikkel PM287

Brackenridge, Anna PM341

Bradley, Elaine PM52

Brailova, Nataliya PM76

Braude, Philip PM50

Braun, Marc P126, P127

Bravo, Alberto Domínguez OC4, P33

Bray, Antonella PM43, PM63, PM203, PM381, PM383

Brennock, Jennifer P23

Bressan, Maria Antonietta P202, P203, P204, P205, P206, P263, PM6, PM7, PM8, PM9, PM10, PM11, PM12, PM13, PM14, PM16, PM16, PM17, PM18, PM19, PM20, PM21, PM22, PM23, PM24, PM25, PM26, РM27

Bretagne, Lisa PM249, PM436

Brewer, Linda P134, P236, PM316

Brigas, Daniela PM112

Brill, Simon Edward P112

Britto, Patrick PM343

Brochado, Ana Isabel P198

Brockhattingen, Kristoffer K. PM441

Brodocianu, Raluca PM439

Brookes, Claire OCM10

Brooks, Fraser OC14, P47

Brown, Peter P130

Bru, Rita López OC3

Brunes, Maria Chiara OCM28

Brunet, Ellen PM213

Bruno, Oquendo PM322

Buchanan, Ryan P167

Buckinx, Fanny PM60

Buckley, Catherine OC32

Buckley, Mary P133, P208, P209, P257

Buglione, Michela P258

Buigues, Cristina PM337

Bülow, Tanja PM449

Burke, Neil PM316

Burkhardt, Heinrich OC20

Burns, Eileen P93

Burst, V. OC36, PM166

Burst, Volker P48

Busonera, Flavio P12, P13

Buttigieg, Sacha PM192

Buurman, Bianca P164, P165, OCM5, PM326

Buurman-van ES, Bianca P123

Buwe, Abubaka P166

Buyck, Griet P55

Buyuk, Alime P235, PM328

Buzaco, R. PM447, PM448

Byrgesen, Stephanie PM226

Byrne, Colm P134, P236

Byrne, Thomas PM79, PM292, PM298, PM302, PM303

Byrne, Amy PM325

Byrne, Sharon PM325

Célarier, Thomas OC52

Céspedes, Almudena Avendaño OC3, OC23

Córcoles, Rubén Alcantud OC3, OC23

Cabral, Catarina P197

Cabrera, Lourdes Del Rosario Evangelista P37

Caby, Claire PM86
Caçola, Rute Lopes PM188

Caiazzo, Andrea OCM28

Cakmak, Guzin P190, P199, PM123, PM228, PM276, PM392,

PM393, PM394, PM470

Çakmak, Güzin PM275

Calderon-Calvo, Gonzalo P191

Calderón-Calvo, Gonzalo PM185

Caljouw, Monique P89

Callus, Claire P63, P252

Calogero, Pietro PM65

Calvo, Pilar Ochoa P46

Calzolaro, Valeria P141

Camacho, Milagritos Margot Vasquez P125

Camartini, Viola PM102, PM103

Cameron, Ewen P113

Camilleri, Lara OC45, P116, P254

Camilleri, Jessica OC45, P116

Camilleri, L. P111

Cammalleri, Lisa PM49

Campbell, Christina D. PM73

Campmany, Mireia Puig PM315

Campos, Luís OC30

Campos, María Gebauer P85, P98

Campos, Maria Gebauer P96

Campos, María GebauerGebauer P183

Campos, Maria José P197

Campos, L. PM447, PM448

Can, Gunay P132

Canais, Jorge P128, PM113, PM114

Canan, Ziylan PM363

Canavan, Michelle D. OC42

Canavan, Michelle P22, P87, P179, PM53, PM212, PM296

Candiani, Tiziana PM224

Cannon, Abigail P168

Cano, Isabel Tejero PM315

Cano, Lourdes Rexach PM445

Cánovas, Carmen PM405

Canteli, Jesús PM46

Caoimh, Ronan O. P57

Caparali, Emine Bilge P50, P207

Capisizu, Ana PM121, PM468

Caraux-Paz, Pauline PM67

Cardenal, Jorge Eduardo Corrales P18, P19, P28, PM100, PM160,

PM291, PM433, PM437

Cardenal, J. Eduardo Corrales P46

Cardoso, Afonso OC48

Cardoso, M. Jorge P166

Carey, David PM208

Carmelo, Lafuente PM322

Carmona, Laura Plaza OC3, OC23

Carnicero, Jose Antonio OCM16

Carolin, Blasius PM144

Carrasco, Cristina PM373

Carrera, Giulia OCM19

Carret, Marie-Christine PM67

Carrieri, Barbara P193

Carrieri, Vito PM43, PM63, PM203, PM381, PM383

Carrillo-García, Pamela P4, P6

Carroll, Carmille OC37

Carroll, Stephanie P168

Cartelle, Beatriz Esteban PM445

Carvalheiro, Licínia P128

Carvalho, António P197

Carvalho, Catarina P215

Carvalho, A.F. PM447, PM448

Caryn, Recto PM322 
Casas-Herrero, Álvaro P30

Casas-Herrero, Álvaro P285, PM47

Casas-Herrero, Alvaro OCM7

Cassagnaud, Pascaline P99

Cassarino, Maria OCM10

Cassinello, Concepción Lobato P37

Cassinello, Concepcion Lobato P157

Castagna, Alberto P189, P287, PM55

Castaldo, Giampiero OC34, P8, P9

Castejon-Hernandez, Santiago PM263, PM264

Castell, Maria Victoria PM285

Castillo, Luana Sandoval PM246

Castillo, Julia PM405

Castro, Maria Elena P43

Castro, Julien Paola Caballero P154

Castro-Tejada, Gelanys OC44

Catalan-Matamoros, Daniel PM350, PM351

Catalano, Antonino PM245, PM402

Catarino, Paula P128

Catherine, Chenailler PM220, PM430, PM431, PM432

Catikkas, Nezahat Muge PM462

Catma, Yunus P50, P207

Cauli, Omar PM337

Cavallin, Anna PM14, PM15, PM16, PM17, PM18, PM19

Cebada, Rocio Toro OC21

Ceballos, Carmen Navarro P37, P157

Cedeño-Veloz, Abel OCM7

Cedervall, Ylva PM140, PM141

Cella, Alberto OC53, P189, P255, OCM4, OCM17, OCM25, PM49,

PM157, PM158, PM247, PM305

Celotto, Stefano PM474

Cenderello, Giovanni P189

Ceolin, Ludovica PM87

Cercos, Purificación Montero PM197

Ceresa, Iride Francesca P202, P203, P204, P205, P206, P263, PM6, PM7, PM8, PM9, PM10, PM11, PM12, PM13, PM14, PM15, PM16, PM17, PM18, PM19, PM20, PM21, PM22, PM23, PM24, PM25, PM26, PM27, PM28

Čerkalová, Monika PM2

Cernomaz, Andrei PM390

Cerra, Rosa Paola PM55

Cerundolo, Nicoletta OC34, P8, P9

Cesari, Valentina P193

Cesari, Matteo PM138, PM277, PM288

Cesaroni, Giulia OC2, P15, P16

Cetin, Sebahat Yaprak P235, PM328

Çetin, Sebahat Yaprak PM331

Ceylan, Aysenur P178

CFAS, MRC OCM29

Chan, Terrence P56, P277

Chan, Andrew T. P166

Chan, Christopher Sin PM42

Chang, Yue OCM10

Chang, Milan PM391

Charlotte, Nouhaud PM322

Charlotte, Therry PM322

Charnley, Karen P95

Charpentier, Anne P99

Chatzaki, Efrosini PM265

Chatzigeorgiadis, Anestis PM101, PM293, PM311, PM312, PM345

Chen, Yaohua P29, P99

Chen, ChenHui P108, P118, P285, OCM7

Chen, Cathy PM93

Chenailler, Catherine P284

Cheng, Samuel E. E. PM5

Chenhuichen, Chenhui PM47
Cheong, Jun Leong PM418

Cherubini, Antonio P193, P224, OCM26

Cherubini, A. PM435

Cheston, Hannah P280

Chevallier, Theodore PM415

Chew, Justin PM406

Chhokar, Gurjit P93

Chiam, Rosalind PM253

Chiaromanni, Federica P12, P13

Chiavalin, Marco P158

Chijiki, Shoko P229

Chilà, Diego PM402

Chin, A. V. P11

Chin, Ai Vyrn P14

Chin, Ai-Vyrn P17, P80, P90, OCM9

Chinnadurai, Rajkumar PM145, PM283

Chiong, Cloie June PM96

Chiong, Cassidy June PM96, PM382

Chiussi, Giulia OC34, P8, P9

Cho, Jea Ho PM77

Chojnicki, Michal P45

Chong, Mei Chan P239

Chowdhury, Samin PM38

Christel, Oasi PM322

Christensen, Julie Braüner PM246

Christine, von Arnim PM151

Christoph, Maurer PM319

Christos, Nikitas PM319

Chroni, Eugenia P223

Chupina, Vilena PM155

Cialic, Ron P61

Ciarrocchi, Erika P193

Ciceri, Luca PM9, PM20, PM21, PM28

Cifuentes, Sara Celaya OC23

Cigiloglu, Ahmet P251, PM392

Cilia, Clarice PM183

Çinçin, Asli Tufan PM172

Ciobanu, Ileana P261, PM149, PM215

Cittadini, Antonio PM99

Claire, Leissing-Desprez OCM14, OCM15

Claire, Roubaud-Baudron PM387

Clancy, Ivan PM52

Clara, João Gorjão PM404

Clarke, Maria PM357

Claus, Jules PM88

Cleary, May PM417

Close, Jacqueline OC37

Cobbaert, Katrien P55

Cocchi, Valentina OC15

Cocchi, Francesca PM359

Coccia, Flaminia OC2, P16

Cocino, Cristina OC36

Cofais, Cécilia PM422

Coin, Alessandra P256, PM143

Cola, Guglielmo PM356

Colameco, Franco P245

Colato-López, Carlos Alfredo P78

Coles, Tanya P136

Coll-Planas, Laura PM472

Colonna, Paolo PM93

Condon, Fibarr P94

Condon, Finbarr PM261

Constantin, Gianina Ioana PM178

Constantin, G.I. PM186

Conway, Aifric PM52

Cooke, John PM79, PM292, PM298, PM302, PM303, PM417 
Cooney, Marie-Therese PM279

Copeta, Silvia P258

Coppolino, Giuseppe PM55

Corbi, Graziamaria PM106

Corcoran, Grace PM52

Cordina, John P63, P252

Corica, Francesco PM245, PM402

Corine, van Maar PM363

Cornally, Nicola OC32

Cornette, Pascale P34

Correa-Pérez, A. PM435

Correia, Fernando PM352

Corsonello, A. PM421

Cosarderelioglu, Caglar P178

Coscarelli, Antonio PM442

Cosco, Lucio P287

Cosimetti, Antonio PM209

Costa, Tiago P150, P151, P152, PM188

Costa, Raffaele P287, PM55

Costa, Célia PM113, PM114

Costa, Ana Paula PM222

Costello, Maria M. OC42

Costello, Maria PM212

Coughlan, Gearoid P22, PM53, PM54, PM212

Couppie, Philippe PM85

Courdier, Guy PM82

Courtel, Tommy PM86

Covens, Bérengère P225

Covington, April PM177

Covinsky, Kenneth P211

Cox, Natalie J PM399

Cozza, Mariagiovanna P193

Crawley, Samantha P136

Crawley, Samatha P169

Crespo, Luis Ignacio Mendibil PM154

Cribbin, Elaine PM332

Crisafulli, Emanuele OC15

Cristiano, Donadio PM322

Cristina, Polidori Maria PM144, PM148, PM151, PM168

Cristini, Carlo P258

Cristofori, Giovanna OC17, OC21, P4, P177

Cristofori, Giovana P282

Crome, P. PM435

Cronin, Finola PM260

Cross, Hanni PM208

Crowley, Patrick P57

Cruz-Grullon, Maricelis PM263, PM264

Cruz-Jentof, Alfonso José PM452

Cruz-Jentoft, Alfonso P290

Cuesta, Gemma P51

Cuesta-Castellón, Gemma P4

Cuevas-Lara, César P30

Cuevas-Lara, César P285, PM47

Čuj, Jakub PM320

Cuocolo, Alberto PM99

Curiati, Pedro P211

Curran, Michael P94

Custodero, Carlo P255, P272, PM49, PM174

Custureri, Romina P189, OCM17, PM49

Cutti, Sara PM8, PM9, PM10, PM14

Cuzzocrea, Fabrizio PM7

Czintner, Dóra P248

D'Agnano, Vito OCM28

D'Amico, Fabio OCM28

D’Andria, Maria Flora PM87, PM94, PM126, PM442
Díaz, Blanca P171

Da Cas, Roberto OCM13

Dağlar, Aynur PM461

Dalal, Gunjan P159

Daliri, Sara OCM5

Dalli, Stephanie OC45

Dalli, S. P111

Dallmeyer, Rebecca P169

Dalton, Kieran PM325

Damulevičienė, Gytė PM396, PM397

Danaee, Mahmoud PM418

Dandoulakis, Michael P146, P149

Danet-Lamasou, Marie PM179

Daniels, Thomas P167

Dauchet, Luc P176

Daunoraviciene, Kristina PM239

Daunt, Ruth P134, P236, PM316, PM444

Dautzenberg, Lauren PM249

Davenport, Rebecca P212

David, Jean-Philippe OC1

David, Beckwée PM286

Davidson, Catriona PM204

Davies, Richard P166

Davies, Franco P228

Davies, Nathan PM221

Dawson, Matthew P42

de Araújo, Ricardo Simões P219

De Benedetti, Pierandrea PM340

de Bergua, Josep P286

De Brauwer, Isabelle P34, P35

de Brouwer, Esther PM88

de Carvalho, Jáíder Freitas Maciel Garcia P162

de Gelder, Jelle PM30

de Groot, Bas PM29, PM30

de Groot, G. Cathrine L. PM31

de Groot, Lisette PM465

De Iaco, Pierandrea PM340

de Jong, Pim PM88

de Jonghe, Annemarieke PM88

de la Puente Rodríguez, Eva P282

de la Puente, Eva Fernáíndez OC21, P177

De La Puente, Eva Fernandez P4

De la Puente, Maria P192

De La Puente Martín, Maria P37

De La Puente Martin, Maria P157

De La Rosa, Ines P163

de Longuemarre, Arnaud Thomasset PM117

de Lurdes Almeida, Maria PM347

de Malglaive, Pauline OC1

de Matos, Leonor Vasconcelos P44

de Molins Peña, Carmen Elías P18, P19

de Molins Peña, Carmen Elias P28

de Molins Peña, Carmen Elías PM100, PM291, PM433, PM437

De Moor, Sabine P186

de Moraes, Edgar Nunes P162

de Moraes, Flavia Lanna P162

de Oliveira, Lélia Mendes Sobrinho OC10, P107

de Oliveira, Vinícius R. PM207

de Oliveira, Vinicius Rosa PM290

de Oliveira, Vinícius R. PM471

de Oliveira Souza Fonseca, Elaine PM70, PM473

de Pascual, Raquel Martín Pozuelo Ruiz P26

De Raes, Eva P55

De Rosario, Lorenza PM92, PM367, PM385

De Schrijver, Lotte P186

de Sousa, Sendes Sobrinholvia Pacheco P131 
de Souto Barreto, Philipe PM288

de Soutter, Marie-Astrid OC1

De Terwangne, Christophe P35

de Tymowski, Christian PM36

de Villelongue, Cédric OC1

de Vogel- van den Bosch, Johan PM466

de Waal, Margot P89

De Wazieres, Benoit PM478

de Wildt, Kelly Kim PM259

Debattista, Daniel P63, P252

Debby L., Gerritsen PM363

Debono, M. P111

Debono, Melanie P116, P254

Dedecker, David P55

Deeg, Dorly J.H. PM285

Deery, James P138

Defebvre, Marguerite-Marie PM33

Defi, Irma Ruslina Defi Irma Ruslina P265

Degli Esposti, Luca OCM19

Degout, Laurence PM478

Dekker, J.W.T. PM344

del Blanco, Ainara Hernández PM153

Del Maestro, Mattia PM6, PM7, PM8, PM11, PM12, PM13, PM28

Del Mar Soguero Perez, Maria PM34

del Nozal, Jesús Mateos P140

del Río, Rocio Gomez P51

Del Rio, Andrea PM174

Del Signore, Susanna P195

Delabrier, Isabelle P99

Delgado, Sagrario Pérez OC17, P60

Delgado, Joao OC33

Demeneopoulou, Sophia PM161

Demurtas, Jacopo OC36, P224, PM138, PM474

Denkinger, Michael PM248

Denkinger, Michael D. PM285

Denkinger, M. PM435

Dennehy, Karen A. OC42

Dennehy, Karen P22, P87, P179, PM53, PM54, PM296

Dennison, Elaine M. PM285

Depraetere, Joke P186

Derejczyk, Jarosíéaw P81, P92

Derejczyk, Grzegorz P81, P92

Derez, Leen P10, P188

Dermott, Clodagh M Flannery Mc PM296

Derniarova, Lubica PM320

Derollez, Céline P29

Deryugina, Natalia OC5, PM414

Deslandes, Cristiana Ceotto P162

Despotovic, Nebojsa PM130

Devakumar, Haran P267

Devita, Maria P256, PM143

Devriendt, Els OC8

Dewi, Juita Sirimanne P14

Dhamija, Sue P159

Di Bari, Mauro P230, OCM19, PM356

Di Iuorio, Ornella OCM28

Di Lena, Luca PM43, PM203, PM381, PM383

Di Nisio, Marcello PM249, PM436

di Rosario, Lorenza PM36

Di Silvestro, Katharine P180

Dias, Sara P184, P216

Dias, Murilo P211

Dias, Paula PM113, PM114

Dias, Mariana PM115

Dias Moura, Raquel PM176

Díaz, Ana Maria Alvarez P290
Diaz-Garelli, Franck OC44

Diaz-Pavon, Alvaro Rodriguez PM233

Dibari, Mauro OCM26

Didier, Armaingaud P147

Didier, Schoevaerdts PM446

Diekmann, Rebecca P242, PM169, PM171

Diem, Peter P136, P173

Diepenbroek, Adry PM156

Dieuveuil, Lisemaine OC44

Dignat-George, Françoise PM111

Dijk, Francina PM465

Diler, Ali Sarper PM461

Dilworth, Lorraine P271

Dimitris, Kikididis PM319

Din, Nathavy Um P84, OC26, PM476

Dinan, Pascale PM193

Ding, Yew Yoong PM406

Dini, Simone P189, PM49

Dismore, Lorelle PM453, PM454, PM455

Dix, Meiberth PM144

Dodds, Richard M. OCM29

Dodds, Richard Matthew PM463

Dokoutsidou, Eleni PM161, PM370

Dolianiti, Foteini P238

Dominguez, Jesabelle OC44

Dominguez, Soledad P192

Dominik, Michiels P66

Dominik, Rudol PM168

Dominique, Huvent PM122

Dominique, Somme PM380

Donadio, Cristiano OC6, OC26, P25, P84, PM36, PM92, PM367, PM385, PM476

Dondi, Giulia PM340

Donlo, Javier Meseguer OC29

Donohue, Sean PM191

Dookhy, Joshi PM146

Dooley, Hannah OC18

Doris-Eva, Bamiou PM319

dos Santos, Rodrigo Ribeiro P162

dos Santos, Fernanda Cajuhy PM473

Douglas, Ian PM243

Doventas, Alper P132

Downs, Thomas OC14

Doyle, Patrick P91, PM332

Draghici, Rozeta P100, P247

Drame, Moustapha PM422

Dratsiou, Ionnna P238

Dratsiou, Ioanna PM353

Drennan, Megan OC14

Drew, David A. P166

Drey, Michael OCM27

Drysdale, Elizabeth P39

du Cadet, Julien Lavigne P166

Duarte, Leila PM115

Duarte, T. PM447, PM448

Dubov, Alexander PM323

Dudinskaya, Ekaterina PM76, PM182, PM237

Duggan, Eoin P52, PM260

Dumoulin, Chloé PM111

Dunnell, Liam PM307

Dunnett-Kane, Victoria P38, P39, P41, P42

Dupont, Jolan P36

Duque, Sofia OC30

Duque, S. PM447, PM448

Durand, Amaury P284

Dureau, Antoine OC1 
Duron, Emmanuelle OC1

Durrieu, Jessica PM179

Durroni, Sonia PM357

Dvoretski, Leonid PM90, PM91, PM236

Dyer, Adam P97, OCM24, PM133, PM134, PM135, PM438, PM443

D'Andria, Maria Flora P230

D’Arcy, Anne PM52

Ebihara, Takae PM451

Efendioglu, Eyyup Murat P251

Efendioglu, Eyyüp Murat PM275

Eichenlaub, Melissa Smith PM32

Eisert, Albrecht PM449

Eizaguirre, Marta Mercedes Gonzalez PM116, PM358

Eizaguirre, Marta Mercedes González PM223, PM375

Ekmann, Anette Addy PM246

El-Sayed Moustafa, Julia S. P166, OC18

Elezi, Brunilda PM268

Elgert, Lena PM169

Eliane, Abraham P153

Eliseeva, Tatyana PM90, PM91, PM236

Ellis, Judith P73

Ellis, Raphael PM69

Emery, Rachel PM42

Emilie, Ferrat OCM15

emilie, Wawrziczny PM122

Emilie, Menand PM380

Emiroğlu, Oya Nuran PM167

Emmanuel, Andres PM271

Emmelot-Vonk, Mariëlle PM88

Emmelot-Vonk, Marielle PM249, PM436

Emmelot-Vonk, M.H. PM435

Encarnação, Sara PM71

England, Bethan OC12

Enting, Deborah PM343

Epelde, Itxaso Marin P108, P118

Erazo, Claudia P. Murillo P46

Erazo, Claudia Patricia Murillo PM160

Erceg, Predrag PM130

Erdincler, Deniz Suna P132

Erel, Suat PM331

Erelel, Mustafa P50

Eriksdotter, Maria PM289

Eriksson, Ann-Sofi PM330

Eruslanova, Ksenia OC5, PM182, PM414

Escribà-Salvans, Anna PM163, PM207, PM368, PM471, PM472

Espaulella-Ferrer, Mariona PM263, PM264, PM426

Espaulella-Panico, Joan OC25

Espaulella-Panicot, Joan PM263, PM264, PM425, PM426

Espinoza, Maria Fernanda Ramon P108

Espinoza, María Fernanda Ramón P118

Esposito, Marcello OC15

Esteves, Ana P44, P215

Estrela Santos, Mariana PM176

Etienne, Audureau OCM15

Eustace, Joe P52

Eva, Peyrusque PM198

Eva, Peyrusqué PM457

Evangelista, Lourdes P192

Evangelista, Vasco P198

Evans, Jo PM208

Evelyn, Van Cleynenbreugel P66

Fabbri, Alessandra P141

Fabian, Dehanne PM446

Fabien, Saint PM469
Fagard, Katleen OC8

Falandry, Claire P278

Falchi, Mario P166

Falcone, Claudia P245

Fall, Tove P166

Faller, Eamonn P52

Fallon, Aoife P97, OCM24

Fallon, Nessa PM262

Falzone, Daniele PM126

Fama, Marcella P272

Fankhauser, Corina P41

Fanny, Buckinx PM198, PM457, PM460

Faria, Marina P215

Farid, Aaseem PM369

Farinella, Sara Tita P189

Farnsworth, Malin PM42

Farrés-Godayol, Pau OCM23, PM163, PM207, PM290, PM368, PM471, PM472

Farrugia, Francesca OC45, P116, P254

Farrugia, F. P111

Fassier, Thomas P180

Fatma, Ben Dahmen PM317, PM372, PM386, PM388

Fattirolli, Francesco PM102, PM103

Fearon, Anna P213

Fedecostante, Massimiliano P193

Federspiel, Carine PM136

Fedyk-Łukasik, Małgorzata P259

Feely, Owen PM444

Feijoó, Maribel Quezada OC17, OC21, P177

Feijoó, Maribel Quezada P282

Felício, Margarida PM113, PM114

Felipe, Casanueva F. OCM1

Femia, Rosetta OCM17, PM49

Fernández-Rius, Ana Perez OC3, OC23

Fernandes, Marilia OC7, P184, P216, PM170, PM409, PM410

Fernandes, Jorge P184

Fernandes, Carla P197

Fernandes, Anna P237

Fernandes, Ricardo PM222

Fernandez, Sofia Solsona P46

Fernandez, Victor P163

Fernandez, Yoana P163

Fernandez, Francisco Javier Balea PM145, PM389

Fernández, Mónica PM46

Fernández, Nuria Cristina Herrera PM109, PM150

Fernández, Francisco Javier Balea PM109, PM150

Fernández, Marta PM110

Fernández, Marta Fernández PM304

Fernández-Garrido, Julio PM337

Ferrara, Nicola PM99, PM106

Ferrari, Uta OCM27

Ferrari, Alberto PM157

Ferreira, Ana Filipa Teixeira P131

Ferreira, Ana Sofia PM419

Ferri, Alberto OCM4, PM158

Ferrucci, Luigi P175

Ferry, Peter PM159, PM192

Ferryman, James PM152

Fevereiro, Teresa PM71

Ficheur, Grégoire PM33

Fidalgo, Mariana P150, P151, P152

Figueiredo, Ines Rego OC7, P184, PM409, PM410

Figueiredo, Inês Rego PM71

Figueiredo, Ines PM170

Figueiredo, Daniela PM175, PM294, PM295, PM365

Figueiredo, B. PM447, PM448 
Fika, Sofia P227

Fikas, Konstantinos P227

FIlardi, Pasquale Perrone PM99

Filip, Charlier P66

Filip, Stanislav PM217

Finne-Soveri, Harriet OCM11

Fiore, Elena Boscolo P68

Fiorentino, Ronald OC45, P116

Fiorentino, R. P111

Fitzgerald, Serena OC32

Fitzgerald, Michelle PM332

Fitzpatrick, Donal PM229

Fiuza, Pedro P184, P216

Flamaing, Johan OC8, P36, PM39

Flanagan, Mary P95

Flanagan, Cliona PM341, PM343

Flatharta, Tomas O. P22

Fleet, James P173

Fleure, Louisa PM343

Flood, Eimear PM332

Flor, Inma Ponz PM197

Florence, Canouï-Poitrineine OCM15

Florence, Pasquier PM122

Flores, Mirea P163

Floris, Annarosa OC53, P272, PM247

Fochs, Sandra Rierola PM458

Fonseca, Cândida OC30, P44

Fonseca, C. PM447

Fontainha, Tania OC7

Forcano, Mercedes P43

Forestier, Emmanuel PM67, PM478

Forini, Guido PM6, PM7

Formilan, Marino P12, P13

Formisano, Roberto PM99

Fortini, Giacomo P230, PM102, PM103

Foss, Catherine Hauerslev OCM6

Fossaert, Romain PM179

Fossey-Diaz, Virginie OC1

Fotiadou, Stavroula PM40

Fourka, Maria- Niki PM165

Fourka, Maria-Niki PM477

Fournaise, Anders PM62, PM287, PM309

Fourrier, Marie-Jeanne P127

Fourriere, Marie-Jeanne P126

Fox, Siobhan PM146

Frade, Luciana P44

Fraisse, Thibault PM478

Françoise, Orsini P153

Francine, Laigle PM380

Franco, Sonia P163

Francois, Puisieux P155, PM122

François, Patrice PM478

François-Xavier, Sibille PM446

Frank, Jessen PM144

Franks, Paul W. P166

Franssen, Casper F.M. P226

Franssen, Casper F. M. PM156

Franziska, Maier PM144

Fratiglioni, Laura P5, P175

Frederic, Bloch PM469

Frédéric, Bloch PM429

Frederick, C. W. Wu OCM1

Freiberger, Ellen PM252

Freidl, Wolfgang OCM20, PM274, PM280

Freire, Gonçalo P32

Freitag, Mathias PM449
Fresco, Ana PM113, PM114

Freydin, Maxim B. OC18, P166

Friedmann, Reuven PM35

Frizzell, Erin OC14, P47

Frutuoso, Ana Filipa PM419

Fuente, Carmelo La P25

Fumagalli, Stefano P195, P230

Fure, Brynjar PM289

Gómez, Raquel Ortés P27

Gómez, Esther Álvarez P125

Gómez-Pavón, Javier P4, P6

Güerri-Fernandez, Robert OC29

Gámez-Asunción, Carla P78

Gabriele, Sara P7

Gagliardone, Chiara PM14, PM15, PM16, PM17, PM18, PM19, PM27

Gaillemin, Olivier PM208

Gala, Carlos P43

Galea, Doriella OC45, P116, P254

Galea, D. P111

Galetti, Caterina PM340

Gallagher, Paul P52

Galluzzo, Lucia OCM13

Galván-Banqueri, Mercedes PM423

Galvin, Rose OCM10

Gambier, Nicolas P126, P127

Gandolfo, Federica OCM17, PM49

Ganesh, Sajaysurya P166

Gantois, Isabelle P117

Ganz, Felipe P86

Garaboldi, Sara OCM17, PM49

Garay-Airagui, Victoria P78

García, Borja Gil OC3, OC23

García, Belén Roldán OC3, OC23

García, Pamela Carrillo OC21

García, Alicia Noguerón OC23

García, Julia Castillo P19, P28

García, Elisabet Sánchez P140

García, Jenifer Peinado P154

García-Baztán, Agurne P30

García-Prendes, Cristina Guirola OC4, P33

Garcez, Flavia P185, P211

Garcia, Pamela Carrillo OC17

Garcia, Julia Castillo P18

Garcia, Pamela Lisette Carrillo P60

Garcia, Maria Muñoz P140

Garcia, Maria Rosario P192

Garcia, Maria Muñoz P290

Garcia, Mercedes Forcano PM197

García, Pamela Carrillo P282

García, Beatriz PM46

García, Ruth Jorge PM72

García, Mercedes Forcano PM375

García, María Muñoz PM445

García-Baztán, Agurne P285

García-Cabrera, Lorena PM445

García-García, Francisco José OCM16

García-Martínezz, Juan PM47

Gareri, Pietro P195

Garmendia-Prieto, Blanca P4, P6

Garnier, Virginie PM266

Garrafa, Emirena P175

Gąsowski, Jerzy P259

Gavana, Magda P144, PM211, PM214

Gavazzi, Gaetan PM67 
Gavazzi, Gaëtan PM266

Gaxatte, Cédric P99

Gebauer-Campos, Maria P78

Gedik, Tugce Emiroglu P132

Geirsdottir, Olof Gudny PM391

Geist, Natalie PM297

Gelatti, Umberto P258

Gemci, Emine P178

Genç, Fatimatüzzehra P243

Gencer, Gokce Yagmur Gunes P235

Genin, Mickael PM33

Gentile, Leandro PM12

Genton, Laurence P180

Gentry, Richard PM38

Gény, Bernard PM85

George, Ryan P213

Georgiadou, Marina P253

Geraci, Francesco PM14, PM15, PM16, PM17, PM18, PM19, PM27

Gereon, Nelles PM148

Geriatric Medicine Research Collaborative, P172, P214

Gerosa, Sebastiano PM6, PM7, PM8, PM9, PM10, PM11, PM12, PM13

Geschwindner, Heike P142

Geurt, Marie-Noëlle P82

Geurts, Marie-Noelle P225

Geyer, Bradley P159

Ghiara, Matteo PM6, PM7

Ghiara, Camilla PM102, PM103

Gianella-Blanco, Cristina P6

Giannakou, Eeleni P223

Giannoni, Paola OCM25

Giansiracusa, Paola P175

Giedraitis, Vilmantas PM140, PM141

Gielen, Evelien OC8, P36

Giger, Ann-Kristine Weber PM342

Gijs, Van Pottelbergh P66

Gil, PedroCaiano PM188

Gil, Isabel PM347

Gilard, Isabelle P34

Gill, Thomas OCM20

Gillibert, André PM272

Gillot, Robert P38

Gillott, Robert P42

Giltay, Erik PM132

Giménez, Ramón P286

Gimeno, Esther Espinosa P96

Giné-Garriga, Maria PM207, PM471

Giordano, Antonella OCM19, PM126, PM356

Giordano, A. PM421

Girardello, Renzo P7

Girardi, Elena PM356

Gires, Catherine P99

Gisca, Eugeniu PM112

Giudici, Kelly Virecoulon PM288

Giulia, Rastrelli OCM1

Giuliani, Giorgio OCM28

Giuliani, Evelina PM87, PM94, PM442

Giusti, Ebony PM159

González-Glaría, Belén P118

Gñi, Saleta P51

Goñi-Roson, Saleta P6

Godaert, Lidvine PM422

Godard-Marceau, Aurélie PM41

Godart, Marie-Christine P117

Godayol, Pau Farrés PM458

Góes, Roberta Pereira PM70, PM473
Goethals, Luc OC52

Goh, Jing Wen P234

Goh, Alicia PM261

Goh, Kiat Sern PM348

Gold, Gabriel P180

Goldbeck, Annelie P48

Golsharifi, Milad PM119

Golüke, Nienke PM88

Gomes, Nildete Pereira OC10, P107

Gomez, Maria F. P166

Gomez R, Karina OC44

Gómez-Pavón, Javier P282

González-Glaría, Belén P30

Gonzales-Montalejo, Nancy Jeanette P30

Gonzalez, Marta Mercedes P43

Gonzalez, Silvia P163

Gonzalez, Marta Pisano PM158

González, Amaia Berrizbeitia PM153

Gonzalez-Bautista, Emmanuel P182

Gonzalez-Bueno, Javier PM426

González-Glaría, Belén P285

Gonzalo-Lázaro, María P30

Gonzalo-Lázaro, María P285

Goodarzi, Zahra PM56

Gordon, Adam OC37

Gorenjak, Vesna OCM3

Gosteva, Elena PM83

Gough, Nick P169

Gouronnec, Adeline PM385

Gouveia, Paulo PM254

Gouveia, C.G. PM447, PM448

Graf, Christophe E. P180

Graham, Mark S. P166

Gramont, Baptiste OC52

Granado, Joana P44

Grande, Giulia P5, PM138

Granic, Antoneta OCM29, PM453, PM454, PM455, PM463

Grant, Emma PM302

Graversen, Susanne Boel OCM6

Gray, Amy PM127

Grech, Michelle P63, P252

Greco, Laura PM55

Gregory, Genna PM319

Greka, Athina P241, PM407, PM467

Gricourt, Cyril P284

Grieco, Fabrizio Vincenzo PM106

Griffin, Claire PM332

Grigorescu, Mihaela Zamfir P261, PM214

Griskevicius, Julius PM239

Grodzicki, Tomasz P259

Groos, Sara PM259

Gross, Ariane OC1

Großhauser, Franz PM403

Grugnetti, Giuseppina PM9, PM21, PM22

Grulli, Francesca P202, P203, P204, P205, P206, P263, PM14, PM15,

PM16, PM17, PM18, PM19

Gruner, Heidi OC7, P184, P216, PM71, PM170, PM346, PM410

Gryglewska, Barbara P259

Guarino, Daniela P141

Guarnone, Roberta PM8, PM9, PM10, PM11, PM12, PM13, PM15,

PM16, PM17, PM18, PM19, PM20, PM21

Gudex, Claire PM287

Gudmundsson, A. PM435

Guérin, Olivier PM478

Guerra, Angela OC34, P8, P9

Guerra, Germano OCM28 
Guerreiro, Joana PM170

Guerrero, Katerin Leslie Quispe OCM4, OCM17, PM305

Gugliucci, Marilyn R. P266

Guida, Loredana OC34, P8, P9

Guillen, Cristina Cazorla PM145, PM389

Guilloton, Céline P284

Guinan, Elaine PM332

Guisado-Gil, Ana Belén P53

Guiteras, Anna Renom PM315

Guo, Chuan-Guo P166

Guo, Suimin PM147

Gussekloo, Jacobijn PM29, PM30

Gutarra, Lizzeth Canchucaja OC29

Gutiérrez-Valencia, M. PM435

Guyssens, Vinciane P55

Guzman, Manuel P163

Gylou, Sotiria P238

Hackbarth, Michel OC41, P240, PM333

Hafizah, Noor PM406

Haghighi, Eduardo Doutel PM164

Haidich, Anna-Bettina P144, PM214

Hall, Emma P169

Hall, K. PM1

Handalage, Celina P93

Hanke, Frank-Christian PM194

Hannah, Weigert PM148

Hanon, Olivier OC1

Hanrahan, Caoimhe P22, P87, P179, PM53, PM212, PM296

Hansen, Rikke Frøslev P72

Hanssen, Michel PM85

Happe, Lisa P242, P246

Haque, Mohammed Ainnul PM44

Harari, Danielle PM341, PM343

Harbarth, Stephan P180

Haris, Muhammad P38

Harnedy, Norma P52

Harrison, Michael PM79, PM292, PM298, PM302, PM303

Hartel, Linda PM326

Hartigan, Irene OC32

Hartikainen, S. PM435

Hasan, Sharjeel OCM21

Hashemi, Nima PM307

Hashmi, Madiha Arslan P270

Hasmu, Kejal P102

Hasmuk, Kejal P14

Hasmukharay, K. P11

Hasmukharay, Kejal P17, P80, P90

Hatter, Lee PM221

Hayes, Mary PM318

Head, Naomi P74, P75, P76

Hein, Andreas P242, P246, PM169

Heinks, Andrea PM171

Helena, Claes P66

Helma W.M.F., Verstraeten PM363

Henderson, David P77, OCM22

Henk, Cuvelier P71

Hennerdal, Britt-Marie PM330

Henrard, Séerine P34, P35

Henrard, Séverine PM434

Henri, Naga OCM14, OCM15

Henriksen, Daniel PM250

Henstra, Marieke PM132

Heppner, Hans Jürgen PM194

Herbst, Andrea PM102, PM103

Herdman, Trent P212
Herghelegiu, Anna Marie P21, P100, P247, PM310, PM439

Herghelegiu, Anne Marie P148

Hermush, Vered PM128

Hernáíndez, Maria Jesús Molina P37

Hernáíndez-Ruiz, Virgilio P221

Hernandez, Issaell OC44

Hernandez, Belinda OCM12

Hernandez, Carmen Dolores Hernandez PM150

Hernandez, Elisa Lasala PM433

Hernández, Itziar Palazuelos PM154

Hernández-Sánchez, Luisa Alejandra PM452

Hernes, Susanne Sørense P49

Herrera-Hidalgo, Laura P53

Herrmann, François P180

Hertogh, Cees P114, P164, P165

Hertogh, C. M. P. M. OCM18

Hesford, James PM50

Hevia, Luis PM46

Hewitt, Jonathan PM127

Heymans, Martijn PM248

Heyns, Arne P36

Hickey, Pamela P94, PM261

Hickey, Paula PM73

Hidalgo, Javier Jaramillo OC17, OC21, P177, P282

Hiemstra, Ytje PM308

Higuet, Sandra P82, P225

Hilde, Servotte P71

Hirsch, Emmanuel P176

Hobson, Peter PM129

Hockle, Jo OCM22

Hockley, Jo P77, P79

Hockleyu, Jo OC22

Hoffmann, Uwe PM107

Holling, Nina P168

Holloway, Mark PM50

Holmerova, Iva PM118

Holten, Aleksander P174

Honda, Miwako PM206

Hoogendijk, Emiel PM274

Hoogendijk, Emiel O. PM280

Hopstaken, R. M. OCM18

Horata, Emel Taşvuran PM331

Horgan, Mary P52

Hormazábal, María José PM420

Horton, Christopher PM213

Hoyas, Maria P163

Hrnciarikova, Dana PM217

Hrycaiczuk, Joshua P168

Hsu, Chen-Yuan PM360, PM361, PM364

Huarte, Beatriz Gamboa P18, P19, P28, PM160, PM437

Huber, Philippe P180

Huisman, Martijn PM285

Hunt, Clare P56, P277

Hurst, Christopher PM453, PM454, PM455, PM463

Hussein, Tunay PM51, PM162

Huvent-Grelle, Domnique P99

Ibar, Olga Váízquez OC29

Ibrahim, Kinda P167, PM399

Ibrahim, Norhayati P234

Ibrahim, Amirah Fatin P239

Ievoli, Riccardo PM65

Iglesias, Ana Lopez PM233

Ignacio, Luis Ignacio Mendibil PM153

Ihle-Hansen, HåÑkon OC9, P170

Ihle-Hansen, Hege PM289 
Ilgar, Mehtap PM255

Ilhan, B. PM435

Ilie, Adina Carmen P21, P148, PM310, PM439

Ilie, Adina P100, P247

Ilnitski, Andrey P222

Ilpo, Huhtaniemi T. OCM1

Immanni, Sudhir PM417

Incalzi, Raffaele Antonelli P195, P230, PM105

Indiano, Ilaria OCM17, PM49

Ingelsson, Martin PM141

Ingo, Beyer P88, PM286

Ingrid, Becker PM144, PM168

Inzitari, Marco P286

Iotti, Giorgio Antonio PM6, PM7, PM8, PM11, PM12, PM13, PM14,

PM23, PM24, PM26

Iqbal, Muhammad P83, P289

Isabell, Fromentin OCM14

Isabelle, Fromentin OCM15

Isabelle, Walz PM319

Isaev, Ruslan PM139, PM412

Isaykina, Olesya PM76

Ishii, Yoko PM349

Islam, Farah P103, PM313

Ivan, Bautmans PM286

Izquierdo, Mikel PM37, PM47

Izquierdo-Zamarriego, Gloria P6

Izumi, Shinichi PM451

Izyumov, Andrey OC5, PM414

Jackman, Catherine OC12

Jackson, Arthur P52

Jackson, S.H.D. PM435

Jacob-Filho, Wilson P211

Jacomine, de Lange PM363

Jacques, Marie-Claire P180

Jaén, Dácil Cabezas PM109

Jan, Ampe P66

Jansen, Ann KristineKristine P162

Jansen, Michael PM324

Janssen-Heijnen, Maryska PM308

Janssens, Wim P55

Janus, Sarah P89

Jaramillo, Diego P163

Jaramillo-Hidalgo, Javier P4, P6

Jarolimova, Eva PM118

Jay, Nicolas P117

Jean-Baptiste, Piedallu PM271

Jean-Claude, Lemper P88

Jean-Claude, Leners PM440

Jean-Philippe, David OCM14, OCM15

Jenneve, Anne PM82

Jenny T., van der Steen PM363

Jensen, Thomas Giver PM246

Jentoft, Alfonso Cruz P140

Jentoft, Alfonso Jose Cruz PM233

Jentoft, Alfonso José Cruz PM445

Jeppesen, Stefan Starup PM342

Jerez-Roig, Javier OCM23, PM163, PM207, PM290, PM368, PM471, PM472

Jeroen, Demarteau PM286

Jesus, Susana P44

Jesus, S. PM447

Jeyaseelan, Arveen PM292, PM298

Jimenez-Armero, S. P156

Jin, James PM93

Jobbágy, Antal P248
Jochen, Klucken PM151

Joel, Belmin PM322

Johan, Flamaing P66, PM416

Johari, Bushra Megat P14

Johdi, Nurul Farhana M. OC39

Johnston, Lucy OC22, P77, P79, OCM22

Jolanta, Slowikowska-Hilczer OCM1

Joly, Laure P126, P127

Jones, Rose P168

Jones, Helen P271

Jonsson, Agnes PM260

Jonsson, Palmi V PM391

Joo, Nam-Seok PM84

Joosten, Hanneke P226, PM156

Jordan, Granet PM198, PM457, PM460

Jos, Tournoy P66, OCM1

Joshi, Amit D. P166

Joshi, Mamta PM341

Jude, Edward PM237

Judge, Conor S. OC42

Julie, Berthou PM380

Jundi, Alice P93

Jung, Theresa OCM27

Jung, Susie PM84

Juozaitis, Mantas PM397

Kłosińska, Urszula P81, P92

Kahraman, Mustafa PM461

Kalafatis, Chris P169

Kalivioti, Xristina PM244

Kallio, Eeva-Liisa OCM8

Kalra, Philip A PM283

Kalsi, Tania P136, P169, P173, OCM21, PM341, PM343

Kaluźniak-Szymanowska, Aleksandra OC49

Kamaruddin, Mohd Zul Amin P234

Kamarulzaman, Adeeba P14

Kamaruzzaman, S. B. P11

Kamaruzzaman, Shahrul Bahyah P14, P17, P80, P90

Kamaruzzaman, Shahrul B. OCM9

Kaplan, Davut Sinan PM123

Karaali, Ridvan P132

Karagianni, Maria P238

Karagiannis, Thomas PM181

Karan, Mehmet AkifAkif P50

Karan, Mehmet Akif P207, PM461, PM462

Karapinar-Carkit, Fatma OCM5

Karbowski, Lukasz P45

Karlatou, Antonia Aravantinou PM281, PM282

Karlsson, Linda Katharina PM227

Karnaushkina, Maria P31

Karyn, Doba PM122

Kasa, Marsida PM268

Kasiukiewicz, Agnieszka P200, P201, P259, PM329

Kassasseya, Christian PM36

Katarzyna, Wieczorowska-Tobis P260, P262

Kateroglou, Stefanos PM204

Katipoglu, Bilal P217, PM398, PM411, PM428

Katleen, Fagard PM416

Katrin, Gillis P71

Katsouli, Aikaterini P238

Kautiainen, Hannu OCM8

Kazantzaki, Ilia PM366

Kazberuk, Miroslaw P200, P201

Kearney-Schwartz, Anna P126, P127

Keena, Alanna P134, P236

Kelliher, StJohn P134, P236, PM316 
Kelly, Lisa P23

Kelly, Rosalind P42

Kelly, Mark PM332

Kemmler, Wolgang PM252

Kendrova, Lucia PM320

Kennelly, Sean OC16, P91, P95, P97, PM133, PM134, PM135, PM146, PM438, PM443

Kennelly, Siobhan P97, PM59

Kennelly, Siobhán OCM24

Kennelly, Seán OCM24

Kenny, Rose Anne OCM12

Kentros, Zikos PM265

Keravec, Héloïse OC6

Kergoat, Marie-Jeanne PM60

Kerif, Marphy PM273

Ketz, Flora OC1

Keygnaert, Ines P186

Khan, Asra P159

Khan, Zahra PM307

Khan, Muhammad PM343

Khani, Aria OC19, P64, P65, P69, P70

Khor, H. M. P11

Khor, Hui Min P14, P17, P80, P90, PM418

Khovasova, Natalia PM251

Kiesswetter, Eva PM403

Kilander, Lena PM141

Kilavuz, Asli OC50, P194, PM66

Kilty, Caroline OC32

Kim, Suji P65

Kim, Nayoung PM77

Kioh, Sheng Hui PM252

Kirk, Charlotte P104

Kirmanidis, Michalis PM101, PM293, PM311, PM312, PM345

Klaus, Jahn PM151

Kleisiaris, Christos PM187, PM199, PM200, PM281, PM282, PM284, PM401

Klijnsma, Petra PM308

Klimek, Ewa P259

Knarren, Lieve PM308

Knašienè, Jurgita PM48, PM396

Knašienè, Jurgita Knašienè PM475

Knight, Megan PM51, PM162

Knol, Wilma PM249, PM436

Knol, W. PM435

Knott, Gareth P104

Knox, Laura OC14, P47

Kobayashi, Masaki PM206

Kocakoç, Nurgül OC47

Kocot, Ewa P103

Kodate, Naonori P110, PM349

Koeberle, Séverine P283, PM41

Koek, Huiberdina PM88

Koek, Dineke PM249, PM436

Koen, Balcaen P66

Koene, Fleur P164, P165

Koenig, Anne PM85

Köhler, Christian P288

Kokkali, Stamatia P144, PM214

Kokkalis, Zinon PM244

Kolesnikova, Olena PM95, PM108, PM155

Kolokithas, Georgios P223

Koloniari, Amalia PM244

Komici, Klara OCM28, PM99, PM106

Komiya, Hitoshi PM424

Konrad, Madejczyk P260

Koopmans, Marion P164
Korkmaz, Busra Yurumez P178

Korkmazer, Bora P132

Koschate, Jessica OC41, P240, P246, PM107, PM333

Kose, Murat P50, P207

Kotovskaya, Natalia OC5, PM414

Kotovskaya, Yulia OC5, PM89, PM104, PM189, PM299, PM323, PM414

Kotsani, Marina P126, P127, P143, PM165, PM211, PM214, PM477

Koukia, Evmorfia PM124, PM125

Koutsouri, Anastasia PM265

Koval, Štefan PM2

Kozaki, Koichi PM451

Kragholm, Kristian PM226

Krause, Olaf PM449

Kravvariti, Evridiki PM40

Kravvariti, Evrydiki PM78, PM165, PM256, PM477

Kropińska, Sylwia OC49

Kruglikova, Anna PM76

Krypciak, Sébastien OC1

Krysiuk, Olga P163

Krzymińska-Siemaszko, Roma OC49

Kuchel, George OC33

Küçük, Ayten PM167

Kukreja, Anjanna P14

Kundracikova, Luboslava PM320

Kuno, Shinya P229

Kuo, Chai-Ling OC33

Kurt, Barbé P88

Kurvers, Roel PM308

Kuskucu, Mert Ahmet P132

Kuwelker, Kanika PM314

Kuzuya, Masafumi PM424

Kyrozis, Andreas PM120

López, Carlos Alfredo Colato P85

López-Graciano, Blanca Estela P221

Laakkonen, Marja-Liisa OCM8, OCM11

Labari, Genoveva PM405

Labat, Carlos P117, P135, OCM3, PM111

Labon, Livia PM385

Lacolley, Patrick PM111

Lacrampe, Mathilde OC1

Ladi, Emma OCM21

Lafaie, Ludovic OC52

Lafarga, Teresa Pujadas PM315

Lafe, Elvis PM8, PM13, PM14, PM15, PM18, PM19

Lafuente-Lafuente, Carmelo OC6, OC26, P84, PM476

Lago, Arlinda P3, PM232, PM238

Lagolio, Erik OC36

Lai, Tsung-Po OCM3, PM111

Lai, Feng-Min PM364

Lakehal, Soumia PM38

Lakomy, Cécile PM111

Lal, Rajni PM415

Lamb, Sarah PM138

Lambertucci, L. PM421

Lamer, Antoine PM33

Lamppu, Pauli OCM11, PM210

Lancaster, Oscar P74, P75, P76

Lancho, Estela Villalba P27

Landi, Francesco OCM26

Landi, F. PM435

Lang, Philippe PM82

Lanoix, Jean-Philippe PM67

Lanthier, Nicolas P34

Lappegard, Knut Tore PM350 
Lapthorne, Susan P52

Lara, César Cuevas PM37

Lasco, Carmen PM245

Latorre, Marina Sanchez P108

Latorre, Marina Sánchez P118

Latorre-Vallbona, Nadina OC25

Latour, Corine OCM5

Lattanzio, Fabrizia P193

Lau, Chun Fatt OC39

Lau, Sandra OC41, P240, P242, P246, PM169, PM171, PM333

Lau, Sabrina PM406

Laure, Joly P153

Lauren, Dautzenberg PM436

Laurentine, Maljean PM446

Laurentius, Thea PM449

Lauriane, Segaux OCM14, OCM15

Lauridsen, Jørgen T. PM287

Laurila, Jouko OCM11

Lava, Massimiliano PM23, PM24, PM25, PM26

Law, Ruth OC12

Law, Caroline PM60

Lawal, Ade P169

Lawlor, Brian PM133, PM134, PM135, PM438, PM443

Lazaridou, Elli PM165, PM477

Le, Phuc-Thien PM272

Leal-Costa, Cesar PM350, PM351

Leander, Julia PM30

Lee, Karla A. OC18, P166

Lee, Eun Ji PM77

Lee, Dong Ho PM77

Leen, Antonio OCM1

Legorburo, Maria Jose Martin P96

Legorburo, Maria José Martín P98

Legorburo, Maria José Martín P183

Lekx, Anita PM308

Lelli, Diana PM105

Lemarié, Nadége OC1

Lems, Willem PM285

Lena, Pickert PM144, PM148, PM168

Lena, Sannemann PM144

Lenehan, Brian P94

Lenne, Bruno PM355

Lente, Alina Katharina PM194

Leroy, Victoire P99

Lesauskaite, Vita PM396, PM397

Lesende, Iñaki Martin PM153, PM154

Lesley K, Fellows PM457

Lewandowicz, Marta OC49

Lewis, Anna P138

Li, Hannah P160, P161

Li, Elaine PM307

Lien, Chris PM4

Liew, Shan Liung P83, P289

Lim, Einly OC39

Lim, W. C. P11

Lim, Wan Chieh P14, P17, P80, P90

Lim, Jing Wei PM4

Lim, Wee Shiong PM406

Lim, Jun Pei PM406

Limodio, Mario P1

Limodio, Marta P1

Lin, Amy P266

Lindenberg, Nitzan OC12

Linge, Magdalena P43, PM223

Linn, Annemiek P233, PM259

Lisa, Van den Eeckhaut P88
Lisk, Clifford OC19, P64, P69, P70

Lisk, Radcliffe P121, P122

Livada, Alexandra PM165, PM477

Liza, De Dobbeleer PM286

Lo, Chun-Han P166

Loïc, Josseran P147

Lobato, Concepcion P192

Lochlainn, Mary Ni OC18, P166

Loh, Siew Ping P14, P90

Loh, Pui San PM418

Longobardo, Luz María Peña OC23

Loor, Genesis Estefania Olaya PM233

Lopes, Arianna Oliveira Santana OC10, P107

Lopes, Vítor Neves P131

Lopes, João Madeira PM115

Lopes, Carla PM404

Lopez-Liria, Remedios PM350, PM351

López-Liria, Remedios PM196

Lopez-Villegas, Antonio PM350, PM351

López-Villegas, Antonio PM196

Lorenzo, Isabel Cirera PM315

Lorenzo-Villalba, Noël PM74, PM82, PM85, PM86, PM272, PM273

Lores, Silvia P171

Lorrigan, David PM417

Louise, Tyvaert P153

Lozano-Montoya, Isabel P4, P6

Luís, Maria Margarida P131

Lucarelli, Moira P193

Lucarelli, Giulia P230, PM102, PM103

Lucien, Weiss PM240

Lucke, Jacinta A. PM30

Ludin, Arimi Fitri Mat P234

Luengo, Iker Llamosas PM153, PM154

Luiking, Yvette PM465, PM466

Luna, Carla P163

Lund, Lars Christian PM250

Lundberg, Caroline PM140

Lunn, Troels Haxholdt PM246

Luzzi, Sabino PM6, PM7, PM8, PM11, PM12, PM13, PM28

Lynch, Amy P218

Lynch, Ciara PM332

Lyons, Carol PM52

Lysenkov, Sergey PM104, PM189, PM299

Méndez, Lourdes Sáez OC3

Ma, Wenjie P166

Maślanka-Seiffert, Barbara P92

Macedo, Ana PM112

Macedo, Cátia PM222

Macedonio, Sarah PM6, PM7, PM8, PM9, PM10, PM11, PM12, PM13, PM20, PM21

Machado, S.B. PM448

Machekhina, Lubov PM182, PM237

Machón, Mónica P268

Mackerrrow, James P271

Mackowiak, Marie-Anne P29

MacLullich, Alasdair OCM10, PM177, PM177

MacNeil-Vroomen, Janet P123, PM326

Maddalena, Gaetano PM105

Madeira, Margarida PM112

Madeira, Teresa PM404

Maertens, Sandra P55

Maestro, Maria Antonietta PM28

Maestro, Laura C. PM29

Maestro, Mareline E. PM29

Magalhaes, Guilherme P184 
Maggi, Stefania P224, OCM4, OCM13, PM138, PM285, PM474

Maggio, Marcello OCM26

Maggioni, Paolo P202, P203, P204, P205, P206, P263, PM23, PM24,

PM25, PM26

Magnani, Dario OC34, P8, P9

Magner, Siobhan PM52

Magnuszewski, Łukasz PM329

Magri, Maurizio OC26, P25, P84, PM92, PM367, PM385

Magrì, Maurizio PM36

Magrini, Stefano M. P258

Mahadzir, Hazlina P234

Maher, Niamh PM262

Mahiat, Cedric P34

Mahiat, Cédric P35

Mahmood, Rashid P121, P122

Maike, Tscheuschler PM144

Mair, A. PM435

Malara, Alba P195

Malcolm, Cari P79

Malek, Muhammad Rabbani Abdul P234

Malek, Hassanine Ben PM74

Malfitano, Antonello PM9, PM10, PM20, PM21

Malik, Irfan Rasool PM59

Mallory, Lindsey PM297

Malloupa, Eleni P213

Mamun, Kaysar PM269

Mancini, Carlotta P230

Mancini, Angela PM106

Mandysova, Petra PM161

Manevich, Tatiana OC5, PM414

Mang, Gordon Pang Hwa P14

Mangino, Massimo OC18

Mangion, Stephen PM371

Mannion, Eileen P23

Mannion, Edel P179, PM53, PM296

Mannion, Stephen P212

Manno, Valerio OCM13

Manolaki, Chrysanthi PM211

Mansinho, Kamal P44, P197

Mantoan, Paola P158

Mantzorou, Marianna PM124, PM125, PM161

Mantzos, Dionysios PM40

Manzoni, Federica PM6, PM7, PM8, PM11, PM12, PM13, PM17,

PM23, PM24, PM25, PM26, PM28

Manzoor, Amir P121

Mapelli, Daniela PM143

Marín-Epelde, Itxaso P30

Marc, Braun P153

Marcel, Rarek PM168

Marchionni, Niccolò OCM19

Marena, Carlo PM9, PM10, PM22

Marengoni, Alessandra P5, P175, P224, PM138

Margari, Nikoletta PM161

Margus, Punab OCM1

Maria, Meyer Anna PM148, PM168

Mariam, Neethu Billy Graham P38, P39, P41, P42

Marian, Dejaeger OCM1

Marie, Laurent OCM14, OCM15

Marie, de Saint-Hubert PM446

Marie-Josée, Brouillette PM457

Marin, Laura P68

Marin, Andreea G. P261, PM149, PM215

Marín-Epelde, Itxaso P285, OCM7, PM47

Maritsa, Aggeliki PM370

Marjolaine, Baude OCM15

Mark, Harriet PM204
Mark Clarfield, A. P220

Markakis, Georgios PM282

Marleen, Verhaegen PM416

Marmaneu, Carmen Oquendo P125, PM116, PM223, PM358, PM375

Marneras, Christos P273, PM201, PM242

Maroua, Mrouki PM317

Marousa, Pavlou PM319

Marques, Lia OC48, P198

Marques, Filipa PM413

Marron, Brooke P120

Martín, Maria del Rosario García P37

Martín, Irene Bartolomé P98, P183

Martín, Magdalena Linge P125, P154

Martínez-Fuerte, Rosario P191

Martens, Han P55

Martial, Coutaz PM240

Martignoni, Alessandra PM14, PM15, PM16, PM17, PM18, PM19

Martin, Finbarr C. OC18

Martin, Ruth P97, OCM24, PM59

Martin, Alan P97, P101, OCM24

Martin, Fionna P136, PM415

Martin, Louise PM229

Martin, Magdalena Linge PM358

Martin, Smalbrugge PM363

Martín, Patricia Mateo PM109, PM150

Martín, Pau Moreno PM458

Martin-Marero, Carmen P270

Martinez, Carlos PM46

Martínez, Beatriz Neira P282

Martínez, Ana Belén Vena PM315

Martínez-Fuerte, Rosario PM185, PM230, PM231, PM235, PM278, PM338, PM339, PM376

Martínez-Velilla, Nicolás PM37, PM47

Martini, Sebastian OCM27

Martino, Gabriella PM245

Martins, Glaucia P237

Martins, Andre PM71

Martins, Rita Tourais PM164

Martins, A. PM447, PM448

Maruthan, Sachan P65

Mary, Morgane OC1

Mascaranhas, Joana PM176

Maselli, Monica P12, P13

Masina, Fabio PM143

Masó-Aguado, Montse PM163

Masoli, Jane OC33

Massalha, Victoria P228

Mastaviciute, Asta PM239

Mastavičiūtè, Asta PM321

Masterton, Jackie P136

Mastrogiannis, Dimos PM124, PM125

Masud, Tahir P72, PM137, PM441

Masurel, Caroline P117

Masuyama, Shigeru P110, PM349

Mat, Sumaiyah OC40, OCM9, PM252, PM257

Mateo-Abad, Maider P268

Mateos-Nozal, Jesús PM445, PM452

Mathieu, Piccoli PM202

Mathieu-Nicot, Florence PM41

Matthew, Liston PM319

Matthews, Fiona E. OCM29

Matthias, Kochanek PM168

Matthissen, Ulla P48

Mattoso, Patricia P58, P62

Matzen, Lars Erik PM62, PM342

Mauricio C, Alicia H. OC44 
Mavridis, Dimitris PM249, PM436

Mawhinney, Jamie PM415

Maya, Ruth Paz PM109

Maya, Abdallah PM317, PM372, PM388

Mayerl, Hannes OCM20, PM274, PM280

Mazanzi, Blessing PM42

Mazelli, Gloria PM43, PM203, PM381, PM383

Mazza, Liliana PM65

Mazzeo, Francesca OC2, P15

Mazzone, Antonino PM224

Mbilo, Céline P142

McCarroll, Kevin PM258, PM262

McCarron, Louise PM332

McCarthy, Julie P2, PM427

McCarthy, Carol P113

McCarthy, Kathryn PM50

McCarthy, Christine Eileen PM191

McCleery, Jenny PM127

Mcdermott, Clodagh P22, P87, PM53, PM212

McDermott, Clodagh Marie P179

McDonough, Anna PM146

McElroy, Morven P113

McFeely, Aoife P95

McGauran, Jane PM318

McGovern, Aine OC14, P47, P113

McGowan, Bernie P208, P209

McGrath, Keith P52

McHale, Cathy PM146

McHugh, Sheena PM260

McIlroy, Kathyrn P113

McKee, Kevin PM141

McKeogh, Anna P40

McLoughlin, Kara PM229

McNeela, Edel PM79

Medetalibeyoglu, Alpay P50, P207

Medlock, Stephanie PM248

Medrano, Martin OC44

Megan, Drennan P47

Megas, Panagiotis PM244

Megaw, Jamie P271

Meirhaeghe, Aline P176

Melgaard, Dorte PM226

Melho, Márcio Ribeiro P107

Melhor, Márcio Ribeiro OC10

Mellaz, Fatima Meksem PM385

Melo, Rita PM68

Melo, Lina PM113, PM114

Melzer, David OC33

Menale, Silvia PM421

Mendes, Filomena P128

Mendes, Aline P180

Mendes, Cristiana Canelas PM115

Mendoza, Soledad Domínguez P37

Mendoza, Soledad Dominguez P157

Menegakis, Emmanouil PM362

Menicagli, Roberto P1

Menni, Cristina P166

Mentis, Manolis P223, PM244

Mercadier, Elise OC1

Merkusheva, Liudmila PM104, PM189, PM299

Merkusheva, Ludmila PM323

Merrick, Cath PM42

Mesa, Karla Diaz OC44

Meschi, Tiziana OC15, OC34, P8, P9

Mese, Sevim P207

Mesquita, Mari P131
Metaxa, Maria P238, PM353

Metz, Millicent OCM21

Metz, François PM86

Meuleman, Yvette PM29

Meya, Abdallah PM386

Meyer, AM. OC36

Meyer, Anna Maria P48

Meyer, A. M. PM166

Meznerics, Fanni P248

Micallef, Clayton PM131

Michael, Atef P2, P3, P59, PM80, PM238, PM427

Michael, A. PM1

Michael, A PM232

Michálková, Helena PM464

Michelozzi, Paola OCM19

Midttun, Mette PM227

Mieke, Deschodt PM416

Migliorini, Marta P230, PM102, PM103

Mihalache, Ruxandra PM121, PM468

Mikołaj, Seostianin P260

Milisen, Koen P103, PM313

Miller, Hazel OC14, P47

Miller, Alan PM96

Miller, Alan B. PM382

Millican, Sarah PM297

Minaya, Dionis Carolina Fernández P125, P154

Minelli, Giada OCM13

Minervini, Sergio P7

Minici, Domenico PM356

Minikavičiūte, Karolina PM475

Minobes-Molina, Eduard PM290, PM471, PM472

Miranda, R. PM447, PM448

Mitchell, Sheena P67

Mitchell, Emma PM50

Mizzi, Jean Marc PM371

Mjahed, Hajare Sarah P135

Mkhitaryan, Elen PM139, PM412

Mo, Xiaocong PM147

Modat, Marc OC18, P166

Mohamed, Belhout PM429

Mohammed, Sajeel PM283

Mohammednoor, Mohammed P168

Molas-Tuneu, Míriam OCM23, PM472

Molas-Tuneu, Miriam PM163, PM207, PM368, PM471

Molina, Rafael García OC23

Molina, Maria Jesus P192

Molina, Eduard Minobes PM458

Molinas, David Molina PM216

Molinos, Maria PM352

Molins, Marta P286

Molins, Maria Teresa P286

Molist-Brunet, Núria OC25

Molist-Brunet, Nuria PM263, PM264

Molist-Brunet, Núria PM425, PM426

Molloy, D. William PM147

Moloney, Elizabeth P52, P57, PM318

Moloney, David OCM12

Monahan, Margaret P. OC32

Money-Coomes, Sally PM283

Mongodi, Silvia PM24, PM25, PM26

Montanari, Enrico PM359, PM359

Montejo, Nancy Jeanette Gonzales P108

Montejo, Nancy Gonzales P118

Monterde, Beatriz PM405

Montero-Errasquin, Beatriz P290

Montero-Errasquín, Beatriz PM452 
Monti, Alexandra OC1

Montoy, Isabel Lozano P177

Montoya, Isabel Lozano OC17, OC21, P282

Monzani, Fabio P141

Mooijaart, Simon P. P226, PM29, PM30, PM156

Mooijaart, Simon PM308

Moola, Riaz P133, P257

Mora, M.A. Caballero PM435

Morabito, Nunziata PM402

Morandi, Alessandro P286

Morano, Pasquale P141

Mørch, Marianne PM226

Moreira, Valter P219

Morel-Corona, Felix-Jorge PM263, PM264

Morelli, Ilaria OC34, P8, P9

Moreno, Juan Oliva OC23

Moreno, Sasha Raquel Botbol P37

Moreno, Maria A. P163

Moreno-Martin, Pau OCM23, PM163, PM207, PM290, РM368, PM471, PM472

Moreno-Martin, Pol PM290

Morgan, Lauren P168

Moriarty, Eileen PM260

Morisco, Carmine PM106

Moroz, Viktoriya PM251

Morris-Smith, Rachael P160, P161

Morrison, Leanne PM399

Morrissey, Y. PM435

Mosconi, Mario PM7

Mossello, Enrico P195, P230, PM87

Mota, Cassio P58, P62

Mota, Camila Barbosa P58, P62

Mougakou, Efterpi PM40

Moura, Raquel P150, P151, P152, PM267, PM408

Mouratidou, Pelagia PM256

Mouton, Veerle P55

Moya, Diego Luis Sepulveda P85

Moynihan, Barry PM444

Mpalta, Maro P143

Msolly, Menel P117

Muñoz, Rocio Ayala OC21

Muño, Beatriz P163

Muñoz, Javier Montero OC4, P33

Muñoz, Rocio Ayala P177

Muela, Francisco Jiménez OC4, P33

Mueller, Christoph PM138

Muir, Peter P168

Mulcahy, Riona PM292, PM298, PM303, PM417

Mulcahy, Ríona PM302

Muller, Majon P54

Müller, Swantje PM241

Muñoz, Rocío Ayala P282

Muñoz, Javier Montero PM110, PM304

Munteanu, Dragos PM390

Munter, Gavriel PM35

Muprhy, Claire PM135

Muraly, Moses Raj OC40

Murphy, Robert P. OC42, PM73

Murphy, Evelyn P. OC42

Murphy, Claire PM133, PM134, PM438, PM443

Murphy, Terence PM417

Murray, Benjamin OC18, P166

Murray, James C. OCM29

Murray, James PM463

Murukesu, Resshaya Roobini P232

Murukesu, Resshaya P234
Musacchio, Clarissa PM49, PM174, PM305

Muslim, Azrin P244, PM261

Muzammil, Bushra OC11, OC13

Muzz, Alba PM9

Muzzi, Alba P202, P203, P204, P205, P206, P263, PM7, PM10,

PM13, PM17, PM22, PM24, PM25

Myint, Phyo Kyaw PM252

Mylène, Radreau PM85

Mylène, Aubertin-Leheudre PM198, PM457, PM460

Myrstad, Marius OC9, P170, PM314

Nadaraja, Sambavy PM342

Nadia, Oubaya OCM14, OCM15

Nagai, Kumiko PM451

Nagaria, Darshan P139

Naharci, Mehmet Ilkin P217, PM398, PM411, PM428

Nair, Sherena P83, P289

Nancy, Mayo PM457

Nardelli, Anna P8, P9

Nasim, Ashraf PM357

Nasrullah, Atif P83, P289

Nathalie, Jobard PM202

Natour, Fidaa P168

Naudó-Molist, Jordi PM163, PM368

Naumov, Anton PM251

Navaldeep, Kaur PM457

Navarrete, Mar P163

Navarro, Maria Victoria Gil P53

Navarro, Carmen P192

Navarro-Martínez, Rut PM337

Nebreda, María Loreto Alvarez PM233

Nee, Rory P133, P257

Neerland, Bjorn Erik P174

Neill, Desmond O. P91

Neira, Beatriz PM373

Nemeth, Frantisek PM320

Nery, Ellen C. H. Pereira PM352

Neumann-Podczaska, Agnieszka P45

Neves, Maria P44

Neves, Hugo PM68, PM113, PM114, PM334, PM335, PM419

Neves, Rui PM352

Newman, Louise OCM12

Newman, Naomi PM51, PM162

Newton, Jessica P38

Ng, C. C. P11

Ng, Chai Chen P14, P17, P80, P90

$\mathrm{Ng}$, Cassandra P212, P213

$\mathrm{Ng}$, Ron PM4

Nghiem, Quoc Duy OC6, OC26, P84, PM476

Nguyen, Long H. P166

Nickel, Christian H. PM287

Nicolas, Gambier P153

Nicosia, Rosa P163, P171

Nicosia, Franco P175

Nieberg, Margot P226

Nielsen, Dorthe S. P72

Nievas, Ginés Sánchez OC3

Nimmons, Danielle PM221

Nissen, Soeren Kabell PM287

Nobels, Anne P186

Noble, Jan P136

Nocella, Pierangela PM106

Noël, Lorenzo-Villalba PM271

Noivo, Emanuel PM409, PM410

Nolan, Marianne P52

Nonoda, Takahiro PM349 
Nossent, Esther J. P54

Nouvenne, Antonio OC15, OC34, P8, P9

Novara, Elena P202, P203, P204, P205, P206, P263, PM14, PM15, PM16, PM17, PM18, PM19

Novelli, Viola P202, P203, P204, P205, P206, P263, PM7, PM9, PM10, PM11, PM13, PM14, PM17, PM22, PM24, PM25

Novello, Cristina P255

Ntouros, Panagiotis PM78

Nuñez, Pedro Ortiz OC24

Nuñez, Pedro Ortiz PM57

Nunziata, Morabito PM245

Nunziata, Stefano PM359

Nuta, Catalina Raluca P21, P100, P148, P247, PM310, PM439

O’Caoimh, Rónán PM318

O'Connor, John OCM12

O'Connor, Aisling PM191

O'Connor, Kieran PM260, PM318

O'Donnell, Martin PM212

O’Donoghue, Margaret PM52

O’Donoghue, Patrick PM292, PM298

O’Donovan, Mark R PM318

O'Hea, Anne PM318

O'Malley, Grainne PM73

O’Neill, Desmond OCM24, PM332

O'Regan, Niamh PM292, PM298, PM303, PM417

O'Sullivan, Cianan P280, P281, PM173

O'Sullivan, Fiona PM73

Obayashi, Kazuko P110, PM349

Oboh, Lelly P130

Oddone, Enrico P202, P203, P204, P205, P206, P263

Odetunde, Temitope P130

Ogedengbe, Onesi PM283

Öhman, Hanna OCM8

Ojardias, Etienne OC52

Okamoto, Yoshimi PM349

Okazaki, Tatsuma PM451

Okoye, Chukwuma P141

Okuwoga, Omolade P281, PM173

Olavsveengen, Theresa P174

Olcott, Flora P56, P277

Olexa, Peter PM2, PM3, PM81

Oliveira, Daniel V. P86

Oliveira, Rosóngela Souza P107

Oliveira, Pedro P150, P151, P152

Oliveira, Agripino P150, P151, P152, PM267, PM408

Oliveira, Sofia A. PM97

Oliveira, Mírcia PM222

Oliveira, Ana Andrade PM254

Oliveira, Sílvia PM254

Oliveras-Fabregas, Albert OCM23, PM207, PM471

Olszewska, Julita P200, P201

Omar, Sharifah Faridah Syed P14

Ommering, Belinda PM195

on behalf of the EFFICHRONIC Consortium, PM158

on behalf of the SELFY-MPI SIGOT Investigators, PM157

Oncul, Mustafa Oral P207

Onder, Graziano P175, OCM13

Onetiu, Vlad PM121

Ong, T. P11

Ong, Terence P14, P90

Ong, Han Cheng P14

Ong, Terence Ing Wei P17, P80

Onieva, Rocio PM405

Onimura, Yuichi P229

Opris, Simona PM178
Opris, S. PM186

Oquendo, Bruno OC6, OC26, P84, PM36, PM385, PM476

Oquendo, Carmen P43

Oren, Meryem Merve P50

Orenstein, Tal PM45

Orion, Yael PM45

Orso, Francesco PM102, PM103

Ortega, Andrés Aranda P276

Ortiz, Jorge Artero OC17, P60

Ortiz, Miguel Sánchez P125

Ortiz, Miguel Sanchez PM34, PM116, PM197, PM223, PM358

Ortiz-Nuñez, Pedro PM61

Ortiz-Ruiz, Francisca P276

Osipova, Olga PM83

Osorio, Luisa Sánchez P282

Ostapenko, Valentina OC5, PM414

Osterdahl, Marc PM243

Otomo, Motoko P74, P75, P76

Ottenheim, Nathaly Rius PM132

Oude Munnik, Bas P164

Oukbir-Ferrag, Sihem OC6

Ourselin, Sebastien OC18

Ourselin, Sébastien P166

Owen-Booth, Bethan PM127

Özgür, Onur PM144

Ozturk, Zeynel Abidin P190, P199, P251, PM123, PM228, PM276,

PM392, PM393, PM394, PM470

Ozturk, Ercument PM228

Öztürk, Gülistan Bahat PM461

Öztürk, Şükrü PM461

O'Brien, John OC37

O’Caoimh, Rónán OC32, P52

O'Connor, Caroline Dalton OC32

O'Connor, Kieran P52

O'Connor, Michael P52

O'Connor, Margaret OCM10

O'Donnell, Elizabeth OC31

O'Donnell, Martin J. OC42

O’Donoghue, Alice P101, P134, P236

O'Donovan, Mark R. OC32

O'Mahony, Denis P52

O'Mahony, D. PM435

O'Neill, Desmond P97, P218

O'Shea, Noreen P23

O'Sullivan, Catherine P52

O'Sullivan, Pádraigín P52

O'Sullivan, Cianan P124

Pîslaru, Anca Iuliana P148

Pépin, Marion OC1

Pérez, Pilar Belén Chaves P177

Pérez-Rodríguez, P. P156

Périvier, Samuel P180

Paccagnella, Gregorio P275

Paccalin, Marc PM67, PM478

Padovani, Andrea P189

Paganas, Aristofanis P144

Pagani, Michele PM23, PM24, PM25, PM26

Paihle, Regis PM266

Paillaud, Elena OC1

Paiva, Sílvia P128

Pallardo-Rodil, Beatriz P6

Palm, Henrik PM246

Palmer, Victoria OCM6

Palmieri, Luigi OCM13

Panagiotakis, Symeon PM199 
Panagiotakis, Simeon PM282, PM284

Panagiotopoulos, Elias P223, P273, PM201, PM242, PM244

Panarra, Antonio OC7, P184, P216

Pandolfini, Valeria OCM25

Pang, G. H. M. P11

Pang, Gordon Hwa Mang P17, P80

Pang, Gordon P90

Paolini, Simone P256

Papadimitriou, Matthaios OC27

Papageorgiou, Dimitra Iosifina P144

Papageorgiou, Dimitra-Iosifina PM211

Papageorgiou, Iosifina-Dimitra PM214

Paraskevopoulou, Eleni P187, P253

Pardo, Patricia López P26

Pardo, Irati Merino PM153

Paredes, Ludovina PM222

Pareja, Carmen Cánovas PM72

Pareja, Carmen Canovas PM433

Pariel, Sylvie P84

Parise, Alberto OC15, OC34, P8, P9, P9

Park, Young Soo PM77

Parkes, Julie P167

Parola, Vítor PM68

Parra, Jorge Eduardo Herrera PM110

Parra, Jorge Eduardo Herrera PM304

Pascal, Jarno PM380

Paschos, Konstantinos PM101, PM293, PM311, PM312, PM345

Pascual-Dapena, Ana OC29

Pasquier, Florence PM355

Passos, Lígia PM175, PM294, PM295, PM365

Patel, Samit OC12

Patel, Jay P136, P169

Patel, Chirag PM42

Patel, Parth PM204

Patelarou, Athina PM187, PM190, PM199, PM200, PM282, PM362, PM377, PM378, PM379, PM401

Patelarou, Evridiki PM190, PM199, PM200, PM281, PM362, PM377, PM378, PM379, PM401

Patelarou, Athina PM284

Paternò, Vincenzo PM43, PM63, PM203, PM381, PM383

Pathania, Monika P231

Patricia, Jepma OCM5

Patrizio, Enrica PM277

Pattyn, Inge P55

Paul-Emile, Hay P147

Pauline, Arnold PM286

Pavón, Javier Gómez OC17, P60

Pavón, Francisco Javier Gómez OC21

Pavón, Francisco-Javier Gómez P177

Pavesi, Claudio PM6, PM7

Pavón, Javier Gómez P249

Payne, Cathy P95

Peña, Melani OC44

Pedersen, Solvejg Henneberg P196

Pedersen, Henrik Schou OCM6

Pedersen, Sidsel Arnspang PM250

Pedersen, Nancy L. PM285

Pederson, Amanda PM208

Pedone, Claudio PM105

Pedreira, Larissa Chaves OC10, OC51, P107, PM70, PM473

Pedreira, Larissa PM175, PM294, PM295

Pedro, Christen San P169

Pedroso, Ana P44

Peers, Koen P36

Peiro, Salvador PM350, PM351

Pek, Kalene PM406
Pelagalli, Giulia P230

Pellitero, Laura PM46

Pembegül, Irem P137

Pena, Barbara Perez OC24, PM57

Pena, Barbara Pérez PM61

Pena, C.M. PM186

Peña, Naiara P268

Penfold, Rose OC18

Peppard, Sarah PM52

Pereira, Gonçalo OC7

Pereira, Gildasio Souza OC10

Pereira, Armando OC48

Pereira, Vâónia P198

Pereira, Olga P198

Pereira, Sílvia PM112

Pereira, Anabela PM450

Perera, Indika Thilan P63

Peres, Susana P44

Perez, Victoria P171

Pérez, Dolors García PM315

Pérez, Eduina PM405

Perez-Abascal, Noelia PM445

Perez-Rodriguez, Patricia P86

Perks, Clarissa P136

Perrone, Anna Myriam PM340

Perrotta, Fabio OCM28

Pers, Yves-Marie PM158

Persiano, Tonia P202, P203, P204, P205, P206, P263

Persico, Alessandra PM14, PM15, PM17, PM18

Pertea, Leonard Iosif PM390

Pétein, Catherine PM434

Peters, Edgar J. G. P54

Peters, Ron OCM5

Petit-Bhoyroo, Helene PM193

Petrauskas, Liudvikas PM239

Petrelis, Alexandros M. OCM3

Petreska-Zovic, Biljana P181, PM301

Petronikolou, Vasileia P238

Petrovic, Mirko P224, PM138, PM441

Petrovic, M. PM435

Peyrusqué, Eva PM60

Pezzella, Alfonso PM196

Phelan, Niamh PM302

Phetnin, Namon PM180, PM336

Philip, Nieberding P71

Phillips, Marianne PM204

Picavet, Hendrika S. J. PM75

Pickert, L. OC36, PM166

Pickert, Lena P48

Picton, Sophie P281, PM173

Piers, Ruth P55

Piette, Anne P188

Pigott, Jennifer S. OC12, P145

Pikčiūnaitè, Evelina PM397

Piling, Luke OC33

Pilotto, Alberto OC53, P158, P189, P255, P272, OCM4, OCM17, OCM25, PM49, PM157, PM158, PM174, PM247, PM305

Pilotto, Andrea P189

Pilotto, A. PM166

Pin, Tan Maw PM253

Pinheiro, Nuno P197

Pinna, Alessandra P255, P272

Pino, Guadalupe Lozano P27

Pinto, Daniela PM65

Piotrowicz, Karolina P259

Pislaru, Anca Iuliana P21, PM310, PM439 
Pislaru, Anca P100, P247

Pitkala, Kaisu OCM8, PM210

Pitkälä, Kaisu OCM2, OCM11

Pitocco, Alessandra P245

Pizzol, Damiano PM474

Plakas, Sotiris PM370

Platon, Izabela Corina PM98

Ploegmakers, Kimberley PM259

Plota, Sofia P223

Podestà, Silvia PM49

Pokrovskaya, Maria PM76

Poli, Stefano OCM25

Polidori, Maria Cristina OC36, P48, OCM4

Polidori, M. C. PM166

Polini, Antonio P141

Pomata, Monica OCM17, PM305

Pommer, Wolfgang PM241

Ponnampalavanar, Sasheela P14

Pons, Jean Carlo Heredia P27

Poon, Mun Hoe P138

Pope, George PM292, PM298, PM303, PM417

Popescu, Ana PM270

Portielje, J.E.A. PM344

Posadas-Alonso, Francisco Javier PM185

Potapenko, Anna PM155

Potic, Bojana PM130

Poularas, John PM219

Pozo-Vico, Ana Sofía P285

Pozo-Vico, Ana Sofia PM47

Pozzobon, Gianfranco P158

Prada, Gabriel Ioan P21, P148, PM310, PM439

Prada, Gabriel-Ioan P100, P247

Pradhan, Sweta PM283

Prado-Mel, Elena P53

Prasad, Ankita P38

Prashchayeu, Kirill P222

Pratesi, Alessandra PM102

Pratesi, Alessandrsa PM103

Prati, Beatrice OC15, OC34, P8, P9

Prendki, Virginie P180

Prescott, David P198

Prestamburgo, Domenico PM224

Prete, Camilla OC53, P255, P272

Prieto, Blanca Garmendia OC17, P60, P177, P282

Psilou, Dimitra PM370

Pugliese, Michela P287

Puigoriol-Juvanteny, Emma OC25, PM425, PM426

Puisieux, François P99

Puisieux, François PM33

Pujol, Joan Capdevila P166

Pujol, Alba Sinfreu PM315

Purcell, Roisin PM142

Puri, Oshin P231

Putallaz, Pauline OC27

Putnaite, Raimonda PM397

Puviani, Filippo Cioli PM340

Puzone, Brunella PM99, PM106

Pytraczyk, Sylvia OC11, OC13

Querin, Giorgia PM367

Quezada-Feijó, Maribel P4, P6

Quiñones, Karina Liz P37

Quiñones, Karina P192

Quinn, Terence OC37, OCM10

Quinn, Terry P113, PM131

Quirke, Michael PM316
Ríos-Germán, Peggy P171

Raat, Hein PM158

Rabe, Sophie PM241

Racha-Pacheco, Ricardo PM112

Račkauskaite, Laura PM475

Radavičius, Benediktas PM48

Radchenko, Anastasiia PM95

Radenkovic, Dina PM415

Rafanelli, Martina PM87

Rafferty, Maire PM258, PM262

Raffin, Jeremy PM288

Raijmann, Renee PM249, PM436

Rainone, Antonio OC6, PM36, PM92, PM367, PM385

Raiteri, Rita PM174

Raivio, Minna OCM8

Ralf-Joachim, Schulz PM148

Ramburuth, Vivek OC11, OC13

Ramel, Alfons PM391

Ramírez, Javier Alonso PM109

Ramírez-Vélez, Robinson PM37

Ramón-Espinoza, Fernanda OCM7

Ramos-Sánchez, Mónica P6

Ramos-Sanchez, Monica P4

Randles, Mary P40

Ranhoff, Anette P15

Ranhoff, Anette Hylen P49

Rarek, Marcel PM166

Rashid, Raazliza P234

Rásky, Éva OCM20

Ratsimbazafy, Fara PM179

Rawle, Mark James P112, PM51, PM162

Rawle, Mark P139

Raymond, Johanna P284

Razali, Shafia S. By PM4

Razzano, Monica PM174

Recto, Caryn PM36, PM92, PM367, PM385

Reddin, Catriona P22, P87, PM53, PM296

Redknapp, Caio P124

Redmond, Anthony P212

Reeves, Neil PM237

Regnault, Véronique PM111

Rego, Paulo OC48

Rego, Ana Rita PM112

Rehbaum, Verena P48

Reidy, Chris PM417

Reilev, Mette PM250

Reimer, Wilma Scholte op OCM5

Reine, NGOULIE P20

Reini, Mertens P66

Remelli, Francesca OCM26

Rémi, Varin PM220, PM430, PM431, PM432

Rengo, Giuseppe PM99, PM106, PM157

Reparaz-Escudero, Imanol PM47

Rhebergen, Didi PM132

Ribe, Anette Riisgaard OCM6

Ribeiro, Andreia Teles P32, P219

Ribeiro, Maritza P128

Ribeiro, Óscar PM450

Ricalde, Tomas P159

Riccioni, Tommaso P141

Ricevui, Giovanni P204

Ricevuti, Giovanni P202, P203, P205, P206, P263, PM6, PM7, PM8, PM9, PM11, PM12, PM15, PM17, PM18, PM19, PM20, PM21, PM22, PM23, PM24, PM25, PM26, PM28

Richard, Dodel PM151

Richardson, Sarah OC37, OCM29 
Richert, Laura PM179

Rickard, Frances PM50

Rico, Leonor P268

Ridolo, Erminia OC34, P8, P9

Rierola-Fochs, Sandra PM163, PM207, PM290, PM368, PM471

Rigamonti, David PM277

Riis, Johannes PM226

Riiser, Sharline P119

Riou, Bruno OC1

Rivan, Nurul Fatin Malek P234

Rivasi, Giulia PM87, PM94, PM126, PM442

Rivasi, G. PM421

Rivera, Marta Martínez OC4, P33

Rivera, Ivón P51

Rizos, Luis Romero OC3

Rizzuto, Debora P5

Robbert, Huijsman PM363

Robert, Egon P186

Roberts, Amy L. OC18

Roberts, Helen P167

Roberts, Helen C PM399

Robey, Rebecca PM208

Robinson, David P133, P208, P209, P257

Robinson, Sian M PM399

Robles-Musso, Emilio PM350, PM351

Roca-Carbonell, Ferran OC25

Rocamora-Perez, Patricia PM350

Rocamora-Pérez, Patricia PM196

Roche, Jean P99

Rocheste, Lynn OC37

Rodger, Michael P120

Rodondi, Nicolas PM249, PM436

Rodríguez, José Gutiérrez OC4, P33

Rodríguez-Sínchez, Isabel P30

Rodrigues, André P198

Rodriguez, Jaime P192

Rodríguez, José Gutiérrez PM110, PM304

Rodriguez Espeso, Erick Arturo PM233

Rodriguez-Lockward, Angel L.L. OC44

Rodriguez-Mañas, Leocadio P86

Rodriguez-Mañas, Leocadio OCM16

Rodríguez-Pérez, Aitana PM423

Rodriguez-Piñera, Mariam PM46

Rodriguez-Sanchez, Isabel P86

Rodríguez-Sánchez, Isabel P285, PM47

Roger, Bouillon OCM1

Rohilla, Jitendra P231

Roig, Clara P286

Roig, Javier Jerez PM458

Rojas, Concepción Jiménez OC24

Rojas, Concepción Jiménez P249

Rojas, Concepción Jiménezn PM57

Rojas, Concepción Jimenezn PM61

Roka, Violeta PM211, PM214

Roldán, J. María Gómez PM315

Rolland, Yves PM288

Roller-Wirnsberger, Regina PM280

Romain, Leguillon PM431

Romanelli, Giuseppe P175

Romano, Antonietta P12, P13

Romanopoulou, Evangelia P238, PM353

Romdhani, Mouna OC1

Romero, Marta Mas OC3, OC23

Romero, Maria Jesus Vicente PM116, PM358, PM375

Romero, Maria Jesús Vicente PM223

Romero-Mas, Montse PM472
Romero-Ortuno, Roman OC16, OCM12, PM279

Romundstad, Luis P174

Roodhooft, Dominique P115

Rooney, Claire PM142

Rosáírio, Vanisa P197

Rosa, Ana Lúcia P128

Roseau-Vincenti, Albane PM67

Rosendahl, Erik PM140, PM141

Rossi, Sandra OC15

Rossi, Paolo Dionigi PM277

Rothenbacher, Dietrich PM248

Rothwell, Nicola PM283

Rottenberg, Yakir OC46

Roubaud, Claire PM478

Roubaud-Baudron, Claire PM67

Roul, Gérald PM82, PM85

Roux, Swasti OC1

Roux, Xavier P180

Rozanov, Alexander OC5, PM414

Rozzini, Renzo OC2, P15, P16

Ruberto, Carmen P287, PM55

Rubio, Yanira Aranda P51

Rubio-Briones, José PM337

Rudi, Doriana P275

Rudol, Dominik PM166

Ruel, Mathilde PM266

Ruiz, Licelore P163

Runichina, Nadezda PM104, PM189, PM299

Ruotolo, Giovanni P189, P287, PM55

Rusina, Robert PM118

Rusu, Alexandra P100, P247

Rutjes, Anne PM249, PM436

Rutten, Jeanine P114

Ryan, Dan P91, PM332

Ryan, Jude P94, P244, PM261

Ryan, Dan James P95

Ryan, Damien OCM10

Ryan, Melissa PM59

Ryg, Jesper P72, PM62, PM137, PM250, PM287, PM309, PM441

Ryg, J. PM435

Sørheim, Inga-Cecilie P119

Sánchez, Mónica Ramos OC17, OC21, P177

Sánchez, Miguel P43

Sánchez-Latorre, Marina P30

Sárdy, Miklós P248

Słowiński, Rafałé P92

Sabatier, Florence PM111

Sabbà, Carlo P272

Sabbe, Marc P103, PM313

Saber, Sadia P124, P280, P281, PM173

Sadlier, Corinna P52

Saedon, N. I. P11

Saedon, Nor Izzati P14, P17, P90

Saedon, Nor ГÇÿIzzati P80

Sáez de Asteasu, Mikel L. PM37, PM47

Şahin, Ayla PM461

Sakakibara, Mikio PM424

Sakian, Noor Ibrahim Mohamed P234

Salazar, Jaime Rodríguez P37

Salazar, Noelia CC PM246

Salazar, Patricia Lizett Vilca PM458

Saldanha, Maria PM170, PM409, PM410

Salem, Mohamed A. P252

Salgueiro, Margarida P197

Sallehuddin, Hakimah P102 
Saller, Thomas OCM10

Salminen, Karoliina PM395

Salvador, Pedro P150, P151, P152

Salvans, Anna Escribà PM458

Samar, Derbal PM317, PM372, PM386, PM388

Sampaio, Rute dos Santos OC10

Sampson, Elizabeth L. PM221

Samsudeen, Amal P39, PM297

Sanchez, Elisabet P290

Sanchez, María Dolores Domingo PM100, PM160, PM291

Sánchez, Mónica Ramos P282

Sánchez, Héctor Villanueva PM315

Sánchez-Cadenas, Vicente PM452

Sanchez-Fidalgo, Susana PM384

Sánchez-García, Elisabet PM452

Sánchez-Latorre, Marina OCM7

Sánchez-Martínez, Vanessa PM337

Sánchez-Sánchez, Juan Luis OCM7

Sancho, Elisa García PM375

Sandbaek, Annelli OCM6

Sander, Rolf Christian PM150

Sandrine, Picard PM122

Sandu, Ioana Alexandra P21, P148, PM310, PM439

Sandu, Ioana P100, P247

Sandu, Câlina Anda P148

Sandven, Irene PM31

Santamaria, Amparo PM93

Santhirapala, Ramai PM415

Santos, Ana Julia P58, P62

Santos, Mariana P150, P151, P152, P215, PM267, PM408

Santos, Catarina PM170

Santos, Andreia PM334, PM335

Santos, Eduardo PM347

Santos-Costa, Paulo PM347

Santos-Ramos, Bernardo PM384, PM423

Santos-Rubio, Maria Dolores PM384

Santova, Tatiana PM320

Sanz-González, Natalia P191

Sanz-González, Natalia PM185, PM230, PM231, PM235, PM278,

PM338, PM339, PM376

Saraiva, Mariana OC30

Sardella, Alberto PM245

Sasson, Rosanna P74, P75, P76

Savas, Sumru OC50, P194, P250, PM66

Saverio, Loddo PM402

Savioli, Gabrieleq P202

Savioli, Gabriele P203, P204, P205, P206, P263, PM6, PM7, PM8, PM9, PM10, PM11, PM12, PM13, PM14, PM15, PM16, PM17, PM18, PM19, PM20, PM21, PM22, PM23, PM24, PM25, PM26, PM27, PM28

Savvakis, Ioannis PM187, PM281, PM282, PM284, PM401

Saw, Kyaw Lin PM64

Saxena, Manish PM93

Sayer, Avan OC37

Sayer, Avan A. OCM29

Sayer, Avan A PM453, PM454, PM455

Sayer, Avan Aihie PM463

Sbrogiò, Luca Gino P68

Scarlett, Siobhan OCM12

Schaap, Laura A. PM75

Schapansky, Evelyn P186

Schedrina, Anna OC5, PM414

Scheel-Hincke, Lasse Lybecker P210

Scheffler, Max P180

Scheisi, Roberto P7

Schena, Federico P275
Schetaki, Stefania PM199, PM200, PM282, PM362, PM377, PM401

Schmidmaier, Ralf OCM27

Schmidt, Daniel P40

Schmidtmann, Marco P288

Schoene, Daniel PM138

Scholten, Rob PM249, PM436

Schrag, Anette PM221

Schwingshackl, Lukas PM138

Sciberras, Philip PM159

Sciberras, Nicole PM159

Sciutti, Fabio PM12, PM28

Scrimieri, Antonia P193

Scurti, Rosa P245

Seabra, Rosmaninho PM352

Sebaux, Alexandre PM272

Seebah, Shelina P134

Seers, Tim P41

Seers, Timothy P212

Seiffert, Piotr P81, P92

Selvaraj, Manoj PM369

Semochkina, Galina PM89

Semushina, Elena P24

Senesi, Barbara OC53, P255, P272, OCM17, OCM25

Senkal, Naci P50, P207

Senn, Laurence OC27

Sepúlveda-Moya, Diego P78

Seppala, Lotta PM248

Seppala, L.J. PM435

Sepulveda-Loyola, Walter P86

Serge, Lievens P71

Sergi, Giuseppe P256, PM143

Serpa, Maria Joúo P198

Serra, Carlos Gala PM197

Serrano, Irene P171

Serrano, Mercè Comas PM315

Serrano-Carrascosa, Maria PM337

Serratrice, Christine P180

Servettaz, Amélie PM86

Sethi, Monica P159

Sevcenko, Viktorija PM239

Severac, François PM82

Sevilla-Sanchez, Daniel PM263, PM264, PM426

Sevilla-Sánchez, Daniel PM425

Sfikakis, Petros P. PM78, PM165, PM477

Sfikakis, Petros P PM256

Shah, Kunal OC12

Shah, Ku P67

Shah, Devesh P74, P75, P76

Shahar, Suzana P232, P234

Shahid Ali, Raja Muhammad PM229

Shahimi, Nur Husna OC39

Shapiro, Dvorah Sara PM35

Shapiro, Dvorah PM69

Sharashkina, Nataliya PM76

Sharashkina, Natalia PM104, PM189, PM299

Shariffuddin, Ina Ismiarti PM418

Sharkey, Jennifer PM146

Sharratt, Phoebe P74, P75, P76

Shaulov, Adir OC46

Shavit, Linda PM35

Shchetkovsky, Denys PM229

Sheldrake, Jonathan OC11, OC13

Shenkin, Susan OC22, OC37, P224, OCM10, PM127, PM138

Shenkin, Susan D. P79

Shepherd, Gillian PM205

Shin, Cheol Min PM77 
Shinners, Orlaith P244, PM261

Shiraishi, Nariaki PM424

Shrestha, Alvin PM218, PM307

Shvedko, Anastasia PM104, PM189, PM299, PM323

Siafi, Eirini P149

Sialino, Lena D. PM75

Sibley, Sarah P212

Siddhartha, Lieten PM286

Sieber, Cornel P224, PM138

Sieber, Cornel C. PM403

Siegrist, Sophie P135

Sierra_martínez, Leticia P191

Sierra-Martínez, Iker P191

Sierra-Martínez, Leticia PM185, PM230, PM231, PM235, PM278, PM338, PM339, PM376

Sierra-Martínez, Iker PM230, PM231, PM235, PM278, PM338, PM339, PM376

Sikkema, Reina P164

Silkiewicz, Marta PM459

Silva, Cláudia Fernanda Trindade OC51

Silva, Luciana P150, P151, P152, PM267, PM408

Silva, Leonor P150, P151, P152, PM267

Silva, Ricardo PM170

Silva, Leonor P. PM176, PM188

Silva, Rosa Carla PM347

Silva, Sofia PM413

Silva, Pedro Henrique Costa PM473

Silva Teixeira, Rafael PM176

Silveira, Eva Delgado P140, P290, PM445

Simón-Talero, Rafaela Sánchez OC3

Simon, Elisa Mateo PM116

Simon, Elisa Garcia PM358

Simón, sa García PM34

Simón, Elisa García PM223

Simoncelli, Anna PM8, PM12, PM14, PM16, PM17

Simoncini, Stéphanie PM111

Simovic, Bénédicte P99

Simpson, Katy P271

Singh, Arpit P231

Singh, Devinder Kaur Ajit P232, P234, PM327

Siniakova, Olga P24

Sinnarajah, Aynharan PM56

Sinnott, Sarah-Jo PM243

Siri, Giacomo P255, OCM17, PM305

Sirmi, Christina P223

Sitkiewicz, Julita P200, P201

Sitta, Rémi PM179

Skaar, Elisabeth P49

Sklavounos, Paolo P146, P149

Skvortsov, Dmitry PM76

Smalbrugge, Martin P114

Small, Kerrin OC18

Smeeth, Liam PM243

Smit, Eline P233

Smith, Jennifer OC11, OC13

Smith, Colette OC19, P64, P69, P70

Smith, Lee P224, PM138

Smith, Lorraine PM417

Smith-Baker, Robert P124, P280

Smithard, David P105

Smorenberg, Annemieke P54

Smorzhevskaya, Marina P31

Smyrnakis, Emmanouil P144, PM211, PM214

Soares, Cadjija P62

Soares, Maria Manuela P197

Soares, Marta PM112
Sohail, Asma P14

Solís, Juan Rodriguez P98

Solís, Juan Rodríguez P183

Soler, Pedro Abizanda OC3, OC23

Solfrizzi, Vincenzo P255, PM49, PM157, PM174

Solis, Juan Rodriguez P96

Solsona, Sofía PM405

Somoano, Arís PM373

Sonja, Rebel P88

Sonnenblick, Moshe PM35

Soric, Gabriela PM374

Souliotis, Vassilis L. PM78

Soulis, George PM211, PM214, PM265

Soulis, G. PM435

Sousa, Joana Pereira P128, PM113, PM114

Souza, Monaliza Lemos OC51

Sowada, Christoph P103

Soysal, Pinar P224, PM138

Soytas, Rabia Bag P132

Spadafora, Laura OC36

Spanakis, Marios PM377

Spector, Tim D. P166

Sperling, Uwe OC20

Spinewine, Anne PM434

Staekenborg, Salka PM88

Stangherlin, Vanni P158

Stanley, Eve P52

Starkel, Peter P34

Starkovaite, Sandra PM396

Stathopoulou, Maria G. OCM3

Steen, Georgina PM262

Stefan, Grund P109

Stefan, Schneider PM148

Stefaniu, Ramona P21, P100, P148, P247, PM310, PM439

Steinert, Anika PM241

Steinmetz, Jean-Paul PM136

Stella, Marco PM174

Stéphane, Baudry PM286

Stephen, Pye R. OCM1

Stevenson, Jennifer M. P130

Stevenson, Emma PM453, PM454, PM455

Steves, Claire J. OC18, P166

Stockmans, Axelle P10

Stolakis, Konstantinos P223, P227, P273, PM201, PM242, PM244

Stolz, Erwin OCM20, PM274, PM280

Strandberg, Timo OCM2, OCM4, OCM8

Stratidaki, Eirini PM187, PM200, PM281, PM284, PM400, PM401

Straus, Sharon PM249

Strazhesko, Irina PM76, PM104, PM139, PM189, PM299, PM323, PM412

Stuart, Beth P167

Stundža, Ernestas PM321

Stuttard, Matthew P168

Suárez, Pablo Enrique Solla OC4, P33

Suarez, Elena PM188

Suárez, Francisco PM46

Suárez, Pablo Enrique Solla PM110, PM304

Subramaniam, Ponnusamy P232, P234

Sudre, Carole H. OC18, P166

Suetta, Charlotte PM246

Sulicova, Andrea PM320

Sun, Kristi OC12, P74, P75, P76

Sungur, Hande P233

Suzan, Veysel P132

Suzuki, Yusuke PM424

Swami, Kavita P231 
Swart, Johnny P74, P75, P76

Swift, Ealish P168, P269

Swift, Orry P168

Sylvie, Bastuji-Garin OCM14, OCM15

Symintiridou, Despoina P144, PM211, PM214

Sytse U., Zuidema PM363

Szczerbińska, K. PM435

Taffet, George OCM10

Talha, Samy PM82, PM85

Talimtzi, Persefoni P144

Tambokon, Rachael T. PM5

Tamir, Yael Adoni P61

Tampaki, Maria PM165, PM477

Tamulaitiene, Marija PM239

Tan, Maw Pin OC39, OC40, P14, P17, P80, P90, P102, P239, OCM9,

PM252, PM257, PM327

Tan, M. P. P11

Tan, K. M. P11

Tan, Kit Mun P14, P17, P80, P90

Tan, Lok YiYi P244

Tan, Lok Yi PM261

Tannou, Thomas P283, PM41

Tarakcioglu, Mehmet PM123

Taranu, Sabinne P21

Tasci, Ilker P217, PM398, PM411, PM428

Tasioudi, Labrini PM281

Taulaigo, Anna PM409, PM410

Tavares, João PM70, PM175, PM294, PM295, PM365, PM413

Tavares, João Paulo Almeida PM473

Tavarez, M. Rodriguez OC44

te Poel, Fam P233

te Witt, Rene P164

Teang, Soon Chen PM418

Teoh, Penelope J. P105

ter Riet, Gerben OCM5

Terbraak, Michel OCM5

Terence, O'Neil W. OCM1

Terry, Quinn P47

Tersenidou, Naili P187, P253

Tersenidou, Riola P187, P253

The COVID-OLD Project Group The COVID-OLD Project Group, OC28

Theresa, Müller PM144

Théry, Charlotte Havreng PM476

Thiam, C. N. P11

Thiam, Chiann Ni P14, P17, P80, P90

Thomas, Caroline OC1

Thomas, Betz PM168

Thomas, Benzing PM168

Thomas, Tannou PM202, PM380

Thomas, Sonia PM249

Thomas, Renoncourt PM469

Thompson, Sanja PM205, PM209, PM213, PM216

Thomsen, Katja PM62, PM342

Thornhill, Louise PM205

Thorsdottir, Inga PM391

Thorsten, Bartsch PM151

Thygesen, Janni PM309

Tiba, Delespierre P147

Ticinesi, Andrea OC15, OC34, P8, P9

Tieges, Zoë OCM10, PM177

Tienari, Pentti OCM2

Tilvis, Reijo OCM8

Tim, Stuckenschneider PM148

Timmermans, Erik J. PM285
Tino, Prell PM151

Tiozzo, Andrea P68

Tkacheva, Olga OC5, PM76, PM89, PM104, PM139, PM182, PM189, PM299, PM323, PM412, PM414

Tobias, Warnecke PM151

Tobis, Sléawomir OC49

Tobis, Slawomir P45

Tognelli, Silvia PM102, PM103

Tojo, Margarida PM71

Toméo, Charlotte OC1

Tomás, Xavier Ichart PM315

Tomic, Snezana PM130

Tomlinson, Laurie PM243

Tommasi, Lorenzo PM385

Ton J.E.M., Bakker PM363

Tonby, Kristian P174

Tonetti, Jerome PM266

Tong, Wing PM195

Topinkova, E. PM435

Topinková, Eva PM464

Topolyanskaya, Svetlana P31, PM90, PM91, PM236

Torbahn, Gabriel P224, PM138, PM403

Torchia, Carlo P287

Torralba, Rocio P86

Torrens, Nicola P130

Torres, Nora Molina PM72

Torres, Elena Rebollar PM72, PM433

Torres-Allepuz, Rosa OC25

Torres-Moreno, Míriam PM472

Torrigiani, Claudio OCM25

Torriglia, Domenico PM174

Torrus, Marta Herrero OC29

Tortù, Virginia P230

Tortù, Virginia PM87

Tortu', Virginia PM94, PM126, PM442

Toscano-Guzmán, María Dolores PM423

Tosounidis, Theodoros P273, PM201, PM242

Tóth, Štefan PM2

Toupance, Simon OCM3, PM111

Tournoy, Jos P36

Trabucchi, Marco P15, P16

Tracey, Eimear P52

Travers, John PM279

Trevisan, Caterina P195

Tricco, Andrea PM249

Tricerri, Francesca OCM17, PM49

Trimaille, Hélène PM41

Tristancho-Perez, Angela PM384

Trivli, Alexandra PM190, PM378, PM379

Trombert, Véronique P180

Tronel, Hubert P117

Trotta, Francesco OCM13

Tsantili, Alexandra PM265

Tsapas, Apostolos PM181

Tsareva, Irina PM89

Tsiligianni, Aggeliki PM161

Tsiou, Chrysoula PM370

Tsokani, Sofia PM249, PM436

Tsukao, Akiko P229

Tsuruzono, Takuya P229

Tsutsumi, Takahiko PM225

Tua, Julia OC45, P116, P254

Tua, J. P111

Tudor, Florina P158

Tuesta, Celia Corral PM233

Tukek, Tufan P50, P207 
Tulha, José PM352

Tunçel, Özlem Kuman OC50

Tuneu, Miriam Molas PM458

Turcu, Ana Maria PM310

Turkmen, Banu Ozulu PM462

Türkmen, Banu Özulu PM461

Turrin, Giada PM87, PM94, PM126, PM442

Turvey, Spencer PM260

Tuur, Helsen P66

Twisk, Johannes W.R. PM75

Tyllianakis, Minos PM244

Tyrrell, Richard PM261

Tyvaert, Louise P126, P127

Tzavara, Vassiliki PM40

Tziraki, Chariklia PM281

Udina, Cristina P286

Ulusal, Hasan PM123

Ulutas, Ozkan PM234

Umurca, Rabia Gökçen PM172

Unal, Damla P132

Une, Saki PM206

Ungar, Andrea P230, PM87, PM94, PM102, PM103, PM126, PM442

Ungar, A. PM421

Unim, Brigid OCM13

Unverdorben, Martin PM93

Uslu, Arzu P243

Utiel, Melisa López OC23

Uwimpuhwe, Germaine OCM29

Vágnerová, Tereza PM464

Vahedi, Ali PM289

Vaitsis, Nikolaos P227

Vakulenko, Olga PM90, PM91, PM236

Valadares, Ana PM346

Valentin, Daucourt PM380

Valentin, Blanchard PM460

Valle, Roberto P68

Vallet, HélèneOC1

Van Braeckel, Eva P55

van Buren, Marjolijn P226, PM156

van Buul, Laura P114, P164, P165

van Buul, L. W. OCM18

van Daele, Paul L. A. P54

van de Loo, Bob PM248

van den Besselaar, Judith P164, P165, PM326

Van Den Noortgate, Nele P55, P115

van der Bol, J.M. PM344

van der Cammen, T.J.M. PM435

van der Hulst, H.C. PM344

van der Mast, Roos PM132

van der Steen, Jenny PM195

van der Velde, Nathalie OC37, PM132, PM248, PM259

van der Velde, N. PM435

van Dijk, Liset P233

van Dijk, Miriam PM465

Van Hecke, Kyri P10, P188

van Heugten, Caroline PM308

van Kooten, Janine P114

Van Leeuwen, Marie Louise P55

van Loon, Anouk P114

van Meer, Leonie J. PM30

van Oevelen, Mathijs P226

van Oostrom, Sandra H. PM75

Van Puyvelde, Katrien P55

van Schoor, Natasja PM248, PM259 van Schoor, Natasja M. PM285

van Tol, Lisa P89

van Tulder, M. W. OCM18

van Weert, Julia PM259

van Wijngaarden, Janneke PM465, PM466

Vanassche, Thomas PM93

Vandaele, Filip P115

Vandeviver, Christophe P186

Vankova, Hana PM118

Vankova, Marketa PM118

Vaqué-Crusellas, Cristina OCM23

Vaquero-Pinto, $\mathrm{M}^{\mathrm{a}}$ Nieves PM452

Vargas-Aleman, Claudia Lorena P182

Varli, Murat P178

Varman, Surendra D. PM4, PM5

Varman, Surendra D PM348

Varsamis, George P146, P149

Varsavsky, Thomas P166

Vasconcelos, Clara Magalhães OC51

Vasilic, Slavica PM130

Vasques, Ana P44

Veedfald, Thomas PM137

Veenhuizen, Miriam PM177

Veerle, Van Parys P66

Veizi, Betül Gülsüm Yavuz PM428

Vella, Antoine OC45, P254, PM371

Vella, A. P111

Vellas, Bruno PM288

Veloso, Ana Isabel PM354

Veríssimo, Rafaela P150, P151, P152

Vergani, Vittoria PM415

Vergara, Itziar P268

Verheij, T. J. M. OCM18

Veríissimo, Manuel PM450

Verissimo, Rafaela PM176, PM188

Veríssimo, Rafaela PM267, PM408

Verloop, David PM33

Veronese, Nicola P12, P13, P68, P158, P189, P224, OCM4, OCM17,

PM138, PM157, PM158, PM305, PM474

Veroniek, Saegeman P71

Verschuren, Monique W. M. PM75

Verweij, Lotte OCM5

Vespertini, Viviana P287

Vetrano, Davide Liborio P175

Vettasseri, Mark OC11, OC13

Viberg, Bjarke PM250

Vico, Ana Sofía Pozo P108, P118

Victoire, Leroy PM202

Vieira, Maria Carolina P58

Vieira, Maria P62

Vigário, Ana PM112

Vighnesh, B. S. P231

Vigo, Martina P272

Vilaró, Jordi P286

Vilca-Salazar, Patricia Lizett OCM23

Vilches-Moraga, Arturo PM38, PM208

Vilela, Maria Elena Castro PM34, PM197

Villain, S. Kenneth PM4

Villalba-Moreno, Angela PM384

Villalba-Moreno, Ángela PM423

Virciglio, Simona PM102, PM103

Visade, Fabien PM33

Visaggi, Lucia P141

Visconti, Alessia OC18, P166

Visser, Marjolein PM75

Visvikis-Siest, Sophie OCM3 
Vitale, Dino Franco PM99, PM106

Vitali, Francesca P275

Vitkus, Dovydas PM239

Vlase, Alexandru PM390

Vogel, Thomas PM74

Volker, Burst PM168

Volkert, Dorothee PM403

Volpato, Stefano P195, OCM26

Volpi, Annalisa OC15

Von Heymann, Christian PM93

Von Hunolstein, Jean-Jacques PM85

Voorend, Carlijn G.N. P226

Voorend, Carlijn PM156

Vorobyeva, Natalya PM89, PM139, PM412

Voskou, Panagiota PM120

Vovk, Kira PM155

Vrotsou, Kalliopi P268

Vygodin, Vladimir PM76

Vyroubal, Pavel PM217

Waddell, Greg P113, P120

Waldorff, Frans PM137

Waldron, Dympna P23

Waldron, Naoise PM52

Wallace, Sophie PM306

Walle-Hansen, Marte Meyer P170

Walsh, J Bernard PM258, PM262

Walsh, Joseph PM303, PM417

Walsh, Ciara PM318

Walter, Maetzler PM151

Walters, Kate PM221

Wammes, Joost David P123

Wammes, Joost PM326

Waters, Ruairi P22, P87, P179, PM53, PM296

Waters, Ruari PM54

Watne, Leiv Otto P174

Watson, Julie OC22

Watters, Hazel P122

Weaver, Shirley A. P266

Webster, Jessica P124, P280

Wehling, M. PM435

Weijs, Peter PM466

Weissma, Yaara Leibovici OC35

Welch, Carly OC18

Wells, Lisa P138

Wensien, Jette P72

Wentworth, Lauren P38, P39, P41, P42, PM297, PM300

Werner, Christian PM171

Westhovens, Ine P55

Whelan, Aisling PM54

Widenfalk, Agnes PM140

Wieczorowska-Tobis, Katarzyna OC49, P45

Wijnhoven, Hanneke A. H. PM75

Wilco, Achterberg P109

Wilco P., Achterberg PM363

Wilhelm, Stahl PM148

Wilkins, Robert PM93

Willem, Van Nuffel P66

Willems, Vanessa P82, P225

William, George P56

Williams, Martin P168

Williams, George P277

Williams, David PM444

Williamson, Elizabeth PM243

Willis, Charlotte PM213

Willoughby, Michael PM316
Wilson, Dan P136, P169

Wim, Tambeur P66

Wimer, Andrew PM14, PM15, PM16, PM17, PM18, PM19

Witham, Miles OC37

Witold, Jarzebowski PM322

Wohlfart, Ján PM2

Woien, Hilde P174

Wojszel, Zyta Beata P200, P201, P259, PM329, PM459

Wolf, Jonathan OC18, P166

Wong, Pui Li P14

Wong, Angel PM243

Woo, Jean OC37

Woodhouse, Natalie P42

Woods, Siobhan P274

Wyatt, Jeremy P167

Wyller, Torgeir Bruun PM289

Xatzipanagiotou, Despoina P187, P253

$\mathrm{Xu}$, Yangfan PM147

Xydakis, Dimitrios PM362

Yahya, Hanis Mastura P234

Yakaryılmaz, Funda Datlı PM255

Yakhno, Nikolay PM139, PM412

Yakimova-Polyzou, Vesela P144

Yalcin, Ahmet P178

Yamada, Minoru OC38

Yamaguchi, Masahiro P229

Yaohua, Chen P155

Yasutake, Sumio PM206

Yavuz, Cemil OC47

Yavuz, Betül Gülsüm Yavuz Veizi PM411

Yavuzer, Hakan P132

Yeong, Keefai P122

Yepez, Viviana Oscullo P96

Yesil, Yusuf P207

Yigit, Seher P178

Yildirim, Caner PM123

Yildirim, Meltem PM207, PM471, PM472

Yldirim, Meltem PM368

Ynes, Punie PM286

Yoruk, Fatma PM428

Yosra, Cherif PM386

Yves, Samson PM322

Zaalberg, Tessel PM308

Zacharias, Antony P67, P76

Zacharias, Antony P. P74, P75

Zaidi, Muhammad Shoaib P264, PM184

Zakharious, Fady PM357

Zambom-Ferraresi, Fabricio P30, P285, PM37, PM47

Zambom-Ferraresi, Fabiola PM37, PM47

Zambon-Ferraresi, Fabricio OCM7

Zamfir, Mihai-Viorel P261, PM149, PM215

Zamfir (Grigorescu), Mihaela PM149

Zamfirescu, Andreea PM121, PM468

Zaniboni, Alberto P15

Zazzara, Maria Beatrice OC18

Zdravkovic, Sanja PM130

Zehlicke, Clarissa P63

Zehlicke, Clarissa-marie P252

Zeimbekis, Doukas PM214

Zekry, Dina P180

Zelenko, Andrey P24

Zerah, Loré OC1

Zevgiti, Stella PM249, PM436 
Zhao, Dan PM147

Zhilevich, Ludmila P24

Zhou, Lan PM147

Zia, Gianluca P195

Ziere, G. PM435

Zieschang, Tania OC41, P240, P242, P246, PM107, PM169, PM171,

PM333

Zigkiri, Helen PM265

Zigoura, Ekaterini OC53, P255, OCM17, OCM25, PM247

Zikos, Laurent PM355

Zilidou, Vasiliki P238

Zini, Elena OCM25
Zisca, Ludovica P141

Zografakis-Sfakianakis, Michail PM362

Zora, Sabrina P255, OCM25, PM157, PM158

Zotkin, Eugeniy PM456

Zotos, Panagiotis PM219

Zucchelli, Alberto P5, P175

Zuidema, Sytse P89

Zuinen, Tatiana P82, P225

Zulfiqar, Abrar-Ahmad PM74, PM82, PM86, PM117, PM272, PM273

Zulueta, Mónica P286

Zúñiga, Rolf Christian Sander PM109 